\title{
HLW FLOWSHEET MATERIAL BALANCE FOR
F RAD OPERATION WITH TANK 51 SLUDGE AND \\ HLW FLOWSHEET MATERIAL BALANCE FOR
DWPF RAD OPERATION WITH TANK 51 SLUDGE AND ITP CYCLE T PRECIPITATE (U)
}

and

April 19, 1995

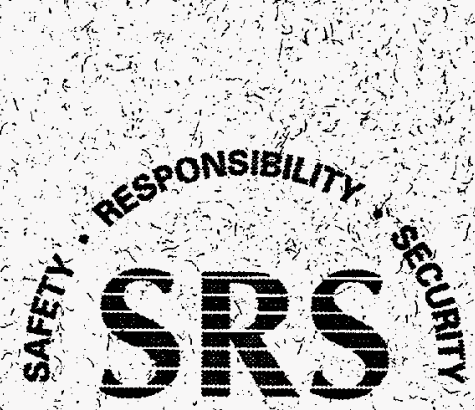

SAVANNAHRIVER SITE

Westinghousé Savannah River Company Savannah River Site Aiken, SC 29808
Alexánder S. Choi Defense Waste Processing Technology Savannah River Technology Center

PREPARED FOR THE USS. DEPARTMENT ÓF ENERGY UNDER CONTRACT NO. DE-ACO9-89SR18035 


\section{DISCLAMMER}

Portions of this document may be illegible in electronic image products. Images are produced from the best available original document. 


\title{
HLW FLOWSHEET MATERIAL BALANCE FOR DWPF RAD OPERATION WITH TANK 51 SLUDGE AND ITP CYCLE 1 PRECIPITATE (U)
}

\section{April 19, 1995}

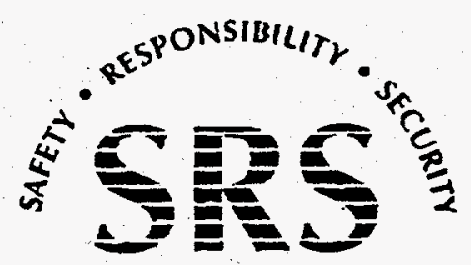

SAVANNAH RIVER SITE

\begin{abstract}
Alexander S. Choi
Defense Waste Processing Technology Savannah River Technology Center Westinghouse Savannah River Company Aiken, SC 29808
\end{abstract}




\section{DISCLAIMER}

This report was prepared as an account of work sponsored by an agency of the United States Government. Neither the United States Government nor any agency thereof, nor any of their employees, makes any warranty, express or implied, or assumes any legal liability or responsibility for the accuracy, completeness, or usefulness of any information, apparatus, product, or process disclosed, or represents that its use would not infringe privately owned rights. Reference herein to any specific commercial product, process, or service by trade name, trademark, manufacturer, or otherwise does not necessarily constitute or imply its endorsement, recommendation, or favoring by the United States Government or any agency thereof. The views and opinions of authors expressed herein do not necessarily state or reflect those of the United States Government or any agency thereof.

This report has been reproduced directly from the best available copy.

Available to DOE and DOE contractors from the Office of Scientific and Technical Information, P.O. Box 62, Oak Ridge, TN 37831; prices available from (615) 576-8401.

Available to the public from the National Technical Information Service, U.S. Department of Commerce; 5285 Port Royal Road, Springfield, VA 22161. 
WESTINGHOUSE SAVANNAH RIVER COMPANY SAVANNAH RIVER TECHNOLOGY CENTER
WSRC-TR-95-0019-TL

(Revision 0)

Keywords: HLW Flowsheet, Material

Balance, DWPF, Tank 51

Sludge, ITP Cycle 1, CPES

Retention Period: Permanent.

April 19, 1995

\author{
D. B. Amerine, Manager / DWPF \\ Westinghouse Savannah River Company \\ Attention: S. F. Piccolo, 704-S \\ Attention: S. F. Piccolo, 704-S
}

\title{
HLW FLOWSHEET MATERIAL BALANCE FOR DWPF RAD OPERATION WITH TANK 51 SLUDGE AND ITP CYCLE 1 PRECIPITATE (U)
}

A HLW flowsheet material balance was calculated for the radioactive operation of DWPF with washed Tank 51 sludge and precipitate to be produced during the first ITP cycle. The CPES integrated HLW flowsheet model used for previous DWPF material balances was updated to produce this material balance. The compositions assumed for the Tank 51 sludge and the salt/supernate feeds for ITP Cycle 1 were all based on analytical results for samples taken recently. Key simulation results include:

- When the Tank 51 sludge was washed between 8.1 and $10.7 \mathrm{wt} \%$ total Na on a dry basis, and $90 \%$ of the insoluble solids present in the Tank 41 saltcake were removed from the ITP feed, the resulting glass satisfied all the glass constraints currently imposed on the PCCS Version 3.0. The maximum sludge loading in glass ranged from 24.6 to $25.6 \mathrm{wt} \%$, and the PHA loading was about $6.0 \mathrm{wt} \%$.

- Assuming that the Tank 51 sludge is washed to $10.0 \mathrm{wt} \%$ total $\mathrm{Na}$ and coupled with precipitates of the same composition as that produced during ITP Cycle 1,

- The total duration that ESP can support 100\% DWPF attainment with the Tank 51 sludge is 1.4 years, excluding the 88,000 gallon heel in Tank 51 .

- The total number of glass canisters produced is 756 . 
- $1.35 \times 10^{6}$ gallons of inhibited wash water is required in ESP to reach the washing endpoint of $10.0 \mathrm{wt} \% \mathrm{Na}$ on a dry basis.

- The volume of DWPF liquid waste recycle generated during Tank 51 sludge processing is $7.4 \times 10^{6}$ gallons at $40 \%$ DWPF attainment.

$-673,000$ gallons of $10 \mathrm{wt} \%$ precipitate and $2.0 \times 10^{6} \mathrm{lb}$ of frit 202 are required to vitrify the sludge in Tank 51, excluding the heel.

- If the Tank 51 sludge were washed to $8.1 \mathrm{wt} \% \mathrm{Na}$, the number of glass canisters produced would be reduced to 747 .

- ITP will produce 155,000 gallons of $10 \mathrm{wt} \%$ precipitate during the first cycle and, theoretically, it can support $100 \%$ DWPF attainment for 4 months.

- The Cs-137 content in the $10 \mathrm{wt} \%$ precipitate slurry produced during the first cycle is projected to be $9.2 \mathrm{Ci} / \mathrm{gal}$. About $5 \%$ of tetraphenylborate precipitate is expected to be decomposed and become soluble during a two-year storage at 9.2 $\mathrm{Ci} / \mathrm{gal}$, compared to about $16 \%$ decomposition at $46 \mathrm{Ci} / \mathrm{gal}$.

- The Saltstone Facility would produce $687,000 \mathrm{ft}^{3}$ of grout from decontaminated supernate generated during the first ITP cycle.

Since the Batch 1 sludge material balance was issued in 1993 (WSRC-TR-93-203, Rev. 0), the CPES integrated HLW flowsheet model and its database have been continuously updated to reflect our improved understanding of the overall HLW processing operation based on the latest bench- and pilot-scale runs. Some of the significant modifications made to the model and its database are summarized in this report.

One significant change made was to allow the model to calculate the required sludge batch size to support the design basis glass production rate with an 86 hour SRAT cycle. The salt workoff rate was maximized by adding 7,900 gallons of PHA at $4.5 \mathrm{wt} \%$ total solids to every sludge batch. The total solids concentration in the PHA was calculated according to the current Precipitate Reactor operating mode. As before, the frit addition was minimized for a maximum waste loading in glass.

After the study was completed, the potential impact of pending changes in PCCS durability and predictability correlations was evaluated, and it was determined that in the composition range dictated by the Tank 51 sludge, there would be no impact on the results of this study. The application of PCCS to this study, however, presumes that errors in sludge sampling and analyses are like those experienced in DWPF vessels, which are the only error statistics available. 
D. B. Amerine

3

WSRC-TR-95-0019-TL

(Revision 0)

A complete listing of material balance calculation results are attached to this report for a sludge feed containing $10 \mathrm{wt} \% \mathrm{Na}$. This comprehensive HLW flowsheet material balance represents the first of its kind computed based on the actual salt and sludge processing schedules.

Finally, the entire contents of this report including the bases used and the complete material balance output were technically reviewed by SRTC and HLWE personnel in February 1995. All comments received have been dispositioned. This report satisfies a partial requirement for TAR\# HLW-DWPF-TAR950016. Please address any questions about this study to A. S. Choir, 725-6429.

Eutbetzehto 5/2/5-

E.W. Holtzscheiter, Manager SRTC - DWPT Section 


\section{APPROVAL SHEET}

\section{Authored by:}

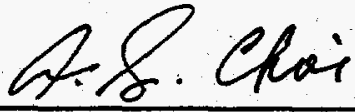

A. S. Choi, 704-1T

Engineer, Process Modeling and Control Group
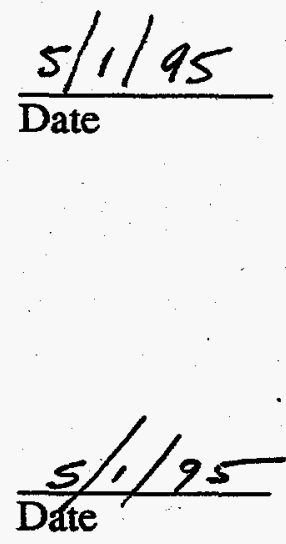

$5 / 2 / 2 T$ Date
E. W. Holtzscheiter, 773-A

Manager, Defense Waste Processing Technology

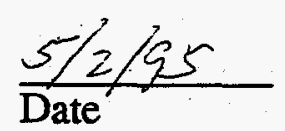

N. E. Bibler, 773-A

Scientist, Glass Technology Group

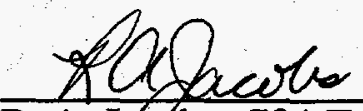

R. A. Jacobs, 704-T

Engineer, Process Technology Development Group

Technically Reviewed by:

fee c. Bible

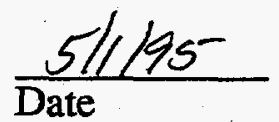


WESTINGHOUSE SAVANNAH RIVER COMPANY

SAVANNAH RIVER TECHNOLOGY CENTER

WSRC-TR-95-0019

(Revision 0)

Keywords: HLW Flowsheet, Material

Balance, DWPF, Tank 51

Sludge, ITP Cycle 1, CPES

April 19, 1995

MEMORANDUM

To: C. T. Randall, 704-T

From: A. S. Choi, 704-1T asc

\author{
HLW FLOWSHEET MATERIAL BALANCE FOR \\ DWPF RAD OPERATION WITH TANK 51 SLUDGE AND \\ ITP CYCLE 1 PRECIPITATE (U)
}

\title{
SUMMARY
}

A HLW flowsheet material balance with the Tanks $42 / 51$ blend sludge feed was issued in 1993 (WSRC-TR-93-203, Rev. 0) to support the radioactive startup of DWPF. Recently, however, ESP revised its operating plan due to inoperable slurry pumps in Tank 42 , and the new plan calls for initially feeding only the sludge in Tank 51 to DWPF for its radioactive startup. Subsequently, HLWM requested that SRTC determine the impact of this Tank 51 only sludge feed on the entire HLW processing operation including the glass quality (HLW-ITP-950058).

The basis for doing such a comprehensive study was the CPES integrated HLW flowsheet model. Since 1993, the model and its database have been continuously updated to reflect the latest results from bench- and pilot-scale runs at SRTC. The most up-to-date CPES model was used in this study to simulate the entire HLW processing operation using the Tank 51 sludge and the salt/supernate feeds for the first ITP cycle. According to the HLW System Plan Revision 4 (HLW-OVP-940145), $100 \%$ of the dissolved saltcake feeds for the first three ITP cycles are to be transferred from Tank 41. Preliminary calculations, however, indicated that the samples taken near the top saltcake layer in Tank 41 contain too high levels of insoluble chromium and phosphate to make acceptable glass. As a result, one of

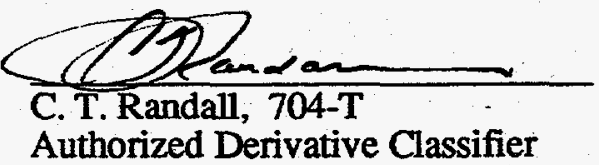

(Cont'd on the next page)

Authorized Derivative Classifier 


\section{SUMMARY (Cont'd)}

the key assumptions made in this study was that $90 \%$ of the insoluble solids present in the Tank 41 saltcake would be removed from the feed. Furthermore, three different ESP washing scenarios were simulated in this study, resulting in $8.1,10.0$ and $10.7 \mathrm{wt} \%$ total $\mathrm{Na}$ in washed sludge feed to DWPF on a dry basis, in order to find the optimum Tank 51 sludge / ITP Cycle 1 precipitate flowsheet which would result in a minimum number of glass canisters. All the major DWPF process bases used in this simulation study are given in Section 6.1 of this report.

One of the key conclusions of this study was that based on the PCCS (Version 3) analysis, the qualities of the glasses produced for all three sludge washing cases were determined to be acceptable. It turned out that the sludge loading in glass was the highest at $25.6 \mathrm{wt} \%$ when the sludge was washed least to $10.7 \mathrm{wt} \% \mathrm{Na}$, and it was the lowest at $24.6 \mathrm{wt} \%$ when the sludge was washed most to $8.1 \mathrm{wt} \%$ $\mathrm{Na}$. These sludge loadings are, however, somewhat misleading, since they include washable salts in the sludge feed. As the sludge was washed more from 10.7 to $8.1 \mathrm{wt} \% \mathrm{Na}$ by using 300,000 gallons of additional wash water, the sludge loading in glass was decreased, but the total number of glass canisters produced was also reduced by 13 .

When the sludge was washed below $8.1 \mathrm{wt} \% \mathrm{Na}$, however, one of the key DWPF process bases used in the model was exceeded, i.e., more than two precipitate batches to the PR were required for one sludge batch to the SRAT. As a result, the optimum washing endpoint for the Tank 51 sludge appears to be about $8 \mathrm{wt} \% \mathrm{Na}$ on a dry basis. For all three sludge washing cases, the PCCS analysis showed that the sludge loading in glass was limited by the liquidus constraint. The calculated PHA loading in glass was constant at $6.0 \mathrm{wt} \%$ for all three washing cases, since the maximum 7,900 gallons of PHA was blended with every sludge batch.

Some of the simulation results for the case of a sludge feed containing $10.0 \mathrm{wt} \%$ $\mathrm{Na}$ are compared in Table 0 to the earlier flowsheet based on the Tanks 42/51 blend sludge. Since the Tanks $42 / 51$ sludge flowsheet used a hypothetical blend salt to make the glass, cumulative quantities such as duration or total volume of precipitate produced per cycle could not be calculated. For comparison purposes, therefore, most of the ITP flows are given in Table 0 in terms of the instantaneous flow rates required to satisfy $100 \%$ DWPF attainment at a given waste loading in glass. The cumulative ITP volumes for the Tank 51/ITP Cycle 1 flowsheet can be calculated simply by multiplying these instantaneous flow rates with the duration for the Cycle 1 .

One interesting point to note is that the instantaneous ITP flow rates for the Cycle 1 are seen to be somewhat higher than those for the blend salt feed case. This is mainly because more washing was required in the LWF for the Cycle 1 precipitate, thus reducing the content of soluble salts in the feed to DWPF. This is further explained later in SUMMARY. 
C. T. Randall

iii

WSRC-TR-95-0019

(Revision 0)

TABLE 0. Comparison of HLW Flowsheet Material Balance Results

Tank 51 Only

Tanks 42/51 Blend

\section{ESP/DWPF:*}

Total Insoluble Sludge Solids (x106 lb)

0.833

1.405

Wash Water Added ( $\times 10^{6}$ gallon)

1.35

0.8

Inhibitors Added (gallon)

- $50 \mathrm{wt} \% \mathrm{NaOH}$

- 40 wt\% $\mathrm{NaNO}_{2}$

29,300

44,900

30,000

57,800

Na Content in Washed Sludge (wt\% dry)

10.0

10.5

Duration@ @ 100\% DWPF Attainment (yr)

1.40

2.31

Total \# of Glass Canisters

756

1,246

Sludge Loading in Glass (wt\%)

25.6

26.4

DWPF Recycle @ 40\% Attainment (x106 gallon)

7.4

13.0

ITP/Late Wash:**

Salt Feed Type

ITP Cycle 1

Blend

Duration@ @100\% DWPF Attainment (mo)

3.93

N/A

33.5 wt\% Feed to Tank 48 (gpm)

13.2

12.7

Total Insoluble Solids Removed (lb)

62,060

0.0

10 wt\% Precipitate to Tank 49 (gpm)

0.913

0.877

Decont'd Supernate to Tank 50 (gpm)

19.27

18.23

Precipitate Feed to Late Wash (gpm)

0.90

0.86

NaTPB Added (gpm)

- ITP

- Late Wash

0.546

0.053

0.565

0.105

0.130

Total Na in Precipitate to DWPF (equiv. molar)

0.687

0.054

Saltstone Produced during ITP Cycle $1\left(x 10^{6} \mathrm{ft}^{3}\right)$

N/A

* All cumulative volumes were calculated per the duration of the sludge feed.

** All instantaneous flow rates represent required flows to satisfy $100 \%$ DWPF attainment. 


\section{SUMMARY (Cont'd)}

(Revision 0)

Table 0 also shows that at the end of ITP Cycle 1, 62,050 lb of insoluble solids would remain. These insoluble solids should be diluted with other sludges in ESP, before being fed to DWPF. By contrast, no insoluble solids remained after the processing of the blend salt, since the reconstituted blend salt solution was assumed to contain only $600 \mathrm{ppm}$ of insoluble solids, compared to over 11,000 ppm measured in the Tank 41 saltcake samples. ${ }^{11}$ It is also noted in Table 0 that the total $\mathrm{Na}$ level in the precipitate feed produced during the ITP Cycle 1 is twice as high as that for the blend salt, because about $40 \mathrm{wt} \%$ of the insoluble solids in the Tank 41 saltcake are due to insoluble $\mathrm{Na}$.

Table $\mathbf{0}$ further shows that although the volume of supernate in Tank 51 is much less than those in Tanks 42 and 51 combined, more wash water was required to reach the same $\mathrm{Na}$ endpoint. This was because the supernate in Tank 42 contains much less $\mathrm{Na}$ than in Tank 51, so it can be used to wash the Tank 51 sludge.

In addition to the updated sludge and salt/supernate feed compositions for ESP and ITP, other significant improvements also have been made to the CPES integrated HLW flowsheet model and its database since 1993, including;

- Increased model capability to manage tank heels in both ESP and ITP.

- Improved radiolysis chemistry of TPB precipitates in Tank 49.

- Modified inhibitor addition algorithm for ESP washing.

- Improved chemistry of $\mathrm{H}_{2}$ and $\mathrm{NH}_{3}$ generation in $\mathrm{CPC}$.

The chemistry used in the model to describe the radiolytic reactions in Tank 49 was revised by including the correlations for the net production rates of organic species and the G-values for the nitrite/nitrate consumption as a function of total dose. These correlations were developed from recent analytical results for the precipitate samples irradiated up to $510 \mathrm{Mrad} .27,28$ The content of Cs-137 in the precipitate to be produced during the ITP Cycle 1 is projected to be $9.2 \mathrm{Ci} / \mathrm{gal}$, and the updated radiolysis chemistry predicts that about $5 \%$ of the first TPB precipitate would be decomposed and become soluble during a 2-year storage in Tank 49, compared to $19 \%$ decomposition at $46 \mathrm{Ci} / \mathrm{gal}$.

The total production rates of $\mathrm{H}_{2}$ and $\mathrm{NH}_{3}$ in one CPC cycle were set in this study at 2.67 and $1.37 \mathrm{lb}$, respectively. These values were obtained by scaling up the rates measured during the IDMS PX-6 run. Since the actual contents of noble metals in the Tank 51 sludge are expected to be considerably lower than those present in the PX-6 feed, these total emission rates assumed in this study should be regarded as very conservative estimates. 


\section{SUMMARY (Cont'd)}

One significant change made to the convergence scheme used in the model was to fix the cycle time for the SRAT at 86 hours and then to calculate the required sludge feed batch size to meet the design basis glass production rate of $228 \mathrm{lb} / \mathrm{hr}$, while satisfying the minimum frit content in glass determined by the PCCS analysis and maximizing the salt workoff rate at the same time.

This report summarizes most of the improvements and the revisions that have been made to the CPES model and its database, and subsequently reflected in this study. Most of the sludge and salt/supernate feed compositions used in this study were developed from the latest analytical data, and they are highlighted in several tables in this report. A complete output of the CPES material balance run including the compositions of blend glass, washed sludge and the first radioactive precipitate to be produced from ITP is also attached to this report for the case of a sludge feed containing $10 \mathrm{wt} \% \mathrm{Na}$. This is the first-ever HLW flowsheet material balance to be issued using the actual salt and sludge feed compositions.

Finally, it is strongly advised that readers use caution when interpreting the numbers given in the material balance tables for any batch process; all the flow rates are given in terms of the instantaneous flow rates required to meet the design glass production rate of $228 \mathrm{lb} / \mathrm{hr}$ or $100 \%$ DWPF attainment. When the total or individual species flow rates given are summed over one cycle period of a batch operation, readers should then obtain estimates of one of the following:

- Required feed batch size for one cycle of operation to support $100 \%$ DWPF attainment.

- Required production rate from one cycle of operation to support $100 \%$ DWPF attainment.

- Total throughput of a specific stream or species during one cycle of operation.

The attached material balance does not provide information on actual instantaneous flow rates during individual batch process steps. Nevertheless, if readers know enough about each batch process, cumulative volumes during each batch step or volumes per cycle time can be estimated from this material balance. Examples are given later in this report. 
C. T. Randall

vi

WSRC-TR-95-0019

(Revision 0)

This page intentionally left blank. 


\section{TABLE OF CONTENTS}

page

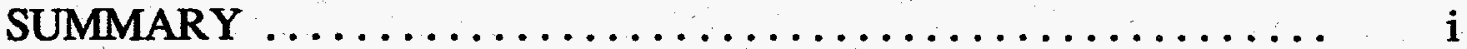

1. SCOPE OF WORK $\ldots \ldots \ldots \ldots \ldots \ldots \ldots \ldots \ldots \ldots \ldots \ldots, 1$

2. INTEGRATED HLW FLOWSHEET MODEL

2.1. Model Scope and Structure $\ldots \ldots \ldots \ldots \ldots \ldots \ldots \ldots \ldots \ldots \ldots$

2.2. Chemical Compounds ......................... 2

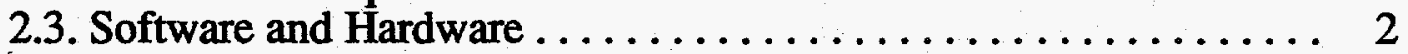

3. STRUCTURE OF MATERIAL BALANCE TABLES $\ldots \ldots \ldots \ldots \ldots, 3$

4. STEADY STATE MATERIAL BALANCE $\ldots \ldots \ldots \ldots \ldots \ldots \ldots, 4$

5. WASTE COMPOSITIONS

5.1. Tank 51 sludge $\ldots \ldots \ldots \ldots \ldots \ldots \ldots \ldots \ldots \ldots \ldots, 5$

5.2. Salt/Supernate Feeds to ITP Cycle $1 \ldots \ldots \ldots \ldots \ldots \ldots \ldots \ldots, 8$

5.3. Concentrate Transfer to ETF $\ldots \ldots \ldots \ldots \ldots \ldots \ldots \ldots \ldots \ldots \ldots \ldots$

6. REVISIONS MADE TO HLW FLOWSHEET MODEL

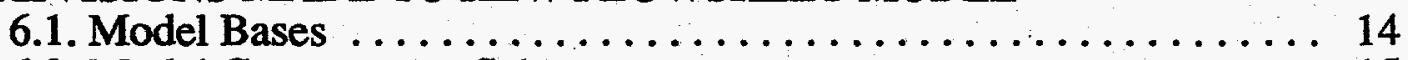

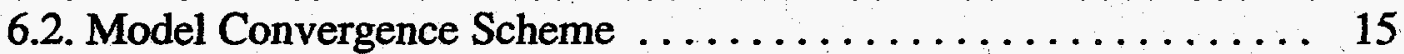

6.3. Extended Sludge Processing $\ldots \ldots \ldots \ldots \ldots \ldots \ldots \ldots \ldots \ldots$

6.4. In-Tank Precipitation $\ldots \ldots \ldots \ldots \ldots \ldots \ldots \ldots \ldots \ldots \ldots \ldots \ldots \ldots$

6.5. Late Wash Facility ......................... 19

6.6. Hydrogen and Ammonia Source Terms in CPC .......... 20

6.7. Ammonia Scrubbers $\ldots \ldots \ldots \ldots \ldots \ldots \ldots \ldots \ldots \ldots \ldots .22$

6.8. Inhibitor Addition to Liquid Recycle/NH3 Release ......... 22

6.9. Miscellaneous Revisions ..................... 23

7. SIMULATION RESULTS AND CONCLUSIONS $\ldots \ldots \ldots \ldots \ldots 23$

8. QUALITY ASSURANCE $\ldots \ldots \ldots \ldots \ldots \ldots \ldots \ldots \ldots \ldots \ldots$

9. ACKNOWLEDGMENT $\ldots \ldots \ldots \ldots \ldots \ldots \ldots \ldots \ldots \ldots \ldots \ldots$

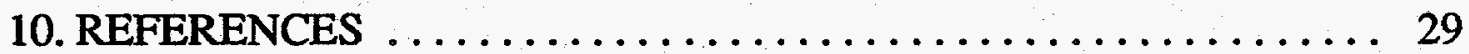

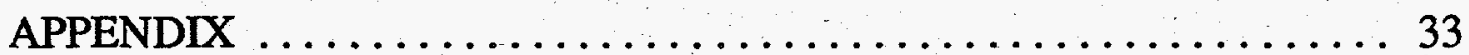

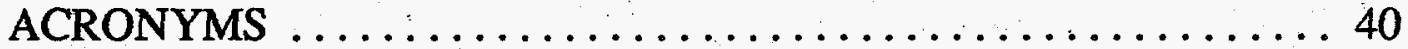




\section{LIST OE TABLES}

page

TABLE 0. Comparison of

HLW Flowsheet Material Balance Results ......... iii

TABLE 1. Partitioned and Components Assumed Soluble

TABLE 2. Components Assumed Insoluble

in the HLW Flowsheet Model ............... 35

in the HLW Flowsheet Model ............. 36

TABLE 3. Composition of Group A and Group B Components ....... 37

TABLE 4. Estimated Composition of

Insoluble Sludge Solids in Tank $51 \ldots \ldots \ldots \ldots 6$

TABLE 5. Estimated Composition of Interstitial Supernate in Tank $51 \ldots \ldots \ldots \ldots \ldots 7$

TABLE 6. Feed Volumes for the First ITP Cycle $\ldots \ldots \ldots \ldots \ldots \ldots \ldots 8$

TABLE 7. Estimated Composition of Saltcake in Tank $41 \ldots \ldots \ldots . .9$

TABLE 8. Estimated Composition of

Insoluble Solids in Tank 41 Saltcake .......... 10

TABLE 9. Estimated Composition of

Supernate Feeds for ITP Cycle $1 \ldots \ldots \ldots \ldots \ldots 11$

TABLE 10. Composition of ETF Concentrate Transfer to Tank $50 \ldots \ldots 13$

TABLE 11. Corrosion Inhibitor Requirements for Waste Tank Farms . . 17

TABLE 12. Results of Optimization of Tank 51 Sludge Washing ...... 24

TABLE 13. Calculated Composition of

Radiolyzed Precipitate Feed to LWF .......... 25

TABLE 14. Calculated Composition of

Washed Tank 51 Sludge Feed .............. 26

TABLE 15. Calculated Composition of Glass Made from

Tank 51 Sludge and ITP Cycle 1 Precipitate ....... 27

\section{LIST OF FIGURES}

FIGURE 1. Calculation Flowchart for Model Convergence . ........ 38

FIGURE 2. Results from PCCS Run for $10.0 \mathrm{wt} \% \mathrm{Na}$ Case ......... 39 


\section{SCOPE OF WORK}

HLWM initially requested that SRTC evaluate the impact for Tank 51 sludge only scenario. ${ }^{1}$ The TAR from DWPF outlines the scope of the work as follows: ${ }^{2}$

- Develop the Tank 51 sludge composition from the latest analytical data for the Tank 51 sludge samples.

- Develop the feed compositions for the ITP Cycle 1 according to the salt removal plan outlined in the HLW System Plan Revision 4.3.

- Calculate the Tank 51 sludge/ITP Cycle 1 flowsheet material balance using the updated CPES integrated HLW flowsheet model.

- Optimize the ESP flowsheet by determining the sludge washing endpoint which will result in a minimum number of glass canisters.

After each simulation run is completed, the quality of the resulting glass must be checked by running the PCCS analysis. For each set of operating conditions used, it must be ensured that the glass produced indeed contains the minimum necessary amount of frit.

\section{INTEGRATED HLW FLOWSHEET MODEL}

All the necessary calculations for this work except the PCCS analysis were made using the CPES integrated HLW flowsheet model. A brief overview of the CPES model is given next. All the major revisions made to this model since the Tanks 42/51 flowsheet material balance was issued in 1993 are discussed later.

\subsection{Model Scope and Structure}

A material balance for the entire $H L W$ processing operation at the Savannah River Site (SRS) is calculated by using the CPES integrated HLW flowsheet model which covers both DWPF and the tank farm operations. ${ }^{3}$ This steady state flowsheet model is made up of several modules each of which describes a specific phase of the HLW processing operation such as ITP and CPC in DWPF. This modular structure is reflected in the way these material balance tables are organized, as shown in Section 2. The model has many internal recycle streams and, if there are changes to be made in the waste feed compositions, all of them must be properly initialized before starting a new simulation run. Once steady state conditions are established, each module can then be run independently of one another. This makes it possible to assess immediate downstream effects promptly without having to run the entire flowsheet. The integrated HLW flowsheet model currently has nearly 1,600 process streams connecting over 650 unit operations. In addition to an extensive use of the CPES software library, the main program of the model is also supported by 45 additional user-added subroutines to describe those unit operations that are unique to the HLW processing operation. 
WSRC-TR-95-0019

(Revision 0)

\subsection{Chemical Compounds}

The actual waste currently stored in large underground tanks at SRS is a complex mixture of many different molecular species and ionic salts. The complexity and diversity make the chemical analysis of waste samples extremely difficult and time consuming. Furthermore, nonhomogeneity within these waste mixtures caused by gravity settling during storage also makes it difficult to obtain representative waste samples. Additional analytical difficulties arise from the fact that although some of the waste species are measured only in insignificantly low concentrations, most of them cannot be ignored in material balance calculations due to radioactivity. As a result, a complete physical and chemical characterization of the waste is virtually impossible at the present time.

In order to minimize the number of species that must be followed throughout the material balance calculations and still retain the key cations, anions, and molecular species identified by chemical analyses, the chemical compounds categorized in Tables 1 and 2 in Appendix were selected to represent the compositions of actual HLW waste at SRS as well as the chemistry for its processing operations. Those trace level species that have radioactive isotopes are identified as either Group A or Group B in Table 3 in Appendix. Group A consists of elements whose oxides are relatively soluble and/or volatile at the operating temperature for the glass melter. Group B consists of elements whose oxides are essentially insoluble and nonvolatile.

Currently, there are 183 chemical compounds in the model database, and species with flow rates as low as $1.0 \mathrm{E}-25 \mathrm{lb} / \mathrm{hr}$ are tracked. Of the 183 compounds, the compositions of salts are represented by 34 compounds, and the four different types of sludge compositions identified so far require additional 46 compounds. The chemistry of salt supernate decontamination and DWPF processes requires additional 17 and 86 compounds, respectively.

The chemistry of the entire HLW processing operation is currently described by over 340 chemical reactions. It is assumed in the model that whenever present, elemental mercury forms a pure liquid phase and excluded from all vapor-liquid equilibrium (VLE) calculations. After a VLE calculation is complete, elemental mercury is split in such a way that the elemental mercury released into the vapor phase always remains conservatively high.

\subsection{Software and Hardware}

The integrated HLW processing model was built by using du Pont's proprietary process simulation software, Chemical Process Evaluation System (CPES). ${ }^{4}$ The CPES version 6.3 which runs on the VAX/VMS system can handle up to 200 components, 2,000 streams, 2,000 unit operation blocks, 1,000 chemical reactions, and 32 recycle loops. The CPES software has a library of programs for most standard chemical engineering unit operations such as nonideal VLE, extraction, heat exchanger, reactor, etc. Data entry is performed interactively. 
The overall convergence scheme used in the model is described later in Section 8. The local convergence scheme used in each module is to monitor one or more recycle streams from one iteration to the next, until a specified tolerance criterion is met. For a typical run with a new feed composition, it takes over 18 hours on a dedicated VAXstation ${ }^{\mathrm{TM}} 4000-60$ to achieve a convergence for-all recycle streams within acceptable tolerance.

\section{STRUCTURE OF MATERIAL BALANCE TABLES}

The material balance tables attached to this report are divided into 10 sections. Of those, 3 sections are further divided into a total of 7 subsections. Section 0 is the overall summary section, which includes all major streams that are interest to most users. As shown below, Sections 1A through 9 each represent specific phases of the HLW processing operation. Due to increased complexity of the salt processing operation, the Late Wash Facility (LWF) operation was separated out from the InTank Precipitation (ITP) section, and all streams related to the LWF are shown in Section 1B. All streams related to the Extended Sludge Processing (ESP) are now shown in Section $1 \mathrm{C}$.

\begin{tabular}{cl}
\hline Section & \multicolumn{1}{c}{ Representation } \\
\hline 0 & major streams in the model \\
1A & $\begin{array}{l}\text { In-Tank Precipitation \& storage } \\
\text { re-precipitation \& Late Washing } \\
\text { 1B }\end{array}$ \\
1C & Saltended Sludge Processing \\
\hline 2 & $\begin{array}{l}\text { precipitate hydrolysis in Salt Cell } \\
\text { boilup to remove C6 } 6 \text { H } 6 \text { in Salt Cell }\end{array}$ \\
3A & $\begin{array}{l}\text { adjustment and blending of melter } \\
\text { feeds \& mercury stripping } \\
\text { frit addition \& feed adjustment }\end{array}$ \\
$4 \mathrm{~B}$ & $\begin{array}{l}\text { vitrification \& off-gas treatment } \\
\text { mercury purification }\end{array}$ \\
$4 \mathrm{~B}$ & $\begin{array}{l}\text { treatment of DWPF liquid waste recycle } \\
\text { canister decontamination } \\
\text { collection of cell vents and sand filter }\end{array}$ \\
\hline 5 & . \\
\hline
\end{tabular}




\section{STEADY STATE MATERIAL BALANCE}

Prior to using the material balance tables in Appendix (Table A-1), readers should know how to interpret the information provided by the tables correctly. Nearly all of the HLW processing operations involved except vitrification and melter off-gas treatment are performed in a batch mode. Ideally, however, regardless of batch or continuous, all the processes must be scheduled and coordinated in such a way that there is always enough quality feed available for the melter to produce acceptable glass continuously at the design basis rate of $228 \mathrm{lb} / \mathrm{hr}$. This is precisely what the CPES model attempts to demonstrate by integrating the entire HLW processing operations into one coherent continuous operation that must maintain its desired production rate.

Therefore, all the flow rates given in the material balance tables actually represent time-averaged values over one cycle period of a specific process or unit operation. Calculation of actual process variables such as batch feed rate and inert purge rate requires additional information on each process itself such as actual feeding time, cycle time, and etc.

For example, Stream 207 in Section 3A of Table A-1 represents the $\mathrm{CO} 2$ purge to PR, and its rate is given as $3.62 \mathrm{cfm}$ or $3.21 \mathrm{scfm}$. Based on a 43-hour PR cycle, the total $\mathrm{CO} 2$ purge required during one cycle is then:

$$
(3.62)(60)(43)=9,340 \mathrm{cfm} \text { or } 8,280 \mathrm{scfm}
$$

In reality, however, much of this purge is required during the periods of precipitate feeding, boilup and cooldown that last for 13 hours total. Therefore, given the baseline purge rate of $2 \mathrm{scfm}$, the actual $\mathrm{CO}_{2}$ purge rate required during the 13hour period is calculated as:

$$
[8,280-(2)(60)(43-13)] / 13 / 60=6 \mathrm{scfm} .
$$

This is somewhat less than twice the time-averaged purge rate of $3.21 \mathrm{scfm}$.

Another example would be to estimate the required sludge feed batch size for the SRAT. Stream 1 in Section 0 or Section 4A.has an instantaneous flow rate of $444.6 \mathrm{lb} / \mathrm{hr}$ or $0.792 \mathrm{gpm}$. Knowing that the cycle time for the SRAT operation is 86 hours, the sludge feed batch size is then simply:

$$
(0.792)(60)(86)=4,090 \text { gallons. }
$$

When the 1,500 gallon heel is added as assumed in the model, the total volume of liquid in the vessel then becomes 5,580 gallons, which is just enough to cover the steam coils completely. It is important to recognize that this sludge feed batch size represents the actual feed volume required in every 86 hour cycle to sustain the glass production rate of $228 \mathrm{lb} / \mathrm{hr}$ and $1 \mathrm{wt} \%$ glass entrainment into the melter offgas system. 
For the tank farm operations that are run on much longer time scales, information useful to the production planning can be extracted from this steady state material balance in addition to the detailed chemical compositions of the final effluents. For example, the instantaneous flow rate of Stream 414 in Section 1A is given as $471.2 \mathrm{lb} / \mathrm{hr}$ or $0.9134 \mathrm{gpm}$. So, if one ITP cycle is to be completed in 3 months including the downtime, then it must produce:

$$
(0.9134)(60)(24)(90)=118,400 \text { gallons of } 10 \mathrm{wt} \% \text { precipitate }
$$

in every 3 months with the given salt feed to support 100\% DWPF attainment. In order to produce precipitate at $0.9134 \mathrm{gpm}$, the average feed rate of the Tank 41 saltcake (Stream 652) must be maintained at $1,767 \mathrm{lb} / \mathrm{hr}$ or $1.68 \mathrm{gpm}$. Since the total volume of $16 \mathrm{wt} \%$ Tank 41 saltcake to be fed during the ITP Cycle 1 is about 287,500 gallons, theoretically the precipitate produced during the first cycle can support $100 \%$ DWPF attainment for:

$$
287,500 / 1.68 / 60 / 24 / 30=3.96 \text { months. }
$$

As stated earlier, the tank farm modules of the CPES integrated HLW flowsheet model can be run decoupled from the DWPF modules in order to enable one to estimate tank farm throughputs based on their actual processing scenarios.

\section{WASTE COMPOSITIONS}

\subsection{Tank 51 Sludge}

The sludge feed to ESP consists of settled sludge and supernatant in Tank 51. An Excel ${ }^{\circledR}$ spreadsheet program developed earlier was used to convert the analytical data given in terms of elemental and ionic distributions into neutral species forms available in the model. The resulting sludge compositions are given in Tables 4 and 5 for insoluble solids and unwashed interstitial supernate, respectively.

The data used to develop the composition of insoluble sludge solids given in Table 4 were obtained from the analysis done on two of the slurry samples taken from Tank 51 during the recent ESP baselining tests.5,6 The elemental and radionuclide analyses of these sludge samples are typically done by first washing the samples in centrifuge tubes several times and then dissolving the remaining solids by $\mathrm{Na}_{2} \mathrm{O}_{2}$ fusion and/or aqua regia for analyses by inductively coupled plasma-atomic emission spectroscopy / mass spectroscopy (ICP-AES/MS) and atomic absorption spectroscopy (AAS). ${ }^{2}$ For some species like Tc, Th, and noble metals, the analytical data were available only on a wt\% dry solids basis. In this case, the wt\% dry basis compositions were converted into those based on the wt\% insoluble solids by multiplying them by an estimated conversion factor of 1.5626 . Furthermore, the analytical data available for Cs in Tank 51 was only for Cs-137.6 As a result, the mass ratio of Cs-137 to the total Cs in Tank 42 was first estimated to be about 0.515 for both soluble and insoluble Cs using the earlier data. ${ }^{8}$ Then, the same mass ratio was assumed to be applicable to the Tank 51 samples and used estimating the wt\% $\mathrm{Cs}_{2} \mathrm{O}$ in Table 4. 
TABLE 4. Estimated Composition of Insoluble Sludge Solids in Tank 51

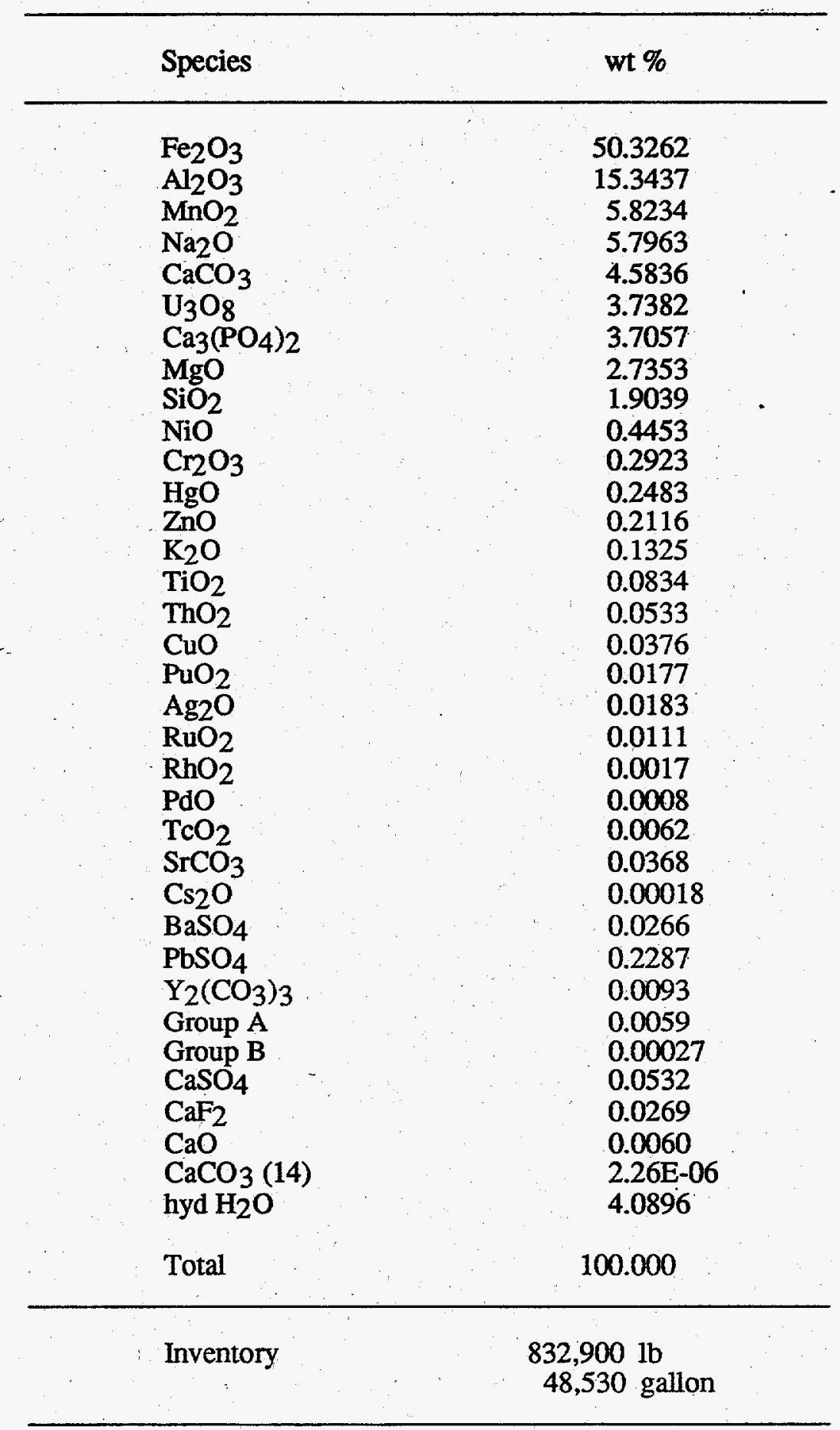


TABLE 5. Estimated Composition of Interstitial Supernate in Tank 51

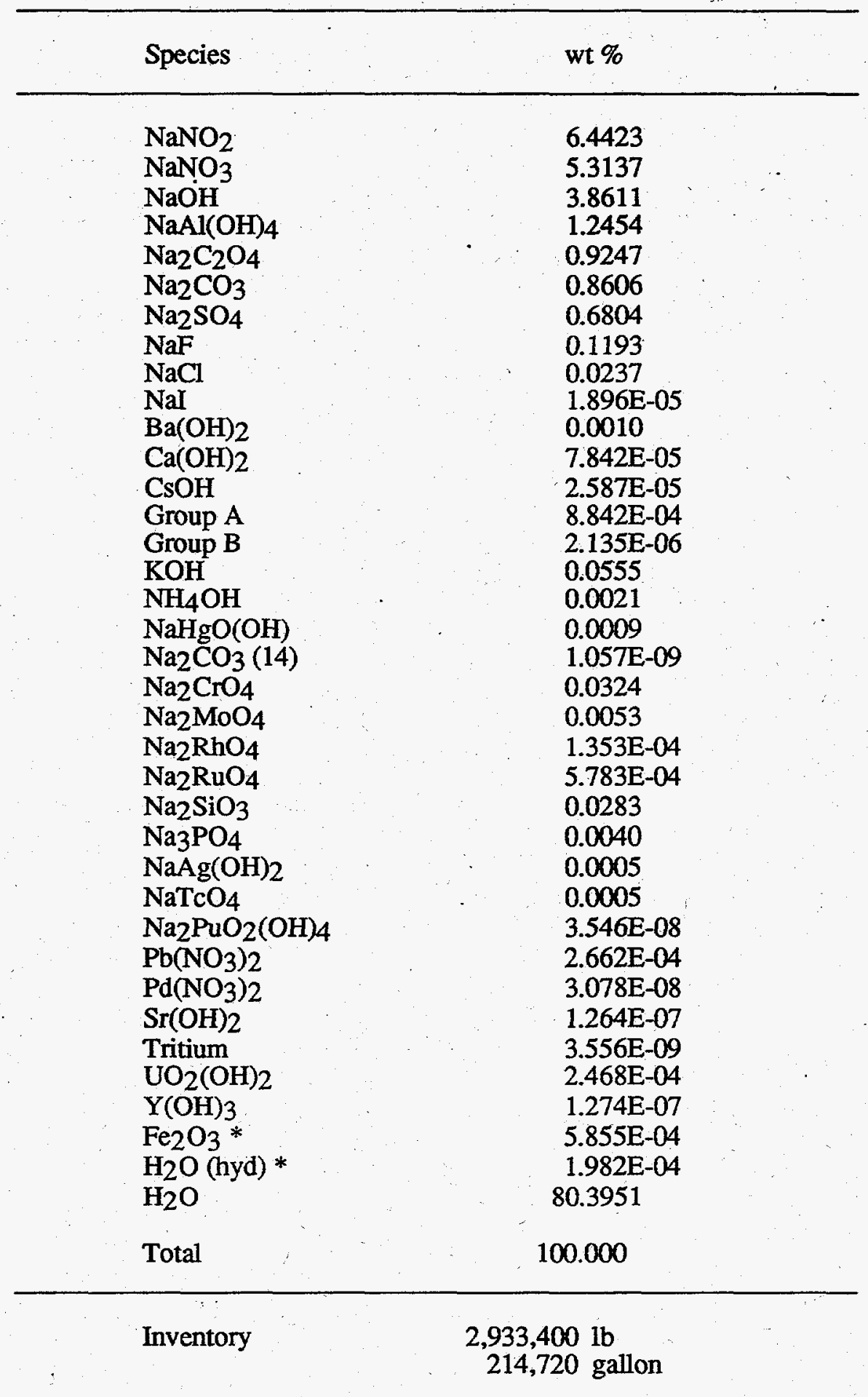

* assumed to exist as $\mathrm{Fe}(\mathrm{OH})_{3}$, since no soluble form of $\mathrm{Fe}$ exists in the database. 
At the time of this study, few data were available for those trace level radioactive species found in Group A or Group B. As a result, the concentrations of Group A and Group B given in Tables 4 and 5 were either estimated by extrapolating the scarce data available for only one or two species to the entire group or assumed to be identical to those in the existing model database. ${ }^{9}$ The concentration of water of hydration in Table 4 was adjusted to be the balance of $100 \%$, after satisfying the required mole balances for all the elements involved.

The data used to construct the Tank 51 supernate composition in Table 5 were also obtained from the analysis done on the same slurry samples taken from Tank 51 during the ESP baselining tests. ${ }^{6}$ Additional soluble species were also added to the list to account for those sodium-containing species that are listed in the CPES model database for the 1988 WAPS Batch 1 sludge supernate but were not analyzed for at this time. The concentrations of these soluble species added were assumed to be the same, on a dry wt\% basis, as those in the database, and are all negligibly small. It was necessary to make modest adjustments to the measured values in order to achieve the overall charge balance.

\subsection{Salt/Sunernate Feeds to ITP Crcle 1}

According to the salt removal schedule outlined in the HLW System Plan Revision $4,{ }^{10}$ four_batches of salt and supernate feeds will be processed during the first ITP cycle lasting 450 days including the washing/concentration step and the projected 92 days of downtime. Table 6 shows the cumulative volumes of dissolved salt and supernates fed from each tank during the first cycle. The compositions of salts and insoluble solids in Tank 41 shown in Tables 7 and 8, respectively, were developed from the recent analytical data taken in 1993.11 The compositions of supernates in each of the remaining feed tanks were also developed from recent analytical data and available tank history data, ${ }^{12-14}$ and they are compared in Table 9.

TABLE 6. Feed Volumes for the First ITP Cycle

\begin{tabular}{ccc}
\hline Tank & Type & Volume (k gal) \\
\hline 41 & dissolved saltcake & 1,150 \\
38 & concentrated supernate & 255 \\
32 & supernate & 200 \\
48 & supernate (heel) & 252 \\
49 & supernate (heel) & 160 \\
- & inhibited water & 280 \\
Total & & 2,297 \\
\hline
\end{tabular}


TABLE 7. Estimated Composition of Saltcake in Tank 41

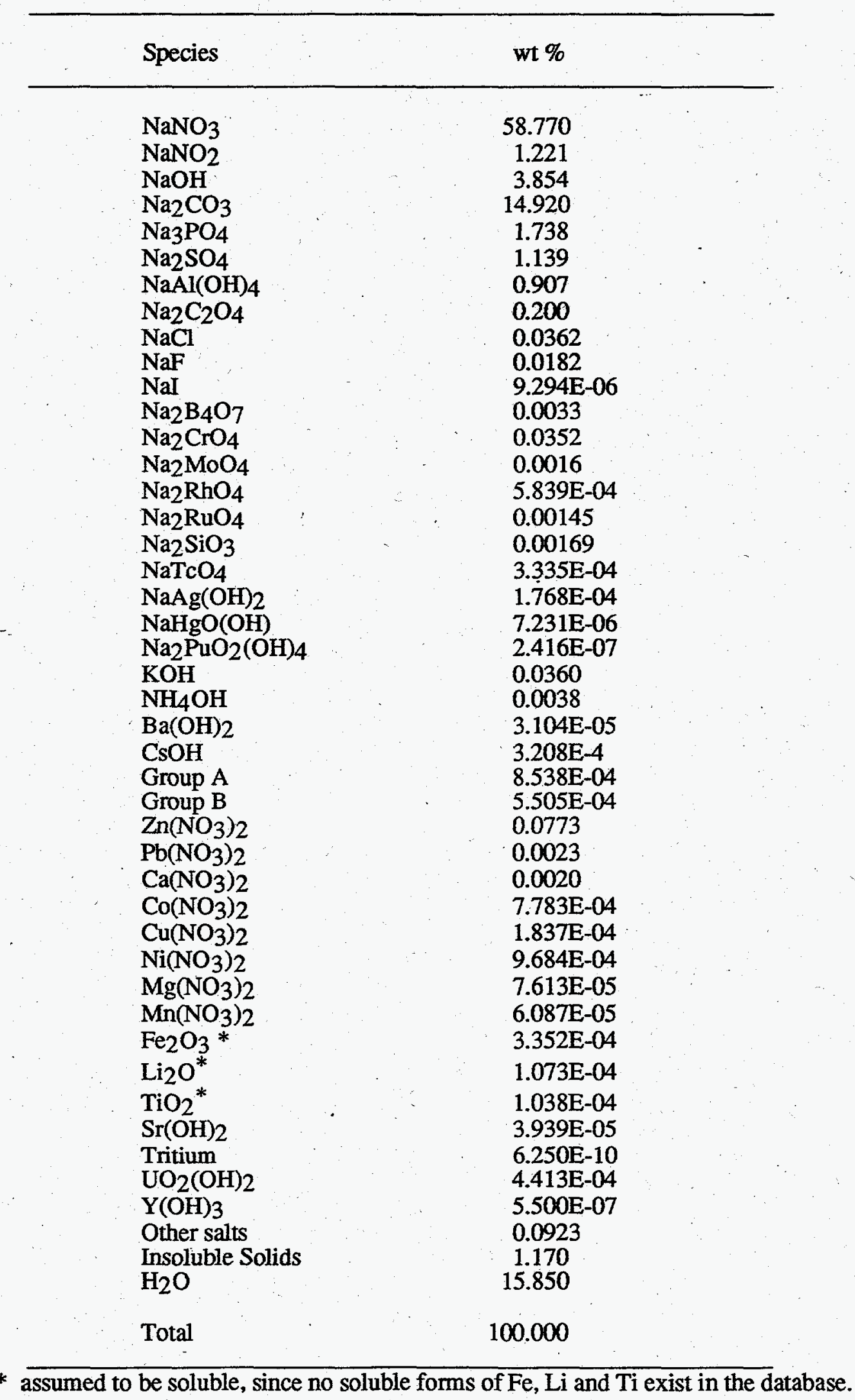


TABLE 8. Estimated Composition of Insoluble Solids in Tank 41 Saltcake

\begin{tabular}{|c|c|}
\hline Species & wt \% \\
\hline $\mathrm{Na}_{2} \mathrm{O}$ & 40.28 \\
\hline $\mathrm{Ca}_{3}\left(\mathrm{PO}_{4}\right)_{2}$ & 22.51 \\
\hline $\mathrm{Cr}_{2} \mathrm{O}_{3}$ & 10.51 \\
\hline $\mathrm{CaSO}_{4}$ & 7.6750 \\
\hline $\mathrm{ZnO}$ & 7.9490 \\
\hline $\mathrm{Fe}_{2} \mathrm{O}_{3}$ & 6.0600 \\
\hline $\mathrm{Al}_{2} \mathrm{O}_{3}$ & 2.6930 \\
\hline $\mathrm{SiO}_{2}$ & 1.1790 \\
\hline $\mathrm{K}_{2} \mathrm{O}$ & 0.2763 \\
\hline $\mathrm{MnO}_{2}$ & 0.1323 \\
\hline $\mathrm{RuO}_{2}$ & 0.1776 \\
\hline $\mathrm{MgO}$ & 0.0693 \\
\hline $\mathrm{B}_{2} \mathrm{O}_{3}$ & 0.0671 \\
\hline $\mathrm{CuO}$ & 0.0426 \\
\hline $\mathrm{NiO}$ & 0.0417 \\
\hline $\mathrm{U}_{3} \mathrm{O}_{8}$ & 0.0302 \\
\hline $\mathrm{TiO}_{2}$ & 0.0215 \\
\hline $\mathrm{HgO}$ & 0.0149 \\
\hline $\mathrm{Ag}_{2} \mathrm{O}$ & 0.0089 \\
\hline $\mathrm{Li}_{2} \mathrm{O}$ & 0.0041 \\
\hline $\mathrm{MoO}_{2}$ & 0.0044 \\
\hline $\mathrm{CoO}$ & 0.0041 \\
\hline $\mathrm{SrCO}_{3}$ & 0.00365 \\
\hline $\mathrm{Cs}_{2} \mathrm{O}$ & 0.00155 \\
\hline $\mathrm{PuO}_{2}$ & 0.0013 \\
\hline $\mathrm{BaSO}_{4}$ & 0.0294 \\
\hline $\mathrm{PbSO}_{4}$ & 0.1752 \\
\hline Group B & 0.0376 \\
\hline Total & 100.000 \\
\hline
\end{tabular}


TABLE 9. Estimated Compositions of Supernate Feeds for ITP Cycle 1 (wt\%)

\begin{tabular}{|c|c|c|c|c|}
\hline Species & Tank 32 & Tank 38 & Tank 48 & Tank 49 \\
\hline $\mathrm{NaNO}_{2}$ & 4.8576 & 9.8372 & 2.4314 & 4.2368 \\
\hline $\mathrm{NaNO}_{3}$ & 16.5920 & 10.7398 & 1.1333 & 4.2500 \\
\hline $\mathrm{NaOH}$ & 3.4530 & 30.5406 & 0.8029 & 0.6314 \\
\hline $\mathrm{NaAl}(\mathrm{OH})_{4}$ & 6.9856 & 4.6243 & 0.6743 & 0.7246 \\
\hline $\mathrm{Na}_{2} \mathrm{C}_{2} \mathrm{O}_{4}$ & 0.0364 & 0.0616 & 0.0230 & 0.1411 \\
\hline $\mathrm{Na}_{2} \mathrm{CO}_{3}$ & 7.6320 & 8.0001 & 3.0286 & 6.7877 \\
\hline $\mathrm{Na}_{2} \mathrm{SO}_{4}$ & 0.5909 & 0.0653 & 0.0514 & 0.3364 \\
\hline $\mathrm{Na}_{3} \mathrm{PO}_{4}$ & 0.0420 & 0.4320 & 0.0250 & 0.0000 \\
\hline $\mathrm{NaF}$ & 0.0437 & 0.1106 & 0.0060 & 0.0000 \\
\hline $\mathrm{NaCl}$ & 0.0655 & 0.0111 & 0.0612 & 0.0359 \\
\hline $\mathrm{NaI}$ & $3.915 \mathrm{E}-05$ & $6.287 \mathrm{E}-05$ & 8.116E-06 & $1.673 \mathrm{E}-05$ \\
\hline $\mathrm{Ba}(\mathrm{OH})_{2}$ & $3.992 \mathrm{E}-06$ & $4.215 \mathrm{E}-06$ & $4.753 \mathrm{E}-06$ & 4.377E-06 \\
\hline $\mathrm{Ca}(\mathrm{OH})_{2}$ & $1.122 \mathrm{E}-04$ & $1.801 \mathrm{E}-04$ & $2.325 \mathrm{E}-05$ & 4.793E-05 \\
\hline $\mathrm{CsOH}$ & 0.0036 & $1.792 \mathrm{E}-04$ & $5.927 \mathrm{E}-06$ & $2.287 \mathrm{E}-05$ \\
\hline Group A & 9.202E-04 & 0.0031 & 0.0011 & 0.0010 \\
\hline Group B & $5.602 \mathrm{E}-07$ & $4.732 \mathrm{E}-07$ & $6.669 \mathrm{E}-07$ & $6.143 E-07$ \\
\hline $\mathrm{KOH}$ & 0.0853 & 0.3146 & 0.1015 & 0.0935 \\
\hline $\mathrm{NH}_{4} \mathrm{OH}$ & 0.0096 & 0.0154 & 0.0020 & 0.0041 \\
\hline $\mathrm{NaHgO}(\mathrm{OH})$ & 0.0084 & 0.0135 & 0.0017 & 0.0036 \\
\hline $\mathrm{Na}_{2} \mathrm{CO}_{3}(14)$ & $2.186 \mathrm{E}-09$ & $3.510 \mathrm{E}-09$ & $4.531 \mathrm{E}-10$ & $9.341 \mathrm{E}-10$ \\
\hline $\mathrm{Na}_{2} \mathrm{CrO}_{4}$ & 0.0292 & 0.1071 & 0.0347 & 0.0320 \\
\hline $\mathrm{Na}_{2} \mathrm{MoO}_{4}$ & 0.0238 & 0.0382 & 0.0049 & 0.0102 \\
\hline $\mathrm{Na}_{2} \mathrm{RhO}_{4}$ & $2.780 \mathrm{E}-04$ & $4.465 \mathrm{E}-04$ & $5.763 \mathrm{E}-05$ & 0.0001 \\
\hline $\mathrm{Na}_{2} \mathrm{RuO}_{4}$ & $2.066 \mathrm{E}-03$ & 0.0023 & 0.00245 & 0.0023 \\
\hline $\mathrm{Na}_{2} \mathrm{SiO}_{3}$ & $2.086 \mathrm{E}-04$ & 0.0573 & 0.0002 & 0.0002 \\
\hline $\mathrm{NaAg}(\mathrm{OH})_{2}$ & $2.445 \mathrm{E}-05$ & $2.375 \mathrm{E}-05$ & $2.911 \mathrm{E}-05$ & $2.681 \mathrm{E}-05$ \\
\hline $\mathrm{NaTcO}_{4}$ & 0.0017 & 0.0015 & 0.0020 & 0.0019 \\
\hline $\mathrm{Na}_{2} \mathrm{PuO}_{2}(\mathrm{OH})_{4}$ & 2.161E-06 & 3.471E-06 & $4.481 \mathrm{E}-07$ & $9.237 \mathrm{E}-07$ \\
\hline $\mathrm{Mn}\left(\mathrm{NO}_{3}\right)_{2}$ & 7.817E-06 & $3.521 \mathrm{E}-05$ & $9.306 \mathrm{E}-06$ & $8.571 \mathrm{E}-06$ \\
\hline $\mathrm{Pd}\left(\mathrm{NO}_{3}\right)_{2}$ & 6.367E-08 & $1.022 \mathrm{E}-07$ & $1.320 \mathrm{E}-08$ & $2.721 \mathrm{E}-08$ \\
\hline $\mathrm{Sr}(\mathrm{OH})_{2}$ & $1.058 \mathrm{E}-07$ & $1.581 \mathrm{E}-08$ & $1.259 \mathrm{E}-07$ & $1.160 \mathrm{E}-07$ \\
\hline Tritium & $2.976 \mathrm{E}-10$ & $4.779 \mathrm{E}-10$ & $6.170 \mathrm{E}-11$ & $1.272 \mathrm{E}-10$ \\
\hline $\mathrm{UO}_{2}(\mathrm{OH})_{2}$ & $5.949 \mathrm{E}-04$ & 0.0012 & 0.0000 & 0.0000 \\
\hline $\mathrm{Y}(\mathrm{OH})_{3}$ & $2.619 \mathrm{E}-07$ & 4.205E-07 & $5.429 \mathrm{E}-08$ & $1.119 \mathrm{E}-07$ \\
\hline $\mathrm{Fe}_{2} \mathrm{O}_{3} *$ & $2.288 \mathrm{E}-05$ & 0.0005 & $2.723 \mathrm{E}-05$ & $2.508 \mathrm{E}-05$ \\
\hline $\mathrm{H}_{2} \mathrm{O}$ (hyd) * & $7.744 \mathrm{E}-06$ & 0.00017 & $9.219 \mathrm{E}-06$ & $8.491 \mathrm{E}-06$ \\
\hline insoluble solids & 0.0122 & 0.0196 & 0.0025 & 0.0052 \\
\hline $\mathrm{H}_{2} \mathrm{O}$ & 59.5233 & 35.002 & 91.6095 & 82.7019 \\
\hline Total & 100.000 & 100.000 & 100.000 & 100.000 \\
\hline Sp. Gr. & 1.25 & 1.48 & 1.05 & 1.14 \\
\hline
\end{tabular}

* assumed to exist as $\mathrm{Fe}(\mathrm{OH})_{3}$, since no soluble form of $\mathrm{Fe}$ exists in the database. 
Table 7 shows the concentration of total insoluble solids in the Tank 41 saltcake at $1.17 \mathrm{wt} \%$, which represents the average value for three saltcake samples. ${ }^{11}$ As a comparison, the insoluble solids concentration in the average $\mathrm{H}$-Area saltcake was estimated earlier to be $2.05 \mathrm{wt} \%, 15$ which is about $75 \%$ higher than that in Tank 41. Table 8 shows that besides sodium, phosphate and chromium are the most abundant insoluble species in the Tank 41 saltcake. If the insoluble phosphate and chromium are fed to the ITP at the same concentrations as shown in Table 8, they all will remain in the precipitate feed to DWPF, and the resulting glass will contain too high levels of $\mathrm{Ca}-\mathrm{P}-\mathrm{O}$ and $\mathrm{Cr}$ to have an acceptable quality. ${ }^{47}$ For this reason, it was assumed in this study that $90 \%$ of the insoluble solids in the Tank 41 saltcake were removed from the ITP feed by gravity settling, and Figure 2 shows that the resulting glass would then contain phosphate and chromium well below their limits, and become acceptable.

The sources for the insoluble phosphate and chromium in Tank 41 are not clearly known at this time. As stated earlier, these high concentrations were estimated based on the latest analytical results for the samples taken near the top saltcake layer in Tank 41,11 and recently there have been concerns about extrapolating these limited data to the entire tank. However, since no samples have been taken from the inner regions of Tank 41 , and analyzed to verify those concerns, these limited data formed a sole basis for developing the Tank 41 saltcake composition during this study.

Since no data were available on the concentrations of insoluble solids present in the supernate feeds, it was assumed that they all contain the same amounts of insoluble solids at $0.03 \mathrm{wt} \%$ on a dry basis as the average $\mathrm{H}$-Area concentrated supernate. 15 The resulting wet-basis concentrations of insoluble solids are shown in Table 9. It was also assumed that the insoluble solids in the supernate feeds have the same composition as that shown in Table 8 for the Tank 41 saltcake. All the chemical species shown in Tables 7 to 9 were developed from the available elemental and ionic data using the neutral species forms present in the CPES model database.

\subsection{Concentrate Transfer to ETF}

The composition and the average transfer rate of the ETF concentrate shown in Table 10 were the same as those used in an earlier material balance. ${ }^{16}$ They were estimated based on the analytical data on the concentrate transfers made from 12/ 89 to 8/91 and the output of the existing ETF spreadsheet model.17-19 It should be noted that the concentrations of Group A and Group B in Table 10 were set solely based on the measured concentrations of Se and Co-60, respectively. 
TABLE 10. Composition of ETF Concentrate Transfer to Tank 50

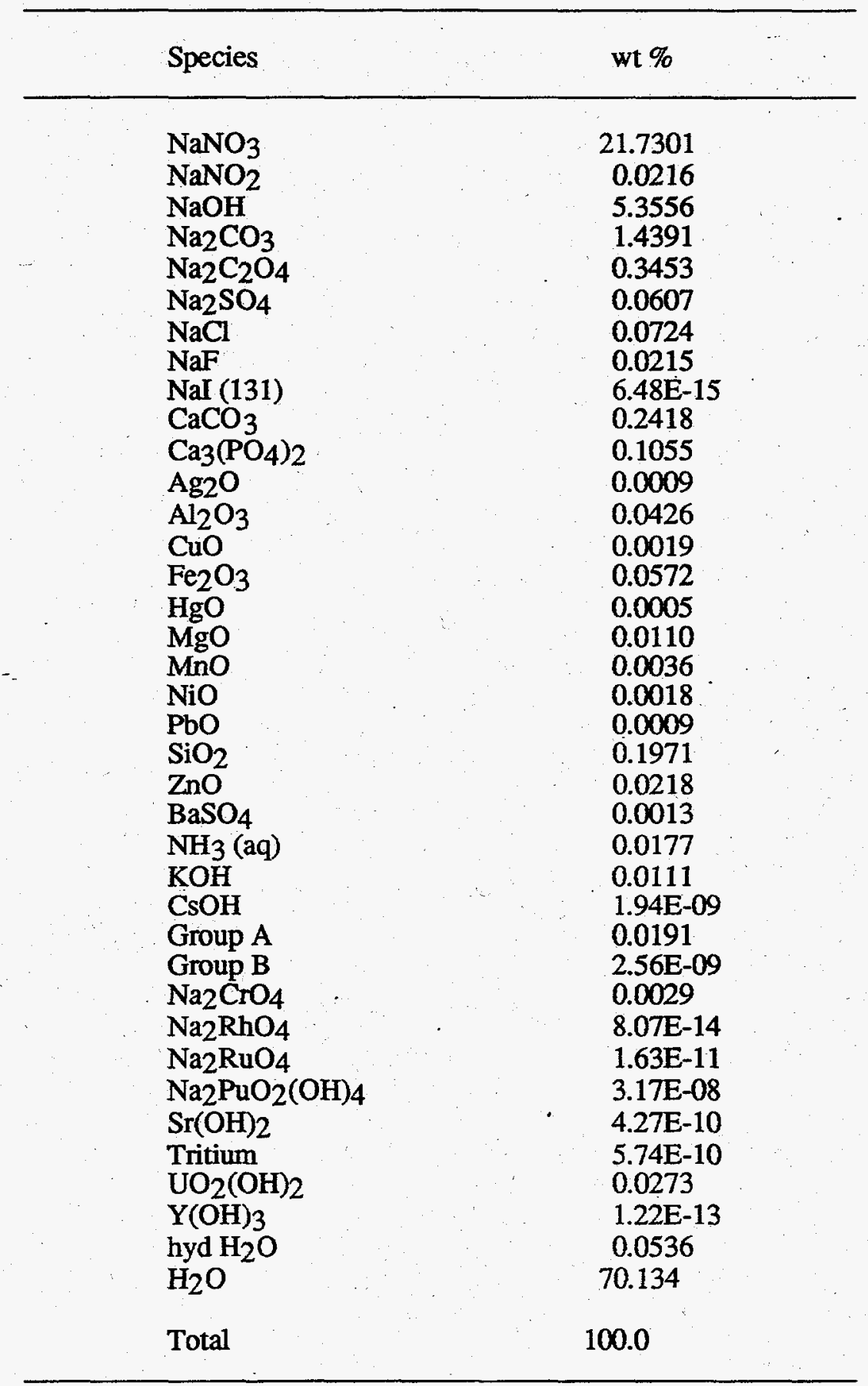

specific gravity $=1.265$

free $\mathrm{OH}=1.70$ molar

wt $\%$ total suspended solids $=0.74$

average transfer rate to Tank $50=0.334 \mathrm{gpm}$ 


\section{REVISIONS MADE TO HLW FLOWSHEET MODEL}

Since the material balance for the Basic Data Report (Revision 138) was issued in $1990,{ }^{20}$ several major revisions were made to the HLW processing operation bases and subsequently implemented into the CPES integrated HLW Flowsheet model. Most of those revisions reflected in the model were summarized in earlier material balances.9,16,21,22 In this section, only the recent revisions made since the Batch 1 sludge material balance was issued in 1993 are summarized. ${ }^{16}$

\subsection{Model Bases}

The primary objective for the CPES integrated HLW flowsheet model is to show the workable flowsheet that produces acceptable glass with the maximum possible waste loading at the design glass production rate of $228 \mathrm{lb} / \mathrm{hr}$. In order to achieve this objective, the CPES model calculates internally the maximum workoff rates of given sludge and salt feeds to ESP and ITP, respectively, that will result in a glass containing just the minimum amount of frit required. While doing so, the model must satisfy numerous process and operating constraints and, finally, it calculates the instantaneous flow rates for the entire HLW flowsheet which, if integrated over a cycle period, should closely represent actual batch sizes or throughputs per cycle of specific operations involved. Listed below are the key process and model variables whose values were fixed in this study to serve as the bases for the HLW flowsheet material balance:

- Instantaneous glass production rate is $228 \mathrm{lb} / \mathrm{hr}$, excluding $1 \mathrm{wt} \%$ glass entrainment into the melter off-gas.

- Sludge feed from ESP contain $15 \mathrm{wt} \%$ total solids.

- Minimum batch size for the sludge feed in SRAT is 4,000 gallons, excluding the 1,500 gallon heel.

- Cycle time for the CPC operation is 86 hours.

- Cycle time for the SPC operation is $1 / 2$ of that for CPC or 43 hours.

- Precipitate products from ITP and LWF contain $10 \mathrm{wt} \%$ insoluble solids.

- Salt workoff rate is to be maximized by maintaining the volume of PHA fed to SRAT in one cycle as closely at 7,900 gallons as possible.

One important basis change made in this revision was to fix the cycle time of CPC at 86 hours and then calculate the required sludge batch size, instead of fixing the transfer rate of sludge feed to SRAT at 5,000 gallons per batch and calculating the required cycle time. 16 The reason for this change was based on the fact that during the actual operation a lot of the cycle time will be spent on analyzing samples, so it would be much easier to control the feed batch size than the cycle time. 
It should be noted that if necessary, all of these bases can assume different values. For example, if the glass production rate is set at $91 \mathrm{lb} / \mathrm{hr}$, the instantaneous flow rates thus calculated can then be integrated to estimate the necessary batch sizes or throughputs to satisfy $40 \%$ DWPF attainment. When the total solids content in the sludge feed is set much higher than $15 \mathrm{wt} \%$, the volume of PHA required to blend with a given sludge batch usually turns out to be greater than 7,900 gallons which is the maximum working volume of PRBT. One way to overcome this difficulty would be to use a concentrated PHA. For a given sludge feed and a nominal PHA, however, the salt workoff is maximized when the volume of PHA added to SRAT per CPC cycle equals 7,900 gallons. The idea of maximizing the salt workoff rate is to minimize the possibility of having to produce glass made out of salts only at the end of sludge campaign.

One key basis missing from the list shown above is that of the glass product, i.e., to minimize the frit content in glass as well as to ensure the quality of the glass produced. This is achieved outside the CPES model by iteration with the PCCS, as explained next.

\subsection{Model Convergence Scheme}

In order to ensure that all the pertinent glass constraints are satisfied in the material balance calculations, the minimum frit content required in glass must be known for a given set of PHA and sludge feed compositions, and this critical information is provided by the PCCS. The overall convergence scheme used in a typical CPES model run can be summarized as follows:

1. If the simulation case to be run involves waste compositions different from those used in the previous run, all recycle streams are properly initialized.

2. Given the compositions of sludge and salt/supernate feeds to ESP and ITP, initial estimates are made for the following variables:

- ESP/ITP/LWF operating conditions such as sludge washing endpoint.

- frit loading (wt\%) in glass.

- sludge workoff rate.

- salt/supernate workoff rates.

3. The ESP, ITP, LWF and SPC modules are run, and the compositions as well as the instantaneous flow rates of PHA and sludge feeds to SRAT are calculated.

4. Model then checks if the difference between the total volume of PHA fed to SRAT in 86 hours and 7,900 gallons is within a specified tolerance. If not, new estimates for the salt/supernate workoff rates are found, and the calculations are repeated from Step \#2 as many times as necessary, until the difference becomes smaller than the tolerance. 
5. Once Step \#4 is completed, the calculations are continued by blending the maximum volume of PHA with washed sludge and the right amount of frit that will result in the same frit loading in glass as assumed in Step \#2, and the remaining DWPF module is run to make glass.

6. Model checks if the difference between the calculated glass production rate and the desired rate of $228 \mathrm{lb} / \mathrm{hr}$ is within a specified tolerance. If not, new estimates are found for the sludge and salt/supernate workoff rates, and the calculations are repeated from Step \#2 as many times as necessary, until the difference becomes smaller than the tolerance.

7. Once Step \#6 is completed, the CPES material balance calculations are converged. PCCS is next run outside CPES using the compositions and the volumes of PHA and sludge fed to SRAT in 86 hours.

8. If PCCS finds that the glass produced will not have an acceptable quality, then the CPES model is rerun from Step \#2 with a new set of operating conditions for ESP/ITP/LWF, new frit loading in glass and new sludge and salt/supernate workoff rates, and the entire calculations up to Step \#7 are repeated as many times as necessary, until PCCS finds that the glass will have an acceptable quality.

9. Once Step \#8 is completed, PCCS then checks if the acceptable glass thus produced contains the minimum amount of frit necessary to ensure quality. If not, the CPES model is again rerun from Step \#2 with a new frit loading in glass and new sludge and salt/supernate workoff rates, and the entire calculations up to Step \#8 are repeated as many times as necessary, until PCCS finds that the glass indeed contains the minimum necessary amount of frit.

10. Once Step \#9 is completed, a convergence between CPES and PCCS runs is achieved, and one simulation case is completed.

In typical simulation runs, the constraints on both glass quality and minimum frit requirement, i.e., Steps \#8 \& \#9, can be satisfied simultaneously. Furthermore, the convergence between CPES and PCCS is usually achieved within 3 overall iterations from Step \#2 to Step \#9. A calculation flowchart for a typical model simulation run is shown in Figure 1 in Appendix.

\subsection{Extended Sludge Processing}

The most significant revision made to the ESP module was to expand the scope of the CPES steady state model so it can manage tank heels. This new capability was tested successfully during the preliminary CPES runs made earlier to simulate the HLW flowsheet with sludge batches 1 through 6.23 The flow diagram for Section $1 \mathrm{C}$ in Table A-1 reflects this change. 
The algorithm used for the addition of corrosion inhibitors during the washing was also modified to meet the Technical Standards requirements shown in Table 11.24 In this study, the actual inhibitor additions were made at $120 \%$ of these minimum requirements. When the measured nitrate level falls below 1.0 molar, two options for adding inhibitors are given in Table 11. When this was the case, it was ensured that the option chosen always resulted in less total sodium in the tank than would with the other option.

TABLE 11. Corrosion Inhibitor Requirements for Waste Tank Farms (all concentrations in molar and temperature in deg. C)

\begin{tabular}{|c|c|c|c|}
\hline$\underset{\left[\mathrm{NO}_{3}\right]}{\text { Measured }}$ & $\underset{[\mathrm{OH}]}{\text { Minimum }}$ & $\begin{array}{c}\text { Minimum } \\
{[\mathrm{OH}]+\left[\mathrm{NO}_{2}\right]}\end{array}$ & $\underset{\left[\mathrm{NO}_{2}\right]}{\text { Minimum }}$ \\
\hline $5.5 \leq\left[\mathrm{NO}_{3}\right]<8.5$ & 0.6 & 1.1 & N/A \\
\hline $2.75 \leq\left[\mathrm{NO}_{3}\right]<5.5$ & 0.3 & 1.1 & N/A \\
\hline $1.0 \leq\left[\mathrm{NO}_{3}\right]<2.75$ & $0.1 *\left[\mathrm{NO}_{3}\right]$ & $0.4 *\left[\mathrm{NO}_{3}\right]$ & N/A \\
\hline $0.02 \leq\left[\mathrm{NO}_{3}\right]<1.0$ & 1.0 & $\begin{array}{l}\text { N/A } \\
\text { or }\end{array}$ & N/A \\
\hline & 0.0001 & N/A & $0.038 *\left[\mathrm{NO}_{3}\right] * 10^{0.041 \mathrm{~T}}$ \\
\hline \multirow[t]{2}{*}[\mathrm{NO}_{3}]{$<0.02$} & 1.0 & $\begin{array}{l}\mathrm{N} / \mathrm{A} \\
\text { or }\end{array}$ & $\mathrm{N} / \mathrm{A}$ \\
\hline & 0.0001 & $\mathrm{~N} / \mathrm{A}$ & $0.00076^{*} 10^{0.041 \mathrm{~T}}$ \\
\hline
\end{tabular}

\subsection{In-Tank Precipitation}

The capability of managing tank heels discussed above was also added to the ITP module, and successfully tested during recent CPES runs made to simulate the proposed ITP startup plans. ${ }^{25}$ As a result, it is now possible to properly model the impact of tank heels during successive batch operations using the CPES model. For example, when a new batch of precipitate slurry is transferred to Tank 49, the model can calculate the composition of the precipitate feed to the LWF.

Since the Late Wash design basis for the Cs- 137 content in Tank 49 was changed from 36 to $46 \mathrm{Ci} / \mathrm{gal}$, experiments have been conducted to evaluate the impact of increased dose rate on the radiolysis of precipitates during storage in Tank 49. From the available data, 27 correlations were developed during this study that can be used to predict the net production rates of organic species during the radiolytic reactions in Tank 49 as a function of total dose. Additional G-value correlations were also developed to predict the consumption rates of nitrate and nitrite. 27,28 The correlations thus developed and incorporated into the model include: 
- $\mathrm{G}\left(\mathrm{NO}_{3}\right)=0.3536 \exp (-0.00346 *$ Dose $)$, molecules $/ 100 \mathrm{eV}$

- $\mathrm{G}\left(\mathrm{NO}_{2}\right)=-0.1024+0.1084 \ln [\mathrm{Cs}-137]$, molecules $/ 100 \mathrm{eV}$

- $\mathrm{R}($ phenol $)=5.1 *$ Dose $-0.0035 *$ Dose $^{2}$, delta ppm

- $\mathrm{R}$ (biphenyl) $=10.14 *$ Dose $-0.0045 *$ Dose $^{2}$, delta ppm

- $\mathrm{R}$ (terphenyl) $=0.36 *$ Dose $+0.0009 * \mathrm{Dose}^{2}$, delta ppm

- $\mathrm{R}(\mathrm{PBA})=0.67 *$ Dose $+0.0014 *$ Dose $^{2}$, delta ppm

- $\mathrm{R}($ Oxalate $)=4.31 *$ Dose $-0.0036 * \operatorname{Dose}^{2}$, delta ppm

- $\mathrm{R}$ (ammonium) $=0.87 *$ Dose $-0.0005 * \mathrm{Dose}^{2}$, delta ppm

- $R$ (phenylphenol) $=130$, delta ppm

- $\mathrm{R}$ (diphenylamine) $=20$, delta ppm

Note that in above correlations, all Dose's are in Mrads, and the concentration of Cs-137 is in Ci/gal. The net production rates given in ppm are based on the total slurry in Tank 49.

These correlations were then used in the model along with a total of 25 radiolytic reactions to describe the Tank 49 chemistry. The conversions for some of these reactions such as radiolytic dissociation of water were calculated iteratively by the model to satisfy the following as well as the desired organic product distributions:

- Total production of $\mathrm{H}_{2}$ based on a G-value for 0.15 .30

- Projected $\mathrm{pH}$ of precipitate feed to the LWF $=11.31$

- Final equilibrium concentration of nitrate $=0.0025$ molar. ${ }^{28}$

In earlier studies,, 916 the calculated $\mathrm{pH}$ of precipitate feed to the LWF was much higher than the projected values of 10-11. In the revised radiolysis chemistry, the lowering of $\mathrm{pH}$ to 11 was achieved by:

- assuming $100 \%$ absorption of $\mathrm{CO}_{2}$ in purge air into Tank 49 slurry.

- allowing radiolysis products such as phenol to react with hydroxide.

It was assumed earlier that ammonium ions were produced at a rate of $460 \mathrm{ppm}$ in two years from the radiolysis of TPB precipitates in Tank 49, and no ammonia was released into the atmosphere. ${ }^{16}$ Based on a recent study, 26 however, it was assumed in this study that: 
- $99.5 \%$ of ammonium formed escapes into the vapor space at an average $\mathrm{pH}$ of 12-13 in the liquid phase of Tank 49.

The concentration of $\mathrm{CO}_{2}$ in purge air was assumed to be 361 ppm. ${ }^{32}$ Compared to $100 \% \mathrm{CO}_{2}$ absorption assumed in this study, earlier data showed that on the average about $77 \%$ of $\mathrm{CO}_{2}$ in purge air entering several waste tanks in H-Area, including Tank 49, was absorbed before exiting those tanks. ${ }^{32}$ During the process of bringing down the $\mathrm{pH}$, a new compound, $\mathrm{K}_{2} \mathrm{CO}_{3}$, was added to the database as the 183rd species.

Finally, as will be shown later in this report, the rate at which the K/CsTPB solids are radiolyzed and go into solution was calculated iteratively. by the model based on the actual Cs-137 content, and the converged solution must satisfy all the process bases stated above.

\subsection{Late Wash Facility}

In support of updating the LWF technical bases, the CPES model was run earlier using the following bases that are also reflected in this study: 33

- Precipitate is stored for 2 years in Tank 49 at the Cs-137 level expected in the actual precipitate, instead of using the LWF design basis of $46 \mathrm{Ci} / \mathrm{gal}$.

- Cycle time for the LWF is 91 hours, assuming that the crossflow filter needs to be cleaned after every cycle.

(With no filter cleaning, cycle time is 60.5 hours.)

- Following each transfer from Tank 49 to the LWPT, the precipitate transfer line is flushed with 300 gallons of water.

- Operating heels in both the LWPT and the LWHT are 650 gallons each.

- The amount of NaTPB slurry to be added is the sum of the stoichiometric amount required to precipitate soluble $\mathrm{K}^{+}, \mathrm{NH}_{4}{ }^{+}$and $\mathrm{Cs}^{+}$plus an excess to maintain the TPB- concentration in the aqueous fraction of the total slurry in the LWPT at $0.004 \mathrm{M}$.

- The DF for Cs is determined by the following solubility product: 34

$\left[\mathrm{Cs}^{+}\right]\left[\mathrm{TPB}^{-}\right]=(0.05+0.014 \mathrm{~T})^{2}\left(10^{-8}\right) \exp \left(-1.34\left[\mathrm{Na}^{+}\right]\right)$,

where $\mathrm{T}=$ slurry temperature in ${ }^{\circ} \mathrm{C}$, and $\left[\mathrm{Na}^{+}\right]=$molar concentration.

- The solubility product of KTPB is calculated by: 34

$\left[\mathrm{K}^{+}\right][\mathrm{TPB}-]=4.87^{2}(0.05+0.014 \mathrm{~T})^{2}\left(10^{-8}\right) \exp \left(-1.34\left[\mathrm{Na}^{+}\right]\right)$ 
- The insoluble solids are concentrated to $10 \mathrm{wt} \%$ before initiating washing.

- Wash water contains $0.004 \mathrm{M} \mathrm{NaTPB}$ and $0.005 \mathrm{M} \mathrm{NaOH}$.

- Washing endpoint is $0.01 \mathrm{M}$ nitrite in the aqueous fraction.

- Total volume of flush water to be added during one filter cleaning cycle is 1,800 gallons. Of this 1,800 gallons, 775 gallons are returned to LWPT, while the remaining 1,025 gallons are returned to LWHT.

- Total volumes of $2 \mathrm{wt} \%$ caustic and $2 \mathrm{wt} \%$ oxalic acid to be added during one filter cleaning cycle are 650 and 450 gallons, respectively, and all of the spent cleaning solutions are returned to the LWHT.

- Hydroxide content in spent wash, spent flush and spent cleaning solution collected in the LWHT is adjusted to $1.2 \mathrm{M}$ by adding $50 \mathrm{wt} \% \mathrm{NaOH}$ before transferring it to Tank 22 .

- Benzene content in spent wash is stripped into the vessel vent system until the residual benzene content is $4 \mathrm{ppm}$. The benzene content in the aqueous fraction of slurry batch from Tank 49 is projected to be $700 \mathrm{mg} / \mathrm{L}$.

- The insoluble solids content in final precipitate slurry transferred to DWPF is $10 \mathrm{wt} \%$.

\subsection{Hydrogen and Ammonia Source Terms in CPC}

The chemistry used in the model to describe the generation of $\mathrm{H}_{2}$ and $\mathrm{NH}_{3}$ during the SRAT cycle was revised to include the following reactions:

$$
\begin{aligned}
& \mathrm{NH}_{4} \mathrm{COOH} \rightarrow \mathrm{NH}_{3}+\mathrm{HCOOH} \\
& 4 \mathrm{NO}+3 \mathrm{O}_{2}+2 \mathrm{H}_{2} \mathrm{O} \rightarrow 4 \mathrm{HNO}_{3} \\
& \mathrm{HCOOH} \rightarrow \mathrm{H}_{2}+\mathrm{CO}_{2} \\
& \mathrm{NaCOOH}+\mathrm{H}_{2} \mathrm{O} \rightarrow \mathrm{NaOH}+\mathrm{H}_{2}+\mathrm{CO}_{2} \\
& \mathrm{KCOOH}+\mathrm{H}_{2} \mathrm{O} \rightarrow \mathrm{KOH}+\mathrm{H}_{2}+\mathrm{CO}_{2} \\
& \mathrm{Fe}_{2} \mathrm{O}_{3}+\mathrm{H}_{2} \mathrm{O}(\text { hyd })+\mathrm{H}_{2}-\rightarrow 2 \mathrm{FeO}+2 \mathrm{H}_{2} \mathrm{O} \\
& \mathrm{NaNO}_{3}+4 \mathrm{H}_{2} \rightarrow \mathrm{NH}_{3}+\mathrm{NaOH}+2 \mathrm{H}_{2} \mathrm{O} \\
& 2 \mathrm{NaOH}+\mathrm{CO}_{2} \rightarrow \mathrm{Na}_{2} \mathrm{CO}_{3}+\mathrm{H}_{2} \mathrm{O} \\
& 2 \mathrm{KOH}+\mathrm{CO}_{2} \rightarrow \mathrm{K}_{2} \mathrm{CO}_{3}+\mathrm{H}_{2} \mathrm{O}
\end{aligned}
$$




$$
\begin{aligned}
& \mathrm{Mn}(\mathrm{COOH})_{2}+2 \mathrm{NaOH} \rightarrow \mathrm{MnO}+\mathrm{H}_{2} \mathrm{O}(\text { hyd })+2 \mathrm{NaCOOH} \\
& \mathrm{Mg}(\mathrm{COOH})_{2}+2 \mathrm{KOH} \rightarrow-\mathrm{MgO}+\mathrm{H}_{2} \mathrm{O}(\text { hyd })+2 \mathrm{KCOOH}
\end{aligned}
$$

These reactions were allowed to take place in the order written, after all the acid/ base and redox reactions were completed. The conversions for these reactions were calculated within the model so that the following bases were satisfied:

- Despite the fact that the projected noble metal content in the Tank 51 only sludge feed is lower than that in the IDMS PX6 feed at least by an order of magnitude, the total production rate of $\mathrm{H}_{2}$ during one SRAT cycle was set at $2.67 \mathrm{lb}$ which was obtained by scaling up the rates measured during the IDMS PX6 run. ${ }^{35}$ This rate is $33 \%$ lower than the design basis of $41 \mathrm{~b} .16,42$

- As in earlier run, ${ }^{16} 75 \%$ of the total $\mathrm{H}_{2}$ produced or $2 \mathrm{lb}$ was released during the SRAT cycle, and the remaining $25 \%$ or $0.67 \mathrm{lb}$ was released during the SME cycle.

- Regardless of whether the PHA contains ammonium ion or not, the total production rate of $\mathrm{NH}_{3}$ during one SRAT cycle was set at $1.74 \times 10^{-4}$ lbmole per gallon of PHA added or about $23 \mathrm{lb}$ for a nominal sludge feed containing $10 \mathrm{wt} \% \mathrm{Na} .{ }^{35}$

- $60 \%$ of $\mathrm{NH}_{3}$ thus produced was released during the SRAT cycle, while the remaining $40 \%$ stayed in the slurry as aqueous $\mathrm{NH}_{3}$ until its release during the SME cycle.

- After completion of a SRAT cycle, the $\mathrm{pH}$ of the product slurry was to be 7 .

- The molar ratio of $\mathrm{CO}_{2}$ to $\mathrm{H}_{2}$ produced during one SRAT cycle was to be between 5 and 10 .

- The net production of nitric oxide after one SRAT cycle was set at $50 \%$ of nitrite contained in the sludge feed on a molar basis.

- The molar ratio of $\mathrm{NO}$ to $\mathrm{NO}_{3}$ produced from nitrite was to be about 1 .

- The conversion for Reaction \#6 was set to give the Fe(II) / Fe(total) molar ratio of 0.1 .

The model database also includes the following reactions to generate nitric oxide:

$$
3 \mathrm{NaNO}_{2}+2 \mathrm{HNO}_{3}->3 \mathrm{NaNO}_{3}+2 \mathrm{NO}+\mathrm{H}_{2} \mathrm{O}
$$$$
3 \mathrm{NaNO}_{2}+2 \mathrm{HCOOH}-\rightarrow \mathrm{NaNO}_{3}+2 \mathrm{NaCOOH}+2 \mathrm{NO}+\mathrm{H}_{2} \mathrm{O}
$$

$$
2 \mathrm{NaNO}_{2}+3 \mathrm{HCOOH} \rightarrow 2 \mathrm{NaCOOH}+2 \mathrm{NO}+\mathrm{CO}_{2}+\mathrm{H}_{2} \mathrm{O}
$$


In order to satisfy the nitric acid addition algorithm used for the nitrite, 36 it turned out that the combined conversion for Reactions \#12 and 13 should be $90 \%$, while that for Reaction \#14 should be $10 \%$, all with respect to nitrite. The last two bases stated above set the conversion for Reaction $\# 2$ at $29 \%$. Nitric acid formed by Reaction \#2 was then used to substitute portions of formic acid in the PHA during the sludge treatment step. The excess formic acid thus formed is next decomposed to produce hydrogen by Reaction \#3.

\subsection{Ammonia Scrubbers}

Three ammonia scrubbers were added to the CPC module earlier; 16 one each downstream of the SRAT condenser and the SME condenser, and one to treat the combined vent from the MFT and the RCT. Listed below are the bases used during this study to model the overall scrubbing efficiency:

- Based on recent scrubber test data obtained during the IDMS PX7 SRAT run, 37 the ammonia concentration at the exit of each scrubber was fixed at $1 \mathrm{ppm}$ by volume. The design basis exit concentration used earlier was $10 \mathrm{ppm} .16$ Ammonia is scrubbed as $\mathrm{NH}_{4} \mathrm{NO}_{3}(\mathrm{~s})$.

- 90 to $95 \%$ of ammonia vented to the SRAT condenser is scrubbed out as $\mathrm{NH}_{4} \mathrm{OH} .38$ The remaining 5 to $10 \%$ ammonia enters the scrubber.

- The design peak generation rates of ammonia are $1.2 \mathrm{lb} / \mathrm{hr}$ in the SRAT, $2.5 \mathrm{lb} / \mathrm{hr}$ in the SME, and $0.655 \mathrm{lb} / \mathrm{hr}$ in the MFT/RCT. The liquid-to-gas mass ratios in each scrubber were fixed at 6.02 for the SRAT, 12.22 for the SME, and 6.97 for the MFT/RCT scrubbers. ${ }^{39}$

- The spent scrubbing solution collected in the SMECT is adjusted to $\mathrm{pH}=2$ by adding $50 \mathrm{wt} \%$ nitric acid.

\subsection{Inhibitor Addition to Liquid Recycle/ $/ \mathrm{NH}_{3}$ Release}

The corrosion inhibitor level and the maximum temperature allowed in the DWPF liquid waste recycle prior to transfer to Tank 43 were set as follows:

- $50 \mathrm{wt} \% \mathrm{NaOH}$ was added to the RCT to maintain the hydroxide level in the liquid recycle at 0.6 molar. 40

- $40 \mathrm{wt} \% \mathrm{NaNO2}$ was added to the RCT to maintain the nitrite level in the liquid recycle at 0.045 molar. 40

- The temperature of the liquid recycle stream was set at $50^{\circ} \mathrm{C}$, below the Process Requirement maximum limit of $55^{\circ} \mathrm{C} .41$ 
- The rate of release of aqueous $\mathrm{NH}_{3}$ from the $\mathrm{RCT}$ was determined by the following equation: 43

$$
\mathrm{R}_{\mathrm{NH} 3(\mathrm{~g})}=0.15896\left[\mathrm{NH}_{3}\right]_{\mathrm{aq}} \exp (0.04834 \mathrm{~T}),
$$

where $\mathrm{R}_{\mathrm{NH}}(\mathrm{g})$ is the release rate in $\mathrm{lb} / \mathrm{hr},\left[\mathrm{NH}_{3}\right]_{\mathrm{aq}}$ the sum of $\mathrm{NH}_{3}(\mathrm{aq})$ and $\mathrm{NH}_{4}$ in molar in the liquid phase, and $\mathrm{T}$ in ${ }^{\circ} \mathrm{C}$.

- Release of aqueous $\mathrm{NH}_{3}$ from Tank 43 was assumed to proceed according to the vapor-liquid equilibrium. The ventilation rate for Tank 43 was set at $300 \mathrm{cfm}$ of air.44

\subsection{Miscellaneous Revisions}

- Based on recent data from the $1 / 200$ th scale SRAT runs, ${ }^{45}$ any excess $\mathrm{Hg}$ that was steam stripped during the SRAT boilup was allowed to dissolve into the condensate up to $100 \mathrm{ppm}$. The current basis for determining the amount of strippable $\mathrm{Hg}$ is that in excess of $0.0045 \mathrm{lb}$ per $\mathrm{lb}$ sludge solids.

- No Hg was allowed to be steam stripped during the SME boilup.

- Despite high acidity in the SMECT, i.e. $\mathrm{pH}=2$, no $\mathrm{Hg}$ was allowed to dissolve and be recirculated to the ammonia scrubbers or sent to the RCT.

- $\mathrm{Cu}(\mathrm{COOH})_{2}$ was replaced with $\mathrm{Cu}\left(\mathrm{NO}_{3}\right)_{2}$ as the new catalyst for the precipitate hydrolysis in the SPC.

\section{SIMULATION RESULTS AND CONCLUSIONS}

Some of the key simulation results from this study were highlighted earlier in SUMMARY. The major conclusions of this study include:

- The quality of glass produced from the Tank 51 sludge and the radioactive precipitate produced during the first ITP cycle can be ensured by removing $90 \%$ of the insoluble solids present in the Tank 41 saltcake.

- Assuming that the sludge feed to the SRAT is diluted from 21 to $15 \mathrm{wt} \%$ total solids, the optimum washing endpoint for the Tank 51 sludge appears to be about $8 \mathrm{wt} \%$ total $\mathrm{Na}$ on a dry basis.

- The total duration that ESP can support 100\% DWPF attainment with the Tank 51 sludge is expected to be 1.4 years, excluding the 88,000 gallon heel, and the total number of glass canisters produced would be at least 747 .

- Based on current operating modes for the SPC and the CPC in DWPF, the maximum PHA loading in glass is estimated to be about $6.0 \mathrm{wt} \%$. 
- ITP is expected to produce 155,000 gallons of $10 \mathrm{wt} \%$ precipitate during its first cycle, and it would support $100 \%$ DWPF attainment for 4 months.

- The Cs-137 content in the precipitate produced during the first ITP cycle is projected to be about $9.2 \mathrm{Ci} / \mathrm{gal}$. At this dose rate, the model predicted that about $5 \%$ of the TPB precipitate would be decomposed and become soluble during a 2-year storage in Tank 49. As a comparison, up to $19 \%$ of TPB precipitate is expected to be decomposed at the LWF design basis Cs-137 level of $46 \mathrm{Ci} / \mathrm{gal}$.

- At $9.2 \mathrm{Ci} / \mathrm{gal}$, the final concentration of nitrite after a 2-year storage is expected to be $0.26 \mathrm{molar}$, compared to $0.16 \mathrm{molar}$ at $46 \mathrm{Ci} / \mathrm{gal}$. As a result, at $9.2 \mathrm{Ci} / \mathrm{gal}$, about $20 \%$ more wash water will be required in the LWF to reach the final nitrite concentration of 0.01 molar than at $46 \mathrm{Ci} / \mathrm{gal}$.

- The Saltstone Facility is expected to produce $687,000 \mathrm{ft}^{3}$ of grout from the decontaminated supernate generated during the first ITP cycle.

- Due to low Hg content in the Tank 51 sludge, there was no net $\mathrm{Hg}$ that was steam stripped during the SRAT boilup and dissolved into the condensate.

The results of the ESP washing optimization are given in Table 12. The calculated compositions of radiolyzed precipitate slurry, washed sludge and glass are given in Tables 13, 14 and 15, respectively, for sludge feed containing $10 \mathrm{wt} \% \mathrm{Na}$. A full output of the CPES HLW flowsheet material balance is given in Table A-1.

\section{TABLE 12. Results of Optimization of Tank 51 Sludge Washing}

\begin{tabular}{|c|c|c|c|c|c|}
\hline \multicolumn{2}{|c|}{ Washed Sludge@ 21wt\% } & \multirow{2}{*}{$\begin{array}{c}\text { Wash } \\
\text { Water (kgal) }\end{array}$} & \multirow{2}{*}{$\begin{array}{l}\text { Duration * } \\
\text { (hr) }\end{array}$} & \multirow{2}{*}{$\begin{array}{c}\text { Sludge Loading } \\
\text { in Glass (wt\%) }\end{array}$} & \multirow{2}{*}{$\begin{array}{c}\# \\
\text { Glass Cans }\end{array}$} \\
\hline wit\% Na (dry) & Volume (kgal) & & & & \\
\hline 10.7 & 492 & 1,200 & 12,325 & 25.6 & 760 \\
\hline 10.0 & 492 & 1,350 & 12,270 & 25.3 & 756 \\
\hline 8.1 & 492 & 1,500 & 12,121 & 24.6 & 747 \\
\hline
\end{tabular}

* at $100 \%$ DWPF attainment, excluding the 88,000 gallon heel in Tank 51 . 


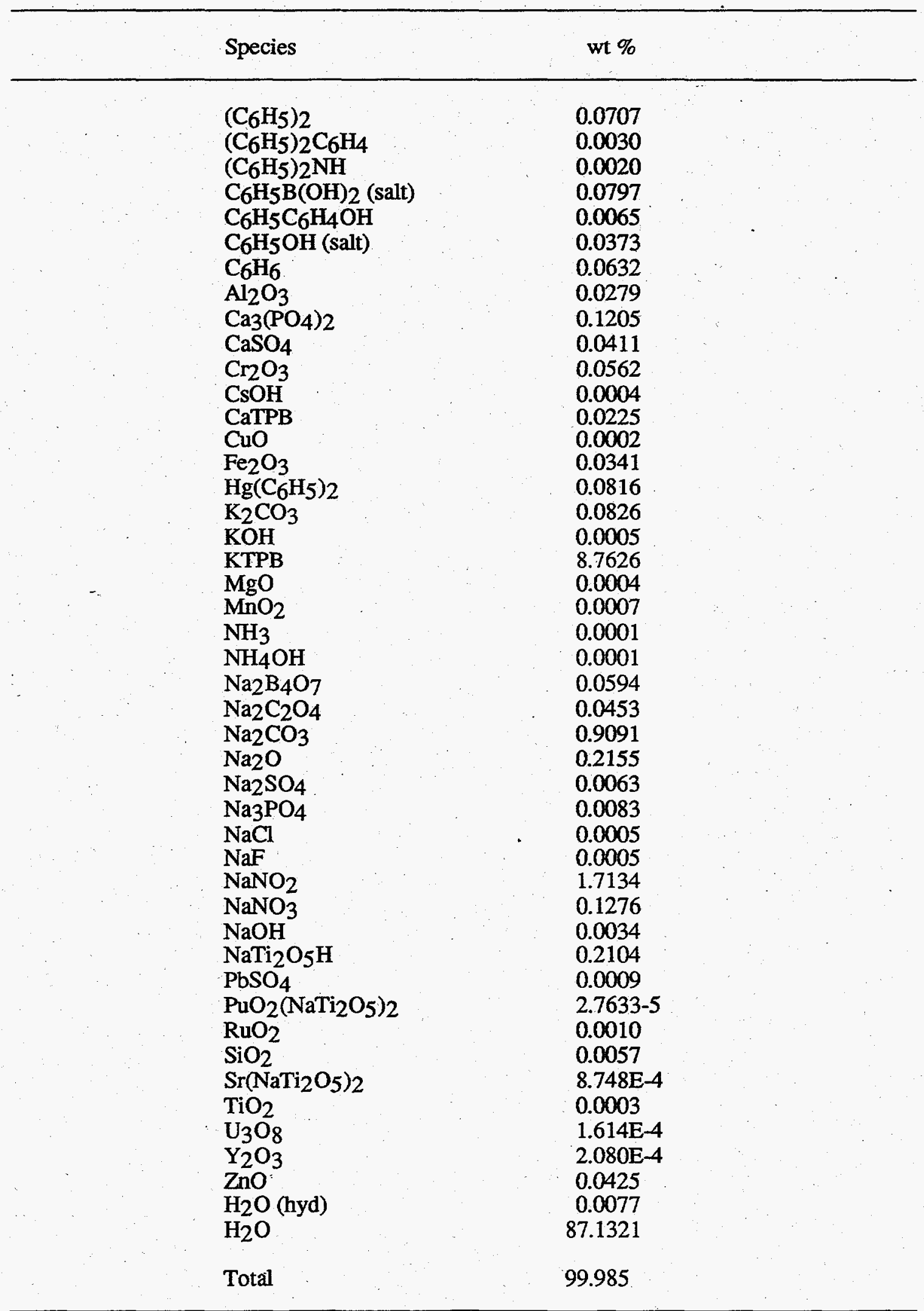


TABLE 14. Calculated Composition of Washed Tank 51 Sludge Feed

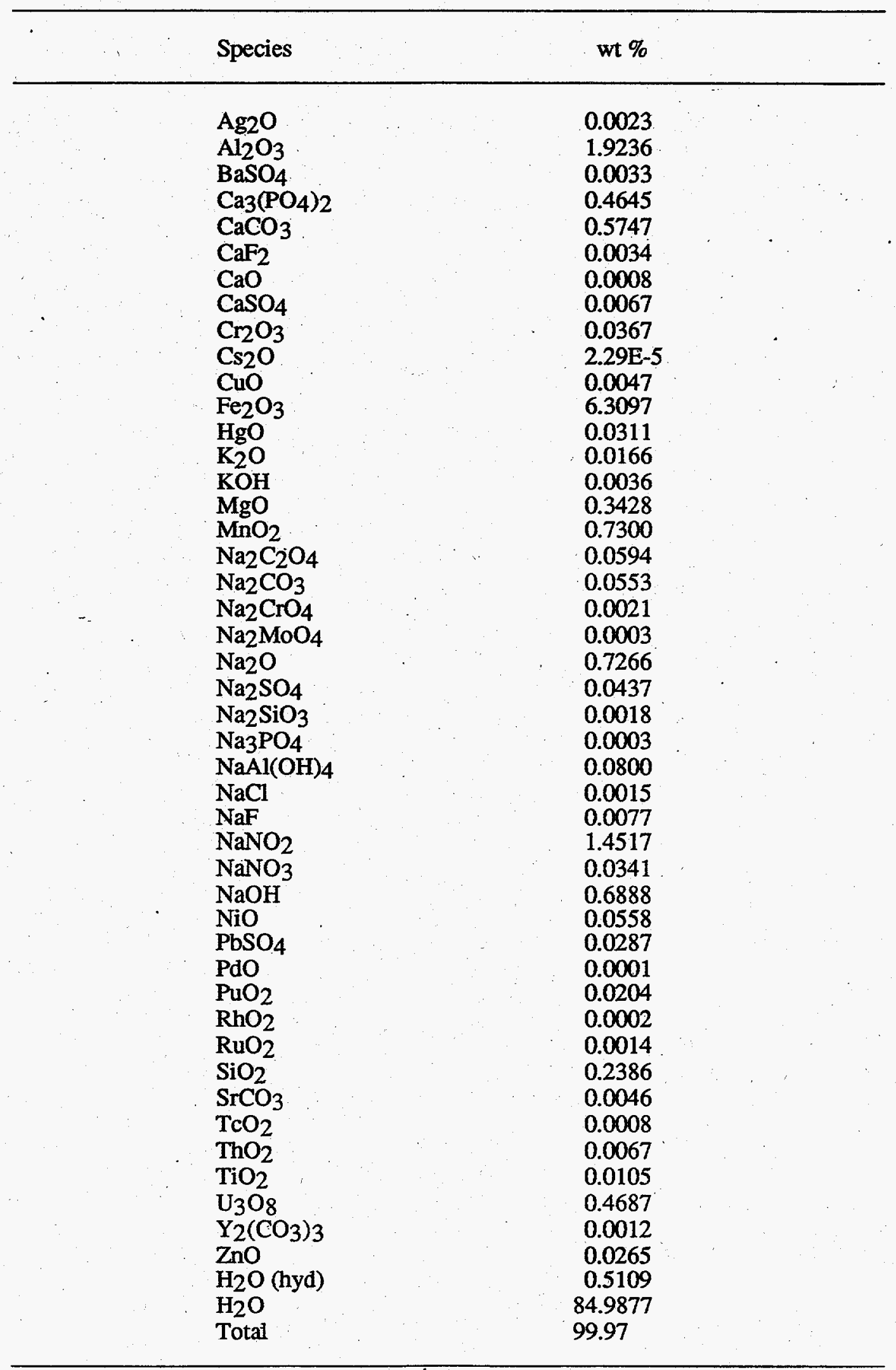


C. T. Randall

TABLE 15. Calculated Composition of Glass Made from Tank 51 Sludge and ITP Cycle 1 Precipitate

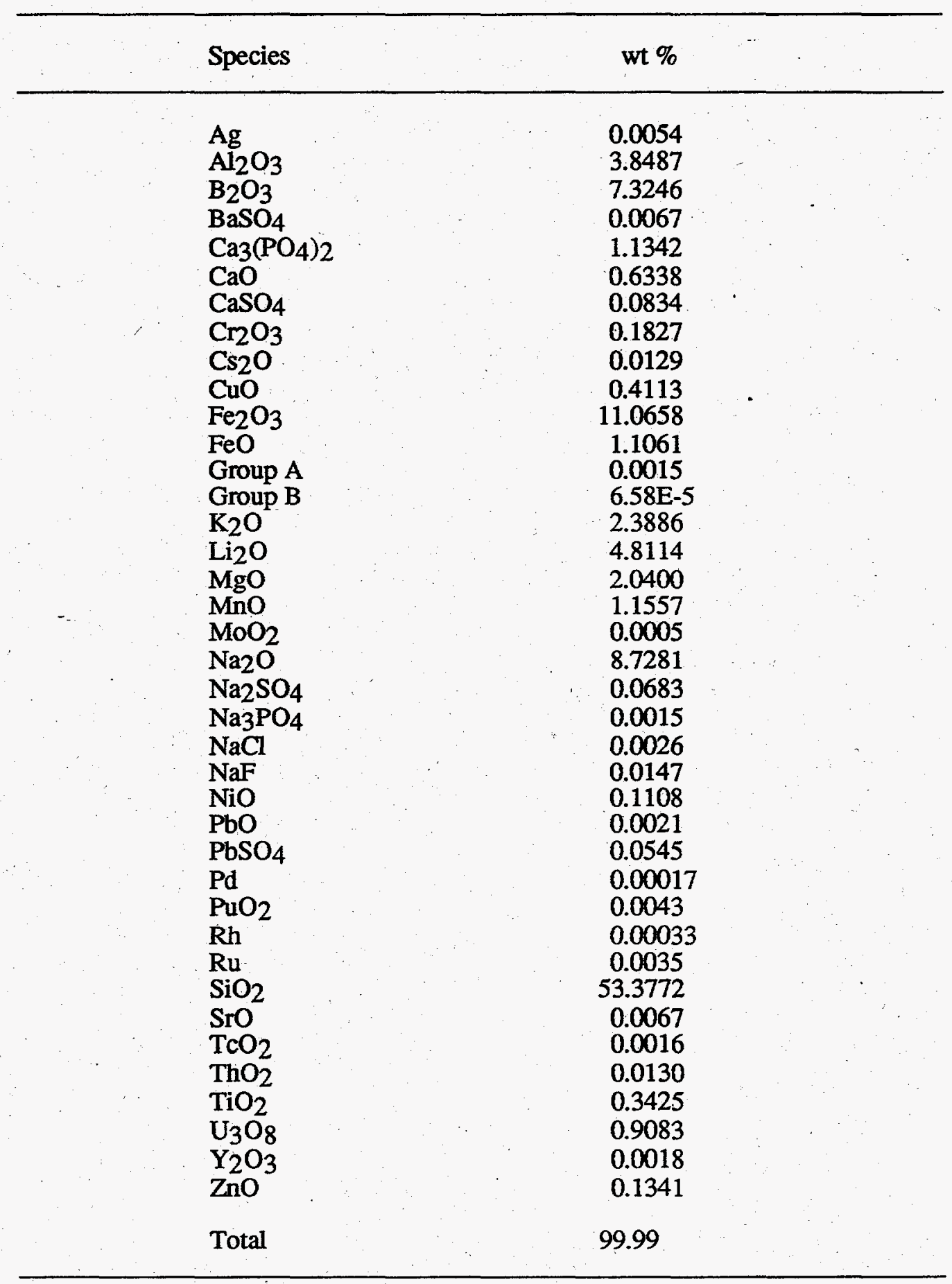




\section{QUALITY ASSURANCE}

The validity of the bases and assumptions made in this study as well as the results of the material balance calculations were technically reviewed by N. E. Bibler and R. A. Jacobs of SRTC-DWPT. Additional review and comments were also made by the following HLWM \& SRTC experts: N. R. Davis, H. H. Elder, J. T. Carter, W. D. Kimball, G. K. Georgeton, E. D. Lee, M. J. Plodinec, L. F. Landon, D. Lambert, M. S. Hay and D. D. Walker.

The minimum frit contents in glass used in this study were all determined from the PCCS Version 3 runs. A typical PCCS output is shown in Figure 2 in Appendix. Recently, the accuracy of the PCCS Version 3 was tested extensively; 46 the entire PCCS code originally written in LISP was rewritten in FORTRAN, and a test case was run to compare the results from the two different compiler versions.

\section{ACKNOWLEDGMENT}

Many of the bases and assumptions used in this study are all based on the results. of experimental and theoretical investigations made by many researchers at SRS who willingly cooperated in implementing their findings into the CPES model. Special thanks are made to N. E. Bibler and M. S. Hay for providing analytical data for sludge samples, to G. K. Georgeton and D. T. Hobbs for providing data for salt/supernate samples, to D. D. Walker for providing insight as well as data for precipitate radiolysis, to $\mathrm{P}$. Monson for providing analytical data for irradiated samples, to C. A. Nash and L. F. Landon for providing the design bases for LWF, to L. M. Lee for providing his calculation results on $\mathrm{NH}_{3}$ release from Tank 49, to M. A. Baich, R. E. Eibling, C. W. Hsu, N. D. Hutson, D. Lambert, J. C. Marek and J. R. Zamecnik for helping update the bases and chemistry for the DWPF processes, and to F. G. Smith, III, and K. G. Brown for performing the PCCS analysis. Valuable comments made by all reviewers whose names were mentioned above are also greatly appreciated. 
10. REFERENCES

1. Lee, E. D., "First Sludge to DWPF," HLW-ITP-950058, January 17, 1995.

2. Technical Assistance Request, HLW/DWPF/TAR-950016, January 17, 1995.

3. Choi, A. S., Fowler, J. R., Edwards, R. E. Jr., and Randall, C. T., "High Level Nuclear Waste Treatment in the Defense Waste Processing Facility Overview and Integrated Flowsheet Model (U)," WSRC-MS-91-401, October 21, 1991.

4. Chemical Process Evaluation System Version 6.3, Engineering Services Division, E. I. du Pont de Nemours, Inc. (August 1988).

5. Hay, M. S., "Estimated Batch 1 Sludge Insoluble Composition Based on the Analysis of ESP Baselining Test Samples from Tank 51 (U)," SRT-LWP-94-086, June 15, 1994.

6. Hay, M. S., "Modeling of Batch 1 Sludge Washing Operations," SRT-LWP-94-088, June 17, 1994.

7. Coleman, C. J., Bibler, N. E., and Dewberry, R. A., "Analyses of High Level Radioactive Glasses and Sludges at the Savannah River Site," Symp. Proc. Waste Management, pp 651-657, Tucson, AZ, February 1990.

8. Bibler, N. E., "Tank 42 Slurry Composition for Batch One CPES Calculations," SRT-GTC-93-0039, May 21, 1993.

9. Choi, A. S., "Material Balance Tables for the DWPF Radioactive Runs with Batch One Sludge/Supernate Feed (U)," WSRC-TR-92-211 (Revision 1), November 9, 1992.

10. HLW System Plan Revision 4, HLW-OVP-94-0145, Westinghouse Savannah River Company, November 30, 1994.

11. Hobbs, D. T., and Coleman, C. J., "Final Report: Analysis of Tank $41 \mathrm{H}$ Saltcake Sample \#2 and Comparison to Sample \#1 (U)," WSRC-TR-94-057, January 26, 1995.

12. Georgeton, G. K., and Pate, T. E., "Recommendations for Alternative ITP Feed (U)," WER-CMD-93-0702, March 17, 1993.

13. Pike, J. A., "Tank Chemistry Report, May 1993 (U)," WER-HLE-930230, June 28, 1993.

14. Georgeton, G. K., Electronic Mail, October 12, 1994.

15. Fowler, J. R., "Composition of H Area and SRP Soluble High-Level Waste," DPST-82-502, April 28, 1982. 
16. Choi, A. S., "Material Balance Tables for the DWPF Coupled Feed Flowsheet with Batch One Sludge (U)," WSRC-TR-93-203 (Revision 0), August 19, 1993.

17. Brown, D. F., "Periodic Update - F/H ETF Waste Concentrate Analysis Results," NMP-WMT-910014, January 7, 1991.

18. Smith, W. P., Memo to A. W. Wiggins on Waste Concentrate Analysis, Document Number and Date not available.

19. Brown, D. F., Unpublished Results from ETF Flowsheet Model Run, July 30, 1991.

20. Choi, A. S., and Fowler, J. R., "Material Balance Tables for the DWPF Basic Data Report (DPSP-80-1033, Revision 138) (U)," WSRC-TR-90-93, February 28, 1990.

21. Choi, A. S., "Preliminary Material Balance Tables for the DWPF Late Wash Flowsheet (U)," WSRC-TR-92-211 (Revision 0), June 15, 1992.

22. Choi, A. S., "Material Balance Tables for the Integrated DWPF Cold Chemical Runs - Test 1 (U)," WRSC-TR-90-408 (Revision 1), October 17, 1992.

23. Choi, A. S., Unpublished Results from CPES Runs made in May 1994.

24. Technical Standards, Waste Tank Farms, DPSTS-241-5.01, November 1988.

25. Choi, A. S., Unpublished Results from CPES Runs made in October 1994.

26. Lee, L. M., "Fate of Ammonia in Tank 49 and Late Washing Facility (U)," WSRC-RP-95-453, April 5, 1995.

27. Monson, P. R., and Eibling, R. E., "Composition of Irradiated KTPB Simulant (U)," SRTC-PTD-94-0084 (Draft), September 20, 1994.

28. Walker, D. D., and Bibler, N. E., "Radiolytic Reactions of Nitrate and Nitrite Solutions for the Coupled In-Tank Precipitation and Late Wash Process (U)," WSRC-RP-94-798, August 19, 1994.

29. Walker, D. D., "Benzene Distribution in Product Streams from In-Tank Processing," DPST-86-390, January 15, 1987,

30. Walker, D. D., "Radiolysis of Tetraphenyl Borates - University of Florida Trip Report," DPST-84-827, October 18, 1984.

31. Walker, D. D., "Comments on Late Wash Material Balance," DON'T SAY IT - WRITE IT, July 8, 1994. 
32. Hobbs, D. T., "Absorption of Carbon Dioxide in Waste Tanks," DPST-87-596, September 3, 1987.

33. Technical Bases - DWPF Late Washing Facility, WSRC-RP-92-793 (Revision 4), July 11, 1994.

34. Walker, D. D., "Calculation of Cesium and Potassium Concentration in Late Wash Filtrate," DON'T SAY IT - WRITE IT, June 10, 1994.

35. Hutson, N. D., and Zamecnik, J. R., "Integrated DWPF Melter System (IDMS) Campaign Report - IDMS Purex-6 (PX6) Operation (U)," WSRC-TR-94-0556 (Rev, 0), November 15, 1994.

36. Hsu, C. W., "Defense Waste Processing Facility Nitric Acid Requirement for Treating Sludge (U)," WSRC-RP-92-1056, September 4, 1992.

37. Lambert, D. P., "Ammonia Scrubber Testing During IDMS SRAT Processing (U)," WSRC-TR-94-8000 (Rev. 0), December 21, 1994.

38. Zamecnik, J. R., "Ammonia and Hydrogen Generation in the CPCSummary," SRTC-LSE-93-DRAFT, February 8, 1993.

39. Cartèr, J. T., Lambert, D. P., and Choi, A. S., "Revised Basis and Calculation Methods for the DWPF Ammonia Scrubbers (U)," WSRC-TR-92-296 June 20, 1992.

40. Zapp, P. E., "Final Report on Nitrite Inhibitor Concentrations for Washed Precipitate at Elevated Temperatures (U)," WSRC-TR-93-198, April 13, 1993.

41. Marra, J. E., "Process Requirements - Materials of Construction," WSRC-IM-91-2, November 1, 1992.

42. Ritter, J. A., Unpublished IDMS Calculations, March 1993.

43. Zamecnik, J. R., Unpublished Calculations, June 1993.

44. Georgeton, G. K., Electronic Mail, March 3, 1993.

45. Baich, M. A., Unpublished Results-1/200th Scale SRAT Runs, October 1994.

46. Brown, K. G., "DWPF PCCS Version 3.0 Test Case (U),". WSRC-RP-94-1332 (Rev. 1), December 17, 1994.

47. Plodinec, M. J., Personal Communication, February 13, 1995. 
This page intentionally left blank. 
C. T. Randall 
C. T. Randall

WSRC-TR-95-0019

(Revision 0)

This page intentionally left blank. 


\section{TABLE 1. Partitioned and Components Assumed Soluble in the HLW Flowsheet Model}

- Components that partition between solubles and insolubles in a stream

Group A, Group B, (NaNO3, NaOH, Na3PO4, Na2SO4, etc.) ${ }^{* *}$

- Components assumed always Soluble:

- Organics: C6H5B(OH)2 (salt), C6H5B $(\mathrm{OH}) 2, \mathrm{C} 6 \mathrm{H} 5 \mathrm{OH}$ (salt), C6H5OH, C6H6, CH3OH, (CH3)2CHOH, C6H5NH2, C6H5NO2, NaB(C6H5)4*

- Hydroxides: $\mathrm{Ba}(\mathrm{OH}) 2, \mathrm{Ca}(\mathrm{OH}) 2, \mathrm{CsOH}, \mathrm{KOH}, \mathrm{NH} 4 \mathrm{OH}, \mathrm{Sr}(\mathrm{OH}) 2, \mathrm{UO} 2(\mathrm{OH}) 2, \mathrm{Y}(\mathrm{OH}) 3$

- Nitrates: $\quad$ AgNO3, KNO3, $\mathrm{Hg}(\mathrm{NO} 3) 2, \mathrm{Pd}(\mathrm{NO} 3) 2, \mathrm{NH} 3 \mathrm{OHNO} 3, \mathrm{Ca}(\mathrm{NO} 3) 2$, $\mathrm{Co}(\mathrm{NO} 3) 2, \mathrm{CsNO} 3, \mathrm{Cu}(\mathrm{NO} 3) 2, \mathrm{Mg}(\mathrm{NO} 3) 2, \mathrm{Mn}(\mathrm{NO} 3) 2, \mathrm{NH} 4 \mathrm{NO} 3$, $\mathrm{Ni}(\mathrm{NO} 3) 2, \mathrm{~Pb}(\mathrm{NO} 3) 2, \mathrm{Sr}(\mathrm{NO} 3) 2, \mathrm{UO} 2(\mathrm{NO} 3) 2, \mathrm{Y}(\mathrm{NO} 3) 2, \mathrm{Zn}(\mathrm{NO} 3) 2$

- Formates: $\quad \mathrm{CsCOOH}, \mathrm{KCOOH}, \mathrm{NaCOOH}, \mathrm{NH} 4 \mathrm{COOH}, \mathrm{Ca}(\mathrm{COOH}) 2, \mathrm{Co}(\mathrm{COOH}) 2$, $\mathrm{Cu}(\mathrm{COOH}) 2, \mathrm{Mg}(\mathrm{COOH}) 2, \mathrm{Mn}(\mathrm{COOH}) 2, \mathrm{Ni}(\mathrm{COOH}) 2, \operatorname{Sr}(\mathrm{COOH}) 2$, $\mathrm{UO} 2(\mathrm{COOH}) 2, \mathrm{Y}(\mathrm{COOH}) 3, \mathrm{Zn}(\mathrm{COOH}) 2$

- Oxy-ions: $\quad \mathrm{KMnO} 4, \mathrm{Na} 2 \mathrm{~B} 4 \mathrm{O} 7, \mathrm{Na} 2 \mathrm{CO} 3, \mathrm{Na} 2 \mathrm{CO} 3$ (C-14), $\mathrm{Na} 2 \mathrm{C} 2 \mathrm{O} 4, \mathrm{Na} 2 \mathrm{CrO} 4$, $\mathrm{NaHCO} 3, \mathrm{Na} 2 \mathrm{MoO} 4, \mathrm{NaNO} 2, \mathrm{Na} 2 \mathrm{RuO} 4, \mathrm{Na} 2 \mathrm{RhO} 4, \mathrm{Na} 2 \mathrm{SiO} 3$, $\mathrm{NaTcO} 4, \mathrm{~K} 2 \mathrm{CO} 3$

- Hydroxy-ions: $\mathrm{Na}[\mathrm{Ag}(\mathrm{OH}) 2], \mathrm{Na}[\mathrm{Al}(\mathrm{OH}) 4], \mathrm{Na}[\mathrm{HgO}(\mathrm{OH})], \mathrm{Na} 2[\mathrm{PuO} 2(\mathrm{OH}) 4]$

- Acids: $\quad \mathrm{H} 3 \mathrm{BO} 3, \mathrm{HCl}, \mathrm{HCOOH}, \mathrm{H} 2 \mathrm{C} 2 \mathrm{O} 4, \mathrm{HF}, \mathrm{HNO} 3, \mathrm{H} 2 \mathrm{SO} 4$

- Gases: $\quad \mathrm{CO}, \mathrm{CO} 2, \mathrm{CO} 2(\mathrm{C}-14), \mathrm{H} 2, \mathrm{I}, \mathrm{N} 2, \mathrm{~N} 2 \mathrm{O}, \mathrm{NO} 2, \mathrm{NO}, \mathrm{O} 2, \mathrm{RuO} 4, \mathrm{SO} 2, \mathrm{SO} 3$, NH3

- Other: all semi-volatiles (8), $\mathrm{CsCl}$, Tritium, water

* Alkali metal salts of tetraphenylborate are shown as MTPB in material balance tables $(\mathrm{M}=\mathrm{Cs}, \mathrm{K}, \mathrm{Na}, \mathrm{NH} 4)$.

** These Na salts are partitioned between solubles and insolubles during ESP washing only for certain sludge feeds. 
TABLE 2. Components Assumed Insoluble in the HLW Flowsheet Model.

- Components assumed always Insoluble:

- organics: (C6H5)2, (C6H5)2C6H4, (C6H5)2NH, C6H5NHCHO, C6H5C6H4OH, C6H5NO, (C6H5)2Hg, CsB(C6H5)4*,KB(C6H5)4*, NH4B(C6H5)4*

- Oxides: $\mathrm{Ag} 2 \mathrm{O}, \mathrm{Al2O} 3, \mathrm{~B} 2 \mathrm{O} 3, \mathrm{BaO}, \mathrm{CaO}, \mathrm{CoO}, \mathrm{Cr} 2 \mathrm{O} 3, \mathrm{Cs} 2 \mathrm{O}, \mathrm{CuO}, \mathrm{FeO}, \mathrm{Fe} 2 \mathrm{O} 3$, $\mathrm{HgO}, \mathrm{K} 2 \mathrm{O}, \mathrm{La} 2 \mathrm{O} 3, \mathrm{Li} 2 \mathrm{O}, \mathrm{MgO}, \mathrm{MnO}, \mathrm{MnO} 2, \mathrm{MoO} 2, \mathrm{Na} 2 \mathrm{O}, \mathrm{NiO}, \mathrm{PbO}, \mathrm{PdO}$, $\mathrm{PuO} 2, \mathrm{RhO} 2, \mathrm{RuO} 2, \mathrm{SiO} 2, \mathrm{SrO}, \mathrm{TcO} 2, \mathrm{ThO} 2, \mathrm{TiO} 2, \mathrm{U} 3 \mathrm{O} 8, \mathrm{Y} 2 \mathrm{O} 3, \mathrm{ZnO}, \mathrm{ZrO} 2$

- Oxy-ions: BaSO4, $\mathrm{CaCO} 3, \mathrm{CaCO} 3$ (C-14), $\mathrm{CaC} 204, \mathrm{Ca} 3(\mathrm{PO} 4) 2, \mathrm{CaSO} 4, \mathrm{PbSO} 4$, NaTi2O5H, PbCO3, PuO2(NaTi2O5)2, SrCO3, Sr(NaTi2O5)2, Y2(CO3)3

- Metals: $\quad \mathrm{Ag}, \mathrm{Hg}, \mathrm{Pd}, \mathrm{Rh}, \mathrm{Ru}$

- $\mathrm{Hg}$ halides: $\mathrm{HgCl} 2, \mathrm{Hg} 2 \mathrm{Cl} 2, \mathrm{Hg} 2 \mathrm{I} 2$

- Other: CaF2, Carbon (coal), Cement, hydrate $\mathrm{H} 2 \mathrm{O}, \mathrm{PbS}$, Zeolite

* Alkali metal salts of tetraphenylborate are shown as MTPB in material balance tables. (M= Cs, K, Na, NH4). 
C. T. Randall

TABLE 3. Composition of Group A and Group B Components

- GROUP A Fission Products:

\begin{tabular}{cccc} 
Component & wt \% & Component & wt \% \\
\hline Se & 1.656 & $\mathrm{Rb}$ & 0.7435 \\
$\mathrm{Mo}$ & 83.37 & $\mathrm{Cd}$ & 0.8694 \\
$\mathrm{Te}$ & 13.35 & &
\end{tabular}

- GROUP B Fission and Activation Products:

\begin{tabular}{clll} 
Component & wt \% & Component & wt \% \\
\hline $\mathrm{Cr}-51$ & $1.98 \mathrm{E}-23$ & $\mathrm{Co}-60$ & 0.0915 \\
$\mathrm{Zr}$ & 30.49 & $\mathrm{Nb}$ & $3.388 \mathrm{E}-6$ \\
$\mathrm{Ag}$ & 0.1135 & $\mathrm{Sn}$ & 0.2785 \\
$\mathrm{Sb}$ & 0.07914 & $\mathrm{La}$ & 8.431 \\
$\mathrm{~Np}$ & 0.2440 & $\mathrm{Am}$ & 0.06184 \\
$\mathrm{Cm}$ & $4.239 \mathrm{E}-5$ & $\mathrm{Ce}$ & 16.19 \\
$\mathrm{Pr}$ & 7.791 & $\mathrm{Nd}$ & 30.01 \\
$\mathrm{Pm}$ & 0.5126 & $\mathrm{Sm}$ & 5.226 \\
$\mathrm{Eu}$ & 0.4763 & $\mathrm{~Tb}$ & 0.00402 \\
$\mathrm{Tl}$ & $7.310 \mathrm{E}-14$ & & \\
\hline
\end{tabular}




\section{OVERALL MODEL CONVERGENCE SCHEME}

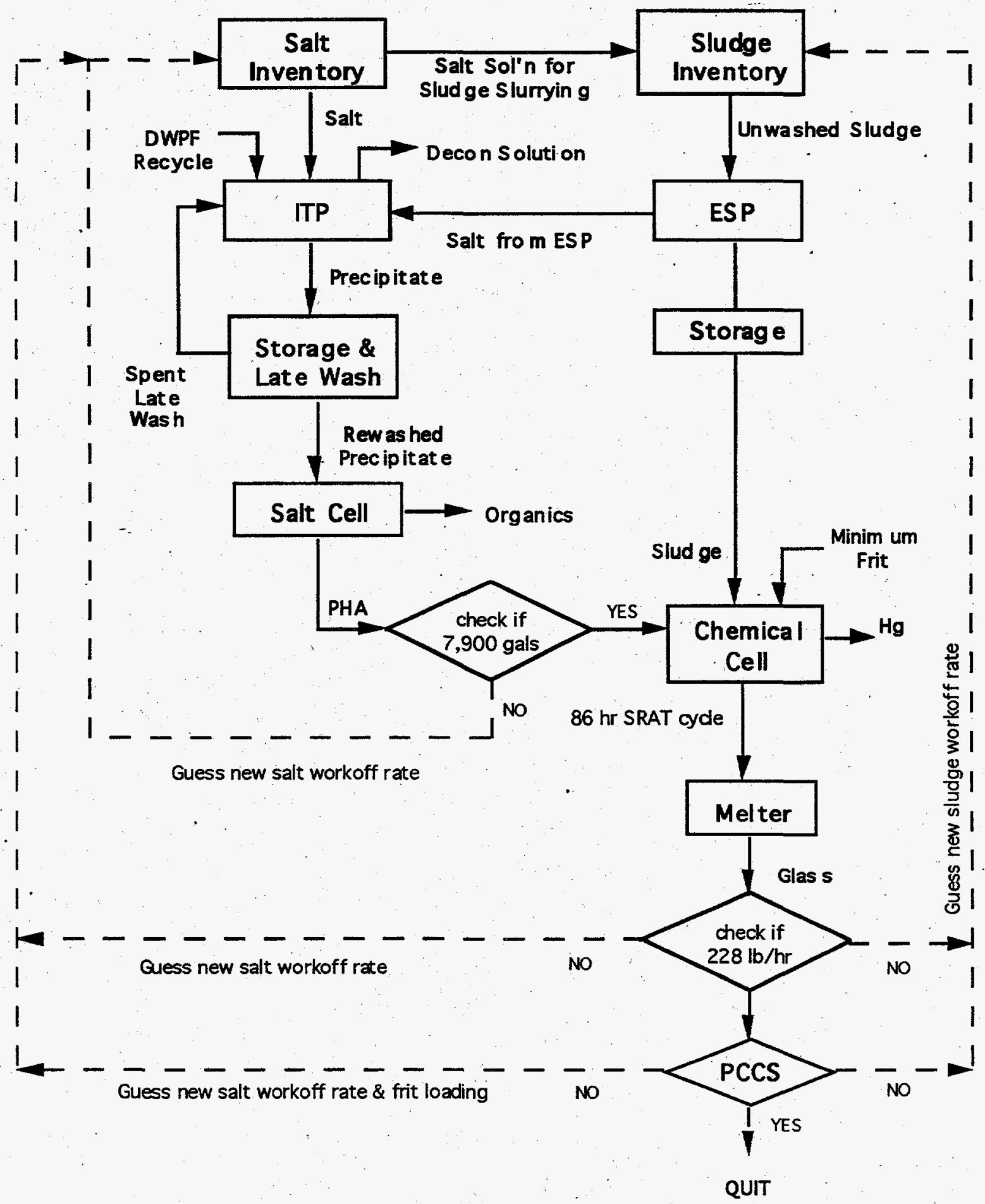

FIGURE 1. Calculation Flowchart for Model Convergence 


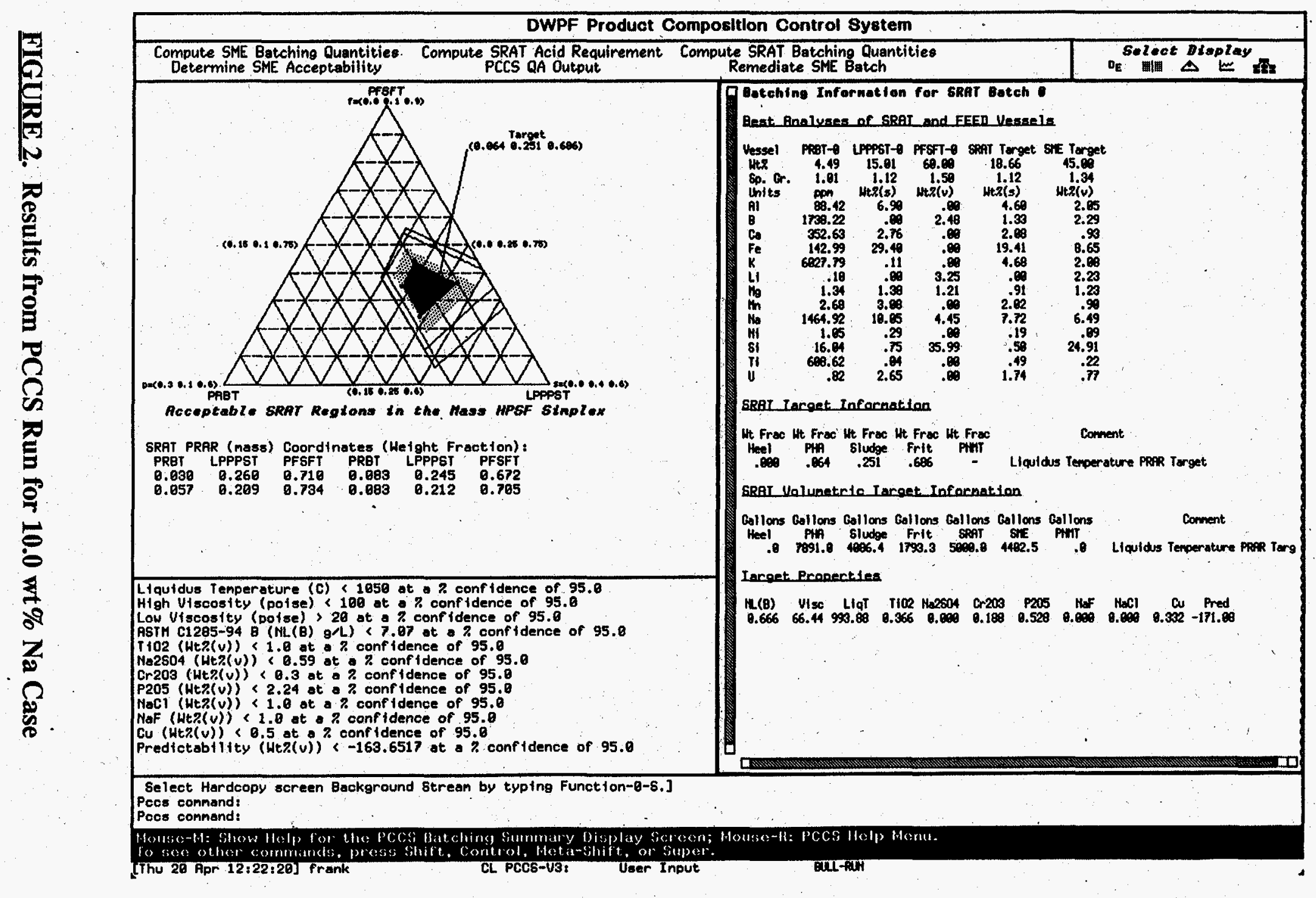




\section{ACRONYMS}

CPC
CPES
DF
DWPF
ESP
ETF
HLW
HLWM
IDMS
ITP
LWF
LWHT
LWPT
MFT
PCCS
PHA
PR
PRBT
RCT
SME
SMECT
SPC
SRAT
SRS
SRTC
TPB
VLE
WAPS
WSRC

Chemical Processing Cell

Chemical Process Evaluation System

Decontamination Factor

Defense Waste Processing Technology

Extended Sludge Processing

Effluent Treatment Facility

High Level Waste

High Level Waste Management

Integrated DWPF Melter System

In-Tank Precipitation

Late Wash Facility

Late Wash Hold Tank

Late Wash Precipitate Tank

Melter Feed Tank

Product Composition Control System

Precipitate Hydrolysis Aqueous

Precipitate Reactor

Precipitate Reactor Bottoms Tank

Recycle Collection Tank

Slurry Mix Evaporator

Slurry Mix Evaporator Condensate Tank

Salt Processing Cell

Sludge Receipt and Adjustment Tank

Savannah River Site

Savannah River Technology Center

Tetraphenyl Borate

Vapor-Liquid Equilibrium

Waste Acceptance Preliminary Specifications

Westinghouse Savannah River Company 
Table A-1. HLW Flowsheet Material Balance (Tank 51/ITP Cycle 1) 


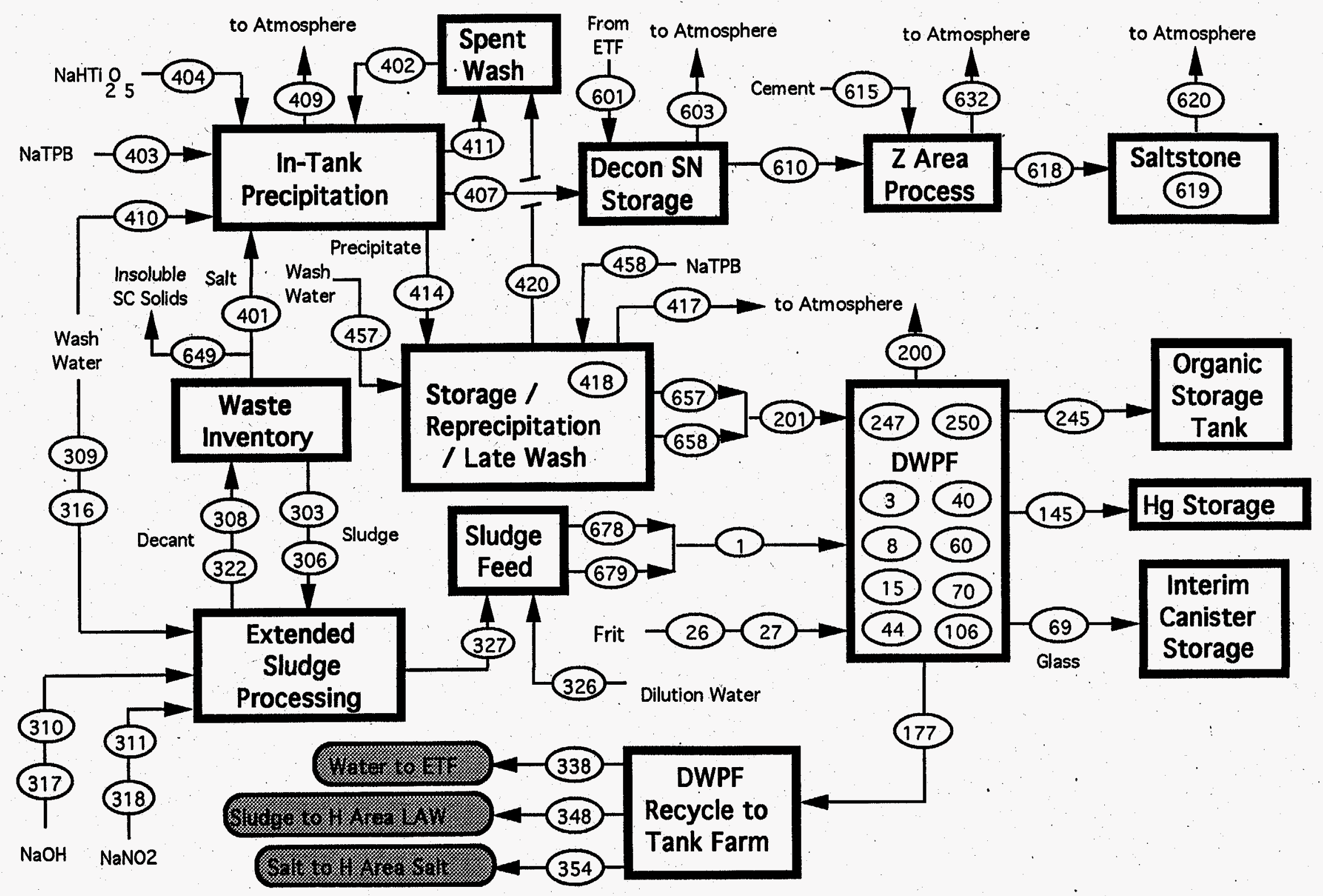

SECTION 0. MAJOR STREAMS IN THE INTEGRATED HLW FLOWSHEET MODEL WSRC-TR-95-0019 (Revision 0) Date: January 1995 Section 0, Page 0 of 37 
Table A-1. HLW Flowsheet Material Balance (Tank 51/ITP Cyole 1 ) section 0. Major streams in the Flowsheet Model

1. STREAM NUMBERS $401,649,402,403,404,410,420 \ldots . . . . . . . . . . . . . .2$

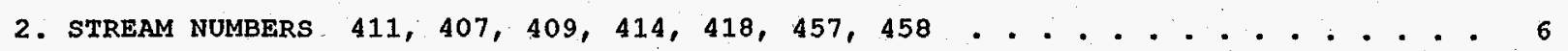

3. STREAM NUMBERS $417,303,309,316,306,308,310 \ldots \ldots \ldots$

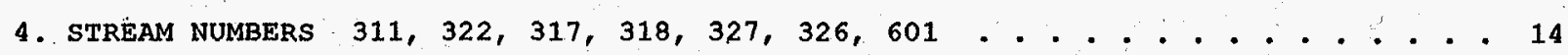

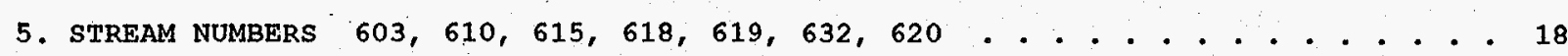

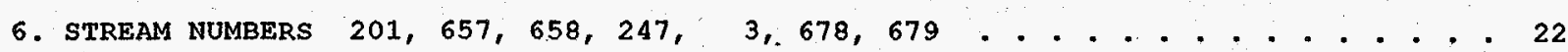

7. STREAM NUMBERS $1,8,15,106,250,40,26 . . . . . . . . . . . .26$

8. STREAM NUMBERS $27,44,60,69,70,200,145 \ldots \ldots$. . . . . . . . . . . 30

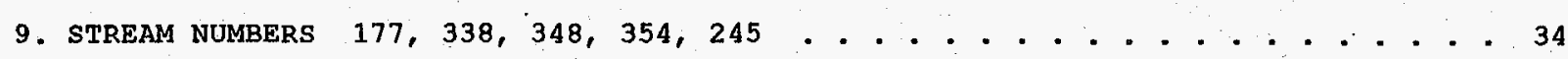


Table A-1. HLW Flowsheet Material Balance (Tank 51/ITP Cycle 1) section 0. Major streams in the Flowsheet Model

STREAM NUMBERS -> STREAM NAME

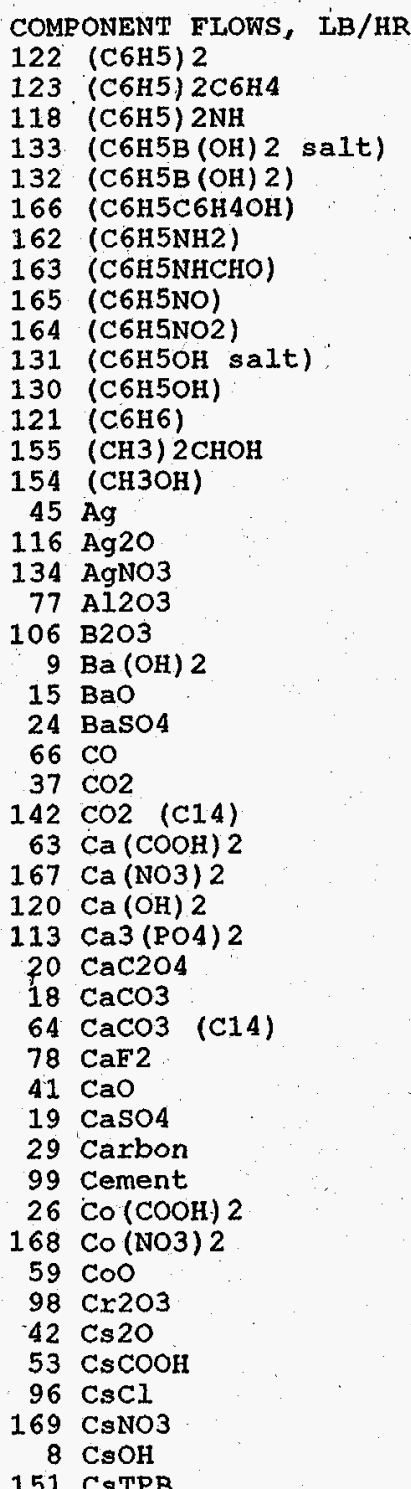

TRC-TR-95-0019 (Revision
Salt/sN settld salt Total spent Feed to ITP Insolubles Wash Reaycle

ZERO
ZERO
ZERO
ZERO
ZERO
ZERO
ZERO
ZERO
ZERO
ZERO
ZERO
ZERO
ZERO
ZERO
ZERO
ZERO

$0.2230 \mathrm{E}-03$

ZERO
$0.6708 E-01$

$0.1670 \mathrm{E}-02$

$0.7011 E-03$

$0.7324 \mathrm{EERO}$

ZERO

ZERO

ZERO

$0.3549 \mathrm{E}-01$

$0.3549 \mathrm{E}-01$
$0.3660 \mathrm{E}-02$

$0.3660 \mathrm{E}-02$
$0.5607 \mathrm{E}+00$

$0.5607 \mathrm{E}+00$

ZERO

ZERO

ZERO

$0.1912 \mathrm{E}+00$

ZERO

$0.1392 \mathrm{E}-01$

$0.1392 \mathrm{E}-01$

$0.1028 \mathrm{E}-03$
$0.2616 \mathrm{E}+00$

$0.3848 \mathrm{E}-04$

ZERO

ZERO

$0.3649 \mathrm{E}-01$
ZERO

$$
649
$$

403

NaTPB

Additions

ZERO

$\begin{array}{lll}\text { ZERO } & \text { ZERO } & \text { ZERO } \\ \text { ZERO } & \text { ZERO } & \text { ZERO } \\ \text { ZERO } & \text { ZERO } & \text { ZERO }\end{array}$

ZERO ZERO $\quad$ ZERO

ZERO ZERO ZERO

ZERO ZERO ZERO

ZERO ZERO ZERO

ZERO ZERO ZERO

ZERO

ZERO

$0.5151 \mathrm{E}+00$

ZERO

$0.2782 \mathrm{E}+01$

$0.1293 \mathrm{E}-03$

$0.4783 E-05$

ZERO $0.3911 \mathrm{E}-06$

$0.1686 \mathrm{E}-02 \quad$ ZERO

ZERO

$0.5073 \mathrm{E}+00$

$0.1263 \mathrm{E}-01$

ZERO

ZERO

$0.4574 \mathrm{E}-09$

ZERO

ZERO

ZERO

ZERO

ZERO ZERO

ZERO $\quad 0.1328 \mathrm{E}-02$

$0.4241 \mathrm{E}+01 \quad$ ZERO

ZERO ZERO

ZERO

ZERO

ZERO

$0.1446 \mathrm{E}+01$

ZERO

ZERO

ZERO

$0.7788 \mathrm{E}-03$

$0.1979 \mathrm{E}+01$

$0.2911 \mathrm{E}-03$

ZERO
ZERO

ZERO

ZERO

ZERO

ZERO

ZERO

ZERO

ZMRO

ZERO

ZERO

ZERO
$0.5208 \mathrm{E}-03$

ZERO
ZERO

ZERO

ZERO

ZERO

ZERO

ZERO

ZERO

ZERO

ZERO

ZERO

ZERO

ZERO

ZERO

ZERO

ZERO

ZERO

ZERO
ZERO

ZERO

ZERO

ZERO

ZERO

ZERO

ZERO

ZERO

ZERO

ZERO

ZERO

ZERO

ZERO

ZERO

E-0 4
ZERO

ZERO

Date: January 1995
404

NaTi205H Inhibited spent Late
Additions Wash Water Wash Recycle

410

420

ZERO ZERO ZERO

ZERO ZERO $\quad$ ZERO

ZERO ZERO ZERO

ZERO ZERO $\quad$ ZERO

ZERO ZERO $\quad$ ZERO

$\begin{array}{lll}\text { ZERO } & \text { ZERO } & \text { ZERO } \\ \text { ZERO } & \text { ZERO } & \text { ZERO }\end{array}$

ZERO ZERO

ZERO ZERO

ZERO ZERO ZERO

ZERO ZERO $0.3998 \mathrm{E}+00$

ZERO ZERO ZERO

$0.1762 \mathrm{E}+00$ ZERO $0.7436 \mathrm{E}-02$

$0.9434 \mathrm{E}-02$ ZERO $0.1448 \mathrm{E}-09$

ZERO ZERO ZERO

ZERO ZERO ZERO

$\begin{array}{lll}\text { ZERO } & \text { ZERO } & \text { ZERO } \\ \text { ZERO } & \text { ZERO } & \text { ZERO }\end{array}$

ZERO

ZERO ZERO $0.1252 \mathrm{E}-14$

ZERO $\quad$ ZERO $\cdots$ ZERO

ZERO ZERO

ZERO ZERO ZERO

ZERO ZERO ZERO

ZERO ZERO ZERO

ZERO ZERO $0.3561 \mathrm{E}-04$

ZERO ZERO $0.3672 \mathrm{E}-05$

ZERO ZERO ZERO

ZERO ZERO ZERO

ZERO ZERO ZERO

ZERO ZERO ZERO

ZERO ZERO ZERO

ZERO ZERO $\quad$ ZERO

ZERO $\quad$ ZERO $\quad$ ZERO

ZERO ZERO ZERO

ZERO

ZERO ZERO $0.1397 \mathrm{E}-04$

ZERO ZERO ZERO

ZERO ZERO $\quad \cdots$ ZERO

ZERO $\quad$ ZERO

ZERO
ZERO
ZERO

$\begin{array}{lll}\text { ZERO } & \text { ZERO } & \text { ZERO } \\ \text { ZERO } & \text { ZERO } & \text { ZERO }\end{array}$

$\begin{array}{rrr}\text { ZERO } & \text { ZERO } & 0.4663 \mathrm{E}-04 \\ \text { ZERO } & \text { ZERO } & \text { ZERO }\end{array}$

ZERO

ZERO

Section 0, Page 2 of 37 
Table A-1. HLW Flowsheet Material Balance (Tank 51/ITP Cycle 1) section 0. Major streams in the Flowsheet Model

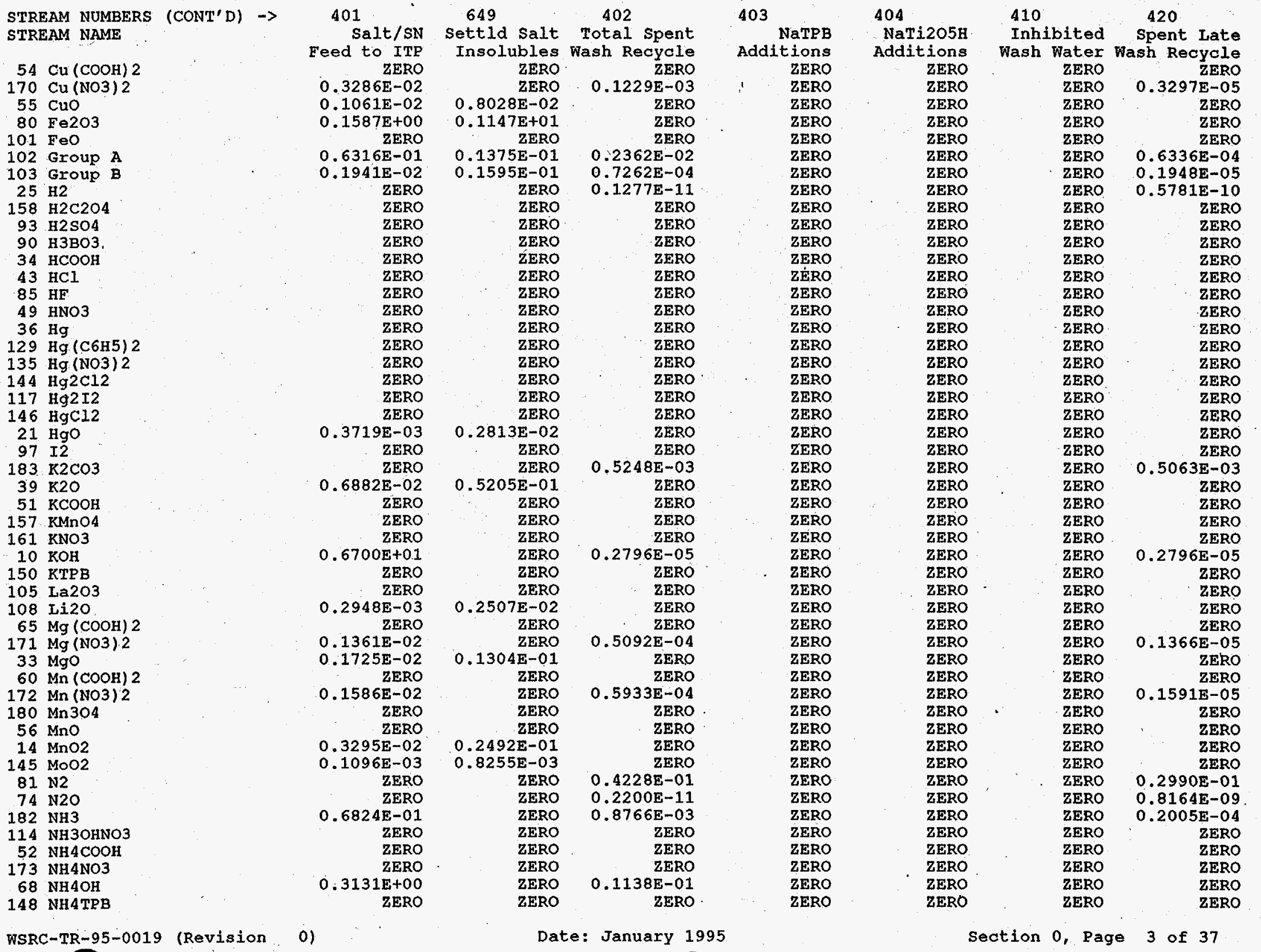

WSRC-TR-95-0019 (Revision

Date: January 1995 
Table A-1. HLW Flowsheet Material Balance (Tank 51/ITP Cycle 1) Section 0 . Major streams in the Flowsheet Model

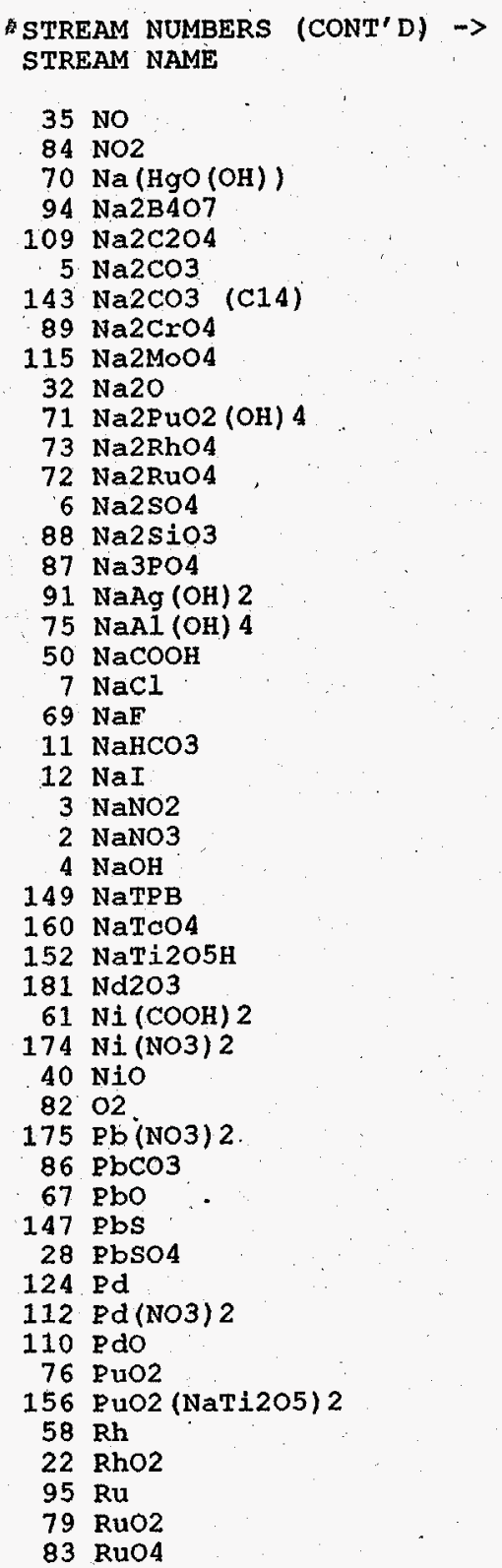

401

Salt/SN Settld Salt Total spent Feed to ITP ZERO
ZERO

$0.2749 \mathrm{E}+00$ $0.5925 \mathrm{E}-01$

$0.2518 \mathrm{E}+01$

$0.4906 \mathrm{E}+03$

$0.7320 \mathrm{E}-05$

$0.2695 \mathrm{E}+01$

$0.8046 \mathrm{E}+00$

$0.1003 \mathrm{E}+01$

$0.7467 \mathrm{E}-04$

$0.1952 \mathrm{E}-01$

$0.1028 \mathrm{E}+00$

$0.2804 \mathrm{E}+02$

$0.7804 \mathrm{E}+00$

$0.3724 \mathrm{E}+02$

$0.1403 \mathrm{E}+03$

ZERO:

$0.1976 \mathrm{E}+01$

$0.2156 \mathrm{E}+01$

ZERO
$0.1444 \mathrm{E}-02$

$0.2311 \mathrm{E}+03$

$0.1353 \mathrm{E}+04$

$0.5045 \mathrm{E}+03$

$0.6478 \mathrm{E}-01$

ZERO

ZERO

ZERO
$0.1732 \mathrm{E}-01$

$0.1037 \mathrm{E}-02$

ZERO

$0.4207 \mathrm{E}-01$

ZERO

ZERO

$0.4362 \mathrm{E}-02$

ZERO
$6 \mathrm{E}-03$

$0.1736 \mathrm{E}-03$

$0.3209 \mathrm{E}-04$

ZERO

ZERO

ZERO

$0.4424 \mathrm{E}-02$

ZERO
$649 \quad 402 \quad 403$

Insolubles Wash Recycle ZERO

ZERO

ZERO

$0.3078 \mathrm{E}+01$

ZERO

ZERO

ZERO
$0.7588 \mathrm{E}+01$

ZERO
ZERO

ZERO

ZERO

ZERO

ZERO

ZERO

ZERO

ZERO

ZERO

ZERO

ZERO

ZERO

ZERO

ZERO

ZERO

ZERO

ZERO

ZERO

$0.7846 \mathrm{E}-02$

ZERO
ZERO

$0.9968 \mathrm{E}-05$
$0.2783 \mathrm{E}+00$

$0.1585 \mathrm{E}+01$

$0.2236 \mathrm{E}+02$

$0.2738 \mathrm{E}-06$

.1008E +00

ZERO

$0.8810 \mathrm{E}-07$

$0.7301 \mathrm{E}-03$

$0.1049 \mathrm{E}+01$

$0.2919 \mathrm{E}-01$

$0.1393 \mathrm{E}+01$

$0.2636 \mathrm{E}-06$
$0.5104 \mathrm{E}+01$

ZERO

$0.1011 \mathrm{E}+00$

$0.8064 \mathrm{E}-01$

ZERO

$0.5401 \mathrm{E}-04$

$0.5136 \mathrm{E}+02$

$0.1508 \mathrm{E}+03$

$.3176 \mathrm{E}+01$

$0.2423 \mathrm{E}-02$

ZERO

$0.6480 \mathrm{E}-03$

ZERO

$0.2474 \mathrm{E}-01$

$0.1573 \mathrm{E}-02$

ZERO

ZERO

ZERO

ZERO

$0.3299 \mathrm{E}-01$

ZERO

ZERO

$0.2433 E-03$

ZERO

$0.6494 \mathrm{E}-05$

ZERO

ZERO

ZERO

ZERO

ZERO

ZERO

ZERO
NaTPB

Additions

ZERO
ZERO

ZERO

ZERO

ZERO

ZERO

ZERO

ZERO

ZERO

ZERO

ZERO

ZERO

ZERO

ZERO

ZERO

ZERO

ZERO

$0.2231 \mathrm{E}+00$

ZERO

ZERO

ZERO

ZERO

$0.1113 \mathrm{E}+01$

$0.4760 \mathrm{E}+02$

ZERO

ZERO

ZERO

ZERO

ZERO

ZERO

ZERO

ZRRO

ZERO

ZERO

ZERO

ZERO

ZERO

ZERO

ZERO

ZERO

ZERO

ZERO

ZERO

ZERO
NaTi205H

Additions

ZERO

ZERO
ZERO

ZERO

ZERO

ZERO

ZERO

ZERO

ZERO

ZERO

ZERO

ZERO

ZERO

ZERO

ZERO

ZERO

ZERO

ZERO

ZERO

ZERO

ZERO

ZERO

ZERO

$0.1272 \mathrm{E}-02$

ZERO

$3141 \mathrm{E}-01$
ZERO

ZERO

$0.9827 \mathrm{E}+00$

ZERO

ZERO

ZERO

ZERO

ZERO

ZERO

ZERO

ZERO

ZERO

ZERO

ZERO

ZERO

ZERO

ZERO

ZERO

ZERO

ZERO

ZERO

ZERO

ZERO

410

420

Inhibited spent Late Wash Water Wash Recycle $\begin{array}{lr}\text { ZERO } & \text { ZERO } \\ \text { ZERO } & \text { ZERO }\end{array}$ ZERO $\quad 0.3027 \mathrm{E}-09$ ZERO $\quad 0.2664 \mathrm{E}+00$ ZERO $0.1441 \mathrm{E}+01$ ZERO $\quad 0.4359 \mathrm{E}+01$ ZERO $0.7343 \mathrm{E}-08$ ZERO $0.2703 E-02$ ZERO $0.8072=03$

ZERO ZERO ZERO $0.3374 \mathrm{E}-10$ ZERO $\quad 0.1958 \mathrm{E}-04$ ZERO $0.1031 \mathrm{E}-03$ 
Table A-1. HLW Flowsheet Material Balance (Tank 51/ITP Cycle 1) Section 0. Major Streams in the Flowsheet Model.

STREAM NUMBERS (CONT'D) $\rightarrow$ STREAM NAME

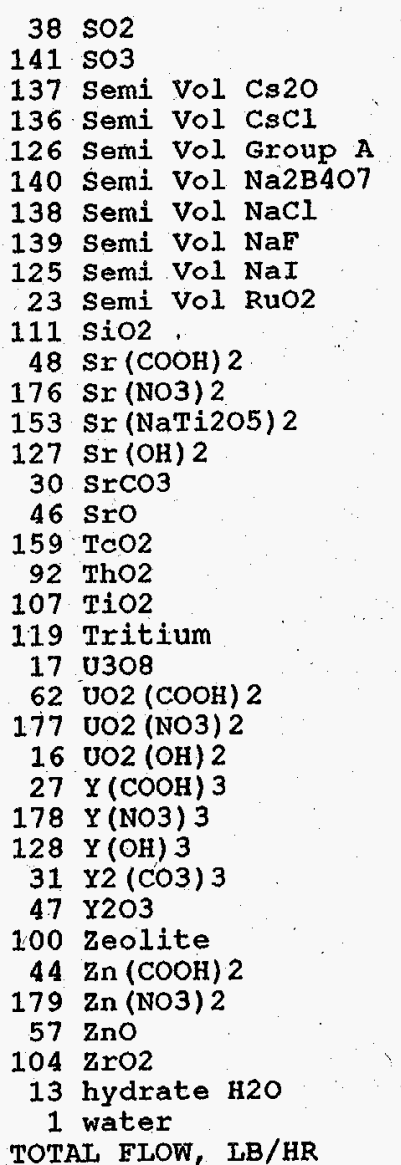

TEMPERATURE, DEG C PRESSURE, ATM PRESSURE， PSIG PRESSURE, MM HG ENTHALPY; PCU/HR VAPOR FLOW, CEM LIQUTD FLOW, GPM DENSITY, LBS/FT3 PHASE
401

salt/SN Feed to ITP 649 402 ettld salt Total spent Insolubles Wash Recycle

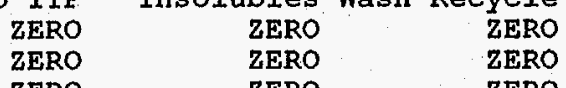
ZERO $\quad$ ZERO $\quad$ ZERO ZERO ZERO $\quad$ ZERO ZERO ZERO $\quad$ ZERO ZERO ZERO Z ZERO ZERO ZERO $\quad$ ZERO ZERO ZERO ZERO ZERO ZERO ZERO

ZERO
$0.2666 \mathrm{E}-01$

$0.2666 \mathrm{E}-01$
ZERO ZERO ZERO

$0.1026 \mathrm{E}-02$

$0.9081 \mathrm{E}-04$ ZERO
ZERO ZERO

$0.1213 \mathrm{E}-02$

$0.4059 \mathrm{E}-07$

$0.4059 \mathrm{E}-07$

0.7512 E-03
ZERO ZERO

$0.2783 E-01$ ZERO ZERO

$0.1206 \mathrm{E}-02$ ZERO ZERO ZERO

$0.1383 \mathrm{E}+01$

$0.1980 \mathrm{E}+00$

$0.1980 \mathrm{E}+00$
ZERO

$0.2434 \mathrm{E}-02$

$0.5573 \mathrm{E}+04$

$0.8380 \mathrm{E}+04$

$0.2992 \mathrm{E}+02$

$0.1000 \mathrm{E}+01$ ZERO

$0.1890 \mathrm{E}+06$

$0.1320 \mathrm{E}+02$

$0.7914 \mathrm{E}+02$ IIQUID

$0.8874 \mathrm{E}-07$

$0.1497 E+01$

ZERO

ZERO

$0.2195 \mathrm{E}+02$

$0.3362 \mathrm{E}+04$

$0.3500 \mathrm{E}+02 \quad 0.3981 \mathrm{E}+02$

$0.1000 \mathrm{E}+01$

$0.9656 \mathrm{E}+00$ $-0.5055 \mathrm{E}+00$ $0.7339 \mathrm{E}+03$

$0.1607 \mathrm{E}+03$

$0.1259 \mathrm{E}+06$

$0.1529 \mathrm{E}-01$

$0.6338 \mathrm{E}+01$

IIQUID

IIQUID
403 NaTPB
Additions ZERO $\therefore \quad$ ZERO ZERO ZERO ZERO

ZERO

ZERO

ZERO

ZERO

ZERO

ZERO

ZERO

ZERO

ZERO

ZERO

ZERO

ZERO

ZERO

ZERO

ZERO

ZERO

ZERO

ZERO

ZERO

ZERO

ZERO

ZERO

ZERO

ZERO

ZERO

ZERO

0.3074 ERO

$0.2265 \mathrm{E}+03$ $0.2782 \mathrm{E}+03$

$0.3500 \mathrm{E}+02$

$0.1000 \mathrm{E}+01$

ZERO

$0.7962 E+04$

$0.5455 \mathrm{E}+00$

$0.6358 \mathrm{E}+02$

LIQUID

NaTi2O5H

410

420

Inhibited spent Late WERO Wash Water Wash Recycle
ZERO ZERO ZERO ZERO

ZERO ZERO ZERO

ZERO ZERO ZERO

ZERO ZERO $\quad$ ZERO

ZERO ZERO Z ZERO

ZERO ZERO — ZERO

ZERO ZRRO

ZERO

ZERO ZERO

$\begin{array}{lll}\text { ZERO } & \text { ZERO } \\ \text { ZERO ZERO } & \text { ZERO }\end{array}$

$\begin{array}{lll}\text { ZERO } & \text { ZERO } \\ \text { ZERO } & \text { ZERO } & \text { ZERO }\end{array}$

$\begin{array}{lll}\text { ZERO } & \text { ZERO } & \text { ZERO } \\ \text { ZERO } & \text { ZERO } & \text { ZERO }\end{array}$

ZERO

ZERO

ZERO

ZERO

ZERO

ZERO

ZERO

ZERO

ZERO

ZERO

ZERO

ZERO

ZERO

ZERO

ZERO

ZERO

ZERO

ZERO

ZERO

ZERO

ZERO

$0.7283 \mathrm{E}+01$

$0.8484 \mathrm{E}+01$

ZERO

ZERO

ZERO

ZERO

ZERO

ZERO

ZERO

ZERO

ZERO

ZERO

ZERO

ZERO

ZERO

ZERO

ZERO

ZERO

ZERO

ZERO

ZERO

ZERO

ZERO

ZERO

$0.1315 \mathrm{E}+04$

$0.1316 \mathrm{E}+04$

ZERO

$0.1926 \mathrm{E}-10$

ZERO

ZERO

ZERO

ZERO

$0.3304 \mathrm{E}-10$

ZERO

ZERO

$0.2792 \mathrm{E}-04$

ZERO

$0.2140 \mathrm{E}-10$

ZERO

ZERO

ZERO

$0.1387 E-02$

$E-02$

ZERO

ZERO

$0.1753 \mathrm{E}+04$

$0.3500 \mathrm{E}+02 \quad 0.2500 \mathrm{E}+02 \quad 0.3989 \mathrm{E}+02$

$0.1000 \mathrm{E}+01$

ZERO

$0.1000 \mathrm{E}+01$

$0.9656 \mathrm{E}+00$

ZERO $-0.5055 \mathrm{E}+00$

$0.7339 \mathrm{E}+03$

$0.2597 \mathrm{E}+03 \quad 0.3301 \mathrm{E}+05 \quad 0.7132 \mathrm{E}+05$

$0.1701 \mathrm{E}-01$

$0.6217 \mathrm{E}+02$

LIQUID

$.2627 \mathrm{E}+01$

$0.3569 \mathrm{E}+01$

$247 \mathrm{E}+02$

$0.6484 \mathrm{E}+02$

LIQUID 
Table A-1. HLW Flowsheet Material Balance (Tank 51/ITP Cycle 1) Section 0. Major Streams in the Flowsheet Model.

STREAM NUMBERS $\rightarrow$

STREAM NAME

COMPONENT FLOWS, LB/HR 122 ( $6 \mathrm{H} 5) 2$

123 ( 6 $6 \mathrm{H} 5) 2 \mathrm{C} 6 \mathrm{H} 4$

118 (C6H5) 2NH

133 (C6H5B (OH) 2 salt)

132 (C6H5B (OH) 2)

166 (C6H5C6H4OH)

162. (C6H5NH2)

163 (C6H5NHCHO)

165 (C6H5NO)

164 (C6H5NO2).

131. (C6H5OH. salt)

130 (C6H5OH)

121 ( 6 $6 \mathrm{H} 6)$

155 (СH3) $2 \mathrm{CHOH}$

154 (CH3OH)

$45 \mathrm{Ag}$

$116 \quad \mathrm{Ag} 2 \mathrm{O}$
$134 \mathrm{AgNO}$

134 AgNO3
77 Al2O3

$106 \mathrm{~B} 203$

$9 \mathrm{Ba}(\mathrm{OH}) 2$

$15 \mathrm{BaO}$

$24 \mathrm{BaSO} 4$

$66 \mathrm{CO}$

$37 \mathrm{CO} 2$

$142 \mathrm{CO} 2$ (C14)

$63 \mathrm{Ca}(\mathrm{COOH}) 2$

$167 \mathrm{Ca}$ (NO3) 2

$120 \mathrm{Ca}(\mathrm{OH}) 2$

$113 \mathrm{Ca} 3(\mathrm{PO} 4) 2$

$20 \mathrm{CaC2O} 4$

$64 \mathrm{CaCO} 3(\mathrm{C} 14)$

78 CaF2

$41 \mathrm{CaO}$

19. CaSO4

29 Carbon

99 Cement

$26 \mathrm{Co}(\mathrm{COOH}) 2$

$168 \mathrm{Co}$
$59 \mathrm{CoO}$

$98 \operatorname{cr} 203$

$42 \mathrm{Cs} 2 \mathrm{O}$

$53 \mathrm{CsCOOH}$

$96 \mathrm{CsCl}$

$169 \mathrm{CsNO} 3$

$\begin{array}{rl}8 & \mathrm{CsOH} \\ 51 & \mathrm{CsTPB}\end{array}$

WSRC-TR-95-0019 (Revision $\begin{array}{ll}407 & 409\end{array}$

414

418

457

Spent. Wash

to storage

Proc Tn

Shed Prec Prec Storage Late Wsh H2O

to Storage Tank bottoms Adjd w/NaTPB

458

NaTPB addn

to LWP T

ZERO

ZERO

ZERO

ZERO

ZERO

ZERO

ZERO

ZERO

TERO

$0.1153 \mathrm{E}+00$

ZERO

$0.1109 \mathrm{E}-03$

$0.7759 \mathrm{E}-05$
$0.5838 \mathrm{E}-06$

ZERO

ZERO

ZERO

$0.4574 \mathrm{E}-09$

ZERO

TERO

$0.2826 \mathrm{E}-04$

ZERO

$0.1292 \mathrm{E}-02$

$0.1332 \mathrm{E}-03$

ZERO
ZERO

ZERO

ZERO
ZERO
ZERO

ZERO

ZERO

ERO

ZERO

ZERO

$0.5068 \mathrm{E}-03$

ZERO

ZERO

ZERO

ZERO

ZERO

$0.5975 \mathrm{E}-07$

ZERO

$0.1653 E-05$

ZERO

$0.5859 \mathrm{E}-11$

Date: January 1995

$\begin{array}{rrr}0.3293 E+00 & \text { ZERO } & \text { ZERO } \\ 0.1399 E-01 & \text { ZERO } & \text { ZERO } \\ 0.9313 E-02 & \text { ZERO } & \text { ZERO } \\ 0.3708 E+00 & \text { ZERO } & \text { ZERO } \\ \text { ZERO } & \text { ZERO } & \text { ZERO } \\ 0.3026 E-01 & \text { ZERO } & \text { ZERO } \\ \text { ZERO } & \text { ZERO } & \text { ZERO } \\ \text { ZERO } & \text { ZERO } & \text { ZERO } \\ \text { ZERO } & \text { ZERO } & \text { ZERO } \\ \text { ZERO } & \text { ZERO } & \text { ZERO }\end{array}$

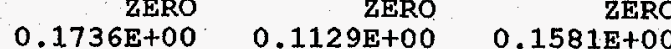

ZERO ZERO ZERO

Z ZERO $\quad$ ZERO

$\begin{array}{rrr}0.7905 E-09 & \text { ZERO } \\ \text { ZERO } & \text { ZERO } & \text { ZERO }\end{array}$

$\begin{array}{lll}0.3054 \mathrm{E}-02 & \text { ZERO } & \text { ZERO }\end{array}$

$0.1297 \mathrm{E}+00 \quad$ ZERO

$0.1670 \mathrm{E}-02$ ZERO

$0.1309 \mathrm{E}-10 \quad$ ZERO ZERO

$\begin{array}{rrr}\text { ZERO } & \text { ZERO } & \text { ZERO } \\ 0.1793 E-02 & \text { ZERO } & \text { ZERO }\end{array}$

ZERO

ZERO

ZERO

ZERO

ZERO

ZERO

ZERO

ZERO

ZERO

ZERO

ZERO

ZERO

ZERO

ZERO

ZERO

ZERO

ZERO

ZERO

ZERO

ZERO

ZERO

ZERO

ZERO

ZERO
O.8484E-08 ZERO ZERO 
Table A-1. HLW Flowsheet Material Balance (Tank 51/ITP Cycle 1)

Section 0. Major streams in the Flowsheet Model

STREAM NUMBERS (CONT'D) -> STREAM NAME

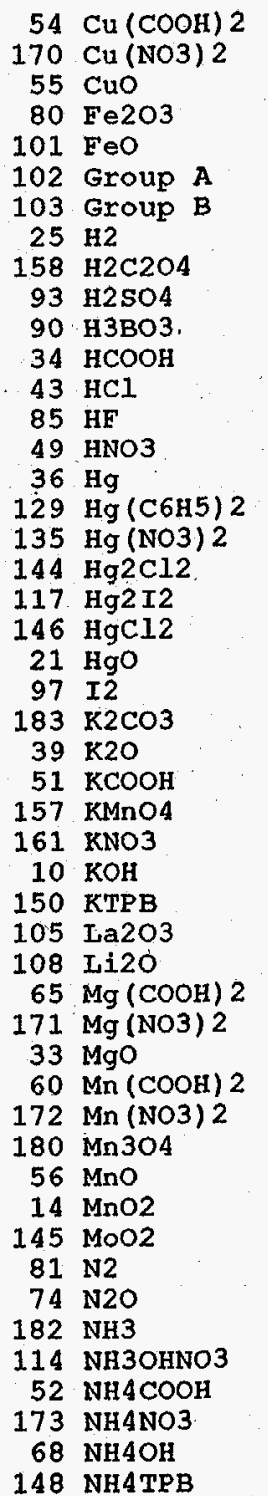

411

Spent Wash to storage

$0.1196 \mathrm{E}-03$ ZERO ZERO ZERO

$0.2299 \mathrm{E}-02$

$0.7067 \mathrm{E}-04$

$0.8835 \mathrm{E}-08$ ZERO ZERO ZERO ZERO ZERO ZERO ZERO ZERO ZERO ZERO ZERO ZERO ZERO ZERO

$0.1841 \mathrm{E}-04$ ZERO ZERO ZERO ZERO ZERO ZERO ZERO ZERO

$0.4956 \mathrm{E}-04$ ZERO

$0.5774 \mathrm{E}-04$ ZERO ZERO ZERO $.5117 \mathrm{E}-02$ $0.1174 \mathrm{E}-15$ $0.2264 \mathrm{E}-02$ ZERO ZERO

$0.1138 \mathrm{E}-01$ ZERO
407

409

41.4

418

457

SN Pro Proc Tnk Washed Prec Pred Storage Late Wsh H2O to Storage Vnt to Atmos to storage Tank bottoms Adjd w/NaTPB ZERO ZERO

$0.3286 \mathrm{E}-02$ $0.5644 \mathrm{E}-13$ ZERO ZERO

$0.6315 \mathrm{E}-01$

$0.1941 \mathrm{E}-02$

$0.2778 \mathrm{E}-06$

ZERO

ZERO

ZERO

ZERO

ZERO

ZERO

ZERO

ZERO

ZERO

ZERO

ZERO

ZERO

$0.5058 \mathrm{E}-03$

ZERO

ZERO

ZERO

ZERO

ZERO

ZERO

ZERO

$5644 \mathrm{E}-13$
$8441 \mathrm{E}-11$

$0.3424 \mathrm{E}-05$

$0.1061 \mathrm{E}-02$

$0.1587 \mathrm{E}+00$

$0.3424 \mathrm{E}-05$

ZERO

ZERO

ZERO

ZERO

$0.1835 \mathrm{E}-11$

$0.6580 \mathrm{E}-04$

ZRRO

$0.2023 \mathrm{E}-05$

$0.6580 \mathrm{E}-04$

$.5640 \mathrm{E}-13$

$0.2529 \mathrm{E}-09$

$0.2023 \mathrm{E}-05$

$.3445 \mathrm{E}-07$

ZERO

ZERO

ZERO

ZERO

ZERO

ZERO

$0.2019 \mathrm{E}-10$

ZERO

ZERO

ZERO

$0.1978 \mathrm{E}-13$ ZERO

$0.3660 \mathrm{E}-12$

ZERO

ZERO

ZERO

$0.2276 \mathrm{E}-08$

ZERO

$0.1568 \mathrm{E}-13$

ZERO

$0.1361 \mathrm{E}-02$

ZERO

$0.1586 \mathrm{E}-02$

$0.9172 \mathrm{E}-13$

ZERO

ZERO
ZERO

ZERO

ZERO

ZERO

$0.1409 \mathrm{E}+00$

. 3232E-14

ZERO.

ZERO

$0.3127 \mathrm{E}+00$

ZERO

ZERO

ZERO

ZERO

ZERO

ZERO

$0.3797 E+00$

ZERO

ZERO

ZERO

$0.3719 \mathrm{E}-03$ ZERO

$0.5270 \mathrm{E}-06$

$0.6882 E-02$

ZERO

ZERO

ZERO

$0.4280 \mathrm{E}+02$

$0.2948 \mathrm{E}-03$

ZERO

$0.1725 \mathrm{E}-02$

ZERO

ZERO

ZERO

$0.17 .52 \mathrm{E}-12$

$0.5828 \mathrm{E}-14$

$0.2207 \mathrm{E}+04$

$0.2197 \mathrm{E}-11$

ZERO

$0.3295 \mathrm{E}-02$

$0.1096 \mathrm{E}-03$

$0.1465 \mathrm{E}-03$

$0.3360 \mathrm{E}-17$

$0.6480 \mathrm{E}-04$

ZERO

ZERO

$0.9085 \mathrm{E}-08$

$$
\text { ZERO }
$$

$0.3258 \mathrm{E}-03$

ZERO

Date: January 1995
458

TPB addn

LWPT

ZERO

ZERO

ZERO

ZERO

ZERO

ZERO

ZERO

ZERO

ZERO

ZERO

ZERO

ZERO

ZERO

ZERO

ZERO

ZERO

ZERO

ZERO

ZERO

ZERO

ZERO

ZERO

ZERO

ZERO

ZERO

ZERO

ZERO

ZERO

ZERO

ZERO

ZERO

ZERO

ZERO

ZERO

ZERO

ZERO

ZERO

ZERO

ZERO

ZERO

ZERO

ZERO

ZERO

ZERO

ZERO 
Table A-1. HLW Flowsheet Material Balánce (Tank 51/ITP Cyole 1) Section 0 . Major streams in the Flowsheet Model

STREAM NUMBERS (CONT'D) $\rightarrow$ STREAM NAME

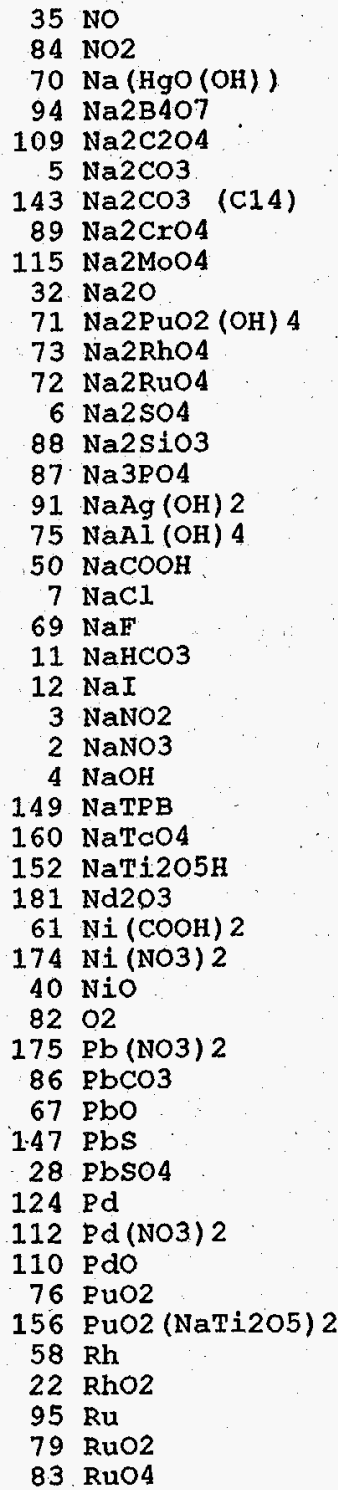

WSRC-TR-95-0019 (Revision
407 409 414 . 418 457 458 Spent Wash Decon SN PrC Proc Tnk Washed Prec Prec Storage Late Wsh H2O to storage to storage Vnt to Atmos to storage Tank bottoms Adjd w/NaTPB ZERO

ZERO
$0.9967 \mathrm{E}-05$ ZERO $0.8198 \mathrm{E}-14$ $0.3253 \mathrm{E}+00 \quad 0.9452 \mathrm{E}-11$ $0.3955 \mathrm{E}+01 \quad 0.1149 \mathrm{E}-09$ $0.1184 \mathrm{E}-01$ $0.1440 \mathrm{E}+00$ $0.1800 \mathrm{E}+02$ $0.2664 \mathrm{E}-06$ $0.9808 \mathrm{E}-01$ $0.2929 \mathrm{E}-01$ $0.8806 \mathrm{E}-07$ $0.7105 \mathrm{E}-03$ $0.3742 \mathrm{E}-02$ $0.1021 \mathrm{E}+01$ $0.1021 \mathrm{E}+01$ $0.2841 \mathrm{E}-01$ $0.1356 \mathrm{E}+01$ $0.2636 \mathrm{E}-06$ $0.5103 \mathrm{E}+01$ ZERO $0.8028 \mathrm{E}-01$ $0.7848 \mathrm{E}-01$ ZERO $0.5257 \mathrm{E}-04$ $0.4368 \mathrm{E}+02$ $0.4921 \mathrm{E}+02$ $0.5621 \mathrm{E}+02$ $0.1051 \mathrm{E}+01$

$0.2358 \mathrm{E}-02$

ZERO ZERO ZERO $0.6306 \mathrm{E}-03$ ZERO
$7 \mathrm{E}-03$ $0.6117 \mathrm{E}-03$ $0.1531 \mathrm{E}-02$ ZERO ZERO ZERO ZERO

$0.6320 \mathrm{E}-05$ ZERO ZERO ZERO ZERO ZERO ZERO ZERO $0.7320 \mathrm{E}-05 \quad 0.2126 \mathrm{E}-15$ $0.2694 \mathrm{E}+01 \quad 0.7828 \mathrm{E}-10$ $0.8046 \mathrm{E}+00 \quad 0.2337 \mathrm{E}-10$ ZERO
$0.1748 \mathrm{E}-05$ $0.1952 \mathrm{E}-01 \quad 0.5670 \mathrm{E}-12$ $0.1028 \mathrm{E}+00 \quad 0.2986 \mathrm{E}-11$ $0.2804 \mathrm{E}+02$ - $0.0146 \mathrm{E}-09$ $0.7803 \mathrm{E} 00$ 0.8146E-09 $0.7803 E+00$ 0.2267E-10 $0.3724 \mathrm{E}+02$ $0.1082 \mathrm{E}-08$ $0.7463 \mathrm{E}-05 \quad 0.2168 \mathrm{E}-15$ $\begin{array}{rr}0.1402 \mathrm{E}+03 & 0.4072 \mathrm{E}-08 \\ \text { ZERO } & \text { ZERO }\end{array}$ $0.2218 \mathrm{E}+01 \quad 0.6445 \mathrm{E}-10$ $0.2156 \mathrm{E}+01$ : $0.6263 \mathrm{E}-10$ $0.1444 \mathrm{E}-02 \quad 0.4195 \mathrm{E}-13$ $0.2723 \mathrm{E}+03 \quad 0.7911 \mathrm{E}-08$ $0.1352 \mathrm{E}+04 \quad 0.392 .7 \mathrm{E}-07$ $0.6376 \mathrm{E}+03 \quad 0.1852 \mathrm{E}-07$

$0.7767 \mathrm{E}+01 \quad 0.4984 \mathrm{E}-09$ to storage Tank bot ZERO ZERO i ZERO $0.2853 \mathrm{E}-06$ $0.3390 \mathrm{E}-03$ $0.4121 \mathrm{E}-02$ $0.5152 \mathrm{E}+00$ $0.7626 \mathrm{E}-08$ $0.2807 \mathrm{E}-02$ $0.8382 \mathrm{E}-03$ $0.1003 \mathrm{E}+01$ $0.2520 E-08$ $0.2033 \mathrm{E}-04$ $0.1071 \mathrm{E}-03$ $0.2921 \mathrm{E}-01$ $0.8130 \mathrm{E}-03$ $0.3880 \mathrm{E}-01$ $0.7545 E-08$ $0.1460 \mathrm{E}+00$ $0.2298 \mathrm{E}-02$ $0.2246 \mathrm{E}-02$ $0.1504 \mathrm{E}-0.5$ $0.7647 \mathrm{E}+01$ $0.1408 \mathrm{E}+01$ $0.2809 \mathrm{E}+01$ $0.3008 \mathrm{E}-01$ $0.6748 E-04$ $0.9793 \mathrm{E}+00$ $0.7902 \mathrm{E}-10$ ZERO ZERO
ZERO
$0.1805 \mathrm{E}-04$ ZERO
$0.1732 \mathrm{E}-01$ $0.1732 \mathrm{E}-01$ $0.1805 \mathrm{E}-04$ $0.1805 \mathrm{E}-04$ $0.1037 \mathrm{E}-02$ $0.4383 \mathrm{E}-04$ ZERO ERO ZERO TERO 马ERO ZERO ZERO ZERO $\quad 0.2320 \mathrm{E}-1$ ZERO

$0.1736 \mathrm{E}-03$ $.2320 \mathrm{E}-12$ ZERO ZERO $\begin{array}{ll}\text { ZERO } & 0.1707 \mathrm{E}-14 \\ \text { ZERO } & 0.6788 \mathrm{E}-14\end{array}$ $\begin{array}{rr}\text { ZERO } & 0.6788 \mathrm{E}-14 \\ \text { ZERO } & \text { ZERO }\end{array}$ ZERO ZERO ZERO ZERO $0.2353 E-12$ ZERO

$\begin{array}{rr}\text { ZERO } & \text { ZERO } \\ \text { ZERO } & \text { ZERO } \\ 0.2853 E-06 & \text { ZERO } \\ 0.2767 E+00 & \text { ZERO } \\ 0.2108 E+00 & \text { ZERO } \\ 0.4232 E+01 & \text { ZERO } \\ 0.7626 E-08 & \text { ZERO } \\ 0.2807 E-02 & \text { ZERO } \\ 0.8382 E-03 & \text { ZERO } \\ 0.1003 E+01 & \text { ZERO } \\ 0.2520 E-08 & \text { ZERO } \\ 0.2033 E-04 & \text { ZERO } \\ 0.1071 E-03 & \text { ZERO } \\ 0.2921 E-01 & \text { ZERO } \\ 0.8130 E-03 & \text { ZERO } \\ 0.3880 E-01 & \text { ZERO } \\ 0.7545 E-08 & \text { ZERO } \\ 0.1006 E-02 & \text { ZERO } \\ \text { ZERO } & \text { ZERO } \\ 0.2298 E-02 & 0.9051 E-02 \\ 0.2246 E-02 & \text { ZERO } \\ \text { ZERO } & \text { ZERO } \\ 0.1504 E-05 & \text { ZERO } \\ 0.7976 E+01 & \text { ZERO } \\ 0.5939 E+00 & \text { ZERO } \\ 0.1572 E-01 & 0.3251 E+0 O \\ \text { ZERO } & 0.1931 E+01 \\ 0.6748 E-04 & \text { ZERO } \\ 0.9792 E+00 & \text { ZERO } \\ \text { ZERO } & \text { ZERO } \\ \text { ZERO } & \text { ZERO } \\ 0.1805 E-04 & \text { ZERO } \\ 0.1037 E-02 & \text { ZERO } \\ 0.7141 E-03 & \text { ZERO } \\ 0.4383 E-04 & \text { ZERO } \\ \text { ZERO } & \text { ZERO } \\ \text { ZERO } & \text { ZERO } \\ \text { ZERO } & \text { ZERO } \\ 0.4424 E-02 & \text { ZERO } \\ \text { ZERO } & \text { ZERO } \\ 0.4362 E-02 & \text { ZERO } \\ \text { ZERO } & \text { ZERO } \\ 0.1809 E-06 & \text { ZERO } \\ \text { ZERO } & \text { ZERO } \\ 0.3209 E-04 & \text { ZERO } \\ 0.1272 E-03 & \text { ZERO } \\ \text { ZERO } & \text { ZERO } \\ \text { ZERO } & \text { ZERO } \\ \text { ZERO } \\ 0.900\end{array}$
to LWPT ZERO ZERO ZERO ZERO ZERO ZERO ZERO ZERO ZERO ZERO ZERO ZERO ZERO ZERO ZERO ZERO ZERO ZERO $0.1268 \mathrm{E}-01$ ZERO ZERO ZERO ZERO ZERO $0.6324 E-01$ $0.2705 E+01$

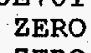
ZERO ZERO ZERO ZERO ZERO ZERO ZERO ZERO ZERO ZERO ZERO ZERO ZERO ZERO ZERO ZERO ZERO ZERO ZERO ZERO ZERO ZERO 
Table A-1. HLW Flowsheet Material Balance (Tank 51/ITP Cycle 1)

Section 0. Major streams in the Flowsheet Model

STREAM NUMBERS (CONT'D) $\rightarrow$ STREAM NAME

$38 \mathrm{SO} 2$

$141 \mathrm{SO} 3$

137 Semi Vol Cs20

136 Semi Vol $\mathrm{CsCl}$

126 Semi Vol Group A

140 Semi Vol Na2B407

138 Semi Vol NaCl

139 Semi Vol NaF

125 Semi Vol NaI

23 Semi Vol RuO2

$111 \mathrm{SiO} 2$

$48 \operatorname{Sr}(\mathrm{COOH}) 2$

$176 \mathrm{Sr}$ (NO3) 2

$153 \mathrm{Sr}(\mathrm{NaTi205)} 2$

$127 \mathrm{Sr}(\mathrm{OH}) 2$

$30 \mathrm{SrCO}_{3}$

46 sro

$159 \mathrm{TCO} 2$

92 ThO2

119 Tritium

119 Tritiu

$62 \mathrm{UO} 2(\mathrm{COOH}) 2$

177 $\mathrm{UO} 2$ (NO3) 2

$16 \mathrm{UO} 2(\mathrm{OH}) 2$

$27 \mathrm{Y}(\mathrm{COOH}) 3$

$178 \mathrm{Y}(\mathrm{NO} 3)$

31 Y2 (CO3) 3

$47 \mathrm{Y} 203$

100 zeolite

$44 \mathrm{Zn}$ (COOH) 2

$179 \mathrm{zn}(\mathrm{NO} 3) 2$

$57 \mathrm{zno}$

$104 \mathrm{zrO} 2$

13 hydrate $\mathrm{H} 2 \mathrm{O}$

1 water

TOTAL FLOW, LB/HR

TEMPERATURE， DEG C

PRESSURE， ATM

PRESSURE， PSIG

PRESSURE, MM HG

ENTHALPY， PCU/RR

VAPOR FLOW, CEM

IIOUID FLOW, GPM

DENSITY， LBS/FT3

PHASE

411

$$
407
$$

409

414

418

457

to Storage Vnt to Atmos to storage Tank bottoms Adjd w/NaTPB ZERO ZERO

ZERO

ZERO

ZERO

ZERO

ZERO

ZERO

ZERO

ZERO

ZERO

$0.2347 \mathrm{E}-06$

ZERO

ZERO

ZERO

ZERO

$0.1465 \mathrm{E}-08$

(1)

ZERO

ZERO

$0.1013 E-02$

ZERO

$0.1095 \mathrm{E}-06$

ZERO

ZERO

ZERO

$0.5033 \mathrm{E}-01$

ZERO

ZERO

$0.1319 \mathrm{E}+04$

$0.1495 \mathrm{E}+04$

$0.2619 \mathrm{E}+02$

$0.1000 \mathrm{E}+01$

ZERO

$0.3588 \mathrm{E}+05$

$0.2757 \mathrm{E}+01$

$.6759 E+02$
LIQUID
zERO
zERO

ZERO ZERO

ZERO

ZERO

ZERO

ZERO

ZERO

ZERO

ZERO

ZERO

ZERO

ZERO

ZERO

ZFRO

$0.4796 \mathrm{E}-05$

ZERO

ZERO

ZERO

ZERO

$0.4026 \mathrm{E}-07$

(1)

ZERO

ZERO

EERO

$0.5683 \mathrm{E}-05$

ZERO

ZERO

ERO

$0.1383 \mathrm{E}+01$

ZERO

ZERO

ZERO

$0.8550 \mathrm{E}+04$

$0.1154 \mathrm{E}+0.5$

ZERO

ZERO

ZERO

ZERO

ZERO

ZERO

ZERO

$0.1418 \mathrm{E}-11$

ZERO

$0.2167 \mathrm{E}-12$

$0.1391 \mathrm{E}-15$

$0.4829 \mathrm{E}-14$

ZERO

ZERO

$0.6453 \mathrm{E}-13$

$0.3193 E-09$

$0.3995 E-13$ ZERO

ZERO

ZERO

$0.1654 \mathrm{E}-15$

ZERO

$0.5145 \mathrm{E}-13$

ZERO

$0.1053 \mathrm{E}-10$

ZERO

$0.1417 \mathrm{E}-12$
$0.6527 \mathrm{E}+02$

$0.6527 \mathrm{E}+02$
$0.2409 \mathrm{E}+04$

$0.3000 \mathrm{E}+02$

$0.4000 E+02$

$0.9656 \mathrm{E}+00$

ZERO

$0.2806 \mathrm{E}+06$

$0.1927 \mathrm{E}+02$

$0.6421 \mathrm{E}+05$

$0.6155 \mathrm{E}+03$

LIQUID

$0.6524 \mathrm{E}-01$

VAPOR
$0.7339 \mathrm{E}+03$ storage

ZERO

ZERO

ZERO

ZERO

ZERO

ZERO

ZERO

$0.2666 \mathrm{E}-01$

ZERO

ZERO

$0.4072 \mathrm{E}-02$

$0.6716 \mathrm{E}-08$

$0.9081 \mathrm{E}-04$

ZERO

ZERO

$0.1213 \mathrm{E}-02$

$0.4194 \mathrm{E}-10$

$0.7512 \mathrm{E}-03$

ZERO

ZERO
$0.2900 \mathrm{E}-04$

$0.2900 \mathrm{E}-04$

ZERO

$0.3135 \mathrm{E}-08$

$0.9683 \mathrm{E}-03$

ZERO

$0.1440 \mathrm{E}-02$

ZERO

$0.2665 \mathrm{E}-02$

$0.4114 \mathrm{E}+03$

$0.4712 \mathrm{E}+03$

ZERO

ZERO

ZERO

ZERO

ZERO

ZERO

ZERO

ZERO

$0.2666 \mathrm{E}-01$

ZERO

$0.4072 \mathrm{E}-02$

$0.6716 \mathrm{E}-08$

$0.9081 E-04$

ZERO

ZERO

$0.1213 \mathrm{E}-02$

$0.3525 \mathrm{E}-10$

$0.3525 E-10$ ZERO

ZERO

ZERO

0.3135 E-08

$0.9683 \mathrm{E}-03$

ZERO

ZERO

ZERO

$0.3589 \mathrm{E}-01$

$0.4655 \mathrm{E}+03$

$0.3000 \mathrm{E}+02$

$0.3846 \mathrm{E}+02$ $-0.5055 \mathrm{E}+00$

$0.7339 \mathrm{E}+03$

$0.1249 \mathrm{E}+05$

$0.1579 \mathrm{E}+05$

$0.9134 \mathrm{E}+00$

$0.9016 \mathrm{E}+00$

$.6437 \mathrm{E}+02$

LIOUID

LIOUID
$0.9656 \mathrm{E}+00$

458

TPB addn

to LWPT

ZERO

ZERO

ZERO

ZERO

ZERO

ZERO

ZERO

ZERO

ZERO

ZERO

ZERO

ZERO

ZERO

ZERO

ZERO

ZERO

ZERO

ZERO

ZERO

ZERO

ZERO

ZERO

ZERO

ZERO

ZERO

ZERO

ZERO

ZERO

ZERO

TERO

zERO 
Table A-1. HLW Flowsheet Material Balance (Tank 51/TTP Cycle 1) Section 0 . Major streams in the Flowsheet Model.

STREAM NUMBERS $\rightarrow$ STREAM NAME

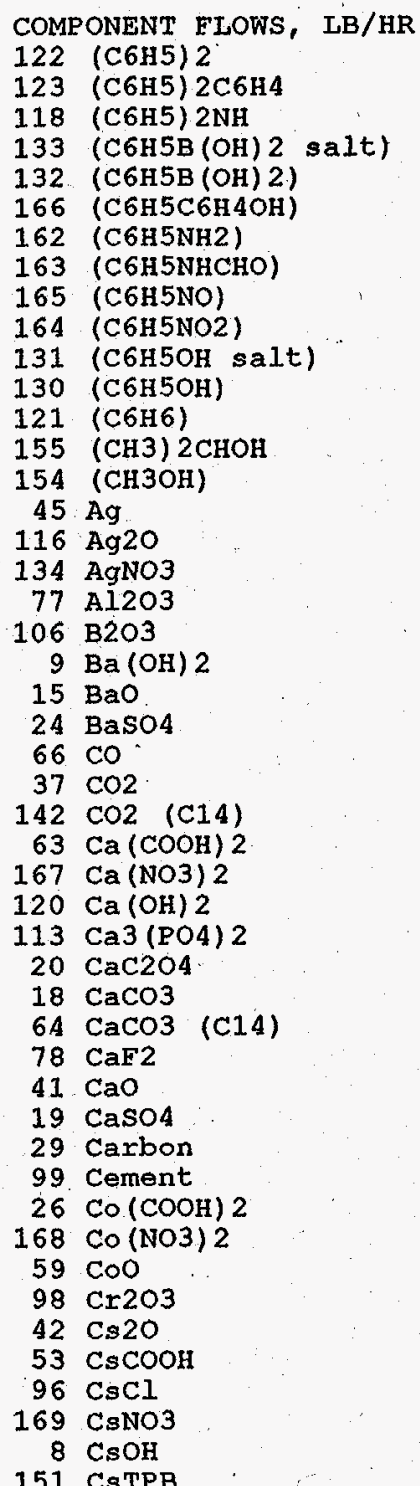

303

309

316

306

Unwshd W/O SN Decant $508 \mathrm{NaOH}$ Vnt to Atmo sldg slurry to A/D Tank to' W/O Tank sldg Slurry frm W/O Tnk to A/D Tank

$$
\begin{array}{r}
0.2059 E-06 \\
0.8746 E-08 \\
0.5823 E-08 \\
0.2319 E-09 \\
\text { ZERO } \\
\text { ZERO } \\
\text { ZERO } \\
\text { ZERO } \\
\text { ZERO } \\
\text { ZERO } \\
0.1086 E-09 \\
\text { ZERO } \\
0.5111 \text { E } 00 \\
0.2136 E-06 \\
0.1592 E-07 \\
\text { ZERO } \\
0.1909 E-11 \\
\text { ZERO } \\
0.8112 E-10 \\
0.1044 E-11 \\
0.8186 E-20 \\
\text { ZERO } \\
0.1121 E-11 \\
\text { ZERO } \\
\text { ZERO } \\
\text { ZERO } \\
\text { ZERO } \\
\text { ZERO } \\
0.2384 E-14 \\
0.3506 E-09 \\
\text { ZERO } \\
\text { ZERO } \\
\text { ZERO } \\
\text { ZERO } \\
\text { ZERO } \\
\text { ZERO } \\
\text { ZERO } \\
\text { ZERO } \\
\text { ZERO } \\
0.6426 E-13 \\
0.1636 E-09 \\
0.2406 E-13 \\
\text { ZERO } \\
\text { ZERO } \\
\text { ZERO } \\
0.1088 E-11 \\
0.6561 E-10 \\
\end{array}
$$

\begin{tabular}{ll} 
ZERO & ZERO \\
ZERO & ZERO \\
ZERO & ZERO \\
ZERO & ZERO \\
ZERO & ZERO \\
ZERO & ZERO \\
ZERO & ZERO \\
ZERO & ZERO \\
ZERO & ZERO \\
ZERO & ZERO \\
ZERO & ZERO \\
ZERO & ZERO \\
ZERO & ZERO \\
ZERO & ZERO \\
ZERO & ZERO \\
ZERO & ZERO \\
ZERO & ZERO \\
ZERO & ZERO \\
ZERO & ZERO \\
ZERO & ZERO \\
ZERO & ZERO \\
ZERO & ZERO \\
ZERO & ZERO \\
ZERO & ZERO \\
ZERO & ZERO \\
ZERO & ZERO \\
ZERO & ZERO \\
ZERO & ZERO \\
ZERO & ZERO \\
ZERO & ZERO \\
ZERO & ZERO \\
ZERO & ZERO \\
ZERO & ZERO \\
ZERO & ZERO \\
ZERO & ZERO \\
ZERO & ZERO \\
ZERO & ZERO \\
ZERO & ZERO \\
ZERO & ZERO \\
ZERO & ZERO \\
ZERO & ZERO \\
ZERO & ZERO \\
ZERO & ZERO \\
ZERO & ZERO \\
ZERO & ZERO \\
ZERO & ZERO \\
ZERO & ZERO \\
ZERO & ZERO \\
\hline
\end{tabular}

ZERO
ZERO
ZERO
ZERO
ZERO
ZERO
ZERO
ZERO
ZERO
ZERO
ZERO
ZERO
ZERO
ZERO
ZERO
ZERO
ZERO
ZERO
ZERO
ZERO
ZERO
ZERO
ZERO
ZERO
ZERO
ZERO
ZERO
ZERO
ZERO
ZERO
ZERO
ZERO
ZERO
ZERO
ZERO
ZERO
ZERO
ZERO
ZERO
ZERO
ZERO
ZERO
ZERO
ZERO
ZERO
ZERO
ZERO
ZERO

Date: January 1995

\begin{tabular}{rrr} 
ZERO & ZERO & ZERO \\
ZERO & ZERO & ZERO \\
ZERO & ZERO & ZERO \\
ZERO & ZERO & ZERO \\
ZERO & ZERO & ZERO \\
ZERO & ZERO & ZERO \\
ZERO & ZERO & ZERO \\
ZERO & ZERO & ZERO \\
ZERO & ZERO & ZERO \\
ZERO & ZERO & ZERO \\
ZERO & ZERO & ZERO \\
ZERO & ZERO & ZERO \\
ZERO & ZERO & ZERO \\
ZERO & ZERO & ZERO \\
ZERO & ZERO & ZERO \\
ZERO & ZERO & ZERO \\
$0.1240 E-01$ & ZERO & ZERO \\
ZERO & ZERO & ZERO \\
$0.1042 E+02$ & ZERO & ZERO \\
ZERO & ZERO & ZERO \\
0.2484 E-02 & $0.2196 E-02$ & ZERO \\
ZERO & ZERO & ZERO \\
$0.1802 E-01$ & ZERO & ZERO \\
ZERO & ZERO & ZERO \\
ZERO & ZERO & ZERO \\
ZERO & ZERO & ZERO \\
ZERO & ZERO & ZERO \\
ZERO & ZERO & ZERO \\
$0.1875 E-03$ & $0.1657 E-03$ & ZERO \\
$0.2516 E+01$ & ZERO & ZERO \\
ZERO & ZERO & ZERO \\
$0.3111 E+01$ & ZERO & ZERO \\
$0.1532 E-06$ & ZERO & ZERO \\
$0.1828 E-01$ & ZERO & ZERO \\
$0.4073 E-02$ & ZERO & ZERO \\
$0.3611 E-01$ & ZERO & ZERO \\
ZERO & ZERO & ZERO \\
ZERO & ZERO & ZERO \\
ZERO & ZERO & ZERO \\
ZERO & ZERO & ZERO \\
ZERO & ZERO & ZERO \\
$0.1984 E+00$ & ZERO & ZERO \\
\hline $1240 E-03$ & ZERO & ZERO \\
ZERO & ZERO & ZERO \\
ZERO & ZERO & ZERO \\
ZERO & ZERO & ZERO \\
ZERO & ZERO \\
$0.5467 E-04$ & ZERO
\end{tabular}

Seation 0, Page 10 of 37 
Table A-1. HLW Flowsheet Material Balance (Tank 51/ITP Cyclo 1 )

Section 0. Major streams in the Flowsheet Model.

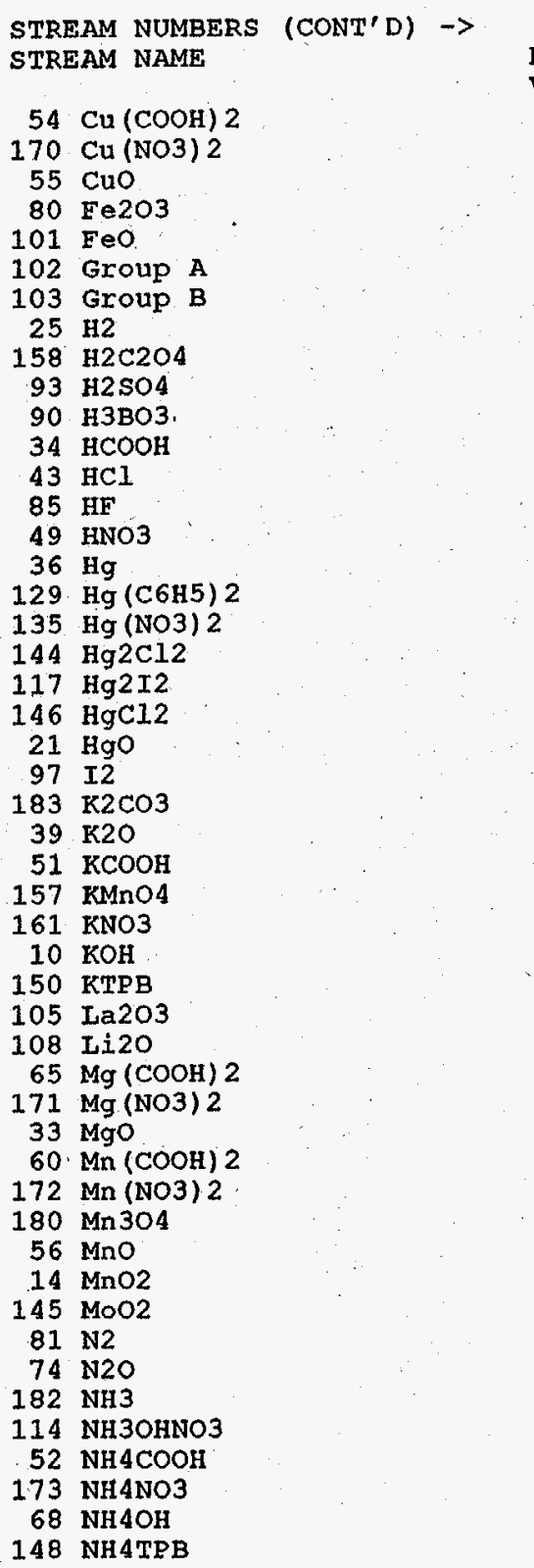

41 303

309

316

$\begin{array}{cccccc}417 & 303 & 309 & 316 & 306 & 308 \\ \text { Precip strg } & \text { Unwshd A/D } & \text { Wsh/Aux H2O } & \text { Makeup H2O } & \text { Unwshd W/O } & \text { SN Decant }\end{array}$

$\begin{array}{cccccc}417 & 303 & 309 & 316 & 306 & 308 \\ \text { Precip strg } & \text { Unwshd A/D } & \text { Wsh/Aux H2O } & \text { Makeup H2O } & \text { Unwshd W/O } & \text { SN Decant }\end{array}$

Sldg Slurry to A/D Tank to W/O Tank sldg Slurry frm W/O Tnk to A/D Tank

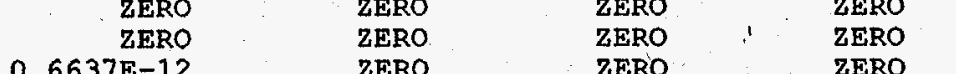

$0.6637 \mathrm{E}-12$

E-10

ZERO ZERO

$0.4114 \mathrm{E}-13$

$0.1265 \mathrm{E}-14$

$0.1045 \mathrm{E}-01$

ZERO

ZERO

ZERO

ZERO

ZERO

ZERO

$0.2374 \mathrm{E}-09$

ZERO

ZERO

ZERO

$0.2326 \mathrm{E}-12$

ZERO

$0.4304 \mathrm{E}-11$

ZERO

ZERO

$0.1328 \mathrm{E}-11$

$0.2551 \mathrm{E}-07$

$$
\text { ZERO }
$$

$0.1843 \mathrm{E}-12$

ZERO

$0.1079 \mathrm{E}-11$

ZERO

ZERO

$2061 \mathrm{E}-11$

$0.6853 \mathrm{E}-13$

$0.2207 \mathrm{E}+04$

$0.7061 \mathrm{E}-01$

$0.2598 \mathrm{E}-01$

ZERO

ZERO

$0.1701 \mathrm{E}-09$

ZERO ZERO

$\begin{array}{ll}\text { ZERO } & \text { ZERO } \\ \text { ZERO } & \text { ZERO }\end{array}$

ZERO ZERO

ZERO ZERO

ZERO ZERO

ZERO ZERO

ZERO

ZERO

ZERO

ZERO

ZERO

ZERO

ZERO

ZERO

ZERO

ZERO

ZERO

ZERO

ZERO

ZERO

ZERO

ZERO

ZERO

ZERO

ZERO

ZERO

ZERO

ZERO

ZERO

ZERO

ZERO

ZERO

ZERO

ZERO

ZERO

ZERO

ZERO

ZERO

ZERO

ZERO

ZERO

ZERO

ZERO

ZERO $\quad 0.3416 \mathrm{E}+02$

ZERO

ZERO

$\begin{array}{rrr}\text { ZERO } & \text { ZERO } & \text { ZERO } \\ 0.6119 \mathrm{E}-02 & 0.1869 \mathrm{E}-02 & \text { ZERO } \\ 0.1884 \mathrm{E}-03 & 0.4512 \mathrm{E}-05 & \text { ZERO }\end{array}$

ZERO

ZERO ZERO

ZERO
$0.1237 \mathrm{E}-02$
ZERO

ZERO

ZERO

ZERO

ZERO

ZERO

ZERO

ZERO
ZERO

ZERO

ZERO

ZERO

ZERO

ZERO

ZERO

ZERO

ZERO

ZERO

ZERO

ZERO

ZERO

ZERO

ZERO

ZERO

ZERO

ZERO

ZERO

ZERO

ZERO

ZERO

ZERO

ZERO

ZERO

ZERO

ZERO

ZERO

ZERO

ZERO

ZERO

ZERO

ZERO

ZERO

ZERO

ZERO

ZERO

ZERO

ZERO

ZERO

ZERO

ZERO

ZERO

ZERO

ZERO

ZERO

ZERO

ZERO

ZERO

ZERO

ZERO

ZERO

ZERO

ZERO

ZERO

ZERO

ZERO

ZERO

ZERO

ZERO

ZERO

ZERO

ZERO

ZERO

Date: January 1995

WSRC-TR-95-0019 (Revision 
Table A-1. HLW Flowsheet Material Balance (Tank 51/ITP Cycle 1) Section 0. Major streams in the Flowsheet. Model

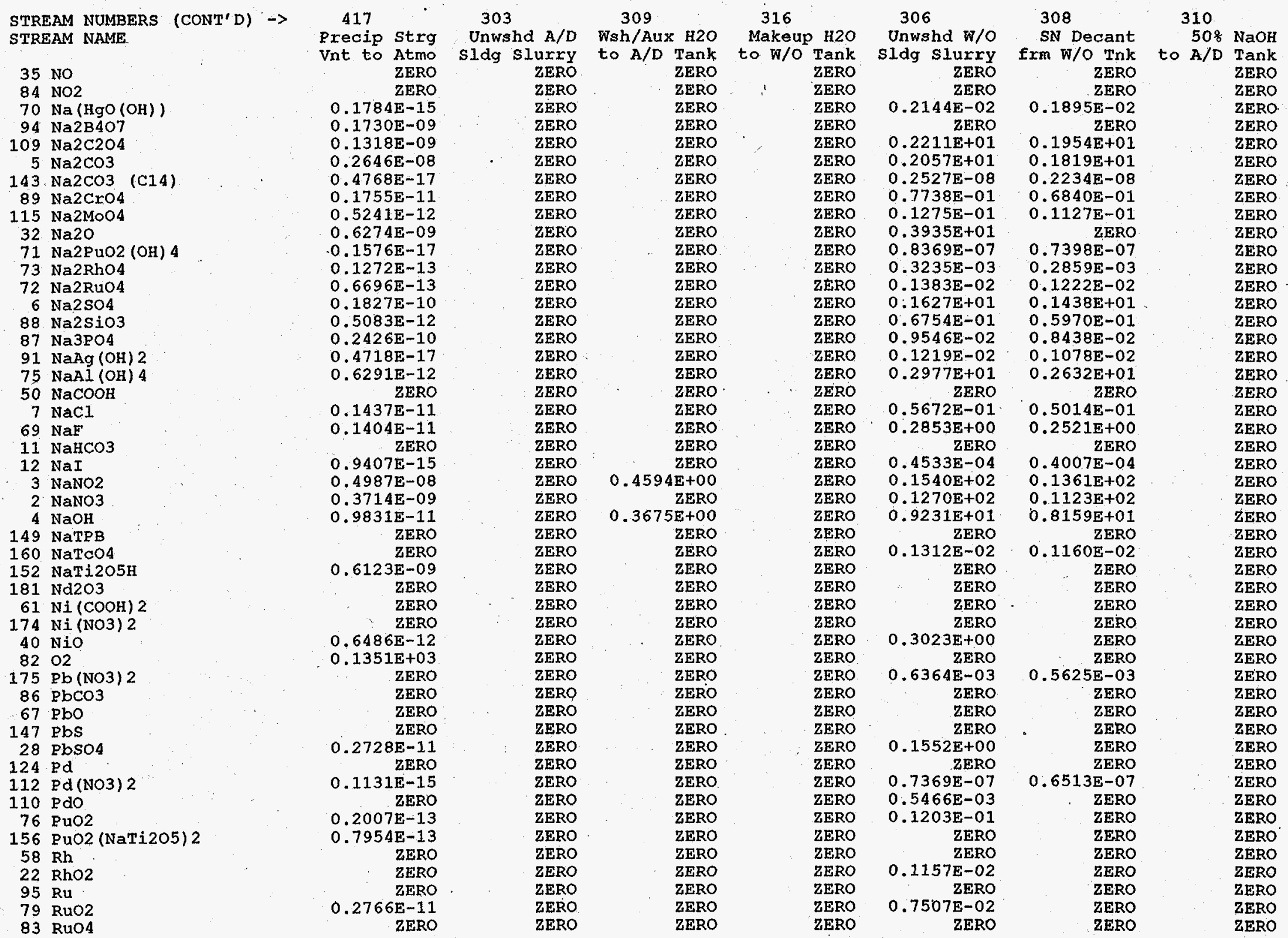

WSRC-TR-95-0019 (Revision 0) $\quad$ Date: January 1995 
Table A-1. HLW Flowsheet Material Balance (Tank 51/ITP Cycle 1) section 0. Major streams in the Flowsheet Model

STREAM NUMBERS (CONT'D) $\rightarrow$ STREAM NAME

$\begin{array}{rr}38 & \mathrm{SO} 2 \\ 141 & \mathrm{SO} 3\end{array}$

137 Semi Vol Cs2O

136 Semi Vol CsCl

126 Semi Vol Group A

140 Semi Vol Na2B4O

139 Semi Vol NaF

125 Semi Vol NaI
23 Semi Vol RuO2

$111 \mathrm{siO2}$

$48 \mathrm{Sr}(\mathrm{COOH}) 2$

$153 \cdot \mathrm{Sr}(\mathrm{NaTi2O5)} 2$

$127 \operatorname{Sr}(\mathrm{OH}) 2$

$30 \mathrm{SrCO}$

$$
46 \text { Sro }
$$

$159 \mathrm{TaO} 2$

92 ThO2

119 Tritium

$17 \mathrm{U} 308$

$62 \mathrm{UO2}$ (COOH) 2

177 UO2 (NO3) 2

$16 \mathrm{VO} 2(\mathrm{OH}) 2$

178 Y (NO3) 3

$128 \mathrm{Y}(\mathrm{OH}) 3$

$31 \mathrm{Y} 2(\mathrm{CO} 3) 3$

47 Y203

100 zeolite

$44 \mathrm{Zn}(\mathrm{COOH}) 2$
$179 \mathrm{Zn}(\mathrm{NO3}) 2$

$57 \mathrm{znO}$

$104 \mathrm{ZxO2}$

13 hydrate H2O

1 water

TOTAL ELOW, LB/HR

TEMPERATURE, DEG C

PRESSURE, ATM

PRESSURE, PSIG

PRESSURE, MM HG

ENTHALPY，PCU/HR

VAPOR FLOW, CFM

ATO

DENSITY, LBS/FT3

PHASE

17

303

Vnt to Atmo

$\begin{array}{ccc}303 & 309 & 316 \\ \text { Unwshd A/D } & \text { Wsh/Aux H2O } & \text { Makeup H2O }\end{array}$

to $A / D$ Tank to W/O Tank

306

$\begin{array}{lll}\text { ZERO } & \text { ZERO } & \text { ZERO } \\ \text { ZERO } & \text { ZERO }, & \text { ZERO } \\ \text { ZERO } & \text { ZERO } & \text { ZERO } \\ \text { ZERO } & \text { ZERO } & \text { ZERO }\end{array}$

ZERO

ZERO

ZERO

ZERO

ZERO

ZERO

ZERO

$0.1667 \mathrm{E}-10$

ZERO

ZERO
$0.2546 \mathrm{E}-11$

$0.4200 \mathrm{E}-17$

$0.5679 \mathrm{E}-13$

ZERO

ZERO

$0.7587 \mathrm{E}-12$

$0.6692 \mathrm{E}-11$

$0.6692 \mathrm{E}-11$
$0.4697 \mathrm{E}-12$

$7 \mathrm{E}-12$
ZERO

ZERO

$0.1813 \mathrm{E}-13$ ZERO

ZERO
$0.1960 \mathrm{E}-17$

ZERO

$0.6054 \mathrm{E}-12$

ZERO

ZERO

$0.1238 \mathrm{E}-09$

ZERO

$0.2244 \mathrm{E}-10$

$0.1631 \mathrm{E}+02$

$0.2359 \mathrm{E}+04$

$0.4000 \mathrm{E}+02$

$0.9656 \mathrm{E}+00$

$-0.5055 \mathrm{E}+00$

$0.7339 \mathrm{E}+03$

$0.3425 \mathrm{E}+05$

$0.5960 \mathrm{E}+03$

$0.6597 \mathrm{E}-01$

VAPOR
ZERO

ZERO.

ZERO

ZERO

ZERO

ZERO

ZERO

ZERO

ZERO

ZERO

ZERO

ZERO

ZERO

ZERO

ZERO

ZERO

ZERO

ZERO

ZERO

ZERO

ZERO

ZERO

ZERO

ZERO

ZERO

ZERO

ZERO

ZERO

ZERO

ZERO

ZERO

ZERO

ZERO

ZERO

$0.5000 \mathrm{E}+02$

$0.1000 \mathrm{E}+01$

ZERO

ZERO

LIQUID

Date: January 1995

ZERO

ZERO

ZERO

ZERO

ZERO

ZERO

ZERO

ZERO

ZERO

ZERO

ZERO

ZERO

ZERO

ZERO

ZERO

ZERO

ZERO

ZERO

ZERO

ZERO

ZERO

ZERO

$0.9180 \mathrm{E}+03$

$0.9188 \mathrm{E}+03$

$0.4800 \mathrm{E}+02$

$0.1000 \mathrm{E}+01$
ZERO

$0.1000 \mathrm{E}+01$

ZERO

ZERO

ZERO

LIQUID
308

310

sldg Slurry frm W/O Tnk to $A / D$ Tank

$\begin{array}{llll} & \text { ZERO } & \text { ZERO } & \text { ZERO } \\ \text { ZERO } & \text { ZERO } & \text { ZERO } & \text { ZERO }\end{array}$

ZERO ZERO $\quad$ ZERO ZERO

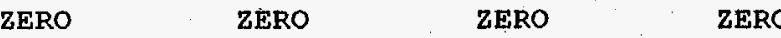

$\begin{array}{llll}\text { ZERO } & \text { ZERO } & \text { ZERO } & \text { ZERO } \\ \text { ZERO }\end{array}$

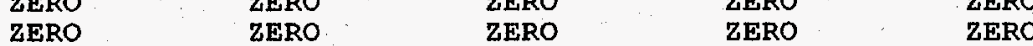

ZERO ZERO ZERO ZERO

ZERO ZERO ZERO ZERO

ZERO ZERO ZERO ZERO

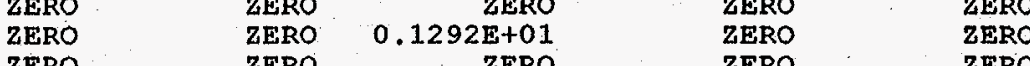

$0.8437 \mathrm{E}+02$

$437 E+02$
LIQUID

$0.3693 \mathrm{E}+00$

$135 \mathrm{E}+02$
IIQUID

ZERO
IIQUID 
Table A-1. HLW Flowsheet Material Balance (Tank 51/ITP Cycle 1) section 0. Major streams in the Flowsheet Model

STREAM NUMBERS $\rightarrow$ STREAM NAME

COMPONENT FLOWS, LB/HR 122 (C6H5) 2

123 (C6H5) 2C6H4

118 (C6H5) $2 \mathrm{NH}$

133 (C6H5B (OH) 2 salt)

132 ( $\mathrm{C} 6 \mathrm{H} 5 \mathrm{~B}(\mathrm{OH}) 2)$

166 (C6H5C6H4O

162 (C6H5NH2)

165 (C6H5NO)

164 (C6H5NO2)

131 (C6H5OH salt)

130 (C6H5OH)

121 (C6H6)
155 (CH3) $2 \mathrm{CHOH}$

154 (СH3OH)

$45 \mathrm{Ag}$

116 Ag20

134 AgNO3

$106 \mathrm{~B} 203$

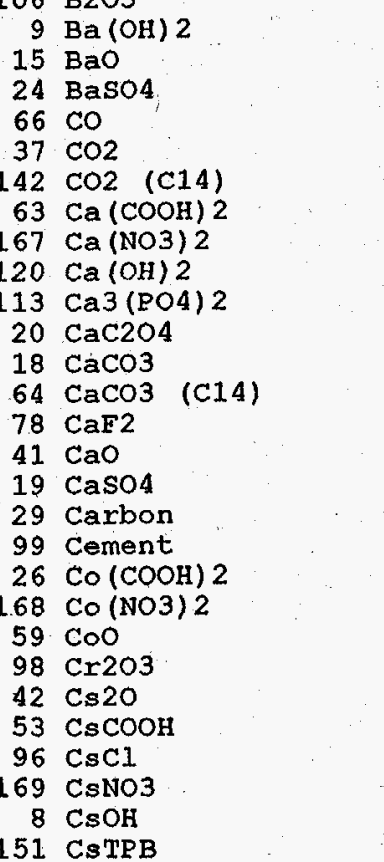

151 CsTPB

WSRC-TR-95-0019 (Revision

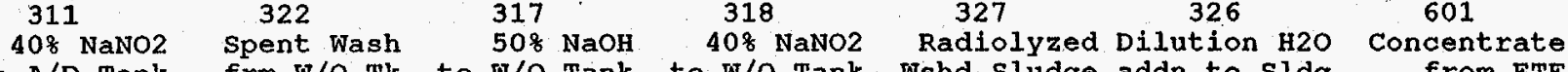

317

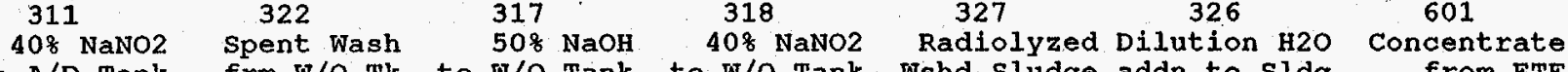

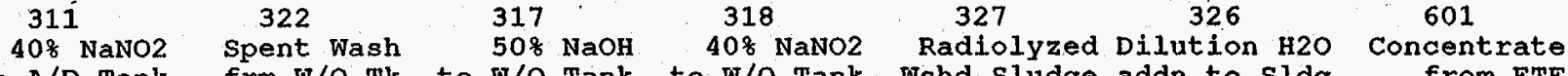

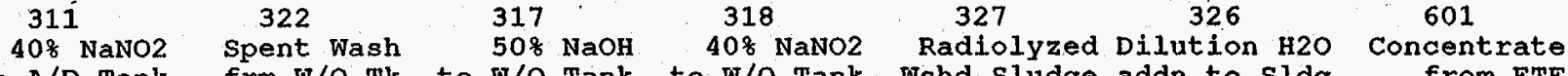

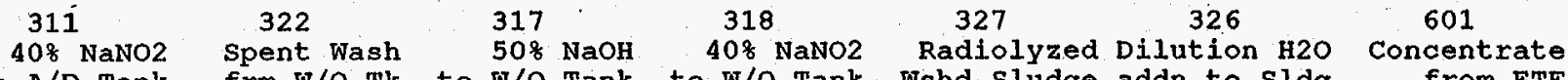
to A/D Tank frm W/O Tk to W/O Tank to W/O Tank Wshd sludge addn to sldg from ETF

\begin{tabular}{|c|c|c|c|c|c|c|}
\hline ZERO & ZERO & ZERO & ZERO & ZERO & ZERO & ZERO \\
\hline ZERO & ZERO & ZERO & ZERO & ZERO & ZERO & ZERO \\
\hline ZERO & ZERO & ZERO & ZERO & ZERO & ZERO & ZERO \\
\hline ZERO & ZERO & ZERO & ZERO & ZERO & ZERO & ZERO \\
\hline ZERO & ZERO & ZERO & ZERO & ZERO & ZERO & ZERO \\
\hline ZERO & ZERO & ZERO & ZERO & ZERO & ZERO & ZERO \\
\hline ZERO & ZERO & ZERO & ZERO & ZERO & ZERO & ZERO \\
\hline ZERO & ZERO & ZERO & ZERO & ZERO & ZERO & ZERO \\
\hline ZERO & ZERO & ZERO & ZERO & ZERO & ZERO & ZERO \\
\hline ZERO & ZERO & ZERO & ZERO & ZERO & ZERO & ZERO \\
\hline ZERO & ZERO & ZERO & ZERO & ZERO & ZERO & ZERO \\
\hline ZERO & ZERO & ZERO & ZERO & ZERO & ZERO & ZERO \\
\hline ZERO & ZERO & ZERO & ZERO & ZERO & ZERO & ZERO \\
\hline ZERO & ZERO & ZERO & ZERO & ZERO & ZERO & ZERO \\
\hline ZERO & ZERO & ZERO & ZERO & ZERO & ZERO & ZERO \\
\hline ZERO & ZERO & ZERO & ZERO & ZERO & ZERO & ZERO \\
\hline ZERO & $0.5206 \mathrm{E}-07$ & ZERO & ZERO & $0.1018 \mathrm{E}-01$ & ZERO & $0.1757 \mathrm{E}-02$ \\
\hline ZERO & ZERO & ZERO & ZERO & ZERO & ZERO & ZERO \\
\hline ZERO & $0.4374 \mathrm{E}-04$ & ZERO & ZERO & $0.8551 \mathrm{E}+01$ & ZERO & $0.8794 \mathrm{E}-01$ \\
\hline ZERO & ZERO & ZERO & ZERO & ZERO & ZERO & ZERO \\
\hline ZERO & $0.2123 \mathrm{E}-02$ & ZERO & ZERO & $0.2968 \mathrm{E}-03$ & ZERO & ZERO. \\
\hline ZERO & ZERO & ZERO & ZERO & ZERO & ZERO & ZERO \\
\hline ZERO & $0.7569 \mathrm{E}-07$ & ZERO & ZERO & $0.1480 \mathrm{E}-01$ & ZERO & $0.2780 \mathrm{E}-02$ \\
\hline ZERO & - ZERO & ZERO & ZERO & ZERO & ZERO & ZERO \\
\hline ZERO & ZERO & ZERO & ZERO & ZERO & ZERO & ZERO \\
\hline ZERO & ZERO & ZERO & ZERO & ZERO & ZERO & ZERO \\
\hline ZERO & ZERO & ZERO & ZERO & ZERO & ZERO & ZERO \\
\hline ZERO & ZERO & ZERO & ZERO & ZERO & ZERO & ZERO \\
\hline ZERO & $0.1602 \mathrm{E}-03$ & ZERO & ZERO & $0.224 \mathrm{OE}-04$ & ZERO & ZERO \\
\hline ZERO & $0.1056 \mathrm{E}-04$ & ZERO & ZERO & $0.2065 \mathrm{E}+01$ & ZERO & $0.2178 \mathrm{E}+00$ \\
\hline ZERO & ZERO & ZERO & ZERO & ZERO & ZERO & ZERO \\
\hline ZERO & $0.1307 \mathrm{E}-04$ & ZERO & ZERO & $0.2554 \mathrm{E}+01$ & ZERO & $0.4990 \mathrm{E}+00$ \\
\hline ZERO & $0.6432 \mathrm{E}-12$ & ZERO & ZERO & $0.1257 \mathrm{E}-06$ & ZERO & ZERO \\
\hline ZERO & $0.7 .677 \mathrm{E}-07$ & ZERO & ZERO & $0.1501 \mathrm{E}-01$ & ZERO & ZERO \\
\hline ZERO & $0.1711 \mathrm{E}-07$ & ZERO & ZERO & $0.3344 \mathrm{E}-02$ & ZERO & ZERO \\
\hline ZERO & $0.1516 \mathrm{E}-06$ & ZERO & ZERO & $0.2964 \mathrm{E}-01$ & ZERO & ZERO \\
\hline ZERO & ZERO & ZERO & ZERO & ZERO & ZERO & ZERO \\
\hline ZERO & ZERO & ZERO & ZERO & ZERO & ZERO & ZERO \\
\hline ZERO & ZERO & ZERO & ZERO & ZERO & ZERO & ZERO \\
\hline ZERO & ZERO & ZERO & ZERO & ZERO & ZERO & ZERO \\
\hline ZERO & ZERO & ZERO & ZERO & ZERO & ZERO & ZERO \\
\hline ZERO & $0.8334 \mathrm{E}-06$ & ZERO & ZERO & $0.1629 \mathrm{E}+00$ & ZERO & ZERO \\
\hline ZERO & $0.5208 \mathrm{E}-09$ & ZERO & ZERO & $0.1018 \mathrm{E}-03$ & ZERO & ZERO \\
\hline ZERO & ZERO & ZERO & ZERO & ZERO & ZERO & ZERO \\
\hline ZERO & ZERO & ZERO & ZERO & ZERO & ZERO & ZERO \\
\hline ZERO & ZERO & ZERO & ZERO & ZERO & ZERO & ZERO \\
\hline ZERO & $0.5285 \mathrm{E}-04$ & ZERO & ZERO & $0.7390 \mathrm{E}-05$ & ZERO & $0.4011 \mathrm{E}-08$ \\
\hline ZERO & ZERO & ZERO & ZERO & ZERO & ZERO & ZERO \\
\hline
\end{tabular}

Date: January 1995 
Table A-1. HLW Flowsheet Material Balance (Tank 51/ITP Cycle 1) section 0. Major streams in the Flowsheet Model

STREAM NUMBERS (CONT'D) -> STREAM NAME

$54 \mathrm{Cu}(\mathrm{COOH}) 2$

$170 \mathrm{Cu}(\mathrm{NO} 3) 2$

55 CuO

$80 \mathrm{Fe} 2 \mathrm{O3}$

101 FeO

102 Group A

$25 \mathrm{H} 2$.

$158 \mathrm{H} 2 \mathrm{C} 2 \mathrm{O} 4$

$93 \mathrm{H} 2 \mathrm{SO}$

$90 \mathrm{H} 3 \mathrm{BO} 3$.

$34 \mathrm{HCOOH}$

$43 \mathrm{HCl}$

$85 \mathrm{HF}$

49 HNO3

$36 . \mathrm{Hg}$

$129 \mathrm{Hg}(\mathrm{C} 6 \mathrm{H} 5) 2$

$135 \mathrm{Hg}(\mathrm{NO} 3) 2$

$144 \mathrm{Hg} 2 \mathrm{Cl}$

$117 \mathrm{Hg} 2 \mathrm{I} 2$

$146 \mathrm{HgCl2}$

$21 \mathrm{HgO}$
$97 \mathrm{I} 2$

$\begin{array}{rl}97 & 12 \\ 183 & \mathrm{~K} 2 \mathrm{CO} 3\end{array}$

39. K2O

$51 \mathrm{KCOOH}$

$157 \mathrm{KMnO} 4$

161 KNO3

$150 \mathrm{KTPB}$

$105 \mathrm{I} a 203$

105 Li20

65. $\mathrm{Mg}(\mathrm{COOH}) 2$

$171 \mathrm{Mg}$ (NO3) 2

$33 \mathrm{MgO}$

$60 \mathrm{Mn}(\mathrm{COOH}) 2$

$172 \mathrm{Mn}(\mathrm{NO} 3) 2$

$180 \mathrm{Mn} 30$

$56 \mathrm{MnO}$

$14 \mathrm{MnO} 2$

$145 \mathrm{MOO2}$

$81 \mathrm{~N} 2$

$74 \mathrm{~N} 2 \mathrm{O}$

114 NH3OHNO3

52. $\mathrm{NH} 4 \mathrm{COOH}$

173 NH4NO3

$68 \mathrm{NH} 4 \mathrm{OH}$

148 NK4TPB

WSRC-TR-95-0019 (Revision

$$
\begin{array}{ccc}
322 & 317 & 318 \\
& 508 &
\end{array}
$$

311

327

326

to A/D Tank

ZERO

ZERO

ZERO

ZERO

ZERO

ZERO

ZERO

ZERO

ZERO

ZERO

ZERO

ZERO

ZERO

ZERO

ZERO

ZERO

ZERO

ZERO

ZERO

ZERO

ZERO

ZERO

ZERO

ZERO

ZERO

ZERO

ZERO

ZERO

ZERO

ZERO

ZERO

ZERO

ZERO

ZERO

ZERO

ZERO

ZERO

ZERO

ZERO

ZERO

ZERO

ZERO
ZERO ZERO

$40 \%$ NaNO2

Radioly

601

$0.1071 \mathrm{E}-06$

$0.1339 \mathrm{E}-02$

ZERO

2ERO

ZERO

$1806 \mathrm{E}-02$

ZERO

$0.4362 \mathrm{E}-05$

ZERO

ZERO

ZERO

ZERO

ZERO

ZERO

ZERO

ZERO

ZERO

ZERO

ZERO

ZERO

$0.7080 \mathrm{E}-06$

ZERO

ZERO
$0.3778 \mathrm{E}-06$

ZERO

ZERO

ZERO

$0.1134 \mathrm{E}+00$

ZERO

ZERO

ZERO

$0.7798 \mathrm{E}-05$

ZERO

ERO

ZERO

$0.1660 \mathrm{E}-04$

ZERO

ZERO

ZERO

ZERO

ZERO

$0.4350 \mathrm{E}-02$

ZERO

ZERO

ZERO

ZERO

ZERO

ZERO

ZERO

ZERO

ZERO

ZERO

ZERO

ZERO

ZERO

ZERO

ZERO

ZERO

ZERO

ZERO

ZERO

ZERO

ZERO

ZERO

ZERO

ZERO

ZERO

ZERO

ZERO

ZERO

ZERO

ZERO

ZERO

ZERO

ZERO

ZERO

ZERO

ZRRO

ZERO

$$
\text { ZERO }
$$

ZERO

ZERO

ZERO

ZERO

ZERO

ZERO

ZERO

ZERO

ZERO
ZERO

ZERO

ZERO

ZERO

ZERO

ZERO

ZERO

ZERO

ZERO

ZERO

ERO

ZERO

ZERO

ZERO

ZERO

ZERO

ZERO

ZERO

ZERO

ZERO

ZERO

ZERO

ZERO

ZERO

ZERO

ZERO

ZERO

ZERO

ZERO

ZERO

ZERO

DRO

ZERO

ZERO

Date: January 1995

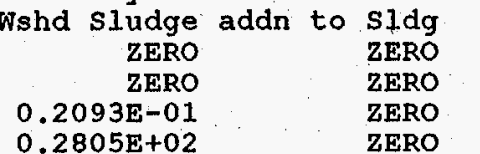

ZERO ZERO

$0.3541 \mathrm{E}-02$ ZERO

ZERO

ZERO

ZERO

ZERO

ZERO

ZERO

ZERO

ZERO

ZERO

ZERO

ZERO

ZERO

ZERO

ZERO

ZERO

ZERO

ZERO

ZERO

ZERO

ZERO

ZERO

ZERO

ZERO

ZERO

ZERO

ZERO

ZERO

ZERO

ZERO

ZERO

ZERO

ZERO

ZERO

ZERO

ZERO

ZERO

ZERO

ZERO

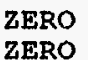

oncentrate

from ETF

ZERO

$0.3968 \mathrm{E}-02$

$0.1181 E+00$

$0.3951 \mathrm{E}-01$

$0.5292 \mathrm{E}-08$ 
Table A-1. HLW Flowsheet Material Balance (Tank 51/ITP Cycle 1) section 0. Major streams in the Flowsheet Model

STREAM NUMBERS (CONT'D) $\rightarrow$ STREAM NAME

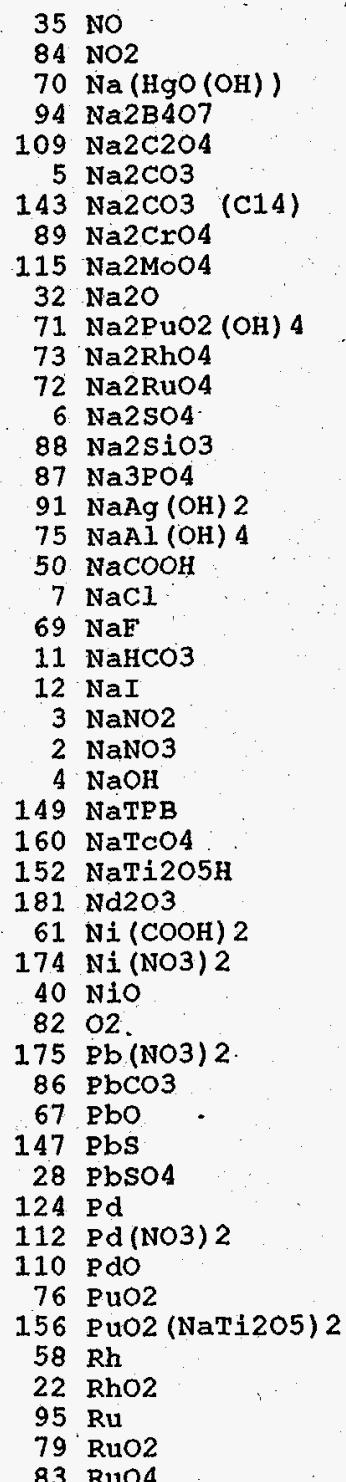

$322 \quad 317$

408 NaNO2 to $\mathrm{A} / \mathrm{D}$ Tank ZERO
ZERO

ZERO

ZERO

ZERO

ZERO

ZERO

ZERO

ZERO

ZERO

ZERO

ZERO

ZERO

ZERO

ZERO

ZERO

ZERO

ZERO

ZERO

ZERO

ZERO

ZERO

ZERO

ZERO

ZERO

ZERO

ZERO

ZERO

ZERO

ZERO

ZERO

ZERO

ZERO

ZERO

ZERO

ZERO

ZERO

ZERO

ZERO

ZERO

ZERO

ZERO

ZERO

ZERO

ZERO

Wash 50 NaOH

318

$40 \%$ NaNO2

327

326

601

Tk to W/O Tank to W/O Tank Wahd Sludge Dilution H2O

Wshd sludge addn to sldg
ZERO

ZERO
ZERO $\quad$ ZERO

$0.1832 E-02$ ZERO

$0.1889 \mathrm{E}+01$

$0.1758 \mathrm{E}+01$

$0.2159 \mathrm{E}-08$

$0.6612 \mathrm{E}-01$

$0.1089 \mathrm{E}-01$

$0.1652 \mathrm{E}-04$

$0.7151 \mathrm{E}-07$

$0.1181 \mathrm{E}-02$

$0.1390 \mathrm{E}+01$

$0.5771 \mathrm{E}-01$

$0.8157 \mathrm{E}-02$

$0.1042 \mathrm{E}-02$

$0.2544 \mathrm{E}+01$

$0.4846 \mathrm{E}-01$

$0.2437 \mathrm{E}+00$

ZERO
$3 E-04$

$0.3873 \mathrm{E}-04$
$0.2590 \mathrm{E}+02$

$0.1085 \mathrm{E}+02$

$0.2116 \mathrm{E}+02$

ZERO
$0.1121 \mathrm{E}-02$

ZERO

ZERO

ZERO

ZERO

ZERO

ZERO

ZERO

ZERO

ZERO

ZERO

ZERO

ZERO

ZERO

ZERO

ZERO

ZERO

ZERO

ZERO

ZERO
ZERO

ZERO

ZERO

$0.1270 \mathrm{E}-05$

- ZERO

$$
\text { ZERO }
$$

ZERO

ZERO

ZERO

$$
\text { ZERO }
$$

ZERO

\section{ZERO}

ZERO

ZERO

$$
\text { ZERO }
$$

ZERO

ZERO

$0.5438 \mathrm{E}-03$

ZERO

ZERO

$0.6519 \mathrm{E}-06$

ZERO

$0.1647 \mathrm{E}+02$

$0.1523 \mathrm{E}+02$

ZERO

ZERO

ZERO

ZERO

ZERO

ZERO

ZERO

ZERO

ZERO

ZERO

ZERO
$0.6296 \mathrm{E}-07$

ZERO
ZERO ZERO

$0.4859 \mathrm{E}-08$ ZERO

$0.3153 \mathrm{E}-07$
ZERO

ZERO

ZERO

ZERO

ZERO

ZERO

ZERO

ZERO
ZERO
ZERO

ZERO

ZERO

ZERO

ZERO
$0.2562 \mathrm{E}-03$

ZERO ZERO

$0.2642 \mathrm{E}+00$

$0.2458 \mathrm{E}+00$

$0.3019 \mathrm{E}-09$

$0.1523 \mathrm{E}-02$

$0.3230 \mathrm{E}+01$

$0.1000 \mathrm{E}-07$

$0.3865 \mathrm{E}-04$

$0.1652 \mathrm{E}-03$

$0.1944 \mathrm{E}+00$

$0.1141 \mathrm{E}-02$
$0.1457 \mathrm{E}-03$

$0.3558 \mathrm{E}+00$

ZERO

$0.6777 \mathrm{E}-02$

$0.3408 \mathrm{E}-01$

ZERO
$0.5416 \mathrm{E}-05$

$0.6393 \mathrm{E}+01$

$0.1518 \mathrm{E}+00$

$0.3013 \mathrm{E}+01$

ZERO

ZERO

ZERO

ZERO

ZERO

ZERO

ZERO

ZERO

ZERO

ZERO

ZERO

ZERO

ZERO

ZERO

ZERO

ZERO

ZERO

ZERO

ZERO

ZERO

ZERO

ZERO

ZERO

ZERO

ZERO
ZERO

ZERO

$0.2482 \mathrm{E}+00$

$0.2571 \mathrm{E}+00$

$0.7604 \mathrm{E}-04$

ZERO

ZERO

$0.1274 \mathrm{E}+00$

ZERO

$0.8804 \mathrm{E}-08$

$0.4488 \mathrm{E}-03$

$.9873 \mathrm{E}-02$

ZERO

$0.9498 \mathrm{E}-03$

ZERO

$0.6163 \mathrm{E}-02$

ZERO

ZERO
$0.9246 \mathrm{E}-02$

$0.8070 \mathrm{E}-02$

Concentrate

from ETF ZERO ZERO ZERO

$\begin{array}{ll}\text { ZERO } & 0.7128 \mathrm{E}+00 \\ \text { ZERO } & 0.2971 \mathrm{E}+01\end{array}$

ZERO $\quad 0.5974 \mathrm{E}-02$

ZERO ZERO

ZERO $0.6451 \mathrm{E}-07$

ZERO $\quad 0.1665 \mathrm{E}-12$

ZERO $0.3364 \mathrm{E}-10$

ZERO $0.1252 E+00$ 
Table A-1. HLW Flowsheet Material Balance (Tank 51/ITP Cyole 1 ) section 0. Major Streams in the Flowsheet Model.

STREAM NUMBERS (CONT'D) $\rightarrow$ STREAM NAME

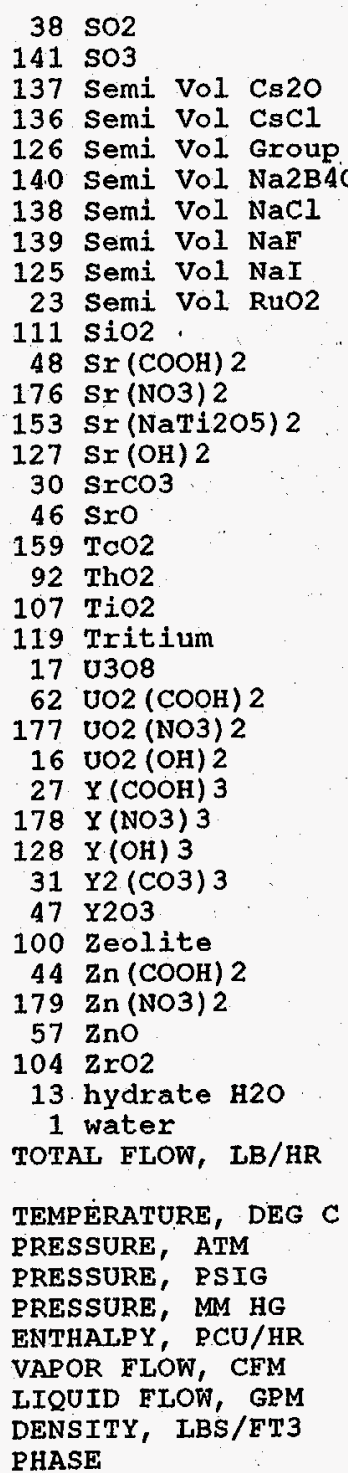

WSRC-TR-95-0019 (Revision
311 $40 \%$ NaNO2
to $A / D$ Tank ZERO frm $w /$

ZERO

ZERO

ZERO

ZERO

ZERO

ZERO

ZERO

ZERO

ZERO

ZERO

ZERO
ZERO

ZERO

ZERO

ZERO

ZERO

ZERO

ZERO

ZERO

ZERO

ZERO

ZERO

ZERO

ZERO

ZERO

ZERO

ZERO

ZERO

ZERO

ZERO

ZERO

ZERO

ZERO

ZERO

$0.4800 \mathrm{E}+02$

$0.1000 \mathrm{E}+01$

ZERO

ZERÓ

ZERO

LIQUID

322 pent Wash

317

318

327

326

601

$\begin{array}{rrrrr}50 \% & \text { NaOH } & 408 \text { NaNO2 } & \text { Radiolyzed Dilution H2O } \\ \text { to } W / 0 \text { Tank } & \text { to W/O Tank } & \text { Wshd Sludge addn to sldg } \\ \text { ZERO } & \text { ZERO } & \text { ZERO } & \text { ZERO }\end{array}$

ZERO

ZERO

ZERO

ZERO

ZERO

ZERO

ZERO

ZERO

ZERO

$0.5428 \mathrm{E}-05$

ZERO

ZERO

$0.2583 \mathrm{E}-06$

$0.1051 \mathrm{E}-06$

ZERO

$0.1768 \mathrm{E}-07$

$0.1520 \mathrm{E}-06$

$0.2378 \mathrm{E}-06$

$0.7312 \mathrm{E}-08$

$0.1066 \mathrm{E}-04$

ZERO

$0.5041 \mathrm{E}-03$

ZERO

ZERO

$0.2603 \mathrm{E}-06$

$0.2644 \mathrm{E}-07$

ZERO

ZERO

ZERO

$0.6033 \mathrm{E}-06$

ZERO

$0.4165 \mathrm{E}-03$

$0.8384 \mathrm{E}+03$
$0.9045 \mathrm{E}+03$

$0.4800 \mathrm{E}+02$

$0.1000 \mathrm{E}+01$

ZERO

$0.4113 \mathrm{E}+05$

$0.1721 \mathrm{E}+01$

IIQUID
ZERO

ZERO

ZERO

ZERO

ZERO

ZERO

ZERO

ZERO

ZERO

ZERO

ZERO

ZERO

ZERO

ZERO

ZERO

ZERO

ZERO

ZERO

ZERO

ZERO

ZERO

ZERO

ZERO

ZERO

ZERO

ZERO

ZERO

ZERO

ZERO

ZERO

ZERO

$0.1523 \mathrm{IERO}$

$0.3046 \mathrm{E}+02$

$0.4800 E+02$

$0.1000 E+01$
ZERO

$0.9707 \mathrm{E}+03$

$0.3984 \mathrm{E}-01$

IIQUID
ZERO

ZERO

ZERO

ZERO

ZERO

ZERO

ZERO

ZERO

ZERO

ZERO

ZERO

ZERO

ZERO

ZERO

ZERO

ZERO

ZERO

ZTRO

ZERO

ZERO

ZERO

ZERO

ZERO

ZERO

ZERO

ZERO

ZERO

ZERO

ZERO

ZERO

ZERO

ZERO

ZERO

$0.4118 \mathrm{E}+02$

$0.4800 \mathrm{E}+02$

$0.1000 \mathrm{E}+01$

ZERO

$0.1371 \mathrm{E}+04$

$0.6093 \mathrm{E}-01$

$0.8426 \mathrm{E}+02$

LIQUID
ZERO

ZERO

ZERO

ZERO

ZERO

ZERO

ZERO

ZERO

$0.1061 \mathrm{E}+01$

ZERO

ZERO

$0.3612 \mathrm{E}-07$

ZERO

$0.3457 \mathrm{E}-02$

$0.2972 \mathrm{E}-01$

$0.4648 \mathrm{E}-01$

$0.1023 \mathrm{E}-08$

$0.2083 \mathrm{E}+01$

ZERO

$0.7050 \mathrm{E}-04$

ZERO

ZERO

$0.3640 \mathrm{E}-07$

$0.5168 \mathrm{E}-02$

ZERO

ZERO

ZERO

$0.1179 \mathrm{E}+00$

ZERO

$0.2279 \mathrm{E}+01$

$0.2559 \mathrm{E}+03$

$0.3226 \mathrm{E}+03$

$0.3500 E+02$

$0.1000 \mathrm{E}+01$

ZERO

$0.9383 \mathrm{E}+04$

$0.5482 \mathrm{E}+00$
$0.7336 \mathrm{E}+02$

$0.7336 \mathrm{E}+02$
LIQUID
$0.2054 \mathrm{E}-01$
ZERO

ZERO

ZERO

ZERO

ZERO

ZERO

ZERO

ZERO

ZERO

ZERO

ZERO

ZERO

ZERO

ZERO

ZERO

ZERO

ZERO

ZERO

ZRRO

ZERO

ZERO

ZERO

ZERO

ZERO

ZERO

ZERO

ZERO

ZERO

ZERO

ZERO

ZERO

ZERO

ZERO

ZERO

$0.1220 \mathrm{E}+\mathrm{O} 3$

$0.2500 \mathrm{E}+02$

$0.1000 \mathrm{E}+01$

$0.3059 \mathrm{E}+04$

$0.2435 E+00$

$0.6247 \mathrm{E}+02$

IIQUID

ncentrate

from ETF

ZERO
ZERO

ZERO

ZERO

ZERO

ZERO

ZERO

ZERO

ZERO

$0.4069 \mathrm{E}+00$

ZERO

ZERO

0.8811 E-09

ZERO

ZERO

ZERO

ZFRO

$0.1193 \mathrm{E}-08$

ZERO ZERO 
Table A-1. HLW Flowsheet Material Balance (Tank 51/ITP Cycle 1) section 0. Major streams in the Flowsheet Model

STREAM NUMBERS -> STREAM NAME

COMPONENT FLOWS, IB/HR 122 (C6H5)

123 (C6H5) $2 \mathrm{C} 6 \mathrm{H}$

118 (C6H5) $2 \mathrm{NH}$

133. (C6H5B (OH) 2 salt)

132 (C6H5B (OH) 2)

166. (C6H5C6H $4 \mathrm{OH})$

$162 \cdot(\mathrm{C} 6 \mathrm{H} 5 \mathrm{NH} 2)$

163 (C6H5NHCHO)

165 (C6H5NO)

164 (C6H5NO2)

131 (C6H5OH salt)

130 (C6H5OH)

121 (C6H6)

155 (CH3) $2 \mathrm{CHOH}$

154 (CH3OH)

$45 \mathrm{Ag}$

116 Ag20

134 AgNO3
77 A1203

106 B203

$9 \mathrm{Ba}(\mathrm{OH}) 2$

$15 \mathrm{BaO}$

$24 \mathrm{BaSO} 4$

$66 \mathrm{CO}$

$142 \mathrm{CO} 2(\mathrm{C} 14$

$142 \mathrm{CO} 2(\mathrm{C} 14)$
$63 \mathrm{Ca}(\mathrm{COOH}) 2$

$63 \mathrm{Ca}(\mathrm{NO} 3) 2$

$120 \mathrm{Ca}(\mathrm{OH}) 2$

$113 \mathrm{Ca} 3(\mathrm{PO} 4) 2$

$20 \mathrm{CaC} 2 \mathrm{O} 4$

$18 \mathrm{CaCO} 3$

$64 \mathrm{CaCO}^{3}$ (C14)

78 CaF2

$41 \mathrm{CaO}$

$19 . \mathrm{CaSO} 4$

29 Carbon

$26 \mathrm{Co}(\mathrm{COOH}) 2$

$168 \mathrm{Co}(\mathrm{NO} 3) 2$

$59 \mathrm{COO}$

$98 \mathrm{Cr} 2 \mathrm{O} 3$
$42 \mathrm{Cs} 2 \mathrm{O}$

$53 \mathrm{CsCOOH}$

$96 \mathrm{CsCl}$

$169 \mathrm{CsNO}$

$8 \mathrm{CsOH}$

$151 \mathrm{CsTPB}$

WSRC-TR-95-0019 (Revision
603

Dcon Str T'k

610

Vnt to Atm

LPPT SN to

Mixr Fd Tk

615

Cement

618

Saltstone

to Trench

$\begin{array}{ll}\text { ZERO } & \text { ZERO } \\ \text { ZERO } & \text { ZERO } \\ \text { ZERO } & \text { ZERO } \\ \text { ZERO } & \text { ZERO } \\ \text { ZERO } & \text { ZERO } \\ \text { ZERO } & \text { ZERO } \\ \text { ZERO } & \text { ZERO } \\ \text { ZERO } & \text { ZERO } \\ \text { ZERO } & \text { ZERO } \\ \text { ZERO } & \text { ZERO }\end{array}$

$0.2887 \mathrm{E}-10$

ZERO

$0.3267 \mathrm{E}-02$

$0.2039 \mathrm{E}-01$

$0.1026 \mathrm{E}-02$

ZERO

$0.1517 \mathrm{E}-13$

ZERO

$0.7593 \mathrm{E}-12$

$0.1118 \mathrm{E}-18$

ZERO

$0.2401 \mathrm{E}-13$

ZERO
$7 \mathrm{E}-03$

$0.7717 \mathrm{E}-03$

ZERO

ZERO

$0.3161 \mathrm{E}-13$

$0.1881 \mathrm{E}-11$

ZERO
$9 E-11$

$0.4309 \mathrm{E}-11$

ZERO

ZERO

ZERO

ZERO

ZERO

ZERO

ZERO

ZERO

ZERO

ZERO

ZERO

ERO

0.1430 E-16
ZERO

$0.3344 \mathrm{E}+01$

ZERO

$0.8805 \mathrm{E}-05$

$0.5497 \mathrm{E}-01$

$0.3571 \mathrm{E}-02$

ZERO
$0.1757 \mathrm{E}-02$

ZERO

$0.8794 \mathrm{E}-01$

$0.1295 \mathrm{E}-07$

$0.2780 \mathrm{E}-02$

ZERO

ZERO
ZERO

ZERO

$0.3549 \mathrm{E}-01$

$0.3660 \mathrm{E}-02$

$0.2178 \mathrm{E}+00$

$0.4990 \mathrm{E}+00$

ZERO

ZERO

ZERO

ZERO

ZERO

25RO

ZERO

ZERO

ZERO

ZERO

$0.1657 \mathrm{E}-05$

ZERO

ZERO : ZERO

ZERO ZERO

ZERO ZERO

ZERO ZERO

ZERO : ZERO

ZERO ZERO

ZERO ZERO

ZERO

ZERO

ZERO

$0.3344 \mathrm{E}+01$

ZERO

ZERO

$0.4456 \mathrm{E}-01$

$0.3018 \mathrm{E}-02$

ZERO

ZERO

ZERO

ZERO

ZERO

ZERO

ZERO

ZERO

ZERO

ZERO

ZERO

ZERO

ZERO

ZERO

ZERO

ZERO

ZERO

ZERO

ZERO

ZERO

$0.1769 \mathrm{E}+05$

ZERO

ZERO

ZERO

ZERO
ZERO

ZERO

ZERO

ZERO

ZERO

ZERO

ZERO
$0.8794 \mathrm{E}-01$

ZERO

$0.1295 \mathrm{E}-07$

$0.2780 \mathrm{E}-02$

$0.7754 \mathrm{E}-10$

$\mathrm{E}-10$

ZERO

$0.3549 E-01$

$0.3660 \mathrm{E}-02$

$0.2178 \mathrm{E}+00$

$0.4990 \mathrm{E}+00$

ZERO

ZERO

ZERO

ZERO

$0.1769 \mathrm{E}+05$

ZERO

$0.1392 \mathrm{E}-01$

ZERO

ZERO

ZERO

ZERO

$0.1657 \mathrm{E}-05$

ZERO
619

620

saltstone

Date: January 1995

Saltstone stack Rels Vapor Relese

$\begin{array}{lll}\text { ZERO } & \text { ZERO } & \text { ZERO } \\ \text { ZERO } & \text { ZERO } & \text { ZERO } \\ \text { ZERO } & \text { ZERO } & \text { ZERO }\end{array}$

ZERO ZERO ZERO

ZERO

ZERO ZERO

$\begin{array}{lll}\text { ZERO } & \text { ZERO } & \text { ZERO } \\ \text { ZERO } & \text { ZERO } & \text { ZERO }\end{array}$

ZERO ZERO

ZERO

0.1523 ZE-10

ZERO

$0.8734 \mathrm{E}-05$

$0.1041 \mathrm{E}-01$

$0.5533 \mathrm{E}-03$

$4456 \mathrm{E}-01$

$.3018 \mathrm{E}-02$

ZERO

ZERO

$0.8794 \mathrm{E}-01$

ZERO

$0.1295 \mathrm{E}-07$

$0.2780 \mathrm{E}-02$

0.7754 ZERO

$4 \mathrm{E}-10$

ZERO
ZERO

$0.3549 \mathrm{E}-01$

$.3660 \mathrm{E}-02$

$.2178 \mathrm{E}+00$

$0.4990 \mathrm{E}+00$

ZERO

'ZERO

ZERO

ZERO

$0.1769 \mathrm{E}+05$

ZERO

$0.1392 \mathrm{E}-01$

ZERO

ZERO

ZERO

ZERO

ZERO

ZERO
$0.1657 \mathrm{E}-05$

ZERO

$0.8005 \mathrm{E}-14$

ZERO

$0.4006 \mathrm{E}-12$

ZERO

ZERO

ZERO

$0.7143 \mathrm{E}-07$

ZERO

ZERO

ZERO

ZERO

ZERO

ZERO

$\begin{array}{rr}0.5899 E-19 & \text { ZERO } \\ \text { ZERO }\end{array}$

ZERO

ZERO

ZERO

ZERO

ZERO

ZERO

ZERO

ZERO
$0.1667 \mathrm{E}-13$

$0.9924 \mathrm{E}-12$ ZERO

ZERO

ZERO

ZERO

ZERO

ZERO
$0.2997 \mathrm{E}-07$

E-07

ZERO

ZERO

ZERO

ZERO

ZERO

ZERO

ZERO
$0.7547 \mathrm{E}-17$ 
Table A-1. HiW Flowsheet Material Balance (Tank 51/ITP Cycle 1) section 0 . Major streams in the Flowsheet Model

STREAM NUMBERS (CONT'D) $\rightarrow$ STREAM NAME

$54 \mathrm{Cu}(\mathrm{COOH}) 2$
$170 \mathrm{Cu}(\mathrm{NO} 3) 2$

$170 \mathrm{Cu}$ (NO3)
$55 \mathrm{CuO}$

80 Fe2O3

$101 \mathrm{FeO}$

102 Group A

$25 \mathrm{H} 2$

158 H2C2O4

$158 \mathrm{H} 2 \mathrm{C} 2 \mathrm{O}$
$93 \mathrm{H} 2 \mathrm{SO} 4$

90 H3BO3

$34 \mathrm{HCOOH}$

$43 \mathrm{HCl}$

$85 \mathrm{HF}$

$36 \mathrm{Hg}$

$129 \mathrm{Hg}$ (C6H5) 2

$135 \mathrm{Hg}$ (NO3) 2

$144 \mathrm{Hg} 2 \mathrm{Cl} 2$

$117 \mathrm{Hg} 2 \mathrm{I} 2$

$146 \mathrm{HgCl2}$

$21 \mathrm{HgO}$

$183 \mathrm{~K} 2 \mathrm{CO}$

$\begin{array}{rl}183 & \mathrm{~K} 2 \mathrm{CO} \\ 39 & \mathrm{~K} 2 \mathrm{O}\end{array}$

$51 \mathrm{KCOOH}$

$157 \mathrm{KMnO4}$

161 KNO3

$10 \mathrm{KOH}$

150 KTPB

108 Li20

$65 \mathrm{Mg}(\mathrm{COOH}) 2$

$171 \mathrm{Mg}$ (NO3) 2

$33 \mathrm{MgO}$

$60 \mathrm{Mn}(\mathrm{COOH}) 2$

$172 \mathrm{Mn}$ (NO3) 2

$180 \mathrm{Mn} 304$

$56 \mathrm{MnO}$

$14 \mathrm{MnO} 2$

$145 \mathrm{MoO} 2$

81 N2

$74 \mathrm{~N} 2 \mathrm{O}$

114 NH3OHNO3

52 NH4 COOH

173 NH 4 NO3

$68 \mathrm{NH} 4 \mathrm{OH}$

148 NH 4 TPB

WSRC-TR-95-0019 (Revision
603

Daon str Tk

Vnt to Atm ZERO

$0.3427 \mathrm{E}-13$

$0.1020 \mathrm{E}-11$

ZERO

$0.8865 \mathrm{E}-12$

$0.1676 \mathrm{E}-13$

$0.2777 \mathrm{E}-06$

ZERO
ZERO

ZERO

ZERO

ZERO

ZERO

ZERO

ZERO

ZERO

ZERO

ZERO

ZERO

$0.8682 \mathrm{E}-14$

ZERO

ZERO

ZERO

ZERO

ZERO

$0.1980 \mathrm{E}-12$

ZERO

ZERO

ZERO

ZERO

$0.1961 \mathrm{E}-12$

ZERO

ZERO

ZERO
$0.6505 \mathrm{E}-13$

ZERO

ZERO

$0.5411 \mathrm{E}+03$

$0.3216 \mathrm{E}-14$

$0.4543 \mathrm{E}-01$

ZERO

ZERO

$0.2700 \mathrm{E}-08$

ZERO

610

LPPT SN to

Mixr Fd Tk

$0.3286 \mathrm{E}-02$

$0.3968 \mathrm{E}-02$

$0.1181 \mathrm{E}+00$

$0.1027 \mathrm{E}+00$

$0.1941 \mathrm{E}-02$

$0.5537 \mathrm{E}-13$

ZERO

ZERO

ZERO

ZERO

ŻERO

ZERO

ZERO

ZERO

ZERO

ZERO

ZERO

ZERO

$0.1005 \mathrm{E}-02$

ZERO

$0.5058 \mathrm{E}-03$

ZERO

ZERO

$0.2293 E-01$

$3 E-01$
ZERO

ZERO

ZERO

$0.1361 \mathrm{E}-02$

$0.2271 \mathrm{E}-01$

$0.1586 \mathrm{E}-02$

ZERO

$0.7533 E-02$

ZERO

$0.1194 \mathrm{E}+00$

$0.2236 \mathrm{E}-18$

$0.4056 \mathrm{E}-01$

ZERO

ZERO

$0.3127 \mathrm{E}+00$

ZERO

618

619

632

Cement

ZERO
ZERO

ZERO $0.3968 \mathrm{E}-02$

ZERO

ZERO

ZERO

ZERO

ZERO

ZERO

ZERO

ZERO

ZERO

ZERO

ZERO

ZERO

ZERO

ZERO

ZERO

ZERO

ZERO

ZERO

ZERO

ZERO

ZERO

ZERO

ZERO

ZERO

ZERO

ZERO

ZERO

ZERO

ZERO

ZERO

ZERO

ZERO

ZERO

ZERO

ZERO

ZERO

ZERO

ZERO

Date: January 1995

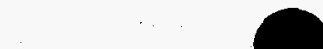

saltstone

to Trench

ZERO

$0.1181 \mathrm{E}+00$

ZERO
$7 \mathrm{E}+00$

$0.1027 \mathrm{E}+00$

$0.3686 \mathrm{E}-19$

Hardened sltston Proc

Hardened sltston Proc Saltstone
saltstone stack Rels vapor Relese

ZERO
$0.3286 \mathrm{E}-02$
ZERO

$\begin{array}{lll}0.3968 \mathrm{E}-02 & 0.1808 \mathrm{E}-13 & \text { ZERO }\end{array}$

$0.1181 \mathrm{E}+00 \quad 0.5382 \mathrm{E}-12 \quad$ ZERO

$\begin{array}{rrr}\text { ZERO } & \text { ZERO } & \text { ZERO } \\ 0.1027 \mathrm{E}+00 & 0.4677 \mathrm{E}-12 & \text { ZERO }\end{array}$

$0.1941 \mathrm{E}-02 \quad 0.8844 \mathrm{E}-14 \quad$ ZERO

$0.3686 \mathrm{E}-19 \quad 0.5537 \mathrm{E}-13 \quad$ ZERO

ZERO

ZERO

ZERO

ZERO

ZERO

ZERO

ZERO

ZERO

ZERO

ZERO

ZERO

$0.1005 \mathrm{E}-02$

$$
\text { ZERO }
$$

$0.5058 \mathrm{E}-03$

ZERO
ZERO

ZERO

ZERO

$0.2293 \mathrm{E}-01$

ZERO

ZERO

ZERO

$0.1361 \mathrm{E}-02$

$0.2271 \mathrm{E}-01$

$0.1586 \mathrm{E}-02$

ZERO

ZERO

$.1201 \mathrm{E}+00$

$0.5104 \mathrm{E}-22$
$0.2462 \mathrm{E}-01$

ZERO

ZERO

$0.3127 \mathrm{E}+00$

ZERO

ZERO
$0.3127 \mathrm{E}+00$

ZERO

Section 0, Page 19 of 37

ZERO

ZERO

ZERO

ZERO

ZERO

ZERO

ZERO

ZERO

ZERO

ZERO

$0.1005 \mathrm{E}-02$

$0.5058 \mathrm{E}-03$

ZERO

ZERO

$0.2293 E-01$

ZERO

ZERO

$0.1361 \mathrm{E}-02$

$0.2271 \mathrm{E}-01$

$0.1586 \mathrm{E}-02$

$0.7533 \mathrm{E}-02$

ZERO

$0.1201 \mathrm{E}+00$

$0.5104 \mathrm{E}-22$

ZERO

$0.5411 \mathrm{E}+03$

$0.1594 \mathrm{E}-01$

ZERO

ZERO

ZERO

ZERO

ZERO

ZERO

ZERO

ZERO

ZERO

ZERO

ZERO

ZERO

ZERO

$\begin{aligned} \text { ZERO } & \text { ZERO } \\ 0.1034 \mathrm{E}-12 & \text { ZERO }\end{aligned}$

ZERO ZERO

ZFRO

ZERO

ZERO

ZERO 
Table A-1. HLW Flowsheet Material Balance (Tank 51/ITP Cycle 1) Section 0 . Major streams in the Flowsheet Model

STREAM NUMBERS (CONT'D) STREAM NAME

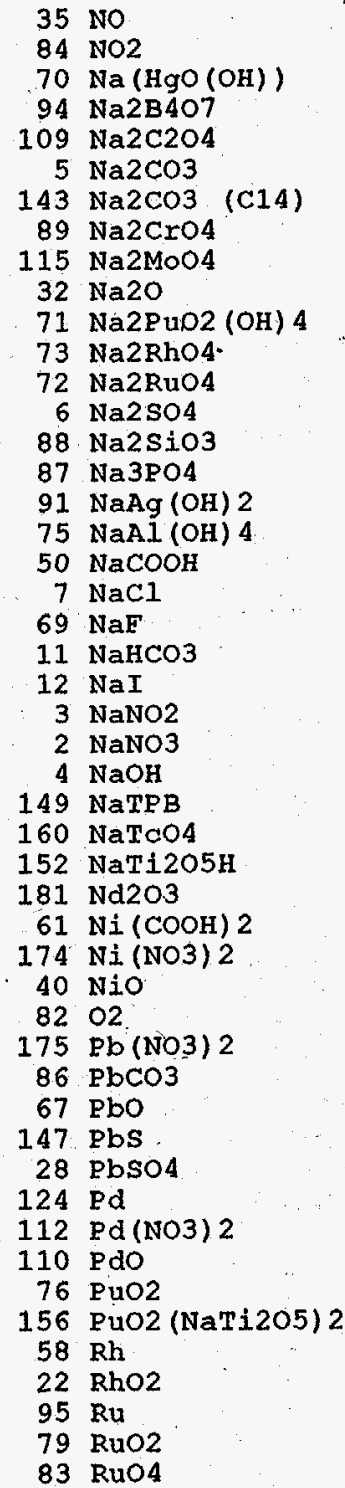

Hardened sltston Proc saltstone Saltstone stack Rels Vapor Relese $\begin{array}{rrr}\text { ZERO } & \text { ZERO } & \text { ZERO } \\ \text { ZERO } & \text { ZERO } & \text { ZERO }\end{array}$ $0.2822 \mathrm{E}-03 \quad 0.1285 \mathrm{E}-14 \quad$ ZERO $0.3253 \mathrm{E}+00 \quad 0.1482 \mathrm{E}-11$ ZERO $0.4668 \mathrm{E}+01 \quad 0.2127 \mathrm{E}-10 \quad$ ZERO $0.4974 \mathrm{E}+03 \quad 0.2266 \mathrm{E}-08 \quad$ ZERO $0.7320 \mathrm{E}-05 \quad 0.3335 \mathrm{E}-16 \quad$ ZERO $0.2700 E+01 \quad 0.1230 E-10 \quad$ ZERO $0.8046 \mathrm{E}+00,0.3665 \mathrm{E}-11 \quad$ ZERO $\begin{array}{rrr}\text { ZERO } & \text { ZERO } & \text { ZERO } \\ 0.1813 \mathrm{E}-05 & 0.8258 \mathrm{E}-17 & \text { ZERO }\end{array}$ $0.1952 \mathrm{E}-01 \quad 0.8892 \mathrm{E}-13 \quad$ ZRRO $0.1028 \mathrm{E}+00 \quad 0.4683 \mathrm{E}-12$ $0.2817 E+02 \quad 0.1283 E-09$ $0.7803 \mathrm{~F}+000.3555 \mathrm{E}-11$ $0.3724 \mathrm{E}+02 \quad 0.1697 \mathrm{E}-09$ $0.7463 \mathrm{D}-05$ 0.3400E-16 $0.1402 \mathrm{E}+03 \quad 0.6386 \mathrm{E}-09$ ZERO ZERO ZERÓ
ZER $0.2367 \mathrm{E}+01 \quad 0.1078 \mathrm{E}-10 \quad$ ZERO $0.2200 \mathrm{E}+01 \quad 0.1002 \mathrm{E}-10 \quad$ ZERO $\begin{array}{lll}0.1444 \mathrm{E}-02 & 0.6579 \mathrm{E}-14 & \text { ZERO }\end{array}$ $0.2723 \mathrm{E}+03 \quad 0.1241 \mathrm{E}-08 \quad$ ZERO $0.1397 \mathrm{E}+04 \quad 0.6363 \mathrm{E}-08 \quad$ ZERO $0.6486 \mathrm{E}+03 \quad 0.2955 \mathrm{E}-08$ $0.7767 \mathrm{E}+01 \quad 0.3538 \mathrm{E}-10 \quad$ ZERO 0.6477 E-01 $\quad$ ZERO $\quad$ ZERO 
Table A-1. HLW Flowsheet Material Balance (Tank 51/ITP Cycle 1)

STREAM NUMBERS (CONT'D) $\rightarrow$ STREAM NAME

$38 \mathrm{SO} 2$

$141 \mathrm{SO3}$

137 Semi Vol Cs2O

136 Semi Vol CsCl

126 Semi Vol Group A

140 Semi Vol Na2B4O?

138 Semi Vol NaCl

139 Semi Vol NaF

125 Semi Vol NaI

23 Semi Vol RuO2

$111 \mathrm{SiO} 2$

$48 \mathrm{Sr}(\mathrm{COOH}) 2$

$176 \mathrm{Sr}(\mathrm{NO} 3) 2$

$153 \mathrm{Sr}$ (NaTi205) 2

$127 \mathrm{Sr}(\mathrm{OH}) 2$

$30 \mathrm{SrCO} 3$

46 Sro

$159 \mathrm{TcO} 2$

92 Tho

119 Tritium

119 Trit
17 U308

$17 \mathrm{U} 308$
$\mathrm{UOO} 2$ (COOH)

177 UO2 (NO3) 2

$16 \mathrm{VO} 2(\mathrm{OH}) 2$

27. $\mathrm{Y}(\mathrm{COOH}) 3$

$178 \mathrm{Y}$ (NO3) 3

128 Y (OH) 3

31 Y $2(\mathrm{CO} 3)$

$47 \mathrm{Y} 203$

100 zeolite

$44 \mathrm{Zn}$ (COOH) 2

$179 \mathrm{zn}(\mathrm{NO} 3) 2$

$57 \mathrm{znO}$

104 zro2

13 hydrate $\mathrm{H} 2 \mathrm{O}$

1 water

TOTAL, FLOW, LB/HR

TEMPERATURE， DEG C

PRESSURE， ATM

PRESSURE， PSIG

PRESSURE, MM HG

ENTHAIPY， PCU/HR

VAPOR FLOW, CFM

IIQUID FLOW, GPM

DENSITY, LBS/ET3

PHASE

603

Dcon Str TK

Vnt to Atm

ZERO

ZERO

ZERO

ZERO

ZERO

ZERO

ZERO

ZERO

ZERO

$0.3514 \mathrm{E}-11$
ZERO

ZERO

ZERO

$0.4142 E-16$

ZERO

ZERO

ZERO

ZERO

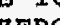

ZERO

ZERO

$0.7262 \mathrm{E}-12$
ZERO

ZERO

$0.4908 \mathrm{E}-16$

ZERO

ZERO

ZERO

IRRO

$0.3878 \mathrm{E}-12$

ZERO

$0.9546 \mathrm{E}-12$

$0.1919 \mathrm{E}+02$

$0.7237 \mathrm{E}+03$

$0.4000 \mathrm{E}+02$

$0.9656 \mathrm{E}+00$

$0.7339 \mathrm{E}+03$

$0.2038 E+05$

$0.1810 \mathrm{E}+03$

$0.6664 \mathrm{E}-01$

VAPOR

Section 0. Major Streams in the Flowsheet Model

610

LPPT SN to

Mixr Fd Tk

ZERO

ZERO

ZERO

ZERO

ZERO

ZERO

ZERO

ZERO

ZERO

$0.4069 \mathrm{E}+00$

ZERO

ZERO

$0.4797 \mathrm{E}-05$

ZERO

ZERO

ZERO

ZERO

ZERO
$0.4132 \mathrm{E}-07$

ZTRO

ZERO

ZERO

$0.8410 \mathrm{E}-01$

ZERO

$0.5683 \mathrm{E}-0.5$

ZERO

ZERO

ZERO

$0.1383 \mathrm{E}+01$

$0.4492 \mathrm{E}-01$

ZERO

$0.1173 \mathrm{E}+05$

$0.3992 \mathrm{E}+02$

$.9656 \mathrm{E}+00$

$0.7339 \mathrm{E}+03$

$0.3793 \mathrm{E}+06$

$0.1959 \mathrm{E}+02$
$0.7467 \mathrm{E}+02$

$67 \mathrm{E}+02$
LIQUID
615

Cement

re-mix

ZERO

ZERO

ZERO

ZERO

ZERO

ZERO

ZERO

ZERO

ZERO

ZERO

ZERO

ZERO

ZERO

ZERO

ZERO

ZERO

ZERO

ZERO

ZERO

ZERO

ZERO

ZERO

ZERO

ZERO

ZERO

ZERO

ZERO

ZERO

ZERO

ZERO

ZERO

$0.1769 \mathrm{E}+05$

618

Saltstone

to Trench

ZERO

ZERO

ZERO

ZERO

ZERO

ZERO

2ERO

ZERO

$0.4069 \mathrm{E}+00$

ZERO

ZERO

.4797 ZERO

ZERO

ZERO
ZERO
ZERO

ZERO

$0.4130 \mathrm{E}-07$

ZERO

ZERO

$0.8410 \mathrm{E}-01$

ZERO

$0.5683 \mathrm{E}-05$

ZERO
ZERO
ZERO

ZERO

$0.1383 \mathrm{E}+01$

$0.4492 \mathrm{E}-01$

ZERO

$0.1106 \mathrm{E}+00$

$0.8768 \mathrm{E}+04$

$0.2951 \mathrm{E}+05$

$0.2500 \mathrm{E}+02 \quad 0.3873 \mathrm{E}+02$

$0.9656 \mathrm{E}+00$

ZERO

$-0.5055 \mathrm{E}+00$

$0.4988 \mathrm{E}+06$

$0.3335 \mathrm{E}+02$

$0.1103 \mathrm{E}+03$
LIQUID

SOLID
619

632

sltston Proo ZERO Vapor Relese ZERO ZERO $\quad$ ZERO ZERO
ZERO

ZRRO

ZERO

ZERO

ZERO

ZERO

ZERO

$0.4069 \mathrm{E}+00$

ZERO

ZERO

$0.4797 \mathrm{E}-05$

ZERO

ZERO

ZERO

ZERO

$0.4087 E-07$

ZERO

ZERO

$0.8410 \mathrm{E}-01$

ZERO

$0.5683 \mathrm{E}-05$

ZERO

ZERO

ZERO

$0.1383 E+01$

'ZERO

$0.1106 \mathrm{E}+00$

$0.7234 \mathrm{E}+04$

$0.2798 \mathrm{E}+05$

$0.3873 \mathrm{E}+02$

$0.9656 \mathrm{E}+00$
$-0.5055 \mathrm{E}+00$

$0.7339 \mathrm{E}+03$

$0.4392 \mathrm{E}+06$

$0.3029 \mathrm{E}+02$

$0.1151 \mathrm{E}+03$

LIQUID

$0.6633 \mathrm{E}-01$
VAPOR 
Table A-1. HLW Flowsheet Material Balance (Tank 51/ITP Cycle 1) section 0. Major streams in the Flowsheet Model

STREAM NUMBERS $\rightarrow$ STREAM NAME

COMPONENT FLOWS, LB/HR $122(\mathrm{C} 6 \mathrm{H} 5) 2$

123 ( $\mathrm{C} 6 \mathrm{H} 5$ ) $2 \mathrm{C} 6 \mathrm{H} 4$

118 (C6H5) 2NH

133 (C6H5B (OH) 2 salt)

132 (C6H5B (OH) 2)

166 (C6H5C6H4

163 (C6H5NHCHO)

165 (C6H5NO)

164 (C6H5NO2)

131 (C6H5OH salt)

130 (C6H5OH)

121 (C6H6)

154 (CH3OH)

$45 \mathrm{Ag}$

116. Ag2O

134 AgNO3
77 A.1203

106 B203

$9 \mathrm{Ba}(\mathrm{OH}) 2$

$\begin{array}{ll}24 & \mathrm{BasO} \\ 66 & \mathrm{CO}\end{array}$

$\begin{array}{ll}66 & \mathrm{CO} \\ 37 & \mathrm{CO} 2\end{array}$

$142 \mathrm{CO} 2$ (C14)

$63 \mathrm{Ca}(\mathrm{COOH}) 2$

167. $\mathrm{Ca}(\mathrm{NO} 3)$
$120 \mathrm{Ca}(\mathrm{OH}) 2$

$113 \mathrm{Ca} 3(\mathrm{PO} 4) 2$

$20 \mathrm{CaC} 204$

$18 \mathrm{CaCO} 3$

$64 \mathrm{CaCO}^{3}$ (C14)

78 CaF2

$41 \mathrm{CaO}$

$19 \mathrm{CaSO} 4$

29 Carbon

$26 \mathrm{Co}(\mathrm{COOH}) 2$

$168 \mathrm{Co}(\mathrm{NO} 3) 2$

$59 \mathrm{COO}$

$98 \mathrm{Cr} 203$
$42 \mathrm{Cs} 2 \mathrm{O}$

$53 \mathrm{CsCOOH}$

$96 \mathrm{CsCl}$

$169 \mathrm{CsNO} 3$

$8 \mathrm{CsOH}$
201

Washd Precip

Feed frm LWE

$0.3293 \mathrm{E}+00$

$0.1399 \mathrm{E}-01$

$0.9313 \mathrm{E}-02$
$0.3712 \mathrm{E}+00$

$0.3712 \mathrm{E}+00$

$0.3026 \mathrm{E}-01$

ZERO

ZERO

ZERO

$0.4503 \mathrm{E}-01$

ZERO

$0.1091 \mathrm{E}-01$

$0.8591 \mathrm{E}-10$
$0.9422 \mathrm{E}-11$

$0.9422 \mathrm{E}-11$

$0.3054 \mathrm{E}-02$
ZERO

ZERO
7E+00

$0.1297 \mathrm{E}+00$

$0.167 .0 \mathrm{E}-02$

$0.4817 \mathrm{E}-16$

$0.1793 \mathrm{E}-02$

ZERO

ZERO

ZERO

ZERO
$0.1370 \mathrm{O}-05$

$0.1413 \mathrm{E}-06$

$0.5607 E+00$

ZERO

ZERO

ZERO

ZERO

$0.1912 \mathrm{E}+00$

ZERO

ZERO

$0.5373 \mathrm{E}-06$

$0.1028 \mathrm{E}-03$

$0.2616 \mathrm{E}+00$

$0.3848 \mathrm{E}-04$

ZERO

ZERO

$0.1794 \mathrm{E}-05$
$0.1100 \mathrm{E}+00$

\begin{tabular}{|c|c|}
\hline $\begin{array}{l}657 \\
\text { Pred Feed } \\
\text { Solubles }\end{array}$ & $\begin{array}{c}658 \\
\text { Prec Feed } \\
\text { Insolubles }\end{array}$ \\
\hline ZERO & $0.3293 E+00$ \\
\hline ZERO & $0.1399 \mathrm{E}-01$ \\
\hline ZERO & $0.9313 E-02$ \\
\hline ZERO & $0.3712 \mathrm{E}+00$ \\
\hline ZERO & ZERO \\
\hline ZERO & $0.3026 \mathrm{E}-01$ \\
\hline ZERO & ZERO \\
\hline ZERO & ZERO \\
\hline ZERO & ZERO \\
\hline ZERO & ZERO \\
\hline $4503 E-01$ & ZERO \\
\hline ZERO & ZERO \\
\hline $91 \mathrm{E}-01$ & ZERO \\
\hline $91 \mathrm{E}-10$ & ZERO \\
\hline $122 \mathrm{E}-11$ & ZERO \\
\hline ZERO & ZERO \\
\hline ZERO & $0.3054 \mathrm{E}-02$ \\
\hline ZERO & ZERO \\
\hline ZERO & $0.1297 \mathrm{E}+00$ \\
\hline ZERO & $0.1670 \mathrm{E}-02$ \\
\hline $4817 E-16$ & ZERO \\
\hline ZERO & ZERO \\
\hline ZERO & $0.1793 \mathrm{E}-02$ \\
\hline ZERO & ZERO \\
\hline ZERO & ZERO \\
\hline ZERO & ZERO \\
\hline ZERO & ZERO \\
\hline $.1370 \mathrm{E}-05$ & ZERO \\
\hline $413 E-06$ & ZERO \\
\hline ZERO & $0.5607 E+00$ \\
\hline ZERO & ZERO \\
\hline ZERO & ZERO \\
\hline ZERO & ZERO \\
\hline ZERO & ZERO \\
\hline ZERO & ZERO \\
\hline ZERO & $0.1912 \mathrm{E}+00$ \\
\hline ZERO & ZERO \\
\hline ZERO & ZERO \\
\hline ZERO & ZERO \\
\hline $.5373 E-06$ & ZERO \\
\hline ZERO & $0.1028 \mathrm{E}-03$ \\
\hline ZERO & $0.2616 \mathrm{E}+00$ \\
\hline ZERO & $0.3848 \mathrm{E}-04$ \\
\hline ZERO & ZERO \\
\hline ZERO & ZERO \\
\hline ZERO & ZERO \\
\hline $.1794 \mathrm{E}-05$ & ZERO \\
\hline ZERO & $0.1100 \mathrm{E}+00$ \\
\hline
\end{tabular}

247
PR Bottoms 3.

to salt Cell

$0 . ! 1022 \mathrm{E}-01$

$0.9245 \mathrm{E}-02$

$0.6901 \mathrm{E}-02$

ZERO

$0.5523 \mathrm{E}-01$

$0.7364 \mathrm{E}-01$

$0.2092 \mathrm{E}+00$

$0.3406 \mathrm{E}-02$

ZERO

$0.9340 \mathrm{E}+00$

$0.1433 \mathrm{E}-01$

$0.8321 \mathrm{E}-10$

$0.8321 \mathrm{E}-10$
$0.8866 \mathrm{E}-11$

$0.2843 \mathrm{E}-02$

ZERO
ZERO

$0.1297 \mathrm{E}+00$
$0.1670 \mathrm{E}-02$

$0.1670 \mathrm{E}-02$

ZERO

$0.1793 \mathrm{E}-02$

ZERO

$0.6183 \mathrm{E}-06$
$0.5975 \mathrm{E}-17$

$0.5975 \mathrm{E}-17$
ZERO

ZERO
0.1370 E-05

ZERO
$0.5607 \mathrm{E}+00$

$0.2442 \mathrm{E}-06$

$2 E-06$
ZERO

ZERO

ZERO

$0.1912 \mathrm{E}+00$

ZERO

$0.1433 \mathrm{E}-03$

$0.1433 \mathrm{E}-03$
$0.5373 \mathrm{E}-06$

$0.5373 \mathrm{E}-06$
$0.3069 \mathrm{E}-04$

$0.2617 \mathrm{E}+00$

$0.3848 \mathrm{E}-04$
$0.4330 \mathrm{E}-01$

ZERO

ZERO

ZERO
678

Sldge Feed

Solubles

$0.1022 \mathrm{E}-01$

$0.9245 \mathrm{E}-02$

$0.6825 \mathrm{E}-02$

ZERO

$0.5523 \mathrm{E}-01$

$0.7162 \mathrm{E}-01$

$0.2092 \mathrm{E}+00$

$0.3406 \mathrm{E}-02$

ZERO
ZERO

$0.9215 \mathrm{E}+00$

$0.3375 \mathrm{E}-03$

$0.8215 \mathrm{E}-10$

$0.8601 \mathrm{E}-11$

$0.2843 \mathrm{E}-02$

ZERO

$0.1297 \mathrm{E}+00$

$0.1670 \mathrm{E}-02$

ZERO

ZERO
$0.1793 \mathrm{E}-02$

ZERO

$0.3621 \mathrm{E}-08$

$0.3499 \mathrm{E}-19$

ZERO
0.1370 E -05

ZERO

$0.5607 \mathrm{E}+00$

$0.2442 \mathrm{E}-06$

ZERO
ZERO

ZERO

ZERO
$0.1912 \mathrm{E}+00$

ZERO

ZERO

$0.1433 \mathrm{E}-03$

$0.5373 \mathrm{E}-06$

$0.3069 \mathrm{E}-04$

$0.2617 \mathrm{E}+00$
$0.3848 \mathrm{E}-04$

$0.4330 \mathrm{E}-01$

ZERO
ZERO

ZERO

ZERO

ZERO
679

Sldge Feed

Insolubles

WSRC-TR-95-0019 (Revision 
Table A-1. HLW Flowsheet Material Balance (Tank 51/ITP Cycle 1 ) Section 0. Major streams in the Flowsheet Model.

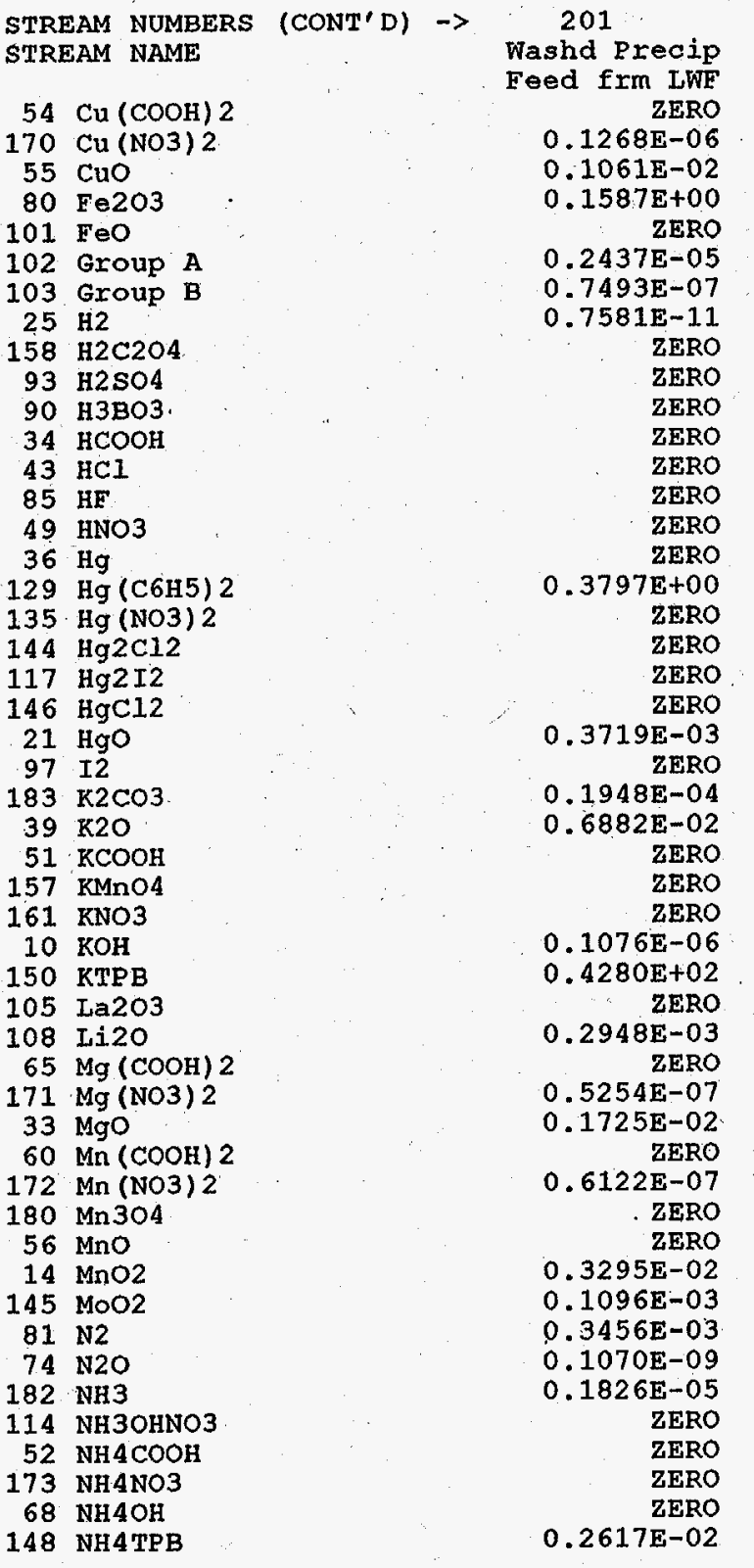

WSRC-TR-95-0019 (Revision

\begin{tabular}{|c|c|c|}
\hline 657 & 658 & 247 \\
\hline $\begin{array}{l}\text { Prec Fèed } \\
\text { Solubles }\end{array}$ & $\begin{array}{l}\text { Prec Feed } \\
\text { Insolubles }\end{array}$ & $\begin{array}{l}\text { PR Bottoms } \\
\text { to PRBT }\end{array}$ \\
\hline ZERO & ZERO & $0.2061 \mathrm{E}-03$ \\
\hline $0.1268 \mathrm{E}-06$ & ZERO & $0: 2179 \mathrm{E}+01$ \\
\hline ZERO & $0.1061 \mathrm{E}-02$ & $.9546 \mathrm{E}-03$ \\
\hline ZERO & $0.1587 \mathrm{E}+00$ & $1587 \mathrm{E}+00$ \\
\hline ZERO & ZERO & ZERO \\
\hline ZERO & $0.2437 \mathrm{E}-05$ & $0.2437 \mathrm{E}-05$ \\
\hline ZERO & $0.7493 \mathrm{E}-07$ & $0.7493 \mathrm{E}-07$ \\
\hline $0.7581 \mathrm{E}-11$ & ZERO & $0.1058 \mathrm{E}-24$ \\
\hline ZERO & ZERO & $0.5247 \mathrm{E}-02$ \\
\hline ZERO & ZERO & ZERO \\
\hline ZERO & ZERO & $0.7707 \mathrm{E}+01$ \\
\hline ZERO & ZERO & $0.8930 \mathrm{E}+01$ \\
\hline ZERO & ZERO & ZERO \\
\hline ZERO & ZERO & ZERO \\
\hline ZERO & ZERO & ZERO \\
\hline ZERO & ZERO & $0.3197 \mathrm{E}-01$ \\
\hline ZERO & $0.3797 \mathrm{E}+00$ & ZERO \\
\hline ZERO & ZERO & ZERO \\
\hline ZERO & ZERO & ZERO \\
\hline ZERO & ZERO & ZERO \\
\hline ZERO & ZERO & ZERO \\
\hline ZERO & $0.3719 \mathrm{E}-03$ & ZERO \\
\hline ZERO & ZERO & ZERO \\
\hline ZERO & ZERO & ZERO \\
\hline ZERO & $0.6882 \mathrm{E}-02$ & $0.6882 \mathrm{E}-02$ \\
\hline ZERO & ZERO & $0.1005 \mathrm{E}+02$ \\
\hline ZERO & ZERO & ZERO \\
\hline ZERO & ZERO & ZERO \\
\hline $0.1076 \mathrm{E}-06$ & ZERO & ZERO \\
\hline ZERO & $0.4280 \mathrm{E}+02$ & ZERO \\
\hline ZERO & ZERO & ZERO \\
\hline ZERO & $0.2948 E-03$ & $0.2948 \mathrm{E}-03$ \\
\hline ZERO & ZERO & $0.3918 \mathrm{E}-02$ \\
\hline $0.5254 \mathrm{E}-07$ & ZERO & $0.5254 \mathrm{E}-07$ \\
\hline ZERO & $0.1725 \mathrm{E}-02$ & $0.3432 E-03$ \\
\hline ZERO & ZERO & $0.3306 \mathrm{E}-02$ \\
\hline $0.6122 E-07$ & ZERO & $0.6122 \mathrm{E}-07$ \\
\hline ZERO & ZERO & ZERO \\
\hline ZERO & ZERO & . ZERO \\
\hline ZERO & $0.3295 \mathrm{E}-02$ & $0.1313 \mathrm{E}-02$ \\
\hline ZERO & $0.1096 \mathrm{E}-03$ & $0.1289 \mathrm{E}-03$ \\
\hline $0.3456 \mathrm{E}-03$ & ZERO & $0.6505 \mathrm{E}-07$ \\
\hline $0.1070 \mathrm{E}-09$ & ZERO & $0.1420 \mathrm{E}-19$ \\
\hline $0.1826 \mathrm{E}-05$ & ZERO & $0.9713 \mathrm{E}-07$ \\
\hline ZERO & ZERO & ZERO \\
\hline ZERO & ZERO & $0.4894 \mathrm{E}-03$ \\
\hline ZERO & ZERO & ZERO \\
\hline ZERO & ZERO & ZERO \\
\hline ZERO & $0.2617 \mathrm{E}-02$ & ZERO \\
\hline
\end{tabular}

Date: January 1995
678

salt Cell

Salt Cell
SRAT

$0.2061 \mathrm{E}-03$

$0.2179 \mathrm{E}+01$

$0.9546 \mathrm{E}-03$

$0.1587 \mathrm{E}+00$

$0.2437 \mathrm{E}-05$

$0.7493 \mathrm{E}-07$

TRACE

$0.5247 \mathrm{E}-02$

ZERO
$0.7707 \mathrm{E}+01$

$0.7707 \mathrm{E}+01$
$0.8914 \mathrm{E}+01$

$0.8914 \mathrm{E}+01$

ZERO

ZERO

$0.3197 \mathrm{E}-01$

ZERO

ZERO

ZERO

ZERO

ZERO

ZERO

ZERO

$0.6882 \mathrm{E}-02$

$0.1005 \mathrm{E}+02$

ZERO
ZERO

ZERO

ZERO

ZERO

$0.2948 \mathrm{E}-03$

$0.3918 \mathrm{E}-02$

$0.5254 \mathrm{E}-07$

$0.3432 \mathrm{E}-03$

$0.3306 \mathrm{E}-02$

$0.6122 \mathrm{E}-07$

ZERO

$0.1313 \mathrm{E}-02$

$0.1289 \mathrm{E}-03$

$0.9541 \mathrm{E}-02$

$0.5114 \mathrm{E}-22$

$0.4404 \mathrm{E}-07$

$0.4894 \mathrm{E}-03$

4E-03

ZERO

ZERO

ldge Feed

679

Insolubles

ZERO

ZERO

ZERO

$0.2093 \mathrm{ERO}$ ZERO

ZERO

ZERO

ZERO

ZERO

ZERO

ZERO

ZERO

ZERO

ZERO

ZERO

ZERO

ZERO

ZERO

ZERO

ZERO

ZERO

ZERO

ZERO

ZERO

ZERO

ZERO

ZERO

ERO

$0.1586 \mathrm{E}-01$

ZERO

ZERO

ZERO

ZERO

ZERO

ZERO

ZERO

ZERO

ZERO

ZERO

ZERO

ZERO

ZERO

ZERO

$.2805 \mathrm{E}+02$

$0.3541 \mathrm{E}-02$

$0.1511 \mathrm{E}-03$

ZERO

ZERO

ZERO

ZERO

ZERO

ZERO

ZERO

ZERO

ZERO

ZERO

ZERO

$0.1384 \mathrm{E}+00$

ZERO

$0.7385 \mathrm{E}-01$

ZERO

ZERO

ZERO

ZERO

ZERO

ZERO

ZERO

ZERO
$0.1524 \mathrm{E}+01$

ZERO

ZERO

ZERO

ZERO

ZERO

ZERO

ZERO

ZERO

ZERO

ZERO
0.6084 TER

ZERO

ZERO

ZERO

section 0, Page 23 of 37 
Table A-1. HLW Flowsheet Material Balance (Tank 51/ITP Cycle 1) Section 0. Major streams in the Flowsheet Model

STREAM NUMBERS (CONT'D) $\rightarrow$ STREAM NAME

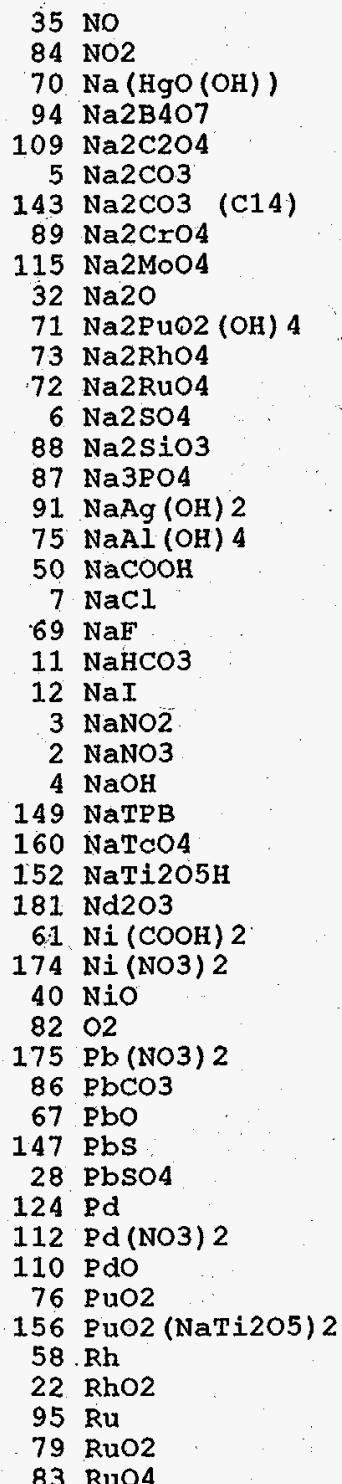

247

3 to PRBT Feed to SRAT $0.3469 \mathrm{E}-11 \quad 0.1771 \mathrm{E}-14$

ZERO
$0.4152 \mathrm{E}-03$ salt Cell

678

Sldge Feed

ZERO InsOLUbles

ZERO
$0.2562 E-03$

ZERO ZERO

ZERO

$0.2458 \mathrm{E}+00$ ZERO

$0.3019 \mathrm{E}-09$ ZERO

$0.9246 \mathrm{E}-02$ ZERO

$3 E+01$

$0.3865 \mathrm{E}-04$

$0.1652 \mathrm{E}-03$

$0.1944 \mathrm{E}+00$ IERO

$0.1141 \mathrm{E}-02$
$0.1457 \mathrm{E}-03$

$0.3558 \mathrm{E}+00$

$0.8321 \mathrm{E}-04$

$0.5573 \mathrm{E}-07$

$0.2200 \mathrm{E}-01$

$0.6777 \mathrm{E}-02$

$0.3408 \mathrm{E}-01$

ZERO
$0.5416 \mathrm{E}-05$

$0.6454 \mathrm{E}+01$

$0.1518 \mathrm{E}+00$

$0.3062 \mathrm{E}+01$

ZERO
$0.1568 \mathrm{E}-03$

ZERO

$0.6686 \mathrm{E}-06$

$0.8288 \mathrm{E}-03$

$0.5537 \mathrm{E}-02$

ZERO
ZERO ZERO

$0.4362 \mathrm{E}-02$

$0.3099 \mathrm{E}-08$ ZERO

ZERO

$0.1272 \mathrm{E}-03$

$0.3642 \mathrm{E}-06$

ZERO

$0.4426 \mathrm{EE}-02$

ZERO

$0.3230 \mathrm{E}+01$

ZERO

ZERO

ZERO

ZERO

ZERO

ZERO

ZERO

ZERO

ZERO

ERO

ZERO

ZERO

$0.9896 \mathrm{~F}-08$

ZERO

ZERO 
Table A-1. HLW Flowsheet Material Balance (Tank 51/ITP Cycle 1) section 0 . Major streams in the Flowsheet Model

STREAM NUMBERS (CONT'D) $\rightarrow$ STREAM NAME

\section{$38 \mathrm{SO} 2$}

141503

137 Semi Vol Cs20

136 Semi Vol $\mathrm{CsCl}$

126 Semi Vol Group A

140 Semi Vol Na2B4O

138 Semi Vol NaCl

139 Semi Vol NaF

125 Semi Vol NaI
23 Semi Vol RuO2

$111 \mathrm{SiO} 2$

$48 \operatorname{sr}(\mathrm{COOH}) 2$

$176 \mathrm{Sr}$ (NO3) 2

153 Sr (NaTi2O5) 2

$127 \mathrm{Sr}(\mathrm{OH}) 2$

$30 \quad \mathrm{SxCO}_{3}$

46 Sro

$159 \mathrm{TcO} 2$

92 ThO2

119 Tritium

$\begin{aligned} & 119 \text { Tritium } \\ & 17 \text { U308 }\end{aligned}$

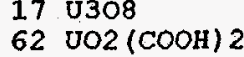

177. UO2 (NO3) 2

$16 \mathrm{UO} 2(\mathrm{OH}) 2$

$27 \mathrm{Y}(\mathrm{COOH}) 3$

178 Y (NO3) 3

$128 \mathrm{Y}(\mathrm{OH}) 3$

$31 \mathrm{Y} 2(\mathrm{CO})$
$47 \mathrm{Y} 2 \mathrm{O}$

100 Zeolite

$44 \mathrm{Zn}(\mathrm{COOH}) 2$

$44 \mathrm{Zn}(\mathrm{COOH})$
$179 \mathrm{Zn}(\mathrm{NO} 3) 2$

$57 \mathrm{zno}$

$104 \mathrm{zrO} 2$

13 hydrate $\mathrm{H} 2 \mathrm{O}$

1 water

TOTAL FLOW, LB/HR

TEMPERATURE， DEG C

PRESSURE, ATM

PRESSURE， PSIG

PRESSURE， MM HG

ENTHALPY, PCU/HR

VAPOR FLOW, CFM

LIQUID FLOW, GPM

DENSITY, LBS/FT3

PHASE

201

Washd Preci

Feed frm LWE

ZERO

ZERO

ZERO

ZERO

ZERO

ZERO

ZERO

ZERO

ZERO

$0.2666 \mathrm{E}-01$

ZERO

$0.4072 \mathrm{E}-02$

$0.7410 \mathrm{E}-12$

$0.9081 \mathrm{E}-04$

$$
\begin{aligned}
& \text { ZERO } \\
& \text { ZERO }
\end{aligned}
$$

$0.1213 \mathrm{E}-02$

$0.1282 \mathrm{E}-11$

$0.7512 \mathrm{E}-03$ ZERO

$0.1074 \mathrm{E}-05$ ZERO

$0.8232 \mathrm{E}-12$

$0.9683 \mathrm{E}-03$ ZERO

$0.5336 \mathrm{E}-04$

$0.1980 \mathrm{E}+00$ ZERO

$0.3589 \mathrm{E}-01$

$0.4274 E+03$

$0.4762 \mathrm{E}+03$

$0.3619 \mathrm{E}+02$

$0.9656 \mathrm{E}+00$

$-0.5055 E+00$
$0.7339 E+03$

$0.1554 \mathrm{E}+05$

$0.9392 \mathrm{E}+00$

$0.6322 \mathrm{E}+02$ LIQUID
657

$\begin{array}{ll}657 & 658 \\ \text { Prec Feed } & \text { Prec }\end{array}$

ZERO

Pred Feed

ZERO

ZERO

ZERO

ZERO

ZERO

ZERO

ZERO

ZERO

ZERO

ZERO

ZERO

$0.7410 \mathrm{E}-12$

ZERO

ZERO

ZERO

ZERO

$0.1282 \mathrm{E}-11$

ZERO
ZERO

ZERO

ZERO

$0.1074 E-05$

ZERO

$0.8232 \mathrm{E}-1$

ZERO

ZERO

ZERO

$0.5336 \mathrm{E}-04$

ZERO

ZERO

$0.4274 \mathrm{E}+03$

$0.4286 \mathrm{E}+03$

$0.3619 \mathrm{E}+02$

$0.9656 \mathrm{E}+00$

$0.7339 \mathrm{E}+03$

$0.1551 E+05$

$0.8551 E+00$

$0.6249 \mathrm{E}+02$

IIQUID

ZERO

ZERO

ZERO

ZERO

ZERO

ZERO

ZERO

ZERO

ZERO

.2666 E-01
ZERO

ZERO

$0.4072 \mathrm{E}-02$

0.9081 ZER-0

ZERO

ZERO

ZERO

$0.1213 \mathrm{E}-02$

ZERO
$0.7512 \mathrm{E}-03$

ZERO

$0.6751 \mathrm{E}-13$

ZERO

ZERO

ZERO

$0.9683 \mathrm{E}-03$

ZERO

ZERO

$0.1980 \mathrm{E}+00$

ZERO

$0.3589 \mathrm{E}-01$

ZERO
$0.4762 \mathrm{E}+02$

$0.3619 \mathrm{E}+02$
$0.9656 \mathrm{E}+00$

$-0.5055 \mathrm{E}+00$

$0.7339 \mathrm{E}+03$

$0.2660 \mathrm{E}+02$

$0.8411 \mathrm{E}-01$

$0.7060 \mathrm{E}+02$

IIQUID
247
PR Bottoms

to PRBT Feed to SRAT ZERO ZERO ZERO ZERO

ZERO

ZERO

ZERO

ZERO

ZERO

ZERO

$0.2667 \mathrm{E}-01$

$0.1093 \mathrm{E}-03$

ZERO
$0.4072 E-02$

ZERO
E-02
ZERO

ZERO

ZERO

$0.1761 \mathrm{E}-05$

ZERO

$0.7844 \mathrm{E}+00$

$0.1281 \mathrm{E}-11$

$0.7512 \mathrm{E}-03$

$0.1272 \mathrm{E}-05$ ZERO

$0.1317 \mathrm{E}-11$

ZERO

TERO

$0.9683 \mathrm{E}-03$

21139RO

$0.5336 \mathrm{~F}-0$

$0.1383 \mathrm{E}+00$

ZERO

$0.7722 \mathrm{E}+03$

$0.5000 \mathrm{E}+02$

$0.9656 \mathrm{E}+00$

$-0.5055 \mathrm{E}+00$

$0.3732 \mathrm{E}+05$

$0.1521 \mathrm{E}+01$

$.6330 \mathrm{E}+02$

LIQUID

$0.2667 \mathrm{E}-01$

ZRO

$0.4072 E-02$

ZERO

ZERO

$0.1761 \mathrm{E}-05$

ZERO

$0.7512 \mathrm{E}-03$

$0.1272 \mathrm{E}-05$

ZERO

$0.1317 \mathrm{E}-11$

ZERO

ZERO

$0.9683 \mathrm{E}-03$

ZERO

$0.1139+00$

$0.1383 \mathrm{E}+00$ ZERO

$0.2176 \mathrm{E}-01$

$0.7415 \mathrm{E}+03$

$0.7763 \mathrm{E}+03$

$0.4566 \mathrm{E}+02$

$0.9656 \mathrm{E}+00$ $-0.5055 \mathrm{E}+00$

$0.7339 \mathrm{E}+03$

$0.3428 \mathrm{E}+05$

$0.1529 \mathrm{E}+01$

$0.6329 \mathrm{E}+02$

LIQUID
678

sldge Feed

Solubles

ZERO

ZERO

ZERO

ZERO

ZERO

ZERO

ZERO

ZERO

ZERO

ZERO

ZERO

ZERO

$0.3612 \mathrm{E}-07$

ZERO

ZERO

ZERO

ZTRO

679

Sldge Eeed

ZERO

ZERO

ZERO

ZERO

ZERO

ZERO

ZERO

$0.1061 \mathrm{E}+01$

ZERO

ZERO 
Table A-1. HLW Flowsheet Material Balance (Tank 51/ITP Cycle 1) Section 0. Major streams in the Flowsheet Model

STREAM NUMBERS -> STREAM NAME

COMPONENT FLOWS, LB/HR 122 (C6H5) 2

$123(\mathrm{C} 6 \mathrm{H} 5) 2 \mathrm{C} 6 \mathrm{H} 4$

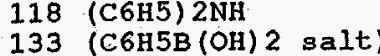

132 ( $6 \mathrm{H} 5 \mathrm{~B}(\mathrm{OH}) 2$ )

166 (C6H5C6H4OH)

162 (C6H5NH2)

163 (C6H5NHCHO)

165 (C6H5NO)

131 (C6H5OH salt)

131 (C6H5OH)

130 (C6H5O
121 (C6H6)

155 (CH3) $2 \mathrm{CHOH}$

154 ( $\mathrm{CH} 3 \mathrm{OH})$

$45 \mathrm{Ag}$

116 Ag2O

134 AgNO3

$106 \mathrm{~B} 203$

$9 \mathrm{Ba}(\mathrm{OH}) 2$

$15 \mathrm{BaO}$

$24 \mathrm{BaSO} 4$

$66 \mathrm{CO}$

$37 \mathrm{CO} 2$

$142 \mathrm{CO} 2$ (C14)

$63 \mathrm{Ca}(\mathrm{COOH}) 2$

$167 \mathrm{Ca}(\mathrm{NO} 3) 2$

$113 \mathrm{Ca} 3(\mathrm{PO} 4) 2$

$20 \mathrm{CaC} 2 \mathrm{O} 4$

$18 \mathrm{CaCO}^{3}$

$64 \mathrm{CaCO}_{3}$ (C14)

78 CaF2

$41 \mathrm{CaO}$

$19 \mathrm{CaSO} 4$

29 Carbon

$26 \mathrm{CO}(\mathrm{COOH}) 2$

$168 \mathrm{Co}$ (NO3)

$59 \mathrm{CoO}$

$98 \mathrm{Cr} 203$

$53 \mathrm{CsCOOH}$

$96 \mathrm{CsCl}$

169 CsNO3

$$
8 \mathrm{CsOH}
$$

151 CsTPB

WSRC-TR-95-0019 (Revision
8

$50 \%$ HNO3 SRAT Trans-
to SRAT fer to SME

1 Feed

to DWPF

ZERO
ZERO
ZERO

ZERO

ZERO

ZERO

ZERO

ZERO

ZERO

ZERO

ZERO

ZERO

ZERO

ZERO

$0.1018 \mathrm{E}-01$

ZERO

$0.8551 \mathrm{E}+01$

ZERO

ZERO

$0.1480 \mathrm{E}-01$

ZERO

ZERO

ZERO

ZERO

$0.2240 \mathrm{E}-04$

$0.2065 \mathrm{E}+01$

ZERO
$4 \mathrm{E}+01$

$0.2554 \mathrm{E}+01$

$0.1257 \mathrm{E}-06$

$0.3344 \mathrm{E}-02$

$0.2964 \mathrm{E}-01$

ZERO

ZERO

ZERO

ZERO

$0.1629 \mathrm{E}+00$

$0.1018 \mathrm{E}-03$

ZERO

ERO

$0.7390 \mathrm{E}-05$

$\begin{array}{lr}\text { ZERO } & 0.2620 \mathrm{E}-06 \\ \text { ZERO } & 0.9245 \mathrm{E}-02 \\ \text { ZERO } & 0.3165 \mathrm{E}-04 \\ \text { ZERO } & \text { ZERO } \\ \text { ZERO } & \text { ZERO } \\ \text { ZERO } & 0.5523 \mathrm{E}-01 \\ \text { ZERO } & 0.2409 \mathrm{E}-02 \\ \text { ZERO } & 0.2092 \mathrm{E}+00 \\ \text { ZERO } & 0.3406 \mathrm{E}-02 \\ \text { ZERO } & 0.6938 \mathrm{E}-02 \\ \text { ZERO } & 0.8234 \mathrm{E}-15 \\ \text { ZERO } & 0.1550 \mathrm{E}+00 \\ \text { ZERO } & 0.1006 \mathrm{E}-09 \\ \text { ZERO } & 0.1017 \mathrm{E}-10 \\ \text { ZERO } & 0.3681 \mathrm{E}-12 \\ \text { ZERO } & 0.1241 \mathrm{E}-01 \\ \text { ZERO } & \text { ZERO } \\ \text { ZERO } & \text { ZERO } \\ \text { ZERO } & 0.8834 \mathrm{E}+01\end{array}$

$0.1670 \mathrm{E}-02$

ZERO $\quad$ ZERO

ZERO $0.1617 \mathrm{E}-19$

ZERO

ZERO

ZERO

ZERO

ZERO

ZERO

ZERO

ZERO

ZERO

ZERO

ZERO

ZERO

ZERO

ZERO

ZERO

ZERO

ZERO

ZERO

ZERO

ZERO

ZERO

ZERO

Date: January 1995

ZERO
ZER

$.2418 \mathrm{E}-07$

ZERO

$0.4188 \mathrm{E}+01$

ZERO

$0.2626 \mathrm{E}+01$

ZERO

ZERO

$0.1501 \mathrm{E}-01$

$.3344 \mathrm{E}-02$

ZERO

$0.5373 \mathrm{E}-06$

$0.3069 \mathrm{E}-04$

$0.4289 \mathrm{E}+00$

$0.4330 \mathrm{E}-01$

ZERO
$0.9608 E-05$

ZERO
$106 \quad 250$

$50 \%$ HNO3 SCVC HEME FAVC HEME Decon Frit

to sMECT Vnt to PVVS Vnt to PVVS slxxy to SME

ZERO
ZERO
ZERO
ZERO
ZERO
ZERO
ZERO
ZERO
ZERO
ZERO
ZERO
ZERO
ZERO
ZERO
ZERO
ZERO
ZERO
ZERO
ZERO
ZERO
ZERO
ZERO
ZERO
ZERO
ZERO
ZERO
ZERO
ZERO
ZERO
ZERO
ZERO
ZERO
ZERO
ZERO
ZERO
ZERO
ZERO
ZERO
ZERO
ZERO
ZERO
ZERO
ZERO
ZERO
ZERO
ZERO
ZERO
ZERO

$0.1905 E-01$
$0.1740 E-15$
0.5247 E-10
ZERO
ZERO
ZERO
ZERO
ZERO
ZERO
ZERO
ZERO

$0.3612 \mathrm{E}-04$

$0.9318 \mathrm{E}+01$

$0.7606 \mathrm{E}-12$

$0.2677 \mathrm{E}-12$

ZERO

ZERO

ZERO

ZERO

ZERO

ZERO

ZERO

$0.6810 \mathrm{E}+02$

$0.1203 \mathrm{E}-09$

$3 E-09$
ZERO

ZERO

ZERO

ZERO

ZERO

ZERO

ZERO

ZERO

ZERO

ZERO

ZERO

ZERO

ZERO

ZERO

ZERO

ZERO

ZERO

ZERO

ZERO

ZERO

$0.3515 \mathrm{E}-10$
$0.7563 \mathrm{E}-04$

$\begin{aligned} & \text { ZERO } \text { ZERO } \\ & \text { ZERO }\end{aligned}$

ZERO ZERO

ZERO ZERO

ZERO ZERO

ZERO ZERO

ZERO ZERO

$0.6223 \mathrm{E}-19 \quad$ ZERO

$0.1138 \mathrm{E}-01$ ZERO

$0.1406 \mathrm{E}-01$ ZERO

ZERO

$0.2173 \mathrm{E}-11$ ZERO

$\begin{aligned} .1404 \mathrm{E}-18 & \text { ZERO } \\ \text { TRACE } & \text { ZERO }\end{aligned}$

ZERO
$0.3136 \mathrm{E}-14$
$0.1137 \mathrm{E}-14$

$0.1137 \mathrm{E}-14 \quad 0.8542 \mathrm{E}+00$

ZERO
$0.1190 \mathrm{E}-23$

$0.6048 \mathrm{E}-17$ : ZERO

ZERO ZERO

$0.5680 \mathrm{E}-07$ ZERO

ZERO

ZERO

ZERO ZERO

$0.8649 \mathrm{E}-22$ ZERO

ZERO ZERO
ZERO

$\begin{aligned} \text { ZERO } & \text { ZERO } \\ 0.5328 \mathrm{E}-17 & \text { ZERO }\end{aligned}$

$0.1189 \mathrm{E}-17$ ZERO

$0.7822 \mathrm{E}-16 \quad$ ZERO

ZERO ZERO

$0.5074 \mathrm{E}-19$

ZERO ZERO

ZERO

$\begin{array}{rr}0.4980 \mathrm{E}-19 & 0.1254 \mathrm{E}-02 \\ \text { ZERO }\end{array}$

$0.1534 \mathrm{E}-16 \quad$ ZERO ZERO ZERO

ZERO
$0.3890 \mathrm{E}-21$

ZERO ZERO

Section 0, Page 26 of 37 
Table A-1. HLW Flowsheet Material Balance (Tank 51/ITP Cyole 1 ) Section 0 . Major Streams in the Flowsheet Model

STREAM NUMBERS (CONT'D) $\rightarrow$ STREAM NAME

$54 \mathrm{Cu}(\mathrm{COOH}) 2$

$170 \mathrm{Cu}(\mathrm{NO} 3)^{2}$

55 CuO

$80 \mathrm{Fe} 203$

$101 \mathrm{E} \in \mathrm{O}$

102 Group $A$

103 Group B

$25 \mathrm{H} 2$

$158 \mathrm{H} 2 \mathrm{C} 2 \mathrm{O} 4$

\begin{tabular}{ll}
93 & $\mathrm{H} 2 \mathrm{SO}$ \\
90 & $\mathrm{H} 3 \mathrm{BO}$ \\
\hline
\end{tabular}

34 . $\mathrm{HCOOH}$

43 HCI

$85 \mathrm{HF}$

49 HNO3

$36 \mathrm{Hg}$

$1.29 \mathrm{Hg}(\mathrm{C} 6 \mathrm{H} 5) 2$

$135 \mathrm{Hg}$ (NO3) 2

$144 \mathrm{Hg} 2 \mathrm{Cl}$

$117 \mathrm{Hg} 2 \mathrm{I} 2$

146 HgCl2

$21 \mathrm{HgO}$

$\begin{array}{rl}97 . & \mathrm{I} 2 \\ 183 \mathrm{KO} & \mathrm{KO}\end{array}$

$39 \mathrm{~K} 2 \mathrm{O}$

$51 \mathrm{KCOOH}$

$157 \mathrm{KMnO4}$

$161 \mathrm{KNO} 3$

$150 \mathrm{KTPB}$

$105 \mathrm{La2O3}$

$\begin{array}{ll}105 & \text { La2O3 } \\ 108 & \mathrm{~L} i 20\end{array}$

$65 \mathrm{Mg}$ (COOH) 2

$171 \mathrm{Mg}$ (NO3) 2

$33 \mathrm{MgO}$

$60 \mathrm{Mn}$ (COOH) 2

$172 \mathrm{Mn}$ (NO3) 2

$180 \mathrm{Mn} 304$

56. MnO

$14 \mathrm{MnO} 2$

$145 \mathrm{MOO} 2$

81 N2

$74 \mathrm{~N} 2 \mathrm{O}$

182 NH3 114 NH3OHNO3

52 NH4 COOH

$173 \mathrm{NH} 4 \mathrm{NO} 3$

$68 \mathrm{NH} 4 \mathrm{OH}$

148 NH 4 TPB

WSRC-TR-95-0019 (Revision

$$
8
$$

sludge Feed

to DWPE

ZERO
ZERO

$0.2093 \mathrm{E}-01$

$0.2805 \mathrm{E}+02$

$0.3541 \mathrm{E}-02$

$0.1511 E-03$

ZERO

ZERO

ZERO

ZERO

ZERO

ZERO

ZERO

ZERO

ZERO

ZERO

ZERO

$0.1384 \mathrm{E}+00$

ZERO

$0.7385 \mathrm{E}-01$

ZERO

ZERO

$0.1586 \mathrm{E}-01$

ZERO

ZERO

ZERO

$0.1524 \mathrm{E}+01$

ZERO

ZERO

ZERO

ZERO

$0.3245 \mathrm{E}+01$

ZERO

ZERO

ZERO

ZERO

ZERO

0. 6084E-03

ZERO
50 HNO3 15

to SRAT

ZERO

ZERO $0.1969 \mathrm{E}-01$

ZERO $0.2539 \mathrm{E}+02$

ZERO $0.2538 \mathrm{E}+01$

ZERO $0.3543 \mathrm{E}-02$

ZERO $\quad 0.1512 \mathrm{E}-03$

ZERO $0.5285 \mathrm{E}-12$

ZERO

ZERO

ZERO
0.7707 E+01
ZERO

ZERO

ZERO

$0.7818 \mathrm{E}+01$

ZERO

ZERO

ZERO

ZERO

ZERO

ZERO

ZERO

ZERO

ZERO

ZERO

ZERO

ZERO

ZERO

ZERO

TERO

ZERO

ZERO

ZERO

ZERO

ZERO

ZERO

ZERO

ZERO

ZERO

TERO

ZERO

ZERO

ZERO

TERO

ZERO

ZERO

ZERO
$0.4360 \mathrm{E}-09$

$0.1604 \mathrm{E}+00$

ZERO
ZERO

ZERO

ZERO

ZERO

ZERO

ZERO

$0.8073 \mathrm{E}-01$

$0.1005 \mathrm{E}+02$

$0.2858 \mathrm{E}-01$

ZERO

ZERO

$0.2948 \mathrm{E}-03$

$0.3463 \mathrm{E}+01$

$0.5254 \mathrm{E}-07$

$0.3049 \mathrm{E}+0.0$

$0.2169 \mathrm{E}+01$

ZERO

$0.3831 \mathrm{E}-16$

$0.1948 \mathrm{E}+01$

$0.1075 \mathrm{E}-02$

.9364E-08

$0.1087 \mathrm{E}+00$

ZERO

$0.1391 \mathrm{E}-02$

ZERO

Date: January 1995
106

$50 \%$ HNO3 SCVC HEME

to SMECT Vnt to PVVS

ZERO ZERO

ZERO

ZERO

ZERO

ZERO

ZERO

ZERO

ZERO

ZERO

ZERO

ZERO

ZERO

$0.5554 \mathrm{E}+01$

ZERO

ZERO

ZERO

ZERO

ZERO

ZERO

ZERO

ZERO

ZERO

ZERO

ZERO

ZERO

ZERO

ZERO

ZERO

ZERO

ZERO

ZERO

ZERO

ZERO

ZERO

ZERO

ZERO

ZERO

ZERO

ZERO

ZERO

ZERO

ZERO

ZERO

$0.7601 \mathrm{E}-04$

ZERO

ZERO

0.4004 ZERO

ZERO

ZERO

ZERO

ZERO

ZERO

ZERO

ZERO

ZERO

ZERO

ZERO

ZERO

ZERO

ZERO

ZERO

ZERO

ZERO

ZERO

ZERO

ZERO

ZERO

ZERO

ZERO

$0.4532 \mathrm{E}+01$

$0.1070 \mathrm{E}-09$

ZERO

ZERO

ZERO

ZERO

FAVC HEME Decon Frit

nt to PVVS slrry to SME

$0.1573 \mathrm{E}-17$

$0.6992 \mathrm{E}-17$

$0.9013 \mathrm{E}-14$

$0.9011 \mathrm{E}-15$

$0.1258 \mathrm{E}-17$

$0.5367 \mathrm{E}-19$

$0.3136 \mathrm{E}-01$

$0.6486 \mathrm{E}-16$

ZERO

ZERO

$0.4880 \mathrm{E}-03$

ZERO

ZERO

ZERO

ZERO

ZERO

ZERO

ZERO ZERO

ZERO
ZERO

ZERO

ZERO

ZERO ZERO

ZERO ZERO

ZERO ZERO

ZERO ZERO

ZERO

ZERO
$0.2866 E-16$

$0.3558 \mathrm{E}-14$ ZERO

$0.1015 \mathrm{E}-16 \quad$ ZERO

$0.1163 E-19$ ZERO

ZERO ZERO


Table A-1. HLW Flowsheet Material Balance (Tank 51/ITP Cycle 1) section 0 . Major streams in the $\mathrm{Flowsheet}$ Model

STREAM NUMBERS (CONT'D) $\rightarrow$ STREAM NAME

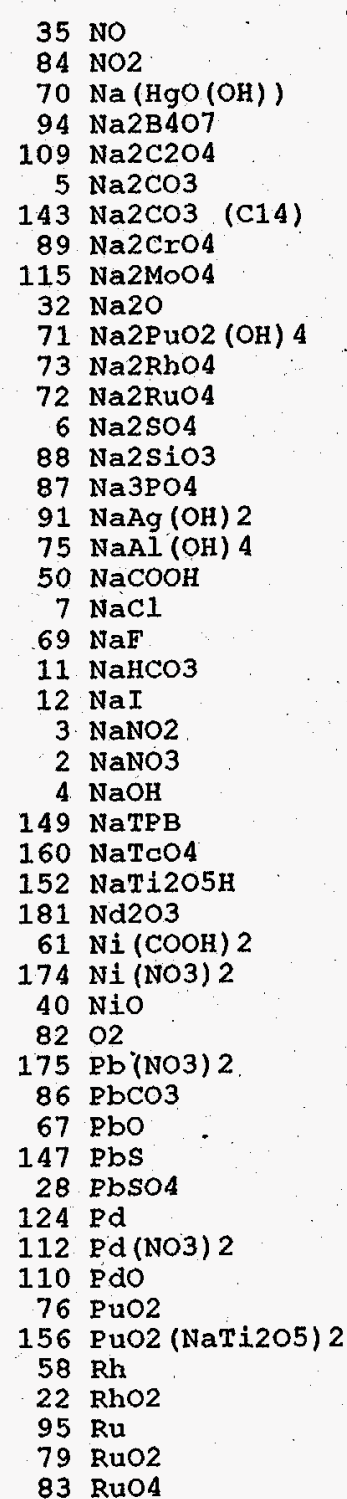

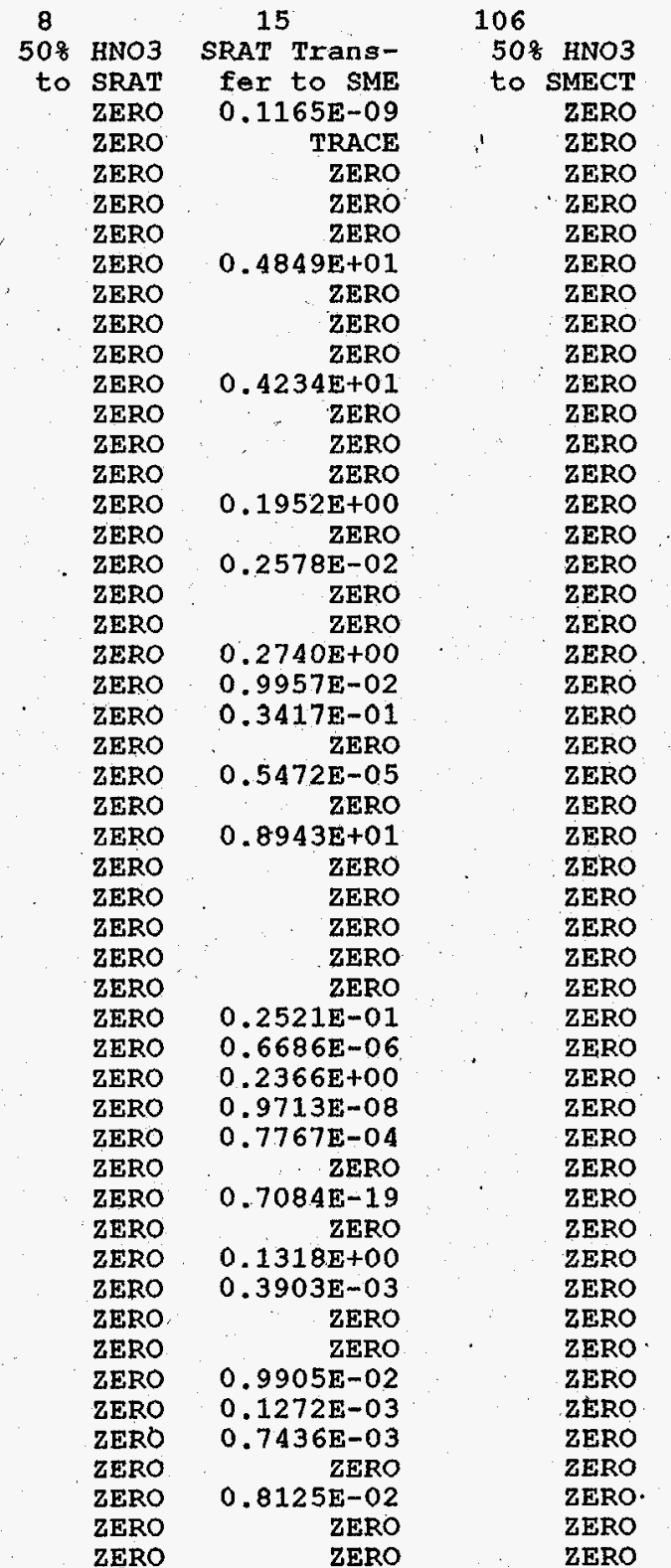

WSRC-TR-95-0019 (Revision
250

SCVC HEME

Vnt to PVVS

$0.3364 \mathrm{E}-01$
$0.2673 \mathrm{E}-08$

0.2673 E-08

ZERO

ZERO

ZERO

ZERO

ZERO

ZERO

ZERO

ZERO

ZERO

ZERO

ZERO

ZERO

ZERO

ZERO

ZERO

ZERO

ZERO

ZERO

ZERO

ZERO

ZERO

ZERO

ZERO

ZERO

ZERO

ZERO

ZERO

$0.1369 \mathrm{~F}+01$

ZERO
ZERO

ZERO

ZERO

ZERO

ZERO

ZERO

ZERO

ZERO

ZERO

ZERO

ZERO

ZERO

ZERO

Date: Januaxy 1995
$40 \quad 26$

FAVC HEME Decon Frit Vnt to PVVS slrry to SME ZERO ZERO $0.2184 \mathrm{E}+01$ ZERO $0.1304 \mathrm{E}-19$ ZERO $0.8409 \mathrm{E}-18$ ZERO $0.1738 \mathrm{E}-14$ ZERO ZERO ZERO ZERO ZERO $0.2259 \mathrm{E}-14 \quad 0.5687 \mathrm{E}+00$ ZERO ZERO ZERO 'ZERO $0.6930 \mathrm{E}-16 \quad$ ZERO $0.9140 \mathrm{E}-18$ ZERO ZERO
ZERO $0.2782 \mathrm{E}-15 \quad 0.1579 \mathrm{E}+00$ $0.3532 \mathrm{E}-17$ ZERO $0.1213 E-16 \quad$ ZERO $0.1943 \mathrm{E}-20 \quad$ ZERO $0.1051 \mathrm{E}-17$ ZERO $0.3203 \mathrm{E}-14$ ZERO ZERO ZERO ZERO ZERO ZERO $\begin{array}{rr}0.8949 \mathrm{E}-1.7 & \text { ZERO } \\ \text { ZERO } & \text { ZERO }\end{array}$ $0.8444 \mathrm{E}-16 \quad 0.5036 \mathrm{E}-02$ $.4007 \mathrm{E}+03$
ZERO ZERO
$0.5210 E-23$
ZERO ZERO ZERO $0.4679 E-16$ ZERO $3964 \mathrm{E}-20$
ZERO $\begin{array}{rr}\text { ZERO } & \text { ZERO } \\ \text { TRACE } & \text { ZERO }\end{array}$ $0.3517 \mathrm{E}-17$ ZERO $0.4505 E-19$ ZERO $0.7554 \mathrm{E}-20 \quad$ ZERO TRACE
$0.2882 \mathrm{E}-17, \quad$ ZERO
ZERO $\begin{array}{lll}\text { ZERO } & \text { ZERO } \\ \text { ZERO } & \text { ZERO }\end{array}$ 
Table A-1. HLW Flowsheet Material Balance (Tank 51/ITP Cycle 1)

Section 0. Major streams in the Flowsheet Model

STREAM NUMBERS (CONT'D) $\rightarrow$ STREAM NAME

$38 \mathrm{SO} 2$

$141 \mathrm{so3}$

137 Semi Vol Cs20

136 Semi Vol CsCl

126 Semi Vol Group A

140 Semi Vol Na2B4O?

138 Semi Vol NaCl

139 Semi Vol NaF

125 Semi Vol NaI

23 Semi Vol RuO2

$111 \mathrm{SiO} 2$

$48 \mathrm{Sr}(\mathrm{COOH}) 2$

$176 \mathrm{Sr}$ (NO3) 2

$153 \mathrm{Sr}(\mathrm{NaTi2O5)} 2$

$127 \mathrm{Sr}(\mathrm{OH}) 2$

$30.5 x \mathrm{sin}$

$\begin{array}{rl}46 & \mathrm{SrO} \\ 159 & \mathrm{TcO}\end{array}$

$159 \mathrm{TcO} 2$

107 TiO2

119 Tritium

170308

$62 \mathrm{VO} 2(\mathrm{COOH}) 2$

177002 (NO3) 2

$16 \mathrm{VO} 2(\mathrm{OH}) 2$

$-27 \mathrm{Y}(\mathrm{COOH}) 3$

$178 \mathrm{Y}(\mathrm{NO} 3)$

$128 \mathrm{Y}(\mathrm{OH}) 3$

$47 \mathrm{Y} 203$

100 Zeolite

$44 \mathrm{Zn}$ (COOH) 2

$179 \mathrm{Zn}(\mathrm{NO} 3)$

$57 \mathrm{zno}$

$104 \mathrm{ZrO} 2$

13 hydrat

TOTAL FLOW, LB/HR

TEMPERATURE, DEG C

PRESSURE; ATM

PRESSURE， PSIG

PRESSURE， MM HG

ENTHATPY, PCU/HR

VAPOR FLOW, CFM

LIQUID FLOW, GPM

DENSITY, LBS/FT3

PHASE

WSRC-TR-95-0019 (Revision
1
sludge Feed $\quad 50$

to D

ZERO

ZERO

ZERO

ZERO

ZERO

ZERO

ZERO

ZERO

$0.1061 E+01$

ZERO
ZERO

ZERO

ZERO

$0.3612 \mathrm{E}-07$

$0.2054 \mathrm{E}-01$

$0.3457 \mathrm{E}-02$

$0.2972 \mathrm{E}-01$

$0.4648 \mathrm{E}-01$

$0.1023 \mathrm{E}-08$

$0.2083 \mathrm{E}+01$

ZERO

$0.7050 \mathrm{E}-04$

ZERO

ZERO
0.364 OE-07

$0.5168 \mathrm{E}-02$

ZERO

ZERO

ZERO

$0.1179 \mathrm{E}+00$

ZERO

$0.2279 \mathrm{E}+01$

$0.3778 \mathrm{E}+03$

$0.4446 \mathrm{E}+03$

$0.6000 \mathrm{E}+02$

$0.1000 E+01$

ZERO

$0.7920 \mathrm{E}+00$

$0.6998 \mathrm{E}+02$

I.IQUID
8

to SRAT SRAT Trans-

SRAT fer to SME

ZERO $\quad$ ZERO

ZERO - ZERO

ZERO. ZERO

ZERO ZERO

ZERO

ZERO ZERO

ZERO ZERO

ZERO ZERO ZERO

ZERO $\quad 0.1092 \mathrm{E}+01$

ZERO $\quad 0.1093 \mathrm{E}-03$

ZERO $\quad 0.2944 \mathrm{E}-01$

ZERO $\quad 0.4072 \mathrm{E}-02$

ZERO ZERO

ZERO $0.2218 \mathrm{E}-18$

ZERO $0.3569 \mathrm{E}-02$

ZERO $0.2972 \mathrm{E}-01$

ZERO $0.8309 \mathrm{E}+00$

ZERO - $0.3022 E-09$

TRRO $0.2084 \mathrm{E}+01$

ZRRO $0.12725-05$

ZERO $\quad 0.9137 \mathrm{E}-04$

TERO

ZERO

ZERO

ZERO

ZERO

ZERO

ZERO

ZERO

ZERO

ZERO

ZERO

$0.7818 \mathrm{E}+01$

$0.1564 \mathrm{E}+02$

$0.2500 \mathrm{E}+02$

$0.1000 E+01$
ZERO

ZERO

ZERO

ZERO

ZERO

O.1499E+01

$0.4401 \mathrm{E}+03$

$0.4959 \mathrm{E}+02$

$0.1000 E+01$
ZERO

$0.2520 \mathrm{E}+03$

$0.1786 \mathrm{E}+05$

$0.2601 \mathrm{E}-01$

$0.7495 \mathrm{E}+02$

$0.7609 \mathrm{E}+00$

$0.7212 \mathrm{E}+02$

IIQUID
$0.7940 \mathrm{E}-02$

106

508 HNO3

250

SCVC HEME

40

Vnt to PVVS slrry to SME

$\begin{array}{lll}\text { ZERO } & \text { ZERO } & \text { ZERO } \\ \text { ZERO } & \text { ZERO }\end{array}$

$\begin{array}{lll}\text { ZERO } & \text { ZERO } & \text { ZERO } \\ \text { ZERO } & \text { ZERO } & \text { ZERO }\end{array}$

ZERO ZERO $\quad$ ZERO

ZERO ZERO ZERO ZERO

ZERO ZERO ZERO ZERO

ZERO ZERO $\quad$ ZERO $\quad$ ZERO

ZERO ZERO $\quad$ ZERO

ZERO

ZERO

ERO

ZERO

ZERO

ZERO

ZERO

ZERO

ZERO

ZERO

ZERO

ZERO

ZERO

ZERO

ZERO

ZERO

ZERO

ZERO

ZERO

ZERO

ZERO

ZERO

ZERO

ZERO

ZERO

ZERO

ZERO

ZERO

$0.5554 \mathrm{E}+01$

$0.1111 \mathrm{E}+02$

$0.2500 \mathrm{E}+02$

$0.1000 \mathrm{E}+01$

ZERO

$0.1790 \mathrm{E}+03$

$0.1848 \mathrm{E}-01$

$0.7495 \mathrm{E}+02$

LIQUID

Date: January, 1995
ZERO

ZERO

ZERO

ZERO

ZERO

ZERO

ZERO

ZERO

$0.7056 \mathrm{E}-15$

ZERO

ZERO

ZERO

ZERO

ZERO

ZERO

ZERO

ZERO

ZERO

ZERO

ZERO

ZERO

$0.3968 \mathrm{E}+00$

$0.8376 \mathrm{E}+02$

$0.1000 \mathrm{E}+02$

$0.9656 \mathrm{E}+00$

$-0.5055 \mathrm{E}+00$

$0.7339 \mathrm{E}+03$

$0.1217 \mathrm{E}+02$

$0.1442 \mathrm{E}-17$

ZERO
ZERO

ZERO

ZERO

$0.1267 \mathrm{E}-17$

$0.1055 \mathrm{E}-16$

$0.2943 \mathrm{E}-15$

$0.4505 \mathrm{E}-21$

ZERO

ZERO

TRACE

ZERO

ERO

$0.3429 \mathrm{E}-18$

0.9241 EERO

¿.6360E-16

ZERO

$0.5323 \mathrm{E}-15$

$0.1281 \mathrm{E}+02$

$0.1000 \mathrm{E}+02$

$0.9263 \mathrm{E}+00$

$-0.1083 \mathrm{E}+01$

$0.4074 \mathrm{E}+03$

$0.1148 \mathrm{E}+00$

$0.7161 \mathrm{E}-01$

VAPOR

ZERO

ZERO

ZERO

ZERO

ZERO

ZERO

ZTRO

ZERO

ZERO

ZERO

ZERO

ZERO

ZERO

ZERO

VAPOR

$0.6412 \mathrm{E}+02$

LIQUID

Seation 0, Page 29 of 37
$0.3870 \mathrm{E}-19$

$0.8221 E+01$ 
WSRC-TR-95-0019 (Revision

0)
Date: January 1995

Section 0, Page 30 of 37

Table A-1. HLW Flowsheet Material Balance (Tank 51/ITP Cyale 1) Section 0. Major streams in the Flowsheet Model

STREAM NUMBERS $\rightarrow$ STREAM NAME

COMPONENT FLOWS, LB/HR 122 (C6H5) 2

123 (C6H5) $2 \mathrm{C} 6 \mathrm{H} 4$

118 (C6H5) $2 \mathrm{NH}$

133 (C6H5B (OH) 2 salt)

132 (C6H5B (OH) 2)

166 (C6H5C6H4OH)

163 (C6H5NHCHO)

165 (C6H5NO)

165 (C6H5NO)
164 (C6H5NO2)

164 (C6H5NO2)

130 (C6H5OH)

121 (C6H6)

154 (СH3OH)

$45 \mathrm{Ag}$

116 Ag20

134 AgNO3

$106 \mathrm{~B} 203$

$9 \mathrm{Ba}(\mathrm{OH}) 2$

$15 \mathrm{BaO}$

$24 \mathrm{BaSO} 4$

$66 \mathrm{CO}$

$\begin{array}{rrr}37 & \mathrm{CO} 2 & \\ 142 & \mathrm{CO} 2 & \text { (C14) }\end{array}$

$63 \mathrm{Ca}(\mathrm{COOH}) 2$

$167 \mathrm{Ca}(\mathrm{NO} 3) 2$

$120 \mathrm{Ca}(\mathrm{OH}) 2$

$20 \mathrm{CaC} 2 \mathrm{O} 4$

$18 \mathrm{CaCO} 3$

$64 \mathrm{CaCO}^{3}$ (C14)

$78 \mathrm{CaF} 2$

$41 \mathrm{CaO}$

19 Caso4

$\begin{array}{ll}19 & \text { Caso4 } \\ 29 & \text { Carbon }\end{array}$

99 Cement

$26 \mathrm{Co}(\mathrm{COOH}) 2$

$168 \mathrm{Co}$ (NO3) 2

$59 \mathrm{CoO}$

98 Cr2O3

$42 \mathrm{Cs} 2 \mathrm{O}$

$53 \mathrm{CsCOOH}$

$96 \mathrm{CsCl}$

$169 \mathrm{CsNO}$

$8 \mathrm{CsOH}$

27 44

resh Frit SME Concensirry to SME trate to MFT

60

Melter Sldge Blend Feed Waste Glass

ZERO
ZERO
ZERO
ZERO
ZERO
ZERO
ZERO
ZERO
ZERO
ZERO
ZERO
ZERO
ZERO
ZERO
ZERO
ZERO
ZERO
ZERO
ZERO

$0.1181 \mathrm{E}+02$

ZERO

ZERO

ZERO

ZERO

ZERO

ZERO

ZERO

ZERO

ZERO

ZERO

ZERO

ZERO

ZERO

ZERO

ZERO

ZERO

ZERO

ZERO

2ERO

ZERO

ZERO

ZERO

ZERO

ZERO

$0.2348 \mathrm{E}-15$

$0.9245 \mathrm{E}-02$

$0.4476 \mathrm{E}-08$

$0.2853 \mathrm{E}-18$

$0.9245 \mathrm{E}-02$

$0.4221 \mathrm{E}-08$.

ZERO

ZERO

$0.5523 \mathrm{E}-01$

$0.1793 \mathrm{E}-03$

$0.2092 \mathrm{E}+00$

$0.3406 \mathrm{E}-02$

$0.6938 \mathrm{E}-02$

$0.8234 \mathrm{E}-15$

$0.5070 \mathrm{E}-01$

$0.3022 \mathrm{E}-22$

$0.2687 \mathrm{E}-11$

$0.4173 \mathrm{E}-13$
$0.1241 \mathrm{E}-01$

$0.1241 \mathrm{E}-01$ ZERO

$0.8834 \mathrm{E}+01$

$0.1266 \mathrm{E}+02$

ZERO

$0.1704 \mathrm{E}-01$

ZERO

$4389 \mathrm{E}-16$ TRACE
ZERO

$0.4188 \mathrm{E}+01$

ZERO

$0.2626 \mathrm{E}+01$
$0.2442 \mathrm{E}-06$ ZERO

$0.1501 \mathrm{E}-01$

$0.3344 \mathrm{E}-02$

$0.5523 E-01$

$0.1648 \mathrm{E}-03$

$0.2092 \mathrm{E}+00$

$0.3406 \mathrm{E}-02$

$0.6938 \mathrm{E}-02$

$0.8234 \mathrm{E}-15$

$0.4876 \mathrm{E}-01$

$0.2534 \mathrm{E}-24$

$0.2588 \mathrm{E}-11$

$0.3832 \mathrm{E}-13$

$0.1241 \mathrm{E}-01$

ZERO
ZERO

$0.8834 \mathrm{E}+01$

$0.1266 \mathrm{E}+02$

ZERO
$0.1617 \mathrm{E}-19$

$0.1704 \mathrm{E}-01$

ZERO

TRACE TRACE
ZERO

$0.4188 \mathrm{E}+01$ ZERO

$0.2626 \mathrm{E}+01$

$0.2442 \mathrm{E}-06$

ZERO

$0.1501 \mathrm{E}-01$

$0.3344 \mathrm{E}-02$

$0.2208 \mathrm{E}+00$

$$
\text { ZERO. }
$$

ZERO

$0.1433 \mathrm{E}-03$

$0.5373 \mathrm{E}-06$

$0.3069 \mathrm{E}-04$
$0.4302 \mathrm{E}+00$

$0.1403 \mathrm{E}-03$

$0.4330 \mathrm{E}-01$

$0.9608 \mathrm{E}-05$

ZERO
ZERO

0.1433E-03

$0.5373 \mathrm{E}-06$

$0.3069 \mathrm{E}-04$

$0.4302 \mathrm{E}+00$

$0.1403 \mathrm{E}-03$

$0.4330 \mathrm{E}-01$

ZERO
$0.9608 \mathrm{E}-05$

ZERO

1 ZERO

ZERO

ZERO

ZERO

ZERO

ZERO

ZERO

ZERO

ZERO

ZERO

ZERO

ZERO

ZERO

$0.1241 \mathrm{E}-01$

ZERO

$0.8746 \mathrm{E}+01$

$0.1675 \mathrm{E}+02$

$0.1108 \mathrm{E}-02$

$0.1518 \mathrm{E}-01$

ZERO

ZERO

ZERO

ZERO
ZERO

ZERO

$0.2600 \mathrm{E}+01$

ZERO

ZERO

ZERO

$0.1440 \mathrm{E}+01$

$0.1967 \mathrm{E}+00$

ZERO
ZERO

ZERO

ZERO

$0.1020 \mathrm{E}-03$

$0.4259 \mathrm{E}+00$
$0.3069 \mathrm{E}-01$

ZERO

ZERO

ZERO

ZERO

Date: January 1995
$200 \quad 145$

Melter

Melter

ZERO

ZERO

ZERO

ZERO

ZERO

ZERO

ZERO
ZERO
EE-03

$0.3469 \mathrm{E}-03$

$0.8234 \mathrm{E}-15$

$.2438 \mathrm{E}-02$

ZERO

ZERO

ZERO

ZERO

$0.8834 \mathrm{E}-01$

$0.1692 \mathrm{E}+00$

ZERO

$0.1119 \mathrm{E}-04$

ZERO

$0.1410 \mathrm{E}+02$

TRACE

ZERO

ZERO

$0.2626 \mathrm{E}-01$

ZERO

ZERO

ZERO

$0.1454 \mathrm{E}-01$
$0.1987 \mathrm{E}-02$

ZERO

ZERO

ZERO

$0.1030 \mathrm{E}-05$

$0.4302 \mathrm{E}-02$

$0.3100 \mathrm{E}-03$

ZERO

ZERO

ZERO

ZERO

Section 0, Page 30 of 37

Stack Purified $\mathrm{Hg}$

$0.2924 \mathrm{E}-01 \quad 0.1893 \mathrm{E}-20$

$0.3515 \mathrm{E}-10 \quad 0.3225 \mathrm{E}-19$

$0.7563 \mathrm{E}-04 \quad 0.3347 \mathrm{E}-15$

$\begin{array}{ll}\text { ZERO } & \text { ZERO } \\ \text { ZERO } & \text { ZERO }\end{array}$

ZERO $0.9397 \mathrm{E}-24$

ZERO $0.3412 \mathrm{E}-20$

ZERO $0.4984 \mathrm{E}-23$

ZERO TRACE

TRACE

$0.1142 \mathrm{E}-01$

$0.0332 \mathrm{E}+01$

$0.8827 \mathrm{E}-11$

$0.2441 \mathrm{E}-11$

TRACE

TRACE

$0.3534 \mathrm{E}-13$

$0.3243 \mathrm{E}-12$

$0.6134 \mathrm{E}-16$

ZERO

$0.8652 \mathrm{E}+02$
$0.5692 \mathrm{E}-07$

ZERO

ZERO

$0.1050 \mathrm{E}-13$

TRACE

ZERO

$0.3463 E-23$$$
\text { ZERO }
$$

ZERO

TRACE

ZERO

$0.4119 \mathrm{E}-18$

$0.1240 \mathrm{E}-15$

$0.9969 \mathrm{E}-23$

$0.5144 \mathrm{E}-11$

ZERO
TRACE

ZERO
$0.4478 \mathrm{E}-17$ 
Table A-1. HLW Flowsheet Material Balance (Tank 51/ITP Cycle 1 ) section 0. Major streams in the Flowsheet Model.

STREAM NOMBERS (CONT'D) $\rightarrow$ STREAM NAME

$54 \mathrm{Cu}(\mathrm{COOH}) 2$

$170 \mathrm{Cu}(\mathrm{NO} 3) 2$

55 CuO

$80 \mathrm{~F} \oplus 203$

$101 \mathrm{FeO}$

102 Group A

103 Group B

$25 \mathrm{H} 2$

$\begin{array}{rl}158 & \mathrm{H} 2 \mathrm{C} 2 \mathrm{O} 4 \\ 93 & \mathrm{H} 2 \mathrm{SO} 4\end{array}$

$90 \mathrm{H} 3 \mathrm{BO} 3$

34 HCOOH

$43 \mathrm{HCl}$

$85 \mathrm{HF}$

49 HNO3

$36 \mathrm{Hg}$

$129 \mathrm{Hg}(\mathrm{C} 6 \mathrm{H} 5) 2$

$135 \mathrm{Hg}$ (NO3) 2

$144 \mathrm{Hg} 2 \mathrm{Cl} 2$

$117 \mathrm{Hg} 2 \mathrm{I2}$

$146 \mathrm{HgCl} 2$

$21 \mathrm{HgO}$

$183 \mathrm{~K} 2 \mathrm{CO} 3$

$39 \mathrm{~K} 20$

$51 \mathrm{KCOOH}$

157 $\mathrm{KMnO} 4$

$161 \mathrm{KNO3}$

$10 \mathrm{KOH}$

$150 \mathrm{KTPB}$

$108 \mathrm{Li} 20$

$65 \mathrm{Mg}(\mathrm{COOH}) 2$

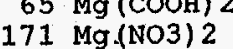

$33 \mathrm{MgO}$

$60 \mathrm{Mn}(\mathrm{COOH}) 2$

$172 \mathrm{Mn}$ (NO3) 2

$180 \mathrm{Mn} 304$

$56 \mathrm{MnO}$

14. $\mathrm{MnO} 2$

$145 \mathrm{MoO2}$

$81 \mathrm{~N} 2$

$74 \mathrm{~N} 2 \mathrm{O}$

$182 \mathrm{NH} 3$

114 NH3OHNO3

$52 \mathrm{NH} 4 \mathrm{COOH}$

173 NH 4 NO

$68 \mathrm{NH} 4 \mathrm{OH}$

148 NH 4 TPB

WSRC-TR-95-0019 (Revision
27 Frit SME Concenslrry to SME trate to MFT ZERO $0.4430 \mathrm{E}-02$ ZERO $0.2179 \mathrm{E}+01$ ZERO $\quad 0.1969 \mathrm{E}-01$ ZERO $\quad 0.2539 \mathrm{E}+02$ ZERO $\quad 0.2538 E+01$ ZERO $\quad 0.3543 \mathrm{E}-02$ ZERO $0.1512 \mathrm{E}-03$ ZERO . $0.3265 \mathrm{E}-22$ ZERO $0.1827 \mathrm{E}+00$ ZERO 0.1827 ZERO ZERO $0.7707 \mathrm{E}+01$ ZERO $0.7707 \mathrm{E}+01$ ZERO $\quad$ ZERO

ZERO

ZERO

ZERO

$0.1603 \mathrm{E}+00$

ZERO

ZERO

ZERO

ZERO

ZERO

ZERO

ZERO

ZERO

ZERO

ZERO

ZERO

ZERO

ZERO

ZERO

$0.1033 \mathrm{E}+02$

ZERO

$0.2952 \mathrm{E}+01$

ZERO

ZERO

ZERO

ZERO

ZERO

ZERO

ZERO

ZERO

ZERO

ZERO

ZERO

ZERO

ZERO

ZERO

ZERO

ZERO

ZERO

$0.1005 \mathrm{E}+02$

ZERO
$0.2858 \mathrm{E}-01$

ZERO

ZERO

$0.1108 \mathrm{E}+02$

$0.3463 \mathrm{E}+01$

$0.5254 \mathrm{E}-07$

$0.3470 \mathrm{E}+01$

$0.2169 \mathrm{E}+01$

$0.6122 \mathrm{E}-07$

ZERO

$0.3831 \mathrm{E}-16$

$0.1075 E-02$

$$
\text { ZERO }
$$

ZERO

ZERO

ZERO

ZERO
60

69
Melter sldge Blend
Feed Waste Glass

$0.4430 \mathrm{E}-02$

$0.2179 \mathrm{E}+01$, ZERO

$0.1969 \mathrm{E}-01 \quad 0.9369 \mathrm{E}+00$

$0.2539 \mathrm{E}+02 \quad 0.2513 \mathrm{E}+02$

$0.2538 \mathrm{E}+01 \quad 0.2513 \mathrm{E}+01$

$0.3543 \mathrm{E}-02: 0.3332 \mathrm{E}-02$

TRACE

$0.1827 \mathrm{E}+00$

ZERO

$0.1496 \mathrm{E}-03$

$0.7707 \mathrm{E}+01$

ZERO
ZERO

ZERO

$0.1318 \mathrm{E}-09$

$0.1506 \mathrm{E}+00$

$0.8073 \mathrm{E}-01$

Date: January 199

ZERO

ZERO

ZERO

ZERO

ZERO

ZERO

$0.1005 \mathrm{E}+02$

ZERO
$0.2858 \mathrm{E}-01$

8E-01
ZERO

ZERO

$0.1108 \mathrm{E}+02$

$0.3463 \mathrm{E}+01$

$0.5254 \mathrm{E}-07$

$0.2169 \mathrm{E}+01$

$0.6122 \mathrm{E}-07$

$0.3831 \mathrm{E}-16$

$0.1948 \mathrm{E}+01$

$0.1075 \mathrm{E}-02$

$0.3859 \mathrm{E}-02$

ZERO

ZERO

ZERO

ZERO

$0.5661 \mathrm{E}+01$

ZERO

ZERO

ZERO

EERO

0. 1097E+02

ZERO

$0.4645 \mathrm{ZER}$

TERO

ZERO

ZERO

0.2624 E+01
ZERO

$0.1065 \mathrm{E}-02$

ZERO

ZERO

ZERO

ZERO

ZERO

ZERO

ZERO
70

Melter

Offgas

ZERO

$0.9463 \mathrm{E}-02$

$0.2539 \mathrm{E}+00$

$0.2538 \mathrm{E}-01$

$0.3366 \mathrm{E}-04$

$0.1512 \mathrm{E}-05$

ZERO

ZERO

ZERO

$0.1242 \mathrm{E}-03$

$0.1978 \mathrm{E}-02$

$0.1318 \mathrm{E}-09$

$0.1506 \mathrm{E}+00$

ZERO

0)

ZERO

$0.5718 \mathrm{E}-01$

ZERO

ZERO

ZERO

ZERO

$0.1108 \mathrm{E}+00$

ZERO

ZERO

ZERO

$0.2651 \mathrm{E}-01$

ZERO

$0.1075 \mathrm{E}-04$

$0.4619 \mathrm{E}+03$

ZERO

ZERO

ZERO

ZERO

ZERO

200

Stack 145

Exhaust

Purified Hg

from Mvs

$0.1814 \mathrm{E}-23$

$0.8912 \mathrm{E}-21$

$\begin{array}{ll}0.3785 \mathrm{E}-14 & 0.8064 \mathrm{E}-23 \\ 0.1017 \mathrm{E}-12 & 0.1039 \mathrm{E}-19\end{array}$

$0.1015 \mathrm{E}-13 \quad 0.1039 \mathrm{E}-20$

$0.2215 \mathrm{E}-12 \quad 0.1451 \mathrm{E}-23$

$0.6046 \mathrm{E}-18$ TRACE

$0.4216 \mathrm{E}-22 \quad 0.7481 \mathrm{E}-22$

ZERO

$0.1774 \mathrm{E}-20$

$0.7601 \mathrm{E}-04$
ZERO

$0.1369 \mathrm{E}-02$

$0.7343 \mathrm{E}+00$

$0.1670 \mathrm{E}-01$

ZERO

$0.5734 \mathrm{E}-14$

$0.4734 \mathrm{E}-19$

$0.4506 \mathrm{E}-24$

TRACE

2E-09

$0.2287 \mathrm{E}-13$

$.2312 \mathrm{E}-20$

$0.6596 \mathrm{E}-23$

TRACE

ZERO

$0.2193 \mathrm{E}-12$

$0.7992 \mathrm{E}-21$

0.73910 REO

ZERO

$0.1060 \mathrm{E}-13$

$0.4496 \mathrm{E}-21$

$0.4301 \mathrm{E}-17$

$0.3655 \mathrm{E}+06$

$0.1070 \mathrm{E}-09$

$0.1078 \mathrm{E}-02$

ZERO

ZERO

TRACE

TRACE
ZERO

ZERO

.ZERO

ZERO

ZERO

$0.2514 \mathrm{E}+00$

$0.7297 \mathrm{E}-13$

ZERO

ZERO

TRACE 
Table A-1. HLW Flowsheet Material Balance (Tank 51/ITP Cyole 1) section 0. Major streams in the Flowsheet Model

STREAM NUMBERS (CONT'D) $\rightarrow$ STREAM NAME

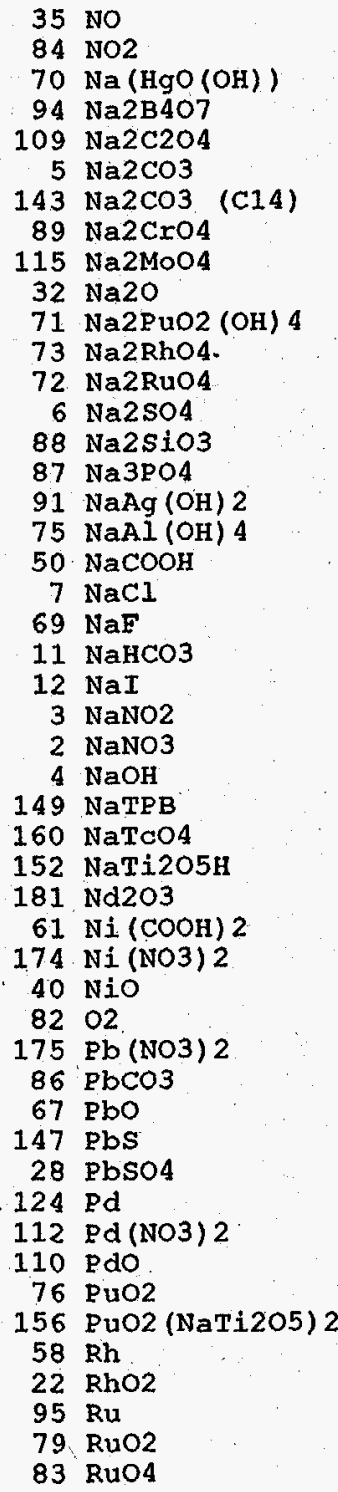

WSRC-TR-95-0019 (Revision
27

44

Frit SME Concenslrry to SME trate to MFT $\begin{array}{ll}\text { ZERO } & \text { TRAC } \\ \text { ZERO } & \text { ZERO } \\ \text { ZERO } & \text { ZERO } \\ \text { ZERO } & \text { ZERO }\end{array}$ ZERO ZERO ZERO ZERO

$0.7861 E+01$

$0.5055 \mathrm{E}+01$ ZERO ZERO $0.1266 \mathrm{E}+02$ ZERO

ZERO

ZERO

ZERO

ZERO

ZERO

ZERO

$0.2182 \mathrm{E}+01$

ZERO
ZERO

ZERO

ZERO

ZERO

ZERO

ZERO

ZERO

ZERO

ZERO

ZERO

ZERO

ZERO

ZERO

ZERO

ZERO

ZERO

ZERO

ZERO

ZERO

ZERO

ZERO

ZERO

ZERO

ZERO

ZERO

ZERO ZERO ZERO

$0.1952 \mathrm{E}+00$ ZERO

$0.2350 \mathrm{E}+01$

$0.9957 \mathrm{E}-02$

0.3417 E-01

ZERO

3E+01

ZERO

ZERO

ZERO

$0.2521 \mathrm{E}-01$

$0.6686 \mathrm{E}-06$

$0.2416 \mathrm{E}+00$
$0.1112 \mathrm{E}-22$

$0.7766 \mathrm{E}-04$

$0.7084 \mathrm{E}-19$

ZERO

$0.1318 \mathrm{E}+00$

$0.3903 E-03$

ZERO

$0.9905 \mathrm{E}-02$

$0.1272 \mathrm{E}-03$

$0.7436 \mathrm{E}-03$
ZERO

$0.8125 \mathrm{E}-02$

ZERO
60

Melter $\quad 69$ Feed Blend TRACE Waste Glass TRACE ZERO ZERO ZERO

$0.5055 \mathrm{E}+01$ ZERO ZERO

$0.1266 \mathrm{E}+02$ ZERO

$0.5472 \mathrm{E}-05$

ZEERO

ZERO

ZERO

ZERO

ZERO

ZERO

ZERO

$0.1972 \mathrm{E}+02$

ZERO

ZERO

$0.1952 \mathrm{E}+00$

ZERO

$0.2578 \mathrm{E}-02$

ZERO

$0.2350 \mathrm{E}+01$

$0.9957 \mathrm{E}-02$

$0.3417 \mathrm{E}-01$

ZERO
$0.5472 \mathrm{E}-05$ ZERO

$0.8943 \mathrm{E}+01$

ZERO

ZERO

ZERO

ZERO

$0.2521 \mathrm{E}-01$

$0.6686 \mathrm{E}-06$

$0.2416 \mathrm{E}+00$
$0.2226 \mathrm{E}-02$

$0.7766 \mathrm{E}-04$

0.7084 ZERO 19

ZERO

$0.1318 \mathrm{E}+00$

$0.3903 \mathrm{E}-03$

ZERO

$0.9905 \mathrm{E}-02$

$0.1272 \mathrm{E}-03$

$0.7436 \mathrm{E}-03$

$0.8125 \mathrm{E}-02$.

ZERO
ZERO

ZERO

ZERO

$0.1546 \mathrm{E}+00$

ZERO

ZRRO

ZERO

ZERO

$0.3337 \mathrm{E}-01$

ZERO

ZERO

ZERO

ZERO

ZERO

ZERO

ZERO

ZERO

ZERO

0.2517

E+0O

ZERO

ZERO

$0.4853 \mathrm{E}-02$

ZERO

$0.1240 \mathrm{E}+00$

ZERO

0.9857 E-02

0.9857 E-02

$0.7436 \mathrm{E}-03$

ZERO

$0.8125 \mathrm{E}-02$

ZERO

Date: January 1995
70

Melter

Offgas

$0.1395 \mathrm{E}+01$

$0.2837 \mathrm{E}+01$

ZERO

ZERO

ZERO

ZERO

ZERO

ZERO

$0.2030 \mathrm{E}+00$

ZERO

ZERO

0.1561E-02

ZERO

$0.2578 \mathrm{E}-04$

ZERO

TERO

$0.4978 \mathrm{E}-04$

$0.3371 \mathrm{E}-03$

ZERO

ZERO

ZERO

ZERO

ZERO.

ZERO

ZERO

ZERO

2ERO

ZERO

$0.2543 \mathrm{E}-02$

$0.1393 \mathrm{E}+03$

ZERO

$0.4902 \mathrm{E}-04$

ZERO

$0.1252 \mathrm{E}-02$

ZERO

ZERO

$0.9957 \mathrm{E}-04$

E-04
ZERO

ZERO

ZERO

ZERO

ZERO

ZERO

200

145

stack Puxified $\mathrm{Hg}$

Exhaust : from MVS

ZERO ZERO

ZERO ZERO

$0.1536 \mathrm{E}-09$ TRACE

$0.5466 \mathrm{E}-24 \quad 0.9699 \mathrm{E}-24$

$0.1130 \mathrm{E}-20 \quad 0.2004 \mathrm{E}-20$

ZERO
ZERO
ZERO

$\begin{array}{rr}\text { ZERO } & \text { ZERO } \\ 0.2732 \mathrm{E}-12 & 0.2605 \mathrm{E}-20\end{array}$

ZERO

ZERO

ZERO

$0.2253 \mathrm{E}-14$

ZERO

$0.1031 \mathrm{E}-16$

ZERO

$0.1991 \mathrm{E}-16$

$0.1557 \mathrm{E}-10$

ZERO

$0.6771 \mathrm{E}-14$

$0.6829 \mathrm{E}-24$

ZERO

ZERO

ZERO

ZERO

ZERO

$0.5817 \mathrm{E}-23$

ZERO

$0.2318 \mathrm{E}-14$

$0.1104 \mathrm{E}+06$

ZERO

$0.1961 \mathrm{E}-16$

ZERO

$0.5008 E-15$

TRACE

ZERO

$0.3983 \mathrm{E}-16$

TRACE

TRACE

$0.1873 \mathrm{E}-23$

ZERO

ZERO

Section 0, Page 32 of 37

ZERO ZERO

$0.4074 \mathrm{E}-23$

$0.1399 \mathrm{E}-22$ TERO

$0.1212 \mathrm{E}-23$

$0.3694 \mathrm{E}-20$

ZERO

ZERO

ZERO

TRACE

TIRRO

TRACE

ZERO

TRACE

$.2540 \mathrm{E}-15$

TRACE

TRACE

$.4841 \mathrm{E}-1$ 
Table A-1. HLW Flowsheet Material Balance (Tank 51/ITP Cycle 1 ) Section 0 . Majox streams in the Flowsheet Model

STREAM NUMBERS (CONT'D) $\rightarrow$ STREAM NAME

$38 \mathrm{SO} 2$

$141 \mathrm{SO}$

137 Semi Vol Cs2O

136 Semi Vol CsCl

126 Semi Vol Group A

138 Semi Vol NaCl

139 Semi Vol NaF

125 Semi. Vol NaI

23 Semi Vol RuO2

111 sio2

$48 \mathrm{Sr}$ (COOH) 2

$\begin{array}{ll}176 & \mathrm{Sr}(\mathrm{NO} 3) 2 \\ 153 & \mathrm{Sr}(\mathrm{NaTi} 2 \mathrm{O5}) 2\end{array}$

$127 \mathrm{Sr}(\mathrm{OH}) 2$

$30 \mathrm{SrCO}$

46 SrO

$159 \mathrm{TCO}_{2}$

92 ThO2

119 Tritium

119 Tritium
17 0308

$62 \mathrm{UO} 2(\mathrm{COOH}) 2$

$177 \mathrm{UO2}$ (NO3) 2

$16 \mathrm{VO} 2(\mathrm{OH}) 2$

$178 \mathrm{Y}(\mathrm{NO3}) 3$

128 Y $(\mathrm{OH}) 3$

$31 \mathrm{Y} 2(\mathrm{CO} 3) 3$

$47 \mathrm{Y} 203$

100 Zeolite

$44 \mathrm{Zn}(\mathrm{COOH}) 2$

$179 \mathrm{Zn}$ (NO3) 2

$57 \mathrm{Zno}$

13 hydrate $\mathrm{H} 2 \mathrm{O}$

13 hydrat

TOTAL FLOW, LB/HR

TEMPERATURE, DEG C

PRESSURE， ATM

PRESSURE， PSIG

PRESSURE， MM HG

ENTHALPY， PCU/HR

VAPOR FLOW, CFM

LIQUID FLOW, GPM

DENSITY, LBS/FT3

PHASE

WSRC-TR-95-0019 (Revision
27 frit 44

slrry to SME trate to MFT

$\begin{array}{ll}\text { ZERO } & \text { ZERO } \\ \text { ZERO } & \text { ZERO }\end{array}$

ZERO ZERO

ZERO $\quad$ ZERO

ZERO ZERO

ZERO — ZERO

ZERO ZERO

ZERO ZERO

7ERO

$0.1136 \mathrm{E}+03$

$0.1230 E+03$

$0.1093 \mathrm{E}-03$

$0.1093 \mathrm{E}-03$
$0.2944 \mathrm{E}-01$

$0.2944 \mathrm{E}-01$
$0.4072 \mathrm{E}-02$

ZERO

ZERO

ZERO

$0.2218 \mathrm{E}-18$

$0.2972 \mathrm{E}-01$

$0.8309 \mathrm{E}+00$

$0.1452 \mathrm{E}-09$

$0.1452 \mathrm{E}-09$

$0.2084 E+01$

$0.1272 \mathrm{E}-05$
$0.9137 \mathrm{E}-04$

$0.9137 \mathrm{E}-04$
ZERO

$0.1317 \mathrm{E}-11$

$0.7940 E-02$

ZERO

$0.9683 \mathrm{E}-03$

ZERO

$0.2607 \mathrm{E}+00$

$0.5336 \mathrm{E}-04$

$0.1794 \mathrm{E}+00$

ZERO

$0.1499 \mathrm{E}+01$

$0.3150 \mathrm{E}+03$
$0.5724 \mathrm{E}+03$

Melter sldge Blend.

Feed Waste Glass

ZERO
ZERO

ZERO ! ZERO

ZERO ZERO

ZERO ZERO

ZERO

ZERO ZERO

ZERO ZERO

ZERO

ZERO

$0.1230 \mathrm{E}+03$

$0.1093 \mathrm{E}-03$

$0.2944 \mathrm{E}-01$

$.4072 \mathrm{E}-02$

ZERO

$0.2218 \mathrm{E}-18$

$0.3569 \mathrm{E}-02$

$0.2972 \mathrm{E}-01$

$0.8309 \mathrm{E}+00$

$0.1424 \mathrm{E}-09$

$0.2084 \mathrm{E}+01$

$0.1272 \mathrm{E}-05$

0.9137 E-04
ZERO

$0.1317 \mathrm{E}-11$

0.794 OE-02

- ZERO

$0.9683 \mathrm{E}-03$

$0.2607 \mathrm{E}+00$

$0.5336 \mathrm{E}-04$

$0.1794 \mathrm{E}+0 \mathrm{O}$

ZERO

$0.1499 \mathrm{E}+01$

$0.3237 \mathrm{E}+03$

$0.9874 \mathrm{E}+02$

$.2475 \mathrm{E}+03$

$0.5000 \mathrm{E}+02$

$0.4987 \mathrm{E}+02$

$0.1340 \mathrm{E}+01$
$0.4997 \mathrm{E}+01$

$0.1000 E+01$
ZERO

$0.9656 \mathrm{E}+00$

$-0.5055 \mathrm{E}+00$

$0.7339 \mathrm{E}+03$

$0.1826 \mathrm{E}+05$

$65 \mathrm{E}+05$

ZERO

ZERO
$\mathrm{E}+03$

ZERO

ZERO

ZERO

ZERO

$0.1519 \mathrm{E}-01$

$0.3534 \mathrm{E}-02$

$0.2943 \mathrm{E}-01$

$0.8253 \mathrm{E}+00$

ZERO

$0.2063 \mathrm{E}+01$

ZERO

ZERO

ZERO

ZERO

ZERO

ZERO

$0.4187 \mathrm{E}-02$

ZERO

ZERO

$0.3128 E+00$

ZERO

ZERO

$0.2280 \mathrm{E}+03$

$0.1050 \mathrm{E}+04$

$0.9840 \mathrm{E}+00$

$-0.2351 \mathrm{E}+00$

$0.7043 \mathrm{E}+05$

$0.3297 \mathrm{E}+00$

$0.8482 \mathrm{E}+00$

$0.8656 \mathrm{E}+00$

$0.1797 \mathrm{E}+00$

$8413 E+02$

$0.8369 \mathrm{E}+02$

LIQUID

$1582 \mathrm{E}+03$

LIQUID
70

Melter

Offgas

ZERO
ZERO

$0.4115 \mathrm{E}-02$

$0.1772 \mathrm{E}-03$

$0.1229 \mathrm{E}+00$

$0.3351 \mathrm{E}-02$

$0.1245 \mathrm{E}-01$

$0.5417 \mathrm{E}-05$

2ERO

ZERO

ZERO

ZERO

ZERO

$0.1535 \mathrm{E}-03$

$0.3569 \mathrm{E}-04$

$0.2972 \mathrm{E}-03$

$0.8337 \mathrm{E}-02$

$0.2084 E-01$

च

ZERO

ZERO

ZERO

ZERO

ZERO

$0.4229 \mathrm{E}-04$

ZERO

ZERO

$0.3159 \mathrm{E}-02$

ZERO

$0.3411 \mathrm{E}+03$

$0.9632 \mathrm{E}+03$

$0.6050 \mathrm{E}+03$

$0.9840 \mathrm{E}+00$

$-0.2351 \mathrm{E}+00$

$0.7478 \mathrm{E}+03$
$0.3967 \mathrm{E}+06$

$0.7863 \mathrm{E}+03$

$0.2042 \mathrm{E}-01$

VAPOR
200 145

stack Purified Hg ZERO ZERO $\begin{array}{ll}\text { ZERO } & \text { ZERO } \\ \text { ZERO } & \text { ZERO }\end{array}$

ZERO ZERO

ZERO ZERO

ZERO ZERO

ZERO : ZERO

ZERO

$0.2552 \mathrm{E}-11$

TRACE

ZERO
$0.9374 \mathrm{E}-24$

TRACE

$0.1205 \mathrm{E}-22$

$0.1663 \mathrm{E}-23$

ZERO

$0.6139 \mathrm{E}-16$

$0.5875 \mathrm{E}-16$

$0.1189 \mathrm{E}-15$

$0.3335 E-14$

$0.1368 \mathrm{E}-09$

36E-14

TRACE

ZERO

TRACE

ZERO

ZERO

ZERO
$0.1692 \mathrm{E}-16$

$0.6006 \mathrm{E}-22$

$0.1264 \mathrm{E}-14$

7no

$0.7965 \mathrm{E}+04$

$0.4840 \mathrm{E}+06$

$0.3873 \mathrm{E}+02$

$0.1000 \mathrm{E}+01$

ZERO

ZERO

TERO

$0.1462 \mathrm{E}-23$

$0.1217 \mathrm{E}-22$

$0.3395 \mathrm{E}-21$

$0.8534 \mathrm{E}-21$

TEA

TRACE

TRACE

TRACE

$0.3251 \mathrm{E}-23$

ZERO

$0.3955 \mathrm{E}-24$

$0.1066 \mathrm{E}-21$

$0.7335 \mathrm{E}-22$

2ERO

$0.6139 \mathrm{E}-21$

$0.2514 \mathrm{E}+00$

$-0.3000 \mathrm{E}+02$

$0.1000 \mathrm{E}+01$

$0.1056 \mathrm{E}+08 \quad-0.2485 \mathrm{E}+00$

$0.1157 \mathrm{E}+06$

$0.6972 \mathrm{E}-01$

$0.37 .07 \mathrm{E}-04$

VAPOR

LIQUID 
Table A-1. HLW Flowsheet Material Balance (Tank 51/ITP Cycle 1) Section 0. Major Streams in the Flowsheet Model

STREAM NUMBERS $\rightarrow$ STREAM NAME

COMPONENT FLOWS, LB/HR 122 ( 6 $6 \mathrm{H} 5) 2$

123 ( (C.6H5) $2 \mathrm{C} 6 \mathrm{H} 4$

118 (C6H5) $2 \mathrm{NH}$

133 (C6H5B (OH) 2 salt)

132 ( $\mathrm{C} 6 \mathrm{H} 5 \mathrm{~B}(\mathrm{OH}) 2$ )

166 (C6H5C6H4

163 (C6H5NHCHO)

165 (C6H5NO)

164 (C6H5NO2).

131 (C6H5OH salt)

130 (C6H5O

155 (CH3) $2 \mathrm{CHOH}$

154 (СH3OH)

$45 \mathrm{Ag}$

$116 \mathrm{Ag} 20$

134 AgNO3

$106 \mathrm{B2O3}$

$9 \mathrm{Ba}(\mathrm{OH}) 2$

$15 \mathrm{BaO}$

$24 \mathrm{BaSO} 4$

$66 \mathrm{CO}$

$37 \mathrm{CO} 2$

$142 \mathrm{CO} 2$ (C14)

$63 \mathrm{Ca}(\mathrm{COOH}) 2$
$167 \mathrm{Ca}(\mathrm{NO} 3)^{2}$

$167 \mathrm{Ca}(\mathrm{NO} 3)^{2}$
$120 \mathrm{Ca}(\mathrm{OH}) 2$

$120 \mathrm{Ca}(\mathrm{OH}) 2$
$113 \mathrm{Ca} 3(\mathrm{POA}) 2$

$20 \mathrm{CaC} 2 \mathrm{O} 4$

$18 \mathrm{CaCO}_{3}$

$64 \mathrm{CaCO}$ (C14)

$78 \mathrm{CaF}^{2}$

$41 \mathrm{CaO}$

$19 \mathrm{CasO} 4$

29 Carbon

$26 \mathrm{CO}(\mathrm{COOH}) 2$

$168 \mathrm{Co}$ (NO3)

$$
\begin{array}{ll}
59 & \mathrm{CoO} \\
98 & \mathrm{Cr} 2 \mathrm{O} \\
42 & \mathrm{Cs} 2 \mathrm{O} \\
53 \mathrm{CsCOOH} & \mathrm{Cs} \\
96 \mathrm{CsC}
\end{array}
$$

$169 \mathrm{CsNO} 3$

$8 \mathrm{CsOH}$

177

Neutr Waste Tnk Frm Evap to Tank Farm

$0.3456 \mathrm{E}-05$

$0.3645 \mathrm{E}-06$

$0.5128 \mathrm{E}-02$

ZERO

$0.2021 \mathrm{E}-06$

$0.5402 \mathrm{E}-01$

$0.7661 \mathrm{E}-06$

$0.8336 \mathrm{E}-08$

$0.6657 \mathrm{E}+00$

ZERO

$0.6288 \mathrm{E}-04$

$0.5514 \mathrm{E}-10$

$0.5226 \mathrm{E}-11$

$0.8863 \mathrm{E}-07$ ZERO
$6 \mathrm{E}-01$

$0.8836 \mathrm{E}-01$

$0.1692 \mathrm{E}+00$

ZERO

$.1119 \mathrm{E}-04$ ZERO

$0.1461 \mathrm{E}-03$

$0.3239 \mathrm{E}-13$

ZERO
$0.1535 \mathrm{E}-04$

ZERO

$0.2627 \mathrm{E}-01$

$0.8930 \mathrm{E}-12$

ZERO

$0.5501 \mathrm{E}-07$

$0.1454 \mathrm{E}-01$

$0.1988 \mathrm{E}-02$

ZERO

ZERO

$0.1965 \mathrm{E}-11$

$0.1030 \mathrm{E}-05$

$0.4303 \mathrm{E}-02$

$0.3099 \mathrm{E}-03$

ZERO
ZERO

$0.3522 \mathrm{E}-10$

$0.3660 \mathrm{E}-02$
338 Aq to ETF

a to

ZERO
ZERO
ZERO
ZERO
ZERO
ZERO

$0.4146 \mathrm{E}-01$

ZERO

ZERO

$0.2077 \mathrm{E}-07$

ZERO

ZERO
$0.2586 \mathrm{E}-06$

$0.5098 \mathrm{E}-10$

$0.4417 \mathrm{E}-11$

ZERO

ZERO
ZERO

ZERO

ZERO

ZERO

ZERO

ZERO

$0.1606 \mathrm{E}-06$

$0.3561 \mathrm{E}-16$

ZERO

ZERO

ZERO

ZERO

ZERO

ZERO

ZERO

ZERO

ZERO

ZERO

ZERO

ZERO

ZERO

ZERO

ZERO

ZERO

ZERO

ZERO
348

354

WPF Recycl

Organic to

$801 \mathrm{E}-08$

$0.3645 \mathrm{E}-06$

$.4560 \mathrm{E}-02$

ZERO

$0.2021 E-06$

ZERO

$0.7661 \mathrm{E}-06$

$0.8336 \mathrm{E}-08$

ZERO

ZERO

ZERO

ZERO

ZERO

ZERO

$0.8863 \mathrm{E}-07$

ZERO

$0.1692 \mathrm{E}+00$

ZERO

$.1119 \mathrm{E}-04$

$0.1534 \mathrm{E}-03$

ZERO

ZERO

ZERO

ZERO

ZERO

$0.2627 \mathrm{E}-01$

$0.8930 \mathrm{E}-12$

ZERO

$0.5501 \mathrm{E}-07$

$0.1454 \mathrm{E}-01$

$0.1988 \mathrm{E}-02$

ZERO

ZERO

ZERO

ZERO

$0.3099 \mathrm{E}-03$

ZERO

ZERO

ZERO

ZERO

$\therefore$ ZERO

ZERO $\quad 0.9976 \mathrm{E}+00$

ZERO $\quad 0.5464 \mathrm{E}-0$

$0.4353 \mathrm{E}-02$

ZERO.

$0.1220 \mathrm{E}-01$

$0.1396 \mathrm{E}-01$

$0.1762 \mathrm{E}-23$

$0.1462 \mathrm{E}-22$

$0.4619 \mathrm{E}-08$

$0.6657 \mathrm{E}+00$

ZERO

0. $038 \mathrm{~B}-09$

$0.1648 \mathrm{E}-12$

$0.7988 \mathrm{E}-14$

ZERO

ZERO

ZERO

ZERO

ZERO

ZERO

ZERO

$0.1387 \mathrm{E}-12$

$0.3074 \mathrm{E}-22$

ZERO

$0.1535 \mathrm{E}-04$

ZERO

ZERO

ZERO

ZERO

ZERO

ZERO

ZERO

ZERO

ZTRO

ZERO

ZERO

ZERO

ZERO

ZERO

ZERO

$0.3522 \mathrm{E}-10$

$0.3660 \mathrm{E}-02$

ZERO

Date: Januaxy 1995
$.9755 \mathrm{E}-24$

$0.127115+02$

11925-11

$.2038 \mathrm{E}-12$

ZERO

ZERO

$0.6831 \mathrm{E}-24$

TRACE

ZERO

TRACE

TRACE

$0.1302 \mathrm{E}+00$

$0.6747 \mathrm{E}-20$

ZERO

TRACE

$0.2952 E-23$

TRACE

ZERO

ZERO

ZERO

$0.1006 \mathrm{E}-23$

ZERO

ZERO

TERO

TRACE

TRACE

TRACE

$0.2280 \mathrm{E}-24$

ZERO

ZERO

ZERO

Section 0, Page 34 of 37 
Table A-1. HLW Flowsheet Material Balance (Tank 51/ITP Cycle 1 ) section 0. Major streams in the Flowsheet Model

STREAM NUMBERS (CONT'D) $\rightarrow$ STREAM NAME

$54 \mathrm{Cu}(\mathrm{COOH}) 2$
$170 \mathrm{Cu}(\mathrm{NO} 3) 2$

$55 \mathrm{CuO}$

$80 \mathrm{Fe} 2 \mathrm{O3}$

$101 \mathrm{FeO}$

102 Group A

103 Group B

$25 \mathrm{H} 2$

$158 \mathrm{H} 2 \mathrm{C} 2 \mathrm{O} 4$

$93 \mathrm{H} 2 \mathrm{SO}_{4}$

$90 \mathrm{H} 3 \mathrm{BO} 3$.

$43 \mathrm{HCl}$

$85 \mathrm{HF}$

49 HNO3

$36 \mathrm{Hg}$

$129 \mathrm{Hg}(\mathrm{C} 6 \mathrm{H} 5) 2$

$135 \mathrm{Hg}(\mathrm{NO} 3) 2$

$144 \mathrm{Hg} 2 \mathrm{Cl}$

$117 \mathrm{Hg} 2 \mathrm{I} 2$

$146 \mathrm{HgCl2}$

$21 \mathrm{Hg}$

$\begin{array}{rl}97 & \mathrm{I} 2 \\ 183 & \mathrm{~K} 2 \mathrm{CO}\end{array}$

$\begin{array}{rl}183 & \mathrm{~K} 2 \mathrm{CO} \\ 39 & \mathrm{~K} 2 \mathrm{O}\end{array}$

$51 \mathrm{KCOOH}$

$157 \mathrm{KMnO} 4$

$161 \mathrm{KNO} 3$

$150 \mathrm{KTPB}$

$105 \mathrm{La} 2 \mathrm{O} 3$

$\begin{array}{ll}105 & \mathrm{La} 2 \mathrm{O} \\ 108 \mathrm{Li2O}\end{array}$

$65 \mathrm{Mg}(\mathrm{COOH}) 2$

$171 \mathrm{Mg}$ (NO3) 2

$33 \mathrm{MgO}$

$60 \mathrm{Mn}(\mathrm{COOH}) 2$

$172 \mathrm{Mn}(\mathrm{NO} 3) 2$

$180 \mathrm{Mn} 304$

$56 \mathrm{MnO}$

$14 \mathrm{MnO} 2$

$145 \mathrm{MoO} 2$

81 N2

74 N2O

182 NH3

114 NH3OHNO3

$52 \mathrm{NH} 4 \mathrm{COOH}$

173 NH4 NO3

$68 \mathrm{NH} 4 \mathrm{OH}$

148 NH 4 TPB

WSRC-TR-95-0019 (Revision
177
Neutr Waste Tnk Frm Evap to Tank Farm Aq to ETF

ZERO

$0.9462 \mathrm{E}-02$

$0.2539 \mathrm{E}+00$

$0.2539 \mathrm{E}-01$

$0.2106 \mathrm{E}-03$

$0.1693 \mathrm{E}-11$

$0.1693 E-11$

ZERO

ZERO

ZERO

ZERO

ZERO

ZERO

$0.6679 \mathrm{E}-01$

ZERO

ZERO

ZERO

ZERO

ZERO

ZERO
ZERO
$0.5718 \mathrm{E}-01$

ZERO
ZERO

ZERO

$0.1094 \mathrm{E}+00$

ZERO

$0.1108 E+00$

ZERO

0.1921 E-12

$0.4692 \mathrm{E}-01$

$0.2239 \mathrm{E}-12$

ZERO
E-01

$0.2651 \mathrm{E}-01$

$0.1695 \mathrm{E}+00$

$0.3857 \mathrm{E}-01$

TRACE

$0.2033 E+00$

ZERO

ZERO

ZERO

ZERO

$$
\begin{aligned}
& \text { ZERO } \\
& \text { ZERO }
\end{aligned}
$$

ZERO

ZERO

ZERO

ZERO

$0.7632 \mathrm{E}-16$

$$
\text { ZERO }
$$

ZERO

ZERO

ZERO

ZERO

ZERO

ZERO

ZERO

ZERO

ZERO

ZERO

ZERO

ZERO

ZERO

ZERO

ZERO

ZERO

ZERO

ZERO

ZERO

ZERO

ZERO

ZERO

ZERO

ZERO

ZERO

ZERO

ZERO

GERO

ZERO

$0.3741 \mathrm{E}-01$

TRACE

$0.2688 \mathrm{E}-01$

ZERO

ZERO

ZERO

ZERO
348 ,

354

Insol frm DWPF Recycl

Salt Cake ZERO
$8 \mathrm{E}-05$

ZERO ZERO

$.9462 \mathrm{E}-02$

$.2539 \mathrm{E}+00$

$.2106 \mathrm{E}-03$

$0.1512 \mathrm{E}-05$

ZERO

ZERO

ZERO

ZERO

ZERO

ZERO
ZERO

ZERO

ZERO

ZERO

ZERO

ZERO

ZERO

ZERO

ZERO

$0.5718 \mathrm{E}-01$

ZERO

ZERO

ZERO

ZERO

ZERO

$0.1108 \mathrm{E}+00$

ZERO

$0.4692 \mathrm{E}-01$

ZERO

ZERO

$0.2651 \mathrm{E}-01$

$0.1695 \mathrm{E}+00$

$0.1076 E-04$

ZERO

ZERO

ZERO

ZERO

ZERO

ZERO

ZERO

Date: January 1995

ERO

ZERO

ERO

$0.1094 \mathrm{E}+00$

ZERO

ZERO

ZERO

$0.1921 \mathrm{E}-12$

ZERO

ZERO
$0.2239 \mathrm{E}-12$

ZERO

ZERO

ZERO

ZERO

$0.1857 \mathrm{E}-08$

ZERO

ZERO

ZERO

ZERO

ZERO
245

Organic to

Org Strg Tk

$0.1148 \mathrm{E}-22$

TRACE

ZERO $0.8357 \mathrm{E}-24$

ZERO ZERO

ZERO TRACE

ZERO TRACE

ZERO

ZERO

ZERO $0.4058 \mathrm{E}-22$

ZERO $\quad 0.2893 \mathrm{E}-05$

ZERO

ZERO : ZERO

ZERO ZERO

ZERO ZERO

ZERO $\quad 0.1326 \mathrm{E}-02$

ZERO ZERO

ZERO ZERO

ZERO - ZERO

ZERO

ZERO

ZERO

ZERO ZERO

0.528 TRACE

TRACE
$89 \mathrm{E}-22$

ZERO

ZERO

TRARE

TRACE

TRACE

TRACE

TRACE

TRACE

ZERO

ZERO

TRACE

$0.1541 \mathrm{E}-03$

$0.8671 \mathrm{E}-21$

ZERO

TRACE

ZERO

ZERO

Section 0, Page 35 of 37 
Table A-1. HLW Flowsheet Material Balance (Tank 51/ITP Cycle 1) Section 0. Major streams in the Flowsheet Model.

STREAM NUMBERS (CONT'D) $\rightarrow$ STREAM NAME

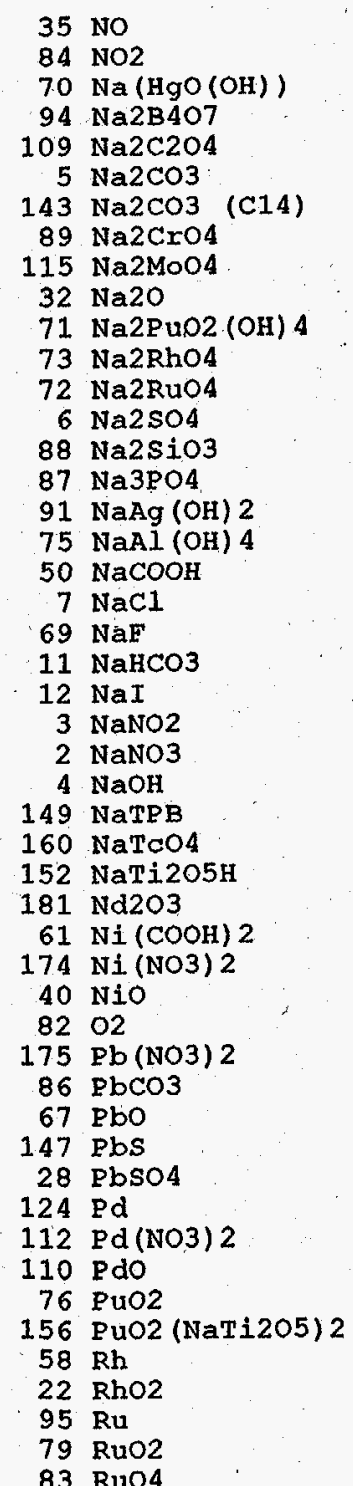

348

Insol frm DWPF Recycl.

DWPF Recyc salt Cake

Organic to

Org Strg Tk

$0.1804 \mathrm{E}-16$
ZERO 0.110 E -17
$1433 \mathrm{E}-23$

ZERO

ZERO
$0.1227 \mathrm{E}+00$

$\begin{array}{lll}\text { ZERO } & 0.1227 \mathrm{E}+00 & \text { ZERO } \\ \text { ZERO } & 0.7909 \mathrm{E}+01 & \text { ZERO }\end{array}$

ZERO O.7909H01 ZERO

ZERO $0.1794 \mathrm{E}-04$ ZERO

ZERO ZERO ZERO

ZERO ZERO

$0.2030 \mathrm{E}+00 \quad$ ZERO $\quad$ ZERO

ZERO ZERO ZERO

$\begin{array}{ll}\text { ZERO ZERO } & \text { ZERO }\end{array}$

ZERO $0.6774 \mathrm{E}-01$ TRACE

ZERO ZERO TERO

ZERO $0.2578 E-04$ TRA

ZERO ZERO ZERO

ZERO ZERO ZERO

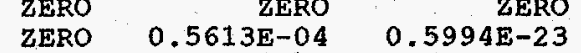

ZERO $0.5026 \mathrm{E}-02$ TRACE

ZERO $0.1405 \mathrm{E}-01$ TRACE

ZERO ZERO $\quad$ ZERO

ZERO $0.5464 \mathrm{E}-05 \quad$ TRACE

ZERO $0.9908 \mathrm{E}+01$ Z $\quad$ ZERO

ZERO $0.1289 \mathrm{E}+02 \quad 0.1158 \mathrm{E}-24$

ZERO

ZERO $\quad$ ZERO $\quad$ ZERO

ZERO ZERO ZERO

IERO ZERO ZERO

ZERO - ZERO ZERO

ZERO ZERO TRACE

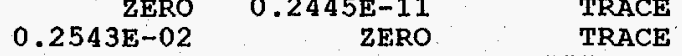

$\begin{array}{rrrr}0.2158 \mathrm{E}-01 & 0.2543 \mathrm{E}-02 & & \\ \text { ZERO } & 0.1926 \mathrm{E}-08 & 0.9555 \mathrm{E}-04\end{array}$

ZERO $0.2847 \mathrm{E}-09$ TRACE

ZERO
$0.4902 \mathrm{E}-04$

ZERO

TRACE

ZERO

ZERO

ZERO

TRACE

TRACE

ZERO

ZERO

TRACE

ZERO 
Table A-1. HLW Flowsheet Material Balance (Tank 51/ITP Cycle 1) section 0 . Major Streams in the Flowsheet Model

STREAM NUMBERS (CONT'D) -> STREAM NAME

$38 \mathrm{SO} 2$

$141 \mathrm{SO}$

137 Semi Vol Cs2O

136 Semi Vol CsCl

126 Semi Vol Group A

140 Semi Vol Na2B4O7

138 Semi Vol NaCl

139 Semi Vol NaF

125 Semi Vol NaI

23 Semi Vol RuO2

$111 \mathrm{SiO} 2$

$48 \mathrm{Sr}$ (COOH) 2

$176 \mathrm{Sr}(\mathrm{NO} 3) 2$

153 Sr(NaTi205) 2

$127 \mathrm{Sr}(\mathrm{OH}) 2$

$30 \mathrm{SrCO} 3$

46 Sro

$159 \mathrm{TcO} 2$

92 ThO2

119 Tritium

$\begin{array}{rl}119 & \text { Trit } \\ 17 & \mathrm{U} 308\end{array}$

$62 \mathrm{UO} 2(\mathrm{COOH}) 2$

$177 \mathrm{UO2}$ (NO3) 2

$16 \mathrm{VO} 2(\mathrm{OH}) 2$

$27 \mathrm{Y}(\mathrm{COOH}) 3$

$178 \mathrm{Y}$ (NO3) 3

$128 \mathrm{Y}(\mathrm{OH}) 3$

$31 \mathrm{Y} 2(\mathrm{CO} 3) 3$

$47 \mathrm{Y} 203$

100 Zeolite

$44 \mathrm{Zn}(\mathrm{COOH}) 2$

$179 \mathrm{zn}(\mathrm{NO} 3) 2$

$57 \mathrm{ZnO}$

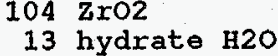

1 water

TOTAL FLOW, IB/HR

TEMPERATURE, DEG C

PRESSURE， ATM

PRESSURE， PSIG.

PRESSURE, MM HG

ENTHALPY, PCU/HR

VAPOR FLOW, CFM

LIOUID FLOW GPM

DENSITY, LBS/FT3

PHASE

WSRC-TR-95-0019 (Revision
177

338

Neutr Waste Tnk Frm Evap

to Tank Farm

ZERO

Aq to ETE

frm DWP Recycl

$\begin{array}{lr}\text { ZERO } & \text { ZERO } \\ \text { ZERO } & \text { ZERO }\end{array}$

ZERO

ZERO

ZERO

ZERO

ZERO

ZERO

ZERO

ZERO

ZERO

ZERO

E +01

$0.1079 \mathrm{E}-06$

$0.1489 \mathrm{E}-07$

ZERO

$0.1535 \mathrm{E}-03$

$0.3570 \mathrm{E}-04$

$0.2973 \mathrm{E}-03$

$0.8338 \mathrm{E}-02$

$0.7859 \mathrm{E}-09$

$0.2085 E-01$

ZERO
$.3349 \mathrm{E}-09$

ZERO

ZERO

$0.2911 \mathrm{E}-07$

ZERO

0.4229 EE-04

ZERO

ZERO

$0.1951 \mathrm{E}-09$

$0.3160 \mathrm{E}-02$

ZERO
$6 \mathrm{E}-05$

$0.3242 \mathrm{E}+04$

$0.3355 \mathrm{E}+04$

$0.4999 \mathrm{E}+02$

$0.9656 \mathrm{E}+00$

$-0.5055 \mathrm{E}+00$

$0.7339 \mathrm{E}+03$

$0.6551 \mathrm{E}+01$

$6385 \mathrm{E}+02$
LIQUID

ZERO

ZERO

ZERO

ZERO

ZERO

ZERO

ZERO

ZERO

$0.7512 E-09$

ZERO

ZERO

ZERO

ZERO

ZERO

ZERO

ZERO

ZERO

ZERO

ZERO

ZERO

ZERO

ZERO

ZERO

$0.3121 \mathrm{E}+04$

$0.3122 \mathrm{E}+04$

$0.1000 \mathrm{E}+03$

$0.1000 \mathrm{E}+01$

ERO

$0.3124 E+06$

$0.6234 \mathrm{E}+01$

$.6243 \mathrm{E}+02$

IIQUID

Date: January 1995
348

245

Org Strg Tk

$\begin{array}{lll}\text { ZERO } & \text { ZERO } & \text { ZERO } \\ \text { ZERO } & \text { ZERO }\end{array}$

ZERO ZERO $\quad$ ZERO

ZERO ZERO ZERO

ZERO ZERO : Z ZERO

ZERO ZERO ZFRO

ZERO ZERO $\quad$ ZERO

ZERO ZERO

ZTRO

ZERO ZERO ZERO

$0.1404 \mathrm{E}-24$

TRACE

$0.1079 \mathrm{E}-06 \quad$ ZERO

ZERO TRACE

ZERO ZERO

ZERO ZERO

ZERO ZERO

$\begin{array}{rrr}0.3570 E-04 & \text { ZERO } & \text { TRACE } \\ 0.2973 E-03 & \text { ZERO } & \text { ZERO }\end{array}$

$0.8338 \mathrm{E}-02$ ZERO $0.4130 \mathrm{E}-23$

$\begin{array}{rrr}\text { ZERO } & 0.4699 \mathrm{E}-11 & 0.4550 \mathrm{E}-19 \\ 0.2085 \mathrm{E}-01 & \text { ZERO } & \text { TRACE }\end{array}$

ZERO TRACE
ZERO TRACE

$0.3349 \mathrm{E}-09$ ZERO

ZERO

ZERO
$0.2911 \mathrm{E}-07$

ZERO ZERO

ZERO ZERO

ZERO TRACE

TRRO

$0.5999 \mathrm{E}-24$

$\begin{array}{rrr}\text { ZERO } & 0.1951 \mathrm{E}-09 & \text { TRACE } \\ 0.3160 \mathrm{E}-02 & \text { ZERO } & 0.7282 \mathrm{E}-24\end{array}$

ZERO
$0.8616 \mathrm{E}-05$

$0.1146 \mathrm{E}-24$

$0.7802 \mathrm{E}+01$

$0.3626 \mathrm{E}+02$

$0.5315 \mathrm{E}+01$

$0.9656 \mathrm{E}+00$

$-0.5055 \mathrm{E}+00$

$0.7339 \mathrm{E}+03$

$\begin{array}{lll}0.2929 \mathrm{E}+02 & 0.5308 \mathrm{E}+04 & 0.1037 \mathrm{E}+03\end{array}$

$0.1907 \mathrm{E}-02 \quad 0.1135 \mathrm{E}+00$

$0.1423 \mathrm{E}+03$

LIQUID

$0.7884 \mathrm{E}-01$

$0.5734 \mathrm{E}+02$

IIQUID 


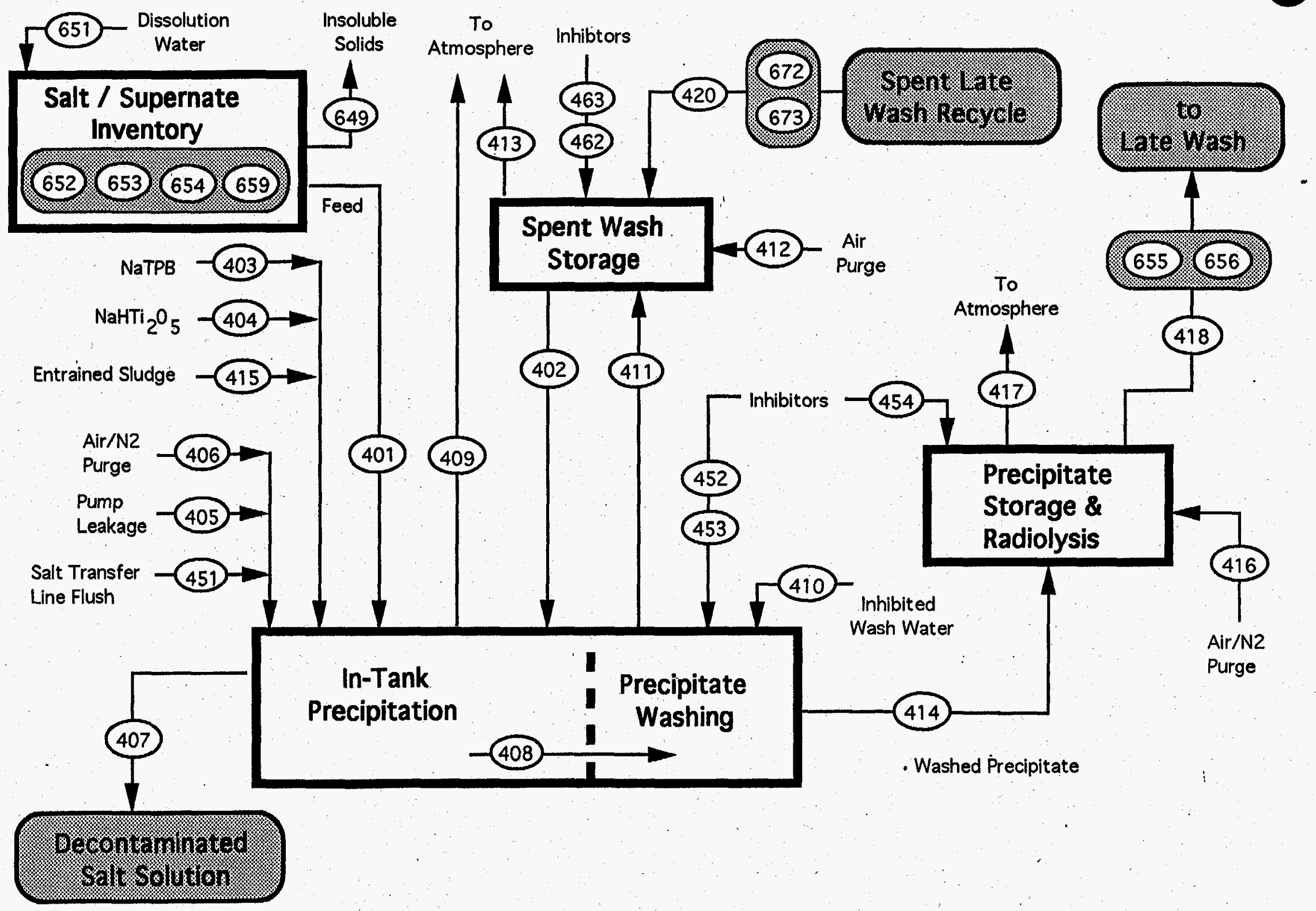

SECTION 1A. IN-TANK PRECIPITATION / SALT DECONTAMINATION WSRC-TR-95-0019 (Revision 0) 
Table A-1. HLW Flowsheet Material Balance (Tank 51/ITP Cycle 1)

Section 1A. In-Tank Precipitation and Salt Decontamination

DESCRIPTION

PAGE

1. STREAM NUMBERS

$652,653,654,659,651,401,649 \ldots \ldots$. . . . . . . . . 2 2

2. STREAM NUMBERS

$402,451,403,404,405,406,415 . . . . . . . . . . . .66$

3. STREAM NUMBERS

$409,407,408,410,411,452,453 \ldots \ldots \ldots$

4. STREEAM NUMBERS

$414,454,416,417,420,672,673 \ldots \ldots . . . . . . . . . .14$

5. STREAM NUMBERS

$412,413,462,463,418,655,656 \ldots \ldots$ 
Table A-1. HLW Flowsheet Material Balance (Tank 51/ITP Cycle 1)

section 1A. In-Tank Precipitation and Salt Decontamination

STREAM NUMBERS $\rightarrow$ STREAM NAME

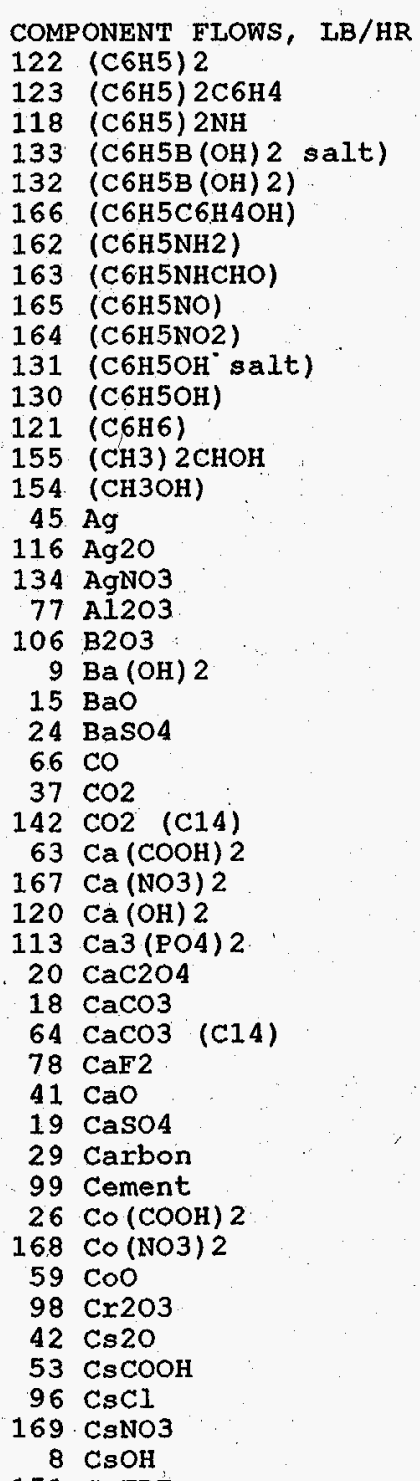

\begin{tabular}{|c|c|}
\hline ZERO & ZERO \\
\hline ZERO & ZERO \\
\hline ZERO & ZERO \\
\hline ZERO & ZERO \\
\hline ZERO & ZERO \\
\hline ZERO & ZERO \\
\hline ZERO & ZERO \\
\hline ZERO & ZERO \\
\hline ZERO & ZERO \\
\hline ZERO & ZERO \\
\hline ZERO & ZERO \\
\hline ZERO & ZERO \\
\hline ZERO & ZERO \\
\hline ZERO & ZERO \\
\hline ZERO & ZERO \\
\hline ZERO & ZERO \\
\hline $0.1873 E-03$ & ZERO \\
\hline ZERO & ZERO \\
\hline $0.5637 \mathrm{E}-01$ & ZERO \\
\hline $0.1404 \mathrm{E}-02$ & ZERO \\
\hline $0.5546 \mathrm{E}-03$ & ZERO \\
\hline ZERO & ZERO \\
\hline $0.6156 \mathrm{E}-03$ & ZERO \\
\hline ZERO & ZERO \\
\hline ZERO & ZERO \\
\hline ZERO & ZERO \\
\hline ZERO & ZERO \\
\hline $0.3549 \mathrm{E}-01$ & ZERO \\
\hline ZERO & ZERO \\
\hline $0.4712 \mathrm{E}+00$ & ZERO \\
\hline ZERO & ZERO \\
\hline ZERO & ZERO \\
\hline ZERO & ZERO \\
\hline ZERO & ZERO \\
\hline ZERO & ZERO \\
\hline $0.1606 \mathrm{E}+00$ & ZERO \\
\hline ZERO & ZERO \\
\hline ZERO & ZERO \\
\hline ZERO & ZERO \\
\hline $0.1392 \mathrm{E}-01$ & ZERO \\
\hline $0.8653 \mathrm{E}-04$ & ZERO \\
\hline $0.2199 \mathrm{E}+00$ & ZERO \\
\hline $0.3234 \mathrm{E}-04$ & ZERO \\
\hline ZERO & ZERO \\
\hline ZERO & ZERO \\
\hline ZERO & ZERO \\
\hline $0.5739 E-02$ & ZERO \\
\hline
\end{tabular}

\begin{tabular}{|c|c|}
\hline ZERO & ZERO \\
\hline ZERO & ZERO \\
\hline ZERO & ZERO \\
\hline ZERO & ZERO \\
\hline ZERO & ZERO \\
\hline ZERO & ZERO \\
\hline ZERO & ZERO \\
\hline ZERO & ZERO \\
\hline ZERO & ZERO \\
\hline ZERO & ZERO \\
\hline ZERO & ZERO \\
\hline ZERO & ZERO \\
\hline ZERO & ZERO \\
\hline ZERO & ZERO \\
\hline ZERO & ZERO \\
\hline ZERO & ZERO \\
\hline ZERO & $0.3570 \mathrm{E}-04$ \\
\hline ZERO & ZERO \\
\hline ZERO & $0.1071 \mathrm{E}-01$ \\
\hline ZERO & $0.2667 \mathrm{E}-03$ \\
\hline ZERO & $0.1465 \mathrm{E}-03$ \\
\hline ZERO & ZERO \\
\hline ZERO & $0.1168 \mathrm{E}-03$ \\
\hline ZERO & ZERO \\
\hline ZERO & ZERO \\
\hline ZERO & ZERO \\
\hline ZERO & ZERO \\
\hline ZERO & ZERO \\
\hline ZERO & $0.3660 \mathrm{E}-02$ \\
\hline ZERO & $0.8951 \mathrm{E}-01$ \\
\hline ZERO & ZERO \\
\hline ZERO & ZERO \\
\hline ZERO & ZERO \\
\hline ZERO & ZERO \\
\hline ZERO & ZERO \\
\hline ZERO & $0.3052 \mathrm{E}-01$ \\
\hline ZERO & ZERO \\
\hline ZERO & ZERO \\
\hline ZERO & ZERO \\
\hline ZERO & ZERO \\
\hline ZERO & $0.1623 \mathrm{E}-04$ \\
\hline ZERO & $0.4178 \mathrm{E}-01$ \\
\hline ZERO & $0.6133 E-05$ \\
\hline ZERO & ZERO \\
\hline ZERO. & ZERO \\
\hline ZERO & ZERO \\
\hline ZERO & $0.3075 \mathrm{E}-01$ \\
\hline ZERO & ZERO \\
\hline
\end{tabular}

Date: January 1995

$\begin{array}{lrr}\text { ZERO } & \text { ZERO } & \text { ZERO } \\ \text { ZERO } & \text { ZERO } & \text { ZERO } \\ \text { ZERO } & \text { ZERO } & \text { ZERO } \\ \text { ZERO } & \text { ZERO } & \text { ZERO } \\ \text { ZERO } & \text { ZERO } & \text { ZERO } \\ \text { ZERO } & \text { ZERO } & \text { ZERO } \\ \text { ZERO } & \text { ZERO } & \text { ZERO } \\ \text { ZERO } & \text { ZERO } & \text { ZERO } \\ \text { ZERO } & \text { ZERO } & \text { ZERO } \\ \text { ZERO } & \text { ZERO } & \text { ZERO } \\ \text { ZERO } & \text { ZERO } & \text { ZERO } \\ \text { ZERO } & \text { ZERO } & \text { ZERO } \\ \text { ZERO } & \text { ZERO } & \text { ZERO } \\ \text { ZERO } & \text { ZERO } & \text { ZERO } \\ \text { ZERO } & \text { ZERO } & \text { ZERO } \\ \text { ZERO } & \text { ZERO } & \text { ZERO } \\ \text { ZERO } & 0.2230 E-03 & 0.1686 E-02 \\ \text { ZERO } & \text { ZERO } & \text { ZERO } \\ \text { ZERO } & 0.6708 E-01 & 0.5073 E+00 \\ \text { ZERO } & 0.1670 E-02 & 0.1263 E-01 \\ \text { ZERO } & 0.7011 E-03 & \text { ZERO } \\ \text { ZERO } & \text { ZERO } & \text { ZERO } \\ \text { ZERO } & 0.7324 E-03 & 0.5540 E-02 \\ \text { ZERO } & \text { ZERO } & \text { ZERO } \\ \text { ZERO } & \text { ZERO } & \text { ZERO } \\ \text { ZERO } & \text { ZERO } & \text { ZERO } \\ \text { ZERO } & \text { ZERO } & \text { ZERO } \\ \text { ZERO } & 0.3549 E-01 & \text { ZERO } \\ \text { ZERO } & 0.3660 E-02 & \text { ZERO } \\ \text { ZERO } & 0.5607 E+00 & 0.4241 E+01 \\ \text { ZERO } & \text { ZERO } & \text { ZERO } \\ \text { ZERO } & \text { ZERO } & \text { ZERO } \\ \text { ZERO } & \text { ZERO } & \text { ZERO } \\ \text { ZERO } & \text { ZERO } & \text { ZERO } \\ \text { ZERO } & \text { ZERO } & \text { ZERO } \\ \text { ZERO } & 0.1912 E+00 & 0.1446 E+01 \\ \text { ZERO } & \text { ZERO } & \text { ZERO } \\ \text { ZERO } & \text { ZERO } & \text { ZERO } \\ \text { ZERO } & \text { ZERO } & \text { ZERO } \\ \text { ZERO } & 0.1392 E-01 & \text { ZERO } \\ \text { ZERO } & 0.1028 E-03 & 0.7788 E-03 \\ \text { ZERO } & 0.3649 E-01 & \text { ZERO } \\ \text { ZERO } & 0.2616 E+00 & 0.1979 E+01 \\ \text { ZERO } & 0.3848 E-04 & 0.2911 E-03 \\ \text { ZERO } & \text { ZERO } & \text { ZERO } \\ \text { ZERO } & \text { ZERO } & \text { ZERO } \\ \text { ZERO } & \text { ZERO } \\ \text { ZERO } & \text { ZERO }\end{array}$

Section 1A, Page 2 of 21

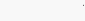


Table A-1. HLW Flowsheet Material Balance (Tank 51/ITP Cycle 1) Section $1 \mathrm{~A}$. In-Tank Precipitation and Salt Decontamination

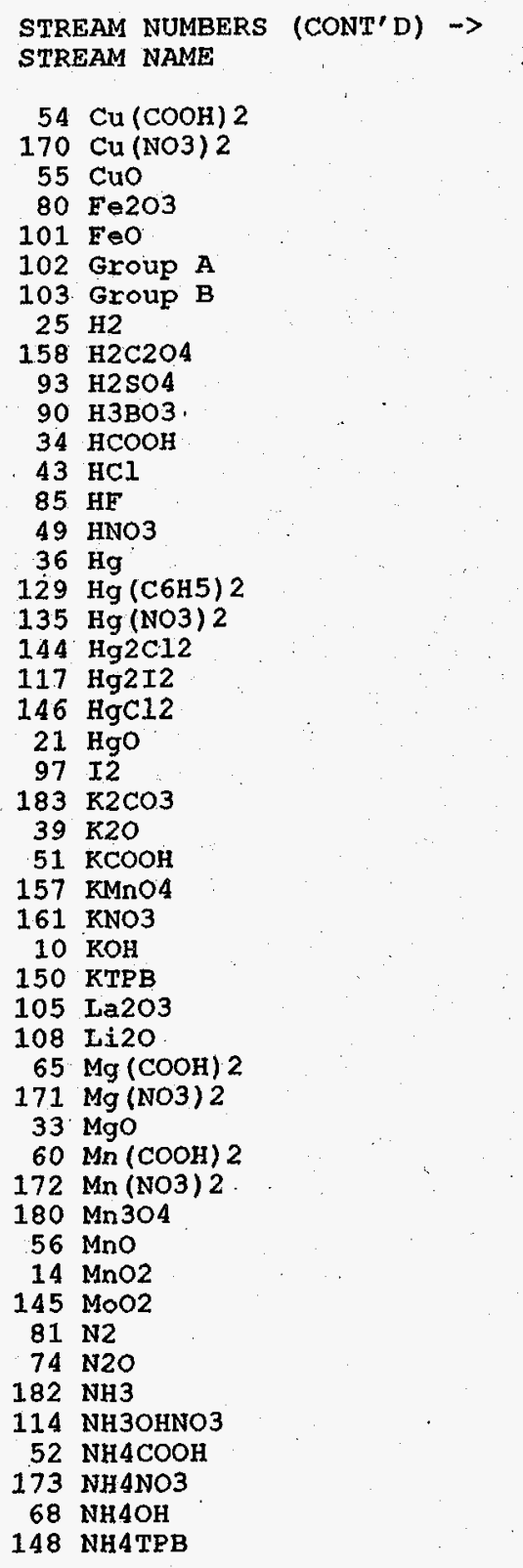

652653 Inv Saltcake Recycle 654 Feed to ITP

ZERO
$0.3286 \mathrm{E}-02$

$0.8920 \mathrm{E}-03$

$0.1274 \mathrm{E}+00$

$0.1527 \mathrm{E}-02$

$0.1773 \mathrm{E}-02$

ZERO
ZERO

ZERO

ZERO

ZERO

ZERO

ZERO

ZERO

ZERO

ZERO

ZERO

ZERO

$0.3125 \mathrm{E}-03$

ZERO

ZERO
$0.5784 \mathrm{E}-02$

ZERO

ZERO

ZTRO

$0.6439 \mathrm{E}+00$

ZERO

$0.2785 \mathrm{E}-03$

ZERO

$0.1361 \mathrm{E}-02$

$0.1449 \mathrm{E}-02$

ZERO
$0.9604 \mathrm{E}-03$

ZERO

$0.2769 \mathrm{E}-02$

$.2769 E-02$
$.9172 E-04$

ZERO

$0.6824 \mathrm{E}-01$

ZERO

ZERO

ZERO

ZERO

ZERO

ZERO

ZERO

ZERO

ZERO

ZERO

ZERO

ZERO

ZRRO

ZERO

DRO

ZERO

ZERO

ZERO

ZERO

ZERO

ZERO

ZERO

ZERO

ZERO

ZERO

ZERO

ZERO

ZERO

ZERO

ZERO

ZERO

ZERO

ZERO

ZERO

ZERO

ZERO

ZERO

ZERO

ZERO

ZERO

ZERO

ZERO

ZERO

ZERO

ZERO

ZERO fole Salt Recycle Salt Tot SN Feed Saltcake rm ESP frm DWRF per ITP Cyc slurry Water ZERO ZERO ZERO Z ZERO

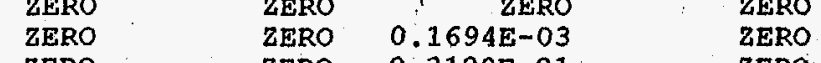

ZERO ZERO $0.3129 \mathrm{E}-01$. ZERO

ZERO ZERO ZERO ZERO

$$
\text { ZERO }
$$

ZERO

ZERO

$0.6163 \mathrm{E}-01$

ZERO

ZERO

ZERO $\quad$ ZERO

ZERO ZERO

ZERO

ZERO

ZERO

ZERO

ZERO

ZERO

ZERO

ZERO

ZERO

ZERO

ZERO

ZERO

ZERO

ZERO

ZERO
ZERO

$$
\begin{aligned}
& \text { ZERO } \\
& \text { ZERO }
\end{aligned}
$$

ZERO

ZERO

ZERO

ZERO

ZERO

ZERO

ZERO

ZERO

ZERO
ZERO
ZERO

ZERO

ZERO

ZERO

ZERO

ZERO

ZERO

ZERO

$0.3131 \mathrm{E}+00$

Date: January 1995

ZERO

$0.5940 \mathrm{E}-04$

ZERO

$0.1099 \mathrm{E}-02$

ZERO

ZERO

$0.6056 \mathrm{E}+01$

ZERO

$0.1623 \mathrm{E}-04$

ZERO

$0.2754 \mathrm{E}-03$

ZERO

ZERO

$0.5260 \mathrm{E}-03$

$0.1787 \mathrm{E}-04$

ZERO

ZERO

ZERO

ZERO
401

Salt/SN
Feed to ITP
ZERO
$0.3286 \mathrm{E}-02$
$0.1061 \mathrm{E}-02$
$0.1587 \mathrm{E}+00$

649

ZERO
$0.1061 \mathrm{E}-02$

$0.1147 \mathrm{E}+01$

$0.6316 \mathrm{E}-01$
ZERO

$0.1941 \mathrm{E}-02 \quad 0.1595 \mathrm{E}-01$

ZERO ZERO

ZERO ZERO

ZERO ZERO

$\begin{array}{ll}\text { ZERO } & \text { ZERO } \\ \text { ZERO } & \text { ZERO }\end{array}$

$\begin{array}{ll}\text { ZERO } & \text { ZERO } \\ \text { ZERO } & \text { ZERO }\end{array}$

ZERO ZERO

ZERO ZERO

ZERO ZERO

ZERO ZERO

ZERO

ZERO ZERO

ZERO

WSRC-TR-95-0019 (Revision

0)
ZERO

ZERO

ZERO

ZERO

ZERO

ZERO

ZERO

ZERO

ZERO

ZERO

ZERO

ZERO

ZERO

ZERO

ZERO

ZERO
ZERO

ZERO

ZERO

ZERO

ZERO

ZERO

ZERO

ZERO

ZERO

ZERO

ZERO

ZERO

ZERO

$0.6700 \mathrm{E}+01$

ZERO

$0.2948 \mathrm{E}-03$

0.1361 E-02

$0.1725 \mathrm{E}-02$

ZERO
$0.1586 \mathrm{E}-02$

$6 \mathrm{E}-02$
ZERO

$0.3295 \mathrm{E}-02$

$0.1096 \mathrm{E}-03$

ZERO

$0.6824 \mathrm{E}-01$

ZERO

ZERO

$0.3131 \mathrm{E}+00$

ZERO
0.3719 ERRO
ZERO
$0.2813 \mathrm{E}-02$

ZERO

ZERO
$0.5205 \mathrm{E}-01$

ZERO

ZERO

ZERO

ZERO

ZERO
$0.2507 \mathrm{E}-02$

ZERO

- 1

ZERO

ZERO

$0.2492 \mathrm{E}-01$

$0.8255 \mathrm{E}-03$

ZERO

ZERO

ZERO

ZERO

ZERO

ZERO

Section 1A, Page 3 of 21
0.6882 ZERO 
Table A-1. HLW Flowsheet Material Balance (Tank 51/ITP Cycle 1)

Section 1A. In-Tank Precipitation and Salt Decontamination

STREAM NUMBERS (CONT'D) $\rightarrow$

652

653.

654

Inv Saltcake Recycle Salt Recycle salt Tot SN Feed ZERO

ZERO
$0.1288 \mathrm{E}-03$

$0.5925 \mathrm{E}-01$

$0.5034 \mathrm{E}+00$

$0.2669 \mathrm{E}+03$

$0.6294 \mathrm{E}+00$

$0.2787 \mathrm{E}-01$

$0.8431 \mathrm{E}+00$

$0.4266 \mathrm{E}-05$

$0.1045 \mathrm{E}-01$

$0.2587 \mathrm{E}-01$

$0.2038 \mathrm{E}+02$

$0.3020 \mathrm{E}-01$

$0.3109 \mathrm{E}+02$

$0.3163 \mathrm{E}-02$

$0.1623 \mathrm{E}+02$

$0.6483 \mathrm{E}+00$

$0.3261 \mathrm{E}+00$

$0.1664 \mathrm{E}-03$

$0.2184 \mathrm{E}+02$

$0.1051 \mathrm{E}+04$

$0.6895 \mathrm{E}+02$

ZERO
ZERO

$0.5966 \mathrm{E}-02$

ZERO

ZERO

ZERO
$0.1732 \mathrm{E}-01$

$0.8718 \mathrm{E}-03$

$0.4207 \mathrm{E}-01$

ZERO

ZERO

ZERO

$0.3666 \mathrm{E}-02$

ZERO
ZERO

ZERO
ZERO

$0.2703 \mathrm{E}-04$

ZERO

ZERO

$0.3717 \mathrm{E}-02$

ZERO

ZERO

ZERO

ZERO

ZERO

ZERO

ZERO

ZERO

ZERO

ZERO

ZERO

ZERO

ZERO

ZERO

ZERO

ZERO

ZERO

ZERO

ZERO

ZERO

ZERO

ZERO

ZERO

ZERO

ZERO

ZERO

ZERO

ZERO

ZERO

ZERO

ZERO

ZERO

ZERO

ZERO

ZERO

ZERO

ZERO

ZERO

ZERO

ZTRO

ZERO

ZERO

ZERO

ZERO

ZERO

ZERO

ZERO

ZERO

ZERO

ZERO

ZERO

$0.2237 \mathrm{E}+03$

$0.7320 \mathrm{E}-05$

$0.2065 \mathrm{E}+01$

$0.7767 \mathrm{E}+00$

$0.1602 \mathrm{E}+00$

$0.7041 \mathrm{E}-04$

$0.9073 \mathrm{E}-02$

$0.7692 \mathrm{E}-01$

$0.7667 \mathrm{E}+01$

$0.7502 \mathrm{E}+00$

$0.7502 \mathrm{E}+00$
$0.6149 \mathrm{E}+01$

$0.6149 \mathrm{E}+01$
$0.8721 \mathrm{E}-03$

$0.1241 \mathrm{E}+03$

ZERO
$0.1328 \mathrm{E}+01$

$0.1830 \mathrm{E}+01$

$0.1278 \mathrm{E}-02$

$0.2077 \mathrm{E}+03$

$0.3013 \mathrm{E}+03$

$0.4343 \mathrm{E}+03$

ZFRO

ZRRO

ZERO

ZERO

ZERO

ZERO

ZERO

ZERO

ZERO

ZERO

ZERO

ZERO

ZERO

ZFRO

ZERO

ZERO

ZERO

ZERO

ZERO

ZERO

ZERO

ZERO

ZERO

ZERO
$0.5881 \mathrm{E}-01$

2mio

ZERO

ZERO

$0.1656 \mathrm{E}-03$

ZERO

ZERO

ZERO

ZERO

$0.6969 \mathrm{E}-03$

ZERO

ZERO

$0.5062 E-05$

ZERO

ZERO

$0.7063 \mathrm{E}-03$

ZERO
651

Saltcake

\begin{tabular}{|c|c|c|c|}
\hline ZERO & ZERO & s.tuIfy & $\begin{array}{r}\text { WaLE } \\
\text { ZERO }\end{array}$ \\
\hline ZERO & ZERO & & ZERO \\
\hline $\mathrm{R}$ & $0.2748 \mathrm{E}+00$ & & ZERO \\
\hline
\end{tabular}

$\begin{array}{lrrr}\text { ZERO } & \text { ZERO } & \text { ZERO } & \text { ZERO } \\ \text { ZERO } & \text { ZERO } & \text { ZERO } & \text { ZERO } \\ \text { ZERO } & \text { ZERO } & 0.2748 E+00 & \text { ZERO }\end{array}$

ZERO ZERO

Date: January 1995
$401 \quad 649$

Salt/sN settld salt

Insolubles

ZERO
ZERO

$0.5925 \mathrm{E}-01 \quad$ ZERO

$0.2518 \mathrm{E}+01,0.3078 \mathrm{E}+01$

$0.4906 \mathrm{E}+03 \quad$ ZERO

$0.7320 \mathrm{E}-05$ ZERO

$0.2695 \mathrm{E}+01$ ZERO

$0.8046 \mathrm{E}+00 \quad$ ZERO

$0.1003 \mathrm{E}+01 \quad 0.7588 \mathrm{E}+01$

$0.7467 \mathrm{E}-04 \quad$ ZERO

$0.1952 \mathrm{E}-01 \quad$ ZERO

$0.1028 \mathrm{E}+00$ ZERO

$0.2804 \mathrm{E}+02$ ZERO

$0.7804 \mathrm{E}+00 \quad$ ZERO

$\begin{array}{lll}0.3724 \mathrm{E}+02 & & \text { ZERO } \\ 0.4035 \mathrm{E}-02 & \text { ZERO }\end{array}$

$0.1403 \mathrm{E}+03$ ZERO

ZERO ZERO

$0.1976 \mathrm{E}+01 \quad$ ZERO

ZERO ZERO

$0.1444 \mathrm{E}-02$ ZERO

$0.1353 \mathrm{E}+04$

$0.5045 \mathrm{E}+03$

ZERO ZERO

$0.647 .8 \mathrm{E}-01 \quad$ ZERO

ZERO ZERO $\quad$ ZERO

ZERO ZERO $\quad$ ZERO

ZERO $0.1732 \mathrm{E}-01 \quad$ ZERO

ZERO $0.1037 \mathrm{E}-02 \quad 0.7846 \mathrm{E}-02$

ZERO ZERO ZERO

ZERO 0.4207 E-01 ZERO

ZERO ZERO $\quad$ ZERO

ZERO ZERO

ZERO $0.4362 \mathrm{E}-02 \quad 0.3299 \mathrm{E}-01$

ZERO ZERO

ZERO $0.1736 \mathrm{E}-03$ ZERO

ZERO ZERO ZERO

ZERO $0.3209 \mathrm{E}-04 \quad 0.2433 \mathrm{E}-03$

ZERO ZERO

ZERO ZERO $\quad$ ZERO

ZERO

$0.4424 \mathrm{E}-02$

$0.3346 \mathrm{E}-01$

ZERO

Seation 1A, Page 4 of 21 
Table A-1. HLW Flowsheet Material Balance (Tank 51/ITP Cycle 1)

section 1A. In-Tank Precipitation and Salt Decontamination

STREAM NUMBERS (CONT'D) $\rightarrow$ STREAM NAME

$\begin{array}{rr}38 & \mathrm{SO} 2 \\ 141 & \mathrm{SO} 3\end{array}$

137 Semi Vol Cs2O

136 Semi Vol CsCl

126 Semi Vol Group A

140 Semi Vol Na2B4O

139 Semi Vol NaF

125 Semi Vol NaI

23 semi Vol RuO2

111 SiO2

$48 \mathrm{Sr}(\mathrm{COOH}) 2$

$176 \mathrm{Sr}$ (NO3) 2

$153 \mathrm{Sr}$ (NaTi2O5) 2

$127 \mathrm{Sr}(\mathrm{OH}) 2$

$30 \mathrm{SrCO}$

46 Sro

$159 \mathrm{TcO} 2$

92 Tho

119 Tritium

170308

62002 (COOH) 2

$177 \mathrm{UO} 2$ (NO3) 2

$16 \mathrm{UO} 2(\mathrm{OH}) 2$

178 Y (NO3) 3

$128 \mathrm{Y}(\mathrm{OH}) 3$

$31 \times 2(\mathrm{CO} 3) 3$

$47 \mathrm{Y} 203$

100 Zeolite

$44 \mathrm{Zn}(\mathrm{COOH}) 2$

$179 \mathrm{zn}(\mathrm{NO} 3) 2$

$\begin{array}{rl}57 & \mathrm{Zno} \\ 104 & \mathrm{zrO} 2\end{array}$

$\begin{array}{rl}104 & \mathrm{ZrO} 2 \\ 13 & \text { hydrate } \mathrm{H} 2 \mathrm{O}\end{array}$

1 water

TOTAL FLOW, LB/HR

TEMRERATURE， DEG C

PRESSURE， ATM

PRESSURE, PSIG

PRESSURE, MM HG

ENTHALPY, PCU/HR

VAPOR FLOW, CFM

IIQUID FLOW, GPM

DENSITY, LBS/FT3

PHASE 652 653 $\begin{array}{cccc}652 & 653 & 654 & 659 \\ \text { Inv Saltcake Recycle Salt Recycle salt } & \text { Tot SN Feed }\end{array}$ 651

\begin{tabular}{|c|c|}
\hline $\begin{array}{l}\text { Feed to ITP } \\
\text { zERO }\end{array}$ & $\begin{array}{r}\text { frm ESP } \\
\text { ZERO }\end{array}$ \\
\hline ZERO & ZERO \\
\hline ZERO & ZERO \\
\hline ZERO & ZERO \\
\hline ZERO & ZERO \\
\hline ZERO & ZERO \\
\hline ZERO & ZERO \\
\hline ZERO & ZERO \\
\hline ZERO & ZERO \\
\hline ZERO & ZERO \\
\hline $0.2467 \mathrm{E}-01$ & ZERO \\
\hline ZERO & ZERO \\
\hline ZERO & ZERO \\
\hline ZERO & ZERO \\
\hline $0.7049 \mathrm{E}-03$ & ZERO \\
\hline $0.7646 \mathrm{E}-04$ & ZERO \\
\hline ZERO & ZERO \\
\hline ZERO & ZERO \\
\hline ZERO & ZERO \\
\hline $0.6354 \mathrm{E}-03$ & ZERO \\
\hline $0.1126 \mathrm{E}-07$ & ZERO \\
\hline $0.6313 \mathrm{E}-03$ & ZERO \\
\hline ZERO & ZERO \\
\hline ZERO & ZERO \\
\hline $0.7895 \mathrm{E}-02$ & ZERO \\
\hline ZERO & ZERO \\
\hline ZERO & ZERO \\
\hline $0.9841 \mathrm{E}-05$ & ZERO \\
\hline ZERO & ZERO \\
\hline ZERO & ZERO \\
\hline ZERO & ZERO \\
\hline ZERO & ZERO \\
\hline $0.1383 \mathrm{E}+01$ & ZERO \\
\hline $0.1664 \mathrm{E}+00$ & ZERO \\
\hline ZERO & ZERO \\
\hline ZERO & ZERO \\
\hline $0.2835 \mathrm{E}+03$ & ZERO \\
\hline $0.1767 \mathrm{E}+04$ & ZERO \\
\hline
\end{tabular}

fret Salt Toltcake

401

Salt/SN settld Salt

Feed to ITP Insolubles $\begin{array}{llll}\text { ZERO } & \text { ZERO } & \text { ZERO } & \text { ZERO }\end{array}$

$\begin{array}{lllll}\text { ZERO } & \text { ZERO } & \text { ZERO } & \text { ZERO } & \text { ZERO } \\ \text { ZERO } & \text { ZERO } & \text { ZERO } & \text { ZERO } & \text { ZERO }\end{array}$

ZERO

ZERO

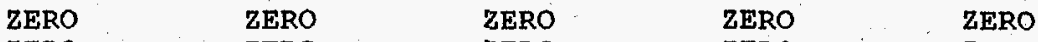

$\begin{array}{llll}\text { ZERO } & \text { ZERO } & \text { ZERO } & \text { ZERO } \\ \text { ZERO }\end{array}$

ZERO ZERO ZERO

ZERO ZERO

ZERO ZERO ZERO

ZERO ZERO

ZERO $0.1985 \mathrm{E}-02 \quad$ ZERO

ZERO ZERO ZERO

ZERO ZERO

ZERO ZERO

ZERO

$0.1435 \mathrm{E}-04$

ZERO

ZERO

ZERO

ZERO

ZERO

ZERO

$0.5780 \mathrm{E}-03$

$0.2933 \mathrm{E}-07$

$0.1199 \mathrm{E}-03$

ZERO ZERO

ZERO

$0.1994 \mathrm{E}-01$

ZERO

ZERO

ZERO

ZERO $0.1196 \mathrm{E}-02$

ZERO ZERO

ZERO ZERO

ZERO ZERO

ZERO ZERO

ZERO ZERO

$0.3160 \mathrm{E}-01$

ZERO ZERO

ZERO $0.2434 \mathrm{E}-02$

ZERO $\quad 0.2080 E+04$

$0.3401 \mathrm{E}+04$

$0.3500 \mathrm{E}+02$

$0.1000 \mathrm{E}+01$

$0.3500 \mathrm{E}+02$

$0.1000 \mathrm{E}+03$

$0.3500 \mathrm{E}+02$

$0.1000 \mathrm{E}+01$

ZERO

ZERO

ZERO

ZERO

ZERO

ZERO

ZERO

ZERO

ZERO

ZERO

ZERO

ZERO

ZERO

ZERO

ZERO

ZERO

ZERO

$0.3209 \mathrm{E}+04$

$0.3212 \mathrm{E}+04$

$0.2500 \mathrm{x}+02$

$0.1000 \mathrm{E}+01$

ZERO

ZERO

ZERO

ZERO

ZERO

$0.2666 \mathrm{E}-01$

ZERO

ZERO

$0.1026 \mathrm{E}-02$

$0.9081 \mathrm{E}-04$

ZERO

ZERO

$0.1213 \mathrm{E}-02$

$0.4059 \mathrm{E}-07$

$0.7512 \mathrm{E}-03$

ZERO
ZERO

$0.2783 \mathrm{E}-01$

ZERO

$0.1206 \mathrm{E}-02$

ZERO

ZERO

ZERO

$0.1383 \mathrm{E}+01$

$0.1980 \mathrm{E}+00$

ZERO
$0.2434 \mathrm{E}-02$

$0.5573 \mathrm{E}+04$

$0.8380 \mathrm{E}+04$

$0.2992 \mathrm{E}+02$

$0.1000 \mathrm{E}+01$

ZERO

ZERO

ZERO

0.2220 E +00

ZERO

ZERO

ZERO

$0.6881 \mathrm{E}-03$

ZERO

ZERO

0.571 ZERO

ZERO

$0.5682 \mathrm{E}-02$

ZERO

ZERO

ZERO

ZERO

ZERO

ZERO

ZERO

ZERO

ZERO

ZERO

ZERO

$0.2195 \mathrm{E}+02$

$\begin{array}{lllll}0.2317 E+05 & \text { ZERO } & \text { ZERO } & 0.8526 \mathrm{E}+05 & 0.8055 \mathrm{E}+05\end{array}$

$0.1890 \mathrm{E}+06$

$0.1000 \mathrm{E}+01$

\section{$0.5113 \mathrm{E}+01 \quad 0.6411 \mathrm{E}+01$}

$0.6411 \mathrm{E}+01$
$0.6247 \mathrm{E}+02$

IIQUID

$0.1320 \mathrm{E}+02$

$0.7914 \mathrm{E}+02$

LIQUID

$0.1607 \mathrm{E}+03$

$0.1529 \mathrm{E}-0.1$

$0.1790 \mathrm{E}+03$

IIQUID 
Table A-1. HLW Elowsheet Material Balance (Tank 51/ITP Cycle 1)

section 1A. In-Tank Precipitation and Salt Decontamination

STREAM NUMBERS $\rightarrow$ STREAM NAME

COMPONENT FLOWS, IB/HR $122(\mathrm{C} 6 \mathrm{H} 5) 2$

123 (C6H5) $2 \mathrm{C} 6 \mathrm{H} 4$

133 (C6H5B (OH) 2 salt)

132 (C6H5B (OH) 2)

166 (C6H5C6H4OH)

162 (C6H5NH2).

163 (C6H5NHCHO)

165 (C6H5NO)

131 (C6H5OH salt)

130 ( $\mathrm{C} 6 \mathrm{H} 5 \mathrm{OH})$

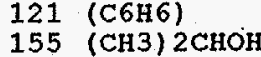

154 ( $\mathrm{CH} 3 \mathrm{OH})$

$45 \mathrm{Ag}$

$116 \mathrm{Ag} 2 \mathrm{O}$

77 A 1203

$106 \mathrm{~B} 203$

$9 \mathrm{Ba}(\mathrm{OH}) 2$

$24 \mathrm{BasO}$

$\begin{array}{ll}66 & \mathrm{CO} \\ 37 & \mathrm{CO} 2\end{array}$

$142 \mathrm{CO} 2$ (C.14)

$63 \mathrm{Ca}(\mathrm{COOH}) 2$

$167 \mathrm{Ca}(\mathrm{NO} 3)^{2} 2$

$120 \mathrm{Ca}(\mathrm{OH}) 2$
$113 \mathrm{Ca} 3$ (PO4)

$20 \mathrm{CaC} 204$

$18 \mathrm{CaCO} 3$

$64 \mathrm{CaCO} 3$ (C14)

78 CaF2

$41 \mathrm{CaO}$

19 CasO4

29 Carbon

99 Cement

$26 \mathrm{Co}(\mathrm{COOH}) 2$
$168 \mathrm{Co}(\mathrm{NO} 3) 2$

$59 \mathrm{COO}$

98 Cr2O3

$42 \mathrm{Cs} 2 \mathrm{O}$

$53 \mathrm{CsCOOH}$

$96 \mathrm{CsCl}$

$169 \mathrm{CsNO3}$

151 CsTPB

WSRC-TR-95-0019 (Revision
451

$$
402
$$

Total spent salt Transfr Wash Recycle Line Flush

ZERO
ZERO
ZERO
ZERO
ZERO
ZERO
ZERO
ZERO
ZERO
ZERO
0.5151 E+00
ZERO
0.1293 E-O3

$0.1293 \mathrm{E}-03$

$0.4783 \mathrm{E}-0.5$
$0.3911 \mathrm{E}-06$

$0.3911 \mathrm{E}-06$

ZERO

ZERO

ZERO

$0.4574 \mathrm{E}-09$

ZERO

ZERO

ZERO

ZERO

ZERO

$0.1328 \mathrm{E}-02$

$0.1369 \mathrm{E}-03$

ZERO

ZERO

ZERO

ZERO

ZERO

ZERO

ZERO

ZERO

ZERO

$0.5208 \mathrm{E}-03$

ZERO

ZERO

ZERO

ZERO

ZERO

$0.4669 E-04$

ZERO

0)
403

NaTPB Additions

ZERO

ZERO

ZERO

ZERO

ZERO

ZERO

ZERO

ZERO

ZERO

ZERO

ZERO

ZERO

ZERO

ZERO

ZERO

ZERO

ZERO

ZERO

ZERO

ZERO

ZERO

ZERO

ZERO

ZERO

ZERO

ZERO

ZERO

ZERO

ZERO

ZERO

ZERO

ZERO

ZERO

ZERO

ZERO

ZERO

ZERO

ZERO

ZERO

ZERO

ZERO

ZERO

ZERO

ZERO

Date: January 1995

ZERO
ZERO
ZERO
ZERO
ZERO
ZERO
ZERO
ZERO
ZERO
ZERO

ZERO

ZERO

ZERO

ZERO

ZERO

ZERO

ZERO

ZERO

ZERO

ZERO

ZERO

ZERO

ZERO

ZERO

ZERO

ZERO

ZERO

ZERO

ZERO

ZERO

ZERO

ZERO

ZERO

ZERO

ZERO

ZERO

ZERO

ZERO

ZERO

ZERO
$404 \quad 405$

NaTi205H

Additions

. ZERO

ZERO

ZERO

ZERO

ZERO

ZERO

ZERO

ZERO

ZERO

ZERO

ZERO

$0.1762 \mathrm{E}+00$

$0.9434 \mathrm{E}-02$

ZERO
ZERO

ZERO

ZERO

ZERO

ZERO

ZFRO

ZTRO

ZTRO

ZERO

ZERO

ZERO

ZERO

ZERO

ZERO

ZERO

ZERO

ZERO

ZERO

ZRRO

ZERO

ZERO

ZERO

ZERO

ZERO

ZERO

ZERO

ZERO

ZERO

ZERO

ZERO
406

Leakage Gas Purge Entrnd sldge

ZERO ZERO

ZERO ZERO ZERO

ZERO ZERO ZERO

$\begin{array}{llr}\text { ZERO } & \text { ZERO } & \text { ZERO } \\ \text { ZERO } & \text { ZERO } & \text { ZERO }\end{array}$

ZERO ZERO ZERO

ZERO ZERO ZERO

ZERO ZERO $\quad$ ZERO

ZERO ZERO $\quad$ ZERO

ZERO ZERO ZERO

ZERO ZERO

ZERO ZERO

ZTRO ZERO ZERO

ZERO ZERO ZERO

ZERO ZERO ZERO

ZERO ZERO ZERO

ZERO ZERO ZERO

ZERO ZERO ZERO

ZERO ZERO ZERO

ZERO ZERO $\quad$ ZERO

ZERO ZERO Z ZERO

ZERO ZERO ZERO

ZERO ZERO ZERO

ZERO ZERO $\quad$ ZERO

ZERO ZERO

ZERO ZERO ZERO

ZERO $0.3320 E+00 \quad$ ZERO

ZERO ZERO ZERO

ZERO ZERO ZERO

ZERO ZERO ZERO

ZERO ZERO ZERO

ZERO $\quad$ ZERO $\cdots$ ZERO

ZERO ZERO ZERO

ZERO ZERO $\quad$ ZERO

ZERO ZERO ZERO

ZERO ZERO $\quad$ ZERO

ZERO ZERO Z ZERO

ZERO ZERO

ZERO ZERO

ZERO ZERO ZERO

ZERO ZERO : ZERO

ZERO ZERO ZERO

ZERO ZERO $\quad$ ZERO.

ZERO ZERO ZERO

$\begin{array}{lll}\text { ZERO } & \text { ZERO } & \text { ZERO } \\ \text { ZERO } & \text { ZERO } & \text { ZERO }\end{array}$

ZERO ZERO ZERO

ZERO ZERO ZERO

ZERO ZERO ZERO

Section 1A, Page 6 of 21 
Table A-1. HLW Flowsheet Material Balance (Tank 51/ITP Cyole 1 )

Section 1A. In-Tank Precipitation and Salt Decontamination

STREAM NUMBERS (CONT'D) $\rightarrow$

402
Total spent salt Transfr STREAM NAME Wash Recycle Iine Flush

$54 \mathrm{Cu}(\mathrm{COOH}) 2$

$170 \mathrm{Cu}(\mathrm{NO} 3) 2$

55 CuO

$80 \mathrm{Fe} 203$

$101 \mathrm{~F} \oplus \mathrm{O}$

102 Group A

103 Grou
252

$\begin{array}{rl}25 & \mathrm{H} 2 \\ 158 & \mathrm{H} 2 \mathrm{C} 2 \mathrm{O} 4\end{array}$

$93 \mathrm{H} 2 \mathrm{SO4}$

$90 . \mathrm{H} 3 \mathrm{BO} 3$

$34 \mathrm{HCOO}$

$43 \mathrm{HCl}$

$85 \mathrm{HF}$
$49 \mathrm{HNO}$

$36 \mathrm{Hg}$

$129 \mathrm{Hg}(\mathrm{C} 6 \mathrm{H} 5) 2$

$135 \mathrm{Hg}$ (NO3) 2

$144 \mathrm{Hg} 2 \mathrm{Cl} 2$

$117 \mathrm{Hg} 2 \mathrm{I2}$

$146 \mathrm{HgCl} 2$

$21 \mathrm{Hg}$

$97 . \mathrm{I} 2$
$183 \mathrm{~K} 2 \mathrm{CO}$

$\begin{array}{rl}183 & \mathrm{~K} 2 \mathrm{CO} \\ 39 & \mathrm{~K} 2 \mathrm{O}\end{array}$

$\begin{array}{ll}39 & \mathrm{~K} 2 \mathrm{O} \\ 51 & \mathrm{KCOO}\end{array}$

$51 . \mathrm{KCOOH}$
$157 \mathrm{KMnO} 4$

161 KNO3

$10 \mathrm{KOH}$

$150 \mathrm{KTPB}$

$105 \mathrm{La} 203$

108 Li20

$65 \mathrm{Mg}(\mathrm{COOH}) 2$

$171 \mathrm{Mg}(\mathrm{NO} 3) 2$

$33 \mathrm{MgO}$

$60 \mathrm{Mn}(\mathrm{COOH}) 2$

$172 \mathrm{Mn}$ (NO3)

$180 \mathrm{Mn} 30$

$56 \mathrm{MnO}$

$14 \mathrm{MnO} 2$

$145 \mathrm{MOO} 2$

81 N2

74 N2O

ZERO
$0.1229 E-03$
ZERO $\begin{aligned} & \text { ZERO } \\ & \text { ZERO } \\ & \text { ZERO }\end{aligned}$

403

NaTPB

404

405

406

415 $\begin{array}{rrrrr}\text { NaTPB } & \text { NaTi2O5H } & \text { Total Pump } & \text { Gas Purge Entrnd sldge } \\ \text { Additions } & \text { Additions } & \text { Leakage } & \text { In-tnk Pptn in Preci Fd } \\ \text { ZERO } & \text { ZERO } & \text { ZERO } & \text { ZERO } & \text { ZERO }\end{array}$

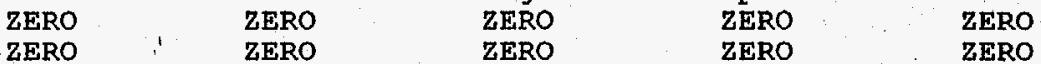

$\begin{array}{llll}\text { ZERO } & \text { ZERO } & \text { ZERO } & \text { ZERO }\end{array}$

ZERO Z ZERO ZERO ZERO

ZERO ZERO

$0.2362 E-02 \quad$ ZERO

$0.7262 \mathrm{E}-04$

ZERO $\quad$ ZERO

ZERO

ZERO

$\begin{array}{ll}\text { ZERO } & \text { ZERO } \\ \text { ZERO } & \text { ZERO }\end{array}$

ZERO ZERO

ZERO ZERO

ZERO ZERO

ZERO ZERO

ZERO

ZERO

ZERO

ZERO ZERO

ZERO -ZERO

ZERO

ZERO - ZERO

$0.5248 \mathrm{E}-03$

ZERO

ZERO ZERO

ZERO

$0.2796 \mathrm{E}-05 \quad$ ZERO

ZERO

ZERO

ZERO

ZERO

ZERO

ZERO

$0.5092 \mathrm{E}-04$ ZERO

$0.5933 E-04$

ZERO

ZERO

$0.4228 \mathrm{E}-01$

$.2200 \mathrm{E}-11$

ZERO

ZERO

TERO

$\begin{array}{rl}114 & \mathrm{NH} 3 \mathrm{OHNO} 3 \\ 52 & \mathrm{NH} 4 \mathrm{COOH}\end{array}$

173 NH4NO3

$68 \mathrm{NH} 4 \mathrm{OH}$

$0.1138 \mathrm{~B}-01$

ZERO

ZERO

ZERO

ZERO

ZERO

ZERO

ZERO

ZERO

ZERO

ZERO

ZERO

ZERO

ZERO

ZERO

ZERO

ZERO

ZERO

ZERO

ZERO

ZERO

ZERO

ZERO

ZERO

ZERO

ZERO

ZERO

ZERO

ZERO

ZERO

ZERO

ZERO

ZERO

ZERO

ZERO

ZERO

ZERO

ZERO

ZERO

ZERO

ZERO

ZERO

ZERO

ZERO

ZERO

ZERO

ZERO

ZERO

ZERO

ZERO

ZERO

ZERO

ZERO

ZERO

ZERO

ZERO

ZERO

ZERO

ZERO

ZERO ZERO

ZERO ZERO

ZERO

ZERO ZERO

ZERO ZERO

ZERO ZERO

$\begin{array}{ll}\text { ZERO } & \text { ZERO } \\ \text { ZERO } & \text { ZERO }\end{array}$

ZERO ZERO

ZERO ZERO

ZERO : ZERO

ZERO ZERO

ZERO ZERO

ZERO ZERO

ZERO ZERO

ZERO

ZERO ZERO

ZERO ZERO

ZERO Z ZERO

ZERO ZERO

ZERO ZERO

ZERO ZERO

ZERO ZERO

ZERO ZERO

ZERO ZERO

ZERO ZERO

ZERO ZERO

ZERO ZRRO

ZERO ZERO

ZERO ZERO

ZERO ZERO

ZERO ZERO

ZERO ZERO

ZERO ZERO

ZERO $\quad$ ZERO

ZERO ZERO

ZERO
$0.2207 \mathrm{E}+04$

ZERO $\quad$ ZERO

ZERO ZERO

ZERO

ZERO - ZERO

ZERO ZERO

ZERO ZERO

WSRC-TR-95-0019 (Revision 
Table A-1. HLW Flowsheet Material Balance (Tank 51/ITP Cycle 1)

Section 1A. In-Tank Precipitation and Salt Decontamination

STREAM NUMBERS (CONT'D) $\rightarrow$ STREAM NAME

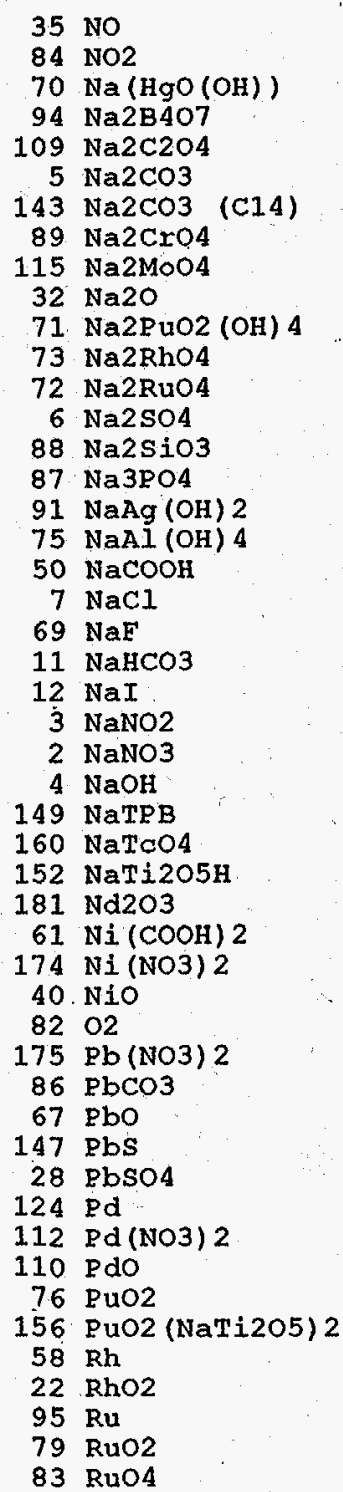

\begin{tabular}{|c|c|}
\hline 403 & 404 \\
\hline NaTPB & NaTi205H \\
\hline Additions & Additions \\
\hline ZERO & ZERO \\
\hline ZERO & ZERO \\
\hline ZERO & ZERO \\
\hline ZERO & ZERO \\
\hline ZERO & ZERO \\
\hline ZERO & ZERO \\
\hline ZERO & ZERO \\
\hline ZERO & ZERO \\
\hline ZERO & ZERO \\
\hline ZERO & ZERO \\
\hline ZERO & ZERO \\
\hline ZERO & ZERO \\
\hline ZERO & ZERO \\
\hline ZERO & ZERO \\
\hline ZERO & ZERO \\
\hline ZERO & ZERO \\
\hline ZERO & ZERO \\
\hline ZERO & ZERO \\
\hline ZERO & ZERO \\
\hline $0.2231 \mathrm{E}+00$ & ZERO \\
\hline ZERO & ZERO \\
\hline ZERO & ZERO \\
\hline ZERO & ZERO \\
\hline ZERO & $0.1272 \mathrm{E}-02$ \\
\hline . ZERO & ZERO \\
\hline $0.1113 \mathrm{E}+01$ & $0.3141 \mathrm{E}-01$ \\
\hline $0.4760 \mathrm{E}+02$ & ZERO \\
\hline ZERO & ZERO \\
\hline ZERO & $0.9827 \mathrm{E}+00$ \\
\hline ZERO & ZERO \\
\hline ZERO & ZERO \\
\hline ZERO & ZERO \\
\hline ZERO & ZERO \\
\hline ZERO & ZERO \\
\hline ZERO & ZERO \\
\hline ZERO & ZERO \\
\hline ZERO & ZERO \\
\hline ZERO & ZERO \\
\hline ZERO & ZERO \\
\hline ZERO & ZERO \\
\hline ZERO & ZERO \\
\hline ZERO & ZERO \\
\hline ZERO & ZERO \\
\hline ZERO & ZERO \\
\hline ZERO & ZERO \\
\hline ZERO & ZERO \\
\hline ZERO & ZERO \\
\hline ZERO & ZERO \\
\hline ZERO & ZERO \\
\hline
\end{tabular}

Date: January 1995
406

405

Total Pump

415 Leakage In-tnk Pptn in Preci Fd ZERO ZERO ZERO ZERO ZERO ZERO ZERO ZERO ZERO ZERO ZZERO - ZERO ZERO ZERO $\quad$ ZERO ZERO ZERO $\quad$ ZERO ZERO $\quad$ ZERO $\quad$ ZERO ZERO ZERO ZERO ZERO ZERO ZERO ZERO ZRRO ZERO ZERO ZERO ZERO ZERO ZERO ZERO $\begin{array}{lll}\text { ZERO } & \text { ZERO } & \text { ZERO } \\ \text { ZERO } & \text { ZERO } & \text { ZERO }\end{array}$ ZERO ZERO ZERO ZERO ZERO ZERO : ZERO ZERO ZERO ZERO ZERO ZERO ZERO ZERO ZERO ZERO ZERO ZERO ZERO ZERO ZERO $\quad$ ZERO

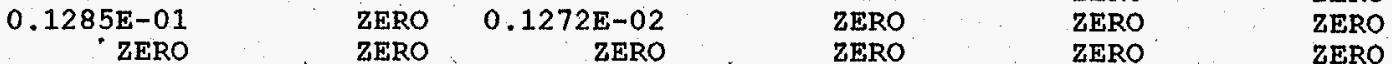
$\begin{array}{llllll}0.1028 \mathrm{E}-01 & 0.1113 \mathrm{E}+01 & 0.3141 \mathrm{E}-01 & 0.1112 \mathrm{E}-01 & \text { ZERO } & \text { ZERO }\end{array}$ ZERO ZERO ZERO ZERO ZERO ZERO $\quad$ ZERO ZERO ZERO ZERO ZERO $\because$ ZERO ZERO ZERO $0.1362 \mathrm{E}+03, \quad$ ZERO ZERO ZERO ZERO ZERO ZFRO ZERO

ZERO

ZERO ZERO

ZERO ZERO

ZERO ZERO ZERO

ZERO ZERO ZERO

ZERO ZERO ZERO

ZERO ZERO ZERO

ZERO ZERO

ZERO ZERO ZERO

ZERO ZERO ZERO

ZERO ZERO ZERO

ZERO * ZERO ZERO

ZERO ZERO

Section IA, Page 8 of 21 
Table A-1. HLW Flowsheet Material Balance (Tank 51/ITP Cycle 1)

Section 1A. In-Tank Precipitation and Salt Decontamination

STREAM NUMBERS (CONT'D) $\rightarrow$ STREAM NAME

$\begin{array}{rr}38 & 502 \\ 141 & 503\end{array}$

137 Semi Vol Cs2O

136 Semi Vol CaCl

126 Semi Vol Group A

140 Semi Vol Na2B407

138 Semi Vol NaCl

139 Semi Vol NaF

125 Semi Vol NaI

23 Semi Vol RuO2

$111 \mathrm{SiO2}$

48 Sr (COOH) 2

$176 \mathrm{Sr}$ (NO3) 2

$153 \mathrm{Sr}$ (NaTi2
$127 \mathrm{Sr}(\mathrm{OH}) 2$

$30 \mathrm{SrCO}$

46 Sro

$159 \mathrm{TCO} 2$

92 ThO2

119 Tritium

170308

$62 \mathrm{OO2}(\mathrm{COOH}) 2$

$177 \mathrm{UO2}(\mathrm{NO3}) 2$
$16 \mathrm{UO2}(\mathrm{OH}) 2$

$\begin{array}{ll}16 & \mathrm{UO} 2(\mathrm{OH}) 2 \\ 27 & \mathrm{Y}(\mathrm{COOH}) 3\end{array}$

178 Y (NO3) 3

128 Y $(\mathrm{OH}) 3$

31 Y2 (CO3) 3

$47 \mathrm{Y} 203$

100 Zeolite

$44 \mathrm{Zn}$ ( $\mathrm{COOH})$

$\begin{array}{rl}179 & \mathrm{Zn}(\mathrm{N} \\ 57 \mathrm{Zno} & \end{array}$

$104 \mathrm{ZrO} 2$

13 hydrate $\mathrm{H} 2 \mathrm{O}$

1 water

TOTAL FLOW, LB/HR

TEMPERATURE, DEG C

PRESSURE， ATM

PRESSURE, PSIG

PRESSURE， MM HG

ENTHALPY， PCU/HR

VAPOR FIOW, CFM

LIQUID FLOW, GPM

DENSITY, IBS/FT3

PHASE

402

Total spent salt Transfr

Wash Recycle Iine Flush

ZERO

ZERO

ZERO

ZERO ZERO

ZERO ZERO

ZERO

ZERO

ZERO

ZERO

ZERO

ZERO

ZERO

ZERO

$0.2347 \mathrm{E}-06$

ZERO

ZERO

ZERO

ZERO

$0.1492 \mathrm{E}-08$

ZERO

ZERO

ZERO

$0.1041 \mathrm{E}-02$

ZERO

$0.1095 \mathrm{E}-06$

ZERO

ZERO

ZERO

$0.5171 \mathrm{E}-01$

ZERO

ZERO

$0.3074 \mathrm{E}+04$

$0.3362 \mathrm{E}+04$

$0.3981 \mathrm{E}+02$

$0.9656 \mathrm{E}+00$

$-0.5055 \mathrm{E}+00$

$0.7339 E+03$

$0.6338 \mathrm{E}+01$.

$0.6613 \mathrm{E}+02$

LIQUID
451

$$
403
$$

NaTPB

Additions

ZERO
ZERO

ZERO
ZERO

ZERO

ZERO

ZERO

ZERO

ZERO

ZERO

ZERO

ZERO

ZERO

ZERO

ZERO

ZERO

ZERO

ZERO

ZERO

ZERO

ZERO

ZERO

ZERO

ZERO

ZERO
ZERO

ZERO

ZERO

ZERO

ZERO

ZERO

ZERO

ZERO

ZERO

ZERO

$0.2265 \mathrm{E}+03$

$0.2570 \mathrm{E}+02$

$0.2500 \mathrm{E}+02$

$0.1000 \mathrm{E}+01$

ZERO

$0.2782 \mathrm{E}+03$

$0.3500 \mathrm{E}+02$

$0.1000 \mathrm{E}+01$

ZERO

404

NaTi205H Additions

$\therefore \quad$ ZERO

ZERO

ZERO

ZERO

ZERO

ZERO

ZERO

ZERO

ZERO

ZERO

ZERO

ZERO

ZERO

ZERO

ZERO

ZERO

ZERO

ZERO

ZERO

ZERO

ZERO

ZERO

ZERO

ZERO

ZERO

ZERO

ZERO

ZERO

ZERO

ZERO

ZERO

ZERO

$0.7283 \mathrm{E}+01$

$0.8484 \mathrm{E}+01$

$0.3500 \mathrm{E}+02$

$0.1000 \mathrm{E}+01$

ZERO

$0.6445 \mathrm{E}+03$

$0.7962 \mathrm{E}+04$

$0.2597 \mathrm{E}+03$

$0.5130 \mathrm{E}-01$

$0.6247 \mathrm{E}+02$

$0.5455 \mathrm{E}+00$

$0.6358 \mathrm{E}+02$

IIQUTD
405

otal Pump

Leakage

ZERO

ZERO

ZERO

ZERO

ZERO

ZERO

ZERO

ZERO

ZERO

ZERO

ZERO

ZERO

ZERO

ZERO

ZERO

ZERO

ZERO

ZERO

ZERO

ZERO

ZERO

ZERO

ZERO

ZERO

ZERO

ZERO

ZERO

ZERO

ZERO

ZERO

ZERO

ZERO

$0.1852 \mathrm{E}+02$

$0.1853 \mathrm{E}+02$

$0.2500 \mathrm{E}+02$

$0.1000 E+O 1$

ZERO

$0.4647 E+03$

$0.3698 \mathrm{E}-01$

$0.6246 \mathrm{E}+02$

IIQUID
406

415

Gas Rurge Entrnd sldge Pptn in Preci Fd
ZERO ZERO ZERO ZERO ZERO

ZERO ZERO

ZERO

ZERO ZERO

ZERO

ZERO ZERO

ZERO ZERO

ZERO ZERO

$\begin{array}{ll}\text { ZERO } & \text { ZERO } \\ \text { ZERO } & \text { ZERO }\end{array}$

$\begin{array}{ll}\text { ZERO } & \text { ZERO } \\ \text { ZERO } & \text { ZERO }\end{array}$

ZERO ZERO

ZERO ZERO

ZERO ZERO

ZERO ZERO

ZERO ZERO

ZERO

ZERO

ZERO -

ZERO ZERO

ZERO ZERO

$\begin{array}{ll}\text { ZERO } & \text { ZERO } \\ \text { ZERO } & \text { ZERO }\end{array}$

ZERO ZERO

ZERO ZERO

ZERO ZERO

ZERO ZERO

ZERO ZERO

ZERO ZERO

ZERO ZERO

ZERO

ZERO

ZERO ZERO

ZERO

$0.9860 \mathrm{E}+01$

$0.3500 \mathrm{E}+02$

$0.1000 \mathrm{E}+01$

ZERO

$0.6000 \mathrm{E}+02$

$0.1000 \mathrm{E}+01$

ZERO

$0.2744 \mathrm{E}+05$

$0.6954 \mathrm{E}-01$

VAPOR

ZERO

IIQUID 
Table A-1. HLW Flowsheet Material Balance (Tank 51/TTP Cycle 1)

Section 1A. In-Tank Precipitation and salt Decontamination

STREAM NUMBERS $\rightarrow$ STREAM NAME

COMPONENT FLOWS, LB/HR $122(\mathrm{C} 6 \mathrm{H} 5) 2$

123 (C6H5) 2 $6 \mathrm{CH} 4$

133 (C6H5B (OH) 2 salt)

132 ( $\mathrm{C} 6 \mathrm{H} 5 \mathrm{~B}(\mathrm{OH}) 2$ )

166 (C6H5C6H4OH)

162 (C6H5NH2)

163 (C6H5NO)

164 (C6H5NO2)

131. (C6H5OH salt)

130 (C6H5OH)

121 (C6H6)

154 ( $\mathrm{CH} 3 \mathrm{OH})$

$45 \mathrm{Ag}$

116 Ag20

$77 \mathrm{Al2O3}$

$106 \mathrm{~B} 203$

$$
\begin{array}{r}
9 \mathrm{Ba}(\mathrm{OH}) 2 \\
25
\end{array}
$$

$15 \mathrm{BaO}$

$24 \mathrm{BaSO}$

$66 \mathrm{CO}$

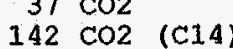

$63 \mathrm{Ca}(\mathrm{COOH}) 2$

$167 \mathrm{Ca}(\mathrm{NO} 3) 2$

$120 \mathrm{Ca}(\mathrm{OH}) 2$

$113 \mathrm{Ca} 3(\mathrm{PO} 4) 2$

$20 \quad \mathrm{CaC}_{2} \mathrm{O} 4$

$18 \mathrm{CaCO} 3$

$64 \mathrm{CaCO}$ (C14)

78 CaF2

$41 \mathrm{CaO}$

19 CasO4

29 Carbon

99 Cement
26 Co $(\mathrm{COOH}) 2$

$168 \mathrm{Co}(\mathrm{NO} 3) 2$

$59 \mathrm{CoO}$

$98 \mathrm{Cr} 2 \mathrm{O3}$

$42 \mathrm{Cs} 2 \mathrm{O}$

$53 \mathrm{CsCOOH}$

$96 \mathrm{CsC1}$

$$
8 \mathrm{CsOH}
$$

409

Prc Proc Tnk Vnt to Atmos

ZERO
ZERO
ZERO
0.1910 E-1O
ZERO
ZERO
ZERO
ZERO
ZERO
ZERO

$0.3553 \mathrm{E}+00$

$0.9414 \mathrm{E}-01$

$0.4493 \mathrm{E}-02$

$0.1624 \mathrm{E}-12$

ZERO

$0.3567 \mathrm{E}-11$

$0.8883 \mathrm{E}-13$

$0.3762 \mathrm{E}-18$

ZERO
$0.9533 \mathrm{E}-13$

ZERO

$0.3312 \mathrm{E}+00$

ZERO

ZERO

$0.1063 \mathrm{E}-12$

$0.2982 \mathrm{E}-10$

ZERO

ZERO

ZERO

ZERO

$0.1017 \mathrm{E}-10$

ZERO

ZERO

ZERO

$0.5465 \mathrm{E}-14$

$0.1391 \mathrm{E}-10$

$0.2046 \mathrm{E}-14$

ZERO

ZERO

$0.4801 \mathrm{E}-16$

$0.5859 \mathrm{E}-11$
407

Decon SN Precipitated

410

Inhibited Tash Water

411

Spent Wash to storage

452

$\mathrm{NaOH}$ add'n NaNO2 add'n

to TK 48 to $\mathrm{TK} 48$

ZERO
ZERO
ZERO
ZERO
ZERO
ZERO
ZERO
ZERO
ZERO
ZERO

$0.3344 \mathrm{E}+01$

ZERO

$0.3374 \mathrm{E}-02$

$0.8203 \mathrm{E}-01$

$0.4940 \mathrm{E}-02$

ZERO

ZERO

ZERO

$\begin{array}{rl}\text { ZERO } & 0.1670 \mathrm{E}-02 \\ 0.1295 \mathrm{E}-07 & 0.4705 \mathrm{E}-09\end{array}$

ZERO
ZERO
ZERO
$0.3451 E+00$
ZERO
ZERO
ZERO
ZERO
ZERO
ZERO

$0.1186 \mathrm{E}+00$

ZERO

$0.1140 \mathrm{E}-03$

$0.7981 \mathrm{E}-05$

$0.6006 \mathrm{E}-06$

ZERO
$0.3054 \mathrm{E}-02$

ZERO

$0.6708 \mathrm{E}-01$

ZERO

ZERO

05E-09

0.1793 E-02

$0.7780 \mathrm{E}-03$

ZERO

$0.2906 \mathrm{E}-04$

ZERO

$0.3549 \mathrm{E}-01$
$0.3660 \mathrm{E}-02$

ZERO

ZERO

$0.1329 \mathrm{E}-02$

$0.1371 \mathrm{E}-03$

$0.5607 \mathrm{E}+00$

ZERO

ZERO

ZERO

ZERO

ZERO

ZERO

ZERO

ZERO

$0.1392 \mathrm{E}-01$

ZERO

ZERO

ZERO

ZERO

ZERO
$0.6146 \pi-07$

ZERO

$0.1102 \mathrm{E}+00$

\begin{tabular}{|c|c|c|c|}
\hline ZERO & ZERO & ZERO & ZERO \\
\hline ZERO & ZERO & ZERO & ZERO \\
\hline ZERO & ZERO & ZERO & ZERO \\
\hline ZERO & ZERO & ZERO & ZERO \\
\hline ZERO & ZERO & ZERO & ZERO \\
\hline ZERO & ZERO & ZERO & ZERO \\
\hline ZERO & ZERO & ZERO & ZERO \\
\hline ZERO & ZERO & ZERO & ZERO \\
\hline ZERO & ZERO & ZERO & ZERO \\
\hline ZERO & ZERO & ZERO & ZERO \\
\hline ZERO & $0.1153 \mathrm{E}+00$ & ZERO & ZERO \\
\hline ZERO & ZERO & ZERO & ZERO \\
\hline ZERO & $0.1109 \mathrm{E}-03$ & ZERO & ZERO \\
\hline ZERO & $0.7759 \mathrm{E}-05$ & ZERO & ZERO \\
\hline ZERO & $0.5838 E-06$ & ZERO & ZERO \\
\hline ZERO & ZERO & ZERO & ZERO \\
\hline ZERO & ZERO & ZERO & ZERO \\
\hline ZERO & ZERO & ZERO & ZERO \\
\hline ZERO & ZERO & ZERO & ZERO \\
\hline ZERO & ZERO & ZERO & ZERO \\
\hline ZERO & $0.4574 \mathrm{E}-09$ & ZERO & ZERO \\
\hline ZERO & ZERO & ZERO & ZERO \\
\hline ZERO & ZERO & ZERO & ZERO \\
\hline ZERO & ZERO & ZERO & ZERO \\
\hline ZERO & $0.2826 \mathrm{E}-04$ & ZERO & ZERO \\
\hline ZERO & ZERO & ZERO & ZERO \\
\hline ZERO & ZERO & ZERO & ZERO \\
\hline ZERO & $0.1292 \mathrm{E}-02$ & ZERO & ZERO \\
\hline ZERO & $0.1332 \mathrm{E}-03$ & ZERO & ZERO \\
\hline ZERO & ZERO & ZERO & ZERO \\
\hline ZERO & ZERO & ZERO & ZERO \\
\hline ZERO & ZERO & ZERO & ZERO \\
\hline ZERO & ZERO & ZERO & ZERO \\
\hline ZERO & ZERO & ZERO & ZERO \\
\hline ZERO & ZERO & ZERO & ZERO \\
\hline ZERO & ZERO & ZERO & ZERO \\
\hline ZERO & ZERO & ZERO & ZERO \\
\hline ZERO & ZERO & ZERO & ZERO \\
\hline ZERO & ZERO & ZERO & ZERO \\
\hline ZERO & $0.5068 \mathrm{E}-03$ & ZERO & ZERO \\
\hline ZERO & ZERO & ZERO & ZERO \\
\hline ZERO & ZERO & ZERO & ZERO \\
\hline ZERO & ZERO & ZERO & ZERO \\
\hline ZERO & ZERO & ZERO & ZERO \\
\hline ZERO. & ZERO & ZERO & ZERO \\
\hline ZERO & ZERO & ZERO & ZERO \\
\hline ZERO & $0.5975 \mathrm{E}-07$ & ZERO & ZERO \\
\hline ZERO & ZERO & ZERO & ZERO \\
\hline
\end{tabular}

Date: January 1995
Section 1A, Page 10 of 21

WSRC-TR-95-0019 (Revision 
Table A-1. HLW Flowsheet Material Balance (Tank 51/ITP Cycle 1)

Section 1A. In-Tank Precipitation and Salt Decontamination

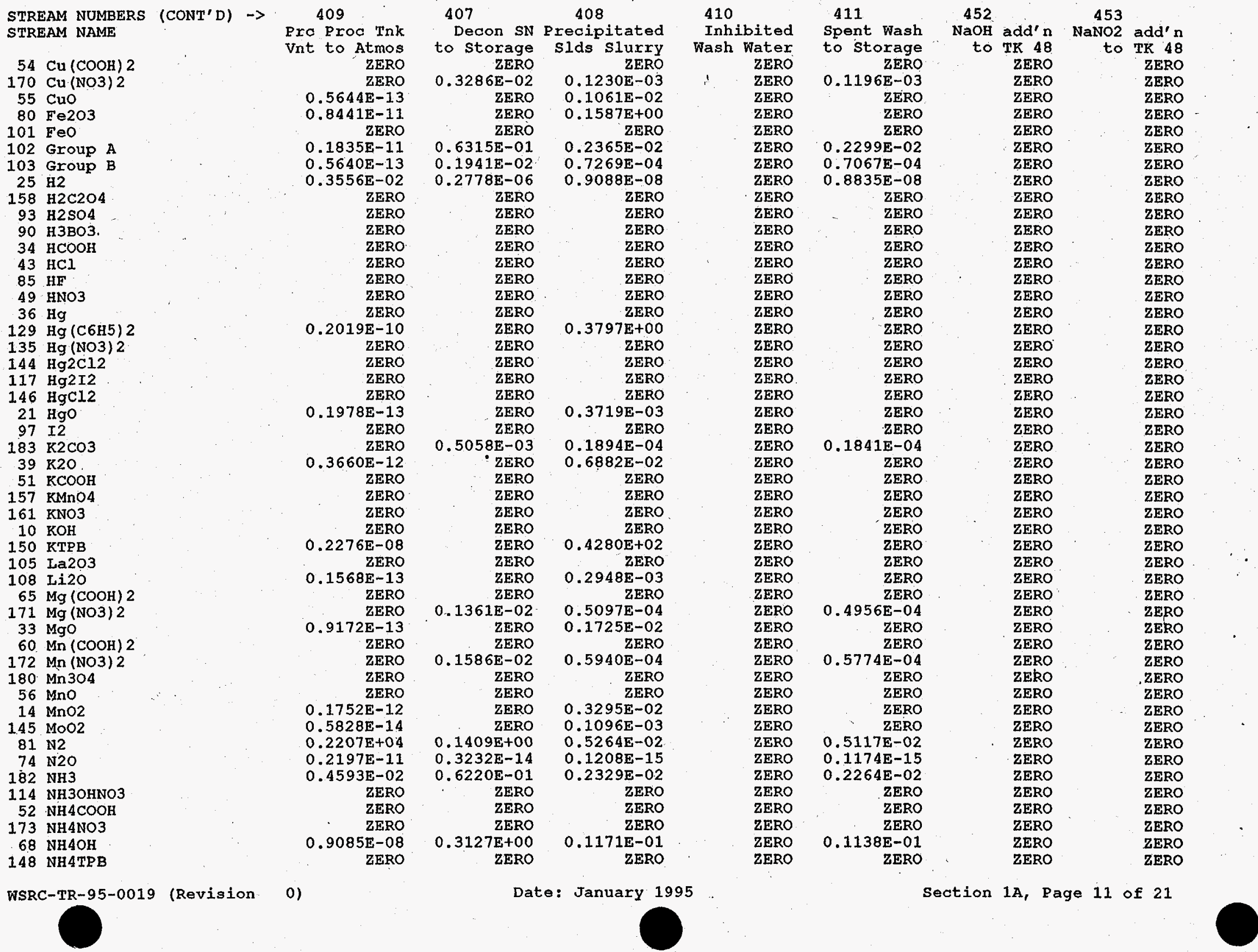


Table A-1. HLW Flowsheet Material Balance (Tank 51/ITP Cycle 1)

Section 1A. In-Tank Precipitation and Salt Decontamination

STREAM NUMBERS (CONT'D) -> STREAM NAME

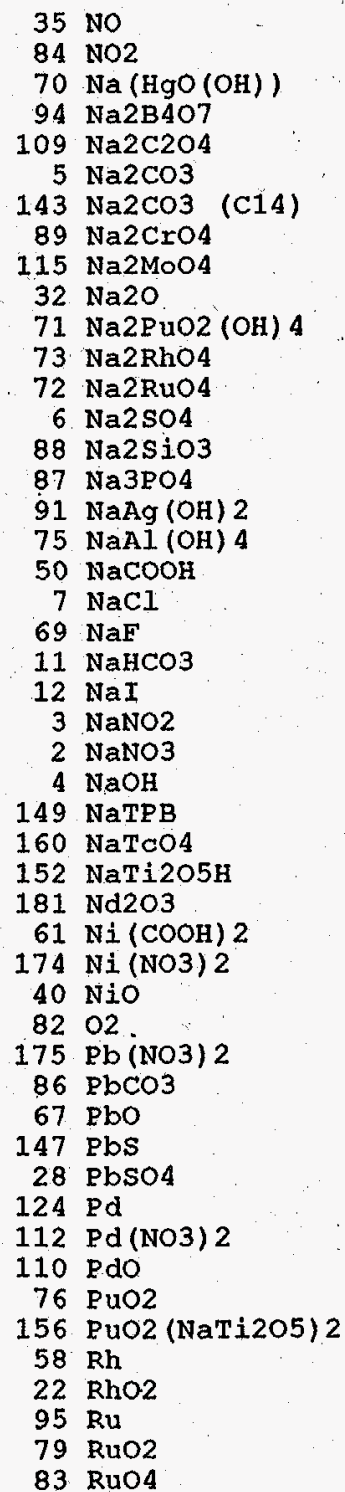

WSRC-TR-95-0019 (Revision

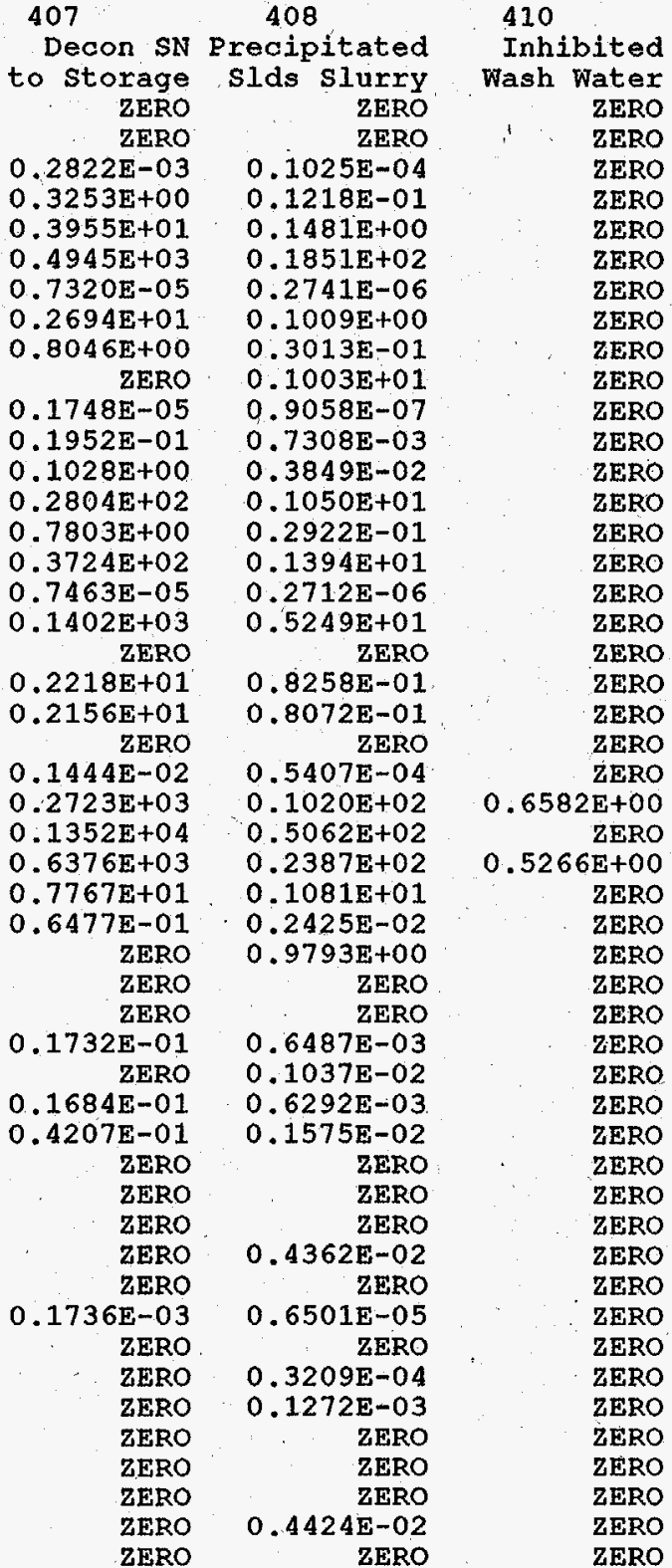

Date: January 1995
411

Spent Wash to storage

ZERo
ZERO ZERO

$0.9967 \mathrm{E}-05$

$0.1184 \mathrm{E}-01$

$0.1440 \mathrm{E}+00$

$0.1800 \mathrm{E}+02$

$0.9808 \mathrm{E}-01$

$0.2929 \mathrm{E}-01$

ZERO

$0.8806 \mathrm{E}-07$

$0.7105 \mathrm{E}-03$

$0.3742 \mathrm{E}-02$

$0.1021 \mathrm{E}+01$

$0.2841 \mathrm{E}-01$
$0.1356 \mathrm{E}+01$

$0.2636 \mathrm{E}-06$

$0.5103 \mathrm{E}+01$

$0.8028 \mathrm{E}-01$

$0.7848 \mathrm{E}-01$

$0.5257 \mathrm{E}-04$

$0.4368 \mathrm{E}+02$

$0.4921 \mathrm{E}+02$

$0.5621 \mathrm{E}+02$

$0.1051 \mathrm{E}+01$

$0.2358 \mathrm{E}-02$

ZERO
ZERO

ZERO

$0.6306 \mathrm{E}-03$ ZERO

$0.6117 \mathrm{E}-03$

$0.1531 \mathrm{E}-02$

ZERO

ZERO

ZERO

ZERO

$0.6320 \mathrm{E}-05$

ZERO

ZERO

ZERO

ZERO

ZERO

ZERO

ZERO
452

add' $n$ NaNO2 add' $n$ TKK 48 to TK 48 $\begin{array}{ll}\text { ZERO } & \text { ZERO } \\ \text { ZERO } & \text { ZERO }\end{array}$ ZERO ZERO ZERO ZERO ZERO ZERO ZERO ZERO ZERO ZERO ZERO ZERO ZERO ZERO ZERO - ZERO ZERO ZTRO ZTRO ZERO ZERO ZERO $\begin{array}{ll}\text { ZERO } & \text { ZERO } \\ \text { ZERO } & \text { ZERO }\end{array}$ ZERO ZERO ZERO ZERO ZERO ZERO ZERO ZERO ZERO ZERO ZERO $\cdots$ ZERO ZERO ZERO ZERO $0.4047 \mathrm{E}+02$ 0.3462 ZERO ZERO $\quad$ ZERO ZERO ZERO ZERO ZERO ZERO ZERO ZERO ZERO ZERO ZERO ZERO ZERO ZERO ZERO ZERO ZERO ZERO ZERO - ZERO ZERO ZERO ZERO ZERO ZERO ZERO $\begin{array}{ll}\text { ZERO } & \text { ZERO } \\ \text { ZERO } & \text { ZERO }\end{array}$ ZERO ZERO ZERO ZERO ZERO ZERO ZERO ZERO ZERO ZERO ZERO ZERO ZERO ZERO

Section 1A, Page 12 of 21 
Table A-1. HLW Flowsheet Material Balance (Tank 51/ITP Cyale 1 ) Section 1A. In-Tank Precipitation and Salt Decontamination

STREAM NUMBERS (CONT'D) $\rightarrow$ STREAM NAME

$\begin{array}{rr}38 & 502 \\ 141 & 503\end{array}$

137 Semi Vol Cs2O

136 Semi Vol CsCl

126 Semi Vol Group A

140 Semi Vol Na2B4O7

138 Semi Vol NaCl

139 Semi Vol NaF

125 Semi Vol NaI
23 Semi Vol RuO2

111 sio2

$48 \mathrm{Sr}(\mathrm{COOH}) 2$

$176 \operatorname{sr}(\mathrm{NO} 3) 2$

$153 \mathrm{Sr}$ (NaTi2O5) 2

$127 \mathrm{Sr}(\mathrm{OH}) 2$

$30 \mathrm{SrCO}$

46 sro

$159 \mathrm{TcO} 2$

92 ThO2

119 Tritiun

$\begin{array}{rl}119 & \text { Triti } \\ 17 & \mathrm{u} 308\end{array}$

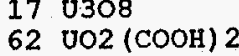

$177 \mathrm{NO2}$ (NO3) 2

$16 \mathrm{UO} 2(\mathrm{OH}) 2$

$27 \mathrm{Y}(\mathrm{COOH}) 3$

$178 \mathrm{Y}$ (NO3)

$128 Y(\mathrm{OH}) 3$

31 Y2 (CO3) 3

$47 \mathrm{Y} 203$

100 zeolite

$44 \mathrm{Zn}(\mathrm{COOH}) 2$
$179 \mathrm{Zn}(\mathrm{NO3}) 2$

$57 \mathrm{ZnO}$

104 ZrO2

13 hydrate $\mathrm{H} 2 \mathrm{O}$

1 water

TOTAL FLOW, LB/HR

TEMPERATURE， DEG C

PRESSURE， ATM

PRESSURE， PSIG

PRESSURE， MM HG

ENTHALPY, PCU/HR

VAPOR FLOW, CFM.

VAPOR FLOW, CEM

DENSITY， LBS/FT3

PHASE

409

Pro Proc Tnk

Vnt to Atmo

ZERO

ZERO

ZERO

ZERO

ZERO

ERO

ERO

ZERO

ZERO

$0.1418 \mathrm{E}-11$

ZERO

$.2167 \mathrm{E}-12$

$0.1391 \mathrm{E}-15$

$0.4829 E-14$

ZERO

ZERO

$0.6453 E-13$

$0.3193 \mathrm{E}-09$

$0.3995 \mathrm{E}-13$

ŻERO

$0.8085 \mathrm{E}-12$ ZERO

ZERO
$0.1654 \mathrm{E}-15$ ZERO

$0.5145 \mathrm{E}-13$ ZERO ZERO

$0.1053 E-10$ ZERO

$0.1417 \mathrm{E}-12$

$0.6527 \mathrm{E}+02$

$0.2409 \mathrm{E}+04$

$0.4000 \mathrm{E}+02$

$0.9656 \mathrm{E}+00$

$-0.5055 \mathrm{E}+00$

$0.7339 \mathrm{E}+03$

$0.6421 \mathrm{E}+05$

$0.6155 \mathrm{E}+03$

$0.6524 \mathrm{E}-01$

VAPOR
407

Decon SN Precipitated

to storage slds slurry

$\begin{array}{ll}\text { ZERO } & \text { ZERO } \\ \text { ZERO } & \text { ZERO } \\ \text { ZERO } & \text { ZERO } \\ \text { ZERO } & \text { ZERO }\end{array}$

ZERO

ZERO

ZERO

ZERO

ZERO

ZERO

ZERO

ZERO

ZERO

$0.4796 \mathrm{E}-05$

ZERO

ZERO

ZERO

ZERO

$0.4026 \mathrm{E}-07$

ZERO

ZERO

$0.2783 \mathrm{E}-01$

ZERO

$0.5683 \mathrm{E}-05$

ZERO

ZERO

ZERO

$0.1383 \mathrm{E}+01$

ZERO

ZERO

$0.8550 \mathrm{E}+04$

$0.1154 \mathrm{E}+05$

$0.3000 \mathrm{E}+02$

$0.1000 \mathrm{E}+01$

ZERO

$0.2806 \mathrm{E}+06$

$0.1927 \mathrm{E}+02$

$0.7463 \mathrm{E}+02$

IIQUID

ZERO

ZERO
ZERO

ZERO

$0.4072 E-02$

$0.2414 \mathrm{E}-06$

$0.9081 \mathrm{E}-04$ ZERO ZERO

$0.1213 \mathrm{E}-02$

$0.1507 \mathrm{E}-08$

$0.7512 \mathrm{E}-03$

ZERO
$0.1042 \mathrm{E}-02$

ZERO

$0.9683 E-03$

3E-03

ZERO
ZERO

$0.5177 \mathrm{E}-01$

$0.1980 \mathrm{E}+00$

$0.2665 \mathrm{E}-02$

$0.3194 \mathrm{E}+03$

$0.4792 \mathrm{E}+03$

$0.3000 E+02$

$0.1000 E+01$
ZERO

$0.1050 \mathrm{E}+05$

$0.8048 \mathrm{E}+00$

$0.7423 \mathrm{E}+02$

LIQUID
$0.1127 \mathrm{E}-06$
410

Inhibited

Wash Water

ZERO

ZERO

ZERO

ZERO

ZERO

ZERO

ZERO

ZERO

411

Spent Wash

to storage ZERO ZERO ZERO ZERO

ZERO

ZERO

ZERO

ZERO

ZERO

ZERO

ZERO

ZERO

ZERO

ZERO

ZERO

ZERO

ZERO

ZERO

ZERO

ZERO

ZERO

ZERO

ZERO

ZERO

ZERO

ZERO

ZERO

ZERO

ZERO

ZERO

ZERO

ZERO

ZERO

ZERO

ZERO

$0.1315 \mathrm{E}+04$

$0.1316 \mathrm{E}+04$

$0.2500 \mathrm{E}+02$

$0.1000 \mathrm{E}+01$

$0.3301 \mathrm{E}+05$

$0.2627 \mathrm{E}+01$.

$0.6247 \mathrm{E}+02$

LIQUID

ZERO

$0.1495 \mathrm{E}+04$

$0.1000 \mathrm{E}+01$

ZERO

$0.3588 \mathrm{E}+05$

$0.2757 \mathrm{E}+01$

$0.6759 \mathrm{E}+02$

IIQUID

452

aOH add'n 453

to TK 48

$\begin{array}{ll}\text { ZERO } & \text { ZERO } \\ \text { ZERO } & \text { ZERO }\end{array}$

ZERO

ZERO ZERO

ZERO ZERO

ZERO ZERO

ZERO

ZERO

ZERO

ZERO

ZERO

ZERO

ZERO

ZERO

ZERO

ZERO

ZERO

ZERO

ZERO

ZERO

ZERO

ZERO

ZERO

ZERO

ZERO

ZERO

ZERO

ZERO

ZERO

ZERO

ZERO

ZERO

ZERO

0.3462

$0.6924 \mathrm{E}+02$

ZERO

$.2500 \mathrm{E}+02$

$0.1000 \mathrm{E}+01$

$0.2500 \mathrm{E}+02$

$0.1000 \mathrm{E}+01$

$0.1150 \mathrm{E}+0 \mathrm{~A}$

$0.1757 \mathrm{E}+04$

$0.9055 \mathrm{E}-01$

$0.1481 \mathrm{E}+00$

$0.9533 \mathrm{E}+02$

LIQUID

$8519 \mathrm{E}+02$

LIQUID 
Table A-1. HiLW Flowsheet Material Balance (Tank 51/ITP Cycle 1) Section 1A. In-Tank Precipitation and Salt Decontamination

STREAM NUMBERS -> STREAM NAME

COMPONENT FLOWS, LB/HR 122 (C6H5) 2

123 (C6H5) 2C6H4

133 (C6H5B(OH) 2 salt)

132 ( $\mathrm{C} 6 \mathrm{H} 5 \mathrm{~B}(\mathrm{OH}) 2$ )

166 (C6H5C6H4OH)

162 (C6H5NH2)

163 (C6H5NHCHO)

165 (C6H5NO)

131 (C6H5OH salt)

130 ( $\mathrm{C} 6 \mathrm{H} 5 \mathrm{OH})$

$\begin{array}{ll}121 & \text { (C6H6) } \\ 155 & \text { (CH3) 2CHOH }\end{array}$

154 ( $\mathrm{CH} 3 \mathrm{OH}$ )

$45 \mathrm{Ag}$

116 Ag20

134 AgNO3

$106 \mathrm{~B} 203$

$9 \mathrm{Ba}(\mathrm{OH}) 2$

$15 \mathrm{BaO}$

$24 \mathrm{BaSO} 4$

$66 \mathrm{CO}$

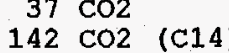

$63 \mathrm{Ca}(\mathrm{COOH}) 2$

$167 \mathrm{Ca}(\mathrm{NO} 3) 2$

$120 \mathrm{Ca}(\mathrm{OH}) 2$

$113 \mathrm{Ca} 3$ (PO4) 2

$20 \mathrm{CaC} 2 \mathrm{O} 4$

$64 \mathrm{CaCO} 3(\mathrm{C} 14)$

78 $\mathrm{CaF}^{2}$

$41 \mathrm{CaO}$

$19 \mathrm{CasO} 4$

29 Carbon

99 Cement

$26 \mathrm{Co}(\mathrm{COOH}) 2$

$168 \mathrm{Co}$ (NO3) 2

$59 \mathrm{COO}$

$98 \mathrm{Cr} 203$

$53 \mathrm{CsCOOH}$

$96 \mathrm{CsCl}$

169 CsNO3

$8 \mathrm{CsOH}$

151 CsTPB

WSRC-TR-95-0019 (Revision $\begin{array}{cc}414 & 454 \\ \text { Washed Prec } & 40 \%\end{array}$

to 4 4orag $\mathrm{NaNO} 2$

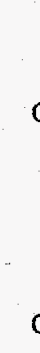

417

420

672

673

In-tnk Pptn Vnt to Atmo Wash Recycle Wash Soluble Wash Insolbl

\begin{tabular}{|c|c|c|c|c|c|}
\hline ZERO & ZERO & $0: 2059 \mathrm{E}-06$ & ZERO & ZERO & ZERO \\
\hline ZERO & ZERO & $0.8746 \mathrm{E}-08$ & ZERO & ZERO & ZERO \\
\hline ZERO & ZERO & $0.5823 E-08$ & ZERO & ZERO & ZERO \\
\hline ZERO & ZERO & $0.2319 \mathrm{E}-09$ & ZERO & ZERO & ZERO \\
\hline ZERO & ZERO & ZERO & ZERO & ZERO & ZERO \\
\hline ZERO & ZERO & ZERO & ZERO & ZERO & ZERO \\
\hline ZERO & ZERO & ZERO & ZERO & ZERO & ZERO \\
\hline ZERO & ZERO & ZERO & ZERO & ZERO & ZERO \\
\hline ZERO & ZERO & ZERO & ZERO & ZERO & ZERO \\
\hline ZERO & ZERO & ZERO & ZERO & ZERO & ZERO \\
\hline ZERO & ZERO & $0.1086 \mathrm{E}-09$ & $0.3998 \mathrm{E}+00$ & $0.3998 \mathrm{E}+00$ & ZERO \\
\hline ZERO & ZERO & ZERO & ZERO & ZERO & ZERO \\
\hline ZERO & ZERO & $0.5111 \mathrm{E}+00$ & $0.7436 \mathrm{E}-02$ & $0.7436 \mathrm{E}-02$ & ZERO \\
\hline ZERO & ZERO & $0.2136 \mathrm{E}-06$ & $0.1232 \mathrm{E}-08$ & $0.1232 \mathrm{E}-08$ & ZERO \\
\hline ZERO & ZERO & $0.1592 \mathrm{E}-07$ & $0.1448 \mathrm{E}-09$ & $0.1448 \mathrm{E}-09$ & ZERO \\
\hline ZERO & ZERO & ZERO & ZERO & ZERO & ZERO \\
\hline ZERO & ZERO & $0.1909 \mathrm{E}-11$ & ZERO & ZERO & ZERO \\
\hline ZERO & ZERO & ZERO & ZERO & ZERO & ZERO \\
\hline ZERO & ZERO & $0.8112 \mathrm{E}-10$ & ZERO & ZERO & ZERO \\
\hline ZERO & ZERO & $0.1044 \mathrm{E}-11$ & ZERO & ZERO & ZERO \\
\hline ZERO & ZERO & $0.8186 \mathrm{E}-20$ & $0.1252 \mathrm{E}-14$ & $0.1252 \mathrm{E}-14$ & ZERO \\
\hline ZERO & ZERO & ZERO & ZERO & ZERO & ZERO \\
\hline ZERO & ZERO & $0.1121 \mathrm{E}-11$ & ZERO & ZERO & ZERO \\
\hline ZERO & ZERO & ZERO & ZERO & ZERO & ZERO \\
\hline ZERO & $0.3320 \mathrm{E}+00$ & ZERO & ZERO & ZERO & ZERO \\
\hline ZERO & ZERO & ZERO & ZERO & ZERO & ZERO \\
\hline ZERO & ZERO & ZERO & ZERO & ZERO & ZERO \\
\hline ZERO & ZERO & ZERO & $0.3561 \mathrm{E}-04$ & $0.3561 \mathrm{E}-04$ & $0.2332 \mathrm{E}-11$ \\
\hline ZERO & ZERO & $0.2384 \mathrm{E}-14$ & $0.3672 E-05$ & $0.3672 \mathrm{E}-05$ & ZERO \\
\hline ZERO & ZERO & $0.3506 \mathrm{E}-09$ & ZERO & ZERO & ZERO \\
\hline ZERO & ZERO & ZERO & ZERO & ZERO & ZERO \\
\hline ZERO & ZERO & ZERO & ZERO & ZERO & ZERO \\
\hline ZERO & ZERO & ZERO & ZERO & ZERO & ZERO \\
\hline ZERO & ZERO & ZERO & ZERO & ZERO & ZERO \\
\hline ZERO & ZERO & ZERO & ZERO & ZERO & ZERO \\
\hline ZERO & ZERO & $0.1195 \mathrm{E}-09$ & ZERO & ZERO & ZERO \\
\hline ZERO & ZERO & ZERO & ZERO & ZERO & ZERO \\
\hline ZERO & ZERO & ZERO & ZERO & ZERO & ZERO \\
\hline ZERO & ZERO & ZERO & ZERO & ZERO & ZERO \\
\hline ZERO & ZERO & ZERO & $0.1397 \mathrm{E}-04$ & $0.1397 \mathrm{E}-04$ & ZERO \\
\hline ZERO & ZERO & $0.6426 \mathrm{E}-13$ & ZERO & ZERO & ZERO \\
\hline ZERO & ZERO & $0.1636 \mathrm{E}-09$ & ZERO & ZERO & ZERO \\
\hline ZERO & ZERO & $0.2406 \mathrm{E}-13$ & ZERO & ZERO & ZERO \\
\hline ZERO & ZERO & ZERO & ZERO & ZERO & ZERO \\
\hline ZERO & ZERO . & ZERO & ZERO & ZERO & ZERO \\
\hline ZERO & ZERO & ZERO & ZERO & ZERO & ZERO \\
\hline ZERO & ZERO & $0.1088 \mathrm{E}-11$ & $0.4663 \mathrm{E}-04$ & $0.4663 \mathrm{E}-04$ & ZERO \\
\hline ZERO & ZERO & $0.6561 \mathrm{E}-10$ & ZERO & ZERO & ZERO \\
\hline
\end{tabular}

Date: January 1995 
Table A-1. HLW Flowsheet Material Balance (Tank 51/ITP Cycle 1 )

Section 1A. In-Tank Precipitation and Salt Decontamination

STREAM NUMBERS (CONT'D) $\rightarrow$ STREAM NAME

$54 \mathrm{Cu}(\mathrm{COOH}) 2$

$170 \mathrm{Cu}(\mathrm{NO} 3) 2$

$55 \mathrm{CuO}$

$80 \mathrm{Fe} 203$

101 FeO

102 Group A

103 Group B

$25 \mathrm{H} 2$

$\begin{array}{rl}158 & \mathrm{H} 2 \mathrm{C} 2 \mathrm{O} \\ 93 & \mathrm{H} 2 \mathrm{SO} 4\end{array}$

\begin{tabular}{ll}
93 & $\mathrm{H} 2 \mathrm{SO} 4$ \\
90 & $\mathrm{H} 3 \mathrm{BO}$ \\
\hline
\end{tabular}

$34 \mathrm{HCOOH}$

$43 \mathrm{HCl}$

$85 \mathrm{HF}$

49 HNO3

$36 \mathrm{Hg}$

$129 \mathrm{Hg}(\mathrm{C} 6 \mathrm{H} 5)^{2}$

$135 \mathrm{Hg}$ (NO3) 2

$144 \mathrm{Hg} 2 \mathrm{Cl}$

$117 \mathrm{Hg} 2 \mathrm{I} 2$

$146 \mathrm{HgCl2}$

$21 \mathrm{Hg}$

97 I2

$183 \mathrm{~K} 2 \mathrm{CO} 3$

$39 \mathrm{~K} 2 \mathrm{O}$

$51 \mathrm{KCOOH}$

$161 \mathrm{KNO}$

$10 \mathrm{KOH}$

$10 \mathrm{KOH}$

$105 \mathrm{La2O3}$

108 Li2O

$65 \mathrm{Mg}$ (COOH) 2

$171 \mathrm{Mg}(\mathrm{NO} 3) 2$

$33 \mathrm{MgO}$

$60 \mathrm{Mn}(\mathrm{COOH}) 2$

$172 \mathrm{Mn}$ (NO3) 2

$180 \mathrm{Mn} 304$

$56 \mathrm{MnO}$

$14 \mathrm{MnO} 2$

$145 \mathrm{MOO} 2$

81 N2

$74 \mathrm{~N} 2 \mathrm{O}$

182 NH3

114 NH3OHNO3

52 NH4 COOH
173 NH 4 NO 3

$\begin{array}{rl}173 & \mathrm{NH} 4 \mathrm{NO} 3 \\ 68 & \mathrm{NH} 4 \mathrm{OH}\end{array}$

148 NH4TPB

WSRC-TR-95-0019 (Revision
414

Tashed Pred

454

416

417

420

672

(a) Lecip strg spent Late spent Late spent Late

Tht to Atmo wash Recycle Wash Soluble Wash Insolbl

ZERO ZERO , ZERO

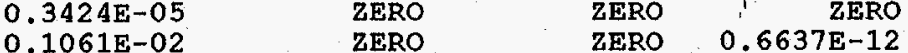

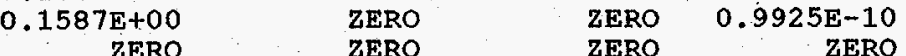

ZERO

$0.6580 \mathrm{E}-04$

$0.2023 \mathrm{E}-05$

$0.2529 \mathrm{E}-09$

ZERO

ZERO

ZERO

ZERO

ZTRO

ZERO

$0.3797 \mathrm{E}+00$

ZERO

ZERO

ZERO

$0.3719 \mathrm{E}-03$

ZERO

$0.5270 \mathrm{E}-06$

$0.6882 \mathrm{E}-02$

ZERO

ZERO

ZERO

$0.4280 \mathrm{E}+02$

ZERO

$0.2948 \mathrm{E}-03$

ZERO

$0.1418 \mathrm{E}-05$

$0.1725 \mathrm{E}-02$

$0.1653 \mathrm{E}-05$

ZERO

$0.3295 \mathrm{E}-02$

$.1096 \mathrm{E}-03$

$0.1465 \mathrm{E}-03$

$0.3360 \mathrm{E}-17$

$0.6480 \mathrm{E}-04$

ZERO

ZRRO

$0.3258 \mathrm{E}-03$ ZERO

o)

ZERO : :

ZERO

ZERO

ZERO

ZERO

ZTRO

TERO

ZERO

ZERO

ZERO

ZERO

ZERO

ZERO

ZERO

ZERO

ZFRO

TERO

ZERO

ZERO

ZERO

ZERO

ZERO

ZERO

ZERO

ZERO

ZERO

ZERO

ZERO

ZERO

ZERO

ZERO

ZERO

ZERO

ZERO

ZERO

ZERO

ZERO

ZERO

ZERO

ZERO

ZERO

ZERO

ERO

ZERO

ZERO

$0.4114 \mathrm{E}-13$

$0.1265 \mathrm{E}-14$

$0.1045 \mathrm{E}-01$

ZERO

ZERO

ZERO

ZERO

ZERO

ZERO

ZERO

$0.2374 \mathrm{E}-09$

ZERO

ZERO

$0.2326 \mathrm{E}-12$

ZERO

ZERO

$0.4304 \mathrm{E}-11$

ZERO

ZERO

0.2328 ZERO

$0.2551 \mathrm{E}-07$

ZERO

$0.1843 \mathrm{E}-12$

ZERO

$0.1079 \mathrm{E}-11$

ZERO

ERO

ZERO

0.32 ZERO

0.3297 ZERO

ZERO

ZERO

ZERO

ZERO

$0.6336 \mathrm{E}-04$

$.1948 \mathrm{E}-05$

$.5781 \mathrm{E}-10$

ZERO

ZERO

ZERO

ZERO

ZERO

ZERO

ZERO

ZERO

ZERO

ZERO

ZERO

ZERO

$0.5063 \mathrm{E}-03$

ZERO

ZERO

ZERO

$0.2796 \mathrm{E}-05$

ZERO

ZERO

ZERO

$0.1366 \mathrm{E}-05$

'ZERO

0.1591 ZRO

$1 E-05$
ZERO

ZERO

ZERO

$0.2061 \mathrm{E}-1$

$0.6853 \mathrm{E}-13$

ZERO $0.7061 \mathrm{E}-01$

$0.2598 \mathrm{E}-01$

ZERO

ZERO

ZERO

ZERO

ZERO

$0.1701 \mathrm{E}-09$

ZERO

$0.2990 E-01$

$0.8164 \mathrm{E}-09$

$0.2005 \mathrm{E}-04$

ZERO

ZERO

ZERO

Date: January 1995

Section $1 A$, Page 15 of 21

ZERO ZERO ZERO ZERO ZERO ZERO $0.6336 \mathrm{E}-04$

ZERO $0.1948 \mathrm{E}-05$

zERO

ZERO

ZERO

ZERO

ZERO

ZERO

ZERO

ZERO

ZERO.

ZERO

ZERO

ZTRO

ZERO

ZERO

ZERO

ZERO

ZERO

$0.2796 \mathrm{E}-05$

ZERO

ZERO

ZERO

$0.1366 \mathrm{E}-05$

ZERO

ZERO

ZERO

ZERO

ZERO

$0.29 .90 \mathrm{E}-01$

$.8164 \mathrm{E}-09$

ZERO

ZERO

ZTRO

ZERO

ZERO

ZERO

ZERO

ZERO

ZERO

ZERO

ZERO

ZERO

ZERO

ZERO

ZERO

ZERO

ZERO

ZERO

ZERO 
Table A-1. HLW Flowsheet Material Balance (Tank 51/ITP Cycle 1)

Section 1A. In-Tank Precipitation and Salt Decontamination.

STREAM NUMBERS (CONT'D) $\rightarrow$ STREAM NAME

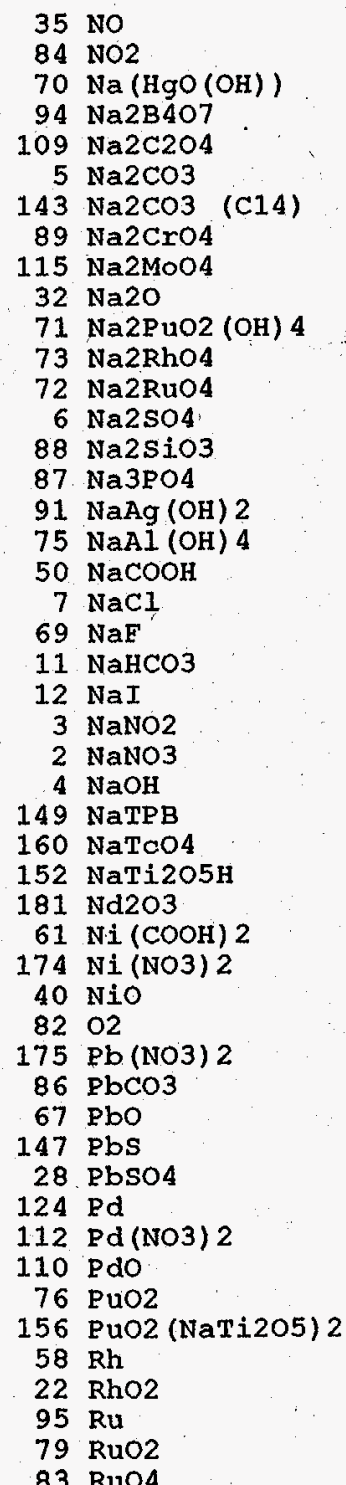

414

454

408 NaNO2

add to TK49 Gas Purge ZERO ZERO

$0.2853 \mathrm{E}-06$

$0.3390 \mathrm{E}-03$

$0.4121 \mathrm{E}-02$

$0.5152 \mathrm{E}+00$

$0.7626 \mathrm{E}-08$

$0.2807 \mathrm{E}-02$

$0.1003 \mathrm{E}+01$

$0.2520 \mathrm{E}-08$

$0.2033 \mathrm{E}-04$

$0.1071 \mathrm{E}-03$

$0.2921 \mathrm{E}-01$

$0.8130 \mathrm{E}-03$
$0.3880 \mathrm{E}-01$

$0.3880 E-01$
$0.7545 E-08$

$0.1460 \mathrm{E}+00$

ZERO

$0.2298 \mathrm{E}-02$

$0.2246 \mathrm{E}-02$

ZERO
$0.1504 \mathrm{E}-05$

$0.7647 \mathrm{E}+01$

$0.1408 \mathrm{E}+01$

$0.2809 \mathrm{E}+01$

$0.3008 \mathrm{E}-01$

$0.6748 \mathrm{E}-04$

$0.9793 E+00$

ZERO
ZERO

$0.1805 \mathrm{E}-0$

$0.1037 \mathrm{E}-02$

$0.1751 \mathrm{E}-04$

$0.4383 \mathrm{E}-04$

ZERO

ZERO

$0.4362 \mathrm{E}-02$

ZERO

$0.1809 \mathrm{E}-06$

ZERO
$9 \mathrm{E}-04$

$0.3209 E-04$
$0.1272 E-03$

ZERO

RO

$0.4424 \mathrm{E}-02$

ZERO
417

420

672

673

recip strg Spent Late spent Late spent Late In-tnk Pptn Vnt to Atmo Wash Recycle Wash Soluble Wash Insolbl

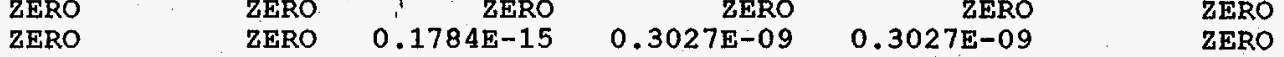

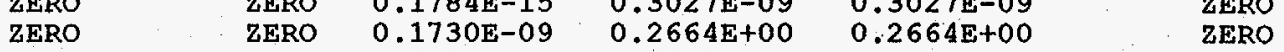
$\begin{array}{llllll}\text { ZERO ZERO } & 0.1318 \mathrm{E}-09 & 0.1441 \mathrm{E}+01 & 0.1441 \mathrm{E}+01 & \text { ZERO }\end{array}$ ZERO ZERO $0.2646 \mathrm{E}-08 \quad 0.4359 \mathrm{E}+01 \quad 0.4359 \mathrm{E}+01 \quad$ ZERO $\begin{array}{llllll}\text { ZERO } & \text { ZERO } & 0.4768 \mathrm{E}-17 & 0.7343 \mathrm{E}-08 & 0.7343 \mathrm{E}-08 & \text { ZERO }\end{array}$ $\begin{array}{llllll}\text { ZERO } & \text { ZERO } & 0.1755 \mathrm{E}-11 & 0.2703 \mathrm{E}-02 & 0.2703 \mathrm{E}-02 & \text { ZERO }\end{array}$

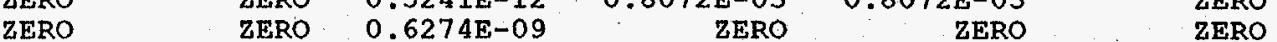
$\begin{array}{llllll}\text { ZERO } & \text { ZERO } & 0.1576 \mathrm{E}-17 & 0.3374 \mathrm{E}-10 & 0.3374 \mathrm{E}-10 & 0.1958\end{array}$ ZERO ZERO $0.1272 \mathrm{E}-13 \quad 0.1958 \mathrm{E}-04 \quad 0.1958 \mathrm{E}-04$ ZERO ZERO $0.6696 \mathrm{E}-13 \quad 0.1031 \mathrm{E}-03$ 0.1031E-03 0

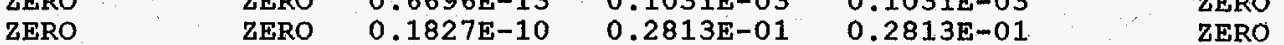
$\begin{array}{llllll}\text { ZERO } & \text { ZERO } & 0.5083 \mathrm{E}-12 & 0.7829 \mathrm{E}-03 & 0.7829 \mathrm{E}-03 & \text { ZERO }\end{array}$

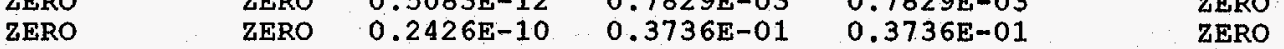

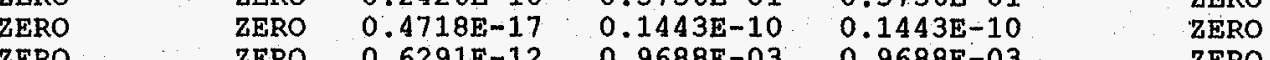
$\begin{array}{llllll}\text { ZERO } & \text { ZERO } & 0.6291 \mathrm{E}-12 & 0.9688 \mathrm{E}-03 & 0.9688 \mathrm{E}-03 & \text { ZERO }\end{array}$ $\begin{array}{rrrrrr}\text { ZERO } & \text { ZERO } & \text { ZERO } & \text { ZERO } & \text { ZERO } & \text { ZERO } \\ \text { ZERO } & \text { ZERO } & 0.1437 \mathrm{E}-11 & 0.2085 \mathrm{E}-01 & 0.2085 \mathrm{E}-01 & \text { ZERO }\end{array}$ $\begin{array}{llllll}\text { ZERO ZERO } & 0.1404 \mathrm{E}-11 & 0.2163 \mathrm{E}-02 & 0.2163 \mathrm{E}-02 & \text { ZERO }\end{array}$

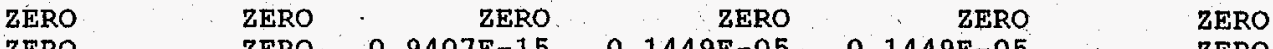
ZERO ZERO ZERO

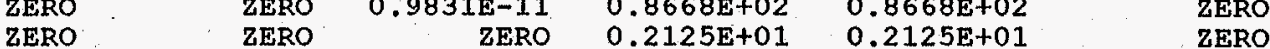

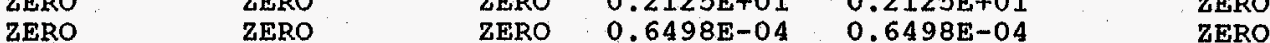
$\begin{array}{lrrrrr}\text { ZERO } & \text { ZERO } & \text { ZERO } & 0.6123 E-09 & \text { ZERO } & \text { ZERO } \\ \text { ZERO } & \text { ZERO } & & \text { ZERO } & \text { ZERO }\end{array}$

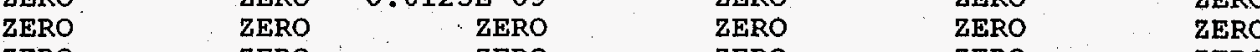
$\begin{array}{llll}\text { ZERO ZERO } & \text { ZERO } & \text { ZERO } & \text { ZERO } \\ \text { ZERO } & \text { ZERO }\end{array}$ $\begin{array}{llllll}\text { ZERO } & \text { ZERO } & \text { ZERO } & 0.1738 \mathrm{E}-04 & 0.1738 \mathrm{E}-04 & 0.1298 \mathrm{E}-11\end{array}$

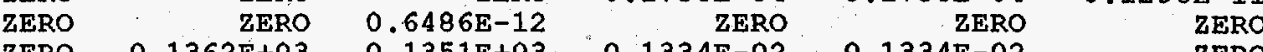
$\begin{array}{llllll}\text { ZERO } & 0.1362 \mathrm{E}+03 & 0.1351 \mathrm{E}+03 & 0.1334 \mathrm{E}-02 & 0.1334 \mathrm{E}-02 & \text { ZERO }\end{array}$

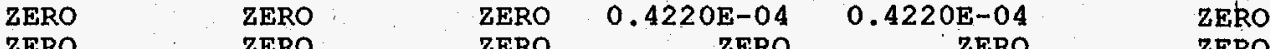
$\begin{array}{llllll}\text { ZERO } & \text { ZERO } & \text { ZERO } & \text { ZERO } & \text { ZERO } & \text { ZERO } \\ \text { ZERO } & \text { ZERO } & \text { ZERO } & \text { ZERO } & \text { ZERO } & \text { ZERO }\end{array}$ ZERO ZERO Z ZERO ZERO Z Z ZERO ZERO ZERO $0.2728 E-11 \quad$ ZERO ZERO ZERO ZERO ZERO ZERO ZERO ZERO $0.1131 \mathrm{E}-15$ ZERO ZTRO $\begin{array}{lrrrrr}\text { ZERO } & \text { ZERO } & \text { ZERO } & \text { ZERO } & \text { ZERO } & \text { ZERO } \\ \text { ZERO } & \text { ZERO } & 0.2007 E-1.3 & \text { ZERO } & \text { ZERO } & \text { ZERO }\end{array}$

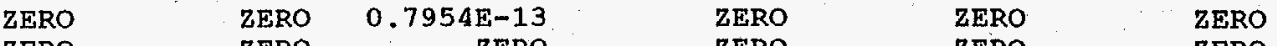

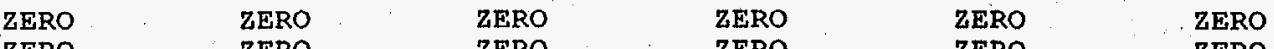

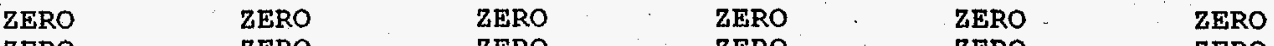
$\begin{array}{lllll}\text { ZERO ZERO } & \text { ZERO } & \text { ZERO } & \text { ZERO } & \text { ZERO } \\ \text { ZERO }\end{array}$ ZERO ZERO $0.2766 \mathrm{E}-11$

\begin{tabular}{lll} 
ZERO & ZERO & ZERO \\
ZERO & ZERO \\
\hline & ZERO & ZERO
\end{tabular}

Section 1A, Page 16 of 21 
Table A-1. HLW Flowsheet Material Balance (Tank 51/ITP Cycle 1) Section 1A. In-Tank Precipitation and Salt Decontamination

STREAM NUMBERS (CONT'D) $\rightarrow$ STREAM NAME

$\begin{array}{rr}38 & 502 \\ 141 & 503\end{array}$

137 Semi Vol Cs2O

136 Semi Vol CsC1

126 Semi Vol Group A

140 Semi Vol Na2B407

138 Semi Vol NaCl

139 Semi Vol NaF

125 Semi Vol NaI

23 Semi Vol RuO2

$111 \mathrm{SiO} 2$

$48 \mathrm{Sr}(\mathrm{COOH}+2$

$176 \mathrm{Sr}(\mathrm{NO} 3) 2$

$153 \mathrm{Sr}(\mathrm{NaTi205)} 2$

$127 \mathrm{Sx}(\mathrm{OH}) 2$

$30 \quad \operatorname{srCO} 3$

46 sro

159. $\mathrm{TCO} 2$

92 ThO2

119 Tritium

17 U308

$62 \mathrm{UO} 2$ ( $\mathrm{COOH}) 2$

$177 \mathrm{UO2}$ (NO3) 2

$16 \mathrm{UO2}(\mathrm{OH}) 2$

$178 \mathrm{Y}$ (NO3) 3

$128 Y(\mathrm{OH}) 3$

31 Y2 $(\mathrm{CO} 3) 3$

47 Y 203

100 Zeolite

$44 \mathrm{Zn}$ (COOH) 2

$179 \mathrm{zn}(\mathrm{NO} 3) 2$

$57 \mathrm{zno}$

104 2rO2

13 hydrate $\mathrm{H} 2 \mathrm{O}$

1 water

TOTAL FLOW, LB/HR

TEMPERATURE， DEG C

PRESSURE， ATM

PRESSURE， PSIG

PRESSURE, MM HG

ENTHALPY, PCU/HR

VAPOR FLOW, CFM

TAPOR FI

DENSITY, IBS/FT3

PHASE

WSRC-TR-95-0019 (Revision
414

Washed Prec

to storage

454

416

417

420

672

673

\begin{tabular}{|c|c|c|c|c|c|c|}
\hline $\begin{array}{r}\text { Storage } \\
\text { ZERO }\end{array}$ & $\begin{array}{l}\text { add to TK49 } \\
\text { ZERO }\end{array}$ & $\begin{array}{r}\text { Ln-tnK } \\
\text { ZERRO }\end{array}$ & $\begin{array}{r}\text { Znt to Atmo } \\
\text { ZERO }\end{array}$ & ZERO & $\begin{array}{r}\text { ZERO } \\
\text { ZESIS }\end{array}$ & $\begin{array}{r}\text { ZERO } \\
\text { ZERII }\end{array}$ \\
\hline ZERO & ZERO & ZERO & $\therefore \quad$ ZERO & ZERO & ZERO & ZERO \\
\hline ZERO & ZERO & ZERO & ZERO & ZERO & ZERO & ZERO \\
\hline ZERO & ZERO & ZERO & ZERO & ZERO & ZERO & ZERO \\
\hline ZERO & ZERO & ZERO & ZERO & ZERO & ZERO & ZERO \\
\hline ZERO & ZERO & ZERO & ZERO & ZERO & ZERO & ZERO \\
\hline ZERO & ZERO & ZERO & ZERO & ZERO & ZERO & ZERO \\
\hline ZERO & ZERO & ZERO & ZERO & ZERO & ZERO & ZERO \\
\hline ZERO & ZERO & ZERO & ZERO & ZERO & ZERO & ZERO \\
\hline ZERO & ZERO & ZERO & ZERO & ZERO & ZERO & ZERO \\
\hline $666 \mathrm{E}-01$ & ZERO & ZERO & $0.1667 \mathrm{E}-10$ & ZERO & ZERO & ZERO \\
\hline ZERO & ZERO & ZERO & ZERO & ZERO & ZERO & ZERO \\
\hline ZERO & ZERO & ZERO & ZERO & ZERO & ZERO & ZERO \\
\hline $4072 \mathrm{E}-02$ & ZERO & ZERO & $0.2546 \mathrm{E}-11$ & ZERO & ZERO & ZERO \\
\hline $6716 \mathrm{E}-08$ & ZERO & ZERO & $0.4200 \mathrm{E}-17$ & $0.1926 \mathrm{E}-10$ & $0.1926 \mathrm{E}-10$ & ZERO \\
\hline $1 \mathrm{E}-04$ & ZERO & ZERO & $0.5679 \mathrm{E}-13$ & ZERO & ZERO & ZERO \\
\hline ZERO & ZERO & ZERO & ZERO & ZERO & ZERO & ZERO \\
\hline ZERO & ZERO & ZERO & ZERO & ZERO & ZERO & ZERO \\
\hline ZERO & ZERO & ZERO & ZERO & ZERO & ZERO & ZERO \\
\hline $1213 \mathrm{E}-02$ & ZERO & ZERO & $0.7587 \mathrm{E}-12$ & ZERO & ZERO & ZERO \\
\hline $1194 \mathrm{E}-10$ & ZERO & ZERO & $0.6692 \mathrm{E}-11$ & $0.3304 \mathrm{E}-10$ & $0.3304 E-10$ & ZERO \\
\hline $12 \mathrm{E}-03$ & ZERO & ZERO & $0.4697 \mathrm{E}-12$ & ZERO & ZERO & ZERO \\
\hline ZERO & ZERO & ZERO & ZERO & ZERO & ZERO & ZERO \\
\hline ZERO & ZERO & ZERO & ZERO & ZERO & ZERO & ZERO \\
\hline $900 \mathrm{E}-04$ & ZERO & ZERO & $0.1813 \mathrm{E}-13$ & $0.2792 \mathrm{E}-04$ & $0.2792 \mathrm{E}-04$ & ZERO \\
\hline ZERO & ZERO & ZERO & ZERO & ZERO & ZERO & ZERO \\
\hline ZERO & ZERO & ZERO & ZERO & ZERO & ZERO & ZERO \\
\hline $3135 \mathrm{E}-08$ & ZERO & ZERO & $0.1960 \mathrm{E}-17$ & $0.2140 \mathrm{E}-10$ & $0.2140 \mathrm{E}-10$ & ZERO \\
\hline ZERO & ZERO & ZERO & ZERO & ZERO & ZERO & ZERO \\
\hline $683 \mathrm{E}-03$ & ZERO & ZERO & $0.6054 \mathrm{E}-12$ & ZERO & ZERO & ZERO \\
\hline ZERO & ZERO & ZERO & ZERO & ZERO & ZERO & ZERO \\
\hline ZERO & ZERO & ZERO & ZERO & ZERO & ZERO & ZERO \\
\hline $1440 \mathrm{E}-02$ & ZERO & ZERO & ZERO & $0.1387 \mathrm{E}-02$ & $0.1387 E-02$ & ZERO \\
\hline $1980 E+00$ & ZERO & ZERO & $0.1238 \mathrm{E}-09$ & ZERO & ZERO & ZERO \\
\hline ZERO & ZERO & ZERO & ZERO & ZERO & ZERO & ZERO \\
\hline $665 \mathrm{E}-02$ & ZERO & ZERO & $0.2244 \mathrm{E}-10$ & ZERO & ZERO & ZERO \\
\hline $114 \mathrm{E}+03$ & ZERO & $60 \mathrm{E}+01$ & $0.1631 \mathrm{E}+02$ & $0.1753 E+04$ & $0.1753 \mathrm{E}+04$ & ZERO \\
\hline $712 E+03$ & ZERO & $0.2354 \mathrm{E}+04$ & $0.2359 \mathrm{E}+04$ & $0.1856 \mathrm{E}+04$ & $0.1856 \mathrm{E}+04$ & $0.6534 \mathrm{E}-04$ \\
\hline $3000 E+02$ & $0.2500 \mathrm{E}+02$ & $0.3500 E+02$ & $0.4000 E+02$ & $0.3989 E+02$ & $0.3989 \mathrm{E}+02$ & $0.3989 \mathrm{E}+02$ \\
\hline $1000 \mathrm{E}+01$ & $0.1000 \mathrm{E}+01$ & $0.1000 E+01$ & $0.9656 \mathrm{E}+00$ & $0.9656 \mathrm{E}+00$ & $0.9656 \mathrm{E}+00$ & $0.9656 \mathrm{E}+00$ \\
\hline ZERO & ZERO & ZERO & $\begin{array}{r}-0.5055 \mathrm{E}+00 \\
0.7339 \mathrm{E}+03\end{array}$ & $\begin{array}{r}-0.5055 \mathrm{E}+00 \\
0.7339 \mathrm{E}+03\end{array}$ & $\begin{array}{r}-0.50 .55 \mathrm{E}+00 \\
0.7339 \mathrm{E}+03\end{array}$ & $\begin{array}{r}-0.5055 \mathrm{E}+00 \\
0.7339 \mathrm{E}+03\end{array}$ \\
\hline $249 E+05$ & ZERO & $\begin{array}{l}0.2744 \mathrm{E}+05 \\
0.5641 \mathrm{E}+03\end{array}$ & $\begin{array}{l}0.3425 \mathrm{E}+05 \\
0.5960 \mathrm{E}+03\end{array}$ & $0.7132 \mathrm{E}+05$ & $0.7132 \mathrm{E}+05$ & $0.1587 \mathrm{E}-03$ \\
\hline $\begin{array}{l}134 \mathrm{E}+00 \\
432 \mathrm{E}+02\end{array}$ & ZERO & $0.6954 \mathrm{E}-01$ & $0.6597 \mathrm{E}-01$ & $\begin{array}{l}0.3569 \mathrm{E}+01 \\
0.6484 \mathrm{E}+02\end{array}$ & $\begin{array}{l}0.3569 E+01 \\
0.6484 E+02\end{array}$ & $\begin{array}{l}0.1771 \mathrm{E}-07 \\
0.4601 \mathrm{E}+03\end{array}$ \\
\hline LIQUID & LIQUID & VAPOR & VAPOR & LIQUID & LIQUID & LIQUID \\
\hline
\end{tabular}


Table A-1. HLW Flowsheet Material Balance (Tank 51/ITP Cycle 1)

Section 1A. In-Tank Precipitation and Salt Decontamination

STREAM NUMBERS $\rightarrow$ STREAM NAME

COMPONENT FLOWS, LB/HR $122(\mathrm{C} 6 \mathrm{H} 5) 2$

1.23 (C6H5) $2 \mathrm{C} 6 \mathrm{H} 4$

118 (C6H5) $2 \mathrm{NH}$

133 (C6H5B (OH) 2 salt)

132 (C6H5B (OH) 2)

166 (C6H5C6H4OH)

162 (C6H5NH2)

163 (C6H5NHCHO)
165 (C6H5NO)

164 (C6H5NO2)

131 (C6H5OH salt)

130 (C6H5OH)

121 (C6H6)

154 ( $\mathrm{CH} 3 \mathrm{OH}$ )

$45 \mathrm{Ag}$

116 Ag20

$134 \mathrm{AgNO} 3$

$106 \cdot \mathrm{B} 203$

$9 \mathrm{Ba}(\mathrm{OH}) 2$

$15 \mathrm{BaO}$

24 BasO4

$66 \mathrm{CO}$

$142 \mathrm{CO} 2$ (C14)

$63 \mathrm{Ca}(\mathrm{COOH}) 2$

$167 \mathrm{Ca}(\mathrm{NO} 3) 2$

$120 \mathrm{Ca}(\mathrm{OH}) 2$

$113 \mathrm{Ca} 3(\mathrm{PO} 4) 2$

$20 \mathrm{CaC} 204$

$18 \mathrm{CaCO} 3$ (C14)

64 Caco

78 CaF2

$19 \mathrm{CaSO} 4$

29 Carbon

99 Cement

$26 \mathrm{Co}(\mathrm{COOH}) 2$

$168 \mathrm{Co}(\mathrm{NO} 3) 2$

$59 \mathrm{COO}$

$98 \mathrm{Cr} 203$

$42 \mathrm{Cs} 2 \mathrm{O}$

$53 \mathrm{CsCOOH}$

$96 \mathrm{CsCl}$

$169 \mathrm{CsNO}^{2}$

151 CsTPB

WSRC-TR-95-0019 (Revision
412

$413 \quad 462 \quad 463$

418

655

656

Spnt Wash Ppt Wsh strg 50 wt 8 NaOH 40 wt: NaNO2 Prec Storage Late Wash Fd Late Wash Fd strge Purge Vnt to Atmo addn to TK22 addn to TK22 Tank bottoms Prec Soluble Prec Tnsolub

\begin{tabular}{|c|c|c|c|}
\hline ZERO & ZERO & ZERO & ZERO \\
\hline ZERO & ZERO & ZERO & ZERO \\
\hline ZERO & ZERO & ZERO & ZERO \\
\hline ZERO & ZERO & ZERO & ZERO \\
\hline ZERO & ZERO & ZERO & ZERO \\
\hline ZERO & ZERO & ZERO & ZERO \\
\hline ZERO & ZERO & ZERO & ZERO \\
\hline ZERO & ZERO & ZERO & ZERO \\
\hline ZERO & ZERO & ZERO & ZERO \\
\hline ZERO & ZERO & ZERO & ZERO \\
\hline ZERO & $0.9168 \mathrm{E}-11$ & ZERO & ZERO \\
\hline ZERO & ZERO & ZERO & ZERO \\
\hline ZERO & $0.7417 \mathrm{E}-02$ & ZERO & ZERO \\
\hline ZERO & $0.2977 \mathrm{E}-05$ & ZERO & ZERO \\
\hline ZERO & $0.1929 \mathrm{E}-06$ & ZERO & ZERO \\
\hline ZERO & ZERO & ZERO & ZERO \\
\hline ZERO & ZERO & ZERO & ZERO \\
\hline ZERO & ZERO & ZERO & ZERO \\
\hline ZERO & ZERO & ZERO & ZERO \\
\hline ZERO & ZERO & ZERO & ZERO \\
\hline ZERO & $0.8141 \mathrm{E}-20$ & ZERO & ZERO \\
\hline ZERO & ZERO & ZERO & ZERO \\
\hline ZERO & ZERO & ZERO & ZERO \\
\hline ZERO & ZERO & ZERO & ZERO \\
\hline $0.2656 \mathrm{E}+00$ & $0.2645 E+00$ & ZERO & ZERO \\
\hline ZERO & ZERO & ZERO & ZERO \\
\hline ZERO & ZERO & ZERO & ZERO \\
\hline ZERO & ZERO & ZERO & .ZERO \\
\hline ZERO & $0.2437 \mathrm{E}-14$ & ZERO & ZERO \\
\hline ZERO & ZERO & ZERO & ZERO \\
\hline ZERO & ZERO & ZERO & ZERO \\
\hline ZERO & ZERO & ZERO & ZERO \\
\hline ZERO & ZERO & ZERO & ZERO \\
\hline ZERO & ZERO & ZERO & ZERO \\
\hline ZERO & ZERO & ZERO & ZERO \\
\hline ZERO & ZERO & ZERO & ZERO \\
\hline ZERO & ZERO & ZERO & ZERO \\
\hline ZERO & ZERO & ZERO & ZERO \\
\hline ZERO & ZERO & ZERO & ZERO \\
\hline ZERO & ZERO & ZERO & ZERO \\
\hline ZERO & ZERO & ZERO & ZERO \\
\hline ZERO & ZERO & ZERO & ZERO \\
\hline ZERO & ZERO & ZERO & ZERO \\
\hline ZERO & ZERO & ZERO & ZERO \\
\hline ZERO & ZERO & ZERO & ZERO \\
\hline ZERO & ZERO & ZERO & ZERO \\
\hline ZERO & $0.8310 \mathrm{E}-15$ & ZERO & ZERO \\
\hline ZERO & ZERO & ZERO & .ZERO \\
\hline
\end{tabular}

\begin{tabular}{|c|c|c|}
\hline $\begin{array}{l}0.3293 \mathrm{E}+00 \\
0.1399 \mathrm{E}-01 \\
0.9313 \mathrm{E}-02 \\
0.3708 \mathrm{E}+00\end{array}$ & $\begin{array}{l}\text { ZERO } \\
\text { ZERO } \\
\text { ZERO } \\
\text { ZERO }\end{array}$ & $\begin{array}{l}0.3293 \mathrm{E}+00 \\
0.1399 \mathrm{E}-01 \\
0.9313 \mathrm{E}-02 \\
0.3708 \mathrm{E}+00\end{array}$ \\
\hline ZERO & ZERO & ZERO \\
\hline $0.3026 \mathrm{E}-01$ & ZERO & $0.3026 \mathrm{E}-01$ \\
\hline ZERO & ZERO & ZERO \\
\hline ZERO & ZERO & ZERO \\
\hline ZERO & ZERO & ZERO \\
\hline ZERO & ZERO & ZERO \\
\hline $0.1736 \mathrm{E}+00$ & $0.1736 \mathrm{E}+00$ & ZERO \\
\hline ZERO & ZERO & ZERO \\
\hline $0.2941 \mathrm{E}+00$ & $0.2941 \mathrm{E}+00$ & ZERO \\
\hline $0.8484 \mathrm{E}-08$ & $84 \mathrm{E}-08$ & ZERO \\
\hline $905 \mathrm{E}-09$ & $5 E-09$ & ZERO \\
\hline ZERO & ZERO & ZERO \\
\hline $0.3054 \mathrm{E}-02$ & ZERO & $0.3054 \mathrm{E}-02$ \\
\hline ZERO & ZERO & ZERO \\
\hline $0.1297 \mathrm{E}+00$ & ZERO & $0.1297 \mathrm{E}+00$ \\
\hline $0.1670 \mathrm{E}-02$ & ZERO & $O E-02$ \\
\hline $.1309 \mathrm{E}-10$ & $0.1309 \mathrm{E}-10$ & ZERO \\
\hline ZERO & ZERO & ZERO \\
\hline $0.1793 E-02$ & ZERO & $0.1793 \mathrm{E}-02$ \\
\hline ZERO & ZERO & ZERO \\
\hline ZERO & ZERO & ZERO \\
\hline ZERO & ZERO & ZERO \\
\hline ZERO & ZERO & ZERO \\
\hline $0.3698 \mathrm{E}-04$ & $0.3698 \mathrm{E}-04$ & ZERO \\
\hline $0.3813 E-05$ & $0.3813 \mathrm{E}-05$ & ZERO \\
\hline $0.5607 \mathrm{E}+00$ & ZERO & $0.5607 \mathrm{E}+00$ \\
\hline ZERO & ZERO & ZERO \\
\hline ZER & ZERO & ZERO \\
\hline ZERO & ZERO & ZERO \\
\hline ZERO & ZERO & ZERO \\
\hline ZERO & ZERO & ZERO \\
\hline $0.1912 \mathrm{E}+00$ & ZERO & $0.1912 \mathrm{E}+00$ \\
\hline ZERO & ZEF & ZERO \\
\hline $\mathrm{ZEF}$ & ZERO & ZERO \\
\hline ZERO & ZERO & ZERO \\
\hline $0.1451 \mathrm{E}-04$ & $0.1451 \mathrm{E}-04$ & ZERO \\
\hline $0.1028 \mathrm{E}-03$ & ZERO & $0.1028 \mathrm{E}-03$ \\
\hline $0.2616 \mathrm{E}+00$ & ZERO & $0.2616 \mathrm{E}+00$ \\
\hline $0.3848 \mathrm{E}-04$ & ZERO & $0.3848 \mathrm{E}-04$ \\
\hline ZERO & ZERO & ZERO \\
\hline ZERO & ZERO & ZERO \\
\hline ZERO & ZERO & ZERO \\
\hline $\mathrm{OE}-02$ & $0.1740 \mathrm{E}-02$ & ZERO \\
\hline$a+00$ & ZERO & $0.1049 \mathrm{E}+00$ \\
\hline
\end{tabular}

Date: January 1995 
Table A-1. HLW Flowsheet Material Balance (Tank 51/ITP Cycle 1 ) Section 1A. In-Tank Precipitation and salt Decontamination

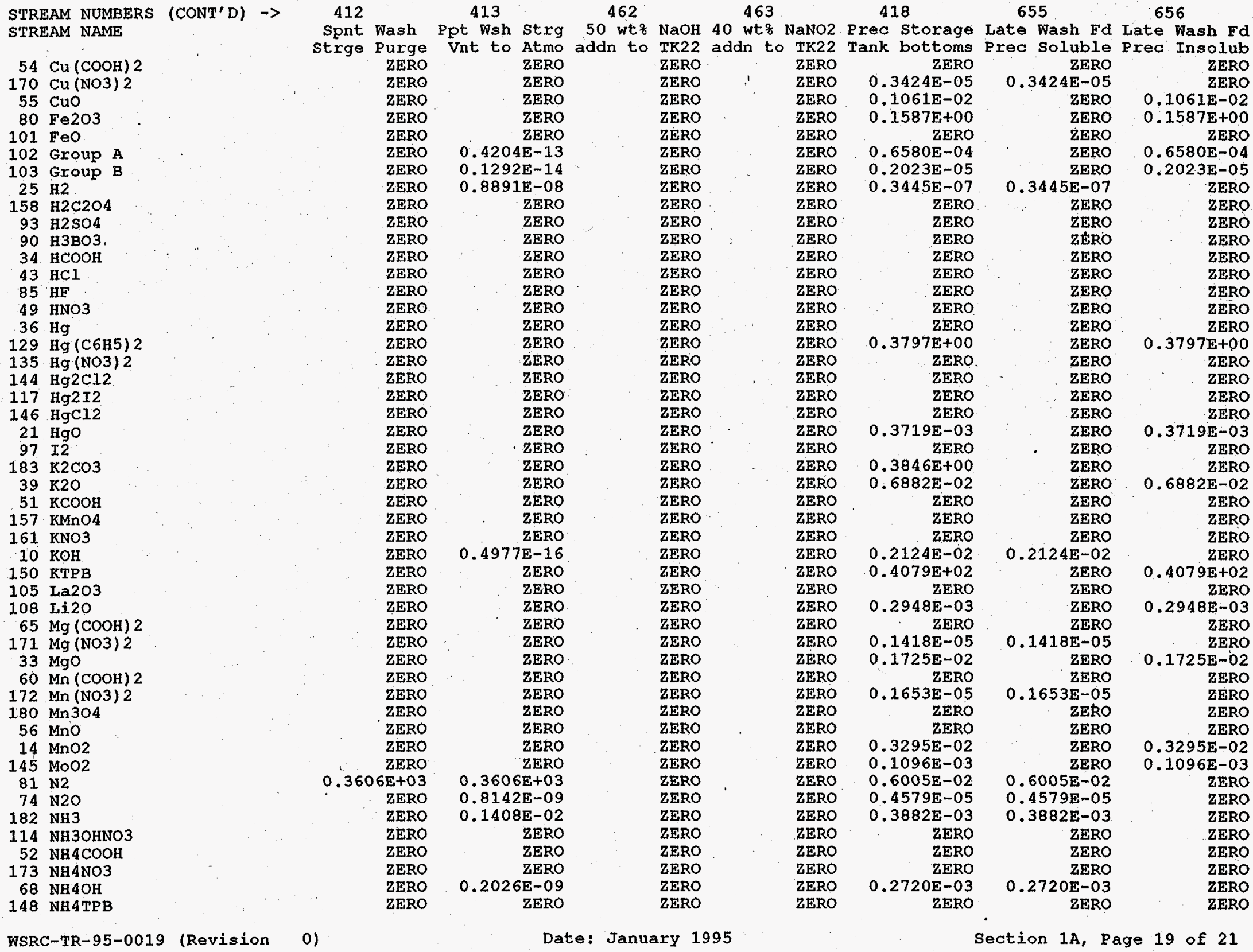


Table A-1. HLW Flowsheet Material Balance (Tank 51/ITP Cycle 1)

Section 1A. In-Tank Precipitation and Salt Decontamination

STREAM NUMBERS (CONT'D) $\rightarrow$ STREAM NAME

\author{
35 NO \\ 84 NO2 \\ $70 \mathrm{Na}(\mathrm{HgO}(\mathrm{OH}))$ \\ $94 \mathrm{Na2B} 407$ \\ $109 \mathrm{Na} 2 \mathrm{C} 2 \mathrm{O} 4$ \\ $5 \mathrm{Na} 2 \mathrm{CO} 3$ \\ $143 \mathrm{Na}_{2 \mathrm{CO} 3}$ (C14) \\ $89 \mathrm{Na} 2 \mathrm{CrO} 4$ \\ $115 \mathrm{Na2MoO}$ \\ 71 Na2PuO2 (OH) 4 \\ $73 \mathrm{Na2RhO4}$ \\ $72 \mathrm{Na2RuO4}$ \\ $6 \mathrm{Na} 2 \mathrm{SO} 4$ \\ $88 \mathrm{Na2} 2 \mathrm{Na} 3 \mathrm{PO} 4$ \\ $91 \mathrm{NaAg}(\mathrm{OH}) 2$ \\ $75 \mathrm{NaAl}(\mathrm{OH}) 4$ \\ $50 \mathrm{NaCOOH}$ \\ $7 \mathrm{NaCl}$ \\ $69 \mathrm{NaF}$ \\ $11 \mathrm{NaHCO} 3$ \\ $12 \mathrm{NaI}$ \\ 3 NaNO2 \\ 2 NaNO3 \\ $\begin{array}{rl}4 & \mathrm{NaOH} \\ 149 & \mathrm{NaTPB}\end{array}$ \\ 160 NaTCO4 \\ $152 \mathrm{NaTi2O5H}$ \\ $181 \mathrm{Nd} 2 \mathrm{O} 3$ \\ $61 \mathrm{Ni}(\mathrm{COOH}) 2$ \\ $174 \mathrm{Ni}(\mathrm{NO} 3) 2$ \\ $40 \mathrm{NiO}$ \\ $175 \mathrm{pb}$ (NO3) 2 \\ $86 \mathrm{PbCO} 3$ \\ $86 \mathrm{PbCO}$
$67 \mathrm{BbO}$ \\ $67 \mathrm{PbO}$
$147 \mathrm{PbS}$ \\ $28 \mathrm{PbSO} 4$ \\ $124 \mathrm{Pd}$ \\ $112 \mathrm{Pd}$ (NO3) 2 \\ $110 \mathrm{PdO}$ \\ 76 PuO2. \\ 156 PuO2 (NaTi2O5) 2 \\ $58 \mathrm{Rh}$ \\ $22 \mathrm{RhO} 2$ \\ 79 RuO2 \\ $83 \mathrm{RuO} 4$
}

462

463

418

655

656

Spnt Wash Ppt Wsh Strg 50 wt: NaOH 40 wt 8 NaNO2 Prec Storage Late Wash Fd Late Wash Fd strge Purge Vnt to Atmo addn to TK22 addn to TK22 Tank bottoms Prec Soluble Prec. Insolub $\begin{array}{lllll}\text { ZERO ZERO } & \text { ZERO } & \text { ZERO } & \text { ZERO } & \text { ZERO } \\ \text { ZERO }\end{array}$

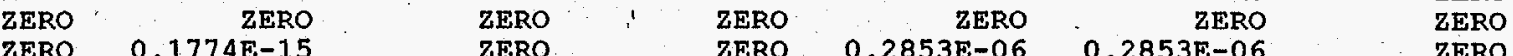
$\begin{array}{lllll}\text { ZERO } & \text { ZERO } & 0.2853 \mathrm{E}-06 & 0.2853 \mathrm{E}-06 & \text { ZERO }\end{array}$

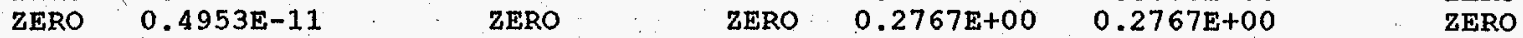
ZERO $0.2108 \mathrm{E}+00 \cdot 0.2108 \mathrm{E}+00 \quad$ ZERO ZERO $0.3979 \mathrm{E}-09 \quad$ ZERO ZERO $0.4873 E-17$ ZERO

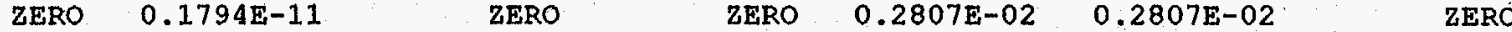
ZERO $0.5356 \mathrm{E}-12 \quad$ ZERO ZERO ZERO $0.1568 \mathrm{E}-17$ ZERO $0.1299 \mathrm{E}-13$ ZERO $0.6843 \mathrm{E}-13$ ZERO $0.6843 \mathrm{E}-13$ ZERO ZERO $0.1867 \mathrm{E}-10$ ZERO ZERO $0.5195 E-12$ ZERO ZERO ZERO ZERO ZERO. ZERO

ZERO

ZERO

ZERO

ZERO

ZERO

ZERO

ZERO

ZERO

ZERO

$0.1089 \mathrm{E}+03$

ZERO

ZERO

ZERO

ZERO

ZERO

ZERO

ZERO

ZERO

ZERO

ZERO

ZERO

ZERO

$0.2479 \mathrm{E}-10$

$0.4692 \mathrm{E}-17$

$0.9084 \mathrm{E}-10$

ZERO

ZERO ZERO

$0.1435 \mathrm{E}-11 \quad$ ZERO

ZERO

$0.914 \mathrm{E}-15$

$0.9142 \mathrm{E}-09$
$0.8860 \mathrm{E}-09$

$0.8860 \mathrm{E}-09$

$0.2684 \mathrm{E}-08$
$0.5652 \mathrm{E}-10$
ZERO

ZERO

ZERO

ZERO

ZERO

$0.1089 \mathrm{E}+03$

ZERO

ZERO

ZERO

ZERO

ZERO

$0.1156 \mathrm{E}-15$

ZERO

ZERO

ZERO

ZERO

ZERO

ZERO

ZERO

ZERO

ZERO

ZRO

$0.7 .931 \mathrm{E}+01$

ZERO

ZERO

ZERO

ZERO

ZERO

ZERO

ZERO

ZERO

ZERO

ZERO

ZERO

ZERO

ZERO

ZERO

ZERO

ZERO

ZERO

ZERO

ZERO

ZERO

ZERO

Date: January 1995
$0.2520 \mathrm{E}-08$ ZERO

$0.2033 E-04 \quad$ ZERO

$0.1071 \mathrm{E}-03$ ZERO

$0.2921 \mathrm{E}-01$ ZERO

$0.8130 \mathrm{E}-03 \quad 0.5550 \mathrm{E}-10$

$0.3880 \mathrm{E}-01 \quad$ ZERO

$0.7545 \mathrm{E}-08$ ZERO

$0.1006 \mathrm{E}-02$ ZERO

ZERO
$0.2298 \mathrm{E}-02$

$0.2246 \mathrm{E}-02 \quad$ ZERO

ZERO

$\begin{array}{ll}0.1504 \mathrm{E}-05 & \text { ZERO } \\ 0.7976 E+01 & \text { ZERO }\end{array}$

$0.5939 \mathrm{E}+00 \quad 0.3959 \mathrm{E}-07$

$0.1572 \mathrm{E}-01$ ZERO

$0.6748 \mathrm{ERO}$

$0.5939 \mathrm{E}+00$

$0.1572 \mathrm{E}-01$

$0.6748 \mathrm{E}-04$

$0.9792 \mathrm{E}+00$

$\begin{array}{lr}\text { ZERO } & \text { ZERO } \\ \text { ZERO } & \text { ZERO } \\ \text { ZERO } & 0.1805 E-04\end{array}$

$0.1805 \mathrm{E}-04$

$0.1037 E-02$

$0.7141 \mathrm{E}-03$

ZERO

ZERO

ZERO

ZERO

ZERO

ZERO

ZERO

ZERO

ZERO

ZERO

ZERO

ZERO

ZERO

$.4383 \mathrm{E}-04$

ZERO

ZERO

$0.4362 \mathrm{E}-02$

ZERO

0.1809 E-06
ZERO

ZERO
$9 \mathrm{E}-04$

ZERO

ZERO

$0.4424 \mathrm{E}-02$

E-02
ZERO

ZERO
ZERO

ZERO

$0.1805 \mathrm{E}-04$

ZERO

$0.7141 \mathrm{E}-03$

$0.4383 \mathrm{E}-04$

ZERO

ZERO

ZERO

ZERO

$0.1809 \mathrm{E}-06$

ZERO

ZERO

ZERO

ZERO

ZERO

ZERO

ZERO

ZERO
$0.9792 \mathrm{E}+00$

ZERO

ERO

0.1037 EERO

ZERO

ZERO

ZERO

ZERO

ZERO

ZERO

$2 \mathrm{E}-03$
ZERO

ZERO

$0.4424 \mathrm{E}-0$

ZERO

Section 1A, Page 20 of 21
ZERO
ZERO

$0.7976 \mathrm{E}+01$ ZERO

$0.1272 \mathrm{E}-03$

$0.1272 \mathrm{n}-03$ 
Table A-1. HLW Flowsheet Material Balance (Tank 51/ITP Cycle 1 )

Section 1A. In-Tank Precipitation and Salt Decontamination

STREAM NUMBERS (CONT'D) $\rightarrow$ STREAM NAME

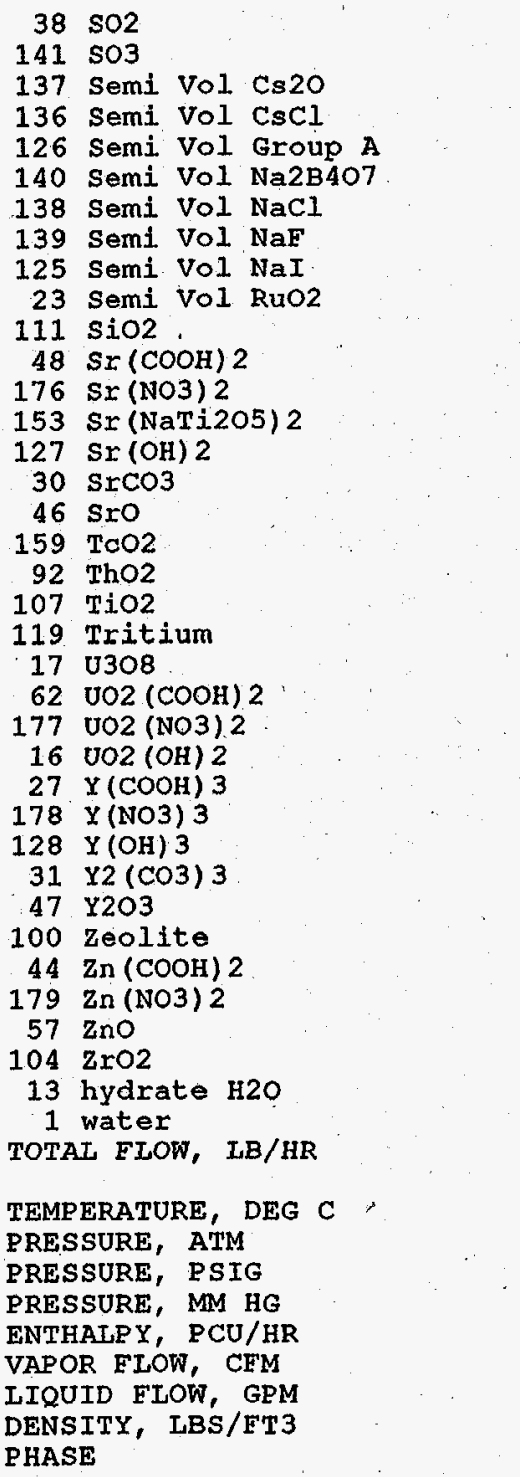

412

\begin{abstract}
413
\end{abstract}
462

418

656

Spnt Wash Ppt Wish Strg 50 wt: NaOH 40 wt 8 NaNO2 Prec Storage Late Wash Fd Late Wash Fd strge Purge Vnt to Atmo addn to TK22 addn to TK22 Tank bottoms Prec soluble Prec Insolub

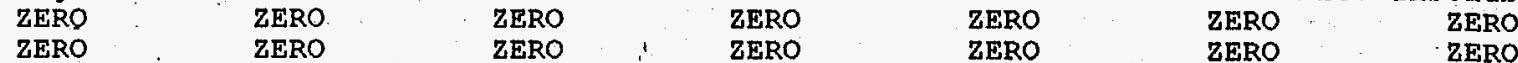

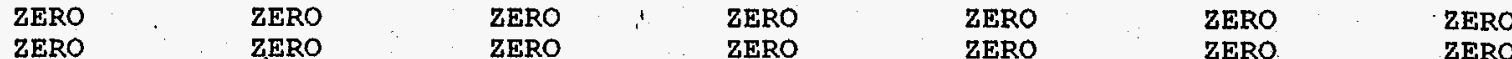

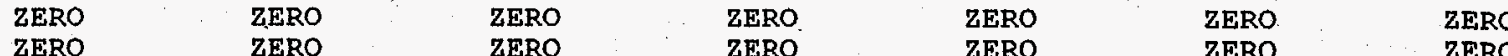

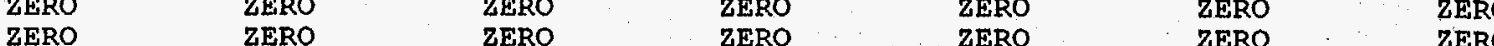
ZERO ZERO ZERO ZERO ZERO Z ZERO ZERO ZERO ZERO ZERO ZERO ZERO ZERO ZERO ZERO

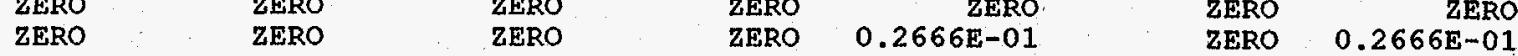

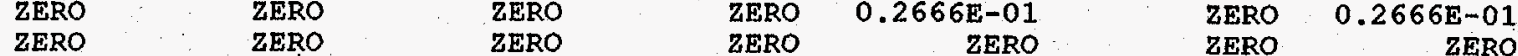

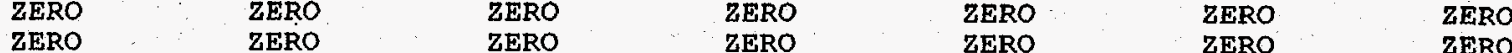

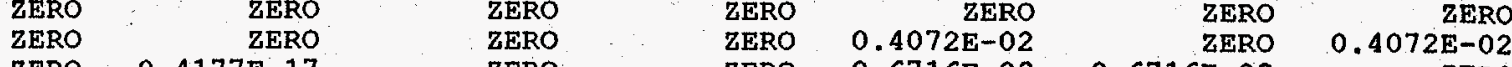
$\begin{array}{lllllll}\text { ZERO } & 0.4177 \mathrm{E}-17 & \text { ZERO } & \text { ZERO } & 0.6716 \mathrm{E}-08 & 0.6716 \mathrm{E}-08 & \text { ZERO }\end{array}$

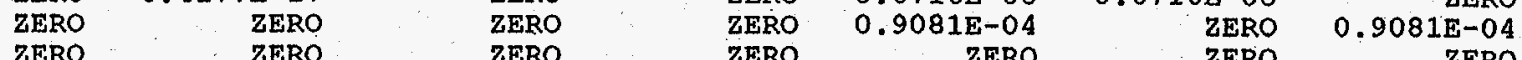

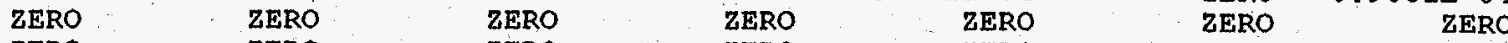
$\begin{array}{lllllll}\text { ZERO } & \text { ZERO } & \text { ZERO } & \text { ZERO } & \text { ZERO } & \text { ZERO } & \text { ZERO }\end{array}$

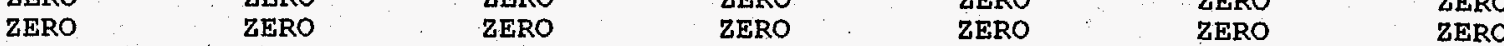

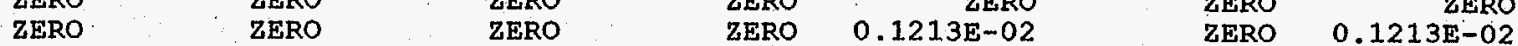

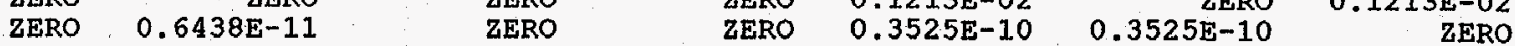
$\begin{array}{rrrrrr}\text { ZERO } & \text { ZERO } & \text { ZERO } & \text { ZERO } & 0.7512 \mathrm{E}-03 & 0.3525 \mathrm{E}-10 \\ \text { ZERO } & 0.7512 \mathrm{E}-03\end{array}$

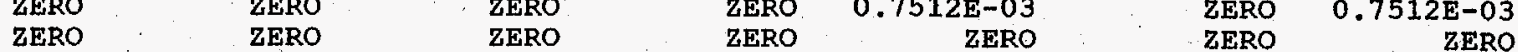

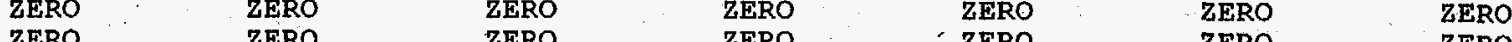

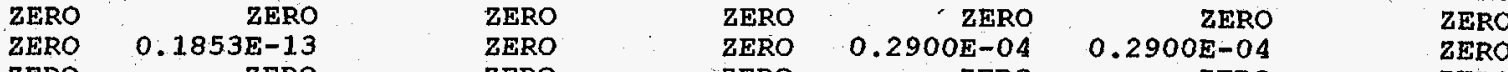

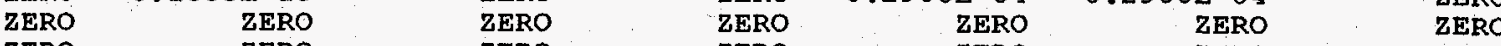

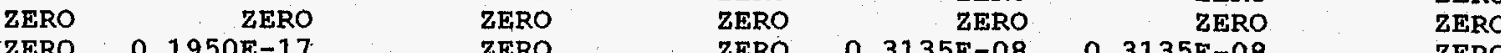
$\begin{array}{lllllll}\text { ZERO } & 0.1950 \mathrm{E}-17 & \text { ZERO } & \text { ZERO } & 0.3135 \mathrm{E}-08 & 0.3135 \mathrm{E}-08 & \text { ZERO }\end{array}$ $\begin{array}{rrrrrr}\text { ZERO } & \text { ZERO } & \text { ZERO } & \text { ZERO } & \text { ZERO } & \text { ZERO }\end{array}$

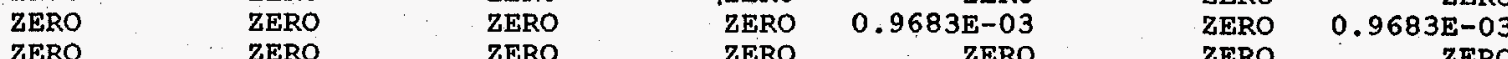
ZERO

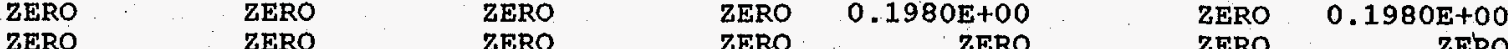

$0.7888 \mathrm{E}+01$ $0.4777 E+03$ ZERO ZERO
ZERO $0.4825 \mathrm{E}+03 \quad 0.1586 \mathrm{E}+02$ ZERO $0.3589 \mathrm{E}-01$ $0.4056 \mathrm{E}+03$ $0.4655 E+03$ ZERO $\begin{array}{rr}\text { ZERO } & 0.3589 \mathrm{E}-01 \\ 0.4056 \mathrm{E}+03 & \text { ZERO } \\ 0.4195 \mathrm{E}+03 & 0.4561 \mathrm{E}+02\end{array}$

$0.3846 \mathrm{E}+02$ $0.9656 \mathrm{E}+00$ $-0.5055 \mathrm{E}+00$ $0.7339 \mathrm{E}+03$

$0.3846 \mathrm{E}+02$ $0.9656 \mathrm{E}+00 \quad 0.3846 \mathrm{E}+02$ $-0.5055 \mathrm{E}+00 \quad 0.9656 \mathrm{E}+00$ $0.7339 \mathrm{E}+03 \quad-0.5055 \mathrm{E}+00$ $0.1576 \mathrm{E}+05 \quad 0.2821 \mathrm{E}+02$

$0.1003 \mathrm{E}+05 \quad 0.7339 \mathrm{E}+03$ $0.1207 \mathrm{E}+03$

$0.2634 \mathrm{E}+03$

ZERO

$0.1579 \mathrm{E}+05$

$0.8208 \mathrm{E}+00$

$0.9016 \mathrm{E}+00$ $0.6372 \mathrm{E}+02$

$0.8042 \mathrm{E}-01$ ZERO
LIQUID LIQUID

$0.7071 E+02$ 


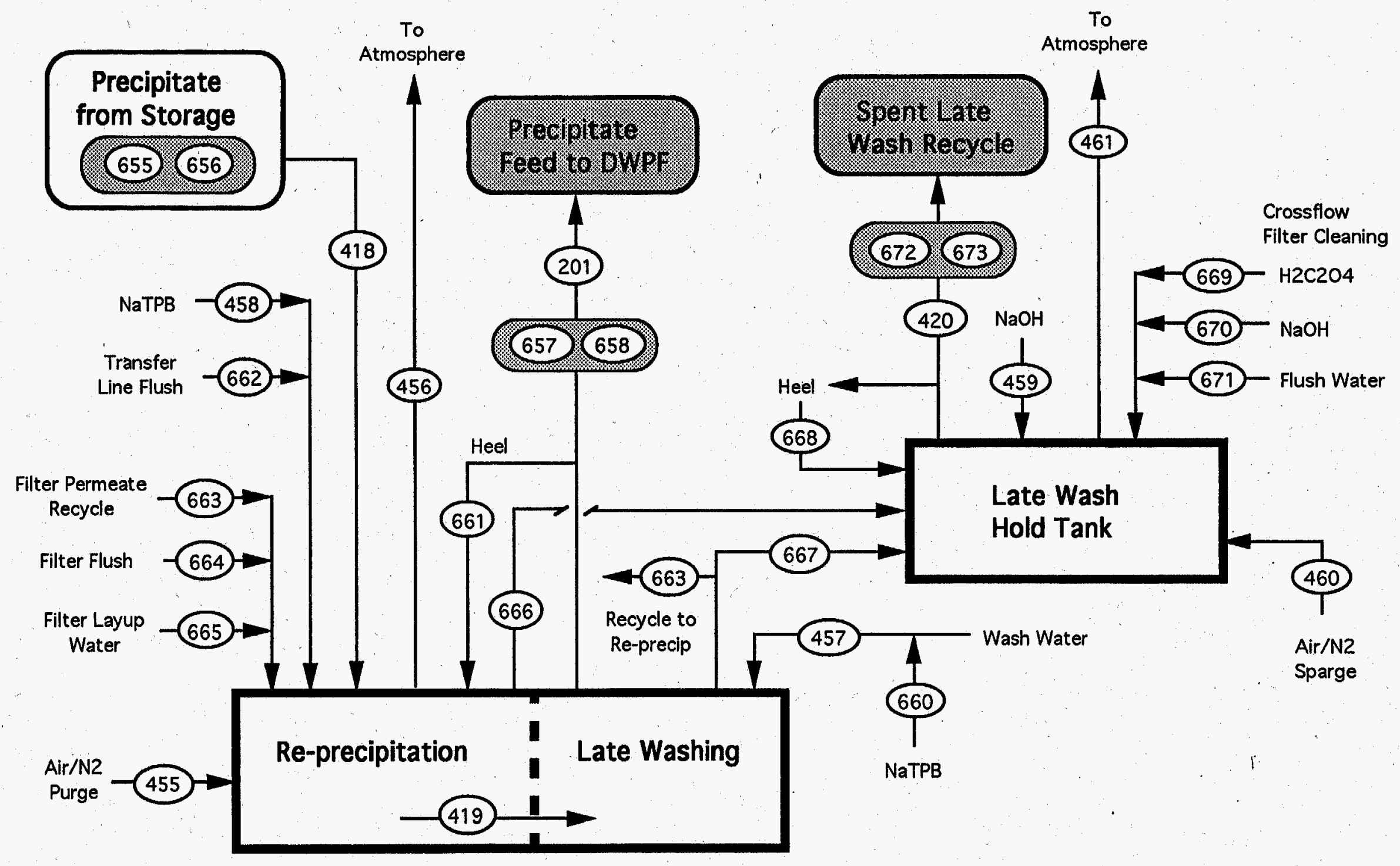

SECTION 1B. RE-PRECIPITATION / LATE WASHING IN AUXILIARY PUMP PIT WSRC-TR-95-0019 (Revision 0) 
Table A-1. HLW Flowsheet Material Balance (Tank 51/ITP Cycle 1) section 1B. Re-precipitation and Late Washing

DESCRIPTION

PAGE

1. STREAM NUMBERS $418,655,656,458,661,662,663 \ldots \ldots$

2. STREAM NUMBERS

$664,665,419,666,660,457,667 \ldots \ldots . . . . . . . . . .66$

3. STREAM NUMBERS

$455,456,668,669,670,671,459$. . . . . . . . . . . 10

4. STREAM NUMBERS

$420,672,673,201,657,658,460 \ldots . . . . . . . . . . . .14$

5. STREAM NUMBERS

461 ................................ 18 
Table A-1. HLW Flowsheet Material Balance (Tank 51/ITP Cycle 1) Section 1B. Re-precipitation and Late Washing

STREAM NUMBERS $\rightarrow$ STREAM NAME

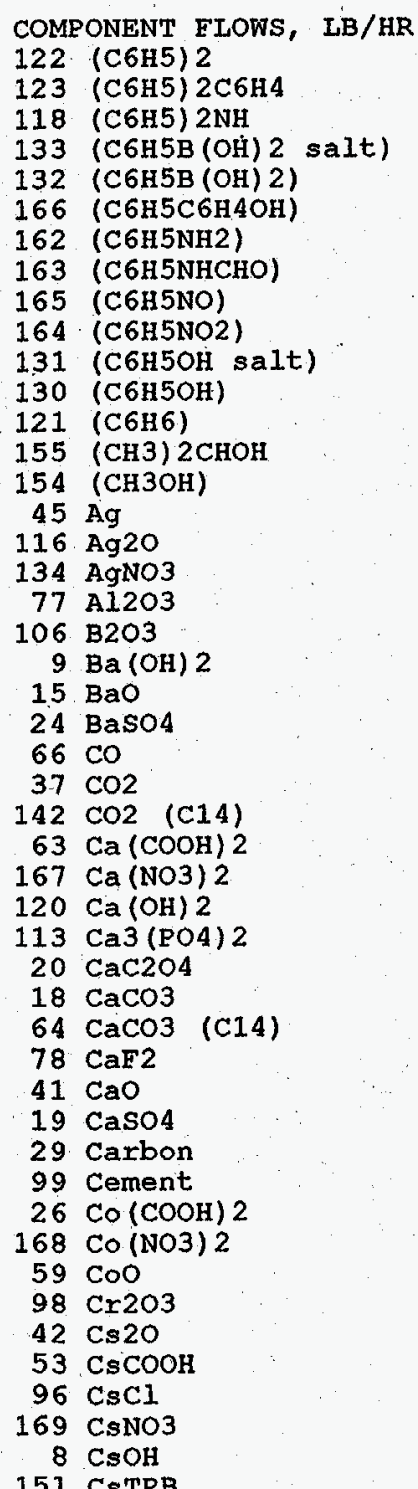

\begin{tabular}{|c|c|}
\hline ZERO & $0.3293 \mathrm{E}+00$ \\
\hline ZERO & $0.1399 \mathrm{E}-01$ \\
\hline ZERO & $0.9313 \mathrm{E}-02$ \\
\hline ZERO & $0.3708 \mathrm{E}+00$ \\
\hline ZERO & ZERO \\
\hline ZERO & $0.3026 \mathrm{E}-01$ \\
\hline ZERO & ZERO \\
\hline ZERO & ZERO \\
\hline ZERO & ZERO \\
\hline ZERO & ZERO \\
\hline $0.1736 \mathrm{E}+00$ & ZERO \\
\hline ZERO & ZERO \\
\hline $0.2941 \mathrm{E}+00$ & ZERO \\
\hline $0.8484 \mathrm{E}-08$ & ZERO \\
\hline $0.7905 \mathrm{E}-09$ & ZERO \\
\hline ZERO & ZERO \\
\hline ZERO & $0.3054 \mathrm{E}-02$ \\
\hline ZERO & ZERO \\
\hline ZERO & $0.1297 \mathrm{E}+00$ \\
\hline ZERO & $0.1670 \mathrm{E}-02$ \\
\hline $0.1309 \mathrm{E}-10$ & ZERO \\
\hline ZERO & ZERO \\
\hline ZERO & $0.1793 \mathrm{E}-02$ \\
\hline ZERO & ZERO \\
\hline ZERO & ZERO \\
\hline ZERO & ZERO \\
\hline ZERO & ZERO \\
\hline $0.3698 \mathrm{E}-04$ & ZERO \\
\hline $0.3813 \mathrm{E}-05$ & ZERO \\
\hline ZERO & $0.5607 \mathrm{E}+00$ \\
\hline ZERO & ZERO \\
\hline ZERO & ZERO \\
\hline ZERO & ZERO \\
\hline ZERO & ZERO \\
\hline ZERO & ZERO \\
\hline ZERO & $0.1912 \mathrm{E}+00$ \\
\hline ZERO & ZERO \\
\hline ZERO & ZERO \\
\hline ZERO & ZERO \\
\hline $0: 1451 \mathrm{E}-04$ & ZERO \\
\hline ZERO & $0.1028 \mathrm{E}-03$ \\
\hline ZERO & $0.2616 \mathrm{E}+00$ \\
\hline ZERO & $0.3848 E-04$ \\
\hline ZERO & ZERO \\
\hline ZERO & ZERO \\
\hline ZERO & ZERO \\
\hline $0.1740 \mathrm{E}-02$ & ZERO \\
\hline ZERO & $0.1049 \mathrm{E}+00$ \\
\hline
\end{tabular}

458

NaTPB addn

to IWPT

661 LWPT Precip Trnsf X-Filtr Tube Heel Line Flush Fltr to LWP

ZERO
ZERO
ZERO
ZERO
ZERO
ZERO
ZERO
ZERO
ZERO
ZERO
ZER 15E+OO
ZERO
ZERO
ZERO
ZERO
ZERO
ZERO
ZERO
ZERO
ZERO
ZERO
ZERO
ZERO
ZERO
ZERO
ZERO
ZERO
ZERO
ZERO
ZERO
ZERO
ZERO
ZERO
ZERO
ZERO
ZERO
ZERO
ZERO
ZERO
ZERO
ZERO
ZERO
ZERO
ZERO
ZERO
ZERO
ZERO
ZERO
ZERO
ZERO
ZERO

$0.1773 \mathrm{E}-02$

$0.1180 \mathrm{E}-02$

$0.4705 \mathrm{E}-01$

$0.3835 \mathrm{E}-02$

ZERO

ZERO

ZERO

0.5707 ZERO

ZERO

$0.1383 \mathrm{E}-02$

$0.1089 \mathrm{E}-10$

$0.1194 \mathrm{E}-11$

$0.3871 \mathrm{E}-03$ ZERO

$0.1644 \mathrm{E}-01$

$0.2117 \mathrm{E}-03$

$0.6105 \mathrm{E}-17$

$0.227 .2 \mathrm{E}-03$

E-03

ZERO

ZERO

ZERO

$0.1736 \mathrm{E}-06$

$0.1736 \mathrm{E}-06$

$0.7107 \mathrm{E}-01$

ZERO

ZERO

ZERO

ZERO

$0.2423 E-01$

ZERO

ZERO

$0.6811 \mathrm{E}-07$

$0.1303 \mathrm{E}-04$

$0.3316 \mathrm{E}-01$

$0.4877 \mathrm{E}-05$

ZERO

ZERO

$0.2274 \mathrm{E}-06$

$0.1395 \mathrm{E}-01$

\begin{tabular}{|c|c|}
\hline ZERO & ZERO \\
\hline ZERO & ZERO \\
\hline ZERO & ZERO \\
\hline ZERO & ZERO \\
\hline ZERO & ZERO \\
\hline ZERO & ZERO \\
\hline ZERO & ZERO \\
\hline ZERO & ZERO \\
\hline ZERO & ZERO \\
\hline ZERO & $\therefore \quad$ ZERO \\
\hline ZERO & $0.1112 \mathrm{E}-02$ \\
\hline ZERO & ZERO \\
\hline ZERO & $0.7552 \mathrm{E}-03$ \\
\hline ZERO & $0.5945 \mathrm{E}-11$ \\
\hline ZERO & $0.6521 \mathrm{E}-12$ \\
\hline ZERO & ZERO \\
\hline ZERO & ZERO \\
\hline ZERO & ZERO \\
\hline ZERO & ZERO \\
\hline ZERO & ZERO \\
\hline ZERO & $0.3333 \mathrm{E}-17$ \\
\hline ZERO & ZERO \\
\hline ZERO & ZERO \\
\hline ZERO & ZERO \\
\hline ZERO & ZERO \\
\hline ZERO & ZERO \\
\hline ZERO & ZERO \\
\hline ZERO & $0.9480 \mathrm{E}-07$ \\
\hline ZERO & $0.9776 \mathrm{E}-08$ \\
\hline ZERO & ZERO \\
\hline ZERO & ZERO \\
\hline ZERO & ZERO \\
\hline ZERO & ZERO \\
\hline ZERO & ZERO \\
\hline ZERO & ZERO \\
\hline ZERO & ZERO \\
\hline ZERO & ZERO \\
\hline ZERO & ZERO \\
\hline ZERO & ZERO \\
\hline ZERO & $0.3719 \mathrm{E}-07$ \\
\hline ZERO & ZERO \\
\hline ZERO & ZERO \\
\hline ZERO & ZERO \\
\hline ZERO & ZERO \\
\hline ZERO & ZERO \\
\hline ZERO & ZERO \\
\hline ZERO & $0.1242 \mathrm{E}-06$ \\
\hline ZERO & ZERO \\
\hline
\end{tabular}

Section 1B, Page 2 of 21 
Table A-1. HLW Flowsheet Material Balance (Tank 51/ITP Cycle 1) section 1B. Re-precipitation and Late Washing

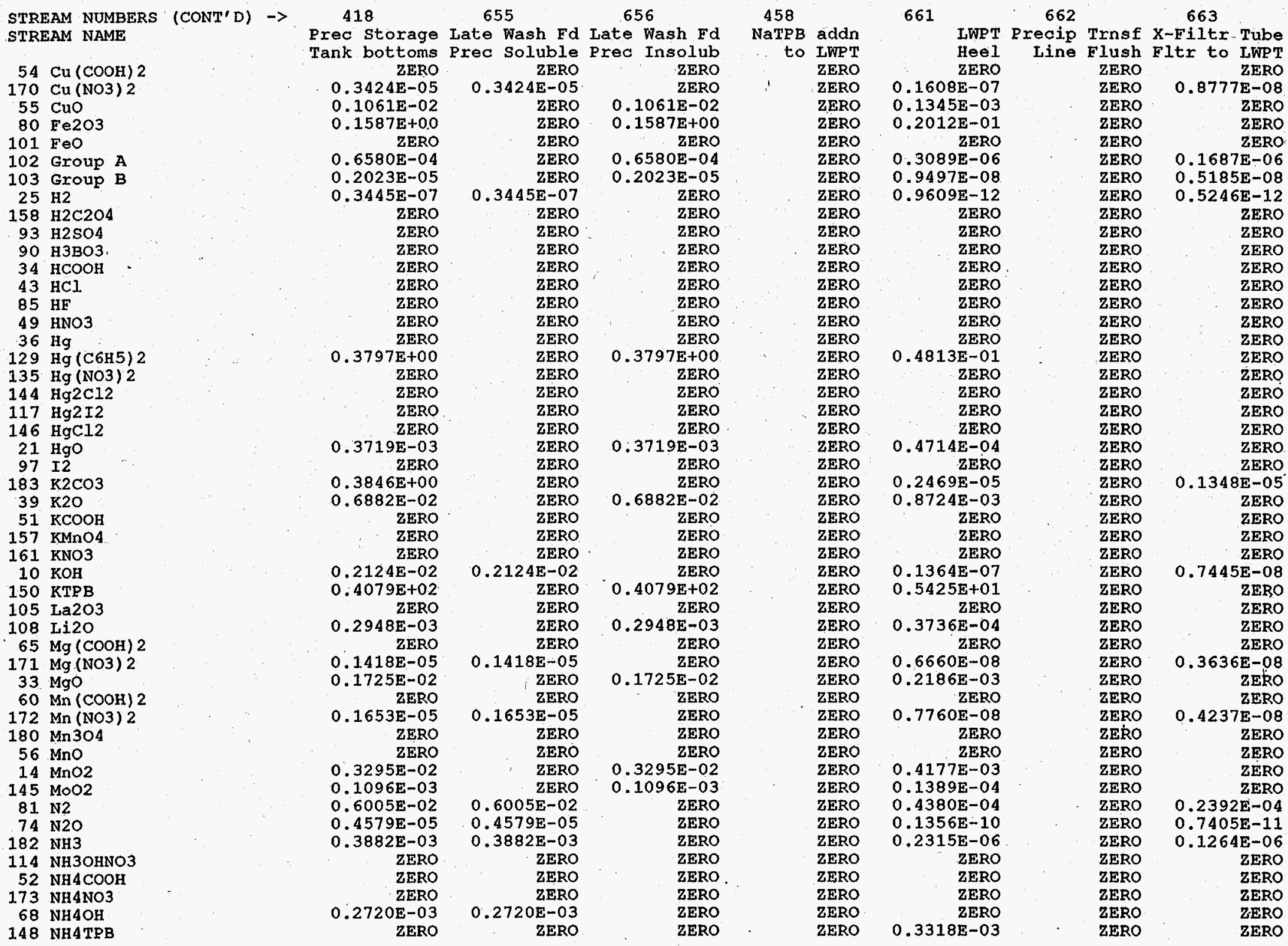


Table A-1. HLW Flowsheet Material Balance (Tank 51/ITP Cycle 1) Section 1B. Re-precipitation and Late Washing

STREAM NUMBERS (CONT'D) $\rightarrow$ STREAM NAME

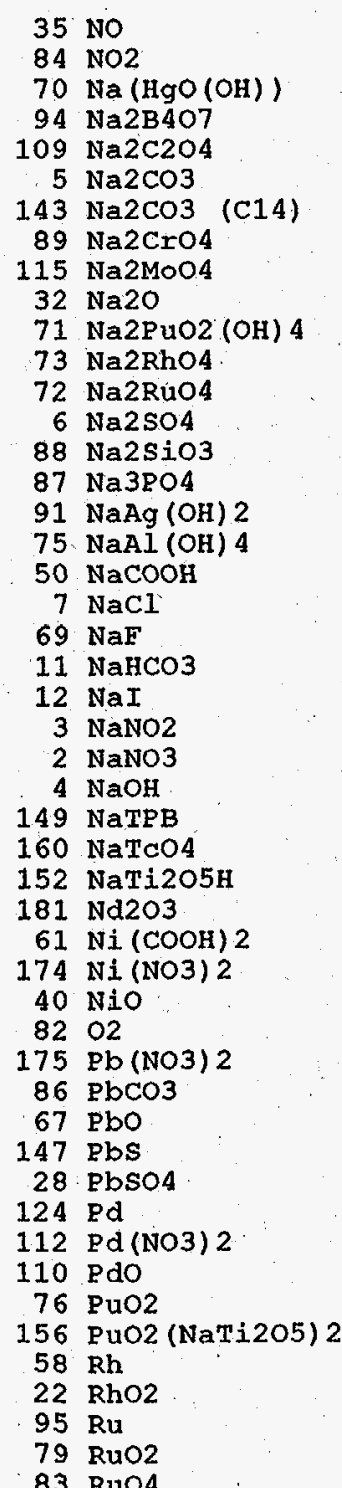

458

$$
\begin{array}{r}
\text { ZERO } \\
\text { ZERO }
\end{array}
$$

ZERO
$0.2853 \mathrm{E}-06$

$0.2767 \mathrm{E}+00$

$0.2108 \mathrm{E}+00$
$0.4232 \mathrm{E}+01$

$0.7626 \mathrm{E}-08$

$0.2807 \mathrm{E}-02$

$0.8382 \mathrm{E}-03$

$0.2520 \mathrm{E}-08$

$0.2033 \mathrm{E}-04$

$0.1071 \mathrm{E}-03$

$0.2921 \mathrm{E}-01$

$0.3880 \mathrm{E}-01$

$0.7545 \mathrm{E}-08$

$0.1006 \mathrm{E}-02$

ZERO
$0.2298 \mathrm{E}-02$

$0.2246 \mathrm{E}-02$

ZERO

$0.7976 \mathrm{E}+01$

$0.5939 \mathrm{E}+00$

$0.1572 \mathrm{E}-01$

ZERO

$0.6748 \mathrm{E}-04$

ZERO

ZERO
$0.1805 \mathrm{E}-04$

ZERO
$1 \mathrm{E}-03$

$0.7141 \mathrm{E}-03$
$0.4383 \mathrm{E}-04$

ZERO
ZERO

ZERO

ZERO

$0.1809 \mathrm{E}-06$

ZERO

ZERO

ZERO $0.1272 \mathrm{E}-03$

ZERO ZERO

ZERO ZERO

$0.4424 \mathrm{E}-02$
ZERO

$\mathrm{PB}$ addn

O LWPT

ZERO

ZERO

ZERO

ZERO

ZERO

ZERO

ZERO

ZERO

ZERO

$0.1003 E+01$

ZERO

ZERO

ZERO

ZERO

ZERO

ZERO

ZERO

ZERO

ZERO

$0.3959 \mathrm{E}-07$

ZERO

ZERO

ZERO

ZERO

$0.1037 \mathrm{E}-02$

ZERO

ZERO.

4362 E-02

ZERO

ZERO

ZERO

ZERO

ZERO

ZERO

ZERO

ZERO

ZERO

$0.1268 \mathrm{E}-01$

ZERO

ZERO

ZERO

ZERO

$0.6324 \mathrm{E}-01$

$0.2705 \mathrm{E}+01$

ZERO

ZEERO

ZERO

ZERO

ZERO

ZERO

ZERO

ZERO

ZERO

ZERO

ZERO

ZERO

ZERO

ZERO

ZERO

ZERO

ZERO

ZERO

ZERO
661

662

662663

LWPT Precip Trnsf X-Filtr Tube Heel Iine Flush Fltr to LWPT ZERO ZERO ZERO

ZERO $0.9900 \mathrm{C}-03$ ZERO $0.7093 \mathrm{E}-03$ ZERO $0.5405 \mathrm{E}-03$ $.3581 \mathrm{E}-10 \quad$ ZERO $0.1955 \mathrm{E}-10$ $0.3936 \mathrm{E}-05 \quad$ ZERO $0.2149 \mathrm{E}-05$ ZERO Z ZERO ZERO $0.8982 \mathrm{E}-13$ $0.5028 \mathrm{E}-06 \quad$ ZERO $0.2745 \mathrm{E}-06$ ZERO $0.7490 \mathrm{E}-04$ $0.3817 \mathrm{E}-05 \quad 0.749 \mathrm{E}-04$ $0.1822 \mathrm{E}-03$ ZERO $0.2084 \mathrm{E}-05$ $0.7034 \mathrm{E}-13$ ZERO $0.9947 \mathrm{E}-04$ $0.4724 \mathrm{E}-05 \quad \mathrm{ZTRO} 0.384 \mathrm{E}-13$ ZERO ZERO $0.4030 \mathrm{E}-03 \quad$ ZERO $0.5935 \mathrm{E}-04$ .1055E-04 ZERO $0.5758 \mathrm{E}-05$ ZERO
$0.7064 E-08$ $0.3745 \mathrm{E}-01 \quad$ ZERO $0.2045 \mathrm{E}-01$ $0.2789 \mathrm{E}-02$ ZERO $0.1523 \mathrm{E}-02$ $0.1243 \mathrm{E}-01 \quad 0.5503 \mathrm{E}-02 \quad 0.1012 \mathrm{E}-02$ $0.7466 \mathrm{E}-01$ O.5503E-02 $0.1012 \mathrm{E}-02$ $0.3169 \mathrm{E}-06$ ZERO $0.1730 \mathrm{E}-06$ $0.1241 \mathrm{E}+00 \quad$ ZERO $\quad$ ZERO ZERO ZERO ZERO ZERO $\begin{array}{lll}0.8475 E-07 & \text { ZERO } 0.4627 \mathrm{E}-07 \\ 0.1315 \mathrm{E}-03 & \text { ZERO } & \end{array}$ $0.4974 \mathrm{E}-05 \quad$ ZERO $0.2716 \mathrm{E}-05$ $0.2058 \mathrm{E}-06 \quad$ ZERO $0.1124 \mathrm{E}-\mathrm{b} 6$ ZERO ZERO ZERO $\begin{array}{lll}\text { ZERO } & \text { ZERO } \\ \text { ZERO }\end{array}$ $\begin{array}{lll}0.5530 \text { Z-03 } & \text { ZERO } & \text { ZERO } \\ \text { ZERO } & \text { ZERO }\end{array}$ ZERO ZERO ZERO 0.4637 ERO ZTRO

$0.4068 \mathrm{E}-05$ $0.1612 \mathrm{E}-04$ ZERO ZERO $0.5607 \mathrm{E}-03$ ZERO
ZERO

ZERO

ZERO

ZERO

ZERO

ZERO

ZERO $7 \mathrm{E}-09$ ZERO
ZERO ZERO ZERO ZERO ZERO ZERO ZERO $0.8060 \mathrm{E}-12$ 
Table A-1. HLW Flowsheet Material Balance (Tank 51/ITP Cycle 1) Section 1B. Re-precipitation and late Washing.

STREAM NUMBERS (CONT'D) $\rightarrow$ STREAM NAME

$38 \mathrm{SO} 2$

141 SO3

137 Semi Vol Cs2O

136 Semi Vol. CsCl

126 Semi Vol Group A

140 Semi Vo1 Na2B4O7

138 Semi Vol NaCl

139. Semi Vol Nat

125 Semi Vol NaI

23 Semi Vol RuO2

$111 \mathrm{siO2}$

$48 \mathrm{Sr}(\mathrm{COOH}) 2$

$176 \mathrm{Sr}$ (NO3) 2

$153 \mathrm{Sr}$ (NaTi2O5) 2

$127 \mathrm{Sr}(\mathrm{OH}) 2$

$30 \quad \operatorname{srcO3}$

46 sxo

$159 \mathrm{TcO} 2$

92 ThO2

119 Tritium

$\begin{array}{rl}119 & \text { Triti } \\ 17 & 0308\end{array}$

$62 \mathrm{VO} 2(\mathrm{COOH}) 2$

$177 \mathrm{UO2}$ (NO3) 2

$16 \mathrm{UO} 2(\mathrm{OH}) 2$

$27 . \mathrm{Y}(\mathrm{COOH})$

$178 \mathrm{Y}(\mathrm{NO} 3)$

$128 \mathrm{Y}(\mathrm{OH}) 3$
31 Y $2(\mathrm{CO} 3) 3$

47 Y 203

100 zeolite

$44 \mathrm{Zn}(\mathrm{COOH}) 2$

$179 \mathrm{Zn}(\mathrm{NO} 3) 2$

$57 \mathrm{nO}$

13 hydrate $\mathrm{H} 2 \mathrm{O}$

1 water

TOTAL FLOW, LB/HR

TEMPERATURE, DEG C

PRESSURE， ATM

PRESSURE, PSIG

PRESSURE, MM HG

ENTHALPY， PCU/HR

VAPOR FLOW, CFM

IIQUID FLOW, GPM

DENSITY， LBS / FT 3

PHASE

WSRC-TR-95-0019 (Revision
418

655

656 Prec Storage Late Wash Fd Late Wash Fd
Tank bottoms Prec Soluble Prec Insolub

ZERO
ZERO
ZERO
ZERO
ZERO
ZERO
ZERO
ZERO
ZERO
$0.2666 E-01$
ZERO
ZERO
$0.4072 E-02$
$0.6716 E-08$
$0.9081 E-04$
ZERO
ZERO
ZERO
$0.1213 E-02$
$0.3525 E-10$
$0.7512 E-03$
ZERO
ZERO
$0.2900 E-04$
ZERO
ZERO
$0.3135 E-08$
ZERO
$0.9683 E-03$
ZERO
ZERO
$0.1440 E-02$
$0.1980 E+00$
ZERO
$0.3589 E-01$
$0.4056 E+03$

$0.4655 \mathrm{E}+03$

$0.3846 \mathrm{E}+02$

$0.9656 \mathrm{E}+00$

$-0.5055 \mathrm{E}+00$

$0.7339 \mathrm{E}+03$

$0.1579 \mathrm{E}+05$

$0.9016 \mathrm{E}+00$

$37 \mathrm{E}+02$
IIOUID ZERO ZERO ZERO

ZERO

ZERO

ZERO

ZERO

ZERO

ZERO

ZERO

ZERO
$0.6716 \mathrm{E}-08$

ZERO
ZERO

ZERO

ZERO

ZERO

$0.3525 \mathrm{E}-10$

ZERO
ZERO

ZERO

$0.2900 \mathrm{E}-04$

ZERO

ZERO
$0.3135 \mathrm{E}-08$

ZERO

ZERO

ZERO

$0.1440 \mathrm{E}-02$

ZERO

ZERO

$0.4056 \mathrm{E}+03$

$0.4195 \mathrm{E}+03$

$0.4072 \mathrm{E}-02$

$0.9081 \mathrm{E}-04$

ZERO

$0.1213 E-$ ZERO

ZERO

$0.1980 \mathrm{E}+00$

ZERO
$0.3589 \mathrm{E}-01$

ZERO
$0.4561 \mathrm{E}+02$

$0.3846 \mathrm{E}+02$

$0.9656 \mathrm{E}+00$

$0.3846 \mathrm{E}+02$

$0.9656 \mathrm{E}+00$

$0.7339 \mathrm{E}+03 \quad 0.7339 \mathrm{E}+03$

$0.1576 \mathrm{E}+05$

$0.2821 \mathrm{E}+02$

$0.8208 \mathrm{E}+00$

$0.8042 E-01$

$6372 \mathrm{E}+02$

$0.7071 E+02$

LIQUID

458

NaTPB addn

to LWPT

ZERO

ZERO

ZERO

ZERO

ZERO

ZERO

ZERO

ZERO

ZERO

ZERO

ZERO

ZERO

ZERO

ZERO

ZERO

ZERO

ZERO

ZERO

ZERO

ZERO

ZERO

ZERO

ZERO

ZERO

ZERO

ZERO

ZERO

ZERO

ZERO

ZERO

ZERO

ZRRO

ZERO

$0.1287 \mathrm{E}+02$

$0.1581 \mathrm{E}+02$

$0.35000+02$

$0.1000 \mathrm{E}+01$

ZERO

$0.4525 \mathrm{E}+03$

$0.6358 \mathrm{E}+02$

LIQUID

Date: January 1995

661 LWPT Precip Trnsf X-Filtr Tube

661 LWPT Precip Trnsf X-Filtr Tube Heel Line Flush Fltr to LWPT ZERO ZERO ZERO ZERO ZERO Z ZERO ZERO ZERO ZERO ZERO ZERO $\quad$ ZERO ZERO ZERO $\quad$ ZERO ZERO ZERO ZERO ZERO ZERO ZERO ZERO ZERO ZERO ZERO ZERO $\quad$ ZERO

$\begin{array}{rrr}0.3379 E-02 & \text { ZERO } & \text { ZERO } \\ 0.02 R O & \text { ZERO }\end{array}$

$\begin{aligned} \text { ZERO } & \text { ZERO ZERO }\end{aligned}$

$\begin{array}{rrr}\text { ZERO } & \text { ZERO } & \text { ZERO } \\ 0.5162 \mathrm{E}-03 & \text { ZERO } & \text { ZERO }\end{array}$

$0.9393 \mathrm{E}-13$ ZERO $0.5128 \mathrm{E}-13$

$0.1151 E-04$ ZERO ZERO

$\begin{array}{lll}\text { ZERO } & \text { ZERO } & \text { ZERO } \\ \text { ZERO } & \text { ZERO } & \text { ZERO }\end{array}$

ZERO

$0.1538 \mathrm{ZERO}$

$0.1625 \mathrm{E}-12$

ZERO

$0.1362 \mathrm{E}-06$

ZERO

$0.1043 \mathrm{E}-12$

ZERO

0.1227 E-03

ZERO

$0.6763 \mathrm{E}-05$

$0.2509 \mathrm{E}-01$

ZERO

$0.549 \mathrm{E}-02$

$0.6037 \mathrm{E}+02$

$0.3619 \mathrm{E}+02$

$0.9656 \mathrm{E}+00$

$-0.5055 E+00$

$0.7339 \mathrm{E}+03$
$0.1969 \mathrm{E}+04$

ZERO

ZERO

ZERO

ZERO

ZERO

ZERO

ZERO

Z ZERO

ZERO

ZERO

ZERO

ZERO

ZERO

ZERO

ZERO

ZERO

$0.275 .1 \mathrm{E}+02$

$0.2752 \mathrm{E}+02$

$0.3500 \mathrm{E}+02$

$0.1000 \mathrm{E}+01$

ZERO

$0.9653 \mathrm{E}+03$

$0.3619 \mathrm{E}+02$

$0.9656 \mathrm{E}+00$

$0.5055 \mathrm{E}+00$

$0.7339 \mathrm{E}+03$
$0.1659 \mathrm{E}+03$

$0.1190 \mathrm{E}+00$

$0.5495 \mathrm{E}-01$

$0.9158 \mathrm{E}-02$

ITOUID

$.6244 \mathrm{E}+02$

$0.9158 \mathrm{E}-02$

LIQUID

Section 1B, Page 5 of 21

. 
Table A-1. HLW Flowsheet Material Balance (Tank 51/ITP Cycle 1) Section 1B. Re-precipitation and Late Washing

STREAM NUMBERS -> STREAM NAME

COMPONENT FLOWS, LB/HR 122 (C6H5)

123. (C6H5) $2 \mathrm{C} 6 \mathrm{H}$

118 (C6H5) $2 \mathrm{NH}$

133 (C6H5B (OH) 2 salt)

132 ( $\mathrm{C} 6 \mathrm{H} 5 \mathrm{~B}(\mathrm{OH}) 2$ )

166 (C6H5C6H4OH)

162 (C6H5NH2)

163 (C6H5NHCHO)

165 (C6H5NO)

131 (C6H5OH salt)

130 ( $6 \mathrm{H} 5 \mathrm{OH})$

121 (C6H6)

155. (СH3) $2 \mathrm{CHOH}$

154 (СН3OH)

$45 \mathrm{Ag}$

116 Ag2O

134 AgNO3
77 Al2O3

$106 \cdot \mathrm{B} 203$

$9 \mathrm{Ba}(\mathrm{OH}) 2$

$15 \mathrm{BaO}$

$24 \mathrm{BaSO} 4$

$66 \mathrm{CO}$

$37 \mathrm{CO} 2$

$142 \mathrm{CO} 2$ ( $\mathrm{C} 14$ )

$63 \mathrm{Ca}(\mathrm{COOH}) 2$

$120 \mathrm{Ca}(\mathrm{OH}) 2$

$113 \mathrm{Ca} 3(\mathrm{PO} 4) 2$

$20 \mathrm{CaC} 204$

$18 \mathrm{CaCO} 3$

$64 \mathrm{CaCO}^{3}$ (C14)

78 CaF2

41 CaO

29 Carbon

99 Cement

$26 \mathrm{Co}(\mathrm{COOH}) 2$

$168 \mathrm{Co}(\mathrm{NO} 3) 2$

$59 \mathrm{COO}$

$98 \quad \mathrm{Cr} 203$

$42 \mathrm{Cs} 2 \mathrm{O}$

$53 \mathrm{CsCOOH}$

$96 \mathrm{CsCl}$

$169 \mathrm{CsNO}_{3}$

151 CsTPB

WSRC-TR-95-0019 (Revision

665

419

666

660

457

667

X-Filtr Tube X-Flow Filtr Precip slrry Permeate dur NaTPB add to Late Wsh H2O Permeate frm H2O Flush H2O Inventry b4 Late Wash Concentrat'n Late Wsh H2O Adjd w/NaTPB Late Wash

ZERO
ZERO
ZERO
ZERO
ZERO
ZERO
ZERO
ZERO
ZERO
ZERO
ZERO
ZERO
ZERO
ZERO
ZERO
ZERO
ZERO
ZERO
ZERO
ZERO
ZERO
ZERO
ZERO
ZERO
ZERO
ZERO
ZERO
ZERO
ZERO
ZERO
ZERO
ZERO
ZERO
ZERO
ZERO
ZERO
ZERO
ZERO
ZERO
ZERO
ZERO
ZERO
ZERO
ZERO
ZERO
ZERO
ZERO
ZERO

ZERO
ZERO
ZERO
ZERO
ZERO
ZERO
ZERO
ZERO
ZERO
ZERO
ZERO
ZERO
ZERO
ZERO
ZERO
ZERO
ZERO
ZERO
ZERO
ZERO
ZERO
ZERO
ZERO
ZERO
ZERO
ZERO
ZERO
ZERO
ZERO
ZERO
ZERO
ZERO
ZERO
ZERO
ZERO
ZERO
ZERO
ZERO
ZERO
ZERO
ZERO
ZERO
ZERO
ZERO
ZERO
ZERO
ZERO
ZERO

$0.3711 \mathrm{E}+00$
$0.1576 \mathrm{E}-01$
$0.1049 \mathrm{E}-01$
$0.4183 \mathrm{E}+00$
ZERO
$0.3409 \mathrm{E}-01$
ZERO
ZERO
ZERO
ZERO

$0.2768 \mathrm{E}+00$

ZERO
E+OO

$0.2424 \mathrm{E}+00$

$0.1908 \mathrm{E}-08$

ZERO
$0.3441 \mathrm{E}-02$

ZE-O2
ZERO

$.1462 \mathrm{E}+00$

$0.1882 \mathrm{E}-02$

$0.1070 \mathrm{E}-14$

$0.2020 \mathrm{E}-02$

$\mathrm{E}-02$

ZERO

ZERO

$0.3042 \mathrm{E}-04$

$0.3138 \mathrm{E}-05$

$0.6318 \mathrm{E}+00$

ZERO
ZERO

ZERO

ZERO

ZERO

$0.2154 \mathrm{E}+00$

ZERO
ZERO

ZERO

$0.1194 \mathrm{E}-04$

$0.1158 \mathrm{E}-03$

$0.2948 \mathrm{E}+00$

$0.4335 \mathrm{E}-04$

ZERO

ZERO

$0.3985 \mathrm{E}-0$

$0.1240 E+00$

Date: January 1995
$0.8932 \mathrm{E}-05$
ZERO

$\therefore \quad$ ZERO ZERO ZERO ZERO ZERO

ZERO

ZERO

ZERO

$0.6204 \mathrm{E}-01$

ZERO

$0.5433 \mathrm{E}-01$

$0.1557 \mathrm{E}-08$

ZERO

ZERO

ZERO

ZERO

$0.2398 \mathrm{E}-15$

ZERO

ZERO

ZERO

ZERO

ZERO

$0.7034 \mathrm{E}-06$

ZERO

ZERO

ZERO

ZERO

ZERO

ZERO

ZERO

ZERO

$0.2676 \mathrm{E}-05$

ZERO

ZERO

ZERO

ZERO

ZERO
ZERO ZERO

ZERO ZERO

$\begin{array}{lll}\text { ZERO } & \text { ZERO } & \text { ZERO } \\ \text { ZERO } & \text { ZERO } & \text { ZERO }\end{array}$

ZERO ZERO ZERO

ZERO ZERO $\quad$ ZERO

ZERO ZERO

ZERO ZERO ZERO

ZERO ZERO ZERO

$\begin{array}{lll}0.1129 \mathrm{E}+00 & 0.1129 \mathrm{E}+00 & 0.3378 \mathrm{E}+00\end{array}$

ZERO ZERO

ZERO

ZERO ZERO

ZERO

ZERO

ZERO

ZERO

ZERO

ZERO

ZERO

ZERO

ZERO

ZERO

ZERO

ZERO

ZERO

ZERO

ZERO

ZERO

ZERO

ZERO

ZERO

ZERO

ZERO

ZERO

ZERO

ZERO

ZERO

ZERO

ZERO

ZERO

ZERO

ZERO

ZERO

ZERO

ZERO

ZERO

ZERO

ZERO

ZERO

ZERO

ZERO

ZERO

ZERO

ZERO

ZERO

ZERO

ZERO

ZERO

ZERO

ZERO

ZERO

ZERO

ZERO

ZERO

ZERO

ZERO

ZERO

ZERO

ZERO

ZERO

ZERO

ZERO

ZERO

ZERO

ZERO

ZERO

$0.1805 \mathrm{E}-08$

$0.1980 \mathrm{E}-0$

ZERO

ZERO

ZERO

ZERO

$0.1012 \mathrm{E}-1$

ZERO

ZERO

ZERO

ZERO

ZERO

ZERO

ZERO

ZERO

ZERO

ZERO

ZERO

ZERO

ZERO

ZERO

$0.1129 \mathrm{E}-04$

zाRO

ZERO

ZERO

ZERO

ZERO

ZERO

Section 1B, Page 6 of 21 
Table A-1. HLW Flowsheet Material Balance (Tank 51/ITP Cycle 1)

Section $1 B$. Re-precipitation and Late Washing

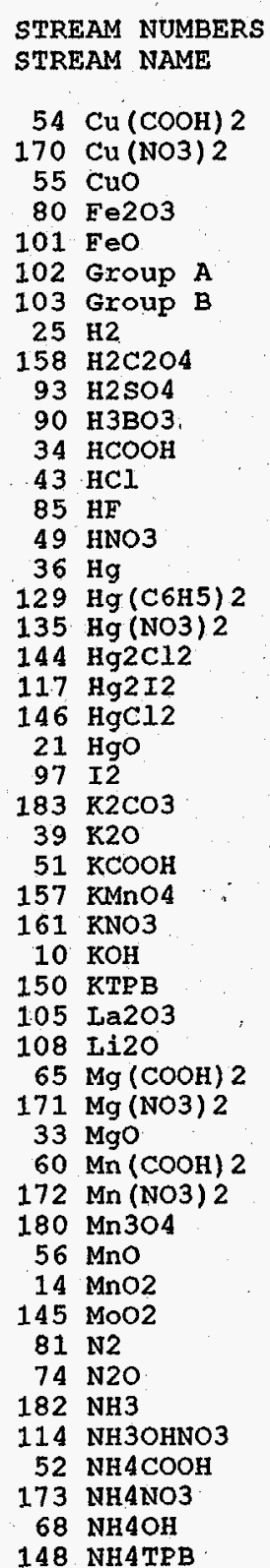

664.665

419

666.

660

457

667

X-Filtr Tube X-Flow Filtr Precip slrry Permeate dur NaTPB add to Late Wsh H2O Permeate frm lush H2O Inventry b4 Late Wash Concentrat'n Late Wsh H2O Adjd w/NaTPB

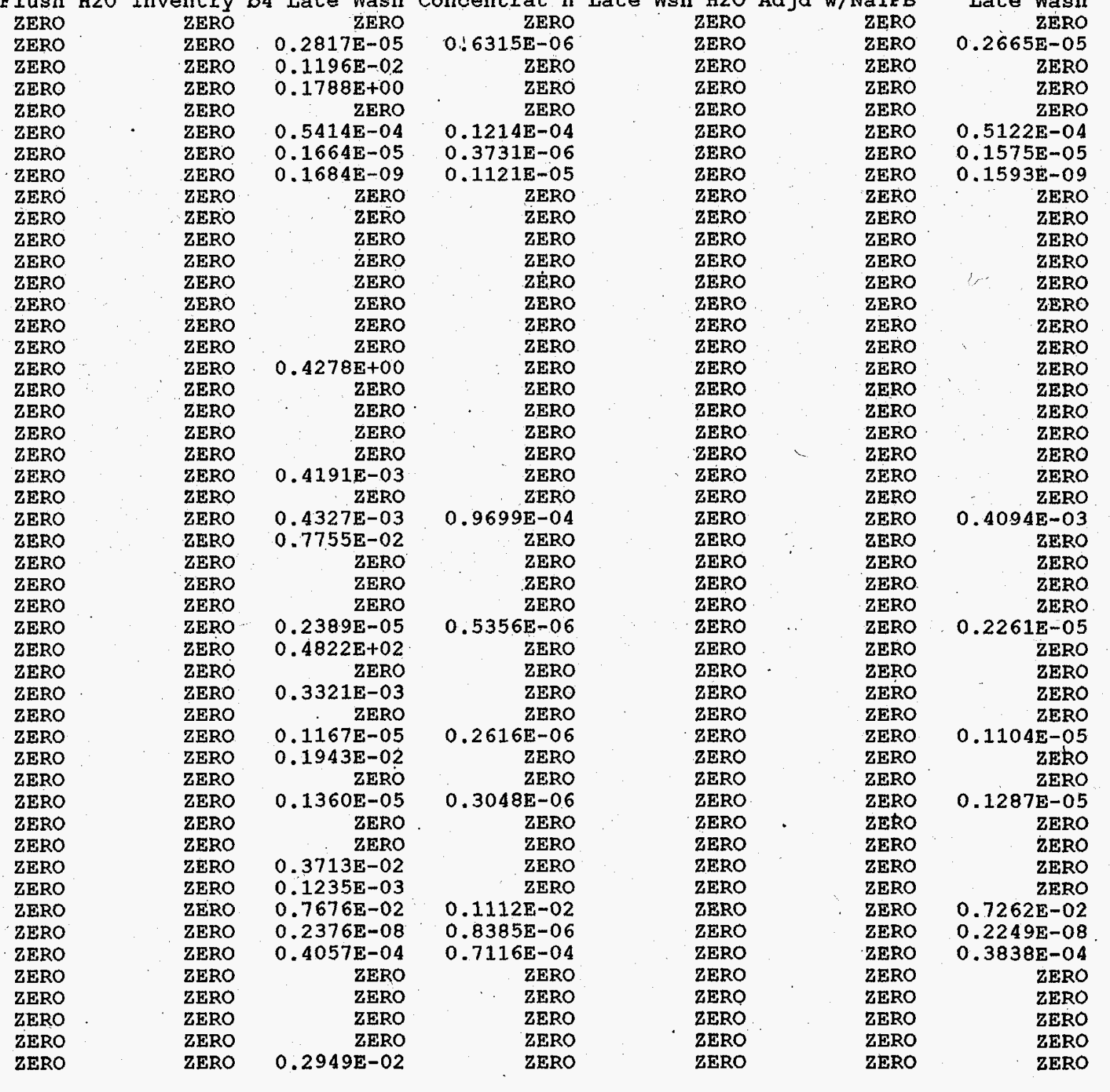


Table A-1. HLW Flowsheet Material Balance (Tank 51/ITP Cycle 1)

Section 1B. Re-precipitation and Late Washing

STREAM NUMBERS (CONT'D) $\rightarrow$ STREAM NAME

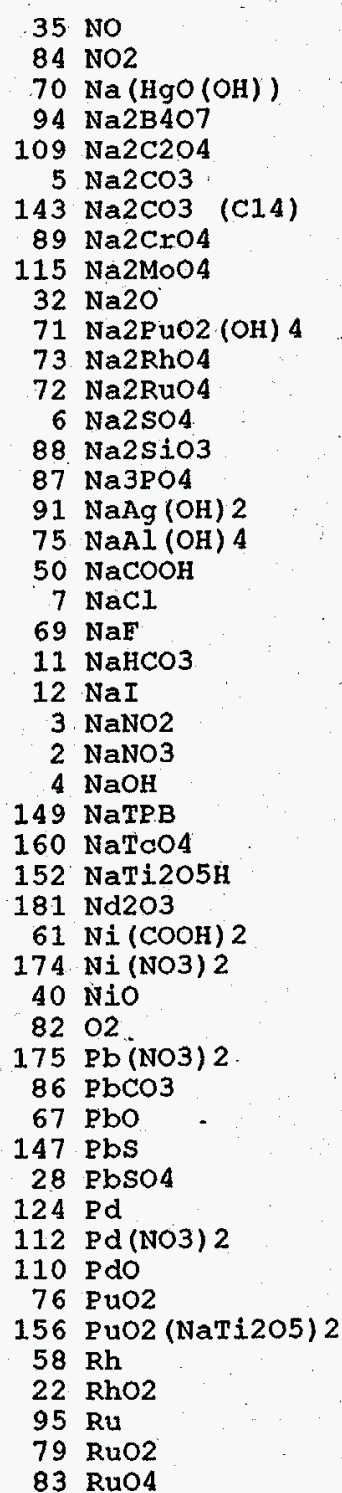


Table A-1. HLW Flowsheet Material Balance (Tank 51/ITP Cycle 1 ) Section 1B. Re-precipitation and Late washing

STREAM NUMBERS (CONT'D) $\rightarrow$ STREAM NAME

$38 \quad 502$

$141 \mathrm{SO} 3$

137 Semi Vol Cs2O

136 Semi Vol Cscl

126 Semi Vol Group A

140 Semi Vol Na2B407

138 Semi Vol NaCl

139 semi Vol NaF

125 Semi Vol NaI
23 semi Vol RuO2

$23 \mathrm{Semi}$
$111 \mathrm{SiO} 2$

$48 \mathrm{Sr}(\mathrm{COOH}) 2$

$176 \operatorname{Sr}$ (NO3) 2

$153 \mathrm{Sr}(\mathrm{NaTi2O5)} 2$

$127 \operatorname{Sr}(\mathrm{OH}) 2$

30 SrCO3

46 Sro

$159 \mathrm{TCO} 2$

92 ThO2

107 TiO2

119 Tritium
17 U308

$62 \mathrm{UO} 2$ ( $\mathrm{COOH}) 2$

177 VO2 (NO3) 2

$16 \mathrm{VO}(\mathrm{OH}) 2$

$178 \mathrm{Y}$ (NO3) 3

$128 \mathrm{Y}(\mathrm{OH}) 3$

$31 \mathrm{Y} 2(\mathrm{CO} 3) 3$

100 Zeolite

$44 \mathrm{Zn}(\mathrm{COOH}) 2$

$179 \mathrm{zn}$ (NO3) 2

$57 \mathrm{Zno}$

$104 \mathrm{zrO} 2$

13 hydrate $\mathrm{H} 2 \mathrm{O}$

1 water

TOTAL FLOW, LB/HR

419

666

660

457

667

Ilt Tube X-Flow Filtr Precip slrry Permeate dur NaTPB add to Late Wsh H2O Permeate frm

H2O Flush H2O Inventry b4 Late Wash Concentrat'n Late Wsh H2O Adjd w/NaTPB Late Wash

$\begin{array}{lllllll}\text { ZERO } & \text { ZERO } & \text { ZERO } & \text { ZERO } & \text { ZERO } & \text { ZERO } & \text { ZERO } \\ \text { ZERO } & \text { ZERO } & \text { ZERO } & \text { ZERO } & \text { ZERO } & \text { ZERO } & \text { ZERO } \\ \text { ZERO } & \text { ZERO } & \text { ZERO } & \text { ZERO } & \text { ZERO } & \text { ZERO } & \text { ZERO }\end{array}$

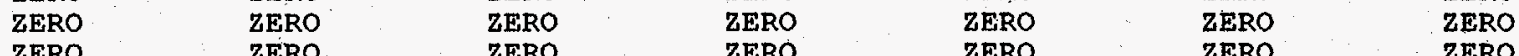

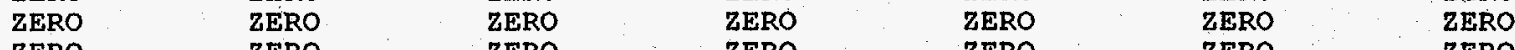

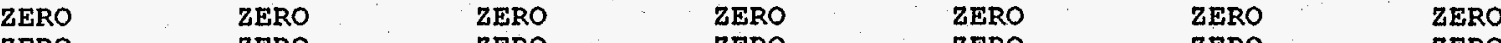

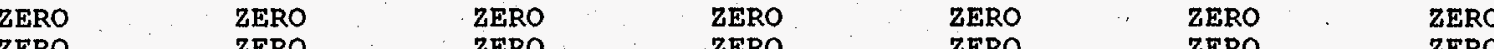

ZERO ZERO

ZERO ZERO Z ZERO ZERO Z ZERO

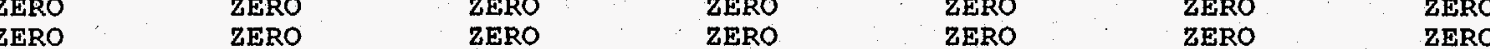

$\begin{array}{llrrrrr}\text { ZERO } & \text { ZERO } & \text { ZERO } & \text { ZERO } & \text { ZERO } & \text { ZERO } & \text { ZERO } \\ \text { ZERO } & \text { ZERO } & 0.3003 \text { EE-01 } & \text { ZERO } & \text { ZERO } & \text { ZERO } & \text { ZERO }\end{array}$

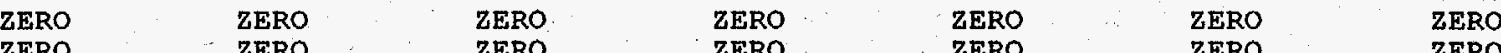

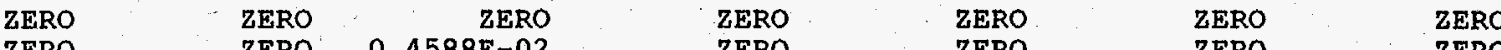

$\begin{array}{llllll}\text { ZERO ZERO } & 0.4588 \mathrm{E}-02 & \text { ZERO } & \text { ZERO } & \text { ZERO } & \text { ZERO }\end{array}$

ZERO ZERO $0.1646 \mathrm{E}-10$ 0.3690E-11 $\quad$ ZERO $\quad$ ZERO $0.1557 \mathrm{E}-10$

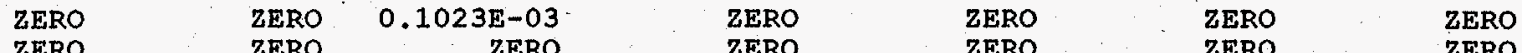

ZERO ZERO $\quad$ ZERO $\quad$ ZERO

ZERO ZERO ZERO

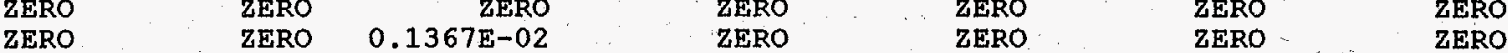

$\begin{array}{lllllll}\text { ZERO } & \text { ZERO } & 0.1367 \mathrm{E}-02 & \text { ZERO } & \text { ZERO } & \text { ZERO } & \text { ZERO } \\ \text { ZERO } & \text { ZERO } & 0.2847 \mathrm{E}-10 & 0.6501 \mathrm{E}-11 & \text { ZERO } & \text { ZERO } & 0.2694 \mathrm{E}-10\end{array}$

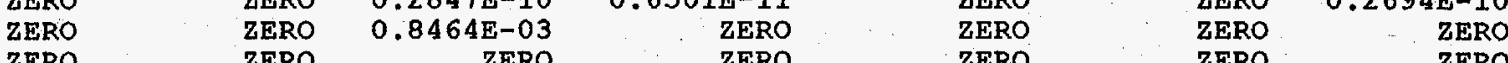

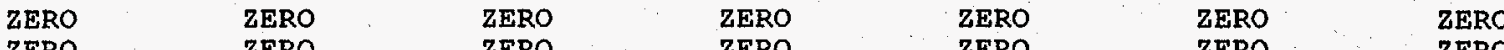

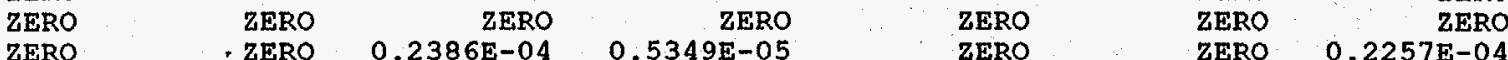

$\begin{array}{lllllll}\text { ZERO } & \text { ZERO } & 0.2386 \mathrm{E}-04 & 0.5349 \mathrm{E}-05 & \text { ZERO } & \text { ZERO } & 0.2257 \mathrm{E}-04\end{array}$

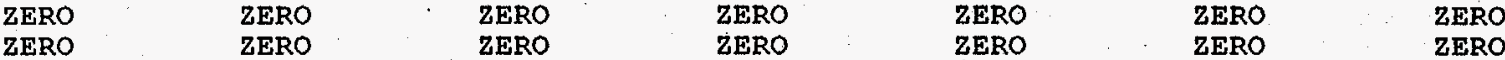

$\begin{array}{llllll}\text { ZERO ZERO } & 0.1828 \mathrm{E}-10 & 0.4099 \mathrm{E}-11 & \text { ZERO } & \text { ZERO } & 0.1730 \mathrm{E}-10\end{array}$

$\begin{array}{llllll}\text { ZERO } & \text { ZERO } & \text { ZERO } & \text { ZERO } & \text { ZERO } & \text { ZERO } \\ \text { ZERO }\end{array}$

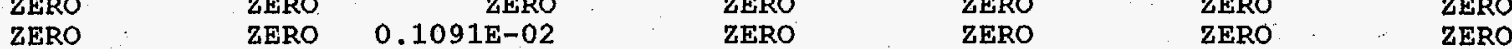

$\begin{array}{llllll}\text { ZERO } & \text { ZERO } & 0.1091 E-02 & \text { ZERO } & \text { ZERO } & \text { ZERO } \\ \text { ZERO } & \text { ZERO } & \text { ZERO } & \text { ZERO } & \text { ZERO } & \text { ZERO }\end{array}$

$\begin{array}{lllll}\text { ZERO } & \text { ZERO } & \text { ZERO } & \text { ZERO } & \text { ZERO }\end{array}$

$\begin{array}{llrl}\text { ZERO } & \text { ZERO } & \text { ZERO } & \text { ZERO }\end{array}$

$\begin{array}{llllll}\text { ZERO } & \text { ZERO } & 0.1185 \mathrm{E}-02 & 0.2657 \mathrm{E}-03 & \text { ZERO } & \text { ZERO } \\ \text { ZERO } & \text { ZERO } & 0.2231 \mathrm{E}+00 & & \text { ZERO } & \text { ZERO }\end{array}$

$\begin{array}{rrrrrr}\text { ZERO } & \text { ZERO } & 0.2231 \mathrm{E}+00 & \text { ZERO } & \text { ZERO } & \text { ZERO } \\ \text { ZERO } & \text { ZERO } & \text { ZERO } & \text { ZERO } & \text { ZERO } & \text { ZERO }\end{array}$

ZERO ZERO $0.4044 \mathrm{E}-01$

$0.7107 \mathrm{E}+02$

$0.7109 \mathrm{E}+02$

ZERO $0.4633 \mathrm{E}+03$

$0.1054 \mathrm{E}+03$

$0.1083 \mathrm{E}+03 \quad 0.1129 \mathrm{E}+02$

$0.1409 \mathrm{E}+04$

$0.1411 \mathrm{E}+\mathrm{b4}$

$0.1121 \mathrm{E}-02$

$1 E-02$
ZERO

ZERO

$0.1386 \mathrm{E}+04$

$0.1400 \mathrm{E}+04$

TEMPERATURE, DEG C

$0.3500 \mathrm{E}+02 \quad 0.3978 \mathrm{E}+02 \quad 0.3000 \mathrm{E}+02$

$0.3500 \mathrm{E}+02$

$0.3500 \mathrm{E}+02$

$0.3619 \mathrm{E}+02$

ZERO

RTOPO

E+01

$-0.5055 \mathrm{E}+00$

PRESSURE, PSIG

PRESSURE, MM HG

VAPOR FLOW, CFM

$0.2494 \mathrm{E}+04$

$0.7339 \mathrm{E}+03$

$0.3192 \mathrm{E}+04$

$0.3230 \mathrm{E}+03$

$0.4943 E+05$

$0.1419 \mathrm{E}+00$

$0.1031 \mathrm{E}+01 \quad 0.2129 \mathrm{E}+00$

ZERO $\quad 0.6406 \mathrm{E}+02$

$0.6339 \mathrm{E}+02$

LIQUID

$0.2213 \mathrm{E}-01$

$0.6358 \mathrm{E}+02$

LIQUID

$0.2817 \mathrm{E}+01$

$0.6245 \mathrm{E}+02$

IIQUID

$0.2781 F+01$

$0.6275 \mathrm{E}+02$

LIQUID 
Table A-1. HLW Flowsheet Material Balance (Tank 51/ITP Cyale 1) Section 1B. Re-precipitation and Late Washing

STREAM NUMBERS -> STREAM NAME

COMPONENT FLOWS, IB/HR 122 (C6H5) 2

123 (C6H5) $2 \mathrm{C} 6 \mathrm{H} 4$

118 (C6H5) $2 \mathrm{NH}$

133 (C6H5B(OH) 2 salt).

132 (C6H5B (OH) 2)

166 (C6H5C6H4OH)

163 (C6H5NHCHO)

165 (C6H5NO)

164 (C6H5NO2)

131 (C6H5OH salt)

130 (C6H5OH)

121 (C6H6)

154 (CH3OH)

$45 \mathrm{Ag}$

116 Ag20

134 AgNO3
77 A12O3

106 B203

$9 \mathrm{Ba}(\mathrm{OH}) 2$

15 BaO

$24 \mathrm{BaSO} 4$

$66 \mathrm{CO}$

$142 \mathrm{CO} 2(\mathrm{C14})$

$63 \mathrm{Ca}(\mathrm{COOH}) 2$

$167 \mathrm{Ca}(\mathrm{NO} 3) 2$
$120 \mathrm{Ca}(\mathrm{OH}) 2$

$113 \mathrm{Ca} 3(\mathrm{PO} 4) 2$

$20 \mathrm{CaC} 2 \mathrm{O} 4$

$18 \mathrm{CaCO} 3$

$64 \mathrm{CaCO}^{3}(\mathrm{C14})$

78 CaF2

$41 \mathrm{CaO}$

$19 \mathrm{CaSO} 4$

29 Carbon

$26 \mathrm{Co}(\mathrm{COOH}) 2$

168 Co (NO3) 2

$59 \mathrm{CoO}$

$98 \mathrm{Cr} 203$

$53 \mathrm{CsCOOH}$

$96 \mathrm{CsCl}$

$169 \mathrm{CsNO} 3$

$8 \mathrm{CsOH}$

CsTPB

WSRC-TR-95-0019 (Revision
455
N2 Purge $\quad 456$
LWPT Vent

to LWPT to stack

668

669

670

671

LWHT 2 wt? H2C2O4 2 wto NaOH X-Flow Filtr Heel addn to LWHT addn to LWHT H2O Flush

459

$\begin{array}{lr}\text { ZERO } & 0.2559 \mathrm{E}-07 \\ \text { ZERO } & 0.1087 \mathrm{E}-08 \\ \text { ZERO } & 0.7235 \mathrm{E}-09 \\ \text { ZERO } & 0.2884 \mathrm{E}-10 \\ \text { ZERO } & \text { ZERO } \\ \text { ZERO } & \text { ZERO } \\ \text { ZERO } & \text { ZERO } \\ \text { ZERO } & \text { ZERO } \\ \text { ZERO } & \text { ZERO } \\ \text { ZERO } & \text { ZERO }\end{array}$

$0.1908 \mathrm{E}-10$

$$
\text { ZERO }
$$

$0.1671 \mathrm{E}-07$

$0.5036 \mathrm{E}-08$

$0.4380 \mathrm{E}-09$

ZERO
$0.2372 \mathrm{E}-12$ $2 \mathrm{E}-12$
ZERO

$0.1008 \mathrm{E}-10$

$0.1298 \mathrm{E}-12$

TRACE

ZERO

ZERO

ZERO

ZERO

ZERO

ZERO

ZERO

$0.1334 \mathrm{E}-01$

ZERO

$0.2480 \mathrm{E}-03$

$0.4111 \mathrm{E}-10$

$.4829 \mathrm{E}-11$
ZERO

ZERO

ZERO

ZERO

$0.4176 \mathrm{E}-16$

ZERO
ZERO

$0.1393 \mathrm{E}-12$

ZERO

ZERO

ZERO

ZERO

$0.2164 \mathrm{E}-15$

$0.4356 \mathrm{E}-10$

ZERO

ZERO

ZERO

$0.1485 \mathrm{E}-10$

ZERO

ZERO

ZERO

ZERO

ZERO

ZERO

ZERO

ZERO

ZERO

ZERO

ZERO

ZERO

ZERO

ZERO

ZERO

$0.7984 \mathrm{E}-14$

$0.2033 \mathrm{E}-10$

$0.2989 \mathrm{E}-14$

ZERO

ZERO

$0.2748 \mathrm{E}-14$

$0.8549 \mathrm{E}-11$

$0.1555 \mathrm{E}-05$

ZERO

ZERO
ZERO
ZERO
ZERO
ZERO
ZERO
ZERO
ZERO
ZERO
ZERO
ZERO
ZERO
ZERO
ZERO
ZERO
ZERO
ZERO
ZERO
ZERO
ZERO
ZERO
ZERO
ZERO
ZERO
ZERO
ZERO
ZERO
ZERO
ZERO
ZERO
ZERO
ZERO
ZERO
ZERO
ZERO
ZERO
ZERO
ZERO
ZERO
ZERO
ZERO
ZERO
ZERO
ZERO
ZERO
ZERO
ZERO
ZERO

Date: January 1995

\begin{tabular}{lll} 
ZERO & ZERO & ZERO \\
ZERO & ZERO & ZERO \\
ZERO & ZERO & ZERO \\
ZERO & ZERO & ZERO \\
ZERO & ZERO & ZERO \\
ZERO & ZERO & ZERO \\
ZERO & ZERO & ZERO \\
ZERO & ZERO & ZERO \\
ZERO & ZERO & ZERO \\
ZERO & ZERO & ZERO \\
ZERO & ZERO & ZERO \\
ZERO & ZERO & ZERO \\
ZERO & ZERO & ZERO \\
ZERO & ZERO & ZERO \\
ZERO & ZERO & ZERO \\
ZERO & ZERO & ZERO \\
ZERO & ZERO & ZERO \\
ZERO & ZERO & ZERO \\
ZERO & ZERO & ZERO \\
ZERO & ZERO & ZERO \\
ZERO & ZERO & ZERO \\
ZERO & ZERO & ZERO \\
ZERO & ZERO & ZERO \\
ZERO & ZERO & ZERO \\
ZERO & ZERO & ZERO \\
ZERO & ZERO & ZERO \\
ZERO & ZERO & ZERO \\
ZERO & ZERO & ZERO \\
ZERO & ZERO & ZERO \\
ZERO & ZERO & ZERO \\
ZERO & ZERO & ZERO \\
ZERO & ZERO & ZERO \\
ZERO & ZERO & ZERO \\
ZERO & ZERO & ZERO \\
ZERO & ZERO & ZERO \\
ZERO & ZERO & ZERO \\
ZERO & ZERO & ZERO \\
ZERO & ZERO & ZERO \\
ZERO & ZERO & ZERO \\
ZERO & ZERO & ZERO \\
ZERO & ZERO & ZERO \\
ZERO & ZERO & ZERO \\
ZERO & ZERO & ZERO \\
ZERO & ZERO & ZERO \\
ZERO & ZERO & ZERO \\
ZERO & ZERO & ZERO \\
ZERO & ZERO & ZERO \\
ZERO & ZERO & ZERO \\
\hline
\end{tabular}


Table A-1. HiW Flowsheet Material Balance (Tank 51/ITP Cycle 1)

section 1B. Re-precipitation and Late Washing

\begin{tabular}{|c|c|c|c|}
\hline \multicolumn{2}{|c|}{$\begin{array}{l}\text { STREAM NUMBERS } \\
\text { STREAM NAME }\end{array}$} & \multirow[t]{2}{*}{ (CONT'D) $\rightarrow$} & $\begin{array}{r}455 \\
\text { Air/N2 Purge } \\
\text { to LWPT }\end{array}$ \\
\hline 54 & $\mathrm{Cu}(\mathrm{COOH}) 2$ & & ZERO \\
\hline 170 & $\mathrm{Cu}(\mathrm{NO} 3)$ & & ZERO \\
\hline 55 & $\mathrm{CuO}$ & & ZERO \\
\hline 80 & $\mathrm{Fe} 2 \mathrm{O}_{3}$ & & ZERO \\
\hline 101 & $\mathrm{FeO}$ & & ZERO \\
\hline 102 & Group A & & ZERO \\
\hline 103 & Group B & & ZERO \\
\hline 25 & $\mathrm{H} 2$ & & ZERO \\
\hline 158 & H $2 \mathrm{C} 204$ & & ZERO \\
\hline 93 & $\mathrm{H} 2 \mathrm{SO} 4$ & & ZERO \\
\hline 90 & Н3ВО3. & & ZERO \\
\hline 34 & $\mathrm{HCOOH}$ & • & ZERO \\
\hline 43 & $\mathrm{HCl}$ & & ZERO \\
\hline 85 & $\mathrm{HF}$ & & ZERO \\
\hline 49 & HNO3 & & ZERO \\
\hline 36 & $\mathrm{Hg}$ & & ZERO \\
\hline 129 & $\mathrm{Hg}(\mathrm{C} 6 \mathrm{H} 5) 2$ & & ZERO \\
\hline 135 & $\mathrm{Hg}(\mathrm{NO} 3)_{2}$ & & ZERO \\
\hline 144 & $\mathrm{Hg} 2 \mathrm{Cl2}$ & & ZERO \\
\hline 117 & $\mathrm{Hg} 2 \mathrm{I} 2$ & & ZERO \\
\hline 146 & $\mathrm{HgCl} 2$ & & ZERO \\
\hline 21 & $\mathrm{HgO}$ & & ZERO \\
\hline 97 & $\mathrm{I} 2$ & . & ZERO \\
\hline 183 & $\mathrm{~K} 2 \mathrm{CO} 3$ & & ZERO \\
\hline 39 & $\mathrm{~K} 2 \mathrm{O}$ & & ZERO \\
\hline 51 & $\mathrm{KCOOH}$ & & ZERO \\
\hline 157 & $\mathrm{KMnO} 4$ & & ZERO \\
\hline 161 & KNO3 & & ZERO \\
\hline 10 & $\mathrm{KOH}$ & & ZERO \\
\hline 150 & KTPB & & ZEERO \\
\hline 105 & $\mathrm{La203}$ & & ZERO \\
\hline 108 & $\mathrm{Li} 20$ & & ZERO \\
\hline 65 & $\mathrm{Mg}(\mathrm{COOH}) 2$ & & ZERO \\
\hline 171 & $\mathrm{Mg}(\mathrm{NO} 3)_{2}$ & & ZERO \\
\hline 33 & Mgò & & ZERO \\
\hline 60 & $\mathrm{Mn}(\mathrm{COOH}) 2$ & & ZERO \\
\hline 172 & $\mathrm{Mn}(\mathrm{NO} 3) 2$ & & ZERO \\
\hline 180 & Mn304 & & ZERO \\
\hline 56 & $\mathrm{MnO}$ & & ZERO \\
\hline 14 & $\mathrm{MnO} 2$ & & ZERO \\
\hline 145 & $\mathrm{MoO2}$ & & ZERO \\
\hline 81 & $\mathrm{~N} 2$ & & $0.2789 \mathrm{E}+03$ \\
\hline 74 & $\mathrm{~N} 2 \mathrm{O}$ & & ZERO \\
\hline 182 & NH3 & & ZERO \\
\hline 114 & NH3OHNO3 & & ZERO \\
\hline 52 & $\mathrm{NH} 4 \mathrm{COOH}$ & & ZERO \\
\hline 173 & NH4NO3 & & ZERO \\
\hline 68 & NH $4 \mathrm{OH}$ & . & ZERO \\
\hline 148 & NH4TPB & & ZERO \\
\hline
\end{tabular}

456

LWPT Vent

to stack

ZERO

$0.8246 \mathrm{E}-13$

$0.1233 \mathrm{E}-10$

ZERO

$.3733 \mathrm{E}-14$

$0.1148 \mathrm{E}-15$

ZERO

ZERO

ZERO

ZERO

ZERO

$0.2950 \mathrm{E}-10$

ZERO

ZERO

ZERO

$0.2890 \mathrm{E}-13$

ZERO

ZERO

$0.5347 \mathrm{E}-12$

$$
\text { ZERO }
$$

ZERO

$0.1648 \mathrm{E}-15$

$0.3325 \mathrm{E}-08$

$0.2290 \mathrm{ZER}$

7ERO

ZERO

$0.1340 \mathrm{E}-12$

ZERO

ZERO

ZERO

$.2560 \mathrm{E}-12$

$0.8515 \mathrm{E}-14$

$0.2789 \mathrm{E}+03$

$.3738 E-05$

ZERÓ

ZERO

ZERO

$0.2034 \mathrm{E}-12$

668

669

670

LWHT 2 wto H2C2O4 2 wt 8 NaOH X-Flow Filtr

Heel addn to LWHT addn to LWHT H2O Flush

$0.1100 \mathrm{E}-06$

ZERO.

ZERO

ZERO

ZERO

ZERO

ZERO

$0.2113 \mathrm{E}-05$

$0.6497 \mathrm{E}-07$

$0.1928 \mathrm{E}-11$

ZERO

ZERO

ZERO

ZERO

ZERO

ZERO

ZERO

ZERO

ZERO

ZERO

ZERO

ZERO

ZERO

$0.1689 \mathrm{E}-04$

ZERO

ZERO

ZERO

$0.9328 \mathrm{E}-07$

ZERO

ZERO

ZERO

$0.4556 \mathrm{E}-07$

ZERO

$0.5308 E-07$

ZERO

ZERO

ZERO

$0.9974 \mathrm{E}-03$

$0.2723 \mathrm{E}-10$

$0.6687 \mathrm{E}-06$

ZERO

ZERO

ZERO

Date: January 1995
0 年 $\mathrm{NaOH}$

to LWHT

ZERO

ZERO

ZERO

ZERO

ZERO

ZERO

ZERO

ZERO

ZERO

ZERO

ZERO

ZERO

ZERO

ZERO

ZERO

ZERO

ZERO

ZERO

ZERO

ZERO

ZERO

ZERO

ZERO

ZERO

ZERO

ZERO

ZERO

ZERO

ZERO

ZERO

ZERO

ZRRO

ZERO

ZERO

ZERO

ZERO

ZERO

ZERO

ZERO

ZERO

ZERO

ZERO

ZERO

ZERO

ZERO

ZERO

ZERO

ZERO 
Table A-1. HLW Flowsheet Material Balance (Tank 51/ITP Cycle 1) section 1B. Re-precipitation and Late Washing

STREAM NUMBERS (CONT'D) STREAM NAME

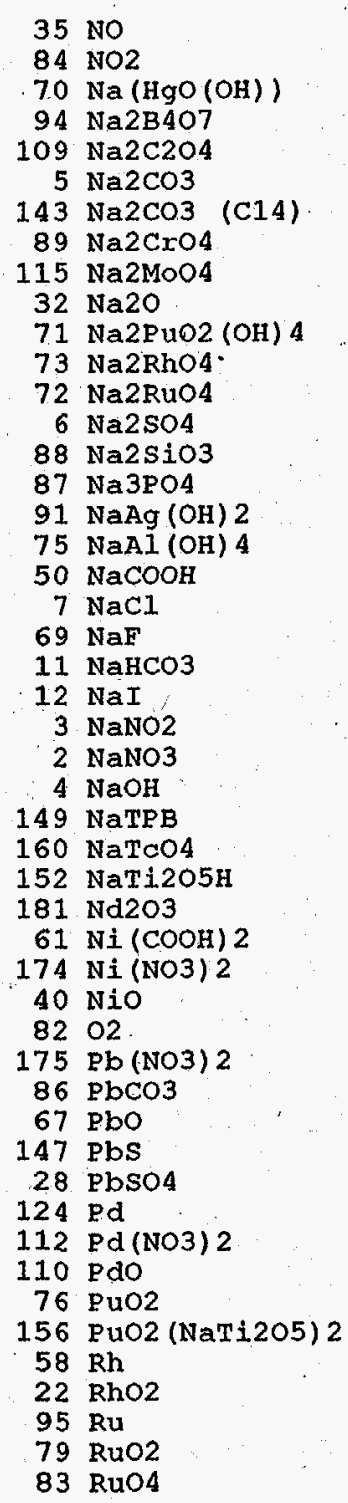

456

Air/N2 Purge IWPT Vent

to IWPT

ZERO

ZERO

$0.1784 \mathrm{E}-19$

$0.1570 \mathrm{E}-10$

$0.1196 \mathrm{E}-10$

$0.2568 \mathrm{E}-09$

$0.4327 \mathrm{E}-18$

$0.1593 \mathrm{E}-12$

$0.4756 \mathrm{E}-13$

$0.7795 \mathrm{E}-10$

$0.1988 \mathrm{E}-20$

$0.1154 \mathrm{E}-14$

$0.6075 \mathrm{E}-14$

$0.1658 \mathrm{E}-11$

$0.4612 \mathrm{E}-13$

$0.8500 \mathrm{E}-21$

$0.5708 \mathrm{E}-13$

ZERO
$6 \mathrm{E}-12$

$0.8696 \mathrm{E}-12$
$0.1274 \mathrm{E}-12$

$0.1274 \mathrm{E}-12$

$0.8536 \mathrm{E}-16$

$0.4525 \mathrm{E}-09$

$0.3370 \mathrm{E}-10$

$0.6450 \mathrm{E}-11$

$0.4865 \mathrm{E}-10$

668

669

670

671

WHT 2 wto $\mathrm{H} 2 \mathrm{C} 2 \mathrm{O} 4$ wto 2 NaOH X-Flow Filtr Heel addn to IWHT addn to IWHT H2O Flush ZERO

ZERO
ZERO
ZERO

ZERO
ZERO
ZERO

$0.1010 \mathrm{E}-10$ ZERO $\quad$ ZERO

Z ZERO ZERO Z ZERO

$0.4808 E-01$ ZERO ZERO

$0.1454 \mathrm{E}+00 \quad$ ZERO ZERO

$0.2449 \mathrm{E}-09$

$.2692 \mathrm{E}-04$

$0.1125 \mathrm{E}-11$

$0.6532 \mathrm{E}-06$

$0.3440 \mathrm{E}-05$

$0.9384 \mathrm{E}-03$

$0.2611 \mathrm{E}-04$

$0.1246 \mathrm{E}-02$

$0.3232 \mathrm{E}-04$

ZERO

$0.6954 \mathrm{E}-03$

$0.7214 \mathrm{E}-04$

ZERO

$0.2562 \mathrm{E}+00$

$0.1908 \mathrm{E}-01$

$0.2891 \mathrm{E}+01$

$0.7088 \mathrm{E}-01$

$0.2168 \mathrm{E}-05$

$0.7608 \mathrm{E}-10$

$0.7608 \mathrm{E}-10$

ZERO

ZERO

ERO

ERO

$0.1634 \mathrm{E}+02$

$0.8059 \mathrm{E}-13$

$0.1634 \mathrm{E}+02$

ZERO

ZERO

ZERO

ZERO

ZERO

ZERO

ZERO

ZERÓ

ZERO

ZERO

ZERO

ZERO

ZERO

ZERO

ZERO

ZERO

$0.3389 \mathrm{E}-12$

$0.1026 \mathrm{E}-16$

ZERO

$0.2493 \mathrm{E}-14$

$0.9883 \mathrm{E}-14$

ZERO

ZERO

ZERO
ZERO

0.5797 E-06

ZERO

$0.4450 \mathrm{E}-04$

$0.1408 \mathrm{E}-05$

ZERO

ZERO

ZERO

ZERO

TERO

ZERO
ZERO

ZERO

ZERO

ZERO

ZERO

$0.3437 \mathrm{E}-12$

ZERO

Date: January 1995

ZERO $\quad$ ZERO

ZERO ZERO

$\begin{array}{rr}\text { ZERO } & \text { ZERO } \\ \text { ZERO } & 0.1209 \mathrm{E}+01\end{array}$

ZERO

ZERO

ZERO

ZERO

ZERO

ZERO

ZERO

ZERO

ZERO

ZERO

ZERO

ZERO

ZERO

ZERO

ZERO

ZERO

ZERO

ZERO

ZERO

ZERO

ZERO

459

$508 \mathrm{NaOH}$

to IWHT

ZERO

ZERO

ZERO

ZERO

ZERO

ZERO

ZERO

ZERO

ZERO

ZERO

ZERO

ZERO

ZTRO

ZERO

ZERO

ZERO

ZERO

ZERO

ZERO

ZERO

ZERO

ZERO

ZERO

ZERO 
Table A-1. HLW Flowsheet Material Balance (Tank 51/ITP Cycle 1) Section 1B. Re-precipitation and Late Washing

STREAM NUMBERS (CONT'D) $\rightarrow$ STREAM NAME

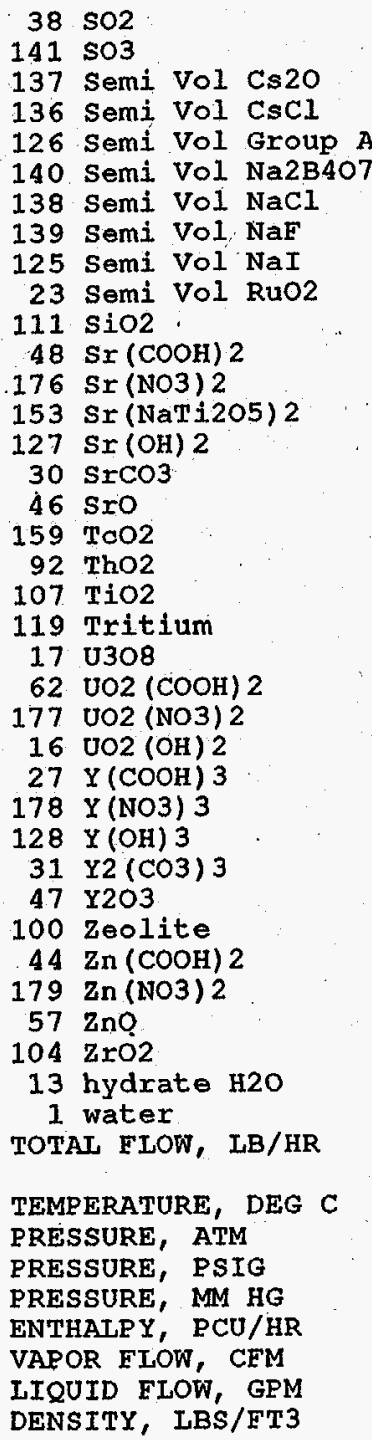

455

Air/N2 Purge
to IWPT
ZERO
ZERO
ZERO
ZERO
ZERO
ZERO
ZERO
ZERO
ZERO
ZERO
ZERO
ZERO
ZERO
ZERO
ZERO
ZERO
ZERO
ZERO
ZERO
ZERO
ZERO
ZERO
ZERO
ZERO
ZERO
ZERO
ZERO
ZERO
ZERO
ZERO
ZERO
ZERO
ZERO
ZERO
ZERO
ZERO
ZERO
ZERO

$0.1184 \mathrm{E}+01$
$0.2965 \mathrm{E}+03$

$0.3500 \mathrm{E}+02$

$0.1000 \mathrm{E}+01$

$$
\text { ZERÓ }
$$

$0.3409 \mathrm{E}+04$ $0.7107 \mathrm{E}+02$

$0.6952 \mathrm{E}-01$
VAPOR
456 LWPT Vent to stack ZERO ZERO ZERO ZERO ZERO ZERO ZERO ZERO ZERO ZERO

$0.2071 E-11$. ZERO

$0.3164 \mathrm{E}-12$ $0.1135 \mathrm{E}-20$ $0.7056 \mathrm{E}-14$

ZERO
ZERO ZERO ZERO

$0.9427 \mathrm{E}-13$

$0.5244 \mathrm{E}-12$

$0.5836 \mathrm{E}-13$ ZERO

$0.1645 \mathrm{E}-14$ ZERO

$0.1261 \mathrm{E}-20$

ZERO

$0.7523 \mathrm{E}-13$ ZERO ZERO

$0.1538 \mathrm{E}-10$ ZERO

$0.2788 \mathrm{E}-11$

$0.3035 \mathrm{E}+03$

ZERO

$0.9656 \mathrm{E}+00$ $-0.5055 \mathrm{E}+00$

$0.5090 \mathrm{E}+04$

$0.6767 \mathrm{E}+02$

$0.7476 \mathrm{E}-01$

VAPOR
668

669

LWHT 2 wt? $\mathrm{H} 2 \mathrm{C} 2 \mathrm{O} 4$

670 2 wt? NaOH X-Flow Filtr H2O Flush ZERO ZERO ZERO ZERO ZERO ZERO ZERO ZERO ZERO ZERO ZERO ZERO ZERO ZERO ZERO

$0.6425 \mathrm{E}-12$ ZERO ZERO ZERO ZERO

ZERO
$0.1102 \mathrm{E}-11$ ZERO ZERO ZERO

$0.9314 \mathrm{E}-06$ ZERO

$0.7137 \mathrm{E}-12$

ZERO

ZERO

ZERO

$0.4627 \mathrm{E}-04$

ZERO

ZERO

$0.5846 \mathrm{E}+02$

$0.6192 \mathrm{E}+02$

$0.3989 \mathrm{E}+02$

$0.9656 \mathrm{E}+00$

$-0.5055 \mathrm{E}+00$

$0.7339 \mathrm{E}+03$

$0.1190 \mathrm{E}+00$

$0.6484 \mathrm{E}+02$

LIQUID $\therefore \quad$ ZERO

ZERO

ZERO

ZERO

ZERO

ZERO

ZERO

ZERO

ZERO

ZERO

ZERO

ZERO

ZERO

ZERO

ZERO

ZERO

ZERO

ZERO

ZERO

ZERO

ZERO

ZERO

ZERO

ZERO

ZERO

ZERO

ZERO

ZERO

ZERO

ZERO

ZERO

ZERO

ZERO

$0.4077 \mathrm{E}+02$

$0.4160 \mathrm{E}+02$

$0.3500 \mathrm{E}+02$

$0.1000 \mathrm{E}+01$

ZERO

$0.1438 \mathrm{E}+04$

$0.8242 \mathrm{E}-01$

$0.6293 \mathrm{E}+02$
LIOUID
ZERO

ZERO
ZERO

ZERO

ZERO

ZERO

ZERO

ZERO

ZERO

ZERO

ZERO

ZERO

ZERO

ZERO

ZERO

ZERO

ZERO

ZERO

ZERO

ZERO

ZERO

ZERO

ZERO

ZERO

ZERO

ZERO

ZERO

ZERO

ZERO

ZERO

ZERO

ZERO

ZERO

ZERO

ZERO

$0.5924 \mathrm{E}+02$

$0.6045 \mathrm{E}+02$

$0.3500 \mathrm{E}+02$

$0.1000 \mathrm{E}+01$

ZERO

$0.2092 \mathrm{E}+04$

$0.1190 \mathrm{E}+00$

$0.6330 \mathrm{E}+02$

LIQUID
67

Zlush

ZERO

ZERO

ZERO

ZERO

ZERO

ZERO

ZERO
ZERO

ZERO

ZERO

ZERO

ZERO

ZERO

ZERO

ZERO

ZERO

ZERO

ZERO

ZERO

ZERO

ZERO

ZERO

ZERO

ZERO

ZERO

ZERO

ZERO

ZERO

ZERO

ZERO

ZERO

ZERO

ZERO

ZERO

$.9400 \mathrm{E}+02$

$0.9402 \mathrm{E}+02$

$0.3500 \mathrm{E}+02$

$0.1000 \mathrm{E}+01$

ZERO

$0.3298 \mathrm{E}+04$

$0.1877 \mathrm{E}+00$

$0.6244 \mathrm{E}+02$

LIQUID 
Table A-1. HLW Flowsheet Material Balance (Tank 51/ITP Cycle 1) Section 1B. Re-precipitation and Late Washing

STREAM NUMBERS $->$ STREAM NAME

COMPONENT FLOWS, LB/HR $122(\mathrm{C} 6 \mathrm{H} 5) 2$

123 ( $66 \mathrm{H} 5) 2 \mathrm{C} 6 \mathrm{H} 4$

118 (C6H5) $2 \mathrm{NH}$

133 (C6H5B (OH) 2 salt)

132 (C6H5B (OH) 2)
166 (C6H5C6H4OH)

166 (C6H5C6H4OH)

163 (C6H5NHCHO)

165 (C6H5NO)

164 (C6H5NO2)

$\begin{array}{ll}131 & \text { (C6H5OH } \\ 130 & \text { (C6H5OH) }\end{array}$

130 (C6H5O
121 (C6H6)

155 (CH3) $2 \mathrm{CHOH}$

154 ( $\mathrm{CH} 3 \mathrm{OH})$

$45 . \mathrm{Ag}$

$116 \mathrm{Ag} 20$

134 AgNO3
77 A1203

$106 \mathrm{~B} 203$

$9 \mathrm{Ba}(\mathrm{OH}) 2$.

$15 \mathrm{BaO}$

$24 \mathrm{BaSO} 4$

$66 \mathrm{CO}$

$37 \mathrm{CO} 2$

$142 \mathrm{CO} 2$ (C14)

$63 \mathrm{Ca}(\mathrm{COOH}) 2$

$120 \mathrm{Ca}(\mathrm{OH}) 2$

$113 \mathrm{Ca} 3$ (PO4) 2

$20 \mathrm{CaC} 204$

$18 \mathrm{CaCO} 3$

$64 \mathrm{CaCO}(\mathrm{C} 14)$

78 CaF2

$41 \mathrm{CaO}$

19 CasO4

29 Carbon

99 Cement

168 Co (NO3) 2

$59 \mathrm{COO}$

98 Cr203

$42 \mathrm{Cs} 2 \mathrm{O}$

$53 \mathrm{CsCOOH}$

$96 \mathrm{CsCl}$

169 CsNO3

$8 \mathrm{CsOH}$.

$151 \mathrm{CsTPB}$

WSRC-TR-95-0019 (Revision
672

673

Spent Late Spent Late Washd Precip Wash Recycle Wash Soluble Wash Insolbl Feed frm LWF

.

ZERO
ZERO
ZERO
ZERO
ZERO
ZERO
ZERO
ZERO
ZERO
ZERO
$0.3998 E+00$
ZERO
0.7436 E-02
0.1232 E-08
$0.1448 E-09$
ZERO
ZERO
ZERO
ZERO
ZERO
$0.1252 E-14$
ZERO
ZERO
ZERO
ZERO
ZERO
ZERO
0.3561 E-04

$0.3561 \mathrm{E}-04$

$0.3672 \mathrm{E}-05$

ZERO

ZERO

ZERO

ZERO

ZERO

ZERO

ZERO

ZERO

$0.1397 \mathrm{E}-04$

$7 E-04$
ZERO

ZERO

ZERO

ZERO

$0.4663 \mathrm{E}-04$

$\begin{array}{rr}\text { E-04 } & 0.4663 \mathrm{E}-04 \\ \text { ZERO } & \text { ZERO }\end{array}$

ZERO
ZERO
ZERO
ZERO
ZERO
ZERO
ZERO
ZERO
ZERO

ZERO

7.1399E-01

ZERO $0.9313 \mathrm{E}-02$

ZERO

ZERO

ZERO

ZERO

ZERO

ZERO

$0.3998 \mathrm{E}+00$

ZERO

$0.7436 \mathrm{E}-02$

$0.1232 \mathrm{E}-08$

$0.1448 \mathrm{E}-09$

ZERO

ZERO

ZERO

ZERO

ZERO
$0.3026 \mathrm{E}-01$

$0.4503 \mathrm{E}-01$

$0.1091 \mathrm{E}-01$

$0.1091 \mathrm{E}-01$

$0.9422 \mathrm{E}-11$

$0.3054 \mathrm{E}-02$

$0.1297 \mathrm{E}+00$

$0.1670 \mathrm{E}-02$

$0.1793 \mathrm{E}-02$

ZERO

ZERO

ZERO

ZERO

$0.3561 \mathrm{E}-04$

$0.3672 \mathrm{E}-05$

ZERO

ZERO

ZERO

ZERO

ZERO

ZERO

ZERO

ZERO

$0.1397 \mathrm{E}-04$

ZERO

ZERO

ZERO

ZERO

ZERO

ZERO
ZERO
$.2332 E-11$
ZERO

ZERO
ZERO

ZERO

ZERO

ZERO

ZERO

ZERO

ZERO

ZERO

ZERO

ZERO

ZERO

ZERO

ZERO

ZERO

ZERO

ZERO

Date: January 1995
201

$0.3712 \mathrm{E}+00$

ZERO

ZERO

ZERO

ZERO

ZERO

$0.4817 \mathrm{E}-16$

$0.1794 \mathrm{E}-05$

657

Prec Feed

Solubles

658

460

Insolubed Air/N2 Sparg

$0.3293 \mathrm{E}+00$

$0.1399 \mathrm{E}-01$ ZERO

$0.9313 \mathrm{E}-02$ ZERO

$0.3712 \mathrm{E}+00 \quad$ ZERO

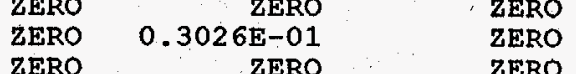

ZERO ZERO $\quad$ ZERO

ZERO $\quad$ ZERO $\quad$ ZERO

ZERO

ZE-01

$0.1091 \mathrm{E}-01$

$0.8591 \mathrm{E}-10$

$0.9422 \mathrm{E}-11$

ZERO

ZERO

ZERO

ZERO

$0.4817 \mathrm{E}-16$

$7 E-16$

ZERO

ZERO

ZERO

ZERO

ZERO

ZERO

$0.1370 \mathrm{E}-05$

$0.1413 \mathrm{E}-06$

$0.5607 \mathrm{E}+00$

$0.1413 \mathrm{E}-06$

ZERO

ZERO

ZERO

ZERO

ZERO

ZERO

ZERO

ZERO
ZERO

$0.5373 \mathrm{E}-06$

$0.1028 \mathrm{E}-03$

$0.2616 \mathrm{E}+00$

$0.3848 \mathrm{E}-04$

ZERO
ZERO

ZERO

ZERO

ZERO

ZERO

0.5373 ZERO

3E-06
ZERO

ZERO

ZERO

ZERO

ZERO

$0.1794 \mathrm{E}-05$

ZERO

ZERO

ZERO

ZERO

ZERO

ZERO

ZERO

$0.3054 \mathrm{E}-02$

ZERO

$0.1297 \mathrm{E}+00$

$0.1670 \mathrm{E}-02$

ZERO

0.1793 ZERO

ZERO

ZERO

ZERO

ZERO

ZERO

$0.5607 \mathrm{E}+00$

ZERO

ZERO

ZERO

ZERO

$0.1912 \mathrm{E}+00$

ZERO

ZERO

ZERO

$0.1028 \mathrm{E}-03$

$0.2616 \mathrm{E}+00$

$0.3848 E-04$

ZERO

ZERO

ZERO

$0.1100 \mathrm{E}+00$

ZERO

ZERO

ZERO

ZERO

ZERO

ZERO

ZERO

ZERO

ZERO

ZERO

ZERO

ZERO

ZERO

ZERO

ZERO

ZERO

ZERO

ZERO

ZERO

ZERO

ZERO

ZERO

ZERO

ZERO

ZERO

ZERO

ZERO

ZERO

ZERO

ZERO

ZERO

ZERO

ZERO

ZERO

ZERO

Section 1B, Page 14 of 21 
Table A-1. HLW Flowsheet Material Balance (Tank 51/ITP Cycle 1) section 1B. Re-precipitation and Late Washing

STREAM NUMBERS (CONT'D) $\rightarrow$ STREAM NAME

$54 \mathrm{Cu}(\mathrm{COOH}) 2$

$170 \mathrm{Cu}(\mathrm{NO} 3) 2$

$55 \mathrm{CuO}$

$80 \mathrm{Fe} 2 \mathrm{O} 3$

101 FeO

102 Group A

$103 \mathrm{Gro}$

158 H2C2O4

93 H2SO4

90 Н 3 BO 3.

$34 \mathrm{HCOOH}$.

$43 \mathrm{HCl}$

85. $\mathrm{HF}$

49 HNO3

$36 \mathrm{Hg}$

$129 \mathrm{Hg}(\mathrm{C} 6 \mathrm{H} 5) 2$

$135 \mathrm{Hg}(\mathrm{NO} 3)^{2}$

$144 \mathrm{Hg} 2 \mathrm{C} 12$

$117 \mathrm{Hg} 2 \mathrm{I} 2$

$146 \mathrm{HgCl}$

$21 \mathrm{HgO}$

$\begin{array}{rl}97 & \text { I } 2 \\ 183 & \mathrm{~K} 2 \mathrm{CO}\end{array}$

39 K20

$51 \mathrm{KCOOH}$

$157 \mathrm{KMnO4}$

$161 \mathrm{KNO} 3$

$10 \mathrm{KOH}$

$150 \mathrm{KTPB}$

$\begin{array}{ll}105 & \mathrm{La2O3} \\ 108 & \mathrm{Li} 2 \mathrm{O}\end{array}$

$65 \mathrm{Mg}$ (COOH) 2

$171 \mathrm{Mg}$ (NO3) 2

$33 . \mathrm{MgO}$

$60 \mathrm{Mn}(\mathrm{COOH}) 2$

$172 \mathrm{Mn}(\mathrm{NO} 3) 2$

$180 \mathrm{Mn} 304$

$56 \mathrm{MnO}$

$14 \mathrm{MnO} 2$

$145 \mathrm{MOO}$

81 N2

74 N2O
182 NH3

114 NH3OHNO3

52 NH4COOH

173 NH4NO

$68 \mathrm{NH} 4 \mathrm{OH}$

148 NH4 TPB

WSRC-TR-95-0019 (Revision
420

672

Spent Late Spent Late Spent Late Washd Precip Wash Recycle Wash Soluble Wash Insolbl Feed frm LWF

\begin{tabular}{|c|c|c|c|}
\hline ZERO & ZERO & ZERO & ZERO \\
\hline $0.3297 \mathrm{E}-05$ & $0.3297 \mathrm{E}-05$ & ZERO & $0.11268 \mathrm{E}-06$ \\
\hline ZERO & ZERO & ZERO & $0.1061 \mathrm{E}-02$ \\
\hline ZERO & ZERO & ZERO & $0.1587 \mathrm{E}+00$ \\
\hline ZERO & ZERO & ZERO & ZERO \\
\hline $0.6336 \mathrm{E}-04$ & ZERO & $0.6336 \mathrm{E}-04$ & $0.2437 \mathrm{E}-0.05$ \\
\hline $0.1948 \mathrm{E}-05$ & ZERO & $0.1948 E-05$ & $0.7493 \mathrm{E}-07$ \\
\hline $.5781 \mathrm{E}-10$ & $0.5781 \mathrm{E}-10$ & ZERO & $0.7581 \mathrm{E}-11$ \\
\hline ZERO & ZERO & ZERO & ZERO \\
\hline ZERO & ZERO & ZERO & ZERO \\
\hline ZERO & ZERO & ZERO & ZERO \\
\hline ZERO & ZERO & ZERO & ZERO \\
\hline ZERO & ZERO & ZERO & ZERO \\
\hline ZERO & ZERO & ZERO & ZERO \\
\hline ZERO & ZERO & ZERO & ZERO \\
\hline ZERO & ZERO & ZERO & ZERO \\
\hline ZERO & ZERO & ZERO & $0.3797 \mathrm{E}+00$ \\
\hline ZERO & ZERO & ZERO & ZERO \\
\hline ZERO & ZERO & ZERO & ZERO \\
\hline ZERO & ZERO & ZERO & ZERO \\
\hline ZERO & ZERO & ZERO & ZERO \\
\hline ZERO & ZERO & ZERO & $0.3719 \mathrm{E}-03$ \\
\hline ZERO & ZERO & ZERO & ZERO \\
\hline $0.5063 \mathrm{E}-03$ & ZERO & ZERO & $0.1948 \mathrm{E}-04$ \\
\hline ZERO & ZERO & ZERO & $0.6882 \mathrm{E}-02$ \\
\hline ZERO & ZERO & ZERO & ZERO \\
\hline ZERO & ZERO & ZERO & ZERO \\
\hline ZERO & ZERO & ZERO & ZERO \\
\hline $0.2796 \mathrm{E}-05$ & $0.2796 \mathrm{E}-05$ & ZERO & $0.1076 \mathrm{E}-06$ \\
\hline ZERO & ZERO & ZERO & $0.4280 \mathrm{E}+02$ \\
\hline ZERO & ZERO & ZERO & ZERO \\
\hline ZERO & ZERO & ZERO & $0.2948 \mathrm{E}-03$ \\
\hline ZERO & ZERO & ZERO & ZERO \\
\hline $0.1366 \mathrm{E}-05$ & $0.1366 \mathrm{E}-05$ & ZERO & $0.5254 \mathrm{E}-07$ \\
\hline ZERO & ZERO & ZERO & $0.1725 E-02$ \\
\hline ZERO & ZERO & ZERO & ZERO \\
\hline $0.1591 \mathrm{E}-05$ & $0.1591 \mathrm{E}-05$ & ZERO & $0.6122 \mathrm{E}-07$ \\
\hline ZERO & ZERO & ZERO & ZERO \\
\hline ZERO & ZERO & ZERO & ZERO \\
\hline ZERO & ZERO & ZERO & $0.3295 \mathrm{E}-02$ \\
\hline ZERO & ZERO & ZERO & $0.1096 \mathrm{E}-03$ \\
\hline $0.2990 \mathrm{E}-01$ & $0.2990 \mathrm{E}-01$ & ZERO & $0.3456 \mathrm{E}-03$ \\
\hline $0.8164 \mathrm{E}-09$ & $0.8164 \mathrm{E}-09$ & ZERO & $0.1070 \mathrm{E}-09$ \\
\hline $0.2005 \mathrm{E}-04$ & $0.2005 \mathrm{E}-04$ & ZERO & $0.1826 \mathrm{E}-05$ \\
\hline ZERO & ZERO & ZERO & ZERO \\
\hline ZERO & ZERO & ZERO. & ZERO \\
\hline ZERO & ZERO & ZERO & ZERO \\
\hline ZERO & ZERO & ZERO & ZERO \\
\hline ZERO & ZERO & ZERO & $0.2617 \mathrm{E}-02$ \\
\hline
\end{tabular}

673

201

\section{7}

Prec Feed

Solubles

$0.1268 \mathrm{E}-06$

ZERO

ZERO

ZERO

ZERO

$0.7581 \mathrm{E}-11$

ZERO

ZERO

ZERO

ZERO

ZERO

ZERO

ZERO

ZERO

ZERO

ZERO

ZERO

ZERO

ZERO

ZERO

ZERO

ZERO

ZERO

$0.1076 \mathrm{E}-06$

ZTRO

ZERO

ZERO

ZERO

$0.5254 \mathrm{E}-07$

ZERO

ZERO
$0.6122 \mathrm{E}-07$

ZERO

ZERO

ZERO

$0.3456 \mathrm{E}-03$

$0.1070 \mathrm{E}-09$

$0.1826 \mathrm{E}-05$

ZERO

ZERO

ZERO
ZERO

Date: January 1995
658

Prec Feed 460

Insolubles Air/N2 Sparg to LWHT

ZERO ZERO

ZERO

ZERO ZERO

2437E-05

7493E-07

ZERO - ZERO

ZERO ZERO

ZERO ZERO

ZERO ZERO

$\begin{array}{ll}\text { ZERO } & \text { ZERO } \\ \text { ZERO } & \text { ZERO }\end{array}$

ZERO ZERO

ZERO ZERO

$0.3797 \mathrm{E}+00 \quad$ ZERO

ZERO

ZERO

ZERO

ZERO ZERO

ZERO ZERO
ZERO

0.68 ZERO ZERO

$.6882 \mathrm{E}-02$ ZERO

ZERO ZERO

ZERO ZERO

ZERO
ZERO

ZERO

$0.2948 \mathrm{E}-03$

ZERO ZERO

$0.1725 \mathrm{E}-02$ ZERO

ZERO ZERO

ZERO ZERO

ZERO ZERO

$0.3295 \mathrm{E}-02$ ZERO

ZERO
10.96E-03

ZERO $0.7099 \mathrm{E}+03$

ZERO ZERO

ZERO ZERO

ZERO ZERO

ZERO ZERO

$0.2617 \mathrm{E}-02$ ZERO

Section 1B, Page 15 of 21 
WSRC-TR-95-0019 (Revision

0)

Date: January 1995

Section 1B, Page 16 of 21

Table A-1. HLW Flowsheet Material Balance (Tank 51/ITP Cycle 1)

Section 1B. Re-precipitation and Late Washing

STREAM NUMBERS (CONT'D) $\rightarrow$ STREAM NAME

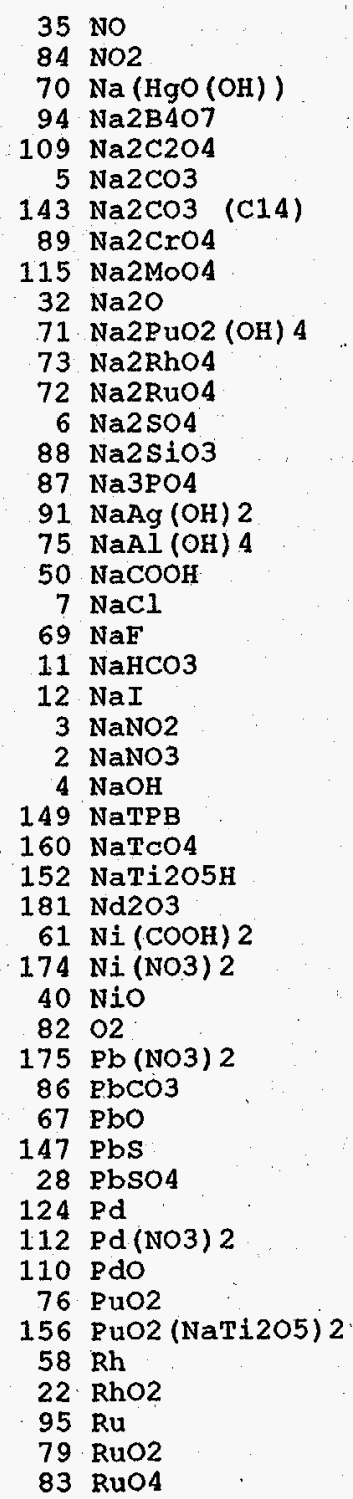

420

Spent Late spent Late Spent Late Washd Precip Wash Recycle Wash Soluble Wash Insolbl Feed frm LWF

\begin{tabular}{|c|c|c|c|}
\hline ZERO & ZERO & ZERO. & ZERO \\
\hline ZERO & ZERO & ZERO & ZERO \\
\hline $0.3027 E-09$ & $0.3027 \mathrm{E}-09$ & ZERO & $0.1165 \mathrm{E}-10$ \\
\hline $0.2664 \mathrm{E}+00$ & $0.2664 \mathrm{E}+00$ & ZERO & $0.1025 \mathrm{E}-01$ \\
\hline $0.1441 \mathrm{E}+01$ & $0.1441 \mathrm{E}+01$ & ZERO & $0.7810 \mathrm{E}-02$ \\
\hline $0.4359 \mathrm{E}+01$ & $0.4359 \mathrm{E}+01$ & ZERO & $0.1677 \mathrm{E}+00$ \\
\hline $0.7343 \mathrm{E}-08$ & $0.7343 \mathrm{E}-08$ & ZERO & $0.2825 \mathrm{E}-09$ \\
\hline $0.2703 E-02$ & $0.2703 \mathrm{E}-02$ & ZERO & $0.1040 \mathrm{E}-03$ \\
\hline $0.8072 E-03$ & $0.8072 \mathrm{E}-03$ & ZERO & $0.3105 \mathrm{E}-04$ \\
\hline ZERO & ZERO & ZERO & $0.1003 E+01$ \\
\hline $0.3374 \mathrm{E}-10$ & $0.3374 \mathrm{E}-10$ & ZERO & $0.1298 \mathrm{E}-11$ \\
\hline $0.1958 E-04$ & $0.1958 \mathrm{E}-04$ & ZERO & $0.7533 \mathrm{E}-06$ \\
\hline $0.1031 \mathrm{E}-03$ & $0.1031 \mathrm{E}-03$ & ZERO & $0.3967 \mathrm{E}-05$ \\
\hline $0.2813 E-01$ & $0.2813 \mathrm{E}-01$ & ZERO & $0.1082 \mathrm{E}-02$ \\
\hline $0.7829 E-03$ & $0.7829 E-03$ & ZERO & $0.3012 \mathrm{E}-04$ \\
\hline $0.3736 \mathrm{E}-01$ & $0.3736 E-01$ & ZERO & $0.1437 \mathrm{E}-02$ \\
\hline $0.1443 E-10$ & $0.1443 \mathrm{E}-10$ & ZERO & $0.5550 \mathrm{E}-12$ \\
\hline $0.9688 \mathrm{E}-03$ & $0.9688 \mathrm{E}-03$ & ZERO & $0.3727 \mathrm{E}-04$ \\
\hline ZERO & ZERO & ZERO & ZERO \\
\hline $0.2085 \mathrm{E}-01$ & $0.2085 \mathrm{E}-01$ & ZERO & $0.3180 \mathrm{E}-02$ \\
\hline $0.2163 \mathrm{E}-02$ & $0.2163 \mathrm{E}-02$ & ZERO & $0.8321 \mathrm{E}-04$ \\
\hline ZERO & ZERO & ZERO & ZERO \\
\hline $0.1449 \mathrm{E}-05$ & $0.1449 \mathrm{E}-05$ & ZERO & $0.5573 \mathrm{E}-07$ \\
\hline $0.7681 \mathrm{E}+01$ & $0.7681 \mathrm{E}+01$ & ZERO & $0.2955 \mathrm{E}+00$ \\
\hline $0.5719 E+00$ & $0.5719 \mathrm{E}+00$ & $0.3959 \mathrm{E}-07$ & $0.2200 \mathrm{E}-01$ \\
\hline $0.8668 \mathrm{E}+02$ & $0.8668 \mathrm{E}+02$ & ZERO & $0.9803 \mathrm{E}-01$ \\
\hline $0.2125 \mathrm{E}+01$ & $0.2125 \mathrm{E}+01$ & ZERO & $0.5890 \mathrm{E}+00$ \\
\hline $0.6498 \mathrm{E}-04$ & $0.6498 \mathrm{E}-04$ & ZERO & $0.2500 \mathrm{E}-05$ \\
\hline ZERO & ZERO & ZERO & $0.9792 \mathrm{E}+00$ \\
\hline ZERO & ZERO & ZERO & ZERO \\
\hline ZERO & ZERO & ZERO & ZERO \\
\hline $0.1738 \mathrm{E}-04$ & $0.1738 E-04$ & $0.1298 \mathrm{E}-11$ & $0.6686 \mathrm{E}-06$ \\
\hline ZERO & ZERO & ZERO & $0.1037 \mathrm{E}-02$ \\
\hline $0.1334 \mathrm{E}-02$ & $0.1334 \mathrm{E}-02$ & ZERO & $0.3924 \mathrm{E}-04$ \\
\hline $0.4220 \mathrm{E}-04$ & $0.4220 \mathrm{E}-04$ & ZERO & $0.1624 \mathrm{E}-05$ \\
\hline ZERO & ZERO & ZERO & , ZERO \\
\hline ZERO & ZERO & ZERO & ZERO \\
\hline ZERO & ZERO & ZERO & ZERO \\
\hline ZERO & ZERO & ZERO & $0.4362 \mathrm{E}-02$ \\
\hline ZERO & ZERO & ZERO & ZERO \\
\hline $0.1742 \mathrm{E}-06$ & $0.1742 \mathrm{E}-06$ & ZERO & $0.6701 \mathrm{E}-08$ \\
\hline ZERO & ZERO & ZERO & ZERO \\
\hline ZERO & ZERO & ZERO & $0.3209 \mathrm{E}-04$ \\
\hline ZERO & ZERO & ZERO & $0.1272 \mathrm{E}-03$ \\
\hline ZERO & ZERO & ZERO & ZERO \\
\hline ZERO & ZERO & ZERO & ZERO \\
\hline ZERO & ZERO & ZERO & ZERO \\
\hline ZERO & ZERO & ZERO & $0.4424 \mathrm{E}-02$ \\
\hline ZERO & ZERO & ZERO & .ZERO \\
\hline
\end{tabular}

WSRC-TR-95-0019 (Revision

\begin{tabular}{|c|c|c|c|}
\hline $\begin{array}{l}657 \\
\text { Pred Feed } \\
\text { Solubles }\end{array}$ & $\begin{array}{l}658 \\
\text { Prec Feed } \\
\text { Insolubles }\end{array}$ & $\begin{array}{r}460 \\
\text { Air } / \mathrm{N} 2 \\
\text { to }\end{array}$ & $\begin{array}{l}\text { sparg } \\
\text { LWHT }\end{array}$ \\
\hline ZERO & ZERO & & ZERO \\
\hline ZERO & ZERO & & ZERO \\
\hline $0.1165 \mathrm{E}-10$ & ZERO & & ZERO \\
\hline $0.1025 E-01$ & ZERÓ & & ZERO \\
\hline $0.7810 \mathrm{E}-02$ & ZERO & & ZERO \\
\hline $0.1677 \mathrm{E}+00$ & ZERO & & ZERO \\
\hline $0.2825 \mathrm{E}-09$ & ZERO & & ZERO \\
\hline $0.1040 \mathrm{E}-03$ & ZERO & & ZERO \\
\hline $0.3105 E-04$ & ZERO & & ZERO \\
\hline ZERO & $0.1003 E+01$ & & ZERO \\
\hline $0.1298 \mathrm{E}-11$ & ZERO & & ZERO \\
\hline $0.7533 \mathrm{E}-06$ & ZERO & & ZERO \\
\hline $0.3967 \mathrm{E}-05$ & ZERO & & ZERO \\
\hline $0.1082 \mathrm{E}-02$ & ZERO & & ZERO \\
\hline $0.3012 \mathrm{E}-04$ & ZERO & & ZERO \\
\hline $0.1437 \mathrm{E}-02$ & ZERO & & ZERO \\
\hline $0.5550 \mathrm{E}-12$ & ZERO & & ZERO \\
\hline $0.3727 E-04$ & ZERO & & ZERO \\
\hline ZERO & ZERO & & ZERO \\
\hline $0.3180 \mathrm{E}-02$ & ZERO & & ZERO \\
\hline $0.8321 \mathrm{E}-04$ & ZERO & & ZERO \\
\hline ZERO & ZERO & & ZERO \\
\hline $0.5573 E-07$ & ZERO & & ZERO \\
\hline $0.2955 \mathrm{E}+00$ & ZERO & & ZERO \\
\hline $0.2200 \mathrm{E}-01$ & ZERO & & ZERO \\
\hline $0.9803 E-01$ & ZERO & & ZERO \\
\hline $0.5890 \mathrm{E}+00$ & ZERO & & ZERO \\
\hline $0.2500 \mathrm{E}-05$ & ZERO & & ZERO \\
\hline ZERO & $0.9792 \mathrm{E}+00$ & & ZERO \\
\hline ZERO & ZERO & & ZERO \\
\hline ZERO & ZERO & & ZERO \\
\hline $0.6686 \mathrm{E}-06$ & ZERO & & ZERO \\
\hline ZERO & $0.1037 \mathrm{E}-02$ & & ZERO \\
\hline $0.3924 \mathrm{E}-04$ & ZERO & 0.163 & $34 \mathrm{E}+02$ \\
\hline $0.1624 E-05$ & ZERO & & ZERO \\
\hline ZERO & ZERO & & ZERO \\
\hline ZERO & ZERO & & ZERO \\
\hline ZERO & ZERO & & ZERO \\
\hline ZERO & $0.4362 \mathrm{E}-02$ & & ZERO \\
\hline ZERO & ZERO & & ZERO \\
\hline $0.6701 E-08$ & ZERO & & ZERO \\
\hline ZERO & ZERO & & ZERO \\
\hline ZERO & $0.3209 \mathrm{E}-04$ & & ZERO \\
\hline ZERO & $0.1272 \mathrm{E}-03$ & & ZERO \\
\hline ZERO & ZERO & & ZERO \\
\hline ZERO & ZERO & & ZERO \\
\hline ZERO & ZERO & & ZERO \\
\hline ZERO & $0.4424 \mathrm{E}-02$ & & ZERO \\
\hline ZERO & ZERO & & ZERO \\
\hline
\end{tabular}

Section 1B, Page 16 of 21 
Table A-1. HLW Flowsheet Material Balance (Tank 51/ITP Cycle 1) Section 1B. Re-precipitation and Late Washing

STREAM NUMBERS (CONT'D) $\rightarrow$ STREAM NAME

$38 \quad 502$

$141 \mathrm{SO} 3$

137 Semi Vol Cs20

136 Semi Vol.CsCl

126 Semi Vol Group A

140 Semi Vol Na2B4

138 Semi Vol NaC.

139 Semi Vol NaF

125 Semi Vol NaI
23 Semi Vol RuO2

$111 \mathrm{SiO} 2$

$48 \mathrm{Sr}(\mathrm{COOH}) 2$

$176 \mathrm{Sr}$ (NO3) 2

153 Sr(NaTi205) 2

$127 \mathrm{Sr}(\mathrm{OH}) 2$

$30 \quad \mathrm{SrCO}_{3}$

46 SrO

$159 \mathrm{TcO} 2$

92 ThO2

$107 \mathrm{TiO2}$

119 Tritium

17 U308

$62 \mathrm{UO2}(\mathrm{COOH}) 2$

177 UO2 (NO3) 2

16. $\mathrm{UO2}(\mathrm{OH}) 2$

$27 \mathrm{Y}(\mathrm{COOH}) 3$

$128 \mathrm{Y}(\mathrm{OH}) 3$

$31 \mathrm{Y} 2(\mathrm{CO} 3) 3$

$31 \mathrm{Y} 2$
$42 \mathrm{O}$

100 Zeolite

$44 \mathrm{Zn}(\mathrm{COOH}) 2$
$179 \mathrm{Zn}(\mathrm{NO} 3) 2$

$57 \mathrm{zno}$

$104 \mathrm{zrO} 2$

13 hydrate $\mathrm{H} 2 \mathrm{O}$

1 water

TOTAL FLOW, LB/HR

TEMPERATURE, DEG $C$

PRESSURE， ATM

PRESSURE， PSIG

PRESSURE， MM HG

ENTHALPY, PCU/HR

VAPOR FIOW, CFM

LIOUID FLOW, GPM

DENSITY, LBS/FT3

PHASE

672

673

201

Spent Late Spent Late Spent Late Washd Precip Wash Recycle Wash Soluble Wash Insolbl Feed frm LWF

\begin{tabular}{|c|c|c|c|}
\hline ŻERO & ZERO & ZERO & ZERO \\
\hline ZERO & ZERO & ZERO & 2ERO \\
\hline ZERO & ZERO & ZERO & ZERO \\
\hline ZERO & ZERO & ZERO & ZERO \\
\hline ZERO & ZERO & ZERO & ZERO \\
\hline ZERO & ZERO & ZERO & ZERO \\
\hline ZERO & ZERO & ZERO & ZERO \\
\hline ZERO & ZERO & ZERO & ZERO \\
\hline ZERO & ZERO & ZERO & ZERO \\
\hline ZERO & ZERO & ZERO & ZERO \\
\hline ZERO & ZERO & ZERO & $0.2666 \mathrm{E}-01$ \\
\hline ZERO & ZERO & ZERO & ZERO \\
\hline ZERO & ZERO & ZERO & ZERO \\
\hline ZERO & ZERO & ZERO & $0.4072 \mathrm{E}-02$ \\
\hline $0.1926 \mathrm{E}-10$ & $0.1926 \mathrm{E}-10$ & ZERO & $0.7410 \mathrm{E}-12$ \\
\hline ZERO & ZERO & ZERO & $0.9081 \mathrm{E}-04$ \\
\hline ZERO & ZERO & ZERO & ZERO \\
\hline ZERO & ZERO & ZERO & ZERO \\
\hline ZERO & ZERO & ZERO & ZERO \\
\hline ZERO & ZERO & ZERO & $0.1213 \mathrm{E}-02$ \\
\hline $0.3304 \mathrm{E}-10$ & $0.3304 \mathrm{E}-10$ & ZERO & $0.1282 \mathrm{E}-11$ \\
\hline ZERO & ZERO & ZERO & $0.7512 \mathrm{E}-03$ \\
\hline ZERO & ZERO & ZERO & ZERO \\
\hline ZERO & ZERO & ZERO & ZERO \\
\hline $0.2792 \mathrm{E}-04$ & $0.2792 \mathrm{E}-04$ & ZERO & $0.1074 \mathrm{E}-05$ \\
\hline ZERO & ZERO & ZERO & ZERO \\
\hline ZERO & ZERO & ZERO & ZERO \\
\hline $0.2140 \mathrm{E}-10$ & $0.2140 \mathrm{E}-10$ & ZERO & $0.8232 \mathrm{E}-12$ \\
\hline ZERO & ZERO & ZERO & ZERO \\
\hline ZERO & ZERO & ZERO & $0.968 .3 \mathrm{E}-03$ \\
\hline ZERO & ZERO & ZERO & ZERO \\
\hline ZERO & ZERO & ZERO & ZERO \\
\hline $0.1387 \mathrm{E}-02$ & $0.1387 \mathrm{E}-02$ & ZERO & $0.5336 \mathrm{E}-04$ \\
\hline ZERO & ZERO & ZERO & $0.1980 \mathrm{E}+00$ \\
\hline ZERO & ZERO & ZERO & ZERO \\
\hline ZERO & ZERO & ZERO & $0.3589 \mathrm{E}-01$ \\
\hline $0.1753 \mathrm{E}+04$ & $0.1753 \mathrm{E}+04$ & ZERO & $0.4274 \mathrm{E}+03$ \\
\hline $0.1856 \mathrm{E}+04$ & $0.1856 \mathrm{E}+04$ & $0.6534 \mathrm{E}-04$ & $0.4762 \mathrm{E}+03$ \\
\hline $\begin{array}{r}0.3989 \mathrm{E}+02 \\
0.9656 \mathrm{E}+00 \\
-0.5055 \mathrm{E}+00 \\
0.7339 \mathrm{E}+03 \\
0.7132 \mathrm{E}+05\end{array}$ & $\begin{array}{r}0.3989 \mathrm{E}+02 \\
0.9656 \mathrm{E}+00 \\
-0.5055 \mathrm{E}+00 \\
0.7339 \mathrm{E}+03 \\
0.7132 \mathrm{E}+05\end{array}$ & $\begin{array}{r}0.3989 \mathrm{E}+02 \\
0.9656 \mathrm{E}+00 \\
-0.5055 \mathrm{E}+00 \\
0.7339 \mathrm{E}+03 \\
0.1587 \mathrm{E}-03\end{array}$ & $\begin{array}{r}0.3619 \mathrm{E}+02 \\
0.9656 \mathrm{E}+00 \\
-0.5055 \mathrm{E}+00 \\
0.7339 \mathrm{E}+03 \\
0.1554 \mathrm{E}+05\end{array}$ \\
\hline $\begin{array}{l}0.3569 \mathrm{E}+01 \\
0.6484 \mathrm{E}+02\end{array}$ & $\begin{array}{l}0.3569 \mathrm{E}+01 \\
0.6484 \mathrm{E}+02\end{array}$ & $\begin{array}{l}0.1771 \mathrm{E}-07 \\
0.4601 \mathrm{E}+03\end{array}$ & $\begin{array}{l}0.9392 \mathrm{E}+00 \\
0.6322 \mathrm{E}+02\end{array}$ \\
\hline LIQUID & LIQUID & IIQUID & LIQUID \\
\hline
\end{tabular}

657

Prec Eeed

Solubles

ZERO

ZERO

ZERO

ZERO

ZERO

ZERO

ZERO

ZERO

ZERO

ZERO

ZERO

ZERO

$0.7410 \mathrm{E}-12$

ZERO

ZERO

ZERO

ZERO

$0.1282 \mathrm{E}-11$

ZERO

ZERO

ZERO

$0.1074 \mathrm{E}-05$

ZERO

$0.8232 \mathrm{E}-12$

ZERO

ZERO

ZERO

ZERO

ZE-04

ZERO

ZERO

$0.4274 \mathrm{E}+03$

$0.4286 \mathrm{E}+03$

$0.3619 \mathrm{E}+02$

$0.9656 \mathrm{E}+00$

$-0.5055 E+00$

$0.7339 \mathrm{E}+03$

$0.1551 \mathrm{E}+05$

$0.8551 \mathrm{E}+00$

$6249 \mathrm{E}+02$
LIQUID
658

rec Feed 460

Insolubles ZERO to LWHT ZERO ZERO ZERO ZERO ZERO ZERO ZERO ZERO ZERO ZERO ZERO ZERO ZERO ZERO

ZERO, ZERO

ZERO
$0.4072 \mathrm{E}-02$

ZERO
$0.9081 \mathrm{E}-04$

ZERO ZERO

ZERO ZERO

$0.7512 \mathrm{E}-03 \quad$ ZERO

ZERO
ZERO ZERO

$0.6751 \mathrm{E}-13 \quad$ ZERO

ZERO ZERO

ZERO ZERO

$\begin{array}{ll}\text { ZERO } & \text { ZERO } \\ \text { ZERO } & \text { ZERO }\end{array}$

$0.9683 E-03 \quad \cdots \quad$ ZERO

ZERO

ZERO ZERO

$0.1980 \mathrm{E}+00$ ZERO

$0.3589 \mathrm{E}-01$ ZERO

$\begin{array}{rl}\text { ZERO } & 0.1184 \mathrm{E}+01 \\ 0.4762 \mathrm{E}+02 & 0.7274 \mathrm{E}+03\end{array}$

$0.3619 \mathrm{E}+02 \quad 0.3500 \mathrm{E}+02$

$0.9656 \mathrm{E}+00 \quad 0.1000 \mathrm{E}+01$

$0.2660 \mathrm{E}+02 \quad 0.7065 \mathrm{E}+04$

$0.1749 \mathrm{E}+03$

Section 1B, Page 17 of 21 
Table A-1. HLW Flowsheet Material Balance (Tank 51/ITP Cycle 1)

STREAM NUMBERS ->

STREAM NAME

COMPONENT FLOWS, IB/HR $122(\mathrm{C} 6 \mathrm{H} 5) 2$

123 ( $6 \mathrm{CH} 5) 2 \mathrm{C} 6 \mathrm{H} 4$

118 ( 6 $6 \mathrm{H} 5) 2 \mathrm{NH}$

133 (C6H5B (OH) 2 salt)

132 (C6H5B (OH) 2)

166 (C6H5C6H4OH)

162 (C6H5NH2)

163 (C6H5NHCHO)

165 (C6H5NO)

164 (C6H5NO2)

131 (C6H5OH salt)

130 ( $6 \mathrm{CH} 5 \mathrm{OH}$ )

121 (C6H6)

155 (CH3) $2 \mathrm{CHOH}$

154 ( $\mathrm{CH} 3 \mathrm{OH})$

$45 \mathrm{Ag}$

$116 \mathrm{Ag} 20$

134 AgNO3

$106 \mathrm{~B} 203$

$9 \mathrm{Ba}(\mathrm{OH}) 2$

$15 \mathrm{BaO}$

$24 \mathrm{BaSO} 4$

$66 \mathrm{CO}$

$37 \mathrm{CO} 2$

$142 \mathrm{CO} 2$ (C14)

$63 \mathrm{Ca}(\mathrm{COOH}) 2$

$167 \mathrm{Ca}$ (NO3) 2

$120 \mathrm{Ca}(\mathrm{OH}) 2$

$113 \mathrm{Ca} 3$ (PO4) 2

$20 \mathrm{CaC} 2 \mathrm{O} 4$

$18 \mathrm{CaCO} 3$

$64 \mathrm{CaCO}$ (C14)

$78 \mathrm{CaF} 2$

$\begin{array}{ll}41 & \mathrm{CaO} \\ 19 & \mathrm{CaSO} 4\end{array}$

19 Caso 4
29 Carbon

99 Cement

$26 \mathrm{Co}(\mathrm{COOH}) 2$

$168 \mathrm{Co}$ (NO3) 2

$59 \mathrm{CoO}$

$98 \quad \mathrm{Cr} 203$

$42 \mathrm{Cs} 2 \mathrm{O}$

$53 \mathrm{CsCOOH}$

$96 \mathrm{CsCl}$

$69 \mathrm{CsNO}^{2}$

$8 \mathrm{CsOH}$
$51 \mathrm{CsTPB}$

WSRC-TR-95-0019 (Revision
Section 1B. Re-precipitation and Late Washing

461

TWHT Htr

HEPA vnt

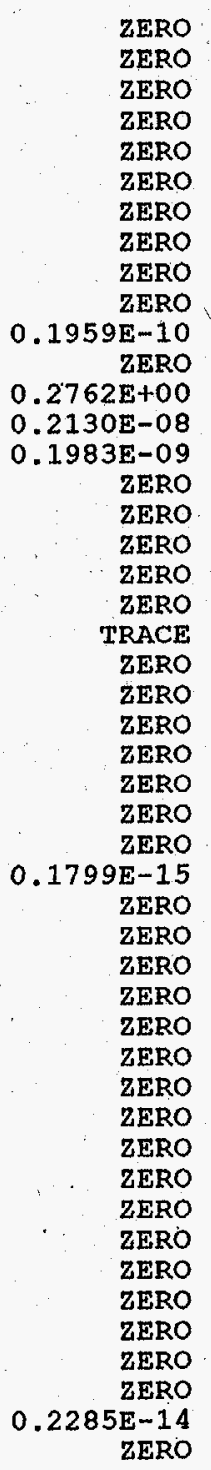

0)
Date: January 1995
Section 1B, Page 18 of 21 
Table A-1. HLW Flowsheet Material Balance (Tank 51/ITP Cycle 1) Section 1B. Re-precipitation and Late Washing

STREAM NUMBERS (CONT'D) $\rightarrow$ STREAM NAME

$54 \mathrm{Cu}(\mathrm{COOH}) 2$

$170 \mathrm{Cu}(\mathrm{NO} 3) 2$

55 CuO

$80 \mathrm{Fe} 203$

$101 \mathrm{FeO}$

102 Group A

103 Group B

$25 \mathrm{H} 2$

$158 \mathrm{H} 2 \mathrm{C} 2 \mathrm{O} 4$

$93 \mathrm{H} 2 \mathrm{SO} 4$

$90 \mathrm{H} 3 \mathrm{BO} 3$

$43 \mathrm{HCl}$

$43 \mathrm{HCl}$

$85 \mathrm{HF}$

49 HNO3

$36 \mathrm{Hg}$
$129 \mathrm{Hg}(\mathrm{C} 6 \mathrm{H} 5)$

$135 \mathrm{Hg}(\mathrm{NO} 3) 2$

$144 \mathrm{Hg} 2 \mathrm{Cl} 2$

$117 \mathrm{Hg} 2 \mathrm{I} 2$

$146 \mathrm{HgCl} 2$

$21 \mathrm{HgO}$

97 I2

$183 \mathrm{~K} 2 \mathrm{CO} 3$

$39 \mathrm{~K} 2 \mathrm{O}$

$51 \mathrm{KCOOH}$

$157 \mathrm{KMnO} 4$

$161 \mathrm{KNO} 3$

$10 \mathrm{KOH}$

150 KTPB

$105 \mathrm{La} 2 \mathrm{O} 3$

$108 \mathrm{Li} 20$

$65 \mathrm{Mg}(\mathrm{COOH}) 2$

$171 \mathrm{Mg}(\mathrm{NO} 3) 2$

$33 \mathrm{MgO}$

$60 \mathrm{Mn}(\mathrm{COOH}) 2$

$172 \mathrm{Mn}(\mathrm{NO} 3) 2$

$180 \mathrm{Mn} 304$

$56 \mathrm{MnO}$

$14 \mathrm{MnO} 2$

$145 \mathrm{MOO2}$

81 N2

74 N2O
182 NH3

114 NH3OHNO3

$52 \mathrm{NH} 4 \mathrm{COOH}$

173 NH4NO3

$68 \mathrm{NH} 4 \mathrm{OH}$

148 NH4TPB

WSRC-TR-95-0019 (Revision
461

LWHT Htr

HEPA vnt

ZERO

ZERO

ZERO

ZERO

$0.3104 \mathrm{E}-14$

$0.9542 \mathrm{E}-16$

$0.1121 \mathrm{E}-05$

ZERO

ZERO

ZERO

ZERO

ZERO

ZERO

ZERO

ZERO

ZERO

ZERO

ZERO

ZERO

ZERO

ZERO

ZERO

ZERO

ZERO

ZERO

ZERO

$0.137 \mathrm{OE}-15$

IRO

TERO

ZERO

ZERO

ZERO

ZERO

ZERO

ZERO

ZERO

ZERO

ZERO

ZERO

ZERO

$0.7098 \mathrm{E}+0$

$0.8400 \mathrm{E}-06$

$0.8949 \mathrm{E}-04$

ZERO

ZERO

ZERO

ZERO

ZERO

0) 
Table A-1. HLW Flowsheet Material Balance (Tank 51/ITP Cycle 1) Section 1B. Re-precipitation and Late Washing

STREAM NUMBERS (CONT'D) $\rightarrow$ STREAM NAME

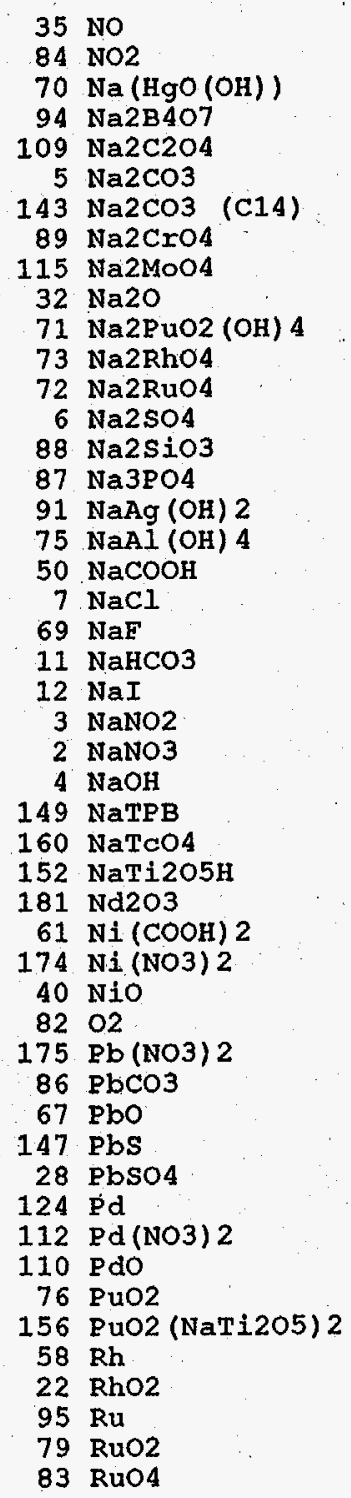

83 RuO4

WSRC-TR-95-0019 (Revision
461

IWHT Htx

HEPA vnt

ZERO

$0.1483 \mathrm{E}-19$

$0.1305 \mathrm{E}-10$

$0.7062 \mathrm{E}-10$

$0.2135 \mathrm{E}-09$

$0.3598 \mathrm{E}-18$

$0.1324 \mathrm{E}-12$

$0.3955 \mathrm{E}-13$

ZERO

$0.1653 \mathrm{E}-20$

$0.9594 \mathrm{E}-15$

$0.5052 \mathrm{E}-14$

$0.1378 \mathrm{E}-11$

$0.3835 \mathrm{E}-13$

$0.1830 \mathrm{E}-11$

$0.7068 \mathrm{E}-21$

$0.4747 E-13$

$0.1021 \mathrm{E}-11$

$0.1060 \mathrm{E}-12$

ZERO

$0.7098 \mathrm{E}-16$

$0.3763 E-0.9$

$0.4247 \mathrm{E}-08$

$0.4241 E-09$
ZERO

ZERO

ZERO

ZERO

ZERO

ZERO

$0.1634 \mathrm{E}+02$

ZERO

ZERO

ZERO

ZERO

ZERO

$0.8534 \mathrm{E}-17$

ZERO

ZERO

ZERO

ZERO

ZERO

ZERO

ZERO

ZERO 
Table A-1. HLW Flowsheet Material Balance (Tank 51/ITP Cycle 1) Section 1B. Re-precipitation and Late Washing

STREAM NUMBERS (CONT'D) $\rightarrow$ STREAM NAME

\section{$38 \quad 502$ \\ $141 \mathrm{SO}$}

137 Semi Vol Cs20

136 Semi Vol Csc1

126 Semi Vol Group A

140 Semi Vol Na2B40

138 Semi Vol NaCl

139 Semi Vol NaF

125 Semi Vol NaI

23 Semi
111 sio2

$48 \mathrm{Sr}(\mathrm{COOH}) 2$

$176 \mathrm{sx}$ (NO3) 2

$153 \mathrm{sx}(\mathrm{NaTi2O5)} 2$

$127 \operatorname{Sr}(\mathrm{OH}) 2$

$30 \mathrm{SrCO} 3$

46 sro

$159 \mathrm{ToO} 2$

92 ThO2

107. TiO2

$\begin{array}{rl}119 & \text { Triti } \\ 17 & 0308\end{array}$

$62 \mathrm{VO} 2(\mathrm{COOH}) 2$

$177 \mathrm{UO2}$ (NO3) 2

$16 \mathrm{VO} 2(\mathrm{OH}) 2$

$27 \mathrm{Y}(\mathrm{COOH}) 3$

178 Y (NO3) 3

128 Y (OH) 3

$31 \mathrm{Y} 2(\mathrm{CO} 3) 3$

$47 \mathrm{Y} 2 \mathrm{O3}$

100 zeolite

$44 \mathrm{Zn}(\mathrm{COOH}) 2$
$79 \mathrm{Zn}(\mathrm{NO}) 2$

$57 \mathrm{ZnO}$

$104 \mathrm{ZrO} 2$

13 hydxate H2O$$
1 \text { water }
$$

TOTAL FLOW, LB/HR

TEMPERATURE， DEG C

PRESSURE, ATM

PRESSURE, PSIG

PRESSURE, MM HG

ENTHALPY, PCU/HR

VAPOR FLOW, CFM

LIQUID FLOW, GPM

DENSITY， LBS/ET3

PHASE

WSRC-TR-95-0019 (Revision
461

LWHT Htr

HEPA vnt

ZERO

ZERO

ZERO

2IRO

ZERO

ZERO

ZERO

ZERO

ZERO

ZERO

ZERO

ZERO

ZERO

ZERO

$0.9438 \mathrm{E}-21$

ZERO

ZERO

ZERO

ZERO

$0.3972 \mathrm{E}-12$

ZERO

ZERO

$0.1368 \mathrm{E}-14$

ZERO

$0.1048 \mathrm{E}-20$

ZERO

ZERO

ZERO

ZERO

ZERO

ZERO

ZERO

ZERO

$0.2031 \mathrm{E}+02$

$0.7468 \mathrm{E}+03$

ZERO

$0.9656 \mathrm{E}+00$

$0.5055 \mathrm{~F}+00$

$0.7339 \mathrm{E}+03$

$0.733 \mathrm{E}+03$

$0.1228 \mathrm{E}+05$

$0.1671 \mathrm{E}+03$

$0.7448 \mathrm{E}-01$

VAPOR 


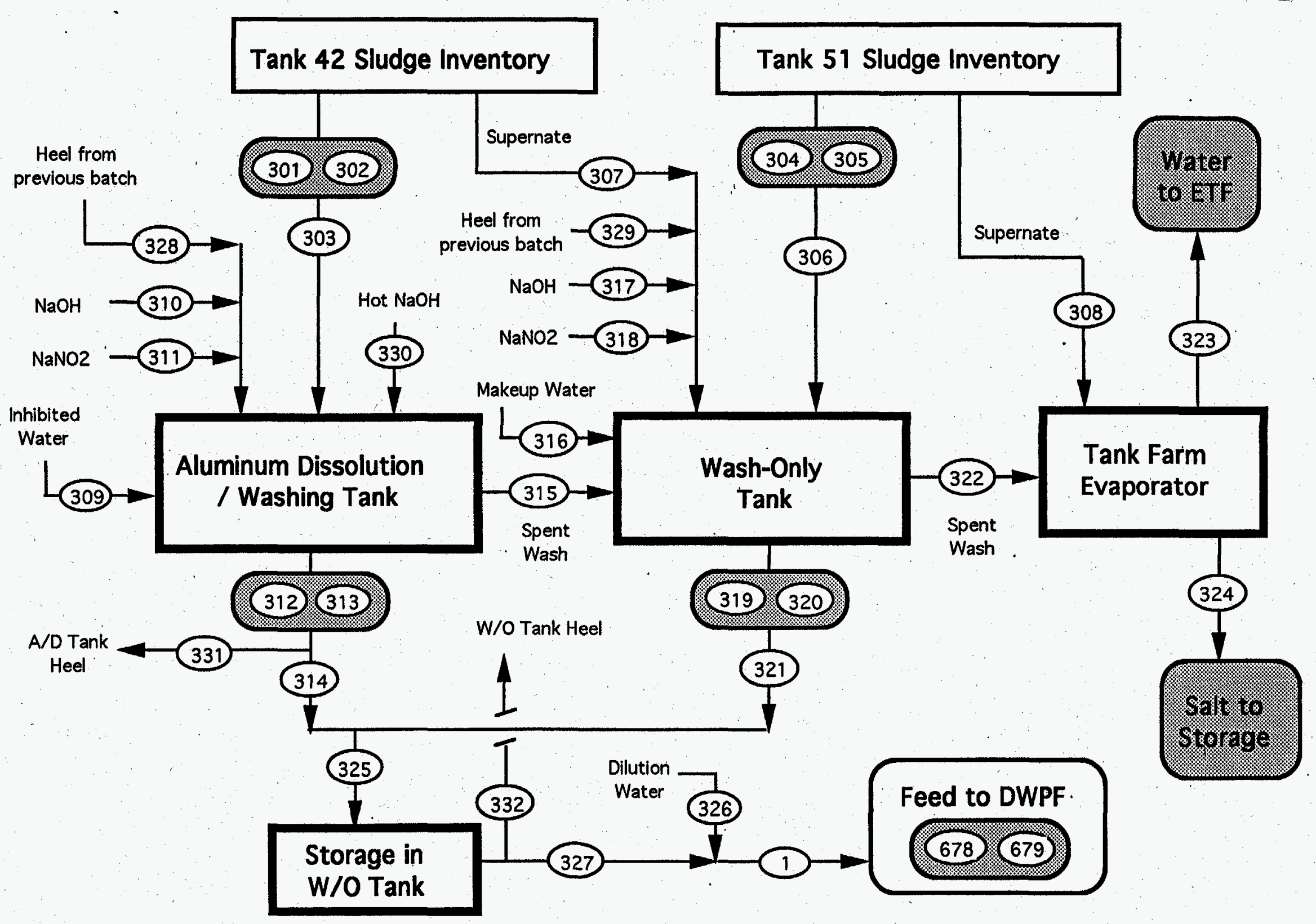

SECTION 1C. EXTENDED SLUDGE PROCESSING 

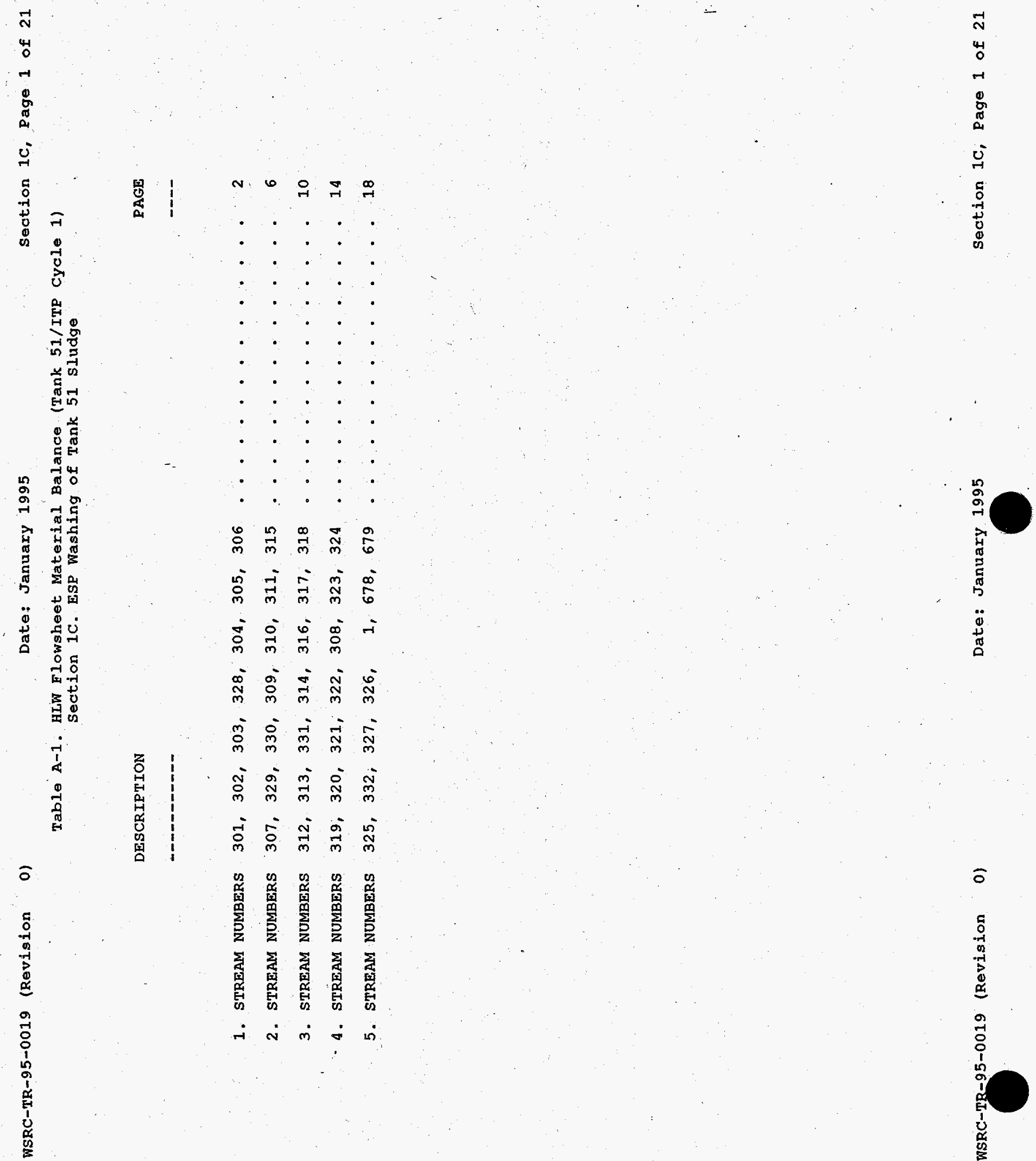

o

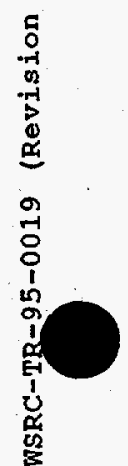


Table A-1. HLW Flowsheet Material Balanoe (Tank 51/ITP Cyole 1)

STREAM NUMBERS $\rightarrow$ STREAM NAME

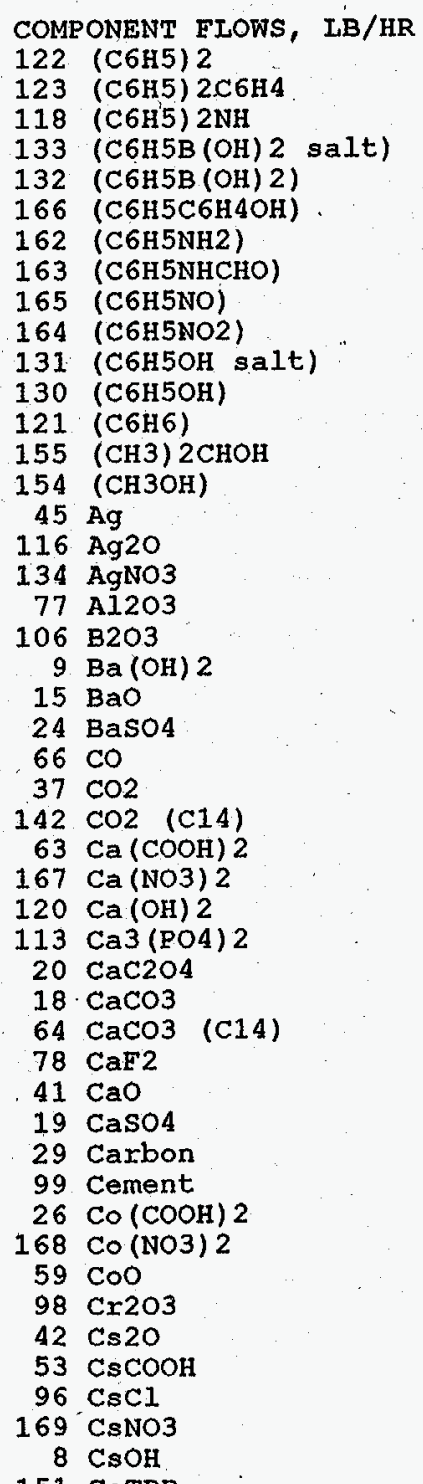

303
Unwshd $A / D$ in $A / D$ Tank solbl in $A / D$ sldg slurry

\begin{tabular}{|c|c|c|}
\hline ZERO & ZERO & ZERO \\
\hline ZERO & ZERO & ZERO \\
\hline ZERO & ZERO & ZERO \\
\hline ZERO & ZERO & ZERO \\
\hline ZERO & ZERO & ZERO \\
\hline ZERO & ZERO & ZERO \\
\hline ZERO & ZERO & ZERO \\
\hline ZERO & ZERO & ZERO \\
\hline ZERO & ZERO & ZERO \\
\hline ZERO & ZERO & ZERO \\
\hline ZERO & ZERO & ZERO \\
\hline ZERO & ZERO & ZERO \\
\hline ZERO & ZERO & ZERO \\
\hline ZERO & ZERO & ZERO \\
\hline ZERO & ZERO & ZERO \\
\hline ZERO & ZERO & ZERO \\
\hline ZERO & ZERO & ZERO \\
\hline ZERO & ZERO & ZERO \\
\hline ZERO & ZERO & ZERO \\
\hline ZERO & ZERO & ZERO \\
\hline ZERO & ZERO & ZERO \\
\hline ZERO & ZERO & ZERO \\
\hline ZERO & ZERO & ZERO \\
\hline ZERO & ZERO & ZERO \\
\hline ZERO & ZERO & ZERO \\
\hline ZERO & ZERO & ZERO \\
\hline ZERO & ZERO & ZERO \\
\hline ZERO & ZERO & ZERO \\
\hline ZERO & ZERO & ZERO \\
\hline ZERO & ZERO & ZERO \\
\hline ZERO & ZERO & ZERO \\
\hline ZERO & ZERO & ZERO \\
\hline ZERO & ZERO & ZERO \\
\hline ZERO & ZERO & ZERO \\
\hline ZERO & ZERO & ZERO \\
\hline ZERO & ZERO & ZERO \\
\hline ZERO & ZERO & ZERO \\
\hline ZERO & ZERO & ZERO \\
\hline ZERO & ZERO & ZERO \\
\hline ZERO & ZERO & ZERO \\
\hline ZERO & ZERO & ZERO \\
\hline ZERO & ZERO & ZERO \\
\hline ZERO & ZERO & ZERO \\
\hline ZERO & ZERO & ZERO \\
\hline ZERO & ZERO & ZERO \\
\hline ZERO & ZERO & ZERO \\
\hline ZERO & ZERO & ZERO \\
\hline ZERO & ZERO & ZERO \\
\hline
\end{tabular}

328 304 306 Tank Insolubles Unwshd Sldg Heel in $W / O$ Tank solbl in $W / O$ Unwshd $w / 0$

$$
\begin{aligned}
& \text { ZERO } \\
& \text { ZERO } \\
& \text { ZERO } \\
& \text { ZERO } \\
& \text { ZERO } \\
& \text { ZERO } \\
& \text { ZERO } \\
& \text { ZERO } \\
& \text { ZERO } \\
& \text { ZERO } \\
& \text { ZERO } \\
& \text { ZERO } \\
& \text { ZERO } \\
& \text { ZERO } \\
& \text { ZERO } \\
& \text { ZERO } \\
& \text { ZERO } \\
& \text { ZERO } \\
& \text { ZERO } \\
& \text { ZERO } \\
& \text { ZERO } \\
& \text { ZERO } \\
& \text { ZERO } \\
& \text { ZERO } \\
& \text { ZERO } \\
& \text { ZERO } \\
& \text { ZERO } \\
& \text { ZERO } \\
& \text { ZERO } \\
& \text { ZERO } \\
& \text { ZERO } \\
& \text { ZERO } \\
& \text { ZERO } \\
& \text { ZERO } \\
& \text { ZERO } \\
& \text { ZERO } \\
& \text { ZERO } \\
& \text { ZERO } \\
& \text { ZERO } \\
& \text { ZERO } \\
& \text { ZERO } \\
& \text { ZERO } \\
& \text { ZERO } \\
& \text { ZERO } \\
& \text { ZERO } \\
& \text { ZERO } \\
& \text { ZERO } \\
& \text { ZERO }
\end{aligned}
$$

\begin{tabular}{|c|c|c|}
\hline ZERO & ZERO & ZERO \\
\hline ZERO & ZERO & ZERO \\
\hline ZERO & ZERO & ZERO \\
\hline ZERO & ZERO & ZERO \\
\hline ZERO & ZERO & ZERO \\
\hline ZERO & ZERO & ZERO \\
\hline ZERO & ZERO & ZERO \\
\hline ZERO & ZERO & ZERO \\
\hline ZERO & ZERO & ZERO \\
\hline ZERO & ZERO & ZERO \\
\hline ZERO & ZERO & ZERO \\
\hline ZERO & ZERO & ZERO \\
\hline ZERO & ZERO & ZERO \\
\hline ZERO & ZERO & ZERO \\
\hline ZERO & ZERO & ZERO \\
\hline ZERO & ZERO & ZERO \\
\hline $0.1240 \mathrm{E}-01$ & ZERO & $0.1240 \mathrm{E}-01$ \\
\hline ZERO & ZERO & , ZERO \\
\hline $0.1042 \mathrm{E}+02$ & ZERO & $0.1042 \mathrm{E}+02$ \\
\hline ZERO & ZERO & ZERO \\
\hline ZERO & $0.2484 \mathrm{E}-02$ & $0.2484 \mathrm{E}-02$ \\
\hline ZERO & ZERO & ZERO \\
\hline $0.1802 \mathrm{E}-01$ & ZERO & $0.1802 \mathrm{E}-01$ \\
\hline ZERO & ZERO & ZERO \\
\hline ZERO & ZERO & ZERO \\
\hline ZERO & ZERO & ZERO \\
\hline ZERO & ZERO & ZERO \\
\hline ZERO & ZERO & ZERO \\
\hline ZERO & $0.1875 E-03$ & $0.1875 \mathrm{E}-03$ \\
\hline $0.2516 \mathrm{E}+01$ & ZERO & $0.2516 \mathrm{E}+01$ \\
\hline ZERO & ZERO & ZERO \\
\hline $0.3111 \mathrm{E}+01$ & ZERO & $0.3111 \mathrm{E}+01$ \\
\hline $0.1532 \mathrm{E}-06$ & ZERO & $0.1532 \mathrm{E}-06$ \\
\hline $0.1828 \mathrm{E}-01$ & ZERO & $0.1828 \mathrm{E}-b_{1}$ \\
\hline $0.4073 \mathrm{E}-02$ & ZERO & $0.4073 \mathrm{E}-02$ \\
\hline $0.3611 \mathrm{E}-01$ & ZERO & $0.3611 \mathrm{E}-01$ \\
\hline ZERO & ZERO & ZERO \\
\hline ZERO & ZERO & ZERO \\
\hline ZERO & ZERO & ZERO \\
\hline ZERO & ZERO & ZERO \\
\hline ZERO & ZERO & ZERO \\
\hline $0.1984 \mathrm{E}+00$ & ZERO & $0.1984 \mathrm{E}+00$ \\
\hline $0.1240 \mathrm{E}-03$ & ZERO & $0.1240 \mathrm{E}-03$ \\
\hline ZERO & ZERO & ZERO \\
\hline ZERO & ZERO & ZERO \\
\hline ZERO & ZERO & ZERO \\
\hline ZERO & $0.6185 \mathrm{E}-04$ & $0.6185 \mathrm{E}-04$ \\
\hline
\end{tabular}

Date: January 1995 section 1C, Page 2 of 21 
Table A-1. HLW Flowsheet Material Balance (Tank 51/ITP Cycle 1) Section 1C. ESP Washing of Tank. 51 sludge

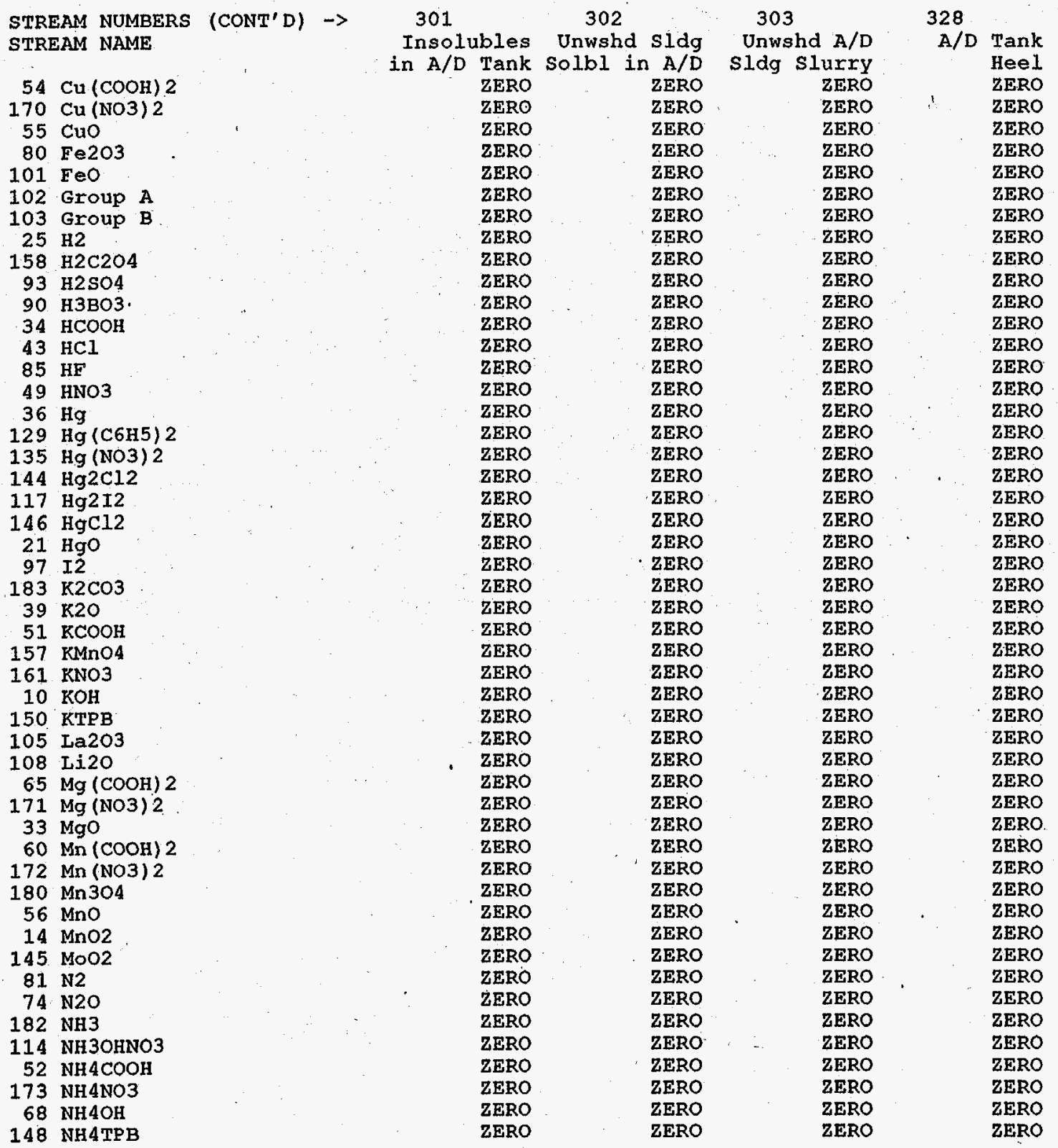

\begin{tabular}{|c|c|c|}
\hline $\begin{array}{l}304 \\
\text { Insolubles }\end{array}$ & $\begin{array}{l}305 \\
\text { Unwshd sldg }\end{array}$ & $\begin{array}{l}306 \\
\text { Unwshd } w / 0\end{array}$ \\
\hline $\begin{array}{r}\text { In W/O Tank } \\
\text { zERO }\end{array}$ & Solbl in $\mathrm{W} / 0$ & $\begin{array}{r}\text { Sldg Sluxxy } \\
\text { zERO }\end{array}$ \\
\hline ZERO & ZERO & ZERO \\
\hline $0.2549 \mathrm{E}-0.1$ & ZERO & $0.2549 \mathrm{E}-0.1$ \\
\hline $0.3416 \mathrm{E}+02$ & $0.1400 \mathrm{E}-02$ & $0.3416 \mathrm{E}+02$ \\
\hline ZERO & ZERO & ZERO \\
\hline $\begin{array}{l}0.4005 E-02 \\
0.1833 E-03\end{array}$ & $\begin{array}{l}0.2114 \mathrm{E}-02 \\
0.5104 \mathrm{E}-05\end{array}$ & $\begin{array}{l}0.6119 \mathrm{E}-02 \\
0.1884 \mathrm{E}-03\end{array}$ \\
\hline ZERO & ZERO & ZERO \\
\hline ZERO & ZERO & ZERO \\
\hline ZERO & ZERO & ZERO \\
\hline ZERO & ZERO & ZERO \\
\hline ZERO & ZERO & ZERO \\
\hline ZERO & ZERO & ZERO \\
\hline ZERO & ZERO & ZERO \\
\hline ZERO & ZERO & ZERO \\
\hline ZERO & ZERO & ZERO \\
\hline ZERO & ZERO & ZERO \\
\hline ZERO & ZERO & ZERO \\
\hline ZERO & ZERO & ZERO \\
\hline ZERO & ZERO & ZERO \\
\hline ZERO & ZERO & ZERO \\
\hline $0.1686 \mathrm{E}+00$ & ZERO & $0.1686 \mathrm{E}+00$ \\
\hline ZERO & ZERO & ZERO \\
\hline ZERO & ZERO & ZERO \\
\hline $0.8995 \mathrm{E}-01$ & ZERO & $0.8995 \mathrm{E}-01$ \\
\hline ZERO & ZERO & ZERO \\
\hline ZERO & ZERO & ZERO \\
\hline ZERO & ZERO & ZERO \\
\hline ZERO & $0.1327 \mathrm{E}+00$ & $0.1327 \mathrm{E}+00$ \\
\hline ZERO & ZERO & ZERO \\
\hline ZERO & ZERO & ZERO \\
\hline ZERO & ZERO & ZERO \\
\hline ZERO & ZERO & ZERO \\
\hline ZERO & ZERO & ZERO \\
\hline $0.1857 \mathrm{E}+01$ & ZERO & $0.1857 \mathrm{E}+\mathrm{b}_{1}$ \\
\hline ZERO & ZERO & ZERO \\
\hline ZERO & ZERO & ZERO \\
\hline ZERO & ZERO & ZERO \\
\hline ZERO & ZERO & ZERO \\
\hline $0.3953 \mathrm{E}+01$ & ZERO & $0.3953 \mathrm{E}+01$ \\
\hline ZERO & ZERO & ZERO \\
\hline ZERO & ZERO & ZERO \\
\hline ZERO & ZERO & ZERO \\
\hline ZERO & ZERO & ZERO \\
\hline ZERO & ZERO & ZERO \\
\hline ZERO & ZERO & ZERO \\
\hline ZERO & ZERO & ZERO \\
\hline ZERO & $0.5091 \mathrm{E}-02$ & $0.5091 \mathrm{E}-02$ \\
\hline ZERO & ZERO & ZERO \\
\hline
\end{tabular}


Table A-1. HLW Flowsheet Material Balance (Tank 51/ITP Cycle 1) - Section 1C. ESP Washing of Tank 51 sludge

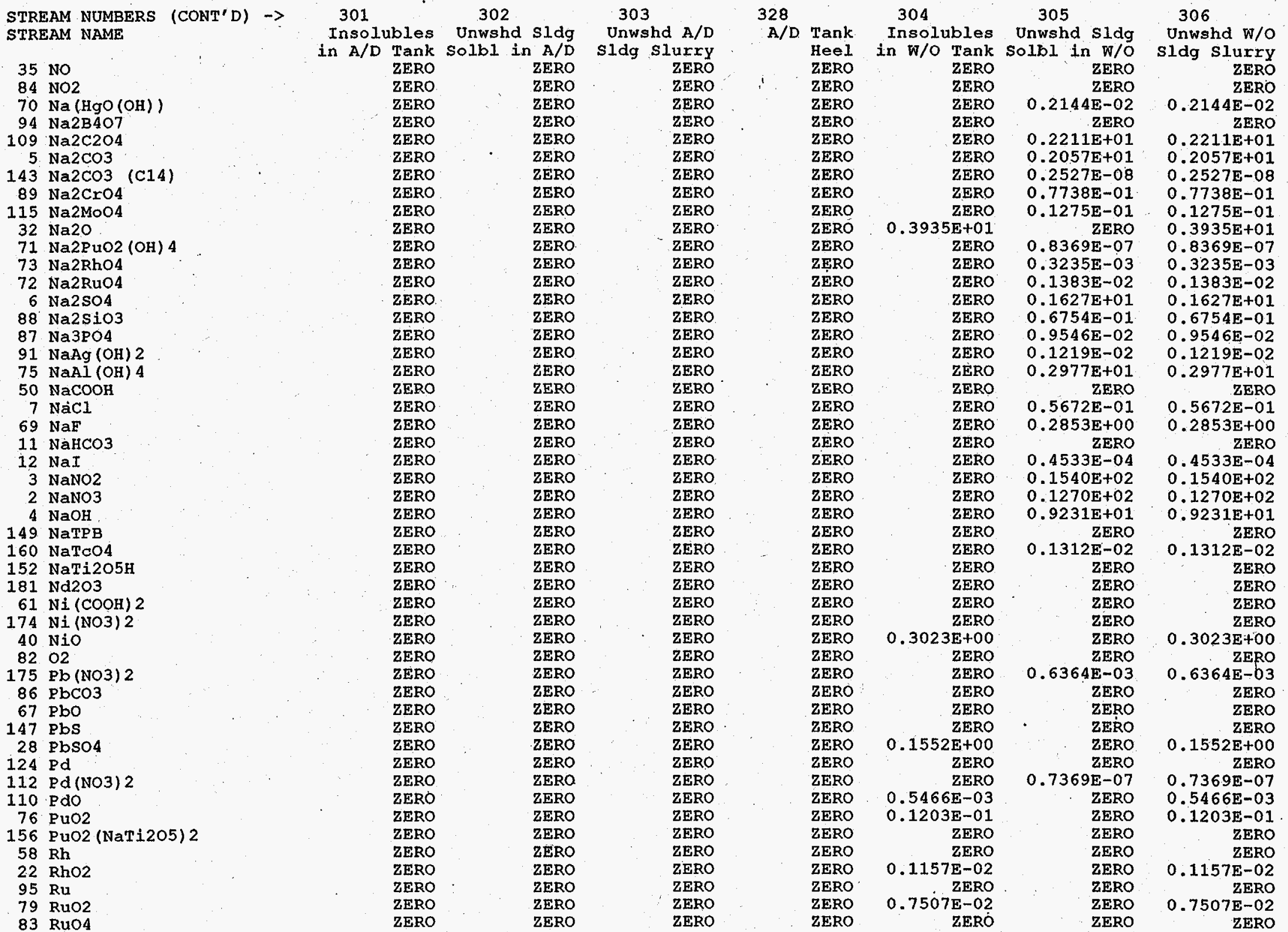


Table A-1. HLW Flowsheet Material Balance (Tank 51/ITP Cycle 1) Section 1C. ESP Washing of Tank 51 sludge

STREAM NUMBERS (CONT'D) $\rightarrow$ STREAM NAME

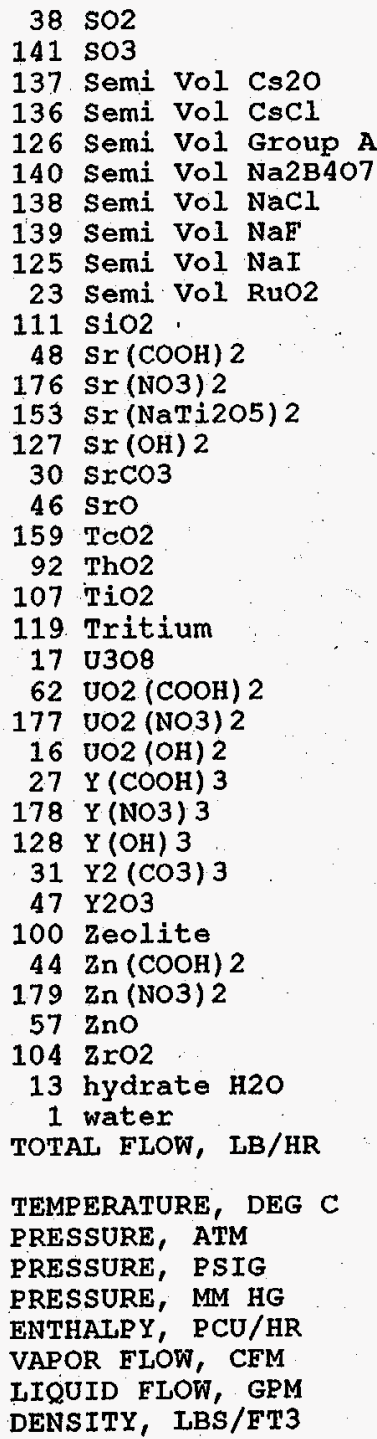

Insolubles Unwshd sldg Unwshd $A / D$ in A/D Tank Solbl in $A / D$ sldg Slurry $\begin{array}{lll}\text { ZERO } & \text { ZERO } & \text { ZERO } \\ \text { ZERO } & \text { ZERO } & \text { ZERO } \\ \text { ZERO } & \text { ZERO } & \text { ZERO }\end{array}$ ZERO ZERO ZERO ZERO ZERO $\begin{array}{lll}\text { ZERO } & \text { ZERO } \\ \text { ZERO } & \text { ZERO } & \text { ZERO }\end{array}$ ZERO 2 ZERO ZERO $\quad$ ZERO ZERO

ZERO ZERO $\quad$ ZERO

ZERO ZERO ZERO

ZERO ZERO $\quad$ ZERO

ZERO ZERO ZERO

ZERO ZERO ZERO

ZERO ZERO ZERO

ZERO ZERO

$\begin{array}{lll}\text { ZERO } & \text { ZERO } & \text { ZERO } \\ \text { ZERO } & \text { ZERO } & \text { ZERO }\end{array}$

ZERO ZERO ZERO

ZERO ZERO $\quad$ ZERO

ZERO

ZERO ZERO

ZERO ZERO ZERO

ZERO ZERO ZERO

ZERO ZERO ZERO

ZERO ZERO ZERO

ZERO ZERO Z ZERO

ZERO ZERO ZERO

ZERO ZERO ZERO

ZERO ZERO $\quad$ ZERO

ZERO ZERO $\quad$ ZERO

ZERO ZERO $\quad$ ZERO

ZERO ZERO ZERO

ZERO ZERO $\quad$ ZERO

ZERO ZERO ZERO

ZERO ZERO Z ZERO

ZERO ZERO $\quad$ ZERO

ZERO $\quad$ ZERO $\quad$ ZERO

$\begin{array}{lll}\text { ZERO } & \text { ZERO } & \text { ZERO } \\ \text { ZERO } & \text { ZERO }\end{array}$

$0.5000 \mathrm{E}+02$

$0.5000 \mathrm{E}+02$

328 304

305

306

A/D Tank Insolubles Unwshd Sldg Unwshd W/O Heel in $W / O$ Tank Solbl in W/O Sldg Slurry ZERO ZERO ZERO Z ZERO ZERO ZERO ZERO ZERO ZERO ZERO ZERO ZERO $\begin{array}{lll}\text { ZERO } & \text { ZERO } & \text { ZERO } \\ \text { ZERO } & \text { ZERO }\end{array}$

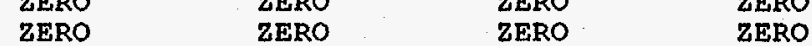
$\begin{array}{lll}\text { ZERO } & \text { ZERO } & \text { ZERO } \\ \text { ZERO } & \text { ZERO } & \text { ZERO }\end{array}$ $\begin{array}{llll}\text { ZERO } & \text { ZERO } & \text { ZERO } & \text { ZERO } \\ \text { ZERO } & \text { ZERO } & \text { ZERO } & \text { ZERO }\end{array}$ $\begin{array}{lll}\text { ZERO } & \text { ZERO } & \text { ZERO } \\ \text { ZERO } & \text { ZERO } & \text { ZERO }\end{array}$ $\begin{array}{llll}\text { ZERO ZERO } & \text { ZERO }\end{array}$ ZERO ZERO ZERO ZERO ZERO $0.1292 \mathrm{E}+01$ ZERO $0.1292 \mathrm{E}+01$ $\begin{array}{llll}\text { ZERO ZERO } & \text { ZERO } \\ \text { ZERO }\end{array}$ ZERO $\quad$ ZERO $\quad$ ZERO $\quad \because \quad$ ZERO $\begin{array}{lrrr}\text { ZERO } & \text { ZERO } & \text { ZERO } & \text { ZERO } \\ \text { ZERO } & \text { ZERO } & 0.3023 E-06 & 0.3023 E-06\end{array}$ ZERO $0.2501 \mathrm{E}-01$ ZERO $0.2501 E-01$ ZERO $0.2501 \mathrm{E}-01$ $\begin{array}{rrrr}\text { ZERO } & \text { ZERO } & \text { ZERO } & \text { ZERO } \\ \text { ZERO } & 0.4211 \mathrm{E}-02 & \text { ZERO } & 0.4211 \mathrm{E}-02\end{array}$

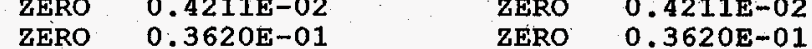
$\begin{array}{llll}\text { ZERO } & 0.3620 \mathrm{E}-01 & \text { ZERO } & 0.3620 \mathrm{E}-01 \\ \text { ZERO } & 0.5662 \mathrm{E}-01 & \text { ZERO } & 0.5662 \mathrm{E}-01\end{array}$ $\begin{array}{rrrr}\text { ZERO } & 0.5662 \mathrm{E}-01 & \text { ZERO } & 0.5662 \mathrm{E}-01 \\ \text { ZERO } & \text { ZERO } & 0.8558 \mathrm{E}-08 & 0.8558 \mathrm{E}-08\end{array}$ ZERO $0.2538 \mathrm{E}+01 \quad$ ZERO $\quad 0.2538 \mathrm{E}+01$ ZERO ZERO $\quad$ ZERO ZERO ZERO ZERO $\quad$ ZERO ZERO ZERO $0.5900 \mathrm{E}-03 \quad 0.5900 \mathrm{E}-03$ ZERO ZERO ZERO ZERO $\begin{array}{llll}\text { ZERO } & \text { ZERO } \\ \text { ZERO } & \text { ZERO }\end{array}$ $\begin{array}{rrrr}\text { ZERO } & 0.3046 \mathrm{E}-06 & 0.3046 \mathrm{E}-06\end{array}$ $\begin{array}{llll}\text { ZERO } & 0.6295 \mathrm{E}-02 & \text { ZERO } & 0.6295 \mathrm{E}-02\end{array}$ $\begin{array}{llll}\text { ZERO } & \text { ZERO } & \text { ZERO } & \text { ZERO } \\ \text { ZERO } & \text { ZERO } & \text { ZERO } & \text { ZERO }\end{array}$ $\begin{array}{llll}\text { ZERO } & \text { ZERO } & \text { ZERO } & \text { ZERO } \\ \text { ZERO } & \text { ZERO } & \text { ZERO } & \text { ZERO }\end{array}$ $\begin{array}{llll}\text { ZERO } & \text { ZERO } & \text { ZERO } & \text { ZERO } \\ \text { ZERO } & \text { ZERO } & \text { ZERO } & \text { ZERO } \\ \text { ZERO } & 1436 \text { E } & & \end{array}$ ZERO $0.1436 \mathrm{E}+00 \quad \because \quad$ ZERO $0.1436 \mathrm{E}+00$ $\begin{array}{rrrr}\text { ZERO } & \text { ZERO } & \text { ZERO } & \text { ZERO } \\ \text { ZERO } & 0.2776 \mathrm{E}+01 & 0.4738 \mathrm{E}-03 & 0.2777 \mathrm{E}+01\end{array}$ $\begin{array}{rrrr}\text { ZERO } & \text { ZERO } & 0.1922 E+03 & 0.1922 E+03 \\ \text { ZERO } & 0.6788 E+02 & 0.2391 E+03 & 0.3069 E+03\end{array}$ $\begin{array}{ll}0.3532 \mathrm{E}+02 & 0.5000 \mathrm{E}+02 \\ 0.1000 \mathrm{E}+01 & 0.1000 \mathrm{E}+01\end{array}$ ZERO $\quad$ ZERO ZERO

ZERO ZERO ZERO

$0.4800 \mathrm{E}+02$

$0.3691 \mathrm{E}+02$ $0.1000 \mathrm{~T}+01$ ZERO

$0.5000 \mathrm{E}+02$

$\begin{array}{ll}0.3621 \mathrm{E}+02 & 0.3691 \mathrm{E}+02 \\ 0.1000 \mathrm{E}+01 & 0.1000 \mathrm{E}+01\end{array}$ $\begin{array}{rrr}\text { ZERO } & 0.1000 E+01 & 0.1000 E+01 \\ \text { ZEERO } & \text { ZERO }\end{array}$ ZERO

$0.5368 \mathrm{E}+03$

$0.7402 E+04$

$0.7939 \mathrm{E}+04$

$0.2362 \mathrm{E}+03$

IIQUID

$0.4178 \mathrm{E}+00$
$0.7135 \mathrm{E}+02$

$135 E+02$
IIOUID

$0.4536 \mathrm{E}+00$

$0.8437 \mathrm{E}+02$

LIQUID 
WSRC-TR-95-0019 (Revision

0)
Date: January 1995

Section 1C, Page, 6 of 21

Table A-1. HLW Flowsheet Material Balance (Tank 51/ITP Cycle 1) section 1C. ESP Washing of Tank 51 sludge STREAM NAME

COMPONENT FLOWS, LB/HR 122 (C6H5) 2

123 (C6H5) 2 $6 \mathrm{CH}$

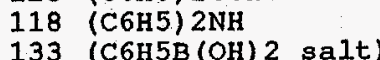

166 (C6H5C6H4OH)

162 (C6H5NH2)

163 (C6H5NHCHO)

131 (C6H5OH salt)

130 (C6H5OH)

121. ( $\mathrm{C} 6 \mathrm{H} 6)$

155 ( $\mathrm{CH} 3) 2 \mathrm{CHOH}$

154 (СH3OH)

$45 \mathrm{Ag}$

$116 \mathrm{Ag} 2 \mathrm{O}$

$73 \mathrm{AgNO}$
$\mathrm{Al} 203$

$106 \mathrm{~B} 203$

$15 \mathrm{BaO}$

$24 \mathrm{BaSO} 4$

$66 \mathrm{CO}$

37
$142 \mathrm{CO} 2$ (C14)

$63 \mathrm{Ca}(\mathrm{COOH}) 2$

$167 \mathrm{Ca}(\mathrm{NO} 3)^{2}$

$120 \mathrm{Ca}(\mathrm{OH}) 2$

$113 \mathrm{Ca} 3(\mathrm{PO} 4) 2$

$20 \mathrm{CaC} 204$

$18 \mathrm{CaCO} 3$

$64 \mathrm{CaCO}$ (C14)

78 CaF́2

$41 \mathrm{CaO}$

19 CasO4.

29 Carbon

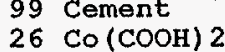

$168 \mathrm{Co}(\mathrm{NO} 3) 2$

$59 \mathrm{COO}$

98 Cr2O3

42 Cs2O

$53 \mathrm{CsCOOH}$

$96 \mathrm{CsCl}$

151 CsTPB

WSRC-TR-95-0019 (Revision
STREAM NUMBERS ->

132 (C6H5B (OH) 2)

165 (C6H5NO)

$9 \mathrm{Ba}(\mathrm{OH}) 2$

169 CsNO3

307

SN Decant

frm $A / D$ Tnk

329

330

309

ank Hot $\mathrm{NaOH}$ Wsh/Aux H2O

310

$508 \mathrm{NaOH}$

311

315

Heel to A/D Tank to A/D Tank to A/D Tank to A/D

ZERO
ZERO
ZERO
ZERO
ZERO
ZERO
ZERO
ZERO
ZERO
ZERO
ZERO
ZERO
ZERO
ZERO
ZERO
ZERO
ZERO
ZERO
ZERO
ZERO
ZERO
ZERO
ZERO
ZERO
ZERO
ZERO
ZERO
ZERO
ZERO
ZERO
ZERO
ZERO
ZERO
ZERO
ZERO
ZERO
ZERO
ZERO
ZERO
ZERO
ZERO
ZERO
ZERO
ZERO
ZERO
ZERO
ZERO
ZERO

ZERO

ZERO

ZERO

ZERO

ZERO

ZERO

ZERO

ZERO

ZERO

ZERO

ZERO

ZERO

ZERO

ZERO

ZERO

ZERO

ZERO

ZERO

ZERO

ZERO

ZERO

ZERO

ZERO

ZERO

ZERO

ZERO

ZERO

ZERO

ZERO

ZERO

ZERO

ZERO

ZERO

ZERO

ZERO

ZERO

ZERO

ZERO

ZERO

ZERO

ZERO

ZERO

ZERO

ZERO

\begin{tabular}{ll} 
ZERO & ZERO \\
ZERO & ZERRO \\
ZERO & ZERO \\
ZERO & ZERO \\
ZERO & ZERO \\
ZERO & ZERO \\
ZERO & ZERO \\
ZERO & ZERO \\
ZERO & ZERO \\
ZERO & ZERO \\
ZERO & ZERO \\
ZERO & ZERO \\
ZERO & ZERO \\
ZERO & ZERO \\
ZERO & ZERO \\
ZERO & ZERO \\
ZERO & ZERO \\
ZERO & ZERO \\
ZERO & ZERO \\
ZERO & ZERO \\
ZERO & ZERO \\
ZERO & ZERO \\
ZERO & ZERO \\
ZERO & ZERO \\
ZERO & ZERO \\
ZERO & ZERO \\
ZERO & ZERO \\
ZERO & ZERO \\
ZERO & ZERO \\
ZERO & ZERO \\
ZERO & ZERO \\
ZERO & ZERO \\
ZERO & ZERO \\
ZERO & ZERO \\
ZERO & ZERO \\
ZERO & ZERO \\
ZERO & ZERO \\
ZERO & ZERO \\
ZERO & ZERO \\
ZERO & ZERO \\
ZERO & ZERO \\
ZERO & ZERO \\
ZERO & ZERO \\
ZERO & ZERO \\
ZERO & ZERO \\
ZERO & ZERO \\
ZERO & ZERO \\
ZERO & ZERO \\
\hline ZERO
\end{tabular}

Date: January 1995

\begin{tabular}{|c|c|c|}
\hline ZERO & ZERO & ZERO \\
\hline ZERO & ZERO & ZERO \\
\hline ZERO & ZERO & ZERO \\
\hline ZERO & ZERO & ZERO \\
\hline ZERO & ZERO & ZERO \\
\hline ZERO & ZERO & ZERO \\
\hline ZERO & ZERO & ZERO \\
\hline ZERO & ZERO & ZERO \\
\hline ZERO & ZERO & ZERO \\
\hline ZERO & ZERO & ZERO \\
\hline ZERO & ZERO & ZERO \\
\hline ZERO & ZERO & ZERO \\
\hline ZERO & ZERO & ZERO \\
\hline ZERO & ZERO & ZERO \\
\hline ZERO & ZERO & ZERO \\
\hline ZERO & ZERO & ZERO \\
\hline ZERO & ZERO & ZERO \\
\hline ZERO & ZERO & ZERO \\
\hline ZERO & ZERO & ZERO \\
\hline ZERO & ZERO & ZERO \\
\hline ZERO & ZERO & ZERO \\
\hline ZERO & ZERO & ZERO \\
\hline ZERO & ZERO & ZERO \\
\hline ZERO & ZERO & ZERO \\
\hline ZERO & ZERO & ZERO \\
\hline ZERO & ZERO & ZERO \\
\hline ZERO & ZERO & ZERO \\
\hline ZERO & ZERO & ZERO \\
\hline ZERO & ZERO & ZERO \\
\hline ZERO & ZERO & ZERO \\
\hline ZERO & ZERO & ZERO \\
\hline ZERO & ZERO & ZERO \\
\hline ZERO & ZERO & ZERO \\
\hline ZERO & ZERO & ZERO \\
\hline ZERO & ZERO & ZERO \\
\hline ZERO & ZERO & ZERO \\
\hline ZERO & ZERO & ZERO \\
\hline ZERO & ZERO & ZERO \\
\hline ZERO & ZERO & ZERO \\
\hline ZERO & ZERO & ZERO \\
\hline ZERO & ZERO & ZERO \\
\hline ZERO & ZERO & ZERO \\
\hline ZERO & ZERO & ZERO \\
\hline ZERO & ZERO & ZERO \\
\hline ZERO & ZERO & ZERO \\
\hline ZERO & ZERO & ZERO \\
\hline ZERO & ZERO & ZERO \\
\hline ZERO & ZERO & ZERO \\
\hline
\end{tabular}

Section 1C, Page 6 of 21 
Table A-1. HLW Flowsheet Material Balance (Tank 51/ITP Cycle 1) Section 1C. ESP Washing of Tank 51 Sludge

\begin{tabular}{|c|c|c|c|}
\hline \multicolumn{2}{|c|}{$\begin{array}{l}\text { STREAM NUMBERS } \\
\text { STREAM NAME }\end{array}$} & $\left(\mathrm{CONT}^{\prime} \mathrm{D}\right) \rightarrow$ & $\begin{array}{l}307 \\
\text { SN Decant } \\
\text { frm A/D Tnk }\end{array}$ \\
\hline 54 & $\mathrm{Cu}(\mathrm{COOH}) 2$ & & ZERO \\
\hline 170 & $\mathrm{Cu}(\mathrm{NO} 3) 2$ & & ZERO \\
\hline 55 & Cuo & & ZERO \\
\hline 80 & $\mathrm{Fe} 2 \mathrm{O} 3$ & & ZERO \\
\hline 101 & Feo & & ZERO \\
\hline 102 & Group A & & ZERO \\
\hline 103 & Group B & & ZERO \\
\hline 25 & $\mathrm{H} 2$ & & ZERO \\
\hline 158 & $\mathrm{H} 2 \mathrm{C} 2 \mathrm{O} 4$ & & ZERO \\
\hline 93 & H2SO4 & & ZERO \\
\hline 90 & Н 3 ВОЗ. & . & ZERO \\
\hline 34 & HCOOH & & ZERO \\
\hline 43 & HCl. & & ZERO \\
\hline 85 & $\mathrm{HF}$ & & ZERO \\
\hline 49 & HNO3 & & ZERO \\
\hline 36 & $\mathrm{Hg}$ & & ZERO \\
\hline 129 & $\mathrm{Hg}(\mathrm{C} 6 \mathrm{H} 5) 2$ & & ZERO \\
\hline 135 & $\mathrm{Hg}(\mathrm{NO} 3) 2$ & & ZERO \\
\hline 144 & $\mathrm{Hg} 2 \mathrm{Cl} 2$ & & ZERO \\
\hline 117 & $\mathrm{Hg} 2 \mathrm{I} 2$ & & ZERO \\
\hline 146 & $\mathrm{HgCl} 2$ & & ZERO \\
\hline 21 & $\mathrm{HgO}$ & & ZERO \\
\hline 97 & I2. & & ZERO \\
\hline 183 & $\mathrm{~K} 2 \mathrm{CO} 3$ & & ZERO \\
\hline 39 & $\mathrm{~K} 20$ & & ZERO \\
\hline 51 & $\mathrm{KCOOH}$ & & ZERO \\
\hline 157 & KMnO4 & & ZERO \\
\hline 161 & KNO3 & & ZERO \\
\hline 10 & KOH & & ZERO \\
\hline 150 & KTPB & & ZERO \\
\hline 105 & La2O3 & & ZERO \\
\hline 108 & Li20 & & ZERO \\
\hline 65 & $\mathrm{Mg}(\mathrm{COOH}) 2$ & & ZERO \\
\hline 171 & $\mathrm{Mg}(\mathrm{NO} 3) 2$ & & ZERO \\
\hline 33 & Mgo & & ZERO \\
\hline 60 & $\mathrm{Mn}(\mathrm{COOH}) 2$ & & ZERO \\
\hline 172 & $\mathrm{Mn}(\mathrm{NO} 3)_{2}$ & 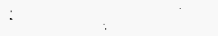 & ZERO \\
\hline 180 & $\mathrm{Mn} 304$ & & ZERO \\
\hline 56 & Mno & & ZERO \\
\hline 14 & $\mathrm{MnO} 2$ & & ZERO \\
\hline 145 & $\mathrm{MoO} 2$ & & ZERO \\
\hline 81 & N2 & & ZERO \\
\hline .74 & $\mathrm{~N} 20$ & & ZERO \\
\hline 182 & NH3 & & ZERO \\
\hline 114 & NH 3 OHNO3 & & ZERO \\
\hline 52 & $\mathrm{NH} 4 \mathrm{COOH}$ & & ZERO \\
\hline 173 & NH 4 NO 3 & & ZERO \\
\hline 68 & NH4OH & & ZERO \\
\hline 148 & NH 4 TPB & & ZERO \\
\hline
\end{tabular}

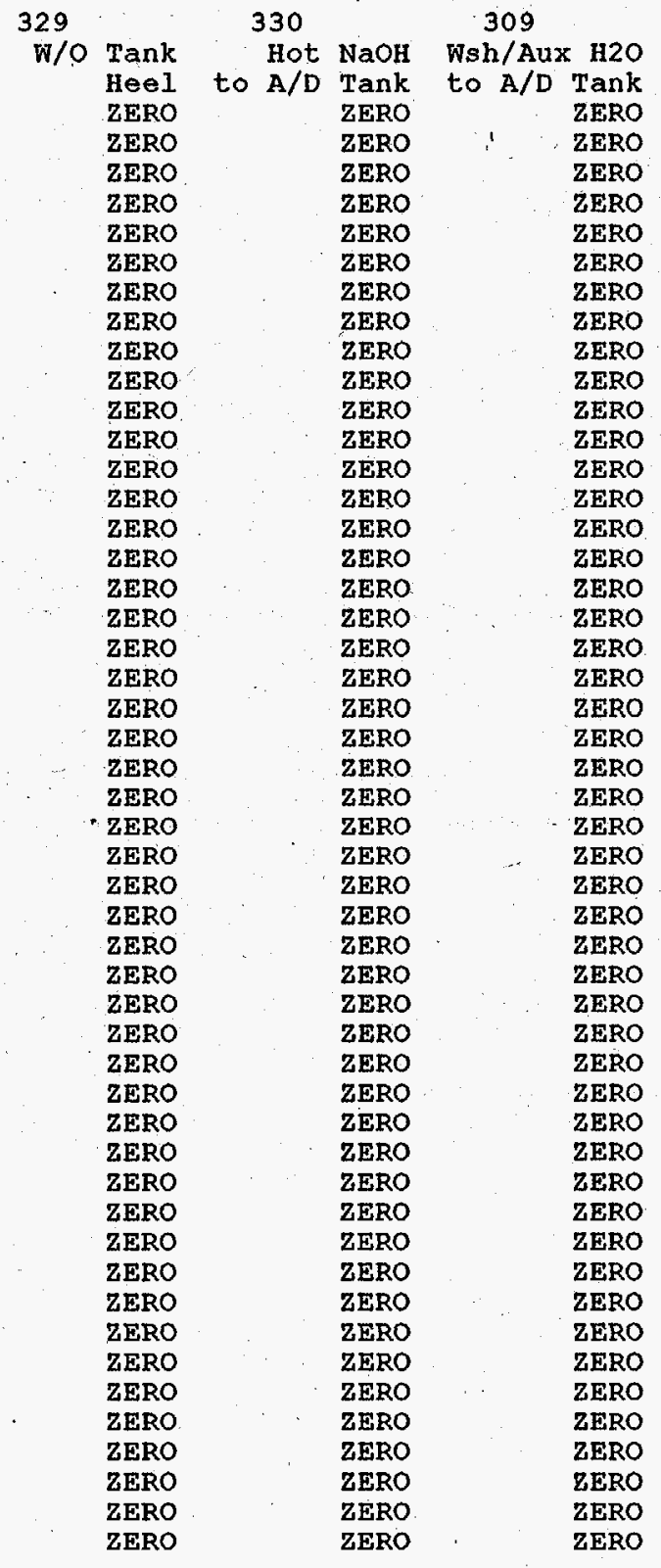

310 to $A / D$ Tank to $40 \% \mathrm{NaNO} 2$ spent Wash to $A / D$ Tank to $A / D$ Tank ZERO frm A/D Wsh $\begin{array}{lll}\text { ZERO } & \text { ZERO } & \text { ZERO } \\ \text { ZERO } & \text { ZERO } & \text { ZERO }\end{array}$ ZERO $\quad$ ZERO ZERO ZERO ZERO ZERO ZERO ZERO ZERO ZERO ZERO ZERO $\quad$ ZERO ZERO ZERO ZERO ZERO ZERO ZERO $\quad$ ZERO ZERO ZERO ZERO ZERO ZERO ZERO ZERO ZERO $\quad$ ZERO $\begin{array}{lll}\text { ZERO } & \text { ZERO } & \text { ZERO } \\ \text { ZERO } & \text { ZERO } & \text { ZERO }\end{array}$ ZERO ZERO ZERO ZERO ZERO $\quad$ ZERO $\quad$ ZERO $\begin{array}{lll}\text { ZERO } & \text { ZERO } & \text { ZERO } \\ \text { ZERO } & \text { ZERO }\end{array}$ ZERO $\quad$ ZERO $\quad$ ZERO ZERO ZERO ZERO ZERO ZERO ZERO ZERO ZERO ZERO ZERO $\quad$ ZERO $\quad$ ZERO ZERO $\quad$ ZERO $\quad$ ZERO ZERO ZERO $\quad$ ZERO ZERO ZERO ZERO ZERO ZERO ZERO ZERO ZERO ZERO ZERO ZERO ZERO ZERO ZERO $\quad$ ZERO ZERO ZERO ZERO ZERO ZERO ZERO ZERO ZERO ZERO ZERO $\quad$ ZERO $\quad$ ZERO ZERO $\quad$ ZERO $\quad$ ZERO ZERO $\cdots$ ZERO ZERO ZERO ZERO ZERO ZRRO ZERO ZERO

ZERO
ZERO ZERO

Date: January 1995 
Table A-1. HLW Flowsheet Material Balance (Tank 51/ITP Cycle 1) Section 1C. ESP Washing of Tank 51 sludge

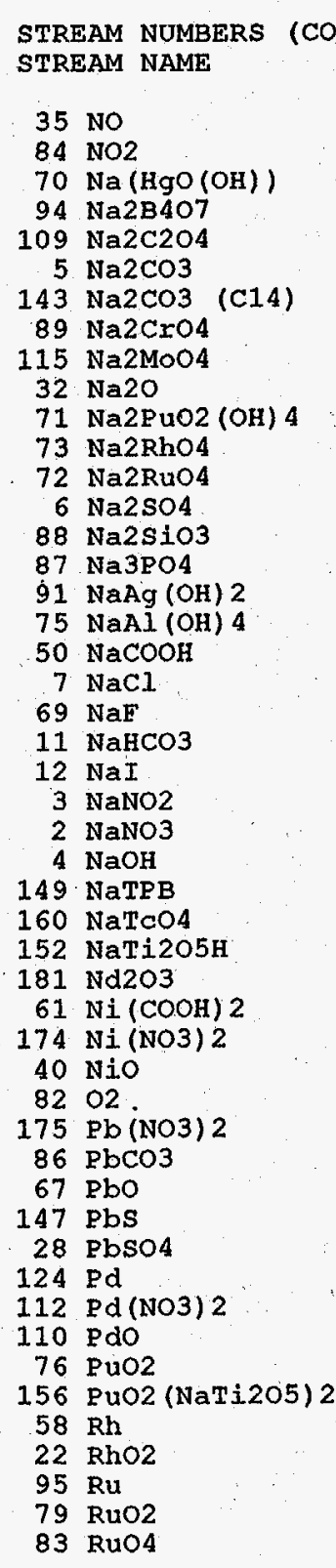

307 SN Decant
frm A/D Tnk Z TnK ZERO ZERO ZERO ZERO

ZERO

ZERO

ZERO

ZERO

ZERO

ZERO

ZERO

ZERO

ZERO

ZERO

ZERO

ZERO

ZERO

ZERO

ZERO

ZERO

ZERO

ZERO

ZERO

ZERO

ZERO

ZERO

ZERO

ZERO

ZERO

ZERO

ZERO

ZERO

ZERO

ZERO

ZERO

ZERO

ZERO

ZERO

ZERO

ZERO

ZERO

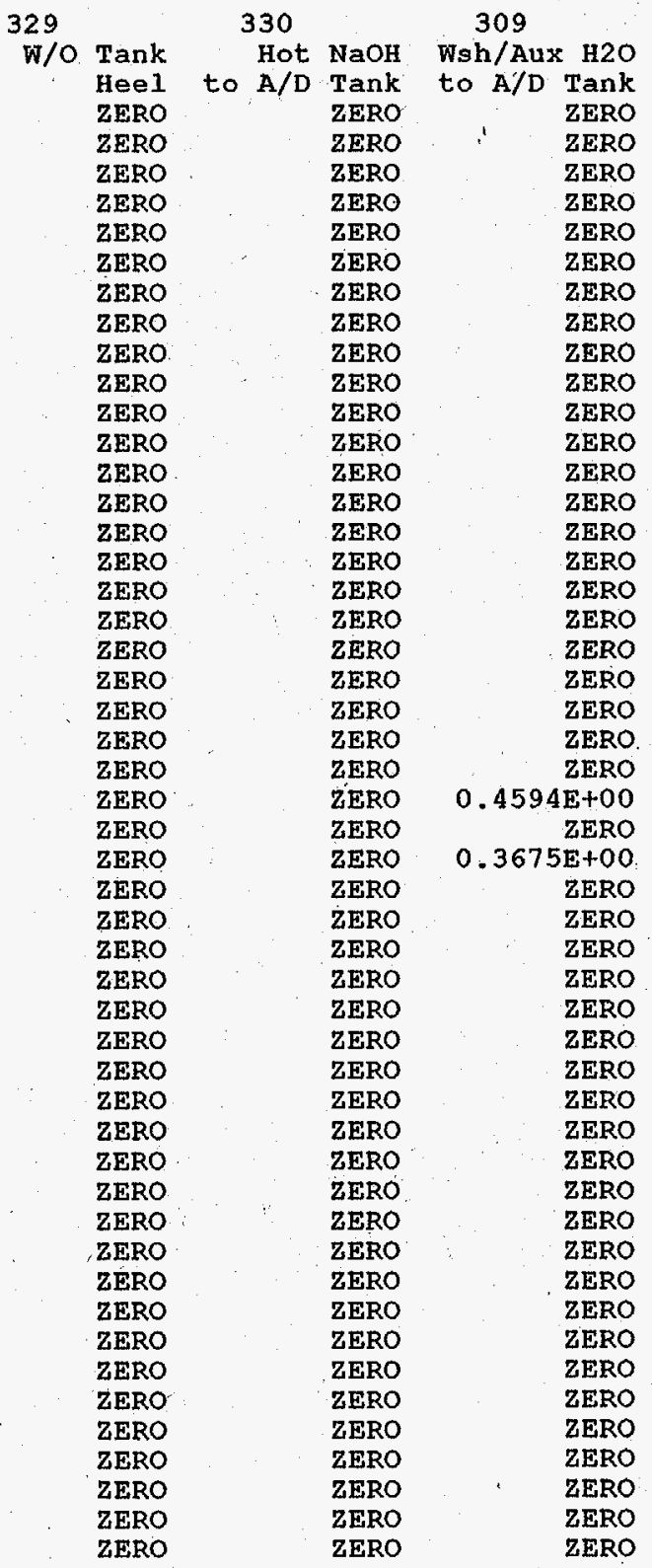

WSRC-TR-95-0019 (Revision

310

$311 \quad 315$

to $\mathrm{A} D$ Nank to $\mathrm{Spent}$ Wash ZERO to A/D Tank frm A/D Wsh
zERO
zERO ZERO ZERO ZERO ZERO ZERO ZERO

ZERO ZERO ZERO

ZERO ZERO ZERO

ZERO ZERO ZERO

ZERO ZERO ZERO

ZERO ZERO ZERO

ZERO ZERO

ZERO

ZERO ZERO

ZERO $\quad$ ZERO

$\begin{array}{lll}\text { ZERO } & \text { ZERO } & \text { ZERO } \\ \text { ZERO } & \text { ZERO } & \text { ZERO }\end{array}$

ZERO ZERO ZERO

ZERO ZERO $\quad$ ZERO

ZERO ZERO ZERO

ZERO ZERO ZERO

ZERO ZERO ZERO

ZERO $\quad$ ZERO $\quad$ ZERO

ZERO ZERO ZERO

ZERO ZERO Z ZERO

ZERO ZERO ZERO

ZERO ZERO $0.4594 \mathrm{E}+00$

ZERO ZERO ZERO

ZERO ZERO $0.3675 \mathrm{E}+00$

ZERO ZERO ZERO

ZERO : - ZERO $\cdots$ ZERO

ZERO ZERO ZERO

ZERO ZERO ZERO

ZERO ZERO ZERO

ZERO ZERO ZERO

ZERO ZERO

ZERO - ZERO

ZERO ZERO

ZERO ZRRO

ZINRO ZERO

ZERO ZERO

$\begin{array}{lll}\text { ZERO } & \text { ZERO } & \text { ZERO } \\ \text { ZERO } & \text { ZERO } & \text { ZERO }\end{array}$

ZERO ZERO ZERO

ZERO Z ZERO ZERO

ZERO ZERO ZERO

ZERO ZERO ZERO

ZERO ZERO ZERO

ZERO ZERO ZERO

ZERO ZERO ZERO

ZERO ZERO ZERO

ZERO ZERO ZERO

ZERO ZERO ZERO

Section 1C, Page 8 of 21 
Table A-1. HLW Flowsheet Material Balance (Tank 51/ITP Cycle 1 ) Section 1C. ESP Washing of Tank 5.1 sludge

STREAM NUMBERS (CONT'D) $\rightarrow$ STREAM NAME

$38 \quad 502$

$141 \mathrm{SO}$

137 Semi Vol Cs2O

136 Semi Vol CsC.

126 Semi Vol Group A

140 Semi Vol Na2B4O

138 Semi Vol NaCl

139 Semi Vol Na

125 Semi Vol NaI

23 Semi Vol

$48 \mathrm{Sr}(\mathrm{COOH}) 2$

$176 \mathrm{Sr}$ (NO3) 2

$153 \mathrm{Sr}$ (NaTi205) 2

$127 \mathrm{Sr}(\mathrm{OH}) 2$

$30 \quad \operatorname{srcO} 3$

46 SrO

159 TCO2

92 ThO2

107 TiO2

$\begin{array}{rl}119 & \text { Tritium } \\ 17 & 0308\end{array}$

$62 \mathrm{UO} 2(\mathrm{COOH}) 2$

177 UO2 (NO3) 2

$16 \mathrm{UO2}(\mathrm{OH}) 2$

$27 \mathrm{Y}(\mathrm{COOH})$

$128 \mathrm{Y}(\mathrm{OH}) 3$

$31 \mathrm{Y} 2(\mathrm{CO} 3) 3$

$47: \mathrm{Y} 203$

100 Zeolite

$44 \mathrm{Zn}(\mathrm{COOH})$
$179 \mathrm{Zn}(\mathrm{NO} 3) 2$

$57 \mathrm{zno}$

$104 \mathrm{ZrO} 2$

13 hydrate $\mathrm{H} 2 \mathrm{O}$

1 water.

TOTAL FIOW, LB/HR

TEMPERATURE， DEG C

PRESSURE, ATM

PRESSURE, PSIG

PRESSURE, MM HG

ENTHALPY， PCU/HR

VAPOR FLOW, CEM

LIQUID FLOW, GPM

DENSITY， LBS/FT3

PHASE

WSRC-TR-95-0019 (Revision
307

SN Decant

frm $A / D$ Tnk

ZERO

ZERO

ZERO

ZERO

ZERO

ZERO

ZERO

ZERO

ZERO

ZERO

ZERO

ZERO

ZERO

ZERO

ZERO

ZERO

ZERO

ZERO

ZERO

ZERO

ZERO

ZERO

ZERO

ZERO

ZERO

ZERO

ZERO

ZERO

ZERO

ZERO

ZERO

ZERO

ZERO

ZERO

$0.3532 \mathrm{E}+02$

$0.1000 \mathrm{E}+01$

ZERO

$0.1000 \mathrm{E}+01$

ZERO

ZERO

ZERO

ZERO

LIQUID

/O Tank
Heel

ZERO

ZERO

ZERO

ZERO

ZERO

ZERO

ZERO

ZERO

ZERO

ZERO

ZERO

ZERO

ZERO

ZERO

ZERO

ZERO

ZERO

ZERO

ZERO

ZERO

ZERO

ZERO

ZERO

ZERO

ZERO

ZERO

ZERO

ZERO

ZERO

ZERO

ZERO

LIQUID
330

309

to A/D Tank Wsh/Aux H2O

ZERO
ZERO A/D Tank
ZERO

ZERO

ZERO

ZERO

ZERO

ZERO

ZERO

ZERO

ZERO

ZERO

ZERO

ZERO

ZERO

ZERO

ZERO

ZERO

ZERO

ZERO

ZERO

ZERO

ZERO

ZERO

ZERO

ZERO

ZERO

ZERO

ZERO

ZERRO

ZERO

ZERO

ZERO

ZERO

ZERO

ZERO

$0.2500 \mathrm{E}+02$

$$
\text { ZERO }
$$

$0.4800 \mathrm{E}+02$

ZERO

ZERO

$0.4413 E+05$

$0.1834 \mathrm{E}+01$.

$\begin{array}{rr}\text { ZERO } & 0.6247 \mathrm{E}+02 \\ \text { IQUID } & \text { LIQUID }\end{array}$

LIQUID
310

$50 \% \mathrm{NaOH}$

311

$40 \%$ NaNO2 Spent Wash

Tank

ZERO

ZERO

ZERO

ZERO

ZERO

ZERO

ZERO

ZERO

ZERO

ZERO

ZERO

ZERO

ZERO

ZERO

ZERO

ZERO

ZERO

ZERO

ZERO

ZERO

ZERO

ZERO

ZERO

ZERO

ZERO

ZERO

ZERO

ZERO

ZERO

ZERO

ZERO

ZERO

ZERO

ZERO

$0.4800 \mathrm{E}+02$

ZERO

$0.4800 \mathrm{E}+02$

ZERO

ZERO

ZERO

ZERO

LIQUID

ZERO

ZERO
LIQUID

.

$0.1834 \mathrm{E}+01$

LIQUID 


\section{0}

WSRC-TR-95-0019 (Revision

0)

Date: January 1995

Section 1C, Page 10 of 21

Table A-1. HLW Flowsheet Material Balance (Tank 51/ITP Cycle 1) Sectiọn 1C. ESP Washing of Tank 51 sludge

STREAM NUMBERS -> STREAM NAME

COMPONENT FLOWS, LB/HR 1.22. (C6H5) 2

123 (C6H5) 2C6H4

118 (C6H5) 2NH

133 (C6H5B (OH) 2 salt)

132 (C6H5B (OH) 2)

166 (C6H5C6H4OH)

163 (C6H5NHCHO)

165 (C6H5NO)

164 (C6H5NO2)

131 (C6H5OH-salt)

130 ( $\mathrm{C} 6 \mathrm{H} 5 \mathrm{OH})$

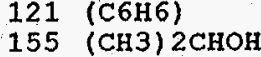

154 (CH3OH)

$45 \mathrm{Ag}$

116 Ag20.

73 Al203
77 AgNo3

$106 \mathrm{~B} 203$

$9 \mathrm{Ba}(\mathrm{OH}) 2$

$15 \mathrm{BaO}$

$24 \mathrm{BasO}$

$66 . \mathrm{CO}$

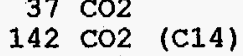

$63 \mathrm{Ca}(\mathrm{COOH}) 2$

$167 \mathrm{Ca}$ (NO3) 2

$120 \mathrm{Ca}(\mathrm{OH}) 2$

$11.3 \mathrm{Ca} 3$ (PO4) 2

$20 \mathrm{CaC2O4}$

$64 . \mathrm{CaCO} 3$ (C14)

78 CaF 2

$41 \mathrm{CaO}$

$19 \mathrm{CaSO} 4$

29 Carbon

99 Cement

$26 \mathrm{Co}(\mathrm{COOH}) 2$

$168 \mathrm{Co}(\mathrm{NO} 3) 2$

$59 \mathrm{COO}$

$98 \quad \mathrm{Cr} 203$

$42 \mathrm{Cs} 2 \mathrm{O}$

$96 \mathrm{CsCl}$

169 CsNO3

$8 \mathrm{CsOH}$

151 CsTPB

WSRC-TR-95-0019 (Revision
$313 \quad 331 \quad 314$

312

313

Insolubles Solubles $A / D$ Tk Heel Wsh A/D sldg Makeup H2O
aftr $A / D$ Wsh aftr $A / D$ Wsh to next Bat to Storage to W/O Tank

$517 \mathrm{NaOH}$

318 aftion

\begin{tabular}{llll} 
ZERO & ZERO & ZERO & ZERO \\
ZERO & ZERO & ZERO & ZERO \\
ZERO & ZERO & ZERO & ZERO \\
ZERO & ZERO & ZERO & ZERO \\
ZERO & ZERO & ZERO & ZERO \\
ZERO & ZERO & ZERO & ZERO \\
ZERO & ZERO & ZERO & ZERO \\
ZERO & ZERO & ZERO & ZERO \\
ZERO & ZERO & ZERO & ZERO \\
ZERO & ZERO & ZERO & ZERO \\
ZERO & ZERO & ZERO & ZERO \\
ZERO & ZERO & ZERO & ZERO \\
ZERO & ZERO & ZERO & ZERO \\
ZERO & ZERO & ZERO & ZERO \\
ZERO & ZERO & ZERO & ZERO \\
ZERO & ZERO & ZERO & ZERO \\
ZERO & ZERO & ZERO & ZERO \\
ZERO & ZERO & ZERO & ZERO \\
ZERO & ZERO & ZERO & ZERO \\
ZERO & ZERO & ZERO & ZERO \\
ZERO & ZERO & ZERO & ZERO \\
ZERO & ZERO & ZERO & ZERO \\
ZERO & ZERO & ZERO & ZERO \\
ZERO & ZERO & ZERO & ZERO \\
ZERO & ZERO & ZERO & ZERO \\
ZERO & ZERO & ZERO & ZERO \\
ZERO & ZERO & ZERO & ZERO \\
ZERO & ZERO & ZERO & ZERO \\
ZERO & ZERO & ZERO & ZERO \\
ZERO & ZERO & ZERO & ZERO \\
ZERO & ZERO & ZERO & ZERO \\
ZERO & ZERO & ZERO & ZERO \\
ZERO & ZERO & ZERO & ZERO \\
ZERO & ZERO & ZERO & ZERO \\
ZERO & ZERO & ZERO & ZERO \\
ZERO & ZERO & ZERO & ZERO \\
ZERO & ZERO & ZERO & ZERO \\
ZERO & ZERO & ZERO & ZERO \\
ZERO & ZERO & ZERO & ZERO \\
ZERO & ZERO & ZERO & ZERO \\
ZERO & ZERO & ZERO & ZERO \\
ZERO & ZERO & ZERO & ZERO \\
ZERO & ZERO & ZERO & ZERO \\
ZERO & ZERO & ZERO & ZERO \\
ZERO & ZERO & ZERO & ZERO \\
ZERO & ZERO & ZERO & ZERO \\
ZERO & ZERO & ZERO \\
ZERO & ZERO & ZERO & ZERO \\
\hline
\end{tabular}

0)

\begin{tabular}{|c|c|c|}
\hline ZERO & ZERO & ZERO \\
\hline ZERO & ZERO & ZERO \\
\hline ZERO & ZERO & ZERO \\
\hline ZERO & ZERO & ZERO \\
\hline ZERO & ZERO & ZERO \\
\hline ZERO & ZERO & ZERO \\
\hline ZERO & ZERO & ZERO \\
\hline ZERO & ZERO & ZERO \\
\hline ZERO & ZERO & ZERO \\
\hline ZERO & ZERO & ZERO \\
\hline ZERO & ZERO & ZERO \\
\hline ZERO & ZERO & ZERO \\
\hline ZERO & ZERO & ZERO \\
\hline ZERO & ZERO & ZERO \\
\hline ZERO & ZERO & ZERO \\
\hline ZERO & ZERO & ZERO \\
\hline ZERO & ZERO & ZERO \\
\hline ZERO & ZERO & ZERO \\
\hline ZERO & ZERO & ZERO \\
\hline ZERO & ZERO & ZERO \\
\hline ZERO & ZERO & ŻERO \\
\hline ZERO & ZERO & ZERO \\
\hline ZERO & ZERO & ZERO \\
\hline ZERO & ZERO & ZERO \\
\hline ZERO & ZERO & ZERO \\
\hline ZERO & ZERO & ZERO \\
\hline ZERO & ZERO & ZERO \\
\hline ZERO & ZERO & ZERO \\
\hline ZERO & ZERRO & ZERO \\
\hline ZERO & ZERO & ZERO \\
\hline ZERO & ZERO & ZERO \\
\hline ZERO & ZERO & ZERO \\
\hline ZERO & ZERO & ZERO \\
\hline ZERO & ZERO & ZERO \\
\hline ZERO & ZERO & ZERO \\
\hline ZERO & ZERO & ZERO \\
\hline ZERO & ZERO & ZERO \\
\hline ZERO & ZERO & ZERO \\
\hline ZERO & ZERO & ZERO \\
\hline ZERO & ZERO & ZERO \\
\hline ZERO & ZERO & ZERO \\
\hline ZERO & ZERO & ZERO \\
\hline ZERO & ZERO & ZERO \\
\hline ZERO & ZERO & ZERO \\
\hline ZERO & ZERO & ZERO \\
\hline ZERO & ZERO & ZERO \\
\hline ZERO & ZERO & ZERO \\
\hline ZERO & ZERO & ZERO \\
\hline
\end{tabular}

Date: January 1995

Section 1C, Page 10 of 21 
Table A-1. HLW Flowsheet Material Balance (Tank 51/ITP Cycle 1) Section 1C. ESP Washing of Tank 51 Sludge

\begin{tabular}{|c|c|c|c|}
\hline \multicolumn{2}{|c|}{$\begin{array}{l}\text { STREAM NUMBERS } \\
\text { STREAM NAME }\end{array}$} & $\left(\operatorname{CONT} T^{\prime} D\right)$ & $->$ \\
\hline 54 & $\mathrm{Cu}(\mathrm{COOH}) 2$ & & \\
\hline 170 & $\mathrm{Cu}(\mathrm{NO}) 2$ & & \\
\hline 55 & $\mathrm{CuO}$ & & \\
\hline 80 & $\mathrm{Fe} 203$ & & \\
\hline 101 & Feo & & \\
\hline 102 & Group A & & \\
\hline 103 & Group B & & \\
\hline 25 & H2 & & \\
\hline 158 & $\mathrm{H} 2 \mathrm{C} 204$ & & \\
\hline 93 & $\mathrm{H} 2 \mathrm{SO}_{4}$ & & \\
\hline $\begin{array}{l}90 \\
34\end{array}$ & Н3BO3. & & \\
\hline $\begin{array}{l}34 \\
43\end{array}$ & $\mathrm{HCOOH}$ & & \\
\hline $\begin{array}{l}43 \\
85\end{array}$ & $\mathrm{HCl}$ & & \\
\hline 85 & $\mathrm{HF}$ & & \\
\hline 49 & HNO3 & & \\
\hline 36 & $\mathrm{Hg}$ & & \\
\hline 129 & $\mathrm{Hg}(\mathrm{C} 6 \mathrm{H} 5)^{2}$ & & \\
\hline 135 & $\mathrm{Hg}(\mathrm{NO} 3)^{2}$ & & \\
\hline 144 & $\mathrm{Hg} 2 \mathrm{C} 12$ & & \\
\hline 117 & $\mathrm{Hg} 2 \mathrm{I} 2$ & & \\
\hline 146 & $\mathrm{HgCl2}$ & & \\
\hline 21 & $\mathrm{HgO}$ & & \\
\hline 97 & I2 & & \\
\hline 183 & $\mathrm{~K} 2 \mathrm{CO} 3$ & & \\
\hline 39 & K2O & & \\
\hline 51 & $\mathrm{KCOOH}$ & & \\
\hline 157 & $\mathrm{KMnO} 4$ & & \\
\hline 161 & KNO3 & & \\
\hline 10 & $\mathrm{KOH}$ & & \\
\hline 150 & KTPB & & \\
\hline 105 & La2O3 & & \\
\hline 108 & I i20 & & \\
\hline 65 & $\mathrm{Mg}(\mathrm{COOH}) 2$ & & \\
\hline 171 & $\mathrm{Mg}(\mathrm{NO}) 3$ & & \\
\hline 33 & Mgo & & \\
\hline 60 & $\mathrm{Mn}(\mathrm{COOH}) 2$ & & \\
\hline 172 & $\mathrm{Mn}(\mathrm{NO} 3) 2$ & & \\
\hline 180 & $\mathrm{Mn} 304$ & & \\
\hline 56 & Mno & & \\
\hline 14 & $\mathrm{MnO} 2$ & & \\
\hline 145 & $\mathrm{MoO} 2$ & & \\
\hline 81 & N2 & & \\
\hline 74 & $\mathrm{~N} 2 \mathrm{O}$ & & \\
\hline 182 & NH3 & & \\
\hline 114 & NH3OHNO3 & & \\
\hline 52 & $\mathrm{NH} 4 \mathrm{COOH}$ & & \\
\hline 173 & NH4NO3 & & \\
\hline 68 & NH4OH & & \\
\hline
\end{tabular}

312 313 Solubles 331

Insolubles Solubles A/D Tk Heel Wsh A/D Sldg
aftr A/D Wsh aftr A/D Wsh to next Bat to Storage ZERO ZERO ZERO ZERO ZERO ZERO ZERO ZERO ZERO ZERO ZERO ZERO ZERO ZERO ZERO ZERO ZERO ZERO ZERO ZERO

ZERO

ZERO

ZERO

ZERO

ZERO

ZERO

ZERO

ZERO

ZERO

ZERO

ZERO

ZERO

ZERO

ZERO

ZERO

ZERO

ZERO

ZERO

ZERO

ZERO

ZERO

ZERO

ZERO

ZERO

ZERO

ZERO

0) 314

ZERO
ZERO
ZERO
ZERO
ZERO
ZERO
ZERO
ZERO
ZERO
ZERO
ZERO
ZERO
ZERO
ZERO
ZERO
ZERO
ZERO
ZERO
ZERO
ZERO
ZERO
ZERO
ZERO
ZERO
ZERO
ZERO
ZERO
ZERO
ZERO
ZERO
ZERO
ZERO
ZERO
ZERO
ZERO
ZERO
ZERO
ZERO
ZERO
ZERO
ZERO
ZERO
ZERO
ZERO
ZERO
ZERO
ZERO
ZERO
ZERO

316

Makeup $\mathrm{H} 2 \mathrm{O}$

to $\mathrm{W} / 0$ Tank

ZERO

ZERO $! \quad$ ZERO

ZERO ZERO

ZERO

ZERO

ZERO

ZERO

ZERO

ZERO

ZERO

ZERO

ZERO

ZERO

ZERO

ZERO

ZERO

ZERO

ZERO

ZERO

ZERO

ZERO

ZERO

ZERO

ZERO

ZERO

ZERO

ZERO

ZERO

ZERO

ZERO

ZERO

ZERO

ZERO

ZERO

ZERO

ZERO

ZERO

ZERO

ZERO

ZERO

ZERO

ZERO

ZERO

ZERO

ZERO

ZERO

ZERO

ZERO

ZERO

Date: January 1995 ZERO

ZERO

ZERO

ZERO

ZERO

ZERO

ZERO

ZERO

ZERO

ZERO

ZERO

ZERO

ZERO

ZERO

ZERO

ZERO

ZERO

ZERO

ZERO

ZERO

ZERO

ZERO

ZERO

ZERO

ZERO

ZERO

ZERO

ZERO

ZERO

ZERO

ZERO

ZERO

ZERO

ZERO

ZERO

ZERO

IERO

ZERO

ZERO

ZERO

ZERO
317

Tank
ZERO
zERO
ZERO
$\mathrm{NaOH} 40 \% \mathrm{NaNO} 2$
Tank to $\mathrm{W} / \mathrm{O}$ Tank

WSRC-TR-95-0019 (Revision
ZERO

ZERO

ZERO

ZERO

ZERO

ZERO

ZERO

ZERO

ZERO

ZERO

ZERO

ZERO

ZERO

ZERO

ZERO

ZERO

ZERO

ZERO

ZERO

ZERO

ZERO

ZERO

ZERO

ZERO

ZERO

ZERO

ZERO

ZERO

ZERO

ZERO

ZERO

ZERO

ZERO

ZERO

ZERO

ZERO

ZERO

ZERO

ZERO

ZERO

ZERO

ZERO

ZERO
ZERO

ZERO
ZERO

ZERO

ZERO

ZERO

ZERO

ZERO

ZERO

ZERO

ZERO

ZERO

ZERO

ZERO

ZERO

ZERO

ZERO

ZERO

ZERO

ZERO

ZERO

ZERO

ZERO

ZERO

ZERO

ZERO

ZEROO

ZERO

ZERO

ZERO

ZERO

ZERO

ZERO

ZEERO

ZEROO

ZERO

ZERO

ZERO

ZERO

ZERO

ZERO

ZERO

ZERO

ZERO

ZERO

Section 1C, Page 11 of 21

TERK
ZERO
ZERO
ZERO
ZERO
ZERO
ZERO
ZERO
ZERO
ZERO
ZERO
ZERO
ZERO
ZERO
ZERO
ZERO
ZERO
ZERO
ZERO
ZERO
ZERO
ZERO
ZERO
ZERO
ZERO
ZERO
ZERO
ZERO
ZERO
ZERO
ZERO
ZERO
ZERO
ZERO
ZERO
ZERO
ZERO
ZERO
ZERO
ZERO
ZERO
ZERO
ZERO
ZERO
ZERO
ZERO
ZERO
ZERO
ZERO
ZERO

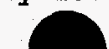


Table A-1. HLW Flowsheet Material Balance (Tank 51/ITP Cyole 1)

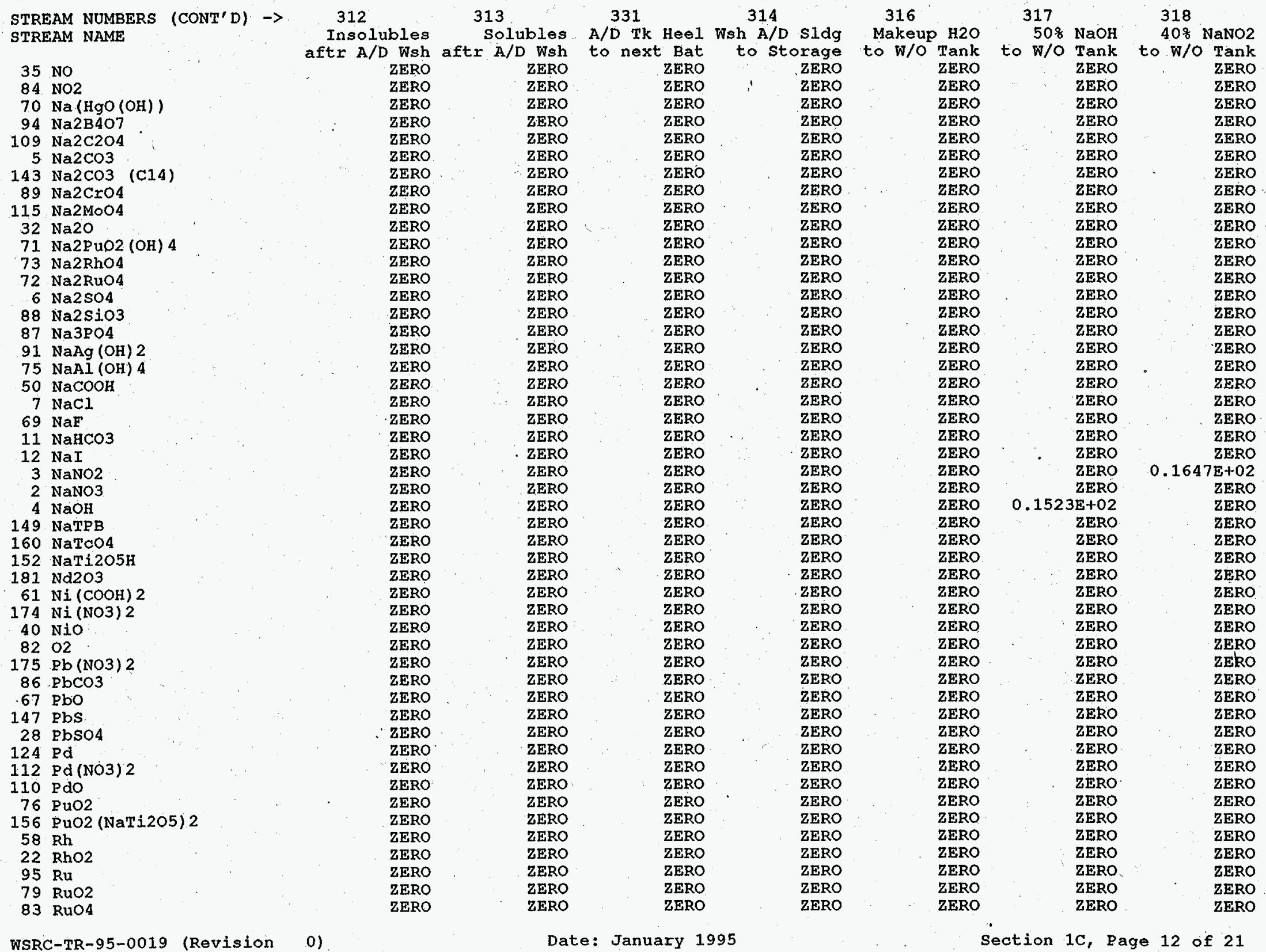


Table A-1. HLW Flowsheet Material Balance (Tank 51/ITP Cycle 1) Section 1C. ESP Washing of Tank 51 sludge STREAM NUMBERS (CONT'D) $\rightarrow$
STREAM NAME

$38 \mathrm{SO} 2$

$141 \mathrm{SO} 3$

137 Semi Vol Cs20

136 Semi Vol CsCl

126 Semi Vol Group A

140 Semi Vol Na2B4O7

138 Semi Vol NaCl

139 Semi VoI NaF

125 Semi Vol NaI

23 Semi Vol

$48 \mathrm{Sr}(\mathrm{COOH}) 2$

$176 \mathrm{Sx}$ (NO3) 2

153 Sr (NaTi2O5) 2

$127 \mathrm{Sr}(\mathrm{OH}) 2$

$30 \mathrm{SxCO} 3$

46 SrO

$159 \mathrm{TcO} 2$

92 ThO2

107 TiO2

17 U308

$62 \mathrm{VO} 2$ ( $\mathrm{COOH}) 2$

$177 \mathrm{OO2}$ (NO3)

$16 \mathrm{VO} 2(\mathrm{OH}) 2$

$27 \mathrm{Y}(\mathrm{COOH}) 3$

$178 \mathrm{Y}(\mathrm{NO}) \mathrm{C}^{3}$

$31 \mathrm{Y} 2(\mathrm{CO} 3) 3$

$31 \mathrm{Y} 2(\mathrm{CO})$
$47 \mathrm{Y} 2 \mathrm{O}$

100 Zeolite

44. $\mathrm{Zn}(\mathrm{COOH}) 2$

$179 \mathrm{Zn}$ (NO3) 2

$57 \mathrm{ZnO}$

104 ZrO2

13 hydrate $\mathrm{H2O}$

1 water

TOTAL FLOW, LB/HR

TEMPERATURE， DEG C PRESSURE， ATM

PRESSURE, PSIG

RRESSURE, MM HG

ENTHALPY, PCU/HR

VAPOR FIOW, CFM

IIQUID FLOW, GPM

DENSITY, IBS/FT 3

PHASE

WSRC-TR-95-0019 (Revision
313

331

314

Insolubles Solubles A/D Tk Heel Wsh A/D Sldg
aftr $A / D$ Wsh aftr A/D.Wsh to next Bat to Storage

aftr. A/D Wsh

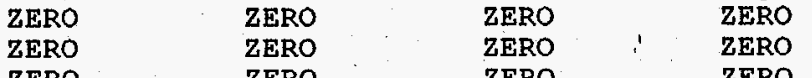

ZERO

ZERO

ZERO ZERO

ZERO

ZERO

ZERO

ZERO

ZERO

ZERO

ZERO

ZERO

ZERO

ZERO

ZERO

ZERO

ZERO

ZERO

ZERO

ZERO

ZERO

ZERO

ZERO

ZERO

ZERO

ZERO

ZERO

ZERO

ZERO

ZERO

ZERO

ZERO

ZERO

ZERO
ZERO

ZERO

ZERO

ZERO

ZERO

ZERO

ZERO

ZERO

ZERO

ZERO

ZERO

ZERO

ZERO

ZERO

ZERO

ZERO

ZERO

ZERO

ZERO

ZERO

ZERO

ZERO

ZERO

ZERO

ZERO

ZERO

ZERO

ZERO

ZERO

$0.4800 \mathrm{E}+02$

$0.1000 \mathrm{E}+01$

$0.4800 \mathrm{E}+02$

$0.1000 \mathrm{E}+01$

ZERO

ZERO

ZERO

ZERO

ZERO

ZERO

ZERO

ZERO

ZERO

ZERO

ZERO

ZERO

ZERO

ZERO

ZERO

ZERO

ZERO

ZERO

ZERO

ZERO

ZERO

ZERO

ZERO

ZERO

ZERO

ZERO

ZERO

ZERO

ZERO

ZERO

ZERO

ZERO

ZERO

ZERO

$0.4800 \mathrm{E}+02$

$0.1000 \mathrm{E}+01$

ZERO

ZERO

ZERO

ZERO

$0.1000 \mathrm{E}+01$

ZERO

ZERO

ZERO

ZERO

LIQUID

ZERO
LIQUID

ZERO

LIQUID

IIQUID

Date: January 1995
316

Makeup $\mathrm{H} 2 \mathrm{O}$

317

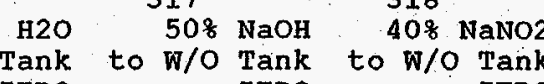

318

$\begin{array}{lll}\text { ZERO } & \text { ZERO } & \text { ZERO } \\ \text { ZERO } & \text { ZERO } & \text { ZERO }\end{array}$

ZERO ZERO Z ZERO

ZERO ZERO, ZERO

ZERO ZERO

ZERO ZERO ZERO

ZERO ZERO ZERO

ZERO ZERO ZERO

ZERO ZERO

ZERO ZERO ZERO

ZERO ZERO ZERO

ZERO ZERO ZERO

$\begin{array}{lll}\text { ZERO } & \text { ZERO } \\ \text { ZERO } & \text { ZERO }\end{array}$

ZERO ZERO

ZERO ZERO

ZERO ZERO ZERO

ZERO ZERO

ZERO Z ZERO

ZERO Z ZERO ZERO

ZERO ZERO $\quad$ ZERO

ZERO ZERO $\quad$ ZERO

ZERO ZERO ZERO

ZERO ZERO ZERO

ZERO ZERO $\quad$ ZERO

ZERO ZERO ZERO

ZERO ZERO ZERO

ZERO ZERO ZERO

ZERO ZERO ZERO

ZERO ZERO

ZERO 2 ZERO $\quad$ ZERO

ZERO 2 ZERO

ZERO $\quad$ ZERO $\quad$ ZERO

$\begin{array}{lll}\text { ZERO } & \text { ZERO } & \text { ZERO } \\ \text { ZERO } & \text { ZERO } & \text { ZERO }\end{array}$

ZERO ZERO ZERO

ZERO ZERO $\quad$ ZERO

$\begin{array}{lll}\text { ZERO } & \text { ZERO } & \text { ZERO } \\ \text { ZERO } & \text { ZERO } & \text { ZERO }\end{array}$

$\begin{array}{lll}\text { ZERO } & 0.1523 \mathrm{E}+02 & 0.2471 \mathrm{E}+02\end{array}$

ZERO $0.3046 \mathrm{E}+02 \quad 0.4118 \mathrm{E}+02$

$0.4800 \mathrm{E}+02 \quad 0.4800 \mathrm{E}+02 \quad 0.4800 \mathrm{E}+02$

$0.1000 \mathrm{E}+01 \quad 0.1000 \mathrm{E}+01 \quad 0.1000 \mathrm{E}+01$

ZERO

ZERO

ZERO

$0.9707 \mathrm{E}+03$

$0.1371 E+04$

$0.3984 \mathrm{E}-01$
$0.9533 \mathrm{E}+02$

IIQUID

$0.6093 \mathrm{E}-01$

$0.8426 \mathrm{E}+02$

IIQUID

Section 1C, Page 13 of 21 
Table A-1. HLW Flowsheet Material Balance (Tank 51/ITP Cycle 1) section 1C. ESP Washing of Tank 51 sludge

STREAM NUMBERS -> STREAM NAME

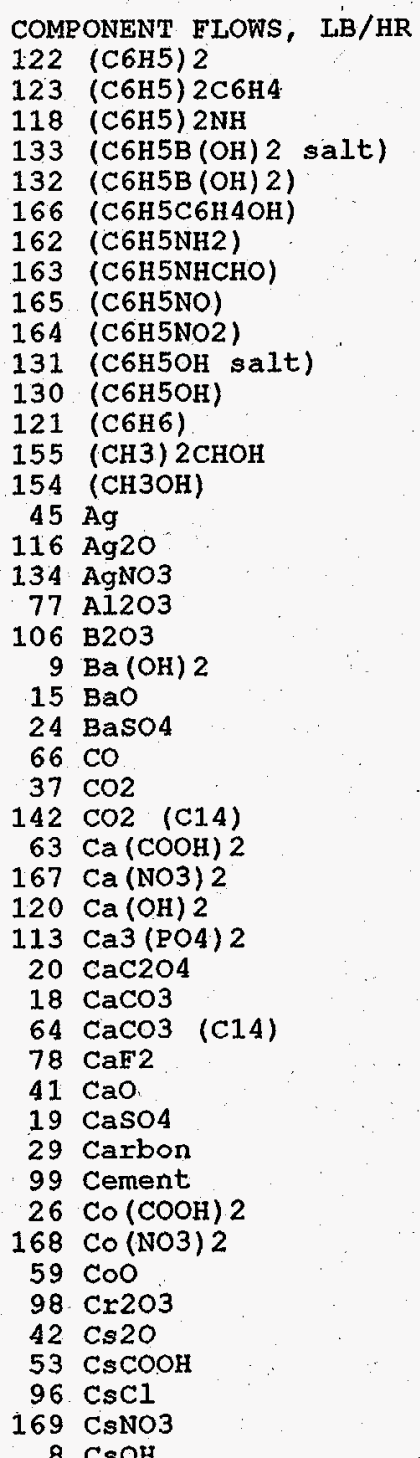

319

Insolubles Solubles Wsh W/O sldg aftr $W / O W s h$ aftr $W / O W s h$ to Storage

ZERO
ZERO
ZERO
ZERO
ZERO
ZERO
ZERO
ZERO
ZERO
ZERO
ZERO
ZERO
ZERO
ZERO
ZERO
ZERO
0.1240 E-01
ZERO
$0.1042 E+02$
ZERO
ZERO
ZERO
$1802 E-01$
ZERO
ZERO
ZERO
ZERO
ZERO
ZERO
$0.2516 E+01$
ZERO
0.3111 E+01
$0.1532 E-06$
$0.1828 E-01$
$0.4073 E-02$
$0.3611 E-01$
ZERO
ZERO
ZERO
ZERO
ZERO
0.1984 E+00
$0.1240 E-03$
ZERO
ZERO
ZERO
ZERO
ZERO

Q. 320 321

322

Spent Wash frm $W / O T k$ Itero

\section{$\therefore \quad$ ZERO}

$$
\text { ZERO }
$$

ZERO$$
\text { ZERO }
$$

ZERO

ZERO

ZERO

ZERO

ZERO

ZERO

ZERO

ZERO
ZERO

ZERO

ZERO

0.2231 E-07

ZERO
$0.1875 \mathrm{E}-04$

ZERO
$0.3615 \mathrm{E}-03$

$5 \mathrm{E}-03$

$0.1240 \mathrm{E}-01$

$0.1042 \mathrm{E}+02$

$0.361 .5 \mathrm{E}-03$

$0.3245 \mathrm{E}-07$

ZERO

ZERO

ZERO
ZERO

$0.4528 \mathrm{E}-05$

ZERO

$0.5601 \mathrm{E}-05$

$0.2757 \mathrm{E}-12$

$0.3291 \mathrm{E}-07$

$0.7332 \mathrm{E}-08$

E-07.
ZERO

ZERO
ZERO

ZERO

ZERO

$0.3572 E-06$

$0.2232 E-09$

ZERO
ZERO

$0.9001 \mathrm{E}-05$

ZERO
ZER
308

323

324

SN Decant Evap Ovrhead $85 \%$ Saltcake frm W/O Tnk frm ESP Rcyc frm ESP Wash

\begin{tabular}{|c|c|c|}
\hline ZERO & ZERO & ZERO \\
\hline ZERO & ZERO & ZERO \\
\hline ZERO & ZERO & ZERO \\
\hline ZERO & ZERO & ZERO \\
\hline ZERO & ZERO & ZERO \\
\hline ZERO & ZERO & ZERO \\
\hline ZERO & ZERO & ZERO \\
\hline ZERO & ZERO & ZERO \\
\hline ZERO & ZERO & ZERO \\
\hline ZERO & ZERO & ZERO \\
\hline ZERO & ZERO & ZERO \\
\hline ZERO & ZERO & ZERO \\
\hline ZERO & ZERO & ZERO \\
\hline ZERO & ZERO & ZERO \\
\hline ZERO & ZERO & ZERO \\
\hline ZERO & ZERO & ZERO \\
\hline ZERO & ZERO & $0.5206 \mathrm{E}-07$ \\
\hline ZERO & ZERO & ZERO \\
\hline ZERO & ZERO & $0.4374 \mathrm{E}-04$ \\
\hline ZERO & ZERO & ZERO \\
\hline $.2196 \mathrm{E}-02$ & ZERO & $0.4318 \mathrm{E}-02$ \\
\hline ZERO & ZERO & ZERO \\
\hline ZERO & ZERO & $0.7569 \mathrm{E}-07$ \\
\hline ZERO & ZERO & ZERO \\
\hline ZERO & ZERO & ZERO \\
\hline ZERO & ZERO & ZERO \\
\hline ZERO & ZERO & ZERO \\
\hline zERO & ZERO & ZERO \\
\hline $0.1657 \mathrm{E}-03$ & ZERO & $0.3259 \mathrm{E}-03$ \\
\hline ZERO & ZERO & $0.1056 \mathrm{E}-04$ \\
\hline ZERO & ZERO & ZERO \\
\hline ZERO & ZERO & $0.1307 \mathrm{E}-04$ \\
\hline ZERO & ZERO & $0.6432 \mathrm{E}-12$ \\
\hline ZERO & ZERO & $0.7677 \mathrm{E}-67$ \\
\hline ZERO & ZERO & $0.1711 \mathrm{E}-07$ \\
\hline ZERO & ZERO & $0.1516 \mathrm{E}-06$ \\
\hline ZERO & ZERO & ZERO \\
\hline ZERO & ZERO & ZERO \\
\hline ZERO & ZERO & ZERO \\
\hline ZERO & ZERO & ZERO \\
\hline ZERO & ZERO & ZERO \\
\hline ZERO & ZERO & $0.8334 \mathrm{E}-06$ \\
\hline ZERO & ZERO & $0.5208 \mathrm{E}-09$ \\
\hline ZERO & ZERO & ZERO \\
\hline ZERO & ZERO & ZERO \\
\hline ZERO & ZERO & ZERO \\
\hline $0.5467 \mathrm{E}-04$ & ZERO & $0.1075 \mathrm{E}-03$ \\
\hline & ZERO & 7FRO \\
\hline
\end{tabular}

WSRC-TR-95-0019 (Revision

ZERO

Section 1C, Page 14 of 21

ZERO

ZERO ZERO

ZERO

$0.2729 \mathrm{E}-04$

ZERO

$.3111 \mathrm{E}+01$

$0.1828 \mathrm{E}-01$

$.3611 \mathrm{E}-01$

ZERO

ZERO

ZERO

ZERO

$0.1984 \mathrm{E}+00$
$0.1240 \mathrm{E}-03$

ZERO

ZERO

ZERO

$0.5285 \mathrm{E}-04$

.ZERO

0.1602 ZERO

$0.1056 \mathrm{E}-04$

$0.1307 \mathrm{E}-04$

$0.6432 \mathrm{E}-12$

$0.7677 \mathrm{E}-07$

$0.1711 \mathrm{E}-07$

ZERO

ZERO

ZERO

ZERO

$0.8334 \mathrm{E}-06$
$0.5208 \mathrm{E}-09$

$0.5467 \mathrm{E}-04$ 
Table A-1. HLW Flowsheet Material Balance (Tank 51/ITP Cycle 1 ) Section 1C. ESP Washing of Tank 51 sludge

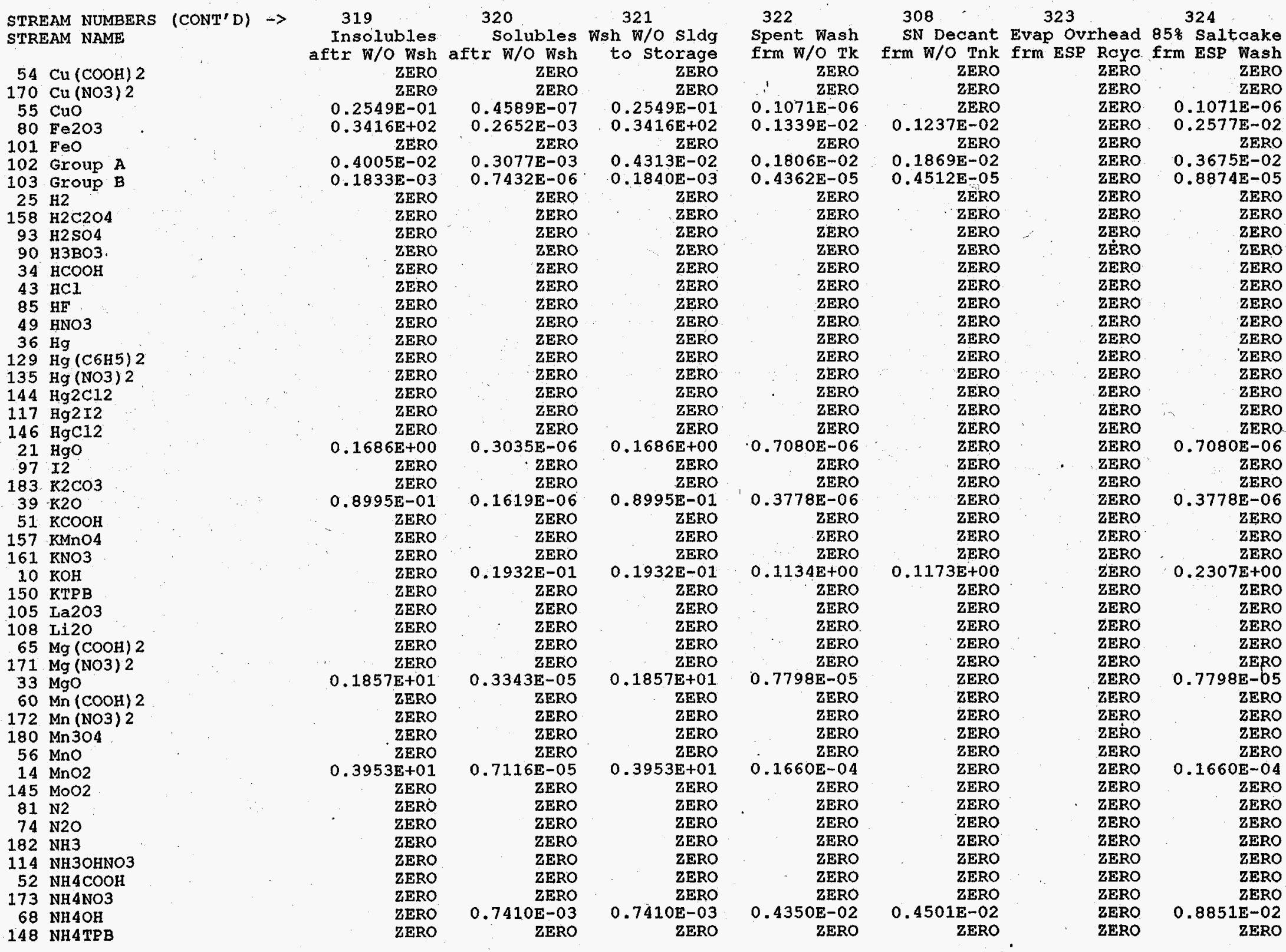


Table $\dot{A}-1$. HLW Flowsheet Material Balance (Tank 51/ITP Cycle 1)

Section 1C. ESP Washing of Tank 51 sludge

STREAM NUMBERS (CONT'D) $\rightarrow$ STREAM NAME

$\begin{array}{ll}35 & \text { No } \\ 84 & \text { NO2 }\end{array}$

$70 \mathrm{Na}(\mathrm{HgO}(\mathrm{OH}))$

$94 \quad \mathrm{Na} 2 \mathrm{~B} 407$

$109 \mathrm{Na} 2 \mathrm{C} 2 \mathrm{O} 4$

$5 \mathrm{Na} 2 \mathrm{CO} 3$

$143 \mathrm{Na} 2 \mathrm{CO} 3$ (C14)

$89 \mathrm{Na} 2 \mathrm{CrO} 4$

$\begin{array}{rl}115 & \mathrm{Na} 2 \mathrm{MOO} \\ 32 & \mathrm{Na} 2 \mathrm{O}\end{array}$

$32 \mathrm{Na} 2 \mathrm{O}$
71. $\mathrm{Na} 2 \mathrm{PuO} 2(\mathrm{OH}) 4$

$73 \mathrm{Na} 2 \mathrm{RhO} 4$

72 Na2RuO4

$6 \mathrm{Na} 2 \mathrm{SO} 4$

$88 \mathrm{Na} 2 \mathrm{SiO}$

$87 \mathrm{Na3PO4}$

$91 \mathrm{NaAg}(\mathrm{OH}) 2$

75 NaAl (OH) 4

$7 \mathrm{NaCl}$

$7 \mathrm{NaCl}$

$69 \mathrm{NaF}$
$11 \mathrm{NaHCO} 3$

$12 \mathrm{NaI}$

3 NaNO2

$2 \mathrm{NaNO} 3$

$4 \mathrm{NaOH}$

149 NaTPB

$160 \mathrm{NaTcO} 4$

152 NaTi20

$61 \mathrm{Ni}(\mathrm{COOH}) 2$

$174 \mathrm{Ni}$ (NO3) 2

$40 \mathrm{NiO}$

8202

$175 \mathrm{~Pb}$ (NO3) 2

$86 \mathrm{PbCO} 3$

$67 \mathrm{PbO}$

$147 \mathrm{PbS}$

$28 \mathrm{PbSO} 4$

$124 \mathrm{Pd}$

$112 \mathrm{Pd}$ (NO3) 2

110 PdO

156 PuO2 (NaTi205) 2

$\begin{array}{rl}156 & \mathrm{Ru} \\ 58 & \end{array}$

$\begin{array}{ll}58 & \mathrm{Rh} \\ 22 & \mathrm{RhO} 2\end{array}$

$\begin{array}{ll}22 & \mathrm{RhO} 2 \\ 95 & \mathrm{Ru}\end{array}$

$\begin{array}{ll}95 & \mathrm{Ru} \\ 79 & \mathrm{RuO} 2\end{array}$

83 RuO4

WSRC-TR-95-0019 (Revision
31

Insolubles Solubles Wsh W/O Sldg
aftr W/O Wsh aftr W/O Wsh to Storage aftr W/O Wsh aftr W/O Wsh to storage
ZERO
ZERO ZERO ZERO ZERO ZERO

ZERO

ZERO

ZERO

ZERO

$0.3935 \mathrm{E}+01$

ZERO

ZERO

ZERO

ZERO

ZERO

ZERO

ZERO

ZERO

ZERO

ZERO

ZERO

ZERO

ZERO

ZERO

ZERO

ZERO

ZERO

ZERO

ZERO

ZERO

$0.3023 \mathrm{E}+00$

ZERO

ZERO

ZERO

ZERO

$0.1552 \mathrm{E}+00$ ZERO
ZERO

$0.5466 \mathrm{E}-03$

$0.1203 E-01$

ZERO

$0.1157 \mathrm{E}-02$

ZERO

$0.7507 \mathrm{E}-02$

ZERO

ZERO

ZERO
$0.1552 \mathrm{E}+00$
ZERO

$0.3120 \mathrm{E}-03$

ZERO

ZERO

$0.1126 \mathrm{E}-01$

$0.1855 \mathrm{E}-02$

$0.1855 \mathrm{E}-02$
0.02

$0.3935 \mathrm{E}+01$

$0.2012 \mathrm{E}-03 \quad 0.2012 \mathrm{E}-03$

. $2012 \mathrm{E}-03$

$0.2367 \mathrm{E}+00$

$0.9830 \mathrm{E}-02$

$0.1775 \mathrm{E}-03$

$0.4333 \mathrm{E}+00$

$0.4152 \mathrm{E}-01$

ZERO

$0.6597 \mathrm{E}-05$

$0.6436 \mathrm{E}+01$

$0.1849 \mathrm{E}+01$

$0.3671 \mathrm{E}+01$

$0.1910 \mathrm{E}-03$

ZERO

ZERO

ZERO

$0.5442 \mathrm{E}-06$

ZnRO

ZERO

ZERO

$0.2794 \mathrm{E}-06$

ZERO

$0.9840 \mathrm{E}-09$

$0.2165 \mathrm{E}-07$

ZERO

E-08

$0.1351 \mathrm{E}-07$

ZERO

Date: January 1995

$0.2367 \mathrm{E}+00$

$0.9830 \mathrm{E}-02$

$0.1775 \mathrm{E}-03$

ZERO

$0.8255 \mathrm{E}-02$

ZERO

$0.6597 \mathrm{E}-05$

$0.6436 \mathrm{E}+01$

$0.3671 \mathrm{E}+01$

$0.1910 \mathrm{OE}-03$

ZERO

ZERO

ZERO

$0.3023 \mathrm{E}+00$

ZERO

$0.9262 \mathrm{E}-0.4$

ZERO
ZERO

ZERO

$0.1072 \mathrm{E}-07$

$0.5466 \mathrm{E}-03$

ZERO

$0.1157 \mathrm{E}-02$

ZERO

$0.7507 \mathrm{E}-02$
$0.3218 \mathrm{E}+00$

$0.3678 \mathrm{E}-09 \quad 0.3678 \mathrm{E}-09$

$0.8255 \mathrm{E}-02$

$0.2083 \mathrm{E}-08$

$322 \quad 308$

323

324

Spent Wash SN Decant Evap Ovrhead $85 \%$ Saltcake

frm W/O Tk frm W/O Tnk frm ESP Rayo frm ESP Wash

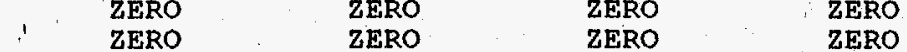

$\begin{array}{rrrr}0.1832 \mathrm{E}-02 & 0.1895 \mathrm{E}-02 & \text { ZERO } & \text { ZERO } \\ 0.3727 \mathrm{E}-02\end{array}$

$0.1889 \mathrm{E}+01$

$0.1758 \mathrm{E}+01$

$0.2159 \mathrm{E}-08$

$0.6612 \mathrm{E}-01$

$0.1089 \mathrm{E}-01$

$0.1652 \mathrm{E}-04$

$0.7151 \mathrm{E}-07$

$0.2764 \mathrm{E}-03$

$0.1181 \mathrm{E}-02$

$0.5771 \mathrm{E}-01$

$0.8157 \mathrm{E}-02$

$0.1042 \mathrm{E}-02$

$0.2544 \mathrm{E}+01$

$0.4846 \mathrm{E}-01$

$0.2437 \mathrm{E}+00$

0.3873 ZERO

$0.3873 \mathrm{E}-04$

$0.2590 \mathrm{E}+02$

$0.2116 \mathrm{E}+02$

ZERO

ZERO

$.1954 \mathrm{E}+01$

$0.2234 \mathrm{E}-08$

$0.6840 \mathrm{E}-01$

0.1127E-01

ZERO

$0.7398 \mathrm{E}-07$

$0.2859 \mathrm{E}-03$

$0.1222 \mathrm{E}-02$

$0.1438 \mathrm{E}+01$

$0.5970 \mathrm{E}-01$

$0.8438 \mathrm{E}-02$

$0.1078 \mathrm{E}-02$

$0.2632 \mathrm{E}+01$

ZERO

$0.2521 \mathrm{E}+00$

ZERO

$0.4007 \mathrm{E}-04$

$0.1361 \mathrm{E}+02$

$0.1123 \mathrm{E}+02$

$0.8159 \mathrm{E}+01$

ZERO
$0.1160 \mathrm{E}-02$

ZERO

ZERO

ZERO

$0.1270 \mathrm{E}-05$

ZERO

$38 \mathrm{E}-03$

ZERO

ZERO

$0.6519 \mathrm{E}-06$

$0.6296 \mathrm{E}-07$

$0.2296 \mathrm{E}-08$

$0.5051 \mathrm{E}-07$

ZERO

$0.4859 \mathrm{E}-08$

ZERO

$0.3153 \mathrm{E}-07$

ZERO

0)
ZERO

ZERO

ZERO

ZERO

ZERO

ZERO

ZERO

ZERO

ZERO

$0.6513 \mathrm{E}-07$

ZERO

ZERO

ZERO

ZERO

ZERO

ZERO

ZERO
$0.3843 E+01$

$0.3577 \mathrm{E}+01$

$0.4393 \mathrm{E}-08$

$0.1345 \mathrm{E}+00$

$0.2216 \mathrm{E}-01$

$0.1652 \mathrm{E}-04$

$0.2403 \mathrm{E}-02$

$0.2828 \mathrm{E}+01$

$0.1174 \mathrm{E}+00$

$0.1660 \mathrm{E}-01$

$0.2120 \mathrm{E}-02$
$0.5176 \mathrm{E}+01$

ZERO $\quad 0.4959 \mathrm{E}+00$

ZERO ZERO

ZERO

ZERO $0.3951 \mathrm{E}+02$

ZERO $\quad 0.2932 \mathrm{E}+02$

ZERO ZERO

ZERO $\quad 0.2281 \mathrm{E}-02$

ZERO ZERO

ZERO ZERO

ZERO ZERO

ZERO $\quad 0.1270 \mathrm{E}-05$

ZERO ZERO

ZERO $0.1106 \mathrm{E}-02$

ZERO ZERO

$\begin{array}{ll}\text { ZERO } & \text { ZERO } \\ \text { ZERO } & \text { ZERO }\end{array}$

ZERO $\quad 0.6519 \mathrm{E}-06$

ZERO ZERO

ZERO $\quad 0.1281 E-06$

ZERO $\quad 0.2296 \mathrm{E}-08$ 
Table A-1. HLW Flowsheet Material Balance (Tank 51/ITP Cycle 1) Section 1C. ESP Washing of Tank 51 sludge

STREAM NUMBERS (CONT'D) $\rightarrow$ STREAM NAME

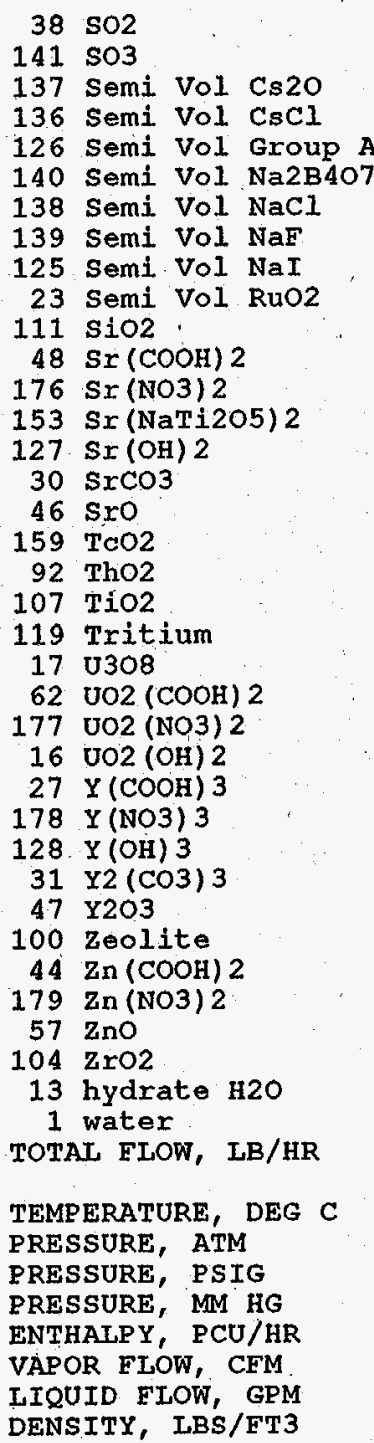

$320 \quad 321$ aftr W/O Wh aftr

322

308

323

324 frm $W / O$ Tnk frm ESP Reyc frm ESP Wash

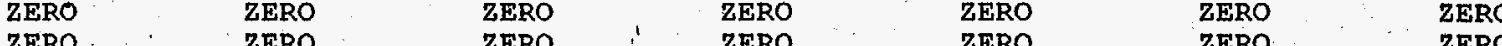
ZERO ZERO ZERO ZERO ZERO ZERO ZERO ZERO ZERO ZERO ZERO ZERO ZERO ZERO $\quad$ ZERO ZERO ZERO $\quad$ ZERO $Z E R O$

$0.2327 \mathrm{E}-0.5$

ZERO

ZERO

ZERO

$0.2501 \mathrm{E}-01$

ZERO

$0.4211 \mathrm{E}-02$

$0.3620 \mathrm{E}-01$

$0.5661 \mathrm{E}-01$

ZERO

$0.2538 \mathrm{E}+01$

ZERO

ZERO

ZERO

ZERO

ZERO

$0.6295 \mathrm{E}-02$

ZERO

ZERO

ZERO

$0.1436 \mathrm{E}+00$

ZERO
$6 \mathrm{E}+01$

$0.2776 \mathrm{E}+01$
ZERO

$0.6788 \mathrm{E}+02$

ZERO

ZERO

ZERO

ZERO

ZERO

ZERO

ZERO

ZERO

$0.1292 \mathrm{E}+0$

$0.5428 \mathrm{E}-05$

ZERO

ZERO

TERO

$0.4400 \mathrm{E}-07$

$0.4503 \mathrm{E}-07$

ZERO

$0.6517 \mathrm{E}-07$

$0.1019 \mathrm{E}-06$

$0.1245 E-08$

$0.4568 \mathrm{E}-05$

ZERO

$0.8587 \mathrm{E}-04$

ZERO

$0.4434 \mathrm{E}-07$

$0.1133 \mathrm{E}-07$

ZERO

ZERO

ZERO
$0.2583 E-06$

$0.2583 \mathrm{E}-06$

$0,1051 \mathrm{E}-06$.

ZERO

$0.1768 \mathrm{E}-07$

$0.4211 \mathrm{E}-02$

$0.3620 \mathrm{E}-01$

$0.5662 \mathrm{E}-01$

$0.1520 \mathrm{E}-06$

$0.2378 \mathrm{E}-06$

$0.2538 \mathrm{E}+01$

$0.7312 \mathrm{E}-08$
$0.1066 \mathrm{E}-04$

ZERO

$0.8587 \mathrm{E}-04$

ZERO

$0.4434 \mathrm{E}-07$

$0.6295 \mathrm{E}-02$

ZERO

ZERO

$0.2586 \mathrm{E}-06$

ZERO

ZERO

$0.1436 \mathrm{E}+00$

$0.7396 \mathrm{E}-04$

$0.3250 \mathrm{E}+03$

$.2776 \mathrm{E}+01$

$0.3117 \mathrm{E}+03$

$0.3929 \mathrm{E}+03$

$0.4800 \mathrm{E}+02$

$0.4800 \mathrm{E}+02$

$0.4800 \mathrm{E}+02$

$0.1000 \mathrm{E}+01$

$0.1000 \mathrm{E}+01$

ZERO

ZERO

ZERO

ZERO

ZERO

ZERO
ZERO

ZERO

ZERO

$0.1000 \mathrm{E}+01$

ZERO

ZERO

ZERO

ZERO

E-06

ZERO

ZERO

ZERO

ZERO

$0.7565 \mathrm{E}-08$

ZERO

ZERO

Z. 504 ZERO

$0.5215 \mathrm{E}-03$

ZERO

$0.26035-06$

$0.2603 \mathrm{E}-06$
$0.2644 \mathrm{E}-07$

$0.2644 \mathrm{E}-07$
ZERO

ZERO

ZERO

ZERO
$0.6033 \mathrm{E}-06$

ZERO

$0.4165 \mathrm{E}-03$

$0.9045 \mathrm{E}+03$

$0.4800 \mathrm{E}+02$

$0.1000 \mathrm{E}+01$

ZERO

ZERO

- ZERO

$.2693 E-06$

ZERO

ZERO
ZERO

ZERO

ZERO

ZERO

$0.4188 \mathrm{E}-03$

$0.1699 \mathrm{E}+03$

$0.2113 \mathrm{E}+03$

$0.3621 \mathrm{E}+02$

$0.1000 \mathrm{E}+01$

ZERO

ZERO ZERO

$0.5147 E+03$

$0.1515 \mathrm{E}+05$

$0.1566 \mathrm{E}+05$

$0.4113 E+05$

$0.6543 \mathrm{E}+04$

$0.1721 \mathrm{E}+01$

$.6318 \mathrm{E}+00$
$0.6414 \mathrm{E}+02$

$0.6677 \mathrm{E}+00$

$.2362 \mathrm{E}+03$

IIQUID

LIQUID
$0.6553 \mathrm{E}+02$

$0.3693 \mathrm{E}+00$

$0.7135 \mathrm{E}+02$

LIQUID
ZERO

ZERO

ZERO

ZERO

ZERO

ZERO

ZERO

ZERO

ZERO

ZERO

ZERO

ZERO

ZERO

ZERO

ZERO

ZERO

ZERO

ZERO

ZERO

ZERO

ZERO

ZERO

ZERO

ZERO

ZERO

ZERO

ZERO

ZERO

ZERO

$0.9893 \mathrm{E}+03$

$0.9893 \mathrm{E}+03$

$0.3500 \mathrm{E}+02$

$0.1000 \mathrm{E}+01$

ZERO

ZERO

ZERO

ZERO

ZERO

ZERO

$0.5428 \mathrm{E}-0.5$

ZERO

ZERO

$0.5255 \mathrm{E}-06$

$0.5255 \mathrm{E}-06$

ZERO

$0.1768 \mathrm{E}-07$

$0.1520 \mathrm{E}-06$

$0.2378 \mathrm{E}-06$

$0.1488 \mathrm{E}-07$

ZERO

$0.1026 \mathrm{E}-02$

ZERO

$0.5296 \mathrm{E}-06$

$0.2644 \mathrm{E}-07$

ZERO

ZERO

ZERO

$0.6033 \mathrm{E}-06$

$0.8354 \mathrm{E}-03$

$0.1897 \mathrm{E}+02$

$0.1265 \mathrm{E}+03$

$0.3500 \mathrm{E}+02$ 
Table A-1. HLW Flowsheet Material Balance (Tank 51/ITP Cyale 1) Section 1C. ESP Washing of Tank 51 sludge

STREAM NUMBERS $\rightarrow$ STREAM NAME

COMPONENT FLOWS, LB/HR 122 (C6H5) 2

123 ( $66 \mathrm{H} 5) 2 \mathrm{C} 6 \mathrm{H}$

118 (C6H5) $2 \mathrm{NH}$

133 (C6H5B (OH) 2 salt)

132 (C6H5B (OH) 2)

166 (C6H5C6H4)

163 (C6H5NHCHO)

165 (C6H5NO)

164 (C6H5NO2)

131 (C6H5OH salt)

130 (C6H5OH)

$\begin{array}{ll}121 & \text { (C6H6) } \\ 155 & \text { (CH3) } 2 \mathrm{CHOH}\end{array}$

154 (СH3OH)

$45 \mathrm{Ag}$

$116 \mathrm{Ag} 20$

134 AgNO3

$77 \mathrm{Al203}$

$106 \mathrm{~B} 2 \mathrm{O} 3$

$9 \mathrm{Ba}(\mathrm{OH}) 2$

$15 \mathrm{BaO}$

$24 \mathrm{BaSO} 4$

$66 \mathrm{CO}$

$142 \mathrm{CO} 2(\mathrm{C14})$

$63 \mathrm{Ca}(\mathrm{COOH}) 2$

$167 \mathrm{Ca}(\mathrm{NO} 3) 2$
$120 \mathrm{Ca}(\mathrm{OH}) 2$

$120 \mathrm{Ca}(\mathrm{OH}) 2$
$113 \mathrm{Ca} 3$ (PO4) 2

$20 \mathrm{CaC} 204$

$18 \mathrm{CaCO}_{3}$

$64 \mathrm{CaCO}(\mathrm{C14})$

78 CaF2

$41 \mathrm{CaO}$

19 CaSO4

29 Carbon

$26 \mathrm{Co}(\mathrm{COOH}) 2$

168 Co (NO3) 2

$59 \mathrm{COO}$

$98 \mathrm{Cr} 203$
$42 \mathrm{Cs} 2 \mathrm{O}$

$53 \mathrm{C}$ COOH

$96 \mathrm{CsCl}$

169 CsNO3

$8 \mathrm{CsOH}$

151 CSTPB

WSRC-TR-95-0019 (Revision
325

Tot Wsh sldg

332

327

326

W/O Tk Heel Radiolyzed Dilution H2O

$$
1
$$

Sludge Feed

to DWPF

678

sldge Feed

Solubles

679

Sldge Feed

Insolubles

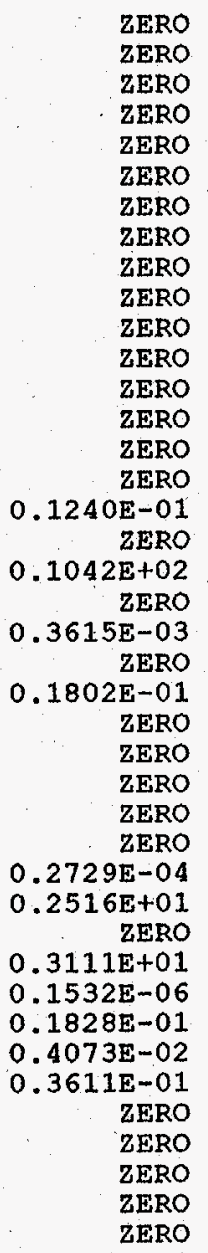

$0.1984 \mathrm{E}+00$

$0.1240 \mathrm{E}-03$

ZERO

ZERO

$0.9001 \mathrm{E}-05$

$\begin{array}{rr}\text { E-05 } & 0.1611 \mathrm{E}-05 \\ \text { ZERO } & \text { ZERO }\end{array}$

$\begin{array}{lll}\text { ZERO } & \text { ZERO } \\ \text { ZERO } & \text { ZERO } & \text { ZERO } \\ \text { ZERO } & \text { ZERO } & \text { ZERO } \\ \text { ZERO } & & \text { ZERO }\end{array}$

ZERO

ZERO

ZERO

ZERO

ZERO

ZERO

ZERO

ZERO

ZERO

ZERO

ZERO

ZERO

$0.2219 \mathrm{E}-02$

ZERO

$0.1864 \mathrm{E}+0.1$

$0.6472 \mathrm{E}-04$

ZERO

$0.3226 \mathrm{E}-02$

- ZERO

ZERO

ZERO

$0.4884 \mathrm{E}-05$

$0.4503 \mathrm{E}+00$

ZERO
$O E+00$

$0.5570 \mathrm{E}+00$

$0.3272 E-02$

$0.7291 \mathrm{E}-03$

$0.6463 \mathrm{E}-02$

ZERO

ZERO

ZERO

$0.3552 \mathrm{E}-01$

$0.3552 \mathrm{E}-01$
$0.2220 \mathrm{E}-04$

ZERO

ZERO

ERRO

$.1018 E-01$
ZERO

$0.8551 \mathrm{E}+01$

$0.2968 \mathrm{EER}-03$

ZERO

ZERO

ZERO

ZERO

ZERO

ZERO

$0.2240 \mathrm{E}-04$

$0.2065 \mathrm{E}+01$

$0.2554 \mathrm{E}+01$

$0.1257 \mathrm{E}-06$

$0.1501 \mathrm{E}-01$

$0.3344 \mathrm{E}-02$

$0.2964 \mathrm{E}-01$

ZERO

ZERO

ZERO

ZERO

$0.1629 \mathrm{E}+00$

$0.1018 \mathrm{E}-03$

ZERO

ZERO
$0.7390 \mathrm{E}-05$

ZERO $\begin{array}{ll}\text { ZERO } & \text { ZERO } \\ \text { ZERO } & \text { ZERO } \\ \text { ZERO } & \text { ZERO }\end{array}$

ZERO ZERO

ZERO ZERO

ZERO ZERO

ZERO $\quad$ ZERO

ZERO ZERO

ZERO

ZERO ZERO

$\begin{array}{ll}\text { ZERO } & \text { ZERO } \\ \text { ZERO } & \text { ZERO }\end{array}$

$\begin{array}{ll}\text { ZERO } & \text { ZERO } \\ \text { ZERO } & \text { ZERO }\end{array}$

$\begin{array}{ll}\text { ZERO } & \text { ZERO } \\ \text { ZERO } & \text { ZERO }\end{array}$

ZERO

ZERO

ZERO $0.8551 \mathrm{E}+01$

$0.8551 \mathrm{E}+01$

ZERO

ZERO

ZERO

ZERO

ZERO

ZERO

ZERO

ZERO

ZERO

ZERO

ZERO

ZERO

ZERO

ZERO

ZERO

ZERO

ZERO

ZERO

ZERO

ZERO

ZERO

ZERO

ZERO

ZERO

ZERO

ZERO

ZERO
$0.2968 \mathrm{E}-03$

ZERO
$.1480 \mathrm{E}-01$

ZERO

ZEERO

ZERO

ZERO

$0.2240 \mathrm{E}-04$

$0.2065 E+01$

$0.2554 \mathrm{EERO}$

$.1257 \mathrm{E}-06$

$0.1501 \mathrm{E}-01$

$.3344 \mathrm{E}-02$

$.2964 \mathrm{E}-01$

ZERO

ZERO

ZERO

ZERO

$0.1629 \mathrm{E}+00$

$0.1018 \mathrm{E}-03$

ZERO

ZERO

$0.7390 \mathrm{E}-05$

ZERO
ZERO ZERO

ZERO
ZERO
ZERO

$\begin{array}{ll}\text { ZERO } & \text { ZERO } \\ \text { ZERO } & \text { ZERO }\end{array}$

ZERO ZERO

ZERO ZERO

ZERO

ZERO ZERO

ZERO

ZERO

ZERO

ZERO

ZERO ZERO

ZERO ZERO

ZERO

$0.1018 \mathrm{E}-01$

$0.8551 \mathrm{E}+01$

ZERO

$0.2968 F-03$

ZERO

0.1480 ZERO

ZERO

ZERO

ZERO

ZERO

ZERO

$0.2240 \mathrm{E}-04$

ZERO

ZERO

ZERO

ZERO

ZERO

ZERO

ZERO

ZERO

ZERO

ZERO

ZERO

ZERO

ZERO

ZERO

ZERO

$0.7390 \mathrm{E}-05$

ZERO

ZERO

ZERO

ZERO

ZERO

ZERO

2065E+O
ZERO

$.2554 \mathrm{E}+01$

$.1501 \mathrm{E}-01$

$0.2964 \mathrm{E}-01$

ZERO

ZERO

ZERO

ZERO

$0.1629 \mathrm{E}+00$

$0.1018 \mathrm{E}-03$

ZERO

ZERO

ZERO

ZERO

Date: January 1995

Section 1C, Page 18 of 21 
Table A-1. HLW Flowsheet Material Balance (Tank 51/ITP Cycle 1 )

- Section 1C. ESP Washing of Tank 51 sludge

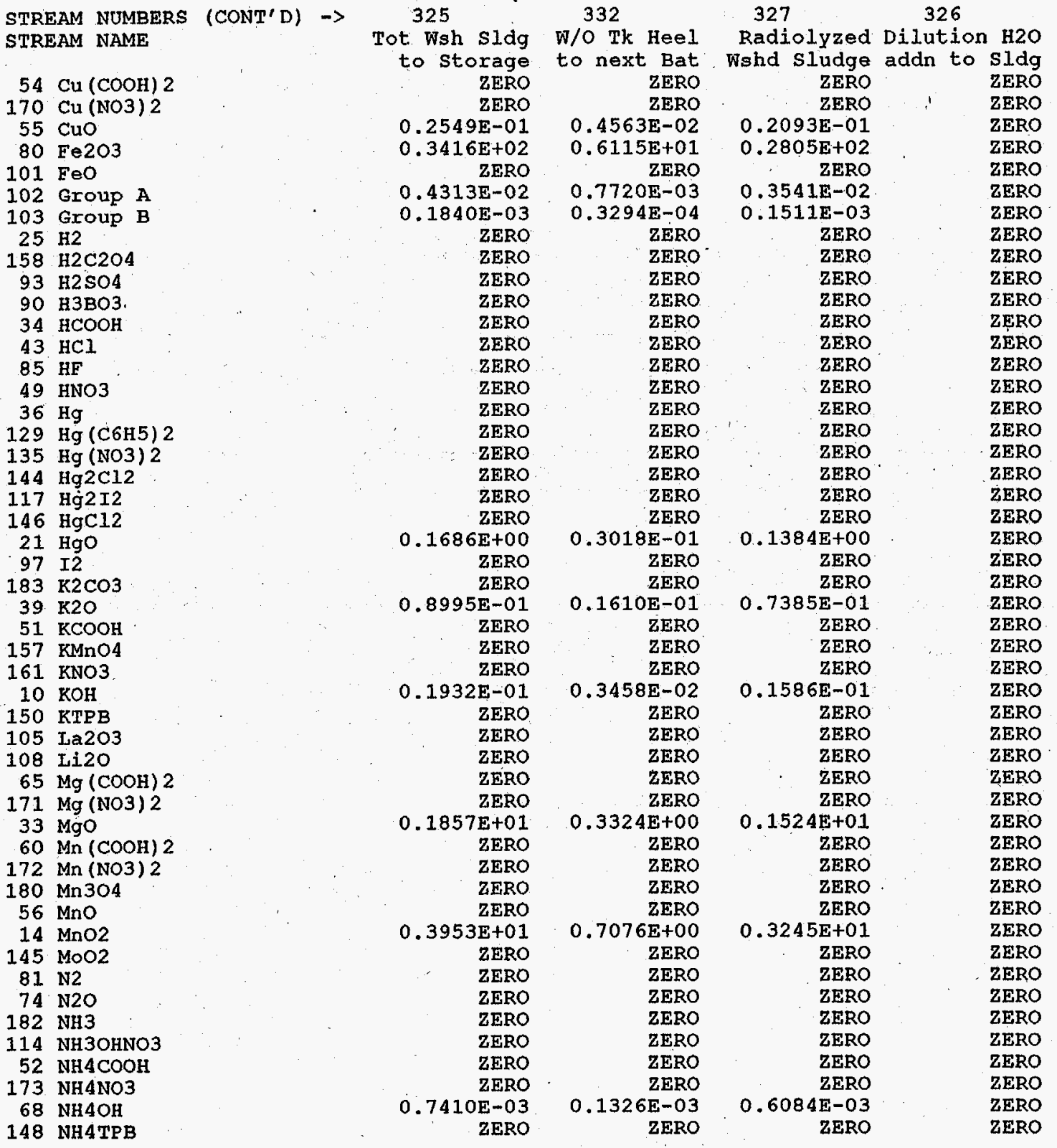

$1 \quad 678$

ludge Feed

DWPF

ZERO

ZERO
ZERO

$\begin{array}{ll}0.2093 \mathrm{E}-01 & \text { ZERO } \\ 0.2805 \mathrm{E}+02 & \text { ZERO }\end{array}$

ZERO

$0.3541 \mathrm{E}-02$ ZERO

$0.1511 \mathrm{E}-03$

ZERO

ZERO
ZERO

ZERO

ZERO

ZERO

ZERO

ZERO

ZERO

ZERO

ZERO

ZERO

ZERO

$0.1384 \mathrm{E}+00$

ZERO
ZERO

$0.7385 \mathrm{E}-01$

ZERO

ZERO

$0.1586 \mathrm{E}-01$

ZERO

ZERO

ZERO

ZERO

$0.1524 \mathrm{E}+01$

ZERO

ZERO

ZERO

$0.3245 E+01$

ZERO

ZERO

ZERO

ZERO

ZERO

$0.6084 \mathrm{E}-03$

$4 \mathrm{E}-03$
ZERO

Date: January 1995
Sldge Feed
solubles

Sldge Feed Insolubles ZERO $0.2093 \mathrm{E}-01$ $0.2805 E+02$ $0.3541 \mathrm{E}-02$ $0.1511 \mathrm{E}-03$ ZERO 0.15 ZERO ZERO ZERO ZERO $\begin{array}{ll}\text { ZERO } & \text { ZERO } \\ \text { ZERO } & \text { ZERO }\end{array}$ ZERO $\quad$ ZERO ZERO ZERO ZERO ZERO ZERO ZERO ZERO ZERO ZERO … ZERO ZERO ZERO ZERO ZERO ZERO ZERO ZERO ZERO 0.1384 ZERO $0.1384 E+00$ ZERO ZERO ZERO ZERO $0.7385 \mathrm{E}-01$
ZERÓ ZERO
ZERO ZERO
ZERO ZERO
$0.1586 \mathrm{E}-01$ ZERO ZERO ZERO ZERO ZERO ZERO ZERO $\quad 0.1524 \mathrm{E}+01$ ZERO ZERO ZERO ZERO $\begin{array}{ll}\text { ZERO } & \text { ZERO } \\ \text { ZERO } & \text { ZERO }\end{array}$ $\begin{array}{rr}\text { ZERO } & \text { ZERO } \\ \text { ZERO } & 0.3245 \mathrm{E}+01\end{array}$ ZERO ZERO ZERO ZERO ZERO ZERO ZERO ZERO ZERO ZERO ZERO ZERO ZERO $0.6084 \mathrm{E}-03$ ZERO ZERO ZERO 
Table A-1. HLW Flowsheet Material Balance (Tank 51/ITP Cycle 1) Section 1C. ESP Washing of Tank 51 sludge

STREAM NUMBERS (CONT'D) $\rightarrow$ STREAM NAME

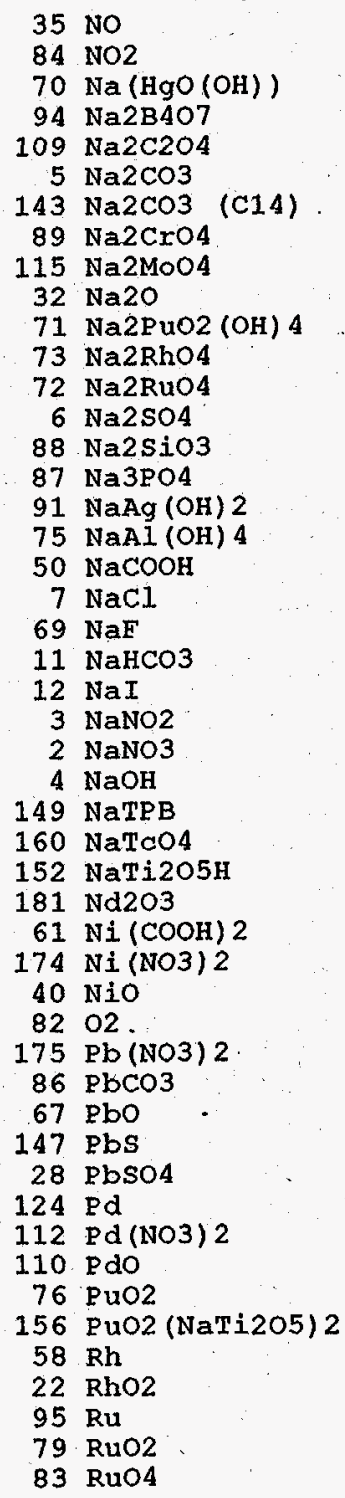
ZERO

i

$\stackrel{1}{1}$ Feed

to DWPF ZERO $0.2562 \mathrm{E}-03$ ZERO $0.2642 \mathrm{E}+00$ $0.2458 \mathrm{E}+00$ $0.3019 \mathrm{E}-09$ $0.9246 \mathrm{E}-02$ $0.1523 \mathrm{E}-02$ $0.3230 \mathrm{E}+01$ . 100 $0.3865 \mathrm{E}-04$ $0.1652 \mathrm{E}-03$ $0.1944 \mathrm{E}+00$ $0.8070 \mathrm{E}-02$ $0.1141 E-02$ $0.1457 \mathrm{E}-03$ $0.3558 \mathrm{E}+00$

0.6777 ZERO

$0.3408 \mathrm{E}-01$

ZERO $0.5416 \mathrm{E}-05$ $0.6454 \mathrm{E}+01$
$0.1518 \mathrm{E}+00$ $0.3062 \mathrm{E}+01$

$0.1568 \mathrm{E}-03$

ZERO

ZERO

ZERO

ZERO

ZERO

ZERO

ZERO

ZERO

ZERO

ZERO

ZERO

ZERO

ZERO

ZERO

ZERO

ZERO

ZERO

ZERO

$0.2482 \mathrm{E}+00$

$0.7604 \mathrm{E}-04$

ZERO
ZERO

ZERO

$0.1274 \mathrm{E}+00$

ZERO
$4 \mathrm{E}-08$

$0.4488 \mathrm{E}-03$

$0.9873 E-02$

ZERO

$0.9498 \mathrm{E}-03$

$$
\begin{array}{r}
\text { ZE-03 } \\
\text { ZERO }
\end{array}
$$

$0.6163 \mathrm{E}-02$
678

Solubles ZERO $0.2562 E-03$

ZERO

$0.2642 E+00$

$0.2458 \mathrm{E}+00$

$0.3019 \mathrm{E}-09$

$0.9246 \mathrm{E}-02$
$0.1523 \mathrm{E}-02$

$0.1523 E-02$
ZERO

$0.1000 \mathrm{E}-07$

$0.3865 \mathrm{E}-04$

$0.1652 \mathrm{E}-03$

$0.1944 \mathrm{E}+00$

$0.8070 \mathrm{E}-02$

$0.1141 \mathrm{E}-02$

$0.1457 \mathrm{E}-03$

$0.3558 \mathrm{E}+00$

$0.6777 \mathrm{E}-02$

$0.3408 \mathrm{E}-01$

ZERO

$0.5416 E-05$

$0.1518 \mathrm{E}+00$

$0.3062 \mathrm{E}+01$

ZERO ZERO ZERO ZERO ZERO

679

Sldge Feed

Insolubles ZERO

ZERO

ZERO

ZERO

ZERO

ZERO

ZERO

$0.3230 \mathrm{E}+01$

ZERO

ZERRO

ZERO

ZERO

ZERO

ZERO

ZERO

ZERO

ZERO

ZERO

ZERO

ZERO

ZERO

$0.9896 \mathrm{E}-08$ 
Table A-1. HLW Flowsheet Material Balance (Tank 51/ITP Cycle 1)

Section 1C. ESP Washing of Tank 51 sludge

STREAM NUMBERS (CONT'D) $\rightarrow$

32.5

STREAM NAME

$38 \quad 502$

141 SO3

137 Semi Vol Cs2O

136 Semi Vol CsCl

126 Semi Vol Group A

140 Semi Vol Na2B4O7

138 Semi Vol NaCl

139 Semi Vol NaF

125 Semi Vol NaI

23 Semi Vol RuO2

111 sio2

$48 \mathrm{Sr}$ (COOH) 2

$176 \mathrm{Sr}$ (NO3) 2

$153 \mathrm{Sr}$ (NaTi205) 2

127. $\mathrm{Sr}(\mathrm{OH}) 2$

$30 \mathrm{SrCO} 3$

46 Sro

159 TcO2

92 Tho2

119 Tritium

17 U308

62.002 ( $\mathrm{COOH}) 2$

177 UO2 (NO3) 2

$16 \mathrm{UO} 2(\mathrm{OH}) 2$
$27 \mathrm{Y}(\mathrm{COOH}) 3$

178 Y (NO3) 3

$128 \mathrm{Y}(\mathrm{OH}) 3$

$31 \mathrm{Y} 2(\mathrm{CO}) 3$

100 Zeolite

$44 \mathrm{Zn}$ (COOH) 2

$179 \mathrm{Zn}(\mathrm{NO} 3) 2$

$\begin{array}{rl}57 & \mathrm{ZnO} \\ 104 & \mathrm{ZrO} 2\end{array}$

$\begin{aligned} 104 & \text { ZrO2 } \\ 13 & \text { hydrate } \mathrm{H} 2 \mathrm{O}\end{aligned}$

1 water

TOTAL ELOW, LB/HR

TEMPERATURE， DEG C

PRESSURE, ATM

PRESSURE, PSIG

PRESSURE， MM HG

ENTHALPY， PCU/HR

VAPOR FLOW, CEM

LIOUID FLOW, GPM

DENSITY, LBS/FT3

PHASE

327

326

1

W/O Tk Heel Radiolyzed Dilution H2O
to storage to next Bat Wshd Sludge addn to Sldg

to stora ZERO

ZERO

ZERO

ZERO

ZERO

ZERO

ZERO

ZERO

ZERO

$0.1292 \mathrm{E}+01$

ZERO

ZERO

$0.4400 \mathrm{E}-07$

$0.2501 \mathrm{E}-01$

ZERO

$0.4211 \mathrm{E}-02$

$0.3620 \mathrm{E}-01$

$0.1245 \mathrm{E}-08$

$0.2538 \mathrm{E}+01$

$0.2538 \mathrm{E}+01$
ZERO ZERO

$0.8587 \mathrm{E}-04$

ZERO
ZERO

ZERO
$0.4434 \mathrm{E}-07$

$.6295 \mathrm{E}-02$

ZERO

ZERO

ZERO

$0.1436 \mathrm{E}+00$ ZERO

$0.2776 \mathrm{E}+01$

$0.3117 \mathrm{E}+03$

$0.3929 \mathrm{E}+03$

$0.4800 \mathrm{E}+02$ 0.1000 E +01
ZERO

$0.1566 \mathrm{E}+05$

$0.6677 \mathrm{E}+00$

$0.7337 \mathrm{E}+02$

LIQUID ZERO
ZERO
ZERO

\section{ZERO}

ZERO

ZERO

ZERO

ZERO

FRO

ZERO

ZERO

ZERO

$0.7876 \mathrm{E}-08$

$0.4478 \mathrm{E}-02$.

$0.7538 \mathrm{E}-03$

. 6481E-02

$0.1013 \mathrm{E}-01$

$0.2230 \mathrm{E}-09$

$0.4542 \mathrm{E}+00$

ZERO

$0.1537 \mathrm{E}-04$

E-O4
ZERO

ERO

$0.7937 \mathrm{E}-0$

$0.1127 \mathrm{E}-02$.

ZERO

ZERO

ERRO

$0.2571 \mathrm{E}-01$

ZERO

$0.4970 \mathrm{E}+00$

$.5580 \mathrm{E}+02$

$0.7034 \mathrm{E}+02$

ZERO

ZERO

ZERO

ZERO

ZERO

ZERO

ZERO

ZERO

0.1061

ZERO
$\mathrm{E}+01$.

ZERO

ZERO

$0.3612 \mathrm{E}-07$

$0.2054 \mathrm{E}-01$

ZERO

$0.3457 \mathrm{E}-02$

$0.2972 \mathrm{E}-01$

$0.4648 \mathrm{E}-01$

$0.1023 \mathrm{E}-08$

$0.2083 \mathrm{E}+01$ ZERO

$0.7050 \mathrm{E}-04$

OE-04
ZERO

ZERO

$0.3640 \mathrm{E}-07$

$0.5168 \mathrm{E}-02$

ZERO

ZERO

0.117 ZERO

E+OO

$0.2279 \mathrm{E}+01$

$0.2559 \mathrm{E}+03$

$0.3226 \mathrm{E}+03$

$0.1220 \mathrm{E}+03$

$0.3500 \mathrm{E}+02$

$0.1000 \mathrm{E}+01$

$0.3500 \mathrm{E}+02$

$0.1000 \mathrm{E}+01$
ZERO

$0.2500 \mathrm{E}+02$

$0.1000 \mathrm{E}+01$

$0.3059 E+04$

$0.2046 \mathrm{E}+04$

$0.5482 E+00$

$0.2435 \mathrm{E}+00$

$0.1195 \mathrm{E}+00$

LIQUID

$0.6247 \mathrm{E}+02$

LIOUID
$0.1219 \mathrm{E}+03$ $\stackrel{1}{\text { Sludge Feed }}$

to DWPF

ZERO

ZERO

ZERO

ZERO

ZERO

ZERO

ZERO

ZERO

ZERO

$0.1061 \mathrm{E}+01$

ZERO

ZERO
$0.3612 \mathrm{E}-07$

$0.2054 \mathrm{E}-01$

ZERO

$0.3457 \mathrm{E}-02$

$0.2972 \mathrm{E}-01$

$0.4648 \mathrm{E}-01$

$0,1023 \mathrm{E}-08$

$0.2083 E+01$ ZERO

$0.7050 \mathrm{E}-04$

ZERO

ZERO

$0.364 \mathrm{OE}-07$

$0.5168 \mathrm{E}-02$

ZERO

ZERO

$0.1179 \mathrm{E}+00$

ZERO

$0.2279 E+01$

$0.3778 \mathrm{E}+03$
$0.4446 \mathrm{E}+03$

$0.6000 \mathrm{E}+02$

$0.1000 E+01$
ZERO

$0.2338 \mathrm{E}+05$

$0.7920 \mathrm{E}+00$

$0.6998 \mathrm{E}+02$

LIOUID

678

ldge Feed

Solubles

ZERO

ZERO

ZERO

ZERO

ZERO

ZERO

ZERO

ZERO

ZERO

TRR

ZERO

ZERO

(2)

$0.3612 \mathrm{E}-07$

ZERO

ZERO

ZERO

ZERO

ZERORO

$0.1023 E-08$

ZERO

ZERO

$0.7050 \mathrm{E}-04$

ZERO

ZERO

$0.3640 \mathrm{E}-07$

ZERO

ZERO

ZERO

ZERO

ZERO

ZERO

ZERO

$0.3778 \mathrm{E}+03$

$0.3888 \mathrm{E}+03$

$0.6000 \mathrm{E}+02$

$0.1000 \mathrm{E}+01$

ZERO

$0.2285 \mathrm{E}+05$

$0.7625 \mathrm{E}+00$

$0.6357 \mathrm{E}+02$

LIOUID

679

ldge Feed

Insolubles

ZERO

ZERO

ZERO

ZERO

ZERO

ZERO

ZERO

ZERO

ŻERO

ZERO

ZERO

$0.2054 \mathrm{E}-01$

ZERO

0. 


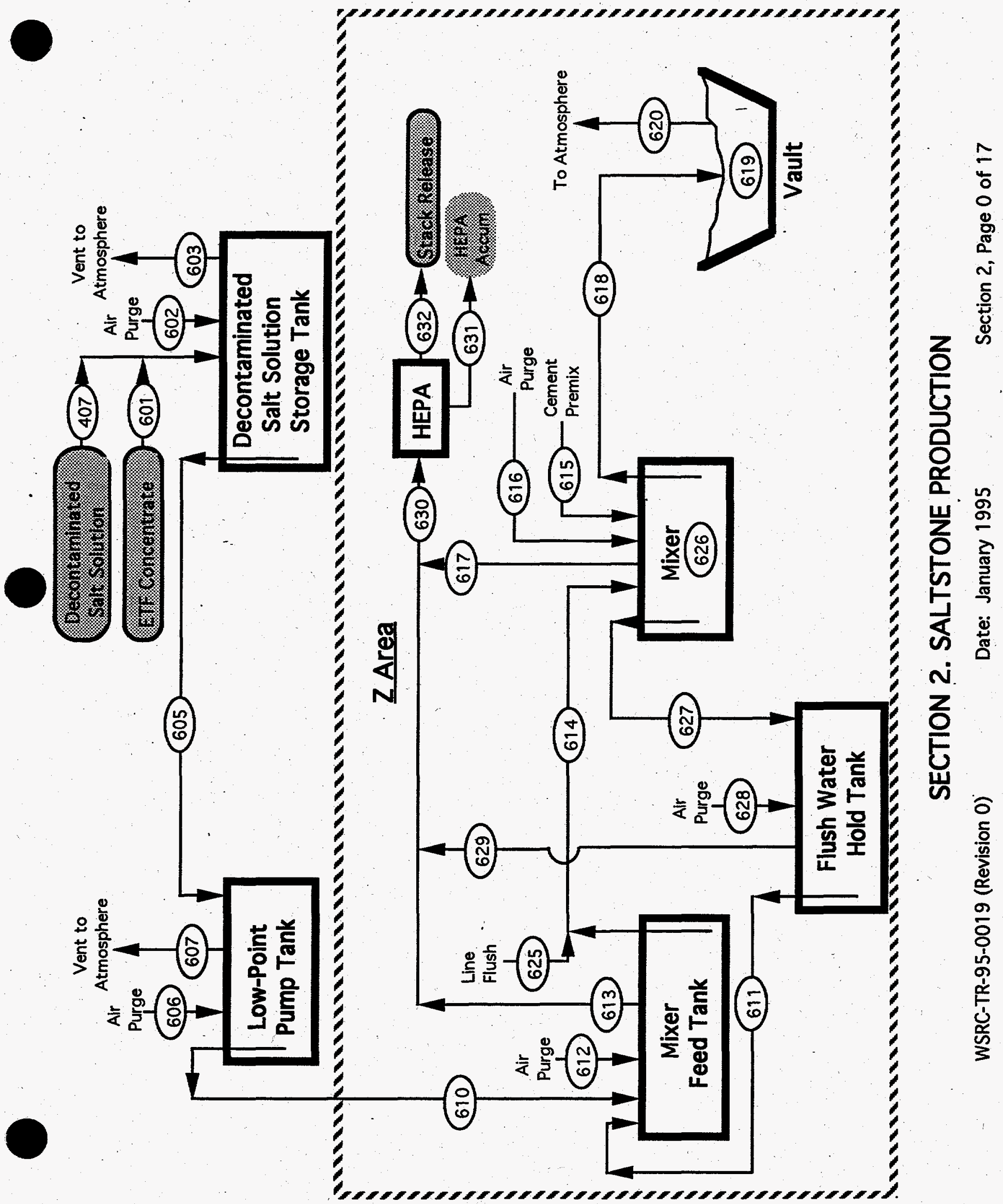



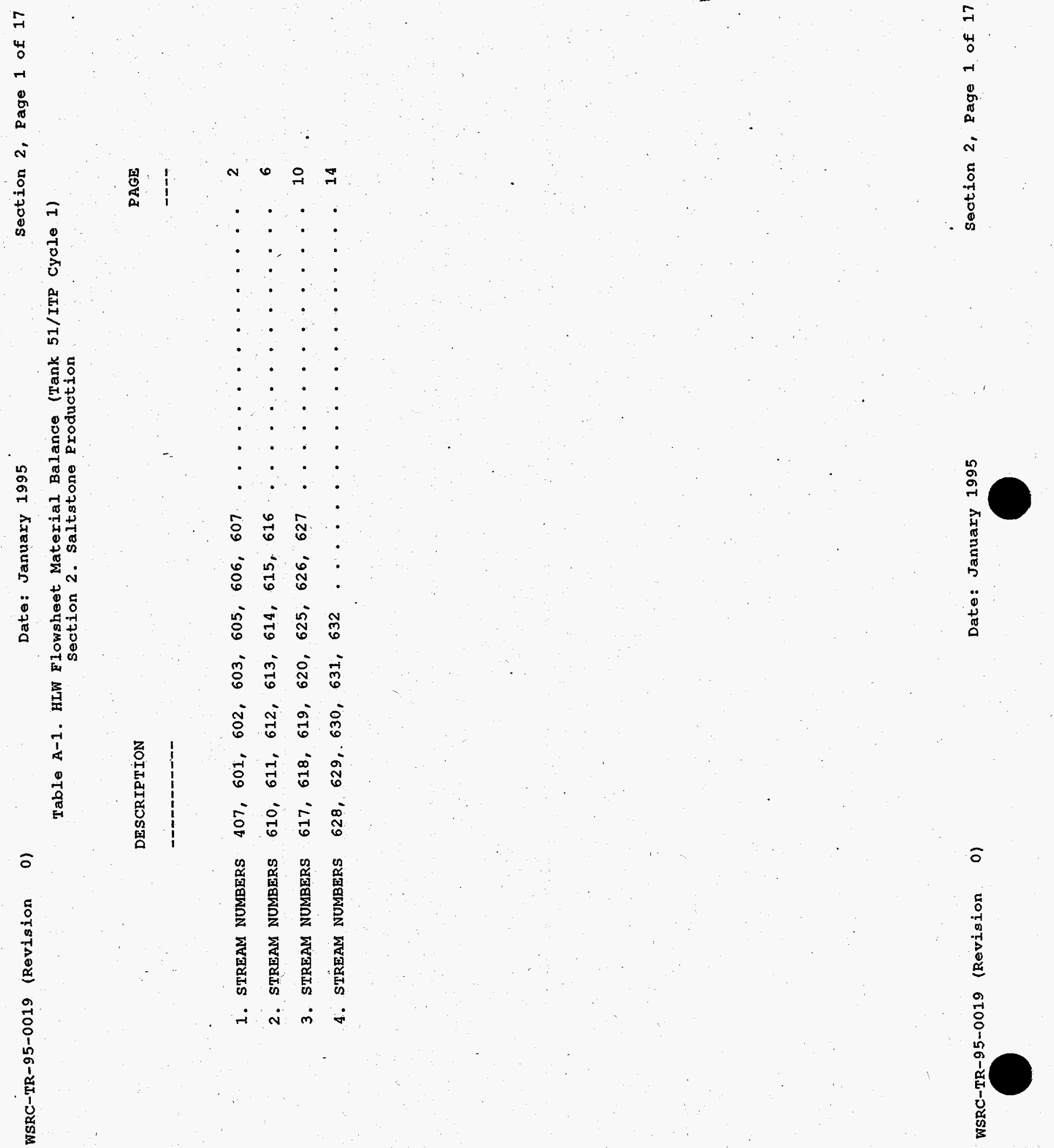
Table A-1. HLW Flowsheet Material Balance (Tank 51/ITP Cycle 1) section 2. Saltstone Production

STREAM NUMBERS -> STREAM NAME

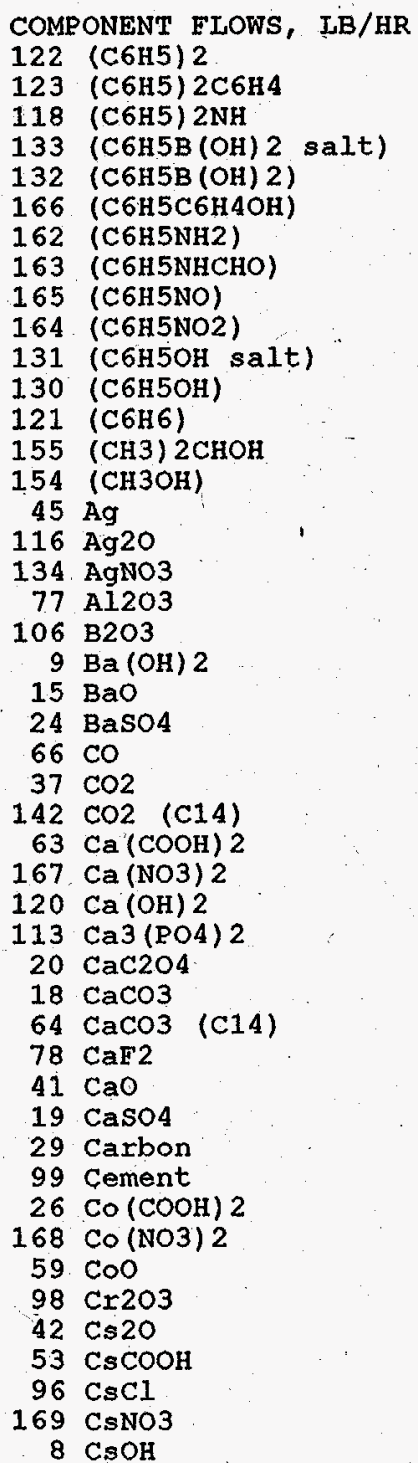

WSRC-TR-95-0019 (Revision $\begin{array}{lccc}407 & 601 & 602 & 603 \\ \text { Decon SN } & \text { Concentrate } & \text { DCon strg } & \text { DCon Str Tk }\end{array}$

to storage from ETF Tank Purge Vnt to Atm

ZERO

ZERO

ZERO

ZERO

ZERO

ZERO

ZERO

ZERO

ZERO

$0.3344 \mathrm{E}+01$

ZERO

$0.3374 \mathrm{E}-02$

$0.8203 \mathrm{E}-01$

$0.494 \mathrm{OE}-02$

ZERO

ZERO

ZERO

$0.1295 \mathrm{E}-07$

ZERO

ZERO

$0.7780 \mathrm{E}-03$

ZERO

$0.3549 \mathrm{E}-01$

$0.3660 \mathrm{E}-02$

ZERO

ZERO

ZERO

ZERO

ZERO

ZERO

ZERO

ZERO

$0.1392 \mathrm{E}-01$

$2 E-01$
ZERO

ZERO

ZERO

ZERO

$0.1653 \mathrm{E}-05$

ZERO

ZERO

ZERO

ZERO

ZERO

ZERO

ZERO

ZERO

ZERO

ZERO

ZERO

ZERO

ZERO

ZERO

ZERO

ZERO

$0.1757 \mathrm{E}-02$

$0.8794 \mathrm{E}-01$

ZERO

ZERO

E-02

ZERO

ZERO

ZERO

ZERO

ZERO

$0.2178 \mathrm{E}+00$

ZERO

$0.4990 \mathrm{E}+00$

ZERO

ZERO

ZERO

ZERO

ZERO

ZERO

ZERO

ZERO

ZERO

ZERO

ZERO

$0.4011 \mathrm{E}-08$

ZERO

$0.1430 \mathrm{E}-16$

$$
605
$$

Dcon $\mathrm{Sn}$ to

LP Pmp Tnk

606

PPT Ai

Purge

$\begin{array}{lr}\text { ZERO } & \text { ZERO } \\ \text { ZERO } & \text { ZERO } \\ \text { ZERO } & \text { ZERO } \\ \text { ZERO } & \text { ZERO } \\ \text { ZERO } & \text { ZERO } \\ \text { ZERO } & \text { ZERO } \\ \text { ZERO } & \text { ZERO } \\ \text { ZERO } & \text { ZERO } \\ \text { ZERO } & \text { ZERO } \\ \text { ZERO } & \text { ZERO }\end{array}$

$0.1059 \mathrm{E}-10$

ZERO $0.1059 E-10$

ZERO
ZERO

$\begin{array}{ll}\text { ZERO } & 0.9844 \mathrm{E}-04 \\ \text { ZERO } & 0.6674 \mathrm{E}-02\end{array}$

ZERO $0.3435 \mathrm{E}-03$

$\begin{array}{lr}\text { ZERO } & \text { ZERO } \\ \text { ZERO } & 0.5565 \mathrm{E}-14\end{array}$

ZERO

ZERO $\quad 0.2785 \mathrm{E}-12$

ZERO

ZERO

ZERO

ZERO

ZERO

ZERO

ZERO

ZERO

ZERO

ZERO

ZERO

ZERO

ZERO

ZERO

ZERO

ZERO

ZERO

ZERO

ZERO

ZERO

ZERO

ZERO

ZERO

ZERO

ZERO

ZERO

ZERO

0.4101 ZERO

ZERO

$.6130 \mathrm{E}-05$

ZERO

$0.1159 \mathrm{E}-13$

$0.6899 \mathrm{E}-12$

$0.1580 \mathrm{E}-11$

ZERO
ZERO
ZERO

ZERO

ZERO

ZERO

ZERO

ZERO

ZERO

ZERO

ZERO

ZERO

ZERO

$0.1657 \mathrm{E}-05$

ZERO
ZERO

Date: January 1995

Section 2, Page 2 of 17 
Table A-1. HLW Flowsheet Material Balance (Tank 51/ITP Cycle 1 )

STREAM NUMBERS (CONT'D) $\rightarrow$ STREAM NAME

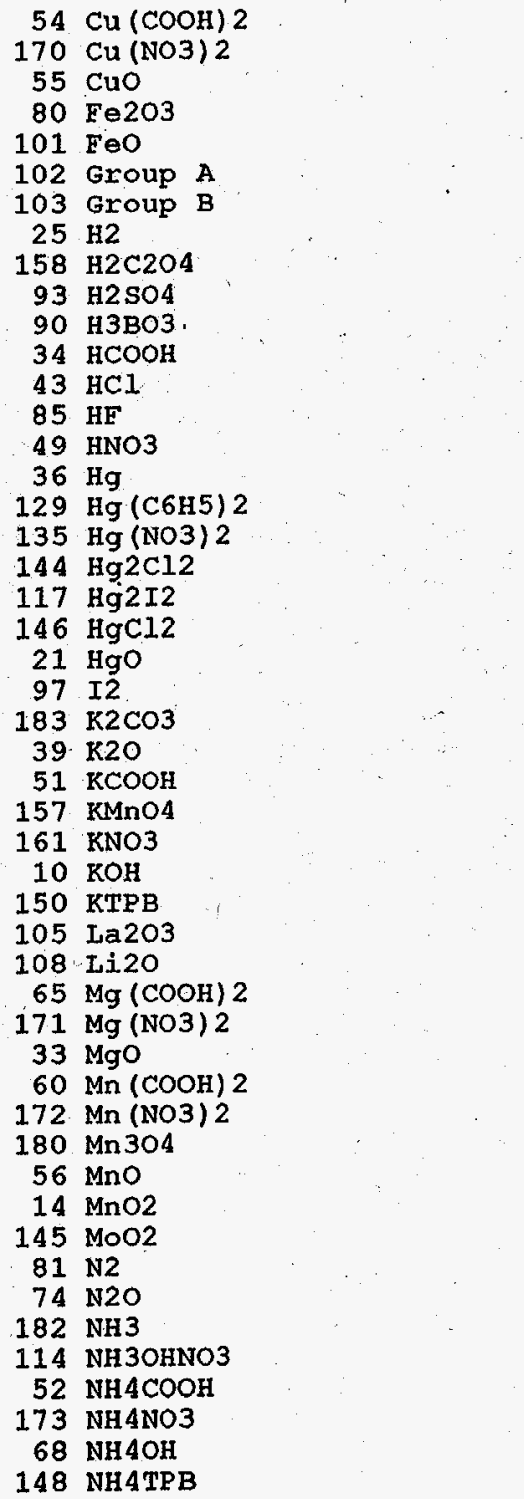

WSRC-TR-95-0019 (Revision
Section 2. Saltstone production

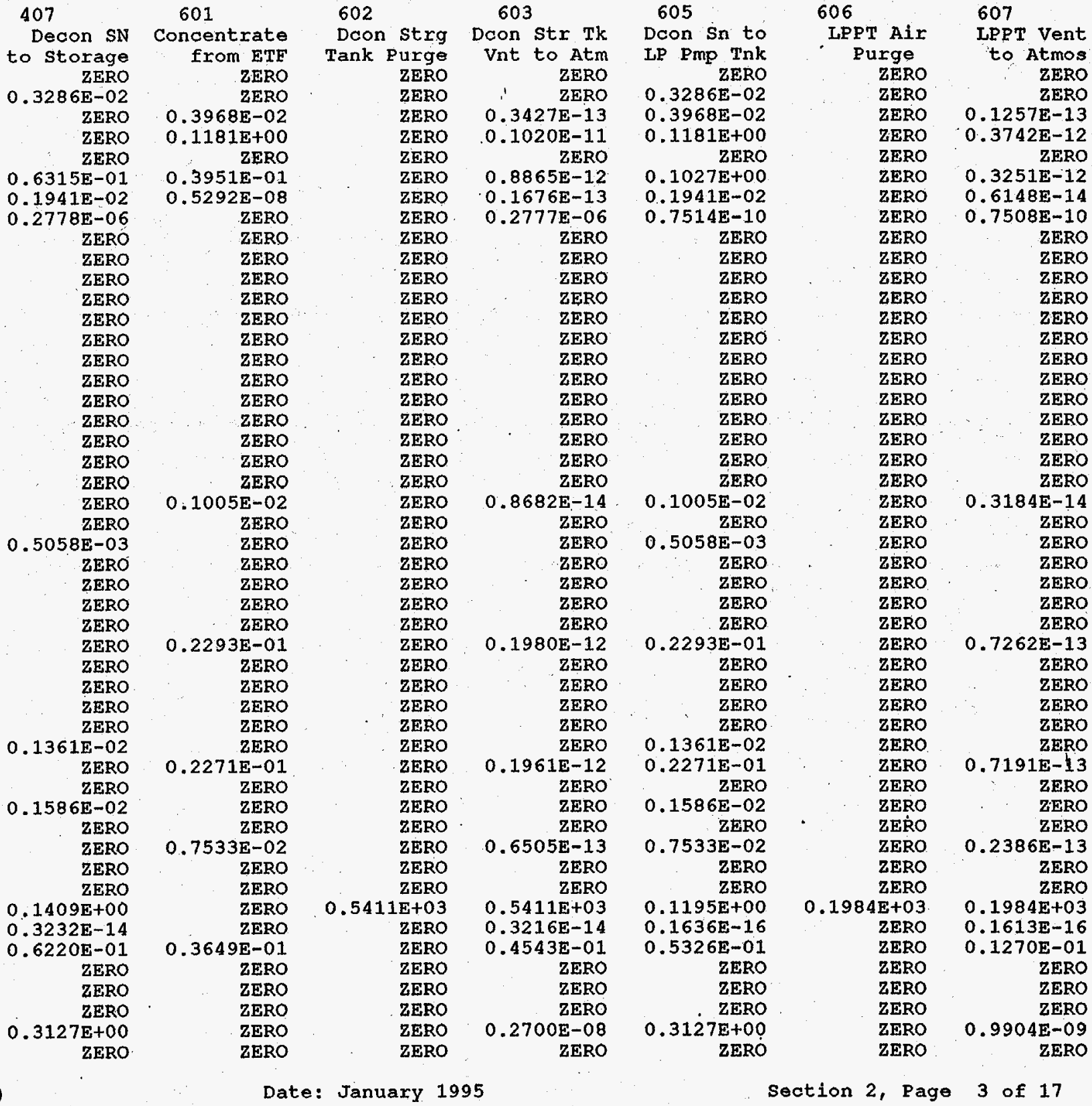

Date: January 1995 


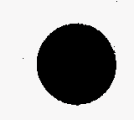

WSRC-TR-95-0019 (Revision

Date: January 1995

Section 2, Page 4 of 17

Table A-1. HLW Flowsheet Material Balance (Tank 51/ITP Cycle 1)
Section 2. Saltstone Production

STREAM NUMBERS (CONT'D) $\rightarrow$ STREAM NAME

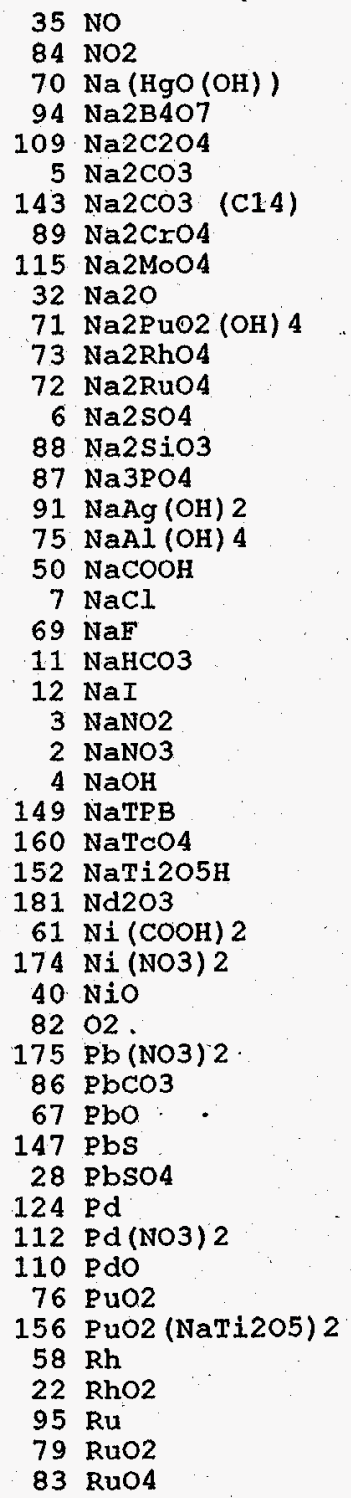

WSRC-TR-95-0019 (Revision

\begin{tabular}{|c|c|c|c|}
\hline 407 & 601 & 602 & 603 \\
\hline Decon SN & Concentrate & Dcon strg & Dcon Str Tk \\
\hline to storage & from ETF & Tank Purge & Vnt to Atm \\
\hline ZERO & ZERO & ZERO & ZERO \\
\hline ZERO & ZERO & ZERO & ZERO \\
\hline $0.2822 \mathrm{E}-03$ & ZERO & ZERO & $0.2436 \mathrm{E}-14$ \\
\hline $0.3253 \mathrm{E}+00$ & ZERO & ZERO & $0.2809 \mathrm{E}-11$ \\
\hline $0.3955 \mathrm{E}+01$ & $0.7128 \mathrm{E}+00$ & ZERO & $031 \mathrm{E}-10$ \\
\hline $0.4945 \mathrm{E}+03$ & $0.2971 \mathrm{E}+01$ & ZERO & $0.4295 \mathrm{E}-08$ \\
\hline $0.7320 \mathrm{E}-05$ & ZERO & ZERO & $0.6320 \mathrm{E}-16$ \\
\hline $0.2694 \mathrm{E}+01$ & $0.5974 \mathrm{E}-02$ & ZERO & $.2332 E-10$ \\
\hline $0.8046 \mathrm{E}+00$ & ZERO & ZERO & $0.6947 \mathrm{E}-11$ \\
\hline ZERO & ZERO & ZERO & ZERO \\
\hline $0.1748 E-05$ & $0.6451 \mathrm{E}-07$ & ZERO & $0.1565 \mathrm{E}-16$ \\
\hline $0.1952 \mathrm{E}-01$ & $0.1665 \mathrm{E}-12$ & ZERO & $0.1685 \mathrm{E}-12$ \\
\hline $0.1028 \mathrm{E}+00$ & $0.3364 \mathrm{E}-10$ & ZERO & $0.8875 \mathrm{E}-12$ \\
\hline $0.2804 \mathrm{E}+02$ & $0.1252 \mathrm{E}+00$ & ZERO & $0.2432 \mathrm{E}-09$ \\
\hline $0.7803 \mathrm{E}+00$ & zERO & ZERO & $0.6738 \mathrm{E}-11$ \\
\hline $0.3724 \mathrm{E}+02$ & ZERO & ZERÓ & $3216 \mathrm{E}-09$ \\
\hline $0.7463 \mathrm{E}-05$ & ZERO & ZERO & $0.6444 \mathrm{E}-16$ \\
\hline $.1402 \mathrm{E}+03$ & ZERO & ZERO & $0.1210 \mathrm{E}-08$ \\
\hline ZERO & ZERO & ZERO & ZERO \\
\hline $0.2218 \mathrm{E}+01$ & $0.1494 \mathrm{E}+00$ & ZERO & $0.2044 \mathrm{E}-10$ \\
\hline $0.2156 \mathrm{E}+01$ & $0.4444 \mathrm{E}-01$ & ZERO & $0.1900 \mathrm{E}-10$ \\
\hline ZERO & ZERO & ZERO & ZERO \\
\hline $0.1444 \mathrm{E}-02$ & $0.1337 \mathrm{E}-13$ & ZERO & $0.1247 \mathrm{E}-13$ \\
\hline $0.2723 E+03$ & $0.4455 \mathrm{E}-01$ & ZERO & $0.2352 \mathrm{E}-08$ \\
\hline $0.1352 \mathrm{E}+04$ & $0.4485 E+02$ & ZERO & $0.1206 \mathrm{E}-07$ \\
\hline $0.6376 E+03$ & $0.1105 \mathrm{E}+02$ & ZERO & $0.5601 \mathrm{E}-08$ \\
\hline $0.7767 \mathrm{E}+01$ & ZERO & ZERO & $0.6707 \mathrm{E}-10$ \\
\hline $0.6477 \mathrm{E}-01$ & ZERO & ZERO & ZERO \\
\hline ZERO & ZERO & ZERO & ZERO \\
\hline ZERO & ZERO & ZERO & ZERO \\
\hline ZERO & ZERO & ZERO & ZERO \\
\hline $0.1732 \mathrm{E}-01$ & ZERO & ZERO & . ZERO \\
\hline ZERO & $0.3657 \mathrm{E}-02$ & ZERO & $0.3158 \mathrm{E}-13$ \\
\hline $0.1684 \mathrm{E}-01$ & ZERO & $0.1634 \mathrm{E}+03$ & $0.1634 \mathrm{E}+03$ \\
\hline $.4207 \mathrm{E}-01$ & ZERO & ZERO & ZERO \\
\hline ZERO & ZERO & ZERO & ZERO \\
\hline ZERO & $0.1762 \mathrm{E}-02$ & ZERO & $0.1522 \mathrm{E}-13$ \\
\hline ZERO & ZERO & ZERO & ZERO \\
\hline ZERO & ZERO & ZERO & ZERO \\
\hline ZERO & ZERO & ZERO & ZERO \\
\hline $0.1736 \mathrm{E}-03$ & ZERO & ZERO & $0.1499 \mathrm{E}-14$ \\
\hline ZERÓ & ZERO & ZERO & ZERO \\
\hline ZERO & ZERO & ZERO & ZERO \\
\hline ZERO & ZERO & ZERO & ZERO \\
\hline ZERO & ZERO & ZERO & ZERO \\
\hline ZERO & ZERO & ZERO & ZERO \\
\hline ZERO & ZERO & ZERO & ZERO \\
\hline ZERO & ZERO & ZERO & ZERO \\
\hline ZERO & ZERO & ZERO & ZERO \\
\hline
\end{tabular}

Date: January 1995

\begin{tabular}{|c|c|c|}
\hline 605 & 606 & 607 \\
\hline Dcon Sn to & IPPT Air & IPPT Vent \\
\hline $\begin{array}{r}\text { LP Pmp Tnk } \\
\text { zERO }\end{array}$ & $\begin{array}{l}\text { Purge } \\
\text { ZERO }\end{array}$ & to Atmos \\
\hline ZERO & ZERO & ZERO \\
\hline $0.2822 \mathrm{E}-03$ & ZERO & $0.8936 \mathrm{E}-15$ \\
\hline $0.3253 \mathrm{E}+00$ & ZERO & $0.1030 \mathrm{E}-11$ \\
\hline $0.4668 \mathrm{E}+01$ & ZERO & $0.1478 \mathrm{E}-10$ \\
\hline $0.4974 \mathrm{E}+03$ & ZERO & $0.1575 \mathrm{E}-08$ \\
\hline $0.7320 \mathrm{E}-05$ & ZERO & $0.2318 \mathrm{E}-16$ \\
\hline $0.2700 \mathrm{E}+01$ & ZERO & $0.8552 \mathrm{E}-11$ \\
\hline $0.8046 \mathrm{E}+00$ & ZERO & $0.2548 \mathrm{E}-11$ \\
\hline ZERO & ZERO & ZERO \\
\hline $0.1813 \mathrm{E}-05$ & ZERO & $0.5740 \mathrm{E}-17$ \\
\hline $0.1952 \mathrm{E}-01$ & ZERO & $0.6181 \mathrm{E}-13$ \\
\hline $0.1028 \mathrm{E}+00$ & ZERO & $0.3255 \mathrm{E}-12$ \\
\hline $0.2817 \mathrm{E}+02$ & ZERO & $0.8920 \mathrm{E}-10$ \\
\hline $0.7803 \mathrm{E}+00$ & ZERO & $0.2471 E-11$ \\
\hline $0.3724 \mathrm{E}+02$ & ZERO & $0.1179 \mathrm{E}-09$ \\
\hline $0.7463 \mathrm{E}-05$ & ZERO & $0.2363 \mathrm{E}-16$ \\
\hline $0.1402 \mathrm{E}+03$ & ZERO & $0.4439 \mathrm{E}-09$ \\
\hline ZERO & ZERO & ZERO \\
\hline $0.2367 \mathrm{E}+01$ & ZERO & $0.7497 \mathrm{E}-11$ \\
\hline $0.2200 \mathrm{E}+01$ & ZERO & $0.6968 \mathrm{E}-11$ \\
\hline ZERO & ZERO & ZERO \\
\hline $0.1444 \mathrm{E}-02$ & ZERO & $0.4573 \mathrm{E}-14$ \\
\hline $0.2723 \mathrm{E}+03$ & ZERO & $0.8625 \mathrm{E}-09$ \\
\hline $0.1397 \mathrm{E}+04$ & ZERO & $0.4423 \mathrm{E}-08$ \\
\hline $0.6486 \mathrm{E}+03$ & ZERO & $0.2054 \mathrm{E}-08$ \\
\hline $0.7767 \mathrm{E}+01$ & ZERO & $0.2460 \mathrm{E}-10$ \\
\hline $0.6477 \mathrm{E}-01$ & ZERO & ZERO \\
\hline ZERO & ZERO & ZERO \\
\hline ZERO & ZERO & ZERO \\
\hline ZERO & ZERO & ZERO \\
\hline $0.1732 \mathrm{E}-01$ & ZERO & ZERO \\
\hline $0.3657 \mathrm{E}-02$ & ZERO & $0.1158 \mathrm{E}-13$ \\
\hline $0.6991 \mathrm{E}-01$ & $0.5993 \mathrm{E}+02$ & $0.5993 \mathrm{E}+02$ \\
\hline $0.4207 \mathrm{E}-01$ & ZERO & ZERO \\
\hline ZERO & ZERO & ZERO \\
\hline $0.1762 \mathrm{E}-02$ & ZERQO & $0.5581 \mathrm{E}-14$ \\
\hline ZERO & ZERO & ZERO \\
\hline ZERO & ZERO & ZERO \\
\hline ZERO & ZERO & ZERO \\
\hline $0.1736 E-03$ & ZERO & $0.5499 \mathrm{E}-15$ \\
\hline ZERO & ZERO & ZERO \\
\hline ZERO & ZERO & ZERO \\
\hline ZERO & ZERO & ZERO \\
\hline ZERO & ZERO & ZERO \\
\hline ZERO & ZERO & ZERO \\
\hline ZERO & ZERO & ZERO \\
\hline ZERO & ZERO & ZERO \\
\hline ZERO & ZERO & ZERO \\
\hline
\end{tabular}




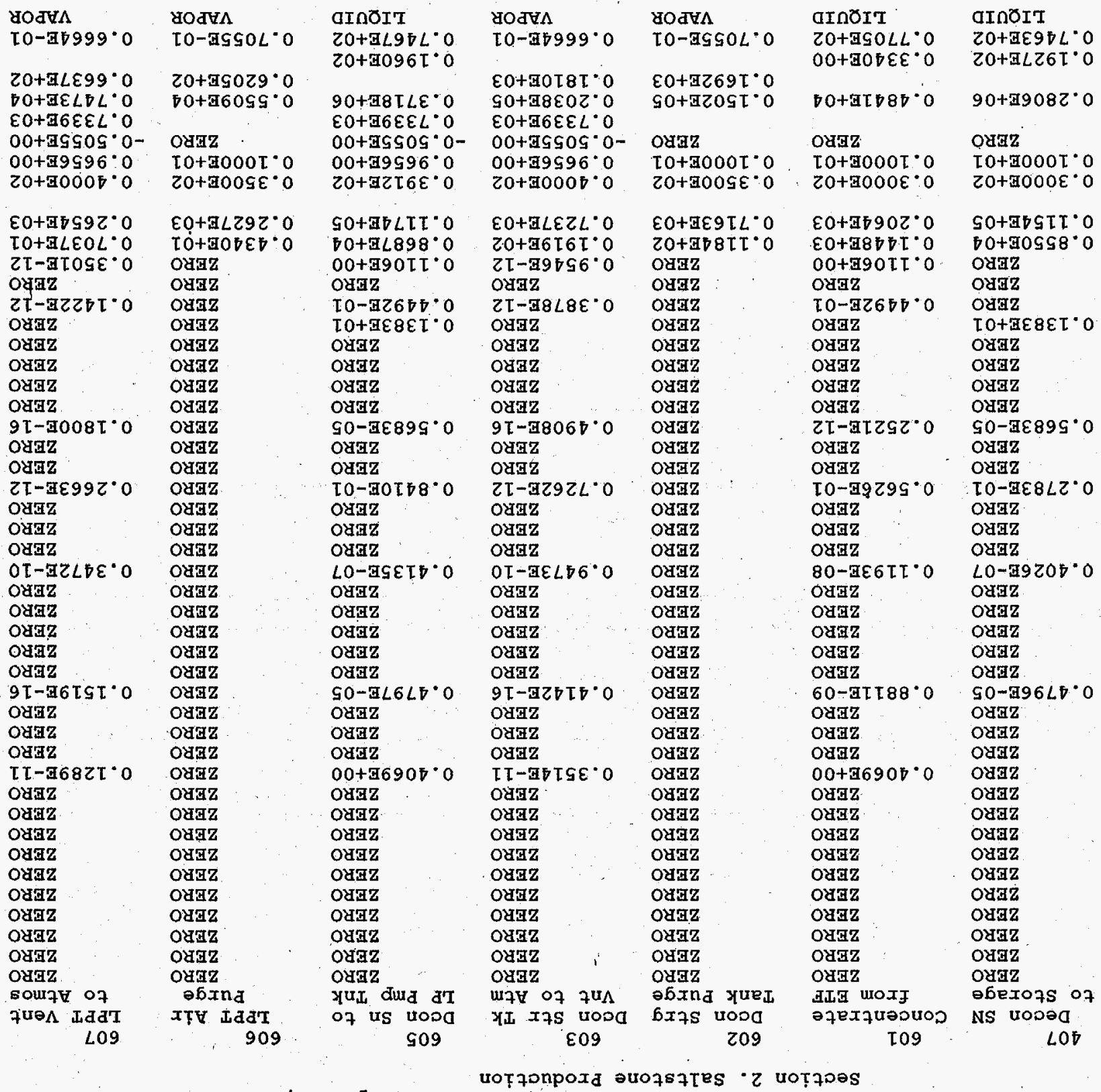
GH/חDd ' $X$ JTHHLNA

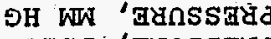

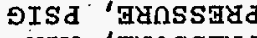

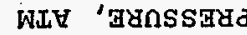

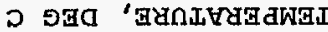

प्वH/घT 'MOTa TVLOI Iอ7eM I OZH ө७еxрКч $\varepsilon T$ zOJz $\begin{aligned} \text { OUz } & L S \\ (\varepsilon O N) \text { uz } & 6 L T\end{aligned}$ $\begin{aligned} Z(E O N) \text { uz } & 6 L \\ z(\text { HOOD) uz } & 00\end{aligned}$ จาTTOəZ 00T EOZX $L b$

$\varepsilon(\varepsilon \circ \supset) 乙 \pi \tau \varepsilon$ $\varepsilon(\mathrm{HO}) \pi 8 Z \mathrm{~L}$ $\varepsilon(\varepsilon O N) \pi 8 L T$ $\varepsilon($ HOOJ) $\pi$ L.Z $z$ (но) $z \circ \cap 9 \tau$ $z$ (EON) ZON LLT $z($ HOOD) zOn $z 9$ $80 \varepsilon \Omega<T$

unกฺาTรน $6 \tau$ ZOTI LOT ZOपू $Z 6$ ZODI $65 \mathrm{~T}$ OXS 90 EODXS $0 \varepsilon$ $Z$ (HO) xS LZT $z$ (SOZTIEN) IS $\varepsilon G$ $Z(\varepsilon O N)$ XS $9 L$ $2($ HOOD) Is 85 zots ITT zond tos tures $\varepsilon z$ IEN TOA TTHOS GZT

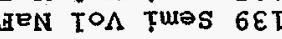
TDEN TOA Tures $8 \varepsilon$ LODgZeN TOA TuSS OD $\forall$ dnoIg TOA țures $9 Z$

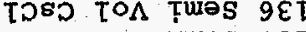

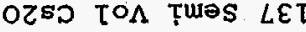
$\operatorname{EOS} T \in I$

THN WH'TYS

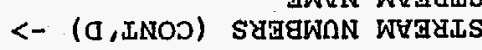

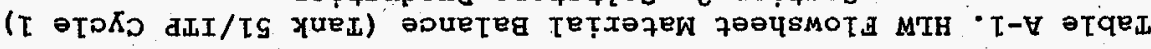


Table A-1. HLW Flowsheet Material Balance (Tank 51/ITP Cycle 1) Section 2. Saltstone production

STREAM NUMBERS $\rightarrow$ STREAM NAME

COMPONENT FLOWS, LB/HR 122 (C6H5) 2

123. (C6H5) 2C6H4

118 (C6H5) 2NH

133 (C6H5B (OH) 2 salt)

132 (C6H5B (OH) 2)

166 (C6H5C6H4O

163 (C6H5NHCHO)

163 (C6H5NHCHO)

164 (C6H5NO2)

131 (C6H5OH salt)

130 (C6H5OH)

$\begin{array}{ll}121 & \text { (C6H6) } \\ 155 & \text { (CH3) } 2 \mathrm{CHOH}\end{array}$

154 (CH3OH)

$45 \mathrm{Ag}$

134 AgNO3

$77 \mathrm{Al2O}$

$106 \mathrm{~B} 203$

9. $\mathrm{Ba}(\mathrm{OH}) 2$

$15 \mathrm{BaO}$

$\begin{array}{ll}24 & \mathrm{BaSO} \\ 66 & \mathrm{CO}\end{array}$

$\begin{array}{ll}66 & \mathrm{CO} \\ 37 & \mathrm{CO} 2\end{array}$

$\begin{array}{rrr}342 & \mathrm{CO} 2 & \text { (C14) }\end{array}$

$63 \mathrm{Ca}(\mathrm{COOH}) 2$

$167 \mathrm{Ca}(\mathrm{NO} 3) 2$

$120 \mathrm{Ca}(\mathrm{OH}) 2$

$113 \mathrm{Ca} 3$ (PO4) 2

$20 \mathrm{CaC} 204$

$18 \mathrm{CaCO} 3$

$64 \mathrm{CaçO} 3$ (c14)

78 CaF́2

$41 \mathrm{CaO}$

19 CasO4

29. Carbon

$26 \mathrm{Co}(\mathrm{COOH}) 2$

$168 \cdot \mathrm{Co}(\mathrm{NO} 3) 2$

$59 \mathrm{CoO}$

$98 \mathrm{Cr} 203$

$42 \mathrm{Cs} 2 \mathrm{O}$

$53 \mathrm{CsCOOH}$

$96 \mathrm{CsCl}$

69 CsNO3

$151 \mathrm{CsTPB}$

WSRC-TR-95-0019 (Revision
610

IPPT SN to 611

Mixr Ed Tk

Recycled

612

613

Feed Tank

i ZERO

ZERO ZERO

ZERO ZERO

ZERO ZERO

ZERO

ZERO

ZERO

ZERO

ZERO

ZERO

$0.3344 \mathrm{E}+0.1$

ZERO

$0.8805 \mathrm{E}-05$

$0.5497 \mathrm{E}-01$

$0.3571 \mathrm{E}-02$

ZERO
$0.1757 \mathrm{E}-02$

ZERO
0.8794 E-O1

ZERO

$0.1295 \mathrm{E}-07$

ZERO

$0.2780 \mathrm{E}-02$

ZERO

$0.1357 E-06$

ZERO

ZERO
$0.3549 \mathrm{E}-01$

$0.3660 \mathrm{E}-02$

$0.2178 \mathrm{E}+00$

ZERO
$0.4990 \mathrm{E}+00$

ZERO

ZERO

ZERO

ZERO

ZERO

ZERO

$0.1392 \mathrm{E}-01$

EROO

ZERO

ZERO

ZERO

$0.1657 \mathrm{E}-05$

ZERO

0)

$\begin{array}{lll}\text { ZERO } & \because & \text { ZERO } \\ \text { ZERO } & \text { ZERO } \\ \text { ZERO } & \text { ZERO } \\ \text { ZERO } & \text { ZERO } \\ \text { ZERO } & \text { ZERO } \\ \text { ZERO } & \text { ZERO } \\ \text { ZERO } & & \text { ZERO }\end{array}$

ZERO ZERO ZERO

ZERO

ZERO

ZERO

ZERO

ZERO

ZERO

ZERO

ZERO

ZERO

ZERO

ZERO

ZERO

ZERO

ZERO

ZERO

ZERO

ZERO

ZERO

ZERO

ZERO

ZERO

ZERO

ZERO

ZERO

ZERO

ZERO

ZERO

ZERO
ZERO
ZERO

ZERO

ZERO

ZERO

ZERO

ZERO

ZERO

ZERO

ZERO

ZERO

ZERO

ZERO

ZERO

ZERO

ZERO

ZERO

ZERO

ZERO

ZERO

ZERO

ZERO

ZTRO

ZERO

ZERO

ZERO

EERO

ZERO

ZERO

ZERO

ZERO

ZERO

ZERO

ZERO

ZERO

ZERO

ZERO

ZERO

ZERO

ZERO

ZERO

ZERO

ZERO

ZERO

ZERO

ZERO

ZERO

Date: January 1995
Feed Tank

614

Aqueous Fd

615

Cement

Pre-mix

616
ZERO
$0.4740 \mathrm{E}-14$

ZERO

9568E-08

$0.5475 \mathrm{E}-02$

$.2882 \mathrm{E}-03$
ZERO

$0.5028 \mathrm{E}-11$

$0.2516 \mathrm{E}-09$

$0.3705 \mathrm{E}-16$

ZERO

ZERO

ZERO

$0.1016 \mathrm{E}-09$

$0.1047 \mathrm{E}-10$

$6233 \mathrm{E}-09$
ZERO

ZERO

ZERO

ZERO

ZERO

ZERO

ZERO

ZERO

ZERO

ZERO

ZERO
0.1657 E-0

ZERO

Section 2, Page
Purge

ZERO

ZERO

ZERO

ZERO

ZERO

ZERO

ZERO

ZERO

ZERO

ZERO

ZERO

ZERO

ERO

ERO

ZERO

ZERO

ZERO

ZERO

ZERO

ZERO

ZERO

ZERO

ZERO

ZERO

ERO

ZERO

ZERO

ZERO

ZERO

ZERO

ZERO

ZERO

ZERO

ZERO

ZERO

ZERO

LRO

ZERO

ZERO

ZERO

ZERO

ERO

ZERO 
Table A-1. HLW Flowsheet Material Balance (Tank 51/ITP Cycle 1)

STREAM NUMBERS (CONT'D) $\rightarrow$ STREAM NAME

$54 \mathrm{Cu}(\mathrm{COOH}) 2$

$170 \mathrm{Cu}(\mathrm{NO} 3) 2$

55 CuO

$80 \mathrm{Fe} 2 \mathrm{O} 3$

101 FeO

102 Group A

103 Grout

158 H2C2O4

$\begin{array}{rl}158 & \mathrm{H} 2 \mathrm{C} 2 \mathrm{O} 4 \\ 93 & \mathrm{HSO} 4\end{array}$

$90 \mathrm{H3BO}$.

$34 \mathrm{HCOOH}$
$43 \mathrm{HCl}$

$\begin{array}{ll}43 & \mathrm{HCl} \\ 85 & \mathrm{HF}\end{array}$

$85 \mathrm{HF}$

$36 \mathrm{Hg}$

$129 \mathrm{Hg}(\mathrm{C} 6 \mathrm{H} 5) 2$

$135 \mathrm{Hg}(\mathrm{NO} 3) 2$

$144 \mathrm{Hg} 2 \mathrm{Cl} 2$

$117 \mathrm{Hg} 2 \mathrm{I} 2$

$146 \mathrm{HgCl}$

$21 \mathrm{HgO}$
$97 \mathrm{I} 2$

$183 \mathrm{~K} 2 \mathrm{CO} 3$

$39 \mathrm{~K} 2 \mathrm{O}$

$51 \mathrm{KCOOH}$

157 KMnO4

161 KNO3

150 KTPB

$105 \mathrm{La} 203$

108 Li2O

$65 \mathrm{Mg}(\mathrm{COOH}) 2$

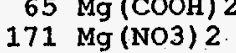

$33 \mathrm{MgO}$

$60 \mathrm{Mn}(\mathrm{COOH}) 2$

$172 \mathrm{Mn}$ (NO3)

$180 \mathrm{Mn} 30$

56 Mno

$14 \mathrm{MnO} 2$

81 N2

74 N2O

$182 \mathrm{NH} 3$

114 NH3OHNO3

52 NH4 COOH

173 NH4NO3

$68 \mathrm{NH} 4 \mathrm{OH}$

148 NH4 TPB

WSRC-TR-95-0019. (Revision
Section 2. Saltstone Production

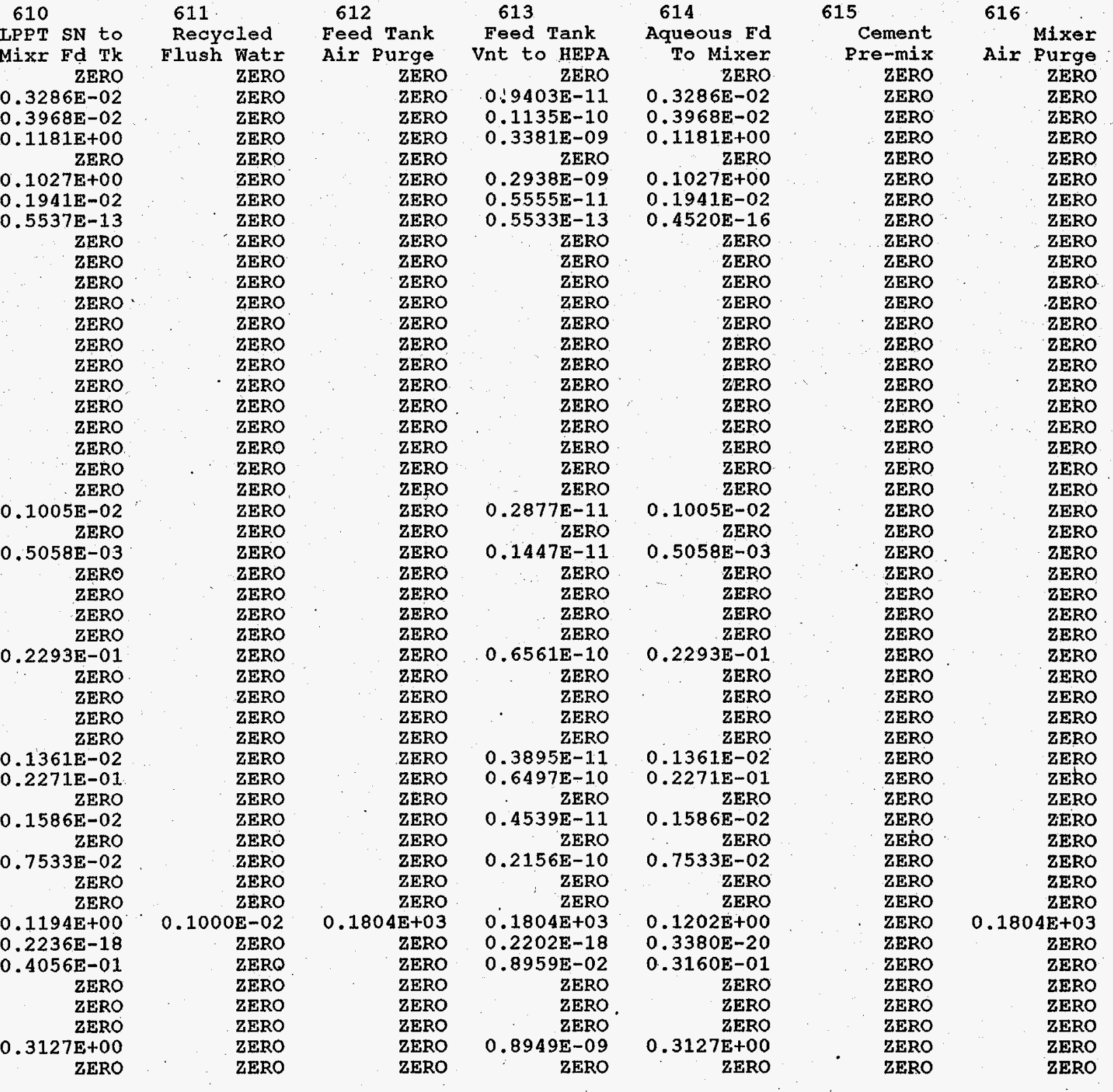

Date: January 1995 
Table A-1. HLW Flowsheet Material Balance (Tank 51/ITP Cycle 1) Section 2 . Saltstone Production

STREAM NUMBERS (CONT'D) $\rightarrow$ STREAM NAME

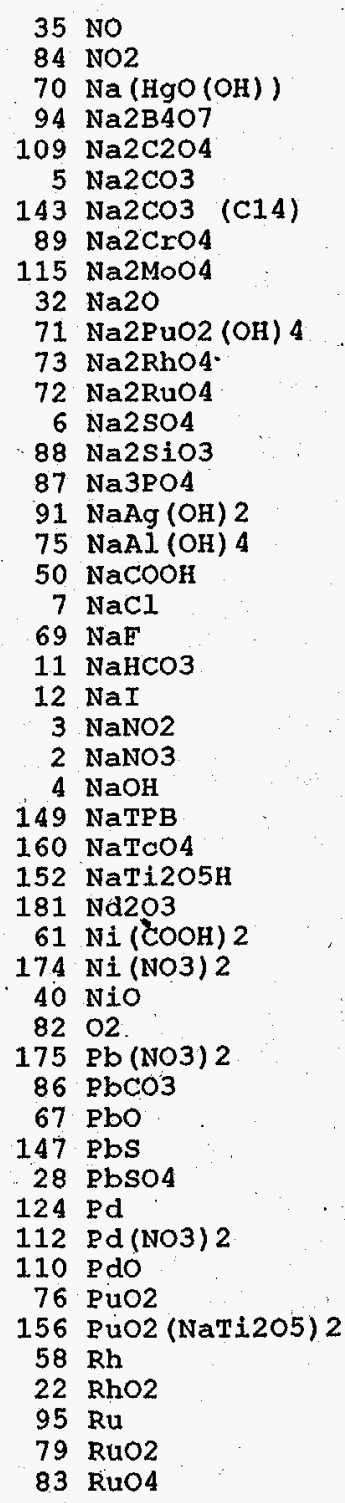

610

LPP'T SN to Mixr Fd Tk

ZERO

$0.2822 \mathrm{E}-03$

$0.3253 \mathrm{E}+00$

$0.4668 \mathrm{E}+01$

$0.4974 \mathrm{E}+03$

$0.2700 \mathrm{E}+01$

$0.8046 \mathrm{E}+00$ ZERO

$0.1813 E-05$

$0.1952 \mathrm{E}-01$

$0.1028 \mathrm{E}+00$
$0.2817 \mathrm{E}+02$

$0.7803 \mathrm{E}+00$

$0.3724 \mathrm{E}+02$

$0.7463 \mathrm{E}-05$

$0.1402 \mathrm{E}+03$

$0.2367 \mathrm{E}+01$

$0.2200 \mathrm{E}+01$

ZERO

$0.1444 \mathrm{E}-02$

$0.2723 \mathrm{E}+03$
$0.1397 \mathrm{E}+04$

$0.6486 \mathrm{E}+03$

$0.7767 \mathrm{E}+01$
$0.6477 \mathrm{E}-01$

$0.6477 \mathrm{E}-01$

ZERO

ZERO

$0.1732 \mathrm{E}-01$

$0.3657 \mathrm{E}-02$

$0.4207 \mathrm{E}-01$

ZERO

$0.1762 E-02$ ZERO

ZERO

$0.1736 \mathrm{E}-03$

ZERO

ZERO

ERO

TERO

ZERO

ZERO

ZERO

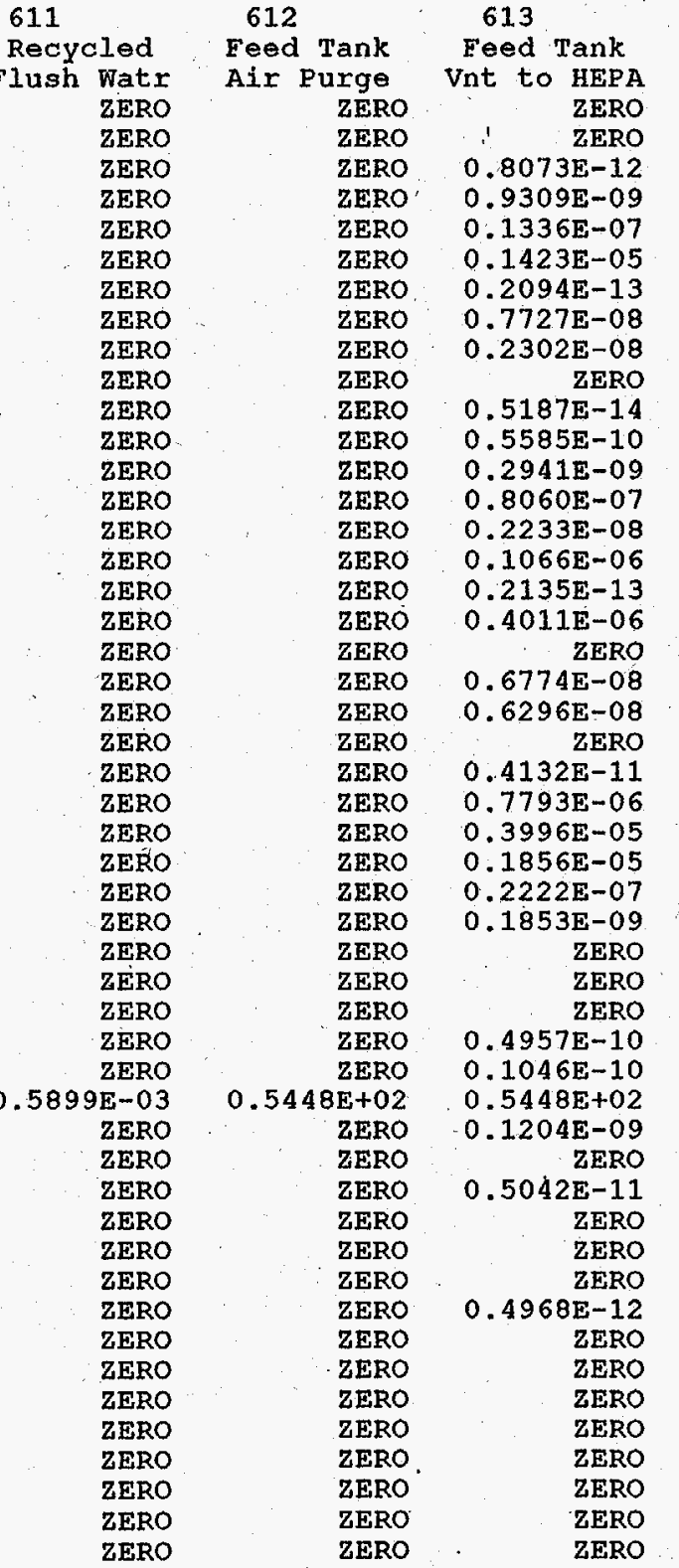

Date: January 1995
$614 \quad 615$

516

ZERO Alr Purge

ZERO ZERO

$\begin{array}{ll}\text { ZERO } & \text { ZERO } \\ \text { ZERO } & \text { ZERO }\end{array}$

ZERO ZERO

ZERO ZERO

ZERO ZERO

ZERO ZERO

ZERO

ZERO ZERO

ZERO ZERO

$\begin{array}{ll}\text { ZERO } & \text { ZERO } \\ \text { ZERO } & \text { ZERO }\end{array}$

ZERO ZERO

ZERO ZERO

ZERO ZERO

ZERO ZERO

ZERO ZERO

ZERO ZERO

ZERO ZERO

ZERO

ZERO ZERO

ZERO ZERO

ZERO ZERO

ZERO ZERO

ZERO ZERO

ERRO

ERO

$0.1732 \mathrm{E}-01$

$0.3657 \mathrm{E}-02$

$0.7037 \mathrm{E}-01$

$0.4207 \mathrm{E}-01$

ZERO

$0.1762 \mathrm{E}-02$

ZERO

ZERO

$0.1736 \mathrm{E}-03$

ZERO

ZERO

ZERO
ZERO

ZERO

ZERO

ZERO

ZERO

ZERO

ZERO

ZERO

ZERO

ZERO

ZERO

ZERO

ZERO

ZERO

ZERO

ZERO

ZERO

ZERO

ZERO

ZERO

ZERO
$0.5448 \mathrm{E}+02$

ZERO

ZERO

ZERO

ZERO

ZERO

ZERO

ZERO

ZERO

ZERO

ZERO

ZERO

ZERO

ZERO

ZERO 
Table A-1. HLW Flowsheet Material Balance (Tank 51/ITP Cycle 1 )

STREAM NUMBERS (CONT'D) $\rightarrow$ STREAM NAME

$38 \mathrm{SO} 2$

$141 \mathrm{SO}$

137 Semi Vol Cs2O

136 Semi Vol CsCl

126 Semi Vol Group A

140 Semi Vol Na2B4O7

138 Semi Vol NaCl

139 Semi Vol NaF

125 Semi Vol NaI

23. Semi Vol RuO2

111 sio

$48 \mathrm{Sr}(\mathrm{COOH}) 2$

$176 \operatorname{Sr}(\mathrm{NO} 3) 2$

$153 \mathrm{Sx}$ (NaTi205) 2

$127 \mathrm{Sr}(\mathrm{OH}) 2$

$30 \quad \operatorname{SrCO} 3$

46 sro

159. $\mathrm{TcO} 2$

92 ThO2

119 Tritium

$\begin{array}{rl}119 & \text { Tritium } \\ 17 & \mathrm{U} 308\end{array}$

$62 \mathrm{VO} 2(\mathrm{COOH}) 2$

$177 \mathrm{UO2}$ (NO3)

$16 \mathrm{UO} 2(\mathrm{OH}) 2$
$27 \mathrm{Y}(\mathrm{COOH}) 3$

178 Y (NO3) 3

$128 \mathrm{Y}(\mathrm{OH}) 3$

$31 \mathrm{Y} 2(\mathrm{CO} 3) 3$

$47 \mathrm{Y} 203$

100 zeolite

$44 \mathrm{Zn}$ (COOH) 2

$179 \mathrm{Zn}(\mathrm{NO} 3) 2$

$\begin{array}{rl}57 & \mathrm{ZnO} \\ 104 & \mathrm{ZrO} 2\end{array}$

13 hydrate $\mathrm{H} 2 \mathrm{O}$

1 water

TOTAL FLOW, LB/HR

TEMPERATURE， DEG C

PRESSURE， ATM

PRESSURE; PSIG

PRESSURE，MM HG

ENTHALPY, PCU/HR

VAPOR FIOW, CFM

LIOUID FLOW, GPM

DENSITY, LBS/ET3

PHASE

610

I.PPT SN to

Mixr Fd

611

Section 2. Saltstone Production

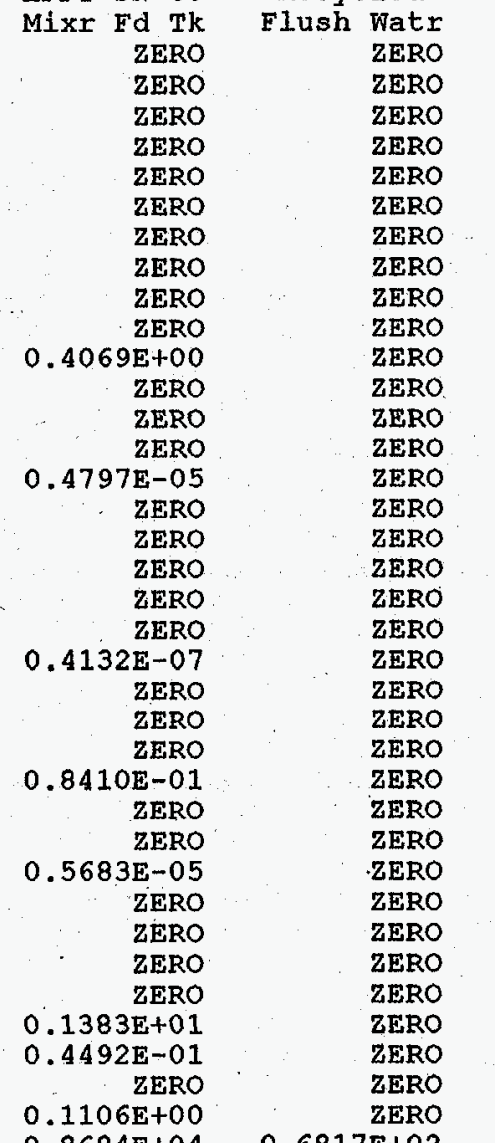

$0.8684 \mathrm{E}+04$

$0.1173 \mathrm{E}+05$

$0.3992 \mathrm{E}+02$

$0.9656 \mathrm{E}+00$

$-0.5055 \mathrm{E}+00$

$0.7339 E+03$

$0.3793 E+06$

$0.6818 \mathrm{E}+02$

$0.9656 \mathrm{E}+00$

$-0.5055 \mathrm{E}+00$

$0.7339 \mathrm{E}+03$

$0.1959 \mathrm{E}+02$

$0.7467 \mathrm{E}+02$

$0.1362 \mathrm{E}+00$

$0.6243 \mathrm{E}+02$

LIQUID

612

Feed Tank

613

ZERO

ZERO
ZERO $: \quad$ ZERO

ZERO ZERO

ZERO

ZERO

ZERO

ZERO

ZERO

ZERO

ZERO

ZERO

ZERO

ZERO

ZERO

ZERO

ZERO

ZERO

ZERO

ZERO

ZERO

ZERO

ZERO

ZERO

ZERO

ZERO

ZERO

ZERO

ZERO

ZERO

ZERO

ZERO

$0.3945 \mathrm{E}+01$

$0.2388 \mathrm{E}+03$

$0.3500 \mathrm{E}+02$

$0.1000 \mathrm{E}+01$

ZERO

$0.5008 \mathrm{E}+04$

ZERO

ZERO

ZERO

ZERO

ZERO

ERO

$0.1164 \mathrm{E}-08$

ZERO

ZERO

$0.1373 \mathrm{E}-13$

ZERO

ZERO

ZERO

ZERO

$0.1182 \mathrm{E}-15$

ZERO

ZERO

ZERO
0.940 .

$6 E-09$
ZERO

ZERO

$0.1626 \mathrm{E}-13$

ZERO

ZERO

ZERO

$0.3956 \mathrm{E}-08$

$0.1285 \mathrm{E}-09$

ZERO

$.3163 E-09$

$0.1164 \mathrm{E}+02$

$0.4000 \mathrm{E}+02$

$0.9656 \mathrm{E}+00$

$0.5055 \mathrm{E}+00$
$0.7339 \mathrm{E}+03$

$0.9999 \mathrm{E}+04$

$0.6240 \mathrm{E}+02$

$0.7055 \mathrm{E}-01$

$0.6583 E-01$

VAPOR
614

queous $\mathrm{Fd}$

To Mixer

ZERO

ZERO

ZERO

ZERO

ZERO

ZERO

ZERO

ZERO

$0.4069 \mathrm{E}+00$

ZERO

ZERO

$0.4797 \mathrm{E}-05$

ZERO

ZERO

ZERO

ZERO

$0.4132 \mathrm{E}-07$

7RRO

ZERO

$0.8410 \mathrm{E}-01$

$.8410 \mathrm{E}-01$

ZERO

0.5683 E-05

ZERO

ZERO

ZERO

$0.1383 \mathrm{E}+01$

$0.4492 \mathrm{E}-01$

ZERO

$0.1179 \mathrm{E}+05$

$0.3999 \mathrm{E}+02$

$0.9656 \mathrm{E}+00$

$-0.5055 \mathrm{E}+00$

$0.7339 \mathrm{E}+03$

$0.1971 \mathrm{E}+02$

$0.7459 \mathrm{E}+02$

IIQUID
615

Cement

re-mix
ZERO

ZERO
ZERO

ZERO

ZERO

ZERO

ZERO

ZERO

ZERO

ZERO

ZERO

ZERO

ZERO

ZERO

ZERO

ZERO

ZERO

ZERO

ZERO

ZERO

ZERO

TERO

ZERO

TERO

ZTRO

ZERO

ZERO

ZERO

ZERO

ZERO

ZERO

ZERO

ZERO

ZERO

ZERO

ZERO

IMRO

$0.1769 \mathrm{E}+05$

$0.2500 \mathrm{E}+02$

$0.1000 \mathrm{E}+01$

ZERO

$0.8227 \mathrm{E}+05$

$0.5008 \mathrm{E}+04$

$0.1622 \mathrm{E}+03$

SOLID

$0.7055 \mathrm{E}-01$

VAPOR 
STREAM NUMBERS $\rightarrow$
STREAM NAME

COMPONENT FLOWS, LB/HR

122 (C6H5) 2

123 (C6H5) $2 \mathrm{C} 6 \mathrm{H} 4$

118 (C6H5) 2NH

133 (C6H5B (OH) 2 salt)

132 (C6H5B (OH) 2)

166 (C6H5C6H4OH)

162 (C6H5NH2)

163 (C6H5NHCHO)

165 (C6H5NO)
164 (C6H5NO2)

131 (C6H5OH salt)

130 (C6H5OH)

121 (С6H6)

155 (CH3) $2 \mathrm{CHOH}$

154 (CH3OH)

$45 \mathrm{Ag}$

116 Ag20

134 AgNO3

$\begin{array}{r}77 \mathrm{Al} 203 \\ \hline\end{array}$

$106 \mathrm{B2O} 3$

$9 \mathrm{Ba}(\mathrm{OH}) 2$

$15 \mathrm{BaO}$

$24 \mathrm{BaSO} 4$

$\begin{array}{ll}66 & \mathrm{CO} \\ 37 & \mathrm{CO} 2\end{array}$

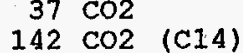

$63 \mathrm{Ca}(\mathrm{COOH}) 2$

$167 \mathrm{Ca}(\mathrm{NO} 3)^{2}$

$120 \mathrm{Ca}(\mathrm{OH}) 2$

$113 \mathrm{Ca} 3(\mathrm{PO} 4) 2$

$20 \mathrm{CaC} 2 \mathrm{O} 4$

$18 \mathrm{CaCO} 3$

$64 \cdot \mathrm{CaCO}$ (C14)

78 CaE2

$41 \mathrm{CaO}$

19. CasO4

29 Carbon

26 Coment

168 Co (NO3) 2 .

$59 \mathrm{CoO}$

98. $\mathrm{Cr} 2 \mathrm{O3}$

$53 \mathrm{CsCOOH}$

$96 \mathrm{CsCl}$

169 CsNO3

$8 \mathrm{CsOH}$

151 CsTPB

WSRC-TR-95-0019 (Revision
Table A-1. HLW Flowsheet Material Balance (Tank 51/ITP Cycle 1)

Table A-1. HLW Flowsheet Material Balance (Tank
Section 2. Saltstone Production
617

to HEPA.

618

Saltstone

to Trench

ZERO

ZERO

ZERO

ZERO

ZERO

ZERO

ZERO

ZERO
$0.5665 \mathrm{E}-08$

ZERO

$0.7220 \mathrm{E}-06$

$0.4934 \mathrm{E}-02$.

$0.2652 \mathrm{E}-03$

$0.2977 \mathrm{E}-11$

ZERO
ZER

ZERO

$0.2194 \mathrm{E}-16$

ZERO
$0.4710 \mathrm{E}-11$

$0.3167 \mathrm{E}-08$

ZERO

$0.6013 \mathrm{E}-10$

$0.6201 \mathrm{E}-11$

$0.3691 \mathrm{E}-09$

$0.8455 \mathrm{E}-09$

ZERO

ZERO

ZERO

ZERO

$0.2997 \mathrm{E}-04$

ZERO
EE-10

$0.2359 \mathrm{E}-10$

ZERO

ZERO

ZERO

ZERO

$0.2807 E-14$

ZERO
ZERO ZERO

$0.4710 \mathrm{E}-11$
ZERO
619 Saltstone Vapor Relese

625

Mixer Feed
Line Flush

626

627

Flush H2O

to HId Tnk
Hardened saltstone

Flush H2O
to sltston

\begin{tabular}{lll} 
ZERO & ZERO & ZERO \\
ZERO & ZERO & ZERO \\
ZERO & ZERO & ZERO \\
ZERO & ZERO & ZERO \\
ZERO & ZERO & ZERO \\
ZERO & ZERO & ZERO \\
ZERO & ZERO & ZERO \\
ZERO & ZERO & ZERO \\
ZERO & ZERO & ZERO \\
ZERO & ZERO & ZERO \\
ZERO & ZERO & ZERO \\
ZERO & ZERO & ZERO \\
ZERO & ZERO & ZERO \\
ZERO & ZERO & ZERO \\
ZERO & ZERO & ZERO \\
ZERO & ZERO & ZERO \\
ZERO & ZERO & ZERO \\
ZERO & ZERO & ZERO \\
ZERO & ZERO & ZERO \\
ZERO & ZERO & ZERO \\
ZERO & ZERO & ZERO \\
ZERO & ZERO & ZERO \\
ZERO & ZERO & ZERO \\
ZERO & ZERO & ZERO \\
ZERO & ZERO & ZERO \\
ZERO & ZERO & ZERO \\
ZERO & ZERO & ZERO \\
ZERO & ZERO & ZERO \\
ZERO & ZERO & ZERO \\
ZERO & ZERO & ZERO \\
ZERO & ZERO & ZERO \\
ZERO & ZERO & ZERO \\
ZERO & ZERO & ZERO \\
ZERO & ZERO & ZERO \\
ZERO & ZERO & ZERO \\
ZERO & ZERO & ZERO \\
ZERO & ZERO & ZERO \\
ZERO & ZERO & ZERO \\
ZERO & ZERO & ZERO \\
ZERO & ZERO & ZERO \\
ZERO & ZERO & ZERO \\
ZERO & ZERO & ZERO \\
ZERO & ZERO & ZERO \\
ZERO & ZERO & ZERO \\
ZERO & ZERO & ZERO \\
ZERO & ZERO & ZERO \\
ZERO & ZERO & ZERO \\
ZERO & ZERO & ZERO \\
\hline
\end{tabular}

$\begin{array}{r}\text { ZERO } \\ \text { ZERO } \\ \text { ZERO } \\ \text { ZERO } \\ \text { ZERO } \\ \text { ZERO } \\ \text { ZERO } \\ \text { ZERO } \\ \text { ZERO } \\ \text { ZERO } \\ \text { ZERO } \\ \text { ZERO } \\ \text { Z14 } 3 \text { E-07 } \\ \text { ZERO } \\ \text { ZERO } \\ \text { ZERO } \\ \text { ZERO } \\ \text { ZERO } \\ \text { ZERO } \\ \text { ZERO } \\ \text { ZERO } \\ \text { ZERO } \\ \text { ZERO } \\ \text { ZERO } \\ \text { ZERO } \\ \text { ZERO } \\ \text { ZERO } \\ \text { ZERO } \\ \text { ZERO } \\ \text { ZERO } \\ \text { ZERO } \\ \text { ZERO } \\ \text { ZERO } \\ \text { ZERO } \\ \text { ZERO } \\ \text { ZERO } \\ \text { ZERO } \\ \text { ZERO } \\ \text { ZERO } \\ \text { ZERO } \\ \text { ZERO } \\ \text { ZERO } \\ \text { ZERO } \\ \text { ZERO } \\ \text { ZERO } \\ \text { ZERO } \\ \text { ZERO } \\ \text { ZERO } \\ \text { ZERO } \\ \text { ZER } \\ \hline\end{array}$

Date: January 1995
Section 2, Page 10 of 17 
Table A-1. HLW Flowsheet Material Balance (Tank 51/ITP Cyale 1) Section 2. Saltstone Production

\begin{tabular}{|c|c|c|c|c|c|c|c|c|}
\hline $\begin{array}{l}\text { STR } \\
\text { STR }\end{array}$ & $\begin{array}{l}\text { EAM NUMBERS } \\
\text { EAM NAME }\end{array}$ & (CONT'D) & $\rightarrow$ & $\begin{array}{l}617 \\
\text { Mixer Vent }\end{array}$ & $\begin{array}{l}618 \\
\text { Saltstone }\end{array}$ & $\begin{array}{l}619 \\
\text { Hardened }\end{array}$ & $\begin{array}{l}620 \\
\text { Sal }\end{array}$ & Ltstone \\
\hline & & & & to HEPA & to Trench & saltstone & Vapor & Relese \\
\hline 54 & $\mathrm{Cu}(\mathrm{COOH}) 2$ & & & ZERO & ZERO & ZERO & & ZERO \\
\hline 170 & $\mathrm{Cu}(\mathrm{NO} 3) 2$ & & & $0.5568 \mathrm{E}-11$ & $0.3286 \mathrm{E}-02$ & $0.3286 \mathrm{E}-02$ & ! & ZERO \\
\hline 55 & $\mathrm{CuO}$ & & & $0.6723 \mathrm{E}-11$ & $0.3968 \mathrm{E}-02$ & $0.3968 \mathrm{E}-02$ & & ZERO \\
\hline 80 & $\mathrm{Fe} 203$ & & & $0.2002 \mathrm{E}-0.9$ & $0.1181 \mathrm{E}+00$ & $0.1181 E+00$ & & ZERO \\
\hline 101 & FeO & & & ZERO & ZERO & ZERO & & ZERO \\
\hline 102 & Group A & & & $0.1739 \mathrm{E}-09$ & $0.1027 \mathrm{E}+00$ & $0.1027 E+00$ & & ZERO \\
\hline 103 & Group B & & & $0.3289 \mathrm{E}-11$ & $0.1941 \mathrm{E}-02$ & $0.1941 E-02$ & & ZERO \\
\hline 25 & $\mathrm{H} 2$ & & & $0.4516 \mathrm{E}-16$ & $0.3686 \mathrm{E}-19$ & $0.3686 \mathrm{E}-19$ & & ZERO \\
\hline 158 & $\mathrm{H} 2 \mathrm{C} 204$ & & & ZERO & ZERO & ZERO & & ZERO \\
\hline 93 & $\mathrm{H} 2 \mathrm{SO} 4$ & & & ZERO & ZERO & ZERO & & ZERO \\
\hline 90 & Н3BO3. & & & ZERO & ZERO & ZERO & & ZERO \\
\hline 34 & $\mathrm{HCOOH}$ & & & ZERO & ZERO & ZERO & & ZERO \\
\hline 43 & HCl & & & ZERO & ZERO & ZERO & & ZERO \\
\hline 85 & $\mathrm{HF}$ & & & ZERO & ZERO & ZERO & & ZERO \\
\hline 49 & HNO3 & & & ZERO & ZERO & ZERO & & ZERO \\
\hline 36 & $\mathrm{Hg}$ & & & ZERO & ZERO & ZERO & & ZERO \\
\hline 129 & $\mathrm{Hg}(\mathrm{C} 6 \mathrm{H} 5) 2$ & & & ZERO & ZERO & ZERO & & ZERO \\
\hline 135 & $\mathrm{Hg}(\mathrm{NO} 3) 2$ & & & ZERO & ZERO & ZERO & & ZERO \\
\hline 144 & $\mathrm{Hg} 2 \mathrm{Cl} 2$ & & & ZERO & ZERO & ZERO & & ZERO \\
\hline 117 & $\mathrm{Hg} 2 \mathrm{I} 2$ & & & ZERO & ZERO & ZERO & & ZERO \\
\hline 146 & $\mathrm{HgCl2}$ & & & ZERO & ZERO & ZERO & & ZERO \\
\hline 21 & $\mathrm{HgO}$ & & & $0.1704 \mathrm{E}-11$ & $0.1005 \mathrm{E}-02$ & $0.1005 \mathrm{E}-02$ & & ZERO \\
\hline 97 & I2 & & & ZERO & ZERO & ZERO & & ZERO \\
\hline 183 & $\mathrm{~K} 2 \mathrm{CO} 3$ & & & $0.8570 \mathrm{E}-1.2$ & $0.5058 \mathrm{E}-03$ & $0.5058 \mathrm{E}-03$ & & ZERO \\
\hline 39 & $\mathrm{~K} 20$ & & & ZERO & ZERO & ZERO & & ZERO \\
\hline 51 & $\mathrm{KCOOH}$ & & & ZERO & ZERO & ZERO & & ZERO \\
\hline 157 & $\mathrm{KMnO4}$ & & & ZERO & ZERO & ZERO & & ZERO \\
\hline 161 & $\mathrm{KNO} 3$ & & & ZERO & ZERO & ZERO & & ZERO \\
\hline 10 & $\mathrm{KOH}$ & & & $0.3885 \mathrm{E}-10$ & $0.2293 \mathrm{E}-01$ & $0.2293 \mathrm{E}-01$ & & ZERO \\
\hline 150 & KTPB & & & ZERO & ZERO & ZERO & & ZERO \\
\hline 105 & $\mathrm{La2O3}$ & & & ZERO & ZERO & ZERO & & ZERO \\
\hline 108 & Li20 & & & ZERO & ZERO & ZERO & & ZERO \\
\hline 65 & $\mathrm{Mg}(\mathrm{COOH}) 2$ & & & ZERO & ZERO & ZERO & & ZERO \\
\hline 171 & $\mathrm{Mg}(\mathrm{NO} 3) 2$ & & & $0.2307 \mathrm{E}-11$ & $0.1361 \mathrm{E}-02$ & $0.1361 \mathrm{E}-02$ & & ZERO \\
\hline 33 & $\mathrm{MgO}$ & & & $0.3847 \mathrm{E}-10$ & $0.2271 \mathrm{E}-01$ & $0.2271 \mathrm{E}-01$ & & ZERO \\
\hline 60 & $\operatorname{Mn}(\mathrm{COOH}) 2$ & & & ZERO & ZERO & ZERO & & ZERO \\
\hline 172 & $\mathrm{Mn}(\mathrm{NO} 3) 2$ & & & $0.2688 \mathrm{E}-11$ & $0.1586 \mathrm{E}-02$ & $0.1586 \mathrm{E}-02$ & & ZERO \\
\hline 180 & Mn 304 & & & ZERO & ZERO & ZERO & & ZERO \\
\hline 56 & Mno & & & $0.1276 \mathrm{E}-10$ & $0.7533 \mathrm{E}-02$ & $0.7533 \mathrm{E}-02$ & & ZERO \\
\hline 14 & $\mathrm{MnO} 2$ & & & ZERO & ZERO & ZERO & & ZERO \\
\hline 145 & $\mathrm{MoO} 2$ & & & ZERO & ZERO & ZERO & & ZERO \\
\hline 81 & $\mathrm{~N} 2$ & & & $0.1804 \mathrm{E}+03$ & $0.1201 \mathrm{E}+00$ & $0.1201 \mathrm{E}+00$ & & ZERO \\
\hline 74 & $\mathrm{~N} 2 \mathrm{O}$ & & & $0.3329 \mathrm{E}-20$ & $0.5104 \mathrm{E}-22$ & $0.5104 \mathrm{E}-22$ & & ZERO \\
\hline 182 & NH3 & & & $0.6985 \mathrm{E}-02$ & $0.2462 \mathrm{E}-01$ & ZERO & 0.24 & $462 \mathrm{E}-01$ \\
\hline 114 & NH 3 OHNO 3 & & & ZERO & . ZERO & ZERO & & ZERO \\
\hline 52 & $\mathrm{NH} 4 \mathrm{COOH}$ & & & ZERO & ZERO & ZERO & & ZERO \\
\hline 173 & NH 4 NO 3 & & & ZERO & ZERO & ZERO & & ZERO \\
\hline 68 & $\mathrm{NH} 4 \mathrm{OH}$ & & & $0.5299 \mathrm{E}-09$ & $0.3127 \mathrm{E}+00$ & $0.3127 \mathrm{E}+00$ & & ZERO \\
\hline 148 & NH4 TPB & & & ZERO & ZERO & ZERO & . & ZERO \\
\hline
\end{tabular}

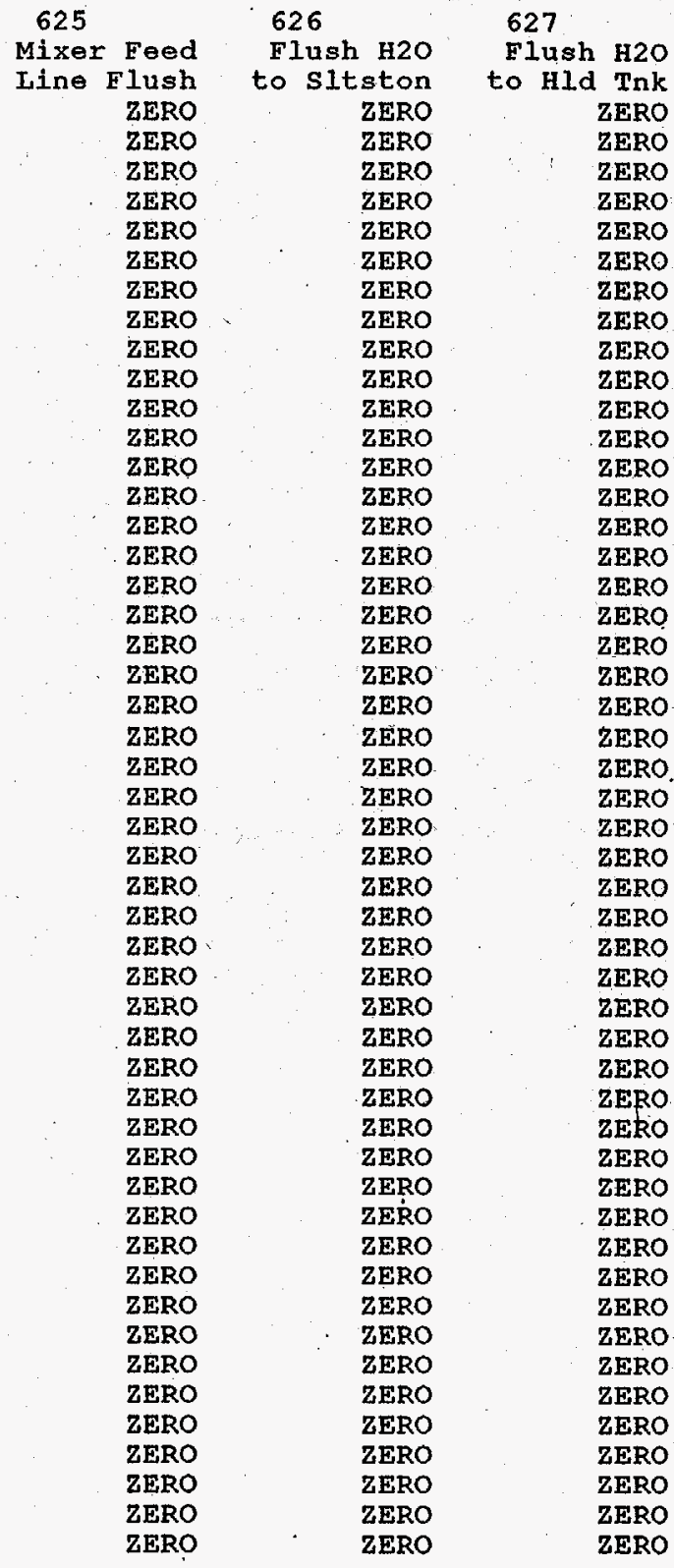


Table A-1. HLW Flowsheet Material Balance (Tank 51/ITP Cycle 1) Section 2. Saltstone Production

STREAM NUMBERS (CONT'D) $\rightarrow$ STREAM NAME

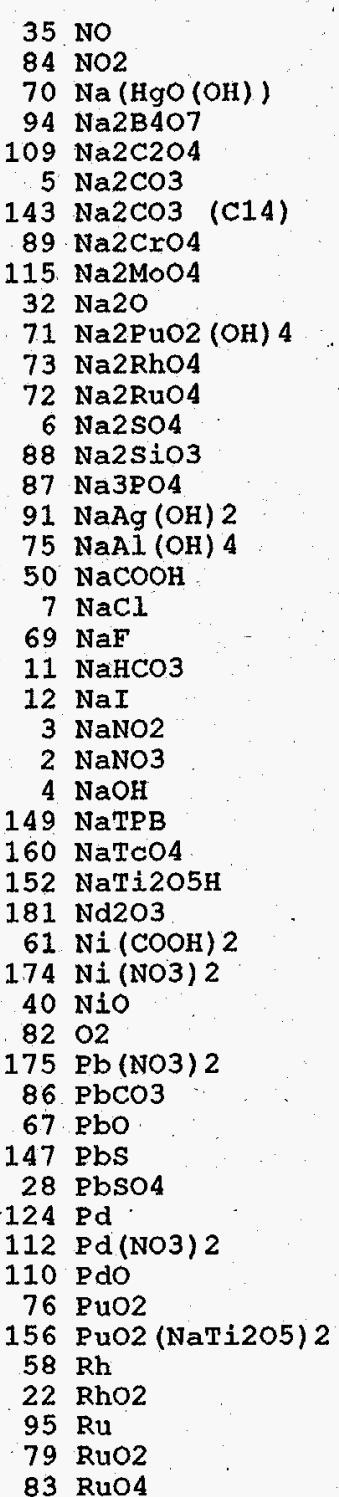

617

Mixex Vent

to HEPA ZERO

$0.4780 \mathrm{E}-12$ $0.5512 \mathrm{E}-09$ $0.7909 \mathrm{E}-08$ $0.8428 \mathrm{E}-06$ $0.1240 \mathrm{E}-13$ $0.4575 \mathrm{E}-08$ $0.1363 \mathrm{E}-08$ ZERO $0.3071 \mathrm{E}-1$ $.3307 \mathrm{E}-10$ $0.1741 \mathrm{E}-09$ $0.4772 \mathrm{E}-07$ $0.1322 \mathrm{E}-08$ $0.6309 \mathrm{E}-07$ $0.1264 \mathrm{E}-13$ $0.2375 \mathrm{E}-06$ $0.4011 \mathrm{E}-08$ $0.3728 E-08$ ZERO $0.2447 \mathrm{E}-11$ $0.4614 \mathrm{E}-0$ $0.2366 \mathrm{E}-05$ $0.1099 \mathrm{E}-05$ $0.1316 \mathrm{E}-07$ $0.1097 \mathrm{E}-09$ ZERO ZERO

$0.2935 \mathrm{E}-10$ $0.6196 \mathrm{E}-11$ $0.5448 \mathrm{E}+02$ $0.7127 E-10$ ZERO $0.2986 \mathrm{E}-11$
ZERO ZERO ZERO

$0.2942 \mathrm{E}-12$ ZERO ZERO ZERO ZERO ZERO ZERO ZERO

\begin{tabular}{|c|c|c|c|}
\hline $\begin{array}{l}618 \\
\text { Saltstone }\end{array}$ & $\begin{array}{l}619 \\
\text { Hardened }\end{array}$ & $\begin{array}{l}620 \\
\mathrm{Sa}\end{array}$ & ltstone \\
\hline $\begin{array}{l}\text { to Trench } \\
\text { ZERO }\end{array}$ & $\begin{array}{r}\text { Saltstone } \\
\text { zERO }\end{array}$ & Vapor & $\begin{array}{r}\text { Relese } \\
\text { ZERO }\end{array}$ \\
\hline ZERO & ZERO & : & ZERO \\
\hline $2822 \mathrm{E}-03$ & $0.2822 \mathrm{E}-03$ & & ZERO \\
\hline $3253 E+00$ & $0.3253 \mathrm{E}+00$ & & ZERO \\
\hline $4668 \mathrm{E}+01$ & $0.4668 \mathrm{E}+01$ & & ZERO \\
\hline $4974 \mathrm{E}+03$ & $0.4974 \mathrm{E}+03$ & & ZERO \\
\hline $0 \mathrm{E}-05$ & $0.7320 \mathrm{E}-05$ & & ZERO \\
\hline $60 \mathrm{E}+01$ & $0.2700 E+01$ & & ZERO \\
\hline $8046 \mathrm{E}+00$ & $0.8046 \mathrm{E}+00$ & & ZERO \\
\hline ZERO & ZERO & & ZERO \\
\hline $1813 E-05$ & $0.1813 E-05$ & & ZERO \\
\hline $1952 \mathrm{E}-01$ & $0.1952 \mathrm{E}-01$ & & ZERO \\
\hline $28 E+00$ & $0.1028 \mathrm{E}+00$ & & ZERO \\
\hline $2817 \mathrm{E}+02$ & $0.2817 \mathrm{E}+02$ & & ZERO \\
\hline $7803 E+00$ & $0.7803 \mathrm{E}+00$ & & ZERO \\
\hline $24 \mathrm{E}+02$ & $0.3724 E+02$ & & ZERO \\
\hline $463 E-05$ & $0.7463 \mathrm{E}-05$ & & ZERO \\
\hline $402 E+03$ & $0.1402 E+03$ & & ZERO \\
\hline ZERO & ZERO & & ZERO \\
\hline $.2367 \mathrm{E}+01$ & $0.2367 \mathrm{E}+01$ & & ZERO \\
\hline $200 E+01$ & $0.2200 \mathrm{E}+01$ & & ZERO \\
\hline ZERO & ZERO & & ZERO \\
\hline $1444 \mathrm{E}-02$ & $0.1444 \mathrm{E}-02$ & & ZERO \\
\hline $723 E+03$ & $0.2723 \mathrm{E}+03$ & & ZERO \\
\hline $397 E+04$ & $0.1397 \mathrm{E}+04$ & & ZERO \\
\hline $6 \mathrm{E}+03$ & $0.6486 \mathrm{E}+03$ & & ZERO \\
\hline $767 E+01$ & $0.7767 E+01$ & & ZERO \\
\hline $477 \mathrm{E}-01$ & $0.6477 \mathrm{E}-01$ & & ZERO \\
\hline ZERO & ZERO & & 'ZERO \\
\hline ZERO & ZERO & & ZERO \\
\hline ZERO & ZERO & & ZERO \\
\hline $732 \mathrm{E}-01$ & $0.1732 \mathrm{E}-01$ & & ZERO \\
\hline $657 \mathrm{E}-02$ & $0.3657 \mathrm{E}-02$ & & ZERO \\
\hline $031 E-01$ & $0.7031 \mathrm{E}-01$ & & ZERO \\
\hline $207 E-01$ & $0.4207 \mathrm{E}-01$ & & ZERO \\
\hline ZERO & ZERO & & ZERO \\
\hline $762 \mathrm{E}-02$ & $0.1762 \mathrm{E}-02$ & & ZERO \\
\hline ZERO & ZERO & & ZERO \\
\hline ZERO & ZERO & & ZERO \\
\hline ZERO & ZERO & & ZERO \\
\hline $1736 \mathrm{E}-03$ & $0.1736 \mathrm{E}-03$ & & ZERO \\
\hline ZERO & ZERO & & ZERO \\
\hline ZERO & ZERO & & ZERO \\
\hline ZERO & ZERO & & ZERO \\
\hline ZERO & ZERO & & ZERO \\
\hline ZERO & ZERO & & ZERO \\
\hline ZERO & ZERO & & ZERO \\
\hline ZERO & ZERO & & ZERO \\
\hline ZERO & ZERO & & -ZERO \\
\hline
\end{tabular}

Date: January 1995
$625 \quad 626$

$626 \quad 627$

Mixer Feed Flush H2O Flush H2O Line Flush to sltston to Hld Tnk. ZERO ZERO ZERO ZERO ZERO ZERO ZERO ZERO Z ZERO ZERO ZERO ZERO ZERO ZERO ZERO ZERO ZERO ZERO ZERO ZERO ZERO ZERO ZERO ZERO ZERO ZERO . ZERO ZERO ZERO - ZERO ZTRO ZRRO $\begin{array}{lll}\text { ZERO ZERO } & \text { ZERO } \\ \text { ZERO } & \text { ZERO }\end{array}$ ZERO ZERO ZERO ZERO ZERO ZERO ZERO ZERO ZERO ZERO ZERO ZERO ZERO ZERO ZERO ZERO ZERO Z ZERO ZERO ZERO ZERO ZERO . ZERO ZERO ZERO ZERO ZERO ZERO ZRRO ZERO ZERO Z ZERO ZERO ZERO ZERO $\begin{array}{lll}\text { ZERO } & \text { ZERO } & \text { ZERO } \\ \text { ZERO } & \text { ZERO } & \text { ZERO }\end{array}$ ZERO ZERO ZERO ZERO ZERO Z ZERO ZERO ZERO ZERO ZERO ZERO ZERO ZERO ZERO ZERO ZERO ZERO Z ZERO ZERO ZERO ZERO ZERO ZERO ZERO ZERO ZERO ZERO $\begin{array}{llll}\text { ZERO } & \text { ZERO } & \text { ZERO }\end{array}$ $\begin{array}{lll}\text { ZERO } & \text { ZERO } & \text { ZERO } \\ \text { ZERO } & \text { ZERO }\end{array}$ ZERO ZERO $\quad$ ZERO ZERO ZERO ZERO ZERO ZERO ZERO ZERO ZERO ZERO ZERO ZERO $\quad$ ZERO ZERO ZERO ZERO ZERO ZERO ZERO ZERO "ZERO $\quad$ ZERO

ZERO ZERO $\quad$ ZERO

ZERO ZERO

Section 2, Page 12 of 17 
Table A-1. HLW Flowsheet Material Balance (Tank 51/TTP Cycle 1)

STREAM NUMBERS (CONT'D) $\rightarrow$ STREAM NAME

$38 \quad 502$

141 SO3

137 Semi Vol Cs2O

136 Semi Vol.CsCl

126 Semi Vol Group A

140 Semi Vol Na2B4O7

138 Semi Vol NaCl

139 Semi Vol NaF

25 Semi Vol NaI

23 Semi Vol RuO2

$111 \mathrm{SiO} 2$

$48 \mathrm{Sr}(\mathrm{COOH}) 2$

$176 \mathrm{Sr}$ (NO3) 2

$153 \mathrm{Sr}$ (NaTi205) 2

$127 \mathrm{Sr}(\mathrm{OH}) 2$

$30 \quad \operatorname{srCO} 3$

$46 \mathrm{SrO}$

$159 \mathrm{TCO} 2$

92 ThO2

119 Tritium

$\begin{array}{rl}119 & \text { Tritiu } \\ 17 & 0308\end{array}$

$62 \mathrm{UO} 2$ ( $\mathrm{COOH}) 2$

177 vO2 (NO3) 2

$16 \mathrm{UO} 2(\mathrm{OH}) 2$

$27 \mathrm{Y}(\mathrm{COOH}) 3$

$178 \mathrm{Y}(\mathrm{NO} 3)$

$31 \mathrm{Y} 2(\mathrm{CO} 3) 3$

$47 \mathrm{Y} 203$

100 zeolite

$44 \mathrm{Zn}(\mathrm{COOH}) 2$

$179 \mathrm{Zn}$ (NO3) 2

$57 \mathrm{Zno}$

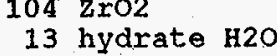

1 water

TOTAL FIOW, LB/HR

TEMPERATURE， DEG C

PRESSURE， ATM

PRESSURE， PSIG

PRESSURE, MM HG

ENTHALPY， PCU/HR

VAPOR FLOW, CEM

I.IQUID FLOW, GPM

DENSITY, LBS/ET3

PHASE

617

to HEPA

ZERO

ZERO

ZERO

ZERO

ZERO

ZERO

ZERO

ZERO

$0.6894 \mathrm{E}-09$

ZERO

ZERO

$0.8127 \mathrm{E}-14$

ZERO

ZERO

ZERO

ZERO

$0.1942 \mathrm{E}-10$

ZERO
ZERO

ZERO

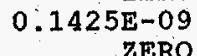

ZERO

$0.9629 \mathrm{E}-14$

ZERO

ZERO

ZERO

$0.2342 \mathrm{E}-08$

$0.7610 \mathrm{E}-10$

ZERO
3E-09

$0.1873 \mathrm{E}-09$
$0.1164 \mathrm{E}+02$

$0.2465 \mathrm{E}+03$

$0.4000 \mathrm{E}+02$

$0.9656 \mathrm{E}+00$

$-0.5055 \mathrm{E}+00$

$0.7339 \mathrm{E}+03$

$0.9998 \mathrm{E}+04$

$0.6583 \mathrm{E}-01$

VAPOR

Section 2. Saltstone Production

\section{8}

Saltstone

to Trench

ZERO

ZERO

ZERO

ZERO

ZERO

ZERO

ZERO

ZERO

0.4069 ERO

ZERO

ZERO

$0.4797 \mathrm{E}-05$

ZERO

ZERO

ZERO

ZERO

$0.4130 \mathrm{E}-07$

ZERO

ZERO

$0.8410 \mathrm{E}-01$

ZERO

$0.5683 \mathrm{E}-05$

ZERO

ZERO

ZERO

$0.1383 \mathrm{E}+01$

$0.4492 \mathrm{E}-01$

ERO

$0.1106 \mathrm{E}+00$

$0.8768 \mathrm{E}+04$
$0.2951 \mathrm{E}+05$

$0.3873 \mathrm{E}+02$

$0.9656 \mathrm{E}+00$

$0.7339 E+03$
$0.4988 E+06$

$0.3335 \mathrm{E}+02$

$0.1103 E+03$

IIQUID
$619 \quad 620$

Hardened Saltstone

Saltstone vapor Relese

ZERO
ZERO $\quad \therefore \quad$ ZERO
ZERO

ZERO ZERO

ZERO ZERO

ZERO

ZERO

ZERO

ZERO

ZERO

$0.4069 \mathrm{E}+00$

ZERO

ZERO

0.4797 E-05

ZERO

ZERO

ZERO

ZERO

$0.4087 \mathrm{E}-07$

ZERO

ZERRO

$0.8410 \mathrm{E}-01$

ZERO

$0.5683 \mathrm{E}-0.5$

ZERO

ZERO

ZERO

$0.1383 \mathrm{E}+01$

$0.4492 \mathrm{E}-01$

ZERO

$0.1106 \mathrm{E}+00$

$0.7234 \mathrm{E}+04$

$0.2798 \mathrm{E}+0.5$

$0.3873 \mathrm{E}+02$

$0.9656 \mathrm{E}+00$

$0.7339 \mathrm{E}+03$

$0.4392 \mathrm{E}+06$

$0.3029 \mathrm{E}+02$

$0.1151 \mathrm{E}+03$

IIQUID
625

Mixer Feed

Line Flush

ZERO

ZERO

ZERO

ZERO

ZERO

ZERO

ZERO

ZERO

ZERO

ZERO

ZERO

ZERO

ZERO

ZERO

ZERO

ZERO

ZERO

ZERO

ZERO

ZERO

ZERO

ZERO

ZERO

ZERO

ZERO

ZERO

ZERO

ZERO

ZERO

ZERO

ZERO

ZERO

$0.1534 \mathrm{E}+04$

(a)

$0.9656 \mathrm{E}+00$

$.5055 \mathrm{E}+00$
$0.7339 \mathrm{E}+03$

$0.9373 \mathrm{E}+06$

$0.4246 \mathrm{E}-01$

VAPOR

$0.1041 \mathrm{E}+03$

$0.2500 \mathrm{E}+02$

$0.1000 \mathrm{E}+01$

$0.2079 \mathrm{E}+00$

$0.6243 \mathrm{E}+02$

IIQUID
626

Flush H2O

to sitston

ZERO ZERO

ZERO ZERO

ZERO ZERO

ZERO

ZERO

ZERO ZERO

ZERO ZERO

ZERO ZERO

ZERO

ZERO ZERO

ZERO

ZERO ZERO

ZERO 'ZERO

ZERO ZERO

ZERO

ZERO

ZERO - ZERO

ZERO ZERO

ZERO ZERO

ZERO ZERO

ZERO

ZERO ZERO

ZERO ZERO

ZERO ZERO

ZERO ZERO

ZERO

ZERO

ZERO

ZERO

ZERO

$0.3124 \mathrm{E}+02$

$0.7289 \mathrm{E}+02$

$0.2500 \mathrm{E}+02$

$0.1000 \mathrm{E}+01$

ZERO

$0.2500 \mathrm{E}$

$0.2500 \mathrm{E}+02$

$0.7838 \mathrm{E}+03$

$0.1829 \mathrm{E}+04$

$0.6238 \mathrm{E}-01$

$0.1456 \mathrm{E}+00$

IIQUID

LIQUID 
Table A-1. HLW Flowsheet Material Balance (Tank 51/ITP Cycle 1) Section 2. Saltstone production

STREAM NUMBERS $\rightarrow$ STREAM NAME

COMPONENT FLOWS, LB/HR $122(\mathrm{C} 6 \mathrm{H} 5) 2$

123 (C6H5) $2 \mathrm{C} 6 \mathrm{H} 4$

118 (C6H5) $2 \mathrm{NH}$

133 (C6H5B (OH) 2 salt)

132 (C6H5B (OH) 2)

166 (C6H5C6H4OH)

162 (C6H5NH2)

163 (C6H5NHCHO)

165 (C6H5NO)

131 (C6H5OH salt)

130 (C6H5OH)

121 (C6H6)

154 (CH3OH)

$45 \mathrm{Ag}$

$116 \mathrm{Ag} 20$

$134 \mathrm{AgNO}$

$106 \mathrm{~B} 203$

$9 \mathrm{Ba}(\mathrm{OH}) 2$

$15 \mathrm{BaO}$

$24 \mathrm{BaSO} 4$

$66 \mathrm{CO}$

$142 \mathrm{CO} 2$ (C14)

$63 \mathrm{Ca}(\mathrm{COOH}) 2$

$167 \mathrm{Ca}(\mathrm{NO} 3) 2$

$120 \mathrm{Ca}(\mathrm{OH}) 2$

$113 \mathrm{Ca} 3(\mathrm{PO} 4) 2$

$20 \mathrm{CaC} 204$

$18 \mathrm{CaCO} 3$
$64 \mathrm{CaCO} 3$ (c14)

$78 \mathrm{CaF} 2$

$41 \mathrm{CaO}$

19 CasO4

29. Carbon

$26 \mathrm{Co}(\mathrm{COOH}) 2$

$168 \mathrm{Co}(\mathrm{NO} 3) 2$

$59 \mathrm{COO}$

98 Cr2O3

$42 \mathrm{Cs} 2 \mathrm{O}$

$53 \mathrm{CsCOOH}$

$96 \mathrm{CsCl}$

169. CsNO3

151 CsTPB

WSRC-TR-95-0019 (Revision
628

Flush Tnk Elush Tnk Air Purge Vnt to HEPA

630

Inlet Vapr

631

sstone Proo

632

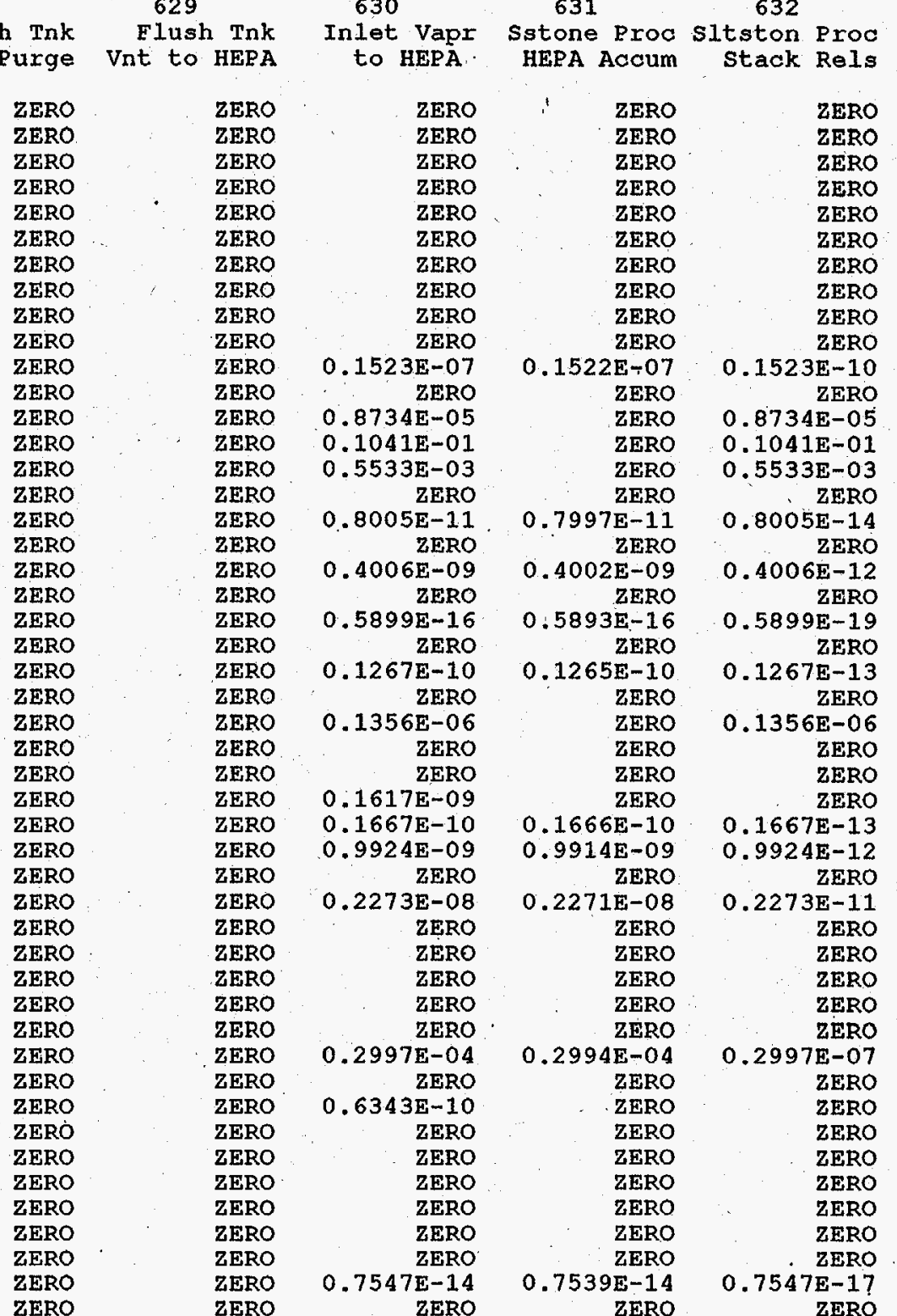

Date: January 1995 
Table A-1. HLW Flowsheet Material Balance (Tank 51/ITP Cycle 1) Section 2. Saltstone Production

STREAM NUMBERS (CONT'D) $\rightarrow$ STREAM NAME

$54 \mathrm{Cu}(\mathrm{COOH}) 2$

$170 \mathrm{Cu}(\mathrm{NO} 3) 2$

$55 \mathrm{CuO}$

$80 \mathrm{Fe} 2 \mathrm{O} 3$

$101 \mathrm{FeO}$

102 Group A

103 Group

25 H2

$158 \mathrm{H} 2 \mathrm{C} 2 \mathrm{O} 4$
$93 \mathrm{H} 2 \mathrm{SO} 4$

$90 \mathrm{H} 3 \mathrm{BO} 3$

$34 \mathrm{HCOOH}$

$43 \mathrm{HCl}$

$85 \mathrm{HF}$

49 HNO 3

$36 \mathrm{Hg}$

$129 \mathrm{Hg}(\mathrm{C} 6 \mathrm{H} 5) 2$

$135 \mathrm{Hg}$ (NO3) 2

$144 \mathrm{Hg} 2 \mathrm{Cl2}$

$117 \mathrm{Hg} 2 \mathrm{I} 2$

$146 \mathrm{HgCl}$

$21 \mathrm{HgO}$

$183 \mathrm{~K} 2 \mathrm{CO} 3$

$39 \mathrm{~K} 20$

$51 \mathrm{KCOOH}$

157 KMnO4

$161 \mathrm{KNO} 3$

$10 \mathrm{KOH}$

150 KTPB

$108 . \mathrm{Li} 20$

$65 \mathrm{Mg}(\mathrm{COOH}) 2$

$65 \mathrm{Mg}(\mathrm{COOH}) 2$
$171 \mathrm{Mg}(\mathrm{NO} 3) 2$

$33 \mathrm{MgO}$

$60 \mathrm{Mn}(\mathrm{COOH}) 2$

$172 \mathrm{Mn}$ (NO3) 2

$180 \mathrm{Mn} 30$
$56 \mathrm{MnO}$

$14 \mathrm{MnO} 2$

$145 \mathrm{MOO2}$

81 N2

$74 \mathrm{~N} 2 \mathrm{O}$

$182 \mathrm{NH} 3$

114 NH3OHNO 3

$52 \mathrm{NH} 4 \mathrm{COOH}$

173 NH4NO3

$68 \mathrm{NH} 4 \mathrm{OH}$

148 NH 4 TPB

WSRC-TR-95-0019 (Revision
628

629

Flush Tnk

630

Inlet Vapr

631

632

Alr Purge Vnt to HEPA to HEPA HEPA Accum Stack Rels

$\begin{array}{rrrrr}\text { ZERO } & \text { ZERO } & \text { ZERO } & \text { ZERO } & \text { ZERO } \\ \text { ZERO } & \text { ZERO } & 0.1497 \mathrm{E}-10 & \text { ZERO } & \text { ZERO }\end{array}$

ZIERO ZERO $0.1808 \mathrm{E}-10 \quad 0.1806 \mathrm{E}-10 \quad 0.1808 \mathrm{E}-13$

ZERO ZERO $0.5382 \mathrm{E}-09 \quad 0.5377 \mathrm{E}-09 \quad 0.5382 \mathrm{E}-12$

ZTRO

ZERO

ZERO

ZERO

ZERO

ZERO

ZERO

ZERO

ZERO

ZERO

ZERO

ZERO

ZERO

ZERO

ZERO

ZERO

ZERO

ZERO

ZERO

ZERO

ZERO

ZERO

ZERO

ZERO

ZERO

ZERO

ZERO

ZERO.

ZERO.

ZERO

ZERO

ZERO

ZERO

ZERO

ZERO

$0.1804 \mathrm{E}+03$

ZERO

ZERO

ZERO

ZERO

ZERO

ZERO

ZERO

ZERO $\quad 0.8844 \mathrm{E}-11$

ZERO

ZERO

ZERO

ZERO

ZERO

ZERO

ZERO

ZERO

ZERO

TERO

TERO

ZERO

ZERO

ZERO

ZERO

ZERO

ZERO

ZERO

ZERO

ZERO

ZERO

ZERO

ZERO

ZERO

ZERO

ZERO

ZERO

ZERO.

ZERO

$0.1804 \mathrm{E}+03$

ZERO $0.5411 \mathrm{E}+03$

ZERO $0.1594 \mathrm{E}-01$

ZERO ZERO

ZERO

ZERO

ZERO

ZERO
ZERO
0.1425 -08

Date: January 1995

ZERO ZERO

ZERO
$537 \mathrm{E}-13$

ZERO

ZERO

$0.5382 \mathrm{E}-12$

$3 E-09$

$0.4677 E-12$

$0.8844 \mathrm{E}-14$

$0.5537 \mathrm{E}-13$

ZERO ZERO

ZERO .

ZERO ZERO

ZERO ZERO

ZERO

ZERO ZERO

ZERO ZERO

ZERO ZERO

ZERO

ZERO

ZERO ZERO

ZERO

-11 $0.4581 E-14$

$\begin{array}{ll}\text { ZERO } & \text { ZERO } \\ \text { ZERO } & \text { ZERO }\end{array}$

ZERO

ZERO

ZERO ZERO

$\begin{array}{r}\text { ZERO } \\ 0.1044 \mathrm{E}-09\end{array} \quad 0.1045 \mathrm{E}-12$

ZERO ZERO

ZERO ZERO

ZERO

ZERO

$6202 \mathrm{E}-11$

$0.1033 \mathrm{E}-09$

34 -12

ZERO

ZERO

$0.3429 \mathrm{E}-10$

ZERO

ZERO

ZERO

ZERO

ZERO

ZERO

ZERO

ZERO

ZERO

.ZERO

ZERO
$0.3432 \mathrm{E}-13$

ZERO

$5411 \mathrm{E}+03$

$0.2236 \mathrm{E}-18$

$0.1594 \mathrm{E}-01$

ZERO

ZERO

ZERO
$0.1425 \mathrm{E}-0 \mathrm{OB}$

ZERO

Seation 2, Page 15 of 17 
Table A-1. HLW Flowsheet Material Balance (Tank 51/ITP Cycle 1)

STREAM NUMBERS (CONT'D) $\rightarrow$ STREAM NAME

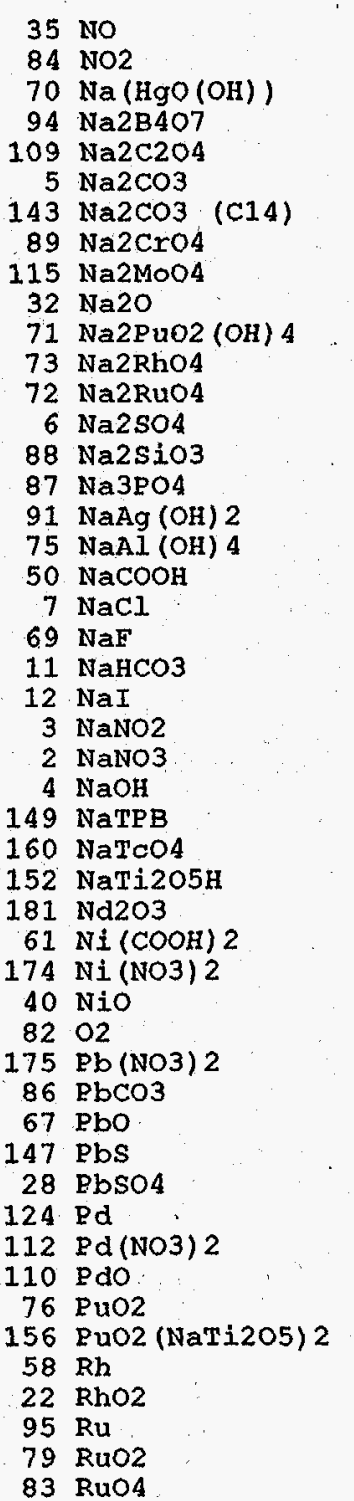

WSRC-TR-95-0019 (Revision

Section 2, Saltstone Production

628

Flush Tnk

Air Purge

ZERO

ZERO

ZERO

ZERO

ZERO

ZERO

ZERO

ZERO

ZERO

ZERO

ZERO

ZERO

ZERO

ZERO

ZERO

ZERO

ZERO

ZERO

ZERO

ZERO

ZERO

ZERO

TERO

ZERO

ZERO

ZERO

ZERO

ZERO

ZERO

ZERO

ERO

ERO

ERO

ZERO
$0.5448 \mathrm{E}+02$

ZERO

ZERO

ZERO

ZERO

ERO

ERO

ZERO

ZERO

ZERO

IERO

ZERO

ZERO

ZERO

ZERO

ZERO

$$
629
$$

Flush Tnk

nt to HEPA

ZERO
ZERO

ZERO

ZERO

ZERO

ZERO

ZERO

ZERO

ZERO

ZERO

ZERO

ZERO

ZERO

ZERO

ZERO

ZERO

ZERO

ZERO

ZERO

ZERO

ZERO

ZERO

ZERO

ZERO

ZERO

ZERO

ZERO

ZERO

ZERO

ZERO

ZERO

$0.5448 E+02$

ZERO

ZERO

ZERO

ZERO

ZERO

ZERO

ZERO

ZERO

ZERO

ZERO

ZERO

ZERO

ZERO

ZERO
631

632

Inlet Vapr Sstone Proc sitston Proc

to HEPA HEPA Accum stack Rels

ZERO ; ZERO $\quad$ ZERO

$0.1285 \mathrm{E}-11 \quad 0.1284 \mathrm{E}-11 \quad 0.1285 \mathrm{E}-14$

$0.1482 \mathrm{E}-08 \quad 0.1481 \mathrm{E}-08 \quad 0.1482 \mathrm{E}-11$

$0.2127 \mathrm{E}-07 \quad 0.2125 \mathrm{E}-07 \quad 0.2127 \mathrm{E}-11$

$0.2127 \mathrm{E}-07$ 0.2125E-07 $0.2127 \mathrm{E}-10$

$0.3335 \mathrm{E}-13 \quad 0.3331 \mathrm{E}-13 \quad 0.3335 \mathrm{E}-16$

$0.1230 \mathrm{E}-07 \quad 0.1229 \mathrm{E}-07 \quad 0.333 \mathrm{E}-16$

$0.3665 \mathrm{E}-08$ 0 $0.3662 \mathrm{E}-08$ $0.1230 \mathrm{E}-10$ $0.3662 \mathrm{E}-08 \quad 0.3665 \mathrm{E}-11$

$0.8258 \mathrm{E}-14 \quad 0.8249 \mathrm{E}-14 \quad 0.8258 \mathrm{E}-17$

$0.8892 \mathrm{E}-10 \quad 0.8883 \mathrm{E}-10 \quad 0.8892 \mathrm{E}-13$

$0.4683 \mathrm{E}-09 \quad 0.4678 \mathrm{E}-09 \quad 0.4683 \mathrm{E}-12$

$0.1283 \mathrm{E}-06 \quad 0.1282 \mathrm{E}-06 \quad 0.1283 \mathrm{E}-09$

$0.3555 \mathrm{E}-08 \quad 0.3551 \mathrm{E}-08 \quad 0.3555 \mathrm{E}-11$

$0.1697 \mathrm{E}-06 \quad 0.1695 \mathrm{E}-06 \quad 0.1697 \mathrm{E}-09$

$0.3400 \mathrm{E}-13 \quad 0.3396 \mathrm{E}-13 \quad 0.3400 \mathrm{E}-16$

$0.6380 \mathrm{E}-06 \quad 0.6386 \mathrm{E}-09$

ZERO

$0.1077 \mathrm{~m}-07 \quad 0.1078 \mathrm{E}-10$

$0.1001-07 \quad 0.1002 \mathrm{E}-10$

ZERO ZERO

$0.6572 \mathrm{E}-11 \quad 0.6579 \mathrm{E}-14$

$0.1239 \mathrm{E}-05 \quad 0.1241 \mathrm{E}-08$

$0.6356 \mathrm{E}-05 \quad 0.6363 \mathrm{E}-08$

$\begin{array}{rrr}0.2955 \mathrm{E}-05 & 0.2952 \mathrm{E}-05 & 0.2955 \mathrm{E}-08 \\ 0.3538 \mathrm{E}-07 & 0.3535 \mathrm{E}-07 & 0.3538 \mathrm{E}-10 \\ 0.2951 \mathrm{E}-09 & \text { ZERO } & \text { ZERO }\end{array}$

$\begin{array}{rrr}0.2955 \mathrm{E}-05 & 0.2952 \mathrm{E}-05 & 0.2955 \mathrm{E}-08 \\ 0.3538 \mathrm{E}-07 & 0.3535 \mathrm{E}-07 & 0.3538 \mathrm{E}-10 \\ 0.2951 \mathrm{E}-09 & \text { ZERO } & \text { ZERO }\end{array}$

$\begin{array}{lll}0.2951 E-09 & \text { ZERO } & \text { ZERO } \\ \text { ZERO } & \text { ZERO }\end{array}$

ZERO

ZERO

$0.1666 \mathrm{E}-10$

$0.1634 \mathrm{E}+03$

$0.1916 \mathrm{E}-09$

ZERO

$28 \mathrm{E}-11$

ZERO
ZERO

ZERO

$0.7910 \mathrm{E}-12$

ZERO

ZERO

ZERO

ZERO

ZERO

ZERO

ZERO

ZERO

$0.1664 E-10$

ZERO

$0.1666 \pi-13$

ZERO

ZERO ZERO

$0.8028 \mathrm{E}-14$

ZERO

ZERO

$0.7902 \mathrm{E}-12 \quad 0.7910 \mathrm{E}-15$

ZERO ZERO

ZERO ZERO

ZERO

ZERO ZERO

ZERO ZERO

ZERO

ZERO ' ZERO 
Table A-1. HLW Flowsheet Material Balance (Tank 51/ITP Cycle 1) Section 2. Saltstone Production

STREAM NUMBERS (CONT'D) $\rightarrow$ STREAM NAME

$38 \mathrm{SO} 2$

137 Semi Vol cs20

136 Semi Vol CsCl

126 Semi Vol Group A

140 Semi Vol Na2B4O7

138 Semi Vol NaCl

139 Semi vol NaF

125 Semi Vol NaI

23 Semi

$48 \mathrm{Sr}(\mathrm{COOH}) 2$

$176 \mathrm{Sr}(\mathrm{NO} 3) 2$

153 Sr (NaTi2O5) 2

$127 \operatorname{Sr}(\mathrm{OH}) 2$

$30 \mathrm{srCO} 3$

46 Sro

$159 \mathrm{TcO} 2$

92 ThO2

119 Tritium

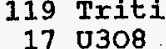

$\begin{array}{ll}17 & 0308 \\ 62 & \mathrm{UO} 2 \\ \text { (COOH) } 2\end{array}$

177 UO2 (NO3) 2

$16 \mathrm{UO2}(\mathrm{OH}) 2$

$27 \mathrm{Y}(\mathrm{COOH}) 3$

$178 \mathrm{Y}$ (NO3) 3

$128 \mathrm{Y}(\mathrm{OH}) 3$

$31 \mathrm{Y} 2(\mathrm{CO} 3) 3$

$47 \mathrm{Y2O3}$

100 Zeolite

$44 \mathrm{Zn}(\mathrm{COOH}) 2$

$179 \mathrm{Zn}(\mathrm{NO} 3) 2$

57 Zno

104 ZrO2

1 water

TOTAL FLOW, LB/HR

TEMPERATURE, DEG C

PRESSURE， ATM

PRESSURE， PSIG

PRESSURE, MM HG

ENTHALPY, PCU/HR

VAPOR ELOW, CFM

LIQUID FLOW, GPM

DENSITY, LBS/FT3

PHASE

WSRC-TR-95-0019 (Revision
628

Flush Tnk Flush Tnk Vnt to HEPA

ZERO Vnt to IERO
ZERO

ZERO ZERO

ZERO ZERO

ZERO ZERO

ZERO - ZERO

ZERO ZERO

$\begin{array}{ll}\text { ZERO } & \text { ZERO } \\ \text { ZERO } & \text { ZERO }\end{array}$

ZERO $\quad$ ZERO

ZERO $\quad$ ZERO

ZERO

ZERO

ZERO

ZERO

ZERO

ZERO

ZERO

ZERO

ZERO

ZERO

ZERO

ZERO

ZERO

ZERO

ZERO

ZERO

ZERO

ZERO

ZERO

ZERO

ZERO

ZERO

ZERO

ZERO

ZERO

$0.3945 \mathrm{E}+01$

$0.2388 \mathrm{E}+03$

$$
\text { ZERO }
$$

ZERO

ZERO

ZERO

ZERO

ZERO

ZERO

ZERO

ZERO

ZERO

ZERO

ZERO

ZERO

ZERO

ZERO

ZERO.

ZERO

ZERO

ZERO

ZERO

ZERO

ZERO

ZERO

ZERO ZERO

$0.8659 \mathrm{E}+01$

$0.2435 \mathrm{E}+03$

630

$631 \quad 632$

Sstone Proc sitston Proc
to HEPA. HEPA Accum stack Rels

ZERO ZERO

ZERO

ZERO

ZERO

ZERO

ZERO

ZERO

ZERO

4E-08

ZERO

$0.2185 E-13$

ZERO

ZERO

ZERO

ZERO

$0.1942 \mathrm{E}-10$

ZERO

ZERO

ZERO

$0.3831 \mathrm{E}-09$

ZERO

$0.2589 \mathrm{E}-13$

ZERO

ZERO

ZERO

2. ZERO

$0.2046 \mathrm{~F}-09$

ZERO

$0.5036 \mathrm{E}-09$

$0.3194 \mathrm{E}+02$

$0.7365 \mathrm{E}+03$

$0.3836 \mathrm{E}+02$

$0.9656 \mathrm{E}+00$

$0.9656 \mathrm{E}+00$

$-0.5055 \mathrm{E}+00$

$0.7339 \mathrm{E}+03$

$0.7339 \mathrm{E}+03$

$0.2788 \mathrm{E}+05$

$0.1851 \mathrm{E}+03$

$0.6024 \mathrm{E}+02$

$0.6633 \mathrm{E}-01$

VAPOR
' ZERO

ZERO

ZERO

ZERO

ZERO

ZERO

ZERO

ZERO

$0.1852 \mathrm{E}-08$

ZERO

ZERO

$0.2183 \mathrm{E}-13$

ZERO

ZERO

ZERO

ZERO

ZERO

ZERO

ZERO

ZERO

$0.38277-09$

ZERO

0.2587 EERO

E-13

ZERO

ZERO

ZERO

ZERO

$0.2044 \mathrm{E}-09$

ZERO

$0.5031 \mathrm{E}-09$

ZERO

$0.4381 \mathrm{E}-04$

$0.3836 \mathrm{E}+02$

$0.3532 \mathrm{E}-03$

$0.1 .644 \mathrm{E}+03$

SOLID
$0.5055 \mathrm{E}+00$

ZERO

ZERO

ZERO

ZTRO

ZERO

ZERO

ZERO

ZERO

$0.1854 \mathrm{E}-11$

ZERO

ZERO

$0.2185 \mathrm{E}-16$

ZERO

ZERO

ZERO

ZERO

$0.1942 \mathrm{E}-10$

ZERO

ZERO

$\mathrm{E}-12$

$0.2589 \mathrm{E}-16$

ZERO

ZERO

ZERO

ZERO

ZERO

ZERO

$\mathrm{E}+02$

$.3836 \mathrm{E}+02$

$-0.5055 \mathrm{E}+00$

$0.7339 \mathrm{E}+03$

$0.2788 \mathrm{E}+05$

$0.1851 \mathrm{E}+03$

VAPOR

VAPOR
Date: January 1995

section 2, Page 17 of 17 


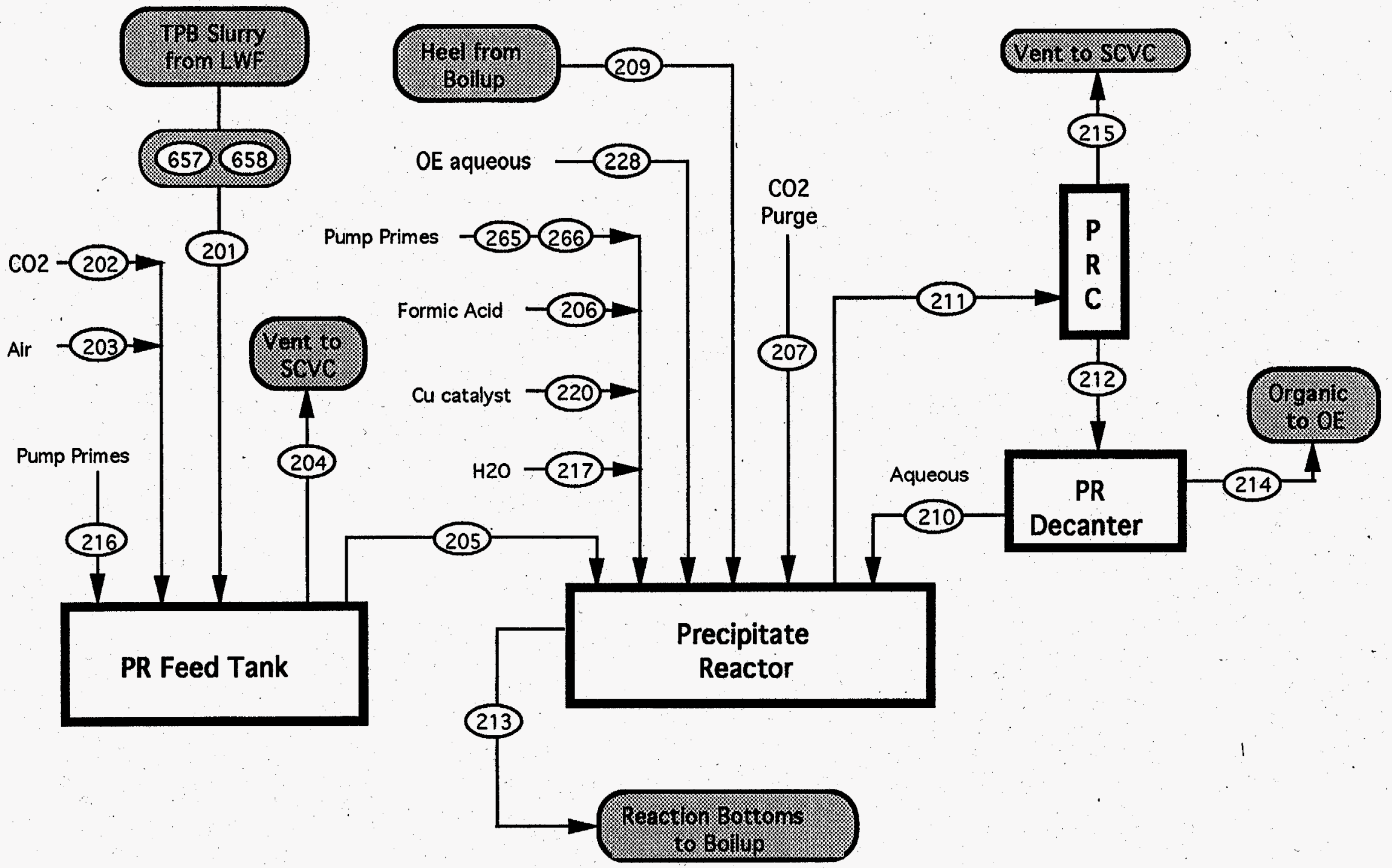

SECTION 3A. PRECIPITATE HYDROLYSIS IN SALT CELL 

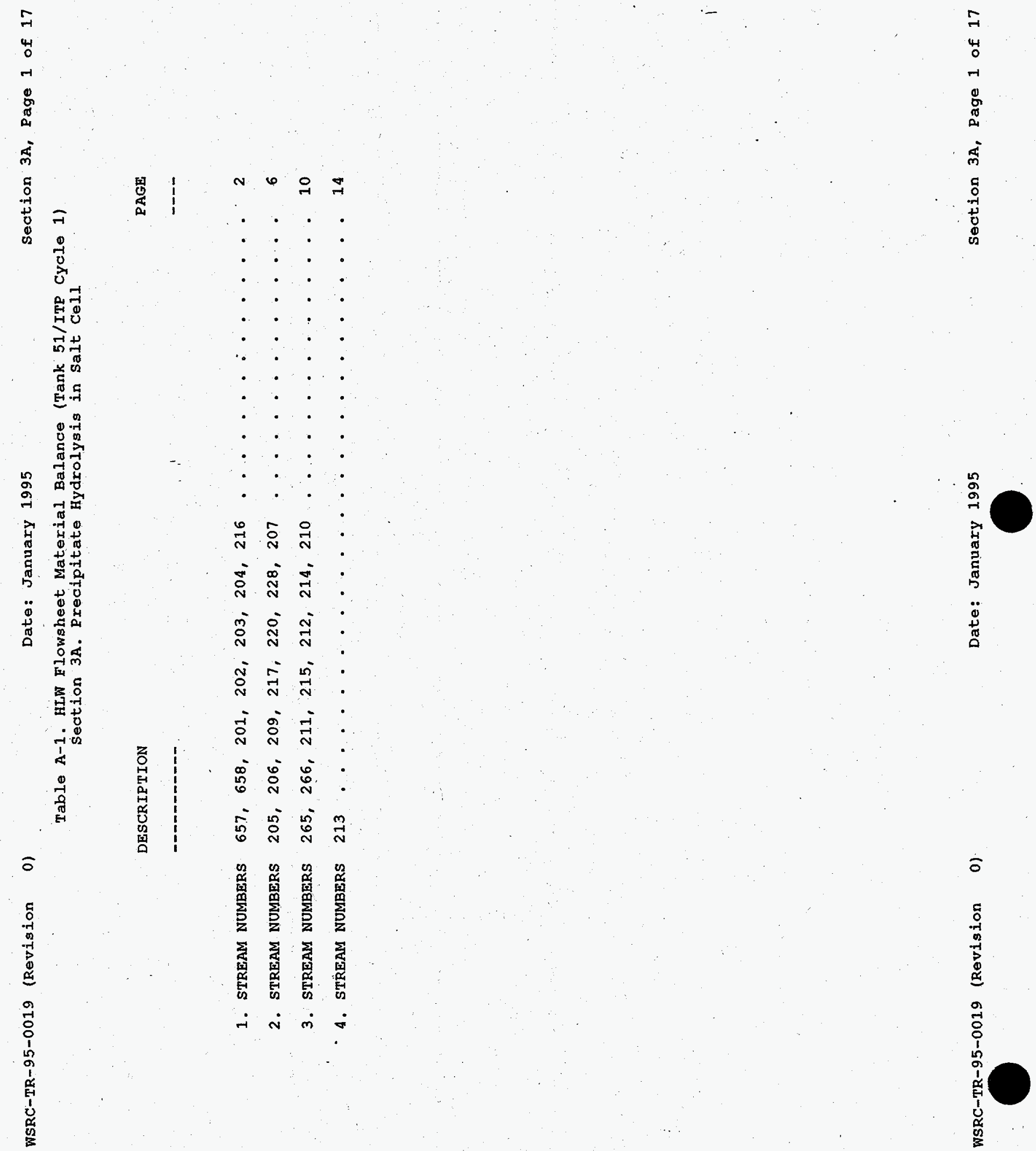

o

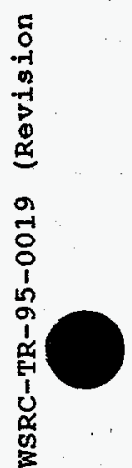


Table A-1. HLW Flowsheet Material Balance (Tank 51/ITP Cycle 1) Section 3A. Precipitate Hydrolysis in Salt Cell

STREAM NUMBERS $\rightarrow$ STREAM NAME

COMPONENT FLOWS, LB/HR 122 ( $\mathrm{C} 6 \mathrm{H} 5) 2$

123 ( $\mathrm{C} 6 \mathrm{H} 5) 2 \mathrm{C} 6 \mathrm{H}$

118 (C6H5) $2 \mathrm{NH}$

133 (C6H5B (OH) 2 salt)

132 (С6H5B (OH) 2)

166 (C6H5C6H4OH)

162 (C6H5NH2)

163 (C6H5NHCHO)

165 (C6H5NO)

164 (C6H5NO2)

131 (C6H5OH salt)

130 (С6H5OH)

1.55 (CH3) $2 \mathrm{CHOH}$

154 ( $\mathrm{CH} 3 \mathrm{OH})$

$45 \mathrm{Ag}$

116 Ag2O

134 AgNO3

$106 \mathrm{~B} 2 \mathrm{O3}$

$$
9 \mathrm{Ba}(\mathrm{OH}) 2
$$

24 BaSO4

$66 \mathrm{CO}$

$1.42 \mathrm{CO} 2$ (C14)

$63 \mathrm{Ca}(\mathrm{COOH}) 2$

$167 \mathrm{Ca}(\mathrm{NO} 3) 2$

$120 \mathrm{Ca}(\mathrm{OH}) 2$
$113 \mathrm{Ca} 3(\mathrm{PO} 4) 2$

$20 \mathrm{CaC} 2 \mathrm{O} 4$

$18 \mathrm{CaCO} 3$

$64 \mathrm{CaCO}^{2}$ (C14)

$78 \mathrm{CaF} 2$

$41 \mathrm{CaO}$

$19 \mathrm{CaSO} 4$

29 Carbon

$26 \mathrm{Co}(\mathrm{COOH}) 2$

$168 \mathrm{Co}(\mathrm{NO} 3) 2$

$59 \mathrm{COO}$

$98 \mathrm{Cr} 203$

$42 \mathrm{Cs} 2 \mathrm{O}$

$53 \mathrm{CsCOOH}$

$96 \mathrm{CsCl}$

$8 \mathrm{CsOH}$

151 CsTPB

WSRC-TR-95-0019 (Revision $\begin{array}{ccc}658 & 201 & 202 \\ \end{array}$

657

Prec Feed Prec Feed Washd Precip
Solubles Insolubles Feed frm LWF

$0.3293 \mathrm{E}+00$

$0.3293 \mathrm{E}+00$

$0.1399 \mathrm{E}-01$

$0.1399 \mathrm{E}-01$

$0.9313 \mathrm{E}-02$
$0.3712 \mathrm{E}+00$

$0.9313 E-02$

ZERO

$0.3712 \mathrm{E}+00$

$0.3712 \mathrm{E}+00$

$0.3026 \mathrm{E}-01$

(2)

ZERO

ZERO

ZERO

ZERO

$0.4503 \mathrm{E}-01$

ZERO

$0.1091 \mathrm{E}-01$

$0.8591 \mathrm{E}-10$

$0.9422 \mathrm{E}-11$

ZERO

ZERO

ZERO

ZERO

$0.4817 \mathrm{E}-16$

ZERO

ZERO

ZERO

ZERO

ZERO

$0.1370 E-05$
$0.1413 E-06$

$0.1413 E-06$

ZERO

ZERO

ZERO

ZERO

ZERO

ZERO

ZERO

ZERO

0.5373 E-06

ZERO

ZERO

ZERO

ZERO

ZERO

$0.1794 \mathrm{E}-05$

ZERO

ZERO

ZERO

ZERO

$0.4503 E-01$

ZERO

ZERO

ZERO

ZERO

$0.1091 \mathrm{E}-01$

$0.8591 \mathrm{E}-10$

ZERO 0.9422 E-11

$0.3054 \mathrm{E}-02 \quad 0.3054 \mathrm{E}-02$

ZERO

$0.1297 \mathrm{E}+00$

ZERO
$0.1297 \mathrm{E}+00$

$0.1670 \mathrm{E}-02$

ZERO $0.4817 \mathrm{E}-16$

ZERO

ZERO
$0.1793 \mathrm{E}-02$

ZERO ZERO

ZERO

ZERO

ZERO

ZERO

ZERO

ZERO

ZERO

$0.1370 \mathrm{E}-05$

$0.5607 \mathrm{E}+00$

$0.1413 \mathrm{E}-06$

ZERO

ZERO

ZERO

$0.1912 \mathrm{E}+00$

ZERO

ZERO

ZERO

$0.1028 \mathrm{E}-03$

$0.261 .6 \mathrm{E}+00$

$0.3848 \mathrm{E}-04$

ZERO
ZERO

ZERO

$0.1100 \mathrm{E}+00$

ZERO

ZERO

ZERO

ZERO

$0.1912 \mathrm{E}+O 0$

ZERO

ZERO

ZERO

$0.2616 \mathrm{E}+00$

$0.3848 \mathrm{E}-04$

ZERO
ZERO

ZERO

$0.1794 \mathrm{E}-05$

203

Purge Air Inlkge

PRF

204

216

vent PRFT Trnsfer

I ZERO

ZERO

ZERO

ZERO

ZERO

ZERO

ZERO

ZERO

ZERO

ZERO

ZERO

ZERO

ZERO

ZERO

ZERO

ZERO

ZERO

ZERO

ZERO

ZERO

ZERO

ZERO

$0.1471 \mathrm{E}+02$

ZERO

ZERO

ZERO

ZERO

ZERO

ZERO

ZERO

ZERO

ZERO

ZERO

ZERO

ZERO

ZERO

ZERO

ZERO

ZERO

ZERO

ZERO

ZERO

ZERO

Date: January 1995

\begin{tabular}{|c|c|c|}
\hline ZERO & ZERO & ZERO \\
\hline ZERO & ZERO & ZERO \\
\hline ZERO & ZERO & ZERO \\
\hline ZERO & ZERO & ZERO \\
\hline ZERO & ZERO & ZERO \\
\hline ZERO & ZERO & ZERO \\
\hline ZERO & ZERO & ZERO \\
\hline ZERO & ZERO & ZERO \\
\hline ZERO & ZERO & ZERO \\
\hline ZERO & ZERO & ZERO \\
\hline ZERO & ZERO & ZERO \\
\hline ZERO & ZERO & ZERO \\
\hline ZERO & $0.9857 \mathrm{E}-02$ & ZERO \\
\hline ZERO & $0.8072 \mathrm{E}-11$ & ZERO \\
\hline ZERO & $0.7180 \mathrm{E}-12$ & ZERO \\
\hline ZERO & ZERO & ZERO \\
\hline ZERO & ZERO & ZERO \\
\hline ZERO & ZERO & ZERO \\
\hline ZERO & ZERO & ZERO \\
\hline ZERO & ZERO & ZERO \\
\hline ZERO & ZERO & ZERO \\
\hline ZERO & ZERO & ZERO \\
\hline ZERO & ZERO & ZERO \\
\hline ZERO & ZERO & ZERO \\
\hline ZERO & $0.1434 \mathrm{E}+02$ & ZERO \\
\hline ZERO & ZERO & ZERO \\
\hline ZERO & ZERO & ZERO \\
\hline ZERO & ZERO & ZERO \\
\hline ZERO & ZERO & ZERO \\
\hline ZERO & ZERO & ZERO \\
\hline ZERO & ZERO & ZERO \\
\hline ZERO & ZERO & ZERO \\
\hline ZERO & ZERO & ZERO \\
\hline ZERO & ZERO & ZERO \\
\hline ZERO & ZERO & ZERO \\
\hline ZERO & ZERO & ZERO \\
\hline ZERO & ZERO & ZERO \\
\hline ZERO & ZERO & ZERO \\
\hline ZERO & ZERO & ZERO \\
\hline ZERO & ZERO & ZERO \\
\hline ZERO & ZERO & ZERO \\
\hline ZERO & ZERO & ZERO \\
\hline ZERO & ZERO & ZERO \\
\hline ZERO & ZERO & ZERO \\
\hline ZERO & ZERO & ZERO \\
\hline ZERO & ZERO & ZERO \\
\hline ZERO & ZERO & ZERO \\
\hline ZERO & ZERO & ZERO \\
\hline
\end{tabular}


Table A-1. HLW Flowsheet Material Balance (Tank 51/ITP Cycle 1) section 3A. Preaipitate Hydrolysis in Salt Cell

STREAM NUMBERS (CONT'D) $\rightarrow$ STREAM NAME

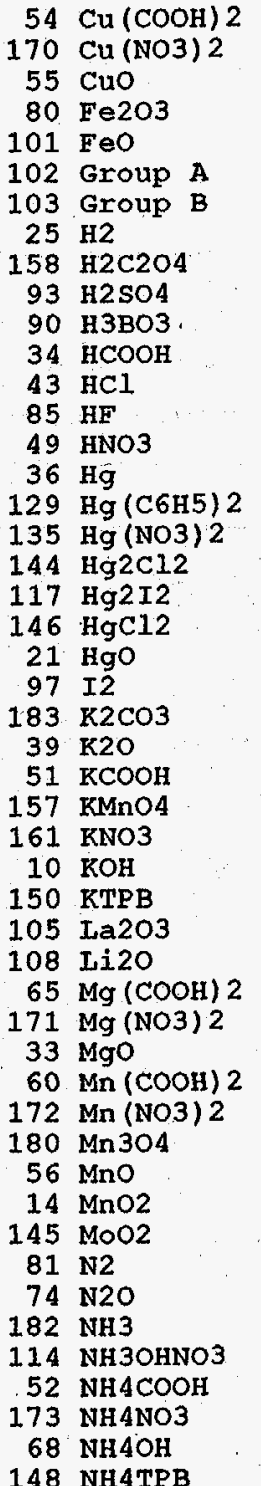

657

Prec

Solubles

ZERO
$0.1268 \mathrm{E}-06$

Z-06

ZERO

ZERO

ZERO

$0.7581 \mathrm{E}-11$

ZERO

ZERO

ZERO

ZERO

ZERO

ZERO

ZERO

ZERO

ZERO

ZERO

ZERO

ZERO

ZERO

ZERO
ZERO

ZERO

ZERO

ZERO

$0.1076 \mathrm{E}-06$

ZERO

ZERO

ZERO

ZERO
$0.5254 \mathrm{E}-07$

ZERO

ZERO

$0.6122 \mathrm{E}-07$

ZERO

ZERO

ZERO

$0.3456 \mathrm{E}-03$

$0.1070 \mathrm{E}-09$

$0.1826 \mathrm{E}-05$

ZERO
ZERO

ZERO

ZERO

ZERO
658

ZERO
ZERO ZERO
ZERO

$0.1061 \mathrm{E}-02$

$0.1587 \mathrm{E}+00$

ZERO

$0.2437 \mathrm{E}-05$

$0.7493 \mathrm{E}-0$

ZERO

ZERO
ZERO

ZERO

ZERO

ZERO

ZERO

ZERO

$0.3797 \mathrm{E}+00$

ZERO

ZERO

ZERO

$0.3719 \mathrm{E}-03$

ZERO

$0.6882 \mathrm{~F}-02$

ZERO

ERRO

ZERO

ZERO

ZERO

$0.2948 \mathrm{E}-03$

ZERO

ZERO
$0.1725 \mathrm{E}-02$

ZE-02
ZERO
ZERO

ZERO

ZERO

$0.3295 \mathrm{E}-02$

$0.1096 \mathrm{E}-03$

$\mathrm{E}-03$

ZERO

ZERO

ZERO

ZERO

ZERO

$0.2617 \mathrm{E}-02$
202

PRFT CO2

Purge
zERO

$0.1268 \mathrm{E}-06$

$0.1061 \mathrm{E}-02$

$0.1587 \mathrm{E}+00$

ZERO
$0.2437 \mathrm{E}-05$

$0.2437 \mathrm{E}-05$
$0.7493 \mathrm{E}-07$

$0.7581 \mathrm{E}-11$

ZERO

ZERO

ZERO

ZERO

ZERO

ZERO

$0.3797 \mathrm{E}+00$

ZERO

ZERO

ZERO

$0.371 .9 \mathrm{E}-03$

ZERO

$0.1948 \mathrm{E}-04$
$0.6882 \mathrm{E}-02$

ZERO

$0.1076 \mathrm{E}-06$

$0.4280 \mathrm{E}+02$

ZERO

ZERO

$0.5254 \mathrm{E}-07$

$0.1725 \mathrm{E}-02$

$0.6122 \mathrm{E}-07$

ZERO

$0.3295 E-02$

$0.1096 \mathrm{E}-03$

$0.3456 \mathrm{E}-03$

$0.1070 \mathrm{E}-09$

ZERO

Date: January 1995
203

PRET

Air Inlkge

ZERO

ZERO

ZERO

ZERO

ZERO

ZERO

ZERO

ZERO

ZERO

ZERO

ZERO

ZERO

ZERO

ZERO

ZERO

ZERO

IERO

ZERO

ZERO

ZERO

ZERO

ZERO

ZERO

ZERO

ZERO

ZERO

ZERO

ZERO

ZERO

ZERO

ZERO

ZERO

ZERO

ZERO

ZERO

ZERO

ZERO

ZERO

$0.1133 \mathrm{E}+01$

ZERO

ZERO

ZERO

ZERO

ZERO

ZERO
ZERO $\begin{array}{ll}204 & 216 \\ \text { PRET vent PRFT Trnsfer }\end{array}$ to sCVC Pump Prime ZERO ZERO ZERO ZERO ZERO ZERO ZERO

ZERO ZERO

ZERO ZERO ZERO ZERO ZERO ZERO ZERO ZERO ZERO ZERO ZERO ZERO ZERO ZERO ZERO ZERO ZERO ZERO ZERO ZERO

ZERO

ZERO ZERO

ZERO ZERO

ZERO ZERO

ZERO ZERO

ZERO ZERO

ZERO ZERO

ZERO ZERO

ZERO ZERO

ZERO ZERO

ZERO ZERO

ZERO : ZERO

ZERO

ZERO

ZERO ZERO

ZERO

ZERO ZERO

ZERO ZERO

ZERO ZERO

ZERO ZERO

ZERO ZERO

ZERO ZERO

ZERO
$0.1133 E+01$

$0.1053 \mathrm{E}-09$

$0.3846 \mathrm{E}-06$

ZERO ZERO

ZERO ZERO

ZERO ZERO

ZERO ZERO

ZERO

ZERO 
Table A-1. HLW Flowsheet Material Balance (Tank 51/ITP Cycle 1) section 3A. Precipitate Hydrolysis in salt cell

STREAM NUMBERS (CONT'D) -> STREAM NAME

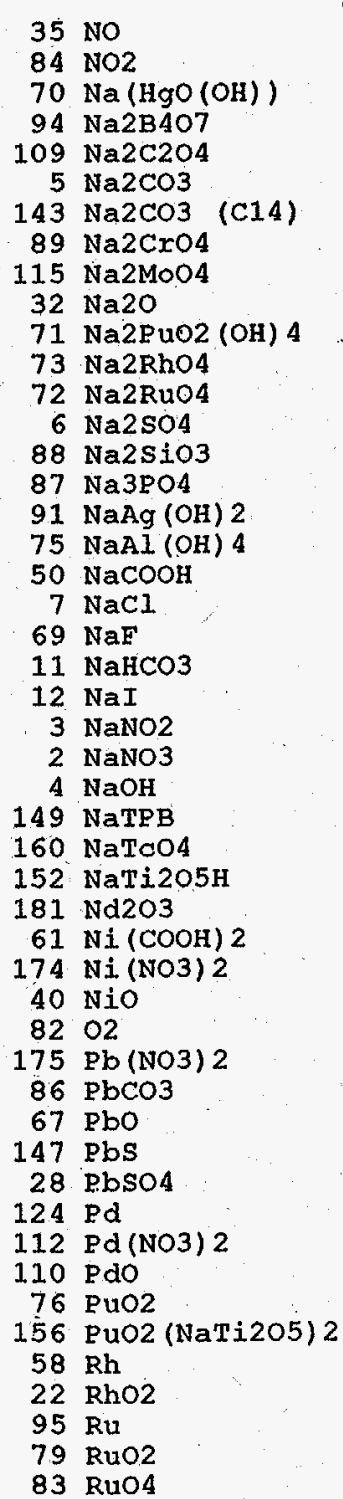

\begin{tabular}{|c|c|c|c|c|}
\hline $\begin{array}{l}657 \\
\text { Prec Feed } \\
\text { Solubles }\end{array}$ & $\begin{array}{l}658 \\
\text { Prec Feed } \\
\text { Insolubles }\end{array}$ & $\begin{array}{l}201 \\
\text { Washd Precip } \\
\text { Feed frm LWF }\end{array}$ & 20 & $\begin{array}{l}\mathrm{CO} 2 \\
\text { Purge }\end{array}$ \\
\hline ZERO & ZERO & ZERO & & ZERO \\
\hline ZERO & ZERO & ZERO & $\therefore$ & ZERO \\
\hline $1165 \mathrm{E}-10$ & ZERO & $0.1165 \mathrm{E}-10$ & & ZERO \\
\hline $1025 \mathrm{E}-01$ & ZERO & $0.1025 \mathrm{E}-01$ & & ZERO \\
\hline $810 \mathrm{E}-02$ & ZERO & $0.7810 \mathrm{E}-02$ & & ZERO \\
\hline $1677 \mathrm{E}+00$ & ZERO & $0.1677 \mathrm{E}+00$ & & ZERO \\
\hline $825 \mathrm{E}-09$ & ZERO & $0.2825 \mathrm{E}-09$ & & ZERO \\
\hline $1040 \mathrm{E}-03$ & ZERO & $0.1040 \mathrm{E}-03$ & & ZERO \\
\hline $.3105 E-04$ & ZERO & $0.3105 E-04$ & & ZERO \\
\hline ZERO & $0.1003 E+01$ & $0.1003 E+01$ & & ZERO \\
\hline $1298 \mathrm{E}-11$ & ZERO & $0.1298 \mathrm{E}-11$ & & ZERO \\
\hline $533 \mathrm{E}-06$ & ZERO & $0.7533 \mathrm{E}-06$ & & ZERO \\
\hline $3967 \mathrm{E}-05$ & ZERO & $0.3967 \mathrm{E}-05$ & & ZERO \\
\hline $082 \mathrm{E}-02$ & ZERO & $0.1082 \mathrm{E}-02$ & & ZERO \\
\hline $3012 E-04$ & ZERO & $0.3012 \mathrm{E}-04$ & & ZERO \\
\hline $.1437 \mathrm{E}-02$ & ZERO & $0.1437 \mathrm{E}-02$ & & ZERO \\
\hline $.5550 E-12$ & ZERO & $0.5550 \mathrm{E}-12$ & & ZERO \\
\hline $727 \mathrm{E}-04$ & ZERO & $0.3727 \mathrm{E}-04$ & & ZERO \\
\hline ZERO & ZERO & ZERO & & ZERO \\
\hline $3180 \mathrm{E}-02$ & ZERO & $0.3180 \mathrm{E}-02$ & & ZERO \\
\hline $0.8321 \mathrm{E}-04$ & ZERO & $0.8321 \mathrm{E}-04$ & & ZERO \\
\hline ZERO & ZERO & ZERO & & ZERO \\
\hline $573 E-07$ & ZERO & $0.5573 \mathrm{E}-07$ & & ZERO \\
\hline $955 E+00$ & ZERO & $0.2955 \mathrm{E}+00$ & & ZERO \\
\hline $2200 \mathrm{E}-01$ & - ZERO & $0.2200 \mathrm{E}-01$ & & ZERO \\
\hline $.9803 \mathrm{E}-01$ & ZERO & $0.9803 \mathrm{E}-01$ & & ZERO \\
\hline $.5890 \mathrm{E}+00$ & ZERO & $0.5890 \mathrm{E}+00$ & & ZERO \\
\hline $0.2500 \mathrm{E}-05$ & ZERO & $0.2500 E-05$ & & ZERO \\
\hline ZERO & $0.9792 E+00$ & $0.9792 \mathbf{E}+00$ & & ZERO \\
\hline ZERO & ZERO & ZERO & & ZERO \\
\hline ZERO & ZERO & ZERO & & ZERO \\
\hline $0.6686 \mathrm{E}-06$ & ZERO & $0.6686 \mathrm{E}-06$ & & ZERO \\
\hline ZERO & $0.1037 \mathrm{E}-02$ & $0.1037 \mathrm{E}-02$ & & ZERO \\
\hline $0.3924 \mathrm{E}-04$ & ZERO & $0.3924 \mathrm{E}-04$ & & ZERO \\
\hline $0.1624 \mathrm{E}-05$ & ZERO & $0.1624 \mathrm{E}-05$ & & ZERO \\
\hline ZERO & ZERO & ZERO & & ZERO \\
\hline ZERO & ZERO & ZERO & & ZERO \\
\hline ZERO & ZERO & ZERO & & ZERO \\
\hline ZERO & $0.4362 \mathrm{E}-02$ & $0.4362 \mathrm{E}-02$ & & ZERO \\
\hline ZERO & ZERO & ZERO & & ZERO \\
\hline $0.6701 \mathrm{E}-08$ & ZERO & $0.6701 \mathrm{E}-08$ & & ZERO \\
\hline ZERO & ZERO & ZERO & & ZERO \\
\hline ZERO & $0.3209 \mathrm{E}-04$ & $0.3209 \mathrm{E}-04$ & & ZERO \\
\hline ZERO & $0.1272 \mathrm{E}-03$ & $0.1272 \mathrm{E}-03$ & & ZERO \\
\hline ZERO & ZERO & ZERO & & ZERO \\
\hline ZERO & ZERO & ZERO & & ZERO \\
\hline ZERO & ZERO & ZERO & & ZERO \\
\hline ZERO & $0.4424 \mathrm{E}-02$ & $0.4424 \mathrm{E}-02$ & & ZERO \\
\hline ZERO & ZERO & ZERO & & ZERO \\
\hline
\end{tabular}

Date: January 1995
204 216

\begin{tabular}{|c|c|c|c|c|}
\hline \multirow{2}{*}{203 PRFT } & \multicolumn{2}{|c|}{204} & \multicolumn{2}{|l|}{216} \\
\hline & PRFT & vent & PRFT $T$ & rnsfer \\
\hline Air Inlkge & & SCVC & Pump & Prime \\
\hline ZERO & & ZERO & & ZERO \\
\hline ZERO & & ZERO & & ZERO \\
\hline ZERO & & ZERO & & ZERO \\
\hline ZERO & & ZERO & & ZERO \\
\hline ZERO & & ZERO & & ZERO \\
\hline ZERO & & ZERO & & ZERO \\
\hline ZERO & & ZERO & & ZERO \\
\hline ZERO & & ZERO & & ZERO \\
\hline ZERO & & ZERO & & ZERO \\
\hline ZERO & & ZERO & & ZERO \\
\hline ZERO & & ZERO & & ZERO \\
\hline ZERO & & ZERO & & ZERO \\
\hline ZERO & & ZERO & & ZERO \\
\hline ZERO & & ZERO & & ZERO \\
\hline ZERO & & ZERO & & ZERO \\
\hline ZERO & & ZERO & & ZERO \\
\hline ZERO & & ZERO & & ZERO \\
\hline ZERO & & ZERO & & ZERO \\
\hline ZERO & & ZERO & & ZERO \\
\hline ZERO & & ZERO & & ZERO. \\
\hline ZERO & & ZERO & & ZERO \\
\hline ZERO & & ZERO & & ZERO \\
\hline ZERO & & ZERO & & ZERO \\
\hline ZERO & & ZERO & & ZERO \\
\hline ZERO & & ZERO & & ZERO \\
\hline ZERO & & ZERO & & ZERO \\
\hline ZERO & & ZERO & & ZERO \\
\hline ZERO & & ZERO & & ZERO \\
\hline ZERO & & ZERO & & ZERO \\
\hline ZERO & & ZERO & & ZERO \\
\hline ZERO & & ZERO & & ZERO \\
\hline ZERO & & ZERO & & ZERO \\
\hline ZERO & & ZERO & & ZERO \\
\hline $0.3423 \mathrm{E}+00$ & 0.3418 & $3 E+00$ & & ZERO \\
\hline ZERO & & ZERO & & ZERO \\
\hline ZERO & & ZERO & & ZERO \\
\hline ZERO & & ZERO & & ZERO \\
\hline ZERO & & ZERO & & ZERO \\
\hline ZERO & & ZERO & & ZERO \\
\hline ZERO & & ZERO & & ZERO \\
\hline ZERO & & ZERO & & ZERO \\
\hline ZERO & & ZERO & & ZERO \\
\hline ZERO & & ZERO & & ZERO \\
\hline ZERO & & ZERO & & ZERO \\
\hline ZERO & & ZERO & & ZERO \\
\hline ZERO & & ZERO & & ZERO \\
\hline ZERO & & ZERO & & ZERO \\
\hline ZERO & & ZERO & & ZERO \\
\hline & & & & \\
\hline
\end{tabular}

Section 3A, Page 4 of 17 
Table A-1. HLW Flowsheet Material Balance (Tank 51/ITP Cycle 1) section 3A. Precipitate Hydrolysis in Salt Cell

STREAM NUMBERS (CONT'D) $\rightarrow$ STREAM NAME

$38 \quad 502$

$141 \mathrm{SO} 3$

137 Semi Vol Cs2O

136 Semi Vol CsC1

126 Semi Vol Group A

140 Semi Vol Na2B407

138 Semi Vol NaCl

139 Semi Vol NaF

125 Semi Vol NaI
23 Semi Vol RuO2

23 Semi

$48 \mathrm{sr}(\mathrm{COOH}) 2$

$176 \mathrm{Sr}$ (NO3) 2

153 Sr (NaTi2O5) 2

$127 \mathrm{Sx}(\mathrm{OH}) 2$

$30 \quad \operatorname{srcO} 3$

46 Sro

159 TCO2

92 ThO2

119 Tritium

$\begin{array}{rl}119 & \text { Tritium } \\ 17 & \mathrm{u} 308\end{array}$

$\begin{array}{ll}17 & \mathrm{U} 308 \\ 62 \mathrm{UO} 2(\mathrm{COOH}) 2\end{array}$

177002 (NO3) 2

$16 \mathrm{VO} 2(\mathrm{OH}) 2$

27 Y (COOH) 3

$178 \mathrm{Y}(\mathrm{NO} 3)$

128. $\mathrm{Y}(\mathrm{OH}) 3$

$31 \mathrm{Y} 2(\mathrm{CO})$

100 Zeolite

$\begin{array}{rl}40 & \mathrm{Zn} \text { (COOH) }\end{array}$

44 Zn (COOH) 2
$179 \mathrm{Zn}(\mathrm{NO} 3)^{2}$

$57 \mathrm{Zno}$

$104 \mathrm{zrO} 2$

13 hydrate $\mathrm{H} 2 \mathrm{O}$

1 water

TOTAL FLOW, LB/HR

TEMPERATURE, DEG C

PRESSURE, ATM

PRESSURE， PSIG

PRESSURE, MM HG

ENTHALPY, PCU/HR

VAPOR FLOW, CFM

LAPOR FLOW, CFM

DENSITY, LBS/FT3

PHASE

657

Prec Feed

Solubles

ZERO

ZERO

ZERO

ZERO

ZERO

ZERO

ZERO

ZERO

ZERO

ZERO

ZERO

$0.7410 \mathrm{OE}-12$

ZERO

ZERO

ZERO

(ERO

$0.1282 \mathrm{E}-11$

ZERO

ZERO

$0.1074 \mathrm{E}-05$

ZERO

$0.8232 \mathrm{E}-12$

ZERO

ZERO

ZERO

$0.5336 \mathrm{E}-04$

ZERO

ZERO

ZERO
$0.4274 \mathrm{E}+03$

$0.4286 \mathrm{E}+03$

$0.3619 \mathrm{E}+02$

$0.9656 \mathrm{E}+00$

$-0.5055 \mathrm{E}+00$

$0.7339 \mathrm{E}+03$

$0.1551 E+05$

$0.8551 E+00$

$6249 E+02$
LIQUID

658

201.

Feed Washd Precip

Insolubles Feed frm LWF $\begin{array}{ll}\text { ZERO } & \text { ZERO } \\ \text { ZERO } & \text { ZERO }\end{array}$ ZERO ZERO ZERO $\quad$ ZERO

ZERO

ZERO

ZERO

ZERO

ZERO

ZERO

$0.2666 \mathrm{E}-01$

ZERO

$0.4072 \mathrm{E}-02$

ZERO

$0.9081 E-04$

ZERO

ZERO

$0.1213 E-02$

ZERO.

ZERO

ZERO

ZERO

ZERO ZERO

$0.2666 \mathrm{E}-01$

ZERO

$0.4072 \mathrm{E}-02$

$0.7410 \mathrm{E}-12$

$0.9081 E-04$

ZERO

ZERO

$0.1213 \mathrm{E}-02$

$0.1282 \mathrm{E}-11$

$0.7512 \mathrm{E}-03 \quad 0.7512 \mathrm{E}-03$

ZERO

$0.6751 \mathrm{E}-13$

ZERO

ZERO

ZERO

$0.9683 \mathrm{E}-03$

ZERO

$0.1980 \mathrm{E}+00$

ZERO

$0.3589 \mathrm{E}-01$

ZERO
$\mathbf{E}+02$

ZERO

$0.1074 \mathrm{E}-05$

ZERO

$0.8232 \mathrm{E}-12$

0.9683 ZERO

ZERO

ZERO

$0.5336 \mathrm{E}-04$

ZERO

$0.3589 \mathrm{E}-01$

$0.4274 \mathrm{E}+03$
$0.4762 \mathrm{E}+03$

$0.3619 \mathrm{E}+02$

$0.9656 \mathrm{E}+00$

$-0.5055 \mathrm{E}+00$

$0.7339 \mathrm{E}+03$
$0.2660 \mathrm{E}+02$

$0.3619 \mathrm{E}+02$

$0.9656 \mathrm{E}+00$

$-0.5055 \mathrm{E}+00$

$0.7339 \mathrm{E}+03$

$0.8411 \mathrm{E}-01$

$0.9392 \mathrm{E}+00$

$0.7060 \mathrm{E}+02$

$0.6322 \mathrm{E}+02$

I.IQUID
- 202

PRFT CO2

Purge
zERO

ZERO

ZERO

ZERO

ZERO

ZERO

ZERO

ZERO

ZERO

ZERO

ZERO

ZERO

ZERO

ZERO

ZERO

ZERO

ZERO

ZERO

ZERO

ZERO

ZERO

ZERO

ZERO

ZERO

ZERO

ZERO

ZERO

ZERO

ZERO

ZERO

ZERO

ZERO

ZERO

ZERO

ZERO

ZERO

$0.1471 \mathrm{E}+02$

$0.3500 \mathrm{E}+02$

$.1000 \mathrm{E}+01$

ZERO

$0.9847 \mathrm{E}+03$

$0.2256 \mathrm{E}+01$

$0.1087 \mathrm{E}+00$

VAPOR
203 PRFT

ir Inlkge

ZERO

ZERO

ZERO

ZERO

ZERO

ZERO

ZERO

ZERO

ZERO

ZERO

ZERO

ZERQ

ZERO

ZERO

ZERO

ZERO

ZERO

ZERO

ZERO

ZERO

ZERO

ZERO

ZERO

ZERO

ZERO

ZERO

ZERO

ZERO

ZERO

ZERO

ZERO

ZERO

ZERO

$0.2478 \mathrm{E}-01$

$0.1500 \mathrm{E}+01$

$0.3500 \mathrm{E}+02$

$0.1000 \mathrm{E}+01$

$$
\text { ZERO }
$$

$0.3146 \mathrm{E}+02$

$0.3543 \mathrm{E}+00$

$0.7055 \mathrm{E}-01$

VAPOR

Section 3A, Page 5 of 17

204

216

RET vent PRFT Trnsfer

sCVC Pump Prime ZERO ZERO ZERO ZERO ZERO

ZERO

ZERO ZERO

ZERO ZERO

$\begin{array}{ll}\text { ZERO } & \text { ZERO } \\ \text { ZERO } & \text { ZERO }\end{array}$

ZERO ZERO

ZERO ZERO

ZERO

ZERO ZERO

ZERO ZERO

ZERO ZERO

ZERO ZERO

ZERO ZERO

ZERO $\quad$ ZERO

ZERO ZERO

ZERO ZERO

ZERO ZERO

ZERO ZERO

ZERO : ZERO

ZERO $\quad$ ZERO

ZERO

ZERO ZERO

ZERO ZERO 
Table A-1. HLW Flowsheet Material Balance (Tank 51/ITP Cyole 1)

STREAM NUMBERS $\rightarrow$ STREAM NAME

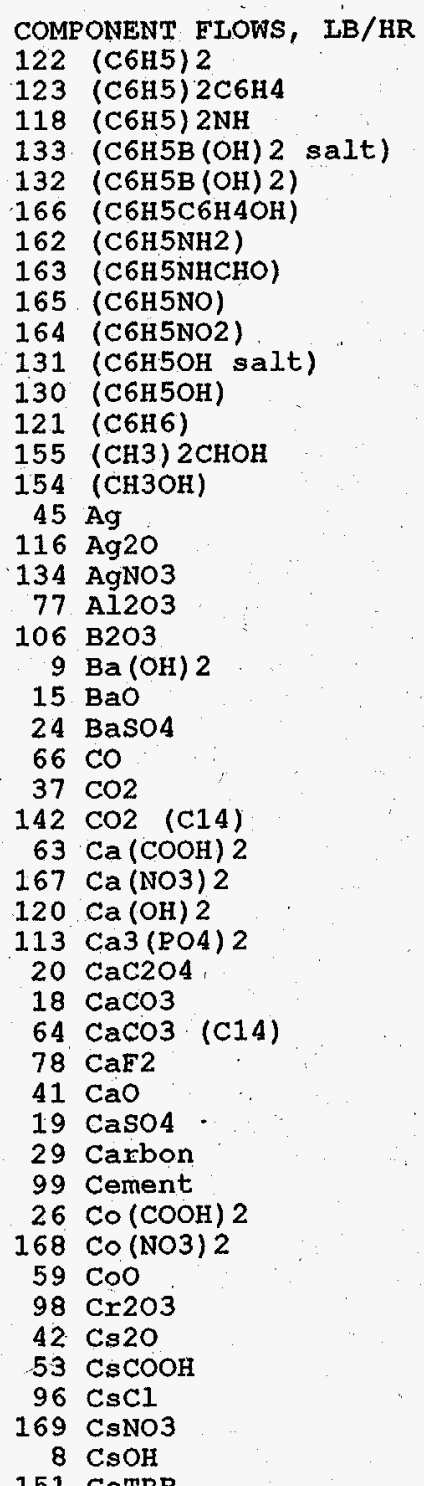


Table A-1. HLW Flowsheet Material Balance (Tank 51/TTP Cycle 1) Seation 3A. Precipitate Hydrolysis in salt Cell

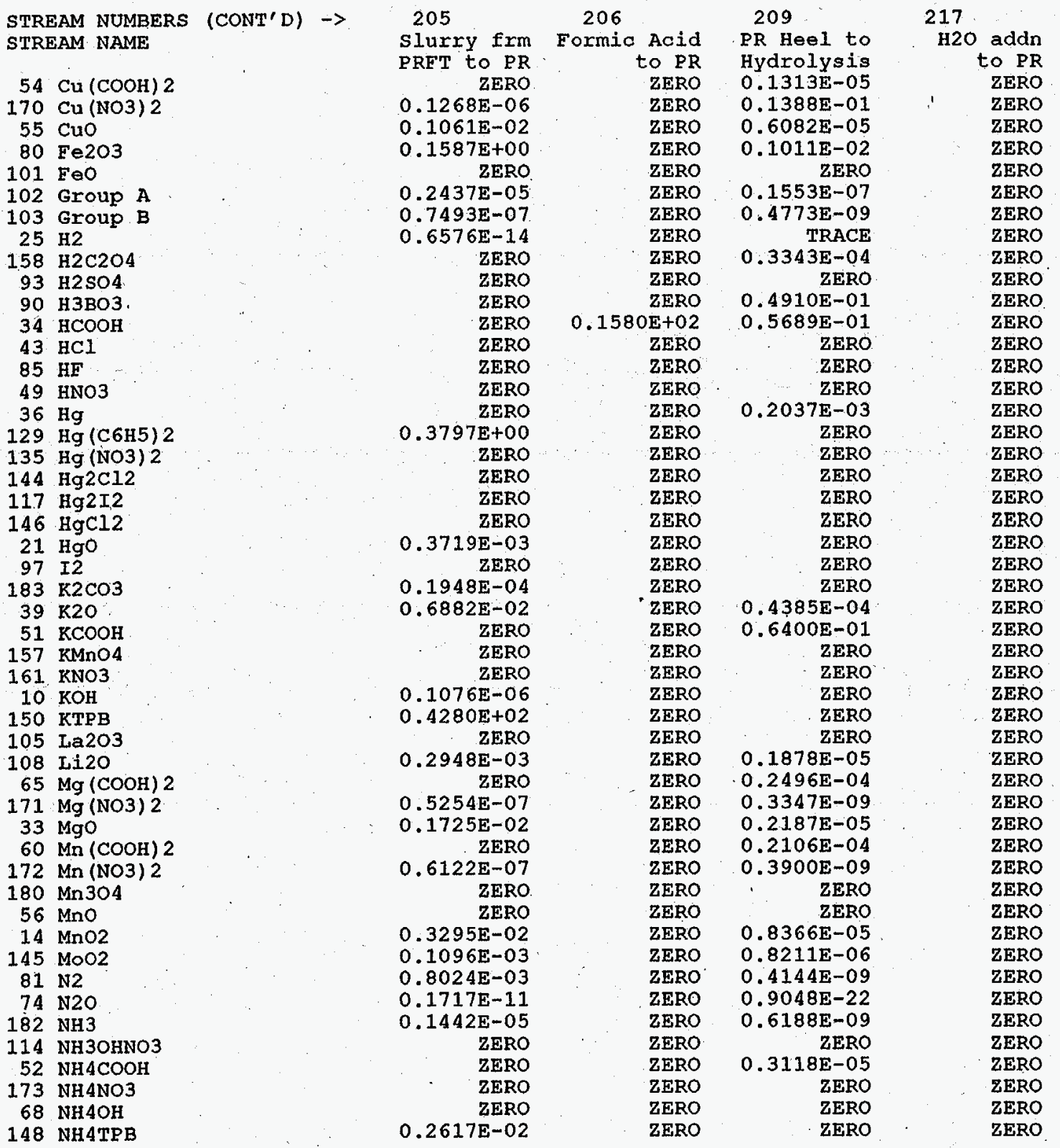

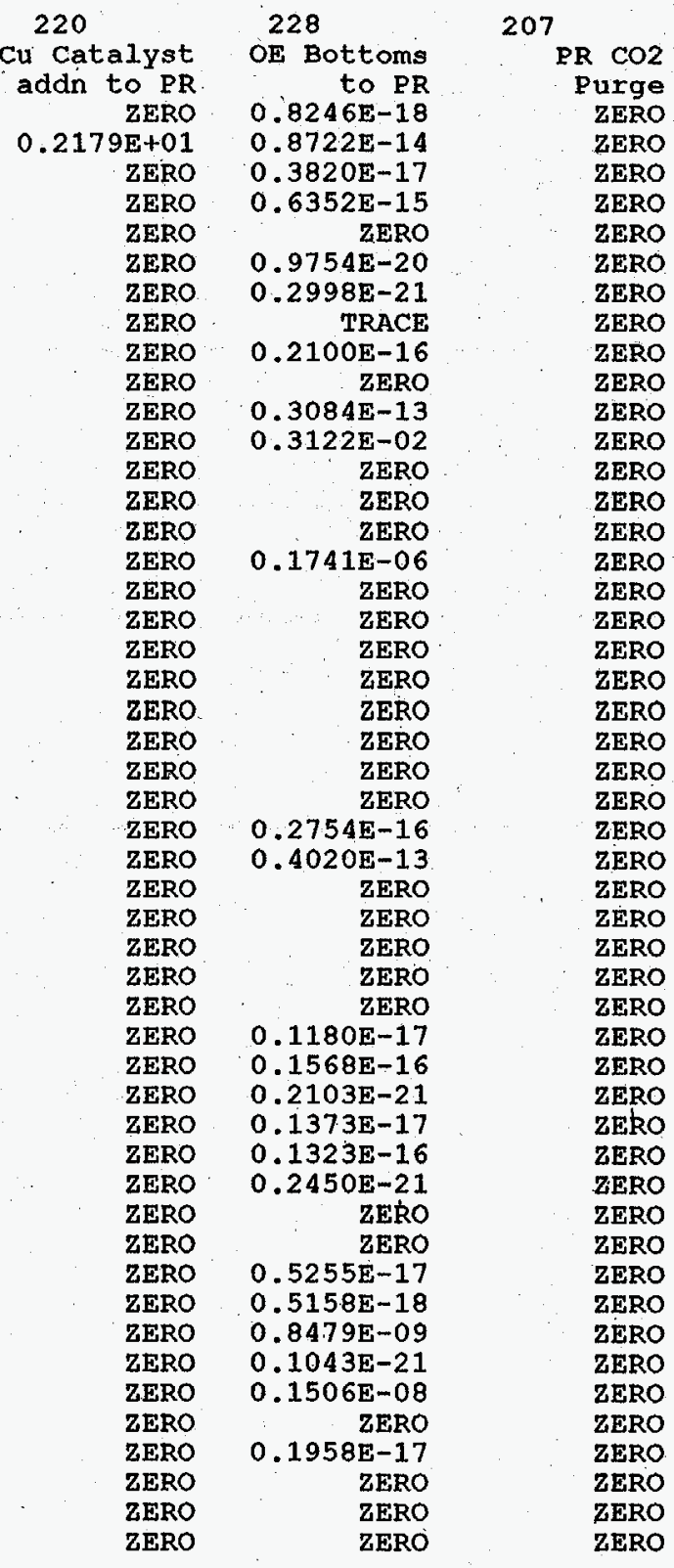


Table A-1. HLW Flowsheet Material Balance (Tank 51/ITP Cycle 1) Section. 3A. Precipitate Hydrolysis in Salt Cell

STREAM NUMBERS (CONT'D) $\rightarrow$ STREAM NAME

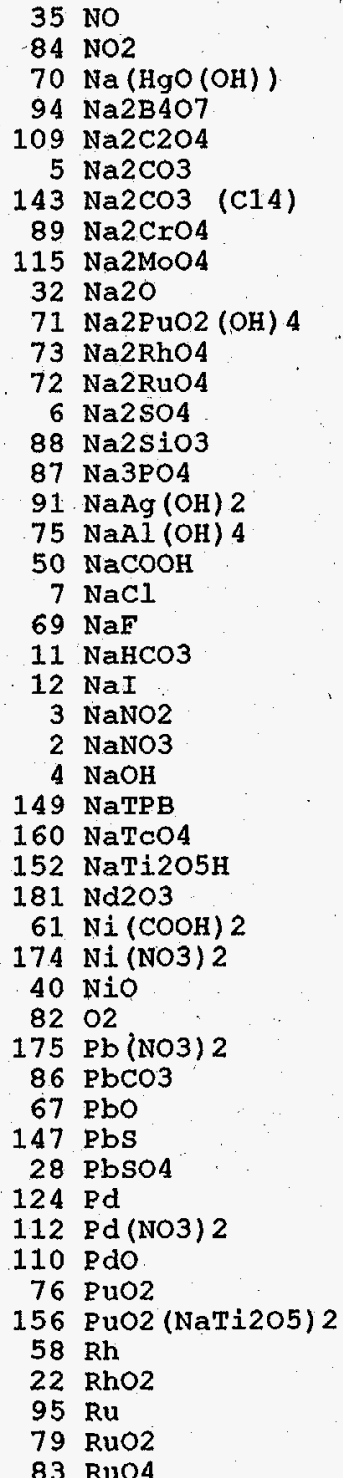

Reel to

$0.2210 \mathrm{E}-13$

$0.1756 \mathrm{E}-20$

ZERO

ZERO

ZERO

ZERO

ZERO

ZERO

ZERO

ZERO

ZERO

ZERO

ZERO

ZERO

ZERO

ZERO

ZERO

ZERO

ZERO

ZERO

ZERO.

ZERO

ZERO

ZERO

ZERO

ZERO

ZERO

ZERO

ZERO

ZERO

ZERO

ZERO

ZERO

ZERO

ZERO

ZERO

ZERO

ZERO

ZERO

ZERO

ZERO

ZERO$$
217
$$

20 addn 220

to $P R$

Cu Catalyst

ZFRO addn to PR

ZERO ZERO

ZERO $\quad$ ZERO

ZERO ZERO

ZERO

ZERO ZERO

ZERO

ZERO ZERO

ZERO ZERO

ZERO ZERO

ZERO ZERO

ZERO ZERO

ZERO ZERO

ZERO ZERO

ZERO ZERO

ZERO ZERO

ZERO $\quad$ ZERO

ZERO

ZERO ZERO

ZERO

ZERO

ZERO

ZERO ZIERO

ZERO ZERO

ZERO ZERO

ZERO ZERO

ZERO ZERO

ZERO : ZERO

ZERO ZERO

$\begin{array}{ll}\text { ZERO } & \text { ZERO } \\ \text { ZERO } & \text { ZERO }\end{array}$

ZERO ZERO

ZERO ZERO

ZERO 'ZERO

ZERO ZERO

ZERO ZERO

ZERO ZERO

ZERO

ZERO ZERO

ZERO ZERO

ZERO.$~ Z E R O$

ZERO

ZERO

ZERO

ZERO

ZERO

ZERO

ZERO
228

E Bottoms

to PR

$0.1256 \mathrm{E}-16$

$0.9981 \mathrm{E}-24$

ZERO

ZERO

ZERO

ZERO

ZERO

$0.4015 \mathrm{E}-14$

ZERO

ZERO

$0.4331 \mathrm{ZERO}$

$\mathrm{E}-17$

$0.5751 \mathrm{E}-17$

ZERO

0.4556

$0.4556 \mathrm{E}-14$

$0.3330 \mathrm{E}-18$

$0.2230 \mathrm{E}-21$

$0.8805 \mathrm{E}-16$

ZERO

ZERO

ZERO

ZERO

ZERO

$0.2676 \mathrm{E}-20$

$0.3317 \mathrm{E}-17$

$0.1454 \mathrm{E}-08$

$0.6497 \mathrm{E}-20$

ZERO

ZERO

$0.1746 \mathrm{E}-16$

$$
\text { ZERO }
$$

ZERO

$0.1284 \mathrm{E}-18$

$0.5090 \mathrm{E}-18$

$0.1456 \mathrm{E}-20$

ZERO

ZERO

ZERO

ZERO

ZERO

207 PR $\mathrm{CO} 2$

Purge

ZERO

ZERO

ZERO

ZERO

ZERO

ZERO

ZERO

ZERO

ZERO

ZERO

ZERO

ZERO

ZERO

ZERO

ZERO

ZERO

2 ERO

ZERO

ZERO

ZERO

ZERO

ZERO

ZERO

ZERO

ZERO

ZERO

ZERO

ZERO

ZERO

ZERO

ZERO

ZERO

ZERO

ZERO

ZERO

ZERO

ZERQ

ZERO 
Table A-1. HLW Flowsheet Material Balance (Tank 51/ITP Cycle 1 ) section 3A. Precipitate Hydrolysis in Salt Cell

STREAM NUMBERS (CONT'D) -> STREAM NAME

$\begin{array}{rr}38 & \mathrm{SO} 2 \\ 141 & \mathrm{SO} 3\end{array}$

137 Semi Vol Cs20

136 Semi Vol CsCl

126 Semi Vol Group A

140 Semi Vol Na2B4O

138 Semi Vol NaCl

139 Semi Vol NaF

$\begin{aligned} 125 & \text { Semi Vol NaI } \\ 23 & \text { Semi Vol RuO2 }\end{aligned}$

111 sio2

$48 \mathrm{Sr}(\mathrm{COOH}) 2$

$176 \mathrm{Sr}$ (NO3) 2

$153 \mathrm{Sr}$ (NaTi205) 2

$127 \mathrm{Sr}(\mathrm{OH}) 2$

$30 \mathrm{SrCO} 3$

46 Sro

$159 \mathrm{TcO} 2$

92 ThO2

119 Tritium

119 v308

$\begin{array}{ll}17 & \mathrm{U} 308 \\ 62 \mathrm{UO} 2 \text { (COOH) } 2\end{array}$

$177 \mathrm{NO2}(\mathrm{NO3}) 2$

$16 \mathrm{VO} 2(\mathrm{OH}) 2$
$27 \mathrm{Y}(\mathrm{COOH}) 3$

$27 \mathrm{Y}(\mathrm{COOH})$

$178 \mathrm{Y}(\mathrm{NO} 3)$

$128 \mathrm{Y}(\mathrm{OH}) 3$

$47 \mathrm{Y} 203$

100 zeolite

$44 \mathrm{Zn}(\mathrm{COOH}) 2$

$179 \mathrm{Zn}$ (NO3) 2

$57 \mathrm{ZnQ}$

104 ZrO2

13 hydrate $\mathrm{H} 2 \mathrm{O}$

1 water

TOTAL FLOW, LB/HR

TEMPERATURE, DEG C

PRESSURE, ATM

PRESSURE， PSIG

PRESSURE， MM HG

ENTHALPY, PCU/HR

VAPOR FLOW, CFM

LIOUID FLOW, GPM

DENSITY, LBS/FT3

PHASE

$\begin{array}{lc}205 & 206 \\ \text { Slurry frm } & \text { Formic Acid } \\ \text { PRET to PR } & \text { to PR }\end{array}$

PRET to PR

ZERO

ZERO

ZERO

ZERO

ZERO

ZERO

ZERO

ZERO

$0.2666 \mathrm{E}-01$

ZERO

ZERO
$0.4072 \mathrm{E}-02$

$0.7410 \mathrm{E}-12$

$0.9081 \mathrm{E}-04$

ZERO

ZERO

$0.1213 \mathrm{E}-02$

$0.1280 \mathrm{E}-11$

$0.7512 \mathrm{E}-03$ ZERO

$0.1074 \mathrm{E}-05$

ZERO

ZERO

$0.8232 \mathrm{E}-12$

ZERO
$0.9683 E-03$
ZERO

ZERO

$0.5336 \mathrm{E}-04$

$0.1980 \mathrm{E}+00$

ZERO

$.3589 E-01$

$0.4308 \mathrm{E}+03$
$0.4800 \mathrm{E}+03$

$0.3999 \mathrm{E}+02$

$0.9656 \mathrm{E}+00$

$-0.5055 \mathrm{E}+00$

$0.7339 \mathrm{E}+03$

$0.1731 \mathrm{E}+05$

$0.9466 \mathrm{E}+00$

$0.6322 \mathrm{E}+02$

IIQUID
209

RR Heel to

Hydrolysis

ZERO

ZERO

ZERO

ZERO

ZERO

ZERO

ZERO

ZERO

ZERO

ZERO

ZERO

ZERO

ZERO

ZERO

ZERO

ZERO

ZERO

ZERO

ZERO

ZERO

ZERO

ZERO

ZERO

ZERO

ZERO

ZERO

ZERO

ZERO

ZERO

ZERO

ZERO

ZERO

$0.1717 \mathrm{E}+01$

$0.1752 \mathrm{E}+02$

$0.2500 \mathrm{E}+02$

$0.1000 \mathrm{E}+01$

ZERO

$0.2457 \mathrm{E}+03$

$0.2930 \mathrm{E}-01$

$7454 \mathrm{E}+02$

LIOUID

$0.3399 E-06$

$0.8812 \mathrm{E}-03$

ZERO
$0.1386 \mathrm{E}-03$

$0.1386 \mathrm{E}-03$
$0.4698 \mathrm{E}+01$

$0.4920 \mathrm{E}+01$

$0.5000 \mathrm{E}+02$

$0.9656 \mathrm{E}+00$ $-0.5055 \mathrm{E}+00$

$0.7339 \mathrm{E}+03$

$0.9690 \mathrm{E}-02$

$0.6330 \mathrm{E}+02$

IIQUID
217

H2O addn Cu Catalyst to $P R$ addn to $P R$

ZERO

ZERO

ZERO

ZERO

ZERO

ZERO

ZERO

ZERO

ZERO

ZERO

ZERO

ZERO

ZERO

ZERO

ZERO

ZERO

ZERO

ZERO

ZERO

ZERO

ZERO

ZERO

ZERO

ZERO

ZERO

ZERO

ZERO

ZERO

ZERO

ZERO

ZERO

ZERO

ZERO

ZERO

ZERO

ZERO
$0.2074 \mathrm{E}+03$ $0.2074 \mathrm{E}+03$

$0.3500 \mathrm{E}+02$

$0.1000 \mathrm{E}+01$

ZERO

$0.7278 \mathrm{E}+04$

$0.6243 \mathrm{E}+02$

LIOUID
220

228

ZERO
ZERO

ZERO

ZERO

ZERO

ZERO

ZERO

ZERO

ZERO

ZERO

ZERO

ZERO

ZERO

ZERO

ZERO

ZERO

ZERO

ZERO

ZERO

ZERO

ZERO

ZERO

ZERO

ZERO

ZERO

ZERO

ZERO

ZERO

ZERO

ZERO

ZERO

ZERO

$0.1274 \mathrm{E}+02$

$0.1492 \mathrm{E}+02$

$0.2500 \mathrm{E}+02$

$0.9690 \mathrm{E}+00$

$-0.4556 \mathrm{E}+00$

$0.7364 \mathrm{E}+03$

$0.2733 \mathrm{E}-01$
$0.6807 \mathrm{E}+02$

IIQUID

E Bottoms

to $P R$

ZERO

ZERO

ZERO

ZERO

ZERO

ZERO

ZERO

ZERO

ZERO

$0.1067 \mathrm{E}-15$

$0.4373 \mathrm{E}-18$

ZERO
$0.1630 \mathrm{E}-16$

ZERO
ZERO

ZERO

ZERO

$0.7046 \mathrm{E}-20$

ZERO

$0.3139 \mathrm{E}-14$

$.4099 \mathrm{E}-14$

$0.3006 \mathrm{E}-17$

$0.5091 \mathrm{E}-20$

ZERO

ZERO

TRACE

ZERO

ZERO

$0.3875 \mathrm{E}-17$

ZERO

$0.4559 \mathrm{E}-15$

$0.2135 E-18$

$0.5535 \mathrm{E}-15$

ZERO

$0.7324 \mathrm{E}+02$

$0.5000 \mathrm{E}+02$

$0.9656 \mathrm{E}+00$

$-0.50 .55 \mathrm{E}+00$

$0.7339 \mathrm{E}+03$

$0.1463 \mathrm{E}+00$

$0.6243 \mathrm{E}+02$

LIQUID

207

PR $\mathrm{CO} 2$
Purge

ZERO

ZERO

ZERO

ZERO

ZERO

ZERO 
Table A-1. HLW Flowsheet Material Balance (Tank 51/ITP Cycle 1) section 3A. Precipitate Hydrolysis in salt Cell

STREAM NUMBERS $\rightarrow$ STREAM NAME

COMPONENT FLOWS, LB/HR $122(\mathrm{C} 6 \mathrm{H} 5) 2$

123 (C6H5) $2 \mathrm{C} 6 \mathrm{H} 4$

118 (C6H5) $2 \mathrm{NH}$

133 (C6H5B (OH) 2 salt)

132 (C6H5B (OH) 2)

166 (C6H5C6H4OH)

162 (C6H5NH2)

163 (C6H5NHCHO)

164 (C6H5NO2)

131 (C6H5OH-salt)

130 ( $6 \mathrm{H} 5 \mathrm{OH})$

121 ( $\mathrm{C} 6 \mathrm{H} 6)$

155 ( $\mathrm{CH} 3) 2 \mathrm{CHOH}$

154 (СН3ОH)

$45 \mathrm{Ag}$

$\begin{array}{ll}116 & \mathrm{Ag} 2 \mathrm{O} \\ 134 & \mathrm{AgNO}\end{array}$

$34 \mathrm{AgNO}$
$77 \mathrm{Al2O}$

$106 \mathrm{~B} 203$

$9 \mathrm{Ba}(\mathrm{OH}) 2$

$15 \mathrm{BaO}$

$24 \mathrm{BaSO} 4$

$66 \mathrm{CO}$

$42 \mathrm{CO} 2(\mathrm{C} 14)$

$142 \mathrm{CO} 2(\mathrm{C14})$
$63 \mathrm{Ca}(\mathrm{COOH}) 2$

$167 \mathrm{Ca}(\mathrm{NO} 3)^{2} 2$

$120 \mathrm{Ca}(\mathrm{OH}) 2$

$113 \mathrm{Ca} 3$ (PO4) 2

$20 \mathrm{CaC} 204$

$18 \mathrm{CaCO} 3$

$64 \mathrm{CaCO} 3(\mathrm{C} 14)$

78 CaF2

$41 \mathrm{CaO}$

19 CaSO4

29 Carbon

26 Co $(\mathrm{COOH}) 2$

$168 \mathrm{Co}(\mathrm{NO} 3) 2$

$59 \mathrm{CoO}$

$98 \mathrm{Cr} 203$
$42 \mathrm{Cs} 2 \mathrm{O}$

$53 \mathrm{CsCOOH}$

$96 \mathrm{CsCl}$

169 CsNO3

$8 \mathrm{CsOH}$

WSRC-TR-95-0019 (Revision
$266 \quad 211215 \quad 212$ 265

PR Trisfer

Pump Prime

RR Sampler

yydrolys/PR Hydroly/PRC

214

210

210

ZERO $0.9887 E+00 \quad 0.1254 E+00-0.8632 E+00$

ZERO ZERO $0.1329 \mathrm{E}-07$

ZERO

ZERO

ZERO

ZERO

ZERO

ZERO

ZERO

ZERO

ZERO

ZERO

ZERO

ZERO

ZERO

ZERO

ZERO

ZERO

ZERO

ZERO

ZERO

ZERO

ZERO

ZERO

ZERO

ZERO

ZERO

ZERO

ZERO

ZERO

ZERO

ZERO

ZERO

ZERO

ZERO

ZERO

ZERO

ZERO

ZERO

ZERO

ZERO

ZERO

ZERO

ZERO

ZERO

$\begin{array}{lll}0.9887 \mathrm{E}+00 & 0.1254 \mathrm{E}+00\end{array}$

$0.1329 \mathrm{E}-07$

$0.1008 \mathrm{E}-14$

$0.8632 \mathrm{E}+00$

$0.1329 \mathrm{E}-07$

$0.8629 \mathrm{E}+00$

$0.7092 \mathrm{E}-09$

R aq to $P R$

ZERO

ZERO ZERO

ZERO

ZERO

ZERO

ZERO

ZERO

ZERO

ZERO

ZERO

ZERO

ZERO

ZERO

ZERO

ZERO

ZERO

ZERO

ZERO

ZERO

ZERO

ZERO

ZERO

ZERO

ZERO

ZERO

ZERO

ZERO

ZERO

ZERO

ZERO

ZERO

ZERO

ZERO

ZERO

ZTRO

ZERO

ZERO

ZERO

ZERO

ZERO

ZERO

ZERO

$0.2055 \mathrm{E}-01$

ZERO

ZERRO

ZERO

$0.1004 \mathrm{E}+00$

$0.1004 \mathrm{E}+00$

$0.3632 \mathrm{E}+02$

$.1529 \mathrm{E}-05$

ZERO

$0.1142 \mathrm{E}-02$

ZERO

ZERO

ZERO

$0.4557 \mathrm{E}-02$

$0.2720 \mathrm{E}+02$

$0.2720 \mathrm{E}+02$
$0.8703 \mathrm{E}-12$

$0.3598 \mathrm{E}-12$

ZERO
ZERO

ZERO

ZERO

ZERO

ZERO

ZERO

ZERO

ZERO

$0.2446 \mathrm{E}+02$

$0.1202 \mathrm{E}-09$

ZERO
ZERO

ZERO

ZERO

ZERO

ZERO

ZERO

ZERO

ZERO

ZERO

ZERO

ZERO

ZERO

ZERO

ZERO

ZERO

ZERO.

ZERO

ZERO

ZERO

ZERO

ZERO

ZERO

ZERO

ZERO

ZERO

$0.2444 \mathrm{E}+02$

ZERO

ZERO

ZERO

ZERO

ZERO

ZERO

ZERO

ZERO

ZERO

ZERO

ZERO

ZERO

ZERO

ZERO

ZERO

ZERO

ZERO

ZERO

ZERO

ZERO
ZERO

Date: January 1995

$0.3319 \mathrm{E}-03$

$.1258 \mathrm{E}-07$

$0.1319 \mathrm{E}-04$

ZERO

ZERO

$0.1941 \mathrm{E}-01$

ZERO

ZERO

ZERO

$0.9583 \mathrm{E}-01$

$0.9123 \mathrm{E}+01$

$.9137 \mathrm{E}-11$

$0.9029 \mathrm{E}+01$

$0.4877 \mathrm{E}-12$

$0.8703 \mathrm{E}-13$

ZERO

ZERO

ZERO

ZERO

ZERO

ZERO

ZERO

ZERO

$0.2206 \mathrm{E}-01$

$0.1084 \mathrm{E}-12$

ZERO

ZERO

ZERO

ZERO

ZERO

ZERO

ZERO

ZERO

ZERO

ZERO

ZERO

ZERO

ZERO

ZERO

ZERO

ZERO

ZERO

ZERO

ZERO

ZERO

ZERO

ZERO

ZERO

ZERO

ZERO

ZERO

$0.1177 \mathrm{E}-02$

$0.1177 E-02$
$0.5787 E-14$

ZERO 
Table A-1. HLW Flowsheet Material Balance (Tank 51/ITP Cyclo 1) Section 3A. Precipitate Hydrolysis in Salt Cell.

STREAM NUMBERS (CONT'D) -> STREAM NAME

\section{$54 \mathrm{Cu}(\mathrm{COOH})$ \\ $170 \mathrm{Cu}(\mathrm{NO} 3) 2$ \\ $55 \mathrm{CuO}$ \\ $80 \mathrm{Fe} 2 \mathrm{O3}$ \\ $101 \mathrm{FeO}$ \\ 103 Group B \\ $25 \mathrm{H} 2$ \\ 158 H2C2O4 \\ $93 \mathrm{H} 2 \mathrm{SO} 4$ \\ $90 \mathrm{H} 3 \mathrm{BO} 3$. \\ 34. $\mathrm{HCOOH}$. \\ 43 HCl \\ $85 \mathrm{HF}$ \\ $36 \mathrm{Hg}$}

$129 \mathrm{Hg}(\mathrm{C} 6 \mathrm{H} 5)^{2}$

$135 \mathrm{Hg}$ (NO3) 2

$144 \mathrm{Hg} 2 \mathrm{Cl} 2$

$117 \mathrm{Hg} 2 \mathrm{I} 2$

$146 \mathrm{HgCl} 2$

$21 \mathrm{HgO}$

$\begin{array}{rl}97 & \mathrm{I} 2 \\ 183 & \mathrm{~K} 2 \mathrm{CO} 3\end{array}$

$39 \mathrm{~K} 2 \mathrm{O}$

$51 \mathrm{KCOOH}$

$157 \mathrm{KMnO} 4$

$161 \cdot \mathrm{KNO}$

$10 \mathrm{KOH}$

150 KTPB

$105 \mathrm{La}_{203}$

$65 \mathrm{Mg}$ (COOH)

$171 \mathrm{Mg}$ (NO3) 2

$33 \mathrm{Mgo}$

$60 \mathrm{Mn}(\mathrm{COOH}) 2$

$172 \mathrm{Mn}(\mathrm{NO} 3) 2$

$180 \mathrm{Mn} 304$

$56 \mathrm{MnO}$

$14 \mathrm{MnO} 2$

$145 \mathrm{MOO} 2$

$81 \mathrm{~N} 2$

74 N2O

114 NH3OHNO3

52 NH4 COOH

173 NH4NO3

$68 \mathrm{NH} 4 \mathrm{OH}$

148 NH4TPB

WSRC-TR-95-0019 (Revision
265

PR Trnsfer

Pump Prime

ZERO

ZERO

ZERO

ZERO

ZERO

ZERO

ZERO

ZERO

ZERO

ZERO

ZERO

ZERO

ZERO

ZERO

ZERO

ZERO

ZERO

ZERO

ZERO

ZERO

ZERO

ZERO

ZERO

ZERO

ZERO

ZERO

ZERO

ZERO

ZERO

ZERO

ZERO

ZERO

ZERO

ZERO

ZERO

ZERO

ZERO

ZERO

ZERO

ZERO

ZERO

ZERO
266

Sampler Hydroly/CO2 215

Hydrolys/PR

212

Purge / PR vnt to sCVC Cndst to PRD ZERO

ZERO

ZERO

ZERO

ZERO

ZERO

ZERO

ZERO

ZTRO

ZERO

ZERO

ZERO

ZERO

ZERO

ZERO

ZERO

ZERO

ZERO

ZERO

ZERO

ZERO

ZERO

ZERO

ZERO

ZERO

ZERO

ZERO

ZERO

ZERO

ZERO

ZERO

ZERO

ZERO

ZERO

ZERO

ZERO

ZERO

ZERO

ZERO

ZERO

ZERO

ZERO

ZERO

ZERO ZERO ZERO ZERO ZERO ZERO ZERO

ZERO

ZERO

ZERO

ZERO

ZERO

ZERO

$0.2297 \mathrm{E}+00$

ZERO

ZERO

$0.3968 \mathrm{E}-05$

ZERO

ZERO

ZERO

ZERO

ZERO

ZERO

ZERO

ZERO

ZERO

ZERO

ZERO

ZERO

ZERO

ZERO

ZERO

ZERO

ZERO

ZERO

ZERO

ZERO

ZERO

ZERO

$0.8024 \mathrm{E}-03$

$0.1716 \mathrm{E}-11$

ZERO

ZERO

ZERO

ZERO

ZERO

Date: January 1995 ZERO ZERO ZERO

$0.3410 \mathrm{E}-02$ ZERO ZERO ZERO

$0.2672 \mathrm{E}-10$

ZERO

ZERO

ZERO

ZERO

ZERO

ZERO

ZERO

ZERO

ZERO

ZERO

ZERO

ZERO

ZERO

ZERO

ZERO

ZERO

ZERO

ZERO

ZERO

ZERO

ZERO

ZERO

ZERO

$0.8023 \mathrm{E}-03$

$0.1715 \mathrm{E}-11$

$8 \mathrm{E}-05$

ZERO

ZERO

ZERO

ZERO

ZERO
$0.2263 E+00$

ZERO

ZERO

$0.3968 \mathrm{E}-05$

$8 E-05$
ZERO
ZERO

ZERO

ZERO

ZERO

ZERO

ZERO

ZERO

ZERO

ZERO

ZERO

ZERO

ZERO

ZERO

ZERO

ZERO

ZERO

ZERO

ZERO

ZERO

ZERO

ZERO

$0.2275 \mathrm{E}-07$

$0.9351 \mathrm{E}-15$

$0.1435 \mathrm{E}-06$

ZERO

ZERO

ZERO

$\begin{array}{cc}214 & 210 \\ \text { Hydroly/PRD } & \text { Hydrolysis }\end{array}$

Org to OE PRD aq to PR ZERO ZERO ZERO ZERO ZERO ZERO ZERO ZERO ZERO ZERO ZERO ZERO

$\begin{array}{llll}\text { ZERO } & \text { ZERO } & \text { ZERO } & \text { ZERO } \\ \text { ZERO } & \text { ZERO } & \text { ZERO } & \text { ZERO }\end{array}$

$0.1298 \mathrm{E}-19$ ZERO ZERO

$0.2652 \mathrm{E}-03$ ZERO

$0.2301 \mathrm{E}-18$ ZERO

0.2261 EROO ZERO

ZERO
$2118 E-06$

ZERO

ZERO

ZERO

ZERO

ZERO

ZERO 


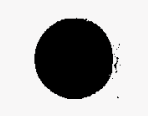

Table A-1. HLW Flowsheet Material Balance (Tank 51/ITP Cycle 1)

Section 3A: Precipitate Hydrolysis in Salt Cell.

STREAM NUMBERS (CONT'D) $\rightarrow$ STREAM NAME

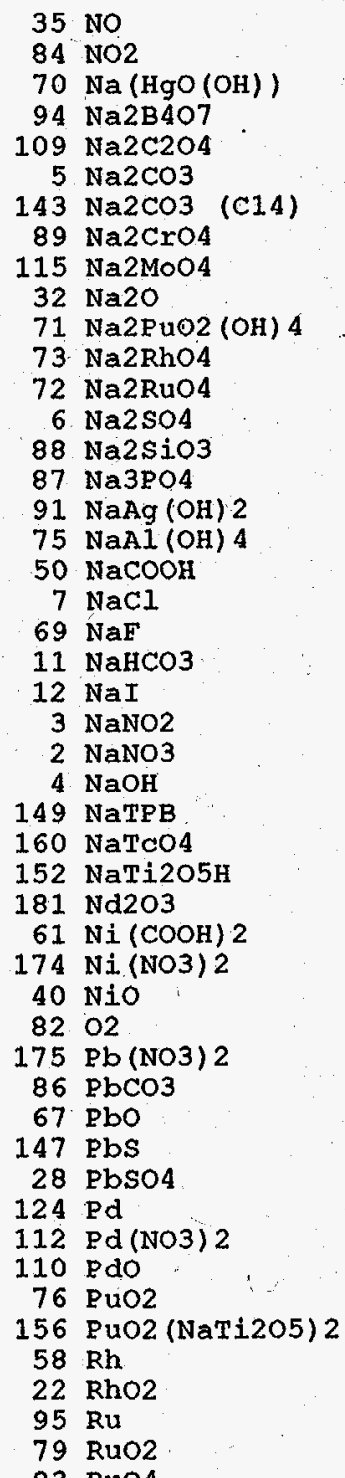

83 RuO4

WSRC-TR-95-0019 (Revision

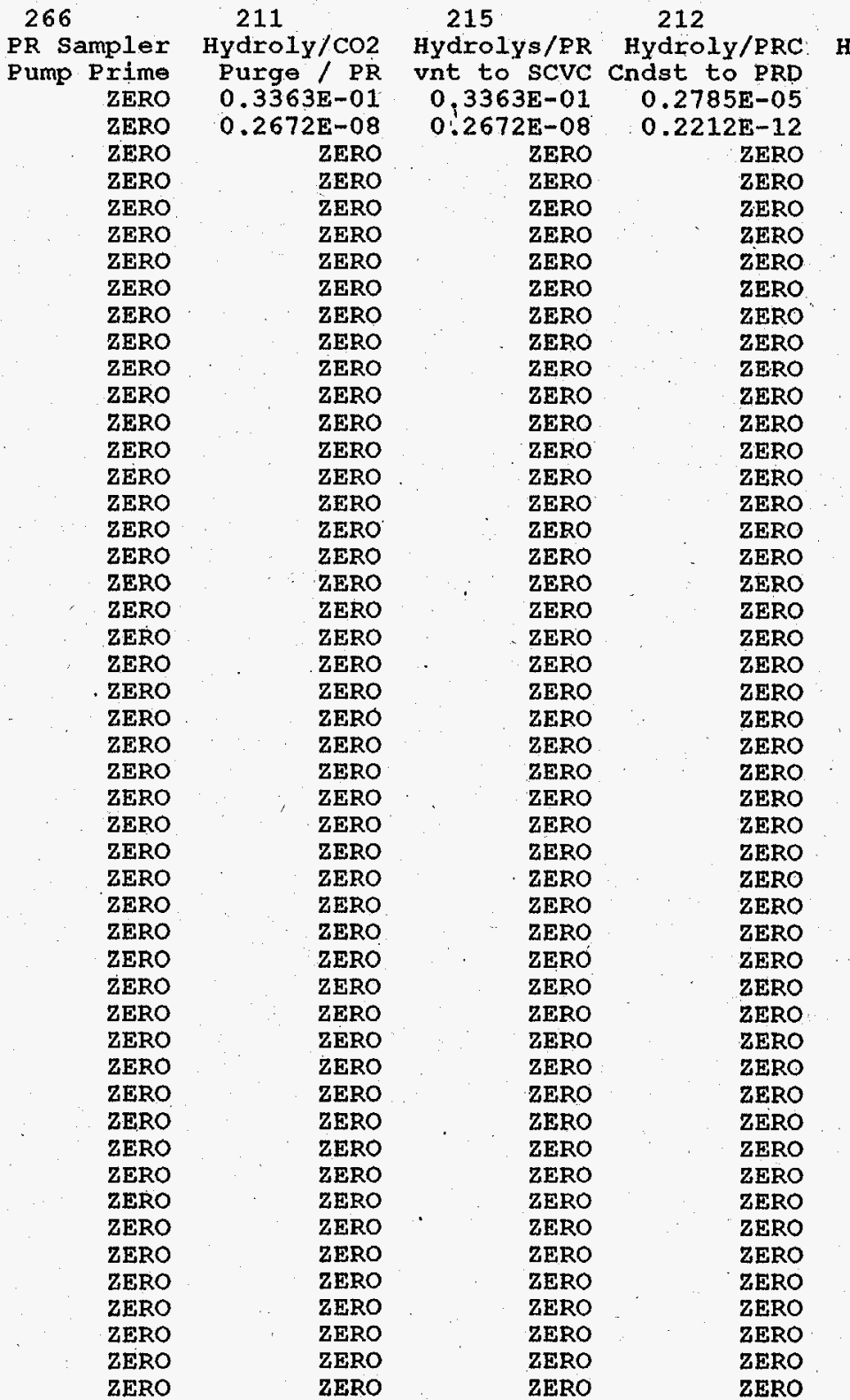


Table A-1. HLW Flowsheet Material Balance (Tank 51/ITP Cycle 1) section 3A. Precipitate Hydrolysis in Salt Cell

STREAM NUMBERS (CONT'D) $\rightarrow$ STREAM NAME

\footnotetext{
$38 \quad \mathrm{SO} 2$.

$141 \mathrm{SO} 3$

137 Semi Vol Cs2O

136 Semi Vol CsCl

126 Semi Vol Group A

140 Semi Vol Na2B4

138 Semi Vol $\mathrm{NaCl}$

139 Semi Vol NaF

$\begin{aligned} 125 & \text { Semi Vol NaI } \\ 23 & \text { Semi Vol RuO2 }\end{aligned}$

23 semi
111 SiO2

$48 \mathrm{sr}(\mathrm{COOH}) 2$

$176 \mathrm{Sr}$ (NO3) 2

$153 \mathrm{Sr}(\mathrm{NaTi2O5)} 2$

$127 \mathrm{Sr}(\mathrm{OH}) 2$

$30 \mathrm{srCO} 3$

46 sro

$159 \mathrm{TcO} 2$

92 ThO2

$107 \mathrm{TiO}$

119 Tritium

170308

$62 \cdot \mathrm{VO} 2(\mathrm{COOH}) 2$

$177 \mathrm{UO2}$ (NO3) 2

$16 \mathrm{VO} 2(\mathrm{OH}) 2$

27. Y ( $\mathrm{COOH}) 3$

178 Y (NO3)

$31 \mathrm{Y} 2(\mathrm{CO} 3) 3$

$31 \mathrm{Y} 2(\mathrm{CO})$
$47 \mathrm{Y} 2 \mathrm{O}$

100 Zeolite

$44 \mathrm{Zn}(\mathrm{COOH}) 2$

$179 \mathrm{Zn}$ (NO3) 2

$57 \mathrm{zno}$

$104 . \mathrm{zrO} 2$

13 hydrate $\mathrm{H} 2 \mathrm{O}$

1 water

TOTAL ELOW, LB/HR

TEMPERATURE， DEG C

PRESSURE, ATM

PRESSURE， PSIG

PRESSURE, MM HG

ENTHALPY, PCU/HR

VAPOR FLOW, CFM

LIQUID FLOW, GPM

DENSITY, LBS/FT3
}

PHASE

265

PR Trnsfer

266

Pump Prime

215

212

ZERO

ZERO

ZERO

ZERO

ZERO

ZERO

ZERO

ZERO

ZERO

ZERO

ZERO

ZERO

ZERO

ZERO

ZERO

ZERO

ZERO

ZERO

ZERO

ZERO

ZERO

ZERO

ZERO

ZERO

ZERO

ZERO

ZERO

ZERO

ZERO

ZERO

ZERO

$0.3900 \mathrm{E}+01$

$0.3900 \mathrm{E}+01$

$0.2500 \mathrm{E}+02$

$0.1000 \mathrm{E}+01$

ZERO

$0.9786 \mathrm{E}+02$

$0.7788 \mathrm{E}-02$

$0.6243 \mathrm{E}+02$
LIQUID rime

ZERO

ZERO

ZERO

ZERO

ZERO

ZERO

ZERO

ZERO

ZERO

ZERO

ZERO

ZERO

ZERO

ZERO

ZERO

ZERO

ZERO

ZERO

ZERO

ZERO

ZERO

ZERO

ZERO

ZERO

ZERO

ZERO

ZERO

ZERO

ZERO

ZERO

ZERO

ZERO

ZERO

$0.7800 \mathrm{E}+01$

$0.7800 \mathrm{E}+01$

$0.2500 \mathrm{E}+02$

$0.1000 \mathrm{E}+01$

ZERO

$0.1957 \mathrm{E}+03$

$0.1558 \mathrm{E}-01$

$0.6243 E+02$

LIQUID
IY $/ \mathrm{CO} 2$
211

ydro

Hydroly/PRC vnt to SCVC Cndst to PRD

$\begin{array}{lll}\text { ZERO } & \text { ZERO } & \text { ZERO } \\ \text { ZERO } & \text { ZERO } & \text { ZERO } \\ \text { ZERO } & \text { ZERO } & \text { ZERO }\end{array}$ ZERO

ZERO

ZERO

ZERO

ZERO

ZERO

ZERO

ZERO

ZERO

ZERO

ZERO

ZERO

ZERO

ZERO

ZERO ZERO

$0.7715 \mathrm{E}-13$

ZERO

ZERO

ZERO

ZERO

ZERO

ZERO

ZERO

ZERO

ZERO

ZERO

ZERO

ZERO

$0.4610 \mathrm{E}+02$

$0.1083 \mathrm{E}+03$

$0.8380 \mathrm{E}+02$

$-0.5055 \mathrm{E}+00$

$0.3593 E+05$

$0.2909 \mathrm{E}+02$

$0.6205 \mathrm{E}-01$

VAPOR

ZERO

ZERO

ZERO

ZERO

ZERO

ZERO

ZERO

ZERO

ZERO

ZERO

ZERO

ZERO

ZERO

ZERO

ZERO

ZERO

ZERO

ZERO

ZERO

ZERO

ZERO

ZERO

ZERO

ZERO

ZERO

ZERO

ZERO

ZERO

ZERO

ZERO

$0.2304 \mathrm{E}+01$

$0.5411 \mathrm{E}+02$

$0.5419 \mathrm{E}+02$

$0.5000 \mathrm{E}+02$

$0.9656 \mathrm{E}+00$

$0.5055 \mathrm{E}+00$

$0.5000 E+02$

$0.9656 \mathrm{E}+00$

$0.7339 \mathrm{E}+03 \quad 0.7339 \mathrm{E}+03$

$0.6408 \mathrm{E}+04$

$0.7575 \mathrm{E}+01$

$0.1191 \mathrm{E}+00$

VAPOR

$0.1115 \mathrm{E}+00$

$0.6060 \mathrm{E}+02$

IIQUID

Date: January 1995

Section 3A, Page 13 of 17 $\begin{array}{lc}214 & 210 \\ \text { Hydroly/PRD } & \text { Hydrolysis }\end{array}$

Org to OE $P R D$ aq to $P R$

ZERO $\cdots$ ZERO

ZFRO

ZERO

ZERO

ZERO

ZERO ZERO

ZERO ZERO

$\begin{array}{ll}\text { ZERO } & \text { ZERO } \\ \text { ZERO } & \text { ZERO }\end{array}$

ZERO ZERO

ZERO ZERO

ZERO ZERO

ZERO ZERO

ZERO ZERO

ZERO

ZERO

ZERO ZERO

$0.9282 \mathrm{ERT}$

ZERO ZERO

ZERO ZERO

ZERO ZERO

ZERO ZERO

ZERO ZERO

ZERO ZERO

ZERO ZERO

ZERO ZERO

ZERO ZERO

ZERO ZERO

$\begin{array}{ll}\text { ZERO } & \text { ZERO } \\ \text { ZERO } & \text { ZERO }\end{array}$

ZERO ZERO

ZERO
$0.1624 \mathrm{E}-01$
$0.4378 \mathrm{E}+02$

$0.1002 \mathrm{E}+02 \quad 0.4417 \mathrm{E}+02$

$0.5000 \mathrm{E}+02 \quad 0.5000 \mathrm{E}+02$

$0.9656 \mathrm{E}+00 \quad 0.9656 \mathrm{E}+00$

$-0.50 .55 E+00 \quad-0.5055 E+00$

$0.2333 \mathrm{E}-01 \quad 0.8816 \mathrm{E}-01$

$0.5352 \mathrm{E}+02 \quad 0.6247 \mathrm{E}+02$

LIQUID

LIQUID 
Table A-1. HLW Flowsheet Material Balance (Tank 51/ITP Cycle 1)

Section 3A. Precipitate Hydrolysis in Salt Cell

STREAM NUMBERS $\rightarrow$

STREAM NAME

213

COMPONENT 122 (C6H5) 2

$\begin{array}{ll}122 & \text { (C6H5) } 2 \\ 123 & \text { (C6H5) 2C6H4 }\end{array}$

118 (C6H5) 2NH

133 (C6H5B (OH) 2 salt)

132 (C6H5B (OH) 2)

166 (C6H5C6H4O

163 (C6H5NHCHO)

165 (C6H5NO)

164 (C6H5NO2)

164 (C6H5NO2)

130 (C6H5OH)

121 ( 155 (CH3) $2 \mathrm{CHOH}$

155 ( $\mathrm{CH} 3) 2 \mathrm{C}$
154 (CH3OH)

$45 \mathrm{Ag}$

$116 \mathrm{Ag} 20$

134 AgNO3

$106 \mathrm{~B} 203$

$9 \mathrm{Ba}(\mathrm{OH}) 2$

$15 \mathrm{BaO}$

$24 \mathrm{BaSO} 4$

$66 \mathrm{CO}$

$\begin{array}{rrr}37 & \mathrm{CO} 2 & \\ 142 & \mathrm{CO} 2 & \text { (C14) }\end{array}$

$63 \mathrm{Ca}$ (COOH) 2

$167 \mathrm{Ca}(\mathrm{NO} 3) 2$

$120 \mathrm{Ca}(\mathrm{OH}) 2$

$113 \mathrm{Ca} 3(\mathrm{PO} 4) 2$

$20 \mathrm{CaC} 204$

$18 \mathrm{CaCO} 3$

$64 \mathrm{CaCO} 3$ (C14)

78 CaF2

$41 \mathrm{CaO}$

19 Caso4

29 Carbon

99 Cement

26 Co (COOH) 2

$26 \mathrm{Co}(\mathrm{COOH}) 2$
$168 \mathrm{Co}(\mathrm{NO} 3) 2$

$59 \mathrm{CoO}$

$98 \mathrm{Cr} 203$

$42 \mathrm{Cs} 2 \mathrm{O}$

$53 \mathrm{CsCOOH}$

$96 \mathrm{CsCl}$

169. $\mathrm{CsNO}^{2}$

$8 \mathrm{CsOH}$

Lig after

Rxn/CO2 Pur
Liq after

$0.4002 \mathrm{E}-0 \mathrm{i}$

$0.1477 \mathrm{E}-01$

$0.3638 \mathrm{E}-01$

ZERO

$0.6779 \mathrm{E}-01$

$0.7438 \mathrm{E}-01$

$0.2105 \mathrm{E}+00$

$0.3427 \mathrm{E}-02$

$0.6983 \mathrm{E}-02$

ZERO

$0.1011 \mathrm{E}+01$

$0.2472 \mathrm{E}+00$

$0.8384 \mathrm{E}-10$

$0.8955 \mathrm{E}-11$
$0.2861 \mathrm{E}-02$

$0.2861 \mathrm{E}-02$
ZERO

- ZERO

$0.1306 \mathrm{E}+00$

$0.1681 \mathrm{E}-02$

ZERO

$0.1804 \mathrm{E}-02$

ZERO

$0.2297 \mathrm{E}-01$

$0.2219 \mathrm{E}-12$

ZERO
$0.1378 \mathrm{E}-05$

ZERO

$0.5643 \mathrm{E}+00$

$0.2458 \mathrm{E}-06$

ZERO

ZERO

ZERO

TERO

$0.1924 \mathrm{E}+00$

ZERO

ZERO

$0.1442 \mathrm{E}-03$

$0.5408 \mathrm{E}-06$

$0.3089 \mathrm{E}-04$

$0.2634 \mathrm{E}+00$

$0.3872 \mathrm{E}-04$

$0.4358 \mathrm{E}-01$

ZERO

ZERO

ZERO

WSRC-TR-95-0019 (Revision 
Table A-1. HLW Flowsheet Material Balance (Tank 51/ITP Cycle 1)

seation $3 A$. Precipitate Hydrolysis in Salt Cell.

STREAM NUMBERS (CONT'D) $\rightarrow$ STREAM NAME

213

$54 \mathrm{Cu}(\mathrm{COOH}) 2$

$170 \cdot \mathrm{Cu}(\mathrm{NO} 3) 2$

55 CuO

$80 \mathrm{Fe} 2 \mathrm{O} 3$

101 FeO

102 Group A

103 Group B

$25 \mathrm{H} 2$

158 H2C2O4

$93 \mathrm{H} 2 \mathrm{SO} 4$

90 Н 3 ВО 3.

$34 \mathrm{HCOOH}$

$43 \mathrm{HCl}$

$85 \mathrm{HF}$

4.9. $\mathrm{HNO}$

$36 \mathrm{Hg}$

$129 \mathrm{Hg}(\mathrm{C} 6 \mathrm{H} 5) 2$

135. $\mathrm{Hg}$ (NO3) 2

$144 \mathrm{Hg} 2 \mathrm{Cl} 2$

$117 \mathrm{Hg} 2 \mathrm{I2}$

$146 \mathrm{HgCl2}$

$21 \mathrm{HgO}$

97 I2

$183 \mathrm{~K} 2 \mathrm{CO} 3$

$39 \mathrm{~K} 2 \mathrm{O}$

51. $\mathrm{KCOOH}$

$157 \mathrm{KMnO} 4$

$161 \mathrm{KNO} 3$

$10 \mathrm{KOH}$

150 KTPB

$105 \mathrm{La} 203$

$108 \mathrm{Li}$
$65 \mathrm{Mg}$ (COOH) 2

$65 \mathrm{Mg}$ (COOH)
$171 \mathrm{Mg}$ (NO3) 2

$33 \mathrm{MgO}$

$60 \mathrm{Mn}$ (COOH) 2

$172 \mathrm{Mn}(\mathrm{NO} 3) 2$

$180 \mathrm{Mn} 304$

$56 \mathrm{MnO}$

56 Mno

$14 \mathrm{MnO} 2$
$145 \mathrm{MOO} 2$

145 M०O2

81 N2

74 N2O

182 NH3

52 NH 4 COOH

173 NH4NO3 Liq after $\mathrm{Rxn} / \mathrm{CO} 2$ Pur $0.2074 \mathrm{E}-03$

$0.2193 \mathrm{E}+01$

$0.9607 \mathrm{E}-03$

$0.1597 E+00$

ZERO

$0.2453 E-05$

$0.7540 \mathrm{E}-07$

$0.1012 \mathrm{E}-17$

$0.5280 \mathrm{E}-02$ ZERO

$0.7756 \mathrm{E}+01$

$0.8986 \mathrm{E}+01$

ZERO

ZERO

$0.2145 \mathrm{E}+00$

$0.1326 \mathrm{E}-02$

ZERO

ZERO

ZERO

ZERO

ZERO

$$
\text { ZERO }
$$

$.6926 \mathrm{E}-02$

$0.1011 \mathrm{E}+02$

ZERO

ZERO

ZERO

ZERO

$0.2966 \mathrm{E}-03$

$0.3943 \mathrm{E}-02$

$0.52875-07$

$0.5287 \mathrm{E}-07$

$0.3327 \mathrm{E}-02$

$0.6161 \mathrm{E}-0.07$ ZERO

$0.1321 \mathrm{E}-02$

$0.1297 \mathrm{E}-03$

$0.7648 \mathrm{E}-07$

$0.1689 \mathrm{E}-14$

$0.2882 \mathrm{E}-06$

$0.4925 \mathrm{E}-03$

ZERO

148 NH4 TPB

ZERO

WSRC-TR-95-0019 (Revision 
Table A-1. HLW Flowsheet Material Balance (Tank 51/ITP Cycle 1)

Section 3A. Precipitate Hydrolysis in Salt Cell

STREAM NUMBERS (CONT'D) $\rightarrow$ STREAM NAME

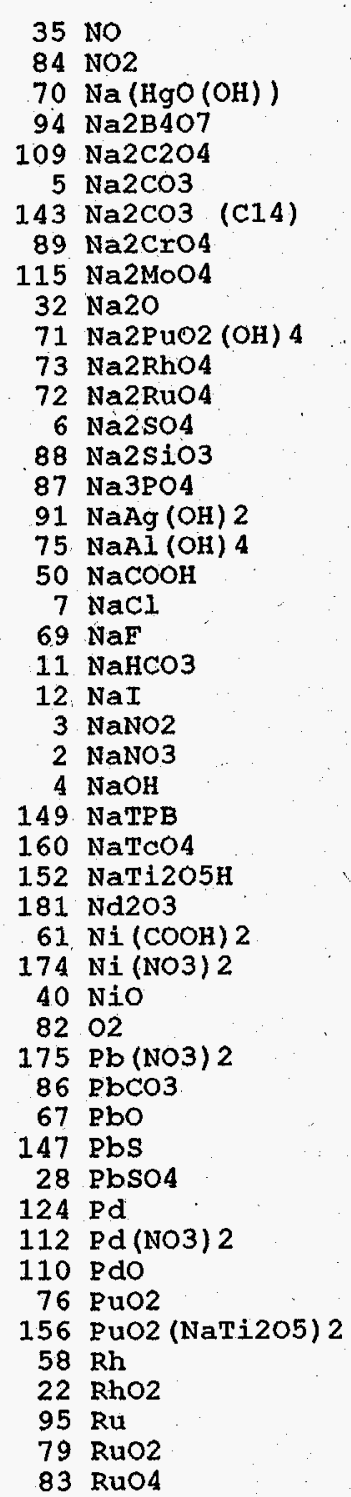

213

PR Liq after

$\mathrm{Rxn} / \mathrm{CO} 2 \mathrm{Pux}$

$0.8978 \mathrm{E}-05$
$0.7133 \mathrm{E}-12$

$0.7133 \mathrm{E}-12$
ZERO

ZERO

ZERO

ZERO

ZERO

ZERO

$0.1010 \mathrm{E}+01$

ZERO

ZERO
ZERO

$0.1089 \mathrm{E}-02$

ZERO

$0.1446 \mathrm{E}-02$.

ZERO

$0.1146 \mathrm{E}+01$

$0.3200 \mathrm{E}-02$

$0.8374 \mathrm{E}-04$

ZERO
$0.5609 E-07$

ZERO

$0.2214 \mathrm{E}-01$

ZERO

ZERO

ZERO

ZERO

$0.4179 \mathrm{E}-03$

$0.6729 \mathrm{E}-06$

$0.8341 \mathrm{E}-03$

ZERO
$0.1634 \mathrm{E}-05$

E-05

ZERO

ZTRO

$0.4390 \mathrm{E}-02$

$0.3119 \mathrm{E}-08$

ZERO

ZERO
$0.3230 E-04$

$0.1280 \mathrm{E}-03$

$0.3665 \mathrm{E}-06$
ZERO

ZERO

$0.4454 \mathrm{E}-02$

$83 \mathrm{RuO} 4$

ZERO 
Table A-1. HLW Flowsheet Material Balance (Tank 51/ITP Cycle 1) section 3A. Precipitate Hydrolysis in Salt Cell

STREAM NUMBERS (CONT'D) $\rightarrow$ STREAM NAME

213

$38 \mathrm{SO} 2$

141 SO3

137 Semi Vol Cs20

$\mathrm{Rxn} / \mathrm{CO} 2$ Pur

ZERO

126 Semi Vol Group A

140 Semi Vol Na2B407 $\quad$ ZERO

138 Semi Vol NaCl ZERO

139 Semi Vol NaF $\quad$ ZERO

$\begin{array}{ll}125 \text { Semi Vol NaI } & \text { ZERO } \\ \text { ZERO }\end{array}$

23 Semi Vol RuO2
111 SiO2
4 ZERO

$48 \mathrm{Sr}(\mathrm{COOH}) 2 \quad 0.1100 \mathrm{E}-03$

176 Sr(NO3) 2 ZERO

$127 \mathrm{Sr}(\mathrm{OH}) 2$ ZERO

$127 \mathrm{Sr}(\mathrm{OH}) 2$

$30 \mathrm{SrCO}$

46 Sro

159 TCO2

92 ThO2

119 Tritium

17 U308

$62 \mathrm{OO} 2(\mathrm{COOH}) 2$

$177 \mathrm{UO2}$ (NO3) 2

$16 \mathrm{UO} 2(\mathrm{OH}) 2$

$27 \mathrm{Y}(\mathrm{COOH}) 3$

$178 Y$ Y (NO3) 3

$128 \mathrm{Y}(\mathrm{OH}) 3$

$31 \mathrm{Y} 2(\mathrm{CO} 3) 3$

$47 \mathrm{Y} 203$

100 Zeolite

$44 \mathrm{Zn}(\mathrm{COOH}) 2$

$179 \mathrm{Zn}(\mathrm{NO} 3) 2$

$57 \mathrm{ZnO}$

$104 \mathrm{ZrO} 2$

13 hydrate $\mathrm{H} 2 \mathrm{O}$

1 water

TOTAL FLOW, LB/HR

\section{TEMPERATURE, DEG C}

PRESSURE, ATM

PRESSURE，'PSIG

PRESSURE, MM HG

ENTHALPY， PCU/HR

VAPOR FLOW, CFM

LIQUID FLOW, GPM

DENSITY， LBS/FT3

PHASE
ZERO
ZERO

$0.1772 \mathrm{E}-05$

ZERO

$0.7894 \mathrm{E}+00$

$0.1289 \mathrm{E}-11$

$0.7560 \mathrm{E}-03$

$0.1280 \mathrm{E}-05$

ZERO

$0.1326 \mathrm{E}-11$

ZERO

ZERO

$0.9744 \mathrm{E}-03$

$0.9744 \mathrm{E}-03$

$0.1147 \mathrm{E}+00$

$0.5370 \mathrm{E}-04$

$0.1392 \mathrm{E}+00$

$0.2190 \mathrm{E}-01$

$0.7422 \mathrm{E}+03$

$0.7778 \mathrm{E}+03$

$0.8999 \mathrm{E}+02$

$0.9656 \mathrm{E}+00$

$-0.5055 \mathrm{E}+00$

$0.73395+03$

$0.7339 \mathrm{E}+03$

$0.6760 \mathrm{E}+05$

$0.1532 \mathrm{E}+01$
$0.6330 \mathrm{E}+02$

LIQUID 


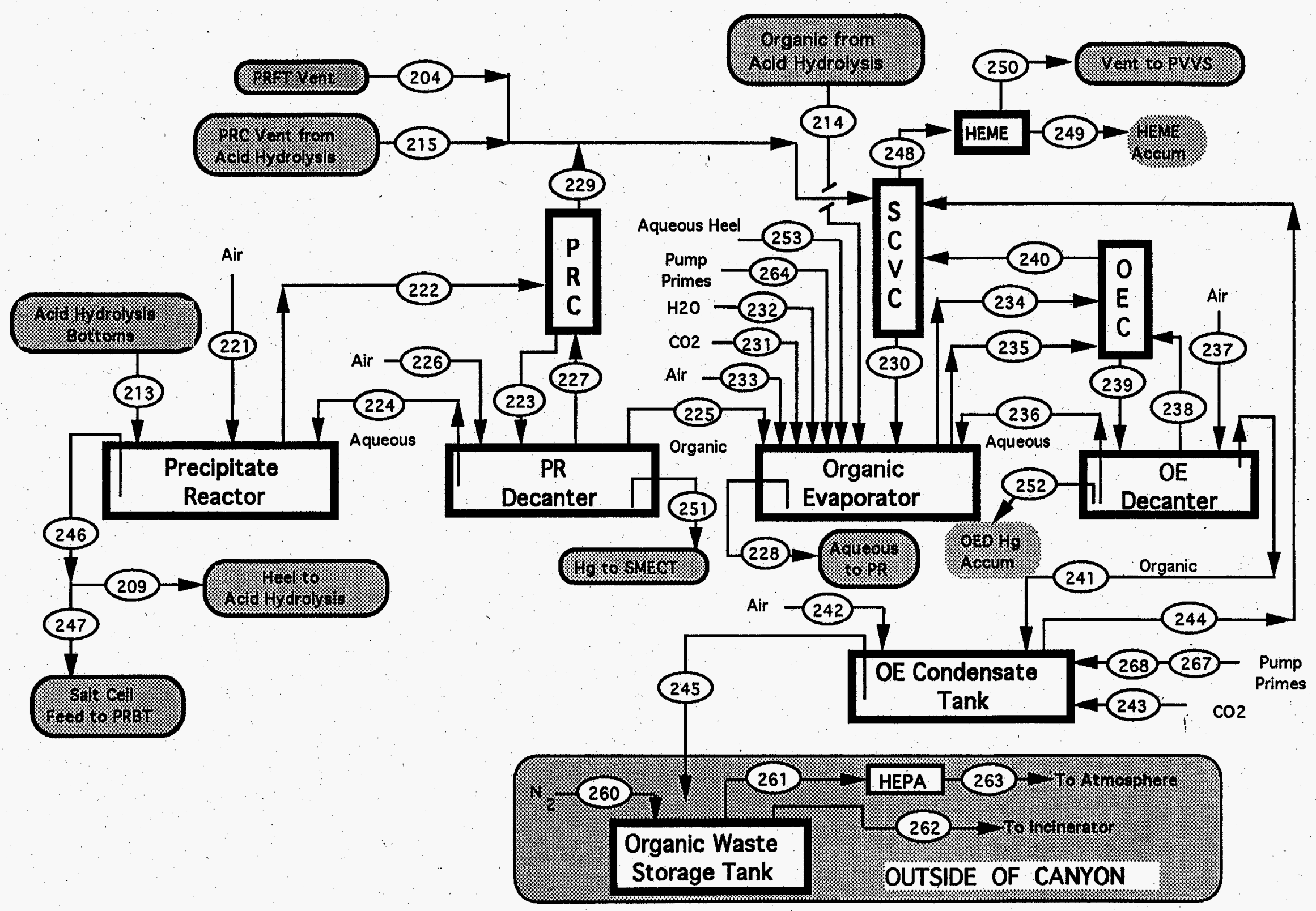

SECTION 3B. BOILUP OF PRECIPITATE HYDROLYSIS PRODUCTS IN SALT CELL

WSRC-TR-95-0019 (Revision 0)

Date: January 1995

Section 3B, Page 0 of 29 
Table A-1. HLW Flowsheet Material Balance (Tank 51/ITP Cycle 1) section 3B. Boilup of Precipitate Hydrolysis Products

DESCRIPTION

PAGE

1. STREAM NUMBERS

$204,209,213,214,215,253,228$. . . . . . . . . . . 2

2. STREAM NUMBERS

$221,222,223,224,225,226,227 \ldots \ldots . . . . . . . . . . .66$

3. STREAM NUMBERS

$229,230,231,232,233,234,235 \ldots \ldots \ldots$

4. STREAM NUMBERS

$236,237,238,239,240,241,242 \ldots \ldots \ldots$

5. STREAM NUMBERS

6. STREAM NUMBERS

$243,244,245,246,247,248,249 \ldots \ldots \ldots \ldots$

7. STREAM NUMBERS

$250,251,252,260,261,262,263 \ldots \ldots 22$

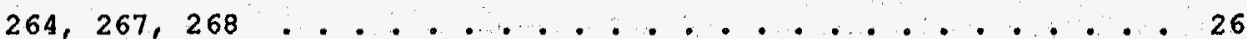


Table A-1. HLW Flowsheet Material Balance (Tank 51/ITP Cycle 1) Section 3B. Boilup of Precipitate Hydrolysis Products

STREAM NUMBERS $\rightarrow$ STREAM NAME

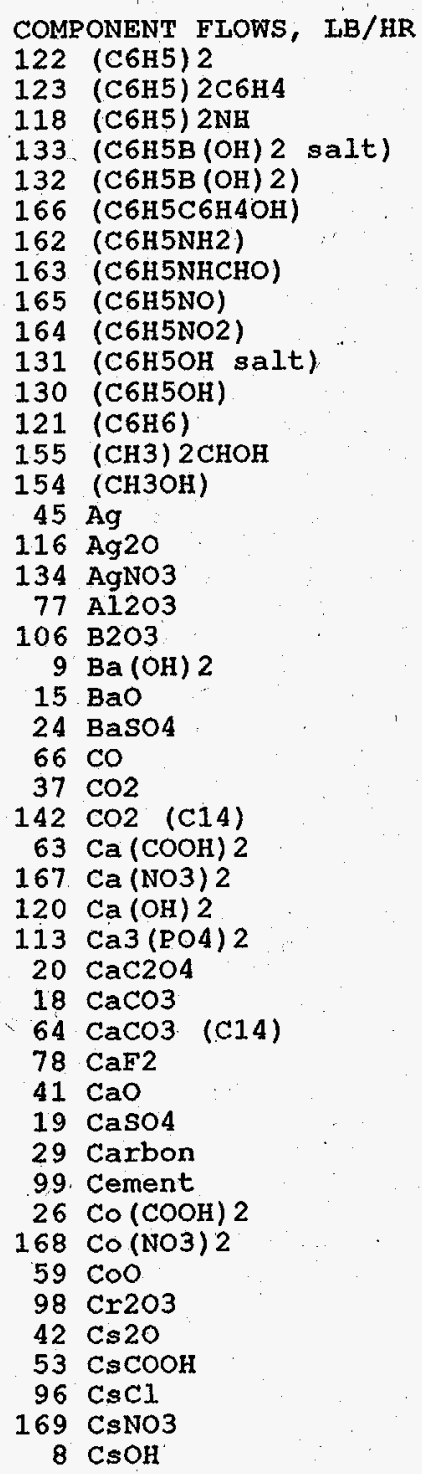

$$
213
$$

204

209

to scVC PR Heel to PR Liq after Hydrolysis Rxn/CO2 Pur

$$
214^{\circ}
$$

Hydroly/PRD org to OE

$$
215
$$

Hydrolys / PR

ZERO

$0.6510 \mathrm{E}-04$

$0.5890 \mathrm{E}-04$

$0.4002 \mathrm{E}-01$

$0.1477 \mathrm{E}-01$

$0: 8629 \mathrm{E}+00$

$0.8629 \mathrm{E}+00$
$0.7092 \mathrm{E}-09$

$0.4079 \mathrm{E}-01$

ZERO

ZERO

ZERO

EERO

ZERO

ZERO

ZERO

ZERO

ZERO

$0.9857 \mathrm{E}-02$

$0.8072 \mathrm{E}-11$

$0.7180 \mathrm{E}-12$

ZERO

ZERO

ZERO

ZERO

ZERO

ZERO

$0.1434 \mathrm{E}+02$

ZERO

ZERO

ZERO

ZERO

ZERO

ZERO

ZERO

ZERO

ZERO

ZERO

ZERO

ZERO

ZERO

ZERO

ZERO

ZERO

ERO

ZERO

ZERO

ZERO

ZERO
$0.3519 \mathrm{E}-03$
$0.6779 \mathrm{E}-01$

$0.4692 \mathrm{E}-03 \quad 0.7438 \mathrm{E}-01$

$0.1333 \mathrm{E}-02 \quad 0.2105 \mathrm{E}+00$

2170E-04

$0.4420 \mathrm{E}-04$

ZERO

$0.5950 \mathrm{E}-02$

$0.9127 \mathrm{E}-04$
$0.5301 \mathrm{E}-1.2$

$0.5648 \mathrm{E}-13$

$0.1811 \mathrm{E}-0.4$

ZERO

$0.8266 \mathrm{E}-03$

$0.1064 \mathrm{E}-04$

ZERO

$0.1142 \mathrm{E}-04$

$0.1142 \mathrm{E}-04$
ZERO

$0.3939 \mathrm{E}-08$

$0.3806 \mathrm{E}-19$

ZERO
$0.8727 \mathrm{E}-08$

ZERO

$0.3572 \mathrm{E}-02$

$0.1556 \mathrm{E}-08$

$0.3427 \mathrm{E}-02$

$0.6983 \mathrm{E}-02$

ZERO

$0.1011 \mathrm{E}+01$

$0.2472 \mathrm{E}+00$

$0.8384 \mathrm{E}-10$
$0.8955 \mathrm{E}-11$

$0.8955 \mathrm{E}-11$
$0.2861 \mathrm{E}-02$

$0.2861 \mathrm{E}-02$ ZERO
ZERO

$0.1306 \mathrm{E}+00$

$0.1681 \mathrm{E}-02$

ZERO

$0.1804 \mathrm{E}-02$

ZERO

$0.2297 \mathrm{E}-01$

$0.2219 \mathrm{E}-12$

$0.1378 \mathrm{E}-05$

ZERO

$0.1430 \mathrm{E}-01$

ZERO

ZERO

ZERO

ZERO

$0.5105 \mathrm{E}-01$

$0.9029 \mathrm{E}+01$

$0.4877 \mathrm{E}-12$

$0.8703 E-13$

ZERO

ZERO

ZERO

ZERO

ZERO

ZERO

ZERO

ZERO
0.1177 E-02

$0.1177 \mathrm{E}-02$

$0.5643 \mathrm{E}+00$

ZERO

ZERO

ZERO

ZERO

ZERO

ZERO

ZERO

ZERO

ZERO

$0.1218 \mathrm{E}-02$

ZERO

ZERO

$0.9128 \mathrm{E}-06$

$0.1955 \mathrm{E}-06$

$0.1667 \mathrm{E}-02$

$0.2451 E-06$

$0.2759 \mathrm{E}-03$

ZERO

ZERO

ZERO

ZERO

ZERO

ZERO

ZERO

$0.1442 \mathrm{E}-03$

$0.3089 \mathrm{E}-04$

$0.2634 \mathrm{E}+00$

$0.3872 \mathrm{E}-04$

$0.4358 \mathrm{E}-01$

ZERO

- ZERO

ZERO

ZERO

\section{$0.1254 \mathrm{E}+00$}

$.1008 \mathrm{E}-14$

$0.1529 \mathrm{E}-05$

ZERO

ZERO

$0.1142 \mathrm{E}-02$

ZERO

ZERO

ZERO

$0.4557 \mathrm{E}-02$

$0.2720 \mathrm{E}+02$

$.8703 E-12$

$0.3598 \mathrm{E}-12$

ZERO
ZERO

ZERO

ZERO

ZERO

ZERO

ZERO

ZERO

ERO

$0.2444 \mathrm{E}+02$

$0.1201 \mathrm{E}-09$

ZERO
ZERO

ZERO

ZERO

ZERO

ZERO

ZERO

ZERO

ZERO

ZERO

ZERO

ZERO

ZERO

ZERO

ZERO

ZERO

ZERO

ZERO

ZERO

ZERO

ZERO

ZERO

Date: January 1995

253

OE Aqueous

Inventory

228

$\begin{array}{rr}\text { ZERO } & \text { ZERO } \\ \text { ZERO } & \text { ZERO } \\ \text { ZERO } & \text { ZERO } \\ \text { ZERO } & \text { ZERO } \\ \text { ZERO } & \text { ZERO } \\ \text { ZERO } & \text { ZERO }\end{array}$

$0.1024 \mathrm{E}-01 \quad 0.1320 \mathrm{E}-02$

$0.6496 \mathrm{E}-14 \quad 0.8373 \mathrm{E}-15$

$0.1057 \mathrm{E}-15 \quad 0.1363 \mathrm{E}-16$

$0.2777 \mathrm{E}-16$

ZERO

$\begin{array}{r}\text { ZERO } \\ 0.1625 \mathrm{E}-01\end{array} \quad 0.2094 \mathrm{E}-02$

$0.5992 \mathrm{E}-03 \quad 0.7723 \mathrm{E}-04$

$0.4820 \mathrm{E}-10 \quad 0.6213 \mathrm{E}-11$

$0.4580 \mathrm{E}-11 \quad 0.5903 \mathrm{E}-12$

$0.8820 \mathrm{E}-16 \quad 0.1137 \mathrm{E}-16$

ZERO

$0.4028 \mathrm{E}-14$

$0.5185 \mathrm{E}-16$

ZERO

0.5565 ZERO

$0.3502 \mathrm{E}-05$

$0.8421 \mathrm{E}-20$

ZERO
$0.4252 \mathrm{E}-19$

$0.1741 \mathrm{E}-13$

$0.7581 \mathrm{E}-20$ 
Table A-1. HLW Flowsheet Material Balance (Tank 51/ITP Cyale 1) A-1. HLW Flowsheet Material Balance (Tank 51/ITP Cycle
Section 3B. Boilup of Precipitate Hydrolysis Products

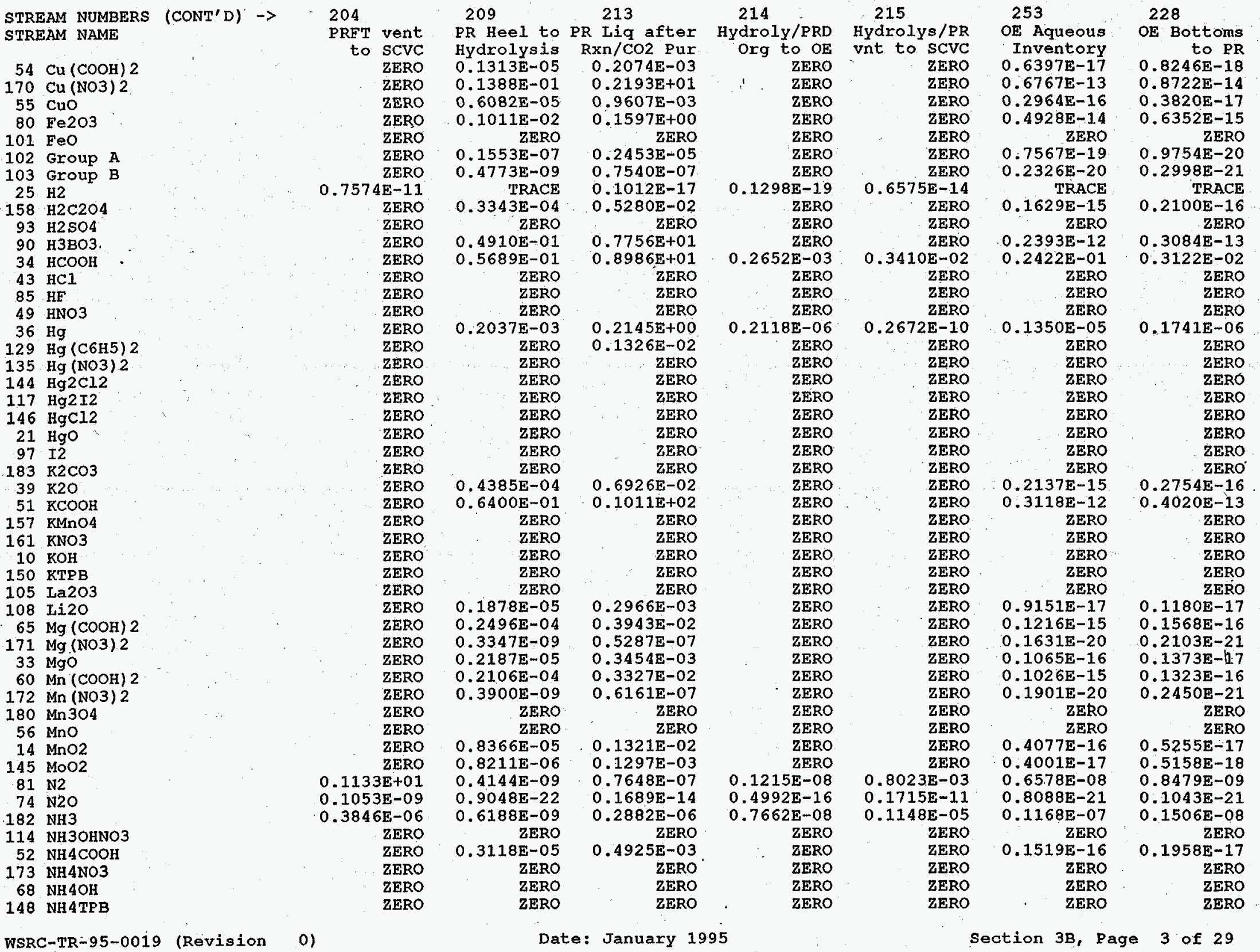


Table A-1. HLW Flowsheet Material Balance (Tank 51/ITP Cycle 1) section 3B. Boilup of Precipitate Hydrolysis Products

STREAM NUMBERS (CONT'D) $\rightarrow$ STREAM NAME

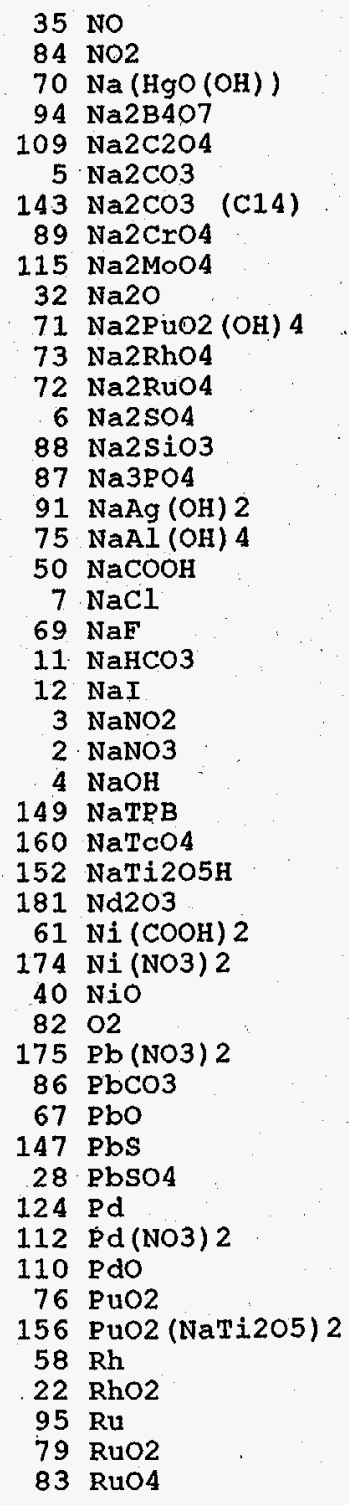


Table A-1. HLW Flowsheet Material Balance (Tank 51/ITP Cycle 1 ) section 3B. Boilup of Precipitate Hydrolysis products

STREAM NUMBERS (CONT'D) $\rightarrow$ STREAM NAME

$38 \mathrm{sO} 2$

$141 \mathrm{SO3}$

137 Semi Vol Cs2O

136 Semi Vol. CsC1

126 Semi Vol Group A

140 Semi Vol Na2B4O7

138 Semi Vol $\mathrm{NaCl}$

139 Semi Vol NaF

125 Semi Vol NaI

23 Semi Vol RuO2

111 sion

$48 \mathrm{Sx}(\mathrm{COOH}) 2$

$176 \mathrm{Sr}$ (NO3) 2

$153 \mathrm{Sr}(\mathrm{NaTi2O5)} 2$

$127 \mathrm{Sr}(\mathrm{OH}) 2$

$30 \mathrm{SrCO} 3$

46 Sro

$159 \mathrm{TCO} 2$

92 ThO2

119. Tritium

119. Tritiu

$62 \mathrm{UO} 2$ ( $\mathrm{COOH}) 2$

$177 \mathrm{VO} 2$ (NO3) 2

16 UO2 (OH) 2

$27 \mathrm{Y}(\mathrm{COOH}) 3$

$178 \mathrm{Y}$ (NO3) 3

$128 \mathrm{Y}(\mathrm{OH}) 3$

$47 \mathrm{Y} 203$

100 zeolite

$44 \mathrm{zn}(\mathrm{COOH}) 2$

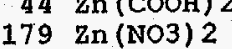

$57 \mathrm{Zno}$

$104 \mathrm{ZxO} 2$

13 hydrate H2O

1 water

TOTAL FLOW, LB/HR

TEMPERATURE, DEG C

PRESSURE， ATM

PRESSURE， PSIG

PRESSURE; $M M$ HG

ENTHALPY, PCU/HR

VAPOR FLOW, CEM

LIOUID FLOW, GPM

DENSITY, LBS/ET3

PHASE

WSRC-TR-95-0019 (Revision $\begin{array}{lr}204 & 209 \\ \text { PRFT vent PR H }\end{array}$

to SCVC

ZERO

ZERO

ZERO

ZERO

ZERO

ZERO

ZERO

ZERO

ZERO

ZERO

ZERO

ZERO

ZERO

ZERO

ZERO

ZERO

ZERO

ZERO

$0.4998 \mathrm{E}-02$

$0.8160 \mathrm{E}-14$

$0.8704 \mathrm{E}-08$

ZERO $0.8104 \mathrm{E}-08$

ZERO

ZERO

ZERO

ZERO

ZERO

ZERO

ZERO

ZERO

ZERO

ZERO

ZERO

ZERO

$0.5397 \mathrm{E}+00$

$0.1636 \mathrm{E}+02$

$0.4000 \mathrm{E}+02$

$0.9656 \mathrm{E}+00$

$-0.5055 \mathrm{E}+00$

$0.733 \mathrm{E}+03$

$0.2891 \mathrm{E}+01$

$0.9434 \mathrm{E}-01$
VAPOR

ZERO
$0.8394 \mathrm{E}-14$

ZERO

ZERO

ZERO

$0.6169 \mathrm{E}-05$

ZERO

$0.7259 E-03$

$0.8812 \mathrm{E}-03$

ZERO

$0.1386 \mathrm{E}-03$

$0.4698 \mathrm{E}+01$
$0.4920 \mathrm{E}+01$

$0.5000 \mathrm{E}+02$

$0.9656 \mathrm{E}+00$

$.5055 \mathrm{E}+00$

$0.9690 \mathrm{E}-02$

$0.6330 \mathrm{E}+02$

213

$\begin{array}{cc}214 & 215 \\ \text { Hydroly/PRD } & \text { Hydrolys/PR }\end{array}$

$\begin{array}{cc}214 & 215 \\ \text { Hydroly/PRD } & \text { Hydrolys/PR }\end{array}$

Org to $O E$ vnt to sCVC

2 Pux

ZERO

ZERO

ZERO

ZERO

ZERO

ZERO

ZERO

ZERO

ZERO

ZERO

ZERO

$0.2684 \mathrm{E}-01$

ZERO

$.1100 \mathrm{E}-03$

ZERO

$0.4098 \mathrm{E}-02$

ZERO

ZERO

ZERO

ZERO

ZERO

$0.1772 \mathrm{E}-05$

ZERO
$0.7894 \mathrm{E}+00$

$0.1289 \mathrm{E}-11$

$0.1289 \mathrm{E}-11$

$0.1280 \mathrm{E}-05$

ZERO

$0.1326 \mathrm{E}-11$

ZERO

ZERO

ZERO
$0.9744 \mathrm{E}-03$

ZERO

$0.1147 \mathrm{E}+00$

$0.5370 \mathrm{E}-04$

$0.1392 \mathrm{E}+00$ ZERO

$0.2190 \mathrm{E}-01$

$0.7422 \mathrm{E}+03$

$0.7778 E+03$

ZERO

ZERO

$0.9282 \mathrm{E}-18$

ZERO

ZERO

ZERO

ZERO

ZERO

ZERO

ZERO

ZERO

ZERO

ZERO

ZERO

ZERO

ZERO

ZERO

$0.1002 \mathrm{E}+02$

$0.8999 E+02$

$0.5000 \mathrm{E}+02$

$0.9656 \mathrm{E}+00$

$0.9656 \mathrm{E}+00$

(

$-0.5055 \mathrm{E}+00$

$0.7339 \mathrm{E}+03$

$.7339 \mathrm{E}+03$

$0.1532 E+01$

$0.2333 \mathrm{E}-01$

$0.6330 \mathrm{E}+02$

$0.5352 \mathrm{E}+02$

IIQUID

ZERO
ZERO
ZERO

ZERO

ZERO

ZERO

ZERO

ZERO

ZERO

ZERO

ZERO

ZERO

ZERO

ZERO

ZERO

ZERO

ZERO

ZERO

$0.3827 \mathrm{E}-14$

ZERO

ZERO

ZERO

ZERO

ZERO

ZERO

ZERO

ZERO

ZERO

ZERO

ZERO

ZERO

ZERO

ZERO

$0.2304 \mathrm{E}+01$

$0.5411 \mathrm{E}+02$

$0.5000 \mathrm{E}+02$

$0.5055 \mathrm{E}+00$

$0.7339 \mathrm{E}+03$

$0.7575 \mathrm{E}+01$

$0.1191 \mathrm{E}+00$

VAPOR

Date: January 1995

253

228

E Aqueous OE Bottoms

Inventory to $P R$ ZERO : ZERO ZERO ZERO

ZERO ZERO

ZERO ZERO

ZERO ZERO

ZERO ZERO

ZERO

ZRRO

$0.820 \div-15$

$0.3393 \mathrm{E}-17$

$0.1067 \mathrm{E}-1.5$

$0.4373 \mathrm{E}-18$

$0.1630 \mathrm{E}-16$

$\mathrm{E}-15$

ZERO

ZERO

ZERO
$0.5467 \mathrm{E}-19$

ZERO

$0.2435 \mathrm{E}-13$

$0.3180 \mathrm{E}-13$

$0.2332 \mathrm{E}-16$

$0.3949 \mathrm{E}-19$

ZERO

ZERO

TRACE

ZERO

ZERO

$0.3006 \mathrm{E}-16$

ZERO

$.3537 \mathrm{E}-14$

ZERO

$0.6755 \mathrm{E}-15$

$.5681 \mathrm{E}+03$

$0.5682 \mathrm{E}+03$

$0.5000 \mathrm{E}+02$

$0.9656 \mathrm{E}+00$

$0.5055 E+00$
$0.7339 E+03$

$0.2844 \mathrm{E}+05$

$0.1135 \mathrm{E}+01$

$0.6243 \mathrm{E}+02$

LIQUID

$0.1463 \mathrm{E}+00$

$0.6243 \mathrm{E}+02$

LIQUID

$0.4099 \mathrm{E}-14$

$0.3006 \mathrm{E}-17$

ZERO 
Table A-1. HLW Flowsheet Material Balance (Tank 51/ITP Cycle 1) Section 3B. Boilup of Precipitate Hydrolysis Products

STREAM NUMBERS $\rightarrow$ STREAM NAME

COMPONENT FLOWS, LB/HR $122(\mathrm{C} 6 \mathrm{H} 5) 2$

123 (C6H5) $2 \mathrm{C} 6 \mathrm{H} 4$

133 (C6H5B (OH) 2 salt)

132 (C6H5B (OH) 2)

166 (C6H5C6H4OH)

162 (C6H5NH2)

163 (C6H5NHCHO)

165 (C6H5NO)

164 (C6H5NO2)

131 (C6H5OH salt)

$130 \cdot(\mathrm{C} 6 \mathrm{H} 5 \mathrm{OH})$

$\begin{array}{ll}121 & \text { ( } \mathrm{CH} 6) \\ 155 & \text { (СH3) } 2 \mathrm{CHOH}\end{array}$

154 (CH3OH)

$45 \mathrm{Ag}$

116 Ag20

134 AgNO3

$106 \mathrm{~B} 203$

$\begin{array}{rl}9 & \mathrm{Ba}(\mathrm{OH}) 2 \\ 15 & \mathrm{BaO} \\ 24 & \mathrm{BasO} 4 \\ 66 & \mathrm{CO} \\ 37 & \mathrm{CO} 2 \\ 142 \mathrm{CO} 2(\mathrm{C} 14) \\ 63 \mathrm{Ca}(\mathrm{COOH}) 2 \\ 167 \mathrm{Ca}(\mathrm{NO} 3) 2 \\ 120 \mathrm{Ca}(\mathrm{OH}) 2 \\ 113 \mathrm{Ca}(\mathrm{PO}) 2 \\ 20 \mathrm{CaC} 2 \mathrm{O} 4 \\ 18 \mathrm{CaCO} 3 \\ 64 \mathrm{CaCO} 3 \text { (C14) } \\ 78 \mathrm{CaF} 2 \\ 41 \mathrm{CaO} \\ 19 \mathrm{CaSO} \\ 29 \mathrm{CarbOn} \\ 99 \mathrm{Cement} \\ 26 \mathrm{Co}(\mathrm{COOH}) 2 \\ 168 \mathrm{Co}(\mathrm{NO} 3) 2 \\ 59 \mathrm{CoO} \\ 98 \mathrm{Cr} 2 \mathrm{O} 3 \\ 42 \mathrm{Cs} 2 \mathrm{O} \\ 53 \mathrm{CsCOOH} \\ 96 \mathrm{CsCl} \\ 169 \mathrm{CsNO3} \\ 8 \mathrm{CsOH} \\ 151 \mathrm{CsTPB}\end{array}$

151 CsTPB

WSRC-TR-95-0019 (Revision
221

222

223

225

$\begin{array}{rrr}\text { to Cndnsr } & \text { to PRD } & \text { to PR PRD Organio } \\ & \text { to OF }\end{array}$

226

PRD Air PRD Vent

ZERO

$0.2974 \mathrm{E}-01$

$0.1385 \mathrm{E}-01$
$0.5464 \mathrm{E}-02$

$0.2938 \mathrm{E}-01$

ZERO

ZERO

ZERO

ZERO

ZERO

ZERO

ZERO

ZERO

ZERO

ZERO

ZERO

ZERO

ZERO

ZERO

ZERO

ZERO

ZERO

ZERO

ZERO

ZERO

ZERO

ZERO

ZERO

ZERO

ZERO

ZERO

ZERO

ZERO

ZERO

ZERO

ZERO

ZERO

ZERO

ZERO

ZERO

ZERO

ZERO

ZERO

ZERO

ZERO

ZERO

$$
\begin{array}{r}
\text { ZERO } \\
\text { ZERO }
\end{array}
$$

$0.1220 \mathrm{E}-01$

$0.1261 \mathrm{E}-06 \quad 0.1261 \mathrm{E}-06$

$0.2053 \mathrm{E}-08$

$0.4182 \mathrm{E}-08$

ZERO

ZERO
ZERO

$0.4664 \mathrm{E}+00$

$0.5010 \mathrm{E}-10$

$0.5808 \mathrm{E}-01$

$0.5005 \mathrm{E}-10$
$0.9561 \mathrm{E}-11$

$0.1713 \mathrm{E}-08$

ZERO
ZERO

$0.1713 \mathrm{E}-08$

ZERO

$0.7820 \mathrm{E}-07$

$0.7820 \mathrm{E}-07$

$0.1007 \mathrm{E}-08$

ZERO

$\begin{array}{r}\text { ZERO } \\ \hline\end{array}$

OOE-08

$0.2439 \mathrm{E}-01$

$0.2356 \mathrm{E}-12$

$0.8256 \mathrm{E}-12$

ZERO

$0.3380 \mathrm{E}-06$

$0.1472 \mathrm{E}-12$

ZERO

ZERO

ZERO ZERO

ZERO

$0.3239 \mathrm{E}-12$

$0.1850 \mathrm{E}-10$

$0.1577 \mathrm{E}-06$

$0.2319 \mathrm{E}-10$

ZERO

$0.1080 \mathrm{E}-08$ ZERO

$0.1418 \mathrm{E}-02$

$0.1370 \mathrm{E}-13$

$0.8256 \mathrm{E}-12$ ZERO

$0.3380 \mathrm{E}-06$

$0.1472 \mathrm{E}-12$

ZERO

ZERO

ZERO

ZERO

ZERO

$0.8636 \mathrm{E}-10$

$0.3239 \mathrm{E}-12$

$0.1850 \mathrm{E}-10$

$0.1577 \mathrm{E}-06$

$0.2319 \mathrm{E}-10$

$0.2610 \mathrm{E}-07$

ZERO

ZERO

ZERO

ZERO

ZERO

ZERO

$0.5464 \mathrm{E}-02$

$0.2938 \mathrm{E}-01$

ZERO

ZERO

ZERO

$0.1220 \mathrm{E}-01$

E-01

$0.1261 \mathrm{E}-06$ ZERO

$0.2053 \mathrm{E}-08$

$0.4182 \mathrm{E}-08$

ZERO

$0.3964 \mathrm{E}+00$

$0.5808 \mathrm{E}-01$

$0.5005 \mathrm{E}-10$
$0.9561 \mathrm{E}-11$

ZERO

ZERO

$0.7820 \mathrm{E}-07$

$0.1007 \mathrm{E}-08$

ZERO

ZERO

$\mathrm{E}-08$

$0.1418 \mathrm{E}-02$

$0.1370 \mathrm{E}-13$

ZERO
$0.8256 \mathrm{E}-12$

ZERO
$0.3380 \mathrm{E}-06$

$0.1472 \mathrm{E}-12$

ZERO

ZERO

ZERO

$0.1152 \mathrm{E}-06$

ZERO

ZERO

$0.8636 \mathrm{E}-10$

$0.1850 \mathrm{E}-10$

0.1577 E-06

$0.2319 \mathrm{E}-10$

$0.2610 \mathrm{E}-07$

ZERO

ZERO

ZERO

Date: January 1995
Inleakage to SCVC

ZERO

ZERO

ZERO

ZERO

ZERO

ZERO

ZERO

ZERO

ZERO

ZERO

ZERO

ZERO

ZERO

ZERO

ZERO

ZERO

ZERO

ZERO

ZERO

ZERO

ZERO

ZERO

ZERO

ZERO

ZERO

ZERO

ZERO

ZERO

ZERO

ZERO

ZERO

ZERO

ZERO

ZERO

ZERO

ZERO

ZERO

ZERO

ZERO

ZERO

ZERO

ZERO

ZERO

ZERO

ZERO

Section 3B, Page 6 of 29 
Table A-1. HLW Flowsheet Material Balance (Tank 51/ITP Cycle 1) section 3B. Boilup of Precipitate Hydrolysis Products

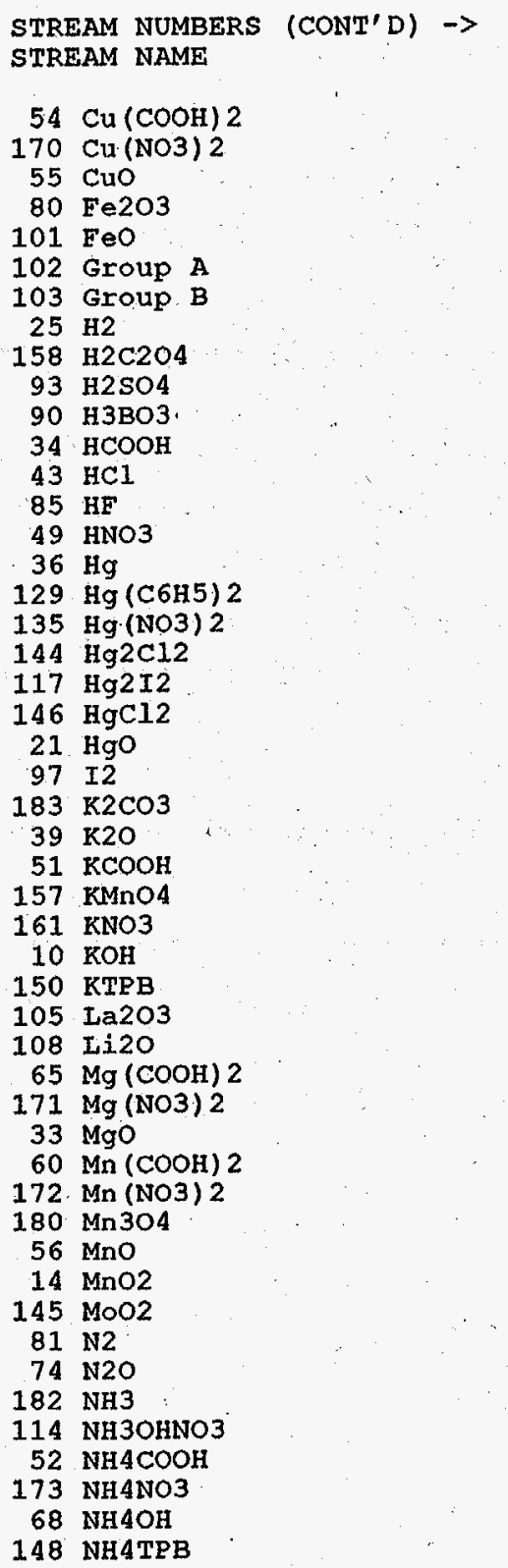

221
PR Air
In leakage
ZERO
ZERO
ZERO
ZERO
ZERO
ZERO
ZERO
ZERO
ZERO
ZERO
ZERO
ZERO
ZERO
ZERO
ZERO
ZERO
ZERO
ZERO
ZERO
ZERO
ZERO
ZERO
ZERO
ZERO
ZERO
ZERO
ZERO
ZERO
ZERO
ZERO
ZERO
ZERO
ZERO
ZERO
ZERO
ZERO
ZERO
ZERO
ZERO
ZERO
ZERO
ZERO
ZERO
ZERO
ZERO
ZERO
ZERO
ZERO
ZERO

\begin{tabular}{|c|c|c|}
\hline 22 & 223 & 224 \\
\hline $\begin{array}{l}\text { PR Boilup } \\
\text { to Cndnsr } \\
0.1242 \mathrm{E}-09 \\
.1313 \mathrm{E}-05 \\
.5754 \mathrm{E}-09 \\
.9567 \mathrm{E}-07\end{array}$ & $\begin{array}{c}\text { PRC Cndnste } \\
\text { to PRD } \\
0.1242 \mathrm{E}-09 \\
0.1313 \mathrm{E}-05 \\
0.5754 \mathrm{E}-09 \\
0.9567 \mathrm{E}-07\end{array}$ & $\begin{array}{r}\text { PRD Aqueous } \\
\text { to PR } \\
0.1242 \mathrm{E}-09 \\
0.1313 \mathrm{E}-05 \\
0.5754 \mathrm{E}-09 \\
0.9567 \mathrm{E}-07\end{array}$ \\
\hline ZERO & ZERO & ZERO \\
\hline $\begin{array}{l}1469 \mathrm{E}-11 \\
4516 \mathrm{E}-13 \\
1014 \mathrm{E}-17\end{array}$ & $\begin{array}{l}0.1469 \mathrm{E}-11 \\
0.4516 \mathrm{E}-13 \\
0.2530 \mathrm{E}-20\end{array}$ & $\begin{array}{l}0.1469 \mathrm{E}-11 \\
0.4516 \mathrm{E}-13 \\
0.2530 \mathrm{E}-20\end{array}$ \\
\hline 3162E-08 & $0.3162 \mathrm{E}-08$ & $0.3162 \mathrm{E}-08$ \\
\hline $4646 \mathrm{E}-05$ & $0.4646 \mathrm{E}-05$ & $0.4646 \mathrm{E}-05$ \\
\hline $1222 \mathrm{E}+0.1$ & $0.1221 \mathrm{E}+01$ & $0.1221 E+01$ \\
\hline ZERO & ZERO & ZERO \\
\hline ZERO & ZERO & ZERO \\
\hline ZERO & ZERO & ZERO \\
\hline $23 \mathrm{E}+00$ & $0.1823 \mathrm{E}+00$ & ZERO \\
\hline $26 \mathrm{E}-02$ & $0.1326 \mathrm{E}-02$ & ZERO \\
\hline ZERO & ZERO & ZERO \\
\hline ZERO & ZERO & ZERO \\
\hline ZERO & ZERO & ZERO \\
\hline ZERO & ZERO & ZERO \\
\hline ZERO & ZERO & ZERO \\
\hline ZERO & ZERO & ZERO \\
\hline ZERO & ZERO & ZERO \\
\hline $\begin{array}{l}148 \mathrm{E}-08 \\
055 \mathrm{E}-05\end{array}$ & $\begin{array}{l}0.4148 \mathrm{E}-08 \\
0.6055 \mathrm{E}-05\end{array}$ & $\begin{array}{l}0.4148 \mathrm{E}-08 \\
0.6055 \mathrm{E}-05\end{array}$ \\
\hline ZERO & ZERO & ZERO \\
\hline ZERO & ZERO & ZERO \\
\hline ZERO & ZERO & ZEERO \\
\hline ZERO & ZERO & ZERO \\
\hline ZERO & ZERO & ZERO \\
\hline $\begin{array}{l}777 E-09 \\
362 E-08\end{array}$ & $\begin{array}{l}0.1777 \mathrm{E}-09 \\
0.2362 \mathrm{E}-08\end{array}$ & $\begin{array}{l}0.1777 \mathrm{E}-09 \\
0.2362 \mathrm{E}-08\end{array}$ \\
\hline $3167 E-13$ & $0.3167 \mathrm{E}-13$ & $0.3167 \mathrm{E}-13$ \\
\hline $2069 \mathrm{E}-09$ & $0.2069 \mathrm{E}-09$ & $0.2069 E-09$ \\
\hline $1993 \mathrm{E}-08$ & $0.1993 \mathrm{E}-08$ & $0.1993 \mathrm{E}-08$ \\
\hline $3690 E-13$ & $0.3690 \mathrm{E}-13$ & $0.3690 \mathrm{E}-13$ \\
\hline ZERO & ZERO & ZERO \\
\hline ZERO & ZERO & ZERO \\
\hline $915 \mathrm{E}-09$ & $0.7915 \mathrm{E}-09$ & $0.7915 \mathrm{E}-09$ \\
\hline $169 E-10$ & $0.7769 \mathrm{E}-10$ & $0.7769 \mathrm{E}-10$ \\
\hline & $0.2620 \mathrm{E}-02$ & $0.2620 \mathrm{E}-02$ \\
\hline $15-14$ & $0.6268 \mathrm{E}-16$ & $0.6268 \mathrm{E}-16$ \\
\hline $2084 E-05$ & $0.18 .94 \mathrm{E}-05$ & $0.1894 \mathrm{E}-05$ \\
\hline ZERO & ZERO & ZERO \\
\hline $950 E-09$ & $0.2950 \mathrm{E}-09$ & $0.2950 \mathrm{E}-09$ \\
\hline ZERO & ZERO & ZERO \\
\hline ZERO & ZERO & ZERO \\
\hline ZERO & ZERO & .ZERO \\
\hline
\end{tabular}

PRD Organic to OE ZERO
ZERO ZERO ZERO ZERO ZERO ZERO ZERO ZERO ZERO ZERO ZERO ZERO ZERO ZERO ZERO $0.1326 \mathrm{E}-02$ ZERO ZERO ZERO ZERO ZERO ZERO ZERO ZERO ZERO ZERO ZERO ZERO ZERO ZERO ZERO ZERO ZERO ZERO ZERO ZERO ZERO ZERO ZERO ZERO ZERO ZERO ZERO ZERO ZERO ZERO ZERO
226 227 ZERO to SCVC ZERO ZERO ZERO ZERO ZERO ZERO ZERO ZERO ZERO ZERO ZERO ZERO ZERO ZERO ZERO ZERO ZERO ZERO ZERO ZERO ZERO ZERO ZERO ZERO ZERO ZERO

ZERO ZERO ZERO ZERO ZERO ZERO ZERO ZERO ZERO ZERO ZERO ZERO ZERO ZERO ZERO ZERO ZERO ZERO … ZERO

ZERO ZERO

ZERO ZERO

ZERO ZERO

ZERO ZERO

ERO

$\begin{array}{ll}\text { ZERO } & \text { ZERO } \\ \text { ZERO } & \text { ZERO }\end{array}$

ZERO ZERO

ZERO

ZERO ZERO

ZERO ZERO

2ERO ZERO

ZERO ZERO

ZERO $\quad$ ZERO

ZERO

ZERO

ZERO ZERO

ZERO 
Table A-1. HLW Flowsheet Material Balance (Tank 51/ITP Cycle 1) seation $3 \mathrm{~B}$. Boilup of Precipitate Hydrolysis Products

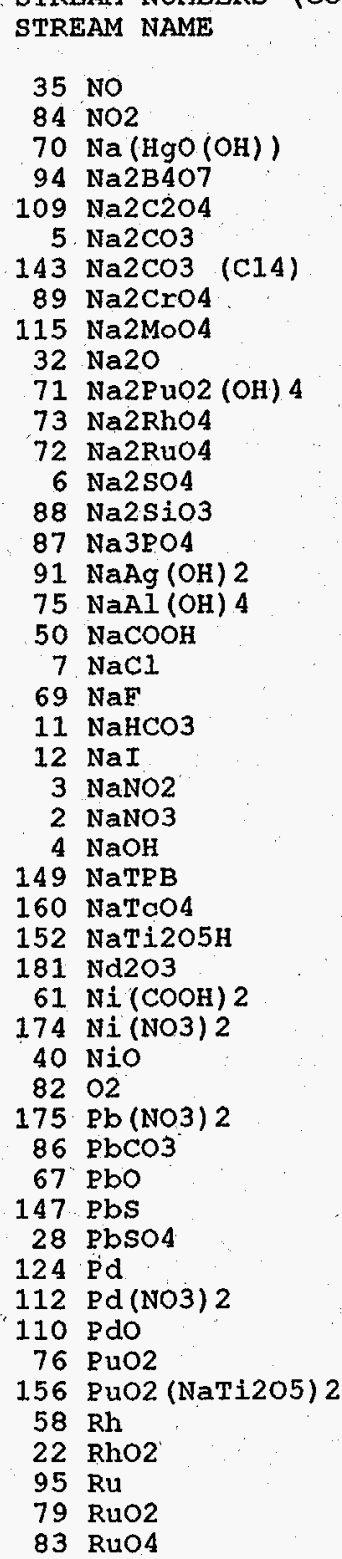

221

PR Air Inleakage

ZERO

ZERO
ZERO

ZERO

ZERO

ZERO

ZERO

ZERO

ZERO

ZERO

ZERO

ZERO

ZERO

ZERO

ZERO :

ZERO

ZERO

ZERO

ZERO

ZERO

ZERO

ZERO

ZERO

ZERO

ZERO

ZERO

ZERO

ERO

ZERO

ZERO

$0.3423 \mathrm{E}+00$

ZERO

ZERO

ZERO

ZERO

ZERO

ZERO

ZERO

ZERO

ZERO

ZERO

ZERO

ZERO

ZERO

\begin{tabular}{|c|}
\hline $\begin{array}{l}222 \\
\text { PR Boilup } \\
\text { to Cndnsr } \\
.9028 \mathrm{E}-05 \\
.7173 \mathrm{E}-12\end{array}$ \\
\hline ZERO \\
\hline ZERO \\
\hline ZERO \\
\hline ZERO \\
\hline ZERO \\
\hline ZERO \\
\hline ZERO \\
\hline $6047 \mathrm{E}-06$ \\
\hline$\therefore \quad$ ZERO \\
\hline ZERO \\
\hline ZERO \\
\hline $.6523 \mathrm{E}-09$ \\
\hline ZERO \\
\hline $8663 E-09$ \\
\hline ZERO \\
\hline ZERO \\
\hline $.6862 \mathrm{E}-06$ \\
\hline $.1916 \mathrm{E}-08$ \\
\hline $.5015 \mathrm{E}-10$ \\
\hline ZERO \\
\hline $3359 E-13$ \\
\hline ZERO \\
\hline $1326 \mathrm{E}-07$ \\
\hline ZERO \\
\hline ZERO \\
\hline ZERO \\
\hline ZERO \\
\hline ZERO \\
\hline $.2503 \mathrm{E}-09$ \\
\hline $\begin{array}{r}4030 \mathrm{E}-12 \\
4996 \mathrm{E}-09\end{array}$ \\
\hline $\begin{array}{l}.4996 \mathrm{E}-09 \\
.3438 \mathrm{E}+00\end{array}$ \\
\hline $.9785 \mathrm{E}-12$ \\
\hline ZERO \\
\hline ZERO \\
\hline ZERO \\
\hline $\begin{array}{r}.2629 \mathrm{E}-08 \\
1868 \mathrm{E}-14\end{array}$ \\
\hline $\begin{array}{r}1868 \mathrm{E}-14 \\
\text { ZERO }\end{array}$ \\
\hline ZERO \\
\hline $.1934 \mathrm{E}-10$ \\
\hline $\begin{array}{r}.7667 \mathrm{E}-10 \\
.2195 \mathrm{E}-12\end{array}$ \\
\hline ZERO \\
\hline ZERO \\
\hline $\begin{array}{r}.2668 \mathrm{E}-08 \\
\text { ZERO }\end{array}$ \\
\hline
\end{tabular}

223

224

225

226

26.227

ZERO
ZERO

ZERO ZERO

ZERO ZERO

ZERO ZERO

ZERO ZERO

ZERO ZERO

ZERO ZERO

ZERO ZERO

ZERO ZERO

ZERO ZERO

ZERO ZERO

ZERO ZERO

ZERO ZERO

ZERO ZERO

ZERO ZERO

ZERO ZERO

ZERO

- TERO

ZERO ZERO

ZERO ZERO

ZERO ZERO

ZERO ZERO

ZERO ZERO

ZERO ZERO

ZERO ZERO

ZERO ZERO

ZERO ZERO

ZERO ZERO

ZERO

ZERO

ZERO

ZERO ZERO

ZERO ZERO

ZERO ZERO

$\begin{array}{ll}\text { ZERO } & \text { ZERO } \\ \text { ZERO } & \text { ZERO }\end{array}$

ZERO ZERO

ZERO ZERO

ZERO ZERO

ZERO ZERO

ZERO $\because$ ZERO

ZERO

ZERO

Date: January 1995
ZERO

8E-08 $\quad 0.2668 \mathrm{E}-08$

ZERO
Section 3B, Page 8 of 29 
Table A-1. HLW Flowsheet Material Balance (Tank 51/ITP Cycle 1 ) section 3B. Boilup of Precipitate Hydrolysis products

STREAM NUMBERS (CONT'D) $\rightarrow$ STREAM NAME

\footnotetext{
$38 \mathrm{SO} 2$

141503

137 Semi Vol Cs2O

136 Semi Vol CsCl

126 Semi Vol Group A

140 Semi Vol Na2B4O7

138 Semi Vol NaCl

139 Semi Vol NaF

125 Semi Vol NaI

23 Semi Vol RuO2

111 sio2

$48 \mathrm{Sr}(\mathrm{COOH}) 2$

$176 \mathrm{Sr}$ (NO3) 2

$153 \mathrm{Sr}$ (NaTi205) 2

$127 \mathrm{Sr}(\mathrm{OH}) 2$

$30 \mathrm{SrCO} 3$

46 Sro

159 TcO2

92
107
TiO

119 Tritium

$\begin{array}{rl}119 & \text { Trit } \\ 17 & \mathrm{U} 308\end{array}$

62 UO2 ( $\mathrm{COOH}) 2$

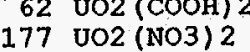

$16 \mathrm{UO2}(\mathrm{OH}) 2$

$16 \mathrm{UO} 2(\mathrm{OH}) 2$
$27 \mathrm{Y}(\mathrm{COOH}) 3$

$178 \mathrm{Y}(\mathrm{NO} 3) 3$

$128 \mathrm{Y}(\mathrm{OH}) 3$

$31 \mathrm{Y} 2(\mathrm{CO} 3) 3$

$47 \mathrm{Y} 203$

100 Zeolite

$44 \mathrm{zn}(\mathrm{COOH}) 2$

$179 \mathrm{Zn}$ (NO3) 2

$\begin{array}{rl}179 & \mathrm{Zn}(\mathrm{N} \\ 57 \mathrm{ZnO}\end{array}$

$104 \mathrm{zrO} 2$

13 hydrate $\mathrm{H} 2 \mathrm{O}$

1 water

TOTAI FLOW, LB/HR

TEMPERATURE， DEG C

PRESSURE, ATM

PRESSURE, PSIG

PRESSURE, MM HG

ENTHALPY, PCU/HR

VAPOR FLOW, CFM

IIOUID FLOW, GPM

DENSITY, LBS/FT3

PHASE
}

\begin{tabular}{|c|c|}
\hline 221 & 222 \\
\hline PR Air & PR Boilup \\
\hline Inleakage & to Cndnsi \\
\hline ZERO & ZERO \\
\hline ZERO & ZERO \\
\hline ZERO & ZERO \\
\hline ZERO & ZERO \\
\hline ZERO & ZERO \\
\hline ZERO & ZERO \\
\hline ZERO & ZERO \\
\hline ZERO & ZERO \\
\hline ZERO & ZERO \\
\hline ZERO & ZERO \\
\hline ZERO & $0.1607 \mathrm{E}-07$ \\
\hline ZERO & $0.6587 \mathrm{E}-10$ \\
\hline ZERO & ZERO \\
\hline ZERO & $0.2454 \mathrm{E}-08$ \\
\hline ZERO & ZERO \\
\hline ZERO & ZERO \\
\hline ZERO & ZERO \\
\hline ZERO & $0.1061 \mathrm{E}-11$ \\
\hline ZERO & ZERO \\
\hline ZERO & $0.4728 \mathrm{E}-06$ \\
\hline ZERO & $0.3875 \mathrm{E}-12$ \\
\hline ZERO & $0.4528 \mathrm{E}-09$ \\
\hline ZERO & $0.7667 \mathrm{E}-12$ \\
\hline ZERO & ZERO \\
\hline ZERO & ZERO \\
\hline ZERO & $0.7941 \mathrm{E}-18$ \\
\hline ZERO & ZERO \\
\hline ZERO & ZERO \\
\hline ZERO & ZERO \\
\hline ZERO & $0.5836 \mathrm{E}-09$ \\
\hline ZERO & ZERO \\
\hline ZERO & $0.6867 \mathrm{E}-07$ \\
\hline ZERO & $0.3216 \mathrm{E}-10$ \\
\hline ZERO & $0.8337 \mathrm{E}-07$ \\
\hline ZERO & ZERO \\
\hline ZERO & $0.1311 E-07$ \\
\hline $.2478 E-01$ & $0.2304 \mathrm{E}+03$ \\
\hline $0.1500 \mathrm{E}+01$ & $0.2342 E+03$ \\
\hline $.3500 \mathrm{E}+02$ & $0.9887 E+02$ \\
\hline $.1000 E+01$ & $0.9656 \mathrm{E}+00$ \\
\hline ZERO & $-0.5055 \mathrm{E}+00$ \\
\hline & $0.7339 \mathrm{E}+03$ \\
\hline $.3146 E+02$ & $0.1476 \mathrm{E}+06$ \\
\hline $.3543 E+00$ & $0.1086 \mathrm{E}+03$ \\
\hline & $0.3594 \mathrm{E}-$ \\
\hline
\end{tabular}

223

224

Cndnste PRD Aqueous PRD Organic
to PRD
to PR

$\begin{array}{lr}\text { PRD } & \text { to PR } \\ \text { ZERO } & \text { ZERO }\end{array}$

ZERO i ZERO

ZERO ZERO

ZERO ZERO

ZERO

ZERO

ZERO

ZERO

ZERO

$0.1607 \mathrm{E}-07$

$0.6587 \mathrm{E}-10$

ZERO

$0.2454 \mathrm{E}-08$

ZERO

ZERO

$0.1061 \mathrm{E}-11$

ZERO

$0.4728 \mathrm{E}-06$

$0.3873 \mathrm{E}-12$

$0.7667 \mathrm{E}-12$

ZERO

$0.7941 \mathrm{E}-18$

$1 E-18$
ZERO

ZERO

ZERO

$0.5836 \mathrm{E}-09$

ZERO

$0.6867 \mathrm{E}-07$

$0.3216 \mathrm{E}-10$

ZERO

$0.1311 \mathrm{E}-07$

$0.2302 \mathrm{E}+03$

$0.2323 E+03$

$0.5000 \mathrm{E}+02$

$0.9656 \mathrm{E}+00$

$-0.5055 \mathrm{E}+00$
$0.7339 \mathrm{E}+03$

$0.1157 \mathrm{E}+05$

$0.4632 \mathrm{E}+00$

$0.6254 \mathrm{E}+02$

$0.2454 \mathrm{E}-08$

0.1061 E-11

ZERO
$0.7941 \mathrm{E}-18$

$0.5836 \mathrm{E}-09$

$0.6867 \mathrm{E}-07$

$-0.5055 \mathrm{E}+00$

225

ZERO

ZERO

ZERO

ZERO

$0.1607 \mathrm{E}-07$

$0.6587 \mathrm{E}-10$

ZÉRO

ZERO

ZERO

ZERO

$0.4728 \mathrm{E}-06$

$0.4528 E-09$

$0.7667 \mathrm{E}-12$

ZERO

$1 E-18$
ZERO

ZERO

ZERO

$0.3216 \mathrm{E}-10$

$0.8337 \mathrm{E}-07$

ZERO
0.1311 E

$0.2302 \mathrm{E}+03$

$0.2320 \mathrm{E}+03$

$0.5000 \mathrm{E}+02$

$0.9656 \mathrm{E}+00$

$0.1157 \mathrm{E}+05$

$0.9656 \mathrm{E}+00$

$-0.5055 E+00$

$0.7339 \mathrm{E}+03$

226

PRD Air

$0.1000 \mathrm{E}+01$

$0.2411 \mathrm{E}-03$

.

IIQUID
ZERO

ZERO

ZERO

ZERO

ZERO

ZERO

ZERO

ZERO

ZERO

ZERO

ZERO

ZERO

ZERO

ZERO

ZERO

ZERO

ZERO

ZERO

ZERO

ZERO

ZERO

ZERO

ZERO

ZERO

ZERO

ZERO

ZERO

ZERO

Inleakage

ZERO
ZERO

ZERO

ZERO

ZERO

ZERO

ZERO

ZERO

ZERO

ZERO

ZERO

ZERO

ZERO

ZERO

ZERO

ZERO

ZERO

ZERO

ZERO

ZERO

ZERO

ZERO

ZERO

ZERO

ZERO

ZERO

ZERO

ZERO

ZERO

ZERO

ZERO

ZERO

ZERO

ZERO

ZERO

ZERO

$0.3500 \mathrm{E}+02$

ZERO

ZERO

$0.9656 \mathrm{E}+00$

$-0.5055 \mathrm{E}+00$

$0.7339 \mathrm{E}+03$

ZERO

VAPOR

ZERO

VAPOR

to. SCVC

ZERO

ZERO

ZERO

ZERO

ZERO

ZERO

ZERO

ZERO

ZERO

ZERO

ZERO

ZERO

ZERO

ZERO

ZERO

ZERO

ZERO

ZERO

ZERO

ZERO

ZERO

ZERO

ZERO

$36 \mathrm{E}+02$
LIQUID

Section 3B, Page 9 of 29 
STREAM NUMBERS -> STREAM NAME

COMPONENT FLOWS, LB/HR $122(\mathrm{C} 6 \mathrm{H} 5) 2$

123 (C6H5) $2 \mathrm{C} 6 \mathrm{H} 4$

118 (C6H5) $2 \mathrm{NH}$

133 (C6H5B (OH) 2 salt)

132 (C6H5B (OH) 2)

166 (C6H5C6H4OH)

162 (C6H5NH2)

163 (C6H5NHCHO)

165 (C6H5NO)

131 (C6H5OH salt)

130 (C6H5OH)

121 (C6H6)

155 (СH3) $2 \mathrm{CHOH}$

154 (СH $30 \mathrm{OH})$

$45 \mathrm{Ag}$

116 Ag20

134 AgNO3

$106 . \mathrm{B} 203$

$9 \mathrm{Ba}(\mathrm{OH}) 2$

15. BaO

24 BasO4

$66 \mathrm{CO}$

$37 \mathrm{CO} 2$

$142 \mathrm{CO} 2$ (C14)

$63 \mathrm{Ca}(\mathrm{COOH}) 2$

$167 \mathrm{Ca}(\mathrm{NO} 3) 2$

$120 \mathrm{Ca}(\mathrm{OH}) 2$

$113 \mathrm{Ca} 3(\mathrm{PO} 4) 2$

$20 \mathrm{CaC} 2 \mathrm{O} 4$

$18 \mathrm{CaCO} 3$
$64 \mathrm{CaCO} 3$ (C1.4)

$64 \mathrm{CaCO}$

$78 \mathrm{CaF} 2$

$\begin{array}{ll}41 & \mathrm{CaO} \\ 19 & \mathrm{CaSO} 4\end{array}$

29 Carbon

99 Cement

$26 \mathrm{Co}(\mathrm{COOH}) 2$

$168 \mathrm{Co}$ (NO3) 2

$59 \mathrm{CoO}$

$98 \mathrm{Cr} 203$

$42 \mathrm{Cs} 2 \mathrm{O}$

$53 \mathrm{CsCOOH}$

$96 \mathrm{CsCl}$

169. CsNO3

$8 \mathrm{CsOH}$

151 CsTPB

WSRC-TR-95-0019 (Revision

Table A-1. HLW Flowsheet Material Balance (Tank 51/ITP Cycle 1) Section 3B. Boilup of Precipitate Hydrolysis Products

$229 \quad 230$

PRC Vent SCVC Cndnste
to SCVC to OE

\section{$0.1589 \mathrm{E}-01$}

$0.4442 \mathrm{E}-10$

$0.5820 \mathrm{E}-04$

ZERO

$0.9919 \mathrm{E}-10$

$0.2603 \mathrm{E}-03$

$0.1025 \mathrm{E}-14$

$0.1669 \mathrm{E}-16$

$0.3400 \mathrm{E}-16$

ZERO

$0.5629 \mathrm{E}-03$

$0.2328 \mathrm{E}+00$

$0.589 .5 \mathrm{E}-13$

$0.2609 \mathrm{E}-1.3$

$0.1393 \mathrm{E}-16$

ZERO

$0.6357 \mathrm{E}-15$

$0.8184 \mathrm{E}-17$

ZERO

ZERO

$0.8783 \mathrm{E}-17$

ZERO

$0.2297 \mathrm{E}-01$
$0.2219 \mathrm{E}-12$

$0.2219 \mathrm{E}-12$

$0.6712 \mathrm{E}-20$

ZERO

$0.2747 \mathrm{E}-14$

$0.1197 \mathrm{E}-20$

ZERO

ZERO

$0.9366 \mathrm{E}-15$

ZERO

ZERO
$0.7020 \mathrm{E}-18$

$0.2633 \mathrm{E}-20$

. $1504 \mathrm{E}-18$

$.1282 \mathrm{E}-14$

$0.2122 \mathrm{E}-15$

ZERO

ZERO

ZERO

$0.1574 \mathrm{E}+00$

$0.4201 \mathrm{E}-09$

$0.1267 \mathrm{E}-03$

ZERO

$0.3099 \mathrm{E}-09$

$0.1460 \mathrm{E}-02$

$0.1025 \mathrm{E}-14$

$0.1788 \mathrm{E}-16$

$0.3400 \mathrm{E}-16$ ZERO

$0.5210 \mathrm{E}-02$

$0.2166 \mathrm{E}+02$

$0.8384 \mathrm{E}-11$
$0.8852 \mathrm{E}-12$

$0.1393 \mathrm{E}-16$

ZERO

$0.6357 \mathrm{E}-15$

$0.8184 \mathrm{E}-17$

$$
\text { ZERO }
$$

ZERO

ZERO

$0.1681 \mathrm{E}-01$
$0.2971 \mathrm{E}-13$

$0.2971 \mathrm{E}-13$

$0.6712 \mathrm{E}-20$

ERO

$0.2747 \mathrm{E}-14$

$0.1197 \mathrm{E}-20$

ZERO

ZERO

ZERO ZE -15
ZERO

$0.2633 \mathrm{E}-20$

$0.1504 \mathrm{E}-18$

$0.1282 \mathrm{E}-14$

$0.1885 \mathrm{E}-18$

$0.2122 \mathrm{E}-15$

ZERO
ZERO
ZERO

ZERO
ZERO
$0.7020 \mathrm{E}-18$

Date: January 1995

ZERO

ZERO

ZERO

ZERO

ZERO

ZERO

ZERO

ZERO

ZERO

ZERO

ZERO

ZERO

ZERO

ZERO

ZERO

ZERO

ZERO

$1 \mathrm{E}+02$
ZERO

ZERO

ZERO

ZERO

ZERO

ZERO

ZERO

ZERO

ZERO

ZERO

ZERO

ZERO

ZERO

ZERO

ZERO

ZERO

ZERO

ZERO

ZERO
233

$\mathrm{CO} 2$ Purge H2O Addtn to OE to Org Evap

ZERO ZERO

ZERO ZERO

ZERO ZERO

ZERO ZERO

ZERO

ZERO

ZERO

ZERO

ZERO

ZERO

ZERO

ZERO

ZERO

ZERO

ZERO

ZERO

ZERO

ZERO

ZERO

ZERO

ZERO

ZERO

ZERO

ZERO

ZERO

ZERO

ZERO

ZERO

ZERO

ZERO

ZERO

$Z$ RRO

ZERO

ZERO

ZERO

ZERO

ZERO

ZERO

ZERO

ZERO

ZERO

ZERO

ZERO

ZERO

ZERO

ZERO

ZERO

o)

Inleakag OE $\mathrm{CO} 2$ Purge

akage Vapor to OEC

$\begin{array}{lr}\text { ZERO } & 0.1614 \mathrm{E}-03 \\ \text { ZERO } & 0.7135 \mathrm{E}-11 \\ \text { ZERO } & 0.1996 \mathrm{E}-08 \\ \text { ZERO } & \text { ZERO } \\ \text { ZERO } & \text { ZERO } \\ \text { ZERO } & 0.6064 \mathrm{E}-08\end{array}$

ZERO

ZERO

ZERO

ZERO

ZERO

ZERO

ZERO

ZERO

ZERO

ZERO

ZERO

ZERO

ZERO

ZERO

ZERO

ZERO

ZERO

ZERO

ZERO

ZERO

ZERO

ZERO

ZERO

ZERO

ZERO

ZERO

ZERO

ZERO

ZERO

ZERO

ZERO

ZERO

ZERO

ZERO

ZERO

ZERO

ZERO

ZERO

ZERO

ZERO

ZERO

ZERO

$0.8781 \mathrm{E}-06$

$0.1258 \mathrm{E}-17$

$0.4074 \mathrm{E}-20$ ZERO

$0.5182 \mathrm{E}-05$

$0.9930 \mathrm{E}-01$

$0.2580 \mathrm{E}-13$

ZERO

ZERO

ZERO

ZERO

ZERO

ZERO 
Table A-1. HLW Flowsheet Material Balance (Tank 51/ITP Cycle 1) section 3B. Boilup of Precipitate Hydrolysis. Products

STREAM NUMBERS (CONT'D) $\rightarrow$ STREAM NAME

$54 \mathrm{Cu}(\mathrm{COOH}) 2$

$170 \mathrm{Cu}$ (NO3) 2

$55 \mathrm{CuO}$

$80 \mathrm{Fe} 203$

101 FeO

102 Group A

103 Group B

$25 \mathrm{H} 2$

$158 \mathrm{H} 2 \mathrm{C} 2 \mathrm{O} 4$

93 H3BO3

34. $\mathrm{HCOOH}$

$43 \mathrm{HCl}$

$85 \mathrm{HF}$

49 . HNO3

$36 \mathrm{Hg}$

$129 \mathrm{Hg}(\mathrm{C} 6 \mathrm{H} 5) 2$

$135 \mathrm{Hg}(\mathrm{NO} 3) 2$

$144 \mathrm{Hg} 2 \mathrm{Cl}$

$117 \mathrm{Hg} 2 \mathrm{I} 2$

$146 \mathrm{HgCl2}$

$21 \mathrm{HgO}$

$183 \mathrm{~K} 2 \mathrm{CO}$

$39 \times 20$

$51 \mathrm{KCOOH}$

$157 \mathrm{KMnO} 4$

$161 \mathrm{KNO}$

$150 \mathrm{KTPB}$

$105 \mathrm{La203}$

$108 \cdot \mathrm{I}$ i20

$65 \mathrm{Mg}(\mathrm{COOH}) 2$

$171 \mathrm{Mg}$ (NO3) 2

$33 \mathrm{Mg}$

$60 \mathrm{Mn}(\mathrm{COOH}) 2$

$172 \mathrm{Mn}$ (NO3)

$180 \mathrm{Mn} 30$

$56 \mathrm{MnO}$

$14 \mathrm{MnO} 2$

$81 \mathrm{~N} 2$

74 N2O

182 NH3

114 NH3OHNO3

52 NH4COOH

173 NH4NO3

148 NH 4 TPB

WSRC-TR-95-0019 (Revision
229 230

PRC Vent sCVC Cndnste to scve

$0.1010 \mathrm{E}-17$

$0.1068 \mathrm{E}-13$

$0.4677 \mathrm{E}-17$

$0.7777 \mathrm{E}-15$

$0.1194 \mathrm{E}-19$

$0.3671 \mathrm{E}-21$

$0.1012 \mathrm{E}-17$

$0.2571 \mathrm{E}-16$

$0.3776 \mathrm{E}-13$

$0.2143 \mathrm{E}-03$

ZERO
ZERO

ZERO

$0.1484 \mathrm{E}-08$

$0.1078 \mathrm{E}-10$

ZERO

ZERO

ZERO

ZERO

ZERO

$0.3372 \mathrm{E}-16$

$0.4922 \mathrm{E}-13$

ZERO

ZERO

ZERO

$0.1444 \mathrm{E}-17$

$0.1920 \mathrm{E}-16$

$0.2574 \mathrm{E}-21$

$0.1682 \mathrm{E}-17$

$0.1620 \mathrm{E}-16$

$0.3000 \mathrm{E}-21$
ZERO ZERO

$0.6434 \mathrm{E}-17$

$0.6315 \mathrm{E}-18$

$0.1133 \mathrm{E}+01$

$0.1897 \mathrm{E}-06$

ZERO

$0.2398 \mathrm{E}-17$

ZERO
ZERO

ZERO
231

232
Puxge
to OE to Org Evap to OE to Org Evap
ZERO

$\begin{array}{ll}\text { ZERO } & \text { ZERO } \\ \text { ZERO } & \text { ZERO }\end{array}$

ZERO ZERO

ZERO

ZERO ZERO

ZERO ZERO

ZERO ZERO

ZERO ZERO

ZERO ZERO

ZERO ZERO

ZERO ZERO

ZERO ZERO

ZERO ZERO

ZERO - ZERO

ZERO ZERO

ZERO ZERO

ZERO ZERO.

ZERO ZERO

ZERO

ZERO

ZERO

ZERO

ZERO
ZERO

ZERO

ZERO

ZERO

ZERO

ZERO

ZERO

ZERO

ZERO

ZERO

ZERO

ZERO

ZERO

ZERO

ZERO

ZERO

ZERO

ZERO

ZERO

ZERO

ZERO

ZERO

ZERO
233

234 kage Vapor to OEC ZERO ZERO ZERO ZERO

ZERO ZERO

ZERO ZERO

ZERO ZERO

ZERO ZERO

ZERO ZERO

ZERO

$\begin{array}{ll}\text { ZERO } & \text { ZERO } \\ \text { ZERO } & \text { ZERO }\end{array}$

ZERO ZERO

ZERO $\quad 0.2688 \mathrm{E}-04$

ZERO

ZERO

ZERO

ZERO

ZERO

ZERO

ZERO

ZERO

ZERO

ZERO

ZERO

ZERO

ZERO

ZERO

ZERO

ZERO

ZERO

ZERO

ZERO

ZERO

ZERO

ZERO

ZERO

ZERO

ZERO

ZERO

ZERO

$0.1133 \mathrm{E}+01$

ZERO

ZERO

ZERO

ZERO

ZERO

ZERO
235

Boilup to

OE Cndnsr

$0.4643 \mathrm{E}-23$

$0.2151 \mathrm{E}-22$
$0.3576 \mathrm{E}-20$

ZERO

TRACE

$0.4387 \mathrm{E}-19$

$0.4387 \mathrm{E}-19$

ZERO

$0.1737 \mathrm{E}-18$

$0.3696 \mathrm{E}-02$

ZERO

ZERO

$0.2087 E-12$

ZERO

ZERO

ZERO

ZERO

ZERO

ZERO

ZERO

ZERO

ZERO

ZERO

ZERO

ZERO

ZERO

ZERO

ZERO

ZERO

ZERO

ZERO

ZERO

ZERO
ZERO

ZERO

ZERO

ZERO

ZERO

$0.1853 \mathrm{E}-04$

$0.1831 E-13$

E-07

ZERO

ZEERO

ZERO

ZERO
ZERO

ZERO

ZERO

$0.1661 \mathrm{E}-09$

$0.1326 \mathrm{E}-02$

ZERO

ZERO

ZERO

ZERO

ZERO

ZERO

$0.1551 \mathrm{E}-21$

$0.2263 \mathrm{E}-18$

ZERO

ZERO

ZERO

ZERO

$0.66417-23$

TRACE

$0.7733 \mathrm{E}-23$

$0.7449 \mathrm{E}-22$
TRACE

ZERO

ZERO

$0.2904 \mathrm{E}-23$

$0.1133 \mathrm{E}+01$

$0.3275 \mathrm{E}-06$

ZERO

ZERO

ZERO 
Table A-1. HLW Flowsheet Material Balance (Tank 51/ITP Cyole 1) Section 3B. Boilup of Precipitate Hydrolysis Products

STREAM NUMBERS (CONT'D) STREAM NAME

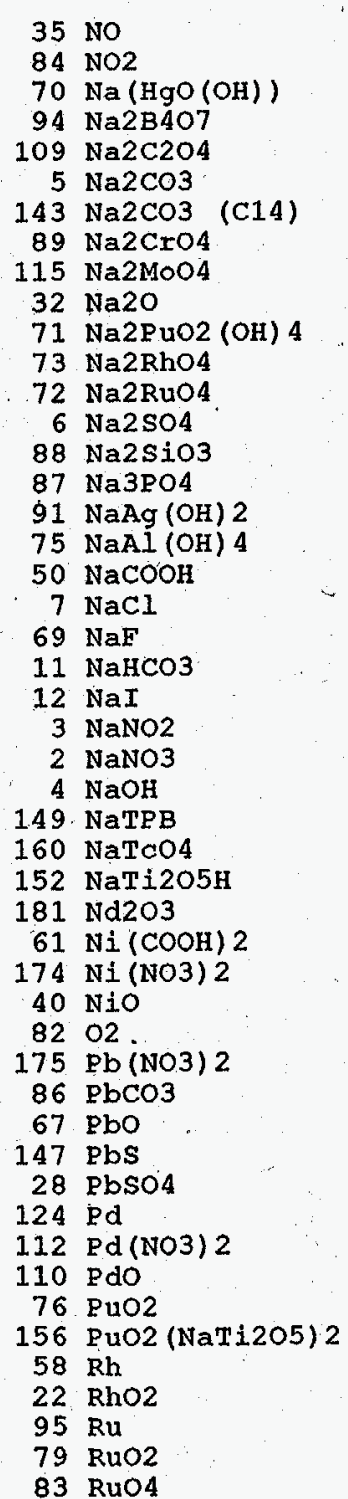

\begin{tabular}{|c|c|c|c|c|c|}
\hline 229 & 230 & 231 & & & \\
\hline PRC Vent & scVC Cndnste & $\mathrm{CO} 2$ & Purge & $\mathrm{H} 2 \mathrm{O}$ & Addtn \\
\hline to SCVC & to $O E$ & & to OE & to org & Evap \\
\hline $0.8978 E-05$ & $0.4251 \mathrm{E}-06$ & & ZERO & & ZERO \\
\hline $0.7133 \mathrm{E}-12$ & $0.3377 \mathrm{E}-13$ & & ZERO & $!$ & ZERO \\
\hline ZERO & ZERO & & ZERO & & ZERO \\
\hline ZERO & ZERO & & ZERO & & ZERO \\
\hline ZERO & ZERO & & ZERO & & ZERO \\
\hline ZERO & ZERO & & ZERO & & ZERO \\
\hline ZERO & ZERO & & ZERO & & ZERO \\
\hline ZERO & ZERO & & ZERO & & ZERO \\
\hline ZERO & ZERO & & ZERO & & ZERO \\
\hline $0.4916 \mathrm{E}-14$ & $0.4916 \mathrm{E}-14$ & & ZERO & & ZERO \\
\hline ZERO & ZERO & & ZERO & & ZERO \\
\hline ZERO & ZERO & & ZERO & & ZERO \\
\hline ZERO & ZERO & & ZERO & & ZERO \\
\hline $0.5303 E-17$ & $0.5303 E-17$ & & ZERO & & ZERO \\
\hline ZERO & ZERO & & ZERO & & ZERC \\
\hline $0.7042 E-17$ & $0.7042 \mathrm{E}-17$ & & ZERO & & ZERC \\
\hline ZERO & ZERO & & ZERO & & ZERC \\
\hline ZERO & ZERO & & ZERO & & ZERO \\
\hline $0.5578 \mathrm{E}-14$ & $0.5578 \mathrm{E}-14$ & & ZERO & & ZERO \\
\hline $0.1558 \mathrm{E}-16$ & $0.1558 \mathrm{E}-16$ & & ZERO & & ZERC \\
\hline $0.4077 E-18$ & $0.4077 \mathrm{E}-18$ & & ZERO & & ZERC \\
\hline ZERO & ZERO & & ZERO & & ZERC \\
\hline $0.2731 \mathrm{E}-21$ & $0.2731 \mathrm{E}-21$ & & ZERO & & ZERC \\
\hline ZERO & ZERO & & ZERO & & ZERO \\
\hline $0.1078 \mathrm{E}-15$ & $0.1078 \mathrm{E}-15$ & & ZERO & & ZERO \\
\hline ZERO & ZERO & & ZERO & & ZERO \\
\hline ZERO & ZERO & & ZERO & & ZERO \\
\hline ZERO & ZERO & & ZERO & & ZERO \\
\hline ZERO & ZERO & & ZERO & & ZERC \\
\hline ZERO & ZERO & & ZERO & & ZERC \\
\hline $0.2034 \mathrm{E}-17$ & $0.2034 \mathrm{E}-17$ & & ZERO & & ZERO \\
\hline $0.3276 \mathrm{E}-20$ & $0.3276 \mathrm{E}-20$ & & ZERO & & ZERC \\
\hline $0.4061 \mathrm{E}-17$ & $0.4061 \mathrm{E}-17$ & & ZERO & & ZERO \\
\hline $0.3423 \mathrm{E}+00$ & $0.1143 \mathrm{E}-04$ & & ZERO & & ZERC \\
\hline $0.7955 \mathrm{E}-20$ & $0.7955 \mathrm{E}-20$ & & ZERO & & ZERC \\
\hline ZERO & ZERO & & ZERO & & ZERC \\
\hline ZERO & ZERO & & ZERO & & ZERO \\
\hline ZERO & ZERO & & ZERO & & ZERC \\
\hline $0.2138 \mathrm{E}-16$ & $0.2138 \mathrm{E}-16$ & & ZERO & & ZERC \\
\hline $0.1518 \mathrm{E}-22$ & $0.1518 \mathrm{E}-22$ & & ZERO & & ZERO \\
\hline ZERO & ZERO & & ZERO & & ZERO \\
\hline ZERO & ZERO & & ZERO & & ZERO \\
\hline $0.1573 \mathrm{E}-18$ & $0.1573 \mathrm{E}-18$ & & ZERO & & ZERO \\
\hline $0.6233 \mathrm{E}-18$ & $0.6233 \mathrm{E}-18$ & & ZERO & & ZERO \\
\hline $0.1784 \mathrm{E}-20$ & $0.1784 \mathrm{E}-20$ & & ZERO & & ZERO \\
\hline ZERO & ZERO & & ZERO & & ZERC \\
\hline ZERO & ZERO & & ZERO & & ZERC \\
\hline $0.2169 \mathrm{E}-16$ & $0.2169 \mathrm{E}-16$ & & ZERO & & ZERC \\
\hline ZERO & ZERO & & ZERO & & ZERC \\
\hline
\end{tabular}

Date: January 1995
233 234

OE Air OE CO2 Purge Inleakage Vapor to OEC $\begin{array}{rr}\text { ZERO } & 0.5708 E-06 \\ \text { ZERO } & 0.4535 E-13 \\ \text { ZERO } & \text { ZERO }\end{array}$ ZERO ZERO ZERO ZERO

ZERO

ZERO

ZERO

ZERO

ZERO

ZERO

ZERO

ZERO

ZERO

ZERO

ZERO.

ZERO,

ZERO

ZERO

ZERO

IERO

ZERO

ZERO

ZERO

ZERO

ZERO

ZERO

ZERO

$0.3423 \mathrm{E}+00$

ZERO

ZERO

ZERO

ZERO

ZERO

ZERO

ZERO

ZERO

ZERO

ZERO

ZERO

ZERO

ZERO

ZERO

ZERO

ZERO

ZERO

ZERO

ZERO

ZERO

ZERO

ZERO

ZERO

ZERO

$$
\text { ZERO }
$$

ZERO

ZERO

ZERO

ZERO

ZERO
ZERO

ZERO

ZERO

ZERO

ZERO

ZERO

ZERO

ZERO

ZE-04
ZERO

ZERO

ZERO

ZERO

ZERO

ZERO

ZERO

ZERO

ZERO

ZERO

ZERO

ZERO

ZERO

235

Boilup to

OE Cndnsr

$0.1489 \mathrm{E}-15$

ZERO

ZERO

ZERO

ZERO

ZERO

ZERO

$0.2261 \mathrm{~F}-19$

ZERO

ZERO

$0.2438 \mathrm{E}-22$

ZERO
$0.3238 E-22$

ZERO

$0.2565 E-19$

$0.7164 \mathrm{E}-22$.

$0.1875 \mathrm{E}-23$

ZERO

PRACE

ZERO
$0.4958 \mathrm{E}-21$

ZERO

ZERO

ZERO

ZERO

$0.9355 \mathrm{E}-23$

TRACE

$0.1867 E-22$

TRACE

ZERO

ZERO

$0.9829 \mathrm{E}-22$

TRACE

ZERO

$0.7231 \mathrm{E}-24$

$0.2866 \mathrm{E}-23$

TRACE

ZERO

$0.9973 \mathrm{E}-22$

ZERO

Section 3B, Page 12 of 29 
Table A-1. HLW Flowsheet Material Balance (Tank 51/ITP Cycle 1) section 3B. Boilup of Precipitate Hydrolysis Products

STREAM NUMBERS (CONT'D) $\rightarrow$ STREAM NAME

\footnotetext{
$38 \mathrm{SO} 2$

$141 \mathrm{SO} 3$

137 Semi Vol Cs20

136 Semi Vol CsCl

126 Semi Vol Group A

140 Semi Vol Na2B4O7

138 Semi Vol NaC1

125 Semi Vol NaI

23 Semi Vol RuO2

111 SiO2:

$48 \mathrm{Sr}(\mathrm{COOH}) 2$

$176 \operatorname{sr}(\mathrm{NO} 3) 2$

$153 \mathrm{Sr}$ (NaTi2O5) 2

127. $\mathrm{Sr}(\mathrm{OH}) 2$

$30 \mathrm{SrCO}$

46 SrO

$159 \mathrm{TcO} 2$

92 ThO2

119 Tritium

$\begin{aligned} 119 & \text { Tritt } \\ 17 & \text { U308 }\end{aligned}$

62 UO2 (COOH) 2

$17.7 \mathrm{OO} 2$ (NO3) 2

16 UO2 (OH) 2

$27 \mathrm{Y}(\mathrm{COOH}) 3$

$178 \mathrm{Y}(\mathrm{NO} 3)$

$128 \mathrm{Y}(\mathrm{OH}) 3$

$47 \mathrm{Y} 203$

100 zeolite

$44 \mathrm{Zn}(\mathrm{COOH})$

$179 \mathrm{zn}(\mathrm{NO} 3)$

$104 \mathrm{ZrO} 2$

13 hydrate $\mathrm{H} 2 \mathrm{O}$

1 water

TOTAL FLOW, LB/HR

TEMPERATURE， DEG C

PRESSURE， ATM

PRESSURE， PSIG

PRESSURE， MM HG

ENTHAT.PY, PCU/HR

VAPOR FLOW, CFM

ITOUID EIOH, CEM

LIQUID FLOW, GPM
}

PHASE

229
PRC

PRC Vent 230

to SCVC SCVC Cndnste

$\begin{array}{lr}\text { SCVC } & \text { to OE } \\ \text { ZERO } & \text { ZERO }\end{array}$

$\begin{array}{ll}\text { ZERO } & \text { ZERO } \\ \text { ZERO } & \text { ZERO }\end{array}$

ZERO ZERO

$\begin{array}{ll}\text { ZERO } & \text { ZERO } \\ \text { ZERO } & \text { ZERO }\end{array}$

ZERO ZERO

ZERO

ZERO

ZERO

$0.1307 \mathrm{E}-15$

$0.5354 \mathrm{E}-18$

ZERO
$5 E-16$

$0.1995 \mathrm{E}-16$

ZERO

ZERO

$0.8628 \mathrm{E}-20$

ZERO

$0.3844 \mathrm{E}-14$

$0.3681 \mathrm{E}-17$

$0.3681 \mathrm{E}-17$

ZERO
ZERO

ZERO

ZERO

$0.4744 \mathrm{E}-17$

$0.5583 \mathrm{E}-15$

$0.2614 \mathrm{E}-18$

$0.6777 \mathrm{E}-15$

ZERO

$0.1066 \mathrm{E}-15$

$0.1407 \mathrm{E}+00$

$0.1889 \mathrm{E}+01$

$0.5000 \mathrm{E}+02$

$0.9656 \mathrm{E}+00$

$-0.5055 \mathrm{E}+00$

$0.7339 \mathrm{E}+03$

$0.1398 \mathrm{E}+03$

$0.4585 \mathrm{E}+00$

$0.6865 \mathrm{E}-01$

VAPOR

$-0.5055 \mathrm{E}+00$
231

Purge
to OE

OE to 2 Addtn

ZERO ZERO

ZERO ' ZERO

ZERO IERO

ZERO ZERO

ZERO

ZERO ZERO

ZERO ZERO

ZERO

ZERO ZERO

ZERO Z ZERO

ZERO

$0.1307 \mathrm{E}-15$

$0.5354 \mathrm{E}-18$

$0.1995 \mathrm{E}-16$

ZERO

ZERO

$0.8628 \mathrm{E}-20$

ZERO

$0.3844 \mathrm{E}-14$

$0.5019 \mathrm{E}-14$

$0.3681 \mathrm{E}-17$

$0.6233 \mathrm{E}-20$

ZERO

TRERO

ZERO

ZERO

ZERO
$0.4744 \mathrm{E}-17$

ZERO

$0.5583 \mathrm{E}-15$

$0.2614 E-18$

ZERO

$-15$

$0.3216 \mathrm{E}+01$

$0.1000 \mathrm{E}+02$

$0.9656 \mathrm{E}+00$

$.7339 \mathrm{E}+03$

$0.5543 \mathrm{E}-01$

$0.5637 \mathrm{E}+02$

LIQUID

$0.8249 \mathrm{E}+02$

$0.8249 \mathrm{E}+02$

$0.35000+02$

$0.1000 \mathrm{E}+01$

ZERO

$0.3500 \mathrm{E}+02$

$0.1000 \mathrm{E}+01$

ZERO

$0.9847 \mathrm{E}+03$

$0.2256 \mathrm{E}+01$

$0.2894 \mathrm{E}+04$

$0.1647 \mathrm{E}+00$

$0.1087 \mathrm{E}+00$

LIQUID
233

23 ZERO ZERO

ZERO

ZERO

ZERO

ZERO

ZERO

ZERO

ZERO

ZERO

ZERO

ZERO

ZERO

ZERO

ZERO

ZERO

ZERO

ZERO

ZERO

ZERO

ZERO

ZERO

ZERO

ZERO

ZERO

2ERO

ZERO

ZERO

ZERO

ZERO

ZERO

ZERO

$0.2478 \mathrm{E}-01$

$0.1500 \mathrm{E}+01$

$0.1000 E+01$

ZERO

$0.3146 \mathrm{E}+0.2$

$0.3543 \mathrm{E}+00$

$0.7055 \mathrm{E}-01$

VAPOR

Section 3B, Page 13 of 29
235

Boilup to

OE Cndnst

ZERO

ZERO

ZERO

ZERO

ZERO

ZERO

ZERO

ZERO

$0.6009 \mathrm{E}-21$

$0.2462 E-23$

$\begin{array}{rr}\text { ZERO } & \text { ZERO } \\ \text { ZERO } & 0.9175 \mathrm{E}-22\end{array}$

ZERO

ZERO

ZERO

ZERO

$0.3757 \mathrm{E}-16$

ZERO

ZERO

ZERO

ZERO

ZERO

ZIERO

ZERO

ZERO

ZERO

ZERO

ZERO

ZERO

IRO

$0.6679 \mathrm{E}+00$

$0.1497 \mathrm{E}+02$

ZERO

ZERO

TRACE

0.1767 E 19

$0.1117 \mathrm{E}-13$

$0.1693 \mathrm{E}-22$

TRACE

ZERO

ZERO
TRACE

ZERO

ZERO

$0.2182 \mathrm{E}-22$

ZERO

$0.2567 \mathrm{E}-20$

$0.1202 \mathrm{E}-23$

ZERO

$0.4902 \mathrm{E}-21$

$0.1999 \mathrm{E}+03$

$0.2342 \mathrm{E}+03$

$0.4652 \mathrm{E}+02$

$\begin{array}{ll}0.4652 \mathrm{E}+02 & 0.9747 \mathrm{E}+02 \\ 0.9656 \mathrm{E}+00 & 0.9656 \mathrm{E}+00\end{array}$

$.5055 \mathrm{E}+00-0.9656 \mathrm{E}+00$

$\begin{array}{ll}0.1405 \mathrm{E}+04 & 0.1320 \mathrm{E}+06 \\ 0.2617 \mathrm{E}+01 & 0.9720 \mathrm{E}+02\end{array}$

$0.4016 \mathrm{E}-01$

VAPOR

VAPOR 
Table A-1. HLW Flowsheet Material Balance (Tank 51/ITP Cycle 1) A-1. HLW Flowsheet Material Balance (Tank $51 /$ ITP Cycl
section 3B. Boilup of Precipitate Hydrolysis Products

STREAM NUMBERS -> STREAM NAME

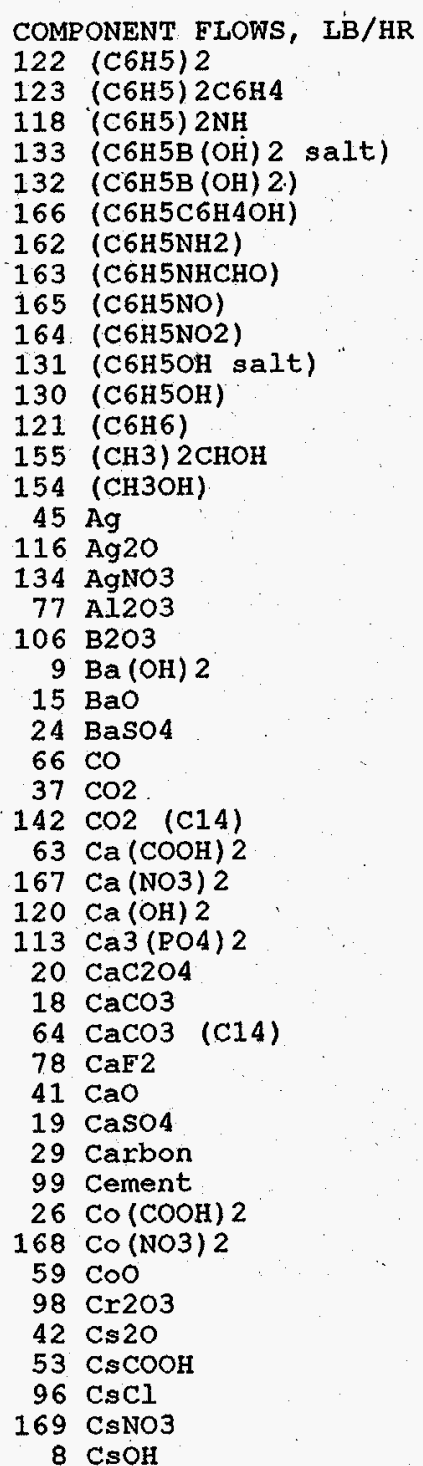

236 OED Aque

$$
\begin{aligned}
& \text { ZERO } \\
& \text { ZERO } \\
& \text { ZERO } \\
& \text { ZERO } \\
& 0.7456 \mathrm{E}-02 \\
& 0.7541 \mathrm{E}-20 \\
& 0.6255 \mathrm{E}-19 \\
& 0.4174 \mathrm{E}-20 \\
& \text { ZERO }
\end{aligned}
$$

237 Inleakage

238

OED Vent

239

OEC Cndnste to OED

$0.5081 \mathrm{E}-04$

$0.9621 \mathrm{E}-040.1004 \mathrm{E}+01$

$0.8983 \mathrm{E}-09$ ZERO ZERO ZERO

$0.3896 \mathrm{E}-06$ ZERO ZERO ZERO

$0.2218 \mathrm{E}-05$ $0.2975 \mathrm{E}-01$ $0.7319 \mathrm{E}-15$ $0.2915 \mathrm{E}-15$ ZERO
- ZERO ZERO ZERO ZERO ZERO ZERO ZERO ZERO

$0.2263 \mathrm{E}-02$ $0.2263 \mathrm{E}-02$
$0.5442 \mathrm{E}-17$ $0.5442 \mathrm{E}-17$
ZERO ZERO
ZERO ZERO ZERO ZERO ZERO ZERO ZERO ZERO ZERO ZERO ZERO ZERO ZERO ZERO ZERO ZERO ZERO ZERO ZERO $0.1004 \mathrm{E}+01$
$0.5464 \mathrm{E}-02$
$0.7022 \mathrm{E}-01$ $0.7022 \mathrm{E}-01$ ZERO

$0.1220 \mathrm{E}-01$ $0.2142 \mathrm{E}-01$ $0.7543 \mathrm{E}-20$ $0.4175 \mathrm{E}-20$ ZERO $0.1650 \mathrm{E}+00$ $0.2931 \mathrm{E}+02$
$0.3301 \mathrm{E}-10$ $0.5675 \mathrm{E}-11$ $0.6401 \mathrm{E}-22$ ZERO $0.2923 \mathrm{E}-20$ $0.3763 \mathrm{E}-22$ ZERO $0.4039 \mathrm{E}-22$ ZERO $0.1103 \mathrm{E}-01$
$0.2653 \mathrm{E}-16$ $0.2653 \mathrm{E}-16$
ZERO ZERO ZERO $0.1263 \mathrm{E}-19$ TRACE ZERO ZERO ZERO $0.4307 \mathrm{E}-20$

ZERO

$0.3228 \mathrm{E}-23$
TRACE $0.6915 \mathrm{E}-24$ $0.5896 \mathrm{E}-20$ $0.8670 \mathrm{E}-24$
$0.9757 \mathrm{E}-21$ ZERO ZERO ZERO
240

OEC Vent

241

to SCVC to OECT

242

OECT Aix

$0.2896 \mathrm{E}-01-0.1004 \mathrm{E}+01$

$0.9346 \mathrm{E}-10 \quad 0.5464 \mathrm{E}-02 \quad$ ZERO

$0.6695 \mathrm{E}-04 \quad 0.7022 \mathrm{E}-01 \quad$ ZERO

ZERO

ZERO
$.2107 \mathrm{E}-09$

$0.5922 \mathrm{E}-04$

$0.7078 \mathrm{E}-24$

$0.1195 \mathrm{E}-17$

$0.5532 \mathrm{E}-22$

ZERO

$0.9094 \mathrm{E}-04$

$0.1794 \mathrm{E}+01$

$0.4758 \mathrm{E}-13$

TRACE

ZERO

TRACE

TRACE

ZERO

TRACE

ZERO

$0.1473 \mathrm{E}+02$

$0.3542 \mathrm{E}-13$
ZERO

ZERO
TRACE

TRACE

TRACE

TRACE

ZERO

ZERO

ZERO

TRACE

ZERO

ZERO

TRACE

TRACE

TRACE

TRACE

TRACE

TRACE

ZERO

ZERO

ZERO

$0.1220 \mathrm{E}-01$

$0.1396 \mathrm{E}-01$

$0.1762 \mathrm{E}-23$

$0.1462 \mathrm{E}-22$

$0.9755 \mathrm{E}+24$

ZERO

$37 \mathrm{E}+00$

$0.2885 \mathrm{E}+02$

$0.1194 \mathrm{E}-11$
$0.2052 \mathrm{E}-12$

ZERO

ZERO

$0.6831 \mathrm{E}-24$

TRACE

ZERO

ZERO

TRACE

$0.3171 \mathrm{E}-03$

$0.7625 \mathrm{E}-18$

ZERO

TRACE

$0.2952 \mathrm{E}-23$

TRACE

ZERO

ZERO

ZERO

$0.1006 \mathrm{E}-23$

ZERO

ZERO

TRACE

TRACE

$0.1378 \mathrm{E}-23$

TRACE

$0.2280 \mathrm{E}-24$

ZERO

ZERO

ZERO

ZERO

ZERO

ZERO

ZERO

ZERO

ZERO

ZERO

ZERO

ZERO

ZERO

ZERO

ZERO

ZERO 
Table A-1. HLW Flowsheet Material Balance (Tank 51/ITP Cycle 1)

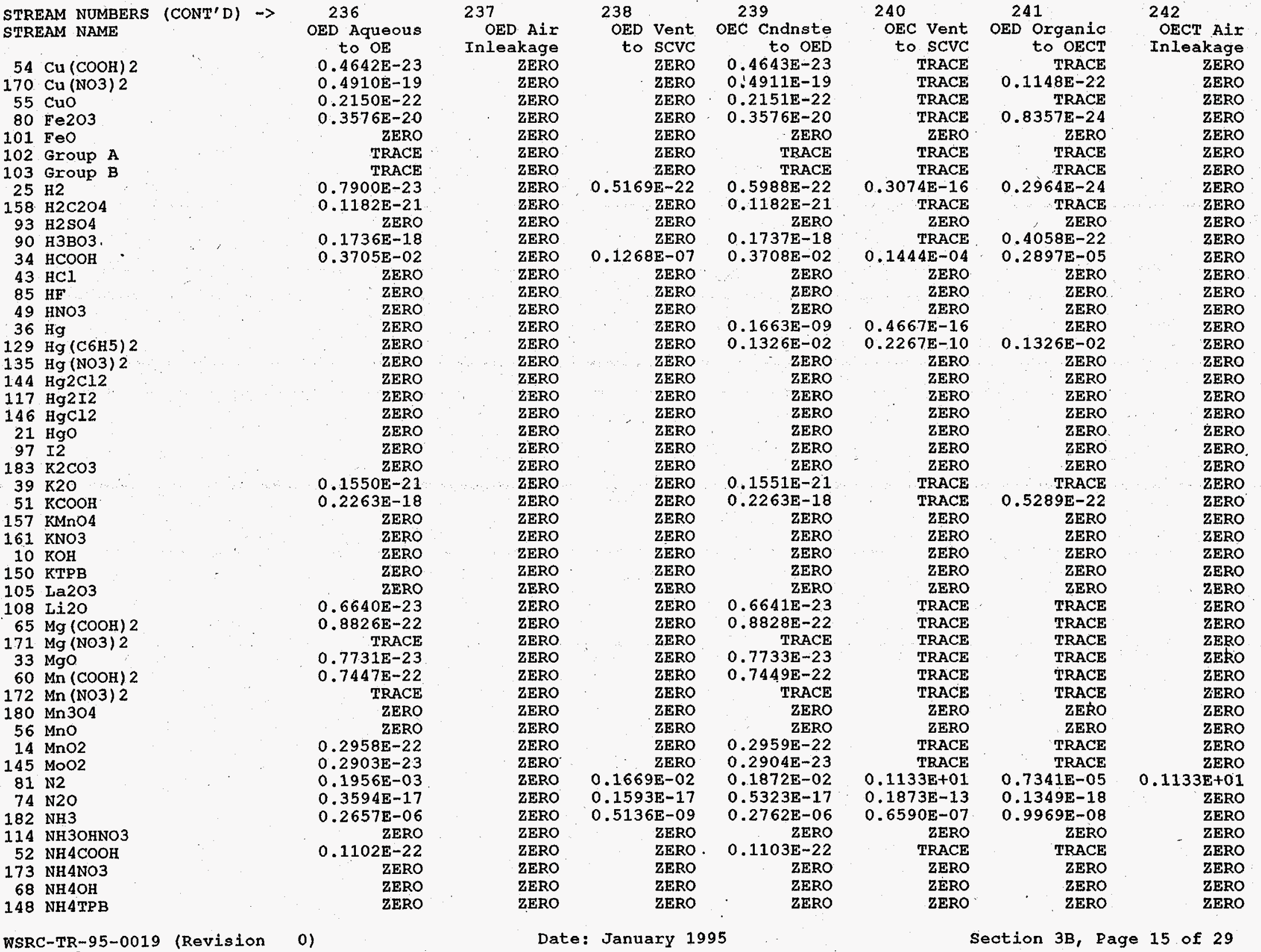


WSRC-TR-95-0019 (Revision

o)

Table A-1. HLW. Flowsheet Material Balance (Tank 51/ITP Cycle 1) A-1. HLW. Flowsheet Material Balance (Tank $51 /$ ITP Cycle
Section 3B. Boilup of Preaipitate Hydrolysis Products

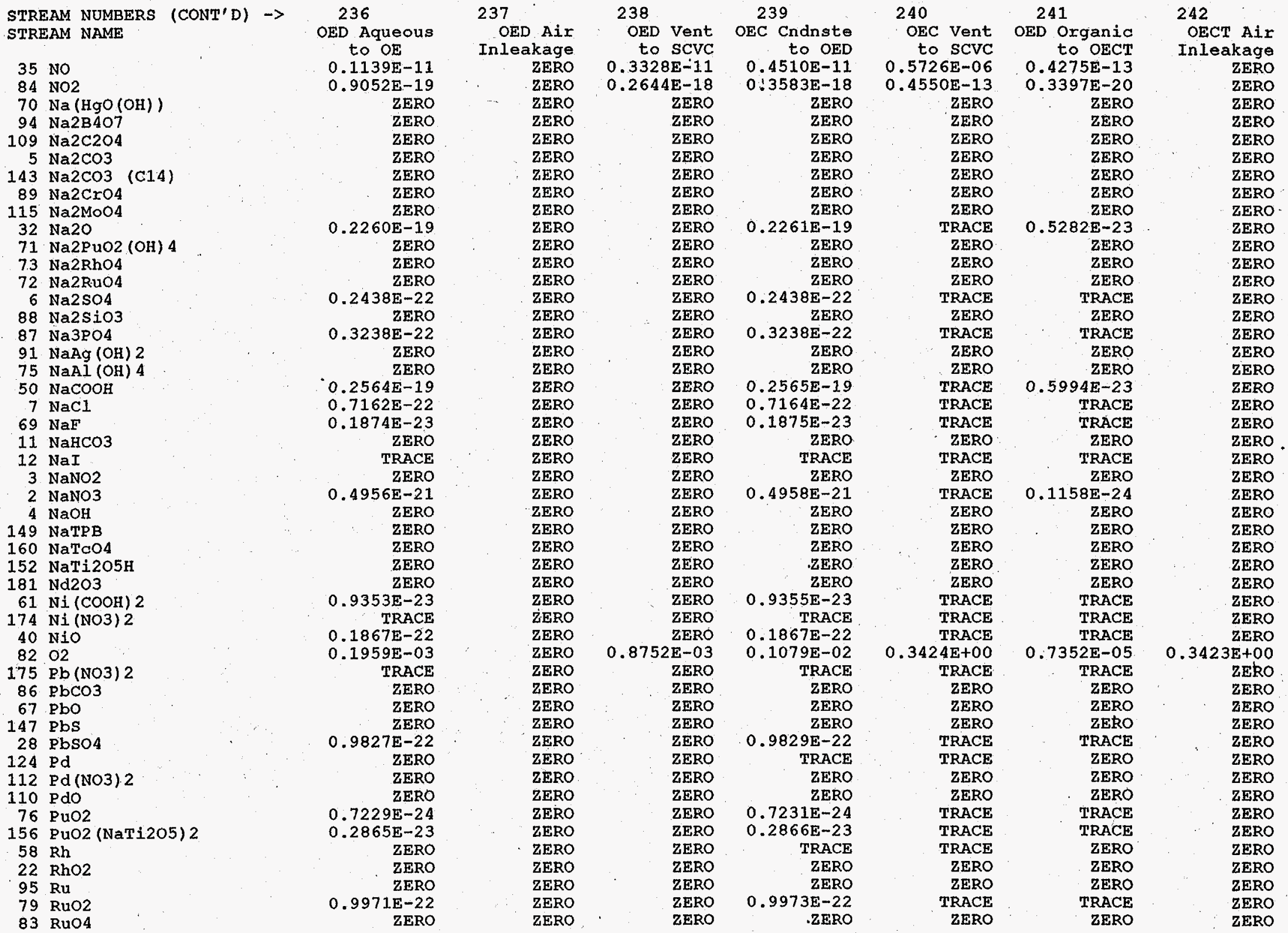

WSRC-TR-95-0019 (Revision 0) Date: January 1995

Seation 3B, Page 16 of 29 
Table A-1. HLW Flowsheet Material Balance (Tank 51/ITP Cycle 1 ) section 3B. Boilup of Precipitate Hydrolysis Products

STREAM NUMBERS (CONT'D) $\rightarrow$ STREAM NAME

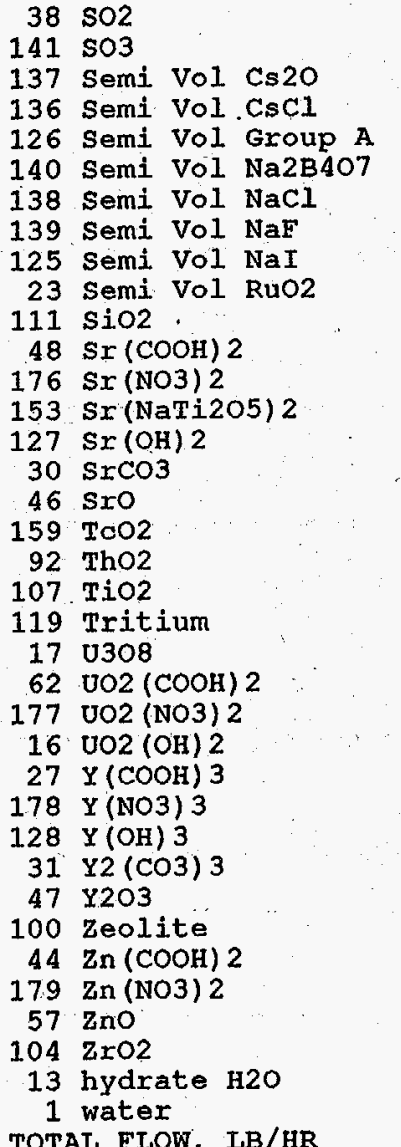

TEMPERATURT, DEG $\mathrm{C}$ PRESSURE， ATM PRESSURE， PSIG PRESSURE, MM HG ENTHALPY, PCU/HR VAPOR FLOW, CFM LIQUID FLOW, GPM DENSITY， LBS/FT3 PHASE

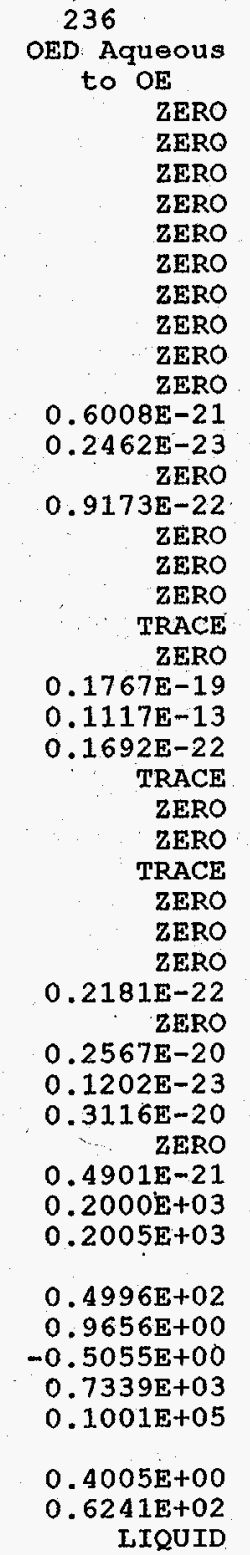

\begin{tabular}{c}
237 \\
OED Air \\
Inleakage \\
ZERO \\
ZERO \\
ZERO \\
ZERO \\
ZERO \\
ZERO \\
ZERO \\
ZERO \\
ZERO \\
ZERO \\
ZERO \\
ZERO \\
ZERO \\
ZERO \\
ZERO \\
ZERO \\
ZERO \\
ZERO \\
ZERO \\
ZERO \\
ZERO \\
ZERO \\
ZERO \\
ZERO \\
ZERO \\
ZERO \\
ZERO \\
ZERO \\
ZERO \\
ZERO \\
ZERO \\
ZERO \\
ZERO \\
ZERO \\
ZERO \\
ZERO \\
ZERO \\
ZERO \\
\\
\hline
\end{tabular}

$$
\begin{aligned}
& 238 \\
& \text { OED }
\end{aligned}
$$

to SCVC

SCVC

ZERO

ZERO

ZERO

ZERO

ZERO

ZERO

ZERO

ZERO

ZERO

ZERO

ZERO

ZERO

ZERO

ZERO

ZERO

ZERO

ZERO

$0.1337 E-18$

ZERO

ZERO

ZERO

ZERO

ZERO

ZERO

ZERO

ZERO

ZERO

ZERO

ZERO

ZERO

ZERO

ZERO

$0.2395 \mathrm{E}-02$

$0.3701 \mathrm{E}-01$

\section{$0.3500 \mathrm{E}+02$}

$0.4997 \mathrm{E}+02$

$0.9656 \mathrm{E}+00$

$0.7339 \mathrm{E}+03$

$0.5248 \mathrm{E}+01$

$0.47,82 \mathrm{E}-02$

$0.1290 \mathrm{E}+00$

VAPOR
239
EC Cnd

Cndnste
to OED

ZERO

ZERO

ZERO

ZERO

ZERO

ZERO

ZERO

ZERO

$0.6009 \mathrm{E}-21$

$0.2462 \mathrm{E}-23$

$0.9175 \mathrm{E}-22$

$5 E-22$

ZERO

ZERO

TRACE

$0.1767 \mathrm{E}-19$

$0.1767 \mathrm{E}-19$

$0.1693 \mathrm{E}-22$

$0.1693 \mathrm{E}-22$

TRACE
ZERO

ZERO

TRACE

ZERO

ZERO

$0.2182 \mathrm{E}-22$

ZERO

$0.2567 \mathrm{E}-20$

$0.1202 \mathrm{E}-23$

$0.3117 \mathrm{E}-20$

$0.4902 \mathrm{E}-21$

$0.2000 \mathrm{E}+03$

$0.2306 \mathrm{E}+03$

$0.4998 \mathrm{E}+02$

$0.9656 \mathrm{E}+00$

$-0.5055 \mathrm{E}+00$

$0.1065 \mathrm{E}+05$

$0.4712 \mathrm{E}+00$

$0.6102 \mathrm{E}+02$

IIQUID
240

OEC Vent

to SCVC

ZERO

ZERO

ZERO

ZERO

ZERO

ZERO

ZERO

ZERO

ZERO

TRACE

TRACE

ZERO

TRACE

ZERO

ZERO

ZERO

TRACE

$0.3155 \mathrm{E}-16$

TRACE

TRACE

ZERO

ZERO

ZERO

ZERO

ZERO

TRACE

ZERO

TRACE

TRACE

TRACE

ZERO

$0.5579 \mathrm{E}+00$

$0.1859 \mathrm{E}+02$

$0.3877 \mathrm{E}+02$

$0.9656 \mathrm{E}+00$

$-0.5055 \mathrm{E}+00$

$0.7339 \mathrm{E}+03$

$0.1569 \mathrm{E}+04$

$0.3113 \mathrm{E}+01$

$0.9953 \mathrm{E}-01$

VAPOR
241

to OECT

ZERO

ZERO

ZERO

ZERO

ZERO

ZERO

ZERO

ZERO

ZERO

$0.1404 \mathrm{E}-24$

TRACE

ZERO

TRACE

ZERO

ZERO

ZERO

TRACE

$0.4130 \mathrm{E}-23$

$0.4584 \mathrm{E}-19$

TRACE

- TRACE

ZERO

ZERO
TRACE

ZERO

ZERO

ZERO

TRACE

ZERO

$0.5999 \mathrm{E}-24$

TRACE

$0.7282 \mathrm{E}-24$

ZERO

$0.1146 \mathrm{E}-24$

$0.4674 \mathrm{E}-01$
$0.3012 \mathrm{E}+02$

$0.4996 \mathrm{E}+02$

$0.9656 \mathrm{E}+00$

$-0.50 .55 \mathrm{E}+00$

$0.7339 \mathrm{E}+03$

$0.6350 \mathrm{E}+03$

$0.7064 \mathrm{E}-01$

$0.5317 \mathrm{E}+02$

LIQUID
242

OECT Air

Inleakage ZERO

ZERO

ZERO

ZERO

ZERO

ZERO

ZERO

ZERO

ZERO

ZERO

ZERO

ZERO

ZERO

ZERO

ZERO

ZERO

ZERO

ZERO

ZERO

ZERO

ZERO

ZERO

ZERO

ZERO

ZERO

ZERO

ZERO

ZERO

ZERO

ZERO

ZERO

ZERO

ZERO

ZERO

ZERO

$0.2478 \mathrm{E}-01$

$0.1500 \mathrm{E}+01$

$0.3500 \mathrm{E}+02$

$0.1000 \mathrm{E}+01$

$$
\text { ZERO }
$$

$0.1520 \mathrm{E}+02$

$0.3449 \mathrm{E}-02$

$0.5422 \mathrm{E}+02$

IIQUID 
Table A-1. HLW Flowsheet Material Balance (Tank 51/ITP Cycle 1) Section 3B. Boilup of Precipitate Hydrolysis Products

STREAM NUMBERS -> STREAM NAME

COMPONENT FLOWS, LB/HR $122(\mathrm{C} 6 \mathrm{H} 5) 2$

123 (C6H5) $2 \mathrm{C} 6 \mathrm{H}$

118 (C6H5) $2 \mathrm{NH}$

133 (C6H5B (OH) 2 salt)

132 (C6H5B (OH) 2 )

166 (C6H5C6H4OH)

162 (C6H5NH2)

163 (C6H5NHCHO)

164 (C6H5NO2)

131 (C6H5OH salt)

130 ( 6 $6 \mathrm{H} 5 \mathrm{OH})$

121 (C6H6)

154 ( $\mathrm{CH} 3 \mathrm{OH}$ )

$45 \mathrm{Ag}$.

116 Ag20

134 AgNO3

$9 \mathrm{Ba}(\mathrm{OH}) 2$

$\begin{array}{rl}9 & \mathrm{Ba}(\mathrm{OH}) \\ 15 \mathrm{BaO}\end{array}$

$24 \mathrm{BaSO} 4$

$66 \mathrm{CO}$

$37, \mathrm{CO} 2$

$142 \mathrm{CO} 2$ (C14)

$63 \mathrm{Ca}(\mathrm{COOH}) 2$

167. $\mathrm{Ca}$ (NO3) 2

$120 \mathrm{Ca}(\mathrm{OH}) 2$

$113 \mathrm{Ca} 3$ (PO4) 2

20. $\mathrm{CaC} 2 \mathrm{O} 4$

$64 \mathrm{CaCO}_{3}(\mathrm{C14})$

$74 \mathrm{CaCO}$

$78 \mathrm{CaF} 2$

$41 \mathrm{CaO}$
$19 \mathrm{CaSO}$

29 Carbon

99 Cement

$26 \mathrm{Co}(\mathrm{COOH}) 2$

168 Co (NO3) 2

$59 \mathrm{COO}$

$98 \mathrm{Cr} 203$

42 Cs 20

$53 \mathrm{CsCOOH}$

96. $\mathrm{CsCl}$

$169 \mathrm{CsNO}$

$8 \mathrm{CsOH}$

151 CsTPB

WSRC-TR-95-0019 (Revision

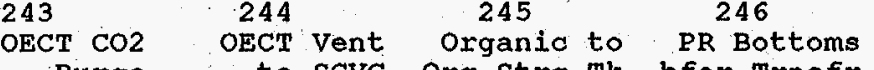

Purge

$$
\text { to SCVC }
$$

$$
\begin{aligned}
& \text { Organic to PR Bottoms } \\
& \text { Org strg Tk bfor Trnsfr }
\end{aligned}
$$

247

Rottoms
to PRBT

248

SCVC Vent SCVC HEME

$0.6137 \mathrm{E}-02$

$0.1022 \mathrm{E}-01$
$0.9245 \mathrm{E}-02$

$0.6901 \mathrm{E}-02$ ZERO

ZERO $\quad 0.3627 \mathrm{E}-08 \quad 0.7022 \mathrm{E}-01 \quad 0.6945 \mathrm{E}-02$

ZERO

ZERO

ZERO

ZERO

ZERO

ZERO

ZERO

ZERO

ZERO

ZERO

ZERO

ŻERO

ZERO

ZERO

ZERO

ZERO

ZERO

ZERO

ZERO

$0.1471 \mathrm{E}+02$

ZERO
ZERO

ZERO

ZERO

ZERO

ZERO

ZERO

ZERO

ZERO

ZERO

ZERO

ZERO

ZERO

ZERO

ZERO

ZERO

ZERO

ERO

ERO

ZERO

ZERO

ZERO

ZERO

$0.1220 \mathrm{E}-01$

$0.1396 \mathrm{E}-01$

$0.1762 \mathrm{E}-23$

$0.1462 \mathrm{E}-22$

$0.9755 \mathrm{E}-24$

ZERO

$0.1741 \mathrm{E}+01$

$0.2457 \mathrm{E}-14$

$0.1484 \mathrm{E}-14$

ZERO
ZERO

ZERO

ZERO

ZERO

ZERO

ZERO

ZERO

$0.1458 \mathrm{E}+02$

$0.7558 \mathrm{E}-18$

ZERO
ZERO

ZERO
ZERO

ZERO

ZERO

ZERO

ZERO

ZERO

ZERO

ZERO

ZERO

ZERO

ZERO

ZERO

ZERO

ZERO

ZERO

ZERO

ZERO

ZERO

$0.1237 \mathrm{E}+00$

$0.2711 \mathrm{E}+02$

$0.2038 \mathrm{E}-12$

ZERO

ZERO

$0.6831 \mathrm{E}-24$

TRACE

ZERO

TRACE

ZERO

$0.1302 \mathrm{E}+00$

$0.6747 \mathrm{E}-20$

ZERO
TRACE

$0.2952 \mathrm{E}-23$

TRACE

ZERO

ZERO

ZRRO

$0.1006 \mathrm{E}-23$

ZERO

ZERO

TRACE

TRACE

TRACE
$78 \mathrm{E}-23$

TRACE

ZERO

$0.5559 \mathrm{E}-01$

$0.7411 \mathrm{E}-01$

$0.2105 \mathrm{E}+00$

$0.3427 \mathrm{E}-02$

$0.6983 \mathrm{E}-02$ ZERO

$0.9400 \mathrm{E}+00$

$0.1442 \mathrm{E}-01$
$0.8374 \mathrm{E}-10$

$0.8922 \mathrm{E}-11$

$0.2861 \mathrm{E}-02$

ZERO

$0.1306 \mathrm{E}+00$

$0.1681 \mathrm{E}-02$

$$
\text { ZERO }
$$

$0.1804 \mathrm{E}-02$

ZERO

$0.6223 \mathrm{E}-06$

$0.6013 \mathrm{E}-17$

ZERO
$0.1378 \mathrm{E}-05$

ZERO

$0.5643 \mathrm{E}+00$

$0.2458 \mathrm{E}-06$

ZERO

ZERO

$0.1924 \mathrm{E}+00$

ZERO

$0.3089 \mathrm{E}-04$

$0.2634 \mathrm{E}+00$

$0.3872 \mathrm{E}-04$

E-24

ZERO

ZERO

ZERO

ZERO

ZERO

Date: Januaxy 1995

$0.5523 \mathrm{E}-01$

$0.7364 \mathrm{E}-01$

$0.2092 \mathrm{E}+00$

$0.3406 \mathrm{E}-02$

$0.6938 \mathrm{E}-02$

ZERO

. $9340 \mathrm{E}+00$

$0.1433 \mathrm{E}-01$
$0.8321 \mathrm{E}-10$

$0.8866 \mathrm{E}-11$

$0.2843 \mathrm{E}-02$

ZERO

$0.1297 \mathrm{E}+00$

$0.1670 \mathrm{E}-02$

ZERO

ZERO

$3 \mathrm{E}-02$

ZERO

$0.6183 \mathrm{E}-06$

$0.5975 \mathrm{E}-17$

ZERO
$0.1370 \mathrm{E}-05$

ZERO

$0.5607 \mathrm{E}+00$
$0.2442 \mathrm{E}-06$

ZERO

ZERO

ZERO

0.1912 ERTO

$0.1905 \mathrm{E}-01$

Accumulatn

$0.1740 \mathrm{E}-15$

ZERO

$0.5247 \mathrm{E}-10 \quad$ ZERO

ZERO

ZERO

ZERO
ZERO ZERO

ZERO ZERO

ZERO

ZERO

ZERO

ZERO
$0.3612 \mathrm{E}-04$

$0.9318 \mathrm{E}+01 \quad$ ZERO

$0.7606 \mathrm{E}-12$ ZERO

ZERO

ZERO

ZERO

ZERO

ZERO

ZERO

ZERO

TERO

ZERO

ZERO
$0.6810 \mathrm{E}+02$

$0.1203 E-09$ ZERO

ZERO

ZERO

ZERO

ZERO

ZERO

ZERO

ZERO

ZERO

ZERO

ZERO

ZERO

$0.1433 \mathrm{E}-03$

$0.5373 \mathrm{E}-06$

$0.2617 \mathrm{E}+00$

$0.3848 \mathrm{E}-04$

$0.3838 \mathrm{E}-04$

ZERO
ZERO

ZERO 
Table A-1. HLW Flowsheet Material Balance (Tank 51/ITP Cyale 1) section 3B. Boilup of Precipitate Hydrolysis Products

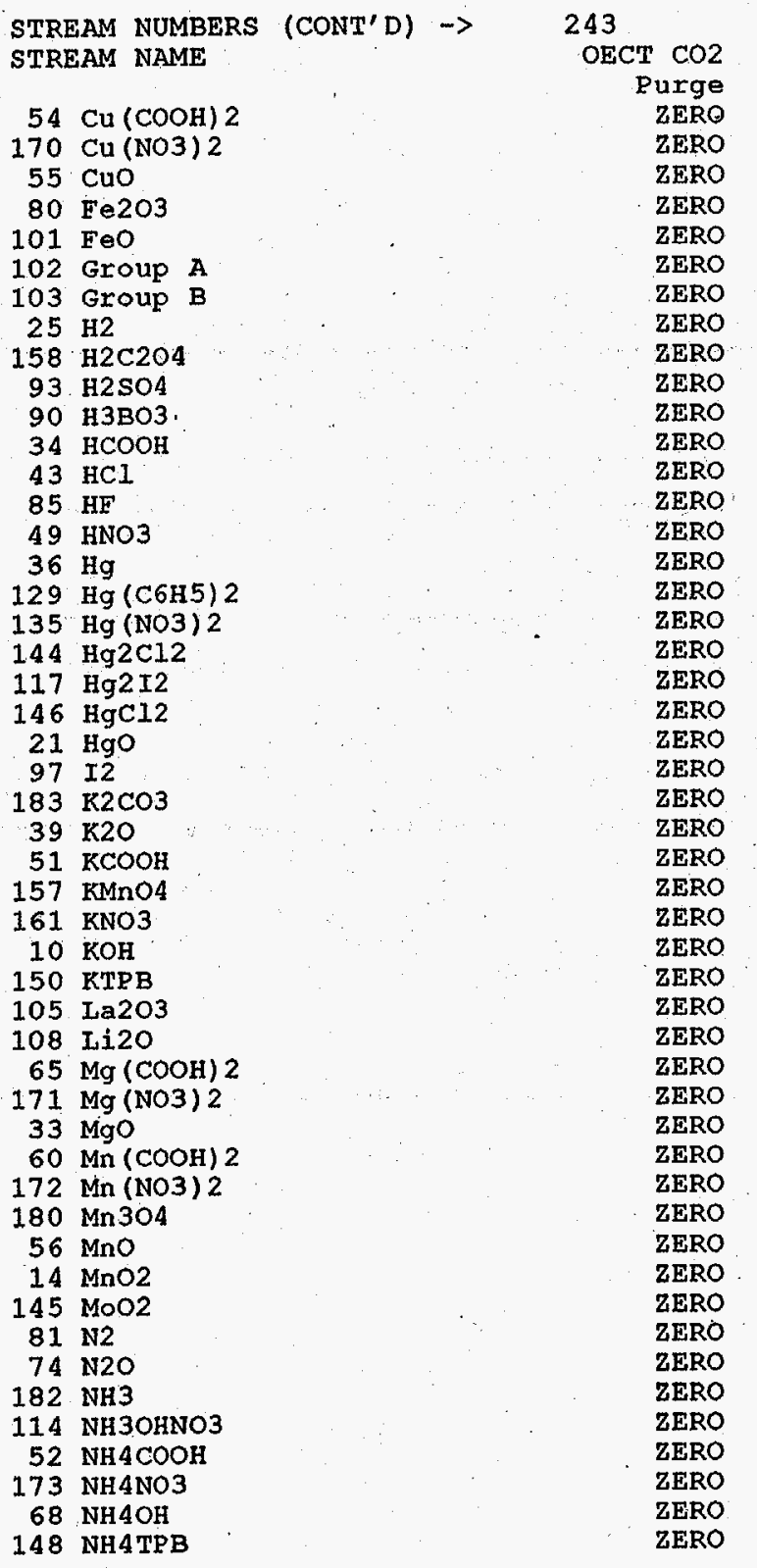

\begin{tabular}{|c|c|c|c|}
\hline \multirow{9}{*}{$\begin{array}{l}244 \\
\text { OECT } \\
\text { to }\end{array}$} & Vent & $\begin{array}{l}245 \\
\text { Organic to }\end{array}$ & $\begin{array}{l}246 \\
\text { PR Bottoms }\end{array}$ \\
\hline & SCVC & Org Strg Tk & bfor Trnsfr \\
\hline & ZERO & TRACE & $0.207 .4 \mathrm{E}-03$ \\
\hline & ZERO & $0.1148 \mathrm{E}-22$ & $0: 2193 \mathrm{E}+01$ \\
\hline & ZERO & TRACE & $0.9607 \mathrm{E}-03$ \\
\hline & ZERO & $0.8357 \mathrm{E}-24$ & $0.1597 \mathrm{E}+00$ \\
\hline & ZERO & ZERO & ZERO \\
\hline & ZERO & TRACE & $0.2453 \mathrm{E}-05$ \\
\hline & ZERO & TRACE & $0.7540 \mathrm{E}-07$ \\
\hline \multicolumn{2}{|c|}{$0.2964 \mathrm{E}-24$} & TRACE & $0.1064 \mathrm{E}-24$ \\
\hline & ZERO & TRACE & $0.5280 \mathrm{E}-02$ \\
\hline & ZERO & ZERO & ZERO \\
\hline & ZERO & $0.4058 \mathrm{E}-22$ & $0.7756 \mathrm{E}+01$ \\
\hline \multicolumn{2}{|c|}{$0.4456 \mathrm{E}-08$} & $0.2893 \mathrm{E}-05$ & $0.8987 \mathrm{E}+01$ \\
\hline \multicolumn{2}{|c|}{$\begin{array}{l}\text { ZERO } \\
\text { ZERO }\end{array}$} & ZERO & ZERO \\
\hline \multirow{2}{*}{\multicolumn{2}{|c|}{$\begin{array}{r}\text { ZERO } \\
\text { ZERO }\end{array}$}} & ZERO & ZERO \\
\hline & & ZERO & ZERO \\
\hline \multirow{2}{*}{\multicolumn{2}{|c|}{$\begin{array}{l}\text { ZERO } \\
\text { ZERO }\end{array}$}} & ZERO & $0.3217 \mathrm{E}-01$ \\
\hline & & $0.1326 \mathrm{E}-02$ & ZERO \\
\hline \multicolumn{2}{|c|}{$\begin{array}{l}\text { ZERO } \\
\text { ZERO }\end{array}$} & ZERO & ZERO \\
\hline \multicolumn{2}{|c|}{ ZERO } & ZERO & ZERO \\
\hline \multirow{2}{*}{\multicolumn{2}{|c|}{$\begin{array}{l}\text { ZERO } \\
\text { ZERO }\end{array}$}} & ZERO & ZERO \\
\hline & & ZERO & ZERO \\
\hline \multirow{2}{*}{\multicolumn{2}{|c|}{ ZERO }} & ZERO & ZERO \\
\hline & ZERO & ZERO & ZERO \\
\hline & ZERO & ZERO & ZERO \\
\hline & ZERO & TRACE & $0.6926 \mathrm{E}-02$ \\
\hline & ZERO & $0.5289 \mathrm{E}-22$ & $0.1011 \mathrm{E}+02$ \\
\hline & ZERO & ZERO & ZERO \\
\hline & ZERO & ZERO & ZERO \\
\hline & ZERO & ZERO & ZERO \\
\hline & ZERO & ZERO & ZERO \\
\hline & ZERO & ZERO & ZERO \\
\hline & ZERO & TRACE & $0.2966 \mathrm{E}-03$ \\
\hline & ZERO & TRACE & $0.3943 \mathrm{E}-02$ \\
\hline & ZERO & TRACE & $0.5287 \mathrm{E}-07$ \\
\hline & ZERO & TRACE & $0.3454 \mathrm{E}-03$ \\
\hline & ZERO & TRACE & $0.3327 \mathrm{E}-02$ \\
\hline & ZERO & TRACE & $0.6161 \mathrm{E}-07$ \\
\hline & ZERO & ZERO & ZERO \\
\hline & ZERO & ZERO & ZERO \\
\hline & ZERO & TRACE & $0.1321 \mathrm{E}-02$ \\
\hline & ZERO & TRACE & $0.1297 \mathrm{E}-03$ \\
\hline \multirow{3}{*}{\multicolumn{2}{|c|}{$\begin{array}{l}0.1133 \mathrm{E}+01 \\
0.1340 \mathrm{E}-18 \\
0.3996 \mathrm{E}-08\end{array}$}} & $0.1541 \mathrm{E}-03$ & $0.6546 \mathrm{E}-07$ \\
\hline & & $0.8671 \mathrm{E}-21$ & $0.1429 \mathrm{E}-19$ \\
\hline & & $0.5973 \mathrm{E}-08$ & $0.9775 \mathrm{E}-07$ \\
\hline & ZERO & ZERO & ZERO \\
\hline & ZERO & TRACE & $0.4925 \mathrm{E}-03$ \\
\hline & ZERO & ZERO & ZERO \\
\hline & ZERO & ZERO & ZERO \\
\hline & ZERO & ZERO & .ZERO \\
\hline
\end{tabular}

247

PR Bottoms to PRBT $0.2061 \mathrm{E}-03$ $0.2179 \mathrm{E}+01$
$0.9546 \mathrm{E}-03$ $0.1587 \mathrm{E}+00$

ZERO $0.2437 E-05$ $0.7493 \mathrm{E}-07$ $0.1058 \mathrm{E}-24$
$0.5247 \mathrm{E}-02$ 0.5247 E-02
ZERO $0.7707 \mathrm{E}+01$ $0.8930 \mathrm{E}+01$ ZERO ZERO $0.3197 \mathrm{E}-01$ ZERO ZERO ZERO ZERO ZERO ZERO ZERO ZERO

$0.6882 \mathrm{E}-02$ $0.1005 \mathrm{E}+02$ ZERO ZERO ZERO ZERO $0.2948 \mathrm{E}-03$ $0.3918 \mathrm{E}-02$ $0.5254 \mathrm{E}-07$
$0.3432 \mathrm{E}-03$ $0.3432 \mathrm{E}-03$
$0.3306 \mathrm{E}-02$ $0.3306 \mathrm{E}-02$
$0.6122 \mathrm{E}-07$ $0.6122 \mathrm{E}-07$ ZERO ZERO
$0.1313 \mathrm{E}-02$ $0.1289 \mathrm{E}-03$ $0.6505 \mathrm{E}-07$ $0.1420 \mathrm{E}-19$ $\begin{array}{r}\text { ZERO } \\ \hline\end{array}$

ZERO
$0.4894 \mathrm{E}-03$ ZERO ZERO

\begin{tabular}{|c|c|c|c|}
\hline $\begin{array}{l}248 \\
\text { SCVC } \\
\text { to }\end{array}$ & $\begin{array}{l}\text { Vent } \\
\text { HEME }\end{array}$ & $\begin{array}{c}249 \\
\text { SCVC } \\
\text { ACcumu }\end{array}$ & $\begin{array}{l}\text { HEME } \\
\text { ilatn }\end{array}$ \\
\hline & ZERO & & ZERO \\
\hline & ZERO & & ZERO \\
\hline & ZERO & & ZERO \\
\hline & ZERO & & ZERO \\
\hline & ZERO & & ZERO \\
\hline & ZERO & & ZERO \\
\hline & ZERO & & ZERO \\
\hline 0.7581 & $E-11$ & & ZERO \\
\hline & ZERO & & ZERO \\
\hline & ZERO & & ZERO \\
\hline & ZERO & & ZERO \\
\hline 0.7601 & $E-04$ & & ZERO \\
\hline & ZERO & & ZERO \\
\hline & ZERO & & ZERO \\
\hline & ZERO & & ZERO \\
\hline 0.4004 & $E-14$ & & ZERO \\
\hline & ZERO & & ZERO \\
\hline & ZERO & & ZERO \\
\hline & ZERO & & ZERO \\
\hline & ZERO & & ZERO \\
\hline & ZERO & & ZERO \\
\hline & ZERO & & ZERO \\
\hline & ZERO & & ZERO \\
\hline & ZERO & & ZERO \\
\hline & ZERO & & ZERO \\
\hline & ZERO & & ZERO \\
\hline & ZERO & & ZERO \\
\hline & ZERO & & ZERO \\
\hline & ZERO & & ZERO \\
\hline & ZERO & & ZERO \\
\hline & ZERO & & ZERO \\
\hline & ZERO & & ZERO \\
\hline & ZERO & & ZERO \\
\hline & ZERO & & ZERO \\
\hline & ZERO & & ZERO \\
\hline & ZERO & & ZERO \\
\hline & ZERO & & ZERO \\
\hline & ZERO & & ZERO \\
\hline & ZERO & & ZERO \\
\hline & ZERO & & ZERO \\
\hline & ZERO & & ZERO \\
\hline 0.4532 & $E+01$ & & ZERO \\
\hline 0.1070 & $E-09$ & & ZERO \\
\hline 0.1723 & $E-05$ & & ZERO \\
\hline & ZERO & & ZERO \\
\hline & ZERO & & ZERO \\
\hline & ZERO & & ZERO \\
\hline & ZERO & & ZERO \\
\hline & ZERO & & ZERO \\
\hline
\end{tabular}

Section $3 B$, Page 19 of 29

Date: January 1995 
Table A-1. HLW Flowsheet Material Balance (Tank 51/ITP Cycle 1) Section 3B. Boilup of Precipitate Hydrolysis Products

STREAM NUMBERS (CONT'D). $\rightarrow$ STREAM NAME

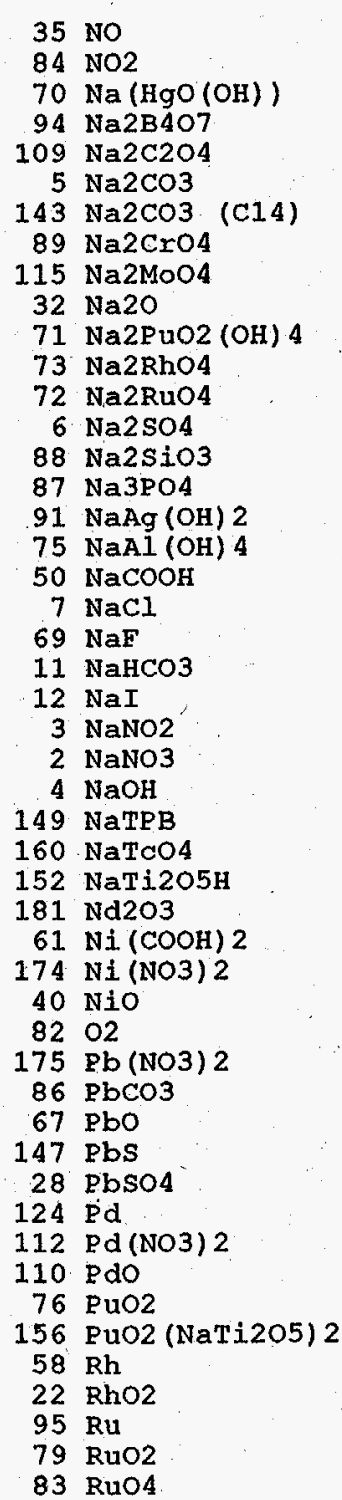

\begin{tabular}{l}
244 \\
OECT VERt \\
to SCVC \\
0.4274 E-13 \\
$0.3395 E-20$ \\
ZERO \\
ZERO \\
ZERO \\
ZERO \\
ZERO \\
ZERO \\
ZERO \\
ZERO \\
ZERO \\
ZERO \\
ZERO \\
ZERO \\
ZERO \\
ZERO \\
ZERO \\
ZERO \\
ZERO \\
ZERO \\
ZERO \\
ZERO \\
ZERO \\
ZERO \\
ZERO \\
ZERO \\
ZERO \\
ZERO \\
ZERO \\
ZERO \\
ZERO \\
ZERO \\
ZERO \\
ZER \\
ZERO \\
ZERO \\
ZERO \\
ZERO \\
ZERO \\
ZERO \\
ZERO \\
ZERO \\
ZERO \\
ZERO \\
ZERO \\
ZERO \\
ZERO \\
ZERO \\
ZERO \\
\hline
\end{tabular}
245 246. Organic to PR Bottoms Org Strg Tk bfor Trnsfr
$0.1804 \mathrm{E}-16$
$0.3491 \mathrm{E}-11$ ZERO
ZERO ZERO

ZERO
$0.4454 \mathrm{E}-02$ 247 R Bottoms to PRBT $0.3469 \mathrm{E}-11$
$0.2756 \mathrm{E}-18$ $0.2756 \mathrm{E}-18$

248

249

to SCVC HEME

$0.3364 \mathrm{E}-01$ Accumulatn

$0.2673 \mathrm{E}-08 \quad$ ZERO

$\begin{array}{ll}\text { ZERO } & \text { ZERO } \\ \text { ZERO } & \text { ZERO }\end{array}$

ZERO ZERO

ZERO ZERO

ZERO ZERO

ZERO ZERO

ZERO

$\begin{array}{rlr}0.1003 E+01 & \text { ZERO } & \text { ZERO } \\ \text { ZERO } & \text { ZERO } & \text { ZERO } \\ \text { ZERO } & \text { ZERO }\end{array}$

$\begin{array}{ll}\text { ZERO } & \text { ZERO } \\ \text { ZERO } & \text { ZERO }\end{array}$

ZERO ZERO

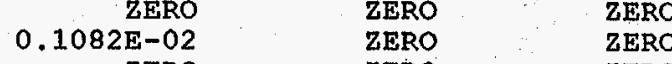

0.1437 ZERO

ZERO ZERO

ZERO ZERO

ZERO
$0.1138 \mathrm{E}+01$

$0.3180 \mathrm{E}-02$ ZERO ZERO

$0.8321 \mathrm{E}-04$ ZERO ZERO

ZERO
$0.5573 E-07$

ZERO ZERO

$0.2200 \mathrm{E}-01 \quad$ ZERO $\quad$ ZERO

ZERO ZERO ZERO

ZERO ZERO ZERO

ZERO ZERO $\quad$ ZERO

ZERO ZERO ZERO

ZERO
ZERO
ZERO

$0.6686 \mathrm{E}-06 \quad$ ZERO $\quad$ ZERO

$0.8288 \mathrm{E}-03$ ZERO ZERO

$0.6751 \mathrm{E}-07 \quad 0.1369 \mathrm{E}+01 \quad$ ZERO

$0.1624 \mathrm{E}-05 \quad$ ZERO ZERO

ZERO ZERO

ZERO ZERO

$\begin{array}{ll}\text { ZERO } & \text { ZERO } \\ \text { ZERO } & \text { ZERO }\end{array}$

$\begin{array}{rrr}0.4362 \mathrm{E}-02 & \text { ZERO } & \text { ZERO } \\ 0.3099 \mathrm{E}-08 & \text { ZERO } & \text { ZERO } \\ \text { ZERO } & \text { ZERO } & \text { ZERO } \\ \text { ZERO } & \text { ZERO } & \text { ZERO }\end{array}$

$\begin{array}{rrr}0.4362 \mathrm{E}-02 & \text { ZERO } & \text { ZERO } \\ 0.3099 \mathrm{E}-08 & \text { ZERO } & \text { ZERO } \\ \text { ZERO } & \text { ZERO } & \text { ZERO } \\ \text { ZERO } & \text { ZERO } & \text { ZERO }\end{array}$

\begin{tabular}{rll}
$0.3209 \mathrm{E}-04$ & ZERO & ZERO \\
\hline & & ZERO
\end{tabular}

$0.1272 \mathrm{E}-03$ ZERO ZERO

$0.3642 \mathrm{E}-06$ 2ERO ZERO

ZERO ZERO

ZERO ZERO

$0.4426 \mathrm{E}-02$ ZERO ZERO 
Table A-1. HLW Flowsheet Material Balance (Tank 51/ITP Cycle 1) Section 3B. Boilup of Precipitate Hydrolysis Products

STREAM NUMBERS (CON'T'D) $\rightarrow$ STREAM NAME

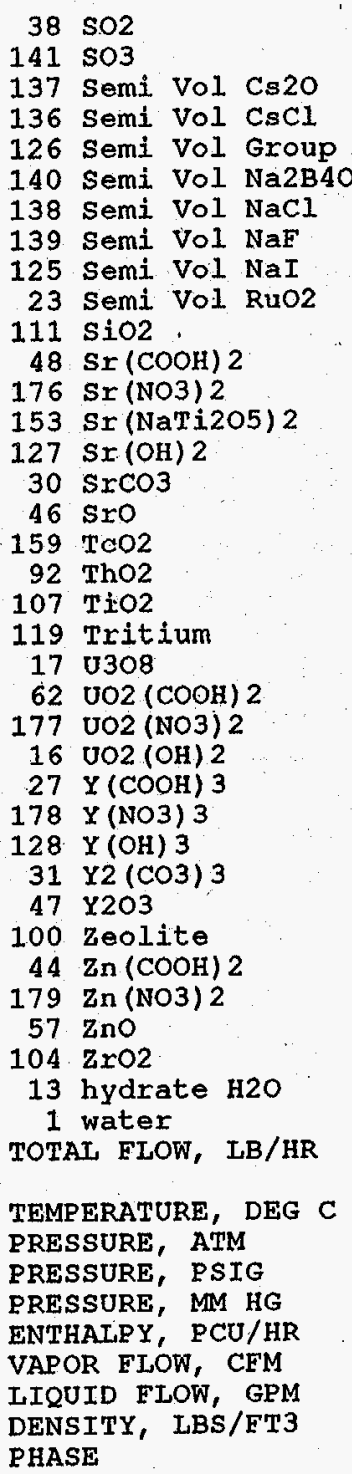

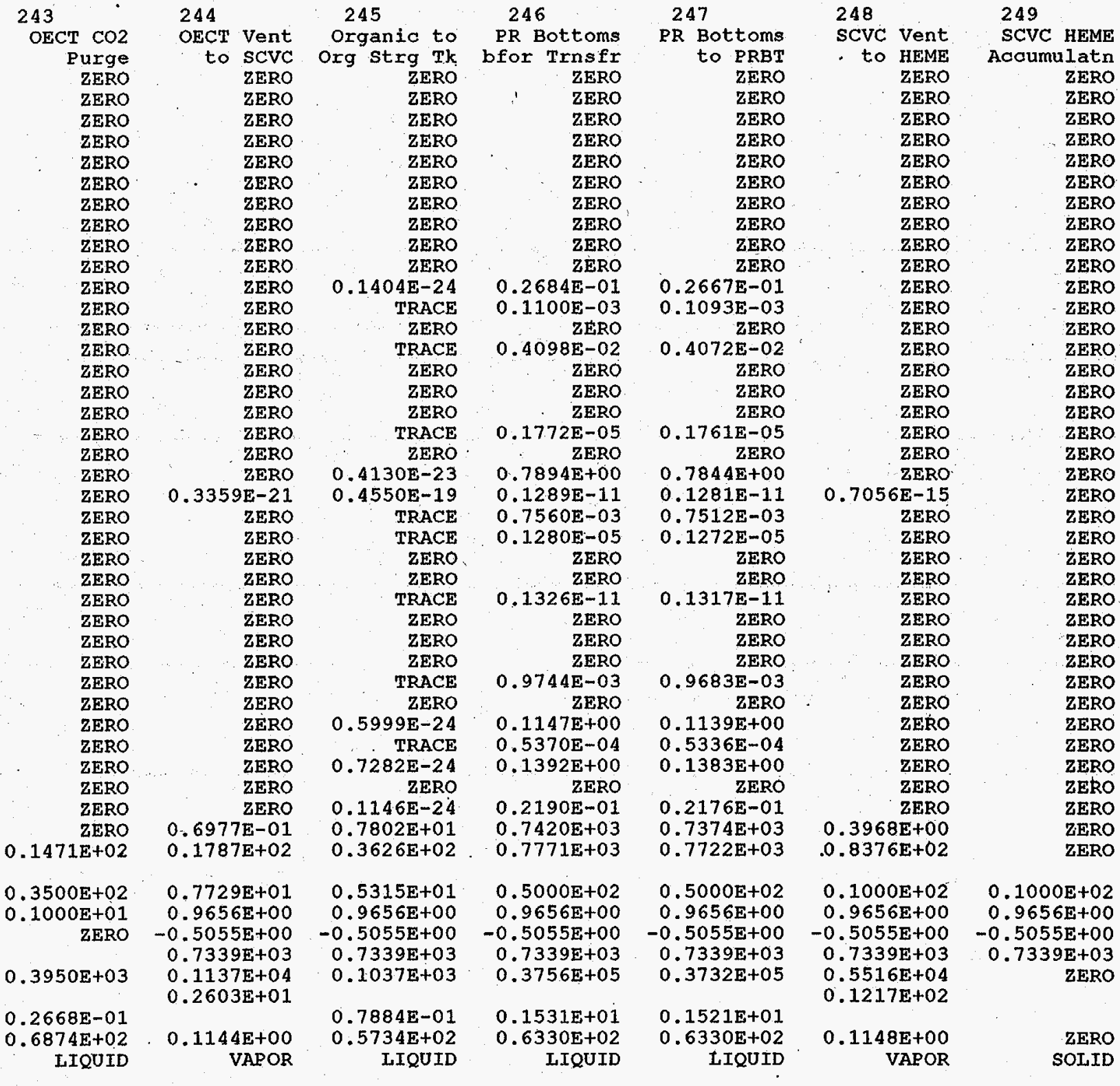


Table A-1: HLW Flowsheet Material Balance (Tank 51/ITP Cycle 1) A-1. HLW Flowsheet Material Balance (Tank 51/ITP Cycl
Section 3B. Boilup of Precipitate Hydrolysis Products

STREAM NUMBERS $\rightarrow$ STREAM NAME

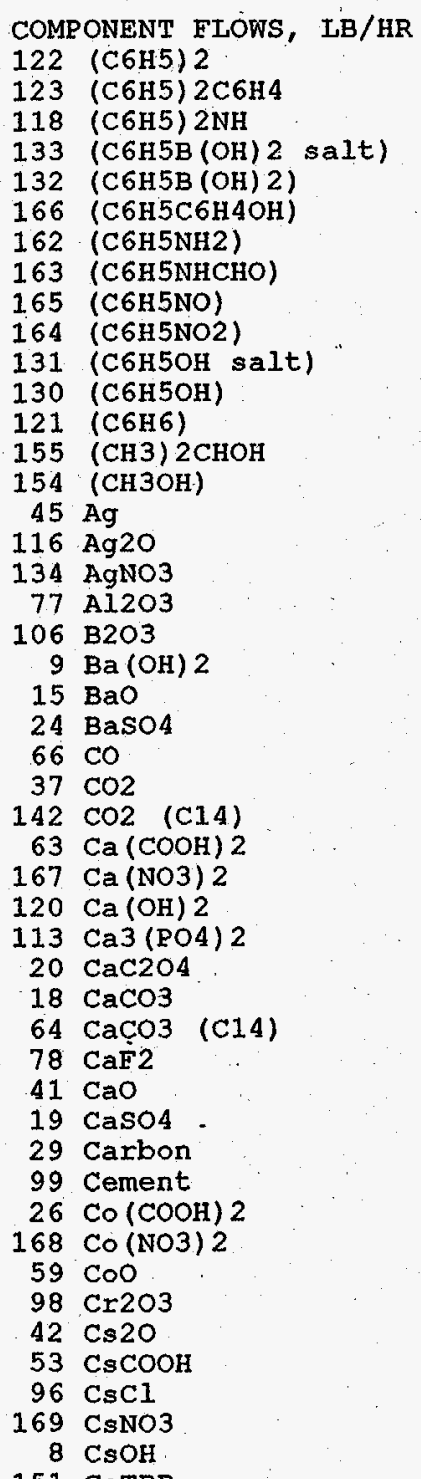
n PRD - PR

ZERO
ZERO
ZERO
ZERO
ZERO
ZERO
ZERO
ZERO
ZERO
ZERO
ZERO
ZERO
ZERO
ZERO
ZERO

$0.1713 \mathrm{E}-08$

ZERO

ZERO

ZERO

ZERO

ZERO

ZERO

ZIRO

ZERO

ZERO

ZERO

ZERO

ZERO

ZERO

ZERO

ZERO

ZERO

ZERO

ZERO

ZRRO

ZERO

ZERO

ZERO

ZERO

ZERO

ZERO

ZERO

ZERO

ZERO
262

261 Hg Accum N2 Purge
in OED

OWST Vent

\section{$0.5230 \mathrm{E}-04$}

$\begin{array}{lll}0.5230 \mathrm{E}-04 & 0.9975 \mathrm{E}+00 & 0.5230 \mathrm{E}-04 \\ 0.1865 \mathrm{E}-11 & 0.5464 \mathrm{E}-02 & 0.1865 \mathrm{E}-11\end{array}$

Fd frm OST

263

ZERO ZERO

ZERO , ZERO

ZERO

ZERO

ZERO

ZERO

ZERO

ZERO

ZERO

ZERO

ZRRO

ZRRO

ZERO

ZERO

ZERO

ZERO

ZERO

ZERO

ZERO

ZERO

ZERO

ZERO

ZERO

ZERO

ZERO

ZERO

ZERO

ZERO

ZERO

ZERO

ZERO

ZERO

ZERO

ZERO

ZERO

ZERO

ZERO

ZERO

ZERO

ZERO

ZERO

ZERO

ZERO

ZERO

\section{ZERO}

ZERO

ZERO

ZERO

ZERO

ZERO

ZERO

ZERO

ZERO

ZERO

ZERO

ZERO

ZERO

ZERO

ZERO

ZERO

ZERO

ZERO

ZERO

ZERO

ZERO

ZERO

ZERO

ZERO

ZERO

ZERO

ZERO

ZERO

ZERO

ZERO

ZERO

ZERO

ZERO

ZERO

ZERO

ZERO

ZERO

ZERO

ZERO

ZERO

ZERO

ZERO

ZERO

ZERO ZERO ZERO

$0.5856 \mathrm{E}-10$

$.5302 \mathrm{E}-08$

TRACE

TRACE

TRACE

$1014 \mathrm{E}-06$

$0.4537 \mathrm{E}-02$

$.6508 \mathrm{E}-16$

$0.5464 \mathrm{E}-02$
$0.7022 \mathrm{E}-01$

$0.7022 \mathrm{E}-01$

ZERO

$0.1220 \mathrm{E}-01$

$0.1396 \mathrm{E}-01$

$0.1762 \mathrm{E}-23$

$0.1457 \mathrm{E}-22$

ZERO

$0.1237 \mathrm{E}+00$

$0.2710 \mathrm{E}+02$

$0.2037 \mathrm{E}-12$

ZERO

ZERO

ZERO

ZERO

ZERO

ZERO

ZERO

Z

$0.9767 \mathrm{E}-01$

$.5062 \mathrm{E}-20$

ZERO

ZERO

ZERO

ZERO

ZERO

ZERO

ZERO

ZERO

ZERO

ZERO

ZERO

ZERO

ZERO

ZERO

ZERO

ZERO

ZERO

ZERO

ZERO

$\mathrm{E}-12$
ZERO
ZERO

ZERO

$0.6831 \mathrm{E}-24$

TRACE

ZERO

ZERO

OERO

$0.3251 \mathrm{E}-01$

$.1685 \mathrm{E}-20$

ZERO

TRACE

$0.2952 \mathrm{E}-23$

TRACE

ZERO

ZERO 
Table A-1. HLW Flowsheet Material Balance (Tank 51/ITP Cycle 1) Seation 3B. Boilup of Precipitate Hydrolysis Products

\begin{tabular}{|c|c|c|c|c|c|c|c|c|c|c|}
\hline \multirow{2}{*}{\multicolumn{2}{|c|}{$\begin{array}{l}\text { STREAM NUMBERS } \\
\text { STREAM NAME }\end{array}$}} & \multirow[t]{2}{*}{ (CONT'D) } & \multirow[t]{2}{*}{$->$} & $\begin{array}{l}250 \\
\text { SCVC HEME }\end{array}$ & 251 & \multirow[t]{2}{*}{$\begin{array}{l}252 \\
\text { Hg Accum } \\
\text { in OED }\end{array}$} & $\begin{array}{l}260 \\
\text { N2 Purge }\end{array}$ & 261 & $\begin{array}{l}262 \\
\text { Incinerator }\end{array}$ & OWs vent to \\
\hline & & & & Vnt to PVVS & in $P R D$ - PR & & of OWST & to HEPA & Fd frm OsT & Atmosphere \\
\hline 540 & $\mathrm{Cu}(\mathrm{COOH}) 2$ & & & ZERO & ZERO & ZERO & ZERO & ZERO & TRACE & ZERO \\
\hline 1700 & $\mathrm{Cu}(\mathrm{NO} 3)_{2}$ & & & ZERO & ZERO & ZERO & ZERO & ZERO & $0.1148 \mathrm{E}-22$ & ZERO \\
\hline 550 & $\mathrm{CuO}$ & & & ZERO & ZERO & ZERO & ZERO & ZERO & TRACE & ZERO \\
\hline $80 \mathrm{E}$ & $\mathrm{F} \in 203$ & & & ZERO & ZERO & ZERO & ZERO & ZERO & $0.8357 \mathrm{E}-24$ & ZERO \\
\hline $101 \mathrm{E}$ & FeO & & & ZERO & ZERO & ZERO & ZERO & ZERO & ZERO & ZERO \\
\hline $102 c$ & Group A & . & & ZERO & ZERO & ZERO & ZERO & ZERO & TRACE & ZERO \\
\hline $103 c$ & Group B & & & ZERO & ZERO & ZERO & ZERO & ZERO & TRACE & ZERO \\
\hline $25 \mathrm{H}$ & $\mathrm{H} 2$ & $:$ & & $0.7581 \mathrm{E}-11$ & ZERO & ZERO & ZERO & TRACE & TRACE & TRACE \\
\hline $158 \mathrm{H}$ & $\mathrm{H} 2 \mathrm{C} 204$ & & & ZERO & ZERO & ZERO & ZERO & ZERO & TRACE & ZERO \\
\hline $93 \mathrm{~F}$ & $\mathrm{H} 2 \mathrm{SO} 4$ & & & ZERO & ZERO & ZERO & ZERO & ZERO & ZERO & ZERO \\
\hline 901 & H3BO3. & & & ZERO & ZERO & ZERO & ZERO & ZERO & $0.4058 \mathrm{E}-22$ & ZERO \\
\hline $34 \mathrm{~F}$ & $\mathrm{HCOOH}$ & & & $0.7601 \mathrm{E}-04$ & ZERO & ZERO & ZERO & $0.8512 \mathrm{E}-10$ & $0.2893 \mathrm{E}-05$ & $0.8512 \mathrm{E}-10$ \\
\hline $43 \mathrm{H}$ & $\mathrm{HCl}$ & & & ZERO & ZERO & ZERO & ZERO & ZERO & ZERO & ZERO \\
\hline $85 \mathrm{H}$ & $\mathrm{HF}$ & & & ZERO & ZERO & ZERO & ZERO & ZERO & ZERO & ZERO \\
\hline $49:$ & HNO3 & & & ZERO & ZERO & ZERO & ZERO & ZERO & ZERO & ZERO \\
\hline $36 \mathrm{~F}$ & $\mathrm{Hg}$ & & & $0.4004 \mathrm{E}-14$ & $0.1823 \mathrm{E}+00$ & $0.1663 \mathrm{E}-09$ & ZERO & ZERO & ZERO & ZERO \\
\hline 129 & $\mathrm{Hg}(\mathrm{C} 6 \mathrm{H} 5) 2$ & & & ZERO & ZERO & ZERO & ZERO & ZERO & $0.1326 \mathrm{E}-02$ & ZERO \\
\hline $135 \mathrm{~F}$ & $\mathrm{Hg}(\mathrm{N} O 3) 2$ & & & ZERO & ZERO & ZERO & ZERO & ZERO & ZERO & ZERO \\
\hline $144 \mathrm{~F}$ & $\mathrm{Hg} 2 \mathrm{Cl} 2$ & & & ZERO & ZERO & ZERO & ZERO & ZERO & ZERO & ZERO \\
\hline $117 \mathrm{~F}$ & $\mathrm{Hg} 2 \mathrm{I} 2$ & & & ZERO & ZERO & ZERO & ZERO & ZERO & ZERO & ZERO \\
\hline $146 \mathrm{~F}$ & $\mathrm{HgCl} 2$ & & & ZERO & ZERO & ZERO & ZERO & ZERO & ZERO & ZERO \\
\hline 21 & $\mathrm{HgO}$ & & & ZERO & ZERO & ZERO & ZERO & ZERO & ZERO & ZERO \\
\hline 97. & I2 & & & ZERO & ZERO & ZERO & ZERO & ZERO & ZERO & ZERO \\
\hline $183 \mathrm{~F}$ & $\mathrm{~K} 2 \mathrm{CO} 3$ & & & ZERO & ZERO & ZERO & ZERO & ZERO & ZERO & ZERO \\
\hline 39.1 & $\mathrm{~K} 20$ & & & ZERO & . ZERO & ZERO & ZERO & ZERO & TRACE & ZERO \\
\hline 51 & $\mathrm{KCOOH}$ & & & ZERO & ZERO & ZERO & ZERO & ZERO & $0.5289 \mathrm{E}-22$ & ZERO \\
\hline $157 \mathrm{~F}$ & $\mathrm{KMnO4}$ & & & ZERO & ZERO & ZERO & ZERO & ZERO & ZERO & ZERO \\
\hline $161 \mathrm{~F}$ & KNO3 & & & ZERO & ZERO & ZERO & ZERO & ZERO & ZERO & ZERO \\
\hline $10 \mathrm{~K}$ & $\mathrm{KOH}$ & & & ZERO & ZERO & ZERO & ZERO & ZERO & ZERO & ZERO \\
\hline $150 \mathrm{~K}$ & KTPB & & & ZERO & ZERO & ZERO & ZERO & ZERO & ZERO & ZERO \\
\hline $105 \mathrm{I}$ & $\mathrm{La} 2 \mathrm{O} 3$ & & & ZERO & ZERO & ZERO & ZERO & ZERO & ZERO & ZERO \\
\hline $108 I$ & $\mathrm{Li} 20$ & & & ZERO & ZERO & ZERO & ZERO & ZERO & TRACE & ZERO \\
\hline $65 \mathrm{~N}$ & $\mathrm{Mg}(\mathrm{COOH}) 2$ & & & ZERO & ZERO & ZERO & ZERO & ZERO & TRACE & ZERO \\
\hline $171 \mathrm{~N}$ & $\mathrm{Mg}(\mathrm{NO} 3) 2$ & & & ZERO & ZERO & ZERO & ZERO & ZERO & TRACE & ZERO \\
\hline $33 \mathrm{~N}$ & Mgo & & & ZERO & ZERO & ZERO & ZERO & ZERO & TRACE & ZERO \\
\hline $60 \mathrm{~N}$ & $\mathrm{Mn}(\mathrm{COOH}) 2$ & & & ZERO & ZERO & ZERO & ZERO & ZERO & TRACE & ZERO \\
\hline $172 \mathrm{~N}$ & $\mathrm{Mn}$ (NO3) 2 & & & ZERO & ZERO & ZERO & ZERO & ZERO & TRACE & ZERO \\
\hline $180 \mathrm{~N}$ & $\mathrm{Mn} 304$ & & & ZERO & ZERO & ZERO & ZERO & ZERO & ZERO & ZERO \\
\hline $56 \mathrm{~N}$ & Mno & & & ZERO & ZERO & ZERO & ZERO & ZERO & ZERO & ZERO \\
\hline $14 \mathrm{~N}$ & $\mathrm{MnO} 2$ & & & ZERO & ZERO & ZERO & ZERO & ZERO & TRACE & ZERO \\
\hline $145 \mathrm{~N}$ & $\mathrm{MoO} 2$ & $\because$ & & ZERO & ZERO & ZERO & ZERO & ZERO & TRACE & ZERO \\
\hline 81 & N2 & & & $0.4532 \mathrm{E}+01$ & ZERO & ZERO & $0.1000 \mathrm{E}-01$ & $0.1010 \mathrm{E}-01$ & $0.5095 \mathrm{E}-04$ & $0.1010 \mathrm{E}-01$ \\
\hline 741 & $\mathrm{~N} 2 \mathrm{O}$ & & & $0.1070 \mathrm{E}-09$ & ZERO & ZERO & ZERO & $0.6984 \mathrm{E}-21$ & $0.1687 \mathrm{E}-21$ & $0.6984 \mathrm{E}-21$ \\
\hline 182 & NH3 & & & $0.1723 \mathrm{E}-05$ & ZERO & ZERO & ZERO & $0.1051 \mathrm{E}-09$ & $0.5868 \mathrm{E}-08$ & $0.1051 \mathrm{E}-09$ \\
\hline 114 & NH3OHNO3 & & & ZERO & ZERO & ZERO & ZERO & ZERO & ZERO & ZERO \\
\hline 52 & $\mathrm{NH} 4 \mathrm{COOH}$ & & & ZERO & ZERO & ZERO & ZERO & ZERO & TRACE & ZERO \\
\hline 173 & $\mathrm{NH} 4 \mathrm{NO} 3$ & & & ZERO & ZERO & ZERO & ZERO & ZERO & ZERO & ZERO \\
\hline 68 & $\mathrm{NH} 4 \mathrm{OH}$ & & & ZERO & ZERO & ZERO & ZERO & ZERO & ZERO & ZERO \\
\hline 148 & NH 4 TPB & & & ZERO & ZERO & ZERO & ZERO & ZERO & ZERO & ZERO \\
\hline WSRC- & $(-2 \pi-0010$ & (Revisi & & 0) & Dat & January 1 & & & ation $3 \mathrm{~B}, \mathrm{P}$ & ge 23 of 29 \\
\hline
\end{tabular}




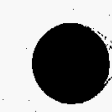

WSRC-TR-95-0019 (Revision

Date: January 1995

Section 3B, Page 24 of 29

Table A-1. HLW Flowsheet Material Balance (Tank 51/ITP Cycle 1) Section 3B. Boilup of Precipitate Hydrolysis Products

STREAM NUMBERS (CONT'D) $\rightarrow$ STREAM NAME

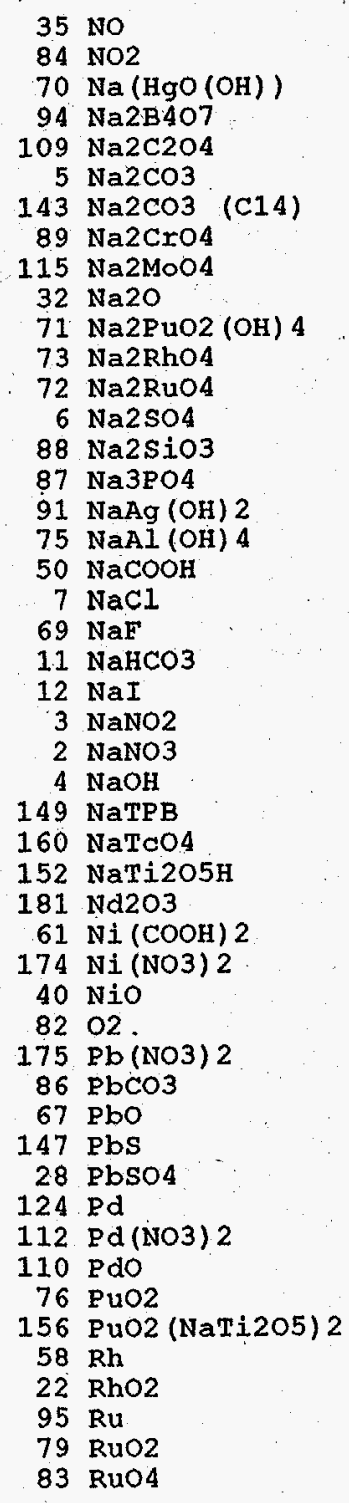

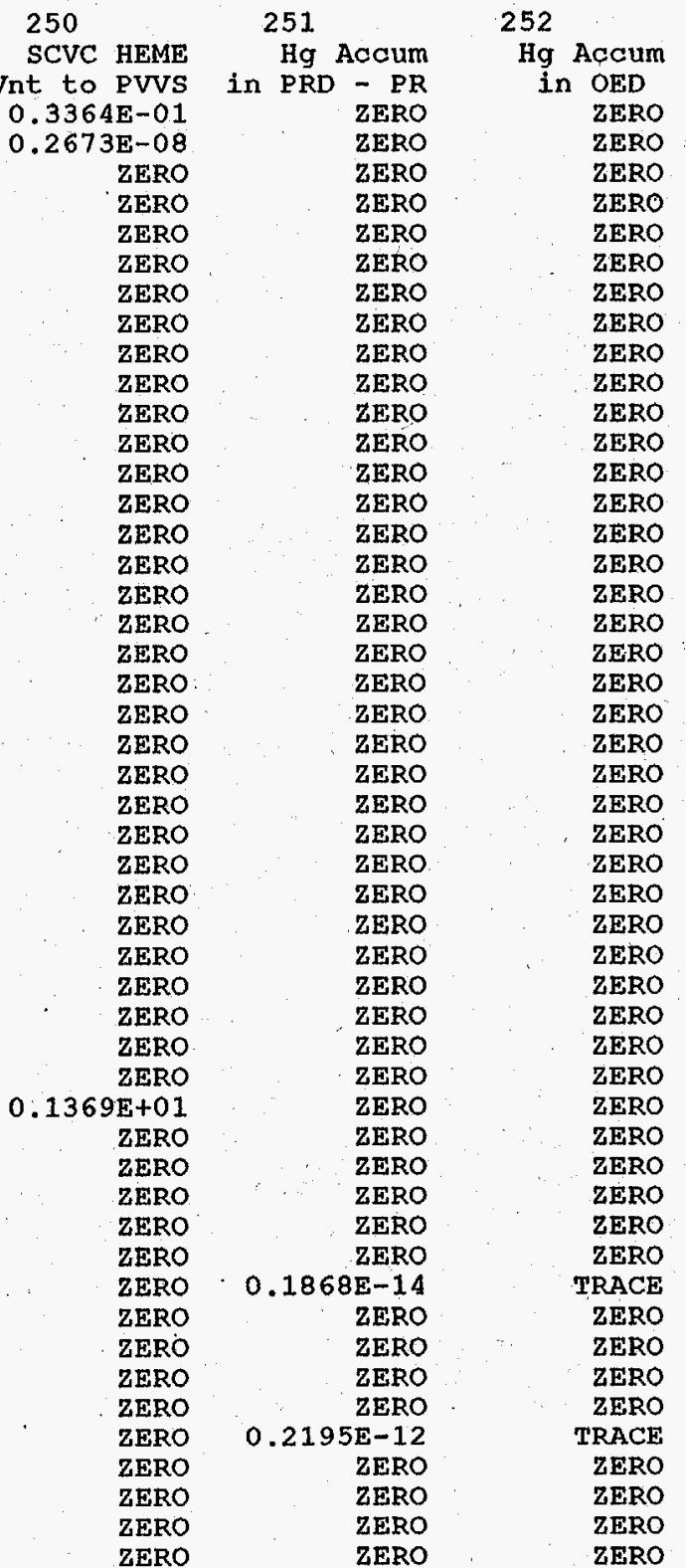

260
N2 PUIge
Of OWST
ZERO
ZERO
ZERO
ZERO
ZERO
ZERO
ZERO
ZERO
ZERO
ZERO
ZERO
ZERO
ZERO
ZERO
ZERO
ZERO
ZERO
ZERO
ZERO
ZERO
ZERO
ZERO
ZERO
ZERO
ZERO
ZERO
ZERO
ZERO
ZERO
ZERO
ZERO
ZERO
ZERO
ZERO
ZERO
ZERO
ZERO
ZERO
ZERO
ZERO
ZERO
ZERO
ZERO
ZERO
ZERO
ZERO
ZERO
ZERO
ZERO

Date: January 1995

\begin{tabular}{|c|c|c|c|}
\hline 261 & & 262 & 263 \\
\hline $\begin{array}{c}\text { OWST } \\
\text { to } \\
.1776 \\
.1411\end{array}$ & $\begin{array}{l}\text { Vent } \\
\text { HEPA } \\
E-16 \\
E-23\end{array}$ & $\begin{array}{r}\text { Incinerator } \\
\text { Fd fxm OST } \\
0.2781 \mathrm{E}-18 \\
\text { TRACE }\end{array}$ & $\begin{array}{l}\text { OWS Vent to } \\
\text { Atmosphere } \\
0.1776 \mathrm{E}-16 \\
0.1411 \mathrm{E}-23\end{array}$ \\
\hline & ZERO & ZERO & ZERO \\
\hline & ZERO & ZERO & ZERO \\
\hline & ZERO & ZERO & ZERO \\
\hline & ZERO & ZERO & ZERO \\
\hline & ZERO & ZERO & ZERO \\
\hline & ZERO & ZERO & ZERO \\
\hline & ZERO & ZERO & ZERO \\
\hline & ZERO & $0.5282 \mathrm{E}-23$ & ZERO \\
\hline & ZERO & ZERO & ZERO \\
\hline & ZERO & ZERO & ZERO \\
\hline & ZERO & ZERO & ZERO \\
\hline & ZERO & TRACE & ZERO \\
\hline & ZERO & ZERO & ZERO \\
\hline & ZERO & TRACE & ZERO \\
\hline & ZERO & ZERO & ZERO \\
\hline & ZERO & ZERO & ZERO \\
\hline & ZERO & $0.5994 \mathrm{E}-23$ & ZERO \\
\hline & ZERO & TRACE & ZERO \\
\hline & ZERO & TRACE & ZERO \\
\hline & ZERO & ZERO & ZERO \\
\hline & ZERO & TRACE & ZERO \\
\hline & ZERO & ZERO & ZERO \\
\hline & ZERO & $0.1158 \mathrm{E}-24$ & ZERO \\
\hline & ZERO & ZERO & ZERO \\
\hline & ZERO & ZERO & ZERO \\
\hline & ZERO & ZERO & ZERO \\
\hline & ZERO & ZERO & ZERO \\
\hline & ZERO & ZERO & ZERO \\
\hline & ZERO & TRACE & ZERO \\
\hline & ZERO & TRACE & ZERO \\
\hline & ZERO & TRACE & ZERO \\
\hline .9457 & $E-04$ & $0.9799 \mathrm{E}-06$ & $0.9457 \mathrm{E}-04$ \\
\hline & ZERO & TRACE & ZERO \\
\hline & ZERO & ZERO & ZERO \\
\hline & ZERO & ZERTO & ZERO \\
\hline & ZERO & ZERO & ZERO \\
\hline & ZERO & TRACE & ZERO \\
\hline & ZERO & ZERO & ZERO \\
\hline & ZERO & ZERO & ZERO \\
\hline & ZERO & ZERO & ZERO \\
\hline & ZERO & TRACE & ZERO \\
\hline & ZERO & TRACE & ZERO \\
\hline & ZERO & ZERO & ZERO \\
\hline & ZERO & ZERO & ZERO \\
\hline & ZERO & ZERO & ZERO \\
\hline & ZERO & TRACE & ZERO \\
\hline & ZERO & ZERO & ZERO \\
\hline
\end{tabular}

Section 3B, Page 24 of 29 
Table A-1. HLW Flowsheet Material Balance (Tank 51/ITP Cycle 1 ) Section 3B. Boilup of Precipitate Hydrolysis Products

STREAM NUMBERS (CONT'D) -> STREAM NAME

$38 \mathrm{SO} 2$

141503

137 Semi Vol Cs2O
136 Semi Vol CsC1

126. Semi Vol Group A

138 Semi Vol NaCl

139 Semi Vol NaF

125 Semi Vol NaI

23 Semi Vol RuO2

$111 \mathrm{SiO} 2$

$48 \mathrm{Sr}$ (COOH) 2

$176 \mathrm{Sr}$ (NO3) 2

$153 \mathrm{Sr}(\mathrm{NaTi205)} 2$

$127 \mathrm{Sr}$ (OH) 2

$30 \mathrm{srcO} 3$

46 sro

$159 \mathrm{TcO} 2$

92 Tho2

119 Tritium

119
17 Tritius

$62 \mathrm{UO} 2$ (COOH) 2

177 UO2 (NO3)

$16 \mathrm{OO} 2(\mathrm{OH}) 2$
$27 \mathrm{Y}(\mathrm{COOH}) 3$

$27 \mathrm{Y}(\mathrm{COOH})$

178 Y (NO3)

$\begin{array}{rl}128 & \mathrm{Y}(\mathrm{OH}) 3 \\ 31 & \mathrm{Y} 2(\mathrm{CO} 3) 3\end{array}$

47 Y2O3

100 Zeolite.

$44 \mathrm{Zn}(\mathrm{COOH}) 2$

$179 \mathrm{Zn}(\mathrm{NO} 3) 2$

$57 \mathrm{ZnO}$

104 ZrO2
13 hydrate $\mathrm{H} 2 \mathrm{O}$

1 water.

TOTAL FLOW, LB/HR

TEMPERATURE， DEG C

PRESSURE, ATM

PRESSURE, PSIG

PRESSURE, MM HG

ENTHALPY, PCU/HR

VAPOR FLOW, CEM

IIQUID FLOW， GPM

DENSITY, LBS/FT3

PHASE

WSRC-TR-95-0019 (Revision
250

SCVC HEME

Vnt to PVV

251

in Accum

PVVS in PRD - PR
ZERO

ZERO

ZERO

ZERO

ZERO

ZERO

ZERO

ZERO

ZERO

ZERO

ZERO

ZERO

ZERO

ZERO

ZERO

ZERO

ZERO

ZERO

$0.7056 \mathrm{E}-15$

ZERO

ZERO

ZERO

ZERO

ZERO

ZERO

ZERO

ZERO

ZERO

ZERO

ZERO

ZERO

ZERO

$0.3968 \mathrm{E}+00$

$0.8376 \mathrm{E}+02$

$0.1000 \mathrm{E}+02$

$0.9656 \mathrm{E}+00$

$-0.5055 \mathrm{E}+00$

$0.7339 \mathrm{E}+03$

$0.5516 \mathrm{E}+04$

$0.1148 \mathrm{E}+00$

VAPOR

$.7339 \mathrm{E}+03$

$0.3004 \mathrm{E}+00$

$0.2688 \mathrm{E}-04$
$0.8457 \mathrm{E}+03$

IIQUID

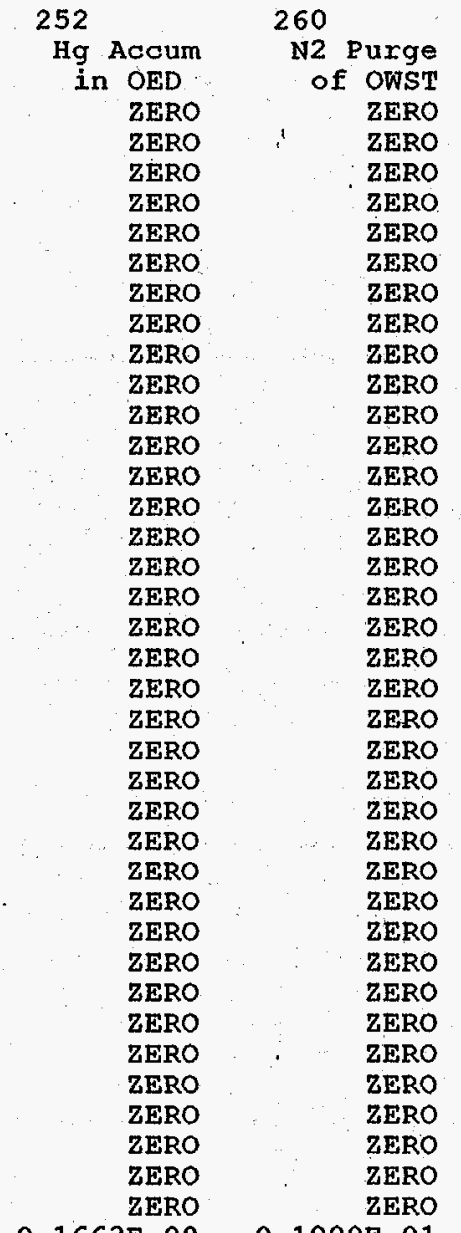

$0.1663 \mathrm{E}-09$

$0.4998 \mathrm{E}+02$

$0.9656 \mathrm{E}+00$

$-0.5055 \mathrm{E}+00$

$0.7339 \mathrm{E}+03$

$0.1000 \mathrm{E}-01$

$0.3500 \mathrm{E}+02$
$0.1000 \mathrm{E}+01$

$0.8482 \mathrm{E}-01$

$0.2452 \mathrm{E}-13$

$0.2409 \mathrm{E}-02$

I IQUID

VAPOR
261

Incinerator

ZERA Fd frm OST

ZERO $\quad$ ZERO

ZERO

ZERO

ZERO

ZERO

ZERO

ZERO

ZERO

ZERO

ZERO

ZERO

ZERO

ZERO

ZERO

ZERO

ZERO

ZERO

ZRRO

$0.1488 \mathrm{E}-23$

ZERO

ZERO

ZERO

ZERO

ZERO

ZERO

ZERO

ZERO

ZERO

ZERO

ZERO

ZERO

ZERO

$0.2176 \mathrm{E}-03$

$0.1127 \mathrm{E}+00$

$0.5016 \mathrm{E}+01$

$0.9656 \mathrm{E}+00$

$-0.5055 \mathrm{E}+00$

$0.7 .339 \mathrm{E}+03$

$0.6602 \mathrm{E}+01$

$0.1674 \mathrm{E}-01$

ZERO

ZERO

ZERO

ZERO

ZERO

2ERO

TRACE

ZERO

TRACE

ZERO

ZERO

ZERO

TRACE

$0.4130 \mathrm{E}-23$

$0.4550 \mathrm{~F}-19$

TRACE

TRACE

ZERO

ZERO
TRACE

ZERO

ZERO

ZERO

TRACE

$0.5999 \mathrm{E}-24$

TRACE

$0.7282 \mathrm{E}-24$

ZERO

$0.1146 \mathrm{E}-24$

$0.7802 \mathrm{E}+01$

$0.3616 \mathrm{E}+02$

$0.4997 \mathrm{E}+01$

$-0.5055 \mathrm{E}+00$

$0.9715 \mathrm{E}+02$

$0.7863 \mathrm{E}-01$

IIQUID

263

Ws Vent to

Atmosphere
zERO

ZERO
ZERO

ZERO

ZERO

ZERO

ZERO

ZERO

ZERO

ZERO

ZERO

ZERO

ZERO

ZERO

ZERO

ZERO

ZERO

ZERO

ZERO

$0.1488 \mathrm{E}-23$

ZERO

ZERO

ZERO

ZERO

ZERO

ZERO

ZERO

ZERO

ZERO

ZERO

ZERO

ZERO

ZERO

ZERO

$0.1127 \mathrm{E}+00$

$0.5016 \mathrm{E}+01$

$0.9656 \mathrm{E}+00$

$-0.5055 \mathrm{E}+00$

$0.7339 \mathrm{E}+03$

$0.1674 \mathrm{E}-01$

VAPOR

$0.1122 \mathrm{E}+00$

VAPOR 
Table A-1. HLW Flowsheet Material Balance (Tank 51/ITP Cycle 1) section $3 \mathrm{~B}$. Boilup of Precipitate Hydrolysis Products

STREAM NUMBERS $\rightarrow$ STREAM NAME

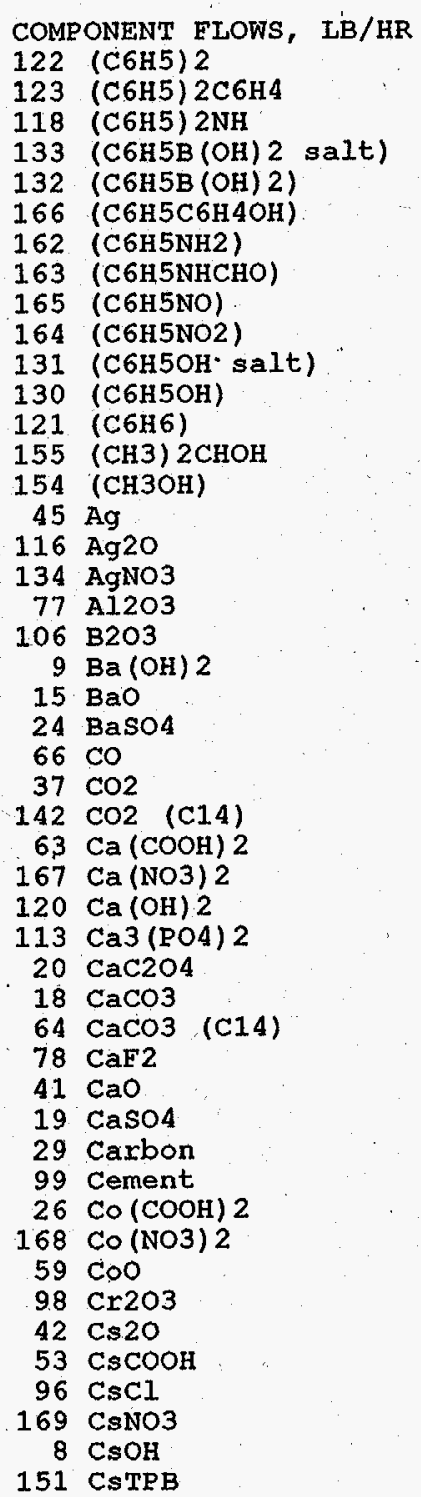

WSRC-TR-95-0019 (Revision

$$
267
$$

268$$
264
$$

OE Trnsfer OECT Trnsfer OECT Sampler

Pump Prime Pump Prime Pump Prime

\begin{tabular}{lll} 
ZERO & ZERO & ZERO \\
ZERO & ZERO & ZERO \\
ZERO & ZERO & ZERO \\
ZERO & ZERO & ZERO \\
ZERO & ZERO & ZERO \\
ZERO & ZERO & ZERO \\
ZERO & ZERO & ZERO \\
ZERO & ZERO & ZERO \\
ZERO & ZERO & ZERO \\
ZERO & ZERO & ZERO \\
ZERO & ZERO & ZERO \\
ZERO & ZERO & ZERO \\
ZERO & ZERO & ZERO \\
ZERO & ZERO & ZERO \\
ZERO & ZERO & ZERO \\
ZERO & ZERO & ZERO \\
ZERO & ZERO & ZERO \\
ZERO & ZERO & ZERO \\
ZERO & ZERO & ZERO \\
ZERO & ZERO & ZERO \\
ZERO & ZERO & ZERO \\
ZERO & ZERO & ZERO \\
ZERO & ZERO & ZERO \\
ZERO & ZERO & ZERO \\
ZERO & ZERO & ZERO \\
ZERO & ZERO & ZERO \\
ZERO & ZERO & ZERO \\
ZERO & ZERO & ZERO \\
ZERO & ZERO & ZERO \\
ZERO & ZERO & ZERO \\
ZERO & ZERO & ZERO \\
ZERO & ZERO & ZERO \\
ZERO & ZERO & ZERO \\
ZERO & ZERO & ZERO \\
ZERO & ZERO & ZERO \\
ZERO & ZERO & ZERO \\
ZERO & ZERO & ZERO \\
ZERO & ZERO & ZERO \\
ZERO & ZERO & ZERO \\
ZERO & ZERO & ZERO \\
ZERO & ZERO & ZERO \\
ZERO & ZERO & ZERO \\
ZERO & ZERO & ZERO \\
ZERO & ZERO & ZERO \\
ZERO & ZERO & ZERO \\
ZERO & ZERO & ZERO \\
ZERO & ZERO & ZERO \\
ZERO & ZERO \\
\hline
\end{tabular}

Date: January 1995 
Table A-1. HLW Flowsheet Material Balance (Tank 51/ITP Cycle 1) Section 3B. Boilup of Precipitate Hydrolysis Products

STREAM NUMBERS (CONT'D) $\rightarrow$ STREAM NAME

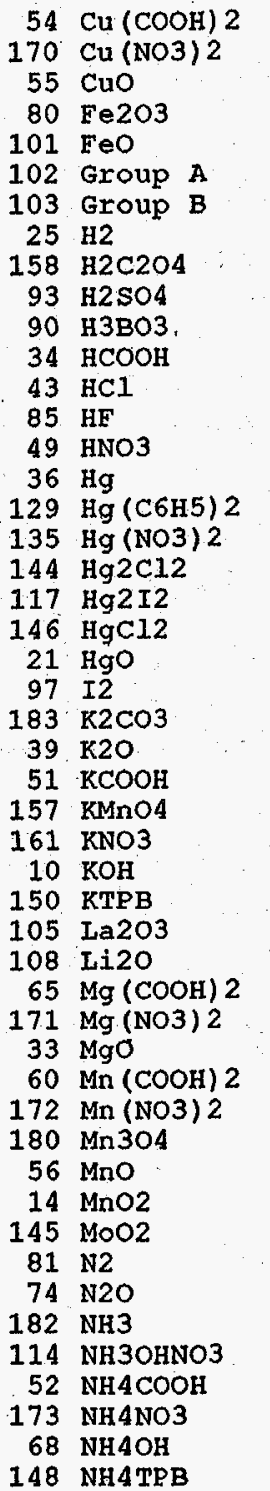

$54 \mathrm{Cu}(\mathrm{COOH}) 2$

$55 \mathrm{CuO}$

$80 \mathrm{Fe} 203$

102 Group A

$25 \cdot \mathrm{H} 2$

H2C2O4

$90 \mathrm{H} 3 \mathrm{BO}$

$5 \mathrm{HF}$

49 HNO3

$129 \mathrm{Hg}(\mathrm{C} 6 \mathrm{H} 5) 2$

$135 \mathrm{Hg}$ (NO3)

$144 \mathrm{Hg} 2 \mathrm{Cl}$

$146 \mathrm{HgCl2}$

97 I2

$183 \mathrm{~K} 2 \mathrm{CO}$

$51 \mathrm{KCOO}$

108 Ii2O

$5 \mathrm{Mg}(\mathrm{COOH}) 2$

$17.1 \mathrm{Mg} \cdot(\mathrm{NO}$

$60 \mathrm{Mn}(\mathrm{COOH}) 2$

$172 \mathrm{Mn}$ (NO3)

$56 \mathrm{MnO}$

$2 \mathrm{NH} 4 \mathrm{COOH}$

$68 \mathrm{NH} 4 \mathrm{OH}$

WSRC-TR-95-0019 (Revision $\begin{array}{ccc}264 & 267 & 268 \\ \text { OE Trnsfer } & \text { OECT } & \text { Trnsfer } \\ \text { OECT Sampler }\end{array}$

Pump Prime Pump Prime Pump Prime

ZERO ZERO $\quad$ ZERO

$\begin{array}{lll}\text { ZERO } & \text { ZERO } & \text { ZERO } \\ \text { ZERO } & \text { ZERO } & \text { ZERO }\end{array}$

ZERO ZERO ZERO

ZERO ZERO $\quad$ ZERO

$\begin{array}{lll}\text { ZERO } & \text { ZERO } & \text { ZERO } \\ \text { ZERO } & \text { ZERO } & \text { ZERO }\end{array}$

ZERO ZERO ZERO

ZERO ZERO ZERO

ZERO ZERO ZERO

ZERO ZERO ZERO

ZERO ZERO

ZERO ZERO ZERO

ZERO ZERO Z ZERO

ZERO — ZERO —.. ZERO

ZERO ZERO ZERO

ZERO ZERO

ZERO ZERO Z ZERO

ZERO $\cdots$ ZERO $\cdots . . . Z$ ZERO

ZERO ZERO

ZERO ZERO ‥ ZERO

ZTRO

ZERO ZERO ZERO

ZERO ZERO

ZERO ZERO ZERO

ZERO ZERO ZERO

ZERO ZERO ZERO

ZERO ZERO ZERO

ZERO ZERO ZERO

ZERO ZERO ZERO

ZERO ZERO ZERO

ZERO ZERO $\quad$ ZERO

ZERO ZERO $\quad$ ZERO

ZERO ZERO

ZERO Z ZERO ZERO

ZERO ZERO

ZERO ZERO

ZERO ZERO ZERO

ZERO ZERO ZERO

ZERO ZERO ZERO

ZERO ZERO ZERO

ZERO ZERO

ZERO

ZERO ZERO

ZERO ZERO ZERO

ZERO ZERO $\quad$ ZERO

ZERO ZERO ZERO

ZERO ZERO $\quad$ ZERO

ZERO ZERO Z ZERO

ZERO ZERO ZERO

Date: January 1995

Section 3B, Page 27 of 29 


\section{-}

WSRC-TR-95-0019 (Revision

Table A-1. HLW Flowsheet Material Balance (Tank 51/ITP Cycle 1) Section 3B. Boilup of Precipitate Hydrolysis Products

STREAM NUMBERS (CONT'D) $\rightarrow$ STREAM NAME

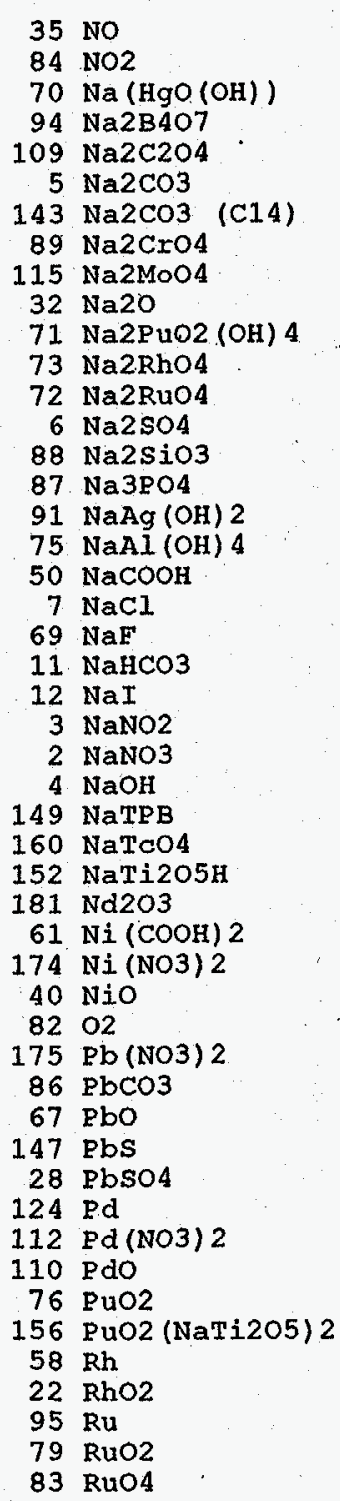

WSRC-TR-95-0019 (Revision
264

OE Trnsfer OECT Trnsfer OECT Sampler

Pump Prime Pump Prime Pump Prime

ZERO

ZERO

ZERO ZERO

ZERO ZERO

ZERO ZERO ZERO

ZERO ZERO ZERO

ZERO ZERO

ZERO

ZERO ZERO

ZERO $\quad$ ZERO $\quad$ ZERO

ZERO

ZERO ZERO ' ZERO

ZERO ZERO ZERO

ZERO ZERO ZERO

ZERO $\quad$ ZERO $\quad$ ZERO

ZERO ZERO ZERO

ZERO ZERO ZERO

ZERO ZERO Z ZERO

ZERO ZERO ZERO

ZERO ZERO ZERO

ZERO ZERO ZERO

$\begin{array}{lll}\text { ZERO } & \text { ZERO } \\ \text { ZERO }\end{array}$

ZERO ZERO ZERO

ZERO ZERO ZERO

ZERO ZERO ZERO

ZERO ZERO ZERO

ZERO ZERO

ZERO ZERO ZERO

ZERO ZERO Z ZERO

ZERO ZERO ZERO

ZERO ZERO $\quad$ ZERO

ZERO

TERO

ZERO :

ZERO ZERO

ZERO ZERO ZERO

$\begin{array}{lll}\text { ZERO } & \text { ZERO } & \text { ZERO } \\ \text { ZERO } & \text { ZERO }\end{array}$

ZERO ZERO

ZERO ZERO ZERO

ZERO ZERO $\quad$ ZERO

ZERO ZERO $\quad$ ZERO

ZERO ZERO ZERO

ZERO ZERO ZERO

ZERO ZERO

ZERO ZERO ZERO 
Table A-1. HLW Flowsheet Material Balance (Tank 51/ITP Cycle 1) Section 3B. Boilup of Precipitate Hydrolysis Products

STREAM NUMBERS (CONT'D) $\rightarrow$ STREAM NAME

$38 \mathrm{SO2}$

$141 \mathrm{SO} 3$

137 Semi Vol Cs2O

136 Semi Vol CsC1

126 Semi Vol Group A

140 Semi Vol Na2B407

138 Semi. Vol NaCl

139 Semi Vol NaE

125 Semi Vol NaI
23 Semi Vol Ruo2

23 Semi
111 Sio2

48 Sr (COOH) 2

$176 \mathrm{Sr}$ (NO3) 2

153. Sr (NaTi205) 2

$127 \operatorname{Sr}(\mathrm{OH}) 2$

$30 \mathrm{SrCO} 3$

46 SrO

$159 \quad \mathrm{TcO} 2$

92 ThO2

119 Tritium

$\begin{aligned} 119 & \text { Trit } \\ 17 & \text { U308 }\end{aligned}$

$62 \mathrm{UO} 2$ ( $\mathrm{COOH}) 2$

$177 \mathrm{UO} 2$ (NO3) 2

$16 \mathrm{UO} 2(\mathrm{OH}) 2$

$27 \mathrm{Y}(\mathrm{COOH}) 3$

178 Y (NO3) 3

$128 \mathrm{Y}(\mathrm{OH}) 3$

31 Y2 ( $\cos )$

100 Zeolite

$44 \mathrm{Zn}$ (COOH) 2

$44 \mathrm{Zn}$ (COOH)
$179 \mathrm{Zn}$ (NO3) 2

$57 \mathrm{ZnO}$

$104 \mathrm{ZrO2}$

13 hydrate $\mathrm{H} 2 \mathrm{O}$

1 water

267

268

26

OE Trnsfer OECT Trnsfer OECT Sampler

Pump Prime Pump Prime Pump Prime

$\begin{array}{lll}\text { ZERO } & \text { ZERO } & \text { ZERO } \\ \text { ZERO } & \text { ZERO } & \text { ZERO }\end{array}$

ZERO ZERO

ZERO ZERO ZERO

ZERO ZERO 2 ZERO

ZERO ZERO ZERO

ZERO ZERO ZERO

ZERO ZERO ZERO

ZERO ZERO W ZERO

ZERO ZERO ZERO

ZERO ZERO Z ZERO

ZERO ZERO $\quad$ ZERO

ZERO ZERO ZERO

ZERO ZERO $\therefore$ ZERO

ZERO ZERO ZERO

ZERO ZERO ZERO

ZERO ZERO ZERO

ZERO
ZERO

ZERO ZERO

$\begin{array}{lll}\text { ZERO ZERO } & \text { ZERO }\end{array}$

ZERO ZERO

ZERO
ZERO
ZERO

ZERO ZERO ZERO

ZERO $\cdots$ ZERO $\therefore$ ZERO

$\begin{array}{lll}\text { ZERO ZERO } & \text { ZERO } \\ & \text { ZERO }\end{array}$

$\begin{array}{lll}\text { ZERO } & \text { ZERO } & \text { ZERO } \\ \text { ERO } & \text { ZERO } & \text { ZERO }\end{array}$

ZERO ZERO ZERO ZERO

ZERO ZERO

ZERO

ZERO ZERO ZERO

ZERO ZERO

ZERO

ZERO

ZERO Z ZERO Z ZERO

$\begin{array}{rrr}\text { ZERO } & \text { ZERO } & \text { ZERO } \\ 0.3900 \mathrm{E}+01 & 0.3900 \mathrm{E}+01 & 0.3900 \mathrm{E}+01\end{array}$

$\begin{array}{lll}0.3900 \mathrm{E}+01 & 0.3900 \mathrm{E}+01 & 0.3900 \mathrm{E}+01\end{array}$

IOTAL FLOW, LB/HR

$0.2500 \mathrm{E}+02 \quad 0.2500 \mathrm{E}+02 \quad 0.2500 \mathrm{E}+02$

$0.1000 \mathrm{E}+01 \quad 0.1000 \mathrm{E}+01 \cdot 0.1000 \mathrm{E}+01$

PRESSURE, ATM

PRESSURE, PSIG

PRESSURE, MM HG

ENTHALPY, PCU/HR

VAPOR FLOW, CFM

LIQUID FLOW, GPM

DENSITY, LBS/FT 3

ZERO

ZERO

$0.9786 \mathrm{E}+02 \quad 0.9786 \mathrm{E}+02 \quad 0.9786 \mathrm{E}+02$

$0.7788 \mathrm{E}-02 \quad 0.7788 \mathrm{E}-02 \quad 0.7788 \mathrm{E}-02$

$0.6243 \mathrm{E}+02 \quad 0.6243 \mathrm{E}+02 \quad 0.6243 \mathrm{E}+02$

IIQUID IIQUID . IIQUID 


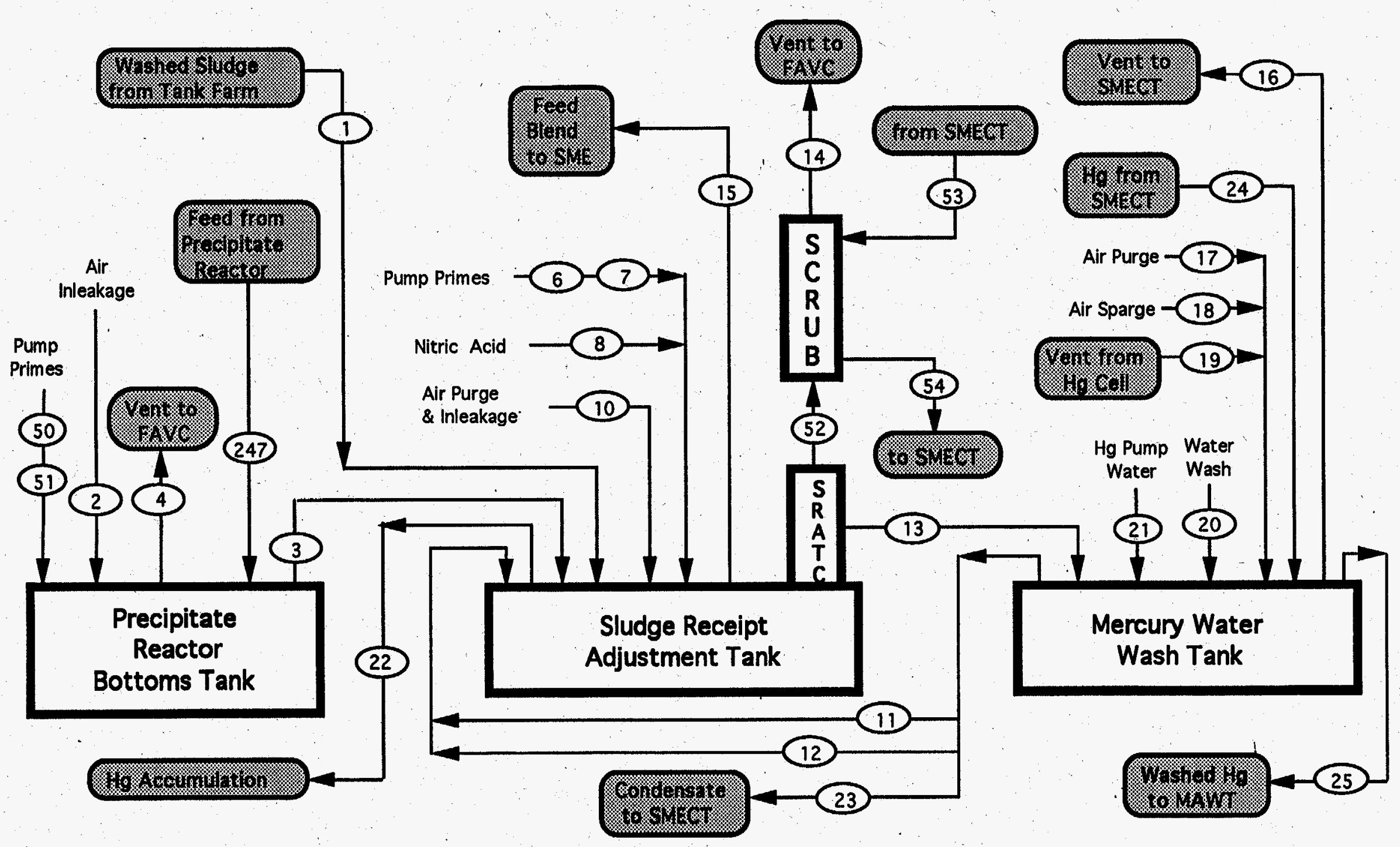

SECTION 4A. MELTER FEED RECEIPT, BLENDING AND ADJUSTMENT

WSRC-TR-95-0019 (Revision 0)

Date: January 1995

Section 4A, Page 0 of 21 
Table A-1. HLW Flowsheet Material Balance (Tank 51/ITP Cycle 1) section 4A. Sludge Receipt, Blending and Adjustment

DESCRIPTION

PAGE

1. STREAM NUMBERS

$247,2,4,107,108,3,1 \ldots \ldots 2$

2. STREAM NUMBERS

$6,7,8,10,11,12,13$.............. . . . . .

3. STREAM NUMBERS

$52,53,54,14,15,16,17 \ldots \ldots \ldots$

4. STREAM NUMBERS

$18,19,20,21,22,23,24 \ldots \ldots . . . . . . . . . .14$

5. STREAM NUMBERS

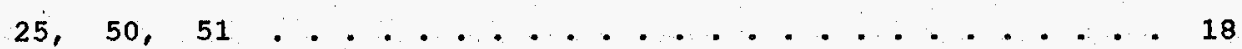


Table A-1. HLW Flowsheet Material Balance (Tank 51/ITP Cycle 1) section 4A. Sludge Receipt, Blending and Adjustment

STREAM NUMBERS -> STREAM NAME

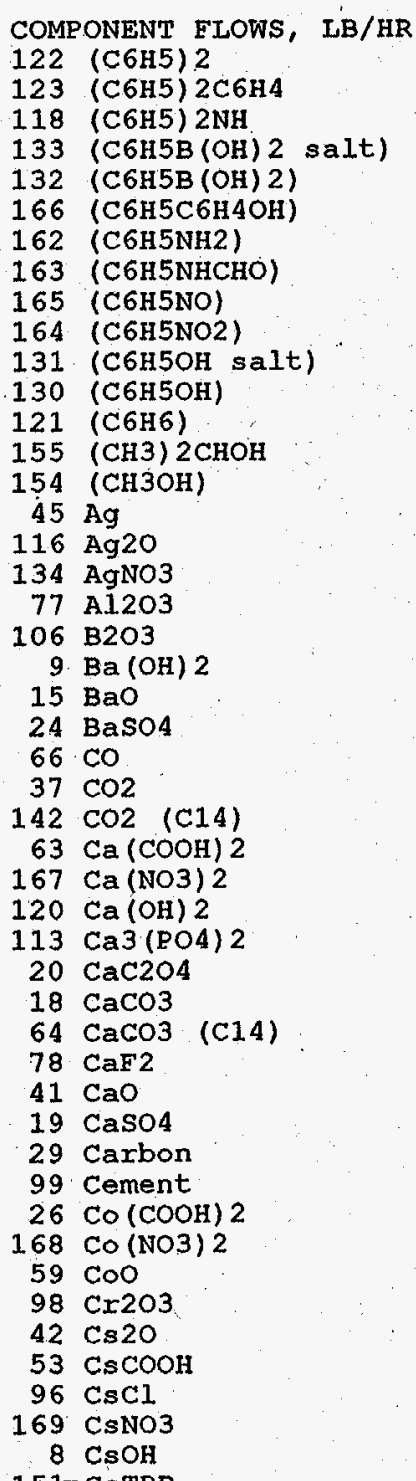

\begin{tabular}{|c|c|c|c|}
\hline $\begin{array}{l}247 \\
\text { PR Bottoms } \\
\text { to PRBT }\end{array}$ & $\begin{array}{c}2 \\
\text { PRBT Air } \\
\text { Inleakage }\end{array}$ & $\begin{array}{r}4 \\
\text { PRBT Vent } \\
\text { to FAVC }\end{array}$ & $\begin{array}{r}107 \text { PHA } \\
\text { Solubles }\end{array}$ \\
\hline $1022 E-01$ & ZERO & ZERO & ZERO \\
\hline $.9245 \mathrm{E}-02$ & ZERO & $0.7022 \mathrm{E}-10$ & ZERO \\
\hline $6901 \mathrm{E}-02$ & ZERO & $0.7624 \mathrm{E}-04$ & ZERO \\
\hline ZERO & ZERO & ZERO & ZERO \\
\hline ZERO & ZERO & ZERO & ZERO \\
\hline $0.5523 \mathrm{E}-01$ & ZERO & ZERO & ZERO \\
\hline $0.7364 E-01$ & ZERO & $0.2021 \mathrm{E}-02$ & $0.7162 \mathrm{E}-01$ \\
\hline $0.2092 \mathrm{E}+00$ & ZERO & ZERO & ZERO \\
\hline $.3406 \mathrm{E}-02$ & ZERO & ZERO & ZERO \\
\hline $.6938 \mathrm{E}-02$ & ZERO & ZERO & $0.6938 \mathrm{E}-02$ \\
\hline ZERO & ZERO & ZERO & ZERO \\
\hline $.9340 \mathrm{E}+00$ & ZERO & $0.1250 \mathrm{E}-01$ & $0.9215 \mathrm{E}+00$ \\
\hline $.1433 \mathrm{E}-01$ & ZERO & $0.1399 \mathrm{E}-01$ & $0.3375 \mathrm{E}-03$ \\
\hline $0.8321 E-10$ & ZERO & $0.1060 \mathrm{E}-11$ & $0.8215 \mathrm{E}-10$ \\
\hline $8866 \mathrm{E}-11$ & ZERO & $0.2651 \mathrm{E}-12$ & $0.8601 \mathrm{E}-11$ \\
\hline $2843 \mathrm{E}-02$ & ZERO & ZERO & ZERO \\
\hline ZERO & ZERO & ZERO & ZERO \\
\hline ZERO & ZERO & ZERO & ZERO \\
\hline $.1297 \mathrm{E}+00$ & ZERO & ZERO & ZERO \\
\hline $.1670 \mathrm{E}-02$ & ZERO & ZERO & ZERO \\
\hline ZERO & ZERO & ZERO & ZERO \\
\hline ZERO & ZERO & ZERO & ZERO \\
\hline $0.1793 E-02$ & ZERO & ZERO & ZERO \\
\hline ZERO & ZERO & ZERO & ZERO \\
\hline $.6183 E-06$ & ZERO & $0.6147 \mathrm{E}-06$ & $0.3621 \mathrm{E}-08$ \\
\hline $.5975 \mathrm{E}-17$ & ZERO & $0.5940 \mathrm{E}-17$ & $0.3499 \mathrm{E}-19$ \\
\hline ZERO & ZERO & ZERO & ZERO \\
\hline $0.1370 \mathrm{E}-05$ & ZERO & ZERO & $0.1370 \mathrm{E}-05$ \\
\hline ZERO & ZERO & ZERO & ZERO \\
\hline $0.5607 \mathrm{E}+00$ & ZERO & ZERO & ZERO \\
\hline $2442 \mathrm{E}-06$ & ZERO & ZERO & ZERO \\
\hline ZERO & ZERO & ZERO & ZERO \\
\hline ZERO & ZERO & ZE: & ZERO \\
\hline ZERO & ZERO & ZERO & ZERO \\
\hline ZER & ZERO & ZERO & ZERO \\
\hline $0.1912 \mathrm{E}+00$ & ZERO & ZERO & ZERO \\
\hline ZERO & ZERO & ZERO & ZERO \\
\hline ZERO & ZERO & ZERO & ZERO \\
\hline $0.1433 E-03$ & ZERO & ZERO & $0.1433 \mathrm{E}-03$ \\
\hline $0.5373 E-06$ & ZERO & ZERO & $0.5373 \mathrm{E}-06$ \\
\hline $0.3069 \mathrm{E}-04$ & ZERO & ZERO & ZERO \\
\hline $0.2617 \mathrm{E}+00$ & ZERO & ZERO & ZERO \\
\hline $0.3848 \mathrm{E}-04$ & ZERO & ZERO & ZERO \\
\hline $0.4330 E-01$ & ZERO & ZERO & $0.4330 \mathrm{E}-01$ \\
\hline ZERO & ZERO & ZERO & ZERO \\
\hline ZERO & ZERO & ZERO & ZERO \\
\hline ZERO & ZERO & ZERO & ZERO \\
\hline ZER & ZERO & ZERO & ZERO \\
\hline
\end{tabular}

108

PHA
Insolubles

3

PHA Feed sludge Feed

$0.1022 \mathrm{E}-01 \quad 0.1022 \mathrm{E}-01$

ZERO

$0.6825 \mathrm{E}-02 \quad$ ZERO

$0.6825 \mathrm{E}-02$ ZERO

ZERO

$\begin{array}{rrr}\text { ZERO } & \text { ZERO } & \text { ZERO } \\ 0.5523 E-01 & 0.5523 E-01 & \text { ZERO } \\ \text { ZERO } & 0.7162 E-01 & \text { ZERO }\end{array}$

ZERO $0.7162 \mathrm{E}-01$ ZERO

$\begin{array}{lll}0.2092 \mathrm{E}+00 & 0.2092 \mathrm{E}+00 & \text { ZERO }\end{array}$

$0.3406 \mathrm{E}-02 \quad 0.3406 \mathrm{E}-02 \quad$ ZERO

ZERO $0.6938 E-02$ ZERO

ZERO $\quad$ ZERO $\quad$ ZERO

ZERO $0.9215 \mathrm{E}+00$ ZERO

ZERO $0.8215 \mathrm{E}-10 \quad$ ZERO

$\begin{array}{rlr}\text { ZERO } & 0.8601 E-11 & \text { ZERO } \\ 0.2843 \mathrm{E}-02 & 0.2843 \mathrm{E}-02 & \text { ZERO }\end{array}$

$0.1018 \mathrm{E}-01$

$\begin{array}{rrr}\text { ZERO } & \text { ZERO } & \text { ZERO } \\ 0.1297 \mathrm{E}+00 & 0.1297 \mathrm{E}+00 & 0.8551 \mathrm{E}+01 \\ 0.1670 \mathrm{E}-02 & 0.1670 \mathrm{E}-02 & \end{array}$

$0.1670 \mathrm{E}-02$
ZERO

$\begin{array}{llr}\text { ZERO } & \text { ZERO } & 0.2968 \mathrm{E}-03 \\ \text { ZERO } & \text { ZERO } & \text { ZERO }\end{array}$

$0.1793 \mathrm{E}-02,0.1793 \mathrm{E}-02 \quad 0.1480 \mathrm{E}-01$

ZERO ZERO ZERO

ZERO $0.3621 E-08$ ZERO

ZERO $0.3499 \mathrm{E}-19 \quad$ ZERO

ZERO : $\begin{aligned} & \text { ZERO } \\ & \text { ZERO }\end{aligned}$

ZERO $0.1370 \mathrm{E}-05$ ZERO

$\begin{array}{rrr}\text { ZERO } & \text { ZERO } & 0.2240 \mathrm{E}-04 \\ 0.5607 \mathrm{E}+00 & 0.5607 \mathrm{E}+00 & 0.2065 \mathrm{E}+01\end{array}$

$0.2442 \mathrm{E}-0.6 \quad 0.2442 \mathrm{E}-06 \quad$ ZERO

ZERO ZERO $0.2554 \mathrm{E}+01$

ZERO ZERO $0.1257 \mathrm{E}-06$

ZERO ZERO $0.1501 \mathrm{E}-01$

ZERO

$0.1912 \mathrm{E}+00 \quad 0.1912 \mathrm{E}+00 \quad 0.2964 \mathrm{E}-01$

ZERO ZERO
ZERO

ZERO ZERO ZERO

ZERO $0.1433 \mathrm{E}-03 \quad$ ZERO

$\begin{array}{rlr}\text { ZERO } & 0.5373 \mathrm{E}-06 & \text { ZERO } \\ 0.3069 \mathrm{E}-04 & 0.3069 \mathrm{E}-04 & \text { ZERO }\end{array}$

$0.2617 \mathrm{E}+00 \quad 0.2617 \mathrm{E}+00 \quad 0.1629 \mathrm{E}+00$

$0.3848 \mathrm{E}-04 \quad 0.3848 \mathrm{E}-04 \quad 0.1018 \mathrm{E}-03$

$0.4330 \mathrm{E}-01$ ZERO

$\begin{array}{ll}\text { ZERO } & \text { ZERO } \\ \text { ZERO } & \text { ZERO }\end{array}$

ZERO $0.7390 \mathrm{E}-05$

ZERO ZERO

Section $4 A$, Page 2 of 21 
Table A-1. HLW Flowsheet Material Balance (Tank 51/ITP Cycle 1 ) Section $4 A$. Sludge Receipt, Blending and Adjustment

\begin{tabular}{|c|c|c|c|c|c|c|c|}
\hline \multirow{2}{*}{\multicolumn{2}{|c|}{$\begin{array}{l}\text { STREAM NUMBERS } \\
\text { STREAM NAME }\end{array}$}} & \multirow[t]{2}{*}{ (CONT'D) } & \multirow{2}{*}{$\rightarrow$} & \multicolumn{2}{|l|}{247} & 4 & 107 \\
\hline & & & & to PRBT & Inleakage & to EAVC & Solubles \\
\hline 54 & $\mathrm{Cu}(\mathrm{COOH}) 2$ & & & $0.2061 \mathrm{E}-03$ & ZERO & ZERO & $0.2061 \mathrm{E}-03$ \\
\hline 170 & $\mathrm{Cu}(\mathrm{NO}) 2$ & & & $0.2179 \mathrm{E}+01$ & ZERO & ZERO & $0: 2179 \mathrm{E}+01$ \\
\hline 55 & Cuo & & & $0.9546 \mathrm{E}-03$ & ZERO & ZERO & ZERO \\
\hline 80 & $\mathrm{Fe} 203$ & & & $0.1587 \mathrm{E}+00$ & ZERO & ZERO & ZERO \\
\hline 101 & $\mathrm{FeO}$ & & & ZERO & ZERO & ZERO & ZERO \\
\hline 102 & Group A & & & $0.2437 \mathrm{E}-05$ & ZERO & ZÈRO & ZERO \\
\hline 103 & Group B & & & $0.7493 \mathrm{E}-07$ & ZERO & ZERO & ZERO \\
\hline 25 & H2 & & & $0.1058 \mathrm{E}-24$ & ZERO & $0.1057 \mathrm{E}-24$ & TRACE \\
\hline 158 & $\mathrm{H} 2 \mathrm{C} 204$ & & & $0.5247 \mathrm{E}-02$ & ZERO & ZERO & $0.5247 \mathrm{E}-02$ \\
\hline 93 & H2SO4 & & & ZERO & ZERO & ZERO & ZERO \\
\hline 90 & $\mathrm{H} 3 \mathrm{BO} 3$. & & & $0.7707 \mathrm{E}+01$ & ZERO & ZERO & $0.7707 \mathrm{E}+01$ \\
\hline 34 & HCOOH & & & $0.8930 \mathrm{E}+01$ & ZERO & $0.1619 \mathrm{E}-01$ & $0.8914 \mathrm{E}+01$ \\
\hline 43 & $\mathrm{HCl}$ & & & ZERO & ZERO & ZERO & ZERO \\
\hline 85 & $\mathrm{HF}$ & & & ZERO & ZERO & ZERO & ZERO \\
\hline 49 & HNO3 & & & ZERO & ZERO & ZERO & ZERO \\
\hline 36 & Hg & & & $0.3197 \mathrm{E}-01$ & ZERO & $0.2660 \mathrm{E}-07$ & ZERO \\
\hline 129 & $\mathrm{Hg}(\mathrm{C} 6 \mathrm{H} 5)_{2} 2$ & & & ZERO & ZERO & ZERO & ZERO \\
\hline 135 & $\mathrm{Hg}(\mathrm{NO} 3)^{2} 2$ & & & ZERO & ZERO & ZERO & ZERO \\
\hline 144 & $\mathrm{Hg} 2 \mathrm{Cl} 2$ & & & ZERO & ZERO & ZERO & ZERO \\
\hline 117 & $\mathrm{Hg} 2 \mathrm{I} 2$ & & & ZERO & ZERO & ZERO & ZERO \\
\hline 146 & $\mathrm{HgCl2}$ & & & ZERO & ZERO & ZERO & ZERO \\
\hline 21 & $\mathrm{HgO}$ & & & ZERO & ZERO & ZERO & ZERO \\
\hline 97 & I2 & & & ZERO & ZERO & ZERO & ZERO \\
\hline 183 & $\mathrm{~K} 2 \mathrm{CO} 3$ & 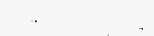 & & ZERO & ZERO & ZERO & ZERO \\
\hline 39 & $\mathrm{~K} 20$ & & & $0.6882 \mathrm{E}-02$ & ZERO & ZERO & ZERO \\
\hline 51 & $\mathrm{KCOOH}$ & & & $0.1005 \mathrm{E}+02$ & ZERO & ZERO & $0.1005 \mathrm{E}+02$ \\
\hline 157 & $\mathrm{KMnO} 4$ & & & ZERO & ZERO & ZERO & ZERO \\
\hline 161 & KNO3 & & & ZERO & ZERO & ZERO & ZERO \\
\hline 10 & $\mathrm{KOH}$ & & & ZERO & ZERO & ZERO & ZERO \\
\hline 150 & KTPB & . & & ZERO & ZERO & ZERO & ZERO \\
\hline 105 & La203 & & & ZERO & ZERO & ZERO & ZERO \\
\hline 108 & Li20 & & & $0.2948 \mathrm{E}-03$ & ZERO & ZERO & $\therefore \quad$ ZERO \\
\hline 65 & $\mathrm{Mg}(\mathrm{COOH}) 2$ & & & $0.3918 \mathrm{E}-02$ & ZERO & ZERO & $0.3918 \mathrm{E}-02$ \\
\hline 171 & $\mathrm{Mg}(\mathrm{NO} 3) 2$ & & & $0.5254 \mathrm{E}-07$ & ZERO & ZERO & $0.5254 \mathrm{E}-07$ \\
\hline 33 & Mgó & & & $0.3432 \mathrm{E}-03$ & ZERO & ZERO & ZERO \\
\hline 60 & $\mathrm{Mn}(\mathrm{COOH}) 2$ & & & $0.3306 \mathrm{E}-02$ & ZERO & ZERO & $0.3306 \mathrm{E}-02$ \\
\hline 172 & Mn (NO3) 2 & & & $0.6122 \mathrm{E}-07$ & ZERO & ZERO & $0.6122 \mathrm{E}-07$ \\
\hline 180 & Mn304 & & & ZERO & ZERO & ZERO & ZERO \\
\hline 56 & Mno & & & ZERO & ZERO & ZERO & ZERO \\
\hline 14 & $\mathrm{MnO} 2$ & & & $0.1313 \mathrm{E}-02$ & ZERO & ZERO & ZERO \\
\hline 145 & $\mathrm{MoO} 2$ & & & $0.1289 \mathrm{E}-03$ & ZERO & ZERO & ZERO \\
\hline 81 & $\mathrm{~N} 2$ & & & $0.6505 E-07$ & $0.5484 \mathrm{E}+02$ & $0.5483 E+02$ & $0.9541 \mathrm{E}-02$ \\
\hline 74 & $\mathrm{~N} 2 \mathrm{O}$ & & & $0.1420 \mathrm{E}-19$ & ZERO & $0.1415 \mathrm{E}-1.9$ & $0.5114 \mathrm{E}-22$ \\
\hline 182 & NH3 & & & $0.9713 \mathrm{E}-07$ & ZERO & $0.5309 \mathrm{E}-07$ & $0.4404 \mathrm{E}-07$ \\
\hline 114 & NH3OHNO3 & & & ZERO & ZERO & ZERO & ZERO \\
\hline 52 & $\mathrm{NH} 4 \mathrm{COOH}$ & & & $0.4894 \mathrm{E}-03$ & ZERO & ZERO & $0.4894 \mathrm{E}-03$ \\
\hline 173 & NH4NO3 & & & ZERO & ZERO & ZERO & ZERO \\
\hline 68 & $\mathrm{NH} 4 \mathrm{OH}$ & & & ZERO & ZERO & ZERO & ZERO \\
\hline 148 & NH 4 TPB & & & ZERO & ZERO & ZERO & ZERO \\
\hline
\end{tabular}

\begin{tabular}{|c|c|c|}
\hline 108 & 3 & 1 \\
\hline PHA & PHA Feed & Sludge Feed \\
\hline Insolubles & to SRAT & to DWPF \\
\hline ZERO & $0.2061 \mathrm{E}-03$ & ZERO \\
\hline ZERO & $0.2179 \mathrm{E}+01$ & ZERO \\
\hline $.9546 \mathrm{E}-03$ & $0.9546 \mathrm{E}-03$ & $0.2093 \mathrm{E}-01$ \\
\hline $.1587 \mathrm{E}+00$ & $.1587 \mathrm{E}+00$ & $0.2805 \mathrm{E}+02$ \\
\hline$\therefore \quad$ ZERO & ZERO & ZERO \\
\hline $0.2437 \mathrm{E}-05$ & $0.2437 \mathrm{E}-05$ & $0.3541 \mathrm{E}-02$ \\
\hline $.7493 \mathrm{E}-07$ & $0.7493 \mathrm{E}-07$ & $0.1511 E-03$ \\
\hline ZERO & TRACE & ZERO \\
\hline ZERO & $0.5247 \mathrm{E}-02$ & ZERO \\
\hline ZERO & ZERO & ZERO \\
\hline ZERO & $0.7707 \mathrm{E}+01$ & ZERO \\
\hline ZERO & $0.8914 \mathrm{E}+01$ & ZERO \\
\hline ZERO & ZERO & ZERO \\
\hline ZERO & ZERO & ZERO \\
\hline ZERO & ZERO & ZERO \\
\hline $0.3197 \mathrm{E}-01$ & $0.3197 \mathrm{E}-01$ & ZERO \\
\hline . $\quad$ ZERO & ZERO & ZERO \\
\hline ZERO & ZERO & ZERO \\
\hline ZERO & ZERO & ZERO \\
\hline ZERO & ZERO & ZERO \\
\hline ZERO & ZERO & ZERO \\
\hline ZERO & ZERO & $0.1384 \mathrm{E}+00$ \\
\hline ZERO & ZERO & ZERO \\
\hline ZERO & ZERO & ZERO \\
\hline $0.6882 \mathrm{E}-02$ & $0.6882 E-02$ & $0.7385 \mathrm{E}-01$ \\
\hline ZERO & $0.1005 E+02$ & ZERO \\
\hline ZERO & ZERO & ZERO \\
\hline ZERO & ZERO & ZERO \\
\hline ZERO & ZERO & $0.1586 \mathrm{E}-01$ \\
\hline ZERO & ZERO & ZERO \\
\hline ZERO & ZERO & ZERO \\
\hline $0.2948 \mathrm{E}-03$ & $0.2948 \mathrm{E}-03$ & ZERO \\
\hline $0.4160 \mathrm{E}-09$ & $0.3918 \mathrm{E}-02$ & ZERO \\
\hline ZERO & $0.5254 \mathrm{E}-07$ & ZERO \\
\hline $0.3432 \mathrm{E}-03$ & $0.3432 \mathrm{E}-03$ & $0.1524 \mathrm{E}+\mathrm{b}_{1}$ \\
\hline ZERO & $0.3306 \mathrm{E}-02$ & ZERO \\
\hline ZERO & $0.6122 \mathrm{E}-07$ & ZERO \\
\hline ZERO & ZERO & ZERO \\
\hline ZERRO & ZERO & ZERO \\
\hline $0.1313 E-02$ & $0.1313 \mathrm{E}-02$ & $0.3245 \mathrm{E}+01$ \\
\hline $0.1289 \mathrm{E}-03$ & $0.1289 \mathrm{E}-03$ & ZERO \\
\hline ZERO & $0.9541 E-02$ & ZERO \\
\hline ZERO & $0.5114 \mathrm{E}-22$ & ZERO \\
\hline ZERO & $0.4404 \mathrm{E}-07$ & ZERO \\
\hline ZERO & ZERO & ZERO \\
\hline ZERO & $0.4894 \mathrm{E}-03$ & ZERO \\
\hline ZERO & ZERO & ZERO \\
\hline ZERO & ZERO & $0.6084 \mathrm{E}-03$ \\
\hline ZERO & ZERO & ZERO \\
\hline
\end{tabular}


Table A-1. HLW Flowsheet Material Balance (Tank 51/ITP Cycle 1) Section 4A. Sludge Receipt, Blending and Adjustment

STREAM NUMBERS (CONT'D) $\rightarrow$ STREAM NAME

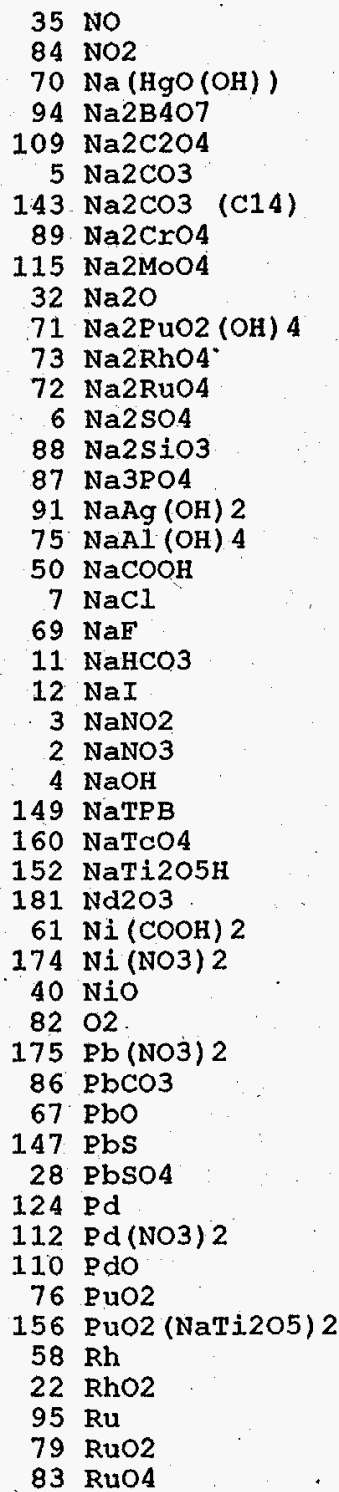

WSRC-TR-95-0019 (Revision

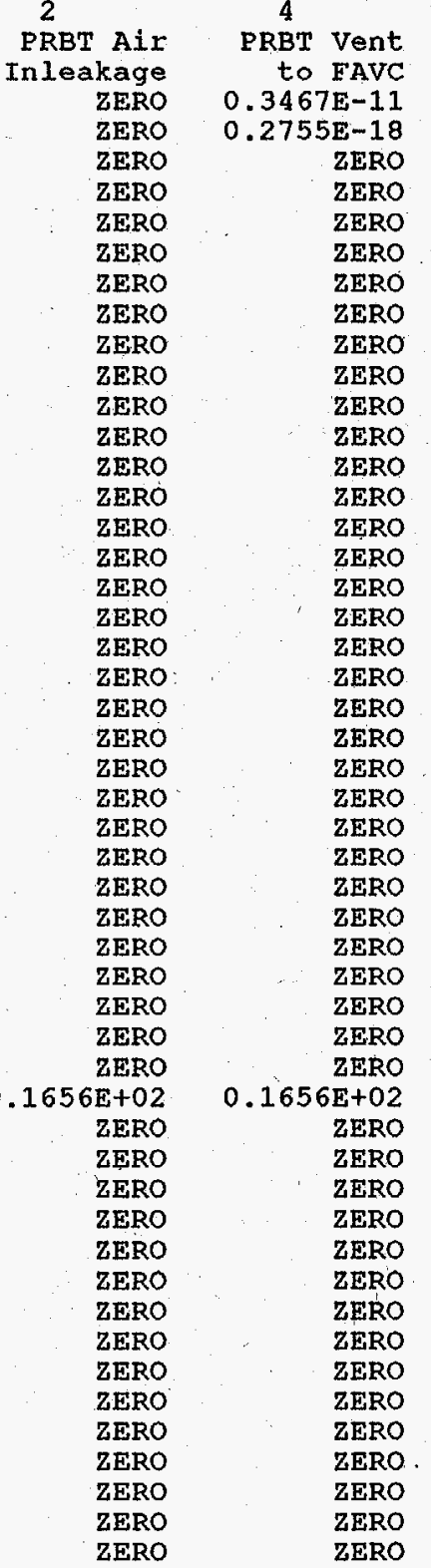

107

PHA
Solubles $0.1771 \mathrm{E}-14$ $0,1771 \mathrm{E}-14$ 108 . PHA
Insolubles 3 reed sludg 1 to SRAT to DWPE $0.1771 \mathrm{E}-14$ ZERO ZERO $0.1407 \mathrm{E}-21$ ZERO ZERO ZERO $0.2562 \mathrm{E}-03$ ZERO ZERO ZERO ZERO ZERO $0.2642 \mathrm{E}+00$ ZERO ZERO $0.2458 \mathrm{E}+00$ ZERO ZERO $0.3019 \mathrm{E}-09$ ZERO ZERO $0.9246 \mathrm{E}-02$ ZERO

$0.1003 \mathrm{E}+01$ ZERO $0.1523 \mathrm{E}-02$

$0.1003 \mathrm{E}+01 \quad 0.3230 \mathrm{E}+01$ zERO ZERO ZERO ZERO ZERO ZERO
ZERO ZERO ZERO ZERO ZERO ZERO ZERO ZERO ZERO TERO ZERO ZERO ZERO ZERO ZERO ZERO ZERO ZERO
ZERO

$0.4152 \mathrm{E}-03$ $0.6686 \mathrm{E}-06$ ZERO
$0.5537 \mathrm{E}-02$ $0.1624 \mathrm{E}-05$ $0.8288 \mathrm{E}-03$ ZERO ZERO ZERO ZERO ZERO ZERO ZERO ZERO ZERO ZERO ZERO ZERO ZERO ZERO ZERO ZERO

\section{$0.4362 \mathrm{E}-02$} $0.3099 \mathrm{E}-08$ ZERO
ZERO

$0.3209 \mathrm{E}-04$ $0.1272 \mathrm{E}-03$ $0.3642 \mathrm{E}-06$ ZERO ZERO

$0.4426 \mathrm{E}-02$ ZERO

\begin{tabular}{|c|c|}
\hline & \\
\hline ERO & $0.3865 \mathrm{E}-04$ \\
\hline ZERO & $0.1652 \mathrm{E}-03$ \\
\hline $1082 \mathrm{E}-02$ & $0.1944 \mathrm{E}+00$ \\
\hline ZERO & $0.8070 \mathrm{E}-02$ \\
\hline $.1437 \mathrm{E}-02$ & $0.1141 \mathrm{E}-02$ \\
\hline ZERO & $.1457 \mathrm{E}-03$ \\
\hline ZERO & $.3558 \mathrm{E}+00$ \\
\hline $38 \mathrm{E}+01$ & ZERO \\
\hline-02 & $0.6777 \mathrm{E}-02$ \\
\hline $21 \mathrm{E}-04$ & $0.3408 \mathrm{E}-01$ \\
\hline ZERO & ŻERO \\
\hline $373 E-07$ & $0.5416 \mathrm{E}-05$ \\
\hline ZERO & $4 E+01$ \\
\hline $00 E-01$ & $8 E+00$ \\
\hline ZERO & $0.3062 \mathrm{E}+01$ \\
\hline ZERO & ZERO \\
\hline ZERO & $0.1568 \mathrm{E}-03$ \\
\hline ZERO & ZERO \\
\hline ZERO & ZERO \\
\hline $152 E-03$ & ZERO \\
\hline $86 \mathrm{E}-06$ & ZERO \\
\hline $8 \mathrm{E}-03$ & $0.2482 E+00$ \\
\hline $37 \mathrm{E}-02$ & $L E+00$ \\
\hline $24 E-05$ & $4 E-64$ \\
\hline ZERO & ZERO \\
\hline & ZERO \\
\hline ZERO & ZERO \\
\hline $0.4362 \mathrm{E}-02$ & $0.1274 \mathrm{E}+00$ \\
\hline $0.3099 \mathrm{E}-08$ & ZERO \\
\hline ZERO & $4 \mathrm{E}-08$ \\
\hline & $0.4488 \mathrm{E}-03$ \\
\hline $0.3209 \mathrm{E}-04$ & $0.9873 \mathrm{E}-02$ \\
\hline $0.1272 \mathrm{E}-03$ & ZERO \\
\hline & ZERO \\
\hline ZERO & $0.9498 \mathrm{E}-03$ \\
\hline & ZERO \\
\hline $126 \mathrm{E}-02$ & $0.6163 \mathrm{E}-02$ \\
\hline
\end{tabular}

Section 4A, Page 4 of 21 
Table A-1. HLW Flowsheet Material Balance (Tank 51/ITP Cycle 1) section 4A. Sludge Receipt, Blending and Adjustment

STREAM NUMBERS (CONT'D) $\rightarrow$ STREAM NAME

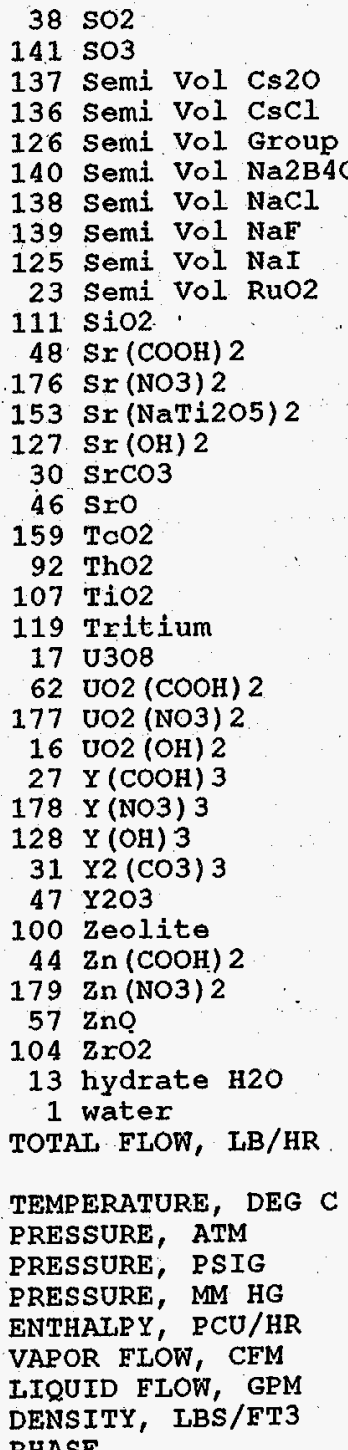

247

PR Bottoms

to PRBT

ZERO

ZERO

ZERO

ZERO

ZERO

ZERÓ

ZERO

ZERO

$0.2667 \mathrm{E}-01$

$0.1093 \mathrm{E}-03$

ZERO
$0.4072 \mathrm{E}-02$

EE-02
ZERO

ZERO

ZERO

$0.1761 \mathrm{E}-05$

ZERO

$0.7844 \mathrm{E}+00$

$0.1281 \mathrm{E}-11$

$0.7512 \mathrm{E}-03$

$0.1272 \mathrm{E}-05$ ZERO

$0.1317 E-11$

ZERO

ZERO

ZERO

$0.9683 E-03$ ZERO
9E+OO

$0.5336 \mathrm{E}-04$

$0.1383 \mathrm{E}+00$ ZERO

$0.7374 \mathrm{E}+03$

$0.7722 \mathrm{E}+03$

$0.5000 E+02$

$0.9656 \mathrm{E}+00$

$-0.5055 \mathrm{E}+00$

$0.7339 \mathrm{E}+03$

$0.3732 \mathrm{E}+05$

$0.1521 \mathrm{E}+01$

IIOUID
2

PRBT Air

nleakage

ZERO

ZERO

ZERO

ZERO

ZERO

ZERO

ZERO

ZERO

ZERO

ZERO

ZERO

ZERO

ZERO

ZERO

ZERO

ZERO

ZERO

ZERO

ZERO

ZERO

ZERO

ZERO

ZERO

ZERO

ZERO

ZERO

ZERO

ZERO

ZERO

ZERO

ZERO

$0.1200 \mathrm{E}+01$

$0.7260 \mathrm{E}+02$

$0.3500 \mathrm{E}+02$

$0.1000 \mathrm{E}+01$

ZERO

$0.7358 \mathrm{E}+03$

$0.1670 \mathrm{E}+00$

$0.5422 \mathrm{E}+02$
${ }^{4}{ }^{2}$ VRT Vent

to FAVC

ZERO

ZERO

ZERO

ZERO

ZERO

ZERO

ZERO

ZERO

ZERO

ZERO

ZERO

ZERO

ZERO

ZERO

ZERO

ZERO

ZERO

$0.8298 \mathrm{E}-14$

ZERO

ZERO

ZERO

ZERO

ZERO

ZERO

ZERO

ZERO

ZERO

ZERO

ZERO

ZERO

ZERO

$0.4886 \mathrm{E}+01$

$0.7632 E+02$

$0.4564 \mathrm{E}+02$

$0.9656 \mathrm{E}+00$

$-0.5055 \mathrm{E}+00$

$0.7339 \mathrm{E}+03$

$0.3975 \mathrm{E}+04$

$0.1985 \mathrm{E}+02$

IIQUID
107

PHA
Solubles

ZERO

ZERO

ZERO

ZERO

ZERO

ZERO

ZERO

ZERO

ZERO

ZERO

$0.1093 \mathrm{E}-03$

ZERO

ZERO

ZERO

ZERO

ZERO

ZERO

ZERO

$0.1272 \mathrm{E}-1$

$0.1272 \mathrm{E}-05$

ZERO

$0.1317 \mathrm{E}-11$

ZERO

ZERO

ZERO

ZERO

ZERO

$0.1139 \mathrm{E}+00$

$0.5336 \mathrm{E}-04$

ZERO

ZERO

$0.7415 \mathrm{E}+03$

$0.7727 \mathrm{E}+03$

$0.4566 \mathrm{E}+02$

$0.9656 \mathrm{E}+00$

$-0.5055 \mathrm{E}+00$

$0.7339 \mathrm{E}+03$

$0.3424 \mathrm{E}+05$

$0.1526 \mathrm{E}+01$

$0.6311 \mathrm{E}+02$
108

Insolubles

ZERO

ZERO

ZERO

ZERO

ZERO

ZERO

ZERO

ZERO

ZERO

0.2667 E-01

ZERO

$0.4072 \mathrm{E}-02$

ZERO

ZERO

$0.1761 \mathrm{E}-05$

ZERO

$0.7844 \mathrm{E}+00$

$0.7512 \mathrm{E}-03$

ZERO

ZERO

ZERO

ZERO

ZERO

ZERO

ZERO

$0.9683 E-03$

ZERO

ZERO

0.1383 ZERO

0 ZERO

$6 \mathrm{E}-01$

$0.3635 \mathrm{E}+01$

$0.4566 \mathrm{E}+02$

$-0.5055 \mathrm{E}+00$

$0.7339 \mathrm{E}+03$

$0.3512 \mathrm{E}+02$

$0.2667 \mathrm{E}-02$

$0.1699 \mathrm{E}+03$

IIQUID
3

to SRAT

ZERO

ZERO

ZERO

ZERO

ZERO

ZERO

ZERO

ZERO

$0.2667 \mathrm{E}-01$

$0.1093 E-03$

$0.4072 \mathrm{E}-02$

ZERO

ZERO

ZERO

$0.1761 \mathrm{E}-05$

ZERO

$0.7844 \mathrm{E}+00$

$0.1272 \mathrm{E}-11$

$.1212 \mathrm{E}-03$

ZERO

$0.1317 \mathrm{E}-11$

ZERO

ZERO

$0.9683 \mathrm{E}-03$ ZERO

$0.1139 \mathrm{E}+00$

$0.5336 \mathrm{E}-04$

$0.1383 \mathrm{E}+00$

$0.2176 \mathrm{E}-01$

$0.7415 \mathrm{E}+03$

$0.7763 \mathrm{E}+03$

$0.4566 \mathrm{E}+02$

$0.9656 \mathrm{E}+00$

$-0.50 .55 \mathrm{E}+00$

$0.7339 \mathrm{E}+03$

$0.3428 \mathrm{E}+05$

$0.1529 E+01$

$0.6329 \mathrm{E}+02$
LIOUID

LIQUID

$0.7920 E+O O$

$0.6998 E+02$

LIQUID 
Table A-1. HLW Flowsheet Material Balance (Tank 51/ITP Cycle 1) Section 4A. Sludge Receipt, Blending and Adjustment

STREAM NUMBERS -> STREAM NAME

COMPONENT FLOWS, LB/HR 122 (C6H5) 2

123 (C6H5) $2 \mathrm{C} 6 \mathrm{H} 4$

118 (C6H5) $2 \mathrm{NH}$

133 (C6H5B (OH) 2 salt)

132 (C6H5B (OH) 2)

166 (C6H5C6H4OH)

162 (C6H5NH2)

163 (C6H5NHCHO)

165 (C6H5NO)
164 (C6H5NO2)

131 (C6H5OH salt)

130 ( $66 \mathrm{H} 5 \mathrm{OH})$

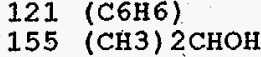

154 ( $\mathrm{CH} 3 \mathrm{OH}$ )

$45 \mathrm{Ag}$

116 Ag2O

134 AgNO3

$106 \mathrm{~B} 203$

$9 \mathrm{Ba}(\mathrm{OH}) 2$

$15 \mathrm{BaO}$

$24 \mathrm{BaSO}$

$66 \mathrm{CO}$

$142 \mathrm{CO} 2$ (C14)

$63 \mathrm{Ca}(\mathrm{COOH}) 2$

$167 \mathrm{Ca}$ (NO3) 2
$120 \mathrm{Ca}$ (OH) 2

$113 \mathrm{Ca}$ (PO4) 2

$20 \mathrm{CaC} 204$

$18 \mathrm{CaCO} 3$

$64 \mathrm{CaCO}$ (C14)

78 CaF2

$41 \mathrm{CaO}$

$19 \mathrm{CaSO} 4$

29 Carbon

99 Cement

$26 \mathrm{Co}(\mathrm{COOH}) 2$
$168 \mathrm{Co}(\mathrm{NO} 3)^{2}$

$59 \mathrm{COO}$

$98 \mathrm{Cr} 203$

42 Cs 20

$93 \mathrm{CsCOOH}$

$169 \mathrm{CsNO3}$

$$
8 \mathrm{CsOH}
$$

151 CsTPB

WSRC-TR-95-0019 (Revision 7

6

SRAT Trnsfer SRAT Sample $50 \%$ HNO3 Pump Prime Pump Prime to SRAT

ZERO
ZERO
ZERO
ZERO
ZERO
ZERO
ZERO
ZERO
ZERO
ZERO
ZERO
ZERO
ZERO
ZERO
ZERO
ZERO
ZERO
ZERO
ZERO
ZERO
ZERO
ZERO
ZERO
ZERO
ZERO
ZERO
ZERO
ZERO
ZERO
ZERO
ZERO
ZERO
ZERO
ZERO
ZERO
ZERO
ZERO
ZERO
ZERO
ZERO
ZERO
ZERO
ZERO
ZERO
ZERO
ZERO
ZERO
ZERO

ZERO

ZERO

ZERO

ZERO

ZERO

ZERO

ZERO

ZERO

ZERO

ZERO

ZERO

ZERO

ZERO

ZERO

ZERO

ZERO

ZERO

ZERO

ZERO

ZERO

ZERO

ZERO

ZERO

ZERO

ZERO

ZERO

ZERO

ZERO

ZERO

ZERO

ZERO

ZERO

ZERO

ZERO

ZERO

ZERO

ZERO

ZERO

ZERO

ZERO

ZERO

ZERO

ZERO

ZERO

ZERO

ZERO

ZERO

ZERO

ZERO

ZERO

ZERO

ZERO

ZERO

ZERO

ZERO

ZERO

ZERO

ZERO

ZERO

ZERO

ZERO

ZERO

ZERO

ZERO

ZERO

ZERO

ZERO

ZERO

ZERO

ZERO

ZERO

ZERO

ZERO

ZERO

ZERO

ZERO

ZERO

ZERO

ZERO

ZERO

ZERO

ZERO

ZERO

ZERO

ZERO

ZERO

ZERO

ZERO

ZERO

ZERO

10

SRAT Air MWWT Spent SRAT Recycle SRATC Cndste

11

12

ZERO

$\begin{array}{rr}\text { ZERO } & 0.4224 \mathrm{E}-09 \\ \text { ZERO } & 0.5464 \mathrm{E}-12 \\ \text { ZERO } & 0.7726 \mathrm{E}-08 \\ \text { ZERO } & \text { ZERO } \\ \text { ZERO } & \text { ZERO } \\ \text { ZERO } & 0.3030 \mathrm{E}-12\end{array}$

ZERO

ZERO

ZERO

ZERO

ZERO

ZERO

ZERO

ZERO

ZERO

ZERO

ZERO

ZERO

ZERO

ZERO

ZERO

ZERO

ZERO

ZERO

ZERO

ZERO

ZERO

ZERO

ZERO

ZERO

ZERO

ZERO

ZERO

ZERO

ZERO

ZERO

ZERO

ZERO

ZERO

ZERO

ZERO

ZERO

ZERO

ZERO

ZERO

ZERO

ZERO

Date: January 1995

Condensate

$0.3578 \mathrm{E}-03$

$0.1481 \mathrm{E}-06$
$0.3298 \mathrm{E}-02$

ZERO

$0.9568 \mathrm{E}-07$

$0.2377 \mathrm{E}-01$

$0.3624 \mathrm{E}-06$

$.8025 \mathrm{E}-07$

. $1204 \mathrm{E}-13$

. $3804 \mathrm{E}-13$

9630E-15

9841 -06

.7271=-09

$0.7840 \mathrm{E}-16$

$.7146 \mathrm{E}-17$

ZERO
$0.1459 \mathrm{E}-21$

ZERO

$0.4854 \mathrm{E}-10$

$0.1759 \mathrm{E}-10$

$0.1841 \mathrm{E}-19$

$0.9359 \mathrm{E}-13$

$$
\text { ZERO }
$$

$.4841 \mathrm{E}-12$

$0.6247 \mathrm{E}-20$

ZERO

IE-10
ZERO

$0.1442 \mathrm{E}-10$

$0.1338 \mathrm{E}-17$

ZERO

$0.8246 \mathrm{E}-13$

$0.1840 \mathrm{E}-13$

$0.1211 \mathrm{E}-11$. ZERO

$0.7853 \mathrm{E}-15$

$0.2945 \mathrm{E}-17$

$0.1682 \mathrm{E}-15$

$0.2355 \mathrm{E}-11$

$73 \mathrm{E}-12$

$0.5279 \mathrm{E}-16$

$0.6020 \mathrm{E}-17$

ZERO

$0.5900 \mathrm{E}-08$

. $1202 \mathrm{E}-07$

$0.1426 \mathrm{E}-20$

$0.3107 \mathrm{E}+00$

$0.2732 \mathrm{E}-10$

$0.2418 \mathrm{E}-11$

ZERO

ZERO

$0.1530 \mathrm{E}-04$

$0.2893 E-08$

ZERO

$0.2951 \mathrm{E}-07$

ZERO

$0.6075 \mathrm{E}-03$

$0.8169 \mathrm{E}-11$

ZERO

$0.7254 \mathrm{E}-05$

$0.4549 \mathrm{E}-05$

$0.4230 \mathrm{E}-12$

ZERO

$0.2600 \mathrm{E}-07$

$0.5792 \mathrm{E}-08$

ZERO

$0.2482 \mathrm{E}-09$

$0.5317 \mathrm{E}-10$

$0.7430 \mathrm{E}-06$

$0.2430 E-09$

$0.7501 E-07$

$0.1664 \mathrm{E}-10$

ZERO

$1094 \mathrm{E}-02$

$0.4530 \mathrm{E}-06$

$0.1008 \mathrm{E}-01$

ZERO

$0.2926 \mathrm{E}-06$

$0.7268 \mathrm{E}-01$

$0.1108 \mathrm{E}-05$

$.2153 \mathrm{E}-05$

$0.8354 \mathrm{E}-10$

$0.7394 \mathrm{E}-11$

$0.6575 \mathrm{E}-07$

ZERO

$0.9026 \mathrm{E}-07$ 
Table A-1. HLW Flowsheet Material Balance (Tank 51/ITP Cycle 1) Section 4A. Sludge Receipt, Blending and Adjustment

STREAM NUMBERS (CONT'D) -> STREAM NAME

\begin{tabular}{|c|c|}
\hline 54 & $\mathrm{Cu}(\mathrm{COOH}) 2$ \\
\hline 170 & $\mathrm{Cu}(\mathrm{NO} 3) 2$ \\
\hline 55 & $\mathrm{CuO}$ \\
\hline 80 & $\mathrm{Fe} 2 \mathrm{O} 3$ \\
\hline 101 & $F \in O$ \\
\hline 102 & Group A \\
\hline 103 & Group B \\
\hline & \\
\hline 158 & $\mathrm{H} 2 \mathrm{C} 2 \mathrm{O} 4$ \\
\hline 93 & H2 504 \\
\hline 90 & $\mathrm{H}_{3} \mathrm{BO} 3$. \\
\hline 34 & $\mathrm{HCOOH}$ \\
\hline 43 & $\mathrm{HCl}$ \\
\hline 85 & $\mathrm{HF}$ \\
\hline 49 & HNO3 \\
\hline 36 & $\mathrm{Hg}$ \\
\hline 129 & $\mathrm{Hg}(\mathrm{C} 6 \mathrm{H} 5) 2$ \\
\hline 135 & $\mathrm{Hg}(\mathrm{NO} 3)_{2} 2$ \\
\hline 144 & Hg2C12 \\
\hline 117 & $\mathrm{Hg} 2 \mathrm{I} 2$ \\
\hline 146 & $\mathrm{HgCl} 2$ \\
\hline 21 & $\mathrm{HgO}$. \\
\hline 97 & $I 2$ \\
\hline 183 & $\mathrm{~K} 2 \mathrm{CO} 3$ \\
\hline 39 & $\mathrm{~K} 2 \mathrm{O}$ \\
\hline 51 & $\mathrm{KCOOH}$ \\
\hline 157 & $\mathrm{KMnO} 4$ \\
\hline 161 & KNO3 \\
\hline 10 & $\mathrm{KOH}$ \\
\hline 150 & KTPB \\
\hline 105 & $\mathrm{~L} a 203$ \\
\hline 108 & Li2o \\
\hline 65 & $\mathrm{Mg}(\mathrm{COOH}) 2$ \\
\hline 171 & $\mathrm{Mg}(\mathrm{NO} 3)_{2}$ \\
\hline 33 & Mgo \\
\hline 60 & $\mathrm{Mn}(\mathrm{COOH}) 2$ \\
\hline 172 & $\mathrm{Mn}(\mathrm{NO} 3) 2$ \\
\hline 180 & $\mathrm{Mn} 304$ \\
\hline 56 & Mno \\
\hline & $\mathrm{MnO} 2$ \\
\hline 145 & $\mathrm{MoO} 2$ \\
\hline 81 & N2 \\
\hline 7 & $\mathrm{~N} 20$ \\
\hline 182 & NH3 \\
\hline 114 & NH3OHNO3 \\
\hline 52 & $\mathrm{NH} 4 \mathrm{COOH}$ \\
\hline 173 & NH4NO3 \\
\hline & NH4OH \\
\hline
\end{tabular}
6 Pump Prime Pump Prime

$\begin{array}{lr}\text { PIIme } & \text { PUMP } \\ \text { ZERO } & \text { ZIIER } \\ \text { ZERO } & \text { ZERO } \\ \text { ZERO } & \text { ZERO } \\ \text { ZERO } & \text { ZERO } \\ \text { ZERO } & \text { ZERO } \\ \text { ZERO } & \text { ZERO } \\ \text { ZERO } & \text { ZERO } \\ \text { ZERO } & \text { ZERO } \\ \text { ZERO } & \text { ZERO } \\ \text { ZERO } & \text { ZERO } \\ \text { ZERO } & \text { ZERO } \\ \text { ZERO } & \text { ZERO } \\ \text { ZERO } & \text { ZERO } \\ \text { ZERO } & \text { ZERO } \\ \text { ZERO } & \text { ZERO } \\ \text { ZERO } & \text { ZERO } \\ \text { ZERO } & \text { ZERO } \\ \text { ZERO } & \text { ZERO } \\ \text { ZERO } & \text { ZERO } \\ \text { ZERO } & \text { ZERO } \\ \text { ZERO } & \text { ZERO } \\ \text { ZERO } & \text { ZERO } \\ \text { ZERO } & \text { ZERO } \\ \text { ZERO } & \text { ZERO } \\ \text { ZERO } & \text { ZERO } \\ \text { ZERO } & \text { ZERO } \\ \text { ZERO } & \text { ZERO } \\ \text { ZERO } & \text { ZERO } \\ \text { ZERO } & \text { ZERO } \\ \text { ZERO } & \text { ZERO } \\ \text { ZERO } & \text { ZERO } \\ \text { ZERO } & \text { ZERO } \\ \text { ZERO } & \text { ZERO } \\ \text { ZERO } & \text { ZERO } \\ \text { ZERO } & \text { ZERO } \\ \text { ZERO } & \text { ZERO } \\ \text { ZERO } & \text { ZERO } \\ \text { ZERO } & \text { ZERO } \\ \text { ZERO } & \text { ZERO } \\ \text { ZERO } & \text { ZERO } \\ \text { ZERO } & \text { ZERO } \\ \text { ZERO } & \text { ZERO } \\ \text { ZERO } & \text { ZERO } \\ \text { ZERO } & \text { ZERO } \\ \text { ZERO } & \text { ZERO } \\ \text { ZERO } & \text { ZERO } \\ \text { ZERO } & \text { ZERO } \\ \text { ZERO } & \text { ZERO } \\ \text { ZERO } & \text { ZERO }\end{array}$

50\%
HNO3
to
ZRAR
ZERO
ZERO
ZERO
ZERO
ZERO
ZERO
ZERO
ZERO
ZERO
ZERO
ZERO
ZERO
ZERO
ZERO
ZERE
ZERO
ZERO
ZERO
ZERO
ZERO
ZERO
ZERO
ZERO
ZERO
ZERO
ZERO
ZERO
ZERO
ZERO
ZERO
ZERO
ZERO
ZERO
ZERO
ZERO
ZERO
ZERO
ZERO
ZERO
ZERO
ZERO
ZERO
ZERO
ZERO
ZERO
ZERO
ZERO
ZERO
ZERO
10 SRAT Air Inleakage , ZERO ZERO ZERO ZERO ZERO ZERO ZERO ZERO ZERO ZERO ZERO ZERO ZERO ZERO ZERO ZERO ZERO ZERO ZERO ZERO ZERO ZERO ZERO ZERO ZERO ZERO ZERO ZERO ZERO ZERO ZERO ZERO ZERO ZERO ZERO ZERO ZERO ZERO ZERO ZERO ZERO $0.8857 \mathrm{E}+03$ ZERO ZERO ZERO ZERO ZERO ZERO ZERO

Date: January 1995
11

12 13

MWWT Spent SRAT Recycle SRATC Cndste Wsh to SRAT Condensate $0.2434 \mathrm{E}-13 \quad 0.7674 \mathrm{E}-08$ $0.1196 \mathrm{E}-10 \quad 0.3774 \mathrm{E}-05$ $0.1082 \mathrm{E}-12 \quad 0.3411 \mathrm{E}-07$ $0.1395 \mathrm{E}-09 \quad 0.4397 \mathrm{E}-04$ $0.1394 \mathrm{E}-10 \quad 0.4396 \mathrm{E}-0.5$ $0.1947 \mathrm{E}-13 \quad 0.6137 \mathrm{E}-08$ $0.8305 \mathrm{E}-15 \quad 0.2618 \mathrm{E}-09$ $0.3206 \mathrm{E}-18 \quad 0.1386 \mathrm{E}-06$ $0.1004 \mathrm{E}-11 \quad 0.3165 \mathrm{E}-06$ $0.3165 \mathrm{E}-06$ $0.4224 \mathrm{E}-10$ ZERO ZERO $0.5594 \mathrm{E}-08$ ZERO ZERO ZERO ZERO ZERO

$0.1082 \mathrm{E}-16$ 2 E-16
ZERO ZERO

$0.4436 \mathrm{E}-12$

$0.5506 \mathrm{E}-10$

$0.1570 \mathrm{~F}-12$

$0.1801 \mathrm{E}-15$

$$
\text { ZERO }
$$

$0.1539 \mathrm{E}-10$ $0.1903 \mathrm{E}-10$ $0.2880 \mathrm{E}-18$ $0.6071 \mathrm{E}-11$ $0.1192 \mathrm{E}-10$ $0.3355 \mathrm{E}-18$ ZERO $0.4360 \mathrm{E}-16$ $0.1070 \mathrm{E}-10$ $0.5906 \mathrm{E}-14$ $0.7403 \mathrm{E}-06$ TRACE $0.1968 \mathrm{E}-10$ ZERO $0.1477 \mathrm{E}-05$ $0.1702 \mathrm{E}-12$ ZERO
ZERO $0.1335 \mathrm{E}=04$ ZERO ZERO ZERO
$0.3921 \mathrm{E}-09$ 1E-09 ZERO ZERO ZERO ZERO ZERO ZERO
ZERO ZERO
ZERO ZERO

$0.1398 \mathrm{E}-06$ $0.1740 \mathrm{E}-04$ ZERO
$0.4951 \mathrm{E}-07$ ZERO ZERO

$0.5106 \mathrm{E}-09$ $0.5999 \mathrm{E}-0.5$ $0.9101 \mathrm{E}-13$ $0.5282 \mathrm{E}-06$ $0.3758 \mathrm{E}-05$ $0.1060 \mathrm{E}-12$ ZERO

$0.6635 \mathrm{E}-22$ $0.3374 \mathrm{E}-05$ $0.1863 \mathrm{E}-08$ $0.4135 \mathrm{E}-02$ TRACE

$0.1884 \mathrm{E}-06$ ZERO $0.2409 \mathrm{E}-08$ ZERO
ZERO $0.2347 \mathrm{E}-07$ $0.1154 \mathrm{E}-04$ $0.1043 \mathrm{E}-06$ $0.1345 \mathrm{E}-03$ $0.1344 \mathrm{E}-04$ $0.1344 \mathrm{E}-04$ $0.1877 \mathrm{E}-07$ $0.8007 \mathrm{E}-09$ $0.4238 \mathrm{E}-06$ $0.9679 \mathrm{E}-06$ $0.4083 \mathrm{E}-04$ ZERO ZERO $0.1199 \mathrm{E}-08$ $0.8494 \mathrm{E}-06$ ZERO ZERO ZERO ZERO ZERO ZERO ZERO $0.4277 \mathrm{E}-06$ $0.5321 \mathrm{E}-04$ ZERO
$0.1514 \mathrm{E}-06$ ZERO ZERO $0.1561 \mathrm{E}-08$ $0.1835 \mathrm{E}-04$ $0.2783 \mathrm{E}-12$ $0.1615 \mathrm{E}-65$ $0.1149 \mathrm{E}-04$ $0.3243 \mathrm{E}-12$ ZERO
$0.2029 \mathrm{E}-21$ $0.1032 \mathrm{E}-04$ $0.5696 \mathrm{E}-08$ $0.1264 \mathrm{E}-01$ TRACE $0.5761 \mathrm{E}-06$ 0.7367 ZERO $0.3022 \mathrm{E}+00$ Section $4 \mathrm{~A}$, Page 7 of 21 
Table A-1. HLW Flowsheet Material Balance (Tank 51/ITP Cycle 1) Section $4 \mathrm{~A}$. Sludge Receipt, Blending and Adjustment

STREAM NUMBERS (CONT'D) $\rightarrow$ STREAM NAME

\begin{tabular}{|c|c|}
\hline 35 & No \\
\hline 84 & NO2 \\
\hline 70 & $\mathrm{Na}(\mathrm{HgO}(\mathrm{OH}))$ \\
\hline 94 & Na2B407 \\
\hline 109 & $\mathrm{Na} 2 \mathrm{C} 2 \mathrm{O} 4$ \\
\hline & $\mathrm{Na} 2 \mathrm{CO} 3$ \\
\hline 143 & $\mathrm{Na} 2 \mathrm{CO} 3(\mathrm{C} 14)$ \\
\hline 89 & $\mathrm{Na} 2 \mathrm{CrO} 4$ \\
\hline 115 & $\mathrm{Na} 2 \mathrm{MoO} 4$ \\
\hline 32 & $\mathrm{Na} 2 \mathrm{O}$ \\
\hline 71 & $\mathrm{Na} 2 \mathrm{PuO} 2(\mathrm{OH}) 4$ \\
\hline 73 & $\mathrm{Na} 2 \mathrm{RhO} 4$ \\
\hline 72 & $\mathrm{Na} 2 \mathrm{RuO} 4$ \\
\hline 6 & $\mathrm{Na} 2 \mathrm{SO}_{4}$ \\
\hline 88 & $\mathrm{Na} 2 \mathrm{SiO} 3$ \\
\hline 87 & $\mathrm{Na} 3 P O 4$ \\
\hline 91 & $\operatorname{NaAg}(\mathrm{OH}) 2$ \\
\hline 75 & $\operatorname{NaAl}(\mathrm{OH}) 4$ \\
\hline 50 & $\mathrm{NaCOOH}$ \\
\hline 7 & Nac1 \\
\hline 69 & $\mathrm{NaF}$ \\
\hline 11 & $\mathrm{NaHCO} 3$ \\
\hline 12 & $\mathrm{NaI}$ \\
\hline 3 & $\mathrm{NaNO} 2$ \\
\hline 2 & NaNO3 \\
\hline 4 & $\mathrm{NaOH}$ \\
\hline 149 & NaTPB \\
\hline 160 & NaToO4 \\
\hline 152 & $\mathrm{NaTi205H}$ \\
\hline 181 & $\mathrm{Nd2O3}$ \\
\hline 61 & $\mathrm{Ni}(\mathrm{COOH}) 2$ \\
\hline 174 & $\mathrm{Ni}(\mathrm{NO} 3) 2$ \\
\hline 40 & NiO \\
\hline 82 & $\mathrm{O} 2$ \\
\hline 175 & $\mathrm{~Pb}(\mathrm{NO} 3) 2$ \\
\hline 86 & $\mathrm{PbCO} 3$ \\
\hline 67 & Pbo : \\
\hline 147 & $\mathrm{PbS}$ \\
\hline 28 & $\mathrm{PbSO} 4$ \\
\hline 124 & $\mathrm{Pd}$ \\
\hline 112 & $\mathrm{Pd}(\mathrm{NO} 3) 2$ \\
\hline 110 & Pdo \\
\hline 76 & $\mathrm{PuO} 2$ \\
\hline 156 & PuO2 (NaTi2O5) 2 \\
\hline 58 & $\mathrm{Rh}$ \\
\hline 22 & RhO2 \\
\hline 95 & Ru \\
\hline 79 & RuO2 \\
\hline 83 & RuO4 \\
\hline
\end{tabular}

WSRC-TR-95-0019 (Revision 7 Pump

P ZIme
ZERO
ZERO
ZERO
ZERO
ZERO
ZERO
ZERO
ZERO
ZERO
ZERO
ZERO
ZERO
ZERO
ZERO
ZERO
ZERO
ZERO
ZERO
ZERO
ZERO
ZERO
ZERO
ZERO
ZERO
ZERO
ZERO
ZERO
ZERO
ZERO
ZERO
ZERO
ZERO
ZERO
ZERO
ZERO
ZERO
ZERO
ZERO
ZERO
ZERO
ZERO
ZERO
ZERO
ZERO
ZERO
ZERO
ZERO
ZERO
ZERO

\begin{tabular}{c}
8 \\
808 \\
HNO \\
to \\
ZRAT \\
ZERO \\
ZERO \\
ZERO \\
ZERO \\
ZERO \\
ZERO \\
ZERO \\
ZERO \\
ZERO \\
ZERO \\
ZERO \\
ZERO \\
ZERO \\
ZERO \\
ZERO \\
ZERO \\
ZERO \\
ZERO \\
ZERO \\
ZERO \\
ZERO \\
ZERO \\
ZERO \\
ZERO \\
ZERO \\
ZERO \\
ZERO \\
ZERO \\
ZERO \\
ZERO \\
ZERO \\
ZERO \\
ZERO \\
ZERO \\
ZERO \\
ZERO \\
ZERO \\
ZERO \\
ZERO \\
ZERO \\
ZERO \\
ZERO \\
ZERO \\
ZERO \\
ZERO \\
ZERO \\
ZERO \\
ZERO \\
ZERO \\
\\
\hline
\end{tabular}

10

SRAT Air 12
11
MWWT Spent SRAT Recycle SRATC Cndste
13
Condensate Inleakage

ZERO
ZERO
ZERO
ZERO
Prime ZERO ZERO ZERO ZERO ZERO ZERO ZERO ZERO ZERO ZERO ZERO ZERO ZERO ZERO ZERO ZERO ZERO ZERO ZERO ZERO ZERO ZERO ZERO ZERO ZERO ZERO ZERO ZERO ZERO ZERO ZERO ZERO ZERO ZERO ZERO ZERO ZERO ZERO ZERO ZERO ZERO ZERO ZERO ZERO ZERO ZERO ZERO ZERO ZERO ZERO ZERO ZERO ZERO ZERO ZERO ZERO ZERO ZERO ZERO ZERO ZERO ZERO ZERO ZERO ZERO ZERO ZERO ZERO ZERO ZERO ZERO ZERO $0.2675 \mathrm{E}+03$ ZERO ZERO ZERO ZERO ZERO ZERO ZERO ZERO ZERO ZERO ZERO ZERO ZERO ZERO 11

$12 \quad \frac{13}{3}$ h to SRAT TRACE
ZERO .2019E-15 $0.1301 \mathrm{E}-13$ $0.2690 \mathrm{E}-10$ ZERO ZERO

$0.3495 E-10$ ZERO ZERO

$0.1072 \mathrm{E}-11$ ZERO

$0.1414 \mathrm{E}-13$ ZERO

$0.4306 \mathrm{E}-11$ $0.5466 \mathrm{E}-13$ $0.1878 \mathrm{E}-12$ ZERO

$0.3007 \mathrm{E}-16$ $0.1626 \mathrm{E}-13$ $0.4957 \mathrm{E}-10$ ZERO ZERO ZERO ZERO

$0.1385 \mathrm{E}-12$ $0.3665 \mathrm{E}-17$ $0.1307 \mathrm{E}-11$ $0.4271 E-06$ $0.4267 \mathrm{E}-15$ $0.8063 \mathrm{E}-19$ ZERO Condensate to MWWT $0.5664 \mathrm{E}-04$ RACE ZERO

0.8400 EERO 05 ZERO ZERO ZERO
$0.7334 \mathrm{E}-05$ ZE-05
ZERO ZERO ZERO
$0.3381 \mathrm{E}-06$

ZERO EE-08
ZERO

$0.4746 \mathrm{E}-06$ $0.1725 \mathrm{E}-07$ $0.5919 \mathrm{E}-07$ $0.9478 \mathrm{E}-11$ ZERO 9E-04 ZERO ZERO ZERO ZERO $0.1158 \mathrm{E}-11$ $0.4098 \mathrm{E}-06$ $0.2384 \mathrm{E}-02$ $0.1345 \mathrm{E}-09$ $0.1227 \mathrm{E}-24$ ZERO ZERO

ZERO

$0.4807 \mathrm{E}-23$

$0.6972 \mathrm{E}-15$ ZERO

$0.4459 \mathrm{E}-13$ ZERO
ZERO ZERO ZERO
ZERO $0.1716 \mathrm{E}-07$ $0.2203 E-09$
ZERO

0.1407 E-07 ZERO
ZERO TRACE
ZERO ZERO 0.2569 E-04 ZERO ZERO $0.2243 \mathrm{E}-04$ ZERO ZERO ZERO $.1034 \mathrm{E}-05$ $0.1366 \mathrm{E}-07$ ZERO $0.1451 \mathrm{E}-05$ $0.5274 \mathrm{E}-07$ $0.1810 \mathrm{E}-06$ $0.2899 \mathrm{E}-10$ $0.4738 \mathrm{E}-04$ 7RRO ZERO ZERO ZERO $0.1335 \mathrm{E}-06$ $0.3542 \mathrm{E}-11$ $0.1253 E-05$ $0.4114 \mathrm{E}-09$ $0.3752 \mathrm{E}-24$ ZERO $0.2067 \mathrm{E}-08$ ZERO $0.5247 \mathrm{E}-07$. $0.6738 \mathrm{E}-09$ $0.3939 \mathrm{E}-08$ $0.4304 \mathrm{E}-07$ ZERO
ZERO 
Table A-1. HLW Flowsheet Material Balance (Tank 51/ITP Cycle 1) section 4A. Sludge Receipt, Blending and Adjustment

STREAM NUMBERS (CONT'D) $\rightarrow$ STREAM NAME

$38 \mathrm{SO} 2$

$141 \mathrm{SO} 3$

137 Semi Vol Cs2O

136 Semi Vol CsCl

126 Semi Vol Group A

140 Semi Vol Na2B407

138 Semi Vol NaCl

139 Semi Vol Na

125 Semi Vol NaI

$\begin{aligned} 23 & \text { Semi } \\ 111 & \text { sio2 }\end{aligned}$

48 Sr (COOH) 2

176 $\mathrm{sr}(\mathrm{NO} 3) 2$

$153 \mathrm{Sr}(\mathrm{NaTi205}) 2$

$127 \mathrm{Sr}(\mathrm{OH}) 2$

$30 \mathrm{SrCO} 3$

46 Sro

$159 \mathrm{TcO} 2$

92 ThO2

119 Tritium

119 Triti

$17 \mathrm{U} 308$
$62 \mathrm{UO}$ (COOH) 2

$177 \mathrm{UO} 2$ (NO3) 2

16. $\mathrm{DO} 2(\mathrm{OH}) 2$

$27 \mathrm{Y}(\mathrm{COOH}) 3$

$178 \mathrm{Y}$ (NO3) 3

$128 \mathrm{Y}(\mathrm{OH}) 3$

$31 \mathrm{Y} 2(\mathrm{CO})$

100 zeolite

$44 \mathrm{Zn}(\mathrm{COOH}) 2$

$\begin{array}{rl}44 & \mathrm{Zn}(\mathrm{COOH}) 2 \\ 179 \mathrm{Zn}(\mathrm{NO} 3) 2\end{array}$

104 Zro2

13 hydrate $\mathrm{H} 2 \mathrm{O}$

1 water

TOTAI FLOW, LB/HR

TEMPERATURE， DEG C

PRESSURE， ATM

PRESSURE， PSIG

PRESSURE, MM HG

ENTHALPY, PCU/HR

VAPOR FLOW, CFM

IIQUID FLOW, GPM

DENSITY, LBS/FT3

PHASE

SRAT Trnsfer 7 .

$\begin{array}{ll} & \\ \text { Pump Prime } & \text { Rump Prim } \\ \text { Ztro } & \end{array}$

ZERO
ZERO

ZERO ZERO

ZERO ZERO

ZERO ZERO

ZERO

ZERO

ZERO

ZERO

ZERO

ZERO

ZERO

ZERO

ZERO

ZERO

ZERO

ZERO

ZERO

ZERO

ZERO

ZERO

ZERO

ZERO

ZERO

ZERO

ZERO

ZERO

ZERO

ZERO

ZERO

ZERO

ZERO

$0.1950 E+01$

$0.1950 \mathrm{E}+01$

$0.2500 \mathrm{E}+02$

$0.1000 \mathrm{E}+01$

ZERO

$0.4893 \mathrm{E}+02$

$0.3894 \mathrm{E}-02$

$.6243 \mathrm{E}+02$

IIQUID
8

508 HNO3

to SRAT

ZERO

ZERO

ZERO

ZERO

ZERO

ZERO

ZERO

ZERO

ZERO

ZERO

ZERO

ZERO

ZERO

ZERO

ZERO

ZERO

ZERO

ZERO

ZERO

ZERO

ZERO

ZERO

ZERO

ZERO

ZERO

ZERO

ZERO

ZERO

ZERO

ZERO

ZERO

ZERO

$0.3890 \mathrm{E}+01$

$0.2500 \mathrm{E}+02$

$0.1000 \mathrm{E}+01$

ZERO

$0.9761 E+02$

$0.7768 E-02$

$0.6243 E+02$

LIOUID

$0.7818 \mathrm{E}+01$

$0.1564 \mathrm{E}+02$

$0.2601 \mathrm{E}-01$

$0.7495 \mathrm{E}+02$
10

SRAT Air

11

12

MWWT Spent SRAT Recycle SRATC Cndste

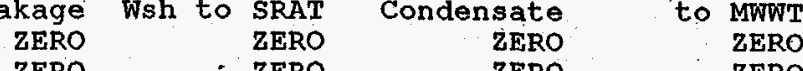
$\begin{array}{llll}\text { ZERO } & \text { ZERO } & \text { ZERO } & \text { ZERO } \\ \text { ZERO } & \text { ZERO } & \text { ZERO } & \text { ZERO }\end{array}$ ZERO ZERO ZERO $\quad$ ZERO $\quad$ ZERO ZERO ZERO ZERO Z ZERO

$\begin{array}{lll}\text { ZERO } & \text { ZERO } & \text { ZERO } \\ \text { ZERO } & \text { ZERO }\end{array}$

ZERO

ZERO

ZERO

ZERO

ZERO

ZERO

ZERO

ZERO

ZERO

ZERO

ZERO

ZER

ZERO

ZERO

ZERO

ZERO

ZERO

ZERO

ZERO

.ZERO

ZERO

ZERO

ZERO

ZERO

ZERO

ZERO

$0.1937 E+02$

$0.1173 \mathrm{E}+04$

$0.3500 \mathrm{E}+02$

$0.1000 \mathrm{E}+01$

ZERO

ZERO

ZERO

$0.1752 \mathrm{E}-09$

$0.5989 \mathrm{E}-15$

$0.1617 \mathrm{E}-12$

$0.2232 \mathrm{E}-13$

ZERO

$0.2524 \mathrm{E}-18$

$0.1961 \mathrm{E}-13$

$0.1633 \mathrm{E}-12$

$0.45550-11$

$0.9347 \mathrm{E}-15$

$0.1145 \mathrm{E}-10$

$0.6972 \mathrm{E}-17$

$0.5020 \mathrm{E}-15$

ZERO

$0.4363 \mathrm{E}-13$

ZERO

$0.5307 \mathrm{E}-14$

ZERO

$0.1430 \mathrm{E}-11$

$0.2925 \mathrm{E}-15$

$0.9842 \mathrm{E}-12$

ZERO

$0.8237 \mathrm{E}-11$

$0.4154 \mathrm{E}-01$

$0.4154 \mathrm{E}-01$

$0.3500 \mathrm{E}+02$

$0.1000 \mathrm{E}+01$

ZERO

$0.1457 \mathrm{E}+01$

$0.2459 \mathrm{E}+05$

IIQUID

$0.7055 \mathrm{E}-01$

$0.8296 \mathrm{E}-04$

$0.6243 \mathrm{E}+02$

LIQUID

ZERO

ZERO

$0.1891 \mathrm{E}-05$

$0.1893 \mathrm{E}-09$

$0.5099 \mathrm{E}-07$

$0.7054 \mathrm{E}-08$

ZERO

$0.3842 \mathrm{E}-24$

$0.6183 \mathrm{E}-08$

$0.5149 \mathrm{E}-07$

$0.1439 E-05$

$0.3072 \mathrm{E}-09$

$0.3610 \mathrm{E}-05$

$0.2204 \mathrm{E}-1$

$0.1583 \mathrm{E}-09$

$0.2282 E-17$

$0.1375 \mathrm{E}-07$

ZERO

$0.1677 \mathrm{E}-08$

ZERO

$0.4517 \mathrm{E}-06$

$0.9243 E-10$

$0.3107 \mathrm{E}-06$

ZERO

$0.2597 \mathrm{E}-05$

$0.3469 \mathrm{E}+03$

$0.3473 \mathrm{E}+03$

$0.5000 \mathrm{E}+02$

$0.9656 \mathrm{E}+00$

$0.7339 \mathrm{E}+03$

$0.1737 \mathrm{E}+05$

VAPOR

$0.6934 \mathrm{E}+00$

$0.6243 \mathrm{E}+02$

I IQUID

ZERO

$0.5783 E-05$

$0.5789 \mathrm{E}-09$

$0.1559 \mathrm{E}-06$
$0.2157 \mathrm{E}-07$

ZERO

$0.1175 \mathrm{E}-23$

$0.1175=23$

$0.4402 E-05$

$0.9394 \mathrm{E}-09$

$0.1104 \mathrm{E}-04$

$0.6739 \mathrm{E}-11$

$0.4840 \mathrm{E}-09$

$0.6979 E-17$

$0.4206 \mathrm{E}-07$

ZERO

$0.5129 \mathrm{E}-08$

ZERO

$0.1381 \mathrm{E}-05$

0.2827 E-09

ZERO

$0.7942 \mathrm{E}-05$

$0.1061 \mathrm{E}+04$

$0.1062 \mathrm{E}+04$

$0.5000 \mathrm{E}+02$

$0.9656 \mathrm{E}+00$

$0.5313 \mathrm{E}+05$

$0.2121 \mathrm{E}+01$ 
Table A-1. HLW Flowsheet Material Balance (Tank 51/ITP Cyale 1) Section 4A. Sludge Receipt, Blending and Adjustment

STREAM NUMBERS -> STREAM NAME

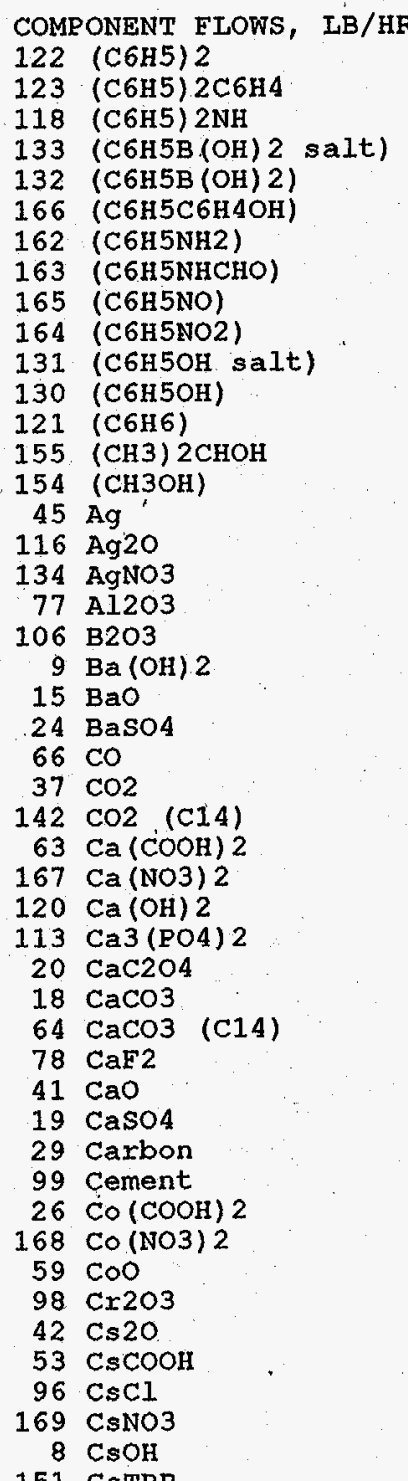

52 53 54 14 15 SRATC Vent Iiquid to Spnt Liq frm SRAT Scrubbr SRAT Transto Scrubber SRAT Scrubbr SRAT Scrubbr Vent to FAVC fer to SME

\begin{tabular}{|c|c|c|c|}
\hline $\begin{array}{l}0.9482 \mathrm{E}-02 \\
0.5373 \mathrm{E}-12 \\
0.6541 \mathrm{E}-05\end{array}$ & $\begin{array}{l}0.1877 \mathrm{E}-02 \\
0.2336 \mathrm{E}-05 \\
0.3306 \mathrm{E}-01\end{array}$ & $\begin{array}{l}0.1133 \mathrm{E}-01 \\
0.2268 \mathrm{E}-05 \\
0.3204 \mathrm{E}-01\end{array}$ & $\begin{array}{l}0.1826 \mathrm{E}-04 \\
0.4628 \mathrm{E}-13 \\
0.1551 \mathrm{E}-07\end{array}$ \\
\hline ZERO & ZERO & ZERO & ZERO \\
\hline ZERO & ZERO & ZERO & ZERO \\
\hline $.3470 E-12$ & $0.1295 \mathrm{E}-05$ & $0.1258 \mathrm{E}-05$ & $0.8321 \mathrm{E}-11$ \\
\hline $.2029 \mathrm{E}-01$ & $.3492 E+00$ & $0.3587 \mathrm{E}+00$ & $0.1626 \mathrm{E}-03$ \\
\hline $14 \mathrm{E}-11$ & $0.4910 \mathrm{E}-05$ & $0.4768 \mathrm{E}-05$ & $0.2540 \mathrm{E}-1$ \\
\hline $40 E-13$ & $0.5342 \mathrm{E}-07$ & $0.4757 \mathrm{E}-07$ & $0.5168 \mathrm{E}-0$ \\
\hline $0.4359 \mathrm{E}-13$ & $0.1627 \mathrm{E}-06$ & $0.1580 \mathrm{E}-06$ & $0.3414 \mathrm{E}-10$ \\
\hline TRACE & $0.4118 \mathrm{E}-08$ & $0.3482 \mathrm{E}-08$ & ZERO \\
\hline $0.1272 \mathrm{E}+00$ & $0.4250 \mathrm{E}+01$ & $0.4248 \mathrm{E}+01$ & $0.1939 \mathrm{E}-0$ \\
\hline $61 \mathrm{E}-03$ & $0.5172 \mathrm{E}-02$ & $0.5266 \mathrm{E}-02$ & $18 \mathrm{E}-0$ \\
\hline $5 E-10$ & $0.3547 \mathrm{E}-09$ & $510 \mathrm{E}-09$ & $5 E-1$ \\
\hline $55 \mathrm{E}-11$ & $0.3379 \mathrm{E}-10$ & $0.3417 \mathrm{E}-10$ & $9 \mathrm{E}-1$ \\
\hline $799 E-13$ & ZERO & $0.779 .9 \mathrm{E}-13$ & ZER \\
\hline ZERO & $0.6236 \mathrm{E}-15$ & $0.5418 \mathrm{E}-15$ & ZER \\
\hline ZERO & ZERO & ZERO & ZERC \\
\hline $551 \mathrm{E}-10$ & $0.2075 \mathrm{E}-03$ & $0.2016 \mathrm{E}-03$ & ZERC \\
\hline $9 \mathrm{E}-13$ & $0.7521 \mathrm{E}-04$ & $0.7301 \mathrm{E}-04$ & ZER \\
\hline ZERO & ZERO & ZERO & ZERC \\
\hline TRACE & $0.7872 \mathrm{E}-13$ & $0.6839 \mathrm{E}-13$ & ZERC \\
\hline $0.1071 \mathrm{E}-12$ & $0.4002 \mathrm{E}-06$ & $.3887 \mathrm{E}-06$ & ZERC \\
\hline ZERO & - ZERO & ZERO & ZER \\
\hline $3 E+01$ & $346 \mathrm{E}-03$ & $12 \mathrm{E}-01$ & $09 \mathbf{E}+0$ \\
\hline $8 E-07$ & $9480 \mathrm{E}-11$ & $99 \mathrm{E}-09$ & $O E-0$ \\
\hline ZERO & ZERO & ZERO & ZER \\
\hline $0.2631 \mathrm{E}-10$ & $0.9838 \mathrm{E}-04$ & $0.9556 \mathrm{E}-04$ & ZER \\
\hline ZERO & ZERO & ZERO & ZER \\
\hline 0.1 & $66 E-04$ & 0.5 & ZER \\
\hline $4 E-17$ & $5723 E-11$ & $7 E-11$ & ZER \\
\hline ZERO & ZERO & ZERO & ZER \\
\hline $\mathrm{ZER}$ & ZERO & ZERO & ZER \\
\hline $0.9430 \mathrm{E}-13$ & $0.3526 \mathrm{E}-06$ & $0.3425 \mathrm{E}-06$ & ZERC \\
\hline $0.2101 \mathrm{E}-13$ & $6 \mathrm{E}-07$ & $9 E-07$ & ZERC \\
\hline $7 \mathrm{E}-11$ & $0.5176 \mathrm{E}-05$ & $0.5026 \mathrm{E}-05$ & ZER \\
\hline ZERO & ZERO & ZERO & ZER \\
\hline $\mathrm{ZEI}$ & ZERO & ZERO & ZERC \\
\hline $0.9002 E-15$ & $0.3358 \mathrm{E}-08$ & $0 E-08$ & ZER \\
\hline $0.3376 E-17$ & $0.1259 \mathrm{E}-10$ & $3 E-10$ & ZER \\
\hline $0.1928 \mathrm{E}-15$ & $0.7193 \mathrm{E}-09$ & $0.6984 \mathrm{E}-09$ & ZERO \\
\hline $0.2695 \mathrm{E}-11$ & $0.1007 \mathrm{E}-04$ & $0.9778 \mathrm{E}-05$ & ZERO \\
\hline $0.8814 \mathrm{E}-15$ & $0.3295 \mathrm{E}-08^{\prime}$ & $0.3200 \mathrm{E}-08$ & ZERO \\
\hline $0.2721 \mathrm{E}-12$ & $0.1015 \mathrm{E}-05$ & $0.9853 \mathrm{E}-06$ & ZERC \\
\hline ZERO & ZE & & ZERC \\
\hline $0.6036 \mathrm{E}-$ & $0.2257 \mathrm{E}-09$ & 99 & ZERC \\
\hline & $0.2574 \mathrm{E}-10$ & $6 \mathrm{E}-10$ & ZER \\
\hline ZERO & ZERO & ZERO & ZERC \\
\hline
\end{tabular}

$0.2620 \mathrm{E}-06$ $0.9245 \mathrm{E}-02$ $0.3165 E-04$ ZERO
ZERO $0.5523 \mathrm{E}-01$ $0.2409 \mathrm{E}-02$ $0.2092 \mathrm{E}+00$ $0.3406 \mathrm{E}-02$ $0.6938 \mathrm{E}-02$ $0.8234 \mathrm{E}-15$ $0.1550 \mathrm{E}+00$ $0.1006 \mathrm{E}-09$ $0.1017 \mathrm{E}-10$ $0.3681 \mathrm{E}-12$ $0.3681 \mathrm{E}-12$
$0.1241 \mathrm{E}-01$ $0.1241 E-01$
ZERO ZERO

$0.8834 \mathrm{E}+01$ $0.1670 \mathrm{E}-02$ 0.1617 ZERO $0.1704 \mathrm{E}-01$ $0.1704 \mathrm{E}-0$ $0.2418 \mathrm{E}-07$ $0.3251 \mathrm{E}-15$ $0.3251 \mathrm{E}-15$ $0.4188 \mathrm{E}+01$ ZERO $0.2626 \mathrm{E}+01$
$0.2442 \mathrm{E}-06$ $0.2442 \mathrm{E}-06$ ZERO $0.1501 \mathrm{E}-01$ $0.3344 \mathrm{E}-02$ $0.2208 \mathrm{E}+00$ ZERO

$0.14335-03$ $0.1433 \mathrm{E}-03$ $0.3069 \mathrm{E}-04$ $0.4289 \mathrm{E}+00$ $0.1403 \mathrm{E}-03$ $0.4330 \mathrm{E}-0$ ZERO
$0.9608 E-05$ ZERO

Date: January 1995
16

to SMECT

17

MWWT Air Purge

$0.1727 \mathrm{E}-10 \quad$ ZERO

$0.5615 \mathrm{E}-17$ ZERO

$0.6828 \mathrm{E}-11$ ZERO

ZERO

ZERO

$0.7119 \mathrm{E}-17$ ZERO

$0.1495 \mathrm{E}-08$ ZERO

$0.2245 \mathrm{E}-16 \quad$ ZERO

$0.4769 \mathrm{E}-15 \quad$ ZERO

$0.1847 \mathrm{E}-16 \quad$ ZERO

ZERO

$0.1041 E-07$ ZERO

$\begin{array}{ll}0.5040 E-09 & \text { ZERO } \\ 0.4770 E-17 & \text { ZERO }\end{array}$

$0.7912 \mathrm{E}-18$ ZERO

$0.7971 E-12 \quad$ ZERO

TRACE ZERO

0.4939 E-15 ZERO

$0.1790 \mathrm{E}-15$

ZERO ZERO

$0.9524 \mathrm{E}-18$ ZERO

ZERO

ZERO

ZERO

ZERO

ZERO

ZERO

ZERO

ZERO

ZERO

ZERO

ZERO

ZERO

ZERO

ZERO

ZERO

ZERO

ZERO

ZERO

ZERO

ZERO

ZERO

ZERO

ZERO

ZERO

ZERO

ZERO 
Table A-1. HLW Flowsheet Material Balance (Tank 51/ITP Cycle 1) Section 4A. Sludge Receipt, Blending and Adjustment

STREAM NUMBERS (CONT'D) $\rightarrow$ STREAM NAME

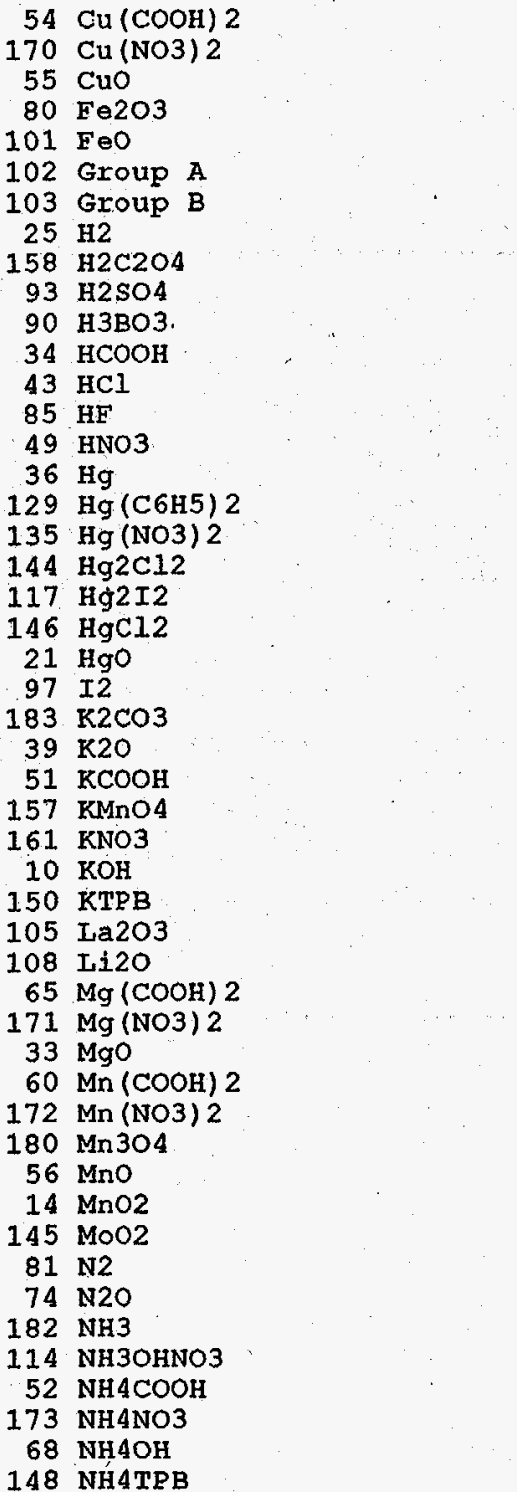
53 54 SRATC Vent Liquid to Spnt Liq frm SRAT Scrubbr to Scrubber SRAT Scrubbr SRAT Scrubbr Vent to FAVC $0.2784 \mathrm{E}-13$ $0.1041 \mathrm{E}-06$
$0.5113 \mathrm{E}-04$

$0.1369 \mathrm{E}-10$ $0.5113 \mathrm{E}-04$

0.1237E-12 $0.4627 \mathrm{E}-06$ $0.59642-03$

$.1595 \mathrm{E}-09$

$.1595 \mathrm{E}-10$

$0.5962 \mathrm{E}-04$

$.2226 \mathrm{E}-13$

$.9497 \mathrm{E}-15$

$0.3551 \mathrm{E}-08$

$0.1184 \mathrm{E}-07$

$0.4292 \mathrm{E}-0.5$

ZERO

$0.1806 \mathrm{E}-0$

ZERO

ZERO

ZERO

$0.3118 \mathrm{E}-09$

$0.1007 \mathrm{E}-11$

ZERO
$0.2408 E+02$

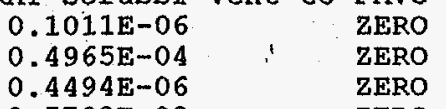

$0.5792 \mathrm{E}-03$

$0.5791 \mathrm{E}-04$

$0.8085 E-07$

$0.3449 \mathrm{E}-08$

$0.3231 \mathrm{E}-05$

$0.4169 \mathrm{E}-05$

$0.1754 \mathrm{E}-03$

ZERO
ZERO

ZERO

ZERO

ZERO

ZERO

ZERO

ZERO

ZERO

$0.5072 \mathrm{E}-12$

$0.6311 \mathrm{E}-10$

$0.1796 \mathrm{E}-12$

$6 \mathrm{E}-12$

ZERO

ZERO

$0.1852 \mathrm{E}-14$

$.2176 \mathrm{E}-10$

$0.3301 \mathrm{E}-18$

$0.1916 \mathrm{E}-11$

$0.1363 \mathrm{E}-10$

ZERO

TRAC

$0.1224 \mathrm{E}-10$

$0.6756 \mathrm{E}-14$

$0.8857 \mathrm{E}+03$

. $5113 \mathrm{E}-22$

$0.5113 \mathrm{E}-22$

ZERO
ZER ZERO

$0.8738 \mathrm{E}-14$

$0.3584 \mathrm{E}-06$

$$
\text { ZERO }
$$

ZERO

ERO

ZERO

ZERO

$0.4628 \mathrm{E}-10$

ZERO

$0.1897 \mathrm{E}-05$

$0.2354 \mathrm{E}-03$

$0.6715 \mathrm{E}-06$

$0.1007 \mathrm{E}-11$

$\mathrm{E}-11$

ZERO

ZERO

ZERO

ZERO

$0.4021 \mathrm{E}-10$

ZERO

$0.1842 \mathrm{E}-05$

$0.2286 \mathrm{E}-03$

ZERO

7699E-09 0.6522E-06

$0.6689 \mathrm{E}-09$$$
\text { ZERO }
$$

$0.6578 \mathrm{E}-04$

$0.8136 \mathrm{E}-04$

$0.1231 \mathrm{E}-11$

$0.2596 \mathrm{E}-04$

$0.1435 \mathrm{E}-11$

ZERO

$0.1864 \mathrm{E}-09$

$0.4577 \mathrm{E}-04$

$0.9021 \mathrm{E}-01$

TRACE

$0.3026 \mathrm{E}-03$ ZERO

$0.6315 \mathrm{E}+01$

$0.9502 \mathrm{E}-18$

ZERO

ZERO

$0.6386 \mathrm{E}-04$

$0.7902 \mathrm{E}-04$

$0.2520 \mathrm{E}-0.4$

$0.4950 \mathrm{E}-04$

$0.1393 \mathrm{E}-11$ ZERO

$0.1620 \mathrm{E}-09$

$0.4445 \mathrm{E}-04$

$0.9336 \mathrm{E}-01$

$0.1034 \mathrm{E}-24$

$0.3545 \mathrm{E}-03$

ZERO

$0.6196 \mathrm{E}+01$

$0.3584 \mathrm{E}-06$

ZERO

Date: Januaxy 1995 1.4

$$
15
$$

SRAT Trans-

fer to SME

$0.4430 \mathrm{E}-02$

$0.2179 \mathrm{E}+01$

$0.1969 \mathrm{E}-01$

$0.2539 \mathrm{E}+02$

$0.3543 \mathrm{E}-02$

$0.1512 \mathrm{E}-03$

$0.5285 \mathrm{E}-12$

$0.1827 \mathrm{E}+00$

$0.7707 \mathrm{E}+01$

ZERO

ZERO

ERO

$0.8203 \mathrm{E}+00$

ZERO

ZERO

ZERO
ZERO

ZERO

ZERO
ZERO

ZERO

ZERO

ZERO

ZERO

ZERO

ZERO

ZERO

ZERO

ZERO

ZERO

ZERO

ZERO

ZERO

ZERO

ZERO

ZERO

ZERO

ZERO

$0.8857 \mathrm{E}+03$

$0.5105 \mathrm{E}-22$

$0.7631 \mathrm{E}-03$

ZERO

ZERO

ZERO

ZERO

$.4360 \mathrm{E}-09$

$0.1604 \mathrm{E}+00$

ZERO

ZERO

ZERO

ZERO

ZERO

ZERO

ZERO

$0.8073 \mathrm{E}-01$

$0.1005 \mathrm{E}+02$

$0.2858 \mathrm{E}-01$

ZERO

ZERO

ZERO

$0.2948 \mathrm{E}-03$

$0.3463 \mathrm{E}+01$

$0.5254 \mathrm{E}-07$
$0.3049 \mathrm{E}+00$

$0.2169 \mathrm{E}+01$

$0.6122 \mathrm{E}-07$

ZERO

$0.1948 \mathrm{E}+01$

$0.1075 \mathrm{E}-02$

酶-08

TRACE

ZERO

$1 \mathrm{E}-02$

ZERO
ZERO

Section $4 A$, Page 11 of 21

17

ZERO

ZERO

$0.1936 \mathrm{E}-15$

$0.2930 \mathrm{E}-23$

$0.6178 \mathrm{E}-16$.

ZERO

$.6010 \mathrm{E}-19$

TRACE

10

ZERO
Purge

ZERO

ZERO

ZERO

ZERO

ZERO

ZERO

ZERO

ZERO

ZERO

ZERO

ZERO

ZERO

ZERO

ZERO

ZERO

ZERO

ZERO

$\begin{array}{rr}\text { ZERO } & \text { ZERO } \\ 0.4514 \mathrm{E}-17 & \text { ZERO } \\ 0.5602 \mathrm{E}-15 & \text { ZERO }\end{array}$

$0.5602 \mathrm{E}-15 \quad$ ZERO

$0.1598 \mathrm{E}-17$ ZERO

$0.1830 E-20$ ZERO

$0.3415 \mathrm{E}-23$

ZERO

ZERO

ZERO

ZERO

ZERO

ZERO

ZERO

ZERO

ZERO

ZERO

WSRC-TR-95-0019 (Revision 
Table A-1. HLW Flowsheet Material Balance (Tank 51/ITP Cycle 1) section 4A. Sludge Receipt, Blending and Adjustment

STREAM NUMBERS (CONT'D) $\rightarrow$ STREAM NAME

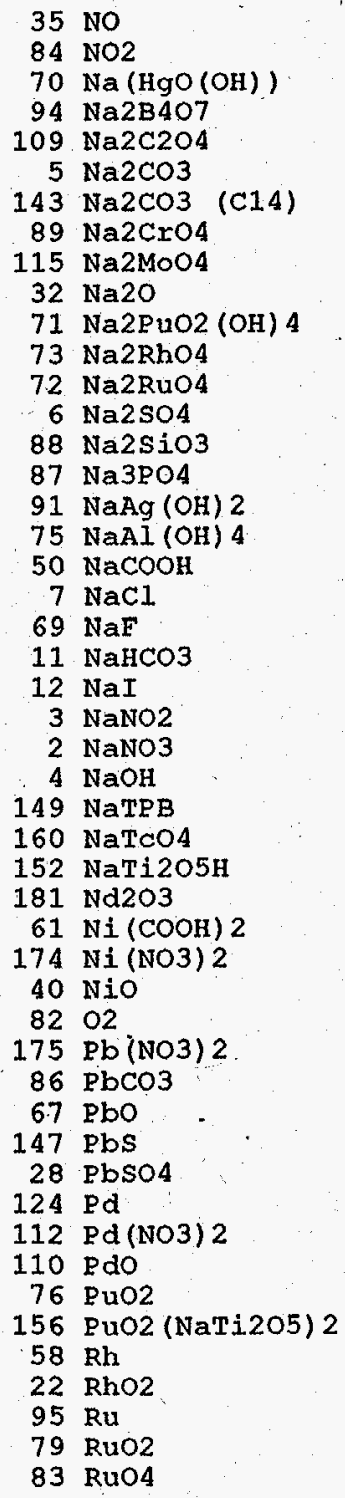

WSRC-TR-95-0019 (Revision
52

SRATC Vent

53

54

14

SRATC Vent Liquid to Spnt Liq frm SRAT Scrubbr SRAT TransSRAT Scrubbr SRAT Scrubbr Vent to FAVC

$0.1403 \mathrm{E}+01$

$0.2100 \mathrm{E}-05$

$0.4318 \mathrm{E}-03$

$0.1403 \mathrm{E}+01$

$0.1165 \mathrm{E}-09$ ZERO ZERO

ZERO

ZERO

$0.8631 \mathrm{E}-09$

ZERO

$0.7499 \mathrm{E}-09$

$0.2986 \mathrm{E}-07$

$0.3047 \mathrm{E}-10$

$0.11505-03$

$0.1117 \mathrm{E}-03$

ZERO

ZERO

$0.2660 \mathrm{E}-10$

ZERO

ZERO

$0.1226 \mathrm{E}-11$

ZERO

$0.1620 \mathrm{E}-13$ ZERO

$0.1721 \mathrm{E}-11$

$0.6256 \mathrm{E}-13$

$0.2147 \mathrm{E}-12$

ZERO
$0.3438 \mathrm{E}-16$

ZERO

$0.5619 \mathrm{E}-10$

ZERO

ZERO

ZERO

$0.1584 \mathrm{E}-12$

$0.1584 \mathrm{E}-12$
$0.4201 \mathrm{E}-17$

$0.1486 \mathrm{E}-11$

$0.2674 \mathrm{E}+03$

$0.4880 \mathrm{E}-15$

ZERO

ZERO

$0.8281 \mathrm{E}-12$

$0.2452 \mathrm{E}-14$ ZERO

$0.6223 \mathrm{E}-13$

$0.7992 \mathrm{E}-15$

$0.4672 \mathrm{E}-14$ ZERO
$5 \mathrm{E}-13$

$0.5105 \mathrm{E}-13$
ZERO

ZERO

ZERO

ZERO

ZERO

$0.1494 \mathrm{E}-03$

ZERO

0.4585 ZERO

ZERO
$0.6048 \mathrm{E}-07$

ZERO

$0.1841 \mathrm{ZERO}$

$0.2337 \mathrm{E}-06$

$0.8028 \mathrm{E}-06$

ZERO
$6 \mathrm{E}-09$

$0.6952 \mathrm{E}-07$

$0.2119 \mathrm{E}-03$

ZERO

ZERO

ZERO

ZERO

$0.5921 \mathrm{E}-06$

$0.1567 \mathrm{E}-10$

$0.5587 \mathrm{E}-05$
$0.5205 \mathrm{E}-01$

$0.1824 \mathrm{E}-08$

ZERO

ZERO

6E-05

ZERO
ZERO

$0.2056 \mathrm{E}-16$

$0.2327 \mathrm{E}-06$

$0.2981 E-08$
ZERO

$0.4465 \mathrm{E}-16$

$0.1907 \mathrm{E}-06$

ZERO
ZERO

Date: January 1995

16

MWWT Vent

17

$0.5130 \mathrm{E}-12$

TRACE

ZERO

ZERO

$0.7298 \mathrm{E}-14$ ZERO

$0.2052 \mathrm{E}-20$

$0.1323 \mathrm{E}-18$

ZERO

$0.4849 \mathrm{E}+01$

ZERO

ZERO

$0.1451 \mathrm{E}-03$

ZERO

ZERO

$0.4454 \mathrm{E}-05$

ZERO

$0.5873 \mathrm{E}-07$ ZERO

$0.1783 \mathrm{E}-04$

$0.2270 \mathrm{E}-06$

$.7797 E-06$

ZERO
E-09

$0.6039 \mathrm{E}-07$

$0.2058 \mathrm{E}-03$

ZERO

ZERO

ZERO

ZERO

ZERO

ZERO

ZERO

ZERO

ZTRO

ZERO

ZERO

ZERO

ZERO

ZERO

ZERO

ZERO

ZERO

ZERO

ZERO

ZERO

ZERO.

ZERO

ZERO

$0.5751 \mathrm{E}-06$

$0.1521 \mathrm{E}-10$

$0.5382 \mathrm{E}-01$

$0.1772 \mathrm{E}-08$

$0.2995 \mathrm{E}-12$

ZERO

$.3007 E-05$

ZERO

$0.1786 \mathrm{E}-16$

$0.2894 \mathrm{E}-08$

$0.4672 \mathrm{E}-14$

$0.3879 \mathrm{E}-16$

$0.1852 \mathrm{E}-06$

ZERO

ZERO

ZERO

ZERO

ZERO

ZERO

ZERO

$0.2674 \mathrm{E}+03$

ZERO

ZERO

ZERO

ZERO

ZTRO

ZRRO

ZERO

ZERO

ZERO

ZERO

ZERO

ZERO

ZERO
ZERO

$0.4234 \mathrm{E}+01$

ZERO

ZERO

$0.1952 \mathrm{E}+00$

$0.2578 \mathrm{E}-02$

ZERO

$0.9957 \mathrm{E}-02$

$0.3417 \mathrm{E}-01$ ZERO

$0.5472 \mathrm{E}-05$

$0.8943 \mathrm{E}+01$

3E+01

ZERO

ZERO

ZERO

$0.2521 \mathrm{E}-01$

$0.6686 \mathrm{E}-06$

$.2366 \mathrm{E}+00$

$.9713 \mathrm{E}-08$

$0.7767 \mathrm{E}-04$

$0.7084 \mathrm{E}-19$

ZERO

$.3318 \mathrm{E}+00$

ZERO

ZERO

$0.9905 \mathrm{E}-02$

$0.1272 \mathrm{E}-03$

$0.7436 \mathrm{E}-03$
ZERO

$0.8125 \mathrm{E}-02$

ZERO
ZERO

ZERO

ZERO

ZERO

$0.3557 \mathrm{E}-15$

ZERO

ZERO

$0.1091 \mathrm{E}-16$

ZERO

$0.1439 \mathrm{E}-18$

ZERO

$0.4382 \mathrm{E}-16$

$0.5563 \mathrm{E}-18$

$0.1911 \mathrm{E}-17$

$0.3060 \mathrm{E}-21$

$0.1653 \mathrm{E}-18$

$0.5044 \mathrm{E}-15$

ZERO

ZERO

ZERO

ZERO

ZERO

$0.1409 \mathrm{E}-17$

$0.1330 \mathrm{E}-16$

$0.1093 \mathrm{E}+01$

$0.4342 \mathrm{E}-20$

ZERO
$0.8196 \mathrm{E}-24$

ZERO

$0.7368 \mathrm{E}-17$

ZERO

0. T538E-18

$0.1091 \mathrm{E}-22$

$0.4538 \mathrm{E}-18$

Section 4 A, Page 12 of 21

ZERO

ZERO

ZERO

ZERO

ZERO

ZERO

ZERO

ZTRO

ZERO

ZERO

ZERO

ZERO

ZERO

ZERO

ZERO

ZERO

ZERO

ZERO
$0.2740 \mathrm{E}+00$

Purge

ZERO

ZERO

ZERO

ZERO

ZERO 
Table A-1. HLW Flowsheet Material Balance (Tank 51/TTP Cycle 1) section 4A. Sludge Receipt, Blending and Adjustment

STREAM NUMBERS (CONT'D) $\rightarrow$ STREAM NAME

38 SO2

$141 \mathrm{SO} 3$

137 Semi Vol Cs2O

136 Semi Vol CsC1

126 Semi Vol Group A

140 Semi Vol Na2B4O

138 Semi Vol NaCl

139 Semi Vol NaF

125 Semi Vol NaI
23 Semi Vol RuO2

$111 \mathrm{SiO2}$

$48 \mathrm{Sr}(\mathrm{COOH}) 2$

$176 \mathrm{Sr}$ (NO3) 2

$153 \operatorname{Sr}(\mathrm{NaTi2O5)} 2$

$127 \mathrm{Sr}(\mathrm{OH}) 2$

$30 \mathrm{SrCO}$

46 Sro

$159 \cdot \mathrm{TCO} 2$

92 ThO2

107 TiO2

119 Tritiu

$17 \mathrm{U} 308$

$62 \mathrm{vO2}(\mathrm{COOH}) 2$

177 UO2 (NO3) 2

$16 \mathrm{UO} 2(\mathrm{OH}) 2$

$27 \mathrm{Y}(\mathrm{COOH}) 3$

$128 \mathrm{Y}(\mathrm{OH}) 3$

31 Y2 (CO3) 3

$47 \mathrm{Y} 203$

100 Zeolite

$44 \mathrm{Zn}(\mathrm{COOH}) 2$

$179 \mathrm{Zn}(\mathrm{NO} 3) 2$

$57 \mathrm{zno}$

104 ZrO2

13 hydrate $\mathrm{H} 2 \mathrm{O}$

1 water

TOTAL FLOW, LB/HR

TEMPERATURE， DEG C

PRESSURE, ATM

PRESSURE， PSIG

PRESSURE, MM HG

ENTHALPY, PCU/HR

VAPOR FIOW, CFM

LIQUID FLOW, GPM

DENSITY， LBS/FT3

PHASE

WSRC-TR-95-0019 (Revision
52

SRATC Vent Liquid to spnt Liq frm SRAT Scrubbr to Scrubber SRAT Scrubbr SRAT Scrubbr Vent to FAVC

ZERO
ZERO
ZERO
ZERO
ZERO
ZERO
ZERO
ZERO
ZERO
ZERO
$0.6859 \mathrm{E}-11$
$0.6866 \mathrm{E}-15$
$0.1850 \mathrm{E}-12$
$0.2559 \mathrm{E}-13$
ZERO
ZERO
TRACE
$0.2243 \mathrm{E}-13$

$0.1867 \mathrm{E}-12$

$0.5221 \mathrm{E}-11$

$0.8940 \mathrm{E}-10$

$0.1309 \mathrm{E}-10$

$0.7992 \mathrm{E}-17$

$0.5741 \mathrm{E}-1.5$

$0.8278 \mathrm{E}-23$

$0.4989 \mathrm{E}-13$

ZERO

$0.6084 \mathrm{E}-14$ ZERO

$0.1638 \mathrm{E}-11$

$0.3353 \mathrm{E}-15$

$0.1127 \mathrm{E}-11$

ZERO
9E -11

$0.9419 \mathrm{E}-11$

$0.1260 \mathrm{E}+04$

$0.5062 \mathrm{E}+02$

$0.9656 \mathrm{E}+00$

$-0.5055 \mathrm{E}+00$

$0.7339 \mathrm{E}+03$

$0.7956 \mathrm{E}+05$

$0.3358 \mathrm{E}+03$

$0.6253 E-01$

VAPOR

$\begin{array}{r}\text { ZERO } \\ \text { ZERO } \\ \text { ZERO } \\ \text { ZERO } \\ \text { ZERO } \\ \text { ZERO } \\ \text { ZERO } \\ \text { ZERO } \\ \text { ZERO } \\ \text { ZERO } \\ \hline\end{array}$

$0.7492 \mathrm{E}-03$

$0.2561 \mathrm{E}-08$

$0.6916 \mathrm{E}-06$

$0,9543 \mathrm{E}-07$

ZERO

$0.1079 \mathrm{E}-11$

$0.8385=-07$

$0.6983 \mathrm{E}-06$

$0.1948 \mathrm{E}-04$

$0.4141 \mathrm{E}-08$

$0.4896 \mathrm{E}-04$

$0.2981 \mathrm{E}-10$

$0.2146 \mathrm{E}-08$

$0.3087 \mathrm{E}-16$

$0.1865 \mathrm{E}-06$

ZERO

$0.22695-07$

ZERO

$0.6114 \mathrm{E}-05$

$0.4208 \mathrm{E}-05$

ZERO

$0.3522 \mathrm{E}-04$

$0.754 .9 \mathrm{E}+04$
$0.7584 \mathrm{E}+04$

$0.4900 \mathrm{E}+02$

$0.1000 \mathrm{E}+01$

$$
\text { ZERO }
$$

ZERO

ZERO

ZERO

ZERO
ZERO

ZERO

ZERO

ZERO

ZERO
$0.7273 E-03$

$0.7273 \mathrm{E}-03$
$0.2487 \mathrm{E}-08$

$0.6717 \mathrm{E}-06$

$0.6717 E-06$
$0.9266 \mathrm{E}-07$

ZERO

$0.9377 \mathrm{E}-12$

$0.8144 \mathrm{E}-07$

$0.6782 \mathrm{E}-06$

$0.1891 \mathrm{E}-04$

$0.4060 \mathrm{E}-08$
$0.4755 \mathrm{E}-04$

$0.4755 \mathrm{E}-04$
$0.2895 \mathrm{E}-10$

$0.2085 \mathrm{E}-08$

ZERO
$0.2998 \mathrm{E}-16$

$0.1812 \mathrm{E}-06$

ZERO

$0.2203 \mathrm{E}-07$

$0.2203 E-07$

ZERO
$0.5937 \mathrm{E}-05$

$0.1214 \mathrm{E}-08$

$0.4087 \mathrm{E}-05$

$0.3421 \mathrm{E}-04$

$0.7552 \mathrm{E}+04$

$0.7586 \mathrm{E}+04$

$0.5000 \mathrm{E}+02$

$0.1000 E+01$

$$
\text { ZERO }
$$

$0.3786 E+06$

$0.1513 \mathrm{E}+02$

$0.6251 \mathrm{E}+02$

IIQUID

$0.1513 \mathrm{E}+02$

$0.6251 \mathrm{E}+02$

LIQUID
15

SRAT Trans -

fer to SME

ZERO

ZERO

ZERO

ZERO

ZERO

ZERO

ZERO

ZERO

Z 1092ERO

$0.1092 \mathrm{E}+01$

$0.1093 \mathrm{E}-03$

$0.2944 \mathrm{E}-01$

ZERO

ZERO

$0.2218 \mathrm{E}-1.8$
$0.3569 \mathrm{E}-02$

$0.3569 \mathrm{E}-02$

$0.2972 \mathrm{E}-01$

$0.8309 \mathrm{E}+00$
$0.3022 \mathrm{E}-09$

$0.2084 \mathrm{E}+01$

$0.1272 \mathrm{E}-05$

$0.9137 \mathrm{E}-04$

$0.1317 \mathrm{E}-11$

ZERO $\quad 0.7940 \mathrm{E}-02$

ZERO

ZERO

ZERO

ZERO

ZERO

ZERO

ZERO

ZERO

ZERO
$0.9829 \mathrm{E}+02$

$0.1258 \mathrm{E}+04$

$0.5000 \mathrm{E}+02$

$0.1000 \mathrm{E}+01$

ZERO

$.9683 \mathrm{E}-03$

ZERO

$0.2607 \mathrm{E}+00$

$0.1794 \mathrm{E}+00$

ZERO

$0.1499 \mathrm{E}+01$

$0.3420 \mathrm{E}+03$
$0.4401 \mathrm{E}+03$

$0.4959 E+02$

$0.1000 E+01$

$$
\text { ZERO }
$$

$0.1786 \mathrm{E}+05$

$0.6498 \mathrm{E}-0$

$0.7609 \mathrm{E}+00$

$0.7212 \mathrm{E}+02$

LIQUID
16

to SMECT

ZERO

ZERO

ZERO

ZERO

ZERO

ZERO

ZERO

ZERO

ZERO

$0.1783 \mathrm{E}-14$

$0.6095 \mathrm{E}-20$

$0.1646 \mathrm{E}-17$

$0.2271 E-18$

ZERO

ZERO
$0.2566 \mathrm{E}-23$

$0.1996 \mathrm{E}-18$

$0.4635 \mathrm{E}-16$

$0.3537 \mathrm{E}-16$

$0.1165 \mathrm{E}-15$

$0.7095 \mathrm{E}-22$

$0.5109 \mathrm{E}-20$

ZERO

TRACE
$0.4440 \mathrm{E}-18$

ZERO

$0.5400 \mathrm{E}-19$

ZERO

$0.1455 \mathrm{E}-16$

$0.1002 \mathrm{E}-16$

ZERO

$0.9516 \mathrm{E}-01$
$0.4807 \mathrm{E}+01$

$0.3500 E+02$

$0.1000 E+01$

ZERO

$0.1102 E+03$

$0.1138 \mathrm{E}+01$

$0.7042 \mathrm{E}-01$

VAPOR

Section 4A, Page 13 of 21

17

Purge

ZERO

ZERO

ZERO

ZERO

ZERO

ZERO

ZERO

ZERO

ZERO

ZERO

ZERO

ZERO

ZERO

ZERO

ZERO

ZERO

ZERO

ZERO

ZERO

ZERO

ZERO

ZERO

ZERO

ZERO

ZERO

ZERO

ZERO

ZERO

ZERO 
Table A-1. HLW Flowsheet Material Balance (Tank 51/ITP Cyole 1) Section 4A. Sludge Receipt, Blending and Adjustment

STREAM NUMBERS $\rightarrow$ STREAM NAME

COMPONENT FLOWS, LB/HR 122 (C6H5) 2

123 (C6H5) 2C6H4

118 (C6H5) 2NH

133 (C6H5B (OH) 2 salt)

132 (C6H5B (OH) 2)

166 (C6H5C6H4O

1.62 (C6H5NH2)

165 (C6H5NO)

164 (C6H5NO2)

131 (C6H5OH salt)

130 ( $66 \mathrm{H} 5 \mathrm{OH})$

121 (C6H6)

155 ( $\mathrm{CH} 3) 2 \mathrm{CHOH}$

154 ( $\mathrm{CH} 3 \mathrm{OH}$ )

$45 \mathrm{Ag}$

116 Ag20

134 AgNO3

$106 \mathrm{~B} 2 \mathrm{O3}$

$9 \mathrm{Ba}(\mathrm{OH}) 2$

$24 \mathrm{BaSO}$

$66 \mathrm{CO}$

$142 \mathrm{CO} 2$ (C14)

$63 \mathrm{Ca}(\mathrm{COOH}) 2$

$167 \mathrm{Ca}(\mathrm{NO} 3) 2$
$120 \mathrm{Ca}(\mathrm{OH}) 2$

$113 \mathrm{Ca} 3$ (PO4) 2

$20 \mathrm{CaC} 204$

$18 \mathrm{CaCO} 3$

$64 \mathrm{CaCO}_{3}(\mathrm{C} 14)$

78 CaF2

$41 \mathrm{CaO}$

$19 \mathrm{CaSO} 4$

29 Carbon

$26 \mathrm{Co}(\mathrm{COOH}) 2$

$168 \mathrm{Co}(\mathrm{NO} 3) 2$

$59 \mathrm{COO}$

$98 \mathrm{Cr} 203$

$42 \mathrm{Cs} 2 \mathrm{O}$

$53 \mathrm{CsCOOH}$

$96 \mathrm{CsCl}$

$69 \mathrm{CsNO}_{3}$
$8 \mathrm{CsOH}$

151 CsTPB

WSRC-TR-95-0019 (Revision
Aix Sparge 19

Hg Cell 20

21

22

Water for SRAT Hg
to MWWT MWWT Hg Pump Accumulation

23

MWWT Vent to MWWT

\begin{tabular}{|c|c|c|c|}
\hline ZERO & $0.1610 \mathrm{E}-10$ & ZERO & ZERO \\
\hline ZERO & $0.1040 \mathrm{E}-18$ & ZERO & ZERO \\
\hline ZERO & $0.6994 \mathrm{E}-11$ & ZERO & ZERO \\
\hline ZERO & ZERO & ZERO & ZERO \\
\hline ZERO & ZERO & ZERO & ZERO \\
\hline ZERO & $0.421 .5 \mathrm{E}-17$ & ZERO & ZERO \\
\hline ZERO & $0.1120 \mathrm{E}-08$ & ZERO & ZERO \\
\hline ZERO & $0.1128 \mathrm{E}-16$ & ZERO & ZERO \\
\hline ZERO & $0.4965 \mathrm{E}-15$ & ZERO & ZERO \\
\hline ZERO & $0.1874 E-16$ & ZERO & ZERO \\
\hline ZERO & $0.1074 \mathrm{E}-21$ & ZERO & ZERO \\
\hline ZERO & $0.8602 E-08$ & ZERO & ZERO \\
\hline ZERO & $0.3236 \mathrm{E}-10$ & ZERO & ZERO \\
\hline ZERO & $0.2399 \mathrm{E}-17$ & ZERO & ZERO \\
\hline ZERO & $0.2787 \mathrm{E}-18$ & ZERO & ZERO \\
\hline ZERO & $0.1794 \mathrm{E}-14$ & ZERO & ZERO \\
\hline ZERO & TRACE & ZERO & ZERO \\
\hline ZERO & $0.3206 E-12$ & ZERO & ZERO \\
\hline ZERO & $0.5412 \mathrm{E}-17$ & ZERO & ZERO \\
\hline ZERO & $0.1961 \mathrm{E}-17$ & ZERO & ZERO \\
\hline ZERO & ZERO & ZERO & ZERO \\
\hline ZERO & TRACE & ZERO & ZERO \\
\hline ZERO & $0.1044 \mathrm{E}-19$ & ZERO & ZERO \\
\hline ZERO & ZERO & ZERO & ZERO \\
\hline ZERO & $0.2156 \mathrm{E}-13$ & ZERO & ZERO \\
\hline ZERO & $0.2782 \mathrm{E}-21$ & ZERO & ZERO \\
\hline ZERO & ZERO & ZERO & ZERO \\
\hline ZERO & $0.2566 \mathrm{E}-17$ & ZERO & ZERO \\
\hline ZERO & ZERO & ZERO & ZERO \\
\hline ZERO & $0.1608 \mathrm{E}-17$ & ZERO & ZERO \\
\hline ZERO & $0.1492 \mathrm{E}-24$ & ZERO & ZERO \\
\hline ZERO & ZERO & ZERO & ZERO \\
\hline ZERO & ZERO & ZERO & ZERO \\
\hline ZERO & $0.9195 \mathrm{E}-20$ & ZERO & ZERO \\
\hline ZERO & $0.2051 E-20$ & ZERO & ZERO \\
\hline ZERO & $0.1350 \mathrm{E}-18$ & ZERO & ZERO \\
\hline ZERO & ZERO & ZERO & ZERO \\
\hline ZERO & ZERO & 'ZERO & ZERO \\
\hline ZERO & $0.8756 \mathrm{E}-22$ & ZERO & ZERO \\
\hline ZERO & $0.3284 \mathrm{E}-24$ & ZERO & ZERO \\
\hline ZERO & $0.1876 \mathrm{E}-22$ & ZERO & ZERO \\
\hline ZERO & $0.2626 \mathrm{E}-18$ & ZERO & ZERO \\
\hline ZERO & $0.8594 \mathrm{E}-22$ & ZERO & ZERO \\
\hline ZERO & $0.2646 \mathrm{E}-19$ & ZERO & ZERO \\
\hline ZERO & ZERO & ZERO & ZERO \\
\hline ZERO & $0.5886 \mathrm{E}-23$ & ZERO & ZERO \\
\hline ZERO & $0.6713 \mathrm{E}-24$ & ZERO & ZERO \\
\hline ZERO & ZERO & ZERO & ZERO \\
\hline
\end{tabular}

Date: January 1995 fr MWWT

$\begin{array}{lrr}\text { ZERO } & 0.7364 \mathrm{E}-03 & 0.4585 \mathrm{E}-09 \\ \text { ZERO } & 0.3049 \mathrm{E}-06 & 0.5707 \mathrm{E}-12 \\ \text { ZERO } & 0.6787 \mathrm{E}-02 & 0.8077 \mathrm{E}-08 \\ \text { ZERO } & \text { ZERO } & \text { ZERO } \\ \text { ZERO } & \text { ZERO } & \text { ZERO } \\ \text { ZERO } & 0.1969 \mathrm{E}-06 & 0.3165 \mathrm{E}-12 \\ \text { ZERO } & 0.4892 \mathrm{E}-01 & 0.8531 \mathrm{E}-07 \\ \text { ZERO } & 0.7458 \mathrm{E}-06 & 0.1199 \mathrm{E}-11 \\ \text { ZERO } & 0.1214 \mathrm{E}-07 & 0.1305 \mathrm{E}-13 \\ \text { ZERO } & 0.2474 \mathrm{E}-07 & 0.3976 \mathrm{E}-13 \\ \text { ZERO } & 0.2935 \mathrm{E}-20 & 0.1006 \mathrm{E}-14 \\ \text { ZERO } & 0.6394 \mathrm{E}+00 & 0.1038 \mathrm{E}-05 \\ \text { ZERO } & 0.1449 \mathrm{E}-05 & 0.1263 \mathrm{E}-08 \\ \text { ZERO } & 0.5622 \mathrm{E}-10 & 0.8666 \mathrm{E}-16 \\ \text { ZERO } & 0.4976 \mathrm{E}-11 & 0.8255 \mathrm{E}-17 \\ \text { ZERO } & \text { ZERO } & 0.1676 \mathrm{E}-07 \\ \text { ZERO } & \text { ZERO } & 0.1523 \mathrm{E}-21 \\ \text { ZERO } & \text { ZERO } & \text { ZERO } \\ \text { ZERO } & 0.3150 \mathrm{E}-04 & 0.5070 \mathrm{E}-10 \\ \text { ZERO } & 0.5955 \mathrm{E}-08 & 0.1837 \mathrm{E}-10 \\ \text { ZERO } & \text { ZERO } & \text { ZERO } \\ \text { ZERO } & \text { TRACE } & 0.1923 \mathrm{E}-19 \\ \text { ZERO } & 0.6074 \mathrm{E}-07 & 0.9776 \mathrm{E}-13 \\ \text { ZERO } & \text { ZERO } & \text { ZERO } \\ \text { ZERO } & 0.1250 \mathrm{E}-02 & 0.1795 \mathrm{E}-09 \\ \text { ZERO } & 0.1681 \mathrm{E}-10 & 0.2316 \mathrm{E}-17 \\ \text { ZERO } & \text { ZERO } & \text { ZERO } \\ \text { ZERO } & 0.1493 \mathrm{E}-04 & 0.2403 \mathrm{E}-10 \\ \text { ZERO } & \text { ZERO } & \text { ZERO } \\ \text { ZERO } & 0.9362 \mathrm{E}-05 & 0.1506 \mathrm{E}-10 \\ \text { ZERO } & 0.8706 \mathrm{E}-12 & 0.1398 \mathrm{E}-17 \\ \text { ZERO } & \text { ZERO } & \text { ZERO } \\ \text { ZERO } & 0.8 \mathrm{ZERO} & \text { ZERO } \\ \text { ZERO } & 0.5351 \mathrm{E}-07 & 0.8613 \mathrm{E}-13 \\ \text { ZERO } & 0.1192 \mathrm{E}-07 & 0.1922 \mathrm{E}-13 \\ \text { ZERO } & 0.7872 \mathrm{E}-06 & 0.1264 \mathrm{E}-11 \\ \text { ZERO } & \text { ZERO } & \text { ZERO } \\ \text { ZERO } & \text { ZERO } & \text { ZERO } \\ \text { ZERO } & 0.5108 \mathrm{E}-09 & 0.8203 \mathrm{E}-15 \\ \text { ZERO } & 0.1916 \mathrm{E}-11 & 0.3076 \mathrm{E}-17 \\ \text { ZERO } & 0.10 .94 \mathrm{E}-09 & 0.1757 \mathrm{E}-15 \\ \text { ZERO } & 0.1529 \mathrm{E}-05 & 0.2460 \mathrm{E}-11 \\ \text { ZERO } & 0.5001 \mathrm{E}-09 & 0.8051 \mathrm{E}-15 \\ \text { ZERO } & 0.1544 \mathrm{E}-06 & 0.2479 \mathrm{E}-12 \\ \text { ZERO } & \text { ZERO } & \text { ZERO } \\ \text { ZERO } & 0.3425 \mathrm{E}-10 & 0.5514 \mathrm{E}-16 \\ \text { ZERO } & \text { ZERO } & 0.6288 \mathrm{E}-17 \\ \text { ZERO } & \text { ZERO } & \text { ZERO } \\ & & \end{array}$

Section 4A, Page 14 of 21

SMECT Hg

to MWWT

RO

$-12$

$-13$

14

08

07

$-21$

$-10$

$-19$

ZERO

17

10

$-17$

$-13$

$-11$

ZERO

ZERO

ZERO

ZERO 
Table A-1. HLW Flowsheet Material Balance (Tank 51/ITP Cycle 1) Section 4A. Sludge Receipt; Blending and Adjustment

STREAM NUMBERS (CONT'D) $\rightarrow$ STREAM NAME

$54 \mathrm{Cu}(\mathrm{COOH}) 2$

$\begin{array}{rl}170 & \mathrm{Cu} \\ 55 \mathrm{CuO}\end{array}$

$80 \mathrm{Fe} 2 \mathrm{O3}$

$101 \mathrm{FeO}$

102 Group A

103 Grou

$\begin{array}{rl}25 & \mathrm{H} 2 \\ 158 & \mathrm{H} 2 \mathrm{C} 2 \mathrm{O} 4\end{array}$

$93 \mathrm{H} 2 \mathrm{SO} 4$

$90 \mathrm{H} 3 \mathrm{BO} 3$

34. $\mathrm{HCOOH}$

$43 \mathrm{HCl}$

49 HNO3

$36 \mathrm{Hg}$

$129 \mathrm{Hg}$ (C6H5) 2

$135 \mathrm{Hg}$ (NO3) 2

$144 \mathrm{Hg} 2 \mathrm{Cl} 2$

$117 \mathrm{Hg} 2 \mathrm{I} 2$

$146 \mathrm{HgCl} 2$

$21 \mathrm{HgO}$

$97 \mathrm{I} 2$
$183 \mathrm{KO} 3$

$39 \mathrm{~K} 20$

$51 \mathrm{KCOOH}$

$157 \mathrm{KMnO} 4$

161 KNO3

$10 \mathrm{KOH}$

150 KTPB

$105 \mathrm{La2O3}$

$108 \mathrm{Li} 20$

$65 \mathrm{Mg}$ (COOH) 2

$171 \mathrm{Mg}(\mathrm{NO}$

$33 \mathrm{MgO}$
$60 \mathrm{Mn}(\mathrm{COOH}) 2$

$172 \mathrm{Mn}$ (NO3) 2

$180 \mathrm{Mn} 304$

$56 \mathrm{Mno}$

$14 \mathrm{MnO} 2$

$145 \mathrm{MOO} 2$

81 N2

74 N2O

$182 \mathrm{NH} 3$

114 NH3OKNO 3

52 NH $4 \mathrm{COOH}$
$173 \mathrm{NH} 4 \mathrm{NO} 3$

68 NH4OH

148 NH4TPB

WSRC-TR-95-0019 (Revision
18

Air sparge

to MWWT vent to MWWT

19.

20

ZERO $\quad 0.2714 \mathrm{E}-20$

ZERO $0.1333 \mathrm{E}-17$

ZERO $0.1207 \mathrm{E}-19$

ZERO $0.1555 \mathrm{E}-16$

ZERO

ZERO $0.2171 \mathrm{E}-20$

ZERO $\quad 0.9261 \mathrm{E}-22$

ZERO $0.1428 \mathrm{E}-19$

ZERO

ZERO

ZERO

ZERO

ZERO

ZERO

ZERO

ZERO

ZERO

2ERO

ZERO

ZERO

ZERO

ZERQ

ZERO

ZERO

ZERO

ZERO

ZERO

ZERO

ZERO

ZERO

ZERO

ZERO

ZERO

ZERO

ZERO

ZERO

$0.1134 \mathrm{E}-01$

ZERO

ZERO

ZERO

ZERO

ZERO

$0.1119 \mathrm{E}-18$
ZERO

$0.4710 \mathrm{E}-17$

ZERO

ZERO

$0.5648 \mathrm{E}-02$

$0.4599 \mathrm{E}-04$

$0.8246 \mathrm{E}-1$

ZERO

ZERO
ZERO

$0.1207 \mathrm{E}-23$

ZERO

$0.4946 \mathrm{E}-19$

$0.6139 \mathrm{E}-17$

ZERO
E-19

$2008 \mathrm{E}-2$

$8 E-22$
ZERO
ZERO

$0.1716 \mathrm{E}-17$

$0.2122 \mathrm{E}-17$

TRACE

$0.6769 \mathrm{E}-18$

$0.1329 \mathrm{E}-17$

ZERO

$0.4862 \mathrm{E}-23$

$0.1193 \mathrm{E}-17$

$0.6586 \mathrm{E}-21$

TRACE

$0.8761 \mathrm{E}-12$

ZERO

$0.1647 \mathrm{E}-12$

$0.1898 \mathrm{E}-19$

ZERO

Date: January 1995
21

23

er Wash Water for SRAT Hg

MWWT MWWT Hg Pump Accumulation

ZERO ZERO ZERO

ZERO $\because \quad$ ZERO $\quad$ ZERO

ZERO , ZERO $\quad$ ZERO

ZERO

ZERO ZERO

ZERO ZERO

ZERO ZERO

ZERO ZERO

ZERO ZERO

ZERO ZERO

ZERO

ZERO

ZERO

ZERO

ZERO

ZERO

ZERO

ZERO

ZERO

ZERO

ZERO

ZERO

ZERO

ZERO

ZERO

ZERO

ZERO

ZERO

ZERO

ZERO

ZERO

ZERO

ZERO

ZERO

ZERO

ZERO

ZERO

ZERO

ZERO

ZERO

ZERO

ZERO

ZERO

ZERO

ZERO

ZERO

ZERO

ZERO

ZERO

ZERO

ZERO

ZERO

ZERO

ZERO

ZERO

ZERO

ZERO

ZERO

ZERO

ZERO

ZERO

ZERO

ZERO

ZERO

ZERO

ZERO

ZERO

ZERO

ZERO

ZERO

ZERO

ZERO

ZERO

ZERO

ZERO

ZERO

ZERO

ZERO

ZERO

ZTRO

ZERO

ZERO

ZERO

ZERO

ZERO

ZERO

ZERO

ZERO

ZERO

ZERO

ZERO

ZERO

ZERO

ZERO

ZERO

ZERO

ZERO

ZERO

ZERO

ZERO

ZERO

ZERO

ZERO

ZERO

ZERO

ZERO

ZERO

ZERO

ZERO

ZERO

ZERO

ZERO

ZERO

ZERO

ZERO

ZERO

ZERO

ZERO

ZERO

ZERO

ZERO

ZERO

ZERO

d fr MWW

$0.1579 \mathrm{E}-07$

$0.7768 \mathrm{E}-05$

$0.7021 \mathrm{E}-07$

$0.9050 \mathrm{E}-04$

$0.9048 \mathrm{E}-05$

$.1263 \mathrm{E}-07$

$0.5389 \mathrm{E}-09$

$0.6514 \mathrm{E}-06$

ZERO

$0.2748 \mathrm{E}-04$ ZERO ZERO

$0.8070 \mathrm{E}-09$

ZERO

ZERO

ZERO

ZERO

ZERO

ZERO

ZERO

ZERO

$0.2878 \mathrm{E}-06$

$0.3581 \mathrm{E}-04$ ZERO

$0.1019 \mathrm{E}-06$

ZERO

ZERO

$0.1051 \mathrm{E}-08$

$0.1235 \mathrm{E}-04$

- $18735-12$

$0.10875-05$

$0.7734 \mathrm{E}-05$

$0.2183 \mathrm{E}-12$ 
Table A-1. HLW Flowsheet Material Balance (Tank 51/ITP Cycle 1) Section 4A. Sludge Receipt, Blending and Adjustment

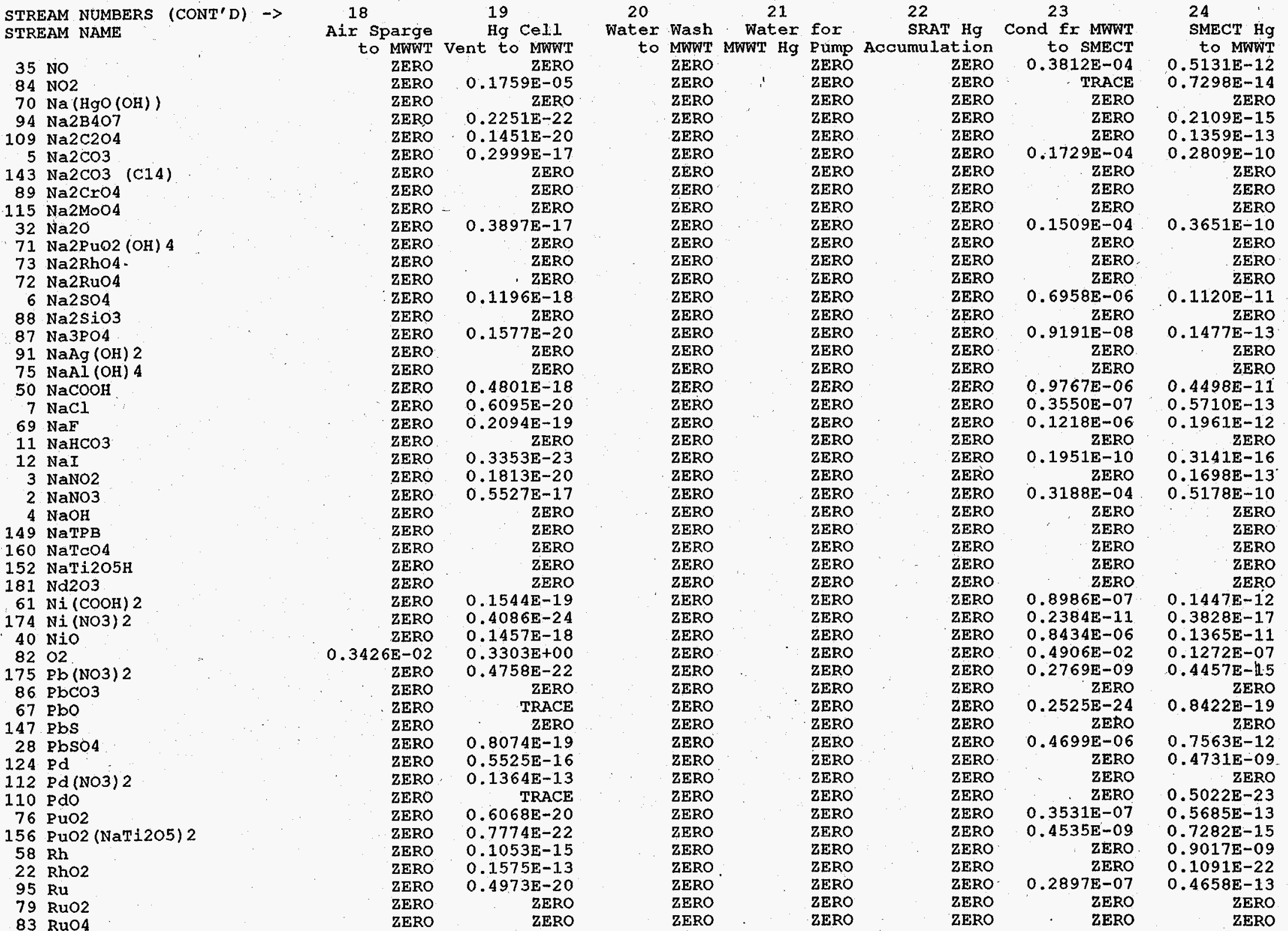


Table A-1. HLW Flowsheet Material Balance (Tank 51/ITP Cycle 1)

Section 4A. Sludge Receipt, Blending and Adjustment

STREAM NUMBERS (CONT'D) $\rightarrow$ STREAM NAME

\footnotetext{
$38 \mathrm{SO} 2$

141 SO3

137 Semi Vol Cs2O

136 Semi Vol CsCl

126 Semi Vol Group A

140 Semi Vol Na2B4O7

138 Semi Vol NaCl

139 Semi Vol NaF

125 Semi Vol NaI

23 Semi vol RuO2

$111 \mathrm{SiO} 2$

$48 \mathrm{Sr}(\mathrm{COOH}) 2$

153 Sr(NaTi2O5) 2

$127 \mathrm{Sr}(\mathrm{OH}) 2$

30 srCO

46 sro

$159 \cdot \mathrm{TcO} 2$

92. ThO2

107 TiO2

$\begin{array}{rl}119 & \text { Tritium } \\ 17 & \mathrm{U} 308\end{array}$

$62 \mathrm{UO} 2$ ( $\mathrm{COOH}) 2$

$177 \mathrm{UO} 2$ (NO3) 2

$16 \mathrm{OO} 2(\mathrm{OH}) 2$

$27 \mathrm{Y}(\mathrm{COOH}) 3$

178 Y (NO3) 3

$128 \mathrm{Y}(\mathrm{OH}) 3$

$31 \mathrm{Y} 2(\mathrm{CO} 3) 3$

$47 \mathrm{Y} 203$

100 zeolite

44. $\mathrm{Zn}(\mathrm{COOH}) 2$

$179 \ln (\mathrm{NO} 3)$

$\begin{array}{rl}57 & \mathrm{ZnO} \\ 104 & \mathrm{ZrO} 2\end{array}$

13 hydrate $\mathrm{H} 2 \mathrm{O}$

1 watex

TOTAL FLOW, LB/HR

TEMPERATURE， DEG C

PRESSURE， ATM

PRESSURE， PSIG

PRESSURE,

PRESSURE, MM HG

ENTHALPY, PCU/KR

VAPOR FLOW, CEM

LIQUID FLOW, GPM

PHASE
}

\begin{tabular}{|c|c|}
\hline${ }^{18}$ Sparge & 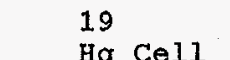 \\
\hline II to MWWT & Vent to MWWT \\
\hline ZERO & ZERO \\
\hline ZERO & ZERO \\
\hline ZERO & ZERO \\
\hline ZERO & ZERO \\
\hline ZERO & ZERO \\
\hline ZERO & ZERO \\
\hline ZERO & ZERO \\
\hline ZERO & ZERO \\
\hline ZERO & ZERO \\
\hline ZERO & ZERO \\
\hline ZERO & $0.1954 \mathrm{E}-16$ \\
\hline ZERO & $0.6678 E-22$ \\
\hline ZERO & $0.1803 E-19$ \\
\hline ZERO & $0.2489 \mathrm{E}-20$ \\
\hline ZERO & ZERO \\
\hline ZERO & ZERO \\
\hline ZERO & TRACE \\
\hline ZERO & $0.2187 \mathrm{E}-20$ \\
\hline ZERO & $0.1821 \mathrm{E}-19$ \\
\hline ZERO & $0.5079 \mathrm{E}-18$ \\
\hline ZERO & $0.2125 \mathrm{E}-16$ \\
\hline ZERO & $0.1277 \mathrm{E}-17$ \\
\hline ZERO & $0.7774 E-24$ \\
\hline ZERO & $0.5598 \mathrm{E}-22$ \\
\hline ZERO & ZERO \\
\hline ZERO & TRACE \\
\hline ZERO & $0.4865 \mathrm{E}-20$ \\
\hline ZERO & ZERO \\
\hline ZERO & ZERO \\
\hline ZERO & $0.5917 \mathrm{E}-21$ \\
\hline ZERO & ZERO \\
\hline ZERO & $0.1595 \mathrm{E}-18$ \\
\hline ZERO & $0.3261 \mathrm{E}-22$ \\
\hline ZERO & $0.1097 \mathrm{E}-18$ \\
\hline ZERO & ZERO \\
\hline ZERO & $0.9185 \mathrm{E}-18$ \\
\hline $0.2481 \mathrm{E}-03$ & $0.4899 \mathrm{E}-01$ \\
\hline $0.1502 \mathrm{E}-01$ & $0.1478 \mathrm{E}+01$ \\
\hline $0.3500 \mathrm{E}+02$ & $0.3800 \mathrm{E}+02$ \\
\hline $0.1000 \mathrm{E}+01$ & $0.9950 E+00$ \\
\hline ZERO & $-0.7348 E-01$ \\
\hline & $0.7562 \mathrm{E}+03$ \\
\hline $0.3150 \mathrm{E}+00$ & $0.4745 \mathrm{E}+02$ \\
\hline $0.3547 \mathrm{E}-02$ & $0.3572 \mathrm{E}+00$ \\
\hline $0.7055 \mathrm{E}-01$ & $0.6899 \mathrm{E}-01$ \\
\hline VAPOR & VAPOR \\
\hline
\end{tabular}

20

21

23

to MWWT MWWT Hg Pump Accumulation to SMECT

$\begin{array}{lrr}\text { ZERO } & \text { ZERO } & \text { ZERO }\end{array}$

ZERO ZERO ZERO

ZERO ZERO $\quad$ ZERO

ZERO

ZERO

ZERO

ZERO ZERO

ZERO ZERO

ZERO

ZERO ZERO

ZERO ZERO

ZERO ZERO

ZERO ZERO

ZERO ZERO

ZERO ZERO

ZERO ZERO

ZERO

ZERO

ZERO ZERO

ZERO ZERO

ZERO ZERO

ZERO ZERO

ZERO ZERO

ZERO ZERO

ZERO ZERO

ZERO ZERO

ZERO ZERO

ZERO ZERO

ZERO ZERO

ZERO ZERO

ZERO ZERO

ZERO ZERO

ZERO ZERO

$\begin{array}{ll}\text { ZERO } & \text { ZERO } \\ \text { ZERO } & \text { ZERO }\end{array}$

$.5551 \mathrm{E}-01$

$0.5551 \mathrm{E}-01$

ZERO

$0.2041 \mathrm{E}-02$

ZERO

ZERO
ZERO

ZERO

ZERO

ZERO

ZERO

ZERO

ZERO

ZERO

ZERO

ZERO

ZERO

ZERO

ZERO

ZERO

ZERO

ZERO

ZERO

ZERO .

ZERO

ZERO

ZERO

ZERO

ZERO

ZERO

ZERO

ZERO

ZERO

ZERO

ZERO

ZERO

ZERO

. 3892E-05

$0.3896 \mathrm{E}-09$

$0.1049 \mathrm{E}-06$

$.1452 \mathrm{E}-07$

ZERO

$0.7906 \mathrm{E}-24$

$0.1273 \mathrm{E}-07$

$0.1060 \mathrm{E}-06$

$0.2962 \mathrm{E}-05$

$0.6322 \mathrm{E}-09$

$.7430 \mathrm{E}-05$

$0.4535 \mathrm{E}-11$

$0.3257 E-09$

ZERO
$.4697 \mathrm{E}-17$

$0.2831 \mathrm{E}-07$

E-07
ZERO

$0.3452 \mathrm{E}-08$ 政

$0.9296 \mathrm{E}-06$

$0.6395 \mathrm{E}-06$ ZERO

$0.5345 \mathrm{E}-05$

$0.7140 \mathrm{E}+03$

$0.7150 \mathrm{E}+03$

24

SMECT Hg

to MWWT

ZERO

ZERO

ZERO

ZERO

ZERO

ZERO

ZERO

ZERO

ZERO

$0.1830 \mathrm{E}-09$

$0.6256 \mathrm{E}-15$

$0.1689 \mathrm{E}-12$

$0.2331 E-13$

ZERO

$0.2637 \mathrm{E}-18$

$0.1706 \mathrm{E}-12$

$0.4758 \mathrm{E}-11$

$0.1012 \mathrm{E}-14$

$0.1196 \mathrm{E}-10$

$0.7283 \mathrm{E}-17$

$0.5244 E-15$

$0.7543 E-23$

$0.4557 \mathrm{E}-13$

ZERO

$0.5543 \mathrm{E}-1.4$

ZERO

$0.1494 \mathrm{E}-11$

$0.3055 \mathrm{E}-1.5$

$0.1028 \mathrm{E}-11$

$0.8604 \mathrm{E}-11$

$0.3886 \mathrm{E}-02$

$0.2554 \mathrm{E}+00$

$\begin{array}{lllll}0.2500 \mathrm{E}+02 & 0.3500 \mathrm{E}+02 & 0.5000 \mathrm{E}+02 & 0.5000 \mathrm{E}+02 & 0.4666 \mathrm{E}+02\end{array}$

ZERO

ZERO

ZERO

$0.9656 \mathrm{E}+00$

$-0.5055 E+00$

$0.1000 \mathrm{E}+01$

$0.1393 \mathrm{E}+01 \quad 0.7162 \mathrm{E}-01$

ZERO

$0.7339 \mathrm{E}+03$

$0.1109 \mathrm{E}-03 \quad 0.407 .7 \mathrm{E}-05$

$.6243 \mathrm{E}+02 \quad 0.6243 \mathrm{E}+02$

$\begin{array}{rr}\text { LIQUID } & \text { LIQUID }\end{array}$

ZERO

$0.1428 \mathrm{E}+01$

$0.6244 \mathrm{E}+02$

IIQUID

$0.4485 \mathrm{E}-04$

$0.7100 \mathrm{E}+03$

LIQUID 
Table A-1. HLW Flowsheet Material Balance (Tank 51/ITP Cycle 1) Section 4A. Sludge Receipt, Blending and Adjustment

STREAM NUMBERS $\rightarrow>$ STREAM NAME

COMPONENT FLOWS, IB/HR 122 (C6H5) 2

123 (C6H5) 2C6H4

118 (C6H5) 2NH

133 (C6H5B (OH) 2 salt)

132 (С. $6 \mathrm{H} 5 \mathrm{~B}(\mathrm{OH}) 2$ )

166 (C6H5C6H4OH)

162 (C6H5NH2)

163 (C6H5NHCHO)

165 (C6H5NO)

164 (C6H5NO2)

131 (C6H5OH salt)
130 ( $6 \mathrm{H} 5 \mathrm{OH})$

130 (C6H5OH)
121 (C6H6)

155 (CH3) $2 \mathrm{CHOH}$

154 (CH3OH)

$45 \mathrm{Ag}$

$116 \mathrm{Ag} 20$

134 AgNO3

$77 \mathrm{Al} 203$

$106 \mathrm{B2O3}$

$9 \mathrm{Ba} \mathrm{(OH)} 2$

$15 \mathrm{BaO}$

$24 \mathrm{BaSO} 4$

$66 \mathrm{CO}$

$142 \mathrm{CO} 2$ (C14)

$63 \mathrm{Ca}(\mathrm{COOH}) 2$

$167 \mathrm{Ca}$ (NO3) 2

$120 \mathrm{Ca}(\mathrm{OH}) 2$

$113 \mathrm{Ca} 3$ (PO4) 2

$20 \mathrm{CaC} 2 \mathrm{O} 4$

$18 \mathrm{CaCO} 3$

$64 \mathrm{CaCO} 3$ (C14)

78 CaF2

$41 \mathrm{CaO}$

19 Caso4.

99 Cement

$26 \mathrm{Co}(\mathrm{COOH}) 2$

$168 \mathrm{Co}$ (NO3) 2

59. $\mathrm{COO}$

$98 \mathrm{Cr} 2 \mathrm{O3}$

$53 \mathrm{CsCOOH}$

$96 \mathrm{CsCl}$

$169 \mathrm{CsNO3}$

$8 \mathrm{CsOH}$

151 CsTPB

WSRC-TR-95-0019 (Revision $\begin{array}{lll}25 & 50 & 51 \\ \text { frm MWWT PRBT } & \text { Trnsfer PRBT Sampler }\end{array}$

$\begin{array}{lll}25 & 50 & 51 \\ \text { frm MWWT PRBT } & \text { Trnsfer PRBT Sampler }\end{array}$

frm MWWT PRBT Trims
to MAWT Pump Prime Pump Prime

\begin{tabular}{|c|c|c|}
\hline $\begin{array}{l}0.1881 \mathrm{E}-10 \\
0.2433 \mathrm{E}-13 \\
0.3441 \mathrm{E}-09\end{array}$ & $\begin{array}{l}\text { ZERO } \\
\text { ZERO } \\
\text { ZERO }\end{array}$ & $\begin{array}{l}\text { ZERO } \\
\text { ZERO } \\
\text { ZERO }\end{array}$ \\
\hline ZERO & ZERO & ZERO \\
\hline ZERO & ZERO & ZERO \\
\hline $0.1349 \mathrm{E}-13$ & ZERO & ZERO \\
\hline $0.3574 E-08$ & ZERO & ZERO \\
\hline $0.5113 E-13$ & ZERO & ZERO \\
\hline $0.5361 E-15$ & ZERO & ZERO \\
\hline $0.1694 \mathrm{E}-14$ & ZERO & ZERO \\
\hline $0.4289 \mathrm{E}-16$ & ZERO & ZERO \\
\hline $0.4383 \mathrm{E}-07$ & ZERO & ZERO \\
\hline $0.3238 \mathrm{E}-10$ & ZERO & ZERO \\
\hline $0.3491 \mathrm{E}-17$ & ZERO & ZERO \\
\hline $0.3182 \mathrm{E}-18$ & ZERO & ZERO \\
\hline $0.8251 \mathrm{E}-07$ & ZERO & ZERO \\
\hline $0.6495 \mathrm{E}-23$ & ZERO & ZERO \\
\hline ZERO & ZERO & ZERO \\
\hline $0.2162 \mathrm{E}-11$ & ZERO & ZERO \\
\hline $0.7834 \mathrm{E}-12$ & ZERO & ZERO \\
\hline ZERO & ZERO & ZERO \\
\hline $0.8199 \mathrm{E}-21$ & ZERO & ZERO \\
\hline $0.4168 \mathrm{E}-14$ & ZERO & ZERO \\
\hline ZERO & ZERO & ZERO \\
\hline $0.2156 \mathrm{E}-13$ & ZERO & ZERO \\
\hline $0.2782 \mathrm{E}-21$ & ZERO & ZERO \\
\hline ZERO & ZERO & ZERO \\
\hline $0.1025 \mathrm{E}-11$ & ZERO & ZERO \\
\hline ZERO & ZERO & ZERO \\
\hline $0.6422 \mathrm{E}-12$ & ZERO & ZERO \\
\hline $0.5961 \mathrm{E}-19$ & ZERO & ZERO \\
\hline ZERO & ZERO & ZERO \\
\hline ZERO & ZERO & ZERO \\
\hline $0.3672 \mathrm{E}-14$ & ZERO & ZERO \\
\hline $0.8192 \mathrm{E}-15$ & ZERO & ZERO \\
\hline $0.5391 \mathrm{E}-13$ & ZERO & ZERO \\
\hline ZERO & ZERO & ZERO \\
\hline ZERO & ZERO & ZERO \\
\hline $0.3497 \mathrm{E}-16$ & ZERO & ZERO \\
\hline $0.1312 \mathrm{E}-18$ & ZERO & ZERO \\
\hline $0.7491 \mathrm{E}-17$ & ZERO & ZERO \\
\hline $0.1049 \mathrm{E}-12$ & ZERO & ZERO \\
\hline $0.3432 \mathrm{E}-16$ & ZERO & ZERO \\
\hline $0.1057 \mathrm{E}-13$ & ZERO & ZERO \\
\hline ZERO & ZERO & ZERO \\
\hline $0.2351 \mathrm{E}-17$ & ZERO & ZERO \\
\hline $0.2681 \mathrm{E}-1.8$ & ZERO & ZERO \\
\hline ZERO & ZERO & ZERO \\
\hline
\end{tabular}

Date: January 1995 
Table A-1. HLW Flowsheet Material Balance (Tank 51/ITP Cycle 1)

Section $4 \mathrm{~A}$. Sludge Receipt, Blending and Adjustment

STREAM NUMBERS (CONT'D) $\rightarrow$ STREAM NAME

\begin{tabular}{|c|c|}
\hline \multirow{2}{*}{\multicolumn{2}{|c|}{ (1) }} \\
\hline & \\
\hline 55 & $\mathrm{CuO}$ \\
\hline 80 & $\mathrm{Fe} 203$ \\
\hline 101 & Feo \\
\hline 102 & Group A \\
\hline 103 & Group B \\
\hline 25 & H2 \\
\hline 158 & $\mathrm{H} 2 \mathrm{C} 2 \mathrm{O} 4$ \\
\hline 93 & H2 SO4 \\
\hline 90 & H3BO3. \\
\hline $\begin{array}{l}34 \\
43\end{array}$ & $\begin{array}{l}\mathrm{HCOOH} \\
\mathrm{HCl}\end{array}$ \\
\hline 85 & $\mathrm{HF}$ \\
\hline 49 & HNO3 \\
\hline 36 & $\mathrm{Hg}$ \\
\hline 129 & $\mathrm{Hg}(\mathrm{C} 6 \mathrm{H} 5)^{2}$ \\
\hline 135 & $\mathrm{Hg}(\mathrm{NO} 3) 2$ \\
\hline 144 & $\mathrm{Hg} 2 \mathrm{Cl} 2$ \\
\hline 117 & $\mathrm{Hg} 2 \mathrm{I} 2$ \\
\hline 146. & $\mathrm{HgCl} 2$ \\
\hline 21 & $\mathrm{HgO}$ \\
\hline 97 & $I \overline{2}$ \\
\hline 183 & $\mathrm{~K} 2 \mathrm{CO} 3$ \\
\hline 39 & $\mathrm{~K} 20$ \\
\hline 51 & $\mathrm{KCOOH}$ \\
\hline 157 & $\mathrm{KMnO} 4$ \\
\hline 161 & KNO3 \\
\hline 10 & KOH \\
\hline 150 & KTPB \\
\hline 105 & $\mathrm{La2O3}$ \\
\hline 108 & Li20 \\
\hline 65 & $\mathrm{Mg}(\mathrm{COOH}) 2$ \\
\hline 171 & $\operatorname{Mg}(\mathrm{NO} 3) 2$ \\
\hline 33 & Mgo \\
\hline 60 & $\mathrm{Mn}(\mathrm{COOH}) 2$ \\
\hline 172 & $\mathrm{Mn}(\mathrm{NO} 3) 2$ \\
\hline 180 & $\mathrm{Mn} 304$ \\
\hline 56 & Mno \\
\hline 14 & $\mathrm{MnO} 2$ \\
\hline 145 & MoO2 \\
\hline 81 & N2 \\
\hline 74 & $\mathrm{~N} 2 \mathrm{O}$ \\
\hline 182 & NH3 \\
\hline $\begin{array}{r}114 \\
52\end{array}$ & NH3OHNO3 \\
\hline $\begin{array}{l}52 \\
173\end{array}$ & $\begin{array}{l}\mathrm{NH} 4 \mathrm{COOH} \\
\mathrm{NH} 4 \mathrm{NO} 3\end{array}$ \\
\hline 68 & $\mathrm{NH} 4 \mathrm{OH}$ \\
\hline & NH4 TPB \\
\hline
\end{tabular}

frm MWWT PRBT Trnsfer PRBT Sampler

to MAWT Pump Prime Pump Prime

$0.1084 \mathrm{E}-14$

$0.5325 \mathrm{E}-12$

$\begin{array}{ll}0.4619 \mathrm{E}-14 & \text { ZERO } \\ 0.6211 \mathrm{E}-11 & \text { ZERO }\end{array}$

$0.6210 \mathrm{E}-12$ ZERO $\quad$ ZERO

$\begin{array}{ll}0.6210 \mathrm{E}-12 & \text { ZERO } \\ 0.8669 \mathrm{E}-15 & \text { ZERO }\end{array}$

$0.3699 \mathrm{E}-16 \quad$ ZERO ZERO

$0.1428 \mathrm{E}-19 \quad$ ZERO ZERO

$0.4470 \mathrm{E}-13 \quad$ ZERO ZERO

$\begin{array}{rrr}\text { ZERO } & \text { ZERO } & \text { ZERO } \\ 0.1881 \mathrm{E}-11 & \text { ZERO } & \text { ZERO }\end{array}$

ZERO ZERO ZERO

ZERO ZERO ZERO

ZERO
ZERO
ZERO

$0.2491 \mathrm{E}-09$

$0.2515 E+00$ ZERO ZERO

ZERO ZERO

$\begin{array}{lll}\text { ZERO } & \text { ZERO } & \text { ZERO } \\ \text { ZERO } & \text { ZERO } & \text { ZERO }\end{array}$

$\begin{array}{lll}\text { ZERO } & \text { ZERO } & \text { ZERO } \\ \text { ZERO } & \text { ZERO } & \text { ZERO }\end{array}$

ZERO $\quad$ ZERO

$\begin{array}{rrr}\text { ZERO } & \text { ZERO } & \text { ZERO } \\ 0.4820 \mathrm{E}-18 & \text { ZERO } & \text { ZERO }\end{array}$

ZERO ZERO ZERO

ZERO
ZERO

ZERO ZERO

ZERO
ZERO ZERO

$0.8018 \mathrm{~B}-17$ ZERO $\quad$ ZERO

ZERO ZERO

ZERO ZERO $\quad$ ZERO

$\begin{array}{lll} & \text { ZERO } & \text { ZERO } \\ 0.6851 \mathrm{E}-12 & \text { ZERO } & \end{array}$

$0.8474 \mathrm{E}-12$ ZERO ZERO

.1282E-19 ZERO $\cdots$ ZERO

$0.2704 \mathrm{E}-12$ ZERO ZERO

$0.5308 \mathrm{E}-12$ ZERO . ZERO

$0.1494 \mathrm{E}-19$ ZERO $\quad$ ZERO

ZERO
$0.1942 \mathrm{E}-17$

$0.4767 \mathrm{E}-12$ ZERO ZERO

ZERO ZERO

$0.3297 \mathrm{E}-07$ ZERO

ZERO ZERO

$\begin{array}{lll}0.8763 \mathrm{E}-12 & \text { ZERE } & \text { ZERO } \\ 0.2 \mathrm{ZERO} & \text { ZERO }\end{array}$

ZERO ZERO

\begin{tabular}{rr} 
ZERO & ZERO \\
ZERO & ZERO \\
$0.6577 \mathrm{E}-07$ & ZERO \\
\hline
\end{tabular}

$\begin{array}{lll}0.7579 \mathrm{E}-14 & \text { ZERO } & \text { ZERO }\end{array}$

ZERO ZERO

WSRC-TR-95-0019 (Revision 0)

Date: January 1995 
Table A-1. HLW Flowsheet Material Balance (Tank 51/ITP Cycle 1) Section 4A. Sludge Receipt, Blending and Adjustment

STREAM NUMBERS (CONT'D) $\rightarrow$ STREAM NAME

\begin{aligned} & 35 NO \\ & 84 NO2 \\ & 70 Na(HgO(OH)) \\ & 94 Na2B4O7 \\ & 109 Na2C2O4 \\ & 5 Na2CO3 \\ & 143 Na2CO3 (C14) \\ & 89 Na2CrO4 \\ & 115 Na2MOO4 \\ & 32 Na2O \\ & 71 Na2PuO2 (OH) 4 \\ & 73 Na2RhO4 \\ & 72 Na2RuO4 \\ & 6 Na2SO4 \\ & 88 Na2SiO3 \\ & 87 Na3PO4 \\ & 91 NaAg (OH) 2 \\ & 75 NaA1 (OH) 4 \\ & 50 NaCOOH \\ & 7 NaC1 \\ & 69 NaF \\ & 11 NaHCO3 \\ & 12 NaI \\ & 3 NaNO2 \\ & 2 NaNO3 \\ & 4 NaOH \\ & 149 NaTPB \\ & 160 NaTCO4 \\ & 152 NaTi2O5H \\ & 181 Nd2O3 \\ & 61 Ni (COOH) 2 \\ & 174 Ni (NO3) 2 \\ & 40 NiO \\ & 82 O2 \\ & 175 Pb (NO3) 2 \\ & 86 PbCO3 \\ & 67 PbO \\ & 147 PbS \\ & 28 PbSO4 \\ & 124 Pd \\ & 112 Pd (NO3) 2 \\ & 110 PdO \\ & 76 PuO2 \\ & 156 PuO2 (NaTi2O5) 2 \\ & 58 Rh \\ & 22 RhO2 \\ & 95 Ru \\ & 79 RuO2 \\ & 83 RuO4 \\ & \\ & \hline\end{aligned}

83 RuO4
25 50

51

Hg frm MWWT PRBT Trnsfer PRBT Sampler

\begin{tabular}{|c|c|c|c|c|}
\hline $\begin{array}{r}\text { to MAWT } \\
0.5671 \mathrm{E}-17\end{array}$ & Pump & $\begin{array}{l}\text { Prime } \\
\text { ZERO }\end{array}$ & Pump & $\begin{array}{l}\text { Prime } \\
\text { zERORO }\end{array}$ \\
\hline TRACE & & ZERO & & ZERO \\
\hline ZERO & & ZERO & & ZERO \\
\hline $0.8990 \mathrm{E}-17$ & & ZERO & & ZERO \\
\hline $0.5796 \mathrm{E}-15$ & & ZERO & & ZERO \\
\hline $.1198 \mathrm{E}-11$ & & ZERO & & ZERO \\
\hline ZERO & & ŻERO & & ZERO \\
\hline ZERO & & ZERO & & ZERO \\
\hline ZERO & & ZERO & & ZERO \\
\hline $0.1557 \mathrm{E}-11$ & & ZERO & & ZERO \\
\hline ZERO & & ZERO & & ZERO \\
\hline ZERO & & ZERO & & ZERO \\
\hline ZERO & & ZERO & & ZERO \\
\hline $0.4776 \mathrm{E}-13$ & & ZERO & & ZERO \\
\hline ZERO & & ZERO & & ZERO \\
\hline $0.6299 \mathrm{E}-15$ & & ZERO & & ZERO \\
\hline ZERO & , & ZERO & & ZERO \\
\hline ZERO & & ZERO & & ZERO \\
\hline $0.1918 E-12$ & & ZERO & & ZERO \\
\hline $0.2434 \mathrm{E}-14$ & & ZERO & & ZERO \\
\hline $0.8361 \mathrm{E}-14$ & & ZERO & & ZERO \\
\hline ZERO & & ZERO & & ZERO \\
\hline $0.1339 E-17$ & & ZERO & & ZERO \\
\hline $0.7240 \mathrm{E}-15$ & & ZERO & & ZERO \\
\hline $0.2207 E-11$ & & ZERO & & ZERO \\
\hline ZERO & & ZERO & & ZERO \\
\hline ZERO & & ZERO & & ZERO \\
\hline ZERO & & ZERO & & ZERO \\
\hline ZERO & & ZERO & & ZERO \\
\hline ZERO & & ZERO & & ZERO \\
\hline $0.6167 \mathrm{E}-14$ & & ZERO & & ZERO \\
\hline $0.1632 \mathrm{E}-18$ & & ZERO & & ZERO \\
\hline $0.5819 \mathrm{E}-13$ & & ZERO & & ZERO \\
\hline $0.1902 E-07$ & & ZERO & & ZERO \\
\hline $0.1900 \mathrm{E}-16$ & & ZERO & & ZERO \\
\hline ZERO & & ZERO & & ZERO \\
\hline $0.3591 \mathrm{E}-20$ & & ZERO & & ZERO \\
\hline ZERO & & ZERO & & ZERO \\
\hline $0.3225 \mathrm{E}-13$ & & ZERO & & ZERO \\
\hline $0.2540 \mathrm{E}-08$ & & ZERO & & ZERO \\
\hline ZERO & & ZERO & & ZERO \\
\hline $0.2141 \mathrm{E}-24$ & & ZERO & & ZERO \\
\hline $0.2424 \mathrm{E}-14$ & & ZERO & & ZERO \\
\hline $0.3105 \mathrm{E}-16$ & & ZERO & & ZERO \\
\hline $0.4841 \mathrm{E}-08$ & & ZERO & & ZERO \\
\hline TRACE & & ZERO & & ZERO \\
\hline $0.1986 \mathrm{E}-14$ & & ZERO & & ZERO \\
\hline ZERO & & ZERO. & & ZERO \\
\hline ZERO & & ZERO & & ZERO \\
\hline
\end{tabular}

Date: January 1995 
Table A-1. HLW Elowsheet Material Balance (Tank 51/ITP Cycle 1)

Section 4A. 'sludge Receipt, Blending and Adjustment

STREAM NUMBERS (CONT'D) $\rightarrow$ STREAM NAME

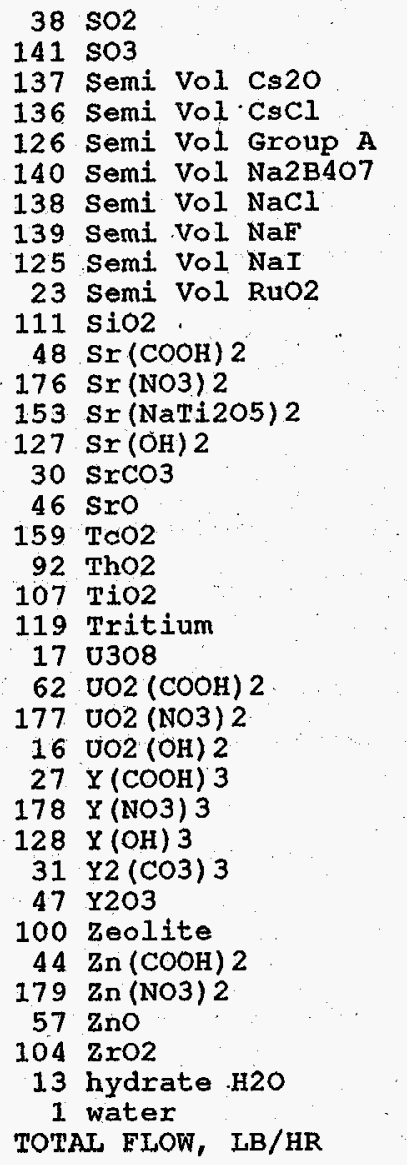

\section{TEMPERATURE, DEG C}

PRESSURE,

PRESSU

PRESSURE， MM HG

ENTHALPY, PCU/HR

VAPOR FLOW, CFM

LIQUID FLOW, GPM

DENSITY, LBS/FT3

PHASE

WSRC-TR-95-0019 (Revision
25

50

51

Hg frm MWWT PRBT Trnsfer PRBT Sampler

to MAWT Pump Prime Pump Prime ZERÓ ZERO $\quad$ ZERO ZERO ZERO ZERO ZERO ZERO ZERO ZERO ZERO..$\quad$ ZERO ZERO ZERO ZERO ZERO ZERO ZERO ZERO ZRRO ZERO

$0.7803 \mathrm{E}-11$ ZERO ZERO

$0.2667 \mathrm{E}-16 \quad$ ZERO ZERO

$0.7203 E-14$ ZERO ZERO

$0.9939 \mathrm{E}-15 \quad$ ZERO ZERO ZERO $\quad$ ZERO $\quad$ ZERO

$\begin{array}{lll}0.1124 \mathrm{E}-19 & \text { ZERO } & \text { ZERO }\end{array}$

$0.8733 E-15$ ZERO ZRRO

$0.8733 \mathrm{E}-15$

$0.7273 E-14$ ZERO

$0.2029-12$ ZERO

$0.5099 \mathrm{E}-12 \quad$ ZERO $\quad$ ZERO

$\begin{array}{lll}0.3105 E-18 & \text { ZERO } & \text { ZERO }\end{array}$

$0.2236 \mathrm{E}-16$ ZERO ZERO

$\begin{array}{rr}\text { ZERO } & \text { ZERO } \\ 0.3216 \mathrm{E}-24 & \text { ZERO }\end{array}$

$0.1943 \mathrm{E}-14 \quad$ ZERO $\quad$ ZERO

ZERO ZERO ZERO

ZERO ZERO
ZERO

$0.2363 \mathrm{E}-15 \quad$ ZERO $\quad$ ZERO

$0.6368 \mathrm{E}-13$ ZERO

$0.1302 \mathrm{E}-16$ ZERO

$\begin{array}{llll}0.4383 \mathrm{E}-13 & \ddots & \text { ZERO } & \text { ZERO }\end{array}$

$\begin{array}{rrr}\text { ZERO } & \text { ZERO } & \text { ZERO } \\ 0.3668 \mathrm{E}-12 & \text { ZERO } & \text { ZERO }\end{array}$

$0.3891 \mathrm{E}-02 \quad 0.3900 \mathrm{E}+01, \quad 0.3900 \mathrm{E}+01$

$0.2554 \mathrm{E}+00 \quad 0.3900 \mathrm{E}+01 \quad 0.3900 \mathrm{E}+01$

$\begin{array}{lll}0.3500 \mathrm{E}+02 & 0.2500 \mathrm{E}+02 & 0.2500 \mathrm{E}+02\end{array}$

$0.1000 \mathrm{E}+01 \quad 0.1000 \mathrm{E}+01, \quad 0.1000 \mathrm{E}+01$

ZERO ZERO ZERO

$0.4265 \mathrm{E}+00 \quad 0.9786 \mathrm{E}+02 \quad 0.9786 \mathrm{E}+02$

$0.4484 \mathrm{E}-04 \quad 0.7788 \mathrm{E}-02 \quad 0.7788 \mathrm{E}-02$

$0.7099 \mathrm{E}+03 \quad 0.6243 \mathrm{E}+02 \quad 0.6243 \mathrm{E}+02$
I.IQUID
LIQUID
LIQUID 


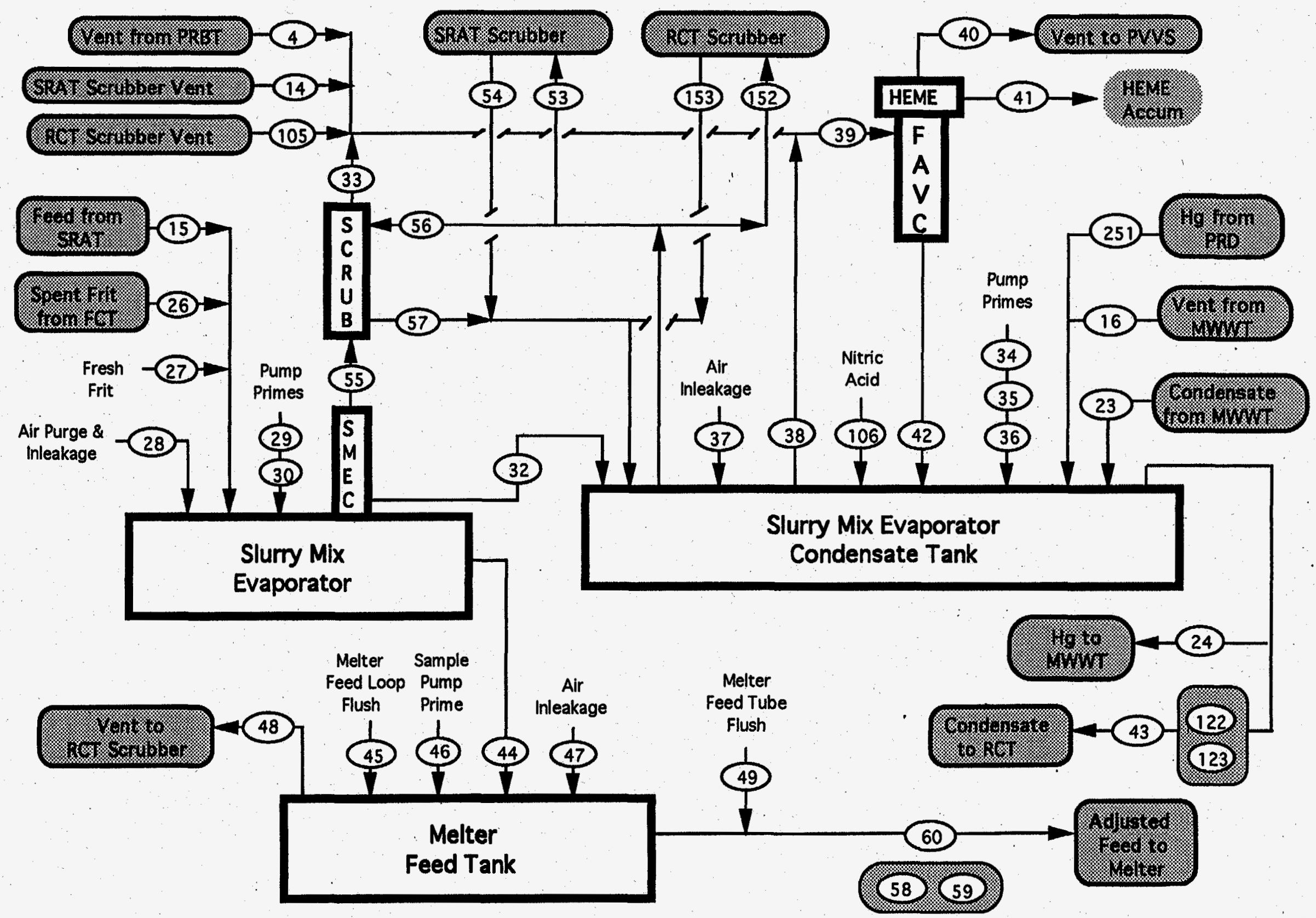

SECTION 4B. FRIT ADJUSTMENT OF MELTER FEED 
Table A-1. HLW Flowsheet Material Balance (Tank 51/ITP Cycle 1) Section 4B. Frit Adjustment of Melter Feed

DESCRIPTION

PAGE

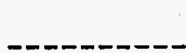

$4,14,105,15,26,27,28 \ldots \ldots$. . . . . . . . . . 2

1. STREAM NUMBERS

$29,30,55,32,33,39,152 \ldots \ldots \ldots$

2. STREAM NUMBERS

$56,57,53,54,153,34,35 \ldots \ldots \ldots$

3. STREAM NUMBERS

$36,37,251,16,23,42,106 \ldots \ldots 14$

4. STREAM NUMBERS

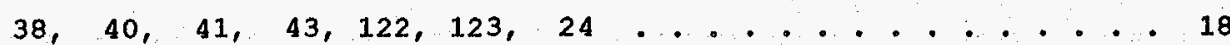

5. STREAM NUMBERS

$44,45,46,47,48,49,60$. . . . . . . . . 22

6. STREAM NUMBERS

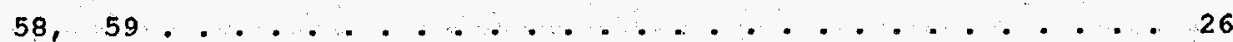


Table A-1. HLW Flowsheet Material Balance (Tank 51/ITP Cycle 1)

Section $4 B$. Frit Adjustment of Melter Feed

STREAM NUMBERS $\rightarrow$ STREAM NAME

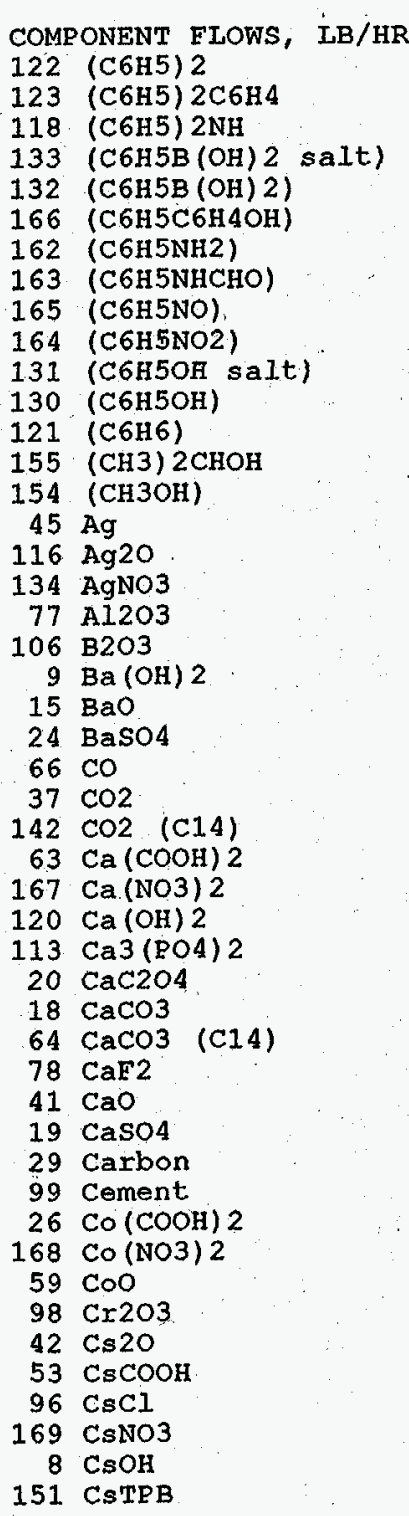

$4 \begin{array}{llr}14 & 105 & 15\end{array}$ $5 \quad 26$

$\begin{array}{lllccc}4 & 14 & 105 & 15 & 26 & 27 \\ \text { PRBT Vent SRAT Scrubbr MFT Scrubber } & \text { SRAT Trans- Decon Frit } & \text { Fresh Frit }\end{array}$

27

to FAVC Vent to FAVC Vent to FAVC fer to SME slrry to SME slrry to SME

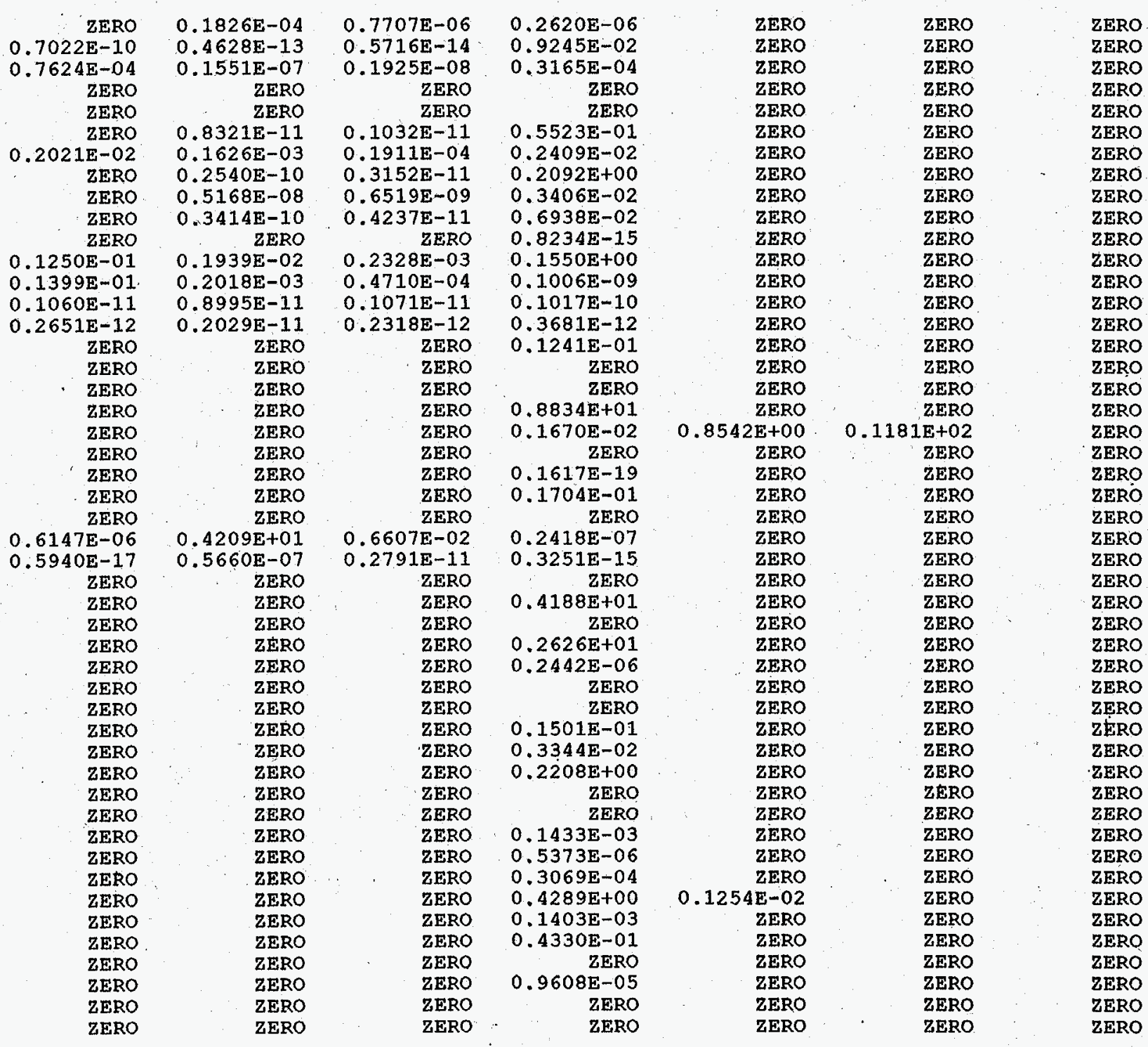

Date: January 1995 
Table A-1. HLW Flowsheet Material Balance (Tank 51/ITP Cycle 1)

Section 4B. Frit Adjustment of Melter Feed

STREAM NUMBERS (CONT'D) -> STREAM NAME

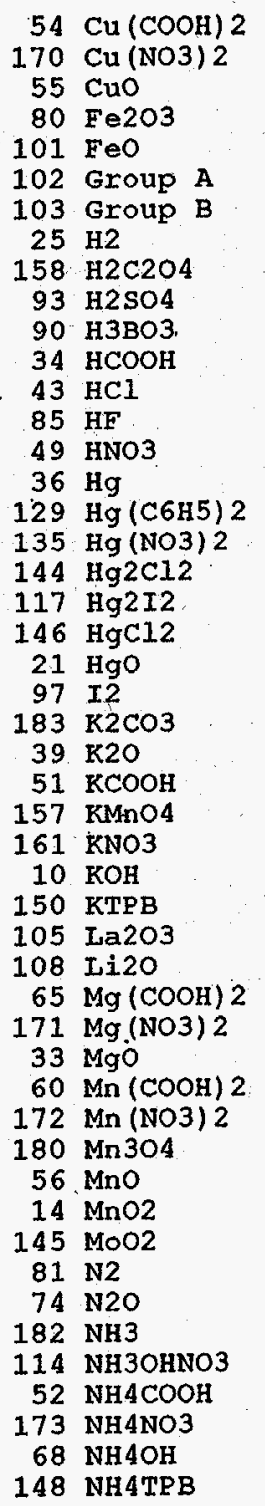

4 14 105

PRBT Vent SRAT Scrubbr MFT Scrubber to FAVC Vent to FAVC Vent to FAVC ZERO 15 26 27 Trans- Decon Frit Fresh Frit fer to SME Slrry to SME slrry to SME fer to SME Slrry to SME slrry to SME
$0.4430 \mathrm{E}-02$
zERO $0 .: 2179 \mathrm{E}+01$ $0.1969 \mathrm{E}-01$ $0.2539 \mathrm{E}+02$ $0.2538 \mathrm{E}+01$ $0.3543 \mathrm{E}-02$ $0.1512 \mathrm{E}-03$ $0.5285 \mathrm{E}-12$ $0.1827 \mathrm{E}+00$ $0.7707 E+01$

$0.1057 \mathrm{E}-24$ ZERO ZERO

$0.1619 \mathrm{E}-01$ ZERO ZERO ZERO

$0.2660 \mathrm{E}-07$ ZERO ZERO ZERO ZERO

ZERO

ZERO

ZERO

ZERO

ZERO

ZERO

ZERO

ZERO
ZERO

ZERO

ZERO

ZERO

ZERO

ZERO

ZERO

ZERO

ZERO

ZERO

ZERO
ZERO

ZERO

$0.5483 \mathrm{E}+02$

$0.1415 \mathrm{E}-19$

$0.5309 \mathrm{E}-07$

ZERO

ZERO

ZERO

ZERO

$0.2351 \mathrm{E}-01$

ZERO

ZERO

ZERO

ZERO

$0.1825 \mathrm{E}-17$

ZERO

ZERO

ZERO

ZERO

ZERO

ZERO

ZERO

ZERO

ZERO

ZERO

ZERO

ZERO

ZERO

ZERO

ZERO

ZERO

ZERO

ZERO

ZERO

ZERO

ZERO

ZERO

ZERO

ZERO

$0.8857 \mathrm{E}+03$

$0.5105 \mathrm{E}-22$
$0.7631 \mathrm{E}-03$

ZERO

ZERO

ZERO

ZERO

9E-08

ZERO

ZERO

ZERO

$0.1012 \mathrm{E}+00$

$0.1208 \mathrm{E}-06$

ZERO

ZERO

ZERO

ZERO

ZERO

ZERO

ZERO

ZERO

ZERO

ZERO

ZERO

ZERO
ZERO

ZERO

ZERO

ZERO

ZERO

ZERO

ZERO

ZERO

ZERO

ZERO

ZERO

ZERO

ZERO

$0.1097 \mathrm{E}+03$

TRACE

$0.9381 E-04$

ZERO

ZERO

ZERO

ZERO

\begin{tabular}{|c|c|}
\hline ZERO & ZERO \\
\hline ZERO & ZERO \\
\hline $0.4880 E-03$ & ZERO \\
\hline ZERO & ZERO \\
\hline ZERO & ZERO \\
\hline ZERO & ZERO \\
\hline ZERO & ZERO \\
\hline ZERO & ZERO \\
\hline ZERO & ZERO \\
\hline ZERO & ZERO \\
\hline ZERO & ZERO \\
\hline ZERO & ZERO \\
\hline ZERO & ZERO \\
\hline ZERO & ZERO \\
\hline ZERO & ZERO \\
\hline ZERO & ZERO \\
\hline ZERO & ZERO \\
\hline ZERO & ZERO \\
\hline ZERO & ZERO \\
\hline ZERO & ZERO \\
\hline ZERO & ZERO \\
\hline ZERO & ZERO \\
\hline ZERO & ZERO \\
\hline ZERO & ZERO \\
\hline ZERO & ZERO \\
\hline ZERO & ZERO \\
\hline ZERO & ZERO \\
\hline ZERO & ZERO \\
\hline ZERO & ZERO \\
\hline ZERO & ZERO \\
\hline $0.7474 \mathrm{E}+00$ & $0.1033 \mathrm{E}+02$ \\
\hline ZERO & ZERO \\
\hline ZERO & ZERO \\
\hline $0.2135 \mathrm{E}+00$ & $0.2952 \mathrm{E}+01$ \\
\hline ZERO & ZERO \\
\hline ZERO & ZERO \\
\hline ZERO & ZERO \\
\hline ZERO & ZERO \\
\hline ZERO & ZERO \\
\hline ZERO & ZERO \\
\hline $0.3223 E-02$ & ZERO \\
\hline ZERO & ZERO \\
\hline ZERO & ZERO \\
\hline ZERO & ZERO \\
\hline ZERO & ZERO \\
\hline ZERO & ZERO \\
\hline ZERO & ZERO \\
\hline ZERO & ZERO \\
\hline
\end{tabular}

28

SME Air

Inleakage

ZERO
ZERO

ZERO

ZERO

ZERO

ZERO

ZERO

ZERO

ZERO

ZERO

ZERO

ZERO

ZERO

ZERO

ZERO

$0.1604 \mathrm{E}+00$

ZERO

ZERO$$
\text { ZERO }
$$

ZERO

ZERO

$0.1005 \mathrm{E}+02$

ZERO

$.2858 E-01$

ZERO

ZERO

$0.2948 \mathrm{E}-03$

$0.3463 \mathrm{E}+01$

$0.3049 \mathrm{E}+00$

$0.2169 \mathrm{E}+01$

$0.6122 \mathrm{E}-07$

ZERO
$0.3831 \mathrm{E}-16$

$0.1948 \mathrm{E}+01$

$0.1075 \mathrm{E}-02$

$0.9364 \mathrm{E}-08$

$0.1087 \mathrm{E}+00$

ZERO

$0.1391 \mathrm{E}-02$

ZERO

Date: January 1995 


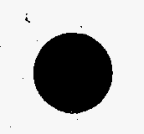

Table A-1. HLW Flowsheet Material Balance (Tank 51/ITP Cycle 1)

section 4B. Frit Adjustment of Melter Feed

STREAM NUMBERS (CONT'D) -> STREAM NAME

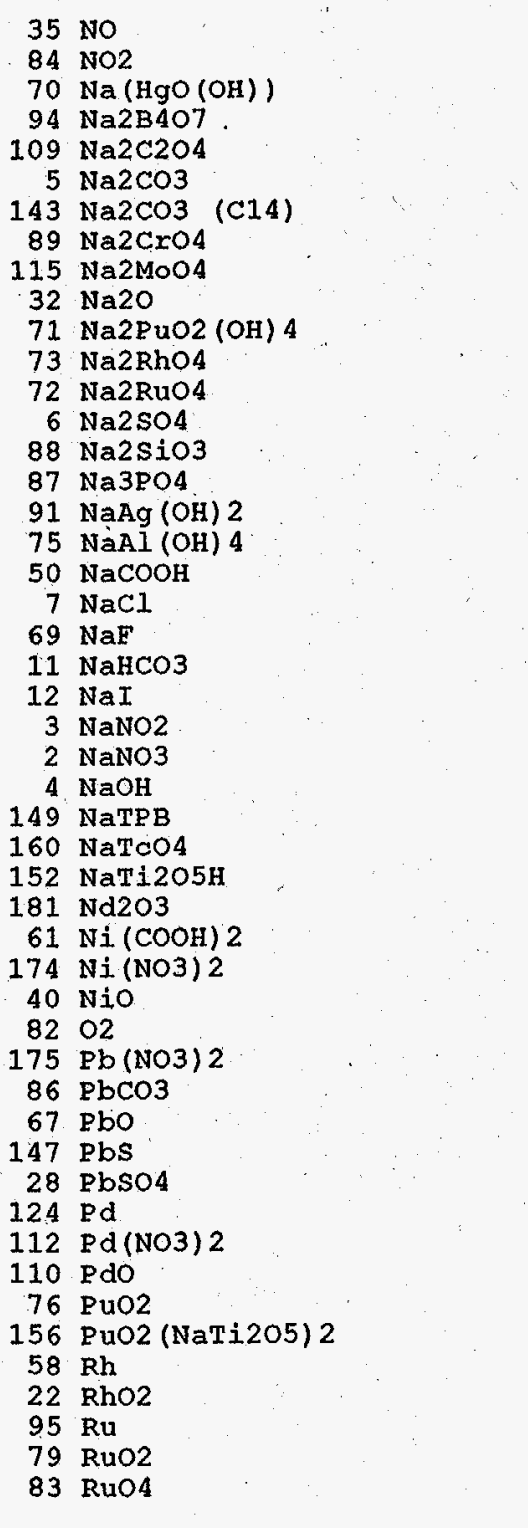

WSRC-TR-95-0019 (Revision
$414 \quad 105$

PRBT Vent SRAT Scrubbr MFT Scrubber

to FAVC Vent to FAVC Vent to FAVC

$0.3467 \mathrm{E}-11$ 1 $0.1403 \mathrm{E}+01$

$0.2755 \mathrm{E}-1.8$

$0.2986 \mathrm{E}-07$

ZERO

$\begin{array}{ll}\text { ZERO } & \text { ZERO } \\ \text { ZERO } & \text { ZERO }\end{array}$

ZERO

ZERO

ZERO

ZERO

ZERO

ZERO

ZERO

ZERO

ZERO

ZERO

ZERO

ZERO

ZERO

ZERO

ZERO

ZERO

ZERO

ZERO

ZERO
ZERO

ZERO

ZERO

ZERO

ZERO

ZERO

ZERO

ZERO

$0.1656 \mathrm{E}+02$

ZERO

ZERO

ZERO

ZERO

ZERO

ZERO

ZERO

ZERO

ZERO

ZERO

ZERO

ZERO

ZERO

$0.7556 \mathrm{E}-04$

ZERO

ZERO

ZERO

ZERO

ZERO

ZERO.

ZERO

ZERO

ZERO

ZERO

ZERO

ZERO

ZERO

ZERO

ZERO

ZERO

ZERO

ZERO

ZERO

ZERO

ZERO

ZERO

ZERO

ZERO

ZERO

ZERO

ZERO

ZERO

$0.2674 \mathrm{E}+03$

ZERO

ZERO

ZERO

ZERO

ZERO

ZERO

ZERO

ZERO

ZERO

ZERO

ZERO

ZERO

ZERO

ZERO

ZERO

ZERO

$$
\text { ZERO }
$$

$$
\text { ZERO }
$$

ZERO$$
\text { ZERO }
$$

ZERO$$
\text { ZERO }
$$

$$
\text { ZERO }
$$$$
\text { ZERO }
$$

$$
\text { ZERO }
$$

ZERO

ZERO

$$
\text { ZERO }
$$

$$
\text { ZERO }
$$$$
\text { ZERO }
$$

ZERO

ZERO

$$
\text { ZERO }
$$

ZERO

ZERO

ZERO

ZERO

ZERO

ZERO
Z +02

ZERO

ZERO

ZERO

ZERO

ZERO

ZERO

ZERO

ZERO

ZERO

ZERO

ZERO

ZERO
ZERO
15

SRAT Trans- Decon Frit Fresh Frit

fer to SME slrry to SME slrry to SME
$0.1165 \mathrm{E}-09$
ZERO

TRACE

ZERO

ZERO
$0.3313 \mathrm{E}+02$

ZERO

$0.4849 \mathrm{E}+01$

ZERO

ZERO

$0.4234 \mathrm{E}+01$

ZERO

ZERO

$0.1952 \mathrm{E}+00$

ZERO

$0.2578 \mathrm{E}-02$

ZERO

$0.2740 \mathrm{E}+00$

$0.9957 \mathrm{E}-02$

$0.3417 \mathrm{E}-01$

ZERO
$0.5472 \mathrm{E}-05$

ZERO

$0.8943 \mathrm{E}+01$
ZERO

ZERO

ZERO

ZERO

$0.2521 \mathrm{E}-01$

$0.6686 \mathrm{E}-06$

$0.2366 \mathrm{E}+00$

$0.9713 \mathrm{E}-08$

ZERO

ZERO
$0.7084 \mathrm{E}-19$

ZERO

$.1318 \mathrm{E}+00$

$0.3903 E-03$

ZERO
ZERO

$0.9905 \mathrm{E}-02$

$0.1272 \mathrm{E}-03$

$0.7436 \mathrm{E}-03$

$0.8125 \mathrm{EERO}$

ZERO

Date: January 1995

ZERO ZERO
26.

ZERO

ZERO

28

kage

ZERO

ZERO

ZERO

ZERO

ZERO

ZERO

ZERO

ZERO

ZERO

ZERO

ZERO

ZERO

ZERO

ZERO

ZERO

ZERO

$\begin{array}{rrr}\text { ZERO } & \text { ZERO } & \text { ZERO } \\ 0.1579 \mathrm{E}+00 & 0.2182 \mathrm{E}+01 & \text { ZERO }\end{array}$

ZERO $0.2182 E$ ZERO

ZERO ZERO

ZERO ZERO

ZERO . ZERO

ZERO ZERO

ZERO ZERO

$\begin{array}{ll}\text { ZERO } & \text { ZERO } \\ \text { ZERO } & \text { ZERO }\end{array}$

ZERO ZERO

ZERO $\therefore$ ZERO

ZERO ZERO

$0.5036 \mathrm{E}-02 \quad$ ZERO

$0.1916 \mathrm{E}-02$

ZRRO

ZERO

ZERO

ZERO

ZERO

ZERO

ZERO

ZERO

ZERO

ZERO

ZERO

ZERO

ZERO

ZERO

section 4B, Page 4 of 29 
Table A-1. HLW Flowsheet Material Balance (Tank 51/ITP Cycle 1)

section 4B. Frit Adjustment of Melter Feed

STREAM NUMBERS (CONT'D) $\rightarrow$ STREAM NAME

$38 \mathrm{SO} 2$

141 SO3

137 Semi Vol cs 20

136 Semi Vol CsCl.

126 Semi Vol Group A

138 Semi Vol NaCl

139 Semi Vol NaF

135 Semi Vol NaI

23 Semi Vol RuO2

$111 \mathrm{SiO2}$

48 Sr (COOH) 2

$176 \mathrm{Sr}(\mathrm{NO} 3) 2$
$153 \mathrm{Sr}(\mathrm{NaTi} 2 \mathrm{O5}) 2$

$127 \mathrm{Sr}(\mathrm{OH}) 2$

$30 \mathrm{srCO}$

46 Sro

$159 \mathrm{TCO} 2$

107 Tio2

119 Tritium

$\begin{aligned} & 17 \text { Trit } \\ & 1708\end{aligned}$

62 UO2 (COOH) 2

$177 \mathrm{UO2}$ (NO3) 2

$16 \mathrm{VO} 2(\mathrm{OH}) 2$

$27 \mathrm{Y}(\mathrm{COOH}) 3$
178 Y (NO3) 3

$128 \mathrm{Y}(\mathrm{OH}) 3$

$31 \mathrm{Y} 2(\mathrm{CO} 3)^{3}$

$47 \mathrm{Y} 203$

100 zeolite

$44 \mathrm{Zn}(\mathrm{COOH}) 2$
$179 \mathrm{Zn}(\mathrm{NO} 3) 2$

179 Zn
57 Zno

$104 \mathrm{zrO2}$

13 hydrate $\mathrm{H} 2 \mathrm{O}$

1 water

TOTAL FLOW, LB/HR

TEMPERATURE， DEG C

PRESSURE, ATM

PRESSUURE，PSIG

PRESSURE, MM HG

ENTHALPY, PCU/HR

VAPOR FLOW, CFM

LIOUID FLOW, GPM

DENSITY， LBS/FT3

PHASE

WSRC-TR-95-0019 (Revision

$414 \quad 105$

PRBT Vent SRAT Scrubbr MFT Scrubber

to FAVC Vent to FAVC Vent to FAVC

ZERO

ZERO

ZERO

ZERO

ZERO

ZERO

ZERO

ZERO

ZERO

ZERO

ZERO

ZERO

ZERO

ZERO

ZERO

ZERO

ZERO

$0.8298 \mathrm{E}-14$

ZERO

ZERO

ZERO

ZERO

ZERO

ZERO

ZERO

ZERO

ZERO

ZERO

ZERO

ZERO

ZERO

$0.4886 \mathrm{E}+01$

$0.7632 \mathrm{E}+02$

$0.4564 \mathrm{E}+02$

$0.9656 \mathrm{E}+00$

$-0.5055 \mathrm{E}+00$

$0.7339 \mathrm{E}+03$

$0.3975 \mathrm{E}+04$

$0.1985 \mathrm{E}+02$

$0.6406 \mathrm{E}-01$

VAPOR

ZERO

ZERO

ZERO

ZERO

ZERO

ZERO

ZERO

ZERO

ZERO

ZERO

ZERO

ZERO

ZERO

ZERO

ZERO

ZERO

ZERO

ZERO

$0.5284 \mathrm{E}-10$

ZERO

ZERO

ZERO

ZERO

ZERO

ZERO

ZERO

ZERO

ZERO

ZERO

ZERO

ZERO

ZERO

$0.9829 \mathrm{E}+02$

$0.1258 \mathrm{E}+04$

$0.5000 \mathrm{E}+02$

$0.1000 E+01$
ZERO

$0.7777 \mathrm{E}+05$

$0.6498 \mathrm{E}-1$

VAPOR

Date: January 1995

ZERO

ZERO

ZERO

ZERO

$0.1212 \mathrm{E}+02$

$0.1550 \mathrm{E}+03$

26

27

Frit . Fresh Frit

$\begin{array}{lll}\text { ZERO } & \text { ZERO } & \text { ZERO } \\ \text { ZERO } & \text { ZERO } & \text { ZERO }\end{array}$

$\begin{array}{lll}\text { ZERO } & \text { ZERO } & \text { ZERO } \\ \text { ZERO } & \text { ZERO } & \text { ZERO }\end{array}$

$\begin{array}{lll}\text { ZERO } & \text { ZERO } & \text { ZERO } \\ \text { ZERO } & \text { ZERO } & \text { ZERO }\end{array}$

ZERO ZERO $\quad$ ZERO

ZERO ZERO ZERO

ZERO ZERO $\quad$ Z ZERO

$\begin{array}{lll}\text { ZERO } & \text { ZERO } & \text { ZERO } \\ \text { ZERO } & \text { ZERO } & \text { ZERO }\end{array}$

ZERO

ZERO

$0.1092 \mathrm{E}+01$

ZERO

ZERO

$0.1093 \mathrm{E}-03$

$0.8221 \mathrm{E}+01$

$0.2944 \mathrm{E}-01$

ZERO

ZERO

ZERO

ZERO

ZERO

ZERO

ZERO

ZERO

ZERO

ZERO

ZERO

ZERO

ZERO

ZERO

ZERO

ZERO

ZERO
ZERO

ZERO

ZERO

ZERO

ZERO

ZERO

ZERO

$0.1794 \mathrm{E}+00$

ZERO

$0.3420 \mathrm{E}+03$

ZERO

$0.4401 \mathrm{E}+03$

$0.2152 \mathrm{E}+03$

$0.2260 \mathrm{E}+03$

$0.4959 \mathrm{E}+02 \quad 0.2980 \mathrm{E}+02$

$0.1000 \mathrm{E}+01 \quad 0.9656 \mathrm{E}+00$

ZERO

$-0.5055 \mathrm{E}+00$

$0.7339 \mathrm{E}+03$

$0.1786 \mathrm{E}+05$

$0.4394 \mathrm{E}+00$

$0.6412 \mathrm{E}+02$

LIQUID

ZERO

ZERO

ZERO

ZERO

ZERO

ZERO

ZERO

ZERO

ZERO

ZERO

ZERO

ZERO

ZERO

ZERO

ZERO

ZERO

ZERO

ZERO

ZERO

ZERO

ZERO

ZERO

ZERO

$0.9874 \mathrm{E}+02$

$0.2475 \mathrm{E}+03$

$0.3000 \mathrm{E}+02$

$0.134 \mathrm{OE}+01$

$0.4997 \mathrm{E}+01$

$0.3866 \mathrm{E}+04$

$0.3297 \mathrm{E}+00$

$0.9360 \mathrm{E}+02$

LIQUID

28

SME Air

Inleakage

ZERO

ZERO

ZERO

ZERO

ZERO

ZERO

ZERO

ZERO

ZERO

ZERO

ZERO

ZERO

ZERO

ZERO

ZERO

ZERO

ZERO

ZERO

ZERO

ZERO

ZERO

ZERO

ZERO

ZERO

ZERO

ZERO

ZERO

ZERO

ZERO

ZERO

ZERO

ZERO

ZERO

ZERO

ZUERO

ZERO

$0.4834 \mathrm{E}+01$

$0.2926 \mathrm{E}+03$

$0.3500 \mathrm{E}+02$

$0.1000 E+01$

$0.6137 \mathrm{E}+04$

$0.6912 \mathrm{E}+02$

$0.7055 \mathrm{E}-01$

VAPOR 
Table A-1. HLW Flowsheet Material Balance (Tank 51/ITP Cycle 1 ) Section:4B. Frit Adjustment of Melter Feed

STREAM NUMBERS $\rightarrow$ STREAM NAME

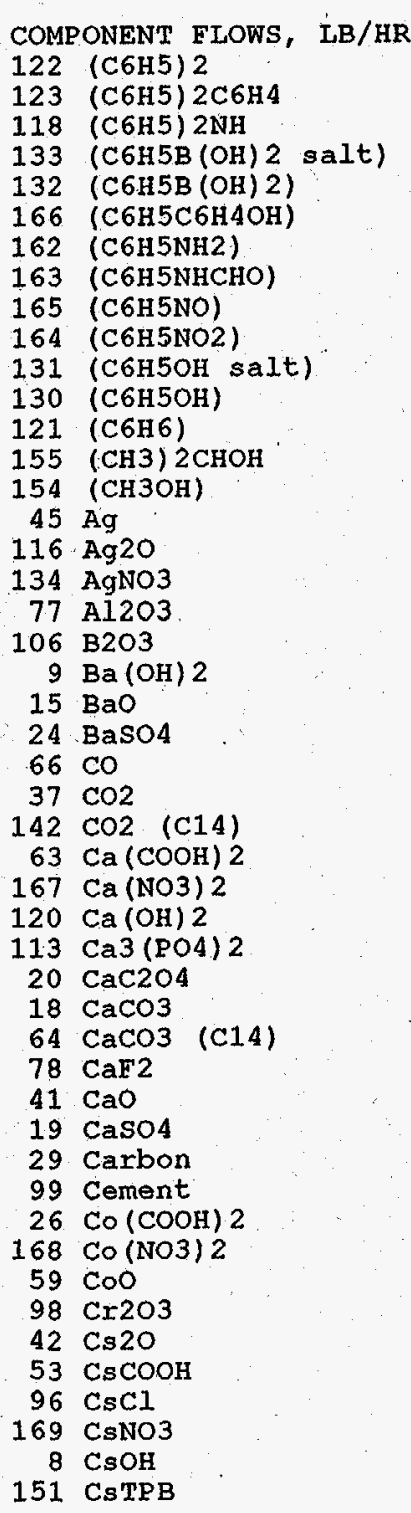

29 30

SME Sample SME Transfer Pump Prime Pump Prime

55 32 33 SMEC Vent SMEC Cndnste SME Sarubbex
to Scrubber to SMECT Vent to FAVC

ZERO
ZERO
ZERO
ZERO
ZERO
ZERO
ZERO
ZERO
ZERO
ZERO
ZERO
ZERO
ZERO
ZERO
ZERO
ZERO
ZERO
ZERO
ZERO
ZERO
ZERO
ZERO
ZERO
ZERO
ZERO
ZERO
ZERO
ZERO
ZERO
ZERO
ZERO
ZERO
ZERO
ZERO
ZERO
ZERO
ZERO
ZERO
ZERO
ZERO
ZERO
ZERO
ZERO
ZERO
ZERO
ZERO
ZERO
ZERO

$0.2619 E-06$

$\begin{array}{ll}0.1873 \mathrm{E}-12 & 0.1712 \mathrm{E}-06 \\ 0.6208 \mathrm{E}-05 & 0.2544 \mathrm{E}-04\end{array}$ ZERO

$0.6439 \mathrm{E}-13$

$0.6439 \mathrm{E}-13$

$0.5886 \mathrm{E}-03$

$0.2439 \mathrm{E}-12$

$0.3971 \mathrm{E}-14$
$0.8089 \mathrm{E}-14$

$0.8089 \mathrm{E}-14$
TRACE

$0.1458 \mathrm{E}-01$

$0.1004 \mathrm{E}-09$

$0.1012 E-11$

$0.8688 \mathrm{E}-13$

$0.1447 \mathrm{E}-13$

$$
\begin{aligned}
& \text { ZERO } \\
& \text { ZERO }
\end{aligned}
$$

$0.1030 \mathrm{E}-10$

$0.1476 \mathrm{E}-10$$$
\text { ZERO }
$$

$0.1986 \mathrm{E}-13$

$0.1986 \mathrm{E}-13$

$0.8535 \mathrm{E}-01$

$0.3249 \mathrm{E}-15$

$0.4882 \mathrm{E}-11$ ZERO

$0.3061 \mathrm{E}-11$

$0.2847 \mathrm{E}-18$ ZERO

$0.1750 \mathrm{E}-13$

$0.3898 \mathrm{E}-14$

$0.3898 \mathrm{E}-14$
$0.2574 \mathrm{E}-12$

$0.2574 \mathrm{E}-12$
ZERO ZERO

$0.1670 \mathrm{E}-15$

$0.6265 \mathrm{E}-18$

$0.3578 \mathrm{E}-16$

$0.5015 \mathrm{E}-12$

$0.1635 \mathrm{E}-15$

$0.5048 E-13$

0. $1120 \mathrm{OE}-16$

ERO

ZERO ZERO

$0.6586 \mathrm{E}-07$

$0.1641 \mathrm{E}-02$

$0.1641 \mathrm{E}-02$

$0.2495 \mathrm{E}-06$

$0.4061 \mathrm{E}-08$

$0.8274 \mathrm{E}-08$

$0.9818 \mathrm{E}-21$

$0.2078 \mathrm{E}-12$

$0.6467 \mathrm{E}-11$

$0.2395 \mathrm{E}-12$

$0.1480 \mathrm{E}-07$

ZERO

$0.1053 E-04$

$0.1510 \mathrm{E}-04$

ZERO

$0.2032 \mathrm{E}-07$

ZERO

$0.4659 \mathrm{E}-04$

$0.1774 \mathrm{E}-18$

ZERO
$0.4994 \mathrm{E}-05$

ZERO

$0.2912 \mathrm{E}-12$

E-12

ZERO

$0.1790 \mathrm{E}-0$

$0.3987 \mathrm{E}-08$

$3 \mathrm{E}-06$ ZERO

$0.1709 \mathrm{E}-09$

$0.6408 \mathrm{E}-12$

$0.3660 \mathrm{E}-10$

$0.5130 \mathrm{E}-06$

$0.1673 \mathrm{E}-09$

ZERO

ZERO

$.1146 \mathrm{E}-10$

ZERO

Date: January 1995
$0.1873 \mathrm{E}-12 \quad 0.8989 \mathrm{E}-10$
$0.7484 \mathrm{E}-06$
$0.1151 \mathrm{E}-13$

$0.3859 \mathrm{E}-08$ ZERO

$0.2070 \mathrm{E}-11$

$0.3830 \mathrm{E}-04$

$0.6319 \mathrm{E}-11$

$0.1353 \mathrm{E}-08$

$0.8495 \mathrm{E}-11$ ZERO

$0.4713 \mathrm{E}-03$

$0.4801 \mathrm{E}-04$

$0.2180 \mathrm{E}-11$

$0.4752 \mathrm{E}-12$

ZERO

ZERO

ZERO

ZERO

ZERO

ZIRO

ZERO

ZERO

$0.8514 \mathrm{E}-01$

$0.4754 \mathrm{E}-11$

ZERO

ZERO

ZERO

ZERO

ZERO

ZERO

ZTRO

ZERO

ZERO

ZERO

ZERO

ZERO

ZERO

ZERO

ZERO

ZERO

ZERO

ZERO

ZERO

ZERO

ZERO
$39 \quad 152$

to FAVC Liquid to (abber

$0.1833 \mathrm{E}-01 \quad 0.2682 \mathrm{E}-03$ $0.7029 \mathrm{E}-10 \quad 0.3339 \mathrm{E}-06$ $0.1513 \mathrm{E}-03 \quad 0.4725 \mathrm{E}-02$ ZERO ZERO $0.1142 \mathrm{E}-10 \quad 0.1851 \mathrm{E}-06$ $0.3620 \mathrm{E}-02 \quad 0.4991 \mathrm{E}-01$ $0.3488 \mathrm{E}-10 \quad 0.7017 \mathrm{E}-06$ $0.7173 \mathrm{E}-08 \quad 0.7635 \mathrm{E}-08$ $0.7173=08$ - $0.7635 \mathrm{E}-08$ $0.4687 \mathrm{E}-10 \mathrm{O}-0.7635 \mathrm{E}-08$ $0.2270 \mathrm{E}-01 \quad 0.6075 \mathrm{E}+00$ $0.4102 \mathrm{E}-01 \quad 0.7392 \mathrm{E}-03$ $0.1393 \mathrm{E}-10 \quad 0.5070 \mathrm{E}-10$ $0.3138 \mathrm{E}-11 \quad 0.4830 \mathrm{E}-11$ $0.1123 \mathrm{E}-16$ ZERO $0.7540 \mathrm{E}-24 \quad 0.8913 \mathrm{E}-16$ $0.2509 \mathrm{E}-12 \quad 0.2966 \mathrm{E}-04$ $0.9093 \mathrm{E}-13 \quad 0.1075 \mathrm{E}-04$ ZERO ZERO $0.9518 \mathrm{E}-22 \quad 0.1125 \mathrm{E}-13$ $\begin{array}{rr}.4838 \mathrm{E}-15 & 0.5720 \mathrm{E}-07 \\ \text { ZERO } & \text { ZERO }\end{array}$ $0.4332 \mathrm{E}+01 \quad 0.1050 \mathrm{E}-03$ $0.5680 \mathrm{E}-07 \quad 0.1355 \mathrm{E}-11$ $\begin{array}{rr}\text { ZERO } & \text { ZERO } \\ 0.1189 \mathrm{E}-12 & 0.1406 \mathrm{E}-04\end{array}$ $\begin{array}{rr}\text { ZERO } & \text { ZERO } \\ 0.7454 \mathrm{E}-13 & 0.8813 \mathrm{E}-05\end{array}$ $0.6919 \mathrm{E}-20 \quad 0.8180 \mathrm{E}-12$ ZERO ZERO

$0.4263 \mathrm{E}-15 \quad 0.5030 \mathrm{~T}-07$ $0.4263 \mathrm{E}-15$ O. $0.6258 \mathrm{E}-14$ $0.1124 \mathrm{E}-07$ ZERO
ZERO $0.4059 \mathrm{E}-17 \quad 0.4799 \mathrm{E}-09$ $0.152 .2 \mathrm{E}-19 \quad 0.1800 \mathrm{E}-11$ $0.8696 \mathrm{E}-18 \quad 0.1028 \mathrm{E}-09$ $0.1217 \mathrm{E}-13 \quad 0.1439 \mathrm{E}-05$ $\begin{array}{ll}0.3984 \mathrm{E}-17 & 0.4710 \mathrm{E}-09 \\ 0.1227 \mathrm{E}-14 & 0.1450 \mathrm{E}-06\end{array}$ $\begin{array}{rr}\text { ZERO } & \text { ZERO } \\ 0.2729 \mathrm{E}-18 & 0.3226 \mathrm{E}-10\end{array}$ $0.3112 \mathrm{E}-19 \quad 0.3679 \mathrm{E}-11$ ZERO ZERO 
Table A-1. HLW Flowsheet Material Balance (Tank 51/ITP Cyale 1)

section 4B. Frit Adjustment of Melter Feed

STREAM NUMBERS (CONT'D) $\rightarrow$ STREAM NAME

\section{$54 \mathrm{Cu}(\mathrm{COOH}) 2$}

$170 \mathrm{Cu}(\mathrm{NO} 3) 2$

$55 \mathrm{CuO}$

$80 \mathrm{Fe} 2 \mathrm{O} 3$

101 FeO

102 Group A

$25 \mathrm{H} 2$

$158 \mathrm{H} 2 \mathrm{C} 204$

$93 \mathrm{H} 2 \mathrm{SO} 4$

$90 \mathrm{H} 3 \mathrm{BO} 3$

$34 \mathrm{HCOOH}$

$43 \mathrm{HCl}$

$85 \mathrm{HF}$

49 HNO3

$36 \mathrm{Hg}$

$129 \mathrm{Hg}(\mathrm{C} 6 \mathrm{H} 5) 2$

$135 \mathrm{Hg}(\mathrm{NO} 3)^{2}$

$144 \mathrm{Hg} 2 \mathrm{Cl} 2$

$117 \mathrm{Hg} 2 \mathrm{I} 2$

$146 \mathrm{HgCl}$

21 HgO

$\begin{array}{rl}97 & \mathrm{I} 2 \\ 183 & \mathrm{~K} 2 \mathrm{CO} 3\end{array}$

$39 \mathrm{~K} 2 \mathrm{O}$

$51 \mathrm{KCOOH}$

161. KNO3

$10 \mathrm{KOH}$.

150. KTPB

$105 \mathrm{Lra2O3}$

108 Li20

$65 \mathrm{Mg}(\mathrm{COOH}) 2$
$171 \mathrm{Mg}(\mathrm{NO} 3) 2$

$33 \mathrm{MgO}$

$60 \mathrm{Mn}(\mathrm{COOH}) 2$

$172 \mathrm{Mn}$ (NO3) 2

$180 \mathrm{Mn} 30$

$56 \mathrm{MnO}$

$14 \mathrm{MnO} 2$

$81 \mathrm{~N} 2$

$\begin{aligned} 74 & \text { N2O } \\ 182 & \text { NH3 }\end{aligned}$

114 NH3OHNO3

173 NH4NO3

68 NH 4 OH
148 NH 4 TPB

WSRC-TR-95-0019 (Revision
29

SME Sample SME Transfer

Pump Prime Pump Prime

ZERO

ZERO

ZERO

ZERO

ZERO

ERO

ZERO

ZERO

ZERO

ZERO

ZERO

ZERO

ZERO

ZERO

ZERO

ZERO

ZERO

ZERO

ZERO

ZERO

ZERO

ZERO

ERO

ZERO

ZERO

ZERO

ZERO

ZERO

ZERO

ZERO

ZERO

ZERO

ZERO

ZERO

ERO

ZERO

ZERO

ZERO

ZERO

ZERO

ZERO

ZEROO

ZERO

ZERO

ZERO

ZRO

ZERO

ZERO

0)

ZERO

ZERO

ZERO

ZERO

ZERO

ZERO

ZERO

ZERO

ZERO

ZERO

ZERO
ZERO

ZERO

ZERO

ZERO

ZERO

ZERO

ZERO

ZERO

ZERO

ZERO
$55 \quad 32$

33

SMEC Vent SMEC Cndnste SME Scrubber

to Scrubber to SMECT Vent to FAVC

$0.5165 \mathrm{E}-14$

$0.2540 \mathrm{E}-11$

$0.2296 \mathrm{E}-13$

$0.2960 \mathrm{E}-10$

$0.2959 \mathrm{E}-11$

$0.4131 E-14$

$0.1762 \mathrm{E}-15$

$0.7839 \mathrm{E}-02$

$0.2130 \mathrm{E}-12$

ZERO

$0.8986 \mathrm{E}-11$

ZERO

ZERO

$0.5277 \mathrm{E}-10$

$0.1869 \mathrm{E}-12$

ZERO

ZERO

ZERO

ZERO

ZERO

ZERO

ZERO

$0.9412 \mathrm{E}-13$

$0.1171 \mathrm{E}-10$

ZERO

$0.3332 \mathrm{E}-13$

ZERO

ZERO

$0.5283 \mathrm{E}-08$

$0^{\prime} .2598 \mathrm{E}-05 \quad$ ZERO

$0.2348 \mathrm{E}-07$ ZERO

$0.3027 \mathrm{E}-04 \quad$ ZERO

$0.3026 \mathrm{E}-05$

$0.4225 \mathrm{E}-08$

$0.1802 \mathrm{E}-09$

$0.1753 \mathrm{E}-06$

$0.2179 \mathrm{E}-06$

ZERO

ZE-05
ZERO

ZERO

ZERO

$0.2445 \mathrm{E}-09$

$0.1912 \mathrm{E}-06$

ZERO

ZERO

ZERO

ZERO

ZERO

ZERO

ZERO

$0.9626 \mathrm{E}-07$

$0.1198 \mathrm{E}-04$

ZERO
$0.3408 \mathrm{E}-07$

ZERO

ZERO

$0.1292 \mathrm{E}-10$

$0.4038 \mathrm{E}-1$

$0.6125 \mathrm{E}-19$

$0.4046 \mathrm{E}-11$

$0.2529 \mathrm{E}-11$

$0.7137 \mathrm{E}-19$

ZERO

$0.2271 \mathrm{E}-11$

$0.1254 \mathrm{E}-14$

$0.2210 \mathrm{E}+03$

TRACE

0.1321 ZERO

$0.1321 \mathrm{E}-04$

$0.4138 \mathrm{E}-05$

$0.2587 \mathrm{E}-05$

$0.7300 \mathrm{E}-13$

$0.4568 \mathrm{E}-22$

$0.2323 \mathrm{E}-05$

$0.1282 \mathrm{E}-08$

$0.3816 \mathrm{E}-02$

$0.5437 \mathrm{E}-02$

ZERO

$0.1621 \mathrm{E}-14$

$0.2079 \mathrm{E}-06$

TRACE

ZERO

ZERO

$0.1658 \mathrm{E}-08$

ZERO

Date: January 1995

ZERO

ZERO

$0.2078 \mathrm{E}+00$

$0.1669 \mathrm{E}-18$

ZERO

ZERO

ZERO

ZERO

ZERO

ZERO

ZERO

ZERO

ZERO

ZERO

ZERO

ZERO

ZERO

ZERO

ZERO

ZERO

ZERO

ZERO

ZERO

ZERO

ZERO

ZERO

ZERO

ZERO

$0.2210 \mathrm{E}+03$

TRACE

$0.1853 \mathrm{E}-03$

ZERO

ZERO

ZERO

ZERO

$39 \quad 152$

Inlet Vapor Iiquid to

to FAVC MFT scrubbe

$0.1258 \mathrm{E}-15 \quad 0.1487 \mathrm{E}-07$

$0.6181 \mathrm{E}-13 \quad 0.7308 \mathrm{E}-05$

$0.5594 \mathrm{E}-15 \quad 0.6613 \mathrm{E}-07$

$0.7210 \mathrm{E}-12 \quad 0.8524 \mathrm{E}-04$

$0.7209 \mathrm{E}-13 \quad 0.8522 \mathrm{E}-05$

$0.1006 \mathrm{E}-15 \quad 0.1190 \mathrm{E}-07$

$0.4293 \mathrm{E}-17 \quad 0.5075 \mathrm{E}-09$

$0.3136 \mathrm{E}-01$ 0. $1693 \mathrm{E}-08$

$0.5189 \mathrm{E}-14 \quad 0.6135 \mathrm{E}-06$

ZERO ZERO

$0.2184 \mathrm{E}-12 \quad 0.2582 \mathrm{E}-04$

ZERO ZERO

ZERO

sect

$0.1143 \mathrm{E}+01$

$0.3442 \mathrm{E}+01$

ZERO

ZERO

ZERO

ZERO

$0.5595 \mathrm{E}-19$

E-19

ZERO

$0.2846 \mathrm{E}-12$ ZERO

$0.8118 E-15$

ZERO

$0.7953 \mathrm{E}-13$

$0.9837 \mathrm{E}-13$

$0.3138 \mathrm{E}-13$

$0.6161 \mathrm{E}-13$ 
Table A-1. HLW Flowsheet Material Balance (Tank 51/ITP Cycle 1)

$$
\text { section 4B. Frit Adjustment of Melter Feed }
$$

STREAM NUMBERS (CONT'D) $\rightarrow$ STREAM NAME

\begin{tabular}{|c|c|}
\hline 35 & \\
\hline 84 & NO2 \\
\hline 70 & $\mathrm{Na}(\mathrm{HgO}(\mathrm{OH}))$ \\
\hline 94 & $\mathrm{Na} 2 \mathrm{~B} 407$ \\
\hline 109 & $\mathrm{Na} 2 \mathrm{C} 2 \mathrm{O} 4$ \\
\hline & $\mathrm{Na} 2 \mathrm{CO} 3$ \\
\hline 143 & $\mathrm{Na} 2 \mathrm{CO} 3$ (C14) \\
\hline & $\mathrm{Na} 2 \mathrm{CrO} 4$ \\
\hline 115 & $\mathrm{Na} 2 \mathrm{MoO} 4$ \\
\hline 32 & $\mathrm{Na} 2 \mathrm{O}$ \\
\hline 71 & $\mathrm{Na} 2 \mathrm{PuO} 2(\mathrm{OH}) 4$ \\
\hline 73 & $\mathrm{Na} 2 \mathrm{RhO} 4$ \\
\hline 72 & $\mathrm{Na} 2 \mathrm{RuO} 4$ \\
\hline 6 & $\mathrm{Na} 2 \mathrm{SO} 4$ \\
\hline $88^{\circ}$ & $\mathrm{Na2SiO3}$ \\
\hline 87 & $\mathrm{Na} 3 \mathrm{PO} 4$ \\
\hline 91 & $\mathrm{NaAg}(\mathrm{OH}) 2$ \\
\hline 75 & NaAI (OH) 4 \\
\hline 50 & $\mathrm{NaCOOH}$ \\
\hline 7 & $\mathrm{NaCl}$ \\
\hline 69 & $\mathrm{NaF}$ \\
\hline 11. & $\mathrm{NaHCO} 3$ \\
\hline 12 & NaI \\
\hline 3 & NaNO2 \\
\hline 2 & NaNO3 \\
\hline 4 & $\mathrm{NaOH}$ \\
\hline 149 & NaTPB \\
\hline 160 & NaTcO4 \\
\hline 152 & NaTi205H \\
\hline 181 & $\mathrm{Nd} 2 \mathrm{O} 3$ \\
\hline 61 & $\mathrm{Ni}(\mathrm{COOH}) 2$ \\
\hline 174 & $\mathrm{Ni}(\mathrm{NO3}) 2$ \\
\hline 40 & $\mathrm{NiO}$ \\
\hline 82 & 02 \\
\hline 175 & $\mathrm{~Pb}(\mathrm{NO} 3) 2$ \\
\hline 86 & $\mathrm{PbCO} 3$ \\
\hline 67 & $\mathrm{Pbo}$ \\
\hline 147 & Pbs \\
\hline 28 & $\mathrm{PbSO} 4$ \\
\hline 124 & Pd \\
\hline 112 & $\mathrm{Pd}\left(\mathrm{NO}_{3}\right)_{2}$ \\
\hline 110 & Pdo \\
\hline 76 & $\mathrm{PuO} 2$ \\
\hline 156 & PuO2 (NaTi205) 2 \\
\hline 58 & $\mathrm{Rh}$ \\
\hline 22 & RhO2 \\
\hline 95 & $\mathrm{Ru}$ \\
\hline 79 & RuO2 \\
\hline & \\
\hline
\end{tabular}

WSRC-TR-95-0019 (Revision
29 30

SME Sample SME Transfer Pump Prime Pump Prime
ZERO

ZERO

ZERO

ZERO

ZERO

ZERO

ZERO

ZERO

ZERO

ZERO

ZERO

ZERO

ZERO

ZERO

ZERO

ZERO

ZERO

ZERO

ZERO

ZERO

ZERO

ZERO

ZERO

ZERO

ZERO

ZERO

ZERO

ZERO

ZERO

ZERO

ZTRO

ZERO

ZERO

ZERO

ZERO

ZERO

ZERO

ZERO

ZERO

ZERO
ZERO

ZERO

ZERO

ZERO

ZERO

ZERO

ZERO

0)

ZERO
ZERO

ZERO

ZERO

ZERO

ZERO

ZERO

ZERO

ZERO

ZERO

ZERO

ZERO

ZERO

ZERO

ZERO

ZERO

ZERO

ZERO.

ZERO

ZERO

ZERO

ZERO

ZERO

ZERO

ZERO

ZERO

ZERO

ZERO

ZERO

ZERO

ZERO

ZERO

ZERO

ZERO

ZERO

ZERO

ZERO

ZERO

ZERO

ZERO

ZERO

ZERO

ZERO

ZERO

ZERO
55 32

$32 \quad 33$

SMEC Vent SMEC Cndnste SME Scrubber

o Scrubber

TRACE

$0.5835 \mathrm{E}-14$

$0.1060 \mathrm{E}-05$
$0.1507 \mathrm{E}-07$

TRACE

$0.1507 E-07$

ZERO

ZERO

$0.5893 E-11$

ZERO

ZERO
$0.6028 \mathrm{E}-05$

ZERO

ZERO

$0.1476 \mathrm{E}-10$

ZERO

ZERO

$0.2275 \mathrm{E}-12$

ZERO

$0.3005 \mathrm{E}-14$

ZERO

$0.2740 E-11$

$0.1161 \mathrm{E}-13$

$0.3983 \mathrm{E}-13$

$0.6379 \mathrm{E}-17$

ZERO

$0.1043 \mathrm{E}-10$

ZERO

ZERO

ZERO

ZERO

$0.2939 \mathrm{E}-13$

$0.7795 \mathrm{E}-18$

$0.2817 \mathrm{E}-12$

$0.6676 \mathrm{E}+02$

ZERO

ZERO.

$0.1537 \mathrm{E}-12$

$0.4550 \mathrm{E}-15$

ZERO

$0.1155 \mathrm{E}-13$

$0.1483 \mathrm{E}-15$

$0.8669 \mathrm{E}-15$

ZERO

$0.9472 \mathrm{E}-14$

ZERO

ZERO

ZERO

ZERO

ZERO

ZERO

ZERO

ZERO

ZERO

ZERO

ZERO

ZERO

ZERO

ZERO

ZERO

ZERO

ZERO

ZERO

ZERO

ZERO

$.2802 E-05$

$0.4074 \mathrm{E}-07$

$0.6525 \mathrm{E}-11$

ZERO

ZERO

ZERO

ZERO
$0.1066 \mathrm{E}-04$

ZERO

ZERO

ZERO

ZERO

ZERO

ZERO

ZERO

ZERO

$0.3006 \mathrm{E}-07$

$0.7973 \mathrm{E}-12$

$0.2881 \mathrm{E}-06$

$0.9261 \mathrm{E}-10$

ZERO

TRACE

$0.1572 \mathrm{E}-06$

$0.4654 \mathrm{E}-09$

ZERO
ZERO

$0.1181 \mathrm{E}-07$

$0.1517 \mathrm{E}-09$

$0.8867 \mathrm{E}-09$

$0.9688 \mathrm{E}-08$

ZERO

$0.6676 \mathrm{E}+02$

ZERO

ZERO

ZERO

ZERO

ZTRO

ZERO

ZERO

ZERO

ZERO

ZERO

ZERO

ZERO

ZERO

ZERO

ZERO

ZERO

Date: January 1995

39

152

Inlet Vapor Liquid to

to FAVC MFT Scrubber

$\begin{array}{ll}0.2184 \mathrm{E}+01 & 0.4270 \mathrm{E}-08\end{array}$

ZERO

$0.1044 \mathrm{E}-17$

$0.6728 \mathrm{E}-16$

$0.1390 \mathrm{E}-12$

ZERO

ZERO

$0.1807 \mathrm{E}-12$

ZERO

ZERO

$0.5544 \mathrm{E}-14$

ZERO

$0.73 .12 \mathrm{E}-16$

ZERO

$.2226 \mathrm{E}-13$

$0.2826 \mathrm{E}-15$

$0.9706 \mathrm{E}-15$

$0.1555 \mathrm{E}-18$

$0.8404 \mathrm{E}-16$

$0.2562 \mathrm{E}-12$

ZERO

ZERO
ZERO

ZERO
ZERO

ZERO

$0.7159 \mathrm{E}-15$

$0.1894 \mathrm{E}-19$

$0.6755 \mathrm{E}-14$

$0.2206 \mathrm{E}-17$

$0.4168 \mathrm{E}-21$

ZERO

$0.3743 \mathrm{E}-14$

$0.3171 \mathrm{E}-18$
ZERO

TRACE

$0.2813 \mathrm{E}-15$

$0.3604 \mathrm{E}-17$

$0.6044 \mathrm{E}-18$

TRACE

$0.2305 \mathrm{E}-15$

ZERO

ZERO
$0.1234 \mathrm{E}-09$

$0.7953 \mathrm{E}-08$

$0.1644 \mathrm{E}-04$

ZERO

ZERO

$0.2136 \mathrm{E}-04$

ZERO

ZERO

ZERO

$0.8644 \mathrm{E}-08$

ZERO

$0.2632 \mathrm{E}-05$

$0.3341 \mathrm{E}-07$

$0.1147 \mathrm{E}-06$

$0.1838 \mathrm{E}-10$

$0.9936 E-08$

ZERO

ZERO

ZERO

ZERO

$0.8463 \mathrm{E}-07$

$0.2239 \mathrm{E}-11$

$0.7439 \mathrm{E}-02$

$0.2608 E+09$ 
Table A-1. HLW Flowsheet Material Balande (Tank 51/ITP Cyole 1) Seation 4B. Frit Adjustment of Melter Feed

STREAM NUMBERS (CON'T'D) $\rightarrow$ STREAM NAME

$38 \quad 502$

141503

137 Semi Vol Cs2O

136 Semi Vol $\mathrm{CsCl}$

126 Semi Vol Group A

140 Semi Vol Na2B407

138 Semi Vol NaCl

139 Semi Vol NaF

125 Semi Vol NaI

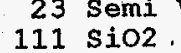

$48 \mathrm{Sx}(\mathrm{COOH}) 2$

176 Sr (NO3) 2

$153 \mathrm{Sr}(\mathrm{NaTi2O5)} 2$

$127 \mathrm{Sr}(\mathrm{OH}) 2$

$30 \mathrm{SxCO} 3$

46 SrO

$159 \cdot \mathrm{TCO} 2$

92 ThO2

119 Tritium
17 v308

$62 \mathrm{UO} 2$ ( $\mathrm{COOH}) 2$

177 UO2 (NO3) 2

$16 \mathrm{VO2}(\mathrm{OH}) 2$

$27 \mathrm{Y}(\mathrm{COOH}) 3$

$178 \mathrm{Y}(\mathrm{NO} 3) 3$
$128 \mathrm{Y}(\mathrm{OH}) 3$

$31 \mathrm{Y} 2(\mathrm{CO} 3)^{3}$

$47 \mathrm{Y} 2 \mathrm{O} 3$

100 Zeolite

$44 \mathrm{Zn}(\mathrm{COOH}) 2$

$179 \mathrm{Zn}(\mathrm{NO} 3) 2$

57 Zno

13 hydrate H2O

1 water

TOTAL ELOW, LB/HR

TEMPERATURE, DEG C

PRESSURE， ATM

PRESSURE， PSIG

PRESSURE, MM HG

ENTHALPY, PCU/HR

VAPOR FLOW, CFM

LIQUID FLOW, GPM

DENSITY， LBS/FT3

PHASE

WSRC-TR-95-0019 (Revision $\begin{array}{ll}29 & 30 \\ \text { SME Sample SME Transfer }\end{array}$

Pump Prime Pump Prime

ZERO

ZERO

ZERO

ZERO

ZERO

ZERO

ZERO

ZERO

ZERO

ZERO

ZERO

ZERO

ZERO

ZERO

ZERO

ZERO

ZERO

ZERO

ZERO

ZERO

ZERO

ZERO
ZERO
ZERO

ZERO
ZERO

ZERO

ZERO

ZERO

ERO

ZERO

ZERO

ZERO

ZERO

$0.1950 \mathrm{E}+01$ $0.1950 \mathrm{E}+01$

$0.2500 \mathrm{E}+02$

$0.1000 \mathrm{E}+01$ ZERO

$0.4893 \mathrm{E}+02$

$0.3894 \mathrm{E}-02$ $.6243 \mathrm{E}+02$

LIQUID

ZERO

ZERO

ZERO

ZERO

ZERO

ZERO

ZERO

ZERO

ZERO

ZERO

ZERO

ZERO

ZERO

ZERO

ZERO

ZERO

ZERO

ZERO

ZERO

ZERO

ZERO

ZERO

ZERO

ZERO

ZERO

ZERO

ZERO

ZERO

ZERO

ZERO

ZERO

ZERO

$0.1950 \mathrm{E}+01$

$0.1950 \mathrm{E}+01$

$0.2500 E+02$

$0.1000 \mathrm{E}+01$

ZERO

$0.4893 \mathrm{E}+02$

$0.3894 \mathrm{E}-02$

$0.6243 \mathrm{E}+02$

LIQUID
$55 \quad 32$

to Scrubber to SMECT Vent to FAVC

$\begin{array}{llll}\text { ZERO } & \text { ZERO } & \text { ZERO } & \text { ZERO } \\ \text { ZERO } & \text { ZERO } & \text { ZERO } & \text { ZERO } \\ \text { ZERO } & \text { ZERO } & \text { ZERO } & \text { ZERO }\end{array}$

ZERO

ZERO

ZERO

ZERO

ZERO

ZERO

0.1434E-09

$0.3432 \mathrm{E}-13$

$0.4748 \mathrm{E}-14$

ZERO
ZERO

$0.3465 \mathrm{E}-13$

$0.9687 \mathrm{E}-12$

$0.1157 \mathrm{E}-10$

$0.2430 \mathrm{E}-11$

$0.1483 \mathrm{E}-17$

$0.1065 \mathrm{E}-15$

ZERO

$.1536 \mathrm{E}-23$

$0.9257 \mathrm{E}-14$

ZERO

$0.1129 \mathrm{E}-14$

ZERO

$0.3040 \mathrm{E}-12$

$0.6221 \mathrm{E}-16$

$0.2091 \mathrm{E}-12$

ZERO

$0.1748 \mathrm{E}-11$

$0.2548 \mathrm{E}+02$

$0.3134 \mathrm{E}+03$

$0.5000 \mathrm{E}+02$

$0.9656 \mathrm{E}+00$

$-0.5055 \mathrm{E}+00$

$0.1990 \mathrm{E}+05$

$0.8351 \mathrm{E}+02$

$0.6254 \mathrm{E}-01$

VAPOR
$0.4161 \mathrm{E}-14$

ZERO

ZERO

ZERO

ZERO

ZERO

ZERO

$0.1466 \mathrm{E}-03$

$0.1303 \mathrm{E}-09$

$0.3510 \mathrm{E}-07$

$0.4856 \mathrm{E}-08$

ZERO

$0.2644 \mathrm{E}-24$

$0.4256 \mathrm{E}-08$

$0.3544 \mathrm{E}-07$

$0.9908 \mathrm{E}-06$

$0.1454 \mathrm{E}-09$

0. 2485E-05

$0.1517 \mathrm{E}-11$

$0.1090 \mathrm{E}-09$

ZERO

$0.1571 \mathrm{E}-17$

$0.9468 \mathrm{E}-08$

ZERO

$0.1155 \mathrm{E}-08$

ZERO

$0.3109 \mathrm{E}-06$

$0.6363 \mathrm{E}-10$

$0.2139 \mathrm{E}-06$

$0.1788 \mathrm{E}-05$

$0.3202 \mathrm{E}+03$

$0.3205 \mathrm{E}+03$

$0.5000 \mathrm{E}+02$

$0.9656 \mathrm{E}+00$

$-0.5055 \mathrm{E}+00$

$0.7339 \mathrm{E}+03$

$0.6400 \mathrm{E}+00$

$0.6244 \mathrm{E}+02$

LIQUID

Date: January 1995

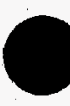


Table A-1. HLW Flowsheet Material Balance (Tank 51/ITR Cycle 1) section 4B. Frit Adjustment of Melter Feed
56 57
54
153

34

35

Liquid to spnt Liq frm Iiquid to spnt Liq frm Spnt Liq frm SMECT Sample SMECT Trnsfr STREAM NAME

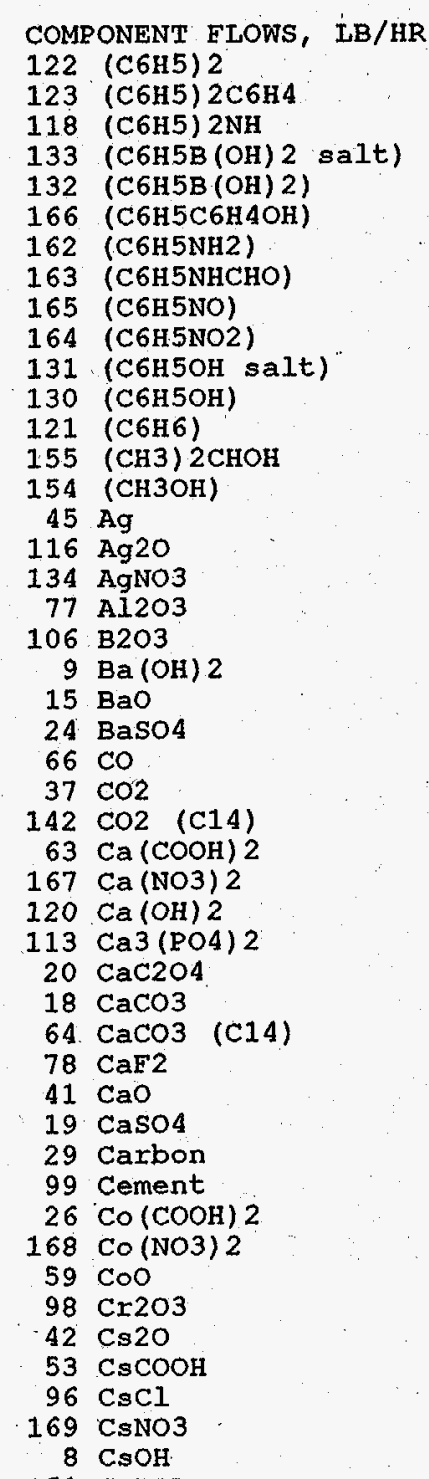
SME Scrubber SME Scrubber SRAT Scrubbr SRAT Scrubbr MFT Scrubber Pump Prime

Pump Prime

\begin{tabular}{|c|c|c|c|c|c|c|}
\hline $\begin{array}{l}0.9475 \mathrm{E}-03 \\
0.1179 \mathrm{E}-05 \\
0.1669 \mathrm{E}-01\end{array}$ & $\begin{array}{l}0.9427 \mathrm{E}-03 \\
0.1145 \mathrm{E}-05 \\
0.1618 \mathrm{E}-01\end{array}$ & $\begin{array}{l}0.1877 \mathrm{E}-02 \\
0.2336 \mathrm{E}-05 \\
0.3306 \mathrm{E}-01\end{array}$ & $\begin{array}{l}0.1133 \mathrm{E}-01 \\
0.2268 \mathrm{E}-05 \\
0.3204 \mathrm{E}-01\end{array}$ & $\begin{array}{l}0.5543 \mathrm{E}-03 \\
0.3246 \mathrm{E}-06 \\
0.4609 \mathrm{E}-02\end{array}$ & $\begin{array}{l}\text { ZERO } \\
\text { ZERO } \\
\text { ZERO }\end{array}$ & $\begin{array}{l}\text { ZERO } \\
\text { ZERO } \\
\text { ZERO }\end{array}$ \\
\hline ZERO & ZERO & ZERO & ZERO & ZERO & ZERO & \\
\hline ZERO & ZERO & ZERO & ZERO & ZERO & ZERO & ZER \\
\hline $0.6540 \mathrm{E}-06$ & $0.6351 \mathrm{E}-06$ & $0.1295 \mathrm{E}-05$ & $0.1258 \mathrm{E}-05$ & $0.1809 \mathrm{E}-06$ & ZERO & ZER \\
\hline $0.1763 \mathrm{E}+00$ & $0.1715 \mathrm{E}+00$ & $0.3492 \mathrm{E}+00$ & $0.3587 \mathrm{E}+00$ & $0.4884 \mathrm{E}-01$ & ZERO & $\mathrm{ZER}$ \\
\hline $0.2479 \mathrm{E}-05$ & $0.2407 \mathrm{E}-05$ & $0.4910 \mathrm{E}-05$ & $0.4768 \mathrm{E}-05$ & $0.6856 \mathrm{E}-06$ & ZERO & ZER \\
\hline $0.2697 \mathrm{E}-07$ & $0.2527 \mathrm{E}-07$ & $0.5342 \mathrm{E}-07$ & $0.4757 \mathrm{E}-07$ & $0.6953 \mathrm{E}-08$ & ZERO & \\
\hline $0.8217 \mathrm{E}-07$ & $0.7979 \mathrm{E}-07$ & $0.1627 \mathrm{E}-06$ & $0.1580 \mathrm{E}-06$ & $0.2272 \mathrm{E}-07$ & ZERO & ZER \\
\hline $0.2079 E-08$ & $0.1758 \mathrm{E}-08$ & $0.4118 \mathrm{E}-08$ & $0.3482 \mathrm{E}-08$ & $0.2188 \mathrm{E}-08$ & ZERO & ZER \\
\hline $0.2146 \mathrm{E}+01$ & $0.2096 \mathrm{E}+01$ & $0.4250 \mathrm{E}+01$ & $0.4248 \mathrm{E}+01$ & $0.5910 \mathrm{E}+00$ & ZERO & ZEF \\
\hline $0.2611 \mathrm{E}-02$ & $0.2543 \mathrm{E}-02$ & $0.5172 \mathrm{E}-02$ & $0.5266 \mathrm{E}-02$ & $0.1425 \mathrm{E}-02$ & ZERO & ZER \\
\hline $0.1791 \mathrm{E}-09$ & $0.1726 \mathrm{E}-09$ & $0.3547 \mathrm{E}-09$ & $0.3510 \mathrm{E}-09$ & $0.4844 \mathrm{E}-10$ & ZERO & ZER \\
\hline $0.1706 \mathrm{E}-10$ & $0.1624 \mathrm{E}-10$ & $0.3379 \mathrm{E}-10$ & $0.3417 \mathrm{E}-10$ & $0.4525 \mathrm{E}-11$ & ZERO & ZEE \\
\hline ZERO & $0.1447 E-13$ & ZERO & $0.7799 \mathrm{E}-13$ & $0.2459 \mathrm{E}-09$ & ZERO & ZER \\
\hline $0.3149 \mathrm{E}-15$ & $0.2736 \mathrm{E}-15$ & $0.6236 \mathrm{E}-1.5$ & $0.5418 \mathrm{E}-15$ & $0.3095 \mathrm{E}-15$ & ZERO & ZEF \\
\hline ZERO & ZERO & ZERO & ZERO & ZERO & ZERO & ZER \\
\hline $0.1048 \mathrm{E}-03$ & $0.1018 \mathrm{E}-03$ & $0.2075 E-03$ & $0.2016 \mathrm{E}-03$ & $0.2899 E-04$ & ZERO & \\
\hline $0.3797 \mathrm{E}-04$ & $0.3686 \mathrm{E}-04$ & $0.7521 \mathrm{E}-04$ & $0.7301 \mathrm{E}-04$ & $0.1069 \mathrm{E}-04$ & ZERO & ZER \\
\hline ZERO & ZERO & ZERO & ZERO & ZERO & ZERO & ZER \\
\hline $0.3975 \mathrm{E}-13$ & $0.3453 \mathrm{E}-13$ & $0.7872 \mathrm{E}-13$ & $0.6839 \mathrm{E}-13$ & $0.3908 \mathrm{E}-13$ & ZERO & ZER \\
\hline $0.2020 \mathrm{E}-06$ & $0.1962 \mathrm{E}-06$ & $0.4002 \mathrm{E}-06$ & $0.3887 \mathrm{E}-06$ & $0.5589 \mathrm{E}-07$ & ZERO & ZER \\
\hline ZERO & - ZERO & ZERO & ZERO & ZERO & ZERO & ZER \\
\hline $0.3709 \mathrm{E}-03$ & $0.5797 \mathrm{E}-03$ & $0.7346 \mathrm{E}-03$ & $0.1412 \mathrm{E}-01$ & $0.2569 \mathrm{E}-04$ & ZERO & ZER \\
\hline $0.4786 \mathrm{E}-11$ & $0.3237 \mathrm{E}-13$ & $0.9480 \mathrm{E}-11$ & $0.1899 \mathrm{E}-09$ & $0.1085 \mathrm{E}-13$ & ZERO & \\
\hline ZERO & ZERO & ZERO & ZERO & ZERO & ZERO & ZER \\
\hline $0.4967 \mathrm{E}-04$ & $0.4825 \mathrm{E}-04$ & $0.9838 \mathrm{E}-04$ & $0.9556 \mathrm{E}-04$ & $0.1374 \mathrm{E}-04$ & ZERO & ZER \\
\hline ZERO & ZERO & ZERO & ZERO & ZERO & ZERO & ZEP \\
\hline $0.3113 \mathrm{E}-04$ & $0.3023 E-04$ & $0.6166 \mathrm{E}-04$ & $0.5988 \mathrm{E}-04$ & $0.8611 \mathrm{E}-05$ & ZERO & ZER \\
\hline $0.2889 \mathrm{E}-11$ & $0.2806 \mathrm{E}-11$ & $0.5723 \mathrm{E}-11$ & $0.5557 \mathrm{E}-11$ & $0.7991 \mathrm{E}-12$ & ZERO & ZER \\
\hline ZERO & ZERO & ZERO & ZERO & ZERO & ZERO & ZER \\
\hline ZERO & ZERO & ZERO & ZERO & ZERO & ZERO & ZER \\
\hline $0.1780 \mathrm{E}-06$ & $0.1729 \mathrm{E}-06$ & $0.3526 \mathrm{E}-06$ & $0.3425 \mathrm{E}-06$ & $0.4924 \mathrm{E}-07$ & ZERO & ZER \\
\hline $0.3971 \mathrm{E}-07$ & $0.3857 E-07$ & $0.7866 \mathrm{E}-07$ & $0.7639 \mathrm{E}-07$ & $0.1102 \mathrm{E}-07$ & ZERO & ZER \\
\hline $0.2613 \mathrm{E}-05$ & $0: 2538 \mathrm{E}-05$ & $0.5176 \mathrm{E}-05$ & $0.5026 \mathrm{E}-05$ & $0.7227 \mathrm{E}-06$ & ZERO & ZER \\
\hline ZERO & ZERO & ZERO & ZERO & ZERO & ZERO & ZER \\
\hline ZERO & ZERO & ZERO & ZERO & ZERO & ZERO & ZER \\
\hline $0.1695 \mathrm{E}-08$ & $0.1646 \mathrm{E}-08$ & $0.3358 \mathrm{E}-08$ & $0.3260 \mathrm{E}-08$ & $0.4688 \mathrm{E}-09$ & ZERO & ZER \\
\hline $0.6358 \mathrm{E}-11$ & $0.6173 \mathrm{E}-11$ & $0.1259 \mathrm{E}-10$ & $0.1223 \mathrm{E}-10$ & $0.1758 \mathrm{E}-11$ & ZERO & ZER \\
\hline $0.3631 \mathrm{E}-09$ & $0.3526 \mathrm{E}-09$ & $0.7193 \mathrm{E}-09$ & $0.6984 \mathrm{E}-09$ & $0.1004 \mathrm{E}-09$ & ZERO & ZER \\
\hline $0.5084 \mathrm{E}-05$ & $0.4937 \mathrm{E}-05$ & $0.1007 \mathrm{E}-04$ & $0.9778 E-05$ & $0.1406 \mathrm{E}-05$ & ZERO & ZER \\
\hline $0.1664 \mathrm{E}-08$ & $0.1616 \mathrm{E}-08$ & $0.3295 \mathrm{E}-08$ & $0.3200 E-08$ & $0.4610 \mathrm{E}-09$ & ZERO & ZER \\
\hline $0.5124 \mathrm{E}-06$ & $0.4975 \mathrm{E}-06$ & $0.1015 \mathrm{E}-0.5$ & $0.9853 \mathrm{E}-06$ & $0.1417 \mathrm{E}-06$ & ZERO & ZER \\
\hline ZERO & ZERO & ZERO & ZERO & ZERO & ZERO & ZER \\
\hline $0.1140 \mathrm{E}-09$ & $0.1107 \mathrm{E}-09$ & $0.2257 E-09$ & $0.2192 \mathrm{E}-09$ & $0.3152 \mathrm{E}-10$ & ZERO & ZER \\
\hline $0.1300 \mathrm{E}-10$ & $0.1129 \mathrm{E}-10$ & $0.2574 \mathrm{E}-10$ & $0.2236 \mathrm{E}-10$ & $0.1278 \mathrm{E}-10$ & ZERO & ZER \\
\hline ZERO & ZERO & ZERO & ZERO & ZERO & ZERO & ZER \\
\hline
\end{tabular}

151 CSTPB 
Table A-1. HLW Flowsheet Material Balance (Tank 51/ITR Cycle 1) . Section 4B. Frit Adjustment of Melter Feed

STREAM NUMBERS (CONT'D) $\rightarrow$ STREAM NAME

\begin{tabular}{|c|c|}
\hline 54 & $\mathrm{Cu}(\mathrm{COOH}) 2$ \\
\hline 170 & $\mathrm{Cu}(\mathrm{NO} 3) 2$ \\
\hline 55 & $\mathrm{CuO}$ \\
\hline 80 & Fe203 \\
\hline 101 & Feo \\
\hline 102 & Group A \\
\hline 103 & Group B \\
\hline 25 & \\
\hline & $\mathrm{H} 2 \mathrm{C} 2 \mathrm{O} 4$ \\
\hline 93 & H2SO4 \\
\hline 90 & $\mathrm{H} 3 \mathrm{BO} 3$ \\
\hline 34 & $\mathrm{HCOOH}$ \\
\hline 43 & $\mathrm{HCl}$ \\
\hline 85 & $\mathrm{HF}$ \\
\hline 49 & HNO3 \\
\hline 36 & $\mathrm{Hg}$ \\
\hline 129 & $\mathrm{Hg}(\mathrm{C} 6 \mathrm{H} 5)^{2}$ \\
\hline 135 & $\mathrm{Hg}(\mathrm{NO} 3)^{2}$ \\
\hline 144 & $\mathrm{Hg} 2 \mathrm{Cl} 2$ \\
\hline 117 & $\mathrm{Hg} 2 \mathrm{I2}$ \\
\hline 146 & $\mathrm{HgCl2}$ \\
\hline 21 & $\mathrm{HgO}$ \\
\hline 97 & \pm 2 \\
\hline 183 & $\mathrm{~K} 2 \mathrm{CO} 3$ \\
\hline 39 & $\mathrm{~K} 2 \mathrm{O}$ \\
\hline 51 & $\mathrm{KCOOH}$ \\
\hline 157. & $\mathrm{KMnO} 4$ \\
\hline 161 & KNO3 \\
\hline 10 & $\mathrm{KOH}$ \\
\hline 150 & KTPB \\
\hline 105 & $\mathrm{La2O3}$ \\
\hline 108 & Li20 \\
\hline 65 & $\mathrm{Mg}(\mathrm{COOH}) 2$ \\
\hline 17.1 & $\mathrm{Mg}(\mathrm{NO} 3) 2$ \\
\hline 33 & Mgo \\
\hline 60 & $\mathrm{Mn}(\mathrm{COOH}) 2$ \\
\hline 172 & $\mathrm{Mn}(\mathrm{NO} 3) 2$ \\
\hline 180 & Mn304 \\
\hline 56 & Mno \\
\hline 14 & $\mathrm{MnO} 2$ \\
\hline 145 & $\mathrm{MoO} 2$ \\
\hline 81 & N2 \\
\hline 74 & $\mathrm{~N} 2 \mathrm{O}$ \\
\hline 182 & NH3 \\
\hline 114 & NH3OHNO3 \\
\hline 52 & $\mathrm{NH} 4 \mathrm{COOH}$ \\
\hline 173 & NH4 4 NO3 \\
\hline 68 & $\mathrm{NH} 4 \mathrm{OH}$ \\
\hline 148 & NH4TPE \\
\hline
\end{tabular}

$$
57
$$$$
53
$$
153 Liquid to Spnt Liq frm Liquid to Spnt Liq frm Spnt Liq frm SMECT Sample SMECT Trnsfr Liquid to Spnt Liq frm Liquid to Spnt Liq frm Spnt Liq frm SMECT Sample SMECT Trnsfr 34

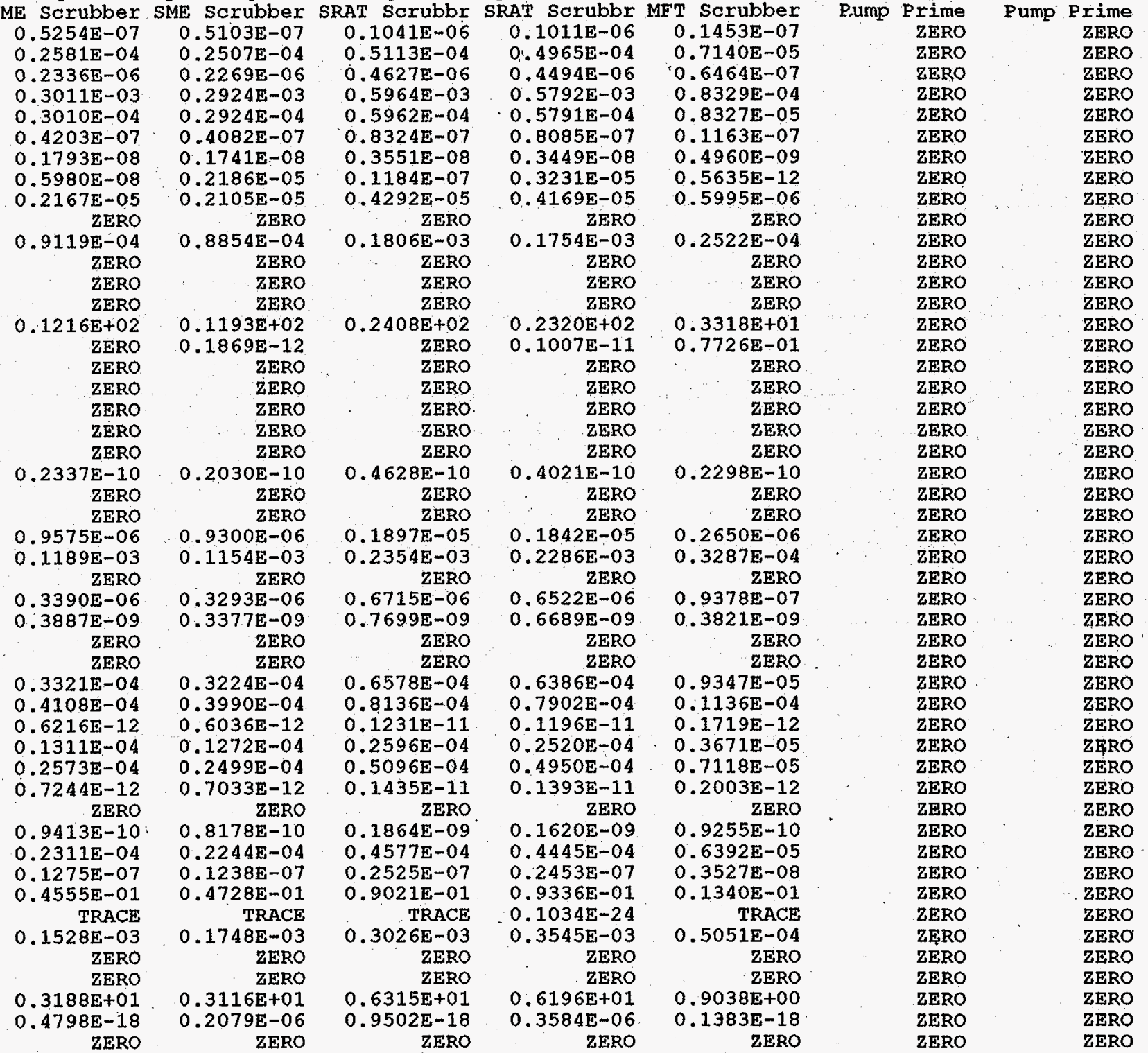


Table A-1. HiW Flowsheet Material Balance (Tank 51/ITP Cycle 1) section 4B. Frit Adjustment of Melter Feed

STREAM NUMBERS (CONT'D) $\rightarrow$ STREAM NAME

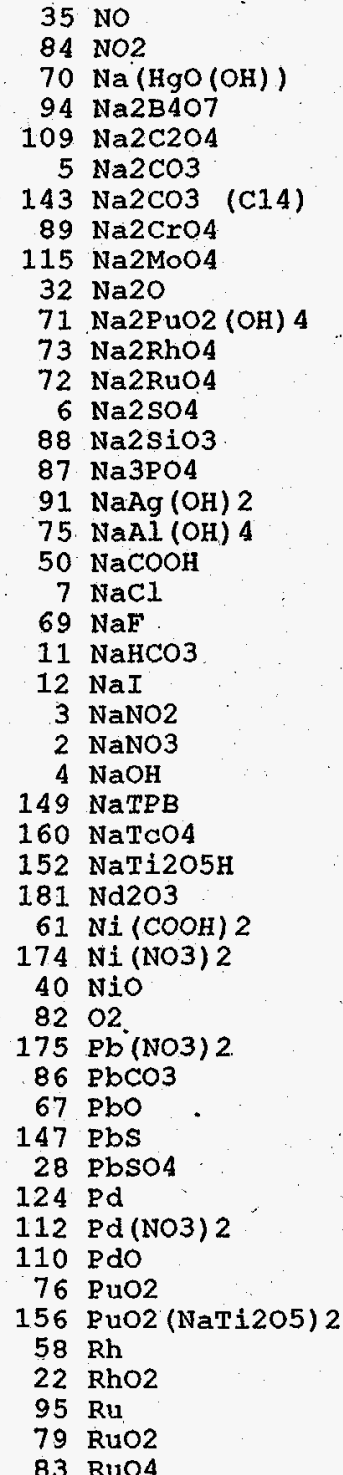


Table A-1. HLW Flowsheet Material Balance (Tank 51/ITP Cycle 1)

Section 4B. Frit Adjustment of Melter Feed

STREAM NUMBERS (CONT'D) $\rightarrow$ STREAM NAME

$38 \mathrm{SO} 2$

$141 \mathrm{SO} 3$

137 Semi Vol Cs2O

136 Semi Vol CsCl

126 Semi Vol Group A

140 Serni Vol Na2B4O

138 Semi Vol NaCl

125 Semi Vol NaI

23 Semi Vol RuO2

$111 \mathrm{SiO} 2$

$48 \mathrm{Sr}(\mathrm{COOH}) 2$

$176 \mathrm{Sr}$ (NO3) 2
$153 \mathrm{Sr}$ (NaTi2O5)

$\begin{array}{rl}153 & \mathrm{Sr}(\mathrm{NaTi} \\ 127 \mathrm{Sr}(\mathrm{OH}) 2 \\ 30 \mathrm{SrCO} 3\end{array}$

46 Sro

$159 \mathrm{TaO} 2$

107 TiO2

11.9 Tritium

17 U308

$62 \mathrm{OO2}(\mathrm{COOH}) 2$

$177 \mathrm{OO} 2$ (NO3) 2

$\begin{array}{ll}16 & \mathrm{OO} 2(\mathrm{OH}) 2 \\ 27 & \mathrm{Y}(\mathrm{COOH}) \cdot 3\end{array}$

$\begin{aligned} 27 & \Psi(\mathrm{COOH}) \cdot 3 \\ 178 & \Psi(\mathrm{NO} 3) 3\end{aligned}$

128 Y (OH) 3

$31 \mathrm{Y} 2$ (CO3) 3

$47 \mathrm{Y} 203$

100 Zeolite

$179 \mathrm{Zn}$ (NO3) 2

$\begin{array}{rl}179 & \mathrm{Zn}(\mathrm{N}) \\ 57 & \mathrm{ZnO}\end{array}$

104 zrO2

13 hydrate $\mathrm{H} 2 \mathrm{O}$

1 water

TOTAL FLOW, LB/HR

TEMPERATURE， DEG C

PRESSURE, ATM

PRESSURE, PSIG

PRESSURE， MM HG

VAPOR FLOW, CFM

VAPOR FLOW, CFM

LIQUID FLOW, GPM
DENSITY, LBS/FT3

PHASE

WSRC-TR-95-0019 (Revision
56 57 53 54 153 34

Liquid to Spnt Liq frm Liquid to spnt Liq frm spnt Liq frm SMECT Sample SMECT Trnsfr SME Scrubber SME Scrubber SRAT Scrubbr SRAT Scrubbr MFT Scrubber. Pump Prime Pump Prime

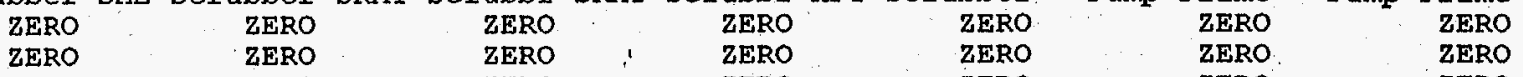
$\begin{array}{lllllll}\text { ZERO } & \text { ZERO } & \text { ZERO } & \text { ZERO } & \text { ZERO } & \text { ZERO } & \text { ZERO } \\ \text { ZERO } & \text { ZERO } & \text { ZERO } & \text { ZERO } & \text { ZERO } & \text { ZERO } & \text { ZERO }\end{array}$

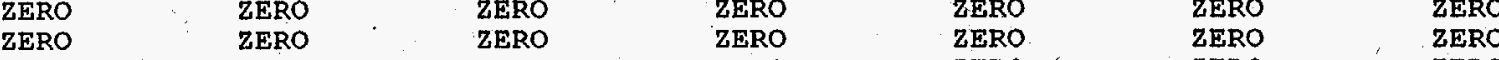

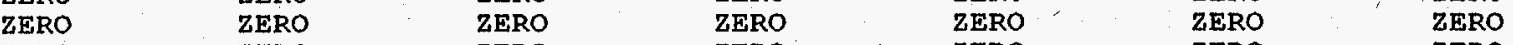

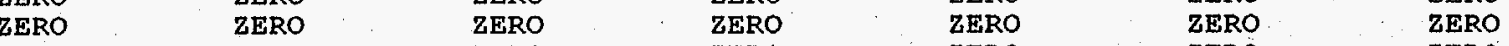

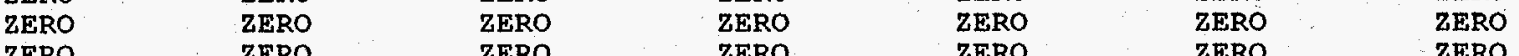

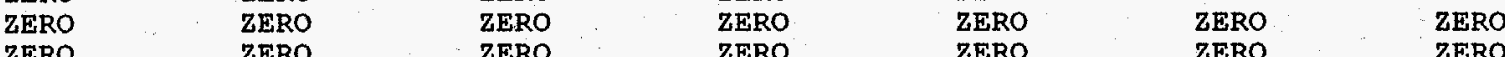

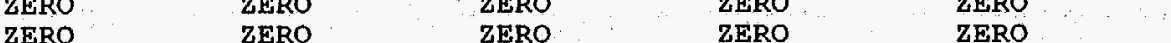

2. ZERO

$0.1293 \mathrm{E}-08$

$0.1293 \mathrm{E}-08$

$0.3492 \mathrm{E}-06$
$0.4818 \mathrm{E}-07$

ZERO
ZERO

$0.3672 \mathrm{E}-03$

$0.1255 \mathrm{E}-08$

$0.3391 \mathrm{E}-06$

$0.2561 \mathrm{E}-08$

$0.6916 \mathrm{E}-0$

$0.6916 \mathrm{E}-06$

$0.2487 \mathrm{E}-08$

$\begin{array}{ll}0.7273 \mathrm{E}-03 & 0.1064 \mathrm{E}-03 \\ 0.2487 \mathrm{E}-08 & 0.3576 \mathrm{E}-09 \\ 0.6717 \mathrm{E}-06 & 0.9659 \mathrm{E}-07\end{array}$

$0.3576 \mathrm{E}-09$

$0.9659 \mathrm{E}-07$ ZERO
ZERO

$0.5450 \mathrm{E}-12$

$0.4234 \mathrm{E}-07$

$0.3525 \mathrm{E}-06$

$0.9833 E-05$

$0.2091 \mathrm{E}-08$

$0.2472 \mathrm{E}-04$

$0.1505 \mathrm{E}-10$

$0.1084 \mathrm{E}-08$

ZERO

$0.1559 \mathrm{E}-16$

$0.9418 \mathrm{E}-07$

ZERO

$0.1146 \mathrm{E}-07$

ZERO

$0.3087 \mathrm{E}-05$

$0.6313 \mathrm{E}-09$

$0.2125 \mathrm{E}-05$

ZERO

$0.1778 \mathrm{E}-04$

$0.3811 \mathrm{E}+04$

$0.3829 \mathrm{E}+04$

$0.4900 \mathrm{E}+02$

$0.1000 \mathrm{E}+01$ ZERO

$0.4734 \mathrm{E}-12$

$0.4112 \mathrm{E}-07$

$0.3424 \mathrm{E}-06$

$0.9548 \mathrm{E}-05$

$0.2030 \mathrm{E}-08$

$0.2401 E-04$

$0.1461 \mathrm{E}-10$

$0.1053 E-08$

- ZERO

$0.1514 \mathrm{E}-16$

$0.9148 \mathrm{E}-07$

ZERO

$0.1112 \mathrm{E}-07$ ZERO
$8 \mathrm{E}-05$

$0.2998 \mathrm{E}-05$

$0.2063 \mathrm{E}-05$

$0.1727 \mathrm{E}-04$

$0.3830 \mathrm{E}+04$

$0.5000 \mathrm{E}+02$

ZERO

$0.1079 \mathrm{E}-11$

$0.8385 \mathrm{E}-07$

$0.6983 \mathrm{E}-06$

$0.1948 \mathrm{E}-04$

$0.4141 \mathrm{E}-08$

$0.4896 \mathrm{E}-04$

$0.2981 \mathrm{E}-10$

$0.2146 \mathrm{E}-08$

ZERO

$0.3087 \mathrm{E}-16$

$.1865 \mathrm{E}-06$

ZERO

$0.2269 \mathrm{E}-07$

ZERO

$0.6114 \mathrm{E}-05$

$0.1250 \mathrm{E}-08$

$0.4208 \mathrm{E}-05$

ZERO

$0.3522 \mathrm{E}-04$

$0.7549 \mathrm{E}+04$

$.7584 \mathrm{E}+0$

$0.4900 \mathrm{E}+02$

$0.1000 \mathrm{E}+01$
ZERO

ZERO

$0.9377 \mathrm{E}-12$

$0.8144 \mathrm{E}-07$

$0.6782 \mathrm{E}-06$

$0.1891 \mathrm{E}-04$

$0.4060 \mathrm{E}-08$

$0.4755 \mathrm{E}-04$

$0.2895 \mathrm{E}-10$

$0.2085 E-08$

ZERO

$0.2998-16$

$0.1812 \mathrm{E}-06$

ZERO

$\begin{array}{r}\text { ZERO } \\ \hline\end{array}$

$0.5937 \mathrm{E}-05$

$0.4087 \mathrm{E}-05$

$0.3421 \mathrm{EERO}$

$0.7552 \mathrm{E}+04$

$0.7586 \mathrm{E}+04$

$0.5000 \mathrm{E}+02$

$0.1000 \mathrm{E}+01$

ZERO

$0.1873 E+06$

$0.1911 E+06$

$0.3709 \mathrm{E}+06$

$0.3786 \mathrm{E}+06$

$0.7639 \mathrm{E}+01$

$0.7637 \mathrm{E}+01$

$0.6251 \mathrm{E}+02$

$0.1513 \mathrm{E}+02$

$0.1513 \mathrm{E}+02$

$0.6251 \mathrm{E}+02$

LIQUID

ZERO
ZERO

$0.5358 \mathrm{E}-12$

$0.1171 \mathrm{E}-07$

$0.9753 \mathrm{E}-07$

$0.2719 \mathrm{E}-05$

$0.5729 \mathrm{E}-09$

$0.6838 \mathrm{E}-05$

$0.4162 \mathrm{E}-11$

ZERO

$0.3168 \mathrm{E}-08$
ZERO

$0.1746 \mathrm{E}-09$

0.5877 E-06

$0.1080 \mathrm{E}+04$

$0.1085 \mathrm{E}+04$

$0.5000 \mathrm{E}+02$

$0.1000 E+01$

ZERO

$0.5412 \mathrm{E}+05$

$0.2163 \mathrm{E}+01$

LIQUID
ZERO

$0.1000 \mathrm{E}+01$
ZERO

ZERO

ZERO

ZERO

ZERO

ZERO

ZERO ZERO

ZERO ZERO

ZERO ZERO

ZERO ZERO

ZERO $\because$ ZERO

ZERO ZERO

ZERO ZERO ZERO

ZERO ZERO

ZERO ZERO

$\begin{array}{rrr}\text { ZERO } & \text { ZERO } & \text { ZERO } \\ 0.4311 \mathrm{E}-17 & \text { ZERO } & \text { ZERO }\end{array}$

ZERO

ZERO

$0.8538 \mathrm{E}-06$

ZERO

ZERO

ZERO

ZERO

ZERO

ZERO

ZERO

$0.3890 \mathrm{E}+01$

$0.3890 \mathrm{E}+01$

$0.2500 \mathrm{E}+02$

$0.1000 \mathrm{E}+01$

ZERO

$0.9761 \mathrm{E}+02$

$0.2459 \mathrm{E}+02$

$0.7768 \mathrm{E}-02$

LIQUID

$0.1957 \mathrm{E}-02$

IIOUID

Section $4 \mathrm{~B}$, Page 13 of 29 
Table A-1. HLW Flowsheet Material Balance (Tank 51/ITP Cycle 1)

STREAM NUMBERS $\rightarrow$ STREAM NAME

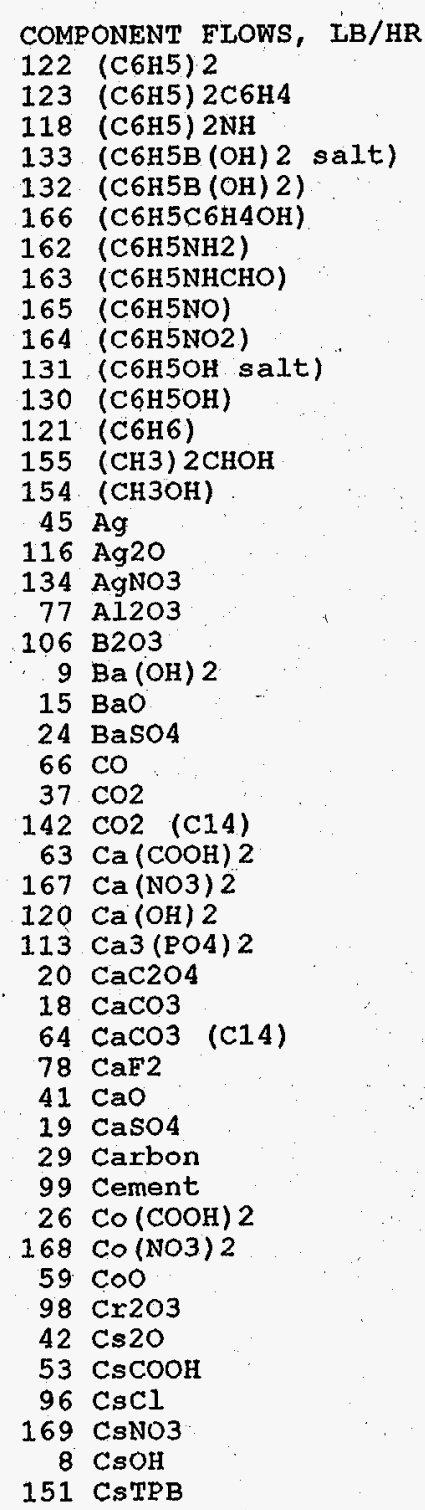

section 4B. Frit Adjustment of Melter Feed
SMECT Air ${ }^{\mathrm{Hg} A c c u m}$ MWWT Vent Cond fr MWWT
Inleakage in PRD - PR Inleakage in $\mathrm{PRD}-\mathrm{PR}$ $\begin{array}{llll}37 & 251 & 16 & 23\end{array}$
$0.1727 \mathrm{E}-10 \quad 0.7364 \mathrm{E}-03$

$0.5615 \mathrm{E}-17 \quad 0.3049 \mathrm{E}-06$

$0.6828 \mathrm{E}-11 \quad 0.6787 \mathrm{E}-02$

ZERO

$0.7119 \mathrm{E}-17$

$0.1495 \mathrm{E}-08$

$0.2245 \mathrm{E}-16$

$0.4769 \mathrm{E}-15$

$0.1847 \mathrm{E}-16$

$0.9786 \mathrm{E}-20$

$0.10 .41 \mathrm{E}-07$

$0.5040 \mathrm{E}-09$
$0.4770 \mathrm{E}-17$

$0.4770 \mathrm{E}-17$
$0.7912 \mathrm{E}-18$

$0.7971 \mathrm{E}-12$

TRACE
ZERÓ

$0.1713 \mathrm{E}-08$

ZERO

ZERO

ZERO

ZERO

ZERO

ZERO

ZERO

ZERO

ZERO

ZERO

ZERO

ZERO

ZERO

ZERO

ZERO

ZERO

ZERO

ZERO

ZERO

ZERO

ZERO

ZERO

ZERO

ZERO

ZERO

ZERO.

ZERO

ZERO

$0.4939 \mathrm{E}-15$

$0.1790 \mathrm{E}-15$

$0.1872 E-24$

$0.9524 \mathrm{E}-18$

ZERO

$0.1790 \mathrm{E}-09$
$0.2309 \mathrm{E}-17$

$0.2309 \mathrm{E}-17$
ZERO

$0.2342 \mathrm{E}-15$

ZERO
$0.1467 \mathrm{E}-15$

$0.1362 \mathrm{E}-22$

ZERO

$0.8391 \mathrm{E}-18$

$0.1872 \mathrm{E}-18$

$0.1232 \mathrm{E}-16$

ZERO

$0.7991 \mathrm{E}-20$

$0.2997 \mathrm{E}-22$

$0.1712 \mathrm{E}-20$
$0.2396 \mathrm{E}-16$

$0.7843 \mathrm{E}-20$

$0.2415 \mathrm{E}-17$

$0.5372 \mathrm{E}-21$

$0.6119 \mathrm{E}-22$

$$
\text { ZERO }
$$

$0.1969 \mathrm{E}-06$

$0.4892 \mathrm{E}-01$

$0.7458 \mathrm{E}-06$

$0.1214 \mathrm{E}-07$

$0.2474 \mathrm{E}-07$

$0.6394 \mathrm{E}+00$

$0.1449 \mathrm{E}-05$

$0.5622 \mathrm{E}-10$

$0.4976 \mathrm{E}-11$

ZERO
ZERO

ZERO

$0.3150 \mathrm{E}-04$

$0.5955 \mathrm{E}-08$

ZERO

TRACE

$0.6074 \mathrm{E}-07$

ZERO

$0.1250 \mathrm{E}-02$

$0.1681 \mathrm{E}-10$
ZERO

$0.1493 \mathrm{E}-04$

ZERO

$0.9362 E-05$

$0.8706 \mathrm{E}-12$

ZERO

$0.5351 \mathrm{E}-07$

$0.1192 \mathrm{E}-07$

$0.7872 \mathrm{E}-06$

ZERO

$0.5108 \mathrm{E}-09$

$0.1916 \mathrm{E}-11$

$0.1094 \mathrm{E}-09$

$0.1529 \mathrm{E}-05$

$0.1544 \mathrm{E}-06$

ZERO

$0.3425 \mathrm{E}-10$

ZERO

$\begin{array}{rl}42 & 106 \\ \text { FAVC } & 508 \\ \text { Condensate } & \text { to }\end{array}$

508 HNO3

$0.8137 \pm-02$

$0.3515 \mathrm{E}-10$

$0.7563 \mathrm{E}-04$

ZERO

$0.5712 \mathrm{E}-11$

$0.1807 \mathrm{E}-02$

$0.1744 \mathrm{E}-10$

$0.3767 \mathrm{E}-09$

$0.2342 \mathrm{E}-10$

$0.2489 \mathrm{E}-17$

$0.1132 \mathrm{E}-01$

$0.2696 \mathrm{E}-01$

$0.5861 \mathrm{E}-11$.

$.9643 \mathrm{E}-12$

$0.5617 \mathrm{E}-17$

$0.3770 \mathrm{E}-24$

$0.1255 \mathrm{E}-12$

$0.4547 \mathrm{E}-13$

$0.4759 \mathrm{E}-22$

$0.2419 \mathrm{E}-15$

ZERO

$0.2579 \mathrm{E}-03$

$0.3381 \mathrm{E}-11$

$0.5947 \mathrm{E}-13$

ZERO
$0.3727 \mathrm{E}-13$

$0.3460 \mathrm{E}-20$

ZERO

$0.2131 \mathrm{E}-15$

$0.4755 \mathrm{E}-16$

$0.3129 \mathrm{E}-14$

ZERO

$0.2030 \mathrm{E}-17$

$0.7612 \mathrm{E}-20$

$0.4348 \mathrm{E}-18$

$0.6087 \mathrm{E}-14$

$0.6134 \mathrm{E}-15$

$0.1364 \mathrm{E}-18$

$0.1556 \mathrm{E}-19$

ZERO

$$
\begin{aligned}
& \text { ZERO } \\
& \text { ZERO } \\
& \text { ZERO } \\
& \text { ZERO } \\
& \text { ZERO } \\
& \text { ZERO } \\
& \text { ZERO } \\
& \text { ZERO } \\
& \text { ZERO } \\
& \text { ZERO } \\
& \text { ZERO } \\
& \text { ZERO } \\
& \text { ZERO } \\
& \text { ZERO } \\
& \text { ZERO } \\
& \text { ZERO } \\
& \text { ZERO } \\
& \text { ZERO } \\
& \text { ZERO } \\
& \text { ZERO } \\
& \text { ZERO } \\
& \text { ZERO } \\
& \text { ZERO } \\
& \text { ZERO } \\
& \text { ZERO } \\
& \text { ZERO } \\
& \text { ZERO } \\
& \text { ZERO } \\
& \text { ZERO } \\
& \text { ZERO } \\
& \text { ZERO } \\
& \text { ZERO } \\
& \text { ZERO } \\
& \text { ZERO } \\
& \text { ZERO } \\
& \text { ZERO } \\
& \text { ZERO } \\
& \text { ZERO } \\
& \text { ZERO } \\
& \text { ZERO } \\
& \text { ZERO } \\
& \text { ZERO } \\
& \text { ZERO } \\
& \text { ZERO } \\
& \text { ZERO } \\
& \text { ZERO } \\
& \text { ZERO } \\
& \text { ZERO }
\end{aligned}
$$


Table A-1. HLW Flowsheet Material Balance (Tank 51/ITP Cycle 1)

A

STREAM NUMBERS (CONT'D) $\rightarrow$ STREAM NAME

$54 \mathrm{Cu}(\mathrm{COOH}) 2$

55 CuO

$80 \mathrm{Fe} 203$

$101 \mathrm{FeO}$

102 Group A

103 Group B

$25 \mathrm{H2}$

$158 \mathrm{H} 2 \mathrm{C} 2 \mathrm{O} 4$
$93 \mathrm{H} 2 \mathrm{SO} 4$

$90 \mathrm{H} 3 \mathrm{BO} 3$

$34 \mathrm{HCOOH}$

$43 \mathrm{HCl}$

$85 \mathrm{HF}$

49 HNO3

$36 \mathrm{Hg}$

$129 \mathrm{Hg}(\mathrm{C} 6 \mathrm{H} 5) 2$

$135 \mathrm{Hg}$ (NO3) 2

$144 \mathrm{Hg} 2 \mathrm{Cl} 2$

$117 \mathrm{Hg} 2 \mathrm{I2}$

$146 \mathrm{HgCl} 2$

$21 \mathrm{HgO}$

97 I2

$183 \mathrm{~K} 2 \mathrm{CO} 3$

$39 \mathrm{~K} 2 \mathrm{O}$

$51 \mathrm{KCOOH}$

$57 \mathrm{KMnO}$

$10 \mathrm{KOH}$

150 KTPB

$105 \mathrm{~L}$ a203

$108 \mathrm{Li}$
$65 \mathrm{Mg}(\mathrm{COOH})$

$171 \mathrm{Mg}$ (NO3) 2

$33 \mathrm{MgO}$

$60 \mathrm{Mn}(\mathrm{COOH}) 2$

$172 \mathrm{Mn}$ (NO3) 2

$180 \mathrm{Mn} 304$

$56 \mathrm{MnO}$

14. $\mathrm{MnO} 2$

$145 \mathrm{MOO}$

81 N2

$\begin{array}{rl}74 & \mathrm{~N} 2 \mathrm{O} \\ 182 & \mathrm{NH} 3\end{array}$

114 NH3OHNO3

$52 \mathrm{NH} 4 \mathrm{COOH}$

173 NH4NO3

$68 \mathrm{NH} 4 \mathrm{OH}$

WSRC-TR-95-0019 (Revision
36

SMECT Hg

Pump Water

ZERO

ZERO

ZERO

ZERO

ZERO

ZERO

ZERO

ZERO

ZERO

ZERO

ZERO

ZERO

ZERO

ZERO

ZERO

ZERO

ZERO

ZERO

ZERO

ZERO

ZERO

ZERO

ZERO

ZERO

ZERO

ZERO

ZERO

ZERO

ZERO

ZERO

ZERO

ZERO

ZERO

ZERO

ERO

ZERO

ZERO

ZERंO

ZERO

ZERO

ZERO

ZERO

ZERO

ZERO

ZERO

\begin{tabular}{|c|c|c|}
\hline $\begin{array}{l}37 \\
\text { SMECT Air }\end{array}$ & $\begin{array}{r}251 \\
\mathrm{Hg} A\end{array}$ & Ácum \\
\hline Inleakage & in $P R D$ & - PR \\
\hline ZERO & & ZERO \\
\hline ZERO & & ZERO \\
\hline ZERO & & ZERO \\
\hline ZERO & & ZERO \\
\hline ZERO & & ZERO \\
\hline ZERO & & ZERO \\
\hline ZERO & & ZERO \\
\hline ZERO & & ZERO \\
\hline ZERO & & ZERO \\
\hline ZERO & & ZERO \\
\hline ZERO & & ZERO \\
\hline ZERO & & ZERO \\
\hline ZERO & & ZERO \\
\hline ZERO & & ZERO \\
\hline ZERO & & ZERO \\
\hline ZERO & 0.1823 & $3 E+00$ \\
\hline ZERO & & ZERO \\
\hline ZERO & & ZERO \\
\hline ZERO & & ZERO \\
\hline ZERO & & ZERO \\
\hline ZERO & & ZERO \\
\hline ZERO & & ZERO \\
\hline ZERO & & ZERO \\
\hline ZERO & & ZERO \\
\hline ZERO & & ZERO \\
\hline ZERO & & ZERO \\
\hline ZERO & & ZERO \\
\hline ZERRO & & ZERO \\
\hline ZERO & & ZERO \\
\hline ZERO & & ZERO \\
\hline ZERO & & ZERO \\
\hline ZERO & & ZERO \\
\hline ZERO & & ZERO \\
\hline ZERO & & ZERO \\
\hline ZERO & & ZERO \\
\hline ZERO & & ZERO \\
\hline ZERO & & ZERO \\
\hline ZERO & & ZERO \\
\hline ZERO & & ZERO \\
\hline ZERO & & ZERO \\
\hline ZERO & & ZERO \\
\hline $.5484 \mathrm{E}+02$ & & ZERO \\
\hline ZERO & & ZERO \\
\hline ZERO & & ZERO \\
\hline ZERO & & ZERO \\
\hline ZERO & & ZERO \\
\hline ZERO & & ZERO \\
\hline ZERO & & ZERO \\
\hline ZERO & & ZERO \\
\hline
\end{tabular}

to SMECT

$0.2477 \mathrm{E}-18$

0'. 1217E-15

$0.1101 \mathrm{E}-17$

$0.1419 \mathrm{E}-14$

$0.1419 \mathrm{E}-15$

$0.1981 \mathrm{E}-18$

$0.8451 \mathrm{E}-20$

$0.2893 E-14$

$0.1021 \mathrm{E}-16$

$0.4299 \mathrm{E}-15$

ZERO

ZERO

$0.5877 \mathrm{E}-05$

$0.4802 \mathrm{E}-04$

ZERO

ZERO

ZERO

ZERO

$0.1100 \mathrm{E}-21$

ZERO

ZERO

$0.4514 \mathrm{E}-17$

$0.5602 \mathrm{E}-15$

ZERO

$0.1830 \mathrm{E}-20$

ZERO

ZERO

$0.1566 \mathrm{E}-15$

$0.1936 \mathrm{E}-15$

$0.2930 \mathrm{E}-23$

$0.1213 \mathrm{E}-15$

$0.3415 \mathrm{E}-23$

ZERO

$0.4432 \mathrm{E}-21$

$0.1089 \mathrm{E}-15$

$0.3618 \mathrm{E}+01$

TRACE

$0.5338 \mathrm{E}-10$ ZERO

$0.1503 \mathrm{E}-10$

$0.1732 \mathrm{E}-17$

ZERO

Date: January 1995
23

42106

FAVC 508 HNO3

Condensate to SMECT

$0.6290 \mathrm{O}-16$ ZERO

$0.2797 \mathrm{E}-15$ ZERO

$0.3605 \mathrm{E}-12$ ZERO

$0.3604 \mathrm{E}-13$

$0.5032 \mathrm{E}-16$

$0.2147 \mathrm{E}-17$

$0.3074 \mathrm{E}-07$ ZERO

$0.2595 \mathrm{E}-14 \quad \ldots \quad$ ZERO

ZERO

ZERO

ZERO

ZERO

$\begin{array}{rr}0.4156 \mathrm{E}+00 \\ 0.8701 \mathrm{E}-01 & 0.5554 \mathrm{E}+01\end{array}$

ZERO

ZERO

ZERO

ZERO

ZERO

ZERO

ZERO

$\begin{array}{rrr}\text { ZERO } & \text { ZERO } & \text { ZERO } \\ 0.2878 \mathrm{E}-06 & 0.1146 \mathrm{E}-14 & \text { ZERO }\end{array}$

$0.3581 \mathrm{E}-04 \quad 0.1423 \mathrm{E}-12 \quad \because \quad$ ZERO

$9 \mathrm{E}-06$

$0.4059 \mathrm{E}-15$

ZERO

ZERO

ZERO

ZERO

ZERO

ZERO

ZERO

ZERO

ZERO

ZERO

ZERO

ZERO

ZERO

ZERO

ZERO

ZERO

ZERO

ZERO

ZERO

ZERO

ERO

ZERO

$0.4958 \mathrm{E}-08$

$0.3022 \mathrm{E}+00$

ZERO

Section 4B, Page 15 of 29 
Table A-1. HLW Flowsheet Material Balance (Tank 51/ITP Cycle 1)

STREAM NUMBERS (CONT'D) $\rightarrow$ STREAM NAME

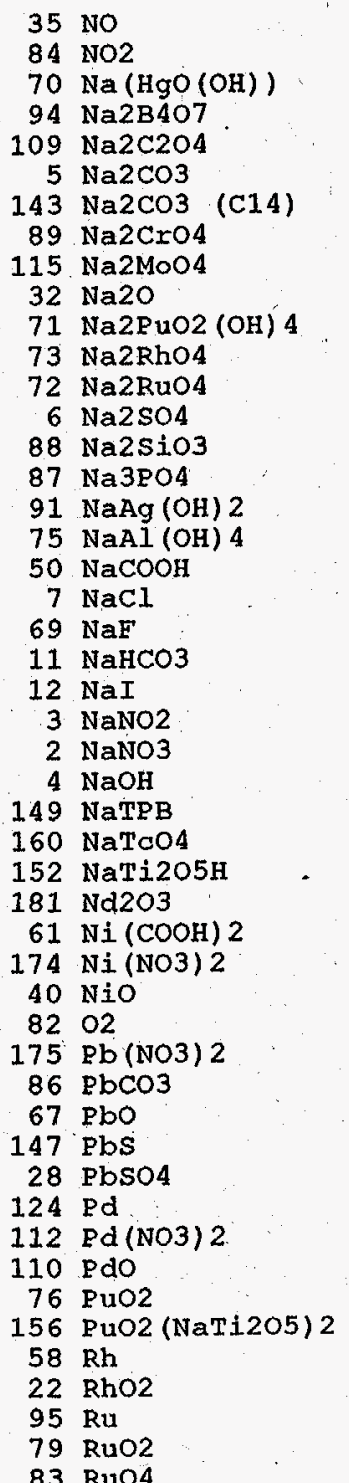

Section 4B. Frit Adjustment of Melter Feed

36

SMECT Hg Pump Water ZER ZERO ZERO ZERO ZERO ZERO ZERO ZERO

ZERO

ZERO

ZERO

ZERO

ZERO

ZERO

ZERO

ZERO

ZERO

ZERO

ZERO

ZERO

ZERO

ZERO

ZERO

ZERO

ZERO

ZERO

ZERO

ZERO

ZERO

ZERO

ZERO

ZERO

ZERO

ZERO

ZERO

ZERO

ZERO

ZERO

ZERO

ZERO

ZERO

ZERO
37

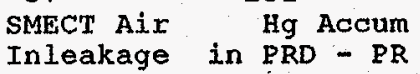

ZERO

ZERO

ZERO

ZERO

ZERO

ZERO

ZERO

ZERO

ZERO

ZERO

ZERO

ZERO

ZERO

ZERO

ZERO

ZERO

ZERO

ZERO

ZERO

ZERO

ZERO

ZERO

ZERO

ZERO

ZERO

ZERO

ZERO

ZERO

ZERO

$0.1656 \mathrm{E}+02$

ZERO

ZERO

ZERO

ZERO

ZERO

ZERO.

ZERO

ZERO

ZERO

ZERO

ZERO

ZERO

ZERO

ZERO

ZERO

ZERO

ZERO

ZERO

ZERO

ZERO

ZERO

ZERO

ZERO

ZERO

ZERO

ZERO

ZERO

ZERO

ZERO

ZERO

ZERO

ZERO

ZERO

ZERO

ZERO

ZERO

ZERO

ZERO

ZERO

ZERO

ZERO

ZERO

ZERO

ZERO

ZERO

$0.2195 \mathrm{E}-12$

ZERO

ZERO

ZERO
23

MWWT Vent Cond fr MWWT

to SMECT to SMECT

$0.7298 \mathrm{E}-14$

ZERO

$0.2052 \mathrm{E}-20$

$0.1323 \mathrm{E}-18$

$0.2737 \mathrm{E}-15$

ZERO ZERO

to SMECT
$0.3812 \mathrm{E}-04$

TRACE

ZERO

ZERO

$0.1729 \mathrm{E}-04$

ZERO

ZERO

$0.3557 \mathrm{E}-15$

ZERO

ZERO

$0.1091 \mathrm{Z}-16$

$0.1091 E-16$
ZERO

$0.1439 \mathrm{E}-18$

ZERO

$.4382 \mathrm{E}-16$

$0.5563 \mathrm{E}-18$

$0.1911 \mathrm{E}-17$

$0.3060 \mathrm{E}-21$

.

. $1653 E-18$

$.5044 E-15$
ZERO

ZERO

ZERO

ZERO

ZERO

$0.1409 \mathrm{E}-17$

$0.3729 \mathrm{E}-22$

$0.1330 \mathrm{E}-16$

$.1093 \mathrm{E}+01$

$.4342 \mathrm{E}-20$

ZERO

$0.8196 \mathrm{E}-24$

$0.7368 \mathrm{E}-17$

$0.2454 \mathrm{E}-13$

ZERO
TRACE

TRACE
$38 \mathrm{E}-18$

$0.7094 \mathrm{E}-20$

$0.4676 \mathrm{E}-13$

$0.1091 \mathrm{E}-22$

$0.4538 \mathrm{E}-18$

ZERO
ZERO

Date: January 1995

\begin{tabular}{|c|c|}
\hline 42 & 106 \\
\hline $\begin{array}{r}\text { FAVC } \\
\text { Condensate }\end{array}$ & $\begin{array}{l}508 \text { HNO3 } \\
\text { to SMECT }\end{array}$ \\
\hline $\begin{array}{r}\text { Condensate } \\
\text { zERO }\end{array}$ & $\begin{array}{l}\text { to SMECT } \\
\text { ZERO }\end{array}$ \\
\hline $0.6656 \mathrm{E}-05$ & ZERO \\
\hline ZERO & ZERO \\
\hline $0.5218 E-18$ & ZERO \\
\hline $0.3364 \mathrm{E}-16$ & ZERO \\
\hline $0.6952 \mathrm{E}-13$ & ZERO \\
\hline ZERO & ZERO \\
\hline ZERO & ZERO \\
\hline ZERO & ZERO \\
\hline $0.9034 \mathrm{E}-13$ & ZERO \\
\hline ZERO & ZERO \\
\hline ZERO & ZERO \\
\hline ZERO & ZERO \\
\hline $0.2772 \mathrm{E}-14$ & ZERO \\
\hline ZERO & ZERO \\
\hline $0.3656 \mathrm{E}-16$ & ZERO \\
\hline ZERO & ZERO \\
\hline ZERO & ZERO \\
\hline $0.1113 \mathrm{E}-13$ & ZERO \\
\hline $0.1413 E-15$ & ZERO \\
\hline $0.4853 \mathrm{E}-15$ & ZERO \\
\hline ZERO & ZERO \\
\hline $0.7773 \mathrm{E}-19$ & ZERO \\
\hline $0.4202 E-16$ & ZERO \\
\hline $0.1281 \mathrm{E}-12$ & ZERO \\
\hline ZERO & ZERO \\
\hline ZERO & ZERO \\
\hline ZERO & ZERO \\
\hline ZERO & ZERO \\
\hline ZERO & ZERO \\
\hline $0.3579 \mathrm{E}-15$ & ZERO \\
\hline $0.9472 \mathrm{E}-20$ & ZERO \\
\hline $0.3378 \mathrm{E}-14$ & ZERO \\
\hline $0.8069 \mathrm{E}-03$ & ZERO \\
\hline $0.1103 \mathrm{E}-17$ & ZERO \\
\hline ZERO & ZERO \\
\hline $0.2084 \mathrm{E}-21$ & ZERO \\
\hline ZERO & ZERO \\
\hline $0.1872 \mathrm{E}-14$ & ZERO \\
\hline $0.1586 \mathrm{E}-18$ & ZERO \\
\hline ZERO & ZERO \\
\hline TRACE & ZERO \\
\hline $0.1407 \mathrm{E}-1.5$ & ZERO \\
\hline $0.1802 \mathrm{E}-17$ & ZERO \\
\hline $0.3022 E-18$ & ZERO \\
\hline TRACE & ZERO \\
\hline $0.1153 \mathrm{E}-15$ & ZERO \\
\hline ZERO & ZERO \\
\hline ZERO & ZERO \\
\hline
\end{tabular}

Section $4 B$, Page 16 of 29 
Table A-1. HLW Flowsheet Material Balance (Tank 51/ITP Cycle 1)

STREAM NUMBERS (CONT'D) $\rightarrow$ STREAM NAME

$38 \mathrm{SO} 2$

$141 \mathrm{SO3}$

137 Semi Vol Cs2O

136 Semi Vol CsCl.

126 Semi Vol Group A

140 Semi Vo1 Na2B4

138 Semi Vol NaCl

135 Semi Vol NaI

23 Semi Vol RuO2

$111 \mathrm{SiO2}$

$48 \mathrm{Sr}$ (COOH) 2

$176 \mathrm{Sr}(\mathrm{NO} 3) 2$

153 Sr(NaTi2O5) 2

$127 \mathrm{Sr}(\mathrm{OH}) 2$

$30 \mathrm{SrCO} 3$

46 sro

$159 \mathrm{TCO} 2$

107 TiO2

119 Tritium

17 Triti

$62 \mathrm{vO} 2(\mathrm{COOH}) 2$

177 UO2 (NO3) 2

$16 \mathrm{VO} 2(\mathrm{OH}) 2$
$27 \mathrm{Y}(\mathrm{COOH}) 3$

$27 \mathrm{Y}(\mathrm{COOH}) 3$
$178 \mathrm{Y}(\mathrm{NO} 3)^{3}$

$128 \mathrm{Y}(\mathrm{OH}) 3$

$31 \mathrm{Y} 2(\mathrm{CO} 3) 3$

$47 . Y 203$

100 Zeolite

$44 \mathrm{Zn}(\mathrm{COOH}) 2$
$179 \mathrm{Zn}(\mathrm{NO} 3)^{2}$

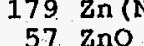

104 Zro2

13 hydrate $\mathrm{H} 2 \mathrm{O}$

1 water

TOTAL FLOW, LB/HR

TEMPERATURE, DEG C

PRESSURE, ATM

PRESSURE， PSIG

PRESSURE, MM HG

ENTHALPY, PCU/HR

VAPOR FLOW, CFM

LIQUID FIOW, GPM

DENSITY, IABS/ET3

PHASE

WSRC-TR-95-0019 (Revision

section 4B. Frit Adjustment of Melter Feed

3

SMECT H

Pump Water

37

SMECT Air

Inleakage

ZERO

ZERO

ZERO

ZERO

ZERO

ZERO

ZERO

ZERO

ZERO

ZERO

ZERO

ZERO

ZERO

ZERO

ZERO

ZERO

ZERO

ZERO

ZERO

ZERO

ZERO

ZERO

ZERO

ZERO

ZERO

ZERO

ZERO

ZERO

ZERO

ZERO

ZERO

ZERO

$0.1200 \mathrm{E}+01$

$0.2042 \mathrm{E}-02$

$0.3500 \mathrm{E}+02$

$0.1000 \mathrm{E}+01$

ZERO

$0.7163 \mathrm{E}-01$

$0.4077 \mathrm{E}-05$

$0.6243 \mathrm{E}+02$

IIQUID
$0.7260 \mathrm{E}+02$

$0.1823 \mathrm{E}+00$

EE+01
ZERO

$0.3004 \mathrm{E}+00$

$0.1715 \mathrm{E}+02$

$0.7055 \mathrm{E}-01$

VAPOR

$0.2688 \mathrm{E}-04$

LIQUID
251

Hg Accum

ZERO

ZERO

ZERO

ZERO

ZERO

ZERO

ZERO

ZERO

ZERO

ZERO

ZERO

ZERO

ZERO

ZERO

ZERO

ZERO

ZERO

ZERO

ZERO

ERRO

ERO

ZERO

ERO

ZERO

ZERO

ZERO

ZERO

ERO

$0.0382 \mathrm{E}-16$

$0.4807 \mathrm{E}+01$

$0.3500 \mathrm{E}+02$

$0.1000 E+01$

$0.1102 E+03$

$0.1138 \mathrm{E}+01$
ZERO

16

23

ZERO SMECT

ZERO

ZERO

ZERO

ZERO

ZERO

ZERO

$.1783 E-14$

, $1646 \mathrm{E}-17$

ZERO

E -23

$.1996 \mathrm{E}-18$

$.4635 \mathrm{E}-16$

$.3537 \mathrm{E}-16$

$.7095 \mathrm{E}-22$

$5109 \mathrm{E}-20$

TRACE

444OE-18

ZERO

ZERO

$.1455 \mathrm{E}-16$

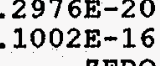

6

.

$0.7140 \mathrm{E}-05$

$0.7150 \mathrm{E}+03$

$0.5000 \mathrm{E}+02$

$0.9656 \mathrm{E}+00$

$-0.5055 \mathrm{E}+00$
$0.7339 \mathrm{E}+03$

$0.3576 \mathrm{E}+05$

$0.1428 \mathrm{E}+01$

$0.6244 \mathrm{E}+02$

IIQUID
$42 \quad 106$

Condensate 50\% HNO3 ZERO ZERO

ZERO
ZERO
ZERO

ZERO ZERO

ZERO ZERO

ZERO ZERO

ZERO

ZERO ZERO

$0.4529 \mathrm{E}-12$

$0.1548 \mathrm{E}-17$ ZERO

$0.4181 \mathrm{E}-15$ ZERO

$0.5769 \mathrm{E}-16 \quad$ ZERO

ZERO

ZERO
$0.6525 \mathrm{E}-21$
ZERO

$0.5069 \mathrm{E}-16$ ZERO

$0.4221 \mathrm{E}-15$ ZERO

$0.1177 \mathrm{E}-13 \quad$ ZERO

$0.3426 \mathrm{E}-10$ ZERO

$0.2960 \mathrm{E}-13$ ZERO

$0.1802 \mathrm{E}-19$

$0.1298 \mathrm{E}-17$ ZRRO

ZERO

ZERO

ZERO

ZERO

ZERO

ZERO

ZERO

ZERO

$0.3695-14$
$0.7559 \mathrm{E}-18$
$0.2544 \mathrm{E}-14$
ZERO

$0.7559 \mathrm{E}-18$
$0.2544 \mathrm{E}-14$
ZERO

$0.7559 \mathrm{E}-18$
$0.2544 \mathrm{E}-14$
ZERO

$0.2129 \mathrm{E}-13 \quad$ ZERO

$0.1337 \mathrm{E}+03 \quad 0.5554 \mathrm{E}+01$

$0.1342 \mathrm{E}+03$

$0.1000 \mathrm{E}+02$

$0.9607 \mathrm{E}+00$

$-0.5776 \mathrm{E}+00$

$0.7301 \mathrm{E}+03$
$0.1346 \mathrm{E}+04$

$0.2500 \mathrm{E}+02$

$0.1000 \mathrm{E}+01$

ZERO

$0.1790 \mathrm{E}+03$

$0.2676 \mathrm{E}+00$

$0.1848 \mathrm{E}-01$

LIQUID 
Table A-1. HLW Flowsheet Material Balance (Tank 51/ITP Cycle 1) Section $4 \mathrm{~B}$. Frit Adjustment of Melter Feed

STREAM NUMBERS $\rightarrow$ STREAM NAME

COMPONENT FLOWS, LB/HR 122 (C6H5) 2

123. (C6H5) $2 \mathrm{C} 6 \mathrm{H} 4$

118 (C6H5) $2 \mathrm{NH}$

133 (C6H5B (OH) 2 salt)

132 ( 6 $6 \mathrm{H} 5 \mathrm{~B}(\mathrm{OH}) 2$ )

166 (C6H5C6H4OH)

162 (C6H5NH2)

163 (C6H5NHCHO)

165 (C6H5NO)

131 (C6H5OH salt)

130 (C6H5OH)

121 ( $\mathrm{C} 6 \mathrm{H} 6)$

155 (CH3) $2 \mathrm{CHOH}$

154 (СH3OH)

$45 \mathrm{Ag}$

$116 \mathrm{Ag} 20$

134 AgNO3

$106 \mathrm{~B} 203$

$9 \mathrm{Ba}(\mathrm{OH}) 2$

$15 \mathrm{BaO}$

$24 \mathrm{BaSO}$

$66 \mathrm{CO}$

$\begin{array}{rrr}37 & \mathrm{CO} 2 & \\ 142 & \mathrm{CO} 2 & (\mathrm{C} 14)\end{array}$

63. $\mathrm{Ca}(\mathrm{COOH}) 2$

$167 \mathrm{Ca}(\mathrm{NO} 3) 2$

$120 \mathrm{Ca}(\mathrm{OH}) 2$

$113 \mathrm{Ca} 3(\mathrm{PO} 4) 2$

$20 \mathrm{CaC2O} 4$

$18 \mathrm{CaCO} 3$
$64 \mathrm{CaCO}^{3}$ (C14)

$78 \mathrm{CaF} 2$

$41 \mathrm{CaO}$

19 Caso 4

29 Carbon

$26 \mathrm{Co}(\mathrm{COOH}) 2$

$168 \mathrm{CO}(\mathrm{NO} 3) 2$

$59 \mathrm{CoO}$

$98 \mathrm{Cr} 203$

$42 \mathrm{Cs} 20$

$96 \mathrm{CsCl}$

$169 \mathrm{CsNO} 3$

$8 \mathrm{CsOH}$

151 CsTPB

WSRC-TR-95-0019 (Revision
38

sMECT Vent

to FAVC Vnt to PVVS

$0.1831 \mathrm{E}-01$

$0.6080 \mathrm{E}-14$

$0.7500 \mathrm{E}-04$

ZERO

$0.1566 \mathrm{E}-14$

$0.1379 \mathrm{E}-02$

$0.5936 \mathrm{E}-14$

$0.6459 \mathrm{E}-16$

$0.1968 \mathrm{E}-15$

$0.4978 \mathrm{E}-17$

$0.7561 \mathrm{E}-02$
$0.2674 \mathrm{E}-01$

$0.2674 \mathrm{E}-01$
$0.6209 \mathrm{E}-12$

$0.6209 \mathrm{E}-12$

$0.1370 \mathrm{E}-12$
$0.1123 \mathrm{E}-16$

$0.7540 \mathrm{E}-24$

$0.2509 \mathrm{E}-12$

$0.9093 \mathrm{E}-13$

ZERO

$0.4838 \mathrm{E}-15$

$0.4838 \mathrm{E}-15$
ZERO

$0.1496 \mathrm{E}-01$

$0.1930 \mathrm{E}-09$

$0.1189 \mathrm{E}-12$

ZERO
$0.7454 \mathrm{E}-13$

$0.6919 \mathrm{E}-20$

ZERO

ZERO

$0.4263 \mathrm{E}-15$

$0.9510 \mathrm{E}-16$

$0.6258 \mathrm{E}-14$ ZERO

$0.4059 \mathrm{E}-17$

$0.1522 \mathrm{E}-19$

$0.8696 \mathrm{E}-18$

$0.1217 \mathrm{E}-13$

$0.3984 \mathrm{E}-17$

$0.1227 \mathrm{E}-14$

$0.2729 \mathrm{E}-18$

$0.3112 \mathrm{E}-19$

$0.1019 \mathrm{E}-01$

$0.3515 \mathrm{E}-10$

$0.7563 \mathrm{E}-04$

ZERO
ZERO

ZERO

ZERO

ZERO

ZERO

$0.6223 \mathrm{E}-19$

$0.1138 \mathrm{E}-01$

$0.1406 \mathrm{E}-01$

$0.8066 \mathrm{E}-11$

$0.2173 \mathrm{E}-11$

$0.1404 \mathrm{E}-18$

TRACE

$0.3136 \mathrm{E}-14$

$0.1137 \mathrm{E}-14$

ZERO

$0.1190 \mathrm{E}-23$

$.6048 \mathrm{E}-17$

ZERO
$0.4331 \mathrm{E}+01$

$0.5680 \mathrm{E}-07$

ZERO
ZERO

ZERO

$0.9318 \mathrm{E}-15$

$0.8649 \mathrm{E}-22$

ZERO

$0.5328 \mathrm{E}-17$

$0.1189 \mathrm{E}-17$

$0.7822 \mathrm{E}-16$

ZERO

ZERO
$0.5074 \mathrm{E}-19$

ZERO

$0.1087 \mathrm{E}-19$

$0.1522 \mathrm{E}-15$

$0.4980 \mathrm{E}-19$

$0.1534 \mathrm{E}-16$

ZERO

$0.3890 \mathrm{E}-21$

ZERO $\begin{array}{llll}40 & 41 & 43 & 122\end{array}$

FAVC HEME FAVC HEME
Vnt to PVVS Accumulatn

$0.2928 \mathrm{E}-03$

$0.3645 \mathrm{E}-06$

$0.5159 \mathrm{E}-02$

ZERO

$0.2021 \mathrm{E}-06$

$0.5449 \mathrm{E}-01$

$0.7661 \mathrm{E}-06$

$0.8336 \mathrm{E}-08$

$0.2539 \mathrm{E}-07$

$0.6425 \mathrm{E}-09$

$0.6632 \mathrm{E}+00$

$0.5535 \mathrm{E}-10$

$0.5273 \mathrm{E}-11$

ZERO
$0.9731 \mathrm{E}-16$

0.5477 RER

$0.5477 \mathrm{E}-17$
$0.3676 \mathrm{E}-24$

ZERO

$0.1223 \mathrm{E}-12$

33E-13

$0.4640 \mathrm{E}-22$

$0.2359 \mathrm{E}-15$

ZERO

ZERO

ZERO

ZERO

$0.3634 \mathrm{E}-13$

$0.3373 \mathrm{E}-20$

ZERO

$0.2078 \mathrm{E}-15$

$0.2078 \mathrm{E}-15$
$0.4636 \mathrm{E}-16$

$0.3051 \mathrm{E}-14$ ZERO

$0.5935 \mathrm{E}-14$ ZERO

ZERO
$0.1517 \mathrm{E}-19$

ZERO

ZERO

$0.1174 \mathrm{E}-04$

ZERO

$0.6244 \mathrm{E}-07$ ZERO

$0.1146 \mathrm{E}-03$

$0.1479 \mathrm{E}-11$

ZERO

ZERO

$0.8930 \mathrm{E}-12$

ZERO

$0.5501 \mathrm{E}-07$

$0.1227 \mathrm{E}-07$

$0.8076 \mathrm{E}-06$ ZERO

0.523 ZERO

$0.5239 \mathrm{E}-09$

$0.1122 \mathrm{E}-09$

$0.1571 \mathrm{E}-05$

$0.5142 \mathrm{E}-09$

$0.1583 \mathrm{E}-06$

$0.3522 \mathrm{E}-10$

$0.4016 \mathrm{E}-11$

ZERO

Date: January 1995
ZERO

$0.9621 \mathrm{E}-05$
SMECT Iiq

24

Solubles
Solu

Insolubles

$0.2928 E-03 \quad 0.4585 E-09$

$0.3645 \mathrm{E}-06 \quad 0.5707 \mathrm{E}-12$

$0.5159 E-02 \quad 0.8077 E-08$

ZERO ZERO ZERO

ZERO ZERO ZERO

$0.5449 \mathrm{E}-01$

$0.2021 \mathrm{E}-06$

$0.3165 \mathrm{E}-12$

$0.1199 \mathrm{E}-11$

$0.8336 \mathrm{E}-08 \quad 0.1305 \mathrm{E}-13$

$0.3976 \mathrm{E}-13$

$0.1006 \mathrm{E}-14$

$0.1038 \mathrm{E}-05$

$0.1263 \mathrm{E}-08$

$0.8666 \mathrm{E}-16$

$\begin{array}{rrr}0.5535 \mathrm{E}-10 & \text { ZERO } & 0.8666 \mathrm{E}-16 \\ 0.5273 \mathrm{E}-11 & \text { ZERO } & 0.8255 \mathrm{E}-17 \\ \text { ZERO } & \text { ZERO } & 0.1676 \mathrm{E}-07 \\ \text { ZERO } & 0.9731 \mathrm{E}-16 & 0.1523 \mathrm{E}-21\end{array}$

$\begin{array}{rrr}0.5535 \mathrm{E}-10 & \text { ZERO } & 0.8666 \mathrm{E}-16 \\ 0.5273 \mathrm{E}-11 & \text { ZERO } & 0.8255 \mathrm{E}-17 \\ \text { ZERO } & \text { ZERO } & 0.1676 \mathrm{E}-07 \\ \text { ZERO } & 0.9731 \mathrm{E}-16 & 0.1523 \mathrm{E}-21\end{array}$

$\begin{array}{rr}0.9731 \mathrm{E}-16 & 0.1523 \mathrm{E}-21 \\ \text { ZERO } & \text { ZERO }\end{array}$

$0.3238 E-04 \quad 0.5070 E-10$

ZERO

$\begin{array}{ll}0.1228 \mathrm{E}-13 & 0.1923 \mathrm{E}-19 \\ 0.6244 \mathrm{E}-07 & 0.9776 \mathrm{E}-13\end{array}$

$0.1146 \mathrm{E}-03$

$0.1479 \mathrm{E}-11$
ZERO

$0.1535 \mathrm{E}-04$

ZERO

ZERO

ZERO

ZERO

ZERO

ZERO

ZERO

ZERO

ZERO

$0.5239 \mathrm{E}-09$

$0.1965 \mathrm{E}-11$

ZERO

ZERO

ZERO
$0.1583 E-06$

ZERO

$0.3522 \mathrm{E}-10$

$0.4016 \mathrm{E}-11$

Section 4B, Page 18 of 29 
Table A-1. HLW Flowsheet Material Balance (Tank 51/ITP Cycle 1) Section 4B. Frit Adjustment of Melter Feed

STREAM NUMBERS (CONT'D) $\rightarrow$ STREAM NAME

$54 \mathrm{Cu}(\mathrm{COOH}) 2$

$170 \mathrm{Cu}(\mathrm{NO} 3) 2$

$55 \mathrm{CuO}$

80 Fe203

101 Feo

102 Group A

$25 \mathrm{H} 2$

$158 \cdot \mathrm{H} 2 \mathrm{C} 2 \mathrm{OA}$

$93 \mathrm{H} 2 \mathrm{SO} 4$

$34 \mathrm{HCOOH}$

$43 \mathrm{HCl}$

$85 \mathrm{HF}$

49 HNO3

$36 \mathrm{Hg}$

$129 \mathrm{Hg}(\mathrm{C} 6 \mathrm{H} 5) 2$

$135 \mathrm{Hg}$ (NO3) 2

$144 \mathrm{Hg} 2 \mathrm{Cl2}$

$117 \mathrm{Hg} 212$

146. $\mathrm{HgCl}$

$21 \mathrm{HgO}$

$98 \mathrm{~K} 2 \mathrm{CO}$

$39 \mathrm{~K} 2 \mathrm{O}$

$51 \mathrm{KCOOH}$

$161 \mathrm{KNO} 3$

$10 \mathrm{KOH}$

150 KTPB

$105 \mathrm{La2O}$

$65 \mathrm{Mg}(\mathrm{COOH}) 2$

171. $\mathrm{Mg}$ (NO3) 2

17. $\mathrm{Mg}(\mathrm{Mg}$

$60 \mathrm{Mn}(\mathrm{COOH}) 2$

$172 \mathrm{Mn}$ (NO3) 2

$180 \mathrm{Mn} 304$

$56 \mathrm{MnO}$

$14 \mathrm{MnO} 2$

$145 \mathrm{MOO2}$

$81 \mathrm{~N} 2$

74 N2O

114 NH3OHNO3

114 NH3OHNO

173 NH4NO3

$68 \mathrm{NH} 4 \mathrm{OH}$

148 NH4TPB

WSRC-TR-95-0019 (Revision
38

SMECT Vent

to EAVC
$1258 \mathrm{E}-15$

$0.1258 \mathrm{E}-15$
$0.6181 \mathrm{E}-13$

$0.5594 \mathrm{E}-15$

$0.7210 \mathrm{E}-12$

$0.7209 \mathrm{E}-13$

$0.1006 \mathrm{E}-15$

$0.4293 \mathrm{E}-17$

$0.5887 \mathrm{E}-05$

$0.5189 E-14$

ZERO
$0.2184 \mathrm{E}-12$

ZERO

ZERO

ZERO
$0.5815 \mathrm{E}-01$

$0.9521 \mathrm{E}-01$

ZERO

ZERO

ZERO

7FRO

$0.5595 \mathrm{E}-19$

ZERO

ZERO
$0.2293 \mathrm{E}-14$

$0.2846 \mathrm{E}-12$

ZERO

$0.8118 \mathrm{E}-15$

$0.9308 \mathrm{E}-18$

ZERO

$0.7953 \mathrm{E}-13$

$0.9837 \mathrm{E}-13$

$0.1489 \mathrm{E}-20$

$0.3138 \mathrm{E}-13$

$0.6161 \mathrm{E}-13$

$0.1735 \mathrm{E}-20$

ZERO

$0.2254 \mathrm{E}-1.8$

$0.5533 \mathrm{E}-1.3$

$0.3053 \mathrm{E}-16$

$0.5846 \mathrm{E}+02$

$0.6761 \mathrm{E}-24$

$0.4449 \mathrm{E}-04$

ZERO

$0.7635 \mathrm{E}-08$

TRACE

ZERO
40

FAVC HEME

nt to PVVS
$0.1573 \mathrm{E}-17$

$0.1573 E-17$

$0.6992 \mathrm{E}-17$

$0.9013 \mathrm{E}-14$

$0.9011 \mathrm{E}-15$

$0.5367 \mathrm{E}-19$

$0.3136 \mathrm{E}-01$

$0.6486 \mathrm{E}-16$

$0.2730 \mathrm{Z}-14$

ZTRO

ZERO

ZERO

$0.7276 \mathrm{E}+00$

$0.8193 \mathrm{E}-02$

ZERO

ZERO

ZERO

0.6994 ZERO

$0.2866 \mathrm{E}-16$

$0.3558 \mathrm{E}-14$

$0.1015 \mathrm{E}-16$

$0.1163 \mathrm{E}-19$

ZERO

$0.9942 \mathrm{E}-15$

$0.1230 \mathrm{E}-14$

$0.3923 E-15$ ZERO

$0.2818 \mathrm{E}-20$

$0.6916 \mathrm{E}-15$

$0.3817 \mathrm{E}-18$

$0.1330 \mathrm{E}+04$

$0.1420 \mathrm{E}-19$

$0.1076 \mathrm{E}-02$

ZERO
ZERO

ZERO

TRACE
41
FAVC HEME

Accumulatn

$0.6133 \mathrm{E}-16$

ZERO
$7 \mathrm{E}-15$

$0.3515 \mathrm{E}-12$

$0.3514 \mathrm{E}-13$

$0.4906 \mathrm{E}-16$

ZERO

ERO

$0.1065 \mathrm{E}-12$

ZERO

ZERO

ZERO

ZERO

ZERO

ZERO

ZERO

ZERO

$0.2728 \mathrm{E}-19$

ZERO

$0.1118 \mathrm{E}-14$

$0.1387 \mathrm{E}-12$

ŻERO

$0.3958 \mathrm{E}-15$
$0.4537 \mathrm{E}-18$

$7 E-18$
ZERO

ZERO

$0.3877 \mathrm{E}-13$

$0.4795 \mathrm{E}-13$

$0.1530 \mathrm{EERO}$

ZERO

ZERO
$0.1099 \mathrm{E}-18$

$0.2697 \mathrm{E}-13$

$0.1488 \mathrm{E}-16$

ZERO

ZERO

ZERO

ZERO

ZERO

ZERO

43
SMECT Liq
to RCT

122

SMECT Liq

Soluble

$0.1624 \mathrm{E}-07$
$0.7978 \mathrm{E}-05$

$.1624 \mathrm{E}-07$

$0.7978 \mathrm{E}-05$

$0.7219 \mathrm{E}-07$

$0.9305 \mathrm{E}-04$

$0.9303 \mathrm{E}-05$

$0.5541 \mathrm{E}-09$

$0.1848 \mathrm{E}-08$

$0.6697 \mathrm{E}-06$

ZERO

ZERO

ZERO

ZERO

$0.3758 \mathrm{E}+01$

ZERO

ZERO

ZERO

ZERO

$0.7222 \mathrm{E}-11$

ZERO

$0.2959 \mathrm{E}-06$

$0.3673 \mathrm{E}-04$

$0.1048 \mathrm{E}-06$

$0.1201 \mathrm{E}-09$

ZERO

$0.1026 \mathrm{E}-04$

$0.1270 \mathrm{E}-04$

$0.1921 \mathrm{E}-12$

$0.4050 \mathrm{E}-05$

$0.7952 \mathrm{E}-05$

ZERO

$0.2909 \mathrm{E}-10$

$0.7141 \mathrm{E}-05$

$0.3941 \mathrm{E}-08$

$0.1408 \mathrm{E}-01$

$0.4722 \mathrm{E}-04$

ZERO

$0.9853 \mathrm{E}+00$

ZERO

Date: January 1995

$0.6697 \mathrm{E}-06$

ZERO

.2818E-04

ZERO

ZERO

$0.3758 E+01$

ZERO

ZERO

ZERO

ZERO

ZERO

ZERO

ZERO

ZERO

$0.3673 \mathrm{E}-04$

ZERO

$0.1048 \mathrm{E}-06$

$0.1201 E-09$

ZERO
ZERO

ZERO

$0.1270 \mathrm{E}-04$

$0.1921 \mathrm{E}-12$

ZERO

$0.7952 \mathrm{E}-05$

$0.2239 \mathrm{E}-12$

ZERO

ZERO

ZERO

$0.1408 \mathrm{E}-01$

TRAC

TRACE
$0.4722 \mathrm{E}-04$

ZERO

$0.9853 \mathrm{E}+00$

ZERO

123

SMECT Liq

Insolubles ZERO

$0.7219 \mathrm{E}-07$

$0.9305 \mathrm{E}-04$

$0.9303 E-05$

$0.1299 \mathrm{E}-07$

ZERO

- ZERO

$0.2959 \mathrm{E}-06$

ZERO

ZERO

ZERO

ZERO

$0.1026 \mathrm{E}-04$ ZERO ERRO

$0.4050 \mathrm{E}-05$

ZERO

ZERO

$0.2909 \mathrm{E}-10$

$0.7141 \mathrm{E}-05$

$0.3941 E-08$

ZERO

ZERO

ZERO

ZERO

ZERO

ZERO 
Table A-1. HLW Flowsheet Material Balance (Tank 51/ITP Cycle 1)

STREAM NUMBERS (CONT'D) $\rightarrow$ STREAM NAME

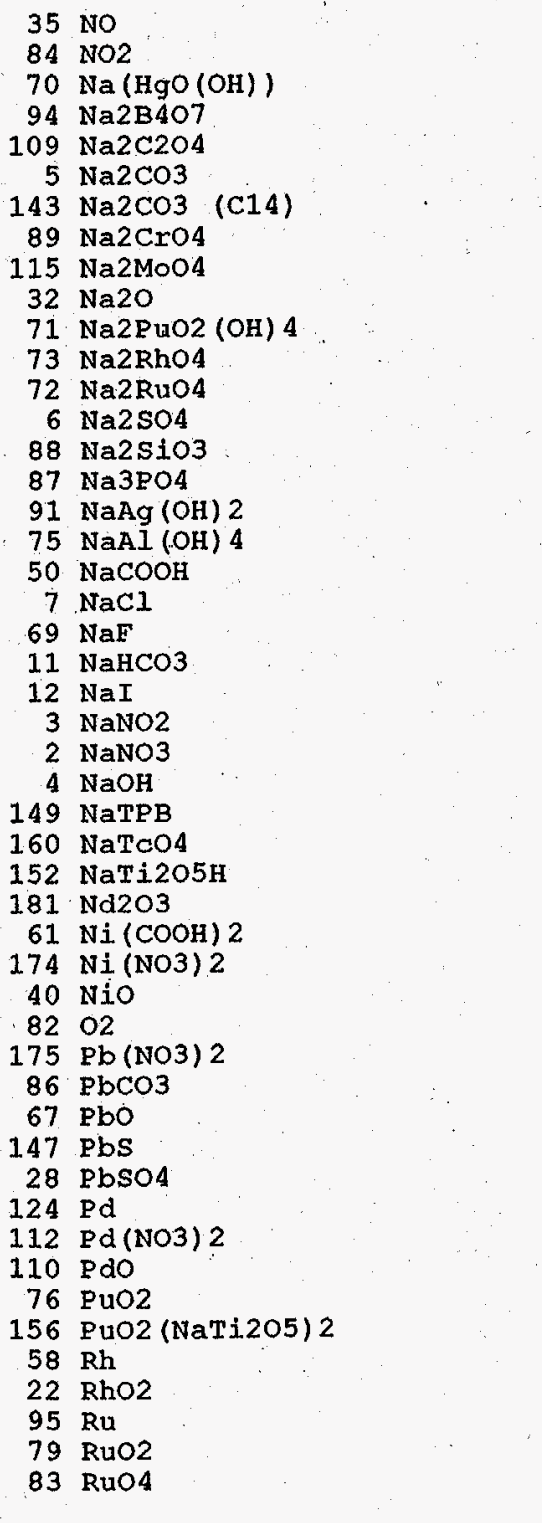

WSRC-TR-95-0019 (Revision

section 4B. Frit Adjustment of Melter Feed

$$
\begin{array}{r}
40 \\
\text { FAVC HEME } \\
\text { Vnt to PVVS } \\
\text { ZERO } \\
0.2184 \text { E+01 } \\
\text { ZERO } \\
0.1304 \mathrm{E}-19 \\
0.8409 \mathrm{E}-18 \\
0: 1738 \mathrm{E}-14 \\
\text { ZERO } \\
\text { ZERO } \\
\text { ZERO } \\
0.2259 \mathrm{E}-14 \\
\text { ZERO } \\
\text { ZERO } \\
\text { ZERO } \\
0.6930 \mathrm{E}-16 \\
\text { ZERO } \\
0.914 \text { OE-18 } \\
\text { ZERO } \\
\text { ZERO }
\end{array}
$$

\begin{tabular}{|c|c|}
\hline & 43 \\
\hline FAVC HEME & SMECT Liq \\
\hline Accumulatn & to $\mathrm{RCT}$ \\
\hline ZERO & $0.3277 \mathrm{E}-06$ \\
\hline ZERO & $0.4661 \mathrm{E}-08$ \\
\hline ZERO & ZERO \\
\hline $0.5087 \mathrm{E}-18$ & $0.1347 \mathrm{E}-09$ \\
\hline $3280 \mathrm{E}-16$ & $0.8683 E-08$ \\
\hline $778 \mathrm{E}-13$ & $0.1794 \mathrm{E}-04$ \\
\hline ZERO & ZERO \\
\hline ZERO & ZERO \\
\hline ZERO & ZERO \\
\hline $0.8808 \mathrm{E}-13$ & $0.2332 \mathrm{E}-04$ \\
\hline ZERO & $\therefore$ ZERO \\
\hline ZERO & ZERO \\
\hline ZERO & ZERO \\
\hline $.2703 E-14$ & $0.7155 \mathrm{E}-06$ \\
\hline ZERO & ZERO \\
\hline $.3565 \mathrm{E}-16$ & $0.9437 \mathrm{E}-08$ \\
\hline ZERO & ZERO \\
\hline ZERO & ZERO \\
\hline $0.1085 \mathrm{E}-13$ & $0.2873 E-05$ \\
\hline $.1378 E-15$ & $0.3647 \mathrm{E}-07$ \\
\hline $.4732 E-15$ & $0.1253 \mathrm{E}-06$ \\
\hline ZERO & ZERO \\
\hline $.7579 E-19$ & $0.2006 \mathrm{E}-10$ \\
\hline $7 E-16$ & $5 E-07$ \\
\hline $.1249 \mathrm{E}-12$ & $0.3307 E-04$ \\
\hline ZERO & ZERO \\
\hline ZERO & ZERO \\
\hline ZERO & ZERO \\
\hline ZERO & ZERO \\
\hline ZERO & ZERO \\
\hline $0.3490 \mathrm{E}-15$ & $0.9239 \mathrm{E}-07$ \\
\hline ZERO & $0.2445 \mathrm{E}-11$ \\
\hline $0.3293 \mathrm{E}-14$ & $0.8718 \mathrm{E}-06$ \\
\hline & $0.8121 \mathrm{E}-02$ \\
\hline ZERQO & $0.2847 E-09$ \\
\hline ZERO & ZERO \\
\hline $0.2032 \mathrm{E}-21$ & $0.5380 \mathrm{E}-13$ \\
\hline ZERO & ZERO \\
\hline $0.1825 \mathrm{E}-14$ & $0.4831 \mathrm{E}-06$ \\
\hline $0.1546 \mathrm{E}-18$ & ZERO \\
\hline ZERO & ZERO \\
\hline TRACE & $0.3207 \mathrm{E}-17$ \\
\hline $0.1371 \mathrm{E}-15$ & $0.3631 \mathrm{E}-07$ \\
\hline & $0.4651 \mathrm{E}-09$ \\
\hline $46 \mathrm{E}-18$ & ZERO \\
\hline TRACE & $0.6966 \mathrm{E}-17$ \\
\hline $0.1124 \mathrm{E}-15$ & $0.2975 \mathrm{E}-07$ \\
\hline ZERO & ZERO \\
\hline ZEKO & ZERO \\
\hline
\end{tabular}

Date: January 1995
122

SMECT Liq

Solubles
$0.3277 \mathrm{E}-06$

$0.3277 \mathrm{E}-06$
$0.4661 \mathrm{E}-08$

0.4661 E-OB
ZERO

$0.1347 \mathrm{E}-09$

$0.8683 \mathrm{E}-08$

$0.1794 \mathrm{E}-04$

ZERO

ZERO

ZERO

ZERO

ZERO

ZERO

$0.7155 \mathrm{E}-06$

$0.9437 \mathrm{E}-08$

ZERO

$0.2873 \mathrm{E}-05$

$0.3647 \mathrm{E}-07$

$0.1253 \mathrm{E}-06$

ZERO

$0.2006 \mathrm{E}-10$

$0.1085 \mathrm{E}-07$

$0.3307 E-04$
ZERO

ZERO

ZERO

ZERO

ZERO
$0.9239 \mathrm{E}-07$

$0.2445 \mathrm{E}-11$

ZERO

$0.8121 \mathrm{E}-02$
$0.2847 \mathrm{E}-09$

ZERO

ZERO

ZERO

ZERO

ZERO

ZERO

ZERO

ZERO

ZERO

ZERO

ZERO

ZERO
123

SMECT Liq

Insolubles

ZERO

ZERO

ZERO

ZERO

ZERO

ZERO

ZERO

$0.2332 \mathrm{E}-04$

ZERO

ZERO

ZERO

ZERO

ZERO

ZERO

ZERO

ZERO

$0.9326 \mathrm{E}-14$

ZERO

ZERO

ZERO

ZERO

ZERO

ZERO

ZERO

ZERO

ZERO

$0.8718 \mathrm{E}-0$

ZERO

ZERO

$0.5380 \mathrm{E}-13$

ZERO

ZERO

$0.3207 \mathrm{E}-17$

$0.3631 \mathrm{E}-07$

$0.4651 \mathrm{E}-09$

$0.6966 \mathrm{E}-17$

$0.2975 \mathrm{E}-07$

ZERO
24

SMECT Hg

to MWW'

$0.5131 \mathrm{E}-12$

ZERO

$0.2109 \mathrm{E}-15$

$0.1359 \mathrm{E}-13$

$0.2809 \mathrm{E}-10$

ZERO

ZERO

$0.3651 \mathrm{E}-10$

ZERO

ZERO

$0.1120 \mathrm{E}-11$

$0.1477 \mathrm{E}-13$

ZERO

ZERO
$0.4498 E-11$

$0.5710 \mathrm{E}-13$

$0.1961 E-12$

0. $3141 \mathrm{EERO}$

$0.1698 \mathrm{E}-13$

$0.1698 \mathrm{E}-13$

ZERO

ZERO

ZERO

ZERO

$0.1447 \mathrm{E}-12$

$0.3828 \mathrm{E}-17$

$0.1365 \mathrm{E}-11$

$0.1272 \mathrm{E}-07$

$0.4457 \mathrm{E}+15$

ZERO
0.8422 E-19

ZERO

$0.7563 \mathrm{E}-12$

$0.4731 \mathrm{E}-09$

$0.5022 \mathrm{E}-23$

$0.5685 \mathrm{E}-13$

$0.7282 \mathrm{E}-15$

$0.9017 \mathrm{E}-09$

$0.1091 \mathrm{E}-22$

$0.4658 \mathrm{E}-13$

ZERO 
Table A-1. HLW Flowsheet Material Balance (Tank 51/ITP Cycle 1)

STREAM NUMBERS (CONT'D), $\rightarrow$ STREAM NAME

$38 \mathrm{SO} 2$

$141 \mathrm{SO3}$

137 Semi Vol Cs2O

136 Semi Vol CsCl

126 Semi Vol Group A

138 Semi Vol NaCl

139 Semi Vol NaF

125 Semi Vol NaI

23. Semi Vol RuO2

111 sio2

$48 \mathrm{Sr}(\mathrm{COOH}) 2$

$\begin{array}{ll}176 & \operatorname{Sr}(\mathrm{NO}) 2 \\ 153 & \mathrm{Sr}(\mathrm{NaT} 2 \mathrm{2O}) \\ 127 & \operatorname{Sr}(\mathrm{OH}) 2\end{array}$

$127 \mathrm{Sr}(\mathrm{OH}) 2$

$30 . \operatorname{srCO} 3$

46 Sro

159 TCO2

92 ThO2

107 TiO2

119 Tritium

170308

$62 \mathrm{UO} 2$ ( $\mathrm{COOH}) 2$

$177 \mathrm{UO} 2(\mathrm{NO} 3)^{2} 2$
$16 \mathrm{UO2}(\mathrm{OH}) 2$

$16 \mathrm{UO} 2(\mathrm{OH}) 2$
$27 \mathrm{Y}(\mathrm{COOH})^{3}$

178 Y (NO3) 3

$128 \mathrm{Y}(\mathrm{OH}) 3$

$31 \mathrm{Y} 2(\mathrm{CO} 3) 3$

$47 \mathrm{Y} 203$

100 Zeolite

$44 \mathrm{Zn}(\mathrm{COOH}) 2$

$179 \mathrm{Zn}(\mathrm{NO} 3) 2$

$57 \mathrm{ZnO}$

$104 \mathrm{ZrO} 2$

13 hydrate $H 20$

TOTAL FLOW, LB/HR

TEMPERATURE, DEG C

PRESSURE, ATM

PRESSURE， PSIG

PRESSURE, MM HG

ENTHALPY, PCU/HR

VAPOR FLOW, CFM

LIQUID FLOW, GRM

DENSITY, LBS/FT3

PHASE

WSRC-TR-95-0019 (Revision

Section 4B. Fit Adjustment of Melter Feed

38 SMECT Vent

to FAVC

ZERO

ZERO

ZERO

ZERO

ZERO

ZERO

ZERO

ZERO

ZERO
$0.9058 \mathrm{E}-12$

$0.3096 \mathrm{E}-17$

$0.8361 \mathrm{E}-15$

$0.1154 \mathrm{E}-15$ ZERO

$0.1305 \mathrm{E}-20$

$0.1014 \mathrm{E}-15$

$0.8442 \mathrm{E}-15$

$0.2355 \mathrm{E}-13$

$0.3691 E-11$

$0.5919 \mathrm{E}-13$

$0.3604 \mathrm{E}-19$

$0.2595 \mathrm{E}-17$

ZERO

TRACE

$0.2255 E-15$

ZERO

$0.2743 \mathrm{E}-16$

ZERO

$0.7392 \mathrm{E}-14$

$0.5088 \mathrm{E}-14$

ZERO

$0.4258 \mathrm{E}-1$

$0.6729 \mathrm{E}+01$

$0.8307 \mathrm{E}+02$

$0.4900 \mathrm{E}+02$

$0.1000 \mathrm{E}+01$

ZERO

$0.5257 \mathrm{E}+04$

$.2126 \mathrm{~B}+02$

$511 \mathrm{E}-01$

VAPOR
40

FAVC HEME

Vnt to PVVS

ZERO

ZERO

ZERO

ZERO

ZERO

ZERO

ZERO

$0.1132 \mathrm{E}-13$

$0.3870 \mathrm{E}-19$

$0.1442 \mathrm{E}-17$

ZERO

$0.1631 \mathrm{E}-22$

$0.1267 \mathrm{E}-17$

$0.1055 \mathrm{E}-16$

$0.2943 \mathrm{E}-15$

$0.4173 \mathrm{E}-10$

$0.7399 \mathrm{E}-15$

$0.4505 \mathrm{E}-21$

ZERO

ZERO

TRACE
ZERO

ZERO

$0.3429 \mathrm{E}-18$

0.9241 ZERO

2ERO

$0.6360 \mathrm{E}-16$

$0.5323 \mathrm{E}-15$

$0.1281 \mathrm{E}+02$

$0.1750 \mathrm{E}+04$

$0.1000 \mathrm{E}+02$

$0.9263 \mathrm{E}+00$

$-0.1083 \mathrm{E}+01$

$0.1745 \mathrm{E}+05$

$0.4074 \mathrm{E}+03$

$0.7161 \mathrm{E}-01$

VAPOR
41

FAVC HEME

Accumulatn

ZERO

ZERO

ZERO

ZERO

ZERO

ZERO

ZERO

ZERO

$0.4416 \mathrm{E}-12$

$0.1509 \mathrm{E}-17$

$0.5625 \mathrm{E}-16$

ZERO

$0.6362 \mathrm{E}-21$

$0.4942 \mathrm{E}-16$

$0.4116 \mathrm{E}-15$

$0.1148 \mathrm{E}-13$

ZERO

$.2886 \mathrm{E}-13$

$0.1757 \mathrm{E}-19$

ZERO

ZERO
TRACE

ZERO

ZERO

$0.1337 \mathrm{E}-16$

$0.3604 \mathrm{E}-14$

ZERO

E-14

ZERO

$.2076 \mathrm{E}-13$
ZERO

$0.1818 \mathrm{E}-11$

$0.1000 \mathrm{E}+02$

$0.9263 \mathrm{E}+00$

$-0.1083 E+01$

$0.3230 \mathrm{E}-11$

$0.1527 \mathrm{E}+03$

SOLID
43

SMECT Liq

to RCT

ZERO

ZERO

ZERO

ZERO

ZERO

ZERO

ZERO

ZERO

$0.1169 \mathrm{E}-03$

0.3996 E-09

$0.1079 \mathrm{E}-06$
$0.1489 \mathrm{E}-07$

ZERO

ZERO

$0.1684 \mathrm{E}-12$

$0.1308 \mathrm{E}-07$

$0.1090 \mathrm{E}-06$

$0.3039 \mathrm{E}-05$

$0.6462 \mathrm{E}-09$

$0.4652 \mathrm{E}-11$

$0.3349 \mathrm{E}-09$

$0.4818 \mathrm{E}-17$

$0.2911 E-07$

ZERO

$0.354 \mathrm{1E}-08$

$0.9541 \mathrm{E}-06$

$0.1951 \mathrm{E}-09$

$0.6566 \mathrm{E}-06$

ZERO
$0.5495 \mathrm{E}-05$

$0.5495 \mathrm{E}-05$
$0.1178 \mathrm{E}+04$

$0.1183 \mathrm{E}+04$

$0.4900 \mathrm{E}+02$

0.1000 E+01
ZERO

$0.5787 \mathrm{E}+05$

$0.2360 \mathrm{E}+01$

$0.6251 \mathrm{E}+02$

IIQUID
122

SMECT Liq
Solubles

ZERO

ZERO

ZERO

ZERO

ZERO

ZERO

ZERO

ZERO

ZERO

ZERO

$0.3996 \mathrm{E}-09$

$0.1079 \mathrm{E}-06$

ZERO
ZERO

2ERO

ZERO

ZERO

ZERO

ZERO

$0.6462 \mathrm{E}-0.9$

ZERO

$0.4652 \mathrm{E}-11$

$0.3349 \mathrm{E}-09$

$0.4818 \mathrm{E}-17$

$0.2911 \mathrm{E}-07$

ZERO

ZERO

ZERO

$0.9541 \mathrm{E}-06$

$0.1951 \mathrm{E}-09$

ZERO

ZERO

ZERO

$0.1178 \mathrm{E}+04$

$0.1183 \mathrm{E}+04$

$0.4900 \mathrm{E}+02$

$0.1000 \mathrm{E}+01$
ZERO

$0.5787 \mathrm{E}+05$

$.2360 \mathrm{E}+01$

$.6251 \mathrm{E}+02$

IIQUID

123

SMECT Iiq

Insolubles ZERO

ZERO

ZERO

ZERO

ZERO

ZERO

ZERO

ZERO

$9 \mathrm{E}-03$

$0.1489 \mathrm{E}-07$ ZERO

$0.1684 \mathrm{E}-12$

1308E-07

. $1090 \mathrm{E}-06$

$0.3039 \mathrm{E}-05$

0.763 ZERO

E-05

ZERO

ZERO

ZERO

ZERO

ZERO

0.3541 EERO

ZERO 
Table A-1. HLW Flowsheet Material Balance (Tank 51/ITP Cyale 1) Section 4B. Frit Adjustment of Melter Feed

STREAM NUMBERS $\rightarrow$ STREAM NAME

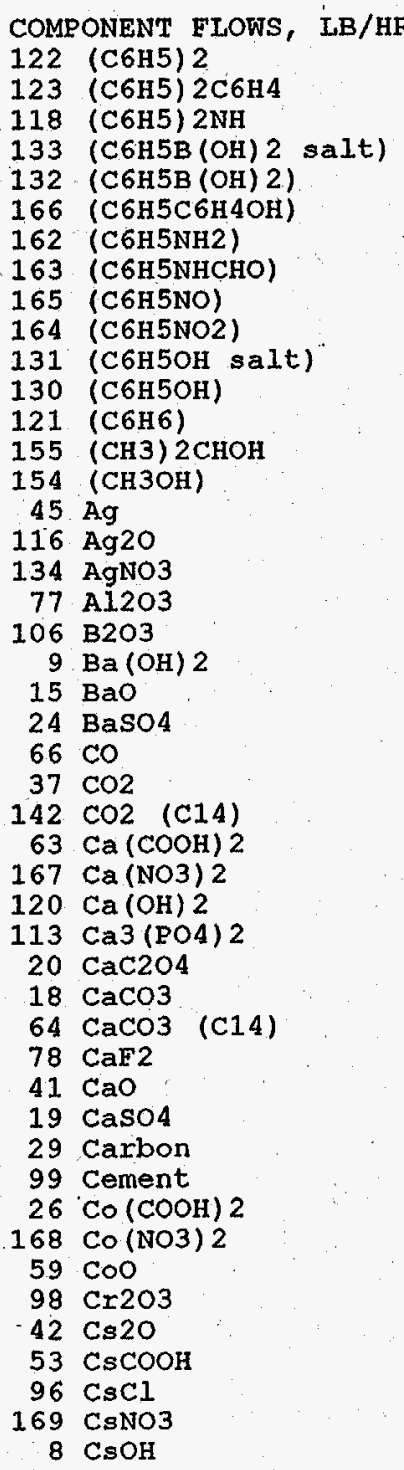

WSRC-TR-95-0019 (Revision
45

44

SME Concen- Melter Loop MFT Sample trate to MFT Water Flush Pump Prime

$0.2348 \mathrm{E}-15$

$0.9245 \mathrm{E}-02$

$0.4476 \mathrm{E}-08$

ZERO

ZERO
$0.5523 E-01$

$0.1793 \mathrm{E}-03$

$0.2092 \mathrm{E}+00$

$0.3406 \mathrm{E}-02$

$0.6938 \mathrm{E}-02$

$0.8234 \mathrm{E}-15$

$0.5070 \mathrm{E}-01$

$0.3022 \mathrm{E}-22$

$0.2687 \mathrm{E}-11$
$0.4173 \mathrm{E}-13$

$0.1241 \mathrm{E}-01$

$0.1241 \mathrm{E}-01$

ZERO

$0.8834 \mathrm{E}+01$

$0.1266 \mathrm{E}+02$

$0.1617 \mathrm{E}-19$

$0.1704 \mathrm{E}-01$

ZERO

TRACE

TRACE

$0.4188 \mathrm{E}+01$

ZERO
$6 \mathrm{E}+01$

$0.2626 \mathrm{E}+01$
$0.2442 \mathrm{E}-06$

ZERO

ZERO
$0.1501 \mathrm{E}-01$

$.3344 \mathrm{E}-02$

$0.2208 \mathrm{E}+00$

ZERO

ZERO

$0.1433 \mathrm{E}-03$

$\mathrm{E}-06$

$0.3069 \mathrm{E}-04$

$0.4302 \mathrm{E}+00$
$0.1403 \mathrm{E}-03$

$0.4330 \mathrm{E}-01$

$0.9608 \mathrm{E}-05$

ZERO
ZERO

0)

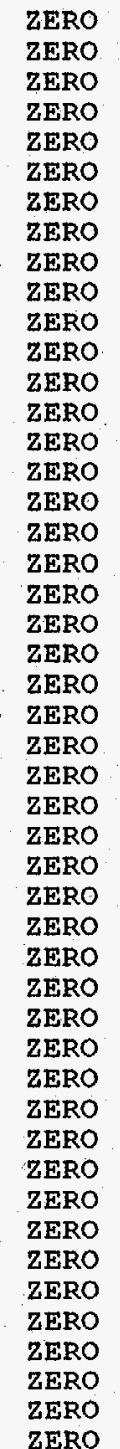

47

48

49

to Scrubber H2O Elush

ZERO
ZERO
ZERO
ZERO
ZERO
ZERO
ZERO
ZERO
ZERO
ZERO
ZERO
ZERO
ZERO
ZERO
ZERO
ZERO
ZERO
ZERO
ZERO
ZERO
ZERO
ZERO
ZERO
ZERO
ZERO
ZERO
ZERO
ZERO
ZERO
ZERO
ZERO
ZERO
ZERO
ZERO
ZERO
ZERO
ZERO
ZERO
ZERO
ZERO
ZERO
ZERO
ZERO
ZERO
ZERO
ZERO
ZERO
ZERO

ZERO
ZERO
ZERO
ZERO
ZERO
ZERO
ZERO
ZERO
ZERO
ZERO
ZERO
ZERO
ZERO
ZERO
ZERO
ZERO
ZERO
ZERO
ZERO
ZERO
ZERO
ZERO
ZERO
ZERO
ZERO
ZERO
ZERO
ZERO
ZERO
ZERO
ZERO
ZERO
ZERO
ZERO
ZERO
ZERO
ZERO
ZERO
ZERO
ZERO
ZERO
ZERO
ZERO
ZERO
ZERO
ZERO
ZERO
ZERO

$0.2345 \mathrm{E}-1.5$

$0.4657 \mathrm{E}-09$

$0.2552 \mathrm{E}-09$

ZERO
ZERO

$0.1094 \mathrm{E}-08$

$0.1449 \mathrm{E}-04$

$0.4145 E-08$

$0.6748 \mathrm{E}-10$

$0.1375 \mathrm{E}-09$

$0.1631 \mathrm{E}-22$

$0.1945 \mathrm{E}-02$

$0.2997 \mathrm{E}-22$

$0.9932 \mathrm{E}-13$

$0.9932 \mathrm{E}-13$

$0.2459 \mathrm{E}-09$

$0.2459 \mathrm{E}-09$

ZERO
$0.1750 \mathrm{OE}-06$

$0.1750 \mathrm{E}-06$
$0.2509 \mathrm{E}-06$

TERO

$0.3376 \mathrm{E}-09$

ZERO

$0.4379 \mathrm{E}-16$

TRACE

ZERO

$0.8297 \mathrm{E}-07$

$0.5203 \mathrm{E}-07$

$0.4838 \mathrm{E}-14$

ZERO

$0.2973 \mathrm{E}-09$

$0.6625 \mathrm{E}-10$

$0.4375 \mathrm{E}-08$

ZERO

$0.2839 \mathrm{E}-11$

$0.1065 \mathrm{E}-13$

$0.6081 \mathrm{E}-12$

$0.8523 \mathrm{E}-08$

$0.2779 \mathrm{E}-11$
$0.8580 \mathrm{E}-09$

$0.8580 \mathrm{E}-09$

ZERO
$0.1904 \mathrm{E}-12$

E -12
ZERO

ZERO

Date: January 1995
60

Melter

$0.2853 \mathrm{E}-18$

$0.9245 \mathrm{E}-02$

$0.4221 \mathrm{E}-08$

ZERO

$0.5523 \mathrm{E}-01$

$0.1648 \mathrm{E}-03$

$0.2092 \mathrm{E}+00$

$0.3406 \mathrm{E}-02$

$0.6938 \mathrm{E}-02$

$0.8234 \mathrm{E}-15$

$0.8234 \mathrm{E}-15$

$0.4876 \mathrm{E}-01$

$0.2534 \mathrm{E}-24$

$0.2588 \mathrm{E}-11$

$0.3832 \mathrm{E}-13$

$0.1241 \mathrm{E}-01$
ZERO

ZERO
$0.8834 \mathrm{E}+01$

$0.1266 \mathrm{E}+02$

0.1617 ZERO

$0.1704 \mathrm{E}-01$

ZERO

$0.9807 E-19$

TRACE

$0.4188 \mathrm{E}+01$

ZERO
$0.2626 \mathrm{E}+01$

$0.2442 \mathrm{E}-06$

ZERO

$0.1501 \mathrm{Et} 01$

$0.3344 \mathrm{E}-02$

$0.2208 \mathrm{E}+00$

ZERO

ZERO

$0.1433 \mathrm{E}-03$

$0.5373 \mathrm{E}-06$

$0.3069 \mathrm{E}-04$

$0.4302 \mathrm{E}+00$

$0.1403 \mathrm{E}-03$

$0.4330 \mathrm{E}-01$

ZERO
$0.9608 \mathrm{E}-05$

ZERO

ZERO 
Table A-1. HLW Flowsheet Matexial Balance (Tank 51/ITP Cycle 1) Section 4B. Frit Adjustment of Melter Feed

STREAM NUMBERS (CONT'D) $\rightarrow$ STREAM NAME

$54 \mathrm{Cu}(\mathrm{COOH}) 2$

$170 \mathrm{Cu}(\mathrm{NO} 3) 2$

$55 \mathrm{CuO}$

$80 \mathrm{Fe} 2 \mathrm{O} 3$

$101 \mathrm{FeO}$

102 Group A

103 Group B

$25 \mathrm{H} 2$

$158 \mathrm{H2} 2204$

$93 \mathrm{H} 2 \mathrm{SO} 4$

$34 \mathrm{HCOOH}$

$43 \mathrm{HCl}$

49 HNO3

$36 \mathrm{Hg}$

$129 \mathrm{Hg}(\mathrm{C} 6 \mathrm{H} 5) 2$

135. $\mathrm{Hg}$ (NO3) 2

$144 \mathrm{Hg} 2 \mathrm{Cl} 2$

$117 \mathrm{Hg} 2 \mathrm{I2}$

$146 \mathrm{HgCl2}$

$21 \mathrm{HgO}$

$183 \mathrm{~K} 2 \mathrm{CO} 3$

$39 \mathrm{~K} 20$.

$51 \mathrm{KCOOH}$

$157 \mathrm{KMnO4}$

161. KNO3

$10 \mathrm{KOH}$

$150 \mathrm{KTPB}$

$105 \mathrm{La}_{203}$

$108 \mathrm{Li} 20$

$65 \mathrm{Mg}$ (COOH) 2

$171 \mathrm{Mg}(\mathrm{NO}$

$60 \mathrm{Mn}(\mathrm{COOH}) 2$

$172 \mathrm{Mn}(\mathrm{NO} 3) 2$

$180 \mathrm{Mn} 304$

$56 \mathrm{MnO}$

$14 \mathrm{MnO} 2$

$145 \mathrm{MoO} 2$

81 N2

$74 \mathrm{~N} 2 \mathrm{O}$

114 NH3OHNO3

$52 \mathrm{NH} 4 \mathrm{COOH}$

173 NH4NO3

$68 \mathrm{NH} 4 \mathrm{OH}$

148 NH4TPB

WSRC-TR-95-0019 (Revision
$44 \cdot 45$

SME Concen- Melter Loop MFT Sample trate to MFT Water Flush $0.4430 \mathrm{E}-02$ ZERO $0.2179 \mathrm{E}+01$

$0.1969 \mathrm{E}-01$

$0.2539 \mathrm{E}+02$

$0.2538 \mathrm{E}+01$

$0.3543 \mathrm{E}-02$

$0.1512 \mathrm{E}-03$

$0.3265 \mathrm{E}-22$

ZERO
ZERO

ZERO
$0.7707 \mathrm{E}+01$

ZERO

ZERO

$0.1388 \mathrm{E}-09$
$0.1603 \mathrm{E}+00$

ZERO
ZERO
ZERO

ZERO

ZERO

ZERO

ZERO

$0.8073 \mathrm{E}-01$

$0.1005 \mathrm{E}+02$

ZERO
$0.2858 \mathrm{E}-01$
ZERO

ZERO
ZERO

ZERO

$0.1108 \mathrm{E}+02$

$0.3463 \mathrm{E}+01$

$0.3470 \mathrm{E}+01$

$0.2169 \mathrm{E}+01$

$0.6122 \mathrm{E}-07$

ZERO

$0.3831 \mathrm{E}-1$

$0.1948 \mathrm{E}+01$

$0.1075 \mathrm{E}-02$

$0.9923 \mathrm{E}-24$

ZERO

ZERO

ZERO

$0.1391 \mathrm{E}-02$

ZERO

Pump Prime

ZERO
ZERO

ZERO

ZERO

ZERO

ZERO

ZERO

ZERO

ZERO

ZERO

ZERO

ZERO

ZERO

ZERO

ZERO

ZERO

ZERO

ZERO

ZERO

ZERO

ZERO

ZERO

ZERO

ZERO

ZERO

ZERO

ZERO

ZERO

ZERO

ZERO

ZERO

ZERO

ZERO

ZERO

ZERO

ZERO

ZRRO

ZERO

ZERO

ZERO

ZERO

ZERO

ZERO

ZERO

ZERO

ZERO

ZERO

Date: January 1995

ZERO

ZERO

ZERO

ZERO

ZERO

ZERO

ZERO

ZERO

ZERO

ZERO

ZERO

ZERO

ZERO

ZERO

ZERO

ZERO

ZERO

ZERO

ZERO

ZERO

IERO

ZERO

ZERO

ZERO

ZERO

ZERO

ZERO

ZERO

ZERO

ZERO

ZERO

ZERO

ZERO

ZERO

ZERO

ZERO

ZERO

ZERO

ZERO

ZERO

ZERO

ZERO

ZERO

ZERO
47

MFT Air

${ }_{\text {MFT Vent Mltr Fd tube }}^{48}$

o)
Inleakage

ZERO
ZERO

Sorubber

$0.8778 \mathrm{E}-10$

$0.4317 \mathrm{E}-07$

$0.3902 E-09$

$0.5030 \mathrm{E}-06$

$0.7020 \mathrm{E}-10$

$0.2995 \mathrm{E}-11$

$0.3620 \mathrm{E}-08$

ZERO

0.15

ZERO

ZERO

ZERO

ZERO

ZERO

ZERO

ZERO

ZERO

ZERO

ZERO

ZERO

ZERO

ZERO

ZERO

ZERO

ZERO

ZERO

ZERO

ZERO

ZERO

ZERO

ZERO

ZERO

ZERO

ZERO

ZERO

ZERO

ZERO

$0.5484 \mathrm{E}+02$

ZERO

ZERO

ZERO

ZERO

ZERO

ZERO ZERO

$0.6978 \mathrm{E}-11$

$0.9784 \mathrm{E}-02$

ZERO

ZERO

ZERO

ZERO

ZERO

ZERO

ZERO

$0.1599 \mathrm{E}-08$

$0.1990 \mathrm{E}-06$

0.5663 ZERO

$3 \mathrm{E}-09$

ZERO

ZERO

$0.2195 \mathrm{E}-06$

$0.6862 \mathrm{E}-07$

$0.1041 \mathrm{E}-14$

$0.4298 \mathrm{E}-07$

ZERO

$0.2130 \mathrm{E}-10$

$0.5483 \mathrm{E}+02$

ZERO

ZERO

ZERO

$0.2755 \mathrm{E}-10$

ZERO

ZERO
$0.5028 \mathrm{E}-07$

60

Melter

$0.4430 \mathrm{E}-02$

$0.2179 \mathrm{E}+01$

$0.1969 E-01$

$0.2539 \mathrm{E}+02$

$0.2538 \mathrm{E}+01$

$0.3543 \mathrm{E}-02$

$0.1512 \mathrm{E}-03$

0.1827 TRACE 


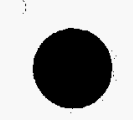

WSRC-TR-95-0019 (Revision

Table A-1. HiW Flowsheet Material Balance (Tank 51/ITP Cycle 1)

$$
\text { Section 4B. Frit Adjustment of Melter Feed }
$$

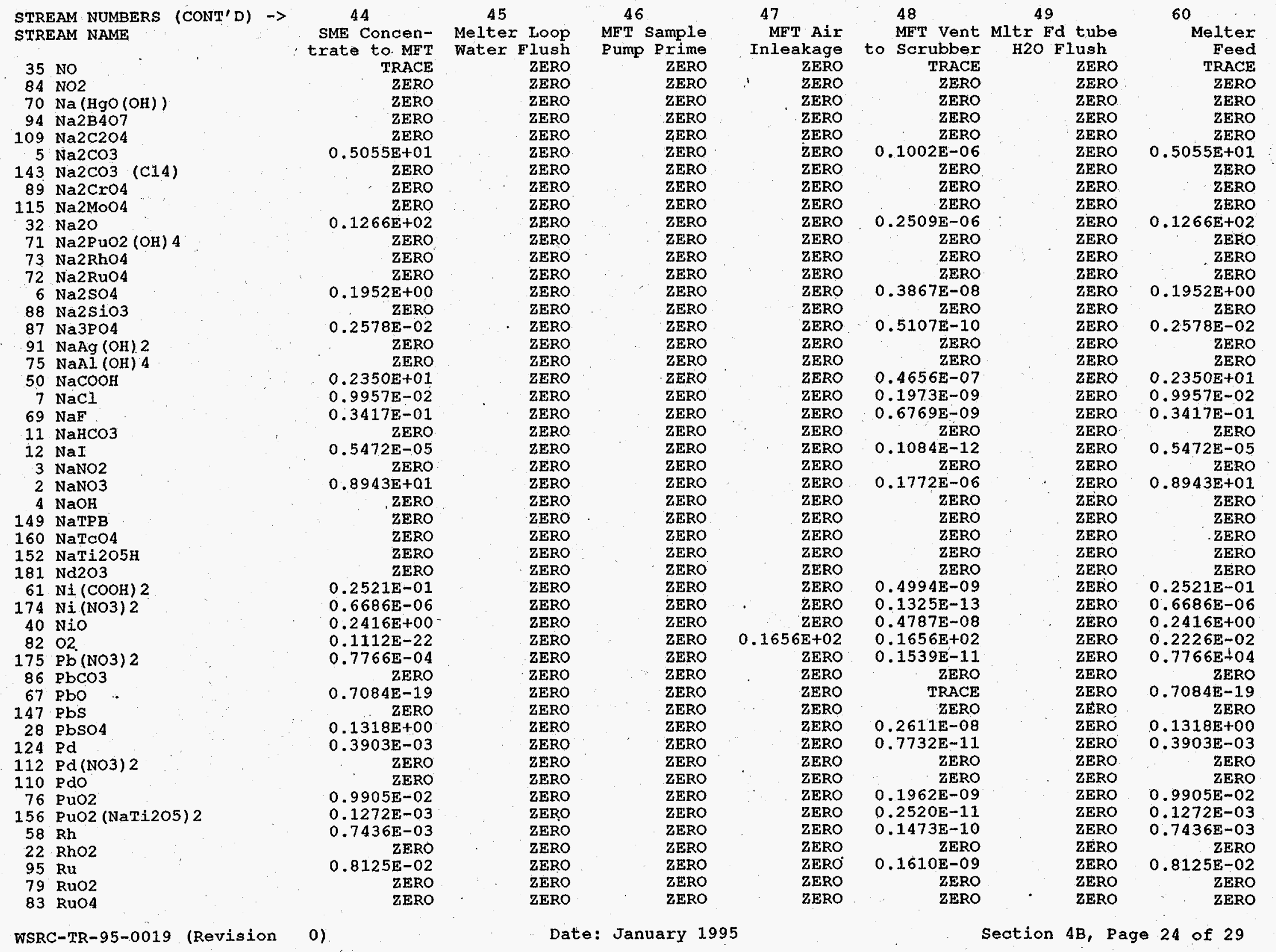


Table A-1. HLW Flowsheet Material Balance (Tank 51/ITP Cycle 1) Section 4B: Frit Adjustment of Melter Feed

STREAM NUMBERS (CONT'D) $\rightarrow$ STREAM NAME

$38 \mathrm{sO} 2$

$141 \mathrm{SO} 3$

137 Semi Vol Cs2O

136 Semi Vol CsC1

126 Semi Vol Group A

138 Semi Vol NaCl

139 Semi Vol NaF

139 Semi Vol Nat

23. Semi Vol $\mathrm{RuO2}$

111 SiO2.

$48 \mathrm{sr}(\mathrm{COOH}) 2$

$176 \mathrm{Sr}$ (NO3) 2

$153 \mathrm{Sr}(\mathrm{NaTi2O5)} 2$

$127 \mathrm{Sr}(\mathrm{OH}) 2$

$30 \mathrm{SrCO} 3$

46. $\mathrm{SrO}$

$159 \mathrm{TCO} 2$

92 ThO2

119 Tritium

$\begin{array}{ll}119 & \text { Triti } \\ 17 & \mathrm{U} 308\end{array}$

$62 \mathrm{UO2}$ ( $\mathrm{COOH}) 2$

$177 \mathrm{UO} 2$ (NO3) 2

$16 \mathrm{UO} 2(\mathrm{OH}) 2$

$27 \mathrm{Y}(\mathrm{COOH}) 3$

$178 \mathrm{Y}^{\mathrm{NO} 3)^{3}}$

$128 \mathrm{Y}(\mathrm{OH}) 3$

$47 \mathrm{Y} 203$

100 zeolite

$44 \mathrm{Zn}(\mathrm{COOH}) 2$

$179 \mathrm{Zn}$ (NO3) 2

$57 \mathrm{ZnO}$

$\begin{array}{rl}104 & \mathrm{ZrO} 2 \\ 13 & \text { hydrate } \mathrm{H} 2 \mathrm{O}\end{array}$

1 water

TOTAL FLOW, IB/HR

TEMPERATURE， DEG C

PRESSURE, ATM

PRESSURE， PSIG

PRESSURE， MM.HG

ENTHALPY, PCU/HR

VAPOR FLOW, CFM

IIQUID FLOW, GPM

DENSITY， LBS/ET3

PHASE

WSRC-TR-95-0019 (Revision
44

SME Concen- Melter Loop trate to MFT Water Flush

\begin{tabular}{|c|c|c|}
\hline ZERO & ZERO & ZERO \\
\hline ZERO & ZERO & ZERO \\
\hline ZERO & ZERO & ZERO \\
\hline ZERO & ZERO & ZERO \\
\hline ZERO & ZERO & ZERO \\
\hline ZERO & ZERO & ZERO \\
\hline ZERO & ZERO & ZERO \\
\hline ZERO & ZERO & ZERO \\
\hline ZERO & ZERO & ZERO \\
\hline ZERO & ZERO & ZERO \\
\hline $0.1230 \mathrm{E}+03$ & ZERO & ZERO \\
\hline $0.1093 \mathrm{E}-03$ & ZERO & ZERO \\
\hline $0.2944 \mathrm{E}-01$ & ZERO & ZERO \\
\hline $0.4072 \mathrm{E}-02$ & ZERO & ZERO \\
\hline ZERO & ZERO & ZERO \\
\hline ZERO & ZERO & ZERO \\
\hline $0.2218 \mathrm{E}-18$ & ZERO & ZERO \\
\hline $0.3569 \mathrm{E}-02$ & ZERO & ZERO \\
\hline $0.2972 \mathrm{E}-01$ & ZERO & ZERO \\
\hline $0.8309 \mathrm{E}+00$ & ZERO & ZERO \\
\hline $0.1452 \mathrm{E}-09$ & ZERO & ZERO \\
\hline $0.2084 \mathrm{E}+01$ & ZERO & ZERO \\
\hline $0.1272 \mathrm{E}-05$ & ZERO & ZERO \\
\hline $0.9137 \mathrm{E}-04$ & ZERO & ZERO \\
\hline ZERO & - ZERO & ZERO \\
\hline $0.1317 \mathrm{E}-11$ & ZERO & ZERO \\
\hline $0.7940 \mathrm{E}-02$ & ZERO & ZERO \\
\hline ZERO & ZERO & ZERO \\
\hline ZERO & ZERO & ZERO \\
\hline $0.9683 \mathrm{E}-03$ & ZERO & ZERO \\
\hline ZERO & ZERO & ZERO \\
\hline $0.2607 \mathrm{E}+00$ & ZERO & ZERO \\
\hline $0.5336 \mathrm{E}-04$ & ZERO & ZERO \\
\hline $0.1794 \mathrm{E}+0.0$ & ZERO & ZERO \\
\hline ZERO & ZERO & ZERO \\
\hline $0.1499 \mathrm{E}+01$ & ZERO & ZERO \\
\hline
\end{tabular}

$0.3150 \mathrm{E}+03$

$0.5724 \mathrm{E}+03$

$0.5000 \mathrm{E}+02$

$0.1000 \mathrm{E}+01$
ZERO

ZERO

$0.1826 \mathrm{E}+05$

$0.8482 \mathrm{E}+00$

$0.8413 \mathrm{E}+02$

LIQUID
$0.2500 \mathrm{E}+02$

$0.1000 \mathrm{E}+01$
ZERO

$0.1746 \mathrm{E}+03$

$0.1390 \mathrm{E}-01$

$0.6243 \mathrm{E}+02$

LIQUID
46 MFT Sample rime

47

$0.1950 \mathrm{E}+01$

$0.1950 \mathrm{E}+01$

$0.2500 \mathrm{E}+02$

$0.1000 \mathrm{E}+01$
ZERO

$0.4893 \mathrm{E}+02$

$0.3894 \mathrm{E}-02$

$0.6243 E+02$

IIQUID
MFT Air

Inleakage

ZERO
ZERO

ZERO

ZERO

ZERO

ZERO

ZERO

ZERO

ZERO

ZERO

ZERO

ZERO

ZERO

ZERO

ZERO

ZERO

ZERO

ZERO

ZERO

ZERO

ZERO

ZERO

ZERO

ZERO

ZERO

ZERO

ZERO

ZERO

ZERO

ZERO

ZERO

ZERO

ZERO

MFT Vent

ZERO

ZERO

ZERO

ZERO

ZERO

ZERO

ZERO

ZERO

ZERO

$0.2436 \mathrm{E}-05$

$0.2165 \mathrm{E}-11$

$0.5832 \mathrm{E}-09$

$0.8068 \mathrm{E}-10$

ZERO

ZERO

$0.7072 \mathrm{E}-10$

$0.5889 \mathrm{E}-09$

$0.1646 \mathrm{E}-07$

$.2778 E-11$

$0.4129 E-07$

$.2520 \mathrm{E}-13$

$.1810 \mathrm{E}-11$

ZERO

$0.1573 E-09$

ZERO

$0.1918 \mathrm{EE}-10$

ZERO

$0.5166 \mathrm{E}-08$

$0.1057 \mathrm{E}-11$

$0.3554 \mathrm{E}-08$

ZERO

$0.1200 \mathrm{E}+01 \quad 0.2970 \mathrm{E}-07$

$0.6313 \mathrm{E}+01$

$0.7772 \mathrm{E}+02$

$0.3500 \mathrm{E}+02$

$0.1000 \mathrm{E}+01$

ZERO

$0.5000 \mathrm{E}+02$

$0.9656 \mathrm{E}+00$
$-0.5055 \mathrm{E}+00$

$0.7339 \mathrm{E}+03$

$\begin{array}{ll}0.1523 \mathrm{E}+04 & 0.4931 \mathrm{E}+04 \\ 0.1715 \mathrm{E}+02 & 0.2070 \mathrm{E}+02\end{array}$

$0.7055 \mathrm{E}-01$

$0.6256 \mathrm{E}-01$

VAPOR
49

tr Fd tube

20 Flush

ZERO

ZERO

ZERO

ZERO

ZERO

ZERO

ZERO

ZERO

ZERO

ZERO

ZERO

ZERO

ZERO

ZERO

ZERO

ZERO

ZERO

ZERO

ZERO

ZERO

ZERO

ZERO

ZERO

ZERO

ZERO

ZERO

ZERO

ZERO

ZERO

ZERO

ZERO

ZERO

ZERO

$0.1000 \mathrm{t}+01$

$0.1000+01$

$0.2500 \mathrm{E}+02$

$0.1000 \mathrm{E}+01$

ZERO

$0.2509 \mathrm{E}+02$

$0.1997 \mathrm{E}-02$

$0.6243 \mathrm{E}+02$

LIQUID
60

Melter

Feed

ZERO

ZERO

ZERO

ZERO

ZERO

ZERO

ZERO

ZERO

0.1230E+03

. $1093 \mathrm{~m}-03$

$0.2944 \mathrm{E}-01$

$0.4072 \mathrm{E}-02$

ZERO

$0.2218 E-18$

$0.3569 \mathrm{E}-02$

$0.2972 \mathrm{E}-01$

$0.8309 \mathrm{E}+00$

$.1424 \mathrm{E}-09$

. $2084 \mathrm{E}+01$ ZERO

$.1317 \mathrm{E}-11$

$0.7940 \mathrm{E}-02$

ZERO

$0.9683 \mathrm{E}-03$ ZERO

$0.2607 \mathrm{E}+00$

$0.1794 \mathrm{E}+00$

0. 1499E+01

$0.4987 \mathrm{E}+02$

$0.9656 \mathrm{E}+00$

$-0.5055 \mathrm{E}+00$

$0.7339 \mathrm{E}+03$
$0.1865 \mathrm{E}+05$

$0.8656 \mathrm{E}+00$

LIQUID 
Table A-1. HLW Flowsheet Material Balance (Tank 51/ITP Cycle 1) Section 4B. Frit Adjustment of Melter Feed

STREAM NUMBERS $\rightarrow$

STREAM NAME

\section{COMPONENT FLOWS, LB/HR}

1.22 . (C6H5) 2

123 (C6H5) 2C6H4

118 (C6H5) $2 \mathrm{NH}$

133 (C6H5B (OH) 2 salt)

132 ( 6 $6 \mathrm{H} 5 \mathrm{~B}(\mathrm{OH}) 2$ )

166 (C6H5C6H4OH)

162 (C6H5NH2)

163 (C6H5NHCHO)

165 (C6H5NO)

164 (C6H5NO2)

131 (C6H5OH salt)

130 (C6H5OH)

121 (C6H6)

155 (CH3) $2 \mathrm{CHOH}$

154 (CH3OH)

$45 \mathrm{Ag}$

$116 \mathrm{Ag} 20$

134 AgNO3

77 A.203

$106 \mathrm{~B} 203$

$9 \mathrm{Ba}(\mathrm{OH}) 2$

$15 \mathrm{BaO}$

$24 \mathrm{BaSO} 4$

$66 \mathrm{CO}$

$142 \mathrm{CO} 2$ (C14)

$63 \mathrm{Ca}(\mathrm{COOH}) 2$

$167 \mathrm{Ca}(\mathrm{NO} 3) 2$

$120 \mathrm{Ca}(\mathrm{OH}) 2$

$113 \mathrm{Ca} 3$ (PO4) 2

$20 \mathrm{CaC2O4}$

$64 . \mathrm{CaCO}^{2}$ (C14)

78 CaF2

$41 \mathrm{CaO}$

19 CaSO4

29 Carbon

$26 \mathrm{Co}(\mathrm{COOH}) 2$

$168 \mathrm{Co}(\mathrm{NO} 3) 2$

$59 \mathrm{CoO}$

98. $\operatorname{Cr} 203$

$52 \mathrm{Cs} 2 \mathrm{O}$

$53 \mathrm{CsCOOH}$

169 CanO3

$8 \mathrm{CsOH}$

151 CsTPB

WSRC-TR-95-0019 (Revision
58

Melter Feed Meiter Feed

Solubles Insolubles

ZERO $0.2853 \mathrm{E}-18$

ZERO $0.9245 \mathrm{E}-02$

ZERO $\quad 0.4221 \mathrm{E}-08$

ZERO ZERO

ZERO ZERO

$0.1648 \mathrm{E}-03 \quad$ ZERO

ZERO $\quad 0.2092 \mathrm{E}+00$

$\begin{array}{rr}\text { ZERO } & 0.3406 \mathrm{E}-02 \\ 0.6938 \mathrm{E}-02 & \text { ZERO }\end{array}$

$0.8234 \mathrm{E}-15 \quad$ ZERO

$0.4876 \mathrm{E}-01$ ZERO

$0.2534 \mathrm{E}-24 \quad$ ZERO

$0.2588 \mathrm{E}-11$ ZERO

$0.3832 \mathrm{E}-13 \quad$ ZERO

$\begin{array}{rr}\text { ZERO } & 0.1241 \mathrm{E}-01 \\ \text { ZERO }\end{array}$

ZERO ZERO

ZERO $\quad 0.8834 \mathrm{E}+01$

ZERO $\quad 0.1266 \mathrm{E}+02$

$\begin{array}{lr}\text { ZERO } & \text { ZERO } \\ \text { ZERO } & 0.1617 \mathrm{E}-19\end{array}$

ZERO $0.1704 \mathrm{E}-01$

ZERO
0.9807 E-19
ZERO

TRACE ZERO

ZERO
$0.4188 \mathrm{E}+01$

ZERO - ZERO

ZERO $\quad 0.2626 \mathrm{E}+01$

ZERO $\quad 0.2442 \mathrm{E}-06$

ZERO ZERO

ZERO ZERO

ZERO $\quad 0.1501 E-01$

ZERO $\quad 0.3344 \mathrm{E}-02$

ZERO $\quad 0.2208 \mathrm{E}+00$

ZERO ZERO

ZERO
0.1433 E-03
ZERO

$0.5373 \mathrm{E}-06$ ZERO

$0.3069 \mathrm{E}-04$

$0.4302 \mathrm{E}+00$

$0.1403 \mathrm{E}-03$

$0.4330 \mathrm{E}-01 \quad 0.2589 \mathrm{E}-08$

ZERO
$0.9608 \mathrm{E}-05$

ZERO ZERO 
Table A-1. HLW Flowsheet Material Balance (Tank 51/ITP Cycle 1) Section 4B. Frit Adjustment of Melter Feed

STREAM NUMBERS (CONT'D) $\rightarrow$ STREAM NAME

$54 \mathrm{Cu}(\mathrm{COOH}) 2$

$170 \mathrm{Cu}(\mathrm{NO} 3)^{2}$

$55 \mathrm{CuO}$

$80 \mathrm{Fe} 2 \mathrm{O3}$

$101 \mathrm{FeO}$

102 Group A

103 Group B

$25 \mathrm{H2}$

158 H2C2O4

$93 \mathrm{H} 2 \mathrm{SO} 4$

$90 \mathrm{H} 3 \mathrm{BO} 3$

$34 \mathrm{HCOOH}$

$43 \mathrm{HCl}$

85 HF

49 HNO3

$129 \mathrm{Hg}(\mathrm{C} 6 \mathrm{H} 5) 2$

$135 \mathrm{Hg}(\mathrm{NO} 3)^{2}$

$144 \mathrm{Hg} 2 \mathrm{C} 12$

$117 \mathrm{Hg} 2 \mathrm{I2}$

$146 \mathrm{HgCl}$

$21 \mathrm{HgO}$

$183 \mathrm{~K} 2 \mathrm{CO} 3$

$39 \mathrm{~K} 20$

$51 \mathrm{KCOOH}$

$157 \mathrm{KMnO} 4$

$161 \mathrm{KNO} 3$

$10 \mathrm{KOH}$

$150 \mathrm{KTPB}$

$105 \mathrm{La} 203$

$65 \mathrm{Mg}$ (COOH) 2

$171 \mathrm{Mg}$ (NO3) 2

$60 \mathrm{Mn}(\mathrm{COOH}) 2$

$172 \mathrm{Mn}(\mathrm{NO} 3) 2$

$180 \mathrm{Mn} 304$

$56 \mathrm{MnO}$

$14 \mathrm{MnO} 2$

$145 \mathrm{MOO} 2$

$81 \mathrm{~N} 2$

74. $\mathrm{N} 2 \mathrm{O}$

182 NH3

$52 \mathrm{NH} 4 \mathrm{COOH}$

$173 \mathrm{NH} 4 \mathrm{NO}$

$68 \mathrm{NH} 4 \mathrm{OH}$

\section{NH 4 TPB}

WSRC-TR-95-0019 (Revision
58

Melter Feed Melter Feed

$\begin{array}{ll}\text { Solubles } & \text { Insolubles } \\ 4430 \mathrm{E}-02 & 0.2794 \mathrm{E}-09\end{array}$

$\begin{array}{rr}0.4430 \mathrm{E}-02 & 0.2794 \mathrm{E}-09 \\ 0.2179 \mathrm{E}+01 & \text { ZERO }\end{array}$

ZERO $0.1969 \mathrm{E}-01$

ZERO $0.2539 \mathrm{E}+02$

ZERO $0.2538 \mathrm{E}+01$

ZERO $0.3543 \mathrm{E}-02$

ZERO $0.1512 \mathrm{E}-03$

TRACE
$0.1827 \mathrm{E}+00$
ZERO

ZERO ZERO

$0.7707 \mathrm{E}+01$ ZERO

ZERO ZERO

ZERO

$\begin{array}{rr}\text { ZERO } & \text { ZERO } \\ 0.1318 E-09 & \text { ZERO }\end{array}$

ZERO $0.1506 \mathrm{E}+00$

ZERO ZERO

ZERO ZERO

ZERO

ZERO

ZERO

ZERO

ZERO

ZERO

ZERO
$0.1005 \mathrm{E}+02$

$\begin{aligned} & \text { ZERO } \\ & \text { ZERO } \text { ZERO }\end{aligned}$

$0.2858 \mathrm{E}-01 \quad$ ZERO

ZERO : ZERO

ZERO ZERO

ZERO

$0.3463 \mathrm{E}+01$

$0.3463 \mathrm{E}+01$

ZERO
ZERO

$0.2169 \mathrm{E}+01 \quad$ ZERO

$\begin{array}{rr}0.6122 \mathrm{E}-07 & \text { ZERO } \\ \text { ZERO } & \text { ZERO }\end{array}$

$\begin{array}{rr}\text { ZERO } & \text { ZERO } \\ \text { ZERO } & 0.3831 \mathrm{E}-16\end{array}$

ZERO $0.3831 \mathrm{E}-16$

ZERO $0.1075 \mathrm{E}-02$

$0.3859 \mathrm{E}-02$ ZERO

ZERO ZERO

ZERO ZERO

ZERO ZERO

0.1391 ZERO

ZERO ZERO

ZERO ZERO

Date: January 1995

Section 4B, Page 27 of 29 
Table A-1. HLW Flowsheet Material Balance (Tank 51/ITP Cycle 1) Section 4B. Frit Adjustment of Melter Feed

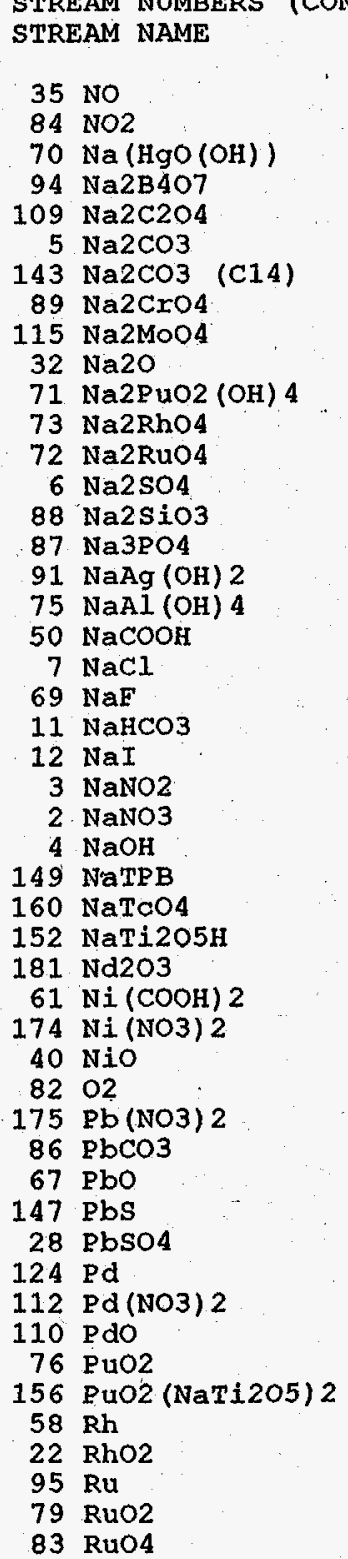

$$
59
$$

$58 \quad 59$

Melter Feed Melter Feed

Solubles. Insolubles

$\begin{array}{ll}\text { ZRACE } & \text { ZERO } \\ \text { ZERO ZERO } & \end{array}$

ZERO ZERO

ZERO ZERO

$\begin{aligned} & 0.5055 E+01 \text { ZERO } \\ & \text { ZERO }\end{aligned}$

ZERO ZERO

ZERO ZERO

ZERO

ZERO $0.1266 \mathrm{E}+02$

ZERO ZERO

ZERO ' ZERO

$0.1952 \mathrm{E}+00$ ZERO

0.2578 ZERO

ZERO

ZERO ZERO

$0.2350 \mathrm{E}+01$ ZERO

$0.9957 \mathrm{E}-02$ ZERO

$0.3417 E-01, \quad$ ZERO

ZERO
$0.5472 \mathrm{E}-05$

$0.5472 E-05$ ZERO

$\begin{aligned} \text { ZERO } & \text { ZERO } \\ 0.8943 \mathrm{E}+01 & \text { ZERO }\end{aligned}$

ZERO ZERO

$\begin{array}{ll}\text { ZERO } & \text { ZERO } \\ \text { ZERO } & \text { ZERO }\end{array}$

ZERO ZERO

ZERO
$0.2521 \mathrm{E}-01$

$0.6686 \mathrm{E}-06$ ZERO

ZERO $\quad 0.2416 \mathrm{E}+00$

$0.2226 \mathrm{E}-02$ ZERO

ZERO ZERO

ZERO $0.7084 E-19$

ZERO ZERO

ZERO $0.1318 \mathrm{E}+00$

ZERO $0.3903 \mathrm{E}-03$

ZERO $\because$ ZERO

ZERO
ZERO

$0.1272 \mathrm{E}-03$

ZERO $\quad 0.7436 \mathrm{E}-03$

ZERO ZERO

$0.8125 \mathrm{E}-02$

$\begin{array}{ll}\text { ZERO } & \text { ZERO } \\ \text { ZERO } & \text { ZERO }\end{array}$ 
Table A-1. HLW Flowsheet Material Balance (Tank 51/ITP Cycle 1)

Section 4B. Frit Adjustment of Melter Feed

STREAM NUMBERS (CONT'D) $\rightarrow$ STREAM NAME

\section{\begin{tabular}{ll}
38 & $\mathrm{SO} 2$ \\
\hline 1 & 503
\end{tabular}}

137 Semi Vol Cs2O

136 Semi Vol CsCl

126 Semi. Vol Group A

140 Semi Vol Na2B4O7

138 Semi Vol NaC1

139 Semi Vol NaF

125 Semi Vol NaI

23 Semi Vol RuO2

111 sio2.

$48 \mathrm{Sr}(\mathrm{COOH}) 2$

$176 \mathrm{Sr}$ (NO3) 2
$153 \mathrm{Sr}$ (NaTi205) 2

$127 \mathrm{Sx}(\mathrm{OH}) 2$

$30 \mathrm{SrCO} 3$

46 Sro

$159 \mathrm{TcO} 2$

92 ThO2

107 TiO2

119 Triti

$17 \mathrm{U} 308$

62002 ( $\mathrm{COOH}) 2$

$177 \mathrm{UO} 2$ (NO3) 2

16. $\mathrm{VO} 2(\mathrm{OH}) 2$

$27 \mathrm{Y}(\mathrm{COOH}) 3$

178 Y (NO3)

$\begin{array}{rl}128 & \mathrm{Y}(\mathrm{OH}) 3 \\ 31 & \mathrm{Y} 2(\mathrm{CO} 3)\end{array}$

$47 \mathrm{Y} 203$

100 Zeolite

$44 \mathrm{2n}(\mathrm{COOH}) 2$

$179 \mathrm{Zn}(\mathrm{NO} 3) 2$

57 Zno

104 zro2

13 hydrate

1 water

TOTAL FLOW, LB/HR

TEMPERATURE, DEG C

PRESSURE， ATM

PRESSURE， PSIG

PRESSURE， MM HG

ENTHALPY, PCU/HR

VAPOR ELOW CFM

LIQUID FLOW, GPM

PHASE

WSRC-TR-95-0019 (Revision
58

Melter Feed Melter Feed

Solubles Insolubles

ZERO ZERO

ZERO ZERO

ZERO

ZERO

ZERO ZERO

ZERO

ZERO

ZERO

ZRRO -

$0.1093 \mathrm{ZERO} \quad 0.1230 \mathrm{E}+03$

$0.2944 \mathrm{E} 01 \quad$ ZERO

$0.4072 \mathrm{E}-02$

$\begin{array}{rr}\text { ZERO } & 0.4072 E-02 \\ \text { ZERO } & \text { ZERO } \\ \text { ZERO } & \text { ZERO }\end{array}$

ZERO $\quad 0.2218 \mathrm{E}-18$

ZERO $0.3569 \mathrm{E}-02$

ZERO $0.2972 \mathrm{E}-01$

ZERO $0.8309 \mathrm{E}+00$

$0.1424 \mathrm{E}-09 \quad$ ZERO

ZERO $\quad 0.2084 \mathrm{E}+01$

$0.1272 \mathrm{E}-05 \quad$ ZERO

$0.9137 \mathrm{E}-04 \quad 0.5600 \mathrm{E}-11$

ZERO
$0.1317 \mathrm{E}-11$

$0.7940 \mathrm{E}-02 \quad$ ZERO

ZERO ZERO

ZERO ZERO

ZERO $\quad 0.9683 \mathrm{E}-03$

ZERO
$0.2607 E+00$

$0.5336 \mathrm{E}-04$ ZERO

ZERO $0.1794 \mathrm{E}+00$

ZERO ZZERO

$\begin{array}{rr}\text { ZERO } & 0.1499 \mathrm{E}+01 \\ 0.3237 \mathrm{E}+03 & \text { ZERO }\end{array}$

$0.3706 \mathrm{E}+03 \quad 0.2104 \mathrm{E}+03$

$0.4987 \mathrm{E}+02.0 .4987 \mathrm{E}+02$

$0.9656 \mathrm{E}+00 \quad 0.9656 \mathrm{E}+00$

$-0.5055 \mathrm{E}+00-0.5055 \mathrm{E}+00$

$0.7339 \mathrm{E}+03 \quad 0.7339 \mathrm{E}+03$

$0.1663 \mathrm{E}+05 \quad 0.2018 \mathrm{E}+04$

$0.6986 \mathrm{E}+00 \quad 0.1670 \mathrm{E}+00$

$0.6615 \mathrm{E}+02 \quad 0.1571 \mathrm{E}+03$

IIQUID LIQUID 


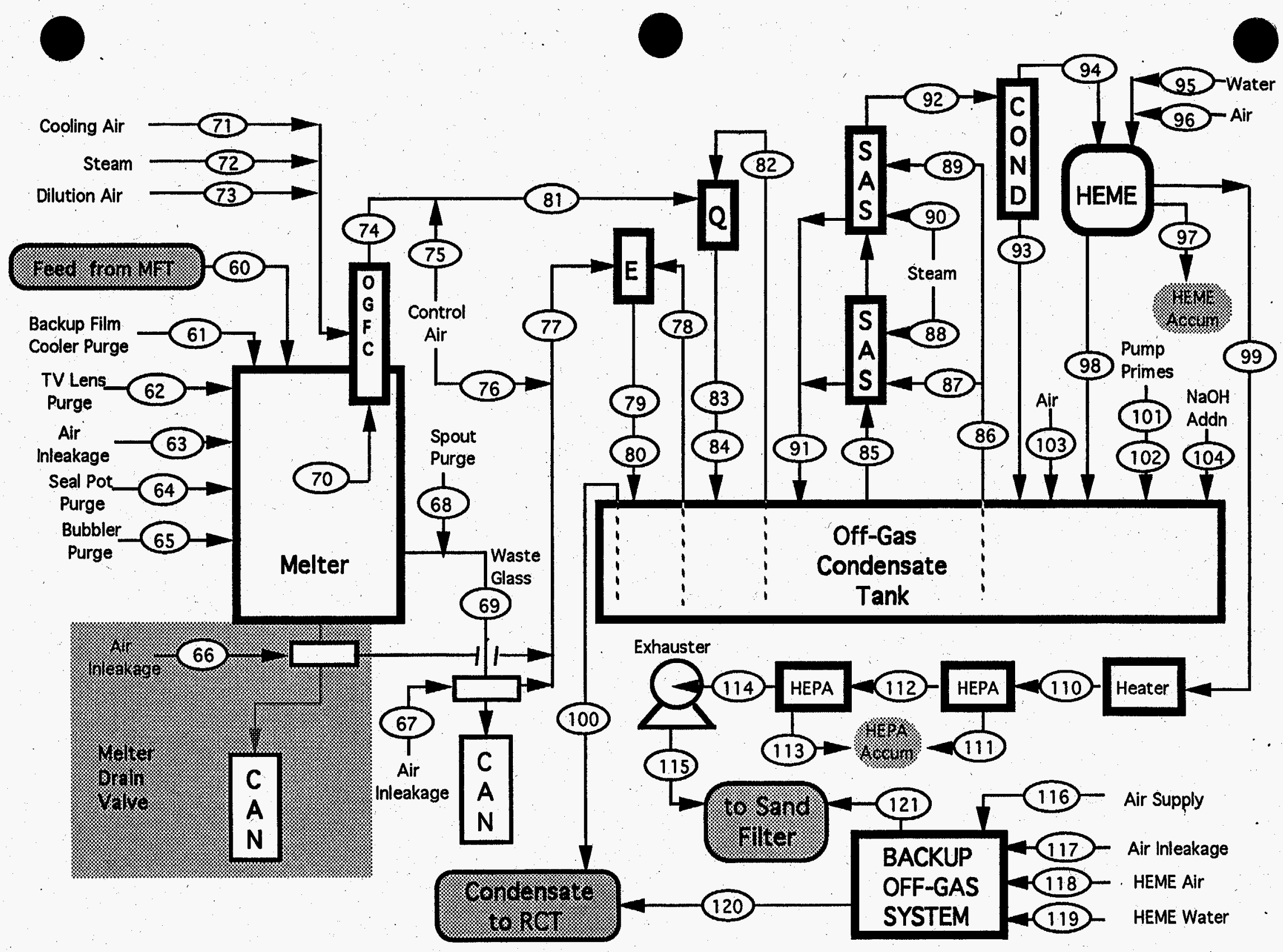

SECTION 5. VITRIFICATION AND MELTER OFF-GAS TREATMENT 
Table A-1. HLW Flowsheet Material Balance (Tank 51/ITP Cycle 1) section 5. Vitrification and Melter off-Gas Treatment

DESCRIPTION

PAGE

1. STREAM NUMBERS

$60,61,62 ; 63,64,65,66 \ldots \ldots \ldots 2$

2. STREAM NUMBERS

$67,68,69,70,71,72,73 \ldots \ldots 6$

3. STREAM NUMBERS

4. STREAM NUMBERS

$74,75,76,77,78,79,80 \ldots \ldots . . . . . . . . . . . . .10$

5. STREAM NUMBERS

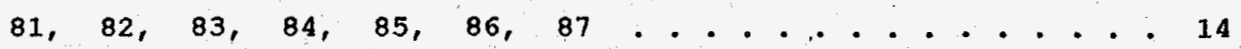

6. STREAM NUMBERS

$88,89,90,91,92,93,94 \ldots \ldots \ldots \ldots$

7. STREAM NUMBERS

$95,96,97,98,99,100,101 \ldots \ldots 22$

$102,103,104,110,111,112,113 \ldots \ldots$

8. STREAM NUMBERS

$114,115,116,117,118,119,120 \ldots \ldots$

9. STREAM NUMBERS

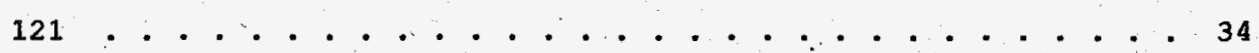


Table A-1. HLW Flowsheet Matexial Balance (Tank 51/ITP Cycle 1) section 5. Vitrification and Melter off-Gas Treatment

STREAM NUMBERS $\rightarrow$ STREAM NAME

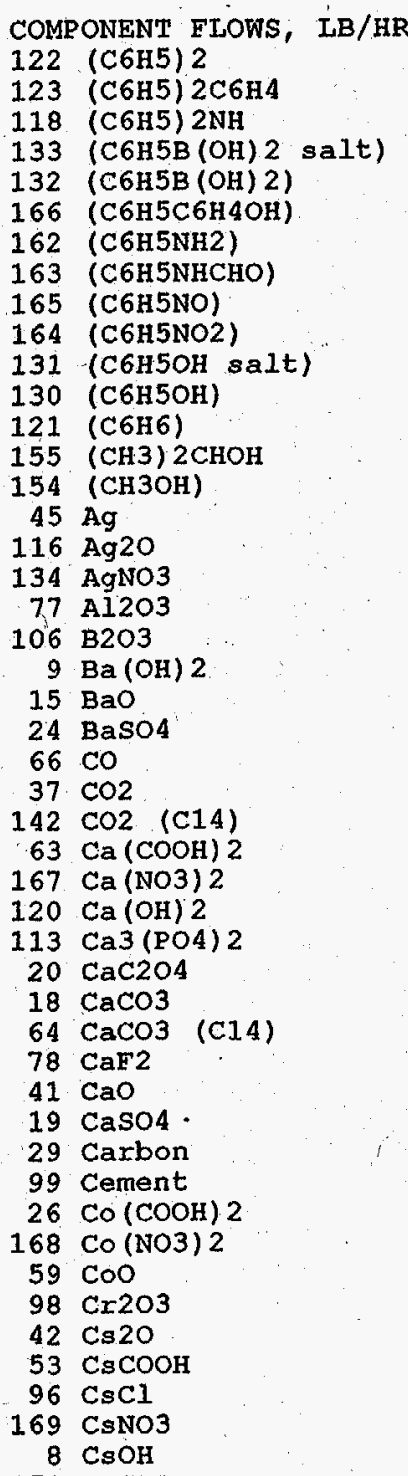

151 CSPB

WSRC-TR-95-0019 (Revision

$$
61
$$

Melter Backup Film Melter CCTV Feed Coolex Flow Lens Purges

$0.2853 \mathrm{E}-18$

$0.9245 \mathrm{E}-02$
$0.4221 \mathrm{E}-08$ ZERO

$0.5523 \mathrm{E}-01$

$0.1648 \mathrm{E}-03$

$0.2092 \mathrm{E}+00$

$0.3406 \mathrm{E}-02$

$0.6938 \mathrm{E}-02$

$0.8234 \mathrm{E}-15$

$0.4876 \mathrm{E}-01$

$0.2534 \mathrm{E}-24$

$0.2588 \mathrm{E}-11$

$0.3832 \mathrm{E}-13$

$0.1241 \mathrm{E}-01$ ZERO

$0.8834 \mathrm{E}+01$

$0.1266 \mathrm{E}+02$$$
\text { ZERO }
$$

$0.1617 \mathrm{E}-19$

$0.1704 \mathrm{E}-01$

ZERO
$0.9807 \mathrm{E}-19$

TE-19
TRACE

ZERO
$0.4188 \mathrm{E}+01$

ZERO

$0.2626 \mathrm{E}+01$

$0.2442 \mathrm{E}-06$

ZERO

$0.1501 \mathrm{E}-01$

$0.3344 \mathrm{E}-02$

$0.2208 \mathrm{E}+00$

ZERO

ZERO
$0.1433 \mathrm{E}-03$

$0.5373 \mathrm{E}-06$

$0.3069 \mathrm{E}-04$

$0.4302 \mathrm{E}+00$

$0.1403 \mathrm{E}-03$

$0.4330 \mathrm{E}-01$

$0.9608 \mathrm{E}-05$

ZERO
63

Melter Air

In leakage

\begin{tabular}{ll} 
ZERO & ZERO \\
ZERO & ZERO \\
ZERO & ZERO \\
ZERO & ZERO \\
ZERO & ZERO \\
ZERO & ZERO \\
ZERO & ZERO \\
ZERO & ZERO \\
ZERO & ZERO \\
ZERO & ZERO \\
ZERO & ZERO \\
ZERO & ZERO \\
ZERO & ZERO \\
ZERO & ZERO \\
ZERO & ZERO \\
ZERO & ZERO \\
ZERO & ZERO \\
ZERO & ZERO \\
ZERO & ZERO \\
ZERO & ZERO \\
ZERO & ZERO \\
ZERO & ZERO \\
ZERO & ZERO \\
ZERO & ZERO \\
ZERO & ZERO \\
ZERO & ZERO \\
ZERO & ZERO \\
ZERO & ZERO \\
ZERO & ZERO \\
ZERO & ZERO \\
ZERO & ZERO \\
ZERO & ZERO \\
ZERO & ZERO \\
ZERO & ZERO \\
ZERO & ZERO \\
ZERO & ZERO \\
ZERO & ZERO \\
ZERO & ZERO \\
ZERO & ZERO \\
ZERO & ZERO \\
ZERO & ZERO \\
ZERO & ZERO \\
ZERO & ZERO \\
ZERO & ZERO \\
ZERO & ZERO \\
ZERO & ZERO \\
ZERO & ZERO \\
ZERO & ZERO \\
& \\
\hline
\end{tabular}

64

Seal Pot

Purge

ZERO

ZERO

ZERO

ZERO

ZERO

ZERO

ZERO

ZERO

ZERO

ZERO

ZERO

ZERO

ZERO

ZERO

ZERO

ZERO

ZERO

ZERO

ZERO

ZERO

ZERO

ZERO

TERO

ZERO

ZERO

ZERO

ZERO

ZERO

ZERO

ZERO

ZERO

ZERO

ZERO

ZERO

ZERO

ZERO

ZERO

ZERO

ZERO

ZERO

ZERO

ZERO

ZERO

ZERO

ZERO

ZERO
65

Melter Dump Can Air Bubbler Inleakage

\begin{tabular}{ll} 
ZERO & ZERO \\
ZERO & ZERO \\
ZERO & ZERO \\
ZERO & ZERO \\
ZERO & ZERO \\
ZERO & ZERO \\
ZERO & ZERO \\
ZERO & ZERO \\
ZERO & ZERO \\
ZERO & ZERO \\
ZERO & ZERO \\
ZERO & ZERO \\
ZERO & ZERO \\
ZERO & ZERO \\
ZERO & ZERO \\
ZERO & ZERO \\
ZERO & ZERO \\
ZERO & ZERO \\
ZERO & ZERO \\
ZERO & ZERO \\
ZERO & ZERO \\
ZERO & ZERO \\
ZERO & ZERO \\
ZERO & ZERO \\
ZERO & ZERO \\
ZERO & ZERO \\
ZERO & ZERO \\
ZERO & ZERO \\
ZERO & ZERO \\
ZERO & ZERO \\
ZERO & ZERO \\
ZERO & ZERO \\
ZERO & ZERO \\
ZERO & ZERO \\
ZERO & ZERO \\
ZERO & ZERO \\
ZERO & ZERO \\
ZERO & ZERO \\
ZERO & ZERO \\
ZERO & ZERO \\
ZERO & ZERO \\
ZERO & ZERO \\
ZERO & ZERO \\
ZERO & ZERO \\
ZERO & ZERO \\
ZERO & ZERO \\
ZERO & ZERO \\
ZERO & ZERO \\
& \\
\hline
\end{tabular}

section 5. Page 2 of 37

Date: January 1995 
Table A-1. HLW Flowsheet Material Balance (Tank 51/ITP Cycle 1) section 5. Vitrification and Melter off-Gas Treatment

STREAM NUMBERS (CONT'D) $\rightarrow$ STREAM NAME

$54 \mathrm{Cu}(\mathrm{COOH}) 2$

$170 \mathrm{Cu}(\mathrm{NO} 3) 2$

$55 \mathrm{CuO}$

$80 \mathrm{Fe} 2 \mathrm{O3}$

$101 \mathrm{FeO}$

102 Group A

103 Group

$25 \mathrm{H} 2$

$158 \mathrm{H} 2 \mathrm{C} 2 \mathrm{O} 4$

93. $\mathrm{H} 2 \mathrm{SO}$

$34 \mathrm{HCOOH}$

$43 \mathrm{HCl}$

$85 \mathrm{HE}$

49 HNO3

$36 \mathrm{Hg}$

$129 \cdot \mathrm{Hg}(\mathrm{C} 6 \mathrm{H} 5)^{2}$

$135 \mathrm{Hg}$ (NO3) 2

$144 \mathrm{Hg} 2 \mathrm{Cl} 2$

$117 \mathrm{Hg} 2 \mathrm{I} 2$

$146 \mathrm{HgCl} 2$

$21 \mathrm{HgO}$

$\begin{array}{rl}97 & \mathrm{I} 2 \\ 183 & \mathrm{~K} 2 \mathrm{CO} 3\end{array}$

$39 \mathrm{~K} 2 \mathrm{O}$

$51 \mathrm{KCOOH}$

$157 \mathrm{KMnO}$

$161 \mathrm{KNO}^{-1}$

$10 \mathrm{KOH}$

$105 \mathrm{La2O3}$

$108 \mathrm{Li} 20$

$65 \mathrm{Mg}(\mathrm{COOH}) 2$

$171 \mathrm{Mg}(\mathrm{NO} 3) 2$

$33 \mathrm{MgO}$

$60 \mathrm{Mn}(\mathrm{COOH}) 2$

$172 \mathrm{Mn}$ (NO3)

$180 \mathrm{Mn} 304$

$56 \mathrm{Mno}$

$14 \mathrm{MnO} 2$

$145 \mathrm{MoO}$

$\begin{array}{ll}81 & \text { N2 } \\ 74 & \text { N2O }\end{array}$

$182 \mathrm{NH} 3$

182 NH3
114 NH3OHNO3

$52 \mathrm{NH} 4 \mathrm{COOH}$

173 NH4NO3

$68 \mathrm{NH} 4 \mathrm{OH}$

WSRC-TR-95-0019 (Revision
60

Melter Backup Film Melter CCTV Feed Cooler Flow Lens Purge $0.4430 \mathrm{E}-02$ $0.2179 \mathrm{E}+01$

$0.1969 \mathrm{E}-01$

$0.1969 \mathrm{E}-01$

$0.2538 \mathrm{E}+01$

$0.3543 \mathrm{E}-02$

$0.1512 \mathrm{E}-03$

TRACE

$0.1827 \mathrm{E}+00$

$\begin{array}{r}0.7707 \mathrm{E}+01 \\ \hline\end{array}$

ZERO
ZERO
ZERO

$0.1318 \mathrm{E}-09$

$0.1506 \mathrm{E}+0.0$

ZERO

ZERO

ZERO

ZERO

ZERO

ZERO

ZERO

$0.8073 \mathrm{E}-01$

$0.1005 \mathrm{E}+02$

$0.2858 \mathrm{E}-01$

ZERO

ZERO

ZERO
$0.1108 E+02$

$0.3463 \mathrm{E}+01$

$0.5254 \mathrm{E}-07$

$0.3470 \mathrm{E}+01$

$0.2169 \mathrm{E}+01$

ZERO

$0.3831 \mathrm{E}-16$

$0.1948 \mathrm{E}+01$

$0.1075 \mathrm{E}-02$

$0.3859 \mathrm{E}-02$

ZERO

ZERO

ZERO

$0: 1391 \mathrm{E}-02$

ZERO $\begin{array}{lr}\text { ZERO } & \text { ZERO } \\ \text { ZERO } & \text { ZERO }\end{array}$

ZERO ZERO

ZERO ZERO

ZERO ZERO

ZERO ZERO

ZERO ZERO

ZERO
ZERO
ZERO

ZERO ZERO

ZERO ZERO

ZERO ZERO

ZERO ZERO

ZERO Z ZERO

ZERO ZERO

ZERO ZERO

ZERO $\cdots$ ZERO

ZERO ZERO

ZERO ZERO

ZERO ZERO

ZERO ZERO

ZERO ZERO

ZERO - ZERO

ZERO ZERO

ZERO ZERO

ZERO ZERO

ZERO ZERO

ZERO ZERO

ZERO ZERO

ZERO ZERO

ZERO ZERO

ZERO ZERO

$\begin{array}{ll}\text { ZERO } & \text { ZERO } \\ \text { ZERO } & \text { ZERO }\end{array}$

ZERO

ZERO

$0.3248 \mathrm{E}+03$

ZERO

ZERO

ZERO

ZERO

ZERO

ZERO

ZERO

ZERO

ZERO

ZERO

ZERO

ZERO
ZERO ZERO

ZERO ZERO
64 elter Aix

Inleakage

ZERO

ZERO

ZERO

ZERO

ZERO

ZERO

ZERO

ZERO

ZERO

ZERO

ZERO

ZERO

ZERO

ZERO

ZERO

ZERO

ZERO

ZERO

ZERO

ZERO

ZERO

ZERO

ZERO

ZERO

ZERO

ZERO

ZERO

ZERO

ZERO

ZERO

ZERO

ZERO

ZERO

ZERO

ZERO

ZERO

ZERO

Seal pot

Purge

ZERO

ZERO.

ZERO

ZERO

ZERO

ZERO

ZERO

ZERO

ZERO

ZERO

ZERO

ZERO

ZERO

ZERO

ZERO

ZERO

ZERO

ZERO

ZERO

ZERO

ZERO

ZERO

ZERO

ZERO

ZERO

ZERO

ZERO

ZERO

ZERO

ZERO

ZERO

ZERO

ZERO

ZERO

ZERO

ZERO

ZERO

ZERO

ZERO

ZERO

ZERO

$0.7553 \mathrm{E}+02$

ZERO

ZERO

ZERO

ZERO

ZERO

$0.1511 \mathrm{E}+02$

ZERO

ZERO

ZERO

ZERO

ZERO

ZERO

ZERO

65

Melter Dump Can Air Bubbler Inleakage ZERO $\quad$ ZERO ZERO ZERO ZERO ZERO

ZERO ZERO

ZERO

ZERO

ZERO ZERO

ZERO

ZERO ZERO

$\begin{array}{ll}\text { ZERO } & \text { ZERO } \\ \text { ZERO } & \text { ZERO }\end{array}$

ZERO ZERO

ZERO ZERO

ZERO ZERO

ZERO ZERO

ZERO ZERO

ZERO ZERO

ZERO

ZERO

ZERO ZERO

ZERO ZERO

ZERO ZERO

ZERO ZERO

ZERO ZERO

ZERO ZERO

ZERO ZERO

ZERO ZERO

ZERO

ZERO

ZERO

ZERO

ZRRO

ZERO

ZERO

ZERO

ZERO

ZERO

$0.1500 \mathrm{E}+00$ 
Table A-1. HLW Flowsheet Material Balance (Tank 51/ITP Cycle 1) section 5 . Vitrification and Melter off-Gas Treatment

STREAM NUMBERS (CONT'D) $\rightarrow$ STREAM NAME

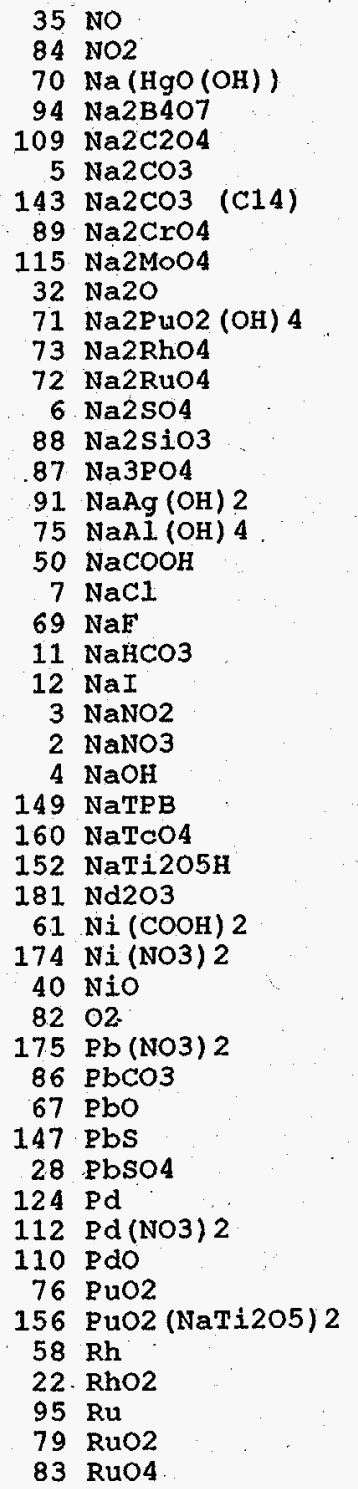

60 lter Backup Film Melter CCTV Melter Air Feed Cooler Flow Lens Purges Inleakage IRACE ZERO $\quad$ ZERO , ZERO ZERO ZERO ZERO ZERO ZERO ZERO ZERO 2ERO

$0.5055 \mathrm{E}+01$ ZERO ZERO

$0.1266 \mathrm{E}+02$ ZERO ZERO

$0.1952 \mathrm{E}+0 \mathrm{O}$ ZERO $0.2578 \mathrm{E}-02$ ZERO

$0.2350 \mathrm{E}+01$

$0.9957 \mathrm{E}-02$

$0.3417 \mathrm{E}-01$ ZERO
$2 E-05$

$0.5472 \mathrm{E}-05$

$0.8943 \mathrm{E}+01$

ZERO

ZERO

ZERO ZERO

0.2521 E-01

$0.6686 \mathrm{E}-06$

$0.2416 \mathrm{E}+00$

$0.2226 \mathrm{E}-02$

$0.7766 \mathrm{E}-04$

$0.7084 \mathrm{E}-19$

ZERO

$0.1318 E+00$

$0.3903 \mathrm{E}-03$ ZERO

$0.9905 \mathrm{E}-02$

$0.1272 \mathrm{E}-03$

$0.7436 \mathrm{E}-03$

$0.8125 \mathrm{E}-02$ ZERO

WSRC-TR-95-0019 (Revision

$0.9811 \mathrm{E}+02$ $61 \quad 62$

64

Seal Pot

Purge

ZERO

ZERO

ZERO

ZERO

ZERO ZERO ZERO

ZERO ZERO ZERO

ZERO ZERO ZERO

ZERO ZERO ZERO

ZERO ZERO

ZERO ZERO ZERO

ZERO

ZERO

ZERO

ZERO

ZERO

ZERO

ZERO

ZERO

ZERO

ZERO

ZERO

ZERO

ZERO

ZERO

ZERO

ZERO

ZERO

ZERO

ZERO

ZERO

ZERO

ZERO

ZERO
ZERO

ZERO

ZERO

ZERO

ZERO

ZERO

ZERO

ZERO

ZERO

ZERO

ZERO

ZERO

ZERO

ZERO

ZERO

ZERO

ZERO

ZERO

ZERO

ZERO

ZERO

ZERO

ZERO

ZERO

ZERO

ZERO

ZERO

ZERO

ZERO

ZERO

ZERO

ZERO

ZERO

$0.1369 \mathrm{E}+02$

ZERO

ZERO

ZERO

ZERO

ZERO

ZERO

ZERO

ZERO

ZERO

ZERO

ZERO

ZERO

ZERO

ZERO

ZERO

ZERO

ZERO

ZERO

ZERO

ZERO

ZERO

ZERO

ZERO

ZERO

ZERO

ZERO

ZERO

ZERO

ZERO

ZERO

ZERO

ZERO

ZERO

$0.2282 \mathrm{E}+02$

ZERO

ZERO

ZERO

ZERO

ZERO

ZERO

ZERO

ZERO

ZERO

ZERO

ZERO

ZERO

ZERO

ZERO

Date: Januaxy 1995
65

66

Melter Dump Can Air Bubbler ZERO ZERO ZERO ZERO ZERO $\cdots$ ZERO ZERO ZERO ZERO ZERO

ZERO ZERO

ZERO ZERO

ZERO ZERO

ZERO ZERO

ZERO ZERO

ZERO ZERO

ZERO ZERO

ZERO ZERO

ZERO ZERO

ZERO

ZERO

ZERO ZERO

ZERO ZERO

ZERO ZERO

ZERO

ZERO ZERO

ZERO ZERO

ZERO ZERO

ZERO ZERO

ZERO ZERO

ZERO ZERO

ZERO $\cdots$ ZERO

ZERO ZERO

ZERO ZERO

ZERO ZERO

ZERO ZERO

ZERO ZERO

ZERO ZERO

ZERO ZERO

ZERO ZERO

ZERO

ZERO

ZERO ZERO

ZERO ZERO

ZERO ZERO

ZERO ZERO

$\begin{array}{ll}\text { ZERO } & \text { ZERO } \\ \text { ZERO } & \text { ZERO }\end{array}$

Section 5 . Page 4 of 37 
Table A-1, HLW Flowshest Material Balance (Tank 51/ITP Cycle 1) section 5 . Vitrification and Melter off-Gas Treatment

STREAM NUMBERS (CONT'D) $\rightarrow$ STREAM NAME

\section{$38 \mathrm{SO} 2$}

$141 \mathrm{SO3}$

137 Semi Vol Cs2O

136 Semi Vol CsCl

126 Semi Vol Group A

140 Semi Vol Na2B407

138 Semi Vol NaCl

139 Semi Vol NaF

125 Semi Vol NaI

23 Semi Vol RuO2

$111 \mathrm{SiO} 2$

$48 \mathrm{Sr}$ (COOH) 2

$176 \mathrm{Sr}$ (NO3) 2

153 Sr (NaTi2O5) 2

$127 \mathrm{Sr}(\mathrm{OH}) 2$

$30 \mathrm{SrCO} 3$

$46 \mathrm{SrO}$

$159 \mathrm{TaO} 2$

92 ThO2

119 Tritium

119 Triti

$62 \mathrm{UO2}(\mathrm{COOH}) 2$

177 VO2 (NO3) 2

$16 \mathrm{UO} 2(\mathrm{OH}) 2$

$27 \mathrm{Y}(\mathrm{COOH}) 3$

$\begin{array}{ll}178 \mathrm{Y}(\mathrm{NO} 3) \\ 128 & \mathrm{Y}(\mathrm{OH}) 3\end{array}$

$31 \mathrm{Y} 2(\mathrm{CO} 3) 3$

47. $\mathrm{Y} 203$

100 zeolite

$44 \mathrm{Zn}(\mathrm{COOH}) 2$

$179 \mathrm{zn}$ (NO3) 2

$57 \mathrm{znO}$

104 zro2

13 hydrate $\mathrm{H} 2 \mathrm{O}$

1 water.

TOTAL ELOW, IBB/HR

TEMPERATURE, DEG C

PRESSURE, ATM

PRESSURE, PSIG

PRESSURE， MM HG

ENTHALPY, PCU/HR

VAPOR FLOW, CFM

LIOUID FLOW, GPM

DENSITY, LBS/FT3

RHASE

60

Me

Melter Backup Film Melter cCrv

Feed Coolex Flow Lens Purges

ZERO ZERO

ZERO

ZERO

ZERO

ZERO

ZERO

ZERO

ZERO

ZERO

ZERO

$0.1230 \mathrm{E}+03$

0.1093E-03

$0.2944 \mathrm{E}-01$
$0.4072 \mathrm{E}-02$

ZERO
ZERO

$0.2218 \mathrm{E}-1$

$0.3569 \mathrm{E}-02$

$0.2972 \mathrm{E}-01$

$0.8309 \mathrm{E}+00$

$0.1424 \mathrm{E}-09$

$0.2084 E+01$
$0.1272 E-05$

$0.1272 E-05$
$0.9137 E-04$

$0.9137 \mathrm{E}-04$

$0.1317 \mathrm{E}-11$

$0.7940 \mathrm{E}-02$

ZERO

$0.9683 E-03$

ZERO

$0.2607 \mathrm{E}+00$

$0.5336 \mathrm{E}-04$

$0.1794 \mathrm{E}+00$

ZERO

$0.1499 \mathrm{E}+01$
$0.3237 \mathrm{E}+03$

$0.3237 \mathrm{E}+03$
$0.5811 \mathrm{E}+03$

$0.4987 \mathrm{E}+02$

$0.9656 \mathrm{E}+00$

$-0.5055 \mathrm{E}+00$

$0.7339 \mathrm{E}+03$

$0.1865 \mathrm{E}+05$

$0.8656 \mathrm{E}+00$

$0.8369 \mathrm{E}+02$

LIQUID

$\begin{array}{ll}\text { ZERO } & \text { ZERO } \\ \text { ZERO } & \text { ZERO } \\ \text { ZERO } & \text { ZERO } \\ \text { ZERO } & \text { ZERO }\end{array}$

ZERO

ZERO

ZERO

ZERO

ZERO
ZERO

ZERO

ZERO

ZERO

ZERO

ZERO

ZERO

ZERO

ZERO

ZERO

ZERO

ZERO

ZERO

ZERO

ZERO
ZERO

ZERO

ZERO

ZERO

ZERO

ZERO

ZERO

ZERO

ZERO

$0.7105 \mathrm{E}+01$

$0.4300 \mathrm{E}+03$

$\begin{array}{ll}0.3500 \mathrm{E}+02 & 0.3500 \mathrm{E}+02 \\ 0.1000 \mathrm{E}+01 & 0.1000 \mathrm{E}+01\end{array}$

ZTRO
63

Melter Air

Inleakage

ZERO

ZERO

ZERO

ZERO

ZERO

ZERO

ZERO

ZERO

ZERO

ZERO

ZERO

ZERO

ZERO

ZERO

ZERO

ZERO

ZERO

ZERO

ZERO

ZERO

ZERO

ZERO

ZERO

ZERO

ZERO

ZERO

ZERO

ZERO

ZERO

ZERO

ZERO

ZERO

ZERO

ZERO

$0.9913 \mathrm{E}+00$

$0.6000 \mathrm{E}+02$

ZERO

ZERO

ZERO

ZERO

0.1652E+01

$0.1000 \mathrm{E}+03$

ZERO

$0.3500 E+02$

$0.1000 \mathrm{E}+01$

ZERO

64

Seal Pot

Purge

ŹERO

ZERO

ZERO

ZERO

ZERO

ZERO

ZERO

ZERO

ZERO

ZERO

ZERO

ZERO

ZERO

ZERO

ZERO

ZERO

ZERO

ZERO

ZERO

ZERO

ZERO

ZERO

ZERO

ZERO

ZERO

ZERO

ZERO

ZERO

ZERO

ZERO

ZERO

ZERO

ZERO

ZERO

ZERO

ZERO

$0.2000 \mathrm{E}+02$

$0.3500 E+02$

$0.1000 \mathrm{E}+01$

ZERO

$0.1258 \mathrm{E}+04$

$0.2097 \mathrm{E}+04$

$0.4195 \mathrm{E}+03$

$0.7055 \mathrm{E}-01$

$0.7055 \mathrm{E}-01$

VAPOR

$7055 \mathrm{E}-01$

VAPOR
65

66

Melter Dump Can Air Bubbler Inleakage ZERO ZERO ZERO ZERO

ZERO

ZERO ZERO

ZERO ZERO

ZERO

ZTRO

ZTRO

ZERO ZERO

ZERO ZERO

ZERO
ZERO
ZERO ZERO

ZERO ZERO

ZERO ZERO

ZERO ZERO

ZERO

ZERO

ZERO

ZERO

ZERO

ZFRO

ZERO

ZERO

ZERO

ZERO

ZERO

ZERO

ZERO

ZERO

ZERO

ZERO

2TRO

RO

ZERO

0.1500

ZERO

0.3500

$0.3500 \mathrm{E}+02$
$0.1000 \mathrm{E}+01$

ZERO $-0.5408 \mathrm{E}+00$

$0.1272 \mathrm{E}+01 \quad 0.7320 \mathrm{E}+03$

$0.3613 \mathrm{E}-01$

$0.6920 \mathrm{E}-01$

VAPOR

ZERO

VAPOR 


\section{-}

Table A-1. HLW Flowsheet Material Balance (Tank 51/ITP Cyole 1) section 5. Vitrification and Melter off-Gas Treatment

STREAM NUMBERS $\rightarrow$ STREAM NAME

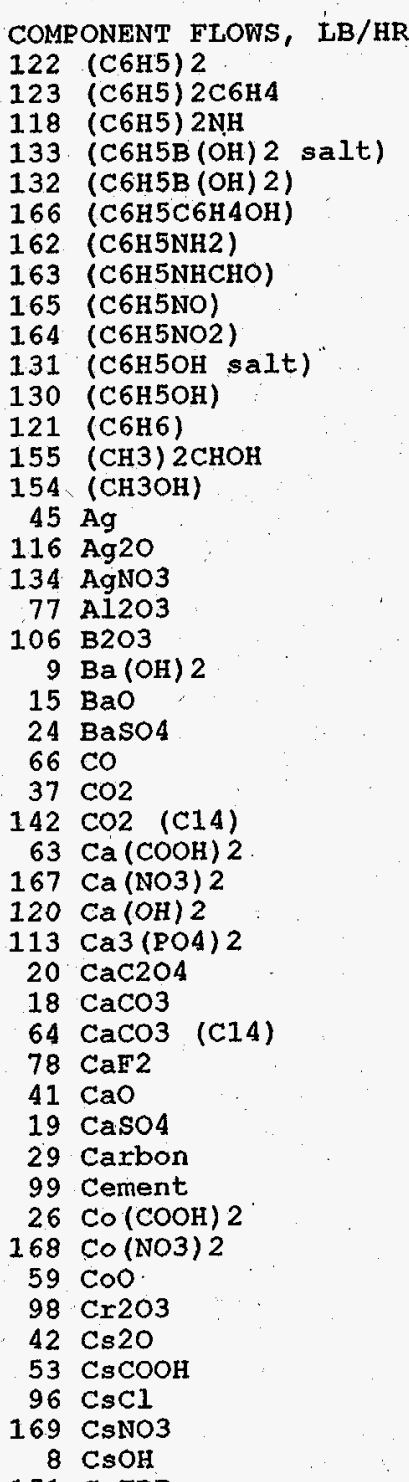


Table A-1. HLW Flowsheet Material Balance (Tank 51/ITP cycle 1) section 5. Vitrification and Melter off-Gas Treatment

STREAM NOMBERS (CONT'D) $\rightarrow$ STREAM NAME

$54 \mathrm{Cu}$ (COOH) 2

$170 \mathrm{Cu}(\mathrm{NO} 3) 2$

$55 \mathrm{CuO}$

$80 \quad \mathrm{Fe} 2 \mathrm{O} 3$

101. $\mathrm{FeO}$

102 Group A

103 Group $B$

$25 \mathrm{H} 2$

$158 \mathrm{H} 2 \mathrm{C} 2 \mathrm{O} 4$
$93 \mathrm{H} 2 \mathrm{SO} 4$

$\begin{array}{ll}93 & \mathrm{H} 2 \mathrm{SO} 4 \\ 90 & \mathrm{H} 3 \mathrm{BO}\end{array}$

$34 \mathrm{HCOOH}$

$43 \mathrm{HCl}$

$85 \mathrm{HF}$

49 HNO3

$36 \mathrm{Hg}$

$129 \mathrm{Hg}(\mathrm{C} 6 \mathrm{H} 5)_{2} 2$

$135 \mathrm{Hg}$ (NO3) 2

$144 \mathrm{Hg} 2 \mathrm{Cl} 2$

$117 \mathrm{Hg} 2 \mathrm{I2}$

$146 \mathrm{HgCl}$
$21 \mathrm{HgO}$

$21 \mathrm{HgO}$
$97 \mathrm{I2}$

$183 \mathrm{~K} 2 \mathrm{CO} 3$

$39 \mathrm{k} 2 \mathrm{O}$

$51 \mathrm{KCOOH}$
$157 \mathrm{KMnO} 4$

161 KNO3

$10 \mathrm{KOH}$

150 KTPB

$105 \mathrm{La}_{203}$

$65 \mathrm{Mg}(\mathrm{COOH}) 2$

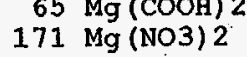

$33 \mathrm{MgO}$

$60 \mathrm{Mn}(\mathrm{COOH}) 2$
$172 \mathrm{Mn}(\mathrm{NO} 3) 2$

$172 \mathrm{Mn}(\mathrm{NO} 3)$
$180 \mathrm{Mn} 304$

$56 \mathrm{MnO}$

$14 \mathrm{MnO} 2$

145 MOO2

81 N2

$74 \mathrm{~N} 2 \mathrm{O}$

114 NH3OHNO 3

52. $\mathrm{NH} 4 \mathrm{COOH}$

173 NH4NO3

$68 \mathrm{NH} 4 \mathrm{OH}$

148 NH4TPB

WSRC-TR-95-0019 (Revision
67

Canister Air Pour Spout sldge Blend

Inleakage Siphon Break Waste Glass

ZERO ZERO

ZERO ZERO

ZERO ZERO $0.2513 E+02$

ZERO

ZERO

ZERO $0.3332 \mathrm{E}-02$

ZERO

ZERO

ZERO

ZERO

ZERO

ZERO

ZERO

ZERO

ZERO

ZERO

ZERO

ZERO

ZERO

ZERO

ZERO

ZERO

ZERO

ZERO

ZERO

ZERO

ZERO

ZERO

ZERO

ZERO

ZERO

ZERO

ZERO

ZERO

ZERO

ZERO

$0.3021 E+02$

ZERO

ZERO

2ERO

ZERO

ZERO

ZERO

0)

ZERO

ZERO

ZERO

ZERO

ZERO

ZERO

ZERO

ZERO.

ZERO

ZERO

ZERO

ZERO

ZERO

ZERO

ZERO

ZERO

ZERO

ZERO

ZERO

ZERO

ZERO

ZERO

ZERO

ZERO

ZERO

ZERO

ZERO

ZERO

ZERO

$0.1500 E+00$

ZERO

ZERO

ZERO

ZFRO

ZERO

ZERO
ZERO $0.9369 \mathrm{E}+00$

70

Melter Cooli. ZERO

$0.9463 \mathrm{E}-02$

$0.2538 \mathrm{E}-01$

$0.3366 \mathrm{E}-04$

$0.1512 \mathrm{E}-05$

ZERO

Date: January 1995

ZERO

ZERO

ZERO

ZERO

ZERO

ZERO

ZERO

ZERO

ZERO

ZERO

ZERO

ZERO

ZERO

ZERO

ZERO

ZERO

ZERO

ZERO

ZERO

ZERO

ZERO

ZERO

ZERO

$0.1108 \mathrm{E}+00$

ZERO

ZERO
$0.4691 \mathrm{E}-01$

ZERO

ZERO

ZERO

$0.1075 E-04$
$0.4619 E+03$

ZERO

ZERO

ZERO

ZERO

ZERO

ZERO

ZERO

ZERO

ZERO

ZERO

ZERO

$0.4154 \mathrm{E}+03$

ZERO

ZERO

ZERO

ZERO

ZERO

ZERO Offgas Cooling Air

ZERO

72

73

ting Air
to OGFC steam
ZERO
ZERO
ZERO

-Gas Dilution Air

$\begin{array}{ll}\text { ZEREY } & \text { to OGFC } \\ \text { ZERO } & \text { ZERO }\end{array}$

ZERO ZERO

ZERO ZERO

ZERO ZERO

ZERO ZERO

ZERO … ZERO

ZERO ZERO

ZERO ZERO

ZERO
ZERO ZERO
ZERO

ZERO $\quad$ ZERO

ZERO ZERO

$0.2651 \mathrm{E}-01$

ZERO

ZERO

ZERO ZERO

ZERO ZERO

ZERO ZERO

ZERO ZERO ZERO

ZERO ZERO

$\begin{array}{ll}\text { ZERO } & \text { ZERO } \\ \text { ZERO } & \text { ZERO }\end{array}$

ZERO $\quad$ ZERO

ZERO $\quad$ ZERO

ZERO ZERO

ZERO ZERO

ZERO ZERO

ZERO ZERO

ZERO ZERO

ZERO ZERO

ZERO ZERO

ZERO ZERO

ZERO

ZERO

ZERO ZERO

ZERO ZRRO

$\begin{array}{ll}\text { ZERO } & \text { ZERO } \\ \text { ZERO } & \text { ZERO }\end{array}$

ZERO ZERO

ZERO ZERO

ZERO ZERO

ZERO $0.7680 \mathrm{Z}-03$

ZERO ZERO

ZERO ZERO

ZERO ZERO

ZERO ZERO

ZERO ZERO

ZERO ZERO

Section 5. Page 7 of 37 
Table A-1. HLW Flowsheet Material Balance (Tank 51/ITP Cycle 1) seation 5 . Vitrification and Melter off-Gas Treatment

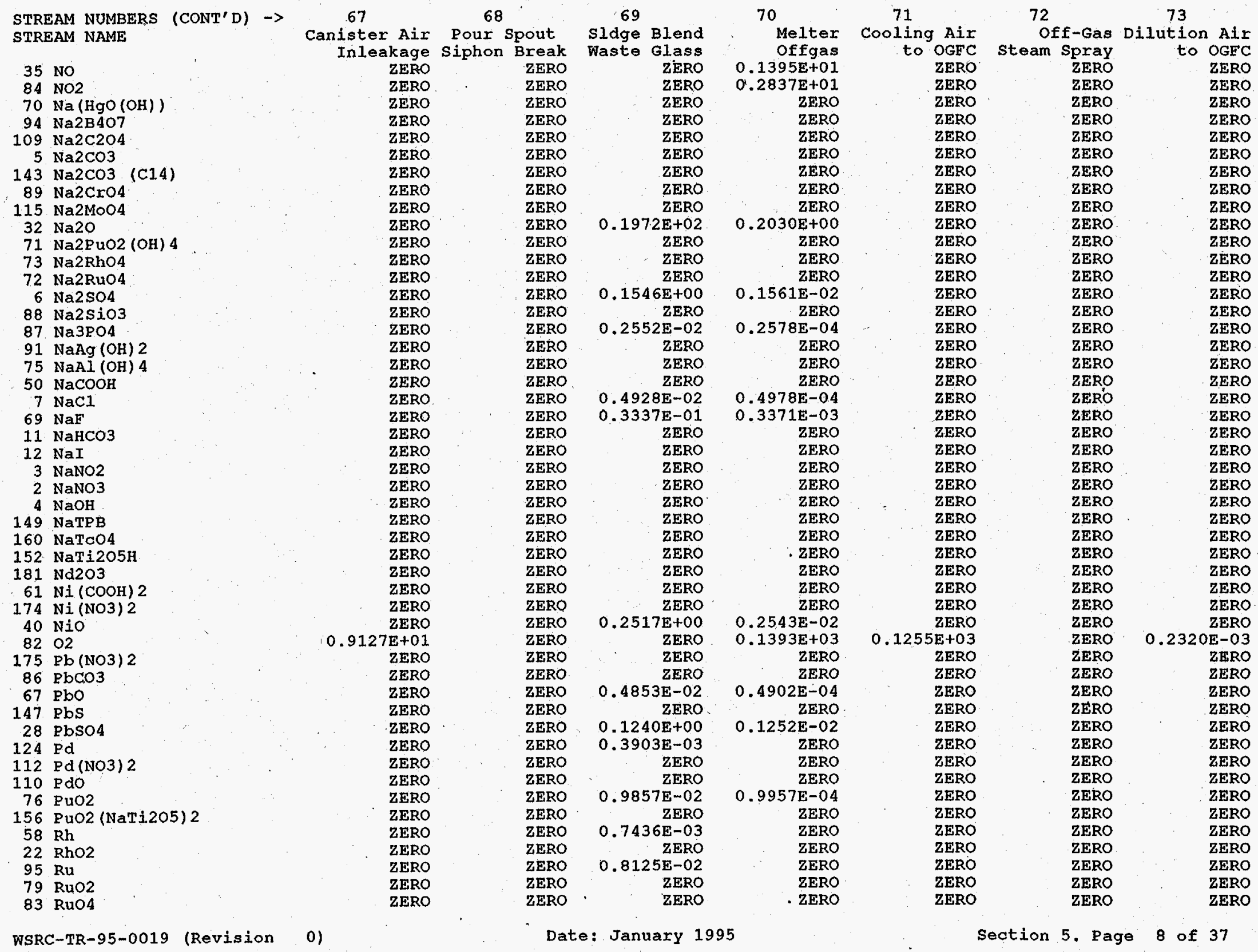


Table A-1. HLW Flowsheet Material Balance (Tank 51/ITP Cycle 1) section 5. Vitrification and Melter off-Gas Treatment

STREAM NUMBERS (CONT'D) -> STREAM NAME

$38 \quad 502$

$141 \mathrm{SO} 3$

137 Semi Vol Cs2O

136 Semi Vol CsCl

126 Semi Vol Group A

140 Semi Vol Na2B407

138 Semi Vol NaCl

139 Semi Vol NaF

125 Semi Vol NaI
23 Semi Vol RuO2

111 SiO2:

4.8 Sr ( $\mathrm{COOH}) 2$

$176 \mathrm{Sr}(\mathrm{NO} 3) 2$

$127 \mathrm{Sr}(\mathrm{OH}) 2$

$30 \mathrm{SrCO} 3$

$46 \mathrm{SrO}$

$159 \mathrm{TCO} 2$

92. ThO2

119 Tritium

$\begin{aligned} 119 & \text { Tritium } \\ 17 & \text { U } 308\end{aligned}$

62. $\mathrm{UO} 2$ ( $\mathrm{COOH}) 2$

177 UO2 (NO3) 2

$16 \mathrm{VO} 2(\mathrm{OH}) 2$

$27 \mathrm{Y}(\mathrm{COOH}) 3$

$178 \mathrm{Y}(\mathrm{NO} 3)$

$31 \mathrm{Y} 2(\mathrm{CO} 3) 3$

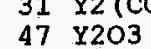

100 Zeolite

$44 \mathrm{Zn}(\mathrm{COOH}) 2$

$179 \mathrm{zn}(\mathrm{NO} 3) 2$

$\begin{array}{rl}57 & \mathrm{ZnO} \\ 104 & \mathrm{ZrO} 2\end{array}$

13 hydrate н2O

1 water

TOTAL ELOW, LB/HR

TEMPERATURE， DEG $\mathrm{C}$

PRESSURE, ATM

PRESSURE, PSIG

PRESSURE, MM HG

ENTHALPY, PCU/HR

VAPOR FLOW, CEM

LIQUID FLOW, GPM

DENSITY， LBS/FT3

PHASE

WSRC-TR-95-0019 (Revision
67

Canister Air Pour spout sldge Blend

Inleakage Siphon

$\begin{array}{rrr}\text { ZERO } & \text { ZERO } & \text { ZERO } \\ \text { ZERO } & \text { ZERO } & \text { ZERO }\end{array}$

$\begin{array}{lll}\text { ZERO } & \text { ZERO } \\ \text { ZERO }\end{array}$

ZERO

ZERO ZERO ZERO

ZERO ZERO ZERO

ZERO ZERO ZERO

ZERO

ZERO

ZTRO

ZERO

ZERO

ZERO

ZERO

ZERO

ZERO

ZERO

ZERO

ZERO

ZERO

ZERO

ZERO'

ZERO

ZERO

ZERO

ZERO

ZERO

ZERO

ZERO

ZERO

ZERO

ZERO

ZERO

ZERO

ZERO

ZERO

$0.6609 \mathrm{E}+00$

$0.4000 \mathrm{E}+02$

$0.3500 \mathrm{E}+02$

$0.9632 \mathrm{E}+00$

$-0.5408 \mathrm{E}+00$

$0.7320 \mathrm{E}+03$

$0.8389 \mathrm{E}+03$

$0.9810 \mathrm{E}+01$

$0.6796 \mathrm{E}-01$

VAPOR

ZERO

ZERO

ZERO

ZERO

ZERO

ZERO

ZERO

ZERO

ZERO

ZERO

ZERO

ZERO

ZERO

ZERO

ZERO

ZERO

ZERO

ZERO

ZERO

ZERO

ZERO

ZERO

ZERO

ZERO

ZERO

$0.1500 \mathrm{E}+00$

$0.3500 \mathrm{E}+02$

$0.1000 \mathrm{E}+01$

ZERO

$0.1272 \mathrm{E}+01$

$0.3613 \mathrm{E}-01$

$0.6920 \mathrm{E}-01$

VAPOR
70

Melter Cooling Air

Offgas
$0.2985 \mathrm{E}-01$

ZERO

ZERO

$0.4115 \mathrm{E}-02$

$0.1772 \mathrm{E}-03$

$0.3351 \mathrm{E}-02$

$0.1245 \mathrm{E}-01$

$0.5417 \mathrm{E}-0.5$

7ERO

- 1217

ZERO

ZERO

ZERO

ZERO

$0.1519 \mathrm{E}-01$

$0.3534 \mathrm{E}-02$

$0.2943 \mathrm{E}-01$

$0.8253 \mathrm{E}+00$

ZERO

$0.2063 E+01$

ZERO

ZERO

ZERO

ZERO

ZERO

ZERO

$0.4187 \mathrm{E}-02$

ZERO

ZERO

$0.3128 E+00$

ZERO

ZERO

$0.2280 \mathrm{E}+03$

$0.1050 \mathrm{E}+04$

$0.9840 \mathrm{E}+00$

$-0.2351 \mathrm{E}+00$

$0.7043 \mathrm{E}+05$

$0.1797 \mathrm{E}+00$

$0.1582 \mathrm{E}+03$

IIQUID
$0.1230 \mathrm{E}+01$

ZERO

ZERO

ZERO

ZERO

$0.1535 \mathrm{E}-03$

$0.3569 \mathrm{E}-04$

$0.2972 \mathrm{E}-03$

$0.1424 \mathrm{E}-09$

ZERO

ZERO

ZERO

ZERO

ZERO

ZERO

$0.4229 \mathrm{E}-04$

ZERO

ZERO

ZERO
$0.3159 \mathrm{E}-02$

E-02

ZERO

$0.3411 \mathrm{E}+03$

$0.9632 \mathrm{E}+03$

$0.6050 \mathrm{E}+03$

$-0.2351 \mathrm{E}+00$

$0.3967 \mathrm{E}+06$

$0.7863 \mathrm{E}+03$

$0.2042 \mathrm{E}-01$

VAPOR
$0.5500 \mathrm{E}+03$
72

Off-Gas Dilution Aix steam spray to OGFC $\begin{array}{lll}\text { ZERO } & \text { ZERO } & \text { ZERO } \\ \text { ZERO } & \text { ZERO } & \text { ZERO }\end{array}$ ZERO ZERO ZERO ZERO ZERO Z ZERO ZERO ZERO $\quad$ ZERO

ZERO ZERO $\quad$ ZERO

ZERO ZERO ZERO

ZERO ZERO ZERO

ZERO $\cdots$ ZERO $\ldots \ldots$ ZERO

ZERO ZERO ZERO
ZERO

ZERO ZERO $\quad$ ZERO

$\begin{array}{lll}\text { ZERO } & \text { ZERO } & \text { ZERO } \\ \text { ZERO } & \text { ZERO }\end{array}$

$\begin{array}{lll}\text { ZERO } & \text { ZERO } & \text { ZERO } \\ \text { ZERO } & \text { ZERO }\end{array}$

ZERO ZERO ZERO

ZERO ZERO Z ZERO

ZERO ZERO ZERO

ZERO Z ZERO W ZERO

ZERO ZERO $\quad$ ZERO

ZERO ZERO

ZERO ZERO

ZERO ZERO ZERO

$\begin{array}{lll}\text { ZERO } & \text { ZERO } & \text { ZERO } \\ \text { ZERO } & \text { ZERO } & \text { ZERO }\end{array}$

$\begin{array}{lll}\text { ZERO } & \text { ZERO } & \text { ZERO } \\ \text { ZERO } & \text { ZERO } & \text { ZERO }\end{array}$

$\begin{array}{lll}\text { ZERO } & \text { ZERO } \\ \text { ZERO } & \text { ZERO }\end{array}$

$\begin{array}{lll}\text { ZERO ZERO ZERO } & \text { ZERO } \\ \text { ZERO ZERO } & \text { ZERO }\end{array}$

ZERO ZERO ZERO

ZERO ZERO ZERO

ZERO ZERO ZERO

ZERO ZERO ZERO

ZERO ZERO ZERO

ZERO ZERO

ZERO ZERO

ZERO ZERO ZRO

ZERO

ZERO

$0.9087 \mathrm{E}+01$

$0.4800 \mathrm{E}+03$

$0.1017 \mathrm{E}-04$

$0.3500 \mathrm{E}+02$

$0.3720 \mathrm{E}+01$

$0.1643 \mathrm{E}+03$

$0.6784 \mathrm{E}+01$

$0.3500 \mathrm{a}+\mathrm{C}$

$0.3997 \mathrm{E}+02$

$0.3997 \mathrm{E}+02$

$0.1154 \mathrm{E}+05$

$0.3215 \mathrm{E}+06$

$0.2133 \mathrm{E}-01$

$0.2625 \mathrm{E}+00$

VAPOR

2127E+00

VAPOR

VAPOR 
STREAM NUMBERS $\rightarrow$ STREAM NAME

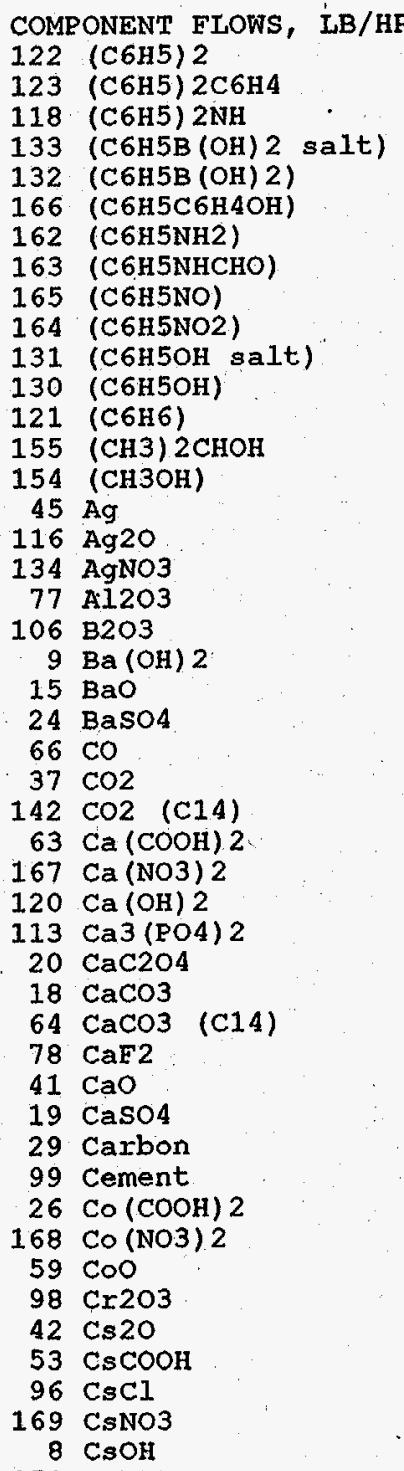

Table A-1. HLW Flowsheet Material Balance (Tank 51/ITP Cycle 1) section 5. Vitrification and Melter off-Gas Treatment

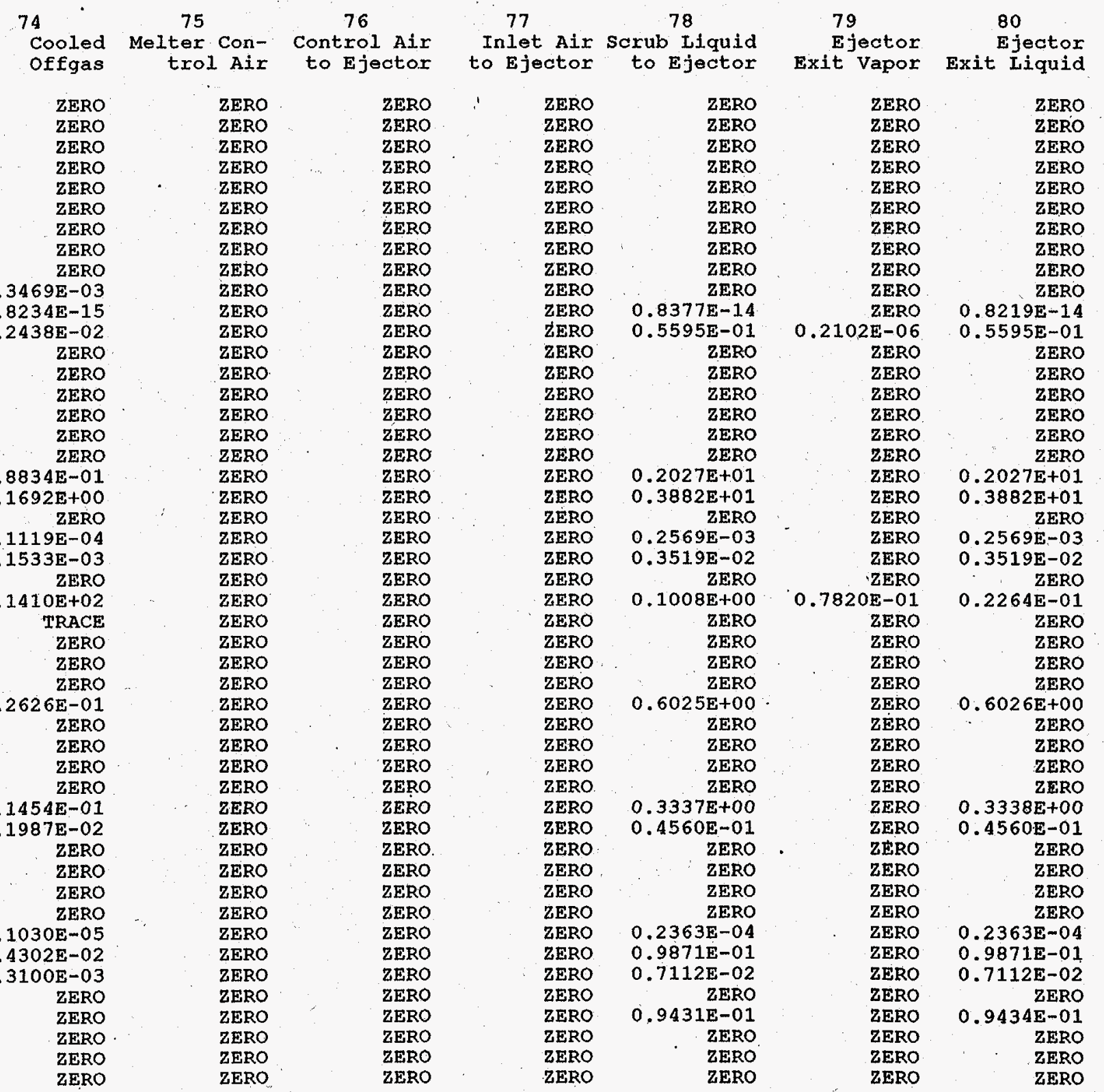

Date: January 1995 
Table A-1. HLW Flowsheet Material Balance (Tank 51/ITP Cycle 1) section 5. Vitrification and Melter off-Gas Treatment

STREAM NUMBERS (CONT'D) $\rightarrow$ STREAM NAME

$54 \mathrm{Cu}(\mathrm{COOH}) 2$
$170 \mathrm{Cu}(\mathrm{NO} 3) 2$

55 CuO

$80 \mathrm{~F} e 203$

$101 \mathrm{FeO}$

102 Group A

103 Group B

$25 \mathrm{H} 2$

$158 \mathrm{H} 2 \mathrm{C} 2 \mathrm{O} 4$

$93 \mathrm{H} 2 \mathrm{SO} 4$

$\begin{array}{ll}90 & \mathrm{H} 3 \mathrm{BO} 3 \\ 34 & \mathrm{HCOOH}\end{array}$

$43 \mathrm{HCl}$

$85 \mathrm{HE}$

49 HNO3

$36 \mathrm{Hg}$

$129 \mathrm{Hg}(\mathrm{C} 6 \mathrm{H} 5) 2$

$135 \mathrm{Hg}$ (NO3) 2

$144 \mathrm{Hg} 2 \mathrm{Cl} 2$

$117 \mathrm{Hg} 2 \mathrm{I2}$

$146 \mathrm{HgCl2}$

21. $\mathrm{HgO}$

$183 \mathrm{~K} 2 \mathrm{CO} 3$

$39 \mathrm{~K} 20$

$51 \mathrm{KCOOH}$
$157 \mathrm{KMnO} 4$

$161 \mathrm{KNO} 3$

$10 \mathrm{KOH}$

$150 \mathrm{KTPB}$

$105 \quad \mathrm{La203}$

$65 \mathrm{Mg}(\mathrm{COOH}) 2$

$171 \mathrm{Mg}$ (NO3) 2

$33 \mathrm{MgO}$

$60 \mathrm{Mn}(\mathrm{COOH}) 2$

$172 \mathrm{Mn}$ (NO3) 2

$180 \mathrm{Mn} 304$

$56 \mathrm{MnO}$

$14 \mathrm{MnO} 2$

$145 \mathrm{MoO} 2$

81 N2

$74 \mathrm{~N} 2 \mathrm{O}$

114 NH3OHNO3

$52 \mathrm{NH} 4 \mathrm{COOH}$

173 NH4NO3

$68 \mathrm{NH} 4 \mathrm{OH}$

148 NH4TPB

WSRC-TR-95-0019 (Revision
74 Cooled Melter Con- Control Air
offgas trol Air to Ejector ZERO

$0.9463 \mathrm{E}-02$

$0.2539 E+00$

$0.2538 \mathrm{E}-01$

$0.3366 \mathrm{E}-04$

$0.1512 \mathrm{E}-05$

ZERO

$0.4289 \mathrm{E}-01$

ZERO

ZERO

ZERO

$0.1978 \mathrm{E}-02$

$0.1318 \mathrm{E}-09$

$0.1384 \mathrm{E}+00$

ZERO

- $0.1434 \mathrm{E}-01$

$0.1183 \mathrm{E}-06$

ZERO

ZERO
$0.4632 \mathrm{E}-09$

ZERO

$0.5718 \mathrm{E}-01$

ZERO

ZERO

ZERO

ZERO

ZERO

$0.1108 \mathrm{E}+00$

ZERO

ZERO

ZERO

ZERO

ZERO

$0.2651 \mathrm{E}-01$

ZERO

$0.8773 \mathrm{E}+03$

ZERO

ZERO

ZERO

ZERO

ZERO

ZERO

0)

$\begin{array}{ll}\text { ZERO } & \text { ZERO } \\ \text { ZERO } & \text { ZERO }\end{array}$

ZERO ZERO

ZERO ZERO

ZERO $:$ ZERO

ZERO ZERO

ZERO ZERO

ZERO

ZERO

ZERO

ZERO

ZERO

ZERO

ZERO

ZERO

ZERO

ZERO

ZERO

ZERO

ZERO

ZERO

ZERO

ZERO

ZERO

ZERO

ZERO

ZERO

ZERO

ZERO

ZERO

ZERO

ZERO

ZERO

ZERO

ZERO

0.3777 E+O3

ZERO

ZERO

ZERO

ZERO

ZERO

ZERO

ZERO

ZERO

ZERO

ZERO

ZERO

ZERO

ZERO

ZERO

ZERO

ZERO

ZERO

ZERO

ZERO

ZERO

ZERO

ZERO

ZERO

ZERO

ZERO

ZERO

ZERO

ZERO

ZERO

ZERO

ZERO

ZERO

ZERO

ZERO

ZERO

ZERO

ZERO
${ }^{77}{ }^{78}$ Inlet Air scrub Liquid

to Ejector to Ejector

ZERO ZERO

ZERO
ZERO
ZERO

ZERO ZERO

ZERO $\quad 0.5825 \mathrm{E}+01$

ZERO $\quad 0.5824 \mathrm{E}+00$

ZERO $0.4833 \mathrm{E}-02$

ZERO $0.3468 \mathrm{E}-04$

ZERO ZERO

ZERO

ZERO

ZERO

ZERO

ZERO

ZERO

$0.3021 \mathrm{E}+02$

Date: January 1995

(2)

ZERO

$0.1396 \mathrm{E}-01$

$0.2417 \mathrm{E}+01$

$0.2980 \mathrm{E}+01$

ZERO

ZERO

ZERO

ZERO $\quad 0.3289 \mathrm{E}+00$

ZERO $0.2716 \mathrm{E}-05$

ZERO $0.1262 \mathrm{E}-11$

ZERO

ZERO

ZERO

ZERO

ZERO

ZERO

- ZERO

ZERO

ZERO

ZERO

ZERO

ZERO

ZERO

ZERO

ZERO

ZERO

$0.6057 \mathrm{E}+02$

ZERO

ZERO

ZERO

ZERO

. ZERO

ZERO

ZERO

$0.1312 \mathrm{E}+01$

ZERO

ZERO

ZERO

ZERO

ZERO

$0.2542 \mathrm{E}+01$

ZERO

0.1077 ZERO

zeno

ZERO

ZERO
$0.6082 \mathrm{E}+00$

ZERO

$0.2467 \mathrm{E}-03$

$0.4388 \mathrm{E}+00$

ZERO

ZERO

ZERO

ZERO

ZERO

79

Ejector

80

Ejector ZERO ZERO

ZERO ZERO

ZERO $0.2172 \mathrm{E}+00$

ZERO $0.5825 \mathrm{E}+01$

$0.5824 \mathrm{E}+00$

ZERO $\quad 0.4834 \mathrm{E}-02$

ZERO ZERO

ZERO ZERO

ZERO

ZERO

$0.8178 \mathrm{E}-03$

$0.5153 \mathrm{E}-02$

ZERO

ZERO

ZERO

$0.3493 \mathrm{E}-17$

ZERO

ZERO

ZERO

ZERO

ZERO

ZERO

ZERO

ZERO

ZERO

ZERO

ZERO

ZERO

ZERO

ZERO

ZERO

ZERO

ZERO

$0.6054 \mathrm{E}+02$

ZERO

ZERO

ZERO

ZERO

ZERO 
Table A-1. HIW Flowsheet Material Balance (Tank 51/ITP Cycle 1) section 5. Vitxification and Melter off-Gas Treatment

STREAM NUMBERS (CONT'D) $\rightarrow$ STREAM NAME

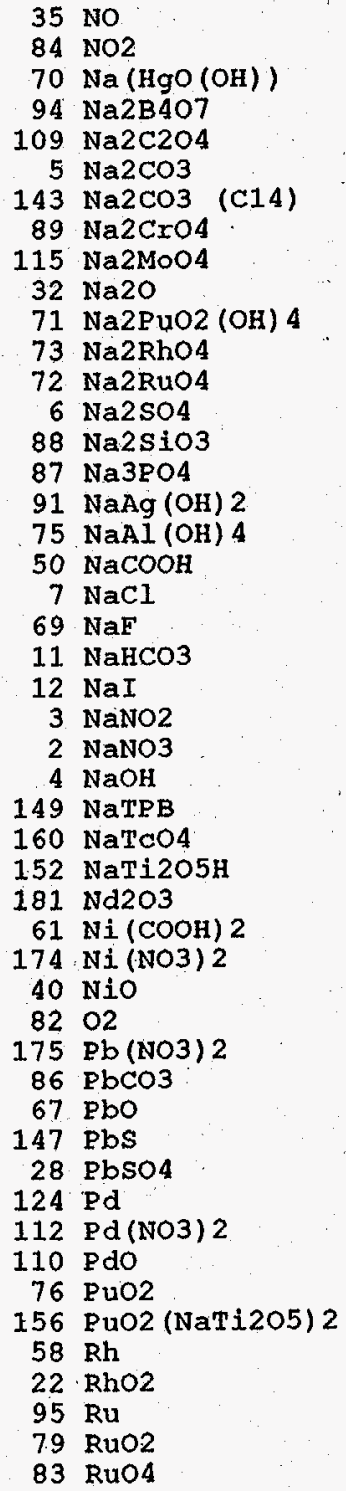

Date: January 1995

ZERO
$0.1125 \mathrm{E}-02$

$0.2873 \mathrm{E}-01$
79

Exit Vapor

$0.8611 \mathrm{E}-03$

$0.1699 \mathrm{E}-02$

ZERO

ZERO $\quad 0.2816 \mathrm{E}+01$

$\begin{array}{ll}\text { ZERO } & \text { ZERO } \\ \text { ZERO } & \text { ZERO }\end{array}$

ZERO $\quad$ ZERO

ZERO ZERO

ZERO ZERO

ZERO $0.4658 \mathrm{E}+01$

ZERO $\quad$ ZERO

ZERO ZERO

ZERO ZERO

ZERO

ZERO

ZERO

ZERO

ZERO

ZERO

ZERO

ZERO

ZERO

ZERO

ZERO

ZERO

ZERO

ZERO

ZERO

ZERO

ZERO

ZERO

ZERO

$0.1825 \mathrm{E}+02$

ZERO

ZERO
ZERO

ZERO

ZERO

ZERO

ZERO

ZERO

ZERO

ZERO

ZERO

ZERO
ZERO

ZERO

ZERO

ZERO

ZERO

ZERO

ZERO
ZERO

$0.1142 \mathrm{E}-02$

$0.2931 \mathrm{E}+00$ ZERO
2E-03

$0.1242 \mathrm{E}-03$

$0.5080 \mathrm{E}-01$

ZERO

ZERO

ZERO

ZERO

ZERO

ZERO
$0.5834 \mathrm{E}-01$

$0.2535 E+00$ ZERO ZERO

ZERO

ZERO

ZERO
$0.2285 \mathrm{E}-02$

ZERO

ZERO

ZERO

ZERO

ZERO

ZERO

$3 \mathrm{E}+02$

ZERO

ZERO

ZERO

ZERO

ZERO

ZERO

ZERO

ZERO

ZERO

ZERO

ZERO

ZERO

ZERO

ZERO

80

Ejector

$0.2003 \mathrm{E}-04$

$0.30525-04$

$0.3952 \mathrm{E}-04$

$0.2817 \mathrm{E}+01$

ZERO ZERO

ZERO ZERO

ZERO ZERO

$\begin{array}{ll}\text { ZERO } & \text { ZERO } \\ \text { ZERO } & \text { ZERO }\end{array}$

ZERO $\quad 0.4658 \mathrm{E}+01$

ZERO ZERO

ZERO ZERO

ZERO ZERO

ZERO ZERO

ZERO ZERO

ZERO $\quad 0.5915 \mathrm{E}-03$

ZERO ZERO

ZERO ZERO

ZERO ZERO

ZERO $0.1142 \mathrm{E}-02$

ZERO $\quad 0.2932 \mathrm{E}+00$

ZERO ZERO

ZERO $0.1242 \mathrm{E}-03$

ZERO $0.8173 \mathrm{E}-01$

ZERO $0.5078 E-01$

ZERO ZERO

ZERO

ZERO ZERO

ZERO ZERO

ZERO ZERO

ZERO
ZERO
ZERO

ZERO . ZERO

$0.5834 \mathrm{E}-01$

$0.2780 \mathrm{E}+00$

$0.1125 \mathrm{E}-02$

$0.2873 \mathrm{E}-01$

ZERO 
Table A-1. HLW Flowsheet Material Balance (Tank 51/ITP Cycle 1) section 5. Vitrification and Melter off-Gas Treatment

STREAM NUMBERS (CONT'D) $\rightarrow$ STREAM NAME

$38 \mathrm{SO} 2$

$141 \mathrm{SO3}$

137 Semi Vol Cs20

136 Semi Vol CsCl

126 Semi Vol Group A

140 Semi Vol Na2B4O7.

138 Semi vol $\mathrm{NaCl}$

139 Semi Vol NaF

$\begin{aligned} 125 & \text { Semi Vol NaI } \\ 23 & \text { Semi Vol RuO2 }\end{aligned}$

23 Semi

$48 \mathrm{Sr}(\mathrm{COOH}) 2$

$176 \operatorname{Sr}$ (NO3) 2

$153 \mathrm{Sr}(\mathrm{NaTi2O5)} 2$

$127 \mathrm{Sx}(\mathrm{OH}) 2$

$30 \mathrm{SrCO} 3$

46 Sro

$159 \cdot \mathrm{TcO} 2$

92 ThO2

119 Tritium

119 Tritiun

$62 \mathrm{UO} 2(\mathrm{COOH}) 2$

177 UO2 (NO3) 2

$16 \mathrm{VO} 2(\mathrm{OH}) 2$
$27 \mathrm{Y}(\mathrm{COOH}) 3$

$178 \mathrm{Y}(\mathrm{NO} 3)^{3}$

128. $\mathrm{Y}(\mathrm{OH}) 3$

31 Y2 (CO3) 3

$47 \mathrm{Y} 203$

100 Zeolite

$44 \mathrm{Zn}$ (COOH) 2

$79 \mathrm{Zn}(\mathrm{NO}) 2$

57 zno

$104 \mathrm{zrO} 2$

13 hydrate $\mathrm{H} 2 \mathrm{O}$

1 water

TOTAL FLOW, LB/HR

TEMPERATURE, DEG $C$

PRESSURE, ATM

PRESSURE， PSIG

PRESSURE， MM HG

ENTHALPY, $\mathrm{PCU} / \mathrm{HR}$

VAPOR FLOW, CFM

LIOUID FLOW, GPM

DENSITY， LBS/ET3

PHASE

WSRC-TR-95-0019 (Revision
74

Cooled Melter Con-

offgas trol Air

ZERO

ZERO

$0.4115 \mathrm{E}-02$

$0.1772 \mathrm{E}-03$

$0.172 \mathrm{E}-03$

ZERO

$0.1245 \mathrm{E}-01$

$0.5417 \mathrm{E}-05$

ZERO

$0.1230 \mathrm{E}+01$

ZERO

ZERO

ZERO

ZERO

$0.1535 \mathrm{E}-03$

$0.3569 \mathrm{E}-04$

$0.3569 \mathrm{E}-04$
$0.2972 \mathrm{E}-03$

$0.2972 \mathrm{E}-03$

$0.1424 \mathrm{E}-09$

$0.2084 \mathrm{E}-01$

ZERO

ZERO

ZERO

ZERO

ZERO

ZERO

$0.4229 \mathrm{E}-04$

ZERO

ZERO

ZERO

$9 E-02$
ZERO

ZERO

$0.8301 \mathrm{E}+03$

$0.1993 \mathrm{E}+04$

$0.3480 \mathrm{E}+03$

$0.9827 E+00$

$-0.2542 E+00$

$0.7469 \mathrm{E}+03$

$.7297 \mathrm{E}+06$

$0.2786 \mathrm{E}-01$

VAPOR
76

Control Air

ZERO

ZERO

ZERO

ZERO

ZERO

ZERO

ZERO

ZERO

ZERO

ZERO

ZERO

ZERO

ZERO

ZERO

ZERO

ZERO

ZERO

ZERO

ZERO

ZERO

ZERO

ZERO

ZERO

ZERO

ZERO

ZERO

ZERO

ZERO

ZERO

ZERO

ZERO

$0.8261 \mathrm{E}+01$

$0.5000 \mathrm{E}+03$

$0.3500 \mathrm{E}+02$

$0.3920 \mathrm{E}+01$

$0.1049 \mathrm{E}+05$

$0.3175 \mathrm{E}+02$

$0.2625 \mathrm{E}+00$

VAPOR

ZERO

$0.4000 \mathrm{E}+02$.

$0.3500 \mathrm{E}+02$

$0.9632 \mathrm{E}+00$

$-0.5408 \mathrm{E}+00$

$0.7320 \mathrm{E}+03$

$0.9810 \mathrm{E}+01$

$0.6796 \mathrm{E}-01$

VAPOR.
77

Inlet Air Scrub Iiquid

to Ejector to Ejector

$\begin{array}{ll}\text { ZERO } & \text { ZERO } \\ \text { ZERO } & \text { ZERO } \\ \text { ZERO } & \text { ZERO }\end{array}$

ZERO ZERO

ZERO ZERO

ZERO ZERO

ZERO ZERO

ZERO

$\begin{array}{ll}\text { ZERO } & \text { ZERO } \\ \text { ZERO } & \text { ZERO }\end{array}$

$\begin{array}{ll}\text { ZERO } & \text { ZERO } \\ \text { ZERO } & \text { ZERO }\end{array}$

ZERO ZERO

ZERO ZERO

ZERO $\quad$ ZERO

ZERO ZERO

ZERO

ZERO

ZERO

ZERO

ZERO

ZERO

ZERO

ZERO

ZERO

ZERO

ZERO

ZERO

ZERO

ZERO

ZERO

ZERO

ZERO

ZERO

ZERO

$0.1322 \mathrm{E}+01$

$0.8015 \mathrm{E}+02$

$0.3500 \mathrm{E}+02$

$-0.1190 \mathrm{E}+01$

$0.6984 \mathrm{E}+03$

$0.2060 \mathrm{E}+02$

$0.6484 \mathrm{E}-01$

VAPOR

$.3522 \mathrm{E}-02$

$0.6820 \mathrm{E}-02$

$0.1913 \mathrm{E}+00$

$0.3242 \mathrm{E}-08$

$.4782 \mathrm{E}+00$

ZERO

ZERO

ZERO

ZERO

ZERO

ERO

$0.9704 \mathrm{E}-03$

ZERO
ZERO

$0.7249 E-01$

ZERO

ZERO

$0.3659 \mathrm{E}+05$

$0.4000 \mathrm{E}+02$

$.3720 \mathrm{E}+01$
$0.3997 \mathrm{E}+02$

$0.1464 \mathrm{E}+07$

$0.7300 \mathrm{E}+02$

$0.6249 \mathrm{E}+02$

LIQUID
ZERO $\quad 0.2821 \mathrm{E}+02$

79

Ejector

80

Ejector apor Exit Liquid ZERO ZERO ZERO ZERO ZERO ZERO ZERO ZERO

ZERO $\quad$ ZERO

ZERO

ZERO ZERO

ZERO
ZERO
ZERO

ZERO $0.2821 \mathrm{E}+02$

ZERO ZERO

ZERO ZERO

ZERO ‥ ZERO

ZERO ZERO

ZERO $0.3522 E-02$

ZERO $\quad 0.8190 \mathrm{E}-03$
ZERO

$0.3711=-12$

12

ZERO

ZERO

ZERO

ZERO

ZERO

ZERO

ZERO

ZERO

ZERO

ZERO

ZERO

ZERO

ZERO

$0.4110 \mathrm{E}+01$

$0.8296 \mathrm{E}+02$

$0.3995 \mathrm{E}+02$

$-0.1190 \mathrm{E}+01$

$0.3482 \mathrm{E}+04$

$0.2209 \mathrm{E}+02$

$0.6260 \mathrm{E}-01$

VAPOR
$0.6820 \mathrm{E}-02$

$0.1913 E+00$

$0.4782 \mathrm{E}+00$

ZERO

ZERO

ZERO

ZERO

TERO

ZERO

$0.9705 \mathrm{E}-0.3$

ZERO

ZERO

ZERO

ZERO

$0.3659 \mathrm{E}+05$

$0.3995 E+02$

$0.1190 \mathrm{E}+01$

$0.1463 \mathrm{E}+07$

$0.7299 \mathrm{E}+02$

$0.6249 \mathrm{E}+02$

LIQUID

Section 5. Page 13 of 37 
Table A-1. HLW Flowsheet Material Balance (Tank 51/ITP Cycle 1) section 5. Vitrification and Melter off-Gas Treatment

STREAM NUMBERS $\rightarrow$ STREAM NAME

COMPONENT FLOWS, LB/HR $122(\mathrm{C} 6 \mathrm{H} 5) 2$

123 ( $\mathrm{C} 6 \mathrm{H} 5) 2 \mathrm{C} 6 \mathrm{H}$

118 (C6H5) $2 \mathrm{NH}$

133. (C6H5B (OH) 2 salt)

132 (C6H5B (OH) 2 )

166 (C6H5C6H4OH)

163 (C6H5NHCHO)

163 (C6H5NHCHO
165 (C6H5NO)

164 (C6H5NO2)

131 (C6H5OH salt)

130 ( $\mathrm{C} 6 \mathrm{H} 5 \mathrm{OH})$

121 ( 155 ( $6 \mathrm{H} 3$ ) $2 \mathrm{CHOH}$

154 (СH3OH)

$45 \mathrm{Ag}$

$116 \mathrm{Ag} 20$

134 AgNO3
77 A12O3

$106 \mathrm{~B} 203$

$9 \mathrm{Ba}(\mathrm{OH}) 2$

$15 \mathrm{BaO}$

24 BasO4

$66 \mathrm{CO}$

$142 \mathrm{CO} 2$ (C14)

$63 \mathrm{Ca}(\mathrm{COOH}) 2$

$167 \mathrm{Ca}(\mathrm{NO} 3)$

$120 \mathrm{Ca}(\mathrm{OH}) 2$

$113 \mathrm{Ca} 3$ (PO4) 2

$20 \mathrm{CaC2} 24$

$18 \mathrm{CaCO} 3$
$64 \mathrm{CaCO} 3$ (C14)

$78 \mathrm{CaF} 2$

$78 \mathrm{CaF} 2$
$41 \mathrm{CaO}$

19. CaSO4.

29 Carbon

99 Cement

$26 \mathrm{Co}(\mathrm{COOH}) 2$

$168 \mathrm{Co}$ (NO3) 2

$59 \mathrm{COO}$

98 Cr203

$42 \mathrm{Cs} 2 \mathrm{O}$

$9.6 \mathrm{CsC} 1$

169 CsNO3

$8 \mathrm{CsOH}$

151 CsTPB

WSRC-TR-95-0019 (Revision
81

Inlet Gas to Quencher 82
Iiquid to 83 Quencher Exit Liquid

Quencher Exit Vapor

ZERO
ZERO
ZERO
ZERO
ZERO
ZERO
ZERO
ZERO
ZERO
$0.3469 \mathrm{E}-03$
$0.8234 \mathrm{E}-15$
$0.2438 \mathrm{E}-02$
ZERO
ZERO
ZERO
ZERO
ZERO
ZERO

$0.8834 \mathrm{E}-01$

$0.1692 \mathrm{E}+00$

ZERO

$0.1119 \mathrm{E}-04$
$0.1533 \mathrm{E}-03$

ZERO

$0.1410 \mathrm{E}+02$

TRACE
ZERO

ZERO

ZERO

$0.2626 \mathrm{E}-01$

ZERO

ZERO

ZERO

$0.1454 \mathrm{E}-01$

$0.1987 \mathrm{E}-02$

ZERO

ZERO

ZERO

$0.1030 \mathrm{E}-05$

$0.4302 \mathrm{E}-02$

$0.3100 E-03$

ZERO

ZERO

ZERO

ZERO

o)

$0.1256 \mathrm{E}-13$

$0.8234 \mathrm{E}-16$

$0.1205 \mathrm{E}-13$

$0.8047 \mathrm{E}-01$

ZERO
ZERO

ZERO

ZERO

ZERO

$0.2916 \mathrm{E}+01$

$0.5584 \mathrm{E}+01$

ZERO

$0.3695 \mathrm{E}-03$

ZERO
$0.1450 \mathrm{E}+00$

ZERO

$0.8290 \mathrm{E}-01$

ZERO

$0.8326 \mathrm{E}-05$

ZERO

ZERO

ZERO

ZERO

$0.2995 \mathrm{E}+01$

$0.5736 \mathrm{E}+01$

$0.3795 \mathrm{E}-03$

$0.5199 \mathrm{E}-02$

ZERO
ZER

ZERO
$0.2222 \mathrm{E}+00$

ZERO

ZERO

ZERO

$0.8903 \mathrm{E}+00$

ZERO

ZERO

ZERO

$0.4800 \mathrm{E}+00$

BE-01

ZERO
ZERO
ZERO

ZERO

$0.3399 \mathrm{E}-04$

$0.1420 \mathrm{E}+00$

ZERO

$0.1357 \mathrm{E}+00$

ZERO
ZERO

ZERO

ZERO
ZERO

ZERO

$0.8834 \mathrm{E}-02$

$0.1692 \mathrm{E}-01$

ZERO

$0.1533 \mathrm{E}-04$

ZERO

TRACE

TRACE
ZERO

$0.2626 \mathrm{E}-02$

ZERO
ZERO

ZERO

ZERO

$0.4931 \mathrm{E}+00$

$0.6738 \mathrm{E}-01$

ZERO

ZERO

ZERO

$0.3492 \mathrm{E}-04$

$0.1459 \mathrm{E}+00$

$0.1051 \mathrm{E}-01$

ZERO
$0.1357 \mathrm{E}+00$

ZERO

ZERO

ZERO

$0.1454 \mathrm{E}-02$

ZERO

ZERO

ZERO

$0.1030 \mathrm{E}-06$

$0.3100 \mathrm{E}-04$

ZERO

ZERO

ZERO

Date: January 1995
85

86

87

Offgas to Liquid to Liquid to Scrubbers Scrbbr Noz's Sarbbr Noz 1

$\begin{array}{lll}\text { ZERO } & \text { ZERO } & \text { ZERO } \\ \text { ZERO } & \text { ZERO } & \text { ZERO } \\ \text { ZERO } & \text { ZERO } & \text { ZERO } \\ \text { ZERO } & \text { ZERO } & \text { ZERO } \\ \text { ZERO } & \text { ZERO } & \text { ZERO } \\ \text { ZERO } & \text { ZERO } & \text { ZERO } \\ \text { ZERO } & \text { ZERO } & \text { ZERO } \\ \text { ZERO } & \text { ZERO } & \text { ZERO } \\ \text { ZERO } & \text { ZERO } & \text { ZERO } \\ \text { ZERO } & \text { ZERO } & \text { ZERO }\end{array}$

$0.8667 \mathrm{E}+00$

ZERO

$0.4302 \mathrm{E}-03$

\section{ZERO}

ZERO

ZERO

ZERO

ZERO

ZERO

ZERO

ZERO

ZERO

$0.8234 \mathrm{E}-16$

$0.8490-16$

ZERO

ZERO

ZERO

ZERO

ZERO

$0.8834 \mathrm{E}-02$

$0.1692 \mathrm{E}-01$

$0.1533 \mathrm{E}-04$

ZERO

$0.1410 \mathrm{E}+02$

TRACE

ZERO

Z ZERO

$6 \mathrm{E}-02$

ZERO

ZERO

ZERO

$0.1454 \mathrm{E}-02$

$$
\begin{aligned}
& \text { ZERO } \\
& \text { ZERO }
\end{aligned}
$$

ZERO

ZERO

ZERO

$0.302 \mathrm{E}-03$

OE: -04
ZERO

ZERO

ZERO

ZERO

ZERO
ZERO
$0.1119 \mathrm{E}-05$

$\begin{array}{lr}\text { ZERO } & \text { ZERO } \\ \text { ZERO } & \text { ZERO } \\ \text { ZERO } & \text { ZERO } \\ \text { ZERO } & \text { ZERO } \\ \text { ZERO } & \text { ZERO } \\ \text { ZERO } & \text { ZERO } \\ \text { ZERO } & \text { ZERO } \\ \text { ZERO } & \text { ZERO } \\ \text { ZERO } & \text { ZERO } \\ \text { ZERO } & \text { ZERO }\end{array}$

$0.3780 \mathrm{E}-14 \quad 0.1890 \mathrm{E}-14$

$0.2525 \mathrm{E}-01 \quad 0.1262 \mathrm{E}-01$ ZERO ZERO ZERO ZERO ZERO ZERO

$\begin{array}{rr}\text { ZERO } & \text { ZERO } \\ 0.9148 \mathrm{E}+00 & 0.4574 \mathrm{E}+00\end{array}$

$0.1752 \mathrm{E}+01 \quad 0.8760 \mathrm{E}+00$

$\begin{array}{rr}\text { ZERO } & \text { ZERO } \\ 0.1159 \mathrm{E}-03 & 0.5796 \mathrm{E}-04\end{array}$

$0.1588 \mathrm{E}-02 \quad 0.7939 \mathrm{E}-03$

ZERO
0.4550 ZERO

5E-01

ZERO

ZERO

$\begin{array}{rr}\text { ZERO } & \text { ZERO } \\ 0.2719 \mathrm{E}+00 & 0.1360 \mathrm{E}+00\end{array}$

ZERO

ZERO

ZERO

$0.1506 \mathrm{E}+00$

$0.2058 \mathrm{E}-01$

ZERO

ZERO

ZERO

$0.1066 \mathrm{E}-04$

$0.4455 \mathrm{E}-01$

$0.4256 \mathrm{E}-01$

E-01

ZERO

ZERO

ZERO

ZERO

$0.7531 \mathrm{E}-01$

$0.1029 \mathrm{E}-01$

ZERO

ZERO

ZERO

$0.5332 \mathrm{E}-05$

$0.2227 E-01$

$0.1605 \mathrm{E}-02$

$0.2128 \mathrm{ZE}-01$

ZERO

ZERO

Section 5. Page 14 of 37 ZERO ZERO

$0.3210 \mathrm{E}-02$ 
Table A-1. HLW Flowsheet Material Balance (Tank 51/ITP Cycle 1) Section 5. Vitrification and Melter off-Gas Treatment

STREAM NUMBERS (CONT'D) $\rightarrow$ STREAM NAME

$54 \mathrm{Cu}(\mathrm{COOH}) 2$
$170 \mathrm{Cu}(\mathrm{NO} 3) 2$

55 CuO

$80 \mathrm{Fe} 203$

$101 \mathrm{FeO}$

102 Group A

103 Grou

$\begin{array}{rl}25 & \mathrm{H} 2 \\ 158 & \mathrm{H} 2 \mathrm{C} 2 \mathrm{O} 4\end{array}$

$93 \mathrm{H} 2 \mathrm{SO} 4$

90
34
$\mathrm{HCOOH}$

$43 \mathrm{HCl}$

$85 \mathrm{HF}$

49 HNO3

$36 \mathrm{Hg}$

$129 \mathrm{Hg}(\mathrm{C} 6 \mathrm{H} 5)^{2}$

$135 \mathrm{Hg}$ (NO3) 2

$144 \mathrm{Hg} 2 \mathrm{Cl} 2$

$117 \mathrm{Hg} 2 \mathrm{I2}$

$146 \mathrm{HgCl2}$

$21 \mathrm{HgO}$

$\begin{array}{rl}97 & \mathrm{I} 2 \\ 83 & \mathrm{~K} 2 \mathrm{CO} 3\end{array}$

$39 \mathrm{~K} 2 \mathrm{O}$

$51 \mathrm{KCOOH}$

$157 \mathrm{KMnO4}$

$161 \mathrm{KNO} 3$

$150 \mathrm{KTPB}$

$105 \mathrm{La} 203$

$108 \mathrm{Li20}$

$65 \mathrm{Mg}(\mathrm{COOH}) 2$

$171 \mathrm{Mg}$ (NO3) 2

$33 \mathrm{MgO}$

$60 \mathrm{Mn}(\mathrm{COOH}) 2$

$172 \mathrm{Mn}(\mathrm{NO} 3) 2$

$\begin{array}{rl}180 & \mathrm{Mn} 304 \\ 56 & \mathrm{MnO}\end{array}$

$14 \mathrm{MnO} 2$

$145 \mathrm{MoO} 2$

81 N2

74
$\mathrm{~N} 2 \mathrm{O}$

114 NH3OHNO3

114 NH3OHNO3

52 NH 4 COOH
173 NH 4 NO3

173 NH4NO3

148 NH4 TPB

WSRC-TR-95-0019 (Revision
81

Inlet Gas

82

Liquid to

83

Liquid to
Quencher Exit Liquid
ZERO

ZERO

$0.9463 \mathrm{E}-02$

$0.2539 \mathrm{E}+00$

$0.2538 \mathrm{E}-01$

$0.3366 \mathrm{E}-04$

$0.1512 \mathrm{E}-05$

ZERO

$0.4289 E-01$

ZERO
ZERO

ZERO

ZERO

$0.1978 \mathrm{E}-02$

$0.1318 \mathrm{E}-09$

$0.1384 \mathrm{E}+00$

ZERO

$0.1434 \mathrm{E}-01$

$0.1183 \mathrm{E}-06$

ZERO

$0.4632 \mathrm{E}-09$

ZERO

$0.5718 \mathrm{E}-01$

ZERO

ZERO

ZERO

ZERO

$0.1108 \mathrm{E}+00$

ZERO

ZERO
$0.4691 \mathrm{E}-01$

ZERO

ZERO

ZERO

$0.2651 \mathrm{E}-01$

ZERO

$0.1255 \mathrm{E}+04$

ZERO

ZERO

ZERO

ZERO

ZERO

ZERO

$0.3123 E+00$

$0.8378 \mathrm{E}+01 \quad 0.8607 \mathrm{E}+01$

$0.8377 \mathrm{E}+00$

$0.451 E-02$

$0.4989-04$
ZERO

ZERO

$0.1544 \mathrm{E}+01$

ZERO

ZERO

$0.2007 \mathrm{E}-01$

$0.3476 \mathrm{E}+01$

$0.4287 \mathrm{E}+01$

ZERO

$0.4731 \mathrm{E}+00$

$0.3906 \mathrm{E}-05$

$0.1816 \mathrm{E}-11$

ZERO

ZERO

$0.1887 \mathrm{E}+01$

ZERO

ZERO

ZERO

ZERO

ZERO

$0.3657 \mathrm{E}+01$

ZERO

$0.1548 \mathrm{E}+01$

ZERO

ZERO

$0.8749 \mathrm{E}+00$

ZERO

$0.3549 \mathrm{E}-03$

$0.6311 \mathrm{E}+00$

ZERO

ZERO

ZERO

ZERO

ZERO

ZERO

$0.8605 \mathrm{E}+00$

$0.6983 \mathrm{E}-02$

$0.5125 \mathrm{E}-04$

ZERO

ZERO
$0.1590 \mathrm{E}+01$

ZERO

ZERO

$0.2078 \mathrm{E}-01$

$0.3562 \mathrm{E}+01$

$0.4185 \mathrm{E}+01$

ZERO

$0.4860 \mathrm{E}+00$

$0.4013 \mathrm{E}-05$

$0.1843 \mathrm{E}-11$

ZERO

ZERO

$0.1939 \mathrm{E}+01$

ZERO

ZERO

ZERO

ZERO

ZERO

$0.3756 \mathrm{E}+01$

ZERO

$0.1591 \mathrm{E}+01$

ZERO

ZERO

$0.8987 \mathrm{E}+00$

ZERO

$0.3646 \mathrm{E}-03$

$0.6352 \mathrm{E}+00$

ZERO

ZERO

ZERO

ZERO

ZERO

ZERO
ZERO

84

Quencher

85

86

ZERO

$\therefore \quad$ ZERO

$0.9463 \mathrm{E}-03$

$0.2539 \mathrm{E}-01$

$0.2538 \mathrm{E}-02$

$0.3366 \mathrm{E}-05$
$0.1512 \mathrm{E}-06$

ZERO

ZERO

ZERO

ZERO

ZERO

$0.1271 \mathrm{E}-02$

$0.2721 \mathrm{E}-01$
$0.2406 \mathrm{E}+00$

ZERO

$0.1434 \mathrm{E}-02$

$0.1183 \mathrm{E}-07$

$0.1222 \mathrm{E}-15$

ZERO
$0.4632 \mathrm{E}-09$

ZERO

$0.5718 \mathrm{E}-02$

ZERO

ZERO

ZERO

ZERO

$0.1108 \mathrm{E}-01$

ZERO

ZERO

ZERO

ZERO

ZERO

$0.2651 E-02$
ZERO

$0.1075 E-05$

$0.1255 \mathrm{E}+04$

ZERO

ZERO

ZERO

ZERO

ZERO

ZERO

Date: January 1995

ZERO

ZERO

ZERO

ZERO

ZERO

$0.1313 \mathrm{E}-02$

$0.2802 \mathrm{E}-01$

$0.2452 \mathrm{E}+\mathrm{O} O$

ZERO

$0.1434 \mathrm{E}-02$

$0.1183 \mathrm{E}-07$

$0.1267 \mathrm{E}-15$

ZERO

$\mathrm{E}-09$

$0.5718 \mathrm{E}-02$

ZERO

ZERO

ZERO

ZERO

ZERO

$0.1108 \mathrm{E}-01$

ZERO

0.4691 ZERO

E-02

ZERO

ZERO

$0.2651 \mathrm{E}-02$

ZERO

$0.1075 \mathrm{E}-05$

ZERO

ZERO

ZERO

ZERO

ZERO

ZERO

ZERO

Offgas to Iiquid to Liquid to Scrubbers Scrbbr Noz's Scrbbr Noz 1

$\begin{array}{rrr}\text { ZERO } & \text { ZERO } & \text { ZERO } \\ 0.9463 \mathrm{E}-03 & 0.9799 \mathrm{E}-01 & 0.4900 \mathrm{E}-01\end{array}$

$\begin{array}{lll}9463 \mathrm{E}-03 & 0.9799 \mathrm{E}-01 & 0.4900 \mathrm{E}-01\end{array}$

$0.2538 \mathrm{E}-02 \quad 0.2628 \mathrm{E}+00 \quad 0.1314 \mathrm{E}+00$

$0.3366 \mathrm{E}-05 \quad 0.2181 \mathrm{E}-02 \quad 0.1090 \mathrm{E}-02$

$0.1512 \mathrm{E}-06 \quad 0.1565 \mathrm{E}-04 \quad 0.7826 \mathrm{E}-05$

ZERO

$0.4844 \mathrm{E}+00$

ZERO

ZERO

$0.2422 \mathrm{E}+00$

$0.6298 \mathrm{E}-02$

ZERO

$0.3149 \mathrm{E}-02$

ZERO

$0.1225 \mathrm{E}-05$

$0.7422 \mathrm{E}-01$

$0.6127=06$

$0.2848 \mathrm{E}-12$

ZERO

ZERO

ZERO

$0.2961 \mathrm{E}+00$

ZERO

ZERO

ZERO 
Table A-1. HLW Flowsheet Material Balance (Tank 51/ITP Cycle 1) section 5. Vitrification and Melter off-Gas Treatment

STREAM NUMBERS (CONT'D) $\rightarrow$ STREAM NAME

\author{
35 NO \\ 84 NO2 \\ $70 \mathrm{Na}(\mathrm{HgO}(\mathrm{OH}))$ \\ $94 \mathrm{Na} 2 \mathrm{~B} 4 \mathrm{O} 7$ \\ $109 \mathrm{Na2C2O4}$ \\ $5 \mathrm{Na} 2 \mathrm{CO} 3$ \\ $143 \mathrm{Na} 2 \mathrm{CO} 3$ (C14) \\ $89 \mathrm{Na} 2 \mathrm{CrO} 4$ \\ $115 \mathrm{Na2MoO}$ \\ 71 Na2PuO2 (OH) 4 \\ $73 \mathrm{Na} 2 \mathrm{RhO} 4$ \\ $72 \mathrm{Na2RuO} 4$ \\ $6 \mathrm{Na2SO} 4$ \\ $\begin{array}{ll}88 & \mathrm{Na} 2 \mathrm{SiO}_{3} \\ 87 \mathrm{Na} 3 \mathrm{PO} 4\end{array}$ \\ . $11 \mathrm{NaAg}(\mathrm{OH}) 2$ \\ $75 \mathrm{NaAl}(\mathrm{OH}) 4$ \\ $50 \mathrm{NaCOOH}$ \\ $7 \mathrm{NaCl}$ \\ $69 \mathrm{NaF}$ \\ $11 \mathrm{NaHCO} 3$ \\ $12 \mathrm{NaI}$ \\ 3. NaNO2 \\ 2 NaNO3 \\ $4 \mathrm{NaOH}$ \\ 149 NaTPB \\ 152. NaTi2O5H \\ $181 \mathrm{Nd} 203$ \\ $61 \mathrm{Ni}(\mathrm{COOH}) 2$ \\ $174 \mathrm{Ni}$ (NO3) 2 \\ $40 \mathrm{NiO}$ \\ $82 \mathrm{O} 2$ \\ $86 \mathrm{PbCO} 3$ \\ $67 \mathrm{PbO}$ \\ $67 \mathrm{PbO}$
$147 \mathrm{PbS}$ \\ 28 PbSO 4 \\ $124 \mathrm{Pd}$ \\ $112 \mathrm{Pd}$ (NO3) 2 \\ $110 \mathrm{PdO}$ \\ $76 \mathrm{PuO} 2$ \\ $156 \mathrm{PuO} 2(\mathrm{NaTi} 205) 2$ \\ $58 \mathrm{Rh}$ \\ 22 RhO2 \\ $95 \mathrm{Ru}$ \\ 79 RuO2
}

83 RuO4

$81 \quad 82$

Inlet Gas o Quencher

$0.1395 \mathrm{E}+01$
$0.2837 \mathrm{E}+01$

0.2837 E +01

ZERO

ZERO

ZERO

ZERO

$0.2030 \mathrm{ZERO}$

ZERO

ZERO

$0.5633 \mathrm{E}-02$

$0.5633 \mathrm{E}-02$
ZERO

$0.2578 \mathrm{E}-04$

ZERO

ZERO

$0.4978 \mathrm{E}-04$

$0.3371 \mathrm{E}-03$

ZERO

ZERO

ZERO

ZERO

ZERO

ZERO

ZERO

ZERO

$0.2543 \mathrm{E}-02$

$0.3788 \mathrm{E}+03$

ZERO

$0.4902 \mathrm{E}-04$

ZERO

$0.1252 \mathrm{E}-02$

ZERO

ZERO

0.9957 E-04

ZERO

ZERO

ZERO

ZERO

ZERO

o)
Iiquid to

Quencher
$0.1267 \mathrm{E}-02$

$0.2500 \mathrm{E}-02$

ZERO
$0.4050 \mathrm{E}+01$

OE+01
ZERO

ZERO

ZERO

ZERO

$0.6700 \mathrm{E}+0$

$83 \quad 84$

Exit Liquid

$0.2057 \mathrm{E}-0.2$

$0.4059 \mathrm{E}-02$
ZERO

$0.4051 \mathrm{E}+01$

ZERO

ZERO

ZERO

$0.6883 \mathrm{E}+01$

ZERO

ZERO

ZERO

ZERO

$0.8508 \mathrm{E}-03$

ZERO
ZERO

ZERO

$0.1643 \mathrm{E}-02$

$0.4216 \mathrm{E}+00$

ZERO

$0.1176 \mathrm{E}+00$

$0.7306 \mathrm{E}-01$

ZERO

ZERO

ZERO

ZERO
ZERO

ZERO

ZERO

$0.8391 \mathrm{E}-01$

$0.3646 \mathrm{E}+00$

ZERO

$0.1618 \mathrm{E}-02$

ZERO

$0.4132 \mathrm{E}-01$

ZERO

ZERO

$0.3286 \mathrm{E}-02$

ZERO
ZERO

ZERO

ZERO

ZERO

ZE+01
ZERO

ZERO

ZERO

ZERO

$0.8740 \mathrm{E}-03$

ZERO

ZERO

ZERO
$0.1688 \mathrm{E}-02$

$0.4221 \mathrm{E}+00$

$0.1786 \mathrm{E}-03$

$0.1212 \mathrm{E}+00$

$0.7464 \mathrm{E}-01$

ZERO

ZERO

ZERO

ZERO

ZERO

ZERO

$0.8620 \mathrm{E}-01$

$0.3656 \mathrm{E}+00$

ZERO

$0.1662 \mathrm{E}-02$

0.424 ZERO

ETRO

ZERO

$0.3376 \mathrm{E}-02$

ZERO

ZERO

ZERO

ZERO

ZERO

ZERO

Date: January 1995
86

Quencher

85

frgas to Liquid to Liquid to ixit Vapor

$13945+01$

$0.2750 \mathrm{E}+01$

ZERO
ZERO

Scrbbr Noz's Scrbbr Noz 1

$\begin{array}{lll}0.1395 \mathrm{E}+01 & 0.3976 \mathrm{E}-03 & 0.1988 \mathrm{E}-03\end{array}$

$0.2752 \mathrm{E}+01 \quad 0.7844 \mathrm{E}-03 \quad 0.3922 \mathrm{E}-03$

ZERO ZERO ZERO

ZERO $0.1271 \mathrm{E}+01 \quad 0.6354 \mathrm{E}+00$

ZERO

ZERO ZERO ZERO

$\begin{array}{lll}\text { ZERO } & \text { ZERO } & \text { ZERO } \\ \text { ZERO } & \text { ZERO } & \text { ZERO }\end{array}$

ZERO

ZERO

$0.2030 \mathrm{E}-01$

$0.2030 \mathrm{Z}-01$

$0.2102 \mathrm{E}+01$

E+01

ZERO

ZERO

$0.5633 \mathrm{E}-03$

ZERO

$0.2578 \mathrm{E}-05$

ZERO

ZERO

$0.4978 \mathrm{E}-05$

$0.3371 \mathrm{E}-04$

ZERO
ZERO

ZERO

ZERO

ZERO

ZERO

ZERO

ZERO

ZERO

ZERO

$0.2543 \mathrm{E}-03$

$0.3788 \mathrm{E}+03$

ZERO

$0.4902 \mathrm{E}-05$

ZERO

$0.1252 \mathrm{E}-03$

ZERO

ZERO

$0.9957 \mathrm{E}-05$

ZERO
ZERO

ZERO

ZERO

ZERO

ZERO

ZERO

ZERO

$0.5633 \mathrm{E}-03$

ZERO

E-05

ZERO

ZERO

$0.4978 \mathrm{E}-05$

$0.3371 \mathrm{E}-04$

ZERO

ZERO

ZERO

ZERO

ZERO

ZERO

ZERO

ZERO

ZERO

$0.2543 \mathrm{E}-03$

$0.4210 \mathrm{E}+03$

- ZERO

$0.4902 \mathrm{E}-05$

ZERO

$0.1252 \mathrm{E}-03$

ZERO

ZERO

$0.9957 \mathrm{E}-05$

ZERO

ZERO

ZERO

ZERO

ZERO

ZERO

Section 5. Page 16 of 37

$0.1051 \mathrm{E}+01$

ZERO

ZERO

ZERO

ZERO

ZERO

$0.2669 \mathrm{E}-03$

ZERO

ZERO

$0.5155 \mathrm{E}-03$

$0.1323 E+00$

ZERO

$0.5603 \mathrm{E}-04$

.3689 $\mathrm{z}-01$

ZERO

ZERO

ZERO

ZERO

ZERO

ZERO

$0.2633 \mathrm{E}-01$

$0.1144 \mathrm{E}+00$

ZERO 
Table A-1. HLW Flowsheet Material Balance (Tank 51/ITP Cycle 1) Section 5. Vitrification and Melter off-Gas Treatment

STREAM NUMBERS (CONT'D) $\rightarrow$ STREAM NAME

38 SO2

$141 \mathrm{SO3}$

137 Semi Vol Cs2O.

136 Semi Vol CsCl

126 Semi Voi Group A

140 Semi Vol Na2B4
138 Semi Vol NaCl

138 Semi Vol NaCl
139 Semi Vol $\mathrm{NaF}$

139 Semi Vol NaF
125 Semi Vol NaI

23 Semi Vol RuO2

111 SiO2.

$48 \mathrm{Sr}(\mathrm{COOH}) 2$

$176 \operatorname{Sr}(\mathrm{NO} 3) 2$

$153 \mathrm{Sr}$ (NaTi2O5) 2

$127 \mathrm{Sr}(\mathrm{OH}) 2$

$30 \mathrm{SrCO} 3$

46 SrO

$159 \mathrm{TaO} 2$

92 ThO2

119 Tritium

17 U308

62 UO2 ( $\mathrm{COOH}) 2$

$177 \mathrm{UO2}$ (NO3) 2

$16 \mathrm{UO} 2(\mathrm{OH}) 2$

$27 \mathrm{Y}(\mathrm{COOH}) 3$

178 Y (NO3)

$31 \mathrm{Y} 2(\mathrm{CO} 3) 3$

$47 \mathrm{Y} 203$

100 Zeolite

$44 \mathrm{Zn}(\mathrm{COOH}) 2$
$179 \mathrm{Zn}(\mathrm{NO3}) 2$

$57 \mathrm{ZnO}$

$104 \mathrm{ZrO} 2$

13 hydrate $\mathrm{H} 2 \mathrm{O}$

1 water.

TOTAL FLOW, LB/HR

TEMPERATURE, DEG C

PRESSURE, ATM

PRESSURE， PSIG

PRESSURE, MM HG

ENTHALPY，PCU/HR

VAPOR FLOW, CFM

LIQUID FLOW, GPM

DENSITY, LBS/FT3

PHASE

WSRC-TR-95-0019 (Revision
81

Inlet Ga

to Quencher

ZERO

ZERO

$0.4115 \mathrm{E}-02$

$0.1772 \mathrm{E}-03$

$0.1229 \mathrm{E}+00$

ZERO

$0.1245 \mathrm{E}-01$

$0.5417 \mathrm{E}-05$

$0.1230 \mathrm{E}+01$

ZERO

ZERO

ZERO

ZERO

$0.1535 \mathrm{E}-03$

$0.3569 \mathrm{E}-04$

$0.2972 \mathrm{E}-03$

$0.8337 \mathrm{E}-02$
$0.1424 \mathrm{E}-09$

$0.1424 \mathrm{E}-09$

0.2084 E-01

ZERO

ZERO

ZERO

ZERO

ZERO

$0.4229 \mathrm{E}-04$

7ero

ZERO

ZERO

$0.3159 \mathrm{E}-02$

ZERO

$0.8384 \mathrm{E}+03$

$0.2493 \mathrm{E}+04$

$0.3014 \mathrm{E}+03$

$0.9754 \mathrm{E}+00$

$-0.3615 \mathrm{E}+00$

$0.7413 \mathrm{E}+03$

$0.7402 \mathrm{E}+0.6$

$0.1337 \mathrm{E}+04$

$0.3109 \mathrm{E}-01$

VAPOR

\begin{tabular}{|c|c|}
\hline $\begin{array}{l}82 \\
\text { Liquid to }\end{array}$ & $\begin{array}{l}83 \\
\text { Quencher }\end{array}$ \\
\hline Quencher & Exit Liquid \\
\hline ZERO & ZERO \\
\hline ZERO & ZERO \\
\hline ZERO. & ZERO \\
\hline ZERO & ZERO \\
\hline ZERO & ZERO \\
\hline ZERO & ZERO \\
\hline ZERO & ZERO \\
\hline ZERO & ZERO \\
\hline ZERO & ZERO \\
\hline ZERO & ZERO \\
\hline $.4058 \mathrm{E}+02$ & $0.4169 \mathrm{E}+02$ \\
\hline ZERO & ZERO \\
\hline ZERO & ZERO \\
\hline ZERO & ZERO \\
\hline ZERO & ZERO \\
\hline ZERO & ZERO \\
\hline & \\
\hline
\end{tabular}

$0.5066 \mathrm{E}-02$

$0.1178 \mathrm{E}-02$

$0.2751 \mathrm{E}+00$
$0.4663 \mathrm{E}-08$

$0.6878 \mathrm{E}+00$

ZERO

ZERO

ZERO

ZERO

ZERO

ZERO

$0.1396 \mathrm{E}-02$

ZERO

ZERO

$0.1043 \mathrm{E}+00$

ZERO

ZERO

$0.5254 \mathrm{E}+05$

$0.5263 \mathrm{E}+05$

$0.4000 \mathrm{E}+02 \quad 0.5118 \mathrm{E}+02$

$\begin{array}{ll}0.9820 \mathrm{E}+01 & 0.989 \mathrm{E}+00\end{array}$

$\mathrm{E}+02-0.1617 \mathrm{E}+00$

$0.7516 \mathrm{E}+03$

$0.1050 \mathrm{E}+03$

$0.6249 \mathrm{E}+02$
LIQUID

$0.1064 \mathrm{E}+03$

$0.6250 \mathrm{E}+02$

IIQUID
84

Quencher

Exit Vapor

$\therefore \quad$ ZERO

$0.4115 \mathrm{E}-02$

$0.4115 \mathrm{E}-02$

$0.1772 \mathrm{E}-03$

$0.1229 \mathrm{E}+00$
ZERO

$0.1245 \mathrm{E}-01$

$0.5417 \mathrm{E}-05$

$0.1230 \mathrm{E}+00$

ZERO

ZERO

ZERO

ZERO

$0.1535 \mathrm{E}-04$

$0.5175 \mathrm{E}-05$

$0.2972 \mathrm{E}-04$

$0.8337 \mathrm{E}-03$

$0.1362 \mathrm{E}-10$

$0.2084 \mathrm{E}-02$

ZERO

ZERO

ZERO

ZERO

ZERO

ZERO

$0.4229 \mathrm{E}-05$

ZERO

ZERO

$0.3159 \mathrm{E}-03$

ZERO

ZERO

$0.1804 \mathrm{E}+04$

$0.5122 \mathrm{E}+02$

$0.9754 \mathrm{E}+00$

$-0.3615 \mathrm{E}+00$

$0.7413 \mathrm{E}+03$

$0.1190 \mathrm{E}+06$

$0.4768 \mathrm{E}+03$

$0.6306 \mathrm{E}-01$

VAPOR
85

Offgas to 86

Liquid to Liquid to Sarbbr Noz's Scrbbr Noz 1

$\begin{array}{rll}\text { ZERO } & \text { ZERO } & \text { ZERO } \\ \text { ZERO } & \text { ZERO } & \text { ZERO }\end{array}$

$0.4115 \mathrm{E}-02$

$0.1772 \mathrm{E}-03$

.1772E-0

$.1229 \mathrm{E}+00$

$0.1245 \mathrm{E}-01$

$.5417 \mathrm{E}-05$

$0.1230 \mathrm{E}+00$

ZERO

ZERO

ZERO

ZERO

$0.1535 \mathrm{E}-04$

$.5175 \mathrm{E}-05$

$0.2972 \mathrm{E}-04$

$0.8337 \mathrm{E}-03$

$0.1399 \mathrm{E}-10$

$0.2084 \mathrm{E}-02$

ZERO

ZERO
ZERO

ZERO

ZERO

ZERO

ZERO

$0.4229 \mathrm{E}-05$

ZERO

ZERO

0.3159E-0

ZERO

ZERO

$0.1573 \mathrm{E}+03$

$0.1992 \mathrm{E}+04$

$0.4999 \mathrm{E}+02$

$0.9866 \mathrm{E}+00$

$0.7498 \mathrm{E}+0$

$0.5172 \mathrm{E}+0$

$0.6420 \mathrm{E}-01$

VAPOR

$0.3294 \mathrm{E}+02$

$0.6249 \mathrm{E}+02$

- LIQUID 
Table A-1. HLW Flowsheet Material Balance (Tank 51/ITP Cycle 1) section 5. Vitrification and Melter off-Gas Treatment

STREAM NOMBERS -> STREAM NAME

COMPONENT FLOKS, LB/HR 122 (C6H5) 2

123 (C6H5) 2C6H4

118 (C6H5) 2NH

133 (C6H5B (OH) 2 salt)

132 (C6H5B (OH) 2)

162 (C6H5NH2)

163 (C6H5NHCHO)

165 (C6H5NO)

164 (C6H5NO2)

131 (C6H5OH salt)

130 (C6H5OH)

121 (C6H6)

154 (СH $3 \mathrm{OH}$ )

$45 \mathrm{Ag}$

116 Ag2O

77 A1203

$106 \mathrm{~B} 2 \mathrm{O} 3$

$9 \mathrm{Ba}(\mathrm{OH}) 2$

$15 \mathrm{BaO}$

$24 \mathrm{BaSO} 4$

$66 \mathrm{CO}$

$142 \mathrm{CO} 2$ (C14)

$63 \mathrm{Ca}(\mathrm{COOH}) 2$

$167 \mathrm{Ca}$ (NO3) 2
$120 \mathrm{Ca}$ (OH) 2

$113 \mathrm{Ca} 3$ (PO4) 2

$20 \mathrm{CaC} 2 \mathrm{O} 4$

$18 \mathrm{CaCO} 3$

$64 \mathrm{CaCO}^{2}$ (C14)

$78 . \mathrm{CaF} 2$

$41 \mathrm{CaO}$

19 CasO4

29 Carbon

$26 \mathrm{Co}(\mathrm{COOH}) 2$

$168 \mathrm{Co}(\mathrm{NO} 3) 2$

$59 \mathrm{CoO}$

98 Cr2O3

$42 \mathrm{Cs} 2 \mathrm{O}$

$53 \mathrm{CsCOOH}$

$96 \mathrm{CsC1}$

$169 \mathrm{CsNO} 3$
$8 \mathrm{CsOH}$

151 CsTPB

WSRC-TR-95-0019 (Revision
89

88

steam Flow Liquid to
to SAS 1 Scrbbr Noz 2

\section{ZERO}

ZERO

ZEROO

ZERO

ZERO

ZERO

ZERO

ZERO

ZERO

ZERO

ZERO

ZERO

ZERO

ZERO

ZERO

ZERO

ZERO

ZERO

ZERO

ZERO

ZERO

ZERO

ZERO

ZERO

ZERO

ZERO

ZERO

ZERO

ZERO

ZERO

ZERO

ZERO

ZERO

7FRO

ZERO

ZERO

ZERO

ZERO

ZERO

ZERO

ZERO

ZERO

ZERO

ZERO

ZERO

\begin{tabular}{|c|c|c|}
\hline ZERO & ZERO & ZERO \\
\hline ZERO & ZERO & ZERO \\
\hline ZERO & ZERO & ZERO \\
\hline ZERO & ZERO & ZERO \\
\hline ZERO & ZERO & ZERO \\
\hline ZERO & ZERO & ZERO \\
\hline ZERO & ZERO & ZERO \\
\hline ZERO & ZERO & ZERO \\
\hline ZERO & ZERO & ZERO \\
\hline ZERO & ZERO & ZERO \\
\hline $0.1890 \mathrm{E}-14$ & ZERO & $0.3790 \mathrm{E}-14$ \\
\hline $0.1262 \mathrm{E}-01$ & ZERO & $0.2524 \mathrm{E}-01$ \\
\hline ZERO & ZERO & ZERO \\
\hline ZERO & ZERO & ZERO \\
\hline ZERO & ZERO & ZERO \\
\hline ZERO & ZERO & ZERO \\
\hline ZERO & ZERO & ZERO \\
\hline ZERO & ZERO & ZERO \\
\hline $0.4574 \mathrm{E}+00$ & ZERO & $0.9235 \mathrm{E}+00$ \\
\hline $0.8760 \mathrm{E}+00$ & ZERO & $0.1769 \mathrm{E}+01$ \\
\hline ZERO & ZERO & ZERO \\
\hline $0.5796 \mathrm{E}-04$ & ZERO & $0.1170 \mathrm{E}-03$ \\
\hline $0.7939 \mathrm{E}-03$ & ZERO & $0.1603 \mathrm{E}-02$ \\
\hline ZERO & ZERO & ZERO \\
\hline $0.2275 \mathrm{E}-01$ & ZERO & $0.4703 \mathrm{E}-01$ \\
\hline ZERO & ZERO & ZERO \\
\hline ZERO & ZERO & ZERO \\
\hline ZERO & ZERO & ZERO \\
\hline ZERO & ZERO & ZERO \\
\hline $0.1360 \mathrm{E}+00$ & ZERO & $0.2745 E+00$ \\
\hline ZERO & ZERO & ZERO \\
\hline ZERO & ZERO & ZERO \\
\hline ZERO & ZERO & ZERO \\
\hline ZERO & ZERO & ZERO \\
\hline $0.7531 \mathrm{E}-01$ & ZERO & $0.1520 \mathrm{E}+00$ \\
\hline $0.1029 \mathrm{E}-01$ & ZERO & $0.2077 \mathrm{E}-01$ \\
\hline ZERO & 2ERO & ZERO \\
\hline ZERO & ZERO & ZERO \\
\hline ZERO & ZERO & ZERO \\
\hline ZERO & ZERO & ZERO \\
\hline $0.5332 \mathrm{E}-05$ & ZERO & $0.1077 \mathrm{E}-04$ \\
\hline $0.2227 \mathrm{E}-01$ & ZERO & $0.4497 \mathrm{E}-01$ \\
\hline $0.1605 \mathrm{E}-02$ & ZERO & $0.3240 \mathrm{E}-02$ \\
\hline ZERO & ZERO & ZERO \\
\hline $0.2128 \mathrm{E}-01$ & ZERO & $0.4661 \mathrm{E}-01$ \\
\hline ZERO & ZERO & ZERO \\
\hline ZERO & ZERO & ZERO \\
\hline ZERO & ZERO & ZERO \\
\hline
\end{tabular}

Date: January 1995.
91

Steam Flow SAS $1 \&$

92

SAS 2

Exit Gas

OGCTC

Condensate

Exit Gas

ZERO ZERO ZERO

$\begin{array}{lll}\text { ZERO } & \text { ZERO } & \text { ZERO } \\ \text { ZERO } & \text { ZERO }\end{array}$

ZERO ZERO $\quad$ ZERO

ZERO : ZERO : ZERO

ZERO ZERO ZERO

ZERO ZERO ZERO

ZERO ZERO ZERO

ZERO ZERO ZERO

ZERO

$\begin{array}{rrr}0.1647 \mathrm{E}-17 & \text { ZERO } & 0.1647 \mathrm{E}-17 \\ 0.1837 \mathrm{E}-04 & 0.1835 \mathrm{E}-04 & 0.2284 \mathrm{E}-07\end{array}$

ZERO

ZERO

ZERO

ZERO

ZERO

$0.1767 \mathrm{E}-03$

$0.3384 \mathrm{E}-03$

ZERO

$0.2239 \mathrm{E}-07$

$0.3067 \mathrm{E}-06$

$0.1410 \mathrm{EERO}$

TRACE

ZERO

ZERO

$0.5252 \mathrm{E}-04$

ZERO

ZERO

ZERO

$0.2909 \mathrm{E}-04$

$0.3974 \mathrm{E}-05$

ZERO

ZERO

ZERO

ZERO

$0.2060 \mathrm{E}-08$

$0.6199 \mathrm{E}-06$

$9 \mathrm{E}-06$
ZERO

ZERO

ZERO

ZERO
ZERO

ZERO

ZERO

ZERO

ZERO

ZERO

$0.1767 \mathrm{E}-03$

$0.3384 \mathrm{E}-03$

ZERO

$0.2239 \mathrm{E}-07$

$0.3067 \mathrm{E}-06$

0.1410 ZERO

TRACF

ZERO

ZERO

$0.5252 \mathrm{E}-04$

ZERO

ZERO

ZERO

ZERO

$0.2909 \mathrm{E}-04$

$0.3974 \mathrm{E}-05$

ZERO

ZERO

ZERO

0.2060 E-08

$0.2060 \mathrm{~B}-08$

$0.8604 \mathrm{E}-05$
$0.619 .9 \mathrm{E}-06$

ZERO

ZERO

ZERO

ZERO

Section 5. Page 18 of 37 
Table A-1. HLW Flowsheet Material Balance (Tank 51/ITP Cycle 1) section 5. Vitrification and Melter off-Gas Treatment

STREAM NUMBERS (CONT'D) $\rightarrow$ STREAM NAME

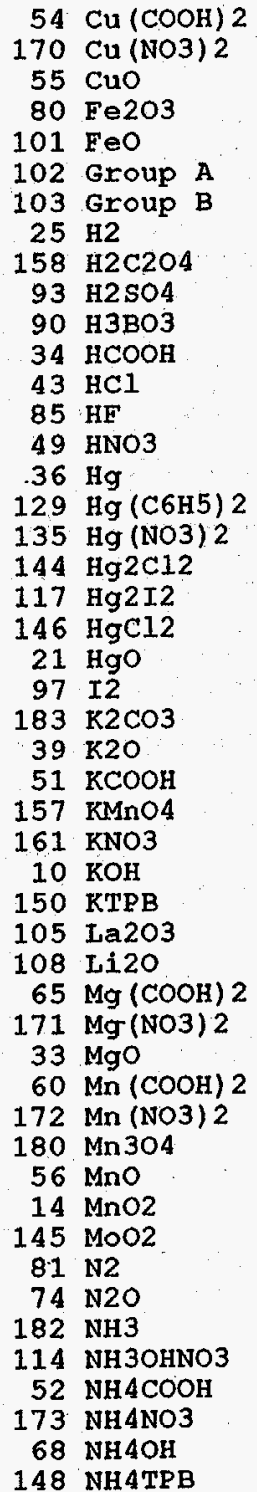

$54 \mathrm{Cu}(\mathrm{COOH}) 2$
$170 \mathrm{Cu}(\mathrm{NO} 3) 2$

55 CuO

80 Fe203

102 Group A

25 H2

58 H2 2204

$93 \mathrm{H} 2504$

45

49 HNO3

$36 \mathrm{Hg}$

29 (C6H5) 2

$144 \mathrm{Hg} 2 \mathrm{Cl2}$

$17 \mathrm{Hg} 2 \mathrm{I} 2$

$21 \mathrm{HgO}$

97 I2

$83 \mathrm{~K} 2 \mathrm{CO}$

$57 \mathrm{KMnO}$

$161 \mathrm{KNO}$

105 La203

$33 \mathrm{MgO}$

$(\mathrm{COOH}) 2$

$172 \mathrm{Mn}(\mathrm{NO3})$

$14 \mathrm{MnO} 2$

(45 MoO2

74 in

182 NH3

$52 \mathrm{NH} 4 \mathrm{COOH}$

$68 \mathrm{NH} 4 \mathrm{OH}$

WSRC-TR-95-0019 (Revision
88

Steam Flow Liquid to

to SAS 1 Scrbbr Noz 2

ZERO
ZERO
ZERO
ZERO
ZERO
ZERO
ZERO
ZERO
ZERO
ZERO
ZERO
ZERO
ZERO
ZERO
ZERO
ZERO
ZERO
ZERO
ZERO
ZERO
ZERO
ZERO
ZERO
ZERO
ZERO
ZERO
ZERO
ZERO
ZERO
ZERO
ZERO
ZERO
ZERO
ZERO
ZERO
ZERO
ZERO
ZERO
ZERO
ZERO
ZERO
ZERO
ZERO
ZERO
ZERO
ZERO
ZERO
ZERO
ZERO

ZERO
$0.4900 E-01$

$0.1314 \mathrm{E}+00$

$0.1090 \mathrm{E}-02$

$0.7826 \mathrm{E}-05$

ZERO

$0.2422 E+00$

ZERO

ZERO

$0.3149 \mathrm{E}-02$

$0.5454 \mathrm{E}+00$

$0.6725 \mathrm{E}+00$

ZERO

$0.7422 \mathrm{E}-01$

$0.6127 \mathrm{E}-06$

$0.6127 \mathrm{E}-06$
$0.2848 \mathrm{E}-12$

$0.2848 \mathrm{E}-12$

ZERO

ZERO

$0.2961 \mathrm{E}+00$

ZERO

ZERO

ZERO

ZERO

ZERO

$0.5736 E+00$

ZERO

$0.2429 \mathrm{E}+00$

ZERO

ZERO

$0.1372 \mathrm{E}+00$

ZERO

$0.5567 \mathrm{E}-04$

$0.9901 \mathrm{E}-01$

ZERO

ZERO

ZERO

ZERO

ZERO
ZERO

90
Steam
ElOW
to
SAS 2
ZERO
ZERO
ZERO
ZERO
ZERO
ZERO
ZERO
ZERO
ZERO
ZERO
ZERO
ZERO
ZERO
ZERO
ZERO
ZERO
ZERO
ZERO
ZERO
ZERO
ZERO
ZERO
ZERO
ZERO
ZERO
ZERO
ZERO
ZERO
ZERO
ZERO
ZERO
ZERO
ZERO
ZERO
ZERO
ZERO
ZERO
ZERO
ZERO
ZERO
ZERO
ZERO
ZERO
ZERO
ZERO
ZERO
ZERO
ZERO
ZERO

91

SAS $1 \& 2$

$\therefore \quad$ ZERO

$0.9892 \mathrm{E}-01$

$0.2654 \mathrm{E}+01$

$0.2653 \mathrm{E}+00$

$0.2358 \mathrm{E}-02$

$0.1580 \mathrm{E}-04$

ZERO

$0.4844 E+00$

ZERO

$0.5671 \mathrm{E}-02$

$0.1069 \mathrm{E}+01$

$0.9264 \mathrm{E}+00$

ZERO

$0.1499 \mathrm{E}+00$

$0.1237 \mathrm{E}-0.5$

$0.5781 \mathrm{E}-12$

ZERO

ZERO

$0.5978 \mathrm{E}+00$

ZERO

ZERO

ZERO

ZERO

ZERO

$0.1158 \mathrm{E}+01$

ZERO

$0.4904 \mathrm{E}+00$

ZERO

ZERO

$0.2771 \mathrm{E}+00$

$0.1124 \mathrm{E}-03$

$0.1690 \mathrm{E}+00$

ZERO

ZERO

ZERO

ZERO

ZERO

ZERO

Date: January 1995
92

93

Exit Gas

ZERO

$0.1893 \mathrm{E}-04$

$0.5077 \mathrm{E}-03$

$0.5076 \mathrm{E}-04$

$.6732 \mathrm{E}-07$

$0.3023 \mathrm{E}-08$

ZERO

ZERO

ZERO

ZERO

ZERO

$0.1940 \mathrm{E}-02$

$0.4971 \mathrm{E}-01$

$0.6639 \mathrm{E}+00$

ZERO

$0.2867 \mathrm{E}-04$

$0.2367 \mathrm{E}-09$

$0.2953 \mathrm{E}-15$ ZERO

$0.4632 \mathrm{E}-09$ ZERO

$0.1144 \mathrm{E}-03$

ZERO

ZERO

ZERO

ZERO

ZERO

ZERO

0.9383 EERO

3E-04
ZERO

ZERO

ZERO

$0.5301 \mathrm{E}-04$

$0.2151 \mathrm{E}-07$

$0.1395 \mathrm{E}+04$

ZERO

ZERO

ZERO

ZERO

ZERO

ZERO

Section 5. Page 19 of 37
OGCTC

94

Exit Gas

ZERO

ZERO

$0.1893 E-04$

$0.5077 \mathrm{E}-03$

$0.5076 \mathrm{E}-04$

$0.6732 \mathrm{E}-07$

$0.3023 E-08$

ZERO

ZERO

ZERO

ZERO

$0.5677 \mathrm{E}-03 \quad 0.1372 \mathrm{E}-02$

$0.4271 \mathrm{E}-01 \quad 0.7001 \mathrm{E}-02$

$0.8511 \mathrm{E}-02$

ZERO

$0.2867 \mathrm{E}-04$

$0.2367 \mathrm{E}-09$

$0.2953 \mathrm{E}-15$

ZERO

ZERO

$0.1144 \mathrm{E}-03$

ZERO

ZERO

ZERO

ZERO

ZERO

ZERO

$0.9383 \mathrm{E}+04$

ZERO

ZERO

$0.5301 \mathrm{E}-04$ ZERO

$0.2151 \mathrm{E}-07$

$0.1395 E+04$

ZERO

ZERO

ZERO

ZERO

ERO 


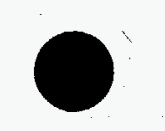

WSRC-TR-95-0019 (Revision

Table A-1. HLW Elowsheet Material Balance (Tank 51/ITP Cycle 1) A-1. HLW Flowsheet Material Balance (Tank 51/ITP Cycle
section 5. Vitrification and Melter off-Gas Treatment

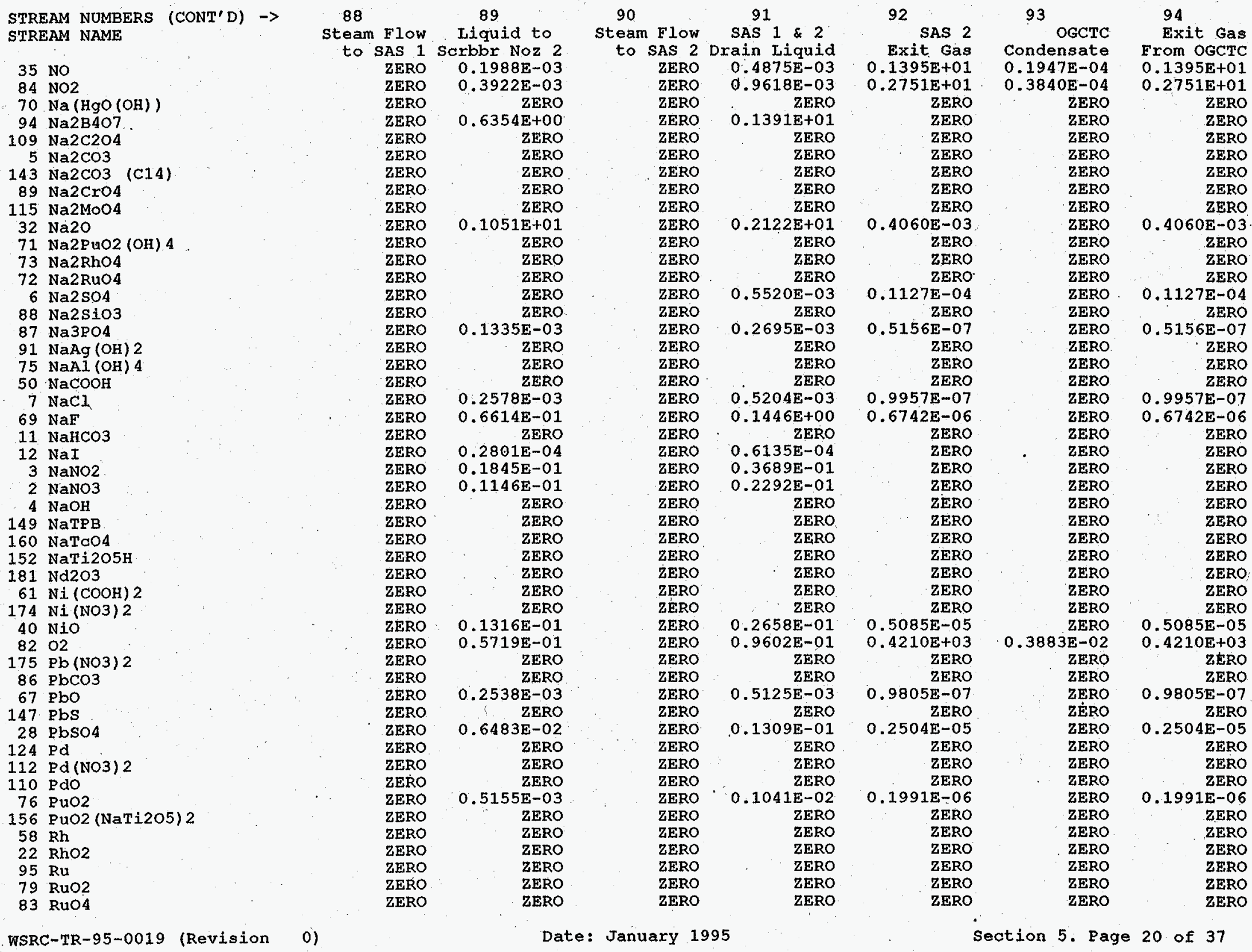




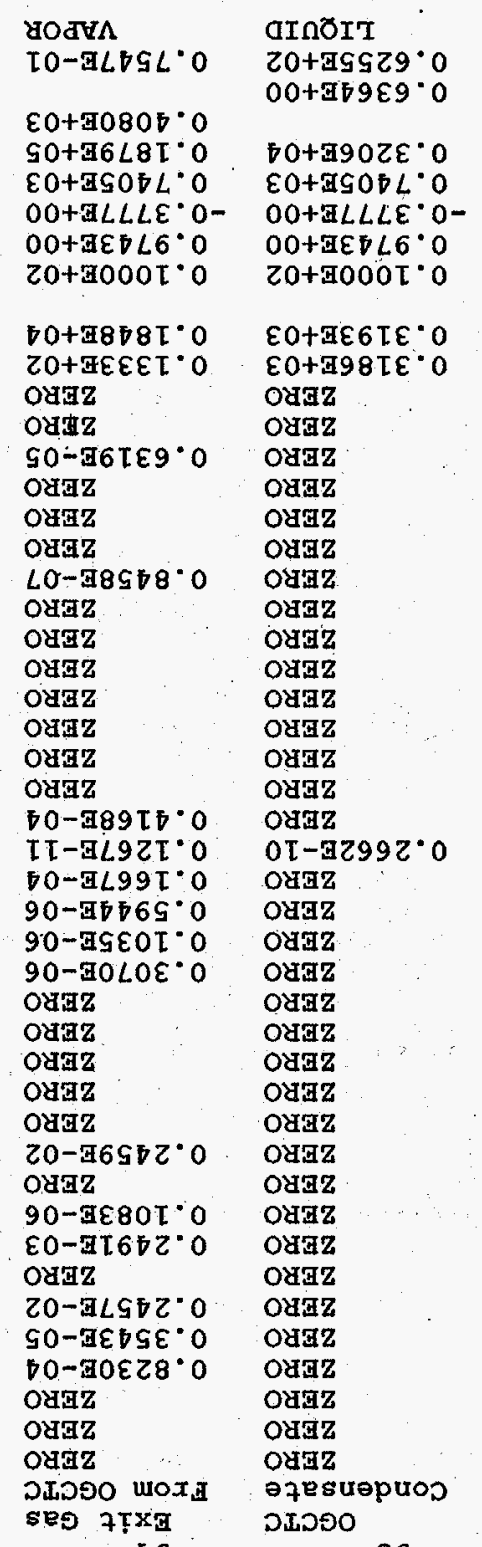

$7 \times \mathbb{G}$
6 I0-'I IS8G. 0 $\varepsilon 0+\pi \varepsilon<\tau 9^{\circ} 0$ $90+996 \varepsilon Z^{\circ} 0$ EO+GGODL $L^{\circ}$ $O O+G L L L E \cdot 0-$ $00+G \varepsilon \cup \angle 6^{\circ} 0$ $Z 0+96929^{\circ} 0$

\section{$0+\mathrm{T} L 9 \tau \mathrm{T} \cdot 0$ $\varepsilon 0+\pi 6$ IE $\varepsilon^{\circ} 0$} Oataz 90-T6 $699 \cdot 0$ OdGz odgz

Oygz $\angle 0-78578^{\circ} 0$ orgz

oytar

oygiz

oxing

Oxin

O४표

0 글

b0-389 T5 0

$0 \mathrm{I}-\mathrm{G} 68 \mathrm{LZ} \cdot 0$ VO-GL99I:0 90-30665.0 90 -GSEOT.0 90-GOLOE'O O४सZ

क्षig?

oxgz

O४t?

Oyg

$20-76 s \nabla 2 \cdot 0$

O4GZ

$90-9 \varepsilon 80 \tau \cdot 0$

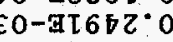

oygu

$Z 0-\pi \angle S D Z \cdot 0$ SO-JEDSE 0

DO-تOEZ8. 0

OdTz

0

O4⿴囗十

sed 7 T⿱X $\times$

$z$ SVS 26
बIกбIT

$Z 0+76 \nabla Z 9^{\circ} 0$
$Z 0+790 \nabla \varepsilon^{\circ} 0$

y० वस

$00+\operatorname{s} L \tau t Z^{\circ} 0$

बIกชIT

$20+06029^{\circ} 0$

$20+1 \angle 59 T \cdot 0$

$\angle 0+G 8 E 0 I^{\circ} 0$

$\varepsilon O+\operatorname{GOE} L L^{\circ} 0$

$00+G L L L \varepsilon^{\circ} 0-\quad Z 0+T 0058 \cdot 0$

$00+B E \nabla \angle 6^{\circ} 0 \quad$ TO+TB $\angle \angle 9^{\circ} 0$

$90+$ ТОЕE. 0

ZO+GTOZE 0

TO+\$08:LE. 0

$20+90005 \cdot 0$

$S O+\operatorname{LLOLT} \cdot 0$

SO+GDOLI. 0

OมTz

OदIz

To- 5

O\&HZ

O४\&47

O४전

EO-GLZ.V $0^{\circ} 0$

oxg7

O४az

oxag

Oytz

oysiz

0

O격

$00+36 \angle \tau 2 \cdot 0$

$80-146$ VI ${ }^{\circ} \circ$
I0-3GI $\angle 8^{\circ} 0$

ZO-GLOTE.

$\varepsilon O-\mathbb{a} \angle \forall \angle E$.

20-5609 I.0

oषga

o⿺辶a

O\&ga

어료

$20+\pi c 8 T^{\circ} 0$

Oytz Oyt

0

oyga

oyga

oygz

०४⿻コ一

oxga

Oyaz

oygz

O\&grz

PŢnbțT uṭexa $Z$ sUs of

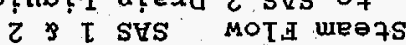

$$
\text { I } 6
$$

$60+\pi \angle c z 8^{\circ} 0 \quad \varepsilon 0+20 \angle 98^{\circ} 0$

$\triangle 0+\pi \angle \$ 28^{\circ} 0 \quad \varepsilon 0+\pi 0 \angle 9 \varepsilon^{\circ} 0$

OdTZ O\&g?

O४प्रZ O४

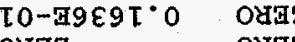

Odaz OAt

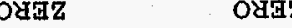

O\&gz Oata

E0-\$06IZ०0 O\&g

O\&!

Oygz O\&g

O\&gz

$\therefore \quad 04 \pi$

Odiz

$0 y$

O\&g

$00+16 \angle 0 T \cdot 0 \quad$ O४

60-g9TEL.0 O४

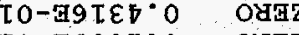

ZO-Z6EST:0 Oस्वस

$\varepsilon 0-\mathrm{G} 88 \mathrm{~T}^{\circ} 0$ O४ O४

$\varepsilon 0-\mathrm{G} L D 6 L \cdot 0$ O\&G

O\&g?

O\&'प

O적

odtz

$0 y$

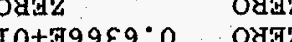

o\&gz oxt?

oym oyg

o\&gz O\&g

O\&अ

OAgZ otg

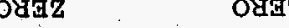

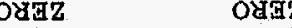

Orसz OdG

O\&gz O\&az

$z$ zON xqqTOS I SYS of

of ptinbț. MOTa ureezs

$68^{\circ} 88$

gStHa

ELA/SET 'XUISNGQ

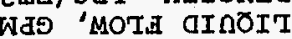

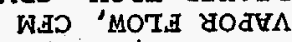
YH/ODd "X JTVHING

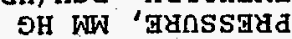
DISd 'Gunss ard

WLY ' gyossagd

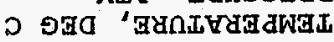

צH/GI 'MOTA TYLOI Хә7ем $\mathrm{T}$ OZH әчеxрКЧ $\varepsilon I$ zOxz b0T $\tau$ (EON) uz $6 L T$ $z$ (HOOS) Uz (HOOD) Uz
ə7?TOOZ OOI EOZX $L F$

$\varepsilon(\varepsilon O D) Z \pi \quad \tau \varepsilon$ $\varepsilon$ (HO) $\pi \quad 8 Z T$ $\varepsilon(\varepsilon O N) \pi 8 L T$ $\varepsilon($ HOOD) $\pi L Z$ $\tau$ (HO) ZON $9 \tau$ $z$ (EON) ZON LLLT $z$ (HOOD) ZON $Z 9$ 8OER LT

UกT7TIL 6IT ZOTI LOT ZOपय Z6 ZODI 6S I OxS 98 EONTS OE $Z$ (HO) XS $L Z \tau$ $z$ (SOZTUEN) xs $\varepsilon S T$ $Z(\varepsilon O N) x S 9 L T$ $Z$ (HOOD) xS 8D ZOTS ITT zony TOL Tüs $\varepsilon z$ IEN TOL TTUOS GZT JEN TOA TUES $6 \varepsilon T$ TOEN TOA TUOS $8 E T$ cotgien ton twes oft Y dnoto tos twes $9 Z T$

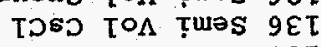

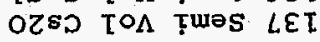
EOS IVI
ZOS $8 \varepsilon$

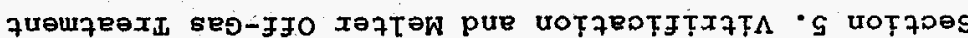

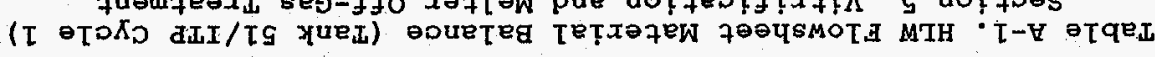


Table A-1. HLW Flowsheet Material Balance (Tank 51/ITP Cycle 1) Section.5. Vitrification and Melter off-Gas Treatment

STREAM NUMBERS $\rightarrow$ STREAM NAME

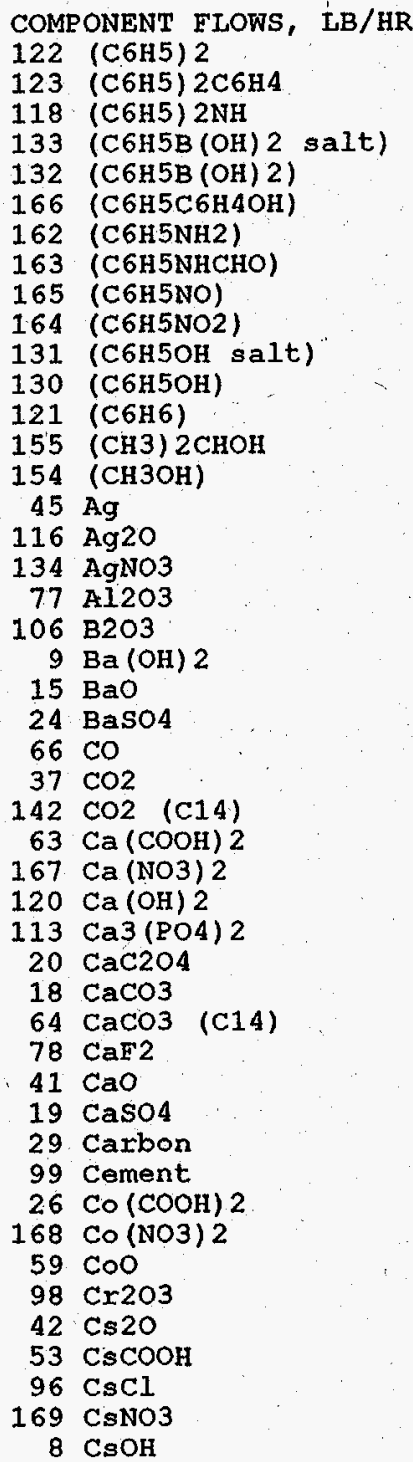

96
Atomizing HEME Solids

to HEME Air to HEME Accumulation

\begin{tabular}{|c|c|c|c|}
\hline ZERO & ZERO & ZERO & ZERO \\
\hline ZERO & ZERO & ZERO & ZERO \\
\hline ZERO & ZERO & ZERO & ZERO \\
\hline ZERO & ZERO & ZERO & ZERO \\
\hline ZERO & ZERO & ZERO & ZERO \\
\hline ZERO & ZERO & ZERO & ZERO \\
\hline ZERO & ZERO & ZÉRO & ZERO \\
\hline ZERO & ZERO & ZERO & ZERO \\
\hline ZERO & ZERO & ZERO & ZERO \\
\hline ZERO & ZERO & ZERO & ZERO \\
\hline ZERO & ZERO & ZERO & ZERO \\
\hline ZERO & ZERO & ZERO & ZERO \\
\hline ZERO & ZERO & ZERO & ZERO \\
\hline ZERO & ZERO & ZERO & ZERO \\
\hline ZERO & ZERO & ZERO & ZERO \\
\hline ZERO & ZERO & ZERO & ZERO \\
\hline ZERO & ZERO & ZERO & ZERO \\
\hline ZERO & ZERO & ZERO & ZERO \\
\hline ZERO & ZERO & ZERO & ZERO \\
\hline ZERO & ZERO & ZERO & ZERO \\
\hline ZERO & ZERO & ZERO & ZERO \\
\hline ZERO & ZERO & ZERO & ZERO \\
\hline ZERO & ZERO & ZERO & ZERO \\
\hline ZERO & ZERO & ZERO & ZERO \\
\hline ZERO & ZERO & ZERO & ZERO \\
\hline ZERO & ZERO & ZERO & ZERO \\
\hline ZERO & ZERO & ZERO & ZERO \\
\hline ZERO & ZERO & ZERO & ZERO \\
\hline ZERO & ZERO & ZERO & ZERO \\
\hline ZERO & ZERO & ZERO & ZERO \\
\hline ZERO & ZERO & ZERO & ZERO \\
\hline ZERO & ZERO & ZERO & ZERO \\
\hline ZERO & ZERO & ZERO & ZERO \\
\hline ZERO & ZERO & ZERO & ZERO \\
\hline ZERO & ZERO & ZERO & ZERO \\
\hline ZERO & ZERO & ZERO & ZERO \\
\hline ZERO & ZERO & ZERO. & ZERO \\
\hline ZERO & ZERO & ZERO & ZERO \\
\hline ZERO & ZERO & ZERO & ZERO \\
\hline ZERO & ZERO & ZERO & ZERO \\
\hline ZERO & ZERO & ZERO & ZERO \\
\hline ZERO & ZERO & ZERO & ZERO \\
\hline ZERO & ZERO & ZERO & ZERO \\
\hline ZERO & ZERO & ZеQRO & ZERO \\
\hline ZERO & ZERO & ZERO & ZERO \\
\hline ZERO & ZERO & ZERO & ZERO \\
\hline ZERO & ZERO & ZERO & ZERO \\
\hline ZERO & ZERO & ZERO & ZERO \\
\hline
\end{tabular}

Date: January 1995
100

$\begin{array}{ccc}99 & 100 & 101 \\ \text { HEME Exit OGCT Liquid } & \text { OGCT Sample }\end{array}$ Vapor - to RCT Pump Prime

\begin{tabular}{|c|c|c|}
\hline ZERO & ZERO & ZERO \\
\hline ZERO & ZERO & ZERO \\
\hline ZERO & ZERO & ZERO \\
\hline ZERO & ZERO & ZERO \\
\hline ZERO & ZERO & ZERO \\
\hline ZERO & ZERO & ZERO \\
\hline ZERO & ZERO & ZERO \\
\hline ZERO & ZERO & ZERO \\
\hline ZERO & ZERO & ZERO \\
\hline ZERO & ZERO & ZERO \\
\hline $0.4117 \mathrm{E}-19$ & $0.3650 \mathrm{E}-15$ & ZERO \\
\hline $0.4250 \mathrm{E}-08$ & $0.2438 \mathrm{E}-02$ & ZERO \\
\hline ZERO & ZERO & ZERO \\
\hline ZERO & ZERO & ZERO \\
\hline ZERO & ZERO & ZERO \\
\hline ZERO & ZERO & ZERO \\
\hline ZERO & ZERO & ZERO \\
\hline ZERO & ZERO & ZERO \\
\hline $0.4417 \mathrm{E}-05$ & $0.8833 \mathrm{E}-01$ & ZERO \\
\hline $0.8459 \mathrm{E}-05$ & $0,1692 \mathrm{E}+00$ & ZERO \\
\hline ZERO & ZERO & ZERO \\
\hline $0.5597 \mathrm{E}-09$ & $0.1119 \mathrm{E}-04$ & ZERO \\
\hline $0.7667 E-08$ & $0.1533 \mathrm{E}-03$ & ZERO \\
\hline ZERO & ZERO & ZERO \\
\hline $0.1410 \mathrm{E}+02$ & $0.4394 \mathrm{E}-02$ & ZERO \\
\hline TRACE & ZERO & ZERO \\
\hline ZERO & ZERO & ZERO \\
\hline ZERO & ZERO & ZERO \\
\hline ZERO & ZERO & ZERO \\
\hline $0.1313 \mathrm{E}-05$ & $0.2626 \mathrm{E}-01$ & ZERO \\
\hline ZERO & ZERO & ZERO \\
\hline ZERO & ZERO & ZERO \\
\hline ZERO & ZERO & ZERO \\
\hline ZERO & ZERO & ZERO \\
\hline $0.7272 \mathrm{E}-06$ & $0.1454 \mathrm{E}-01$ & ZERO \\
\hline $0.9936 \mathrm{E}-07$ & $0.1 .987 \mathrm{E}-02$ & ZERO \\
\hline ZERO & ZERO & ZERO \\
\hline ZERO & ZERO & ZERO \\
\hline ZERO & ZERO & ZERO \\
\hline ZERO & ZERO & ZERO \\
\hline $0.5149 \mathrm{E}-10$ & $0.1030 \mathrm{E}-05$ & ZERO \\
\hline $0.2151 \mathrm{E}-06$ & $0.4301 \mathrm{E}-02$ & ZERO \\
\hline $0.1550 \mathrm{E}-07$ & $0.3099 \mathrm{E}-03$ & ZERO \\
\hline ZERO & ZERO & ZERO \\
\hline ZERO & $0.4110 \mathrm{E}-02$ & ZERO \\
\hline ZERO & ZERO & ZERO \\
\hline ZERO & ZERO & ZERO \\
\hline ZERO & ZERO & ZERO \\
\hline
\end{tabular}

Section 5. Page 22 of 37 
Table A-1. HLW Flowsheet Material Balance. (Tank 51/ITP Cycle 1) section 5. Vitrification and Melter off-Gas Treatment

STREAM NUMBERS (CONT'D) $\rightarrow$ STREAM NAME

54. $\mathrm{Cu}(\mathrm{COOH}) 2$

$170 \mathrm{Cu}(\mathrm{NO} 3) 2$

$55 \mathrm{CuO}$

$80 \mathrm{Fe} 2 \mathrm{O} 3$

$101 . \mathrm{FeO}$

102 Group A

$\begin{array}{rl}25 & \mathrm{H} 2\end{array}$

$158 \mathrm{H} 2 \mathrm{C} 2 \mathrm{O} 4$

$93 \mathrm{H} 2 \mathrm{SO}$

$90 \mathrm{H3BO} 3$

$34 \mathrm{HCOOH}$

$43 \mathrm{HCl}$
$85 \mathrm{HF}$

49 HNO3

$36 \mathrm{Hg}$

$1.29 \mathrm{Hg}(\mathrm{C} 6 \mathrm{H} 5)^{2}$

$135 \mathrm{Hg}$ (NO3) 2

144. $\mathrm{Hg} 2 \mathrm{Cl} 2$

$117 \mathrm{Hg} 2 \mathrm{I} 2$

$146 \mathrm{HgCl2}$

$21 \mathrm{HgO}$

97.12

$\begin{array}{rl}183 & \mathrm{~K} 2 \mathrm{CO} \\ 39 & \mathrm{~K} 2 \mathrm{O}\end{array}$

$51 \mathrm{KCOOH}$

$157 \mathrm{KMnO4}$

$161 \mathrm{KNO}$

$150 \mathrm{KOPB}$

$105 \mathrm{La} 203$

$108 \mathrm{Ii} 20$

$65 \mathrm{Mg}(\mathrm{COOH}) 2$

$171 \mathrm{Mg}(\mathrm{NO} 3) 2$

$33 \mathrm{MgO}$

$60 \mathrm{Mn}(\mathrm{COOH}) 2$

$172 \mathrm{Mn}(\mathrm{NO} 3) 2$

$180 \mathrm{Mn} 304$

$56 \mathrm{MnO}$

$14 \mathrm{MnO} 2$

$145 \mathrm{MoO} 2$

81 N2

$74 \mathrm{~N} 2 \mathrm{O}$

$182 \mathrm{NH} 3$

114 NH3OHNO3

$52 \mathrm{NH} 4 \mathrm{COOH}$

173 NH4 NO3

$\begin{array}{rl}68 & \mathrm{NH} 4 \mathrm{OH} \\ 148 & \mathrm{NH} 4 \mathrm{TPB}\end{array}$

WSRC-TR-95-0019 (Revision
95

Water Flush Atomizing HEME Solids

to HEME Air to HEME Accumulation

ZERO

ZERO

ZERO

ZERO

ZERO

ZERO

ZERO

ZERO

ZERO

ZERO

ZERO

ZERO

ZERO

ZERO

ZERO

ZERO

ZERO

ZERO

ZERO

ZERO

ZERO

ZERO

ZERO

ZERO

ZERO

ZERO

ZERO

ZERO

ZERO

ZERO

ZERO

ZERO

ERO

ZERO

ZERO

ZERO

ZERO

ERO

ZERO

ZERO

ZERO

TRO

RO

ZERO

ZERO

\begin{tabular}{|c|c|}
\hline ZERO & ZERO \\
\hline ZERO & ZERO \\
\hline ZERO & ZERO \\
\hline ZERO & ZERO \\
\hline ZERO & ZERO \\
\hline ZERO & ZERO \\
\hline ZERO & ZERO \\
\hline ZERO & ZERO \\
\hline ZERO & ZERO \\
\hline ZERO & ZERO \\
\hline ZERO & ZERO \\
\hline ZERO & ZERO \\
\hline ZERO & ZERO \\
\hline ZERO & ZERO \\
\hline ZERO & ZERO \\
\hline ZERO & ZERO \\
\hline ZERO & ZERO \\
\hline ZERO & ZERO \\
\hline ZERO & ZERO \\
\hline ZERO & 2ERO \\
\hline ZERO & ZERO \\
\hline ZERO & ZERO \\
\hline ZERO & ZERO \\
\hline ZERO & ZERO \\
\hline ZERO & ZERO \\
\hline ZERO & ZERO \\
\hline ZERO & ZERO \\
\hline ZERO & ZERO \\
\hline ZERO & ZERO \\
\hline ZERO & ZERO \\
\hline ZERO & ZERO \\
\hline ZERO & ZERO \\
\hline ZERO & ZERO \\
\hline ZERO & ZERO \\
\hline ZERO & ZERO \\
\hline ZERO & ZERO \\
\hline ZERO & ZERO \\
\hline ZERO & ZERO \\
\hline ZERO & ZERO \\
\hline ZERO & ZERO \\
\hline ZERO & ZERO \\
\hline $1 \mathrm{E}+00$ & ZERO \\
\hline ZERO & ZERO \\
\hline ZERO & ZERO \\
\hline ZERO & ZERO \\
\hline ZERO & ZERO \\
\hline ZERO & ZERO \\
\hline ZERO & ZERO \\
\hline ZERO & ZERO \\
\hline
\end{tabular}

98

ME Exit

Iiquid

ZERO

ZERO

ZERO

ZERO

ZERO

ZERO

ZERO

ZERO

ZERO

ZERO

ZERO

ZERO

ZERO

ZERO

ZERO

ZERO

ZERO

ZERO

ZERO

ZERO

ZERO

ZERO

ZERO

ZERO

ZERO

ZERO

ZERO

ZERO

ZERO

ZERO

ZERO

ZERO

ZERO

ZERO

ZERO

ZERO

ZERO

ZERO

ZERO

ZERO

ZERO

ZERO

- ZERO

Date: January 1995
99

100

101

HEME Exit OGCT Liquid OGCT Sample Vapor to RCT Pump Prime ZERO

$0.4732 \mathrm{E}-06$

$0.1269 \mathrm{E}-04$

$.1269 \mathrm{E}-05$

$.7558 \mathrm{E}-10$

ZERO

ZERO

ZERO

ZERO
ZERO

ZERO

$0.1369 \mathrm{E}-02$

$0.6776 \mathrm{E}-02$

$6 \mathrm{E}-02$
$1 \mathrm{E}-02$

ZERO

$0.7168 \mathrm{E}-06$

$0.5917 \mathrm{E}-11$

$3 \mathrm{E}-16$

$0.4632 \mathrm{E}-09$

ZERO

$0.2859 \mathrm{E}-05$

ZERO

ZERO

ZERO

ZERO

ZERO

$0.5540 E-05$

ZERO

. $346 E-05$

ZERO

ZERO

ZERO

$0.1325 \mathrm{E}-05$

ZERO

$0.5377 \mathrm{E}-09$

$0.1395 \mathrm{E}+04$

ZERO

ZERO

ZERO

ZERO

ZERO

ZERO

ZERO
$0.9462 \mathrm{E}-02$

ZERO
ZERO

ZERO

$0.2538 \mathrm{E}-01$ ZERO

$0.1511 \mathrm{E}-05$

ZERO ZERO

$0.4677 \mathrm{E}-01$ ZERO

ZERO ZERO

ZERO ZERO

$0.6081 \mathrm{E}-03$ ZERO

$\begin{array}{ll}0.6081 E-03 & \text { ZERO } \\ 0.1053 E+00 & \text { ZERO }\end{array}$

$0.1299 E+00$ ZERO ZERO $\quad$ ZERO

$0.1433 E-01$ ZERO

$0.1183 E-06$ ZERO

$0.5500 E-13$ ZERO

ZERO ZERO

ZERO

ZERO
ZERO
ZERO

ZERO ZERO

ZERO ZERO

ZERO ZERO

ZERO ZERO

ZERO
ZERO
$0.1108 E+00$

ZERO

ZERO ZERO

$0.4691 \mathrm{E}-01 \quad$ ZHRO

ZERO ZERO

0.2650 ZERO ZERO

ZERO
$0.1075 \mathrm{E}-04$

$\begin{array}{ll}0.1075 E-04 & \text { ZERO } \\ 0.1912 E-01 & \text { ZERO }\end{array}$

ZERO ZERO

ZERO ZERO

ZERO ZERO

ZERO

ZERO ZERO

ZERO ZERO

Section 5 . Page 23 of 37 


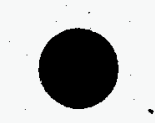

Table A-1. HLW Flowsheet Material Balance (Tank 51/ITP Cycle 1) section 5. Vitrification and Melter off-Gas Treatment

STREAM NUMBERS (CONT'D) $\rightarrow$ STREAM NAME

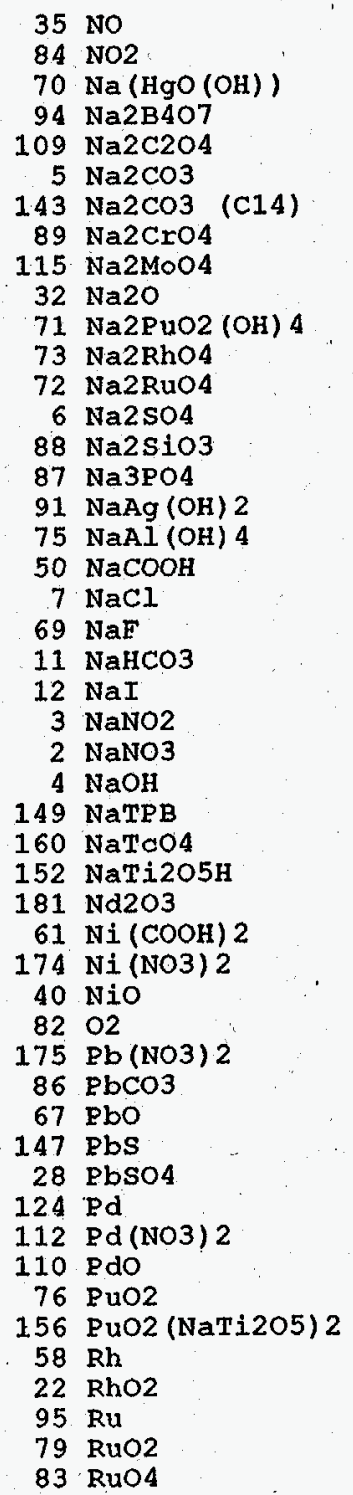

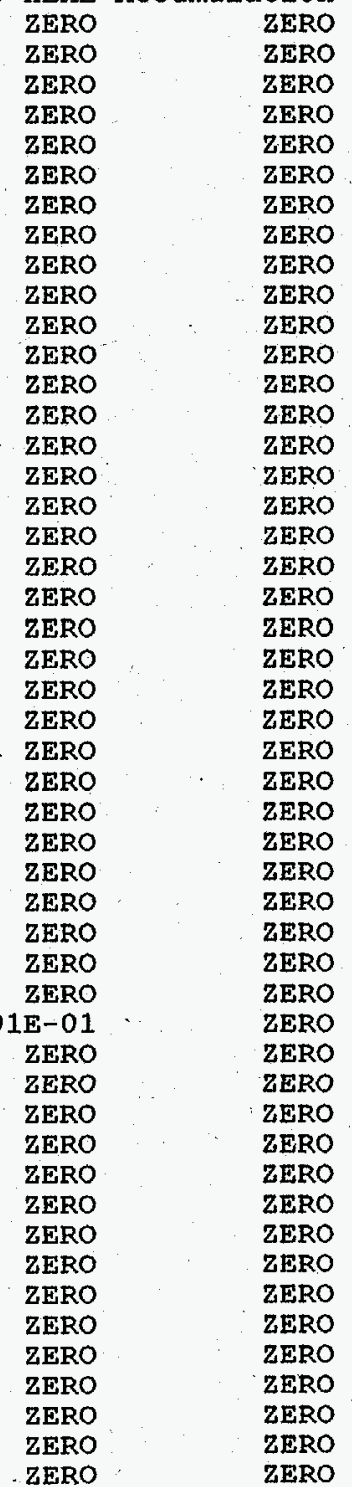

HEME

E Exit

Liquid
zERO

ZERO

ZERO

ZERO

ZERO

ZERO

ZERO

ZERO

ZERO

ZERO

ZERO

ZERO

ZERO

ZERO,

ZERO

ZERO

ZERO

ZERO

ZERO

ZERO

ZERO

ZERO

ZERO

ZERO

ZERO

ZERO

ZERO

ZERO

ZERO

ZERO

ZERO

ZERO

ZERO

ZERO

ZERO

ZERO

ZERO

ZERO

ZERO

ZERO

ZERO

ZERO

ZERO

ZERO

Date: January 1995

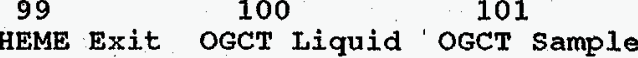

99
HEME Exit OGCT Liquid OGCT Sample Vapor $0.1395 \mathrm{E}+01$ to RCT Pump Prime $0.3839 \mathrm{E}-04$ $0.7574 \mathrm{E}-04$ $0.1227 \mathrm{E}+00$ ZERO ZERO ZERO$$
\text { ZERO }
$$$$
\text { ZERO }
$$

$0.1015 \mathrm{E}-04$ ZERO ZERO ZERO

$0.2816 \mathrm{E}-06$

ZERO

$1289 \mathrm{E}-08$ ZERO
ZERO ZERO
0.2489 E-O8

$0.1685 \mathrm{E}-07$ ZERO ZERO

ZERO

ZERO

ZERO ZERO ZERO ZERO ZERO

$0.1271 \mathrm{E}-06$

$0.4211 \mathrm{E}+03$ ZERO

$0.2451 \mathrm{E}-08$ ZERO

$0.6260 \mathrm{E}-07$ ZERO ZERO

$0.4978 \mathrm{E}-08$ 8E-08
ZERO ZERO ZERO ZERO ZERO
ZERO ZERO ZERO ZERO ZERO ZERO ZERO ZERO ZERO

ZERO BE-04 ZERO
ZERO ZERO

$0.4978 \mathrm{E}-04$

$0.1277 \mathrm{E}-01$

ZERO
$0.5410 \mathrm{E}-05$

$0.3562 \mathrm{E}-02$

$0.2213 \mathrm{E}-02$ ZERO ZERO ZERO ZERO ZERO ZERO

$0.2542 \mathrm{E}-02$ ZERO

$0.4902 \mathrm{E}-04$ ZERO

$0.1252 \mathrm{E}-02$ ZERO ZERO

$0.9956 \mathrm{E}-04$ ZERO ZERO ZERO ZERO. ZERO

$$
\begin{aligned}
& \text { ZERO } \\
& \text { ZERO } \\
& \text { ZERO } \\
& \text { ZERO } \\
& \text { ZERO } \\
& \text { ZERO } \\
& \text { ZERO } \\
& \text { ZERO } \\
& \text { ZERO } \\
& \text { ZERO } \\
& \text { ZERO } \\
& \text { ZERO } \\
& \text { ZERO } \\
& \text { ZERO } \\
& \text { ZERO } \\
& \text { ZERO } \\
& \text { ZERO } \\
& \text { ZERO } \\
& \text { ZERO } \\
& \text { ZERO } \\
& \text { ZERO } \\
& \text { ZERO } \\
& \text { ZERO } \\
& \text { ZERO } \\
& \text { ZERO } \\
& \text { ZERO } \\
& \text { ZERO } \\
& \text { ZERO } \\
& \text { ZERO } \\
& \text { ZERO } \\
& \text { ZERO } \\
& \text { ZERO } \\
& \text { ZERO } \\
& \text { ZERO } \\
& \text { ZERO } \\
& \text { ZERO } \\
& \text { ZERO } \\
& \text { ZERO } \\
& \text { ZERO } \\
& \text { ZERO } \\
& \text { ZERO } \\
& \text { ZERO } \\
& \text { ZERO } \\
& \text { ZERO } \\
& \text { ZERO } \\
& \text { ZERO } \\
& \text { ZERO } \\
& \text { ZERO } \\
& \text { ZERO }
\end{aligned}
$$
$0.3839 \mathrm{E}-04$ $0.2030 \mathrm{E}+00$

$0.1104 \mathrm{E}-01$ 
Table A-1. HLW Flowsheet Material Balance (Tank 51/ITP Cycle 1) seation 5. Vitrification and Melter off-Gas Treatment

STREAM NUMBERS (CONT'D) $\rightarrow$ STREAM NAME

$38 \mathrm{SO2}$

137 Semi Vol Cs2O

136 Semi Vol CsC1

126 Semi Vol Group A

140 Semi Vo1 Na2B4O7

138 Semi Vol NaCl

139 Semi Vol NaF

23 Semi Vol RuO2

$111 \mathrm{SiO2}$

$48 \mathrm{Sr}(\mathrm{COOH}) 2$

$176 \mathrm{Sr}$ (NO3) 2

153 Sr (NaTi2O5) 2

$127 \mathrm{Sr}(\mathrm{OH}) 2$

$30 \mathrm{5xCO}$

46 Sro

159 TCO2

92 ThO2

119 Tritium

170308

$62 \mathrm{UO2}$ ( $\mathrm{COOH}) 2$

177 UO2 (NO3) 2

$16 \mathrm{UO} 2(\mathrm{OH}) 2$

178 Y (NO3) 3

128 Y (OH) 3

31 Y $2\left(\mathrm{CO}_{3}\right) 3$

100 Zeolite

$44 \mathrm{Zn}(\mathrm{COOH}) 2$
$179 \mathrm{Zn}(\mathrm{NO} 3) 2$

104 Zro2

13 hydrate $\mathrm{H} 2 \mathrm{O}$

1 water

TOTAL FLOW, LB/HR

TEMPERATURE, DEG $C$

PRESSURE, ATM

PRESSURE， PSIG

PRESSURE， MM HG

ENTHAIPY, PCU/HR

VAPOR FLOW, CFM

LIQUID ELOW, GPM

DENSTTY, LBS/FT3

PHASE

95

Water Flush Atomizing HEME Solids

to HEME Air to HEME Accumulation

$\begin{array}{llrr}\text { ZERO } & \text { ZERO } & \text { ZERO } & \text { Liquid } \\ \text { ZERO } & \text { ZERO } & \text { ZERO } & \text { ZERO } \\ \text { ZERO } & \text { ZERO } & \text { ZERO } & \text { ZERO } \\ \text { ZERO } & \text { ZERO } & \text { ZERO } & \text { ZERO } \\ \text { ZERO } & \text { ZERO }\end{array}$

ZERO ZERO ZERO $\quad$ ZERO

ZERO $\quad$ ZERO

ZERO

ZERO ZERO ZERO

ZERO Z ZERO Z ZERO

ZERO ZERO ZERO

ZERO ZERO ZERO

ZERO ZERO ZERO

ZERO ZERO ZERO

ZERO

ZERO ZERO $\quad$ ZERO

$\begin{array}{llr}\text { ZERO } & \text { ZERO } \\ \text { ZERO } & \text { ZERO }\end{array}$

ZERO ZERO ZERO ZERO

ZERO ZERO ZERO

ZERO ZERO $\because$ ZERO

ZERO ZERO ZERO

$\begin{array}{lll}\text { ZERO } & \text { ZERO } & \text { ZERO } \\ \text { ZERO } & \text { ZERO } & \text { ZERO }\end{array}$

ZERO ZERO $\quad$ ZERO

ZERO ZERO ZERO

ZERO ZERO $\quad$ ZERO

ZERO ZERO ZERO

ZERO ZERO ZERO

ZERO ZERO

ZERO $\cdots$ ZERO ZERO

ZERO ZERO ZERO

ZERO ZERO ZERO

ZERO ZERO ZERO

ZERO $\quad$ ZERO . ZERO

ZERO $\quad$ ZERO $\quad$ ZERO

ZERO - ZERO

$0.2695 \mathrm{E}+01$

$0.2695 \mathrm{E}+01$

ZERO

$0.3831 \mathrm{E}-02$

ZERO

ZERO

ZERO

ZERO

$0.4000 \mathrm{E}+02$

$0.3180 \mathrm{E}+01$

$0.3500 \mathrm{E}+02$

$0.3720 \mathrm{E}+01$

$0.1000 \mathrm{E}+02$

$0.9165 \mathrm{E}+00$

$0.6965 \mathrm{E}+03$

$0.1080 \mathrm{E}+03$

$0.4863 \mathrm{E}+01$

ZERO

$0.5383 \mathrm{E}-02$

$0.6243 \mathrm{E}+02$

$0.1472 \mathrm{E}-01$

ZERO

SOLID
ZERO

ZERO

ZERO

ZERO

ZERO

ZERO

ZERO

ZERO

ZERO

ZERO

ZERO

ZERO

ZERO

ZERO

ZERO

ZERO

ZERO

ZERO

ZERO

ZERO

ZERO

ZERO

ZERO

ZERO

ZERO

ZERO

ZERO

ZERO

ZERO

$0.1847 \mathrm{E}+01$

$0.1000 \mathrm{E}+02$

$0.9165 \mathrm{E}+00$

$0.6965 \mathrm{E}+03$

$0.1858 \mathrm{E}+02$

$0.3688 \mathrm{E}-02$

$0.6243 \mathrm{E}+02$

LIQUID
$0.1847 \mathrm{E}+01$
99

${ }_{\text {OGCT Liquid OGCT }}^{100}$

Vapor OGCT Liquid OGCT Sample

ZERO ZERO Pump Prime

ZERO ZERO $\quad$ ZERO

\begin{aligned} ZERO ZERO ZERO & ZE \\ \hline & ZERO \end{aligned}

.8858E-07

$0.6143 E-04$

$.2709 \mathrm{E}-08$

E-04

ZERO

ZERO

ZERO

ZERO

$0.7674 \mathrm{E}-08$

$0.2588 \mathrm{E}-08$

$0.1486 \mathrm{E}-07$

$0.4168 \mathrm{E}-06$

$0.1137 \mathrm{E}-11$

$0.1042 \mathrm{E}-0$

ZERO

ZERO

ZERO

ZERO

ZERO

ZRRO

$0.2115 \mathrm{E}-08$

ZERO

ZERO

$0.1580 \mathrm{E}-06$

ZERO
ZER

$0.1418 \mathrm{E}+02$

$0.1849 \mathrm{E}+04$

$0.1000 \mathrm{E}+02$

$-0.1227 \mathrm{E}+01$
$0.6965 \mathrm{E}+03$

$0.1930 \mathrm{E}+05$

$0.4341 \mathrm{E}+03$

$0.7097 \mathrm{E}-01$

VAPOR

ZERO

ZERO

ZERO

ZERO

ZERO
$0.1229 \mathrm{E}+01$

ZERO ZERO

ZERO ZERO

ZERO ZERO

ZERO ZERO

$0.3569 \mathrm{E}-04 \quad$ ZERO

$0.2972 \mathrm{E}-03 \quad$ ZERO

$0.8335 \mathrm{E}-02$ ZERO

$\begin{array}{ll}0.1413 \mathrm{E}-09 & \text { ZERO } \\ 0.2084 \mathrm{E}-01 & \text { ZERO }\end{array}$

ZERO ZERO

ZERO ZERO

ZERO ZERO

ZERO ZERO

ZERO

ZERO $\cdots \quad$ ZERO

E-04 ZERO

ZERO ZERO

$0.31590-02 \quad$ ZERO

$\begin{aligned} \text { ZERO } & \text { ZERO } \\ \text { ZERO } & \text { ZERO }\end{aligned}$

$0.1592 \mathrm{E}+04 \quad 0.6940 \mathrm{E}+01$

$0.1594 \mathrm{E}+04 \quad 0.6940 \mathrm{E}+01$

$0.9650 \mathrm{E}+00 \quad 0.1000 \mathrm{E}+01$

$0.7334 \mathrm{E}+03$

$0.1741 \mathrm{E}+03$

$0.3181 E+01$

$0.6249 \mathrm{E}+02$

IIQUID

$0.1386 \mathrm{E}-01$

$0.6243 \mathrm{E}+02$

IIQUID 


\section{0}

Table A-1. HLW Flowsheet Material Balance (Tank 51/ITP Cycle 1) section 5. Vitrification and Melter off-Gas Treatment

STREAM NUMBERS $\rightarrow$

STREAM NAME

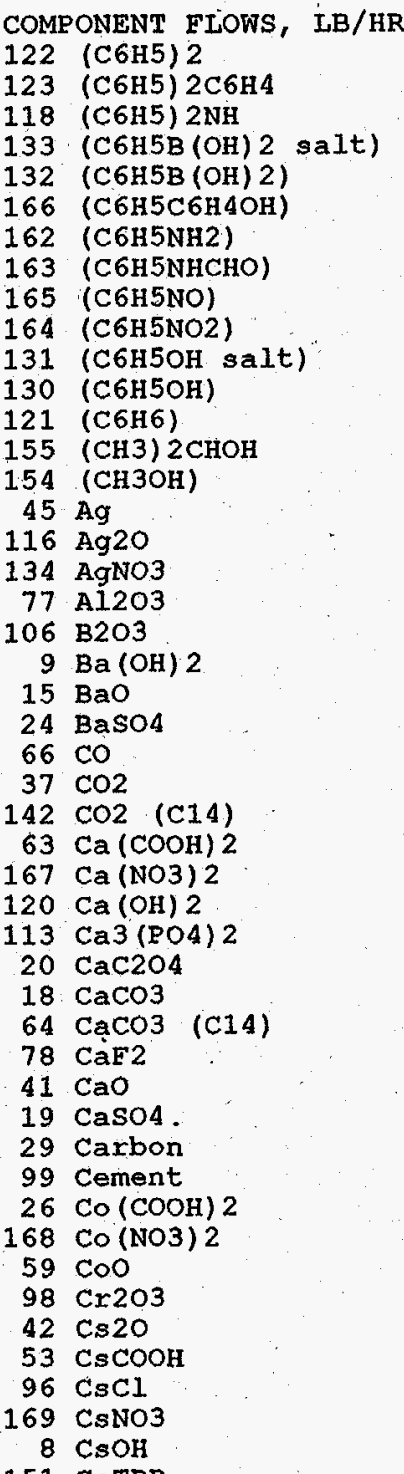

102

OGCT Trnsfr

Pump Prime

ZERO

ZERO

ZERO

ZERO

ZERO

ZERO

ZERO

ZERO

ZERO

ZERO

ZERO

ZERO

ZERO

ZERO

ZERO

ZERO

ZERO

ZERO

ZERO

ZERO

ZERO

ZERO

ZERO

ZERO

ZERO

ZERO

ZERO

ZERO

ZERO

ZERO

ZERO

ZERO

ZERO

ZERO

ZERO

ZERO

ZERO

ZERO

ZERO

ZERO

ZERO
103

OGCT Air 104

104 Add' 110

to OGCT

1m 111

oGCT Gas Feed Aacumulation

112

113

$\begin{array}{lll}\text { ZERO } & \text { ZERO } & \text { ZERO } \\ \text { ZERO } & \text { ZERO } & \text { ZERO } \\ \text { ZERO } & \text { ZERO } & \text { ZERO } \\ \text { ZERO } & \text { ZERO } & \text { ZERO } \\ \text { ZERO } & \text { ZERO } & \text { ZERO } \\ \text { ZERO } & \text { ZERO } & \text { ZERO } \\ \text { ZERO } & \text { ZERO } & \text { ZERO } \\ \text { ZERO } & \text { ZERO } & \text { ZERO } \\ \text { ZERO } & \text { ZERO } & \text { ZERO }\end{array}$

ZERO

ZERO $0.4250 \mathrm{E}-08$

ZERO

ZERO

ZERO

ZERO

ZERO

ZERO

ZERO

ZERO

ZERO

ZERO

ZERO

ZERO

ZERO

ZERO

ZERO

ZERO

ZERO

ZERO

ZERO

ZERO

ZERO

ZERO

ZERO

ZERO

ZERO

ZERO

ZERO

ZERO

ZERO

ZERO

ZERO

ZERO

ZERO
$0.4117 \mathrm{E}-19$

ZERO

ZERO

ZERO

ZERO

ZERO

$0.4417 \mathrm{E}-0.5$

$0.8459 \mathrm{E}-05$

ZERO

$0.7667 \mathrm{E}-08$

ZERO

$0.1410 \mathrm{E}+02$

TRACE

ZERO

ZERO

$0.1313 \mathrm{E}-05$

ZERO

ZERO
ZERO

ZERO

$0.7272 E-06$

$9936 \mathrm{E}-07$

ZERO

ZERO

ZERO

$0.5149 \mathrm{E}-10$

$0.2151 \mathrm{E}-06$

$0.1550 \mathrm{E}-07$

ZERO
ZERO

ZERO

ZERO

ZERO
ZERO
$0.4109 \mathrm{E}-19$

ZERO
ZERO

ZERO

ZERO

ZERO
ZERO
ZERO

RO

ZERO

ZERO

ZERO

ZERO

ZERO

ZERO

$0.4408 \mathrm{E}-05$

$0.8442 \mathrm{E}-05$

ZERO

$0.5586 \mathrm{E}-09$

ZERO

ZERO

ZERO

ZERO

ZERO

$0.1310 \mathrm{E}-05$

ZERO

ZERO

ZERO

$0.7258 \mathrm{E}-06$

$0.9916 \mathrm{E}-07$

ZERO

ZERO

ZERO

$0.5139 \mathrm{E}-10$

$0.2147 \mathrm{E}-06$

0.1547 E-0.7

ZERO

ZERO

ZERO

ZERO

ZERO

ZERO ZERO

ZERO ZERO

ZERO

ZERO

ZERO

ZERO

ZERO ZERO

ZERO
ZERO

$0.42507-08$

ZERO

ZERO ZERO

ZERO ZERO

ZERO ZERO

ZERO ZERO

ZERO
$0.8834 \mathrm{E}-08 \quad 0.8658 \mathrm{E}-08$

$0.1692 \mathrm{E}-07 \quad 0.1658 \mathrm{E}-07$

ZERO

$0.1119 \mathrm{E}-11 \quad 0.1097 \mathrm{E}-11$

ZERO

$0.1410 \mathrm{E}+02$

TRACE

ZERO $\quad$ ZERO

ZERO ZERO

Z

$0.2573 \mathrm{E}-08$

ZERO

ZERO

ZERO

$0.1454 E-08$

ZERO

ZERO

ZERO

ZERO

$0.4302 \mathrm{E}-09$

$0.3100 \mathrm{E}-10$

ZERO

ZERO

ZERO

ZERO

ZERO

Section 5. Page 26 of 37

Date: January 1995 
Table A-1. HLW Flowsheet Material Balance (Tank 51/ITP Cycle 1) section 5 . Vitrification and Melter off-Gas Treatment

STREAM NUMBERS (CONT'D) $\rightarrow$ STREAM NAME

$54 \mathrm{Cu}(\mathrm{COOH}) 2$

$170 \mathrm{Cu}(\mathrm{NO} 3)$

$55 \mathrm{CuO}$

80 Fe2O3

$101 \mathrm{FeO}$

102 Group A

103 Group B

25 H2

158 H2C2O4

93 H2 $\mathrm{SO} 4$

$90 \mathrm{H3BO}$

$34 . \mathrm{HCl}$

$85 \mathrm{HF}$

49 HNO3.

$36 \mathrm{Hg}$

$129 \mathrm{Hg}(\mathrm{C} 6 \mathrm{H} 5)^{2}$

$135 \mathrm{Hg}$ (NO3) 2

$144 \mathrm{Hg} 2 \mathrm{Cl2}$

$117 \mathrm{Hg} 2 \mathrm{I} 2$

$146 \mathrm{HgCl} 2$

$21 \mathrm{HgO}$

$97 \mathrm{I} 2$

183. $\mathrm{K} 2 \mathrm{CO} 3$

39. $\mathrm{K} 2 \mathrm{O}$

$51 \mathrm{KCOOH}$

$61 \mathrm{KNO}$

$10 \mathrm{KOH}$

$105 \mathrm{La} 203$

108 Li20

$65 \mathrm{Mg}(\mathrm{COOH}) 2$

$71 \mathrm{Mg}$ (NO3) 2

$33 \mathrm{MgO}$

$60 \mathrm{Mn}(\mathrm{COOH}) 2$

$172 \mathrm{Mn}(\mathrm{NO} 3) 2$

$180 \mathrm{Mn} 304$

56 Mno

$14 \mathrm{MnO} 2$

$145 \mathrm{MoO} 2$

$81 \mathrm{~N} 2$

$\begin{array}{rl}74 & \mathrm{~N} 2 \mathrm{O} \\ 182 & \mathrm{NH} 3\end{array}$

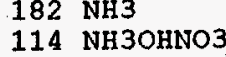

$52 \mathrm{NH} 4 \mathrm{COOH}$

173 NH4NO3

$68 \mathrm{NH} 4 \mathrm{OH}$

WSRC-TR-95-0019 (Revision
103

Inleakag

ZERO

ZERO

ZERO

ZERO

ZERO

ZERO

ZERO

ZERO

ZERO

ZERO

ZERO

ZERO

ZERO

ZERO

ZERO

ZERO

ZERO

ZERO

ZERO

ZERO

ZERO

ZERO

ZERO

ZERO

ZERO

ZERO

ZERO

ZERO

ZERO

ZERO

ZERO

ZERO

ZERO

ZERO

ZERO

ZERO

ZERO

ZERO

ZERO

ZERO

ZERO

ZERO

ZERO

ZERO

ZERO

ZERO

ZERO

$0.7936 \mathrm{E}+02$

ZERO

ZERO

ZERO

ZERO

ZERO

ZERO $\begin{array}{ll}103 & 104 \\ \text { OGCT Air } & \text { NaOH }\end{array}$

OH Add' $n$.

111

112

Gas Feed Accumulation
ZERO ZERO

ZERO I ZERO ZERO

ZERO $\quad 0.4732 \mathrm{E}-06 \quad 0.4722 \mathrm{E}-06$

ZERO $0.1269 \mathrm{E}-04 \quad 0.1267 \mathrm{E}-04$

ZERO $0.1269 \mathrm{E}-05 \quad 0.1266 \mathrm{E}-05$

ZERO $0.1683 \mathrm{E}-08 \quad 0.1680 \mathrm{E}-08$

ZERO $0.7558 \mathrm{E}-10^{\prime} \quad 0.7543 \mathrm{E}-10$

ZRPO

ZERO

ZERO

ZERO

ZERO

ZERO

ZERO

ZERO

ZERO

ZERO

ZERO

ZERO

ZERO

ZERO

ZERO

ZERO

ZERO

ZERO

ZERO

ZERO

ZERO

ZERO

ZTRO

IERO

ZERO

ZERO

ZERO

ZERO

ZERO

ZERO

ZERO

ZERO

ZERO

ZERO

TRO

ZERO

ZERO

ZERO

ZERO

Date: January 1995

$0.6776 \mathrm{E}-02$

$0.8511 \mathrm{E}-02$

ZERO

$0.7168 \mathrm{E}-06$

$0.5917 \mathrm{E}-11$

$0.5633 \mathrm{E}-16$

ZERO

$0.4632 \mathrm{E}-09$

ZERO
$9 \mathrm{E}-05$

ZERO

ZERO

ZERO

ZERO

TERO

$0.5540 \mathrm{E}-05$

ZERO

$0.2346 \mathrm{E}-05$

ZERO

ZERO

ZERO
$0.1325 \mathrm{E}-05$

ZERO

$0.1395 \mathrm{E}+04$

ZERO

ZERO

ZERO

ZERO

ZERO

ZERO

53E-06

$0.5621 \mathrm{E}-16$

$1 \mathrm{E}-16$
ZERO

ZERO

$0.2854 \mathrm{E}-05$

ZERO

ZERO

ZERO

ZERO

ZERO

$0.5529 \mathrm{E}-0.5$

ZERO

$0.2341 \mathrm{E}-05$

ZERO

ZERO

ZERO
$0.1323 E-05$

ZERO

$0.5366 \mathrm{E}-09$

ZERO

ZERO

ZERO

ZERO

ZERO

ZERO

ZERO

113

rim HEPA\#2 Prim HEPA\#2

Gas Feed Accumultion
ZERO ZERO

$0.9463 \mathrm{E}-09$

$0.9274 \mathrm{E}-09$

$0.2538 \mathrm{E}-08 \quad 0.2488 \mathrm{E}-07$

$0.3366 \mathrm{E}-11$ 0.3299E-11

$0.1512 \mathrm{E}-12$ O $0.1481 \mathrm{E}-12$

$\mathrm{E}-12$

ZERO

ZERO

ZERO

ZERO

ZERO

ZERO

ZERO

ZERO

ZERO

$0.1369 \mathrm{E}-02$

$0.8511 \mathrm{E}-02$

ZERO

$0.1434 \mathrm{E}-08$

$0.1183 \mathrm{E}-13$

$0.1127 \mathrm{E}-18$

ZERO

ZERO

$0.5718 \mathrm{E}-08$

ZERO

ZERO

ZERO

ZERO

ZERO

$0.1108 \mathrm{E}-07$

ZERO

$0.4691 \mathrm{E}-08$

ZERO

ZERO

$0.2651 \mathrm{ZERO}-08$

ZERO

$0.1075 \mathrm{E}-11$

ZERO

ZERO

ZERO

ZERO

ZERO

ZERO

$0.1405 \mathrm{E}-08$

$0.1160 \mathrm{E}-13$

$0.1104 \mathrm{E}-18$

ZERO

ZERO

$0.5604 \mathrm{E}-08$

ZERO

ZERO

ZERO

ZERO

ZERO

ZERO

ZERO

ZERO

ZERO
ZERO

ZERO

ZERO
$0.2598 \mathrm{E}-08$

ZERO

ZERO

ZERO

ZERO

ZERO

ZERO

ZERO

ZERO

Section 5. Page 27 of 37 
Table A-1. HLW Flowsheet Material Balance (Tank 51/ITP Cycle 1) Section 5. Vitrification and Melter off-Gas Treatment

STREAM NUMB

$\begin{aligned} & 35 \text { NO } \\ & 84 \text { NO2 } \\ & 70 \text { Na(HOO(OH)) } \\ & 94 \text { Na2B4O7 } \\ & 109 \text { Na2C2O4 } \\ & 5 \text { Na2CO3 } \\ & 143 \text { Na2CO3 (C14) } \\ & 89 \text { Na2CrO4 } \\ & 115 \text { Na2MOO4 } \\ & 32 \text { Na2O } \\ & 71 \text { Na2PuO2 (OH) } 4 \\ & 73 \text { Na2RhO4 } \\ & 72 \text { Na2RuO4 } \\ & 6 \text { Na2SO4 } \\ & 88 \text { Na2SiO3 } \\ & 87 \text { Na3PO4 } \\ & 91 \text { NaAg (OH) } 2 \\ & 75 \text { NaA1 (OH) } 4 \\ & 50 \text { NaCOOH } \\ & 7 \text { NaC1 } \\ & 69 \text { NaF } \\ & 11 \text { NaHCO3 } \\ & 12 \text { NaI } \\ & 3 \text { NaNO2 } \\ & 2 \text { NaNO3 } \\ & 4 \text { NaOH } \\ & 149 \text { NaTPB } \\ & 160 \text { NaTCO4 } \\ & 152 \text { NaTi2O5H } \\ & 181 \text { Nd2O3 } \\ & 61 \text { Ni (COOH) } 2 \\ & 174 \text { Ni(NO3) } 2 \\ & 40 \text { NiO } \\ & 82 \text { O2 } \\ & 175 \text { Pb (NO3) } 2 \\ & 86 \text { PbCO3 } \\ & 67 \text { PbO } \\ & 147 \text { PbS } \\ & 28 \text { PbSO4 } \\ & 124 \text { Pd } \\ & 112 \text { Pd (NO3) } 2 \\ & 110 \text { PdO } \\ & 76 \text { PuO2 } \\ & 156 \text { PuO2 (NaTi2O5) } 2 \\ & 58 \text { Rh } \\ & 22 \text { RhO2 } \\ & 95 \text { Ru } \\ & 79 \text { RuO2 } \\ & 83 \text { RuO4 } \\ &\end{aligned}$

WSRC-TR-95-0019 (Revision
103

Inleakage

110

111

to OGCT

110 HEPA\#1 Prim

OGCT Trnsfr

ZERO

ZERO

ZERO

ZERO

ZERO

ZERO

ZERO

ZERO

ZERO

ZERO

ZERO

ZERO

ZERO

ZERO

ZERO

ZERO

ZERO

ZERO

ZERO

ZERO

ZERO

ZERO

ZERO

ZERO

ZERO

ZERO

ZERO

ZERO

ZERO

ZERO

ZERO

ZERO

ZERO

ZERO

ZERO

ZERO

ZERO

ZERO

ZERO

ZERO

\begin{tabular}{|c|c|}
\hline ZERO & $0.1395 \mathrm{E}+01$ \\
\hline ZERO & $0.2751 \mathrm{E}+01$ \\
\hline ZERO & ZERO \\
\hline ZERO & ZERO \\
\hline ZERO & ZERC \\
\hline ZERO & ZERC \\
\hline ZERO & ZERO \\
\hline ZERO & ZERC \\
\hline ZERO & ZERC \\
\hline ZERO & $0.1015 \mathrm{E}-04$ \\
\hline ZERO & ZERC \\
\hline ZERO & ZERO \\
\hline ZERO & ZERC \\
\hline ZERO & $0.2816 \mathrm{E}-06$ \\
\hline ZERO & ZERO \\
\hline ZERO & $0.1289 \mathrm{E}-08$ \\
\hline ZERO & ZERC \\
\hline ZERO & ZERC \\
\hline ZERO & ZERC \\
\hline ZERO & $0.2489 \mathrm{E}-08$ \\
\hline ZERO & $0.1685 \mathrm{E}-07$ \\
\hline ZERO & ZERC \\
\hline ZERO & ZERC \\
\hline ZERO & ZERC \\
\hline ZERO & ZERC \\
\hline ZERO & ZERC \\
\hline ZERO & ZERC \\
\hline ZERO & ZERC \\
\hline ZERO & ZERO \\
\hline ZERO & ZERO \\
\hline ZERO & ZERC \\
\hline ZERO & ZERC \\
\hline ZERO & $0.1271 \mathrm{E}-06$ \\
\hline ZERO & $0.4211 \mathrm{E}+03$ \\
\hline ZERO & ZERO \\
\hline ZERO & ZERO \\
\hline ZERO & $0.2451 E-08$ \\
\hline ZERO & ZERC \\
\hline ZERO & $0.6260 \mathrm{E}-07$ \\
\hline ZERO & ZERO \\
\hline ZERO & ZERO \\
\hline ZERO & ZERO \\
\hline ZERO & $0.4978 \mathrm{E}-08$ \\
\hline ZERO & ZERC \\
\hline ZERO & ZERC \\
\hline ZERO & ZERC \\
\hline ZERO & ZERC \\
\hline ZERO & ZERC \\
\hline ZERO & ZERO \\
\hline
\end{tabular}

ZERO
ZERO

ZERO

ZERO

ZERO

ZERO

ZERO

ZERO

ZERO

ZERO

ZERO

ZERO

ZERO

ZERO

ZERO

ZERO

ZERO

ZERO

ERO

ZERO

ZERO

ZERO

ZERO

ZERO

ZERO

ZERO

ZERO

ZERO

ZERO

ERO

$0.2397 \mathrm{E}+02$

ZERO

ZERO

ZERO

ZERO

ZERO

ZERO

ZERO

ZERO

ZERO

ZERO

ZERO

ZERO

ZERO

ZERO

Date: January 1995
112

113 rim HEPA\#2 Prim HEPA\#2

Gas Feed Accumultion

$395 \mathrm{E}+01$

ZERO

ZERO

ZERO

ZERO
ZERO

ZERO

ZERO

ZERO

$0.1013 E-04$

ZERO

ZERO

$0.2811 \mathrm{E}-06$

ZERO

$0.1286 \mathrm{E}-08$

ZERO

ZERO
ZERO

$0.2484 \mathrm{E}-08$

$0.1682 \mathrm{E}-07$

ZERO

ZERO
ZERO

ZERO

ZERO

ZERO

ZERO

ZERO

ZERO

ZERO

$0.1269 \mathrm{E}-06$

ZERO

ZERO

$0.2446 \mathrm{E}-08$

ZERO

$0.6248 \mathrm{E}-07$

ZERO

ZERO

$0.4968 \mathrm{E}-08$

ZERO
ZERO

ZERO
ZERO

ZERO

ZERO

ZERO

ZERO

ZERO

Section 5. Page 28 of 37 
Table A-1. HLW Flowsheet Material Balance (Tank 51/ITP Cycle 1) section 5. Vitrification and Melter off-Gas Treatment

STREAM NUMBERS (CONT'D) $\rightarrow$ STREAM NAME

$38 \mathrm{SO} 2$

$141 \mathrm{SO} 3$

137 Semi Vol Cs20

136 Semi Vol CsCl

126 Semi Vol Group A

140 Semi Vol Na2B4O7

138 Semi Vol NaCl

139 Semi Vol NaF

125 Semi Vol NaI
23 Semi Vol RuO2

111 sio2

$48 \mathrm{Sx}$ ( $\mathrm{COOH}) 2$

$176 \mathrm{Sr}$ (NO3) 2

$153 \mathrm{Sr}$ (NaTi2O5) 2

$127 \mathrm{Sr}(\mathrm{OH}) 2$

$30 \cdot \operatorname{srCO} 3$

46 sro

$159 \cdot \mathrm{TcO} 2$

92 ThO2

119 Tritium

$\begin{array}{rl}119 & \text { Triti } \\ 17 & 0308\end{array}$

$62 \mathrm{UO2}(\mathrm{COOH}) 2$

$177002(\mathrm{NO} 3) 2$

$\begin{array}{ll}16 & \mathrm{UO} 2(\mathrm{OH}) 2 \\ 27 & \mathrm{Y}(\mathrm{COOH}) 3\end{array}$

$27 \mathrm{Y}(\mathrm{COOH}) 3$

$178 \mathrm{Y}(\mathrm{NO} 3) 3$
$128 \mathrm{Y}(\mathrm{OH}) 3$

$31 \mathrm{Y} 2(\mathrm{CO} 3)^{3}$

$47 \mathrm{Y} 2 \mathrm{O} 3$

100 Zeolite

$44 \mathrm{Zn}(\mathrm{COOH}) 2$

$179 \mathrm{Zn}$ (NO3) 2

$\begin{array}{rl}104 & \mathrm{ZrO} 2 \\ 13 & \text { hydrate } \mathrm{H} 2 \mathrm{O}\end{array}$

1 water.

TOTAL FLOW, LB/HR

TEMPERATURE, DEG $C$

PRESSURE, ATM

PRESSURE， PSIG

PRESSURE， MM HG

ENTHALPY， PCU/HR

VAPOR FLOW, CFM

IIOUID FLOW, GPM

DENSITY, LBS/FT3

PHASE

102
OGCT Trnsf
Pump Prim

$$
\begin{array}{r}
\text { Prime } \\
\text { ZERO } \\
\text { ZERO }
\end{array}
$$

ZERO

ZERO

ZERO

ZERO

ZERO

ZERO

ZERO

ZERO

ZERO

ZERO

ZERO

ZERO

ZERO

ZERO

ZERO

ZERO

ZERO

ZERO

ZERO

ZERO

ZERO

ZERO

ZERO

ZERO

ZERO

ZERO

ZERO

ZERO

ZERO

ZERO

$0.2090 \mathrm{E}+02$

$0.2090 \mathrm{E}+02$

$0.2500 E+02$

$0.1000 \mathrm{E}+01$

$\mathrm{E}+03$

$0.5244 \mathrm{E}+03$

$0.4174 \mathrm{E}-01$

$0.6243 \mathrm{E}+02$

IIQUID
$103 \quad 104$

eakage
ZERO

ZERO

ZERO

ZERO

ZERO

ZERO

ZERO

ZERO

ZERO

ZERO

ZERO

ZERO

ZERO

ZERO

ZERO

ZERO

ZERO

ZERO

ZERO

ZERO

ZERO

ZERO

ZERO

ZERO

ZERO

ZERO

ZERO

ZERO

ZERO

ZERO

ZERO

ZERO

$0.1736 \mathrm{E}+01$

$0.1051 \mathrm{E}+03$

$0.3500 \mathrm{E}+02$

$0.1000 \mathrm{E}+01$

ZERO

$0.2204 \mathrm{E}+04$

$0.7055 \mathrm{E}-01$

VAPOR
110

111

Add'n

to OGCT

ZERO

ZERO

ZERO

ZERO

ZERO

ZERO

ZERO

ZERO

ZERO

ZERO

ZERO

ZERO

ZERO

ZERO

ZERO

ZERO

ZERO

ZERO

ZERO

ZERO

ZERO

ZERO

ZERO

ZERO

ZERO

ZERO

ZERO

ZERO

ZERO

ZERO

ZERO

ZERO

ZERO

ZERO

$0.2500 \mathrm{E}+02$

$0.1000 \mathrm{E}+01$

ZERO

ZERO

ZERO

LIQUID

ZERO

ZERO

$0.2058 \mathrm{E}-05$

$0.8858 \mathrm{E}-07$

$.6143 \mathrm{E}-04$

$0.2709 \mathrm{E}-08$

ZERO
$0.6148 E-04$

ZERO

ZERO

ZERO

$0.7674 \mathrm{E}-08$

$0.2588 \mathrm{E}-08$

$0.1486 \mathrm{E}-07$

$0.1137 \mathrm{E}-11$

$0.1042 \mathrm{E}-05$

ZERO
ZERO

ZERO

ZERO

ZERO

ZERO

$0.2115 \mathrm{E}-08$

ZERO

ZERO

ZERO

ZERO

$0.1849 \mathrm{E}+04$

$0.2000 \mathrm{E}+02$

$0.9263 \mathrm{E}+00$

$0.7040 \mathrm{E}+03$

$0.2359 \mathrm{E}+05$

$0.6928 \mathrm{E}-01$
$0.6928 E-01$
VAPOR

Gas Feed Accumulation

Date: January 1995

ZERO

$.2047 \mathrm{E}-05$

$.8813 \mathrm{E}-07$

ZERO

ZERO

ZERO

ZERO

$.7659 \mathrm{E}-08$

$0.2582 \mathrm{E}-08$

$0.1483 \mathrm{E}-07$

$0.4160 \mathrm{E}-06$

ZERO

$0.1040 \mathrm{E}-05$

ZERO

ZERO

ZERO

ZERO

ZERO

TERO

$0.2110 \mathrm{E}-08$

ZERO

ZERO

$0.1576 \mathrm{E}-06$

ZERO

ZERO

ZERO

$0.2000 \mathrm{E}+02$

$0.9263 \mathrm{E}+00$

$-0.1083 \mathrm{E}+01$
$0.7040 \mathrm{E}+03$

$0.7709 \mathrm{E}-03$

$112 \quad 113$

Iim HEPA\#2

Gas Feed Accumultion ZERO ZERO ZERO

$0.1029 \mathrm{E}-07$

$0.9259 \mathrm{E}-08$

$0.3986 \mathrm{E}-09$

$0.2764 \mathrm{E}-06$

ZERO

$0.3071 \mathrm{E}-06$

$0.3113 \mathrm{E}-07$

$0.1354 \mathrm{E}-10 \quad 0.1219 \mathrm{E}-10$

ZERO

.

$0.1205 E-06$

ZERO

ZERO

ZERO

ZERO

ZERO

$0.1535 \mathrm{E}-10$

$0.5564 \mathrm{E}-11$

$0.2972 \mathrm{E}-10$

$0.8337 \mathrm{E}-09$

$0.2084 \mathrm{E}-08$

ZERO

ZERO

ZERO

ZERO

ZERO

ZERO

0.4229 E-11 
Table A-1. HLW Flowsheet Material Balance (Tank 51/ITP Cycle 1) Section 5. Vitrification and Melter Off-Gas Treatment

STREAM NUMBERS $\rightarrow$ STREAM NAME

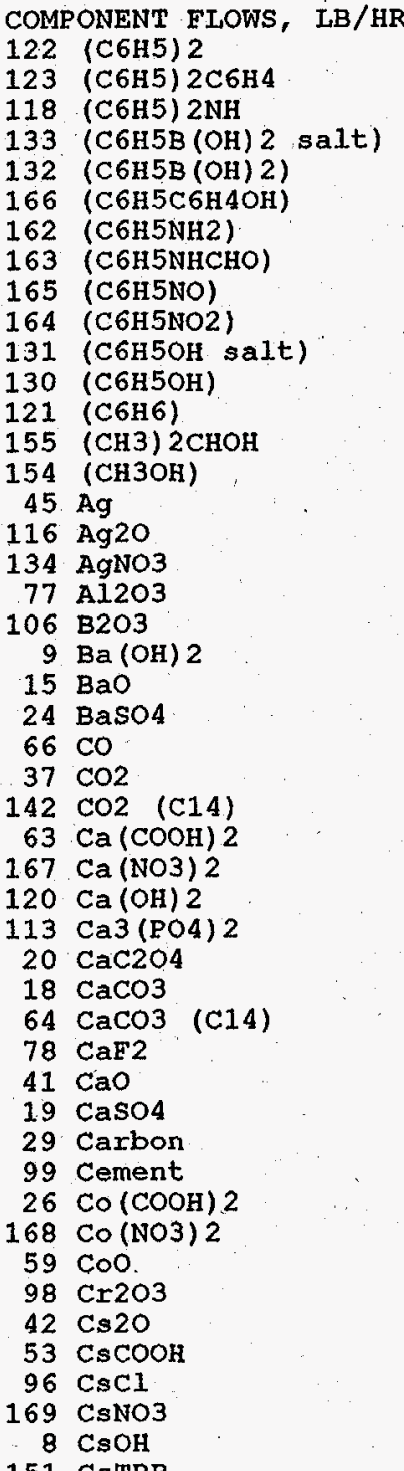

116

117

rim HEPA\#2 Prim Exhstr Bekup Qunchr. BUOGCT Exit Gas Gas to Tunnl

BUOGCT
histr Bckup Qunchr Air to $\begin{array}{r}\text { Water to Backup OGCT } \\ \text { Inlet Air Air Inleakge Backup HEME Backup HEME }\end{array}$

119

\begin{tabular}{|c|c|c|c|}
\hline ZERO & ZERO & ZERO & ZERO \\
\hline ZERO & ZERO & ZERO & ZERO \\
\hline ZERO & ZERO & ZERO & ZERO \\
\hline ZERO & ZERO & ZERO & ZERO \\
\hline ZERO & ZERO & ZERO & ZERO \\
\hline ZERO & ZERO & ZERO & ZERO \\
\hline ZERO & ZERO & ZERO & ZERO \\
\hline ZERO & ZERO & ZERO & ZERO \\
\hline ZERO & ZERO & ZERO & ZERO \\
\hline ZERO & $\quad$ ZERO & ZERO & ZERO \\
\hline $0.1647 \mathrm{E}-23$ & $0.1647 \mathrm{E}-23$ & ZERO & ZERO \\
\hline $0.4250 \mathrm{E}-08$ & $0.4250 \mathrm{E}-08$ & ZERO & ZERO \\
\hline ZERO & ZERO & ZERO & ZERO \\
\hline ZERO & ZERO & ZERO & ZERO \\
\hline ZERO & ZERO & ZERO & ZERO \\
\hline ZERO & ZERO & ZERO & ZERO \\
\hline ZERO & ZERO & ZERO & ZERO \\
\hline ZERO & ZERO & ZERO & ZERO \\
\hline $0.1767 \mathrm{E}-09$ & $0.1767 \mathrm{E}-09$ & ZERO & ZERO \\
\hline $0.3384 \mathrm{E}-09$ & $0.3384 \mathrm{E}-09$ & ZERO & ZERO \\
\hline ZERO & ZERO & ZERO & ZERO \\
\hline $0.2239 \mathrm{E}-13$ & $0.2239 \mathrm{E}-13$ & ZERO & ZERO \\
\hline $0.3067 \mathrm{E}-12$ & $0.3067 \mathrm{E}-12$ & ZERO & ZERO \\
\hline ZERO & ZERO & ZERO & ZERO \\
\hline $0.1410 \mathrm{E}+02$ & $0.1410 \mathrm{E}+02$ & ZERO & ZERO \\
\hline TRACE & TRACE & ZERO & ZERO \\
\hline ZERO & ZERO & ZERO & ZERO \\
\hline ZERO & ZERO & ZERO & ZERO \\
\hline ZERO & ZERO & ZERO & ZERO \\
\hline $0.5252 \mathrm{E}-10$ & $0.5252 \mathrm{E}-10$ & ZERO & ZERO \\
\hline ZERO & ZERO & ZERO & ZERO \\
\hline ZERO & ZERO & ZERO & ZERO \\
\hline ZERO & ZERO & ZERO & ZERO \\
\hline ZERO & ZERO & ZERO & ZERO \\
\hline $0.2909 \mathrm{E}-10$ & $0.2909 E-10$ & ZERO & ZERO \\
\hline $0.3974 \mathrm{E}-11$ & $0.3974 \mathrm{E}-11$ & ZERO & ZERO \\
\hline ZERO & ZERO & ZERO & ZERO \\
\hline ZERO & ZERO & ZERO & ZERO \\
\hline ZERO & ZERO & ZERO & ZERO \\
\hline ZERO & ZERO & ZERO & ZERO \\
\hline $0.2060 \mathrm{E}-1.4$ & $0.2060 \mathrm{E}-14$ & ZERO & ZERO \\
\hline $0.8604 \mathrm{E}-11$ & $0.8604 \mathrm{E}-11$ & ZERO & ZERO \\
\hline $0.6199 \mathrm{E}-12$ & $0.6199 \mathrm{E}-12$ & ZERO & ZERO \\
\hline ZERO & ZERO & ZERO & ZERO \\
\hline ZERO & ZERO & ZERO & ZERO \\
\hline ZERO & ZERO & ZERO & ZERO \\
\hline ZERO & ZERO & ZERO & ZERO \\
\hline ZERO & ZERO & ZERO & ZERO \\
\hline
\end{tabular}

Date: January 1995

\begin{tabular}{|c|c|c|}
\hline ZERO & ZERO & ZERO \\
\hline ZERO & ZERO & ZERO \\
\hline ZERO & ZERO & ZERO \\
\hline ZERO & ZERO & ZERO. \\
\hline ZERO & ZERO & ZERO \\
\hline ZERO & ZERO & ZERO \\
\hline ZERO & ZERO & ZERO \\
\hline ZERO & ZERO & ZERO \\
\hline ZERO & ZERO & ZERO \\
\hline ZERO & ZERO & ZERO \\
\hline ZERO & ZERO & ZERO \\
\hline ZERO & ZERO & ZERO \\
\hline ZERO & ZERO & ZERO \\
\hline ZERO & ZERO & ZERO \\
\hline ZERO & ZERO & ZERO \\
\hline ZERO & ZERO & ZERO \\
\hline ZERO & ZERO & ZERO \\
\hline ZERO & ZERO & ZERO \\
\hline ZERO & ZERO & ZERO \\
\hline ZERO & ZERO & ZERO \\
\hline ZERO & ZERO & ZERO \\
\hline ZERO & ZERO & ZERO \\
\hline ZERO & ZERO & ZERO \\
\hline ZERO & ZERO & ZERO \\
\hline ZERO & ZERO & ZERO \\
\hline ZERO & ZERO & ZERO \\
\hline ZERO & ZERO & ZERO \\
\hline ZERO & ZERO & ZERO \\
\hline ZERO & ZERO & ZERO \\
\hline ZERO & ZERO & ZERO \\
\hline ZERO & ZERO & ZERO \\
\hline ZERO & ZERO & ZERO \\
\hline ZERO & ZERO & ZERO \\
\hline ZERO & ZERO & ZERO \\
\hline ZERO & ZERO & ZERO \\
\hline ZERO & ZERO & ZERO \\
\hline ZERO & ZERO & ZERO \\
\hline ZERO & ZERO & ZERO \\
\hline ZERO & ZERO & ZERO \\
\hline ZERO & ZERO & ZERO \\
\hline ZERO & ZERO & ZERO \\
\hline ZERO & ZERO & ZERO \\
\hline ZERO & ZERO & ZERO \\
\hline ZERO & ZERO & ZERO \\
\hline ZERO & ZERO & ZERO \\
\hline ZERO & ZERO & ZERO \\
\hline ZERO & ZERO & ZERO \\
\hline ZERO & ZERO & ZERO \\
\hline
\end{tabular}

Section 5. Page 30 of 37 
Table A-1. HLW Flowsheet Material Balance (Tank 51/ITP Cycle 1) Section 5. Vitrification and Melter off-Gas Treatment

STREAM NUMBERS (CONT'D) $\rightarrow$
STREAM NAME

$54 \mathrm{Cu}(\mathrm{COOH}) 2$

$170 \mathrm{Cu}(\mathrm{NO} 3)^{2}$

55 CuO

$80 \mathrm{Fe} 203$

$101 \mathrm{FeO}$

102 Group A

$\begin{array}{rl}103 & \text { Group } \\ 25 & \mathrm{H} 2\end{array}$

$\begin{array}{rl}25 & \mathrm{H} 2 \\ 158 & \mathrm{H} 2 \mathrm{C} 2 \mathrm{O} 4\end{array}$

93 H2SO4

$90 \mathrm{H3BO} 3$

$34 \mathrm{HCOOH}$

$43 \mathrm{HCl}$

$85 \mathrm{HF}$

49 HNO3

$36 \mathrm{Hg}$

$129 \mathrm{Hg}(\mathrm{C} 6 \mathrm{H} 5) 2$

$135 \mathrm{Hg}$ (NO3) 2

$144 \mathrm{Hg} 2 \mathrm{Cl} 2$

$117 \mathrm{Hg} 2 \mathrm{I} 2$

$146 \mathrm{HgCl}$

$21 \mathrm{HgO}$

97 I2

$39 \mathrm{~K} 2 \mathrm{O}$

$51 \mathrm{KCOOH}$

$157 \mathrm{KMnO4}$

$161 \mathrm{KNO}$

$10 \mathrm{KOH}$

105 La203

108 Li2o

$65 \mathrm{Mg}(\mathrm{COOH}) 2$

$171 \mathrm{Mg}$.(NO3)

$60 \mathrm{Mn}(\mathrm{COOH}) 2$

$172 \mathrm{Mn}$ (NO3) 2

$180 \mathrm{Mn} 304$

$56 \mathrm{MnO}$

$14 \mathrm{MnO} 2$

$1.45 \mathrm{MOO} 2$

81 N2

74 N2O

182 NH3
114 NH3OHNO3

52 NH4COOH

173 NH4NO3

$68 \mathrm{NH} 4 \mathrm{OH}$

114

Prim HEPA\#2 prim 115 hatr Bcku

117

BUOGCT Gas Gas to Tunnl

ZERO ZERO

$0.1893 \mathrm{E}-10$

$0.5077 \mathrm{E}-09$

$0.5076 \mathrm{E}-10$

$0.6732 \mathrm{E}-13$

$0.3023 \mathrm{E}-14$

ZERO

ZERO

ZERO

ZERO
$0.1369 \mathrm{E}-02$

$0.6776 \mathrm{E}-02$

$0.8511 \mathrm{E}-02$

ZERO

$0.2867 \mathrm{E}-10$

$0.2367 \mathrm{E}-15$

$0.2253 \mathrm{E}-20$

ZERO

$0.4632 \mathrm{E}-09$

ZERO

$0.1144 \mathrm{E}-09$

ZERO

ZERO

ZERO

ZERO

ZERO

$0.2216 \mathrm{E}-0$

ZERO

$0.9383 E-10$

ZERO

ZERO

ZERO
$0.5301 E-10$

ZERO

$0.2151 E-13$

$0.1395 \mathrm{E}+04$

ZERO

ZERO

ZERO

ZERO

ZERO

ZERO

WSRC-TR-95-0019 (Revision Air Air Inleakge
ZERO
ZERO $\quad ! \quad$ ZERO
ZERO

$0.1893 E-10$

$0.5077 \mathrm{E}-09$
$0.5076 \mathrm{E}-10$

$0.6732 \mathrm{E}-13$

$0.3023 \mathrm{E}-14$

Inlet $A j$ $\begin{array}{ll}\text { ZERO } & \text { ZERO } \\ \text { ZERO } & \text { ZERO } \\ \text { ZERO } & \text { ZERO }\end{array}$ ZERO

ZERO

ZERO

ZERO

ZERO

ZERO

ZERO

ZERO

ZERO

$0.1369 \mathrm{E}-02$

$0.6776 \mathrm{E}-02$

$0.8511 E-02$

ZERO

$0.2867 \mathrm{E}-10$

$0.2367 \mathrm{E}-1.5$

$0.2253 \mathrm{E}-20$

$0.4632 \mathrm{E}-09$

ZERO

ZERO

ZERO

ZERO

ZERO

ZERO

$0.2216 \mathrm{E}-09$

ZERO

ZERO
$0.9383 E-10$

ZE-10
ZERO

ZERO

ZERO
$0.5301 \mathrm{E}-10$

$0.1395 \mathrm{E}+04$

ZERO

ZERO

ZERO

ZERO

ZERO

ZERO

ZERO

ZERO

ZERO

ZERO

ZERO

ZERO

ZERO
ZERO

ZERO

ZERO

ZERO

ZERO

ZERO

ZERO

ZERO

ZERO

ZERO

ZERO

ZERO

ZERO

ZERO

ZERO

ZERO.

ZERO

ZERO

ZERO

ZERO

ZERO

ZERO

ZERO
ZERO

ZERO

ZERO

ZERO

ZERO

ZERO

ZERO

ZERO

ZERO

ZERO

ZERO

ZERO

ZERO

ZERO

ZERO

ZERO

ZERO

ZERO

ZERO

ZERO

ZERO

ZERO

ZERO

ZERO

ZERO

ZERO

ZERO

ZERO

ZERO

ZERO

ZERO

ZERO

ZERO

ZERO

ZERO

ZERO

ZERO

ZERO

Date: January 1995
$0.1144 \mathrm{E}-09$

ZERO
$0.2151 \mathrm{E}-13$

ZERO
ZERO
ZERO
ZERO
ZERO
ZERO
ZERO

$0.6042 \mathrm{E}+03$
118

Air to

119

120

HEME Backuter to Backup OGCT ZERO Backup HEME IIq to RCI ZERO ZERO $\quad$ ZERO ZERO ZERO ZERO ZERO ZERO ZERO ZERO ZERO ZERO

ZERO ZERO ZERO

ZERO ZERO

ZERO ZERO

ZERO ZERO ZERO

ZERO ZERO ZERO

ZERO ZERO ZERO

ZERO ZERO

ZERO ZERO ZERO

ZERO ZERO

ZERO ZERO Z ZERO

ZERO ZERO ZERO

ZERO ZERO Z ZERO

ZERO ZERO ZERO

ZERO ZERO Z ZERO

ZERO ZERO

ZERO ZERO

ZERO ZERO ZERO

ZERO ZERO $\quad$ ZERO

ZERO ZERO $\quad$ ZERO

ZERO ZERO $\quad$ ZERO

ZERO ZERO $\quad$ ZERO

$\begin{array}{lll}\text { ZERO } & \text { ZERO } & \text { ZERO } \\ \text { ZERO } & \text { ZERO } & \text { ZERO }\end{array}$

ZERO ZERO ZERO

ZERO : ZERO ZERO

ZERO ZERO ZERO

ZERO ZERO

ZERO ZERO

ZERO

ZERO $\quad$ ZERO

ZERO - ZERO ZERO ZERO

$\begin{array}{lll}\text { ZERO } & \text { ZERO } & \text { ZERO } \\ \text { ZERO } & \text { ZERO } & \text { ZERO }\end{array}$

ZERO ZERO $\quad$ ZERO

ZERO ZERO ZERO

ZERO ZERO ZERO

$\begin{array}{lll}\text { ZERO } & \text { ZERO } & \text { ZERO } \\ \text { ZERO } & \text { ZERO } & \text { ZERO }\end{array}$

ZERO
$0.3023 \mathrm{E}-02$

ZERO

ZERO

ZERO

ZERO

ZERO

ZERO

section 5. Page 31 of 37 
Table A-1. HLW Flowsheet Material Balance (Tank 51/ITP Cycle 1) Section 5. Vitrification and Melter off-Gas Treatment

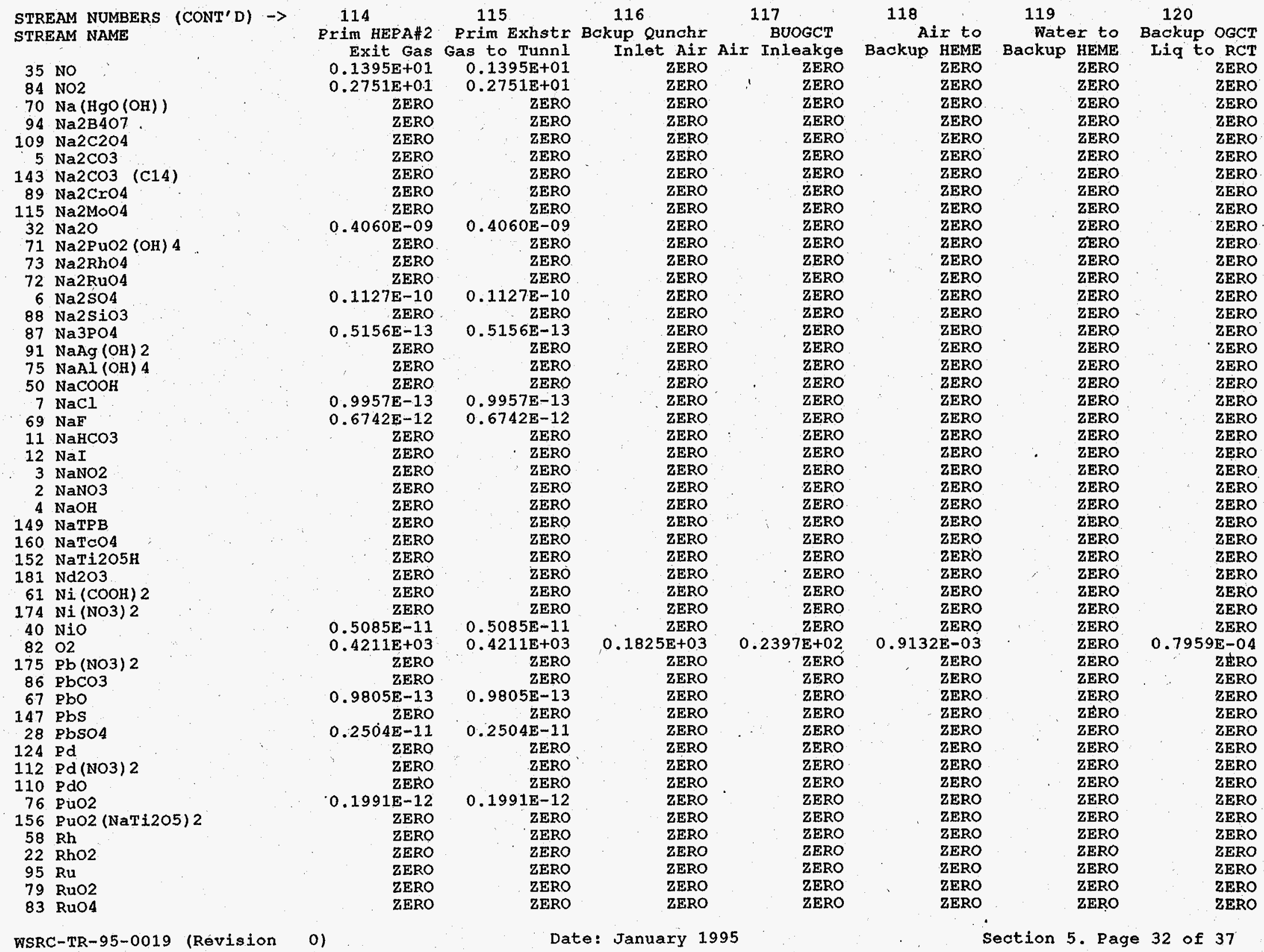


Table A-1. HLW Flowsheet Material Balance (Tank 51/ITP Cycle 1) Section 5. Vitrification and Melter off-Gas Treatment

STREAM NUMBERS (CONT'D) $\rightarrow$ STREAM NAME

$38 \mathrm{SO} 2$

$141 \mathrm{SO} 3$

137 Semi vol cs2O

136 Semi Vol CsCl

126 Semi Vol Group A

138 Semi Vol NaCl

139 Semi Vol NaF

125 Semi Vol NaI

23 Semi Vol RuO2

111 SiO2

$48 \mathrm{Sr}(\mathrm{COOH}) 2$

$176 \mathrm{Sr}(\mathrm{NO} 3)$

$153 \mathrm{Sr}$ (NaTi2O5) 2

$127 \mathrm{Sr}(\mathrm{OH}) 2$

$30 \mathrm{srco} 3$

46 sro

159 $\mathrm{TcO} 2$

92 ThO2

119 Tritium

$\begin{array}{rl}119 & \text { Trititit } \\ 17 & 0308\end{array}$

$62 \mathrm{UO2}(\mathrm{COOH})$

177 UO2 (NO3) 2

$16 \mathrm{VO} 2(\mathrm{OH})$

$27 \mathrm{Y}(\mathrm{COOH}) 3$

$178 \mathrm{Y}(\mathrm{NO} 3)$

$31 \mathrm{Y} 2(\mathrm{CO} 3) 3$

47 Y2O3

100 zeolite

$44 \mathrm{Zn}(\mathrm{COOH}) 2$

$179 \mathrm{Zn}$ (NO3) 2

$57 \mathrm{Zno}$

$104 \mathrm{ZrO} 2$

13 hydrate $\mathrm{H} 2 \mathrm{O}$

1 water

TOTAL ELOW, LB/HR

TEMPERATURE， DEG C

PRESSURE， ATM

PRESSURE， PSIG

PRESSURE， MM HG

ENTHALPY, RCU/HR

VAPOR FLÓW, CFM

IIOUID FLOW, GPM

LIQUID FLOW, GPM
DENSITY, LBS/FT3

PHASE

11

Exit Gas Gas to Tunn1 Inlet Air Air Inleakgo ZERO Gas to Tunn. ZERO

ZERO ZERO

$0.1029 \mathrm{E}-08$

$0.4429 \mathrm{E}-10$

ZERO
$0.1029 E-08$

$0.4429 E-10$

ZERO

$0.3071 \mathrm{E}-07$

$0.3113 \mathrm{E}-08$

$0.3113 \mathrm{E}-08$

$0.1354 \mathrm{E}-11$

$0.1354 \mathrm{E}-11$

ZERO

$0.2459 \mathrm{E}-08$

ZERO

ZERO

ZERO

ZERO

$0.3070 \mathrm{E}-12$

$0.1335 \mathrm{E}-12$

$0.5944 \mathrm{E}-12$

$0.1137 \mathrm{E}-11$

$0.1137 \mathrm{E}-11$

ZERO

ZERO

ZERO

ZERO

$0.8458 \mathrm{E}-13$

ZERO

ZERO

$0.6319 \mathrm{E}-11$

ZERO

$0.1418 \mathrm{E}+02$

$0.1849 \mathrm{E}+04$

ZERO
$0.2459 E-08$

ZERO

ZERO

ZERO

ZERO

$0.1335 \mathrm{E}-12$

$0.5944 \mathrm{E}-12$

$0.1667 \mathrm{E}-10$

$0.1137 \mathrm{E}-11$

$0.4168 \mathrm{E}-10$

ZERO

ZERO

ZERO

ZERO

ZERO

$0.8458 \mathrm{E}-13$

ZERO

ZERO

$0.6319 \mathrm{E}-11$

ZERO

$0.1418 \mathrm{E}+02$

$0.1849 \mathrm{E}+04$

$0.2000 \mathrm{E}+02$

$0.2762 \mathrm{E}+02$

$0.9928 \mathrm{E}+00$

0.926

$-0.1083 \mathrm{E}+01$

$0.7040 \mathrm{E}+03$

$0.7545 \mathrm{E}+03$

$0.2686 \mathrm{E}+05$

$0.4447 \mathrm{E}+03$

$0.4257 \mathrm{E}+03$

$0.6928 \mathrm{E}-01$

VAPOR

$0.7237 \mathrm{E}-01$
VAPOR

Date: January 1995
116

117

118

Air to

119

120.

Backup HEME Backup HEME Backup OGCT Backup HEME Liq to RCT

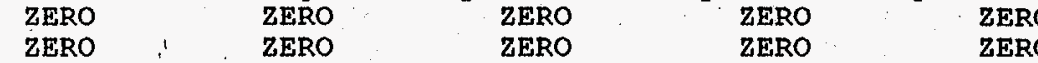

ZERO ZERO ZERO ZERO Z ZERO

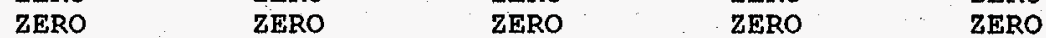

ZERO ZERO $\quad$ ZERO $\quad$ ZERO

ZERO ZERO $\quad$ ZERO ZERO ZERO

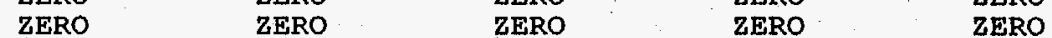

ZERO ZERO ZERO ZERO Z Z Z Z

ZERO

ZERO ZERO ZRO ZERO

$\begin{array}{llll}\text { ZERO } & \text { ZERO } & \text { ZERO } & \text { ZERO } \\ \text { ZERO } & \text { ZERO }\end{array}$

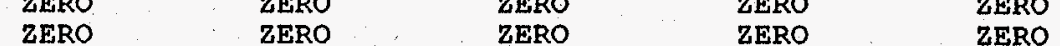

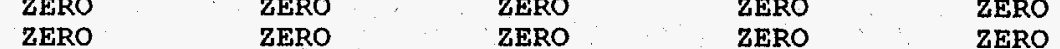

$\begin{array}{llll}\text { ZERO ZERO } & \text { ZERO } & \text { ZERO } & \text { ZERO }\end{array}$

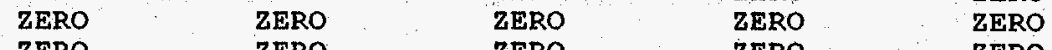

ZERO ZERO ZERO

ZERO ZERO ZERO

ZERO ZERO ZERO

ZERO ZERO ZERO

$\begin{array}{lll}\text { ZERO } & \text { ZERO } \\ \text { ZERO }\end{array}$

ZERO

ZERO ZERO ZERO

$\begin{array}{lll}\text { ZERO } & \text { ZERO } & \text { ZERO } \\ \text { ZERO } & \text { ZERO } & \text { ZERO }\end{array}$

$\begin{array}{lll}\text { ZERO } & \text { ZERO } & \text { ZERO } \\ \text { ZERO } & \text { ZERO } & \text { ZERO }\end{array}$

ZERO ZERO ZERO

ZERO ZERO ZERO

ZERO ZERO Z ZERO

ZERO ZERO $\quad$ ZERO

ZERO ZERO ZERO

ZERO ZERO ZERO

ZERO $\quad$ ZERO $\quad$ ZERO

ZERO ZERO

$\begin{array}{lll}\text { ZERO } & \text { ZERO } \\ \text { ZERO } & \text { ZERO }\end{array}$

ZERO

$0.8000 \mathrm{E}+03$

2.173 ZERO

$0.1051 \mathrm{E}+03$

$0.6613 \mathrm{E}-04$

$0.4003 \mathrm{E}-02$

$0.1329 \mathrm{E}+01$

$0.9646 \mathrm{E}+01$

$0.9853 \mathrm{E}+00 \quad 0.1000 \mathrm{E}+01$

$0.3720 \mathrm{E}+01$

$0.3997 \mathrm{E}+02$

$.4000 \mathrm{E}+02$

$0.3180 \mathrm{E}+01$
$0.3204 \mathrm{E}+02$

$0.4000 \mathrm{E}+02$

$0.1000 \mathrm{E}+01$

$.1678 \mathrm{E}+05$

$0.2204 \mathrm{E}+04 \quad 0.8395 \mathrm{E}-01$

$.2542 \mathrm{E}-03$

$0.5327 E+02$

$0.3866 \mathrm{E}+03$

$0.2655 \mathrm{E}-02$

$0.6243 \mathrm{E}+02$

LIQUID

$0.1926 \mathrm{E}-01$

$0.6243 \mathrm{E}+02$

LIQUID. 
Table A-1. HLW Flowsheet Material Balance (Tank 51/ITP Cycle 1) Section 5. Vitrification and Melter off-Gas Treatment

STREAM NUMBERS $\rightarrow$

STREAM NAME

121

Bkup Exhstr

Gas to Tunnl

\section{COMPONENT FLOWS, LB/HR}

122 ( 6 $6 \mathrm{H} 5) 2$

123 (C6H5) 2C6H4

118 (C6H5) $2 \mathrm{NH}$

133 ( 6 $6 \mathrm{H} 5 \mathrm{~B}(\mathrm{OH}) 2$ salt)

132 (C6H5B (OH) 2)

166 (C6H5C6H4OH)

162 (C6H5NH2)

163 (C6H5NHCHO)

165 (C6H5NO)

164 (C6H5NO2)

131 (C6H5OH sa1t)

131 (C6H5OH

130 (C6H5OH)
121 (C6H6)

121 (C6H6)

154 (CH3OH)

$45 \mathrm{Ag}$

116 Ag20

134 AgNO3
77 Al2O3

$106 \mathrm{~B} 203$

$9 \mathrm{Ba}(\mathrm{OH}) 2$

$15 \mathrm{BaO}$

$\begin{array}{ll}24 & \mathrm{Bas} \\ 66 & \mathrm{CO}\end{array}$

$66 \mathrm{CO}$

$142 \mathrm{CO} 2$ (C14)

$63 \mathrm{Ca}(\mathrm{COOH}) 2$

$167 \mathrm{Ca}(\mathrm{NO} 3) 2$

$167 \mathrm{Ca}(\mathrm{NO} 3) 2$
$120 \mathrm{Ca}(\mathrm{OH}) 2$

$120 \mathrm{Ca}(\mathrm{OH}) 2$
$113 \mathrm{Ca} 3(\mathrm{PO} 4) 2$

$20 \mathrm{CaC2O4}$

$18 \mathrm{CaCO} 3$

$64 \mathrm{CaCO}^{3}$ (C14)

$78 \mathrm{CaF} 2$

$41 \mathrm{CaO}$

19 CasO4

29 Carbon

99 Cement.

$26 \mathrm{Co}(\mathrm{COOH}) 2$

$168 \mathrm{Co}(\mathrm{NO} 3) 2$

$59 \mathrm{CoO}$

$\begin{array}{ll}98 & \mathrm{Cr} 203 \\ 42 & \mathrm{Cs} 2 \mathrm{O}\end{array}$

$\begin{array}{ll}42 & \mathrm{Cs} 2 \mathrm{O} \\ 53 & \mathrm{CsCOO}\end{array}$

$96 \mathrm{CsCl}$.

$169 \mathrm{CsNO3}$

$8 \mathrm{CsOH}$

$151 \mathrm{CsTPB}$

WSRC-TR-95-0019 (Revision 
Table A-1. HLW Flowsheet Material Balance (Tank 51/ITP Cycle 1)

section 5. Vitrification and Melter off-Gas Treatment

STREAM NUMBERS (CONT'D) $\rightarrow$ STREAM NAME

$54 \mathrm{Cu}(\mathrm{COOH}) 2$
$170 \mathrm{Cu}(\mathrm{NO} 3) 2$

$55 \mathrm{CuO}$

80 Fe203

$101 \mathrm{FeO}$

102 Group A

103 Group B

25 H2

$158: \mathrm{H} 2 \mathrm{C} 2 \mathrm{O} 4$

$93 \mathrm{H} 2 \mathrm{SO} 4$

$\begin{array}{ll}90 & \mathrm{H} 3 \mathrm{BO} 3 \\ 34 & \mathrm{HCOOH}\end{array}$

$43 \mathrm{HCl}$

$85 \mathrm{HF}$

$49 . \mathrm{HNO} 3$
$36 \mathrm{Hg}$

$129 \mathrm{Hg}(\mathrm{C} 6 \mathrm{H} 5)^{2}$

$135 \mathrm{Hg}$ (NO3) 2

$144 \mathrm{Hg} 2 \mathrm{Cl} 2$

$117 \mathrm{Hg} 2 \mathrm{I2}$

$146 \mathrm{HgCl2}$

21 Hgo

97 I2

$183 \mathrm{~K} 2 \mathrm{CO}$
39. $\mathrm{K} 2 \mathrm{O}$

$51 \mathrm{KCOOH}$

$157 \mathrm{KMnO4}$

$161 \mathrm{KNO} 3$

$10 \mathrm{KOH}$

$150 \mathrm{KTPB}$

108 Li20

$65 \mathrm{Mg}(\mathrm{COOH}) 2$

$171 \mathrm{Mg}$ (NO3) 2

$33 \mathrm{MgO}$

$60 \mathrm{Mn}(\mathrm{COOH}) 2$

$172 \mathrm{Mn}(\mathrm{NO} 3)$
$180 \mathrm{Mn} 3 \mathrm{O} 4$

$56 \mathrm{MnO}$

$14 \mathrm{MnO} 2$

$145 \mathrm{MoO} 2$

81 N2

$74 \mathrm{~N} 2 \mathrm{O}$

$182 \mathrm{NH}$

114 NH3OHNO3

$52 \mathrm{NH} 4 \mathrm{COOH}$

173 NH4NO3

$68 \mathrm{NH} 4 \mathrm{OH}$

148 NH 4 TP

WSRC-TR-95-0019 (Revision
121

Bkup Exhstr

Gas to Tunnl

ZERO

ZERO

ZERO

ZERO

ZERO

ZERO

ZERO

ZERO

ZERO

ZERO

ZERO

ZERO

ZERO

ZERO

ZERO

ZERO

ZERO

ZERO

ZERO

ZERO

ZERO

ZERO

ZERO

ZERO

ZERO

ZERO

ZERO

ZERO

ZERO

ZERO

ZERO

ZERO

ZERO

ZERO

ZERO

ZERO

ZERO

$0.6836 \mathrm{E}+03$

ZERO

ZERO

ZERO

ZERO

ZERO

ZERO 
Table A-1. HLW Flowsheet Material Balance (Tank 51/ITP Cycle 1) Section 5. Vitrification and Melter off-Gas Treatment

STREAM NUMBERS (CONT'D) $\rightarrow$ STREAM NAME

121

35 NO

$\begin{array}{ll}35 & \text { NO } \\ 84 & \text { NO2 }\end{array}$

$70 \mathrm{Na}(\mathrm{HgO}(\mathrm{OH}))$

$94 \mathrm{Na2B} 407$

$109 \mathrm{Na2C2O4}$

$5 \mathrm{Na} 2 \mathrm{CO} 3$

$143 \mathrm{Na2CO}$ (C14)

$89 \mathrm{Na} 2 \mathrm{CrO} 4$

$115 \mathrm{Na} 2 \mathrm{MOO}$

$32 \mathrm{Na} 2 \mathrm{O}$

$71 \mathrm{Na} 2 \mathrm{PuO} 2(\mathrm{OH}) 4$

73 Na2RhO4

$72 \mathrm{Na2RuO4}$
$6 \mathrm{Na2SO} 4$

$\begin{array}{rl}6 & \mathrm{Na} S \mathrm{SO} 4 \\ 88 & \mathrm{Na} 2 \mathrm{SiO}\end{array}$

$87 \mathrm{Na} 3 \mathrm{PO} 4$

$91 \mathrm{NaAg}(\mathrm{OH}) 2$
$75 \mathrm{NaAl}(\mathrm{OH}) 4$

$50 \mathrm{NaCOOH}$

$7 \mathrm{NaCl}$

$69 \mathrm{NaF}$

$11 \mathrm{NaHCO} 3$

$12 \mathrm{NaI}$

$3 \mathrm{NaNO} 2$

2 NaNO3

$4 \mathrm{NaOH}$

149 NaTPB

152 NaTi205H

$181 \mathrm{Nd} 2 \mathrm{O} 3$

$61 \mathrm{Ni}(\mathrm{COOH}) 2$

$40 \mathrm{NiO}$

$82 \mathrm{Ni}$

$175 \mathrm{~Pb}$ (NO3) 2

$86 \mathrm{PbCO} 3$

$67 \mathrm{pbo}$

$1.47 \mathrm{pbS}$

$28 \mathrm{PbSO}$

$\begin{array}{ll}124 & \mathrm{Pd} \\ 112 \mathrm{Pd}(\mathrm{NO} 3) & 2\end{array}$

$110 \mathrm{PdO}$

156 PuO2 (NaTi205) 2

$58 \mathrm{Rh}$

22 RhO2

$95 \mathrm{Ru}$

79 RuO2

83 RuO4

WSRC-TR-95-0019 (Revision

Bkup Exhstr

Gas to Tunn1

ZERO

ZERO

ZERO

ZERO

ZERO

ZERO

ZERO

ZERO

ZERO

ZERO

ZERO

ZERO

ZERO

ZERO

ZERO

ZERO

ZERO

ZERO

ZERO

ZERO

ZERO

ZERO

ZERO

ZERO

ZERO

ZERO

ZERO

ZERO

ZERO

ZERO

ZERO

$0.2065 \mathrm{E}+03$

ZERO

ZERO

ZERO

ZERO

ZERO

ZERO

ZERO

ZERO

ZERO

ZERO

ZERO

ZERO

ZERO

ZERO

ZERO

ZERO

0) 
Table A-1. HLW Flowsheet Material Balance (Tank 51/ITP Cycle 1) section 5. Vitrification and Melter off-Gas Treatment

STREAM NUMBERS (CONT'D) $\rightarrow$ STREAM NAME

\section{$\begin{array}{rr}38 & \mathrm{SO} 2 \\ 141 & \mathrm{SO} 3\end{array}$}

137 Semi Vol Cs20

136 Semi Vol CsCl

126 Semi Vol Group A

140 Semi Vol Na2B4O7

138 Semi Vol NaCI

139 Semi Vol NaF

125 Semi Vol NaI

23 Semi Vol RuO2

$111 \mathrm{SiO} 2$

$48 \mathrm{Sr}(\mathrm{COOH}) 2$

$176 \mathrm{Sr}(\mathrm{NO} 3) 2$

$153 \mathrm{Sr}(\mathrm{NaTi2O5)} 2$

$127 \mathrm{Sr}(\mathrm{OH}) 2$

$30 \operatorname{srcO} 3$

46 Sro

$159 \mathrm{TcO} 2$

92 ThO2

119 Tritium

$17 \mathrm{v} 308$

$62 . \mathrm{OO} 2(\mathrm{COOH}) 2$

$177 \mathrm{VO} 2$ (NO3) 2

$16 \mathrm{VO} 2(\mathrm{OH}) 2$

$27 \mathrm{Y}(\mathrm{COOH}) 3$

$178 \mathrm{Y}(\mathrm{NO} 3)$

$\begin{array}{rl}128 & \mathrm{Y}(\mathrm{OH}) 3 \\ 31 & \mathrm{Y} 2(\mathrm{CO} 3) 3\end{array}$

$31 Y 2(\mathrm{CO})$
47 Y 203

100 Zeolite

$44 \mathrm{Zn}(\mathrm{COOH}) 2$

$179 \operatorname{zn}(\mathrm{NO} 3) 2$

$57 \mathrm{ZnO}$

13 hydrate $\mathrm{H} 2 \mathrm{O}$

1 water

TOTAL FLOW, LB/HR

TEMPERATURE, DEG C

PRESSURE, ATM

PRESSURE， PSIG

PRESSURE， MM HG

ENTHALPY, PCU/HR

VAPOR FLOW, CFM

LIQUID FLOW, GPM

DENSITY， LBS/FT 3

RHASE

WSRC-TR-95-0019 (Revision
1.2

Bkup Exhst

Gas to Tunn

ZERO

ZERO

ZERO

ZERO

ZERO

ZERO

ZERO

ZERO

ZERO

ERO

ZERO

ZERO

ZERO

ZERO

ZERO

ZERO

ZERO

ZERO

ZERO

ZERO

ZERO

ZERO

ZERO

ZERO

ZERO

ZERO

ZERO

ZERO

ZERO

ZERO

ZERO

ZERO

ZERO

IERO

ZERO

ZERO

$0.6641 \mathrm{E}+01$

$0.8968 \mathrm{E}+03$

$0.2515 \mathrm{E}+02$

$0.9928 \mathrm{E}+00$

$-0.1058 \mathrm{E}+00$

$0.7545 \mathrm{E}+03$

- .7545E+03

$0.1186 \mathrm{E}+05$

$0.7275 \mathrm{E}-01$

VAPOR 


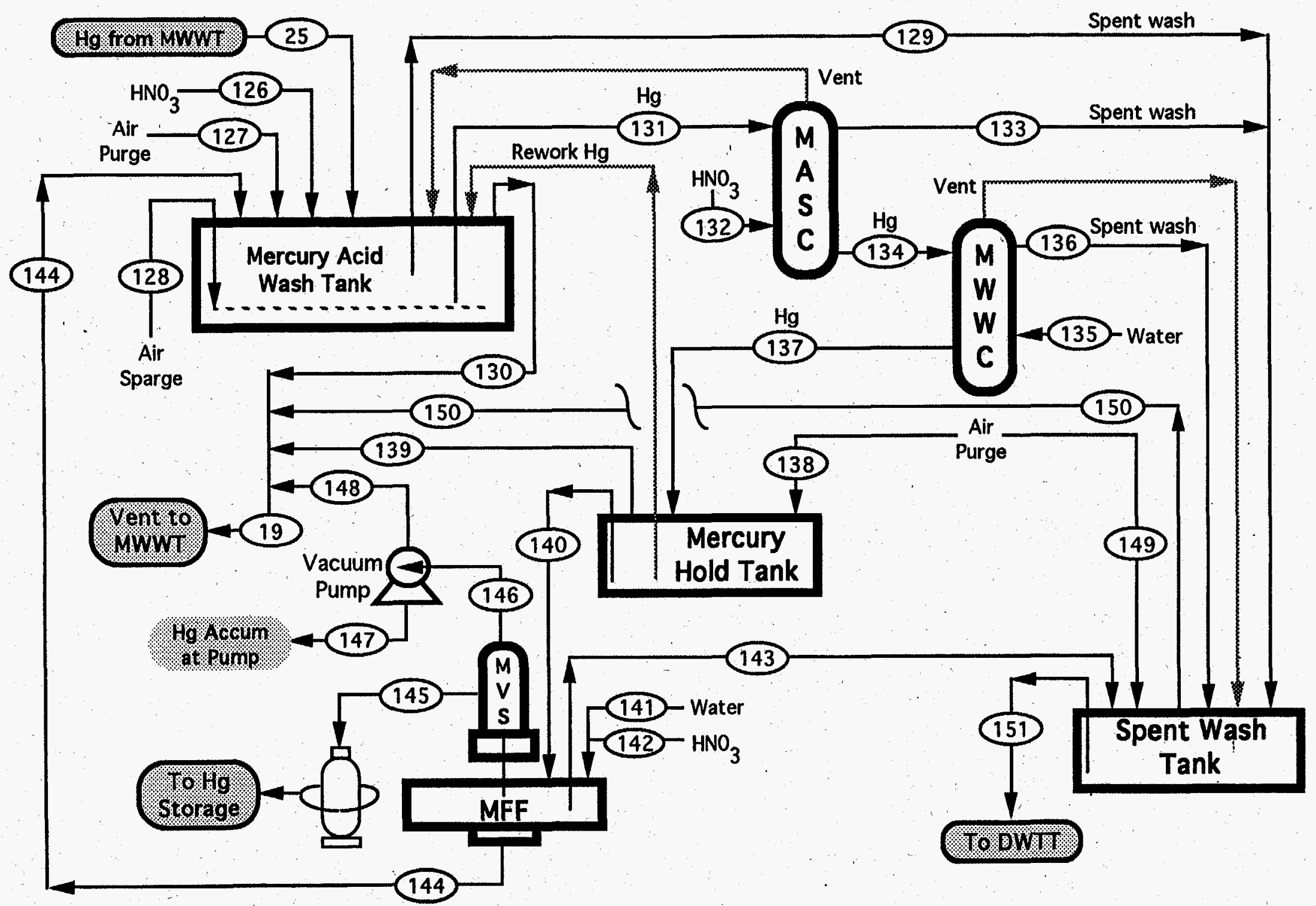

SECTION 6. MERCURY PURIFICATION CELL 
Table A-1. HLW Flowsheet Material Balance (Tank 51/ITP Cycle 1) section 6. Mercury purification Cell

DESCRIPTION

PAGE

1. STREAM NUMBERS

$19,25,126,127,128,129,130 \ldots \ldots$. . . . . . . . . 2

2. STREAM NUMBERS

$131,132,133,134,135,136,137 \ldots \ldots \ldots$

3. STREAM NUMBERS

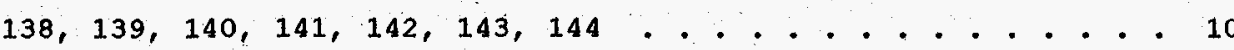

4. STREAM NUMBERS

$145,146,147,148,149,150,151 \ldots . . . . . . . . . . .14$ 
Table A-1. HLW Flowsheet Material Balance (Tank 51/ITP Cycle 1) section 6. Mercury Purification Cell STREAM NAME

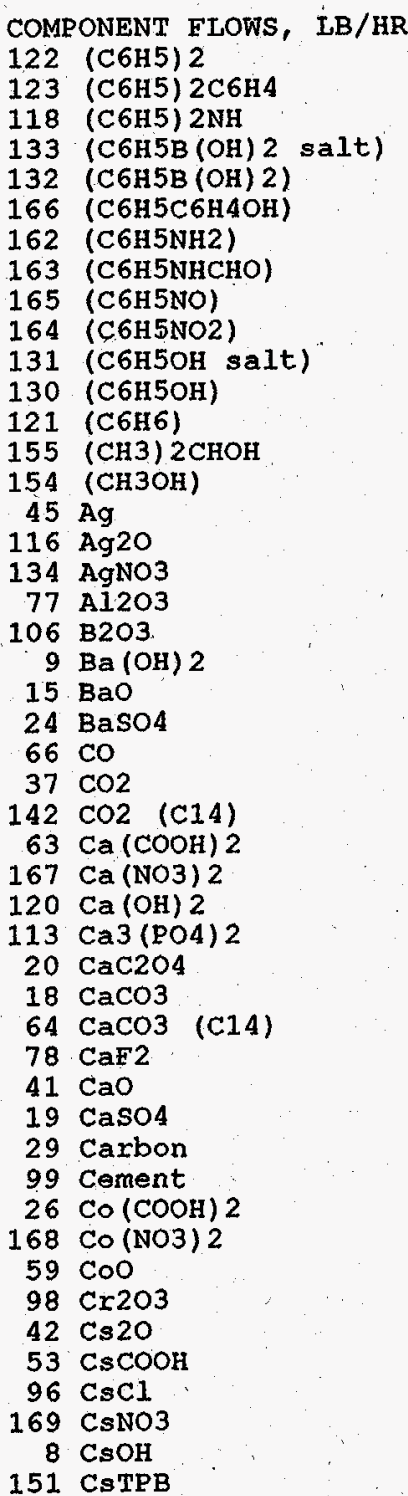

STREAM NUMBERS $\rightarrow$
19 Hg Cell
Vent to MWWT Vent to MWW'

$$
\begin{array}{r}
0.1610 \mathrm{E}-10 \\
0.104 \mathrm{OE}-18 \\
0.6994 \mathrm{E}-11 \\
\text { ZERO }
\end{array}
$$
$0.6713 \mathrm{E}-24$
ZERO
$25 \quad 126 \quad 127$ frm MWWT
to MAWT

\section{$0.1881 \mathrm{E}-10$}

$.2433 \mathrm{E}-13$

$0.3441 \mathrm{E}-09$ ZERO

$0.1349 \mathrm{E}-13$

$0.3574 \mathrm{E}-08$

$0.5113 \mathrm{E}-13$

$0.5361 \mathrm{E}-15$

$0.1694 \mathrm{E}-14$

$0.4289 \mathrm{E}-16$

$.4383 \mathrm{E}-07$

$0.3238 \mathrm{E}-10$

$0.3182 \mathrm{E}-18$

$0.8251 \mathrm{E}-07$

$0.6495 \mathrm{E}-23$ ZERO

$0.2162 \mathrm{E}-11$

$0.7834 \mathrm{E}-12$

$0.8199 \mathrm{E}-21$

$0.4168 \mathrm{E}-14$

$0.2156 \mathrm{E}-13$

$0.2782 \mathrm{E}-21$

$0.1025 \mathrm{E}-11$

ZERO

$.6422 \mathrm{E}-12$

$0.5961 \mathrm{E}-19$ ZERO

$0.3672 \mathrm{E}-14$

$0.8192 \mathrm{E}-15$

$0.5391 \mathrm{E}-13$ ZERO

$0.3497 \mathrm{E}-16$

$0.1312 \mathrm{E}-18$

$0.7491 \mathrm{E}-17$

$0.1049 \mathrm{E}-12$

$0.3432 \mathrm{E}-16$

$0.1057 \mathrm{E}-13$

ZERO

$0.2351 \mathrm{E}-17$

$0.2681 \mathrm{E}-18$
ZERO
HNO3 Wash
to MAWT

ZERO

ZERO

ZERO

ZERO

ZERO

ZERO

ZERO

ZERO

ZERO

ZERO

ZERO

ZERO

ZERO

ZERO

ZERO

ZERO

ZERO

ZERO

ZERO

ZERO

ZERO

ZERO

ZERO

ZERO

ZERO

ZERO

ZERO

ZERO

ZERO

ZERO

ZERO

ZERO

ZERO

ZERO

ZERO

ZERO

ZERO

ZERO

ZERO

ZERO

ZERO

ZERO

ZERO

ZERO

Date: January 1995
$127 \quad 128$ Air Sparge HNO3 Wsh frm
to MAWT MAWT to SWT Purge

ZERO ZERO

ZERO

ZERO

ZERO

ZERO

ZERO

ZERO

ZERO

ZERO

ZERO

ZERO

ZERO

ZERO

ZERO

ZERO

ZERO

ZERO

ZERO

ZERO

ZERO

ZERO

ZERO

ZERO

ZERO

ZERO

ZERO

ZERO

ZERO

ZERO

ZERO

ZERO

ZERO

ZERO

ZERO

ZERO

ZERO

ZERO

ZERO

ZERO

ZERO

ZERO

ZERO

\begin{tabular}{|c|c|c|}
\hline ZERO & $0.1474 \mathrm{E}-10$ & $0.2951 \mathrm{E}-11$ \\
\hline ZERO & $0.2261 \mathrm{E}-13$ & $0.8329 \mathrm{E}-19$ \\
\hline ZERO & $0.3198 \mathrm{E}-09$ & $0.3920 \mathrm{E}-14$ \\
\hline ZERO & ZERO & ZERO \\
\hline ZERO & ZERO & ZERO \\
\hline ZERO & $0.1253 \mathrm{E}-13$ & $0.4189 E-17$ \\
\hline ZERO & $0.3247 \mathrm{E}-08$ & $0.8019 \mathrm{E}-10$ \\
\hline ZEERO & $0.4751 \mathrm{E}-13$ & $0.1118 \mathrm{E}-16$ \\
\hline ZERO & $0.3678 E-16$ & $0.4965 \mathrm{E}-15$ \\
\hline ZERO & $0.1557 \mathrm{E}-14$ & $0.1874 \mathrm{E}-16$ \\
\hline ZERO & $0.3986 \mathrm{E}-16$ & $0.9326 \mathrm{E}-22$ \\
\hline ZERO & $0.3968 \mathrm{E}-07$ & $0.1128 \mathrm{E}-0.8$ \\
\hline ZERO & $0.8016 \mathrm{E}-11$ & $0.2375 \mathrm{E}-10$ \\
\hline ZERO & $0.1260 \mathrm{E}-17$ & $0.2135 E-17$ \\
\hline ZERO & $0.5926 \mathrm{E}-19$ & $0.2545 \mathrm{E}-18$ \\
\hline ZERO & ZERO & $0.1794 \mathrm{E}-14$ \\
\hline ZERO & $0.6036 \mathrm{E}-23$ & TRACE \\
\hline ZERO & $0.1189 \mathrm{E}-06$ & $0.2781 \mathrm{E}-12$ \\
\hline ZERO & $0.2009 \mathrm{E}-11$ & $0.4701 \mathrm{E}-17$ \\
\hline ZERO & $0.7280 \mathrm{E}-12$ & $0.1704 \mathrm{E}-17$ \\
\hline ZERO & ZERO & ZERO \\
\hline ZERO & $0.7620 \mathrm{E}-21$ & TRACE \\
\hline ZERO & $0.3873 \mathrm{E}-14$ & $0.9064 \mathrm{E}-20$ \\
\hline ZERO & ZERO & ZERO \\
\hline ZERO & $0.7697 E-18$ & $0.2156 E-13$ \\
\hline ZERO & TRACE & $0.2782 E-21$ \\
\hline ZERO & ZERO & ZERO \\
\hline ZERO & $0.9523 \mathrm{E}-12$ & $0.2228 \mathrm{E}-17$ \\
\hline ZERO & ZERO & ZERO \\
\hline ZERO & $0.5968 \mathrm{E}-12$ & $0.1397 \mathrm{E}-17$ \\
\hline ZERO & $0.5540 \mathrm{E}-19$ & $0.1296 \mathrm{E}-24$ \\
\hline ZERO & ZERO & ZERO \\
\hline ZERO & ZERO & ZERO \\
\hline ZERO & $0.3413 \mathrm{E}-14$ & $0.7986 \mathrm{E}-20$ \\
\hline ZERO & $0.7614 \mathrm{E}-15$ & $0.1782 \mathrm{E}-20$ \\
\hline ZERO & $0.5010 \mathrm{E}-13$ & $0.1172 \mathrm{E}-18$ \\
\hline ZERO & ZERO & ZERO \\
\hline ZERO & ZERO & ZERO \\
\hline ZERO & $0.3250 \mathrm{E}-16$ & $0.7605 \mathrm{E}-22$ \\
\hline ZERO & $0.1219 \mathrm{E}-18$ & $0.2852 \mathrm{E}-24$ \\
\hline ZERO & $0.6962 \mathrm{E}-17$ & $0.1629 \mathrm{E}-22$ \\
\hline ZERO & $0.9746 \mathrm{E}-13$ & $0.2281 \mathrm{E}-18$ \\
\hline ZERO & $0.3190 \mathrm{E}-16$ & $0.7464 \mathrm{E}-22$ \\
\hline ZERO & $0.9823 \mathrm{E}-14$ & $0.2298 \mathrm{E}-19$ \\
\hline ZERO & ZERO & ZERO \\
\hline ZERO & $0.2185 \mathrm{E}-17$ & $0.5112 \mathrm{E}-23$ \\
\hline ZERO & $0.2492 \mathrm{E}-18$ & $0.5830 \mathrm{E}-24$ \\
\hline ZERO & ZERO & ZERO \\
\hline
\end{tabular}

ZERO

ZTRO

- ZERO
Section 6, Page 2 of 17

$0.2951 \mathrm{E}-11$

$-14$ ZERO

$.4189 \mathrm{E}-17$ $8019 \mathrm{E}-10$ $1874 \mathrm{E}-16$ $0.1128 \mathrm{E}-22$ $0.2375 \mathrm{E}-10$ $4 E-14$ TRACE $701 \mathrm{E}-17$ ZERO $\mathrm{E}-20$ ZERO $2782 \mathrm{E}-21$ ZERO -17
-17 $-24$ TRO
130

MAWT
Vent

WSRC-TR-95-0019 (Revision 
Table A-1, HLW Flowsheet Material Balance (Tank 51/TTP Cycle 1) section 6. Mercury Purification Cell

STREAM NUMBERS (CONT'D) $\rightarrow$ STREAM NAME

$54 \mathrm{Cu}(\mathrm{COOH}) 2$

$170 \mathrm{Cu}(\mathrm{NO} 3) 2$

$55 \mathrm{CuO}$

$80 \mathrm{Fe} 203$

$101 \mathrm{FeO}$

102 Group A

103 Group B

25 H2

$158 \mathrm{H} 2 \mathrm{C} 2 \mathrm{O} 4$

$93 \mathrm{H} 2 \mathrm{SO} 4$

$90 \mathrm{HBO} 3$

43 HC

$85 \mathrm{HF}$

49 HNO3

$36 \mathrm{Hg}$

$129 \mathrm{Hg}(\mathrm{C} 6 \mathrm{H} 5) 2$

$135 \mathrm{Hg}(\mathrm{NO} 3)$

$144 \mathrm{Hg} 2 \mathrm{Cl} 2$

$117 \mathrm{Hg} 2 \mathrm{I2}$
$146 \mathrm{HgCl}$

$21 \mathrm{HgO}$

97 I2

$183 \mathrm{R} 2 \mathrm{CO} 3$

$39 \mathrm{~K} 2 \mathrm{O}$

$51 \mathrm{KCOOH}$

$157 \mathrm{KMnO} 4$

$161 \mathrm{KNO}$

10 kOH

105 La2O3

$108 \mathrm{Li} 20$

$65 \mathrm{Mg}$ (COOH) 2

$171 \mathrm{Mg}$ (NO3) 2

$33 \mathrm{MgO}$

$60 \mathrm{Mn}(\mathrm{COOH}) 2$

$172 \mathrm{Mn}(\mathrm{NO} 3) 2$

$180 \mathrm{Mn} 304$

$56 \mathrm{MnO}$

$14 \mathrm{MnO} 2$

$145 \mathrm{MoO} 2$

81 N2

$74 \mathrm{~N} 2 \mathrm{O}$

$114 \mathrm{NH} 3 \mathrm{OHNO} 3$

52 NH $4 \mathrm{COOH}$

68 NH4O

148 NH4TPB
19

$\mathrm{Hg}$ Cell

\section{Vent to MWWT}

$0.2714 \mathrm{E}-20$

$0.1333 \mathrm{E}-17$

$0.1207 \mathrm{E}-19$

$0.1555 \mathrm{E}-16$

$0.1555 \mathrm{E}-17$

$0.2171 \mathrm{E}-20$

$0.9261 \mathrm{E}-22$

$0.1428 \mathrm{E}-19$

$0.1119 \mathrm{E}-18$

$0.4710 \mathrm{E}-17$

ZERO

ZERO

$0.5648 \mathrm{E}-02$

$0.4599 \mathrm{E}-04$

ZERO
$0.8246 \mathrm{E}-11$ ZERO ZERO

$0.1207 E-23$ ZERO

$0.4946 \mathrm{E}-19$

$0.6139 \mathrm{E}-17$

$0.1751 \mathrm{E}-19$

$0.2008 \mathrm{E}-22$

ZERO ZERO

ZERO
$0.1716 \mathrm{E}-17$

. 2122E-17

TRACE

$0.6769 \mathrm{E}-18$

$329 E-17$
TRACE
ZERO

$0.4862 \mathrm{E}-23$

$0.1193 \mathrm{E}-17$

$0.658 .6 \mathrm{E}-21$

TRACE

$0.8761 \mathrm{E}-12$ ZERO

$0.1647 \mathrm{E}-12$

$0.1898 \mathrm{E}-19$

ZERO
25

frm MWWT

$0.1084 \mathrm{E}-14$

$0.5325 \mathrm{E}-12$

$0.4819 \mathrm{E}-14$

$0.6211 \mathrm{E}-11$

$0.6210 \mathrm{E}-12$

$0.3699 \mathrm{E}-16$

$0.1428 \mathrm{E}-19$

$0.4470 \mathrm{E}-13$

$0.1881 \mathrm{E}-11$

ZERO

ZERO

$0.2491 \mathrm{E}-09$

$0.2515 \mathrm{E}+00$

ZERO

ZERO
ZERO

ZERO

$0.4820 \mathrm{E}-18$ ZERO

$0.1975 \mathrm{E}-13$

$0.2452 \mathrm{E}-11$

$0.6993 \mathrm{E}-14$ ZERO

$0.1282 \mathrm{E}-19$

$0.2704 \mathrm{E}-12$

1494E-19

$0.1942 \mathrm{E}-17$

$0.4767 \mathrm{E}-12$

$0.2630 \mathrm{E}-15$

$0.3297 \mathrm{E}-07$

TRACE

ZERO

$0.6577 \mathrm{E}-07$

$0.7579 \mathrm{E}-14$

ZERO
$126 \quad 127$

HNO3 Wash

T MAWT

ZERO

ZERO

ZERO

ZERO

ZERO

ZERO

ZERO

ZERO

ZERO

ZERO

ZERO

ZERO

ZERO
$0.4626 \mathrm{E}-02$

ZERO

ZERO

ZERO

ZERO

ZERO

ZERO

ZERO

ZERO

ZERO

ZERO

ZERO

ZERO

ZERO

ZERO

ZERO
ZERO
ZERO
ZERO

ZERO

ZERO

ZERO

ZERO

ZERO

ZERO

ZERO

ZERO

ZERO

ZERO

ZERO

ZERO

ZERO

ZERO

ZERO
128

129

Puxge

Purge
ZERO

ZERO

ZERO

ZERO

ZERO

ZERO

ZERO

ZERO

ZERO

ZERO

ZERO

ZERO

ZERO

ZERO

ZERO

ZERO

ZERO

ZERO

ZERO

ZERO

ZERO

ZERO

ZERO

ZERO

ZERO

ZERO

ZERO

ZERO

ZERO

ZERO

ZERO

ZERO

ZERO

ZERO

ZERO

ZERO

ZERO

ZERO

$0.3607 \mathrm{E}+00$

ZERO

ZERO

ZERO

ZERO

ZERO

ZERO
128

parge HNO3 Wsh frm

MAW MAWT to SWT

ZRRO $0.4949 \mathrm{E}-12$

ZTRO $0.4478 \mathrm{E}-14$

ZTRO $0.5772 \mathrm{E}-11$

$0.5771 \pm-12$

2ERO $0.5771 \mathrm{E}-12$

ZERO $0.3437 \mathrm{E}-16$

. 3437 TRACE

ZERO $\quad 0.4155 \mathrm{E}-13$

ZERO $\quad 0.1748 \mathrm{E}-11$

ZERO ZERO

ZERO

ZERO

ZERO

ZERO

ZERO

ZERO

ZERO

ZERO
ZERO

ZERO

ZERO

ZERO

ZERO

ZERO

ZERO

ZERO

ZERO

2ERO

ZERO

ZERO

ZERO

ZERO

ZERO

ZERO

ZERO

ZERO

ZERO

ZERO

ZERO

RER

$0.1134 \mathrm{E}-01$

ZERO

ZERO

ZERO

ZERO

ZERO

ZERO

ZERO 
Table A-1. HLW Flowsheet Material Balance (Tank 51/ITP Cycle 1) section 6 . Mercury purification Cell

STREAM NUMBERS (CONT'D) $\rightarrow$ STREAM NAME

\begin{tabular}{|c|c|}
\hline 35 & NO \\
\hline 84 & NO2 \\
\hline 70 & $\mathrm{Na}(\mathrm{HgO}(\mathrm{OH}))$ \\
\hline 94 & $\mathrm{Na2B} 407$ \\
\hline 109 & $\mathrm{Na} 2 \mathrm{C} 2 \mathrm{O} 4$ \\
\hline 5 & $\mathrm{Na} 2 \mathrm{CO} 3$ \\
\hline 143 & $\mathrm{Na} 2 \mathrm{CO} 3(\mathrm{C} 14)$ \\
\hline 89 & $\mathrm{Na} 2 \mathrm{CrO} 4$ \\
\hline 115 & $\mathrm{Na} 2 \mathrm{MoO} 4$ \\
\hline 32 & $\mathrm{Na2O}$ \\
\hline 71 & $\mathrm{Na} 2 \mathrm{PuO} 2(\mathrm{OH}) 4$ \\
\hline 73 & $\mathrm{Na2RhO4}$ \\
\hline 72 & $\mathrm{Na2RuO} 4$ \\
\hline 6 & $\mathrm{Na} 2 \mathrm{SO} 4$ \\
\hline 88 & $\mathrm{Na} 2 \mathrm{Si} 03$ \\
\hline 87 & $\mathrm{Na} 3 \mathrm{PO} 4$ \\
\hline 91 & $\operatorname{NaAg}(\mathrm{OH}) 2$ \\
\hline 75 & $\operatorname{NaAi}(\mathrm{OH}) 4$ \\
\hline 50 & $\mathrm{NaCOOH}$ \\
\hline 7 & $\mathrm{NaCl}$ \\
\hline 69 & $\mathrm{NaF}$ \\
\hline 11 & $\mathrm{NaHCO} 3$ \\
\hline 12 & NaI \\
\hline 3 & NaNO2 \\
\hline 2 & NaNO3 \\
\hline 4 & $\mathrm{NaOH}$ \\
\hline 149 & NaTPB \\
\hline 160 & $\mathrm{NaTcO} 4$ \\
\hline 152 & $\mathrm{NaTi205H}$ \\
\hline 181 & $\mathrm{Nd} 203$ \\
\hline 61 & $\mathrm{Ni}(\mathrm{COOH}) 2$ \\
\hline 174 & $\mathrm{Ni}(\mathrm{NO} 3) 2$ \\
\hline 40 & NiO \\
\hline 82 & 02 \\
\hline 175 & $\mathrm{~Pb}(\mathrm{NO} 3) 2$ \\
\hline 86 & $\mathrm{PbCO} 3$ \\
\hline 67 & Pbo \\
\hline 147 & $\mathrm{PbS}$ \\
\hline 28 & $\mathrm{PbSO} 4$ \\
\hline 124 & Pd \\
\hline 112 & $\mathrm{Pd}(\mathrm{NO} 3) 2$ \\
\hline 110 & $\mathrm{PdO}$ \\
\hline 76 & $\mathrm{PuO} 2$ \\
\hline 156 & $\mathrm{PuO} 2(\mathrm{NaTi} 205) 2$ \\
\hline 58 & $\mathrm{Rh}$ \\
\hline 22 & RhO2 \\
\hline 95 & Ru \\
\hline 79 & $\mathrm{RuO} 2$ \\
\hline & RuO4 \\
\hline
\end{tabular}
to MWWT

$$
\begin{gathered}
\text { Vent } \\
0 . \\
0 . \\
0 . \\
0 . \\
0 . \\
0 . \\
0 .
\end{gathered}
$$

ZWRO
$0.1759 \mathrm{E}-05$
ZERO
$0.2251 \mathrm{E}-22$
$0.1451 \mathrm{E}-20$

$0.1451 \mathrm{E}-20$

$0.2999 \mathrm{E}-17$
ZERO

ZERO

0.3897 E-17

ZERO

ZERO

$0.1196 \mathrm{E}-18$

ZERO
$7 \mathrm{E}-20$

0.1577 E-20
ZERO

ZERO

$0.4801 \mathrm{E}-18$

$0.6095 \mathrm{E}-20$
$0.2094 \mathrm{E}-19$

$0.2094 \mathrm{E}-19$

$0.3353 \mathrm{E}-23$

$0.1813 \mathrm{E}-20$

$0.5527 \mathrm{E}-17$

ZERO

ZERO

ZERO

ZERO

$0.1544 \mathrm{E}-19$

$0.4086 \mathrm{E}-24$

$0.1457 \mathrm{E}-18$
$0.3303 \mathrm{E}+00$

$0.3303 \mathrm{E}+00$
$0.4758 \mathrm{E}-22$

ZERO
TRACE

ZERO
$0.8074 E-19$

$0.5525 \mathrm{E}-16$

$0.1364 \mathrm{E}-13$ TRACE

$0.6068 \mathrm{E}-20$

$0.7774 \mathrm{E}-22$

$0.1053 \mathrm{E}-15$
$0.1575 \mathrm{E}-13$

$0.4973 \mathrm{E}-20$

ZERO
ZERO

0)

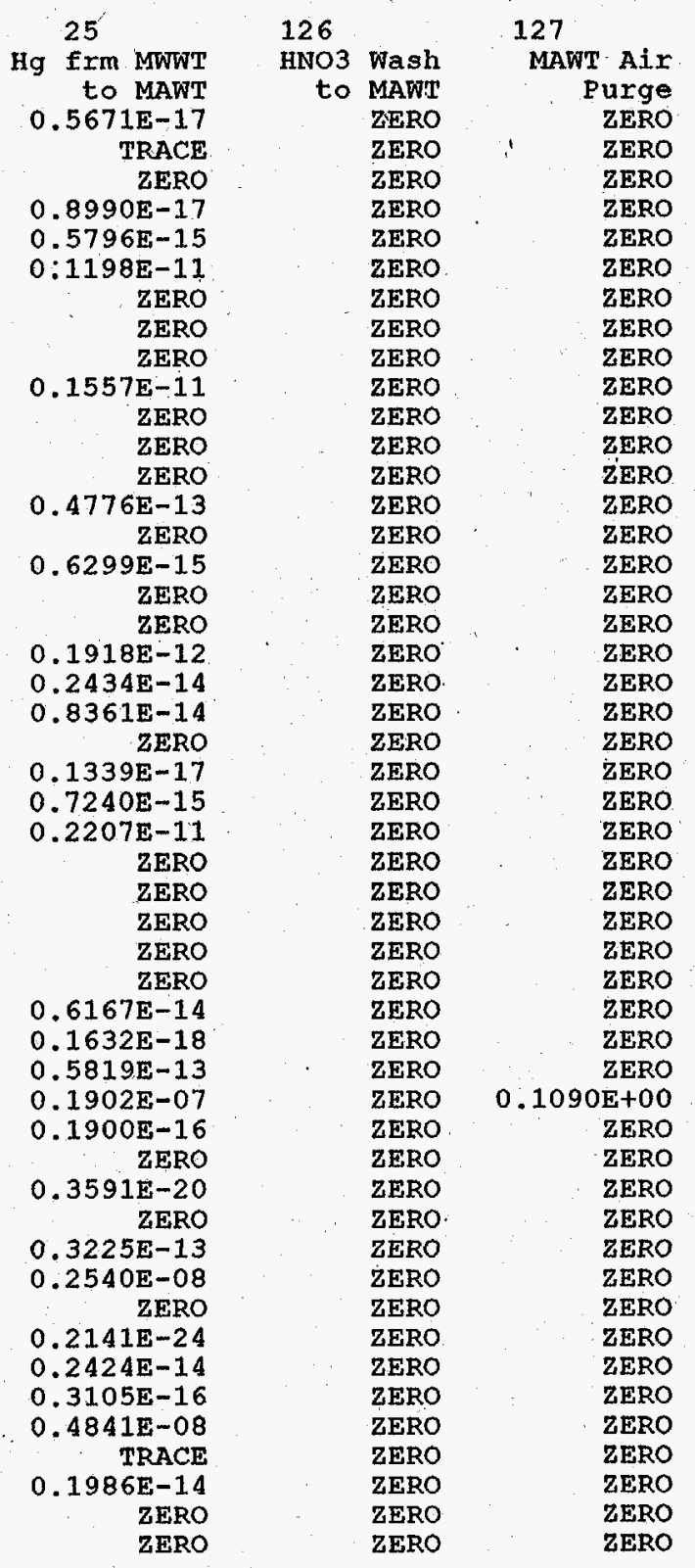

Date: January 1995
$128 \quad 129$

Air Sparge HNO3 Wsh frm to MAWT MAWT to SWT ZERO ZERO ZERO $0.2406 \mathrm{E}-11$

$0.8355 E-17$

ZERO $\quad 0.5386 \mathrm{E}-15$

ZFRO

ZTRO

ZIRO

ZERO

ZERO

ZERO

ZERO

ZERO

ZERO

ZERO

ZERO

ZERO

ZERO

ZERO

ZERO

ZERO

ZERO

ZERO

ZERO

ZERO

ZERO

ZERO

ZERO

ZERO

ZERO

ZERO

ZERO

ZERO

ZERO

$0.3426 \mathrm{E}-02$

ZERO

ZERO

ZERO

ZERO

ZERO

ZERO

ZERO

ZERO

ZERO

ZTRO

ZERO

ZERO

ZERO

ZERO

ZERO

$1113 \mathrm{E}-11$ ZERO $7 \mathrm{E}-11$ ZERO ZERO

$0.4438 \mathrm{E}-13$

ZERO

$\mathrm{E}-15$

ZERO

$0.1782 \mathrm{E}-12$

$0.2262 \mathrm{E}-14$

$0.7770 \mathrm{E}-14$

ZERO

$0.6729 \mathrm{E}-15$

$0.2052 \mathrm{E}-11$

ZERO

ZERO

ZERO

ZERO

$0.5732 \mathrm{~F}-14$

$0.1517 \mathrm{E}-18$

$0.5408 \mathrm{E}-13$

$0.2059 \mathrm{E}-06$

$0.1766 \mathrm{E}-16$

$0.3337 \mathrm{E}-20$

.2997E-13

$0.5054 \mathrm{E}-08$

$0.1990 \mathrm{E}-24$

$0.2252 \mathrm{E}-14$

$0.2885 \mathrm{E}-16$

ZERO

$0.5838 \mathrm{E}-08$

$0.1846 \mathrm{E}-14$

ZERO
ZERO
130

MAWT

Vent

$0.8613 \mathrm{E}-06$

ZERO

$0.1955 E-22$

$0.1260 \mathrm{E}-20$

$0.2605 \mathrm{E}-17$

ZERO

$0.3385 E-17$

ZERO

ZERO

$0.1039 \mathrm{E}-18$

$0.1370 \mathrm{ZERO}$

ZERO

$0.4170 \mathrm{E}-18$

$0.5294 \mathrm{E}-20$

$0.1818 \mathrm{E}-19$ ZERO

$0.2912 \mathrm{E}-23$

$0.1575 \mathrm{E}-20$

$0.4800 \mathrm{E}-17$ ZERO

ZERO

ZERO

ZERO

$0.1341 \mathrm{E}-19$

$0.3549 \mathrm{E}-24$

$0.1265 \mathrm{E}-18$

$0.1124 \mathrm{E}+00$

$0.4132 \mathrm{E}+22$

ZERO

ZERO

$0.7012 \mathrm{E}-19$

$0.5525 \mathrm{E}-16$

$0.1183 \mathrm{E}-13$

0.527 TRACE

$0.5270 \mathrm{E}-20$

$0.6752 \mathrm{E}-22$

$0.1053 \mathrm{E}-15$

$0.1366 \mathrm{E}-13$

$0.4319 \mathrm{E}-20$

ZERO
ZERO 
Table A-1. HLW Flowsheet Material Balance (Tank 51/ITP Cycle 1) section 6. Mercury Purification Cell

STREAM NUMBERS (CONT'D) $\rightarrow$ STREAM NAME

$38 \mathrm{SO} 2$

137 Semi Vol Cs2O

136 Semi Vol CsCl

126 Semi Vol Group A

140 Semi Vol Na2B4O7

138 Semi Vol NaCl

139 Semi Vol NaF

125 Semi Vol NaI

23 Semi Vol RuO2

$111 \mathrm{SiO} 2$

$48 \mathrm{Sr}(\mathrm{COOH}) 2$

$176 \mathrm{Sr}$ (NO3) 2

$153 \mathrm{sr}(\mathrm{NaTi} 2$
$127 \mathrm{sx}(\mathrm{OH}) 2$

$\begin{array}{rl}127 & \mathrm{Sr}(\mathrm{OH}) \\ 30 & \mathrm{SrCO} 3\end{array}$

46 SrO

$159 \mathrm{TCO} 2$

92 Tho2

107 TiO2

119 Tritium

17 v308

$62 \mathrm{VO} 2$ ( $\mathrm{COOH}) 2$

$177 \mathrm{UO2}$ (NO3) 2

$16 \mathrm{UO} 2$ (OH) 2

$27 \mathrm{Y}(\mathrm{COOH})$

$178 \mathrm{Y}(\mathrm{NO} 3)$

$\begin{array}{rl}128 & \mathrm{Y}(\mathrm{OH}) 3 \\ 31 & \mathrm{Y} 2(\mathrm{CO} 3) \\ 4\end{array}$

$47 \Upsilon 203$

100 zeolite

$44 \mathrm{Zn}(\mathrm{COOH}) 2$

$179 \mathrm{Zn}(\mathrm{NO}) 2$

57 Zno

13 hydrate $\mathrm{H} 2 \mathrm{O}$

$$
1 \text { water }
$$

TOTAL FLOW, LB/HR

TEMPERATURE, DEG C

PRESSURE; ATM

PRESSURE， PSIG

PRESSURE， MM HG

ENTHALPY, PCU/HR

VAPOR FLOW, CFM

IIQUID FLOW, GPM

DENSITY， IAS/FT3

PHASE
19

Hg Cell

MWW

ZERO

ZERO

ZERO

ZERO

ZERO

ZERO

ZERO

$0.1954 \mathrm{E}-16$

$0.6678 \mathrm{E}-22$

$0.1803 \mathrm{E}-19$

$0.2489 E-20$

ZERO

ZERO

TRACE

$0.2187 \mathrm{E}-20$

$0.1821 \mathrm{E}-19$

$0.5079 \mathrm{E}-18$

$0.2125 \mathrm{E}-16$

$0.1277 \mathrm{E}-17$

$0.7774 \mathrm{E}-24$

$0.5598 \mathrm{E}-22$

ZERO

TRACE

$$
\text { ZERO }
$$

ZERO

$0.5917 \mathrm{E}-21$

ZERO

$0.1595 \mathrm{E}-18$

$0.3261 \mathrm{E}-22$

$0.1097 \mathrm{E}-18$

$0.9185 \mathrm{E}-18$

$0.4899 \mathrm{E}-01$

$0.1478 \mathrm{E}+01$

$0.3800 \mathrm{E}+02$

$0.9950 \mathrm{E}+00$

$-0.7348 \mathrm{E}-01$

$0.7562 \mathrm{E}+03$

$0.4745 \mathrm{E}+02$

$0.3572 \mathrm{E}+00$

$0.6899 \mathrm{E}-01$
25

fg frm MWWT

to MAWT

ZERO

ZERO

ZERO

ZERO

ZERO

ZERO

ZERO

ZERO

$0.2667 \mathrm{E}-16$

$0.7203 \mathrm{E}-14$

$0.9939 \mathrm{E}-15$ ZERO

$0.1124 \mathrm{E}-19$

$0.8733 \mathrm{E}-15$

$0.7273 \mathrm{E}-14$

$0.2029 \mathrm{E}-12$

$0.4162 \mathrm{E}-16$

$0.5099 \mathrm{E}-12$

$0.3105 \mathrm{E}-18$

ZERO

$0.1943 \mathrm{E}-14$ ZERO

ZERO

ZERO

$0.6368 \mathrm{E}-13$

$0.1302 \mathrm{E}-16$

$0.4383 \mathrm{E}-13$

$0.366 \mathrm{BE}-12$

$0.3891 \mathrm{E}-02$

$0.2554 \mathrm{E}+00$

$0.3500 E+02$

$0.1000 \mathrm{E}+01$

7ERO

$0.4265 \mathrm{E}+00$

$0.4484 \mathrm{E}-04$

$0.7099 \mathrm{E}+03$
126

HNO3 Wash

to MAWT

ZERO

ZERO

ZERO

ZERO

ZERO

ZERO

ZERO

ZERO

ZERO

ZERO

ZERO

ZERO

ZERO

ZERO

ZERO

ZERO

ZERO

ZERO

ZERO

ZERO

ZERO

ZERO

ZERO

ZERO

ZERO

ZERO

ZERO

ZERO

ZERO

ZERO

ZERO

ZERO

ZERO

ZERO

$0.3392 \mathrm{E}-01$

$0.3855 \mathrm{E}-01$

$0.2500 \mathrm{E}+02$

$0.1000 \mathrm{E}+01$

ZERO

$0.8842 \mathrm{E}+00$

$0.7389 \mathrm{E}-04$

$0.6504 \mathrm{E}+02$

IIQUID
127

MADT Air 128

129

to MAWT MAWT to SWT

ZERO

ZERO

ZERO

ZERO

ZERO

ZERO

ZERO

ZERO

ZERO

ZERO

ZERO

ZERO

ZERO

ZERO

ZERO

ZERO

ZERO

ZERO

ZERO

ZERO

ZERO

ZERO

ZERO

ZERO

ZERO

ZERO

ZERO

ZERO

ZERO

ZERO

ZERO

ZERO

$0.7891 \mathrm{E}-02$

$0.4776 \mathrm{E}+00$

$0.3500 \mathrm{E}+02$

$0.1000 \mathrm{E}+01$

ZERO

$0.1002 E+02$

$0.1128 \mathrm{E}+00$

$0.7055 \mathrm{E}-01$

VAPOR
ZERO

7ERO

ZERO

ZERO

ZERO

ZERO

ZERO

ZERO

ZERO

ZERO

ZERO

ZERO

ZERO

ZERO

ZERO

ZERO

IERO

ZERO

ZERO

ZERO

ZERO

ZERO

ZERO

ZERO

ZERO

ZERO

ZERO

ZERO

ZERO

ZERO

ZERO

ZERO

ZERO

ZERO

ZERO

ZERO

$0.2481 \mathrm{E}-03$

$0.1501 \mathrm{E}-01$

$0.3500 \mathrm{E}+02$

$0.1000 \mathrm{E}+01$

ZERO

0. $3149 \mathrm{E}+00$

$0.3547 \mathrm{E}-02$

$0.7055 \mathrm{E}-01$

VAPOR
ZERO

ZERO

ZERO

ZERO

ZERO

ZERO 
Table A-1. HLW Flowsheet Material Balance (Tank 51/ITP Cycle 1) Section 6. Mercury Purification Cell

STREAM NUMBERS $\rightarrow$ STREAM NAME

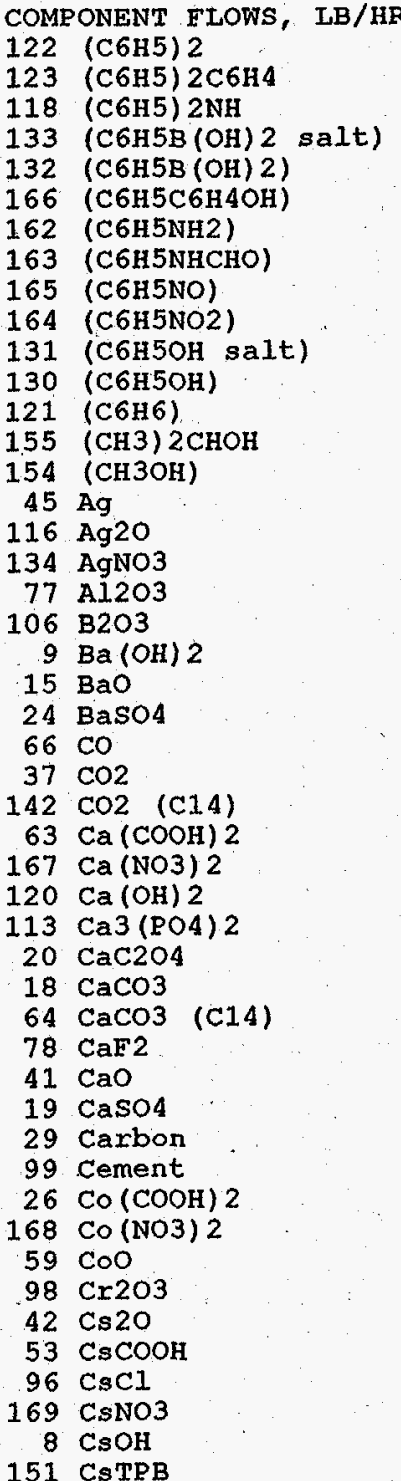

WSRC-TR-95-0019 (Revision
Hg Feed to MASC 132
HNO3 HNO3 Wash

$0.1120 \mathrm{E}-11$ $0.1719 \mathrm{E}-14$ $0.2430 \mathrm{E}-10$ ZERO

ZERO $0.9527 \mathrm{E}-15$ $0.2468 \mathrm{E}-09$ $0.3611 \mathrm{E}-1.4$ $0.2795 \mathrm{E}-17$ $0.1184 \mathrm{E}-15$ $0.3029 \mathrm{E}-17$ $0.3016 \mathrm{E}-0.8$ $0.6093 \mathrm{E}-12$ $0.9578 \mathrm{E}-19$ $0.4505 \mathrm{E}-20$ $0.8251 \mathrm{a}-10$ $0.4588 \mathrm{E}-24$ $0.1527 \mathrm{E}-12$ $0.5534 \mathrm{E}-13$ $0.5792 \mathrm{E}-22$ $0.2944 \mathrm{E}-15$ ZERO RACE

$0.7238 \mathrm{E}-13$ ZERO $0.4536 \mathrm{E}-13$ $0.4211 \mathrm{E}-20$ ZERO

$0.2594 \mathrm{E}-15$ $0.5787 \mathrm{E}-16$ $0.3808 \mathrm{E}-14$ ZERO

$0.2470 \mathrm{E}-17$

$0.9265 \mathrm{E}-20$

$0.5292 \mathrm{E}-1 \mathrm{~B}$

$0.7408 \mathrm{E}-14$

$0.7408 \mathrm{E}-14$

$0.2425 \mathrm{E}-17$

$0.7466 \mathrm{E}-15$
ZERO

$0.1661 \mathrm{E}-18$

$0.1894 \mathrm{E}-19$ ZERO 133
HNO3 from MASC

\begin{tabular}{|c|c|}
\hline ZERO & $0.1115 \mathrm{E}-11$ \\
\hline $\begin{array}{l}\text { ZERO } \\
\text { ZERO }\end{array}$ & $\begin{array}{l}0.1711 \mathrm{E}-14 \\
0.2419 \mathrm{E}-10\end{array}$ \\
\hline ZERO & ZERO \\
\hline ZERO & ZERO \\
\hline ZERO & $0.9482 \mathrm{E}-15$ \\
\hline ZERO & $0.2456 \mathrm{E}-09$ \\
\hline ZERO & $0.3594 \mathrm{E}-14$ \\
\hline ZERO & $0.2782 E-17$ \\
\hline ZERO & $0.1178 \mathrm{E}-15$ \\
\hline ZERO & $0.3015 \mathrm{E}-17$ \\
\hline ZERO & $0.3002 \mathrm{E}-08$ \\
\hline ZERO & $0.6064 \mathrm{E}-12$ \\
\hline ZERO & $0.9532 \mathrm{E}-19$ \\
\hline ZERO & $0.4483 \mathrm{E}-20$ \\
\hline ZERO & ZERO \\
\hline ZERO & $0.4566 \mathrm{E}-24$ \\
\hline ZERO & $0.1026 \mathrm{E}-07$ \\
\hline ZERO & $0.1520 \mathrm{E}-12$ \\
\hline ZERO & $0.5507 \mathrm{E}-13$ \\
\hline ZERO & ZERO \\
\hline ZERO & $0.5764 \mathrm{E}-22$ \\
\hline ZERO & $0.2930 \mathrm{E}-15$ \\
\hline ZERO & $\therefore \quad$ ZERO \\
\hline ZERO & $0.5823 E-19$ \\
\hline ZERO & TRACE \\
\hline ZERO & ZERO \\
\hline ZERO & $0.7204 \mathrm{E}-13$ \\
\hline ZERO & ZERO \\
\hline ZERO & $0.4515 \mathrm{E}-13$ \\
\hline ZERO & $0.4191 \mathrm{E}-20$ \\
\hline ZERO & ZERO \\
\hline ZERO & ZERO \\
\hline ZERO & $0.2582 \mathrm{E}-15$ \\
\hline ZERO & $0.5759 \mathrm{E}-16$ \\
\hline ZERO & $0.3790 \mathrm{E}-14$ \\
\hline ZERO & ZERO \\
\hline ZERO & ZERO \\
\hline ZERO & $0.2459 \mathrm{E}-17$ \\
\hline ZERO & $0.9221 \mathrm{E}-20$ \\
\hline ZERO & $0.5267 \mathrm{E}-18$ \\
\hline ZERO & $0.7373 \mathrm{E}-14$ \\
\hline ZERO & $0.2413 \mathrm{E}-17$ \\
\hline ZERO & $0.7431 \mathrm{E}-1.5$ \\
\hline ZERO & ZERO \\
\hline ZERO & $0.1653 \mathrm{E}-18$ \\
\hline ZERO & $0.1885 \mathrm{E}-19$ \\
\hline ZERO & ZERO \\
\hline
\end{tabular}

1.34
Hg

Hg Feed

$0^{\prime} .5335 \mathrm{E}-14$

$0.8184 \mathrm{E}-17$

$0.1157 \mathrm{E}-12$ ZERO

$0.4537 \mathrm{E}-17$

$0.4537 \mathrm{E}-17$

$0.1175 \mathrm{E}-11$

$0.1720 \mathrm{E}-16$

$0.1331 \mathrm{E}-19$

$0.5636 \mathrm{E}-18$

$0.1443 \mathrm{E}-19$

$0.1436 \mathrm{E}-10$

$0.2901 \mathrm{E}-14$

$0.4561 \mathrm{E}-21$

$0.2145 \mathrm{E}-22$

$0.8251 \mathrm{E}-11$

TRACE

$0.4911 \mathrm{E}-10$

$0.7271 \mathrm{E}-15$

$0.2635 \mathrm{E}-15$

$0.2758 \mathrm{E}-24$

$0.1402 \mathrm{E}-17$

ZERO

TRACE

$0.3447 \mathrm{E}-15$

ZERO

$0.2160 \mathrm{E}-15$

$0.2005 \mathrm{E}-22$

ZERO
ZERO

$0.1235 \mathrm{E}-17$

$0.2756 \mathrm{E}-18$

$0.1813 \mathrm{E}-16$ ZERO

$0.1176 \mathrm{E}-19$

$0.4412 \mathrm{E}-22$

$0.2520 \mathrm{E}-20$

$0.3528 \mathrm{E}-16$

$0.1155 \mathrm{E}-19$

$0.1155 \mathrm{E}-19$

$0.3555 \mathrm{E}-17$

$0.7908 \mathrm{E}-21$

$0.9018 \mathrm{E}-22$ to Wash

$$
\begin{aligned}
& \text { ZERO } \\
& \text { ZERO } \\
& \text { ZERO } \\
& \text { ZERO } \\
& \text { ZERO } \\
& \text { ZERO } \\
& \text { ZERO } \\
& \text { ZERO } \\
& \text { ZERO } \\
& \text { ZERO } \\
& \text { ZERO } \\
& \text { ZERO } \\
& \text { ZERO } \\
& \text { ZERO } \\
& \text { ZERO } \\
& \text { ZERO } \\
& \text { ZERO } \\
& \text { ZERO } \\
& \text { ZERO } \\
& \text { ZERO } \\
& \text { ZERO } \\
& \text { ZERO } \\
& \text { ZERO } \\
& \text { ZERO } \\
& \text { ZERO } \\
& \text { ZERO } \\
& \text { ZERO } \\
& \text { ZERO } \\
& \text { ZERO } \\
& \text { ZERO } \\
& \text { ZERO } \\
& \text { ZERO } \\
& \text { ZERO } \\
& \text { ZERO } \\
& \text { ZERO } \\
& \text { ZERO } \\
& \text { ZERO } \\
& \text { ZERO } \\
& \text { ZERO } \\
& \text { ZERO } \\
& \text { ZERO } \\
& \text { ZERO } \\
& \text { ZERO } \\
& \text { ZERO } \\
& \text { ZERO } \\
& \text { ZERO } \\
& \text { ZERO } \\
& \text { ZERO } \\
&
\end{aligned}
$$

136

fater Wash

$0.5308 \mathrm{E}-14$

$0.8144 \mathrm{E}-17$

$0.1152 \mathrm{E}-12$ ZERO

$0.4514 \mathrm{E}-17$

$0.1169 \mathrm{E}-11$

$0.1711 \mathrm{E}-16$

$0.1324 \mathrm{E}-19$

$0.5608 \mathrm{E}-18$

$0.1435 \mathrm{E}-19$

$0.142 .9 \mathrm{E}-10$

$0.2887 \mathrm{E}-14$

$0.4538 \mathrm{E}-21$

$0.2134 \mathrm{E}-22$

ZERO

$0.4886 \mathrm{E}-10$

$0.7235 \mathrm{E}-15$

$0.2622 \mathrm{E}-15$

$0.2744 \mathrm{E}-24$

$0.1395 \mathrm{E}-17$

$0.2772 \mathrm{E}-21$

TRACE

ZERO

$0.3430 \mathrm{E}-15$

ZERO

$0.1995 \mathrm{E}-22$

$0.1995 \mathrm{E}-22$ ZERO

$0.1229 \mathrm{E}-17$

$0.2742 \mathrm{E}-18$

$0.1804 \mathrm{E}-16$ ZERO

$0.1170 \mathrm{E}-19$

$0.4390 \mathrm{E}-22$

0. 2507F-20

$0.3510 \mathrm{E}-16$

$0.1149 \mathrm{E}-19$

$0.1149 \mathrm{E}-19$

$0.3538 E-17$
ZERO

$0.7868 \mathrm{E}-21$

$0.8973 \mathrm{E}-22$ ZERO
137

Washed Hg

$0.2654 \mathrm{E}-16$ $0.4072 \mathrm{E}-19$ $0.5758 \mathrm{E}-15$ ZERO $0.2257 \mathrm{E}-19$ $0.2257 \mathrm{E}-19$ $0.5846 \mathrm{E}-14$ $0.8555 \mathrm{E}-19$ $.6622 \mathrm{E}-22$ $0.2804 \mathrm{E}-20$ $0.7177 \mathrm{E}-22$ $0.7145 \mathrm{E}-13$ $0.1443 \mathrm{E}-16$ $0.1067 \mathrm{E}-23$ $0.106 \mathrm{E}-24$ TRACE $0.2443 \mathrm{E}-12$ $0.3617 \mathrm{E}-17$ $0.1311 \mathrm{E}-17$ TRACE $0.6975 E-20$ $0.1386 \mathrm{E}-23$ TRACE $0.1715 \mathrm{E}-17$ ZERO $0.1075 \mathrm{E}-17$ TRACE ZERO $0.6146 \mathrm{E}+20$ $0.1371 \mathrm{E}-20$ $0.9022 \mathrm{E}-19$ ZERO $0.5852 E-22$ $0.2195 \mathrm{E}-24$ $0.1755 \mathrm{E}-18$ $0.5744 \mathrm{E}-22$ ZERO $0.4487 \mathrm{E}-24$ ZERO 
Table A-1. HLW Flowsheet Material Balance (Tank 51/ITP Cycle 1) Section 6. Mercury Purification Cell

STREAM NUMBERS (CONT'D) $\rightarrow$ STREAM NAME

$54 \mathrm{Cu}(\mathrm{COOH}) 2$

$170 \mathrm{Cu}(\mathrm{NO} 3) 2$

$55 \mathrm{CuO}$

$80 \mathrm{Fe} 2 \mathrm{O}$
$101 \mathrm{FeO}$

102 Group A

103 Group B

25 H2

$158 \mathrm{H} 2 \mathrm{C} 2 \mathrm{O} 4$

$93 \mathrm{H} 2 \mathrm{SO} 4$

$90 \mathrm{H} 3 \mathrm{BO}$

34 НCOOH

$43 \mathrm{HCl}$

49 HNO3

$39 \mathrm{Hg}$

$129 \mathrm{Hg}(\mathrm{C} 6 \mathrm{H} 5) 2$

$135 \mathrm{Hg}$ (NO3) 2

$144 \mathrm{Hg} 2 \mathrm{Cl} 2$

$117 \mathrm{Hg} 2 \mathrm{I2}$

$146 \mathrm{HgCl}$

$21 \mathrm{Hg}$

$183 \mathrm{~K} 2 \mathrm{CO} 3$

$39 \times 20$

$51 \mathrm{KCOOH}$

$157 \mathrm{KMnO4}$

$10 \mathrm{KOH}$

$10 \mathrm{KOH}$

$105 \mathrm{Ia} 203$

$108 \mathrm{Li} 20$

$65 \mathrm{Mg}(\mathrm{COOH}) 2$

$171 \mathrm{Mg}$ (NO3) 2

$33 \mathrm{MgO}$

$60 \mathrm{Mn}(\mathrm{COOH}) 2$

$172 \mathrm{Mn}(\mathrm{NO} 3) 2$

$180 \mathrm{Mn} 304$

$56 \mathrm{MnO}$

$14 \mathrm{MnO} 2$

$145 \mathrm{MoO} 2$

$81 \mathrm{~N} 2$

$74 \mathrm{~N} 2 \mathrm{O}$

114 NH3OHNO3

52. $\mathrm{NH} 4 \mathrm{COOH}$

173 NH4NO3

$68 \mathrm{NH} 4 \mathrm{OH}$ 131
Hg Feed MASC
$0.7656 \mathrm{E}-16$ $0.3762 \mathrm{E}-13$ $0.3702 \mathrm{1}$ $0.4388 \mathrm{E}-12$ $0.43875-1$ $0.438 \mathrm{E}-13$ $0.2613 \mathrm{E}-17$ $0.3158 \mathrm{E}-14$ ZERO
$0.1329 \mathrm{E}-12$ ZERO ZERO ZERO

$0.9470 E-04$ $0.2540 \mathrm{E}+00$

ZERO
$0.2033 E-06$ ZERO

$0.3405 \mathrm{E}-19$ ZERO

$0.1395 \mathrm{E}-14$ $0.1732 \mathrm{E}-12$ ZERO $0.4940 \mathrm{E}-15$ $0.5664 \mathrm{E}-18$ ZERO ZERO

$0.4840 \mathrm{E}-13$ $0.5986 \mathrm{E}-13$ $0.9059 \mathrm{E}-21$ $0.1910 \mathrm{E}-13$ $0.3749 \mathrm{E}-13$ $0.1056 \mathrm{E}-20$ ZERO $0.1372 \mathrm{E}-18$ $0.3367 \mathrm{E}-13$ $0.1858 \mathrm{E}-16$ $0.2666 \mathrm{E}-07$ $0.3432 \mathrm{E}-15$ ZERO $0.4646 \mathrm{E}-08$ $0.5354 \mathrm{E}-15$
ZERO

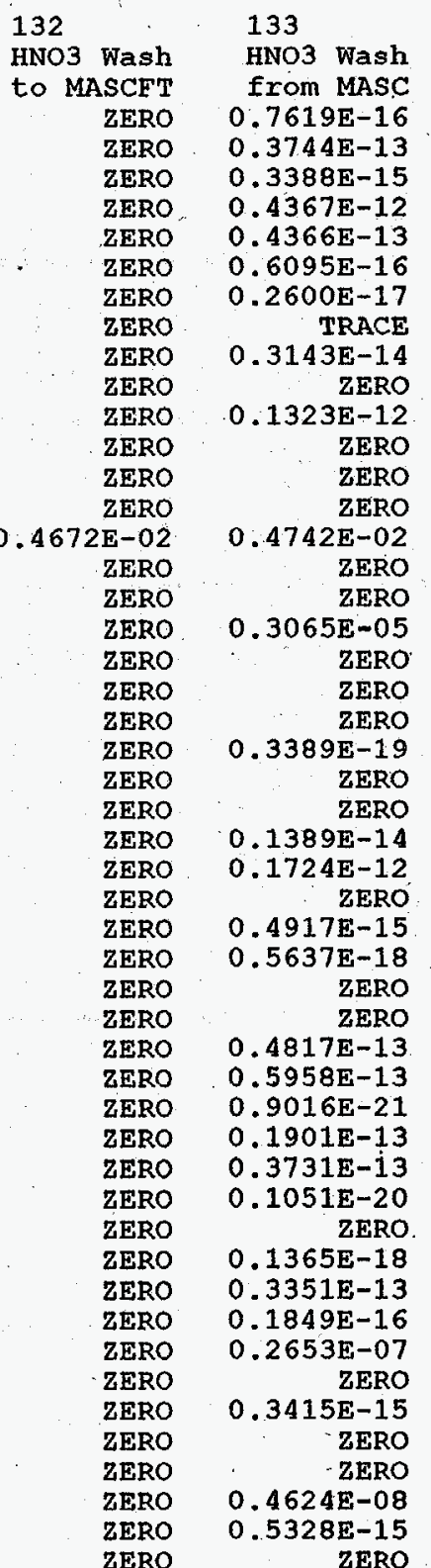

134 to MWWC 135 0 to MWW $0.1791 \mathrm{E}-15$ $0.1621 \mathrm{E}-17$ . 1621E-17 $0.2089 \mathrm{E}-14$ 0.2089 $0.2916 \mathrm{E}-18$ $0.1244 \mathrm{E}-19$ $0.1504 \mathrm{E}-16$ $0.6328 \mathrm{E}-15$ ZERO ZERO ZERO $0.2269 \mathrm{E}-04$ $0.2540 E+00$
ZERO $0.1467 \mathrm{E}-07$ ZERO ZERO $0.1621 \mathrm{E}-21$ ZERO $0.6644 \mathrm{E}-17$ $0.8247 \mathrm{E}-15$ ZERO $0.2352 \mathrm{E}-17$ $0.2697 \mathrm{E}-20$
ZERO ZERO $0.2305 \mathrm{E}-15$ $0.2850 \mathrm{E}-15$ $0.4314 \mathrm{E}-23$ $0.9094 \mathrm{E}-16$ $0.1785 \mathrm{E}-15$ $0.5026 \mathrm{E}-23$

$0.6532 \mathrm{E}-21$

$0.1603 \mathrm{E}-15$

$0.16038-15$

$0.8848 \mathrm{E}-19$

$0.1270 \mathrm{E}-09$ $0.1634 \mathrm{E}-17$ ZERO $0.2212 \mathrm{E}-10$ $0.2549 \mathrm{E}-17$ to MWWC

ZERO

ZERO

ZERO

ZRRO

ZERO

ZERO

ZERO

ZERO

ZERO

ZERO

ZERO

ZERO

ZERO

ZERO

ZERO

ZERO

ZERO

ZERO

ZERO

ZERO

ZERO

ZERO

ZERO

ZERO

ZERO

ZERO

ZERO

ZERO

ZERO

ZERO

ZERO

ZERO

ZERO

ZERO

ZERO

ZERO

ZERO

ZERO

ZERO

ZERO

ZERO

ZERO

ZERO

ZERO

ZERO

ZERO

ZERO

ZERO

ZERO
136

Water Wash

from MWWC

$0.3627 \mathrm{E}-18$

$0.1782 \mathrm{E}-15$

$0.1613 \mathrm{E}-17$

$0.2079 \mathrm{E}-14$

$0.2078 \mathrm{E}-15$

$0.2902 \mathrm{E}-18$

$0.1238 \mathrm{E}-19$

TRACE

$1496 \mathrm{E}-16$
ZERO

$.6296 \mathrm{E}-15$ ZERO ZERO

$0.2258 \mathrm{E}-04$ ZERO

ZERO

$0.1459 \mathrm{E}-07$ ZERO ZERO

$0.1613 \mathrm{E}-21$ ZERO

$0.6611 \mathrm{E}-17$

$0.8206 \mathrm{E}-15$ ZERO

$0.2341 \mathrm{E}-17$

$0.26845-20$ ZERO

0.22935- 15

$0.2836 \mathrm{E}-15$

$0.4292 \mathrm{E}-23$

$0.9049 \mathrm{E}-16$

$0.1776 \mathrm{E}-15$

$.5001 \mathrm{E}-23$

$0.6499 \mathrm{E}-21$

$0.1595 \pi-15$

$0.8804=19$

$0.1263 \mathrm{E}-09$

- 1626 ERO

ZERO

ZERO

$0.2201 \mathrm{E}-10$

0.2537 E-17
ZERO
137

Washed $\mathrm{Hg}$

from MWWC

$0.8912 \mathrm{E}-18$

$0.8064 \mathrm{E}-20$

$.1039 \mathrm{E}-16$

$.1039 \mathrm{E}-17$

$0.1451 E-20$

$0.1451 \mathrm{E}-20$

TRACE

$0.7481 \mathrm{E}-19$

$0.3148 \mathrm{E}-17$

ZERO

ZERO

ZERO

$0.1129 \mathrm{E}-06$

$0.2540 \mathrm{E}+00$.

ZERO
$0.7297 \mathrm{E}-10$

ZERO

ZERO

$0.8067 \mathrm{E}-24$

ZERO

$0.3306 \mathrm{E}-19$

$0.4103 \mathrm{E}-17$

ZERO

$0.1342 \mathrm{E}-2$ ZERO

- $147 \mathrm{E}-17$

$0.1147 \mathrm{E}-17$

TRACE

$8882 \mathrm{E}-18$

ZERO

$0.7977 \mathrm{E}-18$

$0.4402 \mathrm{E}-2$

$0.4402 \mathrm{E}-21$

ZERO

$0.8130 \mathrm{E}-20$

ZERO

$0.1101 \mathrm{E}-12$

$0.1268 \mathrm{E}-19$ 
Table A-1. HitW Flowsheet Material Balance (Tank 51/ITP Cycle 1) section 6. Mercury purification Cell

STREAM NUMBERS (CONT'D) $\rightarrow$

35 NO

84 NO2

$70 \mathrm{Na}(\mathrm{HgO}(\mathrm{OH}))$

$94 \mathrm{Na} 2 \mathrm{~B} 4 \mathrm{O}$

$109 \mathrm{Na} 2 \mathrm{C} 2 \mathrm{O} 4$
$5 \mathrm{Na} 2 \mathrm{CO} 3$

$143 \mathrm{Na2CO} 3$ (C14)

$89 \mathrm{Na} 2 \mathrm{CrO} 4$

$115 \mathrm{Na} 2 \mathrm{MOO} 4$

$32 \mathrm{Na} 2 \mathrm{O}$

$71 \mathrm{Na} 2 \mathrm{PuO} 2(\mathrm{OH}) 4$

73 Na2RhO4

$72 \mathrm{Na2RuO4}$

$6 \mathrm{Na} 2 \mathrm{SO} 4$

$88 \mathrm{Na2SiO} 3$

$87 \mathrm{Na} 3 \mathrm{PO} 4$

$91 \mathrm{NaAg}(\mathrm{OH}) 2$

$75 \mathrm{NaAl}(\mathrm{OH})$
$50 \mathrm{NaCOOH}$.

$7 \mathrm{NaCl}$

$69 \mathrm{NaF}$

$11 \mathrm{NaHCO} 3$

12 NaI

$\begin{array}{ll}3 & \mathrm{NaNO} 2 \\ 2 & \mathrm{NaNO} 3 \\ 4 & \mathrm{NaOH}\end{array}$

149 NaTPB

$160 \mathrm{NaTcO} 4$

$152 \mathrm{NaTi2O}$

$\begin{array}{rl}181 & \mathrm{Nd} 2 \mathrm{O} 3 \\ 61 \mathrm{Ni}(\mathrm{COOH}) 2\end{array}$

$174 \mathrm{Ni}(\mathrm{NO3}) 2$

$40 \mathrm{NiO}$

82 ०2

$175 \mathrm{~Pb}$ (NO3) 2

$86 \mathrm{pbCO} 3$

$67 \mathrm{PbO}$

$147 \mathrm{PbS}$

$28 \mathrm{PbSO}$

$112 \mathrm{Pd}$ (NO3) 2

$110 \mathrm{PdO}$

76 PuO2

156 PuO2 (NaTi2O5) 2

$58 \mathrm{Rh}$

22 RhO2

$95 \mathrm{Ru}$

79. RuO2

131
Hg Feed to

MASC
ZERO
$0.1829 \mathrm{E}-12$

HNO3

ASC

to MASCET

$0.6350 \mathrm{E}-1.8$

$0.4094 \mathrm{E}-16$

$0.8461 \mathrm{E}-13$ ZERO ZERO

$0.1100 \mathrm{E}-12$ ZERO ZERO

$0.3374 \mathrm{E}-14$ ZERO

$0.4450 \mathrm{E}-16$ ZERO

ZERO

$0.1355 \mathrm{E}-13$

$0.1720 \mathrm{E}-15$
$0.5906 \mathrm{E}-15$

$0.9460 \mathrm{E}-19$

$0.5114 \mathrm{E}-16$

$0.1559 \mathrm{E}-12$

ZERO

ZERO

ZERO

ZERO

$0.4356 \mathrm{E}-15$

$0.1153 \mathrm{E}-19$

$0.4111 \mathrm{E}-14$

$0.1565 \mathrm{E}-07$

$0.1342 \mathrm{E}-17$

$0.2537 \mathrm{E}-21$

ZERO

$0.2278 \mathrm{E}-14$

$0.2540 \mathrm{E}-10$

$0.3842 \mathrm{E}-09$

TRACE

$0.1712 \mathrm{E}-15$

$0.2193 \mathrm{E}-17$

$0.4841 \mathrm{E}-10$

$0.4438 \mathrm{E}-09$

$0.1403 \mathrm{E}-15$

ZERO

WSRC-TR-95-0019 (Revision
133

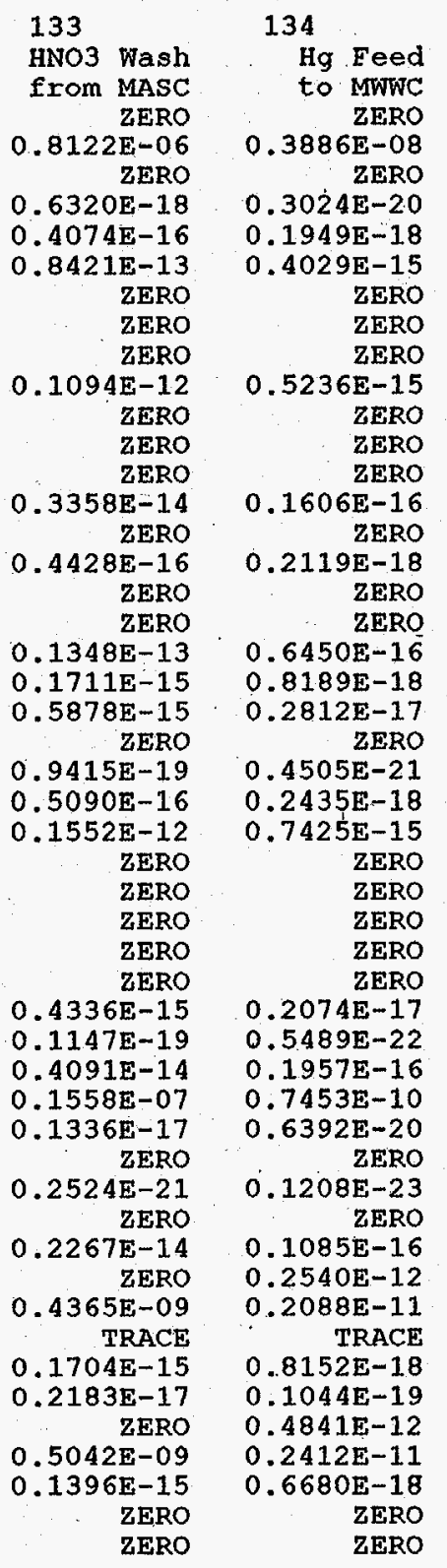

Date: January 1995
135 to MWWC

$$
\begin{aligned}
& \text { ZERO } \\
& \text { ZERO }
\end{aligned}
$$

ZERO

ZERO

ZERO

ZERO

ZERO

ZERO

ZERO

ZERO

ZERO

ZERO

ZERO

ZERO

ZERO

ZERO

ZERO

ZERO

ZERO

ZERO

ZERO

ZERO

ZERO

ZERO

ZERO

ZERO

ZERO

ZERO

ZERO

ZERO

ZERO

ZERO

ZERO

ZERO

ZERO

ZERO

ZERO

ZERO

ZERO

ZERO

ZERO

ZERO

ZERO

ZERO
136

Water Wash

from MWWC

$0.3867 \mathrm{E}-08$

ZERO
3009E-20

$.194 \mathrm{OE}-18$

$0.4009 \mathrm{E}-15$
ZERO

ZERO

ZERO

$0.5210 \mathrm{E}-15$

ZERO

ZERO

$0.1598 \mathrm{E}-16$

ZERO

$0.2108 \mathrm{E}-18$

ZERO

6418E-16

$0.8148 \mathrm{E}-18$

$.2798 \mathrm{E}-17$ ZERO

$.4482 \mathrm{E}-21$

$0.2423 \mathrm{E}-18$

$0.7388 \mathrm{E}-15$

ZERO

ZERO

ZERO

ZERO

$0.2064 \mathrm{E}-17$

$0.5462 \mathrm{~F}-22$

$0.1948 \mathrm{E}-16$

$.7416 \mathrm{E}-10$

$0.6360 \mathrm{E}-20$

$0.1202 \mathrm{E}-23$

0.1079 ZERO $9 \mathrm{E}-16$
ZERO

$0.2078 \mathrm{E}-11$

TRACE

$0.8112 \mathrm{E}-18$

$0.1039 \mathrm{E}-19$ ZERO

$0.2400 \mathrm{E}-11$

$0.6647 \mathrm{E}-18$

ZERO
137

xom MWWC

$0.1933 \mathrm{E}-10$

$0.1504 \mathrm{E}-22$

0 150

$0.2004 \mathrm{E}-17$ ZERO ZERO

$0.2605 \mathrm{E}-17$ ZERO ZERO

$0.7992 \mathrm{E}-19$

0. 105ERO

ZERO

$0.3209 \mathrm{E}-18$

$0.4074 \mathrm{E}-20$

$0.1399 \mathrm{E}-19$

$0.2241 \mathrm{E}-23$

$0.1212 \mathrm{E}-20$

$0.3694 \mathrm{E}-17$ ZERO

ZERO

ZERO

ZERO

$-19$

$0.2731 \mathrm{E}-24$

$0.9739 \mathrm{E}-19$

$0.3708 \mathrm{E}-12$

$0.3180 \mathrm{E}+22$

ZERO
TRACE

ACE

$0.5396 \mathrm{E}-19$

$0.2540 \mathrm{OE}-12$

$0.1039 \mathrm{E}-13$

TRACE

$0.4056 \mathrm{E}-20$

$0.5196 \mathrm{E}-22$

$0.4841 \mathrm{E}-12$

$0.3323 E-20$
ZERO

8 of 17 
Table A-1. HLW Flowsheet Material Balance (Tank 51/ITP Cycle 1) Section 6. Mercury Purification Cell

STREAM NUMBERS (CONT'D) $\rightarrow$ STREAM NAME

\section{$38 \quad 502$}

141.503

137 Semi Vol Cs 20

136 Semi Vol CsC1

126 Semi Vol Group A

140 Semi Vol Na2B4O

138 Semi Vol NaCl

139 Semi Vol NaF

125 Semi Vol NaI

23 Semi Vol RuO2

$111 \mathrm{SiO} 2$

$48 \mathrm{Sr}(\mathrm{COOH}) 2$

153 Sr(NaTi2O5) 2

$127 \mathrm{Sr}(\mathrm{OH}) 2$

$30 \mathrm{srCO} 3$

46. Sro

159 TCO2

92 ThO2

107 TiO2

119 Tritium

17 U308

$62 \mathrm{OO2}(\mathrm{COOH}) 2$

$177 \mathrm{UO2}$ (NO3) 2

16. $\mathrm{UO2}(\mathrm{OH}) 2$

27
178 Y (NO3) 3

$178 \mathrm{Y}$ (NOH) 3

$31 \mathrm{Y} 2(\mathrm{CO} 3) 3$

$\begin{array}{ll}31 & \mathrm{Y} 2(\mathrm{CO} \\ 47 & \mathrm{O} 3\end{array}$

100 Zeolite

$44 \mathrm{Zn}(\mathrm{COOH}) 2$

$179 \mathrm{Zn}$ (NO3) 2

$57 \mathrm{zno}$

$104 \mathrm{zrO} 2$

13 hydrate $\mathrm{H} 2 \mathrm{O}$

1 water

TOTAT FIOW, IB/HR

TEMPERATURE, DEG C

PRESSURE, ATM

PRESSURE， PSIG

PRESSURE， MM HG

ENTHALPY， PCU/HR

VAPOR FLOW, CFM

LIQU ID FLOW，GPM

DENSITY, IBS/FT3

PHASE
131

Hg Feed to

MASC

ZERO

ZERO

ZERO

ZERO

ZERO

ZERO

ZERO

ZERO

TERO

$0.5512 \mathrm{E}-12$

$0.1884 \mathrm{E}-17$

$0.5088 \mathrm{E}-15$

$0.7021 \mathrm{E}-16$

ZERO

ZERO

$0.7941 \mathrm{E}-21$

$0.6169 \mathrm{E}-16$

$0.5137 \mathrm{E}-15$

$0.1433 \mathrm{E}-13$

$0.1619 \mathrm{E}-17$

$0.3602 \mathrm{E}-13$

$0.21 .93 \mathrm{E}-19$

$0.1579 \mathrm{E}-17$

ZERO

$0.1372 \mathrm{E}-1.5$

ZERO

ZERO

$0.1669 \mathrm{E}-16$

ZERO

$0.4499 \mathrm{E}-14$

$0.9200 \mathrm{E}-18$

$0.3096 \mathrm{E}-14$

$0.2591 \mathrm{E}-13$

$0.1806 \mathrm{E}-02$

$0.2559 \mathrm{E}+00$

$0.3800 \mathrm{E}+02$

$0.9950 \mathrm{E}+00$

$-0.7348 \mathrm{E}-01$

$0.7562 \mathrm{E}+03$

$0.3878 \mathrm{E}+00$

$0.4118 \mathrm{E}-04$

$0.7748 \mathrm{E}+03$
132

to MASCFT

ZERO
ZERO

ZERO

ZERO

ZERO

ZERO

ZERO

ZERO

ZERO

ZERO

ZERO

ZERO

ZTRO

ZRRO

ZERO

ZERO

ZERO

ZERO

ZERO

ZERO

ZERO

ZERO

- ZERO

ZERO

ZERO

ZERO

ZERO

ZERO

ZERO

ZERO

ZERO

ZERO

ZERO

$0.3426 \mathrm{E}-01$

$0.3894 \mathrm{E}-01$

$0.2500 \mathrm{E}+02$

$0.1000 \mathrm{E}+01$

ZERO

$0.8931 \mathrm{E}+00$

$0.7464 \mathrm{E}-04$

$0.6504 \mathrm{E}+02$

LIQUID
133
HNO3

from MAsC

ZERO

ZERO

ZERO

ZERO

ZERO

ZERO

ZERO

ZERO

ZERO

$0.5486 \mathrm{E}-12$

$0.1875 \mathrm{E}-17$

$.5064 \mathrm{E}-15$

ZERO

ZERO

$0.7903 \mathrm{E}-21$

$0.6140 \mathrm{E}-16$

$0.5113 \mathrm{E}-15$

$0.1426 \mathrm{E}-13$

$0.3585 \mathrm{E}-13$

$0.2183 \mathrm{E}-19$

$0.1572 \mathrm{E}-17$

ZERO

$0.1366 \mathrm{E}-15$

ZERO

$0.1661 \mathrm{E}-16$

ZERO

$0.4477 \mathrm{E}-14$

$0.9156 \mathrm{E}-18$

$0.3081 \mathrm{E}-14$

$0.257 .9 \mathrm{E}-13$

$0.3590 \mathrm{E}-01$

$0.4064 \mathrm{E}-01$

$0.3800 \mathrm{E}+02$

ZERO

$0.1418 \mathrm{E}+01$

$0.7800 \mathrm{E}-04$

$0.6497 \mathrm{E}+02$

IIQUID
134

Hg Feed

o MWWC

ZERO

ZERO

ZERO

ZERO

ZERO

ZERO

ZERO

ZERO

$0.2625 \mathrm{E}-14$

$0.8972 \mathrm{E}-20$

$0.2423 \mathrm{E}-17$

$0.3343 E-18$

ZERO

ZERO

$0.2938 \mathrm{E}-18$

$0.2446 \mathrm{E}-17$

$0.6823 \mathrm{E}-16$

$0.7711 \mathrm{E}-20$

$0.1044 \mathrm{E}-21$

ZERO

TRACE
$0.6535 \mathrm{E}-18$

ZERO

$0.7950 \mathrm{E}-19$

ZERO

$0.2142 \mathrm{E}-16$

$0.1474 \mathrm{E}-16$

$0.1234 \mathrm{E}-15$

$0.1718 \mathrm{E}-03$

$0.2542 \mathrm{E}+00$

$0.3800 \mathrm{E}+02$

$0.1000 \mathrm{E}+01$

ZERO

$0.3248 \mathrm{E}+00$

$0.3782 \mathrm{E}-04$

$0.8380 \mathrm{E}+03$

LIQUID
135

Watex Wash

to MWWC

ZERO
ZERO

ZERO

ZERO

ZERO

ZERO

ZERO

ZERO

ZERO

ZERO

ZERO

ZERO

ZERO

ZERO

ZERO

ZERO

ZERO

ZERO

ZERO

ZERO

ZERO

ZERO

ZERO

ZERO

ZERO

ZERO

ZERO

ZERO

ZERO

ZERO

ZERO

ZERO

ZERO

ZERO

ZERO

$.3737 \mathrm{E}-0$

$0.3737 \mathrm{E}-01$

$0.2500 \mathrm{E}+02$

$.1340 \mathrm{E}+01$

$0.4997 \mathrm{E}+01$

$0.9378 \mathrm{E}+00$

$0.7464 \mathrm{E}-04$

$0.6243 \mathrm{E}+02$

LIQUID

136

Water Wash

from MWWC

ZERO

ZERO

ZERO

ZERO

ZERO

ZERO

ZERO

ZERO

$0.2612 \mathrm{E}-14$

$0.8927 \mathrm{E}-20$

$.2411 \mathrm{E}-17$ 
Table A-1. HLW Flowsheet Material Balance (Tank 51/ITP Cycle 1)

STREAM NUMBERS $\rightarrow$ STREAM NAME

\begin{tabular}{|c|c|c|}
\hline \multicolumn{3}{|c|}{ 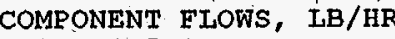 } \\
\hline 122 & (C6H5) 2 & \\
\hline 123 & (C6H5) $2 \mathrm{C} 6 \mathrm{H} 4$ & \\
\hline 118 & (C6H5) $2 \mathrm{NH}$ & \\
\hline 133 & (C6H5B (OH) 2 s & salt) \\
\hline $\begin{array}{l}132 \\
166\end{array}$ & (C6H5B (OH) 2) & \\
\hline $\begin{array}{l}166 \\
162\end{array}$ & $(\mathrm{C} 6 \mathrm{H} 5 \mathrm{C} 6 \mathrm{H} 4 \mathrm{OH})$ & \\
\hline 163 & (C6H5NHCHO) & \\
\hline 165 & (C6H5NO) & \\
\hline 164 & (C6H5NO2) & \\
\hline 131 & (C6H50H salt) & \\
\hline 130 & (C6H5OH) & \\
\hline 121 & $(\mathrm{C} 6 \mathrm{H} 6)$ & \\
\hline 155 & (CH3) $2 \mathrm{CHOH}$ & \\
\hline 154 & (СH3OH) & \\
\hline 45 & $\mathrm{Ag}$ & \\
\hline 116 & Ag20 & $\cdots$ \\
\hline 134 & AgNO3 & \\
\hline 77 & Al203 & \\
\hline 106 & $\mathrm{~B} 203$ & \\
\hline & $\mathrm{Ba}(\mathrm{OH}) 2$ & \\
\hline 15 & $\mathrm{BaO}$ & \\
\hline 24 & BasO4 & \\
\hline 66 & $\mathrm{CO}$ & \\
\hline 37 & $\mathrm{CO} 2$ & \\
\hline 142 & $\mathrm{CO} 2(\mathrm{C} 14)$ & \\
\hline 63 & $\mathrm{Ca}(\mathrm{COOH}) 2$ & \\
\hline 167 & $\mathrm{Ca}(\mathrm{NO} 3) 2$ & \\
\hline 120 & $\mathrm{Ca}(\mathrm{OH}) 2$ & \\
\hline 113 & $\mathrm{Ca} 3(\mathrm{PO} 4) 2$ & \\
\hline 20 & $\mathrm{CaC} 204$ & \\
\hline 18. & $\mathrm{CaCO} 3$ & \\
\hline 64 & $\mathrm{Caco} 3$ (C14) & \\
\hline 78 & $\mathrm{CaF} 2$ & \\
\hline 41 & $\mathrm{CaO}$ & \\
\hline 19 & CasO4 & \\
\hline 29 & Carbon & \\
\hline $\begin{array}{l}99 \\
26\end{array}$ & Cement & \\
\hline $\begin{array}{l}26 \\
168\end{array}$ & $\mathrm{Co}(\mathrm{COOH}) 2$ & \\
\hline $\begin{array}{r}168 \\
59\end{array}$ & $\begin{array}{l}\mathrm{Co}(\mathrm{NO} 3)^{2} \\
\mathrm{CoO}\end{array}$ & \\
\hline 98 & $\mathrm{Cr} 2 \mathrm{O} 3$ & \\
\hline 42 & $\operatorname{cs} 20$ & \\
\hline 53 & $\mathrm{CsCOOH}$ & \\
\hline 96 & $\mathrm{CsCl}$ & \\
\hline 169 & CsNO3 & \\
\hline 8 & $\begin{array}{l}\mathrm{CsOH} \\
\text { CsTPBB }\end{array}$ & \\
\hline
\end{tabular}

WSRC-TR-95-0019 (Revision
138 Purge

ZERO

ZERO

ZERO

ZERO

ZERO

ZERO

ZERO

ZERO

ZERO

ZERO

ZERO

ZERO

ZERO

ZERO

ZERO

ZERO

ZERO

ZERO

ZERO

ZERO

ZERO

ZERO

ZERO

ZERO

ZERO

ZERO

ZERO

ZERO

ZERO

ZERO

ZERO

ZERO

ZERO

ZERO

ZERO

ZERO

ZERO

ZERO

ZERO

ZERO

ZERO

ZERO Section 6. Mercuxy Purification Cell

$\begin{array}{llll}139 & 140 & 141 & 142\end{array}$

Hg Hold
Tank Vent

$0.2654 \mathrm{E}-16$

$0.8144 \mathrm{E}-20$

$0.2377 \mathrm{E}-15$

ZERO

$0.2163 \mathrm{E}-19$

$0.5843 \mathrm{E}-14$

$0.8057 \mathrm{E}-19$

$.6622 \mathrm{E}-22$

$0.2801 \mathrm{E}-20$

ZERO

$0.1443 E-16$

$0.2269 \mathrm{E}-23$

$0.1067 \mathrm{E}-24$

ZERO

ZERO

ZERO

ZERO

ZERO

ZERO

ZERO

ZERO

$0.1386 \mathrm{E}-23$

TRACE

ZERO

ZERO

ZERO

ZERO

ZERO

ZERO

ZERO

ZERO

ZERO

ZERO

ZERO

ZERO

ZERO

ZERO

ZERO

ZERO

ZERO

ZERO

ZERO

Washed $\mathrm{Hg}$

to MFF

Water Rinse
to MFF

HNO3-Rinse

to MFE

$0.1912 \mathrm{E}-20$ ZERO

$0.3257 \mathrm{E}-19$

$0.3381 \mathrm{E}-1.5$

ZERO

$0.9397 \mathrm{E}-21$

$.9397 \mathrm{E}-21$

$0.3412 \mathrm{E}-17$

$0.4984 \mathrm{E}-20$
TRACE

$0.3358 \mathrm{E}-23$

$0.7177 \mathrm{E}-22$

$0.3624 \mathrm{E}-16$

$0.7026 \mathrm{E}-22$

TRACE

$0.8251 \mathrm{E}-11$

TRACE

$0.2443 \mathrm{E}-12$

$0.1311 \mathrm{E}-17$ ZERO

$0.6975 \mathrm{E}-20$

$75 E-20$
ZERO

TRACE

ZERO
$0.1715 \mathrm{E}-17$

ZERO
$0.1075 \mathrm{E}-17$

TRACE

ZERO

ZERO

$0.6146 \mathrm{E}-20$

$0.1371 \mathrm{E}-20$

$0.9022 \mathrm{E}-19$

ZERO

$0.5852 \mathrm{E}-22$

$0.2195 \mathrm{E}-24$

$0.1254 \mathrm{E}-22$

$0.1755 \mathrm{E}-18$

$0.1769 \mathrm{E}-19$

ZERO

ZERO

ZERO
ZERO
ZERO
ZERO
ZERO
ZERO
ZERO
ZERO
ZERO
ZERO
ZERO
ZERO
ZERO
ZERO
ZERO
ZERO
ZERO
ZERO
ZERO
ZERO
ZERO
ZERO
ZERO
ZERO
ZERO
ZERO
ZERO
ZERO
ZERO
ZERO
ZERO
ZERO
ZERO
ZERO
ZERO
ZERO
ZERO
ZERO
ZERO
ZERO
ZERO
ZERO
ZERO
ZERO
ZERO
ZERO
ZERO

Date: January 1995

ZERO
ZERO
ZERO
ZERO
ZERO
ZERO
ZERO
ZERO
ZERO
ZERO
ZERO
ZERO
ZERO
ZERO
ZERO
ZERO
ZERO
ZERO
ZERO
ZERO
ZERO
ZERO
ZERO
ZERO
ZERO
ZERO
ZERO
ZERO
ZERO
ZERO
ZERO
ZERO
ZERO
ZERO
ZERO
ZERO
ZERO
ZERO
ZERO
ZERO
ZERO
ZERO
ZERO
ZERO
ZERO
ZERO
ZERO
ZERO

143

144

MFE Rinses MFF Bottoms

to SWT to MAWT

$0.1912 \mathrm{E}-22$

$0.3257 \mathrm{E}-21$

$0.3380 \mathrm{E}-17$

ZERO

$0.9385 \mathrm{E}-21$

$0.3408 \mathrm{E}-17$

$0.4978 \mathrm{E}-20$

$78 \mathrm{E}-20$
TRACE

$0.3354 \mathrm{E}-23$

$0.7168 \mathrm{E}-22$

$0.3623 \mathrm{E}-18$

$0.7025 \mathrm{E}-24$

TRACE

TRACE

ZERO

TRACE

$0.1302 \mathrm{E}-10$

$0.3613 \mathrm{E}-17$

$0.1309 \mathrm{E}-17$

ZERO

$0.6966 \mathrm{E}-20$

$66 E-20$
ZERO

TRACE

ZERO

$0.1713 \mathrm{EER}-17$

ZERO

$0.1073 \mathrm{E}-17$

TRACE

ZERO

$0.6138 \mathrm{E}-20$

$0.6136 \mathrm{E}-20$

$0.9011 \mathrm{E}-19$

$0.9011 \mathrm{E}-19$
ZERO ZERO

$0.5845 \mathrm{E}-22$

$0.2192 \mathrm{E}-24$

$0.1252 \mathrm{E}-22$

$0.1753 \mathrm{E}-18$

$0.5737 \mathrm{E}-22$

$0.1767 \mathrm{E}-19$

ZERO

$0.3929 \mathrm{E}-23$

ZERO 
Table A-1. HiW Flowsheet Material Balance (Tank 51/ITP Cycle 1) section 6 . Mercury Purification Cell

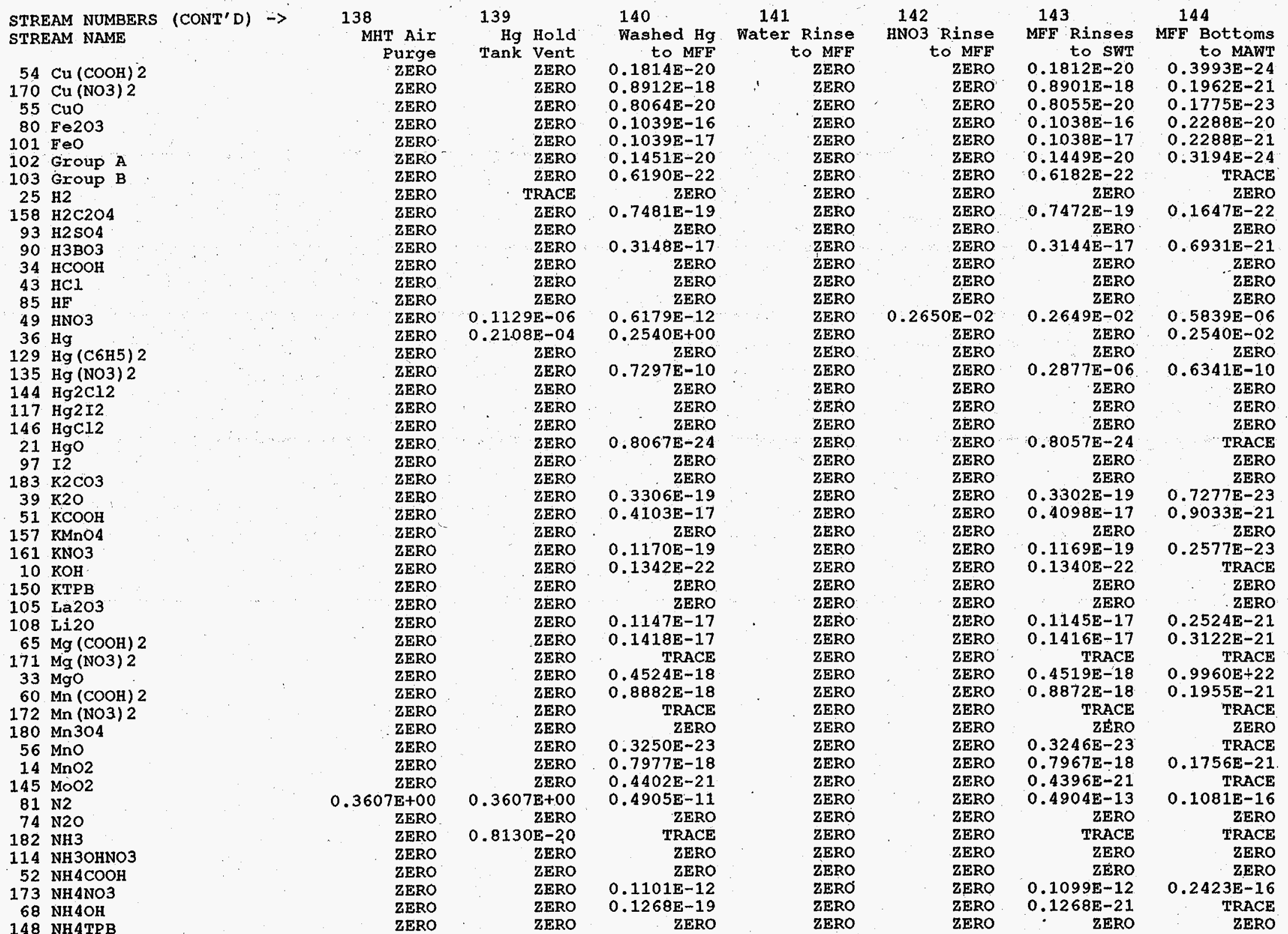


Table A-1. HLW Flowsheet Material Balance (Tank 51/ITP Cycle 1)

STREAM NUMBERS (CONT'D) $\rightarrow \quad 138$ STREAM NAME

$\begin{array}{ll}35 & \text { NO } \\ 84 & \text { NO2 }\end{array}$

70. $\mathrm{Na}(\mathrm{HgO}(\mathrm{OH})$

$94 \mathrm{Na} 2 \mathrm{~B} 4 \mathrm{O} 7$

$109 \mathrm{Na2C} 204$

$5 \mathrm{Na} 2 \mathrm{CO} 3$

$143 \mathrm{Na} 2 \mathrm{CO} 3$ (C14)

$89 \mathrm{Na2CrO} 4$

115 Na2MoO

$\begin{array}{ll}32 & \mathrm{Na} 2 \mathrm{O} \\ 71 & \mathrm{Na} 2 \mathrm{PuO} 2(\mathrm{OH}) 4\end{array}$

73 Na2Rho4

$72 \mathrm{Na2RuO4}$

$6 \mathrm{Na} 2 \mathrm{SO} 4$

$88 \mathrm{Na2SiO3}$

$91 \mathrm{NaAg}(\mathrm{OH}) 2$

$75 \mathrm{NaAl}(\mathrm{OH}) 4$

$50 \mathrm{NaCOOH}$

$7 \mathrm{NaCl}$

$69 \mathrm{NaF}$
$11 \cdot \mathrm{NaHCO} 3$

$12 \mathrm{Na}$

$3 \mathrm{NaNO} 2$

2 NaNO3

$4 \mathrm{NaOH}$

149 NaTPB

152 atis

$181 \mathrm{Nd2O3}$

$61 \mathrm{Ni}(\mathrm{COOH}) 2$

$174 \mathrm{Ni}(\mathrm{NO} 3)^{2} 2$

$40 \mathrm{NiO}$

$175 \mathrm{~Pb}(\mathrm{NO} 3) 2$

$86 \mathrm{PbCO} 3$

$67 \mathrm{PbO}$

$147 \mathrm{PbS}$

$124 \mathrm{Pd}$

$112 \mathrm{Pd}(\mathrm{NO} 3) 2$

110 Pdo

$\begin{array}{rl}76 & \mathrm{PuO} 2 \\ 156 & \mathrm{PuO} 2(\mathrm{NaTi2O5)} 2\end{array}$

\begin{tabular}{rl}
156 & $\mathrm{PuO}$ \\
58 & $\mathrm{Rh}$ \\
\hline
\end{tabular}

$\begin{array}{ll}58 & \mathrm{Rh} \\ 22 & \mathrm{RhO}\end{array}$

$\begin{array}{ll}22 & \mathrm{RhO} \\ 95 \mathrm{Ru}\end{array}$

$\begin{array}{ll}95 & \mathrm{Ru} \\ 79 & \mathrm{RuO}\end{array}$

83 RuO4

WSRC-TR-95-0019 (Revision

section 6. Mercury purification Cell MHT Air

Purge
ZERO

ZERO

ZERO

ZERO

ZERO

ZERO

ZERO

ZERO

ZERO

ZERO

ZERO

ZERO

ZERO

ZERO

ZERO

ZERO

ZERO

ZERO

ZERO

ZERO

ZERO

ZERO

ZERO

ZERO

ZERO

ZERO

ZERO

ZERO

0.1090 EERO

ZERO

ZERO

ZERO

ZERO

ZERO

ZERO

ZERO

ZERO

ZERO

ZERO

ZERO

ZERO
Hg Hold

Tank Vent

$0.1933 E-10$

ZERO

ZERO

ZERO

IERO

ZERO

ZERO

ZERO

ZERO

ZERO
ZERO

ZERO

ZERO

ZERO

ZERO

ZERO

ZERO

ZERO

ZERO

ZERO

ZERO

ZERO

ZERO

ZERO

ZERO

ZERO

ZERO

ZERO

ZERO

$0.1090 \mathrm{E}$

ZERO

ZERO

ZERO

ZERO

ZERO

ZERO

ZERO

ZERO

ZERO

ZERO

ERO

ERO

ERO

ZERO

$$
140
$$

Washed $\mathrm{Hg} 141$ to MFE

$0.7790 \mathrm{E}-21$ ， ZERO

ZERO
$0.1504 \mathrm{E}-22$

$0.9699 \mathrm{E}-21$

$0.2004 \mathrm{E}-17$

ZTRO

ZTRO

ZERO

ZERO

$0.2605 \mathrm{E}-17$

ZERO

ZERO

$0.7992 \mathrm{E}-19$

ZERO
$0.1054 \mathrm{E}-20$

$\mathrm{E}-20$

ZERO

$0.3209 \mathrm{E}-18$

$0.4074 \mathrm{E}-20$

$0.1399 \mathrm{E}-19$

ZERO

$0.2241 \mathrm{E}-23$

$0.1212 \mathrm{E}-20$
$0.3694 \mathrm{E}-17$

ZERO

ZERO

ZERO

ZERO

$0.1032 \mathrm{E}-19$

$0.2731 \mathrm{E}-24$

$0.9739 \mathrm{E}-19$

$0.2880 \mathrm{E}-11$

$0.3180 \mathrm{E}-22$

ZERO
TRACE

ZERO

$0.5396 \mathrm{E}-19$

$0.2540 \mathrm{E}-1.2$

$0.1039 \mathrm{E}-13$

TRACE

$0.4056 \mathrm{E}-20$

$0.5196 \mathrm{E}-22$

$0.4841 \mathrm{E}-12$

$0.1200 \mathrm{E}-13$

$0.3323 \mathrm{E}-20$

ZERO

Date: January 1995
142 No3 Rinse to $\mathrm{MFE}$
ZERO

ZERO

ZERO

ZERO

ZERO

ZERO

ZERO

ZERO

ZERO

ZERO

ZERO

ZERO

ZERO

ZERO

ZERO

ZERO

ZERO

ZERO

ZERO

ZERO

ZERO

ZERO

ZERO

ZERO

ZERO

ZERO

ZERO

ZERO

ZERO

ZERO

ZERO

ZERO

ZERO

ZERO

ZERO

ZERO

ZERO

ZERO

ZERO

ZERO

ZERO

ZERO

ZERO

ZERO
143

F Rinses MFF 144

ZERO
$0.8154 \mathrm{E}-07$

ZERO

$0.9687 \mathrm{E}-21$

$0.2002 E-17$

$$
\text { ZERO }
$$$$
\text { ZERO }
$$

2602E-17

$2 \mathrm{E}-17$
ZERO

ZERO

$0.7983 \mathrm{E}-19$

$0.1053 \mathrm{E}-20$

ZERO

ZERO

$0.3205 \mathrm{E}-18$

$0.4069 \mathrm{E}-20$

$0.1398 \mathrm{E}-19$

$0.1210 \mathrm{E}-20$

$0.3690 \mathrm{E}-17$

ZERO

ZERO

ZERO

ZERO

$0.1031 \mathrm{E}-19$

.

$0.9727 \mathrm{E}-19$

$0.2879 \mathrm{E}-13$

$0.3176 \mathrm{E}-22$

ZERO
TRACE

ZERO

$0.5390 E-19$

$0.5535 \mathrm{E}-12$

TRACE

$0.5189 \mathrm{E}-22$

ZERO

$0.6394 \mathrm{E}-12$

$0.3319 \mathrm{E}-20$

ZERO
ZERO

to MAWT

ZERO

ZERO

$0.2135 \mathrm{E}-24$

$0.2135-24$

$\mathrm{E}-21$

ZERO
ZERO

$0.5735 \mathrm{E}-21$ 
Table A-1. HLW Flowsheet Material Balance (Tank 51/ITP Cycle 1 ) Flowsheet Material Balance (Tank $51 /$
Section 6 . Mercury Purification Cell

STREAM NUMBERS (CONT'D) $\rightarrow$ STREAM NAME

$38 \mathrm{SO} 2$

$141 \mathrm{SO} 3$

137 Semi Vol Cs2o

136 Semi Vol CsCl

126 Semi Vol Group A

140 Semi Vo1 Na2B4O

138 Semi Vol NaCl

139 Semi Vol NaF

125 Semi Vol NaI

23 Semi

$\begin{array}{rl}111 & \mathrm{SiO} 2 \mathrm{C} \\ 48 \mathrm{Sr}(\mathrm{COOH}) 2\end{array}$

$48 \mathrm{Sr}(\mathrm{COOH}) 2$
$176 \mathrm{Sr}(\mathrm{NO} 3) 2$

$153 \mathrm{Sr}$ (NaTi2O5) 2

$127 \operatorname{Sr}(\mathrm{OH}) 2$

$30 \mathrm{srCO}$

46 Sro

$159 \mathrm{TCO} 2$

92 ThO2

107 TiO2

119 Tritium

17 U308

$62 \mathrm{UO} 2(\mathrm{COOH}) 2$

$77 \mathrm{VO} 2$ (NO3) 2

$16 \mathrm{VO} 2(\mathrm{OH}) 2$

$27 \mathrm{Y}(\mathrm{COOH}) 3$
$178 \mathrm{Y}(\mathrm{NO} 3)^{3}$

$178 \mathrm{Y}(\mathrm{NO} 3)$

$128 \mathrm{Y}(\mathrm{OH}) 3$
$31 \mathrm{Y} 2(\mathrm{CO} 3) 3$

$47 \times 203$

100 Zeolite

$44 \mathrm{Zn}(\mathrm{COOH}) 2$

$57 \mathrm{zno}$

104 zio2

13 hydrate H2O

1 water

TOTAL FLOW, LB/HR

TEMPERATURE, DEG C

PRESSURE, ATM

PRESSURE， RSIG

PRESSURE， MM HG

ENTHALPY， PCU/HR

VAPOR FLOW, CFM

ITOUID FLOW, GPM

DENSITY, LBS/FT3

DENSITY
138

MHT Air

Purge

ZERO

ZERO

ZERO

ZERO

ZERO

ZERO

ZERO

ZERO

ZERO

ZERO

ZERO

ZERO

ZERO

ZERO

ZERO

ERO

ZERO

ZERO

ZERO

ZERO

ZERO

ZERO

ZERO

ZERO

ZERO

ZERO

ZERO

ZERO

ZERO

ZERO

ZERO

ZERO

ZERO

ZERO

ZERO

2ERO

$0.7891 \mathrm{E}-02$

$0.4776 \mathrm{E}+00$

$0.3500 \mathrm{E}+02$

$0.1000 \mathrm{E}+01$

ZERO

$0.1002 \mathrm{E}+02$

$0.1128 \mathrm{E}+00$

$0.7055 \mathrm{E}-01$
VAPOR
140

$\mathrm{Hg}$ Hold

ZERO

ZERO

ZERO

ZERO

ZERO

ZERO

ZERO

ZERO

ZERO

ZERO

ZERO

ZERO

ZERO

ZERO

ZERO

ZERO

ZERO

ZERO

$0.3836 \mathrm{E}-22$

ZERO

ZERO

ZERO

ZERO

ZERO

ZERO

ZERO

ZERO

ZERO

ZERO

ZERO

ZERO

$0.8077 \mathrm{E}-02$

$0.4778 \mathrm{E}+00$

$0.3800 \mathrm{E}+02$

$0.9950 \mathrm{E}+00$

$0.7562 \mathrm{E}+03$

$0.1047 \mathrm{E}+02$

$0.1146 \mathrm{E}+00$

$0.6951 \mathrm{E}-01$ $\begin{array}{lcc}140 & 141 & 142\end{array}$

to MFF Water Rinse

to MFE

ZERO

ZERO

ZERO

ZERO

ZERO

ZERO

ZERO

ZERO

ZERO

$0.1306 \mathrm{E}-16$

$0.4464 \mathrm{E}-22$

$0.1205 \mathrm{E}-19$

$0.1663 \mathrm{E}-20$

ZERO

ZERO
TRACE

$0.1462 \mathrm{E}-20$

$0.1217 \mathrm{E}-19$

$0.3395 \mathrm{E}-18$

TRACE

$0.5196 \mathrm{E}-24$

$3741 \mathrm{E}-22$

ZERO

TRACE

$0.3251 \mathrm{E}-20$ ZERO

ZERO
$0.3955 E-21$

ZERO

$0.1066 \mathrm{E}-18$

$0.2180 \mathrm{E}-22$

$0.7335 \mathrm{E}-19$

$0.6139 \mathrm{E}-18$

$0.1356 \mathrm{E}-06$

$0.2540 \mathrm{E}+00$

$0.3800 \mathrm{E}+02$

$0.9950 \mathrm{E}+00$

$-0.7348 \mathrm{E}-01$

$0.7562 \mathrm{E}+03$

$0.3180 \mathrm{E}+00$

$0.3744 \mathrm{E}-04$

$0.8457 \mathrm{E}+03$

IIQUID to MFF

ZERO

ZERO

ZERO

ZERO

ZERO

ZERO

ZERO

ZERO

ZERO

ZERO

ZERO

ZERO

ZRRO

ZERO

ZERO

ZERO

ZERO

ZERO

ZERO

ZERO

ZERO

ZERO

ZERO

ZERO

ZERO

ZERO

ZERO

ZERO

ZERO

ZERO

ZERO

$0.6359 \mathrm{E}-01$

$0.6359 \mathrm{E}-01$

$0.2500 \mathrm{E}+02$

$0.1000 \mathrm{E}+01$

ZERO

$0.1596 \mathrm{E}+01$

$0: 1270 \mathrm{E}-03$

$0.6243 E+02$
LIOUID
HNO3 Rinse

to MFF

ZERO

ZERO

ZERO

ZERO

ZERO

ZERO

ZERO

ZERO

ZERO

ZERO

ZERO

ZERO

ZERO

ZERO

ZERO

ZERO

ZERO

ZERO

ZERO

ZERO

ZERO

ZERO

ZERO

ZERO

ZERO

ZERO

ZERO

ZERO

ZERO

ZERO

ZERO

ZERO

ZERO

ZERO

- ZERO

$0.1943 \mathrm{E}-01$

$0.1943 \mathrm{E}-01$
$0.2208 \mathrm{E}-01$

$0.2500 \mathrm{E}+02$

$0.1000 \mathrm{E}+01$

ZERO

$0.5065 E+00$

$0.4233 \mathrm{E}-04$

$0.6504 \mathrm{E}+02$

LIQUID

143

144

to SWT

ZERO

ZERO

ZERO

ZERO

ZERO

ZERO

ZERO

ZERO 
Table A-1. HLW Flowsheet Material Balance (Tank 51/ITP Cycle 1) Section 6. Mercury Purification Cell

STREAM NUMBERS $\rightarrow$

STREAM NAME

\begin{tabular}{|c|c|}
\hline \multicolumn{2}{|r|}{ 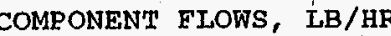 } \\
\hline 122 & $(\mathrm{C} 6 \mathrm{H} 5)^{2}$ \\
\hline 123 & (C6H5) 2C6H 4 \\
\hline 118 & (C6H5) 2NH \\
\hline 133 & (C6H5B (OH) 2 salt) \\
\hline 132 & (C6H5B (OH) 2) \\
\hline 166 & (C6H5C6H4OH) \\
\hline 162 & (C6H5NH2) \\
\hline 163 & (C6H5NHCHO) \\
\hline 165 & (C6H5NO) \\
\hline 164 & (C6H5NO2) \\
\hline 131 & (C6H5OH salt) \\
\hline 130 & (C6H5OH) \\
\hline 121 & (C6H6) \\
\hline 155 & (СH3) $2 \mathrm{CHOH}$ \\
\hline 154 & (СН3ОН) \\
\hline 45 & Ag \\
\hline 116 & $\mathrm{Ag} 20$ \\
\hline 134 & AgNO3 \\
\hline 77 & Al203 \\
\hline 106 & $\mathrm{~B} 203$ \\
\hline $\begin{array}{r}9 \\
15\end{array}$ & $\mathrm{Ba}(\mathrm{OH}) 2$ \\
\hline 24 & BaSO4. \\
\hline 66 & Co \\
\hline 37 & $\mathrm{CO} 2$ \\
\hline 142 & $\mathrm{CO} 2$ (C14) \\
\hline 63 & $\mathrm{Ca}(\mathrm{COOH}) 2$ \\
\hline 167 & $\mathrm{Ca}(\mathrm{NO} 3)^{2}$ \\
\hline 120 & $\mathrm{Ca}(\mathrm{OH}) 2$ \\
\hline 113 & $\mathrm{Ca} 3(\mathrm{PO} 4) 2$ \\
\hline 20 & $\mathrm{CaC} 204$ \\
\hline 18 & $\mathrm{CaCO} 3$ \\
\hline 64 & $\mathrm{CaCO} 3(\mathrm{C} 14)$ \\
\hline 78 & CaF2 \\
\hline 41 & $\mathrm{CaO}$ \\
\hline 19 & CasO4: \\
\hline 29 & Carbon \\
\hline 99 & Cement \\
\hline 26 & $\mathrm{Co}(\mathrm{COOH}) 2$ \\
\hline 168 & $\mathrm{Co}(\mathrm{NO} 3) 2$ \\
\hline 59 & $\mathrm{CoO}$ \\
\hline 98 & $\mathrm{Cr} 203$ \\
\hline 42 & $\operatorname{Cs} 20$ \\
\hline 53 & $\mathrm{CsCOOH}$ \\
\hline 96 & $\mathrm{CsCl}$ \\
\hline 169 & CsNO3 \\
\hline & $\begin{array}{l}\text { CsOH } \\
\text { CsTPB }\end{array}$ \\
\hline
\end{tabular}

151 CsTPB

WSRC-TR-95-0019 (Revision

\begin{tabular}{|c|c|c|c|c|c|}
\hline $\begin{array}{l}145 \\
\text { uxified Hg } \\
\text { from MVS }\end{array}$ & $\begin{array}{l}146 \\
\text { MVs Vent to } \\
\text { Vacuum Pump }\end{array}$ & $\begin{array}{l}\quad 147 \\
\text { Hg Accu } \\
\text { Vacuum }\end{array}$ & $\begin{array}{l}\text { Im at } \\
\text { Pump }\end{array}$ & $\begin{array}{l}14 \\
\text { Mvs } \\
\text { Pump }\end{array}$ & $\begin{array}{l}8 \\
\text { Vacuum } \\
\text { Exhst }\end{array}$ \\
\hline $0.1893 \mathrm{E}-2.0$ & ZERO & & ZERO & $\therefore$ & ZERO \\
\hline $0.3225 \mathrm{E}-19$ & ZERO & & ZERO & & ZERO \\
\hline $347 E-15$ & ZERO & & ZERO & & ZERO \\
\hline ZERO & ZERO & & ZERO & & ZERO \\
\hline ZERO & ZERO & & ZERO & & ZERO \\
\hline $0.9397 \mathrm{E}-24$ & ZERO & & ZERO & & ZERO \\
\hline $0.3412 \mathrm{E}-20$ & ZERO & & ZERO & & ZERO \\
\hline $0.4984 \mathrm{E}-23$ & ZERO & & ZERO & & ZERO \\
\hline TRACE & ZERO & & ZERO & & ZERO \\
\hline TRACE & ZERO & & ZERO & & ZERO \\
\hline TRACE & ZERO & & ZERO & & ZERO \\
\hline $0.3588 \mathrm{E}-16$ & ZERO & & ZERO & & ZERO \\
\hline $0.6956 \mathrm{E}-22$ & ZERO & & ZERO & & ZERO \\
\hline TRACE & ZERO & & ZERO & & ZERO \\
\hline TRACE & ZERO & & ZERO & & ZERO \\
\hline $0.8251 \mathrm{E}-1.4$ & ZERO & & ZERO & & ZERO \\
\hline TRACE & ZERO & & ZERO & & ZERO \\
\hline $0.2443 \mathrm{E}-15$ & ZERO & & ZERO & . & ZERO \\
\hline $0.3617 \mathrm{E}-20$ & ZERO & & ZERO & & ZERO \\
\hline $0.1311 \mathrm{E}-20$ & ZERO & & ZERO & & ZERO \\
\hline ZERO & ZERO & ? & ZERO & & ZERO \\
\hline TRACE & ZERO & & ZERO & & ZERO \\
\hline $0.6975 E-23$ & ZERO & & ZERO & & ZERO \\
\hline ZERO & ZERO & & ZERO & & ZERO \\
\hline ZERO & TRACE & & ZERO & & TRACE \\
\hline ZERO & ZERO & & ZERO & & ZERO \\
\hline ZERO & ZERO & & ZERO & & ZERO \\
\hline $0.1715 \mathrm{E}-20$ & ZERO & & ZERO & & ZERO \\
\hline ZERO & ZERO & & ZERO & & ZERO \\
\hline $0.1075 E-20$ & ZERO & & ZERO & & ZERO \\
\hline TRACE & ZERO & & ZERO & & ZERO \\
\hline ZERO & ZERO & & ZERO & & ZERO \\
\hline ZERO & ZERO & & ZERO & & ZERO \\
\hline $0.6146 \mathrm{E}-23$ & ZERO & & ZERO & & ZERO \\
\hline $0.1371 \mathrm{E}-23$ & ZERO & & ZERO & & ZERO \\
\hline $0.9022 \mathrm{E}-22$ & ZERO & & ZERO & & ZERO \\
\hline ZERO & ZERO & & ZERO & & ZERO \\
\hline ZERO & ZERO & & ZERO & & ZERO \\
\hline TRACE & ZERO & & ZERO & & ZERO \\
\hline TRACE & ZERO & & ZERO & & ZERO \\
\hline TRACE & ZERO & & ZERO & & ZERO \\
\hline $0.1755 \mathrm{E}-21$ & ZERO & & ZERO & & ZERO \\
\hline TRACE & ZERO & & ZERO & & ZERO \\
\hline $0.1769 \mathrm{E}-22$ & ZERO & & ZERO & & ZERO \\
\hline ZERO & ZERO & & ZERO & & ZERO \\
\hline TRACE & ZERO & & ZERO & & ZERO \\
\hline TRACE & ZERO & & ZERO & & ZERO \\
\hline ZERO & ZERO & & ZERO & & ZERO \\
\hline
\end{tabular}

Date: January 1995

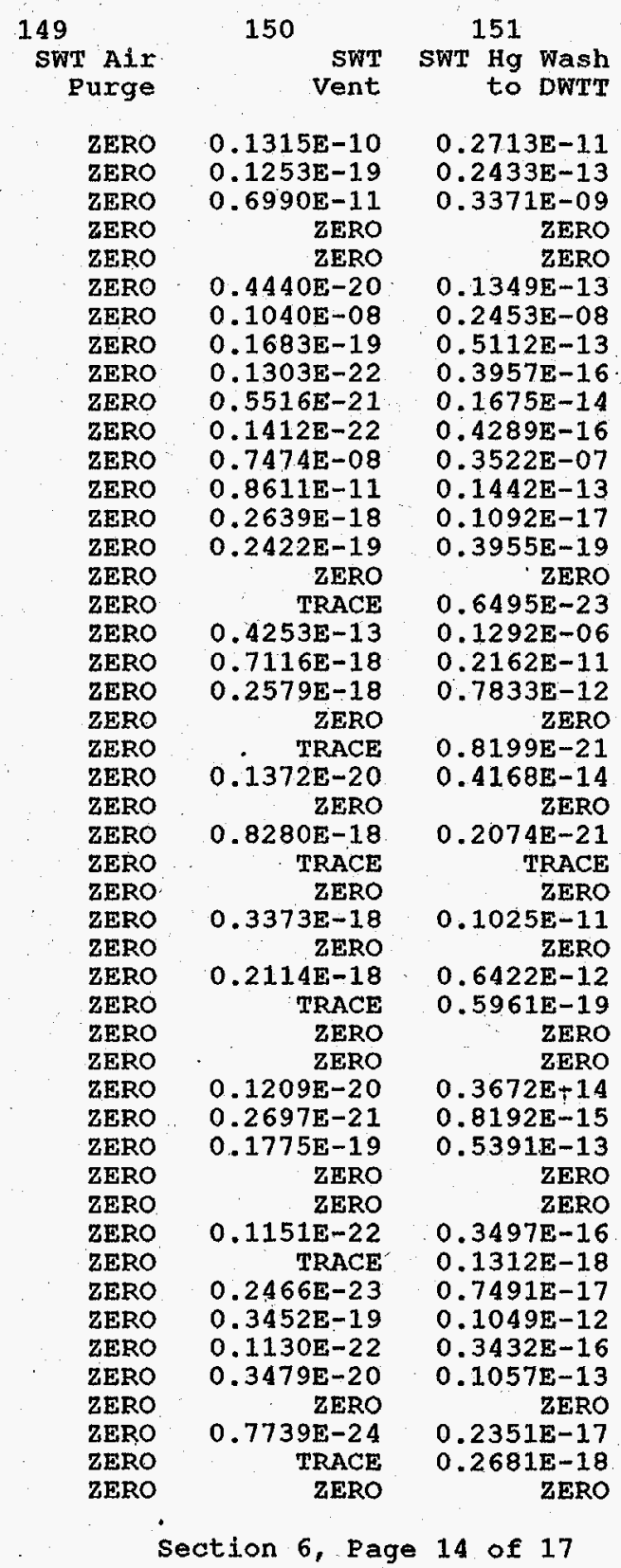


Table A-1. HLW Flowsheet Material Balance (Tank 51/ITP Cycle 1) section 6. Mercury Purification Celi STREAM NUMBERS (CONT'D) $\rightarrow$
STREAM NAME

$54 \mathrm{Cu}(\mathrm{COOH}) 2$

$170 \mathrm{Cu}(\mathrm{NO} 3)^{2}$

$55 \mathrm{CuO}$

$80 \mathrm{Fe} 2 \mathrm{O} 3$

$101 \mathrm{FeO}$

102 Group A

103 Group

$25 \mathrm{H} 2$

93 H2SO4

$90 . \mathrm{H} 3 \mathrm{BO} 3$

$34 \mathrm{HCOOH}$.

$43 \mathrm{HCl}$

$85 \mathrm{HF}$

49 HNO

$36 \mathrm{Hg}$

$129 \mathrm{Hg}$ ( C6H5) 2

$135 \mathrm{Hg}(\mathrm{NO} 3)^{2}$

$144 \mathrm{Hg} 2 \mathrm{Cl} 2$

$117 \mathrm{Hg} 2 \mathrm{I} 2$

$\begin{array}{rl}146 & \mathrm{HgCl} \\ 21 & \mathrm{HgO}\end{array}$

$\begin{array}{ll}21 & \mathrm{HgO} \\ 97 & \mathrm{I} 2\end{array}$

$183 \mathrm{k} 2 \mathrm{CO} 3$

$39 \mathrm{~K} 2 \mathrm{O}$

$51 \mathrm{KCOOH}$

$161 \mathrm{KNO} 3$

$10 \mathrm{KOH}$

150 KTPB

$105 \mathrm{La} 203$

$65 \mathrm{Mg}$ (COOH) 2

$171 \mathrm{Mg}$ (NO3) 2

$33 \mathrm{MgO}$

$60 \mathrm{Mn}(\mathrm{COOH}) 2$

172 Mn (NO3) 2

$180 \mathrm{Mn} 30$

$56 \mathrm{MnO}$

$14 \mathrm{MnO} 2$

81 N2

$74 . \mathrm{N} 2 \mathrm{O}$
$182 \mathrm{NH} 3$

114 NH3OHNO3

$52 \mathrm{NH} 4 \mathrm{COOH}$

173 NH4NO3

$\begin{aligned} 68 & \text { NH4OH } \\ 148 & \text { NH4TPB }\end{aligned}$
145

1456

from MVS

$0.1814 \mathrm{E}-23$

$0.8912 \mathrm{E}-21$

$0.8064 \mathrm{E}-23$

$0.1039 \mathrm{E}-19$

$0.1039 \mathrm{E}-20$

$0.1451 \mathrm{E}-23$

TRACE

$0.7481 \mathrm{E}-22$

ZERO

$0.3148 \mathrm{E}-20$

$$
\text { ZERO }
$$

ZERO

ZERO

$0.2514 \mathrm{E}+00$

ZERO
$0.7297 \mathrm{E}-13$

ZERO

ZERO

ZERO

TRACE

ZERO

$0.3306 \mathrm{E}-22$

$0.4103 \mathrm{E}-20$

$0.1170 \mathrm{E}-22$

TRACE

ZERO

ZERO
$0.1147 \mathrm{E}-20$

$0.1147 \mathrm{E}-20$
$0.1418 \mathrm{E}-20$

TRACE

$0.4524 \mathrm{E}-21$

$0.8882 \mathrm{E}-21$

TRACE

TRACE

$0.7977 \mathrm{E}-21$

$0.4402 \mathrm{E}-24$

ZERO

ZERO

TRACE

ZERO

ZERO

$0.1101 \mathrm{E}-15$

$0.1256 \mathrm{E}-19$

ZERO
146

Vacuum Pump Vacuum Pump Pump Exhst

$\begin{array}{llr}\text { ZERO } & \text { ZERO } & \text { ZERO } \\ \text { ZERO } & \text { ZERO } & \text { ZERO } \\ \text { ZERO } & \text { ZERO } & \text { ZERO }\end{array}$

ZERO ZERO

ZERO Z ZERO ZERO

ZERO ZERO $\quad$ ZERO

ZERO Z ZERO $\quad$ ZERO

ZERO

ZERO ZERO

ZERO ZERO

ZERO ZERO

ZERO

ZERO

$0.2415 \mathrm{E}-04 \quad 0.2415 \mathrm{E}-04$

ZERO ZERO

ZERO ZERO

ZERO $\quad$ ZERO

ZERO

ZERO ZERO

ZERO ZERO

ZERO ZERO

ZERO ZERO

ZERO

ZERO

ZERO ZERO

ZERO ZERO

ZERO

ZERO ZERO

ZERO

ZERO

ZERO

ZERO

ZERO

ZERO

ZERO

ZERO

ZERO

ZERO

ZERO

ZERO

ZERO

ZERO

ZERO

ZERO
149

SWT Air

Purge

ZERO

ZERO

ZERO

ZERO

ZERO

ZERO

ZERO

ZERO

ZERO

ZERO

ZERO

ZERO

ZERO

ZERO

ZERO

ZERO

ZERO

ZERO

ZERO

ZERO

ZERO

ZERO

ZERO

ZERO

ZERO

ZERO

ZERO

ZERO

ZERO

ZERO

ZERO

ZERO

ZERO

ZERO

ZERO

ZERO

ZERO

ZERO

ZERO

ERO

$0.4856 \mathrm{E}-11$

$\mathrm{E}-11$

$0.3607 \mathrm{E}+00$

ZERO

ZERO

ZERO

ZERO

ZERO

ZERO
SWT
Vent
$0.3568 \mathrm{E}-21$
$0.1753 \mathrm{E}-18$

$0.1586 \mathrm{E}-20$

.2044E-18

$0.2854 \mathrm{~F}-21$

$0.1218 \mathrm{E}-22$

TRACE

ZERO

$.6193 \mathrm{E}-18$

ZERO

$0.2365 \mathrm{E}-02$

ZERO

$0.1989 \mathrm{E}-11$

ZERO

ZERO

ZERO

.1587 E-24
ZERO

ZERO

$0.6503 \mathrm{E}-20$

$0.8071 \mathrm{E}-18$

ZERO

$0.2302 \mathrm{E}-20$

ZERO

ZERO

$0.2256 \mathrm{E}-18$

$0.2790 \mathrm{E}-18$

TRACE

$0.8900 \mathrm{E}-19$

$0.1747 \mathrm{E}-18$

RACE

$0.6393 \mathrm{E}-24$

$0.1569 \mathrm{E}-18$

$0.8659 \mathrm{E}-22$

$0.3607 \mathrm{E}+00$

$0.4688 \mathrm{E}-14$

ZERO

ZERO

$0.2495 \mathrm{E}-20$

ZERO
151

WT Hg Wash

to DWTI

$0.5325 \mathrm{E}-12$

$0.4819 \mathrm{E}-14$

$0.6211 \mathrm{E}-11$

$0.6210 \mathrm{E}-12$

$0.8669 \mathrm{E}-15$

$0.3699 \mathrm{E}-16$

TRACE

ZERO

$0.1881 E-11$

ZERO

ZERO

$0.6295 \mathrm{E}-02$

ZERO

$0.6042 E-05$

ZERO

ZERO

ZERO

ZERO

ERO

$.2452 E-11$
ZERO

$0.6993 \mathrm{E}-14$

$0.8018 \mathrm{E}-17$

ZERO

$0.6851 \mathrm{E}-12$

$0.8474 \mathrm{E}-12$

$0.1282 \mathrm{E}-19$

$0.1282 \mathrm{1}-19$

$0.5308 \mathrm{E}-12$

$0.1494 \mathrm{E}-19$

$0.1942 \mathrm{E}-17$

$0.4767 \mathrm{E}-12$

$0.2630 \mathrm{E}-15$

$0.2385 \mathrm{E}-05$

$0.1703 E-15$

ZERO

$0.7579 \mathrm{E}-14$

ZERO 
Table A-1. HLW Flowsheet Material Balance (Tank 51/ITP Cycle 1)

STREAM NUMBERS (CONT'D) $\rightarrow$ STREAM NAME

$\begin{array}{ll}35 & \text { NO } \\ 84 & \text { NO2 }\end{array}$

$70 \mathrm{Na}(\mathrm{HgO}(\mathrm{OH})$

$70 . \mathrm{Na}(\mathrm{HgO}$
$94 \mathrm{Na} \mathrm{B} 4 \mathrm{OH}$

$109 \mathrm{Na2C2O4}$

$5 \mathrm{Na} 2 \mathrm{CO} 3$

$143 \mathrm{Na2CO} 3$ (C14)

$89 \mathrm{Na} 2 \mathrm{CrO} 4$

$115 \mathrm{Na2MOO4}$

$32 \mathrm{Na2O}$

71 Na2PuO2 (OH) 4

$73 \mathrm{Na2RhO} 4$

$72 \mathrm{Na2RuO4}$

$6 \mathrm{Na} 2 \mathrm{SO} 4$

$87 \mathrm{Na2sio3}$

$91 \mathrm{NaAg}(\mathrm{OH}) 2$

$50 \mathrm{NaCOOH}$

$7 \mathrm{NaCl}$

$7 \mathrm{NaCl}$
$69 \mathrm{NaF}$

$11 \mathrm{NaHCO} 3$

$12 \mathrm{NaI}$

3 NaNO2

$4 \mathrm{NaOH}$

$149 \mathrm{NaTPB}$

152 NaTi205H

$181 \mathrm{Nd2O3}$

$61 \mathrm{Ni}$ (COOH) 2

$174 \mathrm{Ni}$ (NO3) 2

$40 \mathrm{NiO}$

8202

$175 \mathrm{~Pb}(\mathrm{NO} 3) 2$

$86 \mathrm{PbCO} 3$

$67 \mathrm{Pbo}$
$147 \mathrm{Pbs}$

$28 \mathrm{PbSO} 4$

$124 \mathrm{Pd}$

$112 \mathrm{Pd}(\mathrm{NO} 3) 2$

$110 \mathrm{PdO}$

$76 \mathrm{PuO}^{2}$

$\begin{array}{rl}156 & \mathrm{PuO} \\ 58 & \mathrm{Rh}\end{array}$

22 RhO2

$95 \mathrm{Ru}$

79 RuO2

83 RuO4 1.45
Purif Section 6. Mercury purification Cell

146

from MVS

MVS Vent to $\mathrm{Hg}$ Accum at ZERO

ZERO

Vacuum Pump Vacuum $\begin{array}{r}\text { Pump } \\ \text { ZERO } \\ \text { ZERO } \\ 0.7712 \mathrm{E}-21\end{array} \quad$ ZERO

TRACE

$E-21$

$0.9699 \mathrm{E}-24$

$0.2004 \mathrm{E}-20$

ZERO

ZERO

$0.2605 \mathrm{E}-20$

ZERO

ZERO

$0.7992 \mathrm{E}-22$

$0.1054 \mathrm{E}-23$

$$
\text { ZERO }
$$

ZERO

$0.3209 \mathrm{E}-21$

$0.4074 \mathrm{E}-23$

$0.1399 \mathrm{E}-22$

ZERO

TRACE
$1212 \mathrm{E}-23$

$0.3694 \mathrm{E}-20$

ZERO

ZERO

ZERO

ZERO

$0.1032 \mathrm{E}-22$

TRACE

$0.9739 \mathrm{E}-22$

ZERO

ZERO

TRACE

ZERO

$0.5396 \mathrm{E}-22$

$0.2540 \mathrm{E}-15$

TRACE

$0.4056 \mathrm{E}-23$

TRACE

$0.4841 \mathrm{E}-15$

$0.1200 \mathrm{E}-16$

$0.3323 \mathrm{E}-23$

ZERO

WSRC-TR-95-0019 (Revision

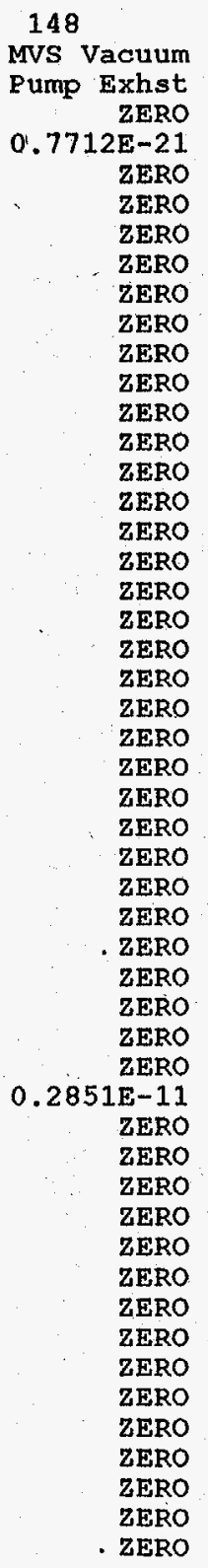

Date: January 1995

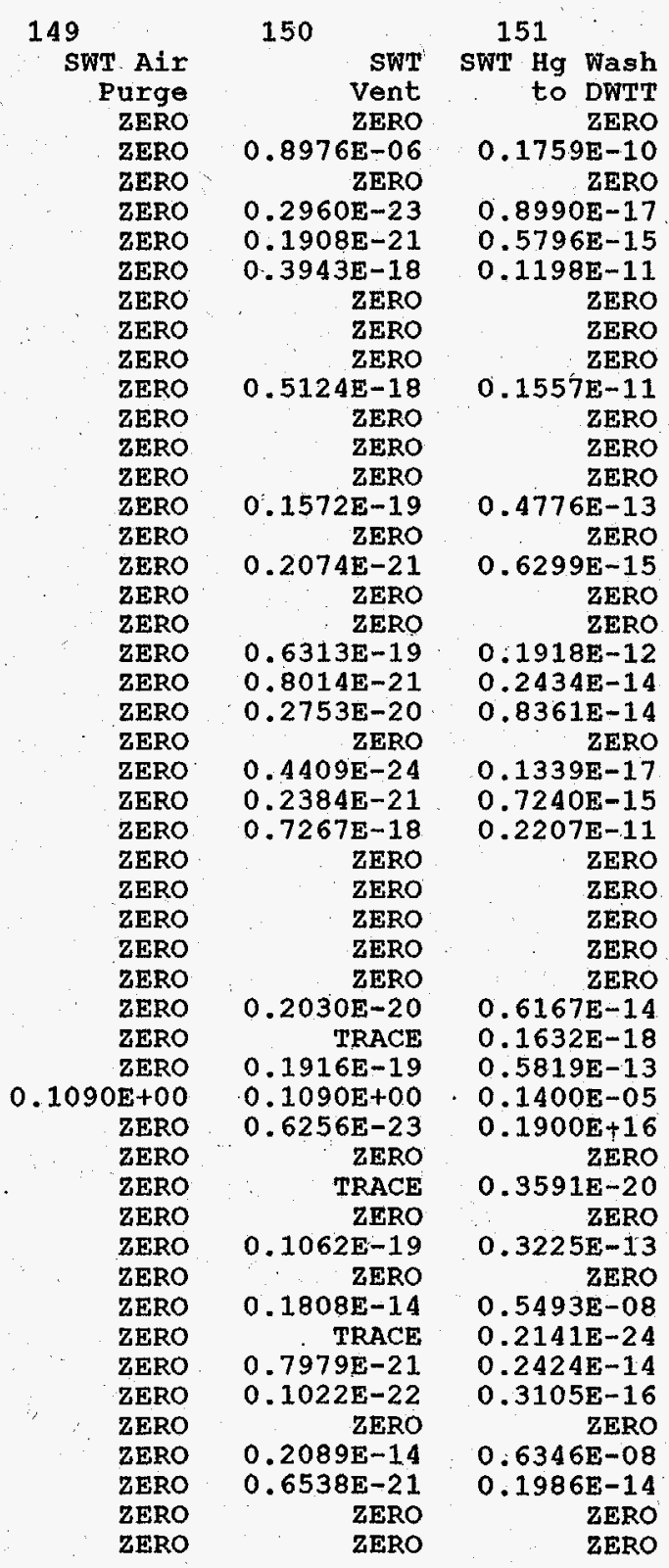

Section 6, Page 16 of 17 
Table A-1. HLW Flowsheet Material Balance (Tank 51/ITP Cycle 1) Section 6. Mercury Purification Cell

STREAM NUMBERS (CONT'D) $\rightarrow$ STREAM NAME

$38 \quad 502$

141503

137 Semi Vol Cs2O

136 Semi Vol $\mathrm{CsCl}$

126 Semi Vol Group A

140 Semi Vol Na2B4O

138 Semi Vol NaCl

139 Semi Vol NaF

125 Semi Vol NaI

$111 \mathrm{SiO2}$

$48 \mathrm{Sr}(\mathrm{COOH}) 2$

$176 \mathrm{Sr}$ (NO3) 2

$153 \mathrm{Sr}(\mathrm{NaTi2O5)} 2$

$127 \mathrm{Sr}(\mathrm{OH}) 2$

$30 \mathrm{SrCO} 3$

46 sro

$159 \mathrm{TCO} 2$

92 ThO2

107. TiO2

119 Tritium

17 U308

$62 \mathrm{UO2}(\mathrm{COOH}) 2$

177. $\mathrm{UO2}$ (NO3) 2

$16 \mathrm{VO} 2(\mathrm{OH}) 2$

27
178 Y (NO3) 3

$128 \mathrm{Y}(\mathrm{OH}) 3$

$31 \mathrm{Y2}(\mathrm{CO} 3) 3$

47 Y2O3

100 Zeolite

$44 \mathrm{Zn}(\mathrm{COOH}) 2$

$179 \mathrm{Zn}(\mathrm{NO} 3) 2$

57 Zno

13 hydrate н2O$$
1 \text { water }
$$

TOTAL FLOW, LB/HR

TEMPERATURE, DEG C

PRESSURE， ATM

PRESSURE， PSIG

PRESSURE; MM HG

ENTHALPY, PCU/HR

VAPOR FLOW, CFM

LIQUID FLOW, GPM

DENSITY, LBS/FT3

PHASE
145

from MVS

147

from MVS

MVs vent to $\mathrm{Hg}$ Accum at

148

MERO
ZERO
ZERORO
ZERO

ZERO

ZERO

ZERO

ZERO

ZERO

ZERO

ZERO

ERO

$0.1306 \mathrm{E}-19$

TRACE

$0.1205 \mathrm{E}-22$

$0.1663 \mathrm{E}-23$

ZERO

TRACE

$0.1462 \mathrm{E}-23$

$0.1462 \mathrm{E}-23$

$0.1217 \mathrm{E}-22$
$0.3395 \mathrm{E}-21$

TRACE

$0.8534 \mathrm{E}-21$

TRACE

TRACE

ZERO

$0.3251 \mathrm{E}-23$

ZERO

ZERO
$0.3955 E-24$

$0.3955 \mathrm{E}-24$
ZERO

$0.1066 \mathrm{E}-21$

TRACE

$0.7335 \mathrm{E}-22$

ZERO
$0.6139 \mathrm{E}-21$
ZERO

$0.2514 \mathrm{E}+00$

$-0.3000 \mathrm{E}+02$

$0.1000 \mathrm{E}+01$

ZERO

$-0.2485 E+00$

$0.3707 \mathrm{E}-04$

$0.8457 \mathrm{E}+03$
ZERO

ZERO

ZERO

ZERO

ZERO

ZERO

ZERO

ZERO

ZERO

ZERO

ZERO

ZERO

ZERO

ZERO

ZERO

ZERO

ZERO

ZERO

ZERO

ZERO

ZERO

ZERO

ZERO

ZERO

ZERO

ZERO

ZERO

ZERO

ZERO

ZERO

ZERO

ZERO

$0.2415 \mathrm{E}-04$

acuum Pump.

ZERP

ZERO

ZERO

ZERO

ZERO

ZERO

ZERO

ZERO

ZERO

ZERO

ZERO

ZERO

ZERO

ZERO

ZERO

ZERO

ZERO

ZERO

ZERO

ZERO

ZERO

ZERO

ZERO

ZERO

ZERO

ZERO

ZERO

ZERO

ZERO

ZERO

ZERO

ZERO

ZERO

ZERO

$0.241 .5 \mathrm{E}-04$

$-0.3000 \mathrm{E}+02$

$-0.3000 \mathrm{E}+02$

$0.9950 \mathrm{E}+00$

$0.6579 \mathrm{E}-04$

$-0.1470 \mathrm{E}+02$
$0.5000 \mathrm{E}-01$

$0.5000 \mathrm{E}-01$

$0.9743 \mathrm{E}-02$

$0.7348 \mathrm{E}-01$

$0.7562 \mathrm{E}+03$

$-0.1439 \mathrm{E}-10$

$0.4131 \mathrm{E}-04$

VAPOR

ZERO
N/A

$0.9147 \mathrm{E}-01$

VAPOR
149

SWT Air

Purge

ZERO

ZERO

ZERO

ZERO

ZERO

ZERO

ZERO

ZERO

ZERO

ZERO

ZERO

ZERO

ZERO

ZERO

ZERO

ZERO

ZERO

TERO

ZERO

ZERO

ZERO

ZERO

ZERO

ZERO

ZERO

ZERO

ZERO

ZERO

ZERO

ZERO

ZERO

ZERO

ZERO

ZERO

$0.7891 \mathrm{E}-02$

$0.4776 \mathrm{E}+00$

$0.3500 \mathrm{E}+02$

$0.1000 \mathrm{E}+01$

ZERO

$0.1128 \mathrm{E}+00$

$0.7055 \mathrm{E}-01$

VAPOR
150

swr

Vent

ZERO

ZERO

ZERO

ZERO

ZERO

ZERO

ZERO

ZERO

ZERO

$0.2569 \mathrm{E}-17$

$0.8781 \mathrm{E}-23$

$2371 \mathrm{E}-20$

2E-21

ZERO

TRACE

0.2875

$0.2394 \mathrm{E}-20$

0.6678E-19

. $6678=19$

$0.1679 \mathrm{E}-18$

$0.1022 \mathrm{E}-24$

$0.7360 \mathrm{E}-23$

ZERO

$0.6396 \mathrm{E}-21$

ZERO

0.7780

ZERO

$0.2097 \mathrm{E}-19$

$0.1443 \mathrm{E}-19$

ZERO

$0.1208 \mathrm{E}-18$

$0.2051 \mathrm{E}-01$
$0.4925 \mathrm{E}+00$

$0.3800 \mathrm{E}+02$

$-0.5055 \mathrm{E}+00$

$0.7339 \mathrm{E}+03$

$0.1837 \mathrm{E}+02$

$0.1232 \mathrm{E}+00$

$0.6665 \mathrm{E}-01$

VAPOR

151

T Hg Wash

to DWTT

ZERO

ZERO

ZERO

ZERO

ZERO

ZERO

ZERO

$0.7803 \mathrm{E}-11$ 


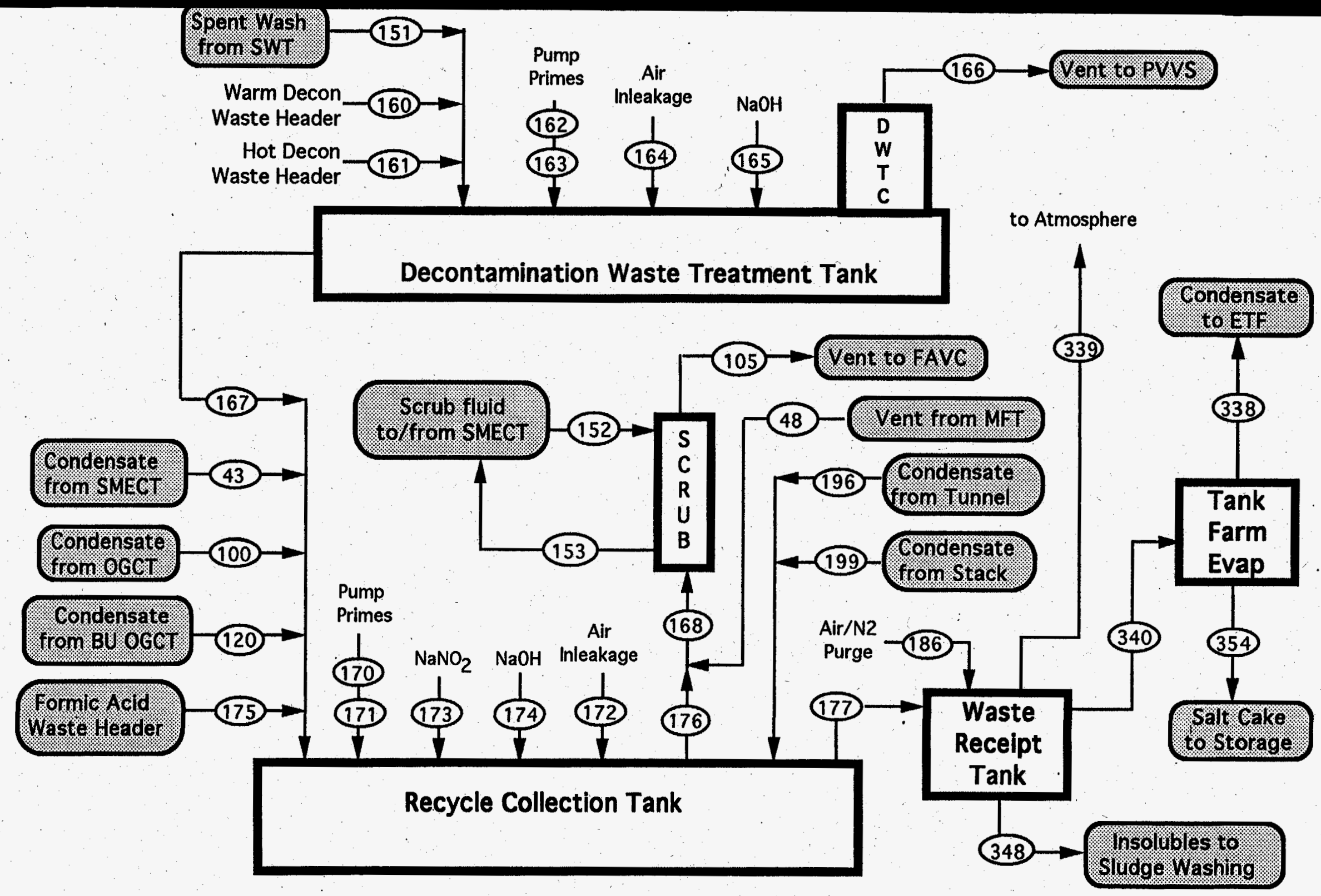

SECTION 7. COLLECTION AND TREATMENT OF WASTE STREAMS 
Table A-1. HLW Flowsheet Material Balance (Tank 51/ITP Cycle 1) Section 7 . Collection and Treatment of Waste Streams

DESCRIPTION

PAGE

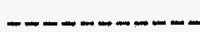

,

$---$

1. STREAM NUMBERS

$151,160,161,162,163,164,165 \ldots \ldots \ldots 2$

2. STREAM NUMBERS

$166,167,43,100,120,170,171 \ldots \ldots 6$

3. STREAM NUMBERS

$173,174,175,196,199,172,48 \ldots \ldots . . . . . . . . . .10$

4. STREAM NUMBERS

$176,168,105,152,153,177,186 \ldots \ldots \ldots \ldots$

5. STREAM NUMBERS

$339,348,340,338,354$

18 
Table A-1. HLW Flowsheet Material Balance (Tank 51/ITP Cycle 1) Section 7. Collection and Treatment of Waste streams

STREAM NUMBERS -> STREAM NAME

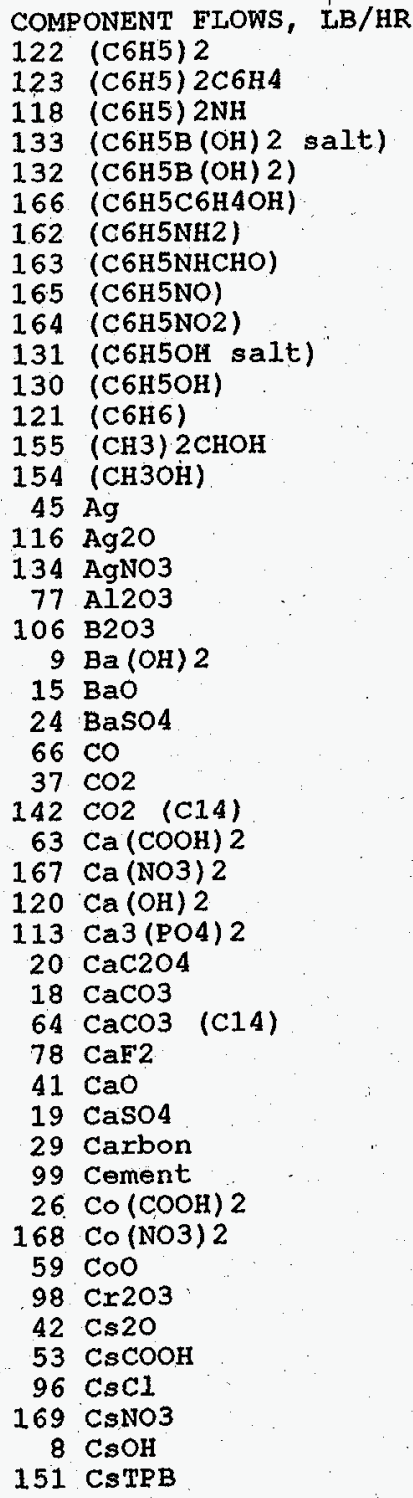
161 to Wash Warm Decon Hot Decon 162 163

$0.2713 E-11$ $0.2433 \mathrm{E}-13$

$0.3371 \mathrm{E}-09$ ZERO

$0.1349 \mathrm{E}-13$ $0.2453 \mathrm{E}-08$ $0.5112 \mathrm{E}-13$ $0.3957 \mathrm{E}-16$

$0.1675 \mathrm{E}-14$

$0.4289 \mathrm{E}-16$

$0.3522 \mathrm{E}-07$

$0.1442 \mathrm{E}-13$

$0.1092 \mathrm{E}-17$

$0.3955 \mathrm{E}-19$ ZERO

$0.6495 \mathrm{E}-23$

$0.1292 \mathrm{E}-06$

$0.2162 \mathrm{E}-11$

$0.7833 \mathrm{E}-12$

$0.8199 \mathrm{E}-21$

$0.4168 \mathrm{E}-1.4$

$0.2074 \mathrm{E}-21$ TRACE

$0.1025 \mathrm{E}-11$ ZERO

$0.6422 \mathrm{E}-12$
$0.5961 \mathrm{E}-19$ ZERO

$0.3672 \mathrm{E}-14$

$0.8192 \mathrm{E}-15$

$0.5391 \mathrm{E}-13$ ZERO

$0.3497 \mathrm{E}-16$

$0.1312 \mathrm{E}-18$

$0.7491 \mathrm{E}-17$

$0.1049 \mathrm{E}-12$

$0.3432 \mathrm{E}-16$

$0.1057 \mathrm{E}-13$

$0.2351 \mathrm{E}-17$ $0.2681 \mathrm{E}-18$
ZERO

\begin{tabular}{ll} 
ZERO & \\
ZERO & ZERO \\
ZERO & ZERO \\
ZERO & ZERO \\
ZERO & ZERO \\
ZERO & ZERO \\
ZERO & ZERO \\
ZERO & ZERO \\
ZERO & ZERO \\
ZERO & ZERO \\
ZERO & ZERO \\
ZERO & ZERO \\
ZERO & ZERO \\
ZERO & ZERO \\
ZERO & ZERO \\
ZERO & ZERO \\
ZERO & ZERO \\
ZERO & ZERO \\
ZERO & ZERO \\
ZERO & ZERO \\
ZERO & ZERO \\
ZERO & ZERO \\
ZERO & ZERO \\
ZERO & ZERO \\
ZERO & ZERO \\
ZERO & ZERO \\
ZERO & ZERO \\
ZERO & ZERO \\
ZERO & ZERO \\
ZERO & ZERO \\
ZERO & ZERO \\
ZERO & ZERO \\
ZERO & ZERO \\
ZERO & ZERO \\
ZERO & ZERO \\
ZERO & ZERO \\
ZERO & ZERO \\
ZERO & ZERO \\
ZERO & ZERO \\
ZERO & ZERO \\
ZERO & ZERO \\
ZERO & ZERO \\
ZERO & ZERO \\
ZERO & ZERO \\
ZERO & ZERO \\
ZERO & ZERO \\
ZERO & ZERO \\
ZERO & ZERO \\
& ZERO \\
\hline & \\
\hline
\end{tabular}

ZERO
ZERO
ZERO
ZERO
ZERO
ZERO
ZERO
ZERO
ZERO
ZERO
ZERO
ZERO
ZERO
ZERO
ZERO
ZERO
ZERO
ZERO
ZERO
ZERO
ZERO
ZERO
ZERO
ZERO
ZERO
ZERO
ZERO
ZERO
ZERO
ZERO
ZERO
ZERO
ZERO
ZERO
ZERO
ZERO
ZERO
ZERO
ZERO
ZERO
ZERO
ZERO
ZERO
ZERO
ZERO
ZERO
ZERO
ZERO

Date: January 1995 DWTT Sample DWTT Trnsfer
Pump Prime Pump Prime 164 DWTT Air 50 Wt: NaOH Inleakage to DWWT

\begin{tabular}{|c|c|c|}
\hline ZERO & ZERO & ZERO \\
\hline ZERO & ZERO & ZERO \\
\hline ZERO & ZERO & ZERO \\
\hline ZERO & ZERO & ZERO \\
\hline ZERO. & ZERO & ZERO \\
\hline ZERO & ZERO & ZERO \\
\hline ZERO & ZERO & ZERO \\
\hline ZERO & ZERO & ZERO \\
\hline ŻERO & ZERO & ZERO \\
\hline ZERO & ZERO & ZERO \\
\hline ZERO & ZERO & ZERO \\
\hline ZERO & ZERO & ZERO \\
\hline ZERO & ZERO & ZERO \\
\hline ZERO & ZERO & ZERO \\
\hline ZERO & ZERO & ZERO \\
\hline ZERO & ZERO & ZERO \\
\hline ZERO & ZERO & ZERO \\
\hline ZERO & ZERO & ZERO \\
\hline ZERO & ZERO & ZERO \\
\hline ZERO & ZERO & ZERO \\
\hline ZERO & ZERO & ZERO \\
\hline ZERO & ZERO & ZERO \\
\hline ZERO & ZERO & ZERO \\
\hline ZERO & ZERO & ZERO \\
\hline ZERO & ZERO & ZERO \\
\hline ZERO & ZERO & ZERO \\
\hline ZERO & ZERO & ZERO \\
\hline ZERO & ZERO & ZERO \\
\hline ZERO & ZERO & ZERO \\
\hline ZERO & ZERO & ZERO \\
\hline ZERO & ZERO & ZERO \\
\hline ZERO & ZERO & ZERO \\
\hline ZERO & ZERO & ZERO \\
\hline ZERO & ZERO & ZERO \\
\hline ZERO & ZERO & ZERO \\
\hline ZERO & ZERO & ZERO \\
\hline ZERO & ZERO & ZERO \\
\hline ZERO & ZERO & ZERO \\
\hline ZERO & ZERO & ZERO \\
\hline ZERO & ZERO & ZERO \\
\hline ZERO & ZERO & ZERO \\
\hline ZERO & ZERO & ZERO \\
\hline ZERO & ZERO & ZERO \\
\hline ZERO & ZERO & ZERO \\
\hline ZERO & ZERO & ZERO \\
\hline ZERO & ZERO & ZERO \\
\hline ZERO & ZERO & ZERO \\
\hline ZERO & ZERO & ZERO \\
\hline
\end{tabular}

Section 7, Page 2 of 21 
Table A-1. HLW Flowsheet Material Balance (Tank 51/ITP Cycle 1) Section 7. Collection and Treatment of Waste Streams

STREAM NUMBERS (CONT'D) $\rightarrow$ STREAM NAME

$54 \mathrm{Cu}(\mathrm{COOH}) 2$
$170 \mathrm{Cu}(\mathrm{NO} 3) 2$

$170 \mathrm{Cu}$
$55 \mathrm{CuO}$

$80 \mathrm{Fe} 2 \mathrm{O3}$

$101 \mathrm{FeO}$

102 Group A

103 Group

$25 \mathrm{H} 2$

$158 \mathrm{H} 2 \mathrm{C} 2 \mathrm{O} 4$

$93 \mathrm{H} 2 \mathrm{SO}_{4}$

$94 \mathrm{HCO}$

$43 \mathrm{HCl}$

$85 \mathrm{HF}$

49 HNO

$36 \mathrm{Hg}$

$129 \mathrm{Hg}$ (C6H5) 2

$135 \mathrm{Hg}$ (NO3) 2

$144 \mathrm{Hg} 2 \mathrm{Cl2}$

$117 \mathrm{Hg} 2 \mathrm{I} 2$

$21 \mathrm{HgO}$

97 I2

$183 \mathrm{~K} 2 \mathrm{CO} 3$

$39 \mathrm{~K} 2 \mathrm{O}$

$51 \mathrm{KCOOH}$

$157 \mathrm{KMnO4}$

161 KNO3

$10 \mathrm{KOH}$

$105 \mathrm{La} 203$

$108 \mathrm{Li} 20$

$65 \mathrm{Mg}(\mathrm{COOH}) 2$

$171 \mathrm{Mg}(\mathrm{NO} 3) 2$

$33 \mathrm{MgO}$

$60 \mathrm{Mn}(\mathrm{COOH}) 2$

$172 \mathrm{Mn}(\mathrm{NO} 3) 2$

$180 \mathrm{Mn} 304$

$56 \mathrm{MnO}$

$14 \quad \mathrm{MnO} 2$

145. MOO2

$81 \mathrm{~N} 2$

74 N2O

$182 \mathrm{NH} 3$

114 NH3OHNO

$52 \mathrm{NH} 4 \mathrm{COOH}$

173 NH4NO3

68 NH4OH
148 NH4TPB
151 to WWTT Warm Decon

$0.1084 \mathrm{E}-14$

$0.5325 \mathrm{E}-12$

$0.5325 \mathrm{E}-12$

$0.6211 \mathrm{E}-11$

$0.6210 \mathrm{E}-12$

$0.8669 \mathrm{E}-15$

$0.3699 \mathrm{E}-16$

TRACE:
$0.4470 \mathrm{E}-13$

$0.1881 \mathrm{E}-11$

ZERO

ZERO

$0.6295 \mathrm{E}-02$

$$
\text { ZERO }
$$

ZERO

$0.6042 \mathrm{E}-05$

ZERO

ZERO

$0.4820 \mathrm{E}-18$ ZERO

ZERO
$0.1975 \mathrm{E}-13$

$0.2452 \mathrm{E}-11$

ZERO

$0.6993 \mathrm{E}-14$

$0.8018 \mathrm{E}-17$ ZERO

$0.6851 \mathrm{E}-12$

$0.8474 \mathrm{E}-12$

$0.1282 \mathrm{E}-19$

$0.2703 \mathrm{E}-12$

$0.5308 \mathrm{E}-12$

$0.1494 \mathrm{E}-19$

$0.1942 \mathrm{E}-17$

$0.4767 \mathrm{E}-12$

$0.2630 \mathrm{E}-15$

$0.2385 \mathrm{E}-05$

ZERO
$0.1703 E-15$

ZERO

$.6577 \mathrm{E}-07$

$0.7579 \mathrm{E}-14$

ZERO

ZERO

ZERO

ZERO

ZERO

ZERO

ZERO

ZERO

ZERO

ZERO

ZERO

ZERO

ZERO

ZERO

ZERO

ZERO

ZERO

ZERO

ZERO

ZERO

ZERO

ZERO

ZERO

ZERO

ZERO

ZERO

ZERO

ZERO

ZERO

ZERO

ZERO

ZERO

ZERO

ZERO

ZERO

ZERO

ZERO

ZERO
161

Hot Decon DWTT Sample DWTT Trnsfer Pump Prime Pump Prime

$\begin{array}{llrr}\text { ZERO } & \text { ZERO } & \text { ZERO } & \text { ZERO } \\ \text { ZERO } & \text { ZERO } & \text { ZERO } & \text { ZERO } \\ \text { ZERO } & \text { ZERO } & \text { ZERO } & \text { ZERO }\end{array}$

$\begin{array}{llll}\text { ZERO } & \text { ZERO } & \text { ZERO } & \text { ZERO }\end{array}$

ZERO ZERO ZERO ZERO

ZERO ZERO ZERO ZERO

ZERO Z ZERO $\quad$ ZERO $\quad$ ZERO

ZERO ZERO $\quad$ ZERO $\cdots$ ZERO

ZERO - ZERO ZERO

$0.5752 \mathrm{E}+01$

$$
\text { EERO }
$$$$
\text { ZERO }
$$

ZERO

ZERO

$0.4931 \mathrm{E}+01$

ZERO

ZTRO

ZERO

ZERO

ZERO

ZERO

ZERO

ZERO

ZERO

ZERO

$0.3082 \mathrm{E}+00$

ZERO

ZERO

ZERO

ZERO

ZERO

ZERO

ZERO

ZERO

ZERO

ZERO

ZERO

ZERO

ZERO

ZERO

ZERO

ZERO

ZERO

ZERO

ZERO

ZERO

ZERO

ZERO

ZERO
ZERO

ZERO

ZERO

ZERO

ZERO

ZERO

ZERO

ZERO

ZERO

ZERO

ZERO

ZERO

ZERO

ZERO

ZERO

ZERO

ZERO

ZERO

ZERO

ZERO

ZERO

ZERO

ZERO

ZERO

ZERO

ZERO

ZERO

ZERO

ZERO

ZERO

ZERO

ZERO

ZERO

ZERO

ZERO

ZERO

ZERO

ZERO
ZERO
164

DNT Air 50 Wt? NaOH

to DWWT

ZERO ZERO

ZERO

ZERO

ZERO ZERO

ZERO ZERO

ZERO ZERO

$\begin{array}{ll}\text { ZERO } & \text { ZERO } \\ \text { ZERO ZERO }\end{array}$

ZERO ZERO

ZERO

ZERO ZERO

ZERO

ZERO ZERO

ZERO

ZERO ZERO

ZERO ZERO

ZERO ZERO

ZERO

ZERO ZERO

ZERO

ZERO ZERO

ZERO ZERO

ZERO

ZERO ZERO

ZERO

$0.5484 \mathrm{E}+02$

ZERO

ZERO

ZERO

ZERO

ZERO

ZERO

ZERO

ZERO

ZERO

ZERO

ZERO

ZERO

ZERO

ZERO 
Table A-1. HLW Flowsheet Material Balance (Tank 51/ITP Cyole 1) section 7. Collection and Treatment of Waste streams

STREAM NUMBERS (CONT'D) $\rightarrow$ STREAM NAME

35 No

$70 \mathrm{Na}(\mathrm{HgO}(\mathrm{OH}))$

$94 \mathrm{Na} 2 \mathrm{~B} 4 \mathrm{O} 7$

$109 \mathrm{Na} 2 \mathrm{C} 204$

$5 \mathrm{Na} 2 \mathrm{CO} 3$

$143 \mathrm{Na} 2 \mathrm{CO} 3$ (C14)

$89 \mathrm{Na} 2 \mathrm{CrO} 4$

115 Na2MoO
32 Na2O

$32 \mathrm{Na2O}$
$71 \mathrm{Na} 2 \mathrm{PuO} 2(\mathrm{OH}) 4$

73 Na2RhO4

$\begin{aligned} 72 & \text { Na2RuO4 } \\ 6 & \text { Na2SO4 }\end{aligned}$

$88 \mathrm{Na} 2 \mathrm{SiO}$

$91 \mathrm{NaAg}(\mathrm{OH}) 2$

$75 \mathrm{NaAl}(\mathrm{OH}) 4$

$50 \mathrm{NaCOOH}$

$7 \mathrm{NaCl}$

$69 \mathrm{NaF}$

$11 \mathrm{NaHCO} 3$

$12 \mathrm{NaI}$

3 NaNO2

$4 \mathrm{NaOH}$

$149 \mathrm{NaTPB}$

$160 \mathrm{NaTCO} 4$

152 NaTi205H

$181 \mathrm{Nd} 2 \mathrm{O} 3$

$61 \mathrm{Ni}$ (COOH) 2

$174 \mathrm{Ni}(\mathrm{NO} 3) 2$

$40 \mathrm{NiO}$

$175 \mathrm{~Pb}(\mathrm{NO} 3) 2$

$175 \mathrm{~Pb}(\mathrm{NO} 3)$
$86 \mathrm{PbCO} 3$

$67 \mathrm{PbO}$

$147 \mathrm{Pbs}$

28 PbSO 4

$124 \mathrm{Pd}$

$112 \mathrm{Pd}(\mathrm{NO} 3) 2$

110 Pdo

$76 \mathrm{PuO} 2$

156 PuO2 (NaTi205) 2

$58 \mathrm{Rh}$

$22 \mathrm{RhO}_{2}$

$95 \mathrm{Ru}$

$\begin{array}{ll}79 & \mathrm{RuO} 2 \\ 83 & \mathrm{RuO} 4\end{array}$

151

$\begin{array}{llll}160 & 161 & 162 & 163\end{array}$

Hot Decon DWTT Sample DWTT Trnsfer
to DWTT Hdr to DWTT Hdr to DWTT Pump Prime Rump Prime

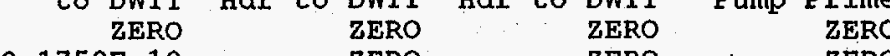

$\begin{array}{rll}0.1759 E-10 & \text { ZERO } & \text { ZERO : } \\ \text { ZERO ZERO }\end{array}$

$0.8990 \mathrm{E}-17$

$0.5796 \mathrm{E}-15$

$0.1198 \mathrm{E}-11$

ZERO

ZERO

$0.1557 \mathrm{E}-11$

ZERO

ZERO

$0.4776 \mathrm{E}-13$

ZERO

$0.6299 E-15$ ZERO

$0.1918 \mathrm{E}-12$

$0.2434 \mathrm{E}-14$

$0.8361 \mathrm{E}-14$ ZERO

$0.1339 \mathrm{E}-17$

$0.1339 \mathrm{E}-17$
$0.7240 \mathrm{E}-15$

$0.2207 \mathrm{E}-11$

ZERO

ZERO

ZERO

$0.6167 \mathrm{E}-14$

$0.1632 \mathrm{E}-18$

$0.5819 \mathrm{E}-13$

$0.1400 \mathrm{E}-05$

$0.1900 \mathrm{E}-16$

$\begin{array}{r}\text { ZERO } \\ \hline\end{array}$

$0.3591 \mathrm{E}-20$

ZERO
$0.3225 \mathrm{E}-13$

ZERO
$3 \mathrm{E}-08$

$0.5493 \mathrm{E}-08$

$0.2141 E-24$

$0.3105 \mathrm{E}-16$

ZERO

$0.6346 \mathrm{E}-08$

$0.1986 \mathrm{E}-14$ ZERO

WSRC-TR-95-0019 (Revision

$\begin{array}{ll}\text { ZERO } & \text { ZERO } \\ \text { ZERO } & \text { ZERO }\end{array}$

ZERO

ZERO

ZERO

ZERO

ZERO

ZERO

ZERO

ZERO

ZERO

ZERO

ZERO

ZERO

ZERO

ZERO

ZERO

ZERO

ZERO

ZERO

ZERO

ZERO

ZERO

ZERO

ZERO

ZERO

ZERO

ZERO

ZERO

ZERO

ZERO

ZERO

ZERO

ZERO

ZERO

ZERO

ZERO

ZERO

ZERO

ZERO

ZERO

ZERO

ZERO

ZERO

ZERO

ZERO

$\begin{array}{lll}\text { ZERO } & \text { ZERO } \\ \text { ZERO } & \text { ZERO } & \text { ZERO } \\ \text { ZERO } & \text { ZERO }\end{array}$

ZERO

Date: January 1995
164

DWTT Air 50 Wt: $\mathrm{NaOH}$

Inleakage to DWWT

ZERO

ZERO

ZERO

ZERO

ZERO

ZERO

ZERO

ZERO

ZERO

ZERO

ZERO

ZERO

ZERO

ZERO

ZERO

ZERO

ZERO

ZERO

ZERO

ZERO

ZERO

ZERO

ZERO

ZERO

ZERO

ZERO

ZERO

ZERO

ZERO

ZERO

ZERO

ZERO

ZERO

ZERO

ZERO

ZERO

ZERO

ZERO

ZERO

ZERO

ZERO

ZERO

ZERO

ZERO

ZERO

ZERO

Section 7, Page 4 of 21 
Table A-1. HLW Flowsheet Material Balance (Tank 51/ITP Cycle 1) Section 7. Collection and Treatment of Waste streams

STREAM NUMBERS (CONT'D) $\rightarrow$ STREAM NAME

\section{$38 \mathrm{SO} 2$}

$141 \mathrm{SO} 3$

137 Semi Vol Cs2O

136 Semi Vol CsCl

126 Semi Vol Group A

140 Semi Vo1 Na2B4O

138 Semi Vol NaCl

139 Semi Vol NaF

125 Semi Vol NaI

23 Semi

$111 \mathrm{SiO} 2 \mathrm{O}$

$176 \mathrm{Sr}(\mathrm{NO} 3) 2$

$153 \mathrm{sr}(\mathrm{NaTi2O5)} 2$

$127 \mathrm{Sr}(\mathrm{OH}) 2$

$30 \mathrm{SrCO}$

46 Sro

159
92
ThO2

92 ThO2

119 Tritium

17 U308

62. $\mathrm{UO} 2(\mathrm{COOH}) 2$

177. $\mathrm{UO2}$ (NO3) 2

$16 \mathrm{UO} 2(\mathrm{OH}) 2$

$27 \mathrm{Y}(\mathrm{COOH})$

$178 \mathrm{Y}(\mathrm{NOH}) 3$

31 Y2(CO3) 3

$47 \mathrm{Y} 203$

100 zeolite

$44 \mathrm{zn}(\mathrm{COOH}) 2$

$179 \mathrm{Zn}(\mathrm{NO} 3) 2$

$57 \mathrm{ZnO}$

104 ZrO2

13 hydrate $\mathrm{H} 2 \mathrm{O}$

1 water

TOTAL FLOW, IB/HR

TEMPERATURE， DEG C

PRESSURE, ATM

PRESSURE, PSIG

PRESSURE, MM HG

ENTHALPY， PCU/HR

VAPOR ELOW, CFM

LIQUID FLOW, GPM

DENSITY, IBS/FT3

PHASE
151
SWT Ho

Hg Wash War

DWTT Hdr to DWTT

ZERO

ZERO

ZERO

ZERO

ZERO

ZERO

ZERO

ZERO

ZERO

$0.7803 E-11$

$0.2667 \mathrm{E}-16$

$0.7203 \mathrm{E}-14$

$0.9939 \mathrm{E}-15$ ZERO

ZERO
$0.1124 \mathrm{E}-19$

$0.1124 \mathrm{E}-19$
$0.8733 \mathrm{E}-15$

$0.8733 E-15$
$0.7273 E-14$

$0.7273 \mathrm{E}-14$

$0.2029 \mathrm{E}-12$

$0.2037 \mathrm{E}-16$

$0.3105 \mathrm{E}-18$

$0.2236 \mathrm{E}-16$

ZERO

$0.3216 \mathrm{E}-24$

$0.1943 \mathrm{E}-14$ ZERO

ZERO
$0.2363 \mathrm{E}-15$ ZERO

$0.6368 \mathrm{E}-13$

$0.1302 E-16$

$0.4383 \mathrm{E}-13$

$0.3668 \mathrm{E}-12$

$0.1674 \mathrm{E}+00$

$0.1737 \mathrm{E}+00$

$0.3800 \mathrm{E}+02$

$0.9656 \mathrm{E}+00$

$-0.5055 E+00$

$0.7339 \mathrm{E}+03$
$0.6443 \mathrm{E}+01$

$0.3427 E-03$

0.632 OE+O2
IIQUID DWTT ZERO ZERO ZERO ZERO ZERO ZERO ZERO ZERO ZERO ZERO ZERO ZERO

ZERO

ZERO

ZERO

ZERO

ZERO

ZERO

ZERO

ZERO

ZERO

ZERO

ZERO

ZERO

ZERO

ZERO

ZERO

ZERO

ZERO

ZERO

ZERO

$0.2840 \mathrm{E}+01$

$0.2840 \mathrm{E}+01$

$0.3500 \mathrm{E}+02$

$0.1000 \mathrm{E}+01$

ZERO

$0.6243 \mathrm{E}+02$
LIQUID 161 162 WTT Sample DWTT Trnsfer

$0.9964 \mathrm{E}+02$

$0.5672 \mathrm{E}-02$ dr to DWTT

ZERO

ZERO

ZERO

ZERO

ZERO

ZERO

ZERO

ZERO

ZERO

ZERO

ZERO

ZERO

ZERO

ZERO

ZERO

ZERO

ZERO

ZERO

ZERO

ZERO

ZERO

ZERO

ZERÓ

ZERO

ZERO

ZERO

ZERO

ZERO

ZERO

ZERO

ZERO

ZERO

ZERO

Z200E+03

$0.3310 \mathrm{E}+03$

$0.3500 \mathrm{E}+02$

$0.1000 \mathrm{E}+01$

ZERO

$0.1133 E+05$

$0.6528 \mathrm{E}+00$

$0.6322 \mathrm{E}+02$

$\begin{array}{lr}\text { ZERO } & \text { ZERO } \\ \text { ZERO } & \text { ZERO } \\ \text { ZERO } & \text { ZERO } \\ \text { ZERO } & \text { ZERO } \\ \text { ZERO } & \text { ZERO }\end{array}$

ZERO ZERO

ZERO

ZERO

ZERO

ZERO

ZERO

ZERO

ZERO

ZERO

ZERO

ZERO

ZERO

ZERO

ZERO

ZERO

ZERO

ZERO

ZERO

ZERO

ZERO

ZERO

ZERO

ZERO

ZERO

ZERO

ZERO

ZERO

ZERO

ZERO

$0.1670 \mathrm{E}+01$

$0.2500 \mathrm{E}+02$

$0.1000 \mathrm{E}+01$

ZERO

$0.4190 \mathrm{E}+02$

$0.3335 \mathrm{E}-02$

$0.6243 E+02$

LIQUID

ZERO

0.8400 ZERO

$0.8400 \mathrm{E}+00$

$0.2500 E+02$

$0.1000 \mathrm{E}+01$

ZERO

$0.2108 \mathrm{E}+02$

$0.1678 \mathrm{E}-02$

$0.6243 E+02$

LIQUID
- 167 ZERO 163

164

DWTT Air 50 Wt: $\mathrm{NaOH}$

Inleakage to DWWT

ZERO ZERO

ZERO ZERO

ZERO ZERO

ZERO ZERO

ZERO $\quad$ ZERO

ZERO ZERO

ZERO

ZERO ZERO

$\begin{array}{ll}\text { ZERO } & \text { ZERO } \\ \text { ZERO } & \text { ZERO }\end{array}$

ZERO ZERO

ZERO ZERO

ZERO

ZERO

ZERO ZERO

ZERO ZERO

ZERO ZERO

ZERO

ZERO

ZERO

ZERO

ZERO

ZERO

ZERO

ZERO

ZERO

ZERO

ZERO

$0.1200 \mathrm{E}+01$

$0.3500 \mathrm{E}+02$

$0.1523 \mathrm{E}+04$

$0.1715 \mathrm{E}+02$

$0.7055 \mathrm{E}-01$

VAPOR

ZERO

ZERO

ZERO

ZERO 
STREAM NUMBERS $->$

STREAM NAME

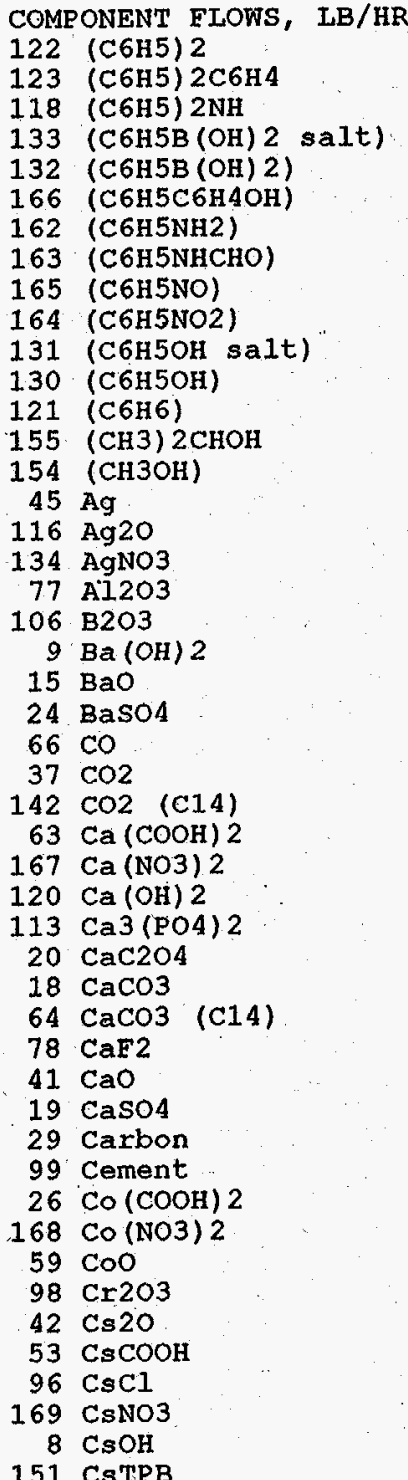

Table A-1. HLW Flowsheet Material Balance (Tank 51/ITP Cycle 1) section 7 . Collection and Treatment of Waste streams

$\begin{array}{rrrrrr}166 & 167 & 43 & 100 & 120 & 170 \\ \text { DWT Vent } & \text { DWTT Liquid } & \text { SMECT Liq } & 171 \\ \text { to PVVS } & \text { to RCT } & \text { to RCT } & \text { to RCT } & \text { Liq to RCT } & \text { RCT Sample RCT Transfer } \\ \text { Prime Pump Prime }\end{array}$

$0.5665 \mathrm{E}-14 \quad 0.2707 \mathrm{E}-11$

$0.53695-20 \quad 0.2433 \mathrm{E}-13$

$0.5369 \mathrm{E}-20.243 \mathrm{E}-13$

$\begin{array}{rr}0.7438 \mathrm{E}-16 & 0.3371 \mathrm{E}-09 \\ \text { ZERO } & \text { ZERO }\end{array}$ ZERO

ZERO
$0.1579 \mathrm{E}-19$
$0.1349 \mathrm{Z}-13$

$0.1887 \mathrm{E}-12 \quad 0.2453 \mathrm{E}-08$

$0.3818 \mathrm{E}-19$

$0.4894 \mathrm{E}-17$

$0.6601 \mathrm{E}-19 \quad 0.1675 \mathrm{E}-14$

$0.3523 E-07$

ZERO

$0.2240 \mathrm{E}-15$

$0.1420 \mathrm{E}-13$

$0.1084 \mathrm{E}-17$

$0.3875 \mathrm{E}-19$

ZERO

$0.1835 \mathrm{E}-13$

$0.4475 \mathrm{E}-18$

$0.1622 \mathrm{E}-18$

ZERO
TRACE

ZERO

$0.8863 \mathrm{E}-07$

$0.2162 \mathrm{E}-11$

$0.7833 \mathrm{E}-12$

$0.8199 \mathrm{E}-21$

$0.4168 \mathrm{E}-14$

ZERO

$0.4269 \mathrm{E}+00$

ZERO
$0.2164 \mathrm{E}-02$

TRACE

$0.2122 \mathrm{E}-18$

$0.1330 \mathrm{E}-18$

TRACE

ZERO

$0.7603 \mathrm{E}-21$

$0.1696 \mathrm{E}-21$

$0.1116 \mathrm{E}-19$

ZERO

ZERO

TRACE

$0.5193 \mathrm{E}-23$

$0.2171 \mathrm{E}-19$

$0.7106 \mathrm{E}-23$ ZERO

$0.4867 \mathrm{E}-24$

$0.1844 \mathrm{E}-20$ to RC.

Lig to RCT

Pump Prime Pump Prime

$0.2928 \mathrm{E}-03 \quad$ ZERO

ZERO

ZERO

ZERO

ZERO

ZERO

$0.2021 \mathrm{E}-06$

$0.5449 \mathrm{E}-01$
$0.7661 \mathrm{E}-06$

$0.8336 \mathrm{E}-08$

$0.2539 \mathrm{E}-07$

$0.6425 \mathrm{E}-09$

$0.6632 \mathrm{E}+00$

$0.8070 \mathrm{E}-03$

$0.5273 \mathrm{E}-11$

$0.9731 \mathrm{E}-16$

$0.9731 \mathrm{E}-16$

$0,3238 \mathrm{E}-04$

$0.1174 \mathrm{E}-04$

$0.1228 \mathrm{E}-13$

$0.6244 \mathrm{E}-07$

ZERO

$0.1146 \mathrm{E}-03$

TRACE

ZERO

$0.1479 \mathrm{E}-11$

ZERO

$0.1535 \mathrm{E}-04$

$0.5961 \mathrm{E}-19$

$0.9621 \mathrm{E}-05$

$0.8930 \mathrm{E}-12$ ZERO ZERO

$0.3672 \mathrm{E}-14$

$0.8192 \mathrm{E}-15$

ZERO
$0.5501 \mathrm{E}-07$

$0.5501 \mathrm{E}-07$
$0.1227 \mathrm{E}-07$

$0.8076 \mathrm{E}-06$

ZERO
ZERO

ZERO

$0.1312 \mathrm{E}-18$

$0.5239 \mathrm{E}-0 \mathrm{~g}$

$0.1965 \mathrm{E}-11$

$0.2508 \mathrm{E}-16$

$0.1122 \mathrm{E}-09$

$0.1571 \mathrm{E}-05$

$.1049 \mathrm{E}-12$

$0.1583 \mathrm{E}-06$ ZERO

0.2351 E-17

$0.8906 \mathrm{E}-14$

ZERO
$0.3522 \mathrm{E}-10$

$0.3522 \mathrm{E}-10$
$0.4016 \mathrm{E}-11$

ZERO

\begin{tabular}{|c|c|c|}
\hline ZERO & ZERO & ZERO \\
\hline ZERO & ZERO & ZERO \\
\hline ZERO & ZERO & ZERO \\
\hline ZERO & ZERO & ZERO \\
\hline ZERO & ZERO & ZERO \\
\hline ZERO & ZERO & ZERO \\
\hline ZERO & ZERO & ZERO \\
\hline ZERO & ZERO & ZERO \\
\hline ZERO & ZERO & ZERO \\
\hline ZERO & ZERO & ZERO \\
\hline ZERO & ZERO & ZERO \\
\hline ZERO & ZERO & ZERO \\
\hline ZERO & ZERO & ZERO \\
\hline ZERO & ZERO & ZERO \\
\hline ZERO & ZERO & ZERO \\
\hline ZERO & ZERO & ZERO \\
\hline ZERO & ZERO & ZERO \\
\hline ZERO & ZERO & ZERO \\
\hline ZERO & ZERO & zERO \\
\hline ZERO & ZERO & ZERO \\
\hline ZERO & ZERO & ZERO \\
\hline ZERO & ZERO & ZERO \\
\hline ZERO & ZERO & ZERO \\
\hline ZERO & ZERO & ZERO \\
\hline ZERO & ZERO & ZERO \\
\hline ZERO & ZERO & ZERO \\
\hline ZERO & ZERO & ZERO \\
\hline ZERO & ZERO & ZERO \\
\hline ZERO & ZERO & ZERO \\
\hline ZERO & ZERO & ZERO \\
\hline ZERO & ZERO & ZERO \\
\hline ZERO & ZERO & ZERO \\
\hline ZERO & ZERO & ZERO \\
\hline ZERO & ZERO & ZERO \\
\hline ZERO & ZERO & ZERO \\
\hline ZERO & ZERO & ZERO \\
\hline ZERO & ZERO & ZERO \\
\hline ZERO & ZERO & ZERO \\
\hline ZERO & ZERO & ZERO \\
\hline ZERO & ZERO & ZERO \\
\hline ZERO & ZERO & ZERO \\
\hline ZERO & ZERO & ZERO \\
\hline ZERO & ZERO & ZERO \\
\hline ZERO & ZERO & ZERO \\
\hline ZERO & ZERO & ZERO \\
\hline ZERO. & ZERO & ZERO \\
\hline ZERO & ZERO & ZERO \\
\hline ZERंO & ZERO & ZERO \\
\hline
\end{tabular}

Date: January 1995

section 7, Page 6 of 21

ZERO

ZERO

ZERO

ZERO

$.3650 \mathrm{E}-15$

$.2438 \mathrm{E}-02$

ZERO

ZERO

ZERO

ZERO

ZERO

$0.88335-01$

ZERO

$0.1533 \mathrm{E}-03$

ZERO

ZERO

ZERO

ZERO

0.2626 ZERO

ZERO

ZERO

ZERO

$0.1454 \mathrm{E}-01$

$0.1987 \mathrm{E}-02$

ZERO

ZERO

ZERO

$0.4301 \mathrm{E}-02$

-3099 -03

ZERO

$0.4110 \mathrm{E}-02$

ZERO

ZERO
ZERO

ERO

ZERO

ZERO

ZERO

ZERO

ZERO

ZERO

EERO

ZERO

ZERO

ZERO

ERO

ZERO

ZERO

ZERO

ZERO

ZFRO

ZERO

ZERO

ERO

ZERO

ERO 
Table A-1. HLW Flowsheet Material Balance (Tank 51/ITP Cyale 1) Section 7 . Collection and Treatment of Waste Streams

STREAM NUMBERS (CONT'D) $\rightarrow$ STREAM NAME

$54 \mathrm{Cu}(\mathrm{COOH}) 2$

$170 \mathrm{Cu}(\mathrm{NO} 3) 2$

$55 \mathrm{CuO}$

$80 \mathrm{Fe} 2 \mathrm{O}$
$101 \mathrm{FeO}$

102 Group A

103 Group B

$25 \mathrm{H} 2$

$158 \mathrm{H} 2 \mathrm{C} 2 \mathrm{O} 4$

$93 \mathrm{H2SO4}$



$43 \mathrm{HCl}$.

$43 \mathrm{HCl}$

85 HF

49 HNO3

$129 \mathrm{Hg}(\mathrm{C} 6 \mathrm{H} 5) 2$

$35 \mathrm{Hg}(\mathrm{NO} 3)$

$144 \mathrm{Hg} 2 \mathrm{C} 12$

$117 \mathrm{Hg} 2 \mathrm{I} 2$

$146 \mathrm{HgCl}$

$21 \mathrm{HgO}$

$183 \kappa 2 \mathrm{CO} 3$

$39 \mathrm{~K} 2 \mathrm{O}$

$51 \mathrm{KCOOH}$

$157 \mathrm{KMnO4}$

161 KNO3

$110 \mathrm{KOH}$

$\begin{array}{ll}150 & \text { KTPB } \\ 105 & \text { La2O3 }\end{array}$

108 Li2O

$65 \mathrm{Mg}(\mathrm{COOH}) 2$

$171 \mathrm{Mg}(\mathrm{NO} 3) 2$

$33 \mathrm{MgO}$

$60 \mathrm{Mn}(\mathrm{COOH}) 2$

172. $\mathrm{Mn}(\mathrm{NO} 3) 2$

$180 \mathrm{Mn} 304$

$56 \mathrm{MnO}$

$14 \mathrm{MnO} 2$

$145 \mathrm{M0O2}$

81 N2

$74 \mathrm{~N} 2 \mathrm{O}$
$182 \mathrm{NH} 3$

114 NH3OHNO

$52 \mathrm{NH} 4 \mathrm{COOH}$

173 NH4 4 NO 3

$68 \mathrm{NH} 4 \mathrm{OH}$
166

DWTC Vent DWTT Liquid

to PVVS to RCT

ZTRO

$0.1103 E-18$

$0.1103 E-18$

$0.1114 \mathrm{E}-20$

$0.1286 \mathrm{E}-1$

$0.1286 \mathrm{E}-18$

$0.1795 \mathrm{E}-2$

$0.7658 \mathrm{E}-23$

TRACE

ZERO

ZERO

ZERO

ZERO

ZERO

ZERO

ZERO

ZERO

ZERO

ZERO

ZERO
$0.8348 \mathrm{E}-12$

ZERO

ZERO

$0.5325 E-12$

$0.6211 \mathrm{E}-11$

$0.6210 \mathrm{E}-12$

$0.8669 \mathrm{E}-15$
$0.3699 \mathrm{E}-16$

TRACE

ZERO

ZERO

ZERO

ZERO

ZTRO

ZERO

ZERO

ZERO

ZERO

ZERO

ZERO

ZERO

$0.4032 \mathrm{E}-05$ ZERO

$0.4090 \mathrm{E}-20$ ZERO

$0.1448 \mathrm{E}-20$

$\mathrm{E}-13$

43 to RCT

$0.1624 \mathrm{E}^{\prime}-07$

$0.7978 \mathrm{E}-05$

$0.7219 \mathrm{E}-07$

$0.9303 \mathrm{E}-0.04$

$0.1299 \mathrm{E}-07$

$0.5541 \mathrm{E}-09$

$0.1848 \mathrm{E}-08$

$0.6697 \mathrm{E}-06$

$0.2818 \mathrm{E}-04$

ZERO

ZERO

$0.3758 \mathrm{E}+01$

120

Backup OGCT

170

171

RCT Sample RCT Transfex

ZERO
ZERO
ZERO
ZERO

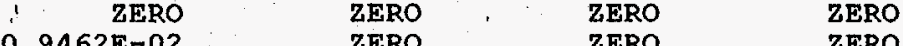

$0.2538 E+00 \quad$ ZERO ZERO ZERO

0.2538 E-01 ZERO $\quad$ ZERO Z ZERO

$\begin{array}{llll}0.2106 \mathrm{E}-03 & \text { ZERO } & \text { ZERO } & \text { ZERO } \\ 0.1511 \mathrm{O}-05 & \text { ZERO } & \text { ZERO } & \text { ZERO }\end{array}$

$\begin{array}{llll}0.1511 E-05 & \text { ZERO } & \text { ZERO } & \text { ZERO }\end{array}$

$\begin{array}{llll}\text { ZERO } & \text { ZERO } & \text { ZERO } & \text { ZERO } \\ \text { ZERO } & \text { ZERO } & \text { ZERO } & \text { ZERO }\end{array}$

$\begin{array}{llll}0.4677 \mathrm{E}-01 & \text { ZERO ZERO ZERO }\end{array}$

ZERO

ZERO ZERO ZERO

$\begin{array}{lll}0.6081 \mathrm{E}-03 & \text { ZERO } & \text { ZERO }\end{array}$

$0.1053 \mathrm{E}+00 \quad$ ZERO ZERO $\quad$ ZERO

$0.1299 \mathrm{E}+00 \quad$ ZERO ZERO $\quad$ ZERO

ZERO

$\begin{array}{rrrrr}\text { ZERO } & \text { ZERO } & \text { ZERO } & \text { ZERO } & \text { ZERO } \\ \text { ZERO } & 0.1433 \text { E-01 } & \text { ZERO } & \text { ZERO } & \text { ZERO }\end{array}$

$\begin{array}{lllll}\text { ZERO } & 0.1433 E-01 & \text { ZERO } & \text { ZERO } & \text { ZERO } \\ \text { ZERO } & 0.1183 E-06 & \text { ZERO } & \text { ZERO } & \text { ZERO }\end{array}$

ZERO $\quad 0.5500 \mathrm{E}-13$

$\begin{array}{lllll}0.7222 \mathrm{E}-11 & \text { ZERO } & \text { ZERO } & \text { ZERO } & \text { ZERO }\end{array}$

ZERO

$\begin{array}{llll}0.2959 \mathrm{E}-06 & 0.5718 \mathrm{E}-01 & \text { ZERO } & \text { ZERO } \\ \text { ZERRO }\end{array}$

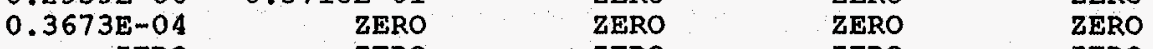

ZERO ZERO

ZERO ZERO

ZERO ZERO

ZERO ZERO

ZERO

ZERO ZERO

ZERO ZERO

ZERO ZERO

ZERO

ZERO ZERO

ZERO ZERO

ZERO ZERO

ZERO ZERO

ZERO

ZERO

ZERO

ZERO

ZERO ZERO

ZERO $\quad$ ZERO

ZERO ZERO

ZERO ZERO

ZERO 
Table A-1. HLW Flowsheet Material Balance (Tank 51/ITP Cyole 1) Section 7 . Collection and Treatment of Waste streams

STREAM NUMBERS (CONT'D) $\rightarrow$ STREAM NAME

35 NO

84 NO2

$70 \mathrm{Na}(\mathrm{HgO}(\mathrm{OH}))$

$94 \mathrm{Na} 2 \mathrm{~B} 4 \mathrm{O} 7$

$109 \mathrm{Na} 2 \mathrm{C2O}_{4}$

$5 \mathrm{Na} 2 \mathrm{CO} 3$

$143 \mathrm{Na} 2 \mathrm{CO} 3(\mathrm{C} 14)$

$89 \mathrm{Na} 2 \mathrm{CrO} 4$

115 Na2MoO

$32 \mathrm{Na2O}$

$71 \mathrm{Na2PuO2}(\mathrm{OH}) 4$

73 Na2RhO4

$\begin{array}{rl}72 & \mathrm{Na} 2 \mathrm{RuO} 4 \\ 6 & \mathrm{Na} 2 \mathrm{SO} 4\end{array}$

$6 \mathrm{Na} 2 \mathrm{SO} 4$
$88 \mathrm{Na} \mathrm{SiO}^{2}$

$87 \mathrm{Na} 3 \mathrm{PO} 4$

$91 \mathrm{NaAg}(\mathrm{OH}) 2$

$50 \mathrm{NaCOOH}$

$7 \mathrm{NaCl}$

$69 \mathrm{NaF}$

$11 \mathrm{NaHCO} 3$

11 NathC

3 NaNO2

2 NaNO3

4. $\mathrm{NaOH}$

149 NaTPB

152 NaTi2O5H

181. $\mathrm{Nd2O} 3$

$61 \mathrm{Ni}(\mathrm{COOH}) 2$

$174 \mathrm{Ni}(\mathrm{NO} 3) 2$

$40 \mathrm{NiO}$

$175 \mathrm{~Pb}$ (NO3) 2

$86 \mathrm{PbCO} 3$

$67 \mathrm{PbO}$

$147 \mathrm{Pbs}$

$28 \mathrm{PbSO}$

$112 \mathrm{Pd}(\mathrm{NO} 3) 2$

110 Pdo

$76 \mathrm{PuO} 2$

$156 \mathrm{PuO} 2$ (NaTi2O5) 2

$58 \mathrm{Rh}$

22. $\mathrm{RhO} 2$

$95 \mathrm{Ru}$

79 RuO2

$83 \mathrm{RuO} 4$

WSRC-TR-95-0019 (Revision $\begin{array}{lc}166 & 167 \\ \text { DWTC Vent } & \text { DWTT Liquid } \\ \text { to PVVS } & \text { to RCT }\end{array}$

to PVVS

$0.1758 \mathrm{E}-10$

ZERO

$0.3170 \mathrm{E}-18$

$0.1637 \mathrm{E}-05$

$0.2480 \mathrm{E}-18$

ZERO

ZERO

$0.3223 \mathrm{E}-18$

ZERO

ZERO

$0.9888 \mathrm{E}-20$

ZERO

$0.1304 \mathrm{E}-21$ ZERO

$0.7757 \mathrm{E}-18$

.504 OE-21 ZTRO

0.2773 ZERO

$0.14997-21$

$0.1379 \mathrm{E}-05$

$0.8178 \mathrm{E}-07$

ZERO

ZERO

ZERO

ZERO

TRACE
69E-19

$0.1653 \mathrm{E}+02$

$0.3934 \mathrm{E}-23$

ZERO

RACE

$0.6676 \mathrm{E}-20$

ZERO

$0.6049 \mathrm{E}-15$

$0.5018 \mathrm{E}-21$

$0.6428 \mathrm{E}-23$

$0.1314 \mathrm{E}-14$

$0,4112 \mathrm{E}-21$

ZERO
ZERO
$0.5568 \mathrm{E}-14$

ZERO

$0.1531 \mathrm{E}-11$

$0.1198 \mathrm{E}-1$

ZERO

ZERO

$0.1557 \mathrm{E}-11$

ZERO

ZERO

ZERO

ZERO

$0.6299 \mathrm{E}-15$

ZERO

$0.3747 \mathrm{E}-11$

$0.2434 \mathrm{E}-14$

$0.8361 \mathrm{E}-14$

$0.1339 \mathrm{ZERO}$

$0.7240 \mathrm{E}-15$

$0.6661 \mathrm{E}+01$

$0.3950 \mathrm{E}+00$

ZERO
ZERO

ZERO

ZERO

ZERO

$0.1632 \mathrm{E}-18$

$0.6129 \mathrm{E}-13$

$.3447 \mathrm{E}-02$

ZERO

$0.3591 \mathrm{E}-20$ ZERO

ZERO
$0.2921 \mathrm{E}-08$

$0.2424 \mathrm{E}-14$

$0.3105 \mathrm{E}-16$

$0.6346 \mathrm{E}-08$

$0.1986 \mathrm{E}-14$

ZERO
ZERO

\section{3 \\ 100}

SMECT Liq

120

$0.7155 \mathrm{E}-06$
$.3277 \mathrm{E}-06$

$.4661 \mathrm{E}-08$

$0.1347 \mathrm{E}-09$

$0.8683 \mathrm{E}-08$

$0.1794 \mathrm{E}-04$

ZERO

ZERO

$0.2332 \mathrm{E}-04$

ZERO

ZERO

ZERO
$0.9437 \mathrm{E}-08$

ZERO

ZERO
$0.2873 \mathrm{E}-05$

$0.3647 \mathrm{E}-07$

$0.1253 \mathrm{E}-06$

ZERO

$0.2006 \mathrm{E}-10$

$0.3307 \mathrm{E}-04$

ZERO
ZERO

ZERO

ZERO

ZERO
$0.9239 E-07$

$0.2445 \mathrm{E}-11$

$0.8718 \mathrm{E}-06$

$0.8121 \mathrm{E}-02$

$0.2847 \mathrm{E}-09$

0.5380 E-13

ZERO

$0.4831 \mathrm{E}-06$
ZERO

ZERO

$0.3207 \mathrm{E}-17$

$0.3631 \mathrm{E}-07$
$0.4651 \mathrm{E}-09$

ZERO
$0.6966 \mathrm{E}-17$

$0.2975 \mathrm{E}-07$

ZERO
ZERO

GCT Liquid

$0.3839 \mathrm{E}-04$

$.7574 \mathrm{E}-04$

ZERO
$0.1227 \mathrm{E}+00$

E+00
ZERO

ZERO

ZERO

ZERO

0.2030 ZERO

E+OO

ZERO

ZERO

ZERO

ZERO

$0.2578 \mathrm{E}-04$

ZERO

ZERO

$0.4978 \mathrm{E}-04$

$0.1277 E-01$

ZERO

$0.5410 \mathrm{E}-05$

$.2213 \mathrm{E}-02$
ZERO

ZERO

ZERO

ZERO

ZERO

ZERO

$0.2542 \mathrm{E}-02$

$0.1104 \mathrm{E}-01$

ZERO

$0.4902 \mathrm{E}-04$

ZERO

$0.1252 \mathrm{E}-02$

ZERO

ZERO

$0.9956 \mathrm{E}-04$

ZERO

ZERO

ZERO

ZERO

ZERO

Date: January 1995
170.

171

120 OGCT

Iig to RCT

ZERO

RCT Sample RCT Transfer

Pump Prime Pump Prime $\begin{array}{ll}\text { ZERO } & \text { ZERO } \\ \text { ZERO } & \text { ZERO }\end{array}$

ZERO ZERO

ZERO ZERO

ZERO ZERO

ZERO ZERO

ZERO

ZERO

ZERO

ZERO

ZERO

ZTRO

ZERO ZERO

ZERO ZERO

ZERO

ZERO ZERO

ZERO ZERO

ZERO ZERO

ZERO ZERO

ZERO

ZERO:

ZERO

ZERO ZERO

ZERO

ZERO

ZERO :

ZERO ZERO

ZERO ZERO

ZERO ZERO

ZERO ZERO

ZERO ZERO

ZERO ZERO

ZERO

7ERO

ZRRO

ZERO

ZERO

ZERO

ZERO

ZERO

ZERO

ZERO

ZERO

ZERO

ZERO

ZERO

ZERO

ZERO

ZERO

ZERO

ZERO

ZERO

ZERO

Section 7, Page 8 of 21

1. 
Table A-1. HLW Flowsheet Material Balance (Tank 51/ITP Cycle 1) section 7 . Collection and Treatment of Waste Streams

STREAM NUMBERS (CONT'D) -> STREAM NAME

$38 \mathrm{SO2}$

$141 \mathrm{SO} 3$

137 Semi Vol Cs2O

136 Semi Vol CsCl

126 Semi Vol Group A

140 Semi Vol Na2B4O

138 Semi Vol NaCl

139 Semi Vol NaF

125 Semi Vol NaI

23 Semi

$48 \mathrm{Sr}(\mathrm{COOH}) 2$

$176 \mathrm{Sr}(\mathrm{NO} 3) 2$

$153 \mathrm{Sr}(\mathrm{NaTi2O5)} 2$

$127 \mathrm{Sr}(\mathrm{OH}) 2$

$30 \mathrm{srCO}$

46 Sro

159 TcO2

107 TiO2

119 Tritium

$17 \mathrm{v} 30 \mathrm{~B}$

$62 \mathrm{UO} 2(\mathrm{COOH}) 2$

177 UO2 (NO3) 2

$16 \mathrm{VO} 2(\mathrm{OH}) 2$

$27 \mathrm{Y}(\mathrm{COOH}) 3$

$178 \mathrm{Y}(\mathrm{NO} 3)$

$128 \mathrm{Y}(\mathrm{OH}) 3$

$31 \mathrm{Y} 2(\mathrm{CO})$
$47 \mathrm{Y} 2 \mathrm{O}$

100 Zeolite

$44 \mathrm{Zn}(\mathrm{COOH}) 2$

$179 \mathrm{Zn}(\mathrm{NO} 3) 2$

57 Zno

13 hydrate $\mathrm{H} 2 \mathrm{O}$

$$
1 \text { water }
$$

TOTAL FLOW, LB/HR

TEMPERATURE， DEG C

PRESSURE, ATM

PRESSURE， PSIG

PRESSURE, MM HG

ENTHALPY, PCU/HR

VAPOR FLOW, CFM

LIQUID FIOW, GPM

DENSITY, IBS/FT3

PHASE
166
DWTC Vent DWTT Iiquid

to pVVS to RCT

ZERO TORO

ZERO

ZERO

ZERO

ZERO

ZERO

ZERO

ZERO

ZERO

ZERO

$0.1616 \mathrm{E}-17$

ZERO
$1 \mathrm{E}-20$

0.14058E-21 ZERO

ZERO

$0.3224 \mathrm{E}-2$

$0.1808 \mathrm{E}-2$

$0.1506 \mathrm{E}-20$

$0.4200 \mathrm{E}-19$

$0.1056 \mathrm{E}-18$

2ERO

$E-23$

ZERO

$0.4023 \mathrm{E}-21$ ZERO ZERO

$0.4893 \mathrm{E}-22$ ZERO

ZERO
$0.2697 \mathrm{E}-23$

$0.1598 \mathrm{E}-19$ ZERO

$0.1220 \mathrm{E}+01$

$0.7301 \mathrm{E}+02$

$0.2250 \mathrm{E}+02$ $0.9656 \mathrm{E}+00$ $0.5055 \mathrm{E}+00$

$0.7339 \mathrm{E}+03$

$0.1348 \mathrm{E}+04$

$0.1710 \mathrm{E}+02$

$0.7114 \mathrm{E}-01$
VAPOR $0.7803 \mathrm{E}-11$

$0.7203 \mathrm{E}-14$

$0.9939 \mathrm{E}-1.5$ ZERO

$0.1557 \mathrm{E}-16$ $0.1557 \mathrm{E}-16$
$0.8733 \mathrm{E}-15$ $0.7273 \mathrm{E}-14$

$0.2029 \mathrm{E}-12$

$0.2029 \mathrm{E}-16$

$0.5099 \mathrm{E}-12$

ZERO

$0.1943 \mathrm{E}-14$

ZERO

$0.2363 \mathrm{E}-15$

0.2363 E-15 ZERO

$0.1302 \mathrm{E}-16$

$0.7718 \mathrm{E}-13$ ZERO
$1 \mathrm{E}-08$ $0.3374 \mathrm{E}+03$

$0.3526 \mathrm{E}+03$

$0.2250 E+02$

$0.9656 \mathrm{E}+00$

$0.7339 \mathrm{E}+03$

$0.7700 \mathrm{E}+04$

$0.6859 \mathrm{E}+00$

$0.6410 \mathrm{E}+02$

LIQUID
43

$\begin{array}{ll}43 & 100 \\ \text { SMECT LIT OGCT }\end{array}$ to RCT RC? ZERO
ZERO ZERO ZERO ZERO ZERO ZERO ZERO ZERO ZERO

$0.1169 \mathrm{E}-03$ $0.3996 \mathrm{E}-09$

$0.1079 \mathrm{E}-06$

$0.1489 \mathrm{E}-07$ ZERO

$0.2236 \mathrm{E}-16$

$-0.5055 \mathrm{E}+00$

ZERO

$0.1684 \mathrm{E}-1.12$

$0.1308 \mathrm{E}-07$
$0.1090 \mathrm{E}-06$

$0.1090 \mathrm{E}-06$
$0.3039 \mathrm{E}-05$

$0.6462 \mathrm{E}-09$

$0.7639 \mathrm{E}-05$

$0.4652 \mathrm{E}-11$

$0.3349 \mathrm{E}-09$

ZERO

$0.4818 \mathrm{E}-17$
$0.2911 \mathrm{E}-07$

ZERO

$0.3541 \mathrm{E}-08$

$0.3541 \mathrm{E}-08$

$0.9541 \mathrm{E}-06$

$0.1951 \mathrm{E}-09$

$0.6566 \mathrm{E}-06$

$0.5495 \mathrm{E}-05$

$0.1178 \mathrm{E}+04$

$0.1183 \mathrm{E}+04$

$0.4900 \mathrm{E}+02$

$0.1000 \mathrm{E}+01$

ZERO

$0.5787 \mathrm{E}+05$

$0.2360 \mathrm{E}+01$

$0.6251 \mathrm{E}+02$ to RCT

RCT
ZERO

$$
\text { ZERO }
$$

$$
\text { ZERO }
$$

ZERO

$$
\text { ZERO }
$$

$$
\text { ZERO }
$$

ZERO

ZERO

ZERO

$0.1229 \mathrm{E}+01$

ZERO

$$
\text { ZERO }
$$

ZERO

ZERO

$1535 \mathrm{E}-03$

$0.3569 \mathrm{E}-04$

$0.2972 \mathrm{E}-03$
$0.8335 \mathrm{E}-02$

$0.1413 \mathrm{E}-09$

$0.2084 \mathrm{E}-01$

ZERO

ZERO

ZERO

ZERO

ZERO

$0.4229 \mathrm{E}-04$

ZERO

ZERO

$0.3159 \mathrm{E}-02$

$0.1592 \mathrm{E}+04$

$0.1594 \mathrm{E}+04$

$0.4000 \mathrm{E}+02$

$0.9650 \mathrm{E}+00$

$-0.5144 \mathrm{E}+00$

$0.7334 \mathrm{E}+03$

$0.6381 \mathrm{E}+05$

$0.3181 \mathrm{E}+01$

$0.6249 \mathrm{E}+02$

IIQUID
120

Backup OGCT

170

171

RCT Sample RCT Transfer

ZERO

ZERO

ZERO

ZERO

ZERO

ZERO

ZERO

ZERO

ZERO

ZERO

ZERO

ZERO

ZERO

ZERO

ZERO

ZERO

ZERO

ZERO

ZERO

ZERO

ZERO

ZERO

ZERO

ZERO

ZERO

ZERO

ZERO

ZERO

ZERO

ZERO

ZERO

ZERO

ZERO

ZERO

ZERO

$0.9646 \mathrm{E}+01$

$0.9646 \mathrm{E}+01$

$0.4000 \mathrm{E}+02$

$0.1000 \mathrm{E}+01$

ZERO

$0.3866 \mathrm{E}+03$

$0.1926 \mathrm{E}-01$

$0.6243 \mathrm{E}+02$

IIQUID

Eump Prime Pump Prime

ZERO ZERO

ZERO ZERO

ZERO

ZERO

ZERO ZERO

ZERO $\quad$ ZERO

$\begin{array}{ll}\text { ZERO } & \text { ZERO } \\ \text { ZERO } & \text { ZERO }\end{array}$

ZERO ZERO

ZERO ZERO

ZERO ZERO

ZERO ZERO

ZERO

ZERO

ZERO

ZERO

ZERO ZERO

ZERO ZERO

ZERO ZERO

ZERO ZERO

ZERO $\quad$ ZERO 
Table A-1. HiW Flowsheet Material Balance (Tank 51/ITP Cycle 1) section 7. Collection and Treatment of Waste streams

STREAM NUMBERS -> STREAM NAME

\begin{tabular}{|c|c|}
\hline \\
\hline & $(\mathrm{C} 6 \mathrm{H} 5) 2$ \\
\hline $\begin{array}{l}123 \\
118\end{array}$ & $\begin{array}{l}\text { (C6H5) 2C6H4 } \\
\text { (C6H5) 2NH }\end{array}$ \\
\hline 133 & (C6H5B (OH) 2 salt) \\
\hline 132 & (C6H5B (OH) 2) \\
\hline 1.66 & (C6H5C6H4OH) \\
\hline 162 & (C6H5NH2) \\
\hline 163 & (C6H5NHCHO) \\
\hline 165 & (C6H5NO) \\
\hline 164 & (C6H5NO2) \\
\hline 131 & (C6H5OH salt) \\
\hline 1.30 & (C6H5OH) \\
\hline 121 & $(\mathrm{C} 6 \mathrm{H} 6)$ \\
\hline 155 & (CH3) $2 \mathrm{CHOH}$ \\
\hline 154 & (CH3OH) \\
\hline 45 & Ag \\
\hline 16 & Ag20 \\
\hline 134 & AgNO3. \\
\hline 77 & $\mathrm{Al2O}$ \\
\hline 106 & B203 \\
\hline & $\mathrm{Ba}(\mathrm{OH}) 2$ \\
\hline 15 & $\mathrm{BaO}$ \\
\hline 24 & $\mathrm{BaSO} 4$ \\
\hline 66 & $\mathrm{CO}$ \\
\hline 37 & $\mathrm{CO} 2$ \\
\hline 142 & $\mathrm{CO} 2$ (C14) \\
\hline 63 & $\mathrm{Ca}(\mathrm{COOH}) 2$ \\
\hline 167 & $\mathrm{Ca}(\mathrm{NO} 3) 2$ \\
\hline 120 & $\mathrm{Ca}(\mathrm{OH}) 2$ \\
\hline 113 & $\mathrm{Ca} 3(\mathrm{PO} 4) 2$ \\
\hline 20 & $\mathrm{CaC2O4}$ \\
\hline 18 & $\mathrm{CaCO} 3$ \\
\hline 64 & $\mathrm{Ca} \mathrm{CO} 3$ (C14) \\
\hline 78 & $\mathrm{CaF2}$ \\
\hline 41 & $\mathrm{CaO}$ \\
\hline 19 & Caso4. \\
\hline 29 & Carbon \\
\hline 99 & Cement \\
\hline 26 & $\mathrm{Co}(\mathrm{COOH}) 2$ \\
\hline 168 & $\mathrm{Co}(\mathrm{NO} 3) 2$ \\
\hline 59 & $\mathrm{COO}$ \\
\hline 98 & Cr203 \\
\hline 42 & $\operatorname{cs} 20$ \\
\hline 53 & $\mathrm{CsCOOH}$ \\
\hline 96 & $\mathrm{CsCl}$ \\
\hline 169 & $\mathrm{CsNO} 3$ \\
\hline & $\begin{array}{l}\mathrm{CsOH} \\
\mathrm{CsTPB}\end{array}$ \\
\hline
\end{tabular}

WSRC-TR-95-0019 (Revision
173

40 wt 8 174 175 Formic Acid Sand Filter
Hdr to RCT Inlet Sump 196 ZERO ZERO ZERO

ZERO

ZERO

ZERO

ZERO

ZERO

ZERO

ZERO

ZERO

ZERO

ZERO

ZERO

ZERO

ZERO

ZERO

ZERO

ZERO

ZERO

ZERO

ZERO

ZERO

ZERO

ZERO

ZERO

ZERO

ZERO

ZERO

ZERO

ZERO

ZERO

ZERO

ZERO

ZERO

ZERO

ZERO

ZERO

ZERO

ZERO

ZERO

ZERO

ZERO

0)

\begin{tabular}{|c|c|c|}
\hline ZERO & ZERO & ZERO \\
\hline ZERO & ZERO & ZERO \\
\hline ZERO & ZERO & ZERO \\
\hline ZERO & ZERO & ZERO \\
\hline ZERO & ZERO & ZERO \\
\hline ZERO & ZERO & ZERO \\
\hline ZERO & ZERO & ZERO \\
\hline ZERO & ZERO & ZERO \\
\hline ZERO & ZERO & ZERO \\
\hline ZERO & ZERO & ZERO \\
\hline ZERO & ZERO & ZERO \\
\hline ZERO & ZERO & ZERO \\
\hline ZERO & ZERO & ZERO \\
\hline ZERO & ZERO & ZERO \\
\hline ZERO & ZERO & ZERO \\
\hline ZERO & ZERO & ZERO \\
\hline ZERO & ZERO & ZERO \\
\hline ZERO & ZERO & ZERO \\
\hline ZERO & ZERO & ZERO \\
\hline ZERO & ZERO & ZERO \\
\hline ZERO & ZERO & ZERO \\
\hline ZERO & ZERO & ZERO \\
\hline ZERO & ZERO & ZERO \\
\hline ZERO & ZERO & ZERO \\
\hline ZERO & ZERO & ZERO \\
\hline ZERO & ZERO & ZERO \\
\hline ZERO & ZERO & ZERO \\
\hline ZERO & ZERO & ZERO \\
\hline ZERO & ZERO & ZERO \\
\hline ZERO & ZERO & ZERO \\
\hline ZERO & ZERO & ZERO \\
\hline ZERO & ZERO & ZERO \\
\hline ZERO & ZERO & ZERO \\
\hline ZERO & ZERO & ZERO \\
\hline ZERO & ZERO & ZERO \\
\hline ZERO & ZERO & ZERO \\
\hline ZERO & ZERO & ZERO \\
\hline ZERO & ZERO & ZERO \\
\hline ZERO & ZERO & ZERO \\
\hline ZERO & ZERO & ZERO \\
\hline ZERO & ZERO & ZERO \\
\hline ZERO & ZERO & ZERO \\
\hline ZERQO & ZERO & ZEERO \\
\hline ZERO & ZERO & ZERO \\
\hline ZERO & ZERO & ZERO \\
\hline ZERO & ZERO & ZERO \\
\hline ZERO & ZERO & ZERO \\
\hline ZERO & ZERO & ZERO \\
\hline
\end{tabular}

Date: January 1995 199

17.2

RCT Air

Sand Filter
Exit Sump

Inleakage to Scrubbex

$\begin{array}{lrr}\text { ZERO } & \text { ZERO } & 0.2345 E-15 \\ \text { ZERO } & \text { ZERO } & 0.4657 \mathrm{E}-09 \\ \text { ZERO } & \text { ZERO } & 0.2552 \mathrm{E}-09 \\ \text { ZERO } & \text { ZERO } & \text { ZERO } \\ \text { ZERO } & \text { ZERO } & \text { ZERO } \\ \text { ZERO } & \text { ZERO } & 0.1094 \mathrm{E}-08 \\ \text { ZERO } & \text { ZERO } & 0.1449 \mathrm{E}-04 \\ \text { ZERO } & \text { ZERO } & 0.4145 \mathrm{E}-08 \\ \text { ZERO } & \text { ZERO } & 0.6748 E-10 \\ \text { ZERO } & \text { ZERO } & 0.1375 E-09 \\ \text { ZERO } & \text { ZERO } & 0.1631 \mathrm{E}-22 \\ \text { ZERO } & \text { ZERO } & 0.1945 \mathrm{E}-02 \\ \text { ZERO } & \text { ZERO } & 0.2997 \mathrm{E}-22 \\ \text { ZERO } & \text { ZERO } & 0.9932 \mathrm{E}-13 \\ \text { ZERO } & \text { ZERO } & 0.3408 \mathrm{E}-14 \\ \text { ZERO } & \text { ZERO } & 0.2459 \mathrm{E}-09 \\ \text { ZERO } & \text { ZERO } & \text { ZERO } \\ \text { ZERO } & \text { ZERO } & \text { ZERO } \\ \text { ZERO } & \text { ZERO } & 0.1750 \mathrm{E}-06 \\ \text { ZERO } & \text { ZERO } & 0.2509 \mathrm{E}-06 \\ \text { ZERO } & \text { ZERO } & \text { ZERO } \\ \text { ZERO } & \text { ZERO } & \text { TRACE } \\ \text { ZERO } & \text { ZERO } & 0.3376 \mathrm{E}-09 \\ \text { ZERO } & \text { ZERO } & \text { ZERO } \\ \text { ZERO } & \text { ZERO } & 0.4379 \mathrm{E}-16 \\ \text { ZERO } & \text { ZERO } & \text { TRRACE } \\ \text { ZERO } & \text { ZERO } & \text { ZERO } \\ \text { ZERO } & \text { ZERO } & 0.8297 \mathrm{E}-07 \\ \text { ZERO } & \text { ZERO } & \text { ZERO } \\ \text { ZERO } & \text { ZERO } & 0.5203 E-07 \\ \text { ZERO } & \text { ZERO } & 0.4838 E-14 \\ \text { ZERO } & \text { ZERO } & \text { ZERO } \\ \text { ZERO } & \text { ZERO } & \text { ZERO } \\ \text { ZERO } & \text { ZERO } & 0.2973 E+09 \\ \text { ZERO } & \text { ZERO } & 0.6625 E-10 \\ \text { ZERO } & \text { ZERO } & 0.4375 E-08 \\ \text { ZERO } & \text { ZERO } & \text { ZERO } \\ \text { ZERO } & \text { ZERO } & \text { ZERO } \\ \text { ZERO } & \text { ZERO } & 0.2839 E-11 \\ \text { ZERO } & \text { ZERO } & 0.1065 E-13 \\ \text { ZERO } & \text { ZERO } & 0.6081 E-12 \\ \text { ZERO } & \text { ZERO } & 0.8523 E-08 \\ \text { ZERO } & \text { ZERO } & 0.2779 E-11 \\ \text { ZERO } & \text { ZERO } & 0.8580 E-09 \\ \text { ZERO } & \text { ZERO } & \text { ZERO } \\ \text { ZERO } & \text { ZERO } & 0.1904 \mathrm{E}-12 \\ \text { ZERO } & \text { ZERO } & \text { ZERO } \\ \text { ZERO } & \text { ZERO } & \text { ZERO } \\ & & \end{array}$

Section 7, Page 10 of 21 
Table A-1. HLW Flowsheet Material Balance (Tank 51/ITP Cyale 1) Section 7. Collection and Treatment of Waste Streams

STREAM NUMBERS (CONT'D) $\rightarrow$ STREAM NAME

$54 \mathrm{Cu}$ ( $\mathrm{COOH}) 2$

$170 \mathrm{Cu}(\mathrm{NO} 3) 2$

55 CuO

$80 \mathrm{Fe} 2 \mathrm{O} 3$

$101 \mathrm{FeO}$

102 Group A

$25 \mathrm{H} 2$

$158 \mathrm{H} 2 \mathrm{C} 2 \mathrm{O} 4$

$93 \mathrm{H} 2 \mathrm{SO} 4$

90 Н $3 \mathrm{BO} 3$

$34 \mathrm{HCOOH}$

$43 \mathrm{HCl}$

$85 \mathrm{HF}$

49 HNO

$36 \mathrm{Hg}$

$129 \mathrm{Hg}$ (C6H5) 2

$135 \mathrm{Hg}$ (NO3) 2

$144 \mathrm{Hg} 2 \mathrm{Cl2}$

$117 \mathrm{Hg} 2 \mathrm{I2}$

$21 \mathrm{HgO}$

$21 \mathrm{Hg}$

$183 \mathrm{~K} 2 \mathrm{CO} 3$

$39 \mathrm{~K} 2 \mathrm{O}$

$51 \mathrm{KCOOH}$

$157 \mathrm{KMnO4}$

161 KNO3

$10 \mathrm{KOH}$

$105 \mathrm{La} 203$

108 Li20

$65 \mathrm{Mg}(\mathrm{COOH}) 2$

$171 \mathrm{Mg}(\mathrm{NO} 3) 2$

33 MgO

$60 \mathrm{Mn}(\mathrm{COOH}) 2$

$172 \mathrm{Mn}(\mathrm{NO} 3) 2$

$180 \mathrm{Mn} 304$

$56 \mathrm{MnO}$

$14 \mathrm{MnO} 2$

$145 \mathrm{MOO} 2$

$81 \mathrm{~N} 2$

$74 \mathrm{~N} 20$

$182 \mathrm{NH} 3$

114 NH3OHNO3

52. $\mathrm{NH} 4 \mathrm{COOH}$

173 NH4NO3

$68 \mathrm{NH} 4 \mathrm{OH}$

$68 \mathrm{NH} 4 \mathrm{OH}$
173 40 wt?

174 $\begin{array}{cc}175 & 196 \\ 0 \text { wto } & \text { Formic Acid sand }\end{array}$ Sand Filter sand Filter ZERO MERO ZERO ZERO $\quad$ ZERO

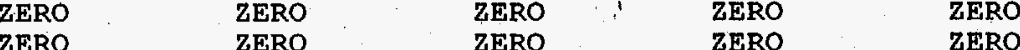

$\begin{array}{lllll}\text { ZERO } & \text { ZERO } & \text { ZERO } & \text { ZERO } & \text { ZERO } \\ \text { ZERO } & \text { ZERO } & \text { ZERO } & \text { ZERO } & \text { ZERO }\end{array}$

ZERO

ZERO

ZERO

ZERO

ZERO

ZERO

ZERO

ZERO

ZERO

ZERO

ZERO

ZERO

ZERO

ZERO

ZERO

ZERO

ZERO

ZERO

ZERO

ZERO

ZERO

ZERO

ZERO

ZERO

ZERO

ZERO

ZERO

ZERO

ZERO

ZERO

ZERO

ZERO

ZERO

ZERO

ZERO

ZERO

ZERO

ZERO

ZERO

ZERO

ZERO

ZERO

ZERO

ZERO

ZERO

URRO
ZERO

ZERO

ZERO

ZERO

ZERO

ZERO

ZERO

ZERO

ZERO

ZERO

ZERO

ZERO

ZERO

ZERO

ZERO

ZERO

ZERO

ZERO

ZERO

ZERO

ZERO

ZERO

2ERO

ZERO

ER

ZERO

ZERO

ZERO

ZERO

ZERO

ZERO

ZERO

ZERO

ZERO

ZERO

ZERO

ZERO

ZERO

ZERO

ZERO

ZERO

ZERO

ZERO

ZERO
172

RCT Air 48

ZERO $0.8778 \mathrm{E}-10$

ZERO $0.4317 \mathrm{E}-07$

ZFRO $0.3902 \mathrm{E}-09$

ZERO $0.5030 \mathrm{E}-06$

ZERO $0.5028 \mathrm{E}=0$

ZERO $0.7020 \mathrm{E}-10$

ZERO $0.7020 \mathrm{E}-10$

ZERO $0.2995 \mathrm{E}-11$

$0.3620 \mathrm{E}-08$

ZERO

ZERO

ZERO

ZERO

ZERO

ZERO

IERO

ZERO

ZERO

ZERO

ZERO

ZERO

ZERO

ZERO

ZERO

ZERO

ZERO

ZERO

ZERO

ZERO

ZERO

ZERO

ZERO

ZERO

ZERO

ZERO

ZERO

ZERO

ZTRO

ZERO

$0.5484 E+02$

ZERO

ZERO

ZERO

ZERO

ZERO

ZERO

ZERO
$0.1527 \mathrm{E}-06$ ZERO ZERO

$0.6978 \mathrm{E}-11$

$0.97847-02$ ZERO ZERO ZERO ZERO ERO ZERO ZERO

$0.1599 \mathrm{E}-0$

$0.1990 \mathrm{E}-06$

ZERO (1) ZERO ZERO.

$0.2195 \mathrm{E}-06$

$0.6862 \mathrm{E}-07$

$0.1041 \mathrm{E}-14$

$.4298 \mathrm{E}-07$

2 ZERO

$0.3860 \mathrm{E}-07$

-

$0.5483 \mathrm{E}+02$ ZERO ZERO ZERO

$0.2755 \mathrm{E}-10$ ZERO 
Table A-1: HLW Flowsheet Material Balance (Tank 51/ITP Cycle 1) section 7 . Collection and Treatment of Waste streams

STREAM NUMBERS (CONT'D) $\rightarrow$ STREAM NAME

\begin{tabular}{|c|c|}
\hline 35 & No \\
\hline 84 & $\mathrm{NO} 2$ \\
\hline 70 & $\mathrm{Na}(\mathrm{HgO}(\mathrm{OH}))$ \\
\hline 94 & $\mathrm{Na} 2 \mathrm{~B} 407$ \\
\hline 109 & $\mathrm{Na} 2 \mathrm{C} 2 \mathrm{O} 4$ \\
\hline & $\mathrm{Na} 2 \mathrm{CO} 3$ \\
\hline 143 & $\mathrm{Na} 2 \mathrm{CO} 3$ (C14) \\
\hline 89 & $\mathrm{Na} 2 \mathrm{CrO} 4$ \\
\hline 115 & $\mathrm{Na} 2 \mathrm{MoO} 4$ \\
\hline 32 & $\mathrm{Na} 2 \mathrm{O}$ \\
\hline 71 & $\mathrm{Na} 2 \mathrm{PuO} 2(\mathrm{OH}) 4$ \\
\hline 73 & $\mathrm{Na} 2 \mathrm{RhO} 4$ \\
\hline 72 & $\mathrm{Na} 2 \mathrm{RuO} 4$ \\
\hline 6 & $\mathrm{Na} 2 \mathrm{SO}_{4}$ \\
\hline 88 & $\mathrm{Na} 2 \mathrm{SiO} 3$ \\
\hline 87. & $\mathrm{Na} 3 \mathrm{PO} 4$ \\
\hline 91 & $\operatorname{NaAg}(\mathrm{OH}) 2$ \\
\hline 75 & NaAl (OH) 4 \\
\hline 50 & $\mathrm{NaCOOH}$ \\
\hline 7 & $\mathrm{NaCl}$ \\
\hline 69 & $\mathrm{NaF}$ \\
\hline 11 & $\mathrm{NaHCO} 3$ \\
\hline 12 & $\mathrm{NaI}$ \\
\hline 3 & NaNO2 \\
\hline 2 & NaNO3 \\
\hline 4 & $\mathrm{NaOH}$ \\
\hline 149 & NaTPB \\
\hline 160 & $\mathrm{NaTcO} 4$ \\
\hline 152. & NaTi205H \\
\hline 181 & $\mathrm{Nd2O} 3$ \\
\hline 61 & $\mathrm{Ni}(\mathrm{COOH}) 2$ \\
\hline 174 & $\mathrm{Ni}(\mathrm{NO} 3) 2$ \\
\hline 40 & NiO \\
\hline 82 & 02 \\
\hline 175 & $\mathrm{~Pb}(\mathrm{NO} 3) 2$ \\
\hline 86 & $\mathrm{PbCO} 3$ \\
\hline 67 & Pbo \\
\hline 147 & $\mathrm{PbS}$ \\
\hline 28 & PbSO4 \\
\hline 124 & Pd \\
\hline 112 & $\mathrm{Pd}(\mathrm{NO} 3) 2$ \\
\hline 110 & Pdo \\
\hline 76 & $\mathrm{PuO} 2$ \\
\hline 156 & PuO2 (NaTi205) \\
\hline 58 & Rh \\
\hline 22 & RhO2 \\
\hline 95 & Ru \\
\hline 79 & $\mathrm{RuO2}$ \\
\hline & RuO4 \\
\hline
\end{tabular}

173

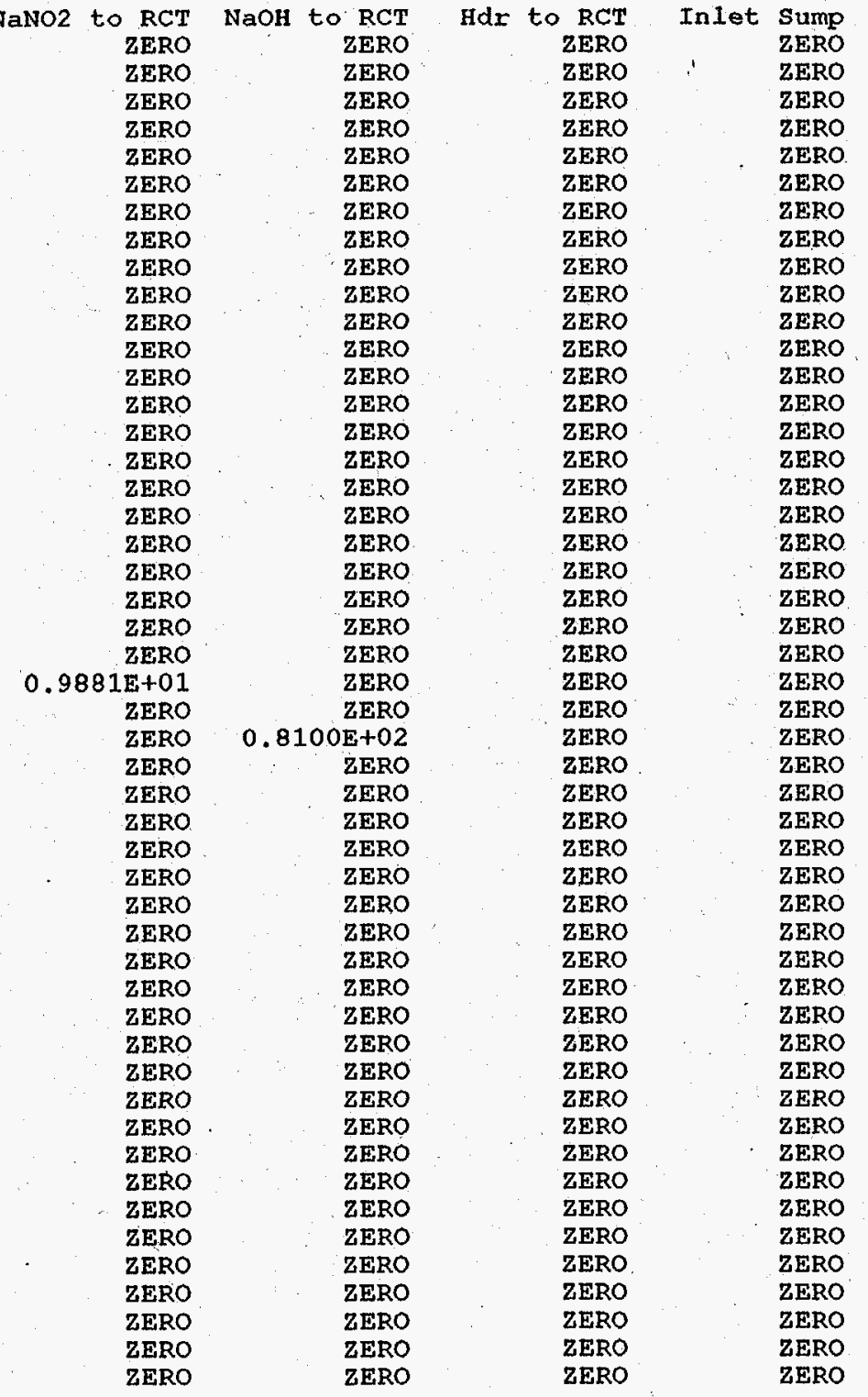

199 and Filter Exit Sump ZERO ZERO ZERO ZERO ZERO ZERO ZERO ZERO ZERO ZERO ZERO ZERO ZERO ZERO ZERO ZERO ZERO ZERO ZERO ZERO ZERO ZERO ZERO ZERO ZERO ZERO ZERO ZERO ZERO ZERO ZERO ZERO ZERO ZERO ZERO - ZERO - ZERO ZERO ZERO ZERO ZERO ZERO ZERO ZERO ZERO ZTRO ZIRO ZERO ZERO ZERO

Date: January 1995
172 Inleakage to Scrubber TRACE ZERO ZERO ZERO ZERO ZERO ZERO ZERO ZERO ZERO ZERO ZERO ZERO ZERO ZERO ZERO ZERO ZERO ZERO ZERO ZERO ZERO ZERO ZERO ZERO ZERO ZERO

$0.1656 \mathrm{E}+02$ ZERO ZERO ZERO ZERO ZERO ZERO ZERO ZERO ZFRO ZERO ZERO 2ERO ZERO ZERO ZERO
ZERO ZERO
$0.1002 E-06$ ZERO
ZERO 0.2509 E-06 9 E-06
ZERO ZERO $0.3867 \mathrm{E}-08$ $0.5107 \mathrm{E}-10$ ZERO ZERO $0.1973=07$ $0.6769 \mathrm{E}-09$ $0.1084 \mathrm{E}-12$ ZERO
$0.1772 \mathrm{E}-06$ ZERO ZERO ZERO ZERO $0.4994 \mathrm{E}-09$ $0.1325 \mathrm{E}-13$ $0.4787=-13$ $0.1656 \mathrm{E}+02$ $0.1539 \mathrm{E}+11$ TRACE ZERO
$0.2611 E-08$ $0.7732 \mathrm{E}-11$ ZERO
ZERO $0.1962 \mathrm{E}-09$ $0.2520 \mathrm{E}-11$ ZERO 0.1610 E-09 ZERO 
Table A-1. HLW Flowsheet Material Balance (Tank 51/ITP Cycle 1) Section 7. Collection and Treatment of Waste streams

STREAM NUMBERS (CONT'D) $\rightarrow$ STREAM NAME

$38 \mathrm{SO} 2$

$141 \mathrm{SO}$

137 Semi Vol Cs2O

136 Semi Vol CsC1

126 Semi Vol Group A
140 Semi Vol Na2B407

138 Semi Vol NaCl

139 Semi. Vol NaF

125 Semi Vol NaI

23 Semi Vol RuO2

$111 \mathrm{SiO}$

$48 \mathrm{Sr}(\mathrm{COOH}) 2$

$176 \mathrm{Sr}$ (NO3) 2

$153 \mathrm{Sr}(\mathrm{NaTi205)} 2$

$127 \operatorname{Sr}(\mathrm{OH}) 2$

$30 \mathrm{srCO}$

159 TcO2

92 ThO2

107 TiO2

119 Tritium

$\begin{array}{lll}17 & \mathrm{v} 308\end{array}$

62002 ( $\mathrm{COOH}) 2$

$177 \mathrm{OO} 2$ (NO3) 2

$16 \mathrm{VO} 2(\mathrm{OH}) 2$

$27 \mathrm{Y}(\mathrm{COOH}) 3$

178. $\mathrm{Y}(\mathrm{NO} 3)^{3}$

$128 \mathrm{Y}(\mathrm{OH}) 3$

31 Y2 (CO3) 3

$47 \mathrm{Y} 203$

100 Zeolite

$44 \mathrm{Zn}(\mathrm{COOH}) 2$

$179 \mathrm{Zn}$ (NO3) 2

$57 \mathrm{zno}$

$104 \mathrm{ZrO} 2$

13 hydrat

TOTAL FLOW, LB/HR

TEMPERATURE， DEG C

PRESSURE， ATM

PRESSURE， PSIG

PRESSURE, MM HG

ENTHALPY, PCU/HR

VAPOR FLOW, CFM

LIQUID FLOW, GPM

DENSITY, LBS/FT3

PHASE
173

174

50 wtz 175

196

Sand Filter 199

Sand Filtex

RCT NaOH to RCT Hdr to RCT Inlet Sump
ZERO
ZERO Exit Sump
ZERO

$\begin{array}{llll}\text { ZERO } & \text { ZERO } & \text { ZERO } \\ \text { ZERO } & \text { ZERO } & \text { ZERO } & \text { ZERO } \\ \text { ZERO } & \text { ZERO } & \text { ZERO }\end{array}$

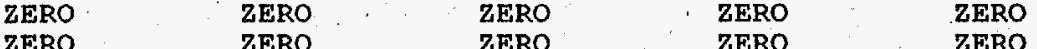

ZERO ZERO ZERO

ZERO ZERO

ZERO

ZERO

ZERO

ZERO ZERO

ZERO ZERO

ZERO ZERO

ZERO $\quad$ ZERO

ZERO ZERO

ZERO

ZERO . ZERO

ZERO $\quad$ ZERO

ZERO ZERO

ZERO ZERO

ZERO ZERO

ZERO ZERO

ZERO ZERO

ZERO ZERO

ZERO

ZERO

ZERO ZERO

ZERO ZERO

ZERO

ZERO

ZERO

ZERO

ZERO

ZERO

ZERO

ZERO

ZERO

$0.1482 \mathrm{E}+02$

$0.2470 \mathrm{E}+02$

$0.2500 \mathrm{E}+02$

$0.1000 \mathrm{E}+01$

ZERO

ZERO

ZERO

ZERO

ZERO

ZERO

ZERO

ZERO

ZERO

$0.8100 \mathrm{E}+02$

$0.1620 \mathrm{E}+03$

ZERO

ZERO

ZERO

ZERO

ZERO

ZERO

ZERO

ZERO

ZERO

ZERO

ZERO

ZERO

ZERO

ZERO

ZERO

ZERO

ZERO

ZERO

ZERO

ZERO

ZERO

ZERO

ZERO

ZERO

ZERO

ZERO

ZERO

ZERO

ZERO

ZERO

$0.2600 \mathrm{E}+02$

$0.2600 \mathrm{E}+02$

$0.2500 \mathrm{E}+02$

$0.1000 \mathrm{E}+01$

$0.3500 \mathrm{E}+02$

$0.1000 \mathrm{E}+01$

ZERO

ZERO

ZERO

ZERO

ZERO

ZERO

ZERO

ZERO

ZERO

ZERO

ZERO

ZERO

ZERO

ZERO

ZERO

ZERO

ZERO

ZERO

ZERO

ZERO

ZERO

ZERO

ZERO

ZERO

ZERO

ZERO

ZERO

ZERO

0.4750 ZERO

$0.4750 \mathrm{E}+00$

$0.3500 \mathrm{E}+02$

$0.1000 \mathrm{E}+01$

7.

ZERO

ZERO

ZERO

ZERO

ZERO

ZERO

ZERO

ZERO

ZERO

ZERO

ZERO

ZERO

ZERO

ZERO

ZERO

ZERO.

ZERO

ZERO

ZERO

ZERO

ZERO

ZERO

ZERO

ZERO

ZERO

ZERO

ZERO

ZERO

ZERO

ZERO

ZERO

$0,4750 \mathrm{E}+00$

$0.3500 \mathrm{E}+02$

$0.1000 E+01$

ZERO

$0.4291 E+03$

$0.2690 \mathrm{E}+\theta 4$

$0.9122 \mathrm{E}+03$

$0.1667 \mathrm{E}+02$

$0.1667 \mathrm{E}+02$

$0.3615 \mathrm{E}-01$

ITOUID

$0.2119 \mathrm{E}+00$

IIOUID

$6243 E+02$

$0.9486 \mathrm{E}-03$

LIOUID

$0.6243 E+02$

IIQUID

172

RCT Air

Inleakage

$\begin{array}{rr}\text { ZERO } & \text { ZERO } \\ \text { ZERO } & \text { ZERO } \\ \text { ZERO } & \text { ZERO } \\ \text { ZERO } & \text { ZERO } \\ \text { ZERO } & \text { ZERO }\end{array}$

ZERO ZERO

$\begin{array}{ll}\text { ZERO } & \text { ZERO } \\ \text { ZERO } & \text { ZERO }\end{array}$

ZERO ZERO

ZERO Z ZERO

ZERO ZERO

ZERO $0.2436 \mathrm{E}-05$

ZERO $\quad 0.216 .5 \mathrm{E}-11$

$0.5832 \mathrm{E}-09$

ZERO

ZERO

ZERO

ZERO

ZERO

ZERO

ZERO

ZERO

ZERO

ZERO

ZERO

ZERO

ZERO

ZERO

ZERO

ZERO

ZERO

ZERO

ZERO

ZERO

$0.1200 \mathrm{E}+01$

$0.7260 \mathrm{E}+02$

$0.3500 \mathrm{E}+02$

$0.1000 \mathrm{E}+01$

ZERO

TRACE

$0.7072 \mathrm{E}-10$

$0.5889 \mathrm{E}-09$

$0.1646 \mathrm{E}-07$

$0.2778 \mathrm{E}-11$

$0.4129 \mathrm{E}-07$

$.2520 E-13$

ZERO

ZERO

1918 EERO
ZERO

$0.1057 \mathrm{E}-11$

$0.2970 \mathrm{E}-07$

$0.6313 \mathrm{E}+01$

$0.7772 \mathrm{E}+02$

$0.5000 E+02$

$0.9656 \mathrm{E}+00$

$-0.5055 \mathrm{E}+00$

$0.7339 E+03$

$0.1523 \mathrm{E}+04 \quad 0.4931 \mathrm{E}+04$

$0.1715 \mathrm{E}+02 \quad 0.207 \mathrm{OE}+02$

$0.7055 \mathrm{E}-01$ 
STREAM NUMBERS $\rightarrow$ STREAM NAME

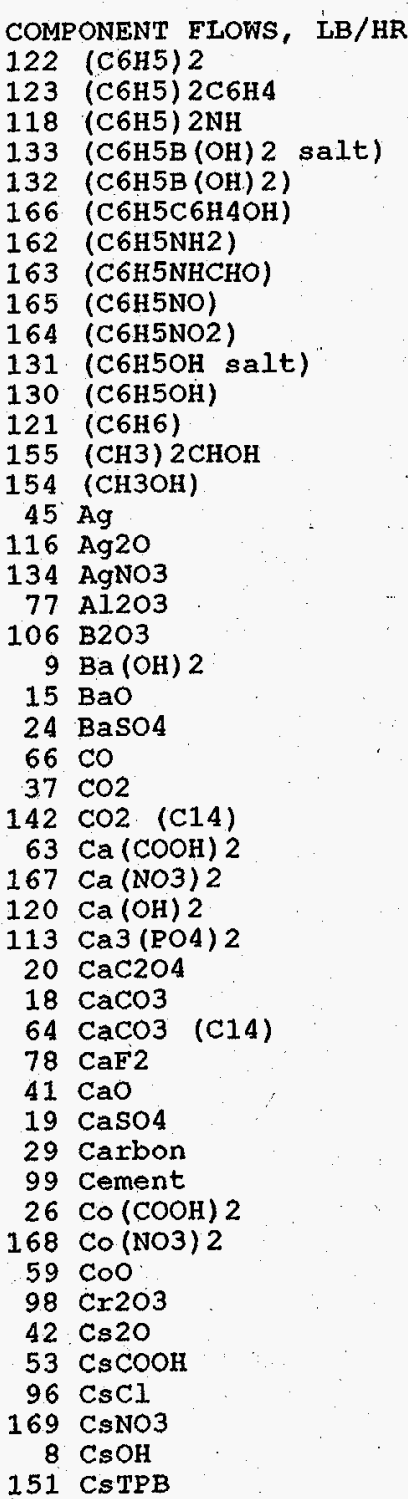

$151 \mathrm{CsTPB}$
Table A-1 HLW Flowsheet Material Balance (Tank 51/ITP Cycle 1) section 7. Colleation and Treatment of Waste Streams

$\begin{array}{llllccc}176 & 168 & 105 & 152 & 153 & 177 & 186\end{array}$ Liquid to Spnt Liq frm Neutr Waste Air Purge to I Scrubber to Tank Farm Recyc Hld Tk

\begin{tabular}{|c|c|c|c|c|c|c|}
\hline $\begin{array}{l}0.2894 \mathrm{E}-03 \\
0.2069 \mathrm{E}-14 \\
0.3109 \mathrm{E}-04\end{array}$ & $\begin{array}{l}0.2881 \mathrm{E}-03 \\
0.4657 \mathrm{E}-09 \\
0.3013 \mathrm{E}-04\end{array}$ & $\begin{array}{l}0.7707 \mathrm{E}-06 \\
0.5716 \mathrm{E}-14 \\
0.1925 \mathrm{E}-08\end{array}$ & $\begin{array}{l}0.2682 \mathrm{E}-03 \\
0.3339 \mathrm{E}-06 \\
0.4725 \mathrm{E}-02\end{array}$ & $\begin{array}{l}0.5543 \mathrm{E}-03 \\
0.3246 \mathrm{E}-06 \\
0.4609 \mathrm{E}-02\end{array}$ & $\begin{array}{l}0.3456 \mathrm{E}-05 \\
0.3645 \mathrm{E}-06 \\
0.5128 \mathrm{E}-02\end{array}$ & $\begin{array}{l}\text { ZERO } \\
\text { ZERO } \\
\text { ZERO }\end{array}$ \\
\hline ZERO & ZERO & ZERO & ZERO & ZERO & ZERO & ZERO \\
\hline ZERO & ZERO & ZERO & ZERO & ZERO & ZERO & ZERO \\
\hline $0.5292 \mathrm{E}-15$ & $0.1094 \mathrm{E}-08$ & $0.1032 \mathrm{E}-11$ & $0.1851 \mathrm{E}-0.6$ & $0.1809 \mathrm{E}-06$ & $0.2021 \mathrm{E}-06$ & ZERO \\
\hline $0.4753 \mathrm{E}-03$ & $0.4752 \mathrm{E}-03$ & $0.1911 \mathrm{E}-04$ & $0.4991 \mathrm{E}-01$ & $0.4884 E-01$ & $0.5402 \mathrm{E}-01$ & ZERO \\
\hline $0.2006 E-14$ & $0.4145 E-08$ & $0.3152 \mathrm{E}-11$ & $0.7017 E-06$ & $0.6856 \mathrm{E}-06$ & $0.7661 \mathrm{E}-06$ & ZERO \\
\hline $0.2182 E-16$ & $0.6748 \mathrm{E}-10$ & $0.6519 \mathrm{E}-09$ & $0.7635 \mathrm{E}-08$ & $0.6953 \mathrm{E}-08$ & $0.8336 \mathrm{E}-08$ & ZERO \\
\hline $0.6648 \mathrm{E}-16$ & $0.1375 \mathrm{E}-09$ & $0.4237 \mathrm{E}-11$ & $0.2326 \mathrm{E}-07$ & $0.2272 \mathrm{E}-07$ & $0.2539 \mathrm{E}-07$ & ZERO \\
\hline $0.1743 \mathrm{E}-08$ & $0.1691 \mathrm{E}-08$ & ZERO & $0.5885 \mathrm{E}-09$ & $0.2188 \mathrm{E}-08$ & $0.6657 \mathrm{E}+00$ & ZERO \\
\hline ZERO & $0.1945 \mathrm{E}-02$ & $0.2328 \mathrm{E}-03$ & $0.6075 \mathrm{E}+00$ & $0.5910 \mathrm{E}+00$ & ZERO & ZERO \\
\hline $0.7441 \mathrm{E}-03$ & $0.7383 \mathrm{E}-03$ & $0.4710 \mathrm{E}-04$ & $0.7392 \mathrm{E}-03$ & $0.1425 \mathrm{E}-02$ & $0.6288 \mathrm{E}-04$ & ZERO \\
\hline $0.2117 \mathrm{E}-12$ & $0.3048 \mathrm{E}-12$ & $0.1071 \mathrm{E}-11$ & $0.5070 \mathrm{E}-10$ & $0.4844 \mathrm{E}-10$ & $0.5514 \mathrm{E}-10$ & ZERO \\
\hline $0.4649 \mathrm{E}-13$ & $0.4873 \mathrm{E}-13$ & $0.2318 \mathrm{E}-12$ & $0.4830 \mathrm{E}-11$ & $0.4525 \mathrm{E}-11$ & $0.5226 \mathrm{E}-11$ & ZERO \\
\hline ZERO & $0.2459 \mathrm{E}-09$ & ZERO & ZERO & $0.2459 \mathrm{E}-09$ & ZERO & ZERO \\
\hline $0.2320 \mathrm{E}-15$ & $0.2321 \mathrm{E}-15$ & ZERO & $0.8913 E-16$ & $0.3095 \mathrm{E}-15$ & $0.8863 \mathrm{E}-07$ & ZERO \\
\hline ZERO & ZERO & ZERO & ZERO & ZERO & ZERO & ZERO \\
\hline $0.2313 \mathrm{E}-09$ & $0.1753 \mathrm{E}-0.6$ & ZERO & $0.2966 \mathrm{E}-04$ & $0.2899 \mathrm{E}-04$ & $0.8836 \mathrm{E}-01$ & ZERO \\
\hline $0.4429 \mathrm{E}-09$ & $0.2513 \mathrm{E}-06$ & ZERO & $0.1075 \mathrm{E}-04$ & $0.1069 \mathrm{E}-04$ & $0.1692 \mathrm{E}+00$ & ZERO \\
\hline ZERO & ZERO & ZERO & ZERO & ZERO & ZERO & ZERO \\
\hline $0.2930 \mathrm{E}-13$ & $0.2930 \mathrm{E}-13$ & ZERO & $0.1125 \mathrm{E}-13$ & $0.3908 \mathrm{E}-13$ & $0.1119 \mathrm{E}-04$ & ZERO \\
\hline $0.4016 \mathrm{E}-12$ & $0.3380 E-09$ & ZERO & $0.5720 \mathrm{E}-07$ & $0.5589 \mathrm{E}-07$ & $0.1534 \mathrm{E}-03$ & ZERO \\
\hline ZERO & ZERO & ZERO & ZERO & $\therefore \quad$ ZERO & ZERO & ZERO \\
\hline $0.6526 \mathrm{E}-02$ & $0.6527 \mathrm{E}-02$ & $0.6607 \mathrm{E}-02$ & $0.1050 \mathrm{E}-03$ & $0.2569 \mathrm{E}-04$ & $0.1461 E-03$ & ZERO \\
\hline $0.1447 \mathrm{E}-11$ & $0.1447 \mathrm{E}-11$ & $0.2791 \mathrm{E}-11$ & $0.1355 \mathrm{E}-11$ & $0.1085 \mathrm{E}-13$ & $0.3239 \mathrm{E}-13$ & ZERO \\
\hline ZERO & ZERO & ZERO & ZERO & ZERO & ZERO & ZERO \\
\hline $0.4019 \mathrm{E}-13$ & $0.8297 \mathrm{E}-07$ & ZERO & $0.1406 \mathrm{E}-04$ & $0.1374 \mathrm{E}-04$ & $0.1535 \mathrm{E}-04$ & ZERO \\
\hline ZERO & ZERO & ZERO & ZERO & ZERO & ZERO & ZERO \\
\hline $0.6876 \mathrm{E}-10$ & $0.5210 \mathrm{E}-07$ & ZERO & $0.8813 \mathrm{E}-0.5$ & $0.8611 \mathrm{E}-05$ & $0.2627 \mathrm{E}-01$ & ZERO \\
\hline $0.2338 \mathrm{E}-20$ & $0.4838 \mathrm{E}-14$ & ZERO & $0.8180 \mathrm{E}-12$ & $0.7991 \mathrm{E}-12$ & $0.8930 \mathrm{E}-12$ & ZERO \\
\hline ZERO & ZERO & ZERO & ZERO & ZERO & ZERO & ZERO \\
\hline ZERO & ZERO & ZERO & ZERO & ZERO & ZERO & ZERO \\
\hline $0.1440 \mathrm{E}-15$ & $0.2973 E-09$ & ZERO & $0.5039 \mathrm{E}-07$ & $0.4924 \mathrm{E}-07$ & $0.5501 \mathrm{E}-07$ & ZERO \\
\hline $0.3807 \mathrm{E}-10$ & $0.1043 E-09$ & ZERO & $0.1124 \mathrm{E}-07$ & $0.1102 E-07$ & $0.1454 \mathrm{E}-01$ & ZERO \\
\hline $0.5204 \mathrm{E}-11$ & $0.4380 \mathrm{E}-08$ & ZERO & $0.7398 \mathrm{E}-06$ & $0.7227 \mathrm{E}-06$ & $0.1988 E-02$ & ZERO \\
\hline ZERO & ZERO & ZERO & ZERO & ZERO & ZERO & ZERO \\
\hline ZERO & ZERO & ZERO & ZERO & ZERO & ZERO & ZERO \\
\hline ZERO & $0.2839 \mathrm{E}-11$ & ZERO & $0.4799 \mathrm{E}-09$ & $0.4688 \mathrm{E}-09$ & ZERO & ZERO \\
\hline $0.5144 \mathrm{E}-20$ & $0.1065 \mathrm{E}-13$ & ZERO & $0.1800 \mathrm{E}-11$ & $0.1758 \mathrm{E}-11$ & $0.1965 \mathrm{E}-11$ & ZERO \\
\hline $0.2697 \mathrm{E}-14$ & $0.6108 \mathrm{E}-12$ & ZERO & $0.1028 \mathrm{E}-09$ & $0.1004 \mathrm{E}-09$ & $0.1030 \mathrm{E}-05$ & ZERO \\
\hline $0.1127 \mathrm{E}-10$ & $0.8535 \mathrm{E}-08$ & ZERO & $0.1439 \mathrm{E}-05$ & $0.1406 \mathrm{E}-05$ & $0.4303 \mathrm{E}-02$ & ZERO \\
\hline $0.8114 \mathrm{E}-12$ & $0.3591 \mathrm{E}-11$ & ZERO & $0.4710 \mathrm{E}-09$ & $0.4610 \mathrm{E}-09$ & $0.3099 E-03$ & ZERO \\
\hline ZERO & $0.8580 \mathrm{E}-09$ & ZERO & $0.1450 \mathrm{E}-06$ & $0.1417 \mathrm{E}-06$ & ZERO & ZERO \\
\hline ZERO & ZERO & ZERO & ZERO & ZERO & ZERO & ZERO \\
\hline $0.9220 \mathrm{E}-19$ & $0.1904 \mathrm{E}-12$ & ZERO & $0.3226 \mathrm{E}-10$ & $0.3152 \mathrm{E}-10$ & $0.3522 \mathrm{E}-10$ & ZERC \\
\hline $0.9581 \mathrm{E}-11$ & $0.9582 \mathrm{E}-11$ & ZERO & $0.3679 \mathrm{E}-11$ & $0.1278 \mathrm{E}-10$ & $0.3660 \mathrm{E}-02$ & ZERO \\
\hline ZERO & ZERO & ZERO & ZERO & ZERO & ZERO & ZERO \\
\hline
\end{tabular}


Table A-1. HLW Flowsheet Material Balance (Tank 51/ITP Cycle 1) Section 7. Collection and Treatment of Waste streams

STREAM NUMBERS (CONT'D) $\rightarrow$ STREAM NAME

$54 \mathrm{Cu}(\mathrm{COOH}) 2$
$170 \mathrm{Cu}(\mathrm{NO} 3) 2$

$70 \mathrm{Cu}(\mathrm{NO}$

$80 \mathrm{Fe} 203$

$\begin{array}{rl}80 & \mathrm{Fe} \\ 101 \mathrm{FeO} & \end{array}$

102 Group A

103 Group

$25 \mathrm{H} 2$

$158 \mathrm{H} 2 \mathrm{C} 2 \mathrm{O} 4$

$93 \mathrm{H} 2 \mathrm{SO} 4$

90 H3BO3

$34 \mathrm{HCOO}$

$85 \mathrm{HF}$

49 HNO3

$49 \mathrm{HNO}$
$36 \mathrm{Hg}$

$129 \mathrm{Hg}$ (C6H5) 2

$135 \mathrm{Hg}$ (NO3)

$144 \mathrm{Hg} 2 \mathrm{Cl} 2$

$117 \mathrm{Hg} 2 \mathrm{I2}$

$21 \mathrm{HgO}$

97 I2

$183 \mathrm{~K} 2 \mathrm{CO} 3$

$39 \mathrm{~K} 2 \mathrm{O}$

$51 \mathrm{KCOOH}$

$157 \mathrm{KMnO} 4$

$161 \mathrm{KNO} 3$

$10 \mathrm{KOH}$

$\begin{array}{ll}150 & \mathrm{KTPB} \\ 105 & \mathrm{La} 203\end{array}$

$108 \mathrm{Li} 20$

$65 \mathrm{Mg}(\mathrm{COOH}) 2$

$171 \mathrm{Mg}(\mathrm{NO} 3) 2$

$33 \mathrm{MgO}$

$60 \mathrm{Mn}(\mathrm{COOH}) 2$

$172 \mathrm{Mn}(\mathrm{NO} 3) 2$

$180 \mathrm{Mn} 304$

$56 \mathrm{MnO}$

$14 \mathrm{MnO} 2$

$145 \mathrm{MoO} 2$

$81 \mathrm{~N} 2$

$\begin{array}{rl}74 & \mathrm{~N} 2 \mathrm{O} \\ 182 & \mathrm{NH} 3\end{array}$

182 NH 3

114 NH 3 OHNO3

$52 \mathrm{NH} 4 \mathrm{COOH}$

173 NH 4 NO3

148 NH
176 168 105 152 153

17.7 186 RCT Vent MFT/RCT Vent MFT Scrubber Liquid to Spnt Liq frm Neutr Waste Air Purge to o Scrubber to Sent to FAVC MFT Scrubber MFT Sarubber to Tank Farm Recyo HId Tk

\begin{tabular}{|c|c|c|c|c|c|c|}
\hline ZERO & $0.8778 \mathrm{E}-10$ & ZERO & $0.1487 \mathrm{E}-07$ & $0.1453 \mathrm{E}-07$ & ZERO & ZERO \\
\hline $0.2089 \mathrm{E}-13$ & $0.4317 \mathrm{E}-07$ & ZERO & $0.7308 \mathrm{E}-05$ & $0.7140 \mathrm{E}-05$ & $0.7978 \mathrm{E}-05$ & ZERO \\
\hline $0.2477 \mathrm{E}-10$ & $0.4150 \mathrm{E}-09$ & ZERO & $0.6613 \mathrm{E}-07$ & $0.6464 \mathrm{E}-07$ & $0.9462 \mathrm{E}-02$ & ZERO \\
\hline $0.6648 \mathrm{E}-09$ & $0.5036 \mathrm{E}-06$ & ZERO & $0.8524 \mathrm{E}-04$ & $0.8329 \mathrm{E}-04$ & $0.2539 \mathrm{E}+00$ & ZERO \\
\hline $0.6646 \mathrm{E}-10$ & $0.5035 \mathrm{E}-07$ & ZERO & $0.8522 \mathrm{E}-05$ & $0.8327 \mathrm{E}-05$ & $0.2539 \mathrm{E}-01$ & ZERO \\
\hline $0.5514 \mathrm{E}-12$ & $0.7075 \mathrm{E}-10$ & ZERO & $0.1190 \mathrm{E}-07$ & $0.1163 \mathrm{E}-07$ & $0.2106 \mathrm{E}-03$ & ZERO \\
\hline $0.3958 \mathrm{E}-1.4$ & $0.2999 \mathrm{E}-11$ & ZERO & $0.5075 \mathrm{E}-09$ & $0.4960 \mathrm{E}-09$ & $0.1512 \mathrm{E}-05$ & ZERO \\
\hline $0.1846 E-08$ & $0.1846 \mathrm{E}-08$ & $0.3539 E-08$ & $0.1693 E-08$ & $0.5635 \mathrm{E}-12$ & $0.1693 \mathrm{E}-11$ & ZERO \\
\hline ZERO & $0.3620 \mathrm{E}-08$ & ZERO & $0.6135 \mathrm{E}-06$ & $0.5995 \mathrm{E}-06$ & ZERO & ZERO \\
\hline ZERO & ZERO & ZERO & ZERO & ZERO & ZERO & ZERO \\
\hline ZERO & $0.1527 \mathrm{E}-06$ & ZERO & $0.2582 \mathrm{E}-04$ & $0.2522 \mathrm{E}-04$ & ZERO & ZERO \\
\hline ZERO & ZERO & ZERO & $\therefore \quad$ ZERO & ZERO & ZERO & ZERO \\
\hline ZERO & ZERO & ZERO & ZERO & ZERO & ZERO & ZERO \\
\hline ZERO & ZERO & ZERO & ZERO & ZERO & ZERO & ZERO \\
\hline ZERO & $0.6978 \mathrm{E}-11$ & $0.1012 \mathrm{E}+00$ & $0.3442 \mathrm{E}+01$ & $0.3318 \mathrm{E}+01$ & ZERO & ZERO \\
\hline $0.6917 \mathrm{E}-01$ & $0.7726 \mathrm{E}-01$ & $0.1208 \mathrm{E}-06$ & ZERO & $0.7726 \mathrm{E}-01$ & $0.6679 \mathrm{E}-01$ & ZERO \\
\hline ZERO & ZERO & ZERO & ZERO & ZERO & ZERO & ZERO \\
\hline ZERO & ZERO & ZERO & ZERO & ZERO & ZERO & ZERO \\
\hline ZERO & ZERO & ZERO & ZERO & ZERO & ZERO & ZERO \\
\hline ZERO & ZERO & ZERO & ZERO & ZERO & ZERO & ZERO \\
\hline ZERO & ZERO & ZERO & ZERO & ZERO & ZERO & ZERO \\
\hline $0.1723 \mathrm{E}-10$ & $0.1723 \mathrm{E}-10$ & ZERO & $0.6615 \mathrm{E}-11$ & $0.2298 \mathrm{E}-10$ & $0.6580 \mathrm{E}-02$ & ZERO \\
\hline ZERO & ZERO & ZERO & ZERO & ZERO & ZERO & ZERO \\
\hline ZERO & ZERO & ZERO & ZERO & ZERO & ZERO & ZERO \\
\hline $0.1497 \mathrm{E}-09$ & $0.1749 \mathrm{E}-08$ & ZERO & $0.2711 E-06$ & $0.2650 \mathrm{E}-06$ & $0.5718 \mathrm{E}-01$ & ZERO \\
\hline ZERO & $0.1990 \mathrm{E}-06$ & ZERO & $0.3365 \mathrm{E}-04$ & $0.3287 E-04$ & ZERO & ZERO \\
\hline ZERO & ZERO & ZERO & ZERO & ZERO & ZERO & ZERO \\
\hline ZERO & $0.5663 \mathrm{E}-09$ & ZERO & $0.9597 \mathrm{E}-07$ & $0.9378 \mathrm{E}-07$ & ZERO & ZERO \\
\hline $0.2865 \mathrm{E}-09$ & $0.2865 \mathrm{E}-09$ & ZERO & $0.1100 \mathrm{E}-09$ & $0.3821 \mathrm{E}-09$ & $0.1094 \mathrm{E}+00$ & ZERO \\
\hline ZERO & ZERO & ZERO & ZERO & ZERO & ZERO & ZERO \\
\hline . ZERO & ZERO & ZERO & ZERO & ZERO & ZERO & ZERO \\
\hline $0.2900 \mathrm{E}-09$ & $0.2198 \mathrm{E}-06$ & ZERO & $0.9402 \mathrm{E}-05$ & $0.9347 \mathrm{E}-05$ & $0.1108 E+00$ & ZERO \\
\hline ZERO & $0.6862 \mathrm{E}-07$ & ZERO & $0.1163 \mathrm{E}-04$ & $0.1136 \mathrm{E}-04$ & ZERO & ZERO \\
\hline $0.5030 \mathrm{E}-21$ & $0.1041 \mathrm{E}-14$ & ZERO & $0.1760 \mathrm{E}-12$ & $0.1719 \mathrm{E}-12$ & $0.1921 \mathrm{E}-12$ & ZERO \\
\hline $0.1228 \mathrm{E}-09$ & $0.6888 \mathrm{E}-07$ & ZERO & $0.3710 \mathrm{E}-05$ & $0.3671 \mathrm{E}-05$ & $0.4692 \mathrm{E}-01$ & ZERO \\
\hline ZERO & $0.4298 \mathrm{E}-07$ & ZERO & $0.7284 \mathrm{E}-05$ & $0.7118 \mathrm{E}-05$ & ZERO & ZERO \\
\hline $0.5861 \mathrm{E}-21$ & $0.1213 E-14$ & ZERO & $0.2051 \mathrm{E}-12$ & $0.2003 \mathrm{E}-12$ & $0.2239 \mathrm{E}-12$ & ZERO \\
\hline ZERO & ZERO & ZERO & ZERO & ZERO & ZERO & ZERO \\
\hline $0.6940 \mathrm{E}-10$ & $0.6940 \mathrm{D}-10$ & ZERO & $0.2665 \mathrm{E}-10$ & $0.9255 \mathrm{E}-10$ & $0.2651 \mathrm{E}-01$ & ZERO \\
\hline $0.4438 \mathrm{E}-09$ & $0.3904 \mathrm{E}-07$ & ZERO & $0.6541 \mathrm{E}-05$ & $0.6392 \mathrm{E}-05$ & $0.1695 \mathrm{E}+00$ & ZERO \\
\hline $0.2816 \mathrm{E}-13$ & $0.2133 \mathrm{E}-10$ & ZERO & $0.3610 \mathrm{E}-08$ & $0.3527 \mathrm{E}-08$ & $0.1076 \mathrm{E}-04$ & ZERO \\
\hline $0.5484 \mathrm{E}+02$ & $0.1097 \mathrm{E}+03$ & $0.1097 E+03$ & $0.1289 \mathrm{E}-01$ & $0.134 \mathrm{OE}-01$ & $0.3857 \mathrm{E}-01$ & $0.1082 E+04$ \\
\hline TRACE & TRACE & TRACE & TRACE & TRACE & TRACE & ZERO \\
\hline $0.6390 \mathrm{E}-02$ & $0.6196 \mathrm{E}-02$ & $0.9381 \mathrm{E}-04$ & $0.4325 \mathrm{E}-04$ & $0.5051 E-04$ & $0.2033 \mathrm{E}+00$ & ZERO \\
\hline ZERO & ZERO & ZERO & ZERO & ZERO & ZERO & ZERO \\
\hline ZERO & ZERO & ZERO & ZERO & ZERO & ZERO & ZERO \\
\hline ZERO & $0.2755 \mathrm{E}-10$ & ZERO & $0.9026 \mathrm{E}+00$ & $0.9038 \mathrm{E}+00$ & ZERO & ZERO \\
\hline ZERO & ZERO & ZERO & $0.1358 \mathrm{E}-18$ & $0.1383 E-18$ & ZERO & ZERO \\
\hline ZERO & ZERO & ZERO & ZERO & ZERO & ZERO & ZERO \\
\hline
\end{tabular}


Table A-1. HLW Flowsheet Material Balance (Tank 51/ITP Cycle 1) Section 7. Collection and Treatment of Waste streams

STREAM NUMBERS (CONT'D) $\rightarrow$ STREAM NAME

\begin{tabular}{|c|c|}
\hline & \\
\hline $\begin{array}{l}35 \\
84\end{array}$ & $\begin{array}{l}\text { NO } \\
\text { NO2 }\end{array}$ \\
\hline 70 & $\mathrm{Na}(\mathrm{HgO}(\mathrm{OH}))$ \\
\hline 94 & $\mathrm{Na2B} 407$ \\
\hline 109 & $\mathrm{Na} 2 \mathrm{C} 204$ \\
\hline & $\mathrm{Na} 2 \mathrm{CO} 3$ \\
\hline 143 & $\mathrm{Na} 2 \mathrm{CO} 3(\mathrm{C} 14)$ \\
\hline 89 & $\mathrm{Na} 2 \mathrm{CrO} 4$ \\
\hline 115 & $\mathrm{Na2MoO4}$ \\
\hline 32 & $\mathrm{Na} 2 \mathrm{O}$ \\
\hline 71 & $\mathrm{Na} 2 \mathrm{PuO} 2(\mathrm{OH}) 4$ \\
\hline 73 & $\mathrm{Na} 2 \mathrm{RhO} 4$ \\
\hline 72 & $\mathrm{Na2RuO4}$ \\
\hline 6 & $\mathrm{Na} 2 \mathrm{SO} 4$ \\
\hline 88 & Na2sio3 \\
\hline 87 & $\mathrm{Na3PO4}$ \\
\hline 91 & $\mathrm{NaAg}(\mathrm{OH}) 2$ \\
\hline 75 & NaAl (OH) 4 \\
\hline 50 & $\mathrm{NaCOOH}$ \\
\hline & $\mathrm{NaCl}$ \\
\hline 69 & $\mathrm{NaF}$ \\
\hline 11 & $\mathrm{NaHCO} 3$ \\
\hline 12 & NaI \\
\hline & $\mathrm{NaNO} 2$ \\
\hline 2 & NaNO3 \\
\hline 4 & $\mathrm{NaOH}$ \\
\hline 149 & NaTPB \\
\hline 160 & $\mathrm{NaTcO} 4$ \\
\hline 152 & NaTi205H \\
\hline 181 & $\mathrm{Nd2O} 3$ \\
\hline 61 & $\mathrm{Ni}(\mathrm{COOH}) 2$ \\
\hline 174 & $\mathrm{Ni}(\mathrm{NO} 3) 2$ \\
\hline 40 & Nio \\
\hline 82 & $\mathrm{O} 2$ \\
\hline 175 & $\mathrm{~Pb}(\mathrm{NO} 3) 2$ \\
\hline 86 & $\mathrm{PbCO} 3$ \\
\hline 67 & Pbo \\
\hline 147 & $\mathrm{PbS}$ \\
\hline 28 & $\mathrm{PbSO} 4$ \\
\hline 124 & Pd \\
\hline 112 & $\mathrm{Pd}(\mathrm{NO} 3) 2$ \\
\hline 76 & $\begin{array}{l}\mathrm{PdO} \\
\mathrm{PuO} 2\end{array}$ \\
\hline 156 & PuO2 (NaTi2O5) 2 \\
\hline 58 & $\mathrm{Rh}$ \\
\hline 22 & $\mathrm{RhO} 2$ \\
\hline 95 & $\mathbf{R u}$ \\
\hline & $\mathrm{RuO} 2$ \\
\hline & RuO4 \\
\hline
\end{tabular}

WSRC-TR-95-0019 (Revision
176

$$
168
$$

105

152

153

177

Liquid to Spnt Iiq frm Neutr Waste Air Purge to to Scrubber to Scrubber Vent to FAVC MFT Scrubber MFT Scrubber to Tank Farm Recyc HId Tk $0.7559 \mathrm{E}-04$ $0.3213 E-09$ $0.2071 \mathrm{E}-07$

$0.4698 \mathrm{E}-13$ $0.7556 \mathrm{E}-04$ ZERO $0.3213 \mathrm{E}-09$ $0.2071 E-07$ $0.1002 \mathrm{E}-0$ ZERO ZERO ZERO

$0.5315 \mathrm{E}-0$ ZERO ZERO ZERO
$4 \mathrm{E}-09$

$0.1774 \mathrm{E}-09$

$0.6751 \mathrm{E}-13$ ZERO

$0.1470 \mathrm{E}-12$

$0.1470 \mathrm{E}-12$

$0.1316 \mathrm{E}-10$

$0.3678 E-10$
ZERO

$0.1431 \mathrm{E}-13$

$0.2588 \mathrm{E}-07$

$0.3383 \mathrm{E}-07$

$0.2053 E-06$ ZERO ZERO ZERO ZERO

$0.6401 \mathrm{E}-20$ $0.6658 \mathrm{E}-11$ $0.1657 \mathrm{E}+02$

$0.7453 \mathrm{E}-18$

ZERO
$0.1283 \mathrm{E}-12$

$0.1283 \mathrm{E}-12$

$0.3279 \mathrm{E}-11$ ZERO

$0.7648 \mathrm{E}-17$ $0.2607 \mathrm{E}-12$ $0.1218 \mathrm{E}-17$ ZERO

$0.1661 \mathrm{E}-16$ ZERO ZERO
ZERO ZERO
ZERO $0.2514 \mathrm{E}-06$ ZERO ZERO $0.4044 \mathrm{E}-08$ ZERO
$0.5114 \mathrm{E}-10$ E-10 ZERO

$0.4656 \mathrm{E}-07$ $0.2104 \mathrm{E}-09$ $0.7137 \mathrm{E}-09$ ZERO $0.1227 \mathrm{E}-12$ $0.2588 \mathrm{E}-07$ $0.2109 \mathrm{E}-06$
$0.2053 \mathrm{E}-06$ $0.2053 \mathrm{E}-06$ ZERO ZERO ZERO

0.4994 ZERO $0.1325 \mathrm{E}-13$ $0.4793 \mathrm{E}-08$ $0.3313 \mathrm{E}+02$ $0.1539 \mathrm{E}-11$ ZERO $0.1283 \mathrm{E}-12$ $0.2614 \mathrm{E}-08$ $0.7732 \mathrm{E}-11$ 0.7649 ZERO $0.1965 \mathrm{E}-09$ $0.2520 \mathrm{E}-11$ $0.1473 \mathrm{E}-10$ $0.1661 \mathrm{E}-16$ $0.1610 \mathrm{~F}-09$ ZERO ZERO

\section{$0.3002 \mathrm{E}-06$ \\ $0.1388 \mathrm{E}-07$}

0. $4270 \mathrm{E}-08$

$0.1234 \mathrm{E}-09$

$0.7953 \mathrm{E}-08$

$0.1644 \mathrm{E}-04$

ZERO

ZERO

ZERO

ZERO

ZERO

ZERO

ZERO

ZERO

ZERO

ZERO

ZERO

ZERO

ZERO

ZERO

ZERO

ZERO

ZERO

ZERO

ZERO

ZERO

ZERO

ZERO

ZERO

ZERO

ZERO

ZERO

ZFRO

ZERO

ZERO

ZERO

ZERO

ZERO

ZERO

ZERO

ZERO

ZERO

ZERO

ZERO

ZERO

ZERO

ZERO

Tank Farm Recyc HId Tk
$0.7938 \mathrm{E}-07$
$0.1553 \mathrm{E}-06$

$\begin{array}{rrr}\text { ZERO } & 0.1553 E-06 & \text { ZERO } \\ \text { ZERO } & \text { ZERO }\end{array}$

$\begin{array}{lll}0.4285 \mathrm{E}-09 & 0.1227 \mathrm{E}+00 & \text { ZERO }\end{array}$

$0.2762 \mathrm{E}-07 \quad 0.7909 \mathrm{E}+01 \quad$ ZERO

$0.1606 \mathrm{E}-04 \quad 0.1794 \mathrm{E}-04$

ZERO

ZERO

$0.2136 \mathrm{E}-04$

$36 \mathrm{E}-04$
ZERO

ZERO

$0.6554 \mathrm{E}-06$

ZERO

$0.8644 \mathrm{E}-08$

ZERO

$0.2632 \mathrm{E}-05$

$3341 \mathrm{E}-07$

$0.1147 \mathrm{E}-06$ ZERO

$0.1838 \mathrm{E}-10$

$.9936 \mathrm{E}-08$

$0.3029 \mathrm{E}-04$

ZERO

ZERO

ZERO

$0.8463 \mathrm{E}-07$

$0.2239 \mathrm{E}-11$

$0.7986 \mathrm{E}-06$

$0.7439 \mathrm{E}-02$

$0.2608 \mathrm{E}-09$

ZERO
$0.4928 \mathrm{E}-13$

ZERO

$0.4425 \mathrm{E}-06$

ZERO

$.2938 \mathrm{E}-17$

$0.3326 \mathrm{E}-07$

$.4261 \mathrm{E}-09$

$0.6381 \mathrm{E}-17$

ZERO

Date: January 1995
ZERO

ZERO

ZERO

$0.2099 \mathrm{E}-04$ ZERO

ZERO

$0.6406 \mathrm{E}-06$ ZERO

$0.8446 \mathrm{E}-08$ ZERO

$0.2595 \mathrm{E}-05$

$0.2595 E-05$
$0.3265 E-07$

$0.1122 \mathrm{E}-06$

$0.1797 \mathrm{E}-10$

$0.3451 \mathrm{E}-07$

$0.2963 \mathrm{E}-04$
$0.2053 \mathrm{E}-06$

ZERO

ZERO

ZERO

$0.8270 \mathrm{E}-07$

$0.7804 \mathrm{E}-06$

$0.7728 \mathrm{E}-02$

$0.2548 E-09$

ZERO

ZERO
ZER

$0.4324 \mathrm{E}-06$

$0.7732 \mathrm{E}-11$

ZERO

$0.1020 \mathrm{E}-16$

$0.4162 \mathrm{E}-09$

$0.1473 \mathrm{E}-10$

$0.2216 \mathrm{E}-16$

$0.2663 \mathrm{E}-07$

ZERO

$1794 \mathrm{E}-04$

ZERO

ZERO

ZERO

ZERO ZERO

ZERO
ZERO
ZERO

$0.6774 \mathrm{E}-01$

$0.2578 \mathrm{E}-04$

ZERO

ZERO

ZERO ZERO

$0.1405 \mathrm{E}-01$

ZERO

$0.9885 \mathrm{E}+01$

$0.1292 \mathrm{E}+02$

$0.7840 \mathrm{E}+02$

ZERO

ZERO

ZERO

0.2445 ZERO

$0.2543 \mathrm{E}-02$

$0.2225 \mathrm{E}-01$

$0.2847 \mathrm{E}-09$

$0.4902 \mathrm{EERO}$

ZERO

$0.1252 \mathrm{E}-02$

ZERO

$0.2921 \mathrm{E}-08$

$0.9959 \mathrm{E}-04$

$0.4651 \mathrm{E}-09$

$0.6346 \mathrm{E}-08$

$0.2975 \mathrm{E}-07$

$$
\text { ZERO }
$$

Section 7, Page 16 of 21
ZERO 
Table A-1. HLW Flowsheet Material Balance (Tank 51/ITP Cycle 1) section 7. Collection and Treatment of Waste Streams

STREAM NUMBERS (CONT'D) $\rightarrow$ STREAM NAME

38. $\mathrm{SO} 2$

$141 \mathrm{SO} 3$

137 Semi Vol Cs20

136 Semi Vol CsCl

126 Semi vol Group A

140 Semi Vol Na2B4O7

138 Semi Vol NaCl

139 Semi Vol NaF

125 Semi Vol NaI

23 Semi

$48 \mathrm{Sr}(\mathrm{COOH}) 2$

$176 \mathrm{Sr}$ (NO3) 2

153 Sx (NaTi2O5) 2

$127 \mathrm{Sr}(\mathrm{OH}) 2$

$30 \mathrm{SrCO}$

46 Sro

159
92
ThOO2

107 TiO2

119 Tritium

$17 \mathrm{v} 308$

62002 (COOH) 2

$177 \mathrm{VO} 2(\mathrm{NO3})$

$16 \mathrm{OO} 2(\mathrm{OH}) 2$
$27 \mathrm{Y}(\mathrm{COOH}) 3$

$178 \mathrm{Y}$ (NO3) 3

$128 \mathrm{Y}(\mathrm{OH}) 3$

31 Y2 (CO3) 3

$47 \mathrm{Y} 2 \mathrm{O} 3$

100 Zeolite
44 Zn (COOH)

$179 \mathrm{Zn}$ (NO3) 2

$57 \mathrm{znO}$

104 ZrO2

13 hydrate $\mathrm{H} 2 \mathrm{O}$

$$
1 \text { water }
$$

TOTAL FLOW; LB/HR

TEMPERATURE, DEG C

PRESSURE， ATM

PRESSURE, PSIG

PRESSURE, MM HG

ENTHALPY, PCU/HR

VAPOR FLOW, CFM

LIQUID FLOW, GPM

DENSITY, IBS/FT3

PHASE
RCT Vent MFT/RCT Vent MFT Scrubber Liquid to Spnt Liq frm Neútr Waste Air Purge to to Scrubber to Scrubber Vent to FAVC MFT Scrubber MFT Scrubber to Tank Farm Recyc Hld Tk

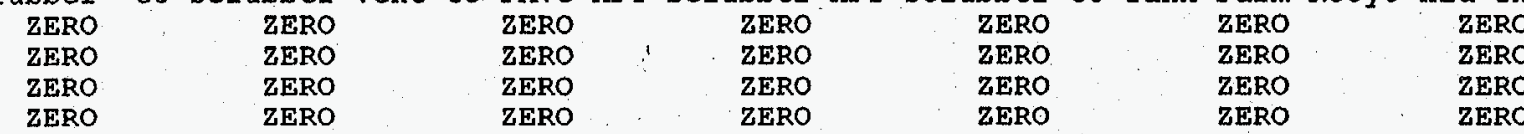

ZERO

ZERO

$\begin{array}{llll}\text { ZERO } & \text { ZERO } & \text { ZERO } & \text { ZERO } \\ \text { ZERO } & \text { ZERO } & \text { ZERO } & \text { ZERO }\end{array}$

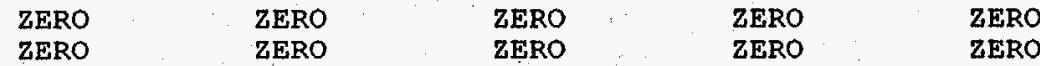

ZERO ZERO

ZERO

$0.3219 \mathrm{E}-08$

ZERO $\quad 0.1071 \mathrm{E}-03$

$0.1064 \mathrm{E}-03$

$0.3576 \mathrm{E}-09$

$0.9659 \mathrm{E}-07$

$0.1332 \mathrm{E}-07$

ZERO $0.9884 \mathrm{E}-07$

$0.2825 \mathrm{E}-15$

ZERO

ZERO

ZERO

$0.4018 \mathrm{E}-12$

$9346 \mathrm{E}-13$

$0.7783 \mathrm{E}-12$

$0.7081 \mathrm{E}-10$

$0.5897 \mathrm{E}-09$

$0.1648 \mathrm{E}-07$

$0.4275 \mathrm{E}-11$

$0.4135 \mathrm{E}-07$

$0.2520 \mathrm{E}-13$

ZERO

ZERO

$0.7620 \mathrm{E}-16$

ZERO

ZERO

$0.2610 \mathrm{E}-19$

$0.1107 \mathrm{E}-12$

$0.1929 \mathrm{E}-10$
ZERO

$0.5166 \mathrm{E}-08$
$0.1057 \mathrm{E}-11$

$0.3562 \mathrm{E}-08$

ZERO

ZE -1

$0.2256 \mathrm{E}-13$

$0.2970 \mathrm{E}-07$

$0.1555 \mathrm{E}+03$

$0.7781 \mathrm{E}+02$

$0.1555 \mathrm{E}+03$

$0.5000 \mathrm{E}+02$

$0.5000 \mathrm{E}+02$

$0.9656 \mathrm{E}+00$

$-0.5055 \mathrm{E}+00$
$0.7339 \mathrm{E}+03$

$0.9873 \mathrm{E}+04$

$0.4142 \mathrm{E}+02$

$0.2072 \mathrm{E}+02$

$0.6258 \mathrm{E}-01$

VAPOR

VAPOR
$0.15435-12$

$0.1543 \mathrm{E}-12$

$0.1198 \mathrm{E}-07$

$0.9980 \mathrm{E}-07$

$0.2784 \mathrm{E}-05$

$0.5919 \mathrm{E}-09$

$0.6998 \mathrm{E}-05$

$0.4261 \mathrm{E}-11$

$0.4413 \mathrm{E}-17$

$0.2666 E-07$

ZERO

ZTRO

ZERO

ZERO

RRO

ZERO

ZERO

ZERO

ZERO

$0.1212 \mathrm{E}+02$

$0.1550 \mathrm{E}+03$

$0.5000 \mathrm{E}+02$

$0.1000 \mathrm{E}+01$

ZERO

$0.3243 E-08$
ZERO

$0.8739 \mathrm{E}-06$

$0.1787 \mathrm{E}-09$

$0.6015 \mathrm{E}-06$

$0.5034 \mathrm{E}-05$

$0.1079 \mathrm{E}+04$

$0.1084 \mathrm{E}+04$

$0.4900 \mathrm{E}+02$

$0.1000 \mathrm{E}+01$

$0.9563 \mathrm{E}+04$

$0.3980 \mathrm{E}+02$

$0.5301 \mathrm{E}+05$

VAPOR
$0.6492 \mathrm{E}-01$

$0.2162 \mathrm{E}+01$
ZERO

ZERO

ZERO ZERO

ZERO

ZERO

$0.5358 \mathrm{E}-12$

$0.5358 \mathrm{E}-12$

$0.1171 \mathrm{E}-07$

$0.2719 \mathrm{E}-05$

$0.5729 \mathrm{E}-09$

$0.6838 \mathrm{E}-05$

$0.4162 \mathrm{E}-11$

$0.2998 \mathrm{E}-09$

$0.4311 \mathrm{E}-17$

$0.2605 \mathrm{E}-07$

ZERO

ZERO

$0.8538 \mathrm{E}-06$

$0.1746 \mathrm{E}-09$

$0.5877 \mathrm{E}-06$

ZERO
$19 E-05$

$.1080 \mathrm{E}+04$

$0.1085 \mathrm{E}+04$

$0.5000 \mathrm{E}+02$

$0.1000 \mathrm{E}+01$

ZERO

$0.5412 \mathrm{E}+05$

$0.6251 \mathrm{E}+02$
LIQUID

LIOUID
$0.2163 \mathrm{E}+01$
ZERO

ZERO$$
\text { ZERO }
$$

ZERO

ZERO

$0.1230 \mathrm{E}+01$

ZERO

$0.1079 \mathrm{E}-06$

ZERO

$0.1535 \mathrm{E}-03$

$0.3570 \mathrm{E}-04$
$0.2973 \mathrm{E}-03$

$0.8338 \mathrm{E}-02$

$0.7859 \mathrm{E}-09$

$0.2085 \mathrm{E}-01$

ZERO
$0.3349 \mathrm{E}-09$

ZERO

ZERO
0.2911 ER-07

ZERO

ZERO
ZERO

0.1951 EERO

$0.3160 \mathrm{D}-02$

ZERO
$0.8616 \mathrm{E}-05$

$0.3242 E+04$

$0.3355 \mathrm{E}+04$

$0.1433 \mathrm{E}+04$

$0.4999 \mathrm{E}+02$

$0.9656 \mathrm{E}+00$

$-0.5055 \mathrm{E}+00$

$0.7339 \mathrm{E}+03$

$0.1639 \mathrm{E}+06$

$0.6551 E+01$

$0.6385 \mathrm{E}+02$

LIQUID

$0.3500 \mathrm{E}+02$

$0.1000 \mathrm{E}+01$

ZERO

$0.3005 \mathrm{E}+05$

$0.3384 \mathrm{E}+03$

$0.7055 E-01$

VAPOR
ZERO

ZERO

$0.2367 \mathrm{E}+02$ 
Table A-1. HLW Elowsheet Material Balance (Tank 51/ITP Cycle 1) Section 7. Collection and Treatment of Waste streams

STREAM NUMBERS $\rightarrow$ STREAM NAME

COMPONENT FLOWS, LB/HR

123 (C6H5) $2 \mathrm{C} 6 \mathrm{H} 4$

118 (C6H5) 2NH

133 ( $6 \mathrm{CH} 5 \mathrm{~B}(\mathrm{OH}) 2$ salt)

132 (С6H5B (OH) 2 )

166 (C6H5C6H40H)

162 (C6H5NH2)

163 (C6H5NHCHO)

165 (C6H5NO)

131 (C6H5OH salt)

$130 \cdot(\mathrm{C} 6 \mathrm{H} 5 \mathrm{OH})$

121 ( 6 $6 \mathrm{H} 6$ )

155 (CH3) $2 \mathrm{CHOH}$

154 (СH3OH)

$45 \mathrm{Ag}$

116 Ag20

34 AgNO3
77 Al2O3

$\begin{array}{rl}77 & \mathrm{Al} 2 \mathrm{O} \\ 106 & \mathrm{~B} 203\end{array}$

$9 \mathrm{Ba}$ (OH) 2

$15 \mathrm{BaO}$

$24 \mathrm{BaSO} 4$

$66 \mathrm{CO}$

$37 \mathrm{CO} 2$

$142 \mathrm{CO} 2$ (C14)

$63 \mathrm{Ca}(\mathrm{COOH})$

$167 \mathrm{Ca}(\mathrm{NO} 3)$

$120 \mathrm{Ca}(\mathrm{OH}) 2$

$113 \mathrm{Ca} 3(\mathrm{PO} 4) 2$

$20 \mathrm{CaC2O}$

$18 \mathrm{CaCO} 3$
$64 \mathrm{CaCO} 3$ (C14)

$78 \mathrm{CaF} 2$

$41 \mathrm{CaO}$

19 CasO4

29 Carbon

$26 \mathrm{Co}(\mathrm{COOH}) 2$

$168 \mathrm{Co}$ (NO3) 2

$59 \mathrm{COO}$

$98 \quad \mathrm{Cr} 2 \mathrm{O}$

$\begin{array}{ll}42 & \mathrm{Cs} 2 \mathrm{O} \\ 53 & \mathrm{CsCOOH}\end{array}$

$96 \mathrm{CsCl}$

169. CsNO3

$8 \mathrm{CsOH}$

151 CsTPB

WSRC-TR-95-0019 (Revision
339

Vent from Recyc HId Tk

$$
\begin{array}{r}
0.2267 \mathrm{E}-13 \\
0.5680 \mathrm{E}-03 \\
\text { ZERO } \\
\text { ZERO } \\
\text { ZERO } \\
0.8206 \mathrm{E}-02 \\
\text { ZERO } \\
\text { ZERO } \\
\text { ZERO } \\
\text { ZERO } \\
\text { ZERO }
\end{array}
$$

$0.6262 \mathrm{E}-04$

$0.3996 \mathrm{E}-11$

$0.8010 \mathrm{E}-12$

ZERO

ZLRO

ZERO

ZERO

ZERO

ZERO

ITRO

$0.1459 \mathrm{E}-03$

$0.3236 \mathrm{E}-13$

ZERO

ZERO

ZERO

ZERO

ZERO

ZERO

ZERO

ZERO

ZERO

ZERO

ZERO

ZERO

ZERO

ZERO

ZERO

ZERO

ZERO

ZERO

ZERO

0)
$348 \quad 340 \quad 338$

Insol frm Recy Soln to Tnk Frm Evap DWPF Recyc Tnk Frm Evap

Aq to ETF

354

Salt Cake

$0.1881 \mathrm{E}-08$

$36457-06$

ZERO

$0.2021 \mathrm{E}-06$

$.7661 \mathrm{E}-06$

$0.8336 \mathrm{E}-08$

ZERO

ZERO

ZERO

ZERO

ZERO

ZTRO

$0.8863 \mathrm{E}-07$ ZERO

$0.8836 \mathrm{E}-01$

$.1692 \mathrm{E}+00$

ZERO

$0.111 .9 \mathrm{E}-04$

$0.1534 \mathrm{E}-03$

ZERO

ZERO

ZERO

ZERO

ZERO

ZERO

$.2627 \mathrm{E}-0$

ZERO

ZERO
$0.5501 \mathrm{E}-07$

$.1454 \mathrm{E}-01$

$0.1988 \mathrm{E}-02$

ZERO

ZERO

ZERO

$0.1030 \mathrm{E}-05$

$0.4303 E-02$

$0.3099 \mathrm{E}-03$

ZERO
ZERO

ZERO

ZERO

ZERO

ZERO

ZERO

ZERO

ZERO

ZERO

DRO

ZERO

$.4146 \mathrm{E}-01$

ZERO

$0.2539 \mathrm{E}-07$

$0.6657 \mathrm{E}+00$

$0.2077 \mathrm{E}-07$

ZERO

$0.2595 \mathrm{E}-06$

$0.5114 \mathrm{E}-10$

$0.4425 \mathrm{E}-11$

$0.2586 \mathrm{E}-06$

$0.5098 \mathrm{E}-10$

$0.4417 \mathrm{E}-11$

ZERO

ZERO

ZERO

ZERO

ZERO

ZERO

ZERO

ZERO

$0.1606 \mathrm{E}-06$

$0.3561 \mathrm{E}-16$

ZERO

$0.1535 \mathrm{E}-04$

ZERO

ZERO

ZERO

ZERO

ZERO

ZERO

ZERO

ZERO

ZERO

ZERO

$0.1965 \mathrm{E}-11$

列

ZERO

ZERO

ZERO

ZERO

ZERO

ZERO

ZERO

$0.1606 \mathrm{E}-06$

$0.3561 \mathrm{E}-16$

ZERO
. ZERO

ZERO

ZERO

ZERO

ZERO

ZERO

ZERO

ZERO

ZERO

ZERO

ZERO

ZERO

ZERO

ZERO

ZERO

ZERO

ZERO

$0.3522 \mathrm{E}-10$

ZERO

ZERO

ZERO

Date: January 1995 \begin{tabular}{r} 
ZERO \\
ZERO \\
ZERO \\
ZERO \\
ZERO \\
ZERO \\
$0.4353 E-02$ \\
ZERO \\
ZERO \\
$0.4619 E-08$ \\
$0.6657 E+00$ \\
ZERO \\
$0.9038 E-09$ \\
$0.1648 E-12$ \\
$0.7988 E-14$ \\
ZERO \\
ZERO \\
ZERO \\
ZERO \\
ZERO \\
ZERO \\
ZERO \\
ZERO \\
ZERO \\
$0.1387 E-12$ \\
$0.3074 E-22$ \\
ZERO \\
$0.1535 E-04$ \\
ZERO \\
ZERO \\
ZERO \\
ZERO \\
ZERO \\
ZERO \\
ZERO \\
ZERO \\
ZERO \\
ZERO \\
ZERO \\
$0.3522 E-10$ \\
ZERO \\
ZEERO \\
ZER \\
\hline $.1965 E-11$ \\
ZERO \\
ZERO \\
ZERO \\
ZERO \\
0
\end{tabular}

Section 7, Page 18 of 21 
Table A-1. HLW Flowsheet Material Balance (Tank 51/ITP Cycle 1) section 7. Collection and Treatment of Waste streams

STREAM NUMBERS (CONT'D) $\rightarrow$ STREAM NAME

\begin{tabular}{|c|c|}
\hline \multicolumn{2}{|c|}{$54 \mathrm{Cu}(\mathrm{COOH}) 2$} \\
\hline 170 & $\mathrm{Cu}(\mathrm{NO} 3) 2$ \\
\hline $\begin{array}{l}55 \\
80\end{array}$ & Cuo \\
\hline 80 & $\mathrm{Fe} 2 \mathrm{O} 3$ \\
\hline 101 & $\mathrm{FeO}$ \\
\hline 102 & Group A \\
\hline 103 & Group B \\
\hline 25 & $\mathrm{H} 2$ \\
\hline 158 & $\mathrm{H} 2 \mathrm{C} 204$ \\
\hline 93 & H2SO4 \\
\hline 90 & Н3ВОЗ \\
\hline 34 & $\mathrm{HCOOH}$ \\
\hline 43 & $\mathrm{HCl}$ \\
\hline 85 & $\mathrm{HF}$ \\
\hline 49 & HNO3 \\
\hline 36 & $\mathrm{Hg}$ \\
\hline 129 & $\mathrm{Hg}(\mathrm{C} 6 \mathrm{H} 5)_{2} 2$ \\
\hline 135 & $\mathrm{Hg}(\mathrm{NO} 3)_{2} 2$ \\
\hline 144 & $\mathrm{Hg} 2 \mathrm{Cl2}$ \\
\hline 117 & $\mathrm{Hg} 2 \mathrm{I} 2$ \\
\hline 146 & $\mathrm{HgCl}$ \\
\hline 21 & $\mathrm{HgO}$ \\
\hline 97 & I2 \\
\hline 183 & $\mathrm{~K} 2 \mathrm{CO} 3$ \\
\hline 39 & $\mathrm{~K} 2 \mathrm{O}$ \\
\hline 51 & $\mathrm{KCOOH}$ \\
\hline 157 & $\mathrm{KMnO4}$ \\
\hline 161 & $\mathrm{KNO} 3$ \\
\hline 10 & $\mathrm{KOH}$ \\
\hline 150 & KTPB \\
\hline 105 & La2O3 \\
\hline 108 & Li2O \\
\hline 65 & $\mathrm{Mg}(\mathrm{COOH}) 2$ \\
\hline 171 & $\mathrm{Mg}(\mathrm{NO} 3) 2$ \\
\hline 33 & $\mathrm{MgO}$ \\
\hline 60 & $\operatorname{Mn}(\mathrm{COOH}) 2$ \\
\hline 172 & $\mathrm{Mn}(\mathrm{NO}) 2$ \\
\hline 180 & Mn304 \\
\hline 56 & Mno \\
\hline 14 & $\mathrm{MnO} 2$ \\
\hline 145 & MoO2 \\
\hline 81 & N2 \\
\hline 74 & $\mathrm{~N} 2 \mathrm{O}$ \\
\hline 182 & NH3 \\
\hline 114 & NH3OHNO3 \\
\hline 52 & $\mathrm{NH} 4 \mathrm{COOH}$ \\
\hline 173 & NH4NO3 \\
\hline 68 & $\mathrm{NH} 4 \mathrm{OH}$ \\
\hline
\end{tabular}

339 Recyc Hld Tk

348

340 DWPF Recyc Tnk Frm Evap Aq to ETF

ZERO

ZERO

$0.2539 \mathrm{E}-01$

ZERO 0.21

$0.1693 \mathrm{E}-11$

ZERO

ZERO

ZERO

ZERO

ZERO

ZERO

ZERO

ZERO

ZERO

ZERO

ZERO

ZERO

ZERO

ZERO

ZERO

ZERO

ZERO

ZERO

ZERO

ZERO

ZERO

ZERO

ZERO

ZERO

ZERO

ZERO

ZERO

ZERO

ZERO

$0.1082 \mathrm{E}+04$

TRACE

$0.1764 \mathrm{E}+00$

ZERO

ZERO

ZERO

ZERO

$$
\begin{array}{r}
\text { ZERO } \\
\text { ZERO }
\end{array}
$$

ZERO

$0.9462 \mathrm{E}-02$

$0.2539 \mathrm{E}+00$

$0.1512 \mathrm{E}-05$

ZERO

$0.7870 \mathrm{E}-01$

ZERO

ZERO

$0.5718 E-01$

ZERO

ZERO

ZERO

ZERO

ZERO
ZERO

$0.1108 \mathrm{E}+00$

ZERO

$0.4692 \mathrm{E}-01$

ZERO

ZERO

0.2651 ZERO

$0.2651 \mathrm{E}-01$

$0.1076 \mathrm{E}-04$

ZERO

ZERO

ZERO

ZERO

ZERO

ZERO
ZERO

$0.7978 \mathrm{E}-05$

ZERO

ZERO

ZERO

ZERO

$0.7632 \mathrm{E}-16$

$$
\begin{aligned}
& \text { ZERO } \\
& \text { ZERO }
\end{aligned}
$$

ZERO

ZERO

ZERO

ZERO

ZERO

ZERO

ZERO

ZERO

ZERO

ZERO

ZERO

Aq to ETF

ZERO

ZERO

ZERO

ZERO

ZERO

$0.7632 \mathrm{E}-16$

ZERO

ZERO

ZERO

ZERO

ZERO

ZERO

ZERO

ZERO

ZERO

ZERO

ZERO
ZERO

ZERO

ZERO
ZERO

ZERO
ZERO
ZERO

ZERO

ZERO

ZERO

ZERO

ZERO

$0.1094 \mathrm{E}+00$

(DRO

ZERO

ZERO

$0.1921 \mathrm{~F}-12$

1E-12

IRRO

$0.2239 \mathrm{E}-12$

ZERO

ZERO

ZERO

TRACE

$0.2689 \mathrm{E}-01$

ZERO

ZERO

ZERO

ZERO

ZERO

ZERO

ZERO

ZERO

ZERO

ZERO

ZERO

ZERO

ZERO

ZERO

ZERO

ZERO

ZERO

ZERO

$0.3741 \mathrm{E}-01$

TRACE

$0.2688 \mathrm{E}-01$

ZERO

ZERO

ZERO

ZERO

ZERO
338

354

DWPF Recycl

Salt Cake

$0.7978 \mathrm{E}-05$

ZERO

ZERO

ZERO

ZERO

$0.6408 \mathrm{E}-23$

ZERO

ZERO

ZERO

ZERO

ZERO

ZERO

ZERO

ZERO

ZERO

ZERO

ZERO

ZERO

ZERO

ZERO

ZERO

ZERO

ZERO

ZERO

ZERO

ZERO
ZERO
0.1094 E+0O

促O

ZERO

ZERO

$0.1921 \mathrm{E}-12$

ZERO

$0.2239 \mathrm{E}-12$

ZERO

ZERO

ZERO

$0.1857 \mathrm{E}-08$

ZERO

E-O5

ZERO

ZERO

ZERO 
Table A-1. HLW Flowsheet Material Balance (Tank 51/TTP Cycle 1) Section 7. Colleation and Treatment of Waste streams

STREAM NUMBERS (CONT'D) $\rightarrow$ STREAM NAME

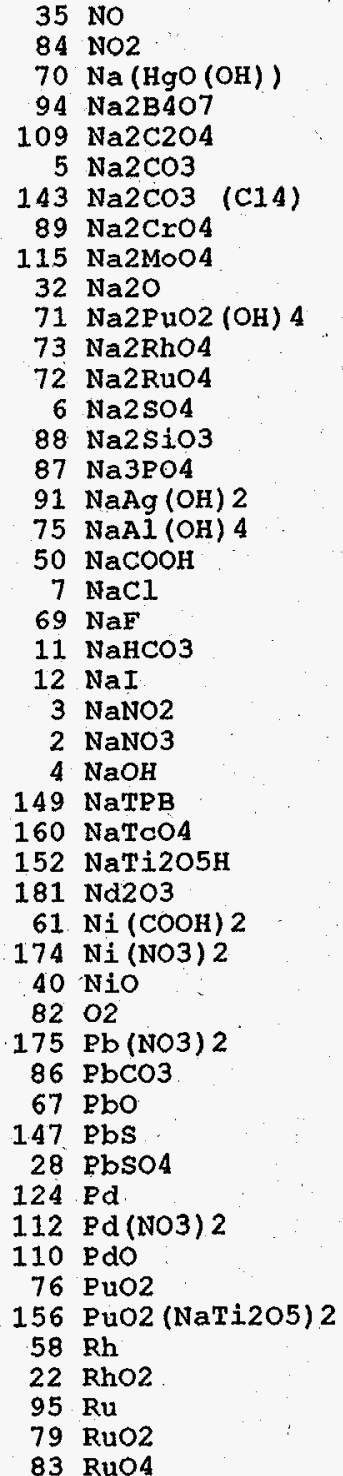

339
Vent

$\begin{array}{llr}339 & 348 & 340 \\ \text { Vent from } & \text { Insol frm Recy So }\end{array}$ Recyc HId TK

$0.7937 \mathrm{E}-07$

$0.1553 \mathrm{E}-06$

338

Insol frm Recy Soln to Tnk Frm Evap $\begin{array}{lll}\text { Recyc Tnk Frm Evap } & \text { Aq to ETF } \\ \text { ZERO } & 0.8013 \mathrm{E}-11 & 0.8013 \mathrm{E}-11\end{array}$ ZERO $0.1567 \mathrm{E}-10 \quad 0.1567 \mathrm{E}-10$

ZERO

ZERO

ZERO

ZERO

ZERO

ZERO

ZERO

ZERO

ZERO

ZERO

ZERO

ZERO

ZERO

ZERO

ZERO

ZERO

ZERO

ZERO

ZERO

ZERO

ZERO

ZERO

ZERO

ZERO

ZERO

$0.3269 \mathrm{E}+03$

ZERO

ZERO

ZERO

ZERO

ZERO

ZERO

ZERO

ZERO

ZERO

ZERO

ZERO

ZERO ZERO

$\begin{array}{rr}0.1567 \mathrm{E}-10 & 0.1567 \mathrm{E}-10 \\ \text { ZERO } & \text { ZERO }\end{array}$

$0.1227 \mathrm{E}+00$ ZERO

ZERO $0.7909 \mathrm{E}+01$

ZERO $\quad 0.1794 E-04$

ZERO

ZERO

ZERO

$0.2030 \mathrm{E}+00$

ZERO

ZERO

ZERO

ZERO

ZERO

ZERO

ZERO

ZERO

ZERO

ZERO

ZERO

ZERO

ZERO

ZERO

ZERO

ZERO

ZERO

ZERO

ZERO

ZERO

$0.2543 \mathrm{E}-02$

ZERO

ZERO

$0.4902 \mathrm{E}-04$

ZERO

$0.1252 \mathrm{E}-02$

ZERO
ZERO

$0.2921 \mathrm{E}-08$

$0.9959 \mathrm{E}-04$

$0.4651 \mathrm{E}-09$

ZERO
$0.6346 \mathrm{E}-08$

$.6346 \mathrm{E}-08$

ZERO

ZERO
ZERO

ZERO

ZERO

ZERO

ZERO

$0.6774 \mathrm{E}-01$

0.2578 EE-04

ZERO

ZERO

$0.5613 \mathrm{E}-04$

$0.5026 \mathrm{E}-02$

$0.1405 \mathrm{E}-01$

ZERO

$0.5464 \mathrm{E}-05$

$0.9908 \mathrm{E}+01$
$0.1289 \mathrm{E}+02$

$0.7840 \mathrm{E}+02$

ZERO
ZERO

ZERO

ZERO

0.2445 E-11

ZERO

$0.2158 \mathrm{E}-01$

$0.2847 \mathrm{E}-09$

ZERO

ZERO

ZERO
ZERO

ZERO

ZERO

ZERO

ZERO

ZERO

ZERO

ZERO
ZERO

ZERO

ZERO

ZERO

ZERO

ZERO

ZERO

ZERO

ZERO

ZERO

ZERO

ZERO

ZERO

ZERO

ZERO

ZERO

ZERO

ZERO

ZERO

ZERO

ZERO

ZERO

ZERO

ZERO

ZERO

ZERO

ZERO

ZERO

$0.2158 \mathrm{E}-01$

BE-01

ZERO

ZERO

ZERO

ZERO

ZERO

ZERO

ZERO

ZERO

ZERO

ZERO

ZERO

ZERO

Date: January 1995
354

DWPF Recycl

salt Cake

$.1104 \mathrm{E}-17$

2160E-17

$0.1227 \mathrm{E}+00$

. 7909E+01

ZERO

ZERO

ZERO

ZERO

ZERO

ZERO

ZERO$$
\begin{aligned}
& \text { ZERO } \\
& \text { ZERO }
\end{aligned}
$$

$0.5613 \mathrm{E}-04$

$0.5026 \mathrm{E}-02$

$0.1405 \mathrm{E}-01$

$0.5464 E-05$

$0.9908 \mathrm{E}+01$

$0.1289 \mathrm{E}+02$

$0.7840 E+02$

ZERO

ZERO

ZERO

$0.2445 \mathrm{EERO}$

ZERO

$0.1926 \mathrm{E}-08$

$47 \mathrm{E}-09$

ZERO

ZERO

ZERO

ZERO

ZERO

ZERO

ZERO

ZERO

ZERO

ZERO

2ERO

ZERO

Section 7, Page 20 of 21 
Table A-1. HLW Flowsheet Material Balance (Tank 51/ITP Cyale 1) Section 7. Collection and Treatment of Waste streams

STREAM NUMBERS (CONT'D) $\rightarrow$ STREAM NAME

$38 \mathrm{SO} 2$

141 SO3

137 Semi Vol Cs20

136 Semi Vol CsCl

126 Semi Vol Group A

138 Semi Vol NaCl

139 Semi Vol NaF

125 Semi Vol NaI

23 Semi Vol RuO2

$111 \mathrm{SiO2}$

$48 \mathrm{Sr}(\mathrm{COOH}) 2$

$176 \mathrm{Sr}$ (NO3) 2

$153 \mathrm{Sr}(\mathrm{NaTi2O5)} 2$

$127 \mathrm{Sr}(\mathrm{OH}) 2$

$30 \mathrm{SrCO} 3$

46 sro

$159 \mathrm{TcO} 2$

92 ThO2

107 TiO2

119 Tritium

170308

$62 \mathrm{VO} 2(\mathrm{COOH}) 2$

177 UO2 (NO3) 2

$16 \mathrm{UO2}(\mathrm{OH}) 2$

$27 \mathrm{Y}(\mathrm{COOH}) 3$

$178 \mathrm{Y}(\mathrm{NO} 3) 3$

$128 \times(\mathrm{OH}) 3$

31 Y $2(\mathrm{CO} 3) 3$

47 Y 203

100 zeolite

$44 \mathrm{Zn}(\mathrm{COOH}) 2$

$179 \mathrm{zn}(\mathrm{NO} 3) 2$

$57 \mathrm{zno}$

$104 \mathrm{ZrO} 2$

13 hydrate $\mathrm{H} 2 \mathrm{O}$

TOTAL FLOW, IB/HR

TEMPERATURE, DEG $C$

PRESSURE, ATM

PRESSURE， PSIG

PRESSURE, MM HG

ENTHALPY, PCU/HR

VAPOR FLOW, CEM

LIOUID FLOW， GPM

DENSITY, IBS/FT3

PHASE $\begin{array}{llr}339 & 348 & 340 \\ \text { Vent from } & \text { Insol frm Recy So }\end{array}$

Inso

340

Frm 354

Recyc Hid Tk DWPF Recyc Tnk Frm Evap Aq to FTF

$\begin{array}{lll}\text { ZERO } & \text { ZERO } & \text { ZERO } \\ \text { ZERO } & \text { ZERO } & \text { ZERO }\end{array}$

ZERO

ZERO

ZERO

ZERO

ZERO

ZERO

ZERO

ZERO

ZERO

ZERO

ZERO

ZERO

ZERO

$0.2999 \mathrm{E}-10$

ZERO

ZERO

ZERO

ZERO

ZERO

ZERO

ZERO

ZERO

ZERO

ZERO

ZERO

ZERO

ZERO

$0.1246 \mathrm{E}+03$

$0.1534 \mathrm{E}+04$

$0.5000 \mathrm{E}+02$

$0.9656 \mathrm{E}+00$

$-0.5055 \mathrm{E}+00$

$0.7339 \mathrm{E}+03$

$0.9737 \mathrm{E}+05$
$0.4087 \mathrm{E}+03$

$0.6255 \mathrm{E}-01$

VAPOR
ZERO

ZERO
ZERO

ZERO

ZERO

ZERO

ZERO

$0.1230 \mathrm{E}+01$

ZERO

ZERO

$.1489 \mathrm{E}-07$ ZERO

$0.1535 \mathrm{E}-03$

$0.3570 \mathrm{E}-04$

$0.2973 \mathrm{E}-03$

$.8338 \mathrm{E}-02$

$0.2085 \mathrm{E}-01$

E-01

ZERO

ZERO

ZERO

ZERO

ZERO

0.422 9E-04

ZERO

ZERO

0. 3160E-02

$0.8616 \mathrm{E}-05$

ZERO

$0.6000 \mathrm{E}+02$

$0.1000 \mathrm{E}+01$

ZERO

0.107 ZERO

$0.7559 \mathrm{E}-09$

$0.3349 \mathrm{E}-09$

$0.2911 \mathrm{E}-07$

0.1951 E-O

$0.3141 \mathrm{E}+04$

$0.3251 \mathrm{E}+04$

$0.6000 \mathrm{E}+02$

$0.1000 \mathrm{E}+01$

$0.2929 \mathrm{E}+02$

$0.1905 \mathrm{E}+06$

$0.1907 \mathrm{E}-02$

$0.6347 \mathrm{E}+01$

$.1672 \mathrm{E}+03$

$0.6387 \mathrm{E}+02$
ZERO

ZERO

ZERO

ZERO

ZERO

ZERO

ZERO

ZERO

210

ZERO

ZERO

ZERO

ZERO

ZERO

ZERO

ZERO

ZERO

ZERO

ZERO

ZERO

ZERO

ZERO

ZERO

ZERO

ZERO

ZERO

ZERO

ZERO

ZERO

ZERO

ZERO

ZERO

ZERO

ZERO

ZERO

ZERO

ZERO

ZERO

ZERO

ZERO

$0.7512 \mathrm{E}-09$

ZERO

ZERO

ZERO

ZERO

ZERO

ZERO

ZERO

ZERO

ZERO

ZERO

ZERO

ZERO

$0.3121 \mathrm{E}+04$

$0.3122 \mathrm{E}+04$

$0.1000 \mathrm{E}+03$

$0.1000 \mathrm{E}+01$

ZERO

$0.3124 \mathrm{E}+06$

$0.6234 \mathrm{E}+01$

$0.6243 E+02$

LIQUID

WPF Recycl

salt Cake

ZERO

ZERO

ZERO

ZERO

ZERO

ZERO

ZERO

ZERO

ZERO

ZERO

$0.1079 \mathrm{E}-06$

ZERO

ZERO

ZERO

ZERO

ZERO

4699E-11

ZERO

$0.3349 \mathrm{E}-09$

ZERO

ZRRO

0.2911 07

ZERO

ERO

ZERO

ZERO

$0.1951 \mathrm{E}-09$

ZERO

ZERO

ZERO

$0.1952 \mathrm{E}+02$

$0.1296 \mathrm{E}+03$

$0.1000 \mathrm{E}+03$

$0.1000 \mathrm{E}+01$

$0.5308 \mathrm{E}+04$

IIOUTD

LIQUID 


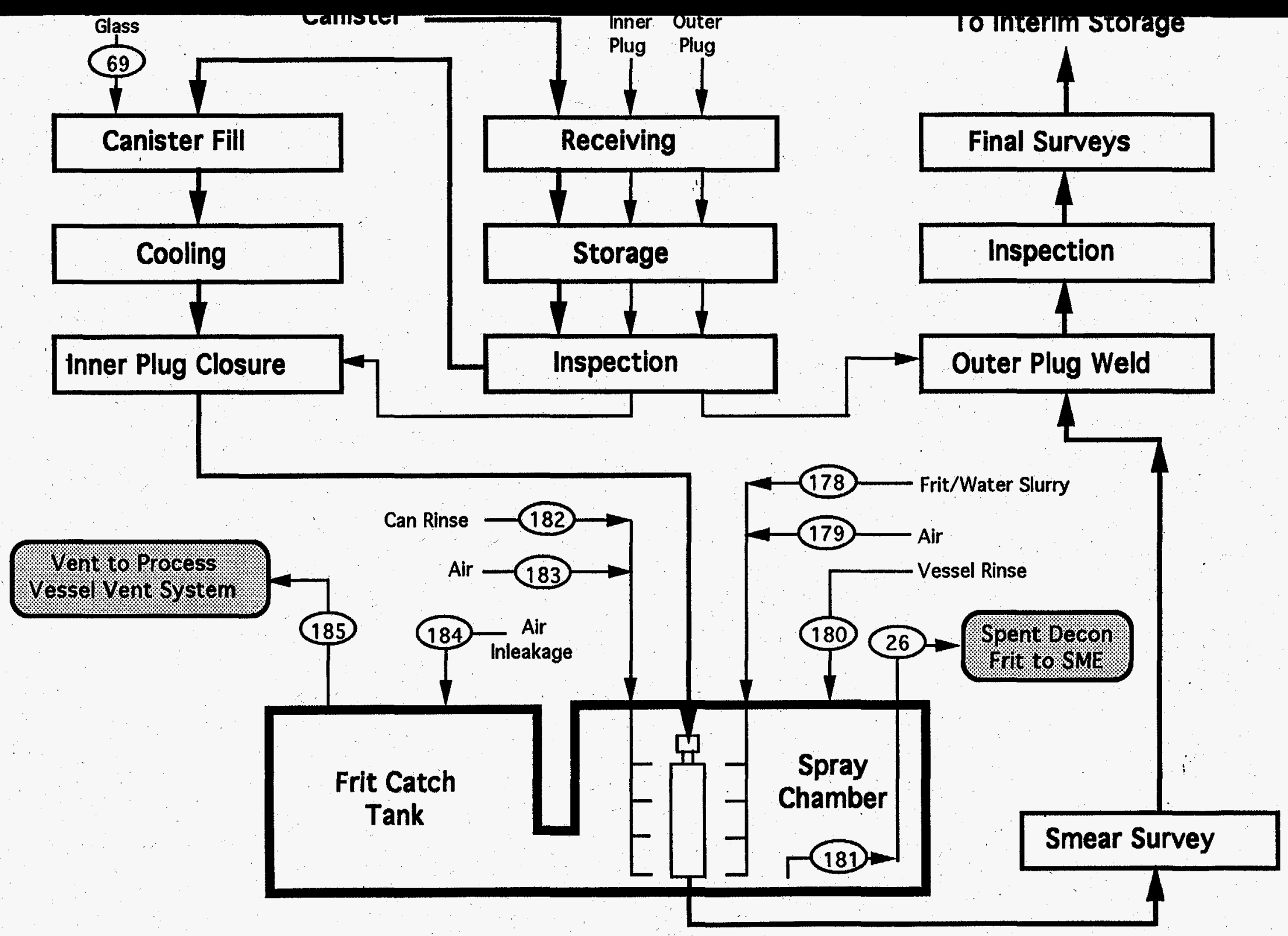

SECTION 8. CANISTER DECONTAMINATION 
Table A-1. HLW Flowshet Material Balance (Tank 51/ITR Cycle 1) section 8. Canister Decontamination

DESCRIPTION

PAGE

1. STREAM NUMBERS

$69,178,179,180,181,182,183 \ldots \ldots \ldots \ldots$

2. STREAM NUMBERS

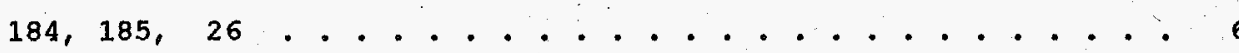


Table A-1. HLW Flowsheet Material Balance (Tank 51/ITP Cycle 1) section 8. Canister Decontamination

STREAM NUMBERS $\rightarrow$ STREAM NAME

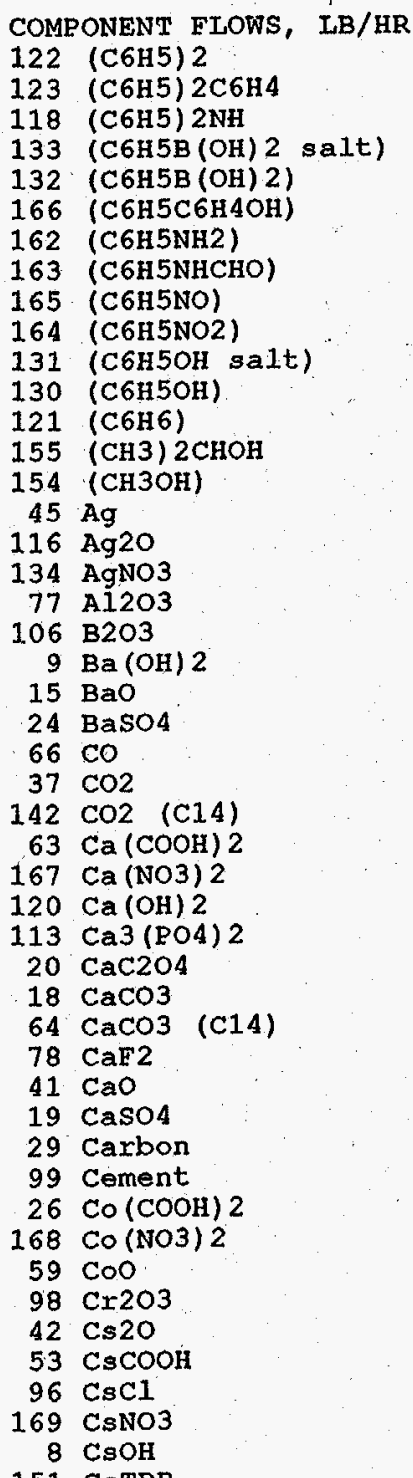

69 178 179 180 181 182 183 Sldge Blend Frit Slry to Atomzg Air Spray Chambr Oxide from Rinse H2O to Atomzg Air Waste Glass Can Decon to Can Decon Water Rinse Can Cleaning Can Decon to Can Rinse

\begin{tabular}{|c|c|c|c|c|c|c|}
\hline ZERO & ZERO & ZERO & ZERO & ZERO & ZERO & ZERO \\
\hline ZERO & ZERO & ZERO & ZERO & ZERO & ZERO & ZERO \\
\hline ZERO & ZERO & ZERO & ZERO & ZERO & ZERO & ZERO \\
\hline ZERO & ZERO. & ZERO & ZERO & ZERO & ZERO & ZERO \\
\hline ZERO & ZERO & ZERO & ZERO & ZERO & ZERO & ZERO \\
\hline ZERO & ZERO & ZERO & ZERO & ZERO & ZERO & ZERO \\
\hline ZERO & ZERO & ZERO & ZERO & ZERO & ZERO & ZERO \\
\hline ZERO & ZERO & ZERO & ZERO & ZERO & ZERO & ZERO \\
\hline ZERO & ZERO & ZERO & ZERO & ZERO & ZERO & ZERO \\
\hline ZERO & ZERO & ZERO & ZERO & ZERO & ZERO & ZERO \\
\hline ZERO & ZERO & ZERO & ZERO & ZERO & ZERO & ZERO \\
\hline ZERO & ZERO & ZERO & ZERO & ZERO & ZERO & ZERO \\
\hline ZERO & ZERO & ZERO & ZERO & ZERO & ZERO & ZERO \\
\hline ZERO & ZERO & ZERO & ZERO & ZERO & ZERO & ZERO \\
\hline ZERO & ZERO & ZERO & ZERO & ZERO & ZERO & ZERO \\
\hline $0.1241 \mathrm{E}-01$ & ZERO & ZERO & ZERO & ZERO & ZERO & ZERO \\
\hline ZERO & ZERO & ZERO & ZERO & ZERO & ZERO & ZERO \\
\hline ZERO & ZERO & ZERO & ZERO & ZERO & ZERO & ZERO \\
\hline $0.8746 \mathrm{E}+01$ & ZERO & ZERO & ZERO & ZERO & ZERO & ZERO \\
\hline $0.1675 \mathrm{E}+02$ & $0.8542 \mathrm{E}+00$ & ZERO & ZERO & ZERO & ZERO & 2ERO \\
\hline ZERO & ZERO & ZERO & ZERO & ZERO & ZERO & ZERO \\
\hline $0.1108 \mathrm{E}-02$ & ZERO & ZERO & ZERO & ZERO & ZERO & ZERO \\
\hline $0.1518 \mathrm{E}-01$ & ZERO & ZERO & ZERO & 2ERO & ZERO & ZERO \\
\hline ZERO & ZERO & ZERO & ZERO & ZERO & ZERO & ZERO \\
\hline ZERO & ZERO & ZERO & ZERO & ZERO & ZERO & ZERO \\
\hline ZERO & ZERO & ZERO & ZERO & ZERO & ZERO & ZERO \\
\hline ZERO & ZERO & ZERO & ZERO & ZERO & ZERO & ZERO \\
\hline ZERO & ZERO & ZERO & ZERO & ZERO & ZERO & ZERO \\
\hline ZERO & ZERO & ZERO & ZERO & ZERO & ZERO & ZERO \\
\hline $0.2600 \mathrm{E}+01$ & ZERO & ZERO & ZERO & ZERO & ZERO & ZERO \\
\hline ZERO & ZERO & ZERO & ZERO & ZERO & ZERO & ZERO \\
\hline ZERO & ZERO & ZERO & ZERO & ZERO & ZERO & ZERO \\
\hline ZERO & ZERO & ZERO & ZERO & ZERO & ZERO & ZERO \\
\hline ZERO & ZERO & ZERO & ZERO & ZERO & ZERO & ZERO \\
\hline $0.1440 \mathrm{E}+01$ & ZERO & ZERO & ZERO & ZERO & ZERO & ZERO \\
\hline $0.1967 \mathrm{E}+00$ & ZERO & ZERO & ZERO & ZERO & ZERO & ZERO \\
\hline ZERO & ZERO & ZERO & ZERO & ZERO & ZERO & ZERO \\
\hline ZERO & ZERO & ZERO & ZERO & ZERO & ZERO & ZERO \\
\hline ZERO & ZERO & ZERO & ZERO & ZERO & ZERO & ZERO \\
\hline ZERO & ZERO & ZERO & ZERO & ZERO & ZERO & ZERO \\
\hline $0.1020 \mathrm{E}-03$ & ZERO & ZERO & ZERO & ZERO & ZERO & ZERO \\
\hline $0.4259 \mathrm{E}+00$ & ZERO & ZERO & ZERO & $0.1254 \mathrm{E}-02$ & ZERO & ZERO \\
\hline $0.3069 \mathrm{E}-01$ & ZERO & ZERO & ZERO & ZERO & ZERO & ZERO \\
\hline ZERO & ZERO & ZERO & ZERO & ZERO & ZERO & ZERO \\
\hline ZERO & ZERO & ZERO & ZERO & ZERO & ZERO & ZERO \\
\hline ZERO & ZERO & ZERO & ZERO & ZERO & ZERO & ZERO \\
\hline ZERO & ZERO & ZERO & ZERO & ZERO & ZERO & ZERO \\
\hline ZERO & ZERO & ZERO & ZERO & ZERO & ZERO & ZERO \\
\hline
\end{tabular}


Table A-1. HLW Flowsheet Material Balance (Tank 51/ITP Cycle 1) section 8. Canister Decontamination

STREAM NUMBERS (CONT'D) $\rightarrow$ STREAM NAME

$54 \mathrm{Cu}(\mathrm{COOH}) 2$
$170 \mathrm{Cu}(\mathrm{NO} 3) 2$

$\begin{array}{rl}70 & \mathrm{Cu} \\ 55 & \mathrm{CuO}\end{array}$

$80 \mathrm{Fe} 2 \mathrm{O}$

$101 \mathrm{FeO}$

102 Group A

103 Group

$25 \mathrm{H} 2$

158 H2C2O4

$93 \mathrm{H} 2 \mathrm{SO} 4$

90. $\mathrm{H} 3 \mathrm{BO} 3$

$43 \mathrm{HCl}$

$85 \mathrm{HF}$

4.9 HNO3

$36 \mathrm{Hg}$

129. Hg (C6H5) 2

$135 \mathrm{Hg}$ (NO3) 2

$144 \mathrm{Hg} 2 \mathrm{Cl} 2$

$117 \mathrm{Hg} 2 \mathrm{I} 2$

$21 \mathrm{HgO}$

97 I2

$183 \mathrm{~K} 2 \mathrm{CO} 3$

$39 \mathrm{~K} 2 \mathrm{O}$

$51 \mathrm{KCOOH}$

$157 \mathrm{KMnO} 4$

$161 \mathrm{KNO} 3$

$10 \mathrm{KOH}$

150 RTPB

$\begin{array}{ll}105 & \mathrm{La} 203 \\ 108 & \mathrm{Li} 20\end{array}$

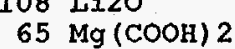

$171 \mathrm{Mg}$ (NO3) 2

$33 \mathrm{MgO}$

$60 \mathrm{Mn}$ (COOH) 2

$172 \mathrm{Mn}(\mathrm{NO} 3) 2$

$180 \mathrm{Mn} 304$

$56 \mathrm{MnO}$

$14 \mathrm{MnO} 2$

$145 \mathrm{MoO} 2$

$81 \mathrm{~N} 2$

74 N2O
$182 \mathrm{NH} 3$

114 NH3OHNO3

$52 \mathrm{NH} 4 \mathrm{COOH}$

173 NH4NO3

$68 \mathrm{NH} 4 \mathrm{OH}$
69 178

179 180 181

182 183 Sldge Blend Frit Slry to Atomz Air Spray Chambr oxide from Rinse H2O to Atomzg Air Waste Glass Can Decon to Can Decon Water Rinse Can Cleaning Can Decon to Can Rinse

\begin{tabular}{|c|c|c|c|c|c|c|c|}
\hline ZERO & ZERO & ZERO & & ZERO & ZERO & ZERO & ZERO \\
\hline ZERO & ZERO & ZERO & $\because$ & ZERO & ZERO & ZERO & ZERO \\
\hline $0.9369 E+00$ & ZERO & ZERO & & ZERO & ZERO & ZERO & ZERO \\
\hline $0.2513 \mathrm{E}+02$ & ZERO & ZERO & & ZERO & $0.4880 \mathrm{E}-03$ & ZERO & ZERO \\
\hline $0.2513 \mathrm{E}+01$ & ZERO & ZERO & & ZERO & ZERO & ZERO & ZERO \\
\hline $0.3332 \mathrm{E}-02$ & ZERO & ZERO & & ZERO & ZERO & ZERO & ZERO \\
\hline $0.1496 \mathrm{E}-03$ & ZERO & ZERO & & ZERO & ZERO & ZERO & ZERO \\
\hline ZERO & ZERO & ZERO & & ZERO & ZERO & ZERO & ZERO \\
\hline ZERO & ZERO & ZERO & & ZERO & ZERO & ZERO & ZERO \\
\hline ZERO & ZERO & ZERO & & ZERO & ZERO & ZERO & ZERO \\
\hline ZERO & ZERO & ZERO & & ZERO & ZERO & ZERO & ZERO \\
\hline ZERO & ZERO & ZERO & & ZERO & ZERO & ZERO & ZERO \\
\hline ZERO & ZERO & ZERO & & ZERO & ZERO & ZERO & ZERO \\
\hline ZERO & ZERO & ZERO & & ZERO & ZERO & ZERO & ZERO \\
\hline ZERO & ZERO & ZERO & & ZERO & ZERO & ZERO & ZERO \\
\hline ZERO & ZERO & ZERO & & ZERO & ZERO & ZERO & ZERO \\
\hline ZERO & ZERO & ZERO & & ZERO & ZERO & ZERO & ZERO \\
\hline ZERO & ZERO & ZERO & & ZERO & ZERO & ZERO & ZERO \\
\hline ZERO & ZERO & ZERO & & ZERO & ZERO & ZERO & ZERO \\
\hline ZERO & ZERO & ZERO & & ZERO & ZERO & ZERO & ZERO \\
\hline ZERO & ZERO & ZERO & & ZERO & ZERO & ZERO & ZERO \\
\hline ZERO & ZERO & ZERO & & ZERO & ZERRO & ZERO & ZERO \\
\hline ZERO & ZERO & ZERO & & ZERO & ZERO & ZERO & ZERO \\
\hline ZERO & ZERO & ZERO & & ZERO & ZERO & ZERO & ZERO \\
\hline $0.5661 \mathrm{E}+01$ & ZERO & ZERO & & ZERO & ZERO & ZERO & ZERO \\
\hline ZERO & ZERO & ZERO & & ZERO & ZERO & ZERO & ZERO \\
\hline ZERO & ZERO & ZERO & & ZERO & ZERO & ZERO & ZERO \\
\hline ZERO & ZERO & ZERO & & ZERO & ZERO & ZERO & ZERO \\
\hline ZERO & ZERO & ZERO & & ZERO & ZERO & ZERO & ZERO \\
\hline ZERO & ZERO & ZERO & & ZERO & ZERO & ZERO & ZERO \\
\hline ZERO & ZERO & ZERO & & ZERO & ZERO & ZERO & ZERO \\
\hline $0.1097 \mathrm{E}+02$ & $0.7474 \mathrm{E}+00$ & ZERO & & ZERO & ZERO & ZERO & ZERO \\
\hline ZERO & ZERO & ZERO & & ZERO & ZERO & ZERO & ZERO \\
\hline ZERO & ZERO & ZERO & & ZERO & ZERO & ZERO & ZERO \\
\hline $0.4645 \mathrm{E}+01$ & $0.2135 \mathrm{E}+00$ & ZERO & & ZERO & ZERO & ZERO & ZERO \\
\hline ZERO & ZERO & ZERO & & ZERO & ZERO & ZERO & ZERO \\
\hline ZERO & ZERO & ZERO & & ZERO & ZERO & ZERO & ZERO \\
\hline ZERO & ZERO & ZERO & & ZERO & ZERO & ZERO & ZERO \\
\hline $0.2624 \mathrm{E}+01$ & ZERO & ZERO & & ZERO & ZERO & ZERO & ZERO \\
\hline ZERO & ZERO & ZERO & & ZERO & ZERO & ZERO & ZERO \\
\hline $0.1065 \mathrm{E}-02$ & ZERO & ZERO & & ZERO & ZERO & ZERO & ZERO \\
\hline ZERO & ZERO & $0.1424 \mathrm{E}+03$ & & ZERO & ZERO & ZERO & $0.1379 \mathrm{E}+02$ \\
\hline ZERO & ZERO & ZERO & & ZERO & ZERO & ZERO & ZERO \\
\hline ZERO & ZERO & ZERO & & ZERO & ZERO & ZERO & ZERO \\
\hline ZERO & ZERO & ZERO & & ZERO & ZERO & ZERO & ZERO \\
\hline ZERO & ZERO & ZERO & & ZERO & ZERO & ZERO & ZERO \\
\hline ZERO & ZERO & ZERO & & ZERO & ZERO & ZERO & ZERO \\
\hline ZERO & ZERO & ZERO & & ZERO & ZERO & ZERO & ZERO \\
\hline ZERO & ZERO & ZERO & & ZERO & ZERO & ZERO & ZERO \\
\hline
\end{tabular}


Table A-1. HLW Flowsheet Material Balance (Tank 51/ITP Cycle 1 ) section 8. Canister Decontamination

STREAM NUMBERS (CONT'D) $\rightarrow$ STREAM NAME

\begin{tabular}{|c|c|}
\hline 35 & No \\
\hline 84 & NO2 \\
\hline 70 & $\mathrm{Na}(\mathrm{HgO}(\mathrm{OH}))$ \\
\hline 94 & $\mathrm{Na} 2 \mathrm{~B} 407$ \\
\hline 109 & $\mathrm{Na} 2 \mathrm{C} 2 \mathrm{O} 4$ \\
\hline & $\mathrm{Na} 2 \mathrm{CO} 3$ \\
\hline 143 & $\mathrm{Na} 2 \mathrm{CO} 3(\mathrm{C} 14)$ \\
\hline 89 & $\mathrm{Na} 2 \mathrm{CrO} 4$ \\
\hline 115 & $\mathrm{Na} 2 \mathrm{MoO} 4$ \\
\hline 32 & $\mathrm{Na} 2 \mathrm{O}$ \\
\hline 71 & $\mathrm{Na} 2 \mathrm{PuO} 2(\mathrm{OH}) 4$ \\
\hline 73 & $\mathrm{Na} 2 \mathrm{RhO} 4$ \\
\hline 72 & $\mathrm{Na} 2 \mathrm{RuO} 4$ \\
\hline 6 & $\mathrm{Na} 2 \mathrm{SO}_{4}$ \\
\hline 88 & $\mathrm{Na} 2 \mathrm{SiO} 3$ \\
\hline 87 & $\mathrm{Na3PO4}$ \\
\hline 91 & $\mathrm{NaAg}(\mathrm{OH}) 2$ \\
\hline 75 & $\operatorname{NaAl}(\mathrm{OH}) 4$ \\
\hline 50 & $\mathrm{NaCOOH}$ \\
\hline 7 & $\mathrm{NaCl}$ \\
\hline 69 . & $\mathrm{NaF}$ \\
\hline 11 & $\mathrm{NaHCO} 3$ \\
\hline 12 & NaI \\
\hline 3 & NaNO2 \\
\hline 2 & NaNO3 \\
\hline 4 & $\mathrm{NaOH}$ \\
\hline 149 & NaTPB \\
\hline 160 & $\mathrm{NaTcO} 4$ \\
\hline 152 & NaTi205H \\
\hline 181 & $\mathrm{Nd} 203$ \\
\hline 61 & $\mathrm{Ni}(\mathrm{COOH}) 2$ \\
\hline 174 & $\mathrm{Ni}(\mathrm{NO} 3)_{2}$ \\
\hline 40 & NiO \\
\hline 82 & 02 \\
\hline 175 & $\mathrm{~Pb}(\mathrm{NO} 3) 2$ \\
\hline 86 & $\mathrm{PbCO} 3$ \\
\hline 67 & $\mathrm{PbO}$ \\
\hline 147 & $\mathrm{PbS}$ \\
\hline 28 & $\mathrm{PbSO} 4$ \\
\hline 124 & $\mathrm{Pd}$ \\
\hline 112 & $\mathrm{Pd}(\mathrm{NO} 3) 2$ \\
\hline 110 & Pdo \\
\hline 76 & $\mathrm{PuO} 2$ \\
\hline 156 & PuO2 (NaTi205) 2 \\
\hline 58 & $\mathrm{Rh}$ \\
\hline 22 & RhO2 \\
\hline 95 & $\mathrm{Ru}$ \\
\hline 79 & RuO2 \\
\hline & \\
\hline
\end{tabular}

WSRC-TR-95-0019 (Revision
69 178 179 . 180 181 182 183 sldge Blend Frit slry to Atomzg Air Spray Chambr Oxide from Rinse H2O to Atomzg Air

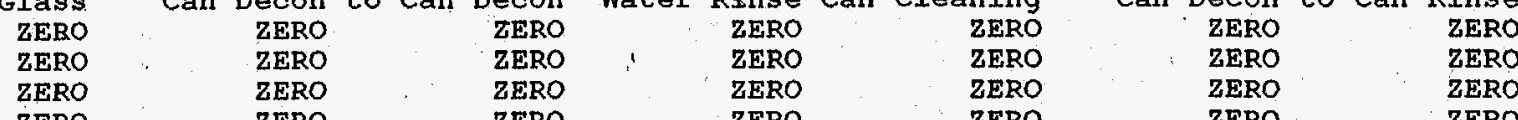
ZERO ZERO ZERO ZERO ZERO ZERO ZERO ZERO

$0.1972 \mathrm{E}+02$ ZERO ZERO $0.1546 \mathrm{E}+00$ ZERO ZERO
ZERO ZERO

$0.4928 \mathrm{E}-02$ $0.3337 \mathrm{E}-01$ ZERO ZERO ZERO ZERO ZERO ZERO ZERO ZERO ZERO ZERO

$0.2517 \mathrm{E}+00$ ZERO ZERO

ZERO
$0.4853 \mathrm{E}-02$ ZERO

$0.1240 \mathrm{E}+00$

$0.3903 \mathrm{E}-03$ ZERO

$0.9857 \mathrm{E}-02$ ZERO

$0.7436 \mathrm{E}-03$ ZERO
$0.8125 \mathrm{E}-02$ ZERO
ZERO ZERO ZERO ZERO ZERO
E+OO

$$
\text { ZERO }
$$$$
\text { ZERO }
$$$$
\text { ZERO }
$$

ZERO

ZERO

ZERO

ZERO

ZERO
$0.1579 E+00$ ZERO

ZERO

ZERO

ZERO

ZERO

ZERO

ZERO

ZERO

ZERO

ZERO

ZERO

ZERO

ZERO

ZERO

ZERO

ZERO

ZERO

ZERO

ZERO

ZERO

ZERO

ZERO

ZERO

ZERO
ZERO

ZERO

ZERO

ZERO

ZERO

ZERO

ZERO

ZERO

ZERO

ZERO

ZERO

ZERO

ZERO

ZERO

ZERO

ZERO

ZERO

ZERO

ZERO

ZERO

ZERO

ZERO

ZERO

ZERO

ZERO

ZERO

$0.4303 E+02$

ZERO

ZERO

ZERO

ZERO

ZERO

ZERO

ZERO

ZERO

ZERO

ZERO

ZERO

ZERO

ZERO

ZERO

ZERO

ZERO

ZERO

ZERO

ZERO

ZERO

ZERO

ZERO

ZERO

ZERO

ZERO

ZERO

ZERO

ZERO

ZERO

ZERO

ZERO

ZERO

ZERO

ZERO

ZERO

ZERO

ZERO

ZERO

ZERO

ZERO

ZERO

ZERO

ZERO

ZERO

ZERO

ZERO

ZERO

ZERO

ZERO

ZERO

ZERO

ZERO

ZERO

ZERO

Date: January 1995
ZERO ZERO

ZERO ZERO ZERO

ZERO ZERO

ZERO

ZERO ZERO ZERO

ZERO ZERO ZERO

ZERO

ZERO ZERO

ZERO ZERO

ZERO ZERO ZERO

ZERO ZERO $\quad$ ZERO

ZERO ZERO ZERO

ZERO ZERO ZERO

ZERO ZERO

ZERO ZERO

ZERO ZERO ZERO

ZERO ZERO

ZERO ZERO

$\begin{array}{lll}\text { ZERO } & \text { ZERO } & \text { ZERO } \\ \text { ZERO } & \text { ZERO }\end{array}$

$\begin{array}{lll}\text { ZERO } & \text { ZERO } \\ \text { ZERO } & \text { ZERO } & \text { ZERO }\end{array}$

$\begin{array}{lll}\text { ZERO } & \text { ZERO } & \text { ZERO } \\ \text { ZERO } & \text { ZERO } & \text { ZERO }\end{array}$

ZERO ZERO Z ZERO

ZERO ZERO

ZERO ZERO

ZERO ZERO

ZERO

ZERO ZERO ZERO

ZERO
ZERO
ZERO

ZERO ZERO

ZERO

ZERO

ZERO

ZERO

ZERO

ZERO

ZERO

ZERO

ZERO

ZERO

ZERO

ZERO

ZERO

$\begin{array}{ll}\text { ZERO } & \text { ZERO } \\ \text { ZERO } & \text { ZERO } \\ \text { ZERO } & \text { ZERO }\end{array}$

section 8, Page 4 of 9

$$
\begin{aligned}
& \text { 6E+01 } \\
& \text { ZERO } \\
& \text { ZERO } \\
& \text { ZEERO } \\
& \text { ZERO } \\
& \text { ZERO } \\
& \text { ZERO } \\
& \text { ZERO } \\
& \text { ZERO } \\
& \text { ZERO } \\
& \text { ZERO } \\
& \text { ZERO } \\
& \text { ZERO } \\
& \text { ZERO } \\
& \text { ZERO } \\
& \text { ZERO }
\end{aligned}
$$

ZERO ZERO $\quad$ ZERO


Table A-1. HLW Flowsheet Material Balance (Tank 51/ITP Cycle 1) Section 8. Canister Decontamination

STREAM NUMBERS (CONT'D) $\rightarrow$ STREAM NAME

$38 \mathrm{SO} 2$

$141 \mathrm{SO} 3$

137 Semi Vol Cs2O

136 Semi Vol $\mathrm{CsCl}$

126 Semi Vol Group A

140 Semi vol Na2B4O

138 Semi Vol NaCl

139 Semi Vol NaE
125 Semi Vol NaI

23 Semi Vol RuO2

$111 \mathrm{SiO} 2$

$48 \mathrm{Sr}(\mathrm{COOH}) 2$

$176 \mathrm{Sr}$ (NO3) 2

$153 \mathrm{Sr}(\mathrm{NaTi2O5)} 2$

$127 \mathrm{Sr}(\mathrm{OH}) 2$

$30 \quad \operatorname{SrCO} 3$

46. $\mathrm{sro}$

$159 \mathrm{TCO} 2$

92 ThO2

107 TiO2

119 Tritium

$\begin{array}{ll}17 & \mathrm{U} 308 \\ 62 \mathrm{UO} 2 \text { (COOH) } 2\end{array}$

177 UO2 (NO3) 2

$16 \mathrm{UO} 2(\mathrm{OH}) 2$

$27 \mathrm{X}(\mathrm{COOH}) 3$

178. $\mathrm{Y}(\mathrm{NO} 3)^{3}$

$128 \mathrm{Y}(\mathrm{OH}) 3$

$31 \mathrm{Y2}(\mathrm{CO} 3) 3$

100 Zeolite

$\begin{aligned} 44 \mathrm{Zn}(\mathrm{COOH}) & 2 \\ 179 \mathrm{Zn}(\mathrm{NO} 3) & 2\end{aligned}$

$57 \mathrm{ZnO}$

$104 \mathrm{zrO} 2$

13. hydrate $\mathrm{H} 2 \mathrm{O}$

1 water

TOTAL FLOW, LB/HR

TEMPERATURE, DEG C

PRESSURE， ATM

PRESSURE, PSIG

PRESSURE, MM HG

ENTHALPY， PCU/HR

VAPOR FLOW, CEM

IAPOR FLOW, CEM

LIQUID FLOW, GPM
DENSITY, LBS / FT3

PHASE
178

179

180

181

182

183

Sldge Blend Frit Slry to Atomzg Air Spray Chambr Oxide from Rinse H2O to Atomzg Air Waste Glass Can Decon to Can Decon Water Rinse Can Cleaning Can Decon to Can Rinse ZERO

ZERO

ZERO

ZERO

ZERO

ZERO

ZERO

ZERO

ZERO

ZTRO

$0.1217 \mathrm{E}+03$

ZERO

ZERO

ZERO

ZERO

ZERO

$0.1519 \mathrm{E}-01$

$0.3534 \mathrm{E}-02$

$0.2943 \mathrm{E}-01$
$0.8253 \mathrm{E}+00$

$0.8253 \mathrm{E}+00$

$0.2063 \mathrm{E}+01$

ZERO

ZERO

ZERO

ZERO

ZERO

TFRO

$0.4187 \mathrm{E}-02$

ZERO

ZERO

ZERO

$0.3128 \mathrm{E}+00$

ZERO

IERO

$0.2280 \mathrm{E}+03$

$0.1050 \mathrm{E}+04$

$0.9840 \mathrm{E}+00$

$-0.2351 \mathrm{E}+00$

$0.7478 \mathrm{E}+03$

$0.7043 \mathrm{E}+05$

$0.1797 \mathrm{E}+00$

$0.1582 \mathrm{E}+03$

LIQUID
ZERO

ZERO

ZERO

ZERO

ZERO

ZERO

ZERO

ZERO

ZERO

ZERO

ZERO

ZERO

ZERO

ZERO

ZERO

ZERO

ZERO

ZERO

ZERO

ZERO

ZERO

ZERO

ZERO

ZERO

ZERO

ZERO

ZERO

ZERO

ZERO

$0.1081 \mathrm{E}+03$

$0.1188 \mathrm{E}+03$

$0.3000 \mathrm{E}+02$

$0.4497 \mathrm{E}+02$

$0.3317 \mathrm{E}+04$

$0.2254 \mathrm{E}+00$

$0.6573 \mathrm{E}+02$

IIQUID
$0.8221 \mathrm{E}+01$
ZERO

ZERO

ZERO

ZERO

ZERO

ZERO

ZERO

ZERO

ZERO

ZERO

ZERO

ZERO

ZERO

ZERO

ZERO

ZERO

ZERO

ZERO

ZERO

ZERO

ZERO

ZERO

ZERO

ZERO

ZERO

ZERO

ZERO

ZERO

ZERO

ZERO

ZERO

$0.3116 \mathrm{E}+01$

$0.1886 \mathrm{E}+03$

$0.3000 \mathrm{E}+02$

$0.6780 \mathrm{E}+01$

$0.8494 \mathrm{E}+02$

$0.3733 \mathrm{E}+04$

$0.6464 \mathrm{E}+01$

$0.4862 \mathrm{E}+00$

VAPOR
ZERO

ZERO ZERO

ZERO ZERO

ZTRO

ZERO

ZERO

ZERO

ZERO
ZERO

ZERO

ZERO

ZERO

ZERO

ZERO

ZERO

ZERO

ZERO

ZERO

ZERO

ZERO

ZERO

ZERO

ZERO

ZERO

ZERO

ZERO

ZERO

ZERO

ZERO

ZERO

ZERO.

ZERO

ZERO

ZERO
$0.1286 \mathrm{E}+02$

$0.1286 \mathrm{E}+02$

$0.3000 \mathrm{E}+02$

$0.4060 \mathrm{E}+01$

$0.4497 \mathrm{E}+02$

$0.9656 \mathrm{E}+00$

$0.3869 \mathrm{E}+03$

$-0.5055 \mathrm{E}+00$

$0.2568 \mathrm{E}-01$

$0.6243 \mathrm{E}+02$

IIQUID

$0.3302 \mathrm{~g}-01$

$0.2017 \mathrm{E}-05$

$0.4189 \mathrm{E}+03$

IIQUID
$0.1849 \mathrm{E}+00$
$0.6243 \mathrm{E}+02$

$0.6243 \mathrm{E}+02$
IIQUID

ZERO

ZERO : ZERO

ZERO ZERO

ZERO ZERO

ZERO ZERO

ZERO

$\begin{array}{ll}\text { ZERO } & \text { ZERO } \\ \text { ZERO } & \text { ZERO }\end{array}$

$\begin{array}{ll}\text { ZERO } & \text { ZERO } \\ \text { ZERO } & \text { ZERO }\end{array}$

ZERO

ZERO ZERO

ZERO

ZERO ZERO

ZERO ZERO

ZERO ZERO

ZERO

ZERO

ZERO ZERO

ZERO ZERO

ZERO ZERO

ZERO

ZERO

ZERO ZERO

ZERO ZERO

ZTRO

ZERO

ZERO

ZFRO ZERO

ZERO ZERO

ZERO ZERO

ZERO

$0.9257 \mathrm{E}+02$

$0.1826 \mathrm{E}+02$

$0.3000 \mathrm{E}+02 \quad 0.3500 \mathrm{E}+02$

$0.4060 \mathrm{E}+01 \quad 0.6780 \mathrm{E}+01$

$0.8494 \mathrm{E}+02$

$0.3830 \mathrm{E}+03$

$0.6362 \mathrm{E}+00$

$0.4784 \mathrm{E}+00$

VAPOR 
STREAM NUMBERS $\rightarrow$

STREAM NAME

COMPONENT FLOWS, IB/HR

122 (C6H5) 2

123 (C6H5) $2 \mathrm{C} 6 \mathrm{H} 4$

118 (C6H5) $2 \mathrm{NH}$

133 (C6H5B (OH) 2 salt)

132 (C6H5B (OH) 2 )

166 (C6H5C6H4OH)

162 (C6H5NH2)

163 (C6H5NHCHO)

165 (C6H5NO)

164 (C6H5OH salt)

130 (C6H5OH)

121 (C6H6)

155 (CH3) $2 \mathrm{CHOH}$

154 (CH3OH)

$45 \mathrm{Ag}$

116 Ag2O

$134 \mathrm{AgNO}$
$77 \mathrm{Al2O3}$

$106 \mathrm{~B} 203$

$9 \mathrm{Ba}(\mathrm{OH}) 2$

$15 \mathrm{BaO}$

$24 \mathrm{BaSO} 4$

$66 \mathrm{CO}$

$\begin{array}{lll}37 & \mathrm{CO} 2\end{array}$

$142 \mathrm{CO} 2$ (C14)

$63 \mathrm{Ca}(\mathrm{COOH}) 2$

167. $\mathrm{Ca}(\mathrm{NO} 3)^{2}$

$120 \mathrm{Ca}(\mathrm{OH}) 2$

$113 \mathrm{Ca} 3$ (PO4) 2

$20 \mathrm{CaC2O4}$

$18 \mathrm{CaCO}^{6}$ (C14)
$64 \mathrm{CaCO}^{2}$ (C14)

$78 \mathrm{CaE} 2$

$41 \mathrm{CaO}$

19 CaSO4

29 Carbon

$26 \mathrm{CO}(\mathrm{COOH}) 2$

$168 \mathrm{Co}(\mathrm{NO}) 2$

$59 \mathrm{COO}$

$98 \quad \mathrm{Cr} 203$

$42 \mathrm{Cs} 2 \mathrm{O}$

$53 \mathrm{CsCOOH}$

$96 \mathrm{CsCl}$

169 CsNO3

$8 \mathrm{CsOH}$

151 CsTPB

WSRC-TR-95-0019 (Revision

Table A-1. HLW Flowsheet Material Balance (Tank 51/ITP Cyole 1)

Section 8. Canister Decontamination

184

FCT Air

Inleakage

185

Vent Decon Frit

ZERO ZERO $\quad$ ZERO

ZERO $\quad$ ZERO ZERO

ZERO ZERO ZERO

ZERO : ZERO ZERO

ZERO ZERO ZERO

ZERO ZERO ZERO

ZERO ZERO ZERO

ZERO ZERO $\quad$ ZERO

ZERO ZERO $\quad$ ZERO

ZERO ZERO $\quad$ ZERO

ZERO ZERO : ZERO

ZERO ZERO ZERO

ZERO ZERO ZERO

ZERO ZERO Z ZERO

ZERO ZERO ZERO

ZERO ZERO ZERO

ZERO ZERO ZERO

ZERO ZERO

ZERO $\quad 0.2284 \mathrm{E}-06 \quad 0.8542 \mathrm{E}+00$

ZERO ZERO ZERO

ZERO ZERO ZERO

ZERO ZERO ZERO

ZERO Z ZERO ZERO

ZERO ZERO ZERO

ZERO ZERO ZERO

ZERO ZERO ZERO

ZERO ZERO

ZRRO

ZERO

ZERO

ZERO ZERO $\because$ ZERO

ZERO ZERO $\quad$ ZERO

ZERO ZERO $\quad$ ZERO

ZERO $\cdots$ ZERO ZERO

ZERO ZERO $\quad$ ZERO

ZERO ZERO

ZERO ZERO

ZERO ZERO ZERO

ZERO ZERO $\quad$ ZERO

ZERO
ZERO

$0.1254 \mathrm{E}-02$

ZERO ZERO

$\begin{array}{lll}\text { ZERO } & \text { ZERO } \\ \text { ZERO } & \text { ZERO } & \text { ZERO }\end{array}$

ZERO ZERO ZERO

ZERO ZERO ZERO

ZERO

ZERO

ZERO

Date: January 1995 
Table A-1. HLW Flowsheet Material Balance (Tank 51/ITP Cycle 1) Section 8 . Canister Decontamination

STREAM NUMBERS (CONT'D) $\rightarrow$ STREAM NAME

$54 \mathrm{Cu}(\mathrm{COOH}) 2$

$55 \mathrm{CuO}$

80 Fe203

$101 \mathrm{~F} e \mathrm{O}$

102 Group A

103 Group

$25 \mathrm{H2}$

158 H $2 \mathrm{C} 2 \mathrm{O} 4$

93 H2SO4

$90 \mathrm{H} 3 \mathrm{BO}$

34 HCOOH

$43 \mathrm{HCl}$

$85 \mathrm{HF}$

49 HNO3

$36 \mathrm{Hg}$

$129 \mathrm{Hg}(\mathrm{C} 6 \mathrm{H} 5) 2$

$135 \mathrm{Hg}$ (NO3) 2

$144 \mathrm{Hg} 2 \mathrm{Cl} 2$

$1.17 \mathrm{Hg} 2 \mathrm{I} 2$

21 HgO

97 I2

$183 \mathrm{~K} 2 \mathrm{CO} 3$

$39 \mathrm{~K} 2 \mathrm{O}$

$51 \mathrm{KCOOH}$

$157 \mathrm{KMnO4}$

$161 \mathrm{KNO}$
$10 \mathrm{KOH}$

$150 \mathrm{TPPB}$

$150 \mathrm{KTPB}$

$\begin{array}{ll}105 & \mathrm{La} 203 \\ 108 & \mathrm{Li} 20\end{array}$

$65 \mathrm{Mg}$ (COOH) 2

$171 \mathrm{Mg}$ (NO3) 2

$33 \mathrm{MgO}$

$60 \mathrm{Mn}(\mathrm{COOH}) 2$

$172 \mathrm{Mn}$ (NO3) 2

$180 \mathrm{Mn} 304$

$56 \mathrm{MnO}$

$14 \quad \mathrm{MnO} 2$

$145 \mathrm{MoO2}$

81 N2

74 N2O

182 NH3

114 NH3OHNO3

$52 \mathrm{NH} 4 \mathrm{COOH}$

173 NH4NO3

68 NH4OH
148 NH 4 TPB
184

FCT Air

Inleakag

ZERO

ZERO

ZERO

ZERO

ZERO

ZERO

ERO

ZERO

ERO

ZERO

ZERO

ZERO

ZERO

ZERO

ZERO

ZERO

ZERO

ZERO

ERO

ZERO

ZERO

ZERO

EERO

ZERO

ZERO

ZERO

ZERO

ZERO

ZERO

ZERO

FRO

ZERO

ZERO

ZERO

ERO

ZERO

ERO

ERO

ERO

$0.3607 \mathrm{E}+03$

ZERO

ZERO

ZERO

ZERO

ZERO

ZERO
185

CT Vent Decon

o PVVS Slrry to SME

ZERO ZERO

ZERO

$0.1305 \mathrm{E}-09 \quad 0.4880 \mathrm{E}-03$

$$
\text { ZERO ZERO }
$$

ZERO

ZERO

ZERO ZERO

ZERO $\quad$ ZERO

ZERO ZERO

ZERO ZERO

ZERO ZERO

ZERO ZERO

ZERO ZERO

ZERO ZERO

ZERO ZERO

ZTRO

ZERO

ZERO ZERO

ZERO ZERO

2ERO ZERO

ZERO ZERO

ZERO ZERO

ZERO ZERO

ZERO ZERO

ZERO ZERO

ZERO ZERO

0.7474 ZERO

ZERO $0.7474 \mathrm{E}$

ZERO

ZERO

ZERO

ZERO

ZERO

ZERO

$0.5169 \mathrm{E}+03 \quad 0.3223 \mathrm{E}-02$

ZRRO

ZERO ZERO

ZERO

ZERO ZERO

ZERO ZERO

ZERO

ZERO , ZERO 
Table A-1. HLW Flowsheet Material Balance (Tank 51/ITP Cyole 1) Section 8. Canister Decontamination

STREAM NUMBERS (CONT'D) $\rightarrow$ STREAM NAME

\begin{tabular}{|c|c|}
\hline 35 & \\
\hline 84 & $\mathrm{NO} 2$ \\
\hline 70 & $\mathrm{Na}(\mathrm{HgO}(\mathrm{OH}))$ \\
\hline 94 & $\mathrm{Na} 2 \mathrm{~B} 407$ \\
\hline 109 & $\mathrm{Na} 2 \mathrm{C} 2 \mathrm{O} 4$ \\
\hline & $\mathrm{Na} 2 \mathrm{CO} 3$ \\
\hline 143 & $\mathrm{Na} 2 \mathrm{CO} 3$ (C14) \\
\hline 89 & $\mathrm{Na2CrO4}$ \\
\hline 115 & $\mathrm{Na} 2 \mathrm{MoO} 4$ \\
\hline 32 & $\mathrm{Na2O}$ \\
\hline $\begin{array}{l}71 \\
73\end{array}$ & $\begin{array}{l}\mathrm{Na} 2 \mathrm{PuO} 2(\mathrm{OH}) 4 \\
\mathrm{Na} 2 \mathrm{RhO}^{4}\end{array}$ \\
\hline 72 & $\mathrm{Na} 2 \mathrm{RuO} 4$ \\
\hline & $\mathrm{Na} 2 \mathrm{SO} 4$ \\
\hline 88 & $\mathrm{Na2SiO} 3$ \\
\hline 87 & $\mathrm{Na3PO} 4$ \\
\hline 91 & $\mathrm{NaAg}(\mathrm{OH}) 2$ \\
\hline 75 & $\operatorname{NaAl}(\mathrm{OH}) 4$ \\
\hline 50 & $\mathrm{NaCOOH}$ \\
\hline & $\mathrm{NaCl}$ \\
\hline 69 & $\mathrm{NaF}$ \\
\hline 11 & $\mathrm{NaHCO} 3$ \\
\hline 12 & NaI \\
\hline 3 & NaNO2 \\
\hline 2 & NaNO3. \\
\hline & $\mathrm{NaOH}$ \\
\hline 149 & NaTPB \\
\hline 160 & $\mathrm{NaTcO} 4$ \\
\hline 152 & NaTi205H \\
\hline 181 & $\mathrm{Nd2O} 3$ \\
\hline .61 & $\mathrm{Ni}(\mathrm{COOH}) 2$ \\
\hline 174 & $\mathrm{Ni}(\mathrm{NO} 3) 2$ \\
\hline 40 & $\mathrm{NiO}$ \\
\hline 82 & $\mathrm{O} 2$ \\
\hline 175 & $\mathrm{~Pb}(\mathrm{NO} 3) 2$ \\
\hline 86 & $\mathrm{PbCO} 3$ \\
\hline 67 & $\mathrm{Pbo}$ \\
\hline 147 & $\mathrm{PbS}$ \\
\hline 28 & $\mathrm{PbSO} 4$ \\
\hline 124 & Pd \\
\hline 112 & $\mathrm{Pd}(\mathrm{NO} 3) 2$ \\
\hline 110 & Pdo \\
\hline .76 & PuO2 \\
\hline 156 & $\mathrm{PuO} 2(\mathrm{NaTi} 205) / 2$ \\
\hline 58 & $\mathrm{Rh}$ \\
\hline 22 & RhO2 \\
\hline & Ru \\
\hline$y$ & RuO2 \\
\hline & RuOA \\
\hline
\end{tabular}

WSRC-TR-95-0019 (Revision
184

FCT Air

Inleakage

ZERO

ZERO

ZERO

ZERO

ZERO

ZERO

ZERO

ZERO

ZERO

ZERO

ZERO

ZERO

ZERO

ZERO

ZERO

ZERO

ZERO

ZERO

ZERO

ZERO

ZERO

ZERO

ZERO

ZERO

ZERO

ZERO

ZERO

ZERO

ZERO

$0.1090 \mathrm{E}+03$

ZERO

ZERO

ZERO

ZERO

ZERO

ZERO

ZERO

ZERO

ZERO

ZERO

ZERO

ZERO

ZERO

0)
185 Vent 26

to PVVS Sl rry to SME

PURO Slrry to SME

$\begin{array}{ll}\text { ZERO } & \text { ZERO } \\ \text { ZERO } & \text { ZERO }\end{array}$

ZERO $\quad$ ZERO

ZERO

ZERO

$\begin{array}{ll}\text { ZERO } & \text { ZERO } \\ \text { ZERO } & \text { ZERO }\end{array}$

ZERO ZERO

ZERO ZERO

$\begin{array}{rr}\text { ZERO } \\ 0.1521 \mathrm{E}-06 & 0.5687 \mathrm{E}+00\end{array}$

ZERO

ZERO ZERO

ZERO ZERO

ZERO ZERO

ZERO . ZERO

ZERO ZERO

ZERO ZERO

ZERO

$0.1579 \mathrm{E}+00$

ZERO : ZERO

ZERO ZERO

ZERO ZERO

ZERO ZERO

ZERO $\because$ ZERO

ZERO ZERO

ZERO ZERO

ZERO ZERO

ZERO ZERO

ZERO

ZERO ZERO

ZERO ZERO

$0.1347 \mathrm{E}-08 \quad 0.5036 \mathrm{E}-02$

$0.1562 \mathrm{E}+03 \quad 0.1916 \mathrm{E}-02$

ZERO ZERO

ZERO ZERO

ZERO ZERO

ZERO $\because$ ZERO

ZERO

ZERO

ZERO

ZERO

ZERO ZERO

ZERO ZERO

ZERO ZERO

$\begin{array}{ll}\text { ZERO } & \text { ZERO } \\ \text { ZERO } & \text { ZERO }\end{array}$

ZERO ZERO

ZERO ZERO

Date: January 1995 
Table A-1. HLW Flowsheet Material Balance (Tank 51/ITP Cycle 1)

STREAM NUMBERS (CONT'D) $\rightarrow$ STREAM NAME

\section{$38 \quad 502$}

141503

137 Semi Vol cs2o

136 Semi Vol CsCl

126 Semi Vol Group A

140 Semi Vol Na2B4O
138 Semi Vol NaCl.

138 Semi Vol NaCl

139 Semi Vol NaI

23 Semi Vol RuO2

111. SiO2

$48 \mathrm{Sr}(\mathrm{COOH}) 2$

$176 \mathrm{Sr}$ (NO3) 2

153 Sr (NaTi2O5) 2

$127 \mathrm{sr}(\mathrm{OH}) 2$

$30 \mathrm{SrCO} 3$

46 Sro

$159 \mathrm{TcO} 2$

92 ThO2

107 TiO2

119 Tritium

17 v308

$62 \mathrm{OO} 2(\mathrm{COOH}) 2$

177 UO2 (NO3) 2

$16 \mathrm{UO} 2(\mathrm{OH}) 2$

$27 \mathrm{Y}(\mathrm{COOH})$

$178 \mathrm{Y}$ (NO3)

$\begin{array}{rl}128 & \mathrm{Y}(\mathrm{OH}) 3 \\ 31 & \mathrm{Y} 2(\mathrm{CO} 3)\end{array}$

$31 \mathrm{Y} 2(\mathrm{CO})$
$47 \mathrm{Y} 2 \mathrm{O}$

47 Y203
100 Zeolite

$44 \mathrm{Zn}(\mathrm{COOH}) 2$

$179 \mathrm{zn}(\mathrm{NO}) 2$

57 Zno

13 hydrate $\mathrm{H} 2 \mathrm{O}$

$$
1 \text { water }
$$

TOTAL FLOW, IB/HR

TEMPERATURE， DEG C

PRESSURE, ATM

PRESSURE, PSIG

PRESSURE, MM HG

ENTHALPY， PCU/HR

VAPOR FLOW, CEM

LIQUID FLOW, GPM

DENSITY, IBS/FT3

DHASE

\begin{tabular}{|c|c|c|c|}
\hline 184 & 185 & & 26 \\
\hline $\begin{array}{r}\text { FCT Air } \\
\text { Inleakace }\end{array}$ & $\begin{array}{r}\mathrm{FCT} \\
\text { to }\end{array}$ & $\begin{array}{l}\text { Vent } \\
\text { PVVS }\end{array}$ & $\begin{array}{l}\text { Decon Frit } \\
\text { slrry to SME }\end{array}$ \\
\hline ZERO & & ZERO & ZERO \\
\hline ZERO & & ZERO & ZERO \\
\hline ZERO & & ZERO & ZERO \\
\hline ZERO & & ZERO & ZERO \\
\hline ZERO & & ZERO & ZERO \\
\hline ZERO & . & ZERO & ZERO \\
\hline ZERO & & ZERO & ZERO \\
\hline ZERO & & ZERO & ZERO \\
\hline ZERO & & ZERO & ZERO \\
\hline ZERO & & ZERO & ZERO \\
\hline ZERO & 0.2198 & $8 E-05$ & $0.8221 \mathrm{E}+01$ \\
\hline ZERO & & ZERO & ZERO \\
\hline ZERO & & ZERO & ZERO \\
\hline ZERO & & ZERO & ZERO \\
\hline ZERO & & ZERO & ZERO \\
\hline ZERO & & ZERO & ZERO \\
\hline ZERO & & ZERO & ZERO \\
\hline ZERO & & ZERO & ZERO \\
\hline ZERO & & ZERO & ZERO \\
\hline ZERO & & ZERO & ZERO \\
\hline ZERO & & ZERO & ZERO \\
\hline ZERO & & ZERO & ZERO \\
\hline ZERO & & ZERO & ZERO \\
\hline ZERO & & ZERO & ZERO \\
\hline ZERO & & ZERO & ZERO \\
\hline ZERO & & ZERO & ZERO \\
\hline ZERO & & ZERO & ZERO \\
\hline ZERO & & ZERO & ZERO \\
\hline ZERO & & ZERO & ZERO \\
\hline ZERO & & ZERO & ZERO \\
\hline ZERO & & ZERO & ZERO \\
\hline ZERO & & ZERO & ZERO \\
\hline ZERO & & ZERO & ZERO \\
\hline ZERO & & ZERO & ZERO \\
\hline ZERO & & ZERO & ZERO \\
\hline ZERO & & ZERO & ZERO \\
\hline $\begin{array}{l}0.7891 \mathrm{E}+01 \\
0.4776 \mathrm{E}+03\end{array}$ & $\begin{array}{l}0.1834 \\
0.6914\end{array}$ & $\begin{array}{l}4 \mathrm{E}+02 \\
4 \mathrm{E}+03\end{array}$ & $\begin{array}{l}0.2152 \mathrm{E}+03 \\
0.2260 \mathrm{E}+03\end{array}$ \\
\hline $\begin{array}{l}0.3500 \mathrm{E}+02 \\
0.1000 \mathrm{E}+01\end{array}$ & $\begin{array}{l}0.3000 \\
0.9656\end{array}$ & $\begin{array}{l}\mathrm{OE}+02 \\
6 \mathrm{E}+00\end{array}$ & $\begin{array}{l}0.2980 \mathrm{E}+02 \\
0.9656 \mathrm{E}+00\end{array}$ \\
\hline ZERO & $\begin{array}{r}-0.5055 \\
0.7330\end{array}$ & $\begin{array}{l}5 \mathrm{E}+00 \\
9 \mathrm{E}+03\end{array}$ & $\begin{array}{r}-0.5055 \mathrm{E}+00 \\
0.7339 \mathrm{E}+03\end{array}$ \\
\hline $\begin{array}{l}0.1002 \mathrm{E}+05 \\
0.1128 \mathrm{E}+03\end{array}$ & $\begin{array}{l}0.1781 \\
0.1674\end{array}$ & $\begin{array}{l}1 \mathrm{E}+05 \\
4 \mathrm{E}+03\end{array}$ & $0.6497 \mathrm{E}+04$ \\
\hline $\begin{array}{r}7055 E-01 \\
\text { VAPOR }\end{array}$ & 0.6884 & $\begin{array}{l}4 E-01 \\
\text { VAPOR }\end{array}$ & $\begin{array}{r}0.4394 \mathrm{E}+00 \\
0.6412 \mathrm{E}+02 \\
\text { IIQUID }\end{array}$ \\
\hline
\end{tabular}




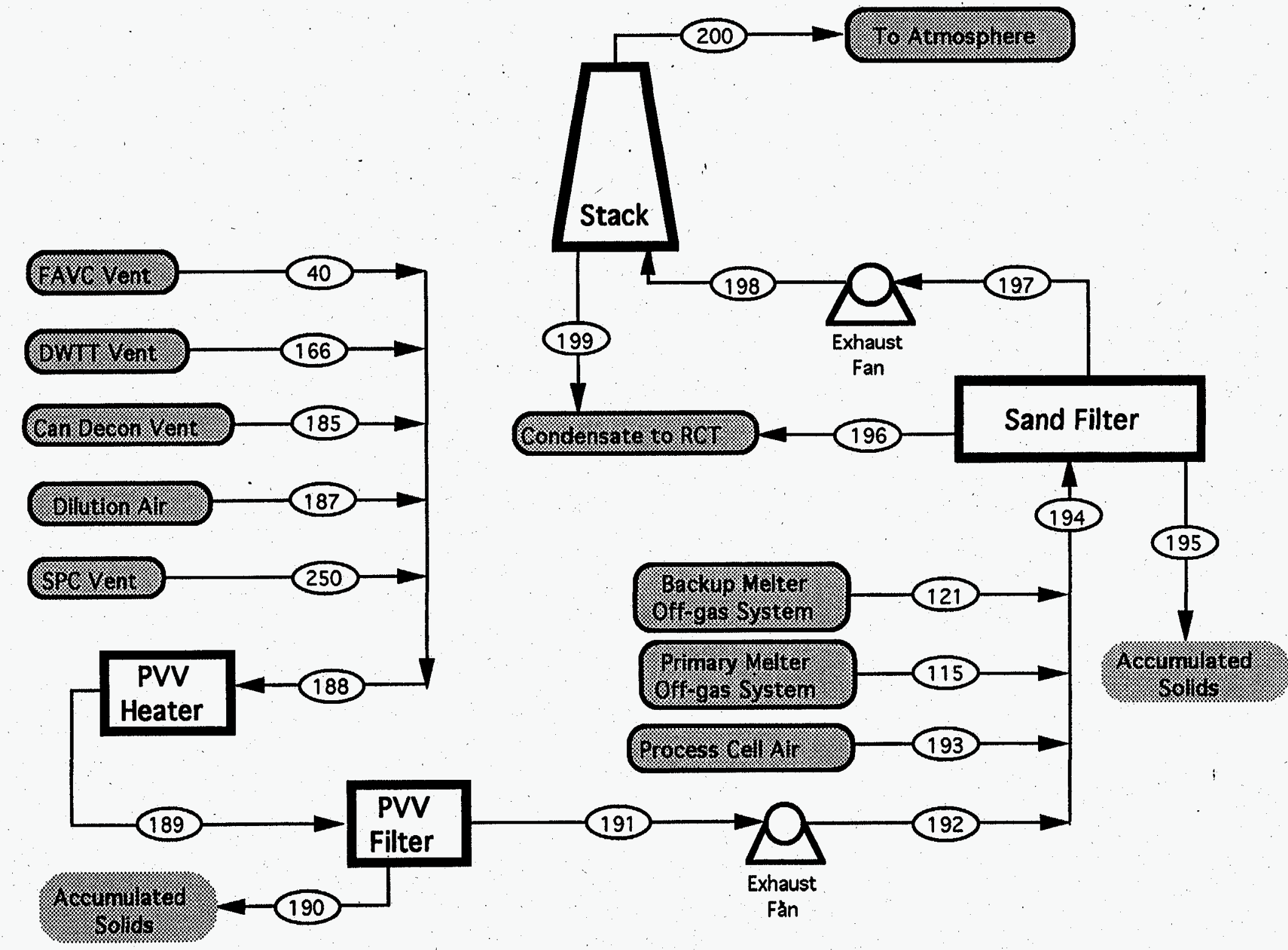

SECTION 9. COLLECTION OF CELL VENTS AND SAND FILTER 


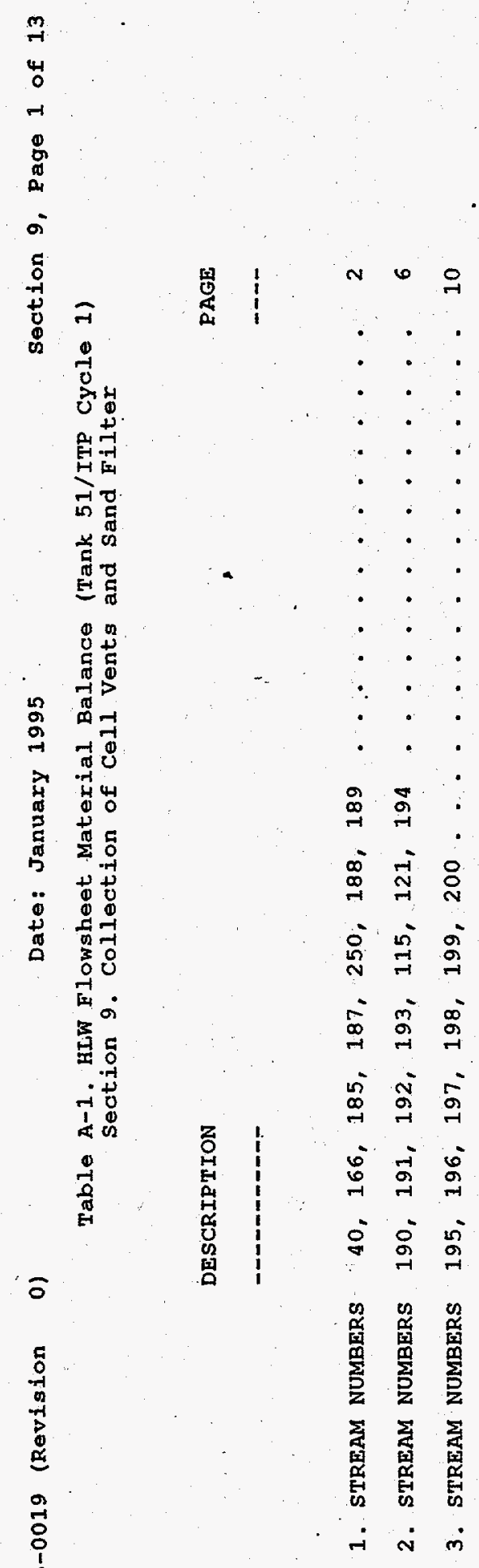


Table A-1. HLW Flowsheet Material Balance (Tank 51/ITP Cycle i) section 9. Collection of Cell Vents and Sand Filter

STREAM NUMBERS $\rightarrow$ STREAM NAME

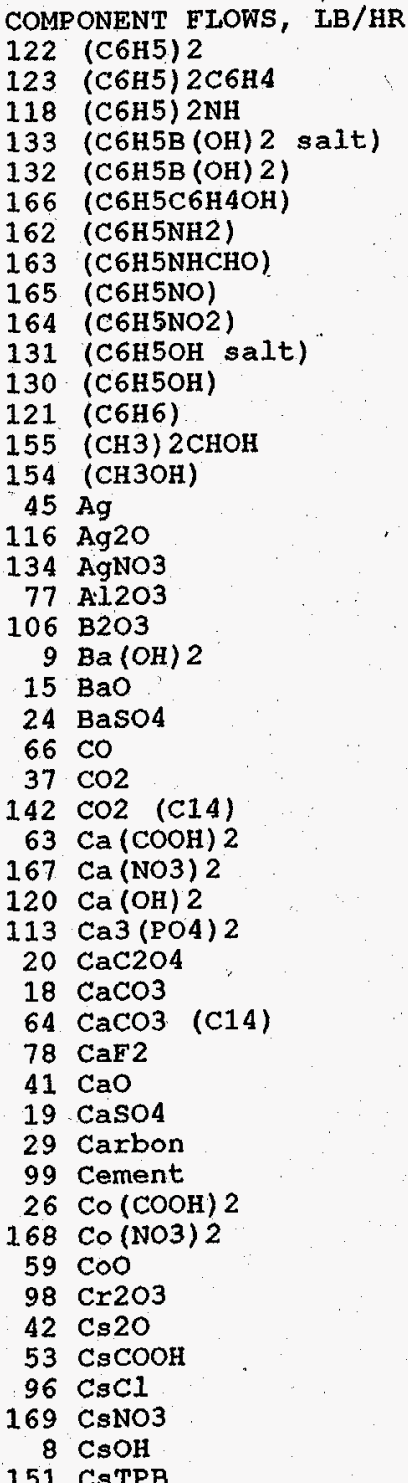

WSRC-TR-95-0019 (Revision

40

FAVC HEM Vnt to PVVs

$0.1019 \mathrm{E}-01$

$0.3515 \mathrm{E}-10$

$0.7563 \mathrm{E}-04$

ZERO

ZERO

ZERO

ZERO

ZERO
$3 E-19$

$0.1138 \mathrm{E}-01$

$0.1406 \mathrm{E}-01$

$0.8066 \mathrm{E}-11$

$0.2173 \mathrm{E}-11$

$0.1404 \mathrm{E}-18$

$$
\text { TRACE }
$$

$0.3136 \mathrm{E}-14$

$0.1137 \mathrm{E}-14$

ZERO
$0.1190 \mathrm{E}-23$

$0.6048 \mathrm{E}-17$

$0.4331 \mathrm{E}+01$

$0.5680 \mathrm{E}-07$

$$
\text { ZERO }
$$

ZERO

$0.9318 \mathrm{E}-15$

$0.8649 \mathrm{E}-22$ ZERO

ZERO
$0.5328 \mathrm{E}-17$

$0.1189 \mathrm{E}-17$

$0.7822 \mathrm{E}-16$

ZERO

ZERO
$0.507 .4 \mathrm{E}-19$

ZERO

$0.1087 \mathrm{E}-19$

$0.1522 \mathrm{E}-15$

$0.4980 \mathrm{E}-19$

$0.1534 \mathrm{E}-16$ ZERO

$0.3890 \mathrm{E}-21$ ZERO 166
DWTC Vent
to PVVS

$0.5665 \mathrm{E}-14$

$0.5369 \mathrm{E}-20$

$0.7438 \mathrm{E}-16$

ZERO

- 1579

$0.1579 \mathrm{E}-19$

$0.3818 \mathrm{E}-19$

$0.4894 \mathrm{E}-17$

$0.6601 \mathrm{E}-19$

$0.7293 \mathrm{E}-14$ ZERO

$0.2240 \mathrm{E}-15$

$0.7726 \mathrm{E}-20$

$0.8037 \mathrm{E}-21$

$0.1835 \mathrm{E}-13$

ZERO

$0.4475 \mathrm{E}-18$

$0.1622 \mathrm{E}-18$

ZERO
TRACE

$0.8629 \mathrm{E}-21$

ZERO

$0.4269 \mathrm{E}+00$

TRACE

$0.2122 \mathrm{E}-18$

ZERO

TRACE

ZERO

ZERO
$0.7603 \mathrm{E}-21$

$0.1696 \mathrm{E}-21$

$0.1116 \mathrm{E}-19$

ZERO

ZERO

TRACE

$0.2171 \mathrm{E}-19$

$0.2171 \mathrm{E}-19$

$0.7106 \mathrm{E}-23$
ZERO ZERO

$0.4867 \mathrm{E}-24$

$0.1844 \mathrm{E}-20$
185
FCT Vent Dil Air

to pvvs Cil Alr frm

ZERO I ZERO

ZERO

ZERO

ZERO

ZERO

ZERO

ZERO

ZERO

ZERO

ZERO

ZERO

ZERO

ZERO

ZERO

ZERO

ZERO

ZERO

$0.2284 \mathrm{E}-06$

ZERO

ZERO

ZERO

ZERO

ZERO

ZERO

ZERO

ZERO

ZERO

ZERO

ZERO

ZERO

ZERO

ZERO

ZERO

ZERO

ZERO

ZERO

$0.3353 \mathrm{E}-09$

ZTRO

ZERO

ZERO

ZERO

ZERO

ZERO

Date: January 1995

ZERO
ZERO
ZERO
ZERO
ZERO
ZERO
ZERO
ZERO
ZERO
ZERO
ZERO
ZERO
ZERO
ZERO
ZERO
ZERO
ZERO
ZERO
ZERO
ZERO
ZERO
ZERO
ZERO
ZERO
ZERO
ZERO
ZERO
ZERO
ZERO
ZERO
ZERO
ZERO
ZERO
ZERO
ZERO
ZERO
ZERO
ZERO
ZERO
ZERO
ZERO
ZERO
ZERO
ZERO
ZERO
ZERO
ZERO
ZERO

250 Vnt to PVVS

$0.1905 \mathrm{E}-01$

$0.1740 \mathrm{E}-15$

$0.5247 \mathrm{E}-10$

ZERO

ZERO

ZERO

ZERO

ZERO

ZERO

ZERO

$0.361 .2 \mathrm{E}-04$

$0.9318 \mathrm{E}+0.1$

$0.7606 \mathrm{E}-12$

$0.2677 \mathrm{E}-12$

ZERO

ZERO

ZERO

ZERO

ZERO

ZERO

ZERO

ZERO

$0.6810 \mathrm{E}+02$

$0.1203 E-09$

ZERO

ZERO

ZERO

ZERO

ZERO

ZERO

ZERO

ZERO

ZERO

ZERO

ZERO

ZERO

ZERO

ZERO

ZERO

ZERO

ZERO

ZERO

ZERO

ZERO

ZERO
188

to PVVH
to

$0.2924 \mathrm{E}-01$

$0.3515 \mathrm{E}-10$

$0.7563 \mathrm{E}-04$

ZERO

ZERO

ZERO

ZERO

ZERO

ZERO

$0.6223 \mathrm{E}-19$

$0.1142 \mathrm{E}-01$

$0.9332 \mathrm{E}+01$

$0.8827 \mathrm{E}-11$

$0.1404 \mathrm{E}-18$

TRACE

$0.3136 \mathrm{E}-14$

$0.3949 \mathrm{E}-06$

ZERO

$0.6048 \mathrm{E}-17$

ZERO
$0.7243 \mathrm{E}+02$

$0.5692 \mathrm{E}-07$

ZERO

ZERO

$0.9318 \mathrm{E}-15$

$0.8649 \mathrm{E}-22$

ZERO

ZERO

$.1189 \mathrm{E}-17$

$0.7822 \mathrm{E}-16$ ZERO

$0.5074 \mathrm{E}-1$ ZERO

$0.1087 \mathrm{E}-19$

$0.4982 E-09$

$0.4980 \mathrm{E}-19$ ZERO

section ZERO
189

PVVH Exit Gas

$0.2924 \mathrm{E}-01$

$0.3515 E-10$

$0.7563 \mathrm{E}-04$ ZERO ZERO ZERO ZERO ZERO ZERO

$0.6223 \mathrm{E}-19$

$0.1142 \mathrm{E}-01$

$0.9332 \mathrm{E}+01$

$0.8827 \mathrm{E}-11$

$0.2441 \mathrm{E}-11$

$$
\text { TRACE }
$$

$0.3136 \mathrm{E}-14$

ZERO

$0.1190 \mathrm{E}-23$

$0.6048 \mathrm{E}-17$

$0.7243 \mathrm{E}+02$

$0.5692 \mathrm{E}-07$ ZERO ZERO

$0.9318 \mathrm{E}-15$

$0.8649 \mathrm{E}-22$ ZERO

ZERO

$0.5328 \mathrm{E}-17$

$0.1189 \mathrm{E}-17$
$0.7822 \mathrm{E}-16$

$$
\text { ZERO }
$$

$0.5074 \mathrm{E}-19$

ZERO

$0.1087 \mathrm{E}-19$

$0.1534 \mathrm{E}-16$ ZERO

$0.3890 \mathrm{E}-21$

ZERO 
Table A-1. HLW Flowsheet Material Balance (Tank 51/ITP Cycle 1) Section 9. Collection of Cell Vents and Sand Filter

STREAM NUMBERS (CONT'D) $\rightarrow$ STREAM NAME

$54 \mathrm{Cu}(\mathrm{COOH}) 2$

$170 \mathrm{Cu}(\mathrm{NO} 3) 2$

$55 \mathrm{CuO}$

$80 \mathrm{Fe2O}$

102 Group A

103 Group B

$25 \mathrm{H} 2$

158. $\mathrm{H} 2 \mathrm{C} 2 \mathrm{O} 4$

$93 \mathrm{H} 2 \mathrm{SO} 4$

$90 \mathrm{H} 3 \mathrm{BO} 3$

$34 \mathrm{HCOOH}$

$43 \mathrm{HCl}$

$85 \mathrm{HF}$

49 HNO3

$36 \mathrm{Hg}$

$129 \mathrm{Hg}(\mathrm{C} 6 \mathrm{H} 5) 2$

$135 \mathrm{Hg}$ (NO3) 2

$144 \mathrm{Hg} 2 \mathrm{Cl2}$

$117 \mathrm{Hg} 2 \mathrm{I2}$

$146 \mathrm{HgCl}$

21 Ho

$183 \mathrm{~K} 2 \mathrm{CO} 3$

$39 \mathrm{~K} 2 \mathrm{O}$

$51 \mathrm{KCOOH}$

$157 \mathrm{KMnO}$

$161 \mathrm{KNO} 3$

$10 \mathrm{KOH}$

$105 \mathrm{La203}$

$108 \mathrm{Li2O}$

$65 \mathrm{Mg}(\mathrm{COOH}) 2$

$171 \mathrm{Mg}$ (NO3) 2

$33 \mathrm{MgO}$

$60 \mathrm{Mn}(\mathrm{COOH}) 2$

$172 \mathrm{Mn}$ (NO3) 2

$180 \mathrm{Mn} 304$

$56 \mathrm{MnO}$

$14 \mathrm{MnO} 2$

$145 \mathrm{MoO} 2$

81 N2

$74 \mathrm{~N} 2 \mathrm{O}$
$182 \mathrm{NH} 3$

182 NH3

114 NH3OHNO3

$52 \mathrm{NH} 4 \mathrm{COOH}$

173 NH4NO3

148 NH4TPB
FAVC HEME Vnt to PVVS

$0.1573 \mathrm{E}-1.7$

ZERO

$0.6992 \mathrm{E}-1$

$0.9013 \mathrm{E}-14$

$0.912 \mathrm{E}-15$

$0.5367 \mathrm{E}-19$

$0.3136 \mathrm{E}-01$

$0.6486 \mathrm{E}-16$

$0.2730 \mathrm{E}-14$

ZERO

ZERO

ZERO

$0.8193 E-02$

तथRO

ZERO

ZERO

ZTRO

$0.6994 \mathrm{E}-21$

ZERO

$0.2866 \mathrm{E}-16$

$0.3558 \mathrm{E}-14$

ZERO

$0.1015 \mathrm{E}-16$

ZERO

ZERO

$0.9942 \mathrm{E}-15$

$0.1230 \mathrm{E}-14$

ZERO

$0.3923 \mathrm{E}-15$

ZERO

$0.2818 \mathrm{E}-20$

$0.6916 \mathrm{E}-15$

$0.3817 \mathrm{E}-18$

$0.1330 \mathrm{E}+04$

$0.1420 \mathrm{E}-19$

$0.1076 \mathrm{E}-02$

ZERO.
ZERO

ZERO

TRAC

ZERO
DWTC Vent

to PVVS

ZERO

$0.1114 \mathrm{E}-20$

$0.1286 \mathrm{E}-17$

$0.1795 E-21$

$0.7658 \mathrm{E}-23$

TRACE

ZERO

ZERO

ZERO

ZERO

ZERO

ZERO

ZERO

ZERO

ZERO

ZERO

ERO

$0.8348 \mathrm{E}-12$ ZERO

$0.4090 \mathrm{E}-20$

$$
\text { ZERO }
$$

ZERO

$0.1448 \mathrm{E}-20$

$0.2265 \mathrm{E}-07$
ZERO

- 1419 ERO

$0.1419 \mathrm{E}-18$ ZERO

$0.1178 \mathrm{E}-18$ ZERO TRACE

$0.5376 \mathrm{E}-19$

$0.3510 \mathrm{E}-07$

$0.5446 \mathrm{E}-22$

$0.5483 \mathrm{E}+02$

ZERO
$0.9544 \mathrm{E}-16$ ZERO
ZERO

$0.1362 \mathrm{E}-13$

$0.1569 \mathrm{E}-20$

ZERO
185 187

PVVS Crane Decon

ZERO C DERO

ZERO

ZERO

ZERO

ZERO

ZERO

ZERO

ZERO

ZERO

ZERO

ZERO

ZERO

ZERO

ZERO

ZERO

ZERO

ZERO

ZERO

ZERO

ZERO

ZERO

ZERO

ZERO

ZERO

ZERO

ZERO

ZERO

ZERO

ZERO

$0.1998 \mathrm{E}-06$

ZERO

ZERO
$0.5710 \mathrm{E}-07$

ZERO

ZERO

ZERO

ZERO

ZERO

ZERO

ZERO

ZERO

ZERO

ZERO

ZERO
250 SCVC HEME ZERO :

ZERO

ZERO

ZERO

ZERO

ZERO

ZERO

ZERO

ZERO

ZERO

ZERO

ZERO

ZERO

ZERO

ZERO

ZERO

ZERO

ZERO

ZERO

ZERO

ZERO

ZERO

ZERO

ZERO

ERO

ZERO

ZERO

ZERO

ZERO

ZERO

ZERO

ZERO

ZERO

ZERO

ZERO

ZERO

ZERO

ZFRO

ZERO

ZERO

ZERO

ZERO

ZERO

ZERO

ZERO

ZERO

ZERO

ZERO

ZERO

$0.7581 \mathrm{E}-11$

ZERO

ZERO

ZERO

$0.7601 \mathrm{E}-04$

ZERO

ZERO

$0.4004 \mathrm{E}-14$

ZERO

ZERO

ZERO

ZERO

ZERO

ZERO

ZERO

ZERO

ZERO

ZERO

ZERO

ZERO

ZERO

ZERO

ZERO

ZERO

ZERO

ZERO

ZERO

ZERO

ZERO

ZERO

ZERO

ZERO

$0.4532 \mathrm{E}+01$

$0.1070 \mathrm{E}-09$

$0.1723 E-05$

ZERO
ZERO

ZERO

ZERO

ZERO

ZERO
Tot Vs 1 Vnt

to PVVH 
Table A-1. HLW Flowsheet Material Balanoe (Tank 51/ITP Cyole 1) section 9. Collection of Cell vents and Sand Filter

STREAM NUMBERS (CONT'D) $\rightarrow$ STREAM NAME

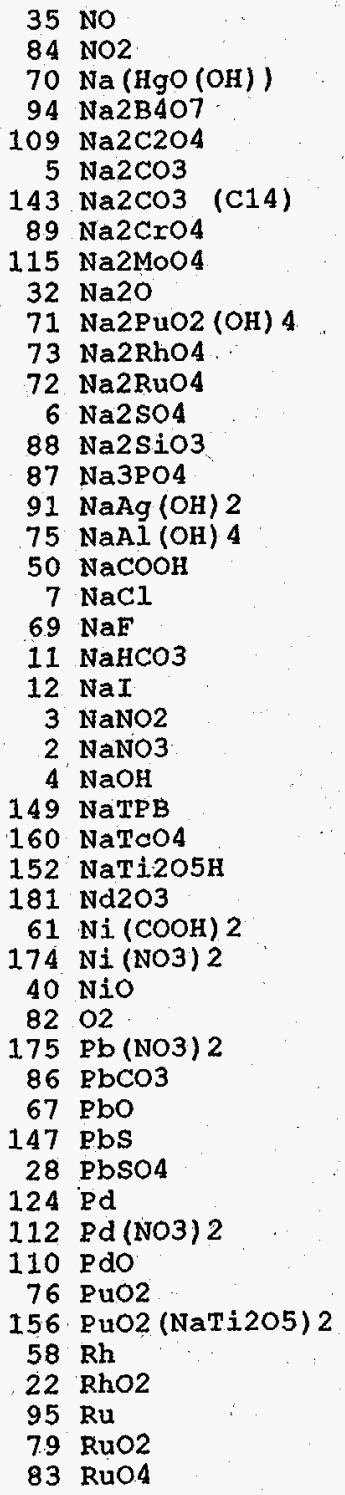

WSRC-TR-95-0019 (Revision 4

FAVC 166 Vnt to PVVS ZERO
$0.2184 \mathrm{E}+01$ $0.1304 \mathrm{E}-19$ $0.8409 \mathrm{E}-18$ $0.1738 \mathrm{E}-14$ ZERO
ZERO ZERO

$0.2259 \mathrm{E}-14$ ZERO ZERO

$0.6930 \mathrm{E}-16$

ZERO

$0.9140 E-18$
ZERO ZERO

$0.2782 \mathrm{E}-15$

$0.3532 \mathrm{E}-17$

$0.1213 \mathrm{E}-16$
ZERO

$0.1943 \mathrm{E}-20$

$0.1051 \mathrm{E}-17$

$0.3203 E-14$

$$
\text { ZERO }
$$

ZERO ZERO

$0.8949 \mathrm{E}-17$ ZERO

$0.8444 \mathrm{E}-16$
$0.4007 \mathrm{E}+03$

$0.4007 \mathrm{E}+03$
ZERO

$0.5210 \mathrm{E}-23$

$0.4679 \mathrm{E}-16$

$0.3964 \mathrm{E}-20$

$$
\text { ZERO }
$$

TRACE

$0.3517 \mathrm{E}-17$

$0.4505 \mathrm{E}-19$

$0.7554 E-20$

$0.2882 \mathrm{E}-17$

ZERO
ZERO

o)

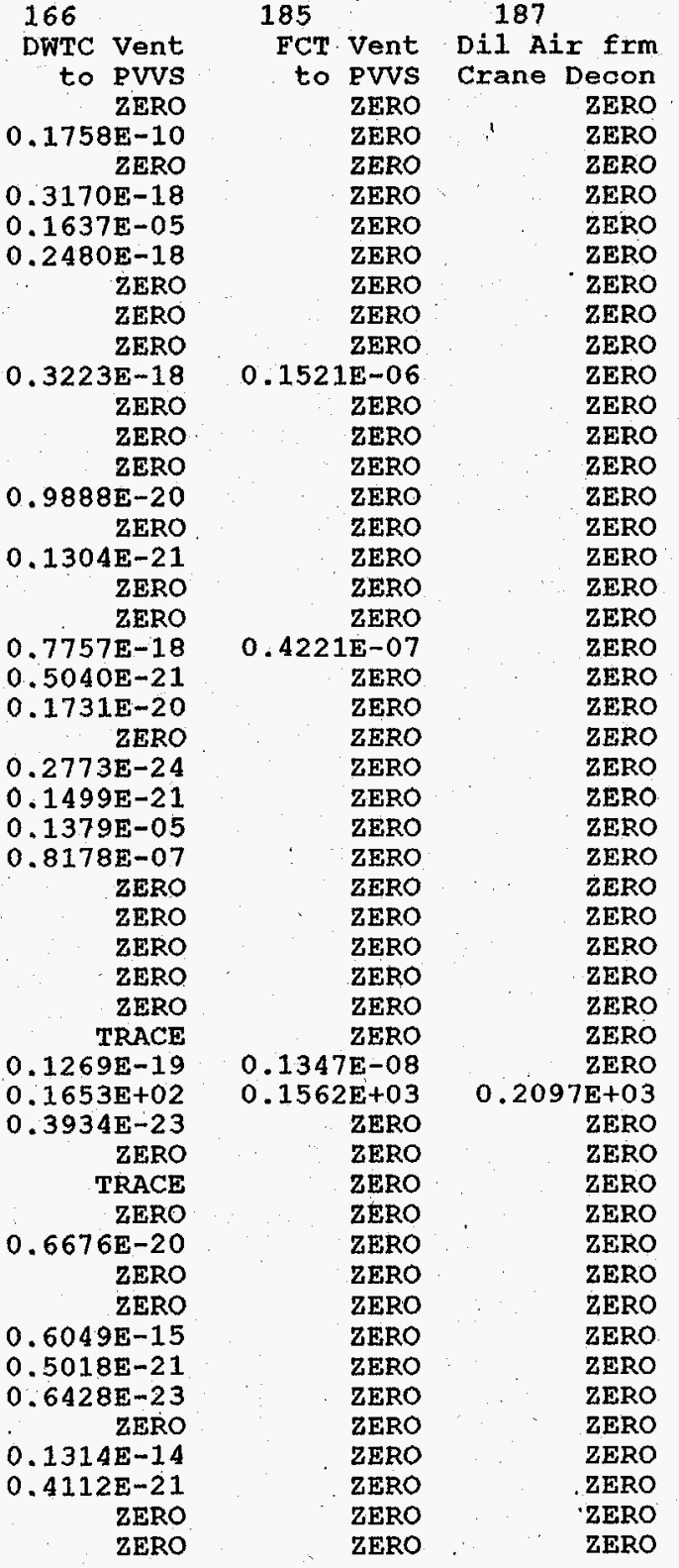

Date: January 1995
SCVC HEME Vnt to PVVS

$0.3364 \mathrm{E}-01$

$0.2673 \mathrm{E}-08$

ZERO

ZERO

ZERO

ZERO

ZERO

ZERO

ZERO

ZERO

ZERO

ZERO

ZERO

ZERO

ZERO

ZERO

ZERO

ZERO

ZERO

ZERO

ZERO

ZERO

ZERO

ZERO

ZERO

ZERO

ZERO

ZERO

ZERO

ZERO

ZERO

ZERO

$0.1369 \mathrm{E}+01$

ZERO
ZERO

ZERO

ZERO

ZERO

ZERO

ZERO

ZERO

ZERO

ZERO

ZERO

ZERO

ZERO

ZERO

ZERO

Seation 9, Page
188

189

PVVH Exit Gas $.2235 \mathrm{E}+01$ $1304 \mathrm{E}-19$ (18 $0.1738 \mathrm{E}-14$ ZERO
ZERO 3E-06 ZERO ZERO

$0.6930 \mathrm{E}-16$ ZERO

$0.6272 \mathrm{E}-07$

$.3532 \mathrm{E}-17$

$0.1213 E-16$
ZERO

$0.1943 \mathrm{E}-20$

$.1051 \mathrm{E}-17$ ZERO ZERO ZERO ZERO

$0.8949 \mathrm{E}-17$

ZERO $0.8278 \mathrm{E}+03$

ZERO

$0.5210 \mathrm{E}-23$ ZERO

$0.4679 \mathrm{E}-16$ ZERO $0.3517 \mathrm{E}-17$ $0.4505 \mathrm{E}-19$ $.7554 \mathrm{E}-20$ TRACE ZERO $0.2235 \mathrm{E}+01$ ZERO $0.1304 \mathrm{E}-19$ $0.8409 \mathrm{E}-18$ $0.1738 \mathrm{E}-14$
ZERO ZERO
ZERO ZERO $0.2953 \mathrm{E}-06$ ZERO ZERO $0.6930 \mathrm{E}-16$ ZERO ZERO 0.6272 E-07 $0.3532 \mathrm{E}-17$ $0.1213 \mathrm{E}-16$ $0.1943 \mathrm{E}-20$ $0.1051 \mathrm{E}-17$ $0.3203 \mathrm{E}-14$ ZERO ZERO ZERO ZERO ZERO $0.2001 \mathrm{E}-08$ $0.8278 \mathrm{E}+03$
ZERO ZERO
$0.5210 \mathrm{E}-23$ ZERO
$0.4679 \mathrm{E}-16$ $0.3964 \mathrm{E}-20$ ZERO $0.3517 \mathrm{E}-17$ $0.3517 \mathrm{E}-17$
$0.4505 \mathrm{E}-19$ $0.4505 \mathrm{E}-19$ $0.7554 \mathrm{E}-20$ $0.2882 \mathrm{E}-17$ ZERO ZERO 
Table A-1. HLW Flowsheet Material Balance (Tank 51/ITP Cycle 1) Section 9: Collection of Cell Vents and Sand Filter

STREAM NUMBERS (CON'T'D) $\rightarrow$ STREAM NAME

$38 \quad 502$

141 so3

137 Semi Vol Cs20

136 Semi Vol CsCl

126 Semi Vol Group A

140 Semi Vol Na2B4O

138 Semi Vol NaCl

139 Semi. Vol NaF

125 Semi Vol NaI

111 SiO2

$48 \mathrm{Sr}(\mathrm{COOH}) 2$

$176 \mathrm{Sr}(\mathrm{NO} 3) 2$

$153 \mathrm{Sr}(\mathrm{NaTi} 2 \mathrm{O}) 2$

$127 \operatorname{Sr}(\mathrm{OH}) 2$

$30 \mathrm{SrCO}$

$4.6 \mathrm{SrO}$

$\begin{array}{rl}159 & \mathrm{TcO} 2 \\ 92 & \mathrm{ThO} 2\end{array}$

107 TiO2

119 Tritium

170308

62. $\mathrm{UO} 2(\mathrm{COOH}) 2$

$177 \mathrm{UO} 2(\mathrm{NO} 3) 2$

$16 \mathrm{UO} 2(\mathrm{OH}) 2$

$27 \mathrm{Y}(\mathrm{COOH}) 3$

$178 \quad \mathrm{Y}(\mathrm{NO} 3)^{3}$

128. $Y(\mathrm{OH}) 3$

31. $\mathrm{Y2}(\mathrm{CO} 3)$

$47 \mathrm{Y} 203$

100 Zeolite

$44 \mathrm{Zn}(\mathrm{COOH}) 2$
$179 \mathrm{Zn}(\mathrm{NO}) 2$

$57 \mathrm{zno}$

$104 \mathrm{zrO} 2$

13 hydrate $\mathrm{H} 2 \mathrm{O}$

1 water

TOTAL FLOW, LB/HR

TEMPERATURE， DEG C

PRESSURE， ATM

PRESSURE, PSIG

PRESSURE; MM HG

ENTHALPY， PCU/HR

VAPOR FLOW, CFM

LIQUID FLOW, GPM

DENSITY, LBS/ET3

PHASE
166

FAVC HEME

Vnt to PVVS

ZERO

ZERO

ZERO

ZERO

ZERO

ZERO

ZERO

ZERO

$0.1132 \mathrm{E}-13$

$0.3870 \mathrm{E}-19$

ZERO

ZERO

ZERO

$0.1631 \mathrm{E}-22$

$0.1267 \mathrm{E}-17$

$0.1055 \mathrm{E}-16$
$0.2943 \mathrm{E}-15$

$0.4173 \mathrm{E}-10$

$0.7399 \mathrm{E}-15$

$0.4505 \mathrm{E}-21$

ZERO

TRACE

ZERO

ZERO

$0.3429 \mathrm{E}-18$

$0.3429 \mathrm{E}-18$
ZERO

$0.9241 \mathrm{E}-16$

ZERO

ZERO

$0.5323 E-15$

$0.1281 \mathrm{E}+02$

$0.1750 E+04$

$0.1000 \mathrm{E}+02$

$0.9263 \mathrm{E}+00$

$-0.1083 \mathrm{E}+01$

$0.7040 \mathrm{E}+03$

$0.1745 \mathrm{E}+05$

$0.4074 \mathrm{E}+03$

$0.7161 \mathrm{E}-01$
$166 \quad 185$

to PVVS

ZERO

ZERO

ZERO

ZERO

ZERO

ZERO

ZERO

ZERO

$0.1616 \mathrm{E}-17$

ZERO

$0.1491 \mathrm{E}-20$

$0.2058 \mathrm{E}-21$

ZERO

$0.3224 \mathrm{E}-23$

$0.1808 \mathrm{E}-21$

$0.1506 \mathrm{E}-20$

$0.4200 \mathrm{E}-19$

$0.7863 \mathrm{E}-19$

$0.1056 \mathrm{E}-18$

ZERO
$0.4629 \mathrm{E}-23$

EE-23
ZERO

$0.4023 \mathrm{EERO}$

$.4023 \mathrm{E}-21$ ZERO

$0.4893 \mathrm{E}-22$ ZERO

ZERO
$0.2697 \mathrm{E}-23$

$0.1598 \mathrm{E}-19$

$0.1516 \mathrm{E}-14$

$0.1220 \mathrm{E}+01$

$0.7301 \mathrm{E}+02$

$0.2250 \mathrm{E}+02$

$0.9656 \mathrm{E}+00$

$-0.5055 \mathrm{E}+00$

$0.7339 \mathrm{E}+03$

$0.1348 \mathrm{E}+04$

$0.1710 \mathrm{E}+02$

$0.7114 \mathrm{E}-01$

VAPOR to Vent Dil Air frm

ZFRO Crane Decon

ZERO

ZERO

ZERO

ZERO

ZERO

ZERO

ZERO

ZERO

ZERO
$0.2198 \mathrm{E}-05$

ZERO

ZERO

ZERO

ZERO

ZERO

ZERO

ZERO

ZERO

ZERO

ZERO

ZERO

ZERO

ZERO

ZERO

ZERO

ZERO

ZERO

ZERO

ZERO

ZERO

ZERO

ZERO

ZERO

ZERO

ZERO

$0.1834 \mathrm{E}+02$

$0.6914 \mathrm{E}+03$

$0.3000 \mathrm{E}+02$

$0.9656 \mathrm{E}+00$

$-0.5055 \mathrm{E}+00$

$0.1781 \mathrm{E}+05$

$0.1674 \mathrm{E}+03$

$0.6884 \mathrm{E}-0.1$

VAPOR

ZERO

ZERO

ZERO

ZERO

ZERO

ZERO

ZERO

ZERO

ZERO

ZERO

ZERO

ZERO

ZERO

ZERO

ZERO

ZERO

ZERO

ZERO

ZERO

ZERO

ZERO

ZERO

ZERO

ZERO

ZERO

ZERO

ZERO

ZERO

ZERO

ZERO

ZERO

ZERO

$0.1518 \mathrm{E}+02$

$0.9189 \mathrm{E}+03$

$0.3500 \mathrm{E}+02$

$0.9263 \mathrm{E}+00$

$-0.1083 \mathrm{E}+01$

$0.7040 \mathrm{E}+03$

$0.1927 \mathrm{E}+05$

$0.2344 \mathrm{E}+03$

$0.6535 \mathrm{E}-01$

VAPOR
250 SCVC HEME

Vnt to PVVS

ZERO

ZERO

ZERO

ZERO

ZERO

ZERO

ZERO

ZERO

ZERO

ZERO

ERO

ERO

ZERO

$0.3968 E+00$

$0.8376 \mathrm{E}+02$

$0.1000 \mathrm{E}+02$

$0.9656 \mathrm{E}+00$

$0.5055 \mathrm{E}+00$

$0.7339 \mathrm{E}+03$

$0.5516 \mathrm{E}+04$

$0.1217 \mathrm{E}+02$

$0.1148 \mathrm{E}+00$.

VAPOR
188

ot Vsl Vnt

to PVVH

ZERO

ZERO

ZERO

ZERO

ZERO

ZERO

ZERO

ZERO

$0.3169 \mathrm{E}-05$

$0.3870 \mathrm{E}-19$

$0.1442 \mathrm{E}-17$

ZERO

0.1631

$0.1267 \mathrm{E}-17$

$0.1055 \mathrm{E}-16$
$0.2943 \mathrm{E}-15$

$0.7056 \mathrm{E}-15 \quad 0.4173 \mathrm{E}-10$

$0.4173 \mathrm{E}-1.0$
$0.7399 \mathrm{E}-15$

ZERO $\quad 0.4505 \mathrm{E}-21$

ZERO

ZERO

ZERO

ZERO

$0.3429 \mathrm{E}-18$

ZERO

ZERO

$.6360 \mathrm{E}-16$

$0.5323 \mathrm{E}-15$

$0.5376 \mathrm{E}+02$

$0.3710 \mathrm{E}+04$

$0.9214 \mathrm{E}+00$ 
STREAM NUMBERS $\rightarrow$ STREAM NAME

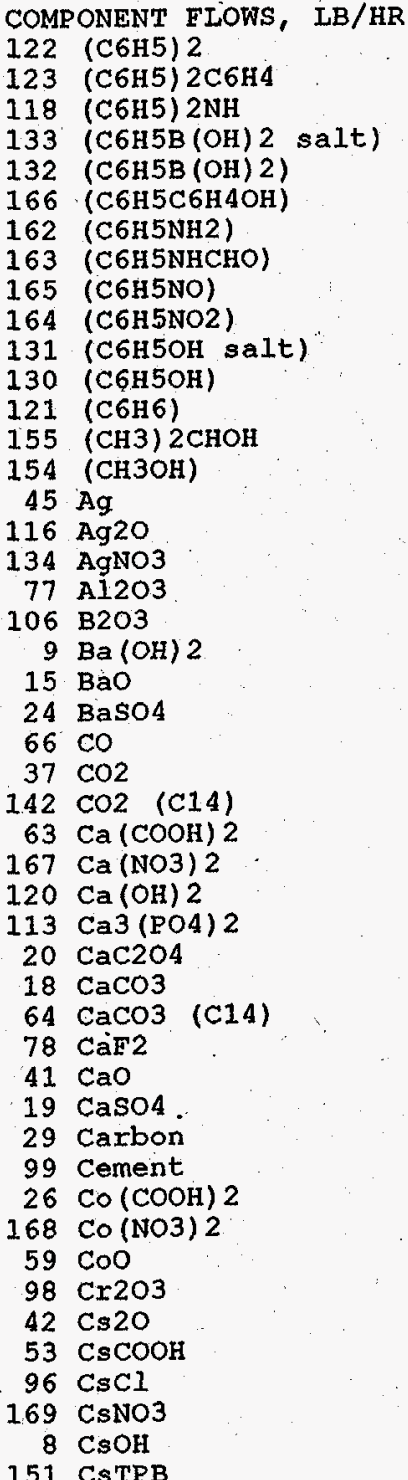

Table A-1. HLW Flowsheet Material Balance (Tank 51/ITP Cycle 1) section 9. Collection of Cell vents and Sand Filtex
190 192 192 193 115 121 194 PVVF Exit PVVF Exhst Proces Cell Prim Exhstr Bkup Exhstr Inlet Gas to
Gas to Tunnel Exh to Tunnl Gas to Tunnl Gas to Tunnl Sand Filter

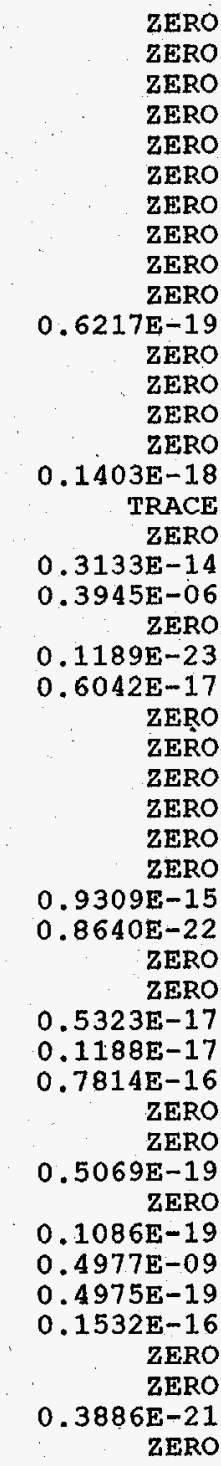

\begin{tabular}{|c|c|c|}
\hline $\begin{array}{l}0.2924 \mathrm{E}-01 \\
0.3515 \mathrm{E}-10 \\
0.7563 \mathrm{E}-04\end{array}$ & $\begin{array}{l}0.2924 \mathrm{E}-01 \\
0.3515 \mathrm{E}-10 \\
0.7563 \mathrm{E}-04\end{array}$ & $\begin{array}{l}\text { ZERO } \\
\text { ZERO } \\
\text { ZERO }\end{array}$ \\
\hline ZERO & ZERO & ZERO \\
\hline ZERO & ZERO & ZERO \\
\hline ZERO & ZERO & ZERO \\
\hline ZERO & ZERO & ZERO \\
\hline ZERO & ZERO & ZERO \\
\hline ZERO & ZERO & ZERO \\
\hline ZERO & ZERO & ZERO \\
\hline $.6223 E-22$ & $0.6223 \mathrm{E}-22$ & ZERO \\
\hline $0.1142 \mathrm{E}-01$ & $0.1142 \mathrm{E}-01$ & ZERO \\
\hline $0,9332 \mathrm{E}+01$ & $0.9332 \mathrm{E}+01$ & ZERO \\
\hline $0.8827 \mathrm{E}-11$ & $0.8827 \mathrm{E}-11$ & ZERO \\
\hline $441 \mathrm{E}-11$ & $0.2441 \mathrm{E}-11$ & ZERO \\
\hline $404 \mathrm{E}-21$ & $0.1404 \mathrm{E}-21$ & $0.3160 \mathrm{E}-21$ \\
\hline TRACE & TRACE & TRACE \\
\hline ZERO & ZERO & ZERO \\
\hline $0.3136 \mathrm{E}-17$ & $0.3136 \mathrm{E}-17$ & $0.7057 \mathrm{E}-17$ \\
\hline $0.3949 \mathrm{E}-09$ & $0.3949 \mathrm{E}-09$ & $0.8885 \mathrm{E}-09$ \\
\hline ZERO & ZERO & ZERO \\
\hline TRACE & TRACE & TRACE \\
\hline $0.6048 \mathrm{E}-20$ & $0.6048 E-20$ & $0.1361 \mathrm{E}-19$ \\
\hline ZERO & ZERO & ZERO \\
\hline $3 E+02$ & $0.7243 E+02$ & ZERO \\
\hline $2 E-07$ & $0.5692 E-07$ & ZERO \\
\hline ZERO & ZERO & ZERO \\
\hline ZERO & ZERO & ZERO \\
\hline ZERO & ZERO & ZERO \\
\hline $0.9318 \mathrm{E}-18$ & $0.9318 \mathrm{E}-18$ & $0.2097 \mathrm{E}-17$ \\
\hline TRACE & TRACE & $0.1946 \mathrm{E}-24$ \\
\hline ZERO & ZERO & ZERO \\
\hline ZERO & ZERO & ZERO \\
\hline $0.5328 \mathrm{E}-20$ & $0.5328 \mathrm{E}-20$ & $0.1199 \mathrm{E}-19$ \\
\hline $\begin{array}{l}0.1189 \mathrm{E}-20 \\
0.7822 \mathrm{E}-19\end{array}$ & $\begin{array}{l}0.1189 \mathrm{E}-20 \\
0.7822 \mathrm{E}-19\end{array}$ & $0.2675 \mathrm{E}-20$ \\
\hline $\begin{array}{l}\text { ZERO } \\
\text { ZER }\end{array}$ & ZERO & ZERO \\
\hline ZERO & ZERO & ZERO \\
\hline $0.5074 \mathrm{E}-22$ & $0.5074 \mathrm{E}-22$ & $0.1142 \mathrm{E}-21$ \\
\hline ZERO & ZERO & ZERO \\
\hline $0.1087 \mathrm{E}-22$ & $0.1087 \mathrm{E}-22$ & $0.2446 \mathrm{E}-22$ \\
\hline $0.4982 \mathrm{E}-12$ & $0.4982 \mathrm{E}-12$ & $0.1121 \mathrm{E}-11$ \\
\hline $0.4980 \mathrm{E}-22$ & $0.4980 \mathrm{E}-22$ & $0.1121 \mathrm{E}-21$ \\
\hline $0.1534 \mathrm{E}-19$ & $0.1534 \mathrm{E}-19$ & $0.3451 \mathrm{E}-19$ \\
\hline ZERO & ZERO & ZERO \\
\hline ZERO & ZERO & ZERO \\
\hline $0.3890 \mathrm{E}-24$ & $0.3890 \mathrm{E}-24$ & $0.8753 \mathrm{E}-24$ \\
\hline ZERO & ZERO & ZERO \\
\hline
\end{tabular}

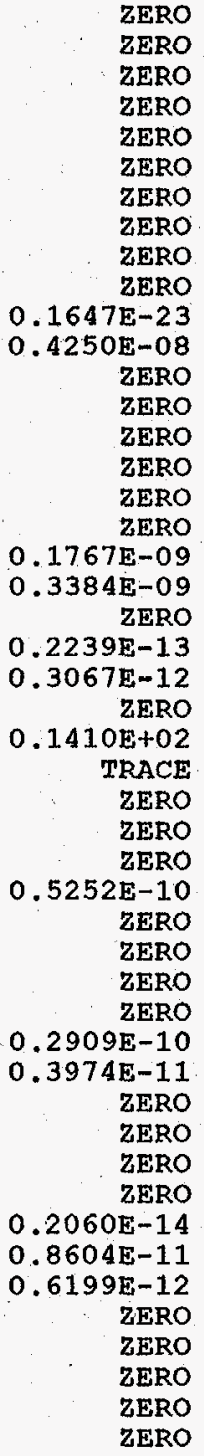

\begin{tabular}{lr} 
ZERO & $0.2924 \mathrm{E}-01$ \\
ZERO & $0.3515 \mathrm{E}-10$ \\
ZERO & $0.7563 \mathrm{E}-04$ \\
ZERO & ZERO \\
ZERO & ZERO \\
ZERO & ZERO \\
ZERO & ZERO \\
ZERO & ZERO \\
ZERO & ZERO \\
ZERO & ZERO \\
ZERO & $0.6387 \mathrm{E}-22$ \\
ZERO & $0.1142 \mathrm{E}-01$ \\
ZERO & $0.9332 \mathrm{E}+01$ \\
ZERO & $0.8827 \mathrm{E}-11$ \\
ZERO & $0.2441 \mathrm{E}-11$ \\
ZERO & $0.4564 \mathrm{E}-21$ \\
ZERO & TRACE \\
ZERO & ZERO \\
ZERO & $0.1767 \mathrm{E}-09$ \\
ZERO & $0.1622 \mathrm{E}-08$ \\
ZERO & ZERO \\
ZERO & $0.2239 \mathrm{E}-13$ \\
ZERO & $0.3067 \mathrm{E}-12$ \\
ZERO & ZERO \\
ZERO & $0.8652 \mathrm{E}+02$ \\
ZERO & $0.5692 \mathrm{E}-07$ \\
ZERO & ZERO \\
ZERO & ZERO \\
ZERO & ZERO \\
ZERO & $0.5252 \mathrm{E}-10$ \\
ZERO & $0.2811 \mathrm{E}-24$ \\
ZERO & ZERO \\
ZERO & ZERO \\
ZERO & $0.1732 \mathrm{E}+19$ \\
ZERO & $0.2909 \mathrm{E}-10$ \\
ZERO & $0.3974 \mathrm{E}-11$ \\
ZERO & ZERO \\
ZERO & ZERO \\
ZERO & $0.1649 \mathrm{E}-21$ \\
ZERO & ZERO \\
ZERO & $0.2060 \mathrm{E}-14$ \\
ZERO & $0.1022 \mathrm{EE-10}$ \\
ZERO & $0.6199 \mathrm{E}-12$ \\
ZERO & $0.4984 \mathrm{E}-19$ \\
ZERO & ZERO \\
ZERO & ZERO \\
ZERO & $0.1264 \mathrm{E}-23$ \\
ZERO & ZERO \\
& \\
ZER & 0.07 \\
\hline
\end{tabular}


Table A-1. HLW Flowsheet Material Balance (Tank 51/ITP Cycle 1) section 9. Collection of Cell Vents and Sand Filter

STREAM NUMBERS (CONT'D) $\rightarrow$ STREAM NAME

$54 \mathrm{Cu}(\mathrm{COOH}) 2$
$70 \mathrm{Cu}(\mathrm{NO} 3) 2$

$170 \mathrm{Cu}($

$80 \mathrm{Fe} 203$

$101 \mathrm{FeO}$

102 Group A

103 Group $B$

$25 . \mathrm{H} 2$

$158 \mathrm{H} 2 \mathrm{C} 2 \mathrm{O} 4$

$93 \mathrm{H} 2 \mathrm{SO} 4$

90 H3BO3

$34 \mathrm{HCOOH}$

$43 \mathrm{HCl}$

$8.5 \mathrm{HF}$

49 HNO

$36 \mathrm{Hg}$

$129 \mathrm{Hg}$ (C6H5) 2

$135 \mathrm{Hg}(\mathrm{NO} 3)$

$144 \mathrm{Hg} 2 \mathrm{Cl} 2$

$146 \mathrm{HgCl2}$

$21 \mathrm{HgO}$

97 I2

$183 \mathrm{~K} 2 \mathrm{CO} 3$

$39 \mathrm{~K} 2 \mathrm{O}$

$51 \mathrm{KCOOH}$

$157 \mathrm{KMnO} 4$

161 KNO3

$10 \mathrm{KOH}$

150 KTPB

$105 \mathrm{La203}$

$\begin{array}{rl}108 & \mathrm{Li} 2 \mathrm{O} \\ 65 \mathrm{Mg}(\mathrm{COOH}) 2\end{array}$

$171 \mathrm{Mg}(\mathrm{NO} 3) 2$

$33 \mathrm{MgO}$

$60 \mathrm{Mn}(\mathrm{COOH}) 2$

$172 \mathrm{Mn}(\mathrm{NO} 3) 2$

$180 \mathrm{Mn} 304$

$56 \mathrm{MnO}$

$14 \mathrm{MnO} 2$

$145 \mathrm{MoO} 2$

$81 \mathrm{~N} 2$

74 N2O
$182 \mathrm{NH3}$

114 NH3OHNO3

$52 \mathrm{NH} 4 \mathrm{COOH}$

173 NH4NO3

$68 \mathrm{NH} 4 \mathrm{OH}$
190

PVVF

Accumulation

$0.1571 \mathrm{E}-17$

ZERO

$0.6985 \mathrm{E}-1$

$0.1937 \mathrm{E}-09$

$0.9002 \mathrm{E}-15$

$0.1257 \mathrm{E}-17$

$0.5361 \mathrm{E}-19$

ZERO

$0.6480 \mathrm{E}-16$

$0.2727 \mathrm{E}-14$

ZERO

ZERO

ZERO

ZERO

ZERO

ZERO

ZERO

ZERO

ZERO

$0.6959 \mathrm{E}-21$

ZERO

$0.2863 \mathrm{E}-16$

$0.3554 \mathrm{E}-14$

ZERO

$0.1014 \mathrm{E}-16$

ZERO

$0.2689 \mathrm{E}-06$

$0.1228 \mathrm{E}-14$

ZERO

$0.7694 \mathrm{E}-15$

ZERO

$0.2815 \mathrm{E}-20$

$0.6909 \mathrm{E}-15$

$0.3813 \mathrm{E}-18$

ZERO

ZERO

ZERO

ZERO

ZERO
$191 \quad 192$

$192 \quad 193$

115

121

194

Exhst Proces Cell Prim Exhstr Bkup Exhstr Inlet Gas to to Tunnel Exh to Tunnl Gas to Tunnl Gas to Tunnl sand Filter $\begin{array}{llll}0.1573 E-20 & 0.1573 E-20 & 0.3538 E-20 & \text { ZERO }\end{array}$

$69927-20$ $.6992 \mathrm{E}-20$ $0.1939 \mathrm{E}-12$ $0.1258 \mathrm{E}-20$ $0.5367 \mathrm{E}-22$ $0.3136 \mathrm{E}-01$ $0.6486 \mathrm{E}-19$ $0.2730 \mathrm{E}-17$

$0.7601 \mathrm{E}-04$ ZERO

$0.7276 \mathrm{E}+00$ $0.7276 \mathrm{E}+00$

ZERO

$0.6992 \mathrm{E}-20$

$0.1939 \mathrm{E}-12$

$0.9011 \mathrm{E}-18$

$0.5367 \mathrm{E}-22$

$0.3136 \mathrm{E}-01$

$0.6486 \mathrm{E}-19$

ZERO
$0.2730 \mathrm{E}-17$

$0.7601 \mathrm{E}-04$

ZERO

$0.3538 \mathrm{E}-20$

$0.1573 \mathrm{E}-19$

$0.4363 \mathrm{E}-12$

$0.2027 \mathrm{E}-17$

$0.2830 \mathrm{E}-20$

$0.1207 \mathrm{E}-21$

$0.1459 \mathrm{E}-18$

ZERO

ZERO

$0.1893 \mathrm{E}-10$

$0.5077 \mathrm{E}-09$

$0.5076 \mathrm{E}-10$

$0.6732 \mathrm{E}-13$

$3 E-14$

ZERO

ZERO

$0.6142 \mathrm{E}-17$

ZERO

$0.7276 \mathrm{E}+00$

ZERO

$8193 \mathrm{E}-02$

ZERO

ZERO

ZERO

ZERO

ZERO

ZERO

$0.3497 \mathrm{E}-23$

ZERO

$0.3497 \mathrm{E}$

$7 \mathrm{E}-23$

ZERO

$0.2866 \mathrm{E}-19$

$0.3558 \mathrm{E}-17$

$0.1015 \mathrm{E}-19$ ZERO

$0.2692 \mathrm{E}-09$

$0.1230 \mathrm{E}-17$

$0.8484 \mathrm{E}-10$

$0.7701 \mathrm{E}-18$

ZERO

$0.2818 \mathrm{E}-23$

$0.6916 \mathrm{E}-18$

$0.3817 \mathrm{E}-21$

$0.2744 \mathrm{E}+04$

$0.1070 \mathrm{E}-09$

$0.1078 \mathrm{E}-02$

ZERO
ZERO
ZERO

TRACE

ZERO
$0.2866 \mathrm{E}-19$

$0.3558 \mathrm{E}-17$

$0.1015 \mathrm{E}-19$ ZERO

$0.2692 \mathrm{~F}-09$

$0.1230 \mathrm{E}-17$

ZERO

$0.7701 \mathrm{E}-18$

ZERO

$0.2818 \mathrm{E}-23$

$0.6916 \mathrm{E}-18$

$0.3817 \mathrm{E}-21$

$0.2744 \mathrm{E}+04$

$0.1070 \mathrm{E}-09$

$0.1078 \mathrm{E}-02$

ZERO

ZERO

ZERO
TRACE
$0.8484 \mathrm{E}-10$
ZERO

ZERO

ZERO

ZERO

ZERO

$0.7869 \mathrm{E}-23$

ZERO

ZERO

$0.6449 \mathrm{E}-19$

$0.8004 \mathrm{E}-17$

$0.2618 \mathrm{E}-22$

$$
\text { ZERO }
$$

0.6057 T 09

$0.2767 \mathrm{E}-17$

ZERO

$0.1909 \mathrm{E}-09$

$0.1733 \mathrm{E}-17$

ZERO

$0.634 \mathrm{OE}-23$

$0.1556 \mathrm{E}-17$

$0.8587 \mathrm{E}-21$

$0.3607 \mathrm{E}+06$

ZERO
ZERO
ZERO
ZERO
ZERO
ZERO
ZERO
ZERO
ZERO

ZERO

$0.1369 \mathrm{E}-02$

$0.6776 \mathrm{E}-02$

.8511E-02

ZERO

ZERO

$0.2867 \mathrm{E}-10$

$0.2253 \mathrm{E}-20$

ZERO
$0.4632 \mathrm{E}-09$

ZERO
$0.1144 \mathrm{E}-09$

ZERO

ZERO

ZERO

ZERO

ZERO

$0.2216 \mathrm{E}-09$ ZERO

ZERO
0.9383 E-10

ZERO

ZERO

ZERO
$0.5301 \mathrm{E}-10$

ZERO

$0.2151 \mathrm{E}-13$

$0.1395 \mathrm{E}+04$

ZERO

ZERO

ZERO

ZERO

ZERO

ZERO
ZERO

ZERO

ZERO

ZRRO

ZERO

IERO

ZERO

ZERO

ZERO

ZERO

ZERO

ZERO

ZERO

ZERO

ZERO

ZTRO

ZERO

TERO

ZERO

ZERO

ZERO

ZERO

ZERO

ZERO

ZERO

ZERO

ZERO 
Table A-1. HLW Flowsheet Material Balance (Tank 51/ITP Cycle 1) section 9. Collection of Cell Vents and Sand Filter

STREAM NUMBERS (CONT'D) - $\rightarrow$ STREAM NAME

$\begin{array}{ll}35 & \text { NO } \\ 84 & \text { NO2 }\end{array}$

$70 \mathrm{Na}(\mathrm{HgO}(\mathrm{OH}))$

$94 \mathrm{Na} 2 \mathrm{~B} 4 \mathrm{O} 7$

$109 \mathrm{Na} 2 \mathrm{C} 204$

$5 \mathrm{Na} 2 \mathrm{CO} 3$

$143 \mathrm{Na} 2 \mathrm{CO} 3$ (C14)

$89 \mathrm{Na} 2 \mathrm{CrO} 4$

115 Na2MoO

$\begin{array}{ll}32 & \mathrm{Na} 2 \mathrm{O} \\ 71 & \mathrm{Na} 2 \mathrm{PuO} 2(\mathrm{OH})\end{array}$

$73 \mathrm{Na2RhO4}$

72 Na2RuO4

$6 \mathrm{Na2SO4}$

$88 \mathrm{Na2SiO3}$

$91 \mathrm{NaAg}(\mathrm{OH}) 2$

75 NaAl (OH) 4

$50 \mathrm{NaCOOH}$

$7 \mathrm{NaCl}$

$69 \mathrm{NaF}$

$11 \mathrm{NaHCO} 3$

12. $\mathrm{NaI}$

3 NaNO2

$4 \mathrm{NaOH}$

149 NaTPB

$160 \mathrm{NaTCO}$

$181 \mathrm{Nd} 203$

$61 \mathrm{Ni}$ (COOH) 2

$174 \mathrm{Ni}$ (NO3) 2

40. NiO

$175 \mathrm{~Pb}$ (NO3) 2

$86 \mathrm{PbCO} 3$

$67 \mathrm{PbO}$

28 PbSO 4

$124 \mathrm{Pd}$

$112 \mathrm{Pd}(\mathrm{NO} 3) 2$

110 Pdo

$\begin{array}{rl}76 & \mathrm{PuO} 2 \\ 156 & \mathrm{PuO} 2(\mathrm{NaTi} 205) 2\end{array}$

$\begin{array}{ll}156 & \mathrm{PuO} \\ 58 \mathrm{Rh}\end{array}$

22 RhO2

$95 \mathrm{Ru}$

$\begin{array}{ll}79 & \text { RuO2 } \\ 83 & \text { RuO4 }\end{array}$

WSRC-TR-95-0019 (Revision
190 PVVF Accumulation ZERO ZERO

$0.1303 \mathrm{E}-19$

$0.8401 \mathrm{E}-18$

$0.8401 \mathrm{H}-18$

$0.1736 \mathrm{E}-14$ ZERO ZERO

$0.2950 \mathrm{E}-06$ ZERO
ZERO ZERO

$0.6923 \mathrm{E}-16$ ZERO

$0.9131 \mathrm{E}-18$ ZERO

ZERO

$0.3529 \mathrm{E}-17$

$0.1212 \mathrm{E}-16$
ZERO

$0.1941 \mathrm{E}-20$

$0.1050 \mathrm{E}-17$

$0.3200 \mathrm{E}-14$ ZERO ZERO ZERO

$0: 8940 \mathrm{E}-17$

ZERO

$0.1999 \mathrm{E}-08$ ZERO ZERO

$0.5205 \mathrm{E}-23$ ZERO

$0.4674 \mathrm{E}-16$

$0.3960 \mathrm{E}-20$ ZERO

$0.3513 \mathrm{E}-17$

$0.4500 \mathrm{E}-19$

$0.7547 \mathrm{E}-20$

TRACE
$0.2879 \mathrm{E}-17$ ZERO
191

PVVF Exit Gas

$0.2235 \mathrm{E}+01$

ZERO

$0.8409 \mathrm{E}-21$

$0.8409 \mathrm{E}-21$

$8 \mathrm{E}-17$ ZERO
ZERO

$0.2953 \mathrm{E}-09$

ZERO ZERO
ZERO

$0.6930 \mathrm{E}-19$

$0.9140 E-21$

ZERO

ZERO

$0.6272 \mathrm{E}-10$

$0.3532 \mathrm{E}-20$

$0.1213 E-19$

ZERO

$0.1943 \mathrm{E}-23$

$0.1051 \mathrm{E}-20$
$0.3203 \mathrm{E}-17$

. 3203E-17
ZERO

ZERO

ZERO

ZERO

$0.8949 \mathrm{E}-20$

ZERO

$0.2001 \mathrm{E}-11$

$0.8278 \mathrm{E}+03$

ZERO

TRACE

$0.4679 \mathrm{E}-19$

$0.3964 \mathrm{E}-23$

ZERO

$0.3517 \mathrm{E}-20$

$0.4505 \mathrm{E}-22$

$0.7554 \mathrm{E}-23$

TRACE

$0.2882 \mathrm{E}-20$ ZERO
ZERO to Exhst Proces Cell Prim Exhstr Bkup Exhstr Inlet Gas to to Tunnel Exh to Tunnl Gas to Tunnl Gas to Tunnl sand Filtex ZERO ZERO $0.1395 \mathrm{E}+01$. ZERO $0.2235 \mathrm{E}+01 \quad$ ZERO $0.2751 \mathrm{E}+01 \quad$ ZERO

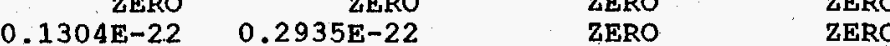
$0.8409 \mathrm{E}-21 \quad 0.1892 \mathrm{E}-20 \quad$ ZERO $\quad$ ZERO

$\begin{array}{llll}0.1738 \mathrm{E}-17 & 0.3910 \mathrm{E}-17 & \text { ZERO } & \text { ZERO }\end{array}$ ZERO

$0.2953 \mathrm{E}-09$ ZERO ZERO

$0.6930 \mathrm{E}-19$ ZERO

$0.9140 \mathrm{OE}-21$ ZERO

$0.6272 \mathrm{E}-10$

$0.6272 \mathrm{E}-10$

$0.1213 \mathrm{E}-19$

$0.1213 \mathrm{E}-19$ ZERO

$0.1051 \mathrm{E}-20$

$0.3203 \mathrm{E}-17$

ZERO

ZERO

ZERO

ZERO

$0.8949 \mathrm{E}-20$ ZERO

$0.2001 \mathrm{E}-11$

$0.8278 \mathrm{E}+03$ ZERO
ZERO ZERO
TRACE TRACE

$0.4679 \mathrm{E}-19$

$0.3964 \mathrm{E}-23$ ZERO
TRACE

$0.3517 \mathrm{E}-20$

$0.4505 \mathrm{E}-22$

$0.7554 \mathrm{E}-23$

TRACE
$0.2882 \mathrm{E}-20$

ZERO
ZERO

$0.1395 \mathrm{E}+01$

$0.4987 \mathrm{E}+01$

$0.4239 \mathrm{E}-22$

$0.2733 \mathrm{E}-20$

$0.5648 \mathrm{E}-17$

ZERO

7TRO

ZERO

ZERO

ZERO

ZERO

ZERO

ZERO

ZERO

ZERO

$0.1559 \mathrm{E}-18$

$0.2056 \mathrm{E}-20$

ZERO

$0.1411 \mathrm{E}-09$

$0.1411 E-09$

$0.2730 \mathrm{E}-19$

ZERO

$.4372 \mathrm{E}-23$

$0.2364 \mathrm{E}-20$

$0.7207 \mathrm{E}-17$

ZERO

ZERO

ZERO

ZERO

$0.2013 E-19$ ZERO

$0.4502 \mathrm{E}-11$

$0.1090 \mathrm{E}+06$

ZERO
ZERO

TRACE

ZERO

$0.1053 \mathrm{E}-18$

$0.8919 \mathrm{E}-23$

ZERO

$0.7912 \mathrm{E}-20$

$0.1014 \mathrm{E}-21$

$0.1700 \mathrm{E}-22$

TRACE
$0.6484 \mathrm{E}-20$

ZERO

$0.1127 \mathrm{E}-10$

ZERO

$0.5156 \mathrm{E}-13$

ZERO

ZERO

$0.9957 \mathrm{E}-13$

$0.6742 \mathrm{E}-12$

ZERO

ZERO

ZERO

ZERO

ZERO

ZERO

ZERO

ZERO

ZERO

ZERO

0.5085 ZERO

$0.5085 E-11$ - ZERO

ZERO
$0.9805 \mathrm{E}-13$

ZERO

$0.2504 \mathrm{E}-11$

ZERO

ZERO

0.1991E-12

ZERO

ZERO

ZERO

ZERO

ZERO

ZERO

ZERO

ZERO

ZTRO

ZERO

ZERO

ZERO

ZERO

ZERO

ZERO

ZERO

ZERO

ZERO

ZERO

ZERO

ZERO

ZERO

$0.2065 \mathrm{E}+03$

ZERO

ZERO

ZERO

ZERO

ZERO

ZERO

ZERO

ZERO

ZERO

ZERO

ZERO

ZERO 
Table A-1. HLW Flowsheet Material Balance (Tank 51/ITP Cycle 1) section 9. Collection of Cell vents and sand Filter

STREAM NUMBERS (CONT'D) $\rightarrow$ STREAM NAME

$38 \mathrm{SO} 2$.

$141 \mathrm{SO} 3$

137 Semi Vo1 Cs20

136 Semi Vol CsCl

126 Semi Vol Group A

140 Semi vo1 Na2B4O7

138 Semi Vol NaCl

139 Semi Vol NaF

125 Semi Vol NaI

23 Semi Vol RuO2

111 SiO2

$48 \mathrm{Sr}(\mathrm{COOH}) 2$

$176 \mathrm{Sr}$ (NO3) 2

$153 \mathrm{Sr}(\mathrm{NaTi2O5)} 2$

$127 \mathrm{Sr}(\mathrm{OH}) 2$

30
$46 \mathrm{SrCO}$

$\begin{array}{rl}46 & \mathrm{SrO} \\ 159 & \mathrm{TaO} 2\end{array}$

92 Tho2

107 Ti.02

119 Tritium

$17 \mathrm{U} 308$

$62 \mathrm{UO2}$ ( $\mathrm{COOH}) 2$

177.002 (NO3) 2

$16 \mathrm{VO} 2(\mathrm{OH}) 2$

$27 \mathrm{Y}(\mathrm{COOH}) 3$

178 Y (NO3) 3

$128 \times(\mathrm{OH}) 3$

31. Y2 (CO3) 3

$\begin{array}{rl}47 & 1203 \\ 100 & \text { Zeolit }\end{array}$

$\begin{aligned} & 100 \text { zeolite } \\ & 44 \mathrm{Zn}(\mathrm{COOH}) 2\end{aligned}$

$44 \mathrm{Zn}(\mathrm{COOH}) 2$
$179 \mathrm{Zn}(\mathrm{NO}) 2$

$57 \mathrm{zmo}$

1042502

13 hydrate $\mathrm{H} 20$

1 water

TOTAL FLOW, LB/HR

TEMPERATURE, DEG C

PRESSURE， ATM

PRESSURE, PSIG

PRESSURE, MM HG
ENTHALPY, PCU/HR

VAPOR FLOW, CFM

LIQUID FLOW, GPM

DENSITY, LBS/FT3

PHASE

191
PVVF EXIt
Gas
ZERO
ZERO
ZERO
ZERO
ZERO
ZERO
ZERO
ZERO
ZERO
ZERO
$0.3169 E-08$
$0.3870 E-22$
ZERO
$0.1442 E-20$
ZERO
ZERO
TRACE
$0.1521 E-20$
$0.1055 E-19$
$0.2943 E-18$
$0.4173 E-10$
$0.7399 E-18$
$0.4505 E-24$
ZERO
ZERO
TRACE
ZERO
ZERO
ZERO
$0.3429 E-21$
ZERO
$0.9241 E-19$
ZERO
$0.6360 E-19$
ZERO
$0.5323 E-18$
$0.5376 E+02$
$0.3710 E+04$

$0.3146 E+02$
$0.9018 E+00$
$0.1443 E+01$
$0.6854 E+03$
$0.7556 E+05$
$0.9514 E+03$
$0.6499 E-01$
VAPOR

192

193

115

121

PVVF
Accumu lation

ZERO

ZERO

ZERO

ZERO

ZERO

ZERO

ZERO

0. $166 E-05$

$0.3866 \mathrm{E}-19$

ZERO

$0.1441 \mathrm{E}-17$
ZERO ZERO

$0.1630 \mathrm{E}-22$

$0.1266 \mathrm{E}-17$

$0.1054 \mathrm{E}-16$

$0.2940 \mathrm{E}-15$

$0.7392 \mathrm{E}-15$

$0.4501 \mathrm{E}-21$

ZERO

ZERO

TRACE

ZERO

ZRRO

$0.3426 \mathrm{E}-18$ ZERO

$0.9231 \mathrm{E}-16$

$0.6354 \mathrm{E}-16$

$0.5317 \mathrm{E}-15$

ZERO

$.4275 \mathrm{~K}-05$

$0.3146 \mathrm{E}+02$

$0.9165 \mathrm{E}+00$

$-0.1227 E+01$
$0.6965 E+03$

$0.2705 E-04$

$0.1397 E+03$

SOLID

VAPOR

VVF Exhst proces Cell ZERO $\because \quad$ ZERO

$0.3071 \mathrm{E}-07$

$0.1667 \mathrm{E}-10$ to ZERO TXRO Sand Filter ZERO . ZERO

in:

ZERO

ZERO

ZERO

ZERO

ZERO

$0.3169 \mathrm{E}-08$

$0.3870 \mathrm{E}-22$

$$
\text { ZERO }
$$

$0.1442 \mathrm{E}-20$

ZERO

TRARE

$0.1521 \mathrm{E}-20$

$0.1055 \mathrm{E}-19$

$0.2943 \mathrm{E}-18$

$0.4173 \mathrm{E}-10$

$0.7399 \mathrm{E}-18$

$0.4505 \mathrm{E}-24$

ZERO

TRACE

ZERO

ZERO

$0.3429 \mathrm{E}-21$

0.3429 E-21
ZERO

$0.9241 \mathrm{E}-19$

ZERO
$0.6360 \mathrm{E}-19$

ZERO
$0.5323 \mathrm{E}-18$

$0.5376 \mathrm{E}+02$

$0.3710 \mathrm{E}+04$$$
\because \text { ZERO }
$$

ZERO

ZERO

ZERO

ZERO

ZERO

$0.7131 \mathrm{E}-08$

$0.8708 \mathrm{E}-22$$$
\begin{array}{r}
\text { ZERO } \\
\text { ZERO }
\end{array}
$$

$0.3245 \mathrm{E}-20$ ZERO ZERO

TRACE
$0.3422 \mathrm{E}-20$

$0.2374 \mathrm{E}-19$

$0.6623 \mathrm{E}-18$

$0.9389 \mathrm{E}-10$

$0.1665 \mathrm{E}-17$

$4 \mathrm{E}-23$
ZERO

ZERO

TRACE

ZERO ZERO

$0.7716 \mathrm{E}-21$

$0.7716 \mathrm{E}-21$
ZERO

$0.2079 \mathrm{E}-18$

ZERO
$0.1431 \mathrm{E}-18$

ZERO

$0.1198 \mathrm{E}-17$

$0.7891 \mathrm{E}+04$

$0.4776 \mathrm{E}+06$

$0.4238 \mathrm{E}+02$

$0.9928 \mathrm{E}+00$

$0.3500 \mathrm{E}+02$

$0.9928 \mathrm{E}+00$

$-0.1058 \mathrm{E}+00-0.1058 \mathrm{E}+00$

$0.7545 \mathrm{E}+03 \quad 0.7545 \mathrm{E}+0$

$0.1002 \mathrm{E}+08$

$0.6907 \mathrm{E}-01$

$0.7005 \mathrm{E}-01$

VAPOR

VAPOR

ZERO
ZERO

$0.1029 \mathrm{E}-08$

$0.4429 \mathrm{E}-10$

$0.3113 \mathrm{E}-08$

$0.1354 \mathrm{E}-11$

$0.2459 \mathrm{EERO}$

$9 \mathrm{E}-08$

ZERO

ZERO

ZERO ZERO

$0.3070 \mathrm{E}-12$

$0.1335 \mathrm{E}-12$

$0.5944 \mathrm{E}-12$

$0.1137 \mathrm{E}-11$

$0.4168 \mathrm{E}-10$

ZERO

ZERO

ZERO

ZERO

ZERO ZERO

$0.8458 \mathrm{E}-13$

ZERO
ZERO
ZERO

$0.6319 \mathrm{E}-11$

$0.1418 \mathrm{E}+02$

$0.1849 \mathrm{E}+04$

$0.2762 \mathrm{E}+02$

$0.9928 \mathrm{E}+00$

$-0.1058 \mathrm{E}+00$

$0.7545 \mathrm{E}+03$

$0.2686 \mathrm{E}+05$

$0.4257 \mathrm{E}+03$

$0.9928 \mathrm{E}+00$

$-0.1058 \mathrm{E}+00$

$0.1186 \mathrm{E}+05$

$0.7237 E-01$

VAPOR

$0.7275 \mathrm{E}-01$
$0.7545 \mathrm{E}+03$

VAPOR

$\begin{array}{ll}\text { ZERO } & \text { ZERO } \\ \text { ZERO } & \text { ZERO }\end{array}$

ZERO

ZERO $0.1029 \mathrm{E}-08$

ZERO $0.4429 \mathrm{E}-10$

$0.3071 E-07$

ZERO $0.3113 E-08$

ZERO $\quad 0.1354 \mathrm{E}-11$

ZERO

ZERO

ZERO

ZERO

ZERO

ZERO

ZERO
ZERO

ZERO

ZERO

ZERO

ZERO

ZERO

ZERO

ZERO

ZERO

ZERO

ZERO

ZERO

ZERO

ERO

$.6641 E+01$

ZERO

$0.12588-21$

ZERO

$0.4687 \mathrm{E}-20$ ZERO

(.) $3070 \mathrm{E}-12$

$0.5944 \mathrm{E}-12$

$0.1667 \mathrm{E}-10$

$0.1368 \mathrm{E}-09$

$0.4168 \mathrm{E}-10$

ZERO

ZERO

TRACE

TRACE

ZERO

ZERO

$0.3003 \mathrm{E}-18$

$0.6319 \mathrm{E}-11$

$0.1730 \mathrm{E}-17$

$0.7965 E+04$

$0.4840 \mathrm{E}+06$

$0.3501 \mathrm{E}+02$

$0.9926 \mathrm{E}+00$

$-0.1088 \mathrm{E}+00$

$0.7544 \mathrm{E}+03$

$0.1014 \mathrm{E}+08$

$0.1152 \mathrm{E}+06$

0.7004 E-01
VAPOR 
Table A-1. HLW Flowsheet Material Balance (Tank 51/ITP Cycle 1) section 9. Collection of Cell vents and Sand Filter

STREAM NUMBERS $\rightarrow$ STREAM NAME

COMPONENT FLOWS, LB/HR 122 (C6H

123 (C6H5) $2 \mathrm{C} 6 \mathrm{H} 4$

(C6H5) 2NH

(C6H5B (OH) 2 salt)

132 (C6H5B (OH) 2)

166 (C6H5C6H4O

162 (C6H5NH2)

165 (C6H5NO)

164 (C6H5NO2)

131 (C6H5OH salt)

130 (C6H5OH)

121 (C6H6)

155. (СH3) $2 \mathrm{CHOH}$

154 (СH3OH)

$45 \mathrm{Ag}$

$116 \mathrm{Ag} 20$

134 AgNO3
77 A1203

$77 \mathrm{Al2O3}$

9. $\mathrm{Ba}(\mathrm{OH}) 2$

$15 \mathrm{BaO}$

24 BasO4

$66 \mathrm{CO}$

$37 \mathrm{CO} 2$

$63 \mathrm{Ca}(\mathrm{COOH}) 2$

$167 \mathrm{Ca}(\mathrm{NO} 3) 2$

$120 \mathrm{Ca}$ (OH) 2

$113 \mathrm{Ca}(\mathrm{POA}) 2$

$20 \mathrm{CaC} 2 \mathrm{O} 4$

$18 \mathrm{CaCO} 3$

$64 \mathrm{CaCO}^{2}$ (C14)

$78 \mathrm{CaF} 2$

19 Caso

29 Carbon

9ement

$168 \mathrm{CO}(\mathrm{NO} 3) 2$

$59 \mathrm{COO}$

98 Cr203

42 C 20

$53 \mathrm{CsCOOH}$

$96 \mathrm{CsCl}$

$169 \mathrm{CsNO}^{2}$

151 CsTPB

195
and $F$ Sand Filter Sand Filter sand Filter
ccumulation Inlet Sump aftr SV rem$$
\text { ZERO }
$$$$
\text { ZERO }
$$$$
\text { ZERO }
$$$$
\text { ZERO }
$$$$
\text { ZERO }
$$$$
0.6386 \mathrm{E}-22
$$$$
\text { ZERO }
$$$$
\text { ZERO }
$$$$
\text { ZERO }
$$$$
0.4563 E-21
$$$$
\text { TRACE }
$$$$
\text { ZERO }
$$$$
0.1767 \mathrm{E}-09
$$$$
0.1621 \mathrm{E}-08
$$$$
\text { ZERO }
$$$$
0.2238 \mathrm{E}-13
$$$$
0.3066 \mathrm{E}-12
$$$$
\text { ZERO }
$$$$
\text { ZERO }
$$$$
\text { ZERO }
$$$$
\text { ZERO }
$$$$
0.5251 \mathrm{E}-10
$$$$
0.5251 \mathrm{E}-10
$$$$
\text { ZERO }
$$$$
0.1731 \mathrm{E}-19
$$$$
0.2908 \mathrm{E}-10
$$$$
0.3974 \mathrm{E}-11
$$$$
\text { ZERO }
$$$$
0.1649 \mathrm{E}-21
$$$$
\text { ZERO }
$$$$
0.2059 \mathrm{E}-14
$$$$
0.1022 \mathrm{E}-10
$$$$
0.6198 \mathrm{E}-12
$$$$
0.4983 \mathrm{E}-19
$$$$
\text { ZERO }
$$

$0.1264 \mathrm{E}-23$ ZERO aftr sV rem

$$
\begin{array}{lr}
\text { ZERO } & 0.2924 \mathrm{E}-01 \\
\text { ZERO } & 0.3515 \mathrm{E}-10 \\
\text { ZERO } & 0.7563 \mathrm{E}-04 \\
\text { ZERO } & \text { ZERO } \\
\text { ZERO } & \text { ZERO } \\
\text { ZERO } & \text { ZERO } \\
\text { ZERO } & \text { ZERO } \\
\text { ZERO } & \text { ZERO } \\
\text { ZERO } & \text { ZERO } \\
\text { ZERO } & \text { ZERO } \\
\text { ZERO } & \text { TRACE } \\
\text { ZERO } & 0.1142 E-01
\end{array}
$$

$0.1142 \mathrm{E}-01$

$0.8827 \mathrm{E}-11$

$0.2441 E-11$

TRACE

TRACE

ZERO

$0.3534 \mathrm{E}-13$

$0.3243 \mathrm{E}-12$
ZERO

$0.4478 \mathrm{E}-17$

$0.6134 \mathrm{E}-16$ ZERO

$0.8652 \mathrm{E}+02$

$0.5692 \mathrm{E}-07$

ZERO

ZERO

$0.1050 \mathrm{E}-13$

TRACE ZERO ZERO

$0.3463 \mathrm{E}-23$

$0.5818 \mathrm{E}-14$

$0.7949 \mathrm{E}-15$ ZERO ZERO TRACE

ZERO

ZERO

ZERO

ZERO

ZERO

ZERO

ZERO

ZERO

ZERO

ZERO

198

199

Sand Filter
Exit Sump

to stack

$0.2924 \mathrm{E}-01$

$5 \mathrm{E}-10$

$0.7563 \mathrm{E}-04$

ZERO
ZERO

ZERO

ZERO

ZERO

ZERO

2ERO

TRACE
$0.1142 \mathrm{E}-01$

$0.9332 \mathrm{E}+01$

$0.8827 \mathrm{E}-11$

$0.2441 \mathrm{E}-11$

TRACE

TRACE

0.3534

$0.3534 \mathrm{E}-13$

$.3243 \mathrm{E}-12$
ZERO

$0.4478 \mathrm{E}-17$

$0.6134 \mathrm{E}-16$

$0.8652 \mathrm{E}+02$

$0.5692 \mathrm{E}-07$

ZERO

ZERO

$0.1050 \mathrm{E}-13$

TRACE

ZERO

ZERO
$0.3463 \mathrm{E}-23$

$0.5818 \mathrm{E}-14$

$0.7949 \mathrm{E}-15$

ZERO

ZERO

TRACE

$0.4119 \mathrm{E}-18$

$0.2045 \mathrm{E}-14$

$0.1240 \mathrm{E}-15$

$0.9969 \mathrm{E}-23$

$0.5144 \mathrm{E}-11$

ZERO

TRACE
ZERO

$0.4119 \mathrm{E}-18$

$0.2045 \mathrm{E}-14$

$0.1240 \mathrm{E}-15$

$0.9969 \mathrm{E}-23$

$.5144 \mathrm{E}-11$

ZERO
TRACE

ZERO

Date: January 1995
200

$\begin{array}{lr}\text { ZERO } & 0.2924 \mathrm{E}-01 \\ \text { ZERO } & 0.3515 \mathrm{E}-10 \\ \text { ZERO } & 0.7563 \mathrm{~T}-04 \\ \text { ZERO } & \text { ZERO } \\ \text { ZERO } & \text { ZERO } \\ \text { ZERO } & \text { ZERO } \\ \text { ZERO } & \text { ZERO } \\ \text { ZERO } & \text { ZERO } \\ \text { ZERO } & \text { ZERO } \\ \text { ZERO } & \text { ZERO } \\ \text { ZERO } & \text { TRACE } \\ \text { ZERO } & 0.1142 \mathrm{E}-01 \\ \text { ZERO } & 0.9332 \mathrm{E}+01 \\ \text { ZERO } & 0.8827 \mathrm{E}-11 \\ \text { ZERO } & 0.2441 \mathrm{11}-11 \\ \text { ZERO } & \text { TRACE } \\ \text { ZERO } & \text { TRACE } \\ \text { ZERO } & \text { ZERO } \\ \text { ZERO } & 0.3534 \mathrm{E}-13 \\ \text { ZERO } & 0.3243 \mathrm{E}-12 \\ \text { ZERO } & \text { ZERO } \\ \text { ZERO } & 0.4478 \mathrm{E}-17 \\ \text { ZERO } & 0.6134 \mathrm{E}-16 \\ \text { ZERO } & \text { ZERO } \\ \text { ZERO } & 0.8652 \mathrm{E}+02 \\ \text { ZERO } & 0.5692 \mathrm{E}-07 \\ \text { ZERO } & \text { ZERO } \\ \text { ZERO } & \text { ZERO } \\ \text { ZERO } & \text { ZERO } \\ \text { ZERO } & 0.1050 \mathrm{E}-13 \\ \text { ZERO } & \text { TRACE } \\ \text { ZERO } & \text { ZERO } \\ \text { ZERO } & \text { ZERO } \\ \text { ZERO } & 0.3463 \mathrm{E}-23 \\ \text { ZERO } & 0.5818 \mathrm{E}-14 \\ \text { ZERO } & 0.7949 \mathrm{E}-15 \\ \text { ZERO } & \text { ZERO } \\ \text { ZERO } & \text { ZERO } \\ \text { ZERO } & \text { TRACE } \\ \text { ZERO } & \text { ZERO } \\ \text { ZERO } & 0.4119 \mathrm{E}-18 \\ \text { ZERO } & 0.2045 \mathrm{E}-14 \\ \text { ZERO } & 0.1240 \mathrm{E}-15 \\ \text { ZERO } & 0.9969 \mathrm{E}-23 \\ \text { ZERO } & 0.5144 \mathrm{E}-11 \\ \text { ZERO } & \text { ZERO } \\ \text { ZERO } & \text { TRACE } \\ \text { ZERO } & \text { ZERO } \\ & \\ & \end{array}$

Section 9, Page 10 of 13 
Table A-1. HLW Flowsheet Material Balance (Tank 51/ITP Cycle 1) Section 9. Collection of Cell Vents and sand Filter

STREAM NUMBERS (CONT'D) -> STREAM NAME

$54 \mathrm{Cu}(\mathrm{COOH}) 2$
$70 \mathrm{Cu}(\mathrm{NO} 3)^{2}$

$170 \mathrm{Cu}($

80. $\mathrm{Fe} 2 \mathrm{O3}$

101 Feo

102 Group A

103 Group B

$25 \mathrm{H} 2$

$158 \mathrm{H} 2 \mathrm{C} 2 \mathrm{O} 4$

$93 \mathrm{H} 2 \mathrm{SO} 4$

$34 \mathrm{HCOOH}$

$43 \mathrm{HCl}$

$85 \mathrm{HF}$

49 , HNO3

$36^{\prime} \mathrm{Hg}$

$129 \mathrm{Hg}(\mathrm{C} 6 \mathrm{H} 5) 2$

$135 \mathrm{Hg}(\mathrm{NO} 3)$

$144 \mathrm{Hg} 2 \mathrm{Cl}$

$117 \mathrm{Hg} 2 \mathrm{I} 2$

$21 \mathrm{HgO}$

97 I

$183 \mathrm{~K} 2 \mathrm{CO} 3$

$39 \mathrm{~K} 20$

$51 \mathrm{KCOOH}$

$157 \mathrm{KMnO} 4$

161. KNO3

$10 \mathrm{KOH}$

$\begin{array}{ll}150 & \mathrm{KTPB} \\ 105 \mathrm{La} 203\end{array}$

$108 \mathrm{Li2O}$

$65 \mathrm{Mg}(\mathrm{COOH}) 2$

$171 \mathrm{Mg}(\mathrm{NO} 3) 2$

$33 \mathrm{MgO}$

$60 \mathrm{Mn}(\mathrm{COOH}) 2$

$172 \mathrm{Mn}(\mathrm{NO} 3) 2$

$180 \mathrm{Mn} 304$

$56 \mathrm{MnO}$

$14 \mathrm{MnO} 2$

$145 \mathrm{MOO} 2$

81 N2

$\begin{array}{rl}74 & \mathrm{~N} 2 \mathrm{O} \\ 182 & \mathrm{NH} 3\end{array}$

182 NH3 114 NH3OHNO3

$52 \mathrm{NH} 4 \mathrm{COOH}$

173 NH 4 NO 3

$68 \mathrm{NH} 4 \mathrm{OH}$
195 Accumulater sand Filter sand Filter Inlet Sump aftr SV rem

E-20

2 2 ZERO

$0.1892 \mathrm{E}-10$

$0.5082 \mathrm{E}-09$

$0.5075 \mathrm{E}-10$

$0.6730 \mathrm{E}-13$

$0.3023 \mathrm{E}-14$

$0.2108 \mathrm{E}-18$

$0.8869 \mathrm{E}-17$

ZERO

ZERO

ZERO

ZERO

ZERO

$0.2866 \mathrm{E}-10$

$0.2366 \mathrm{E}-15$

$0.2253 \mathrm{E}-20$

$0.1131 \mathrm{E}-22$ ZERO

$0.1143 \mathrm{E}-09$

$0.1156 \mathrm{E}-16$ ZERO

$0.3297 \mathrm{E}-19$

$0.3780 \mathrm{E}-22$ ZERO ZERO

$0.1096 \mathrm{E}-08$

$0.3995 \mathrm{E}-17$ ZERO
E-09

$0.2502 \mathrm{E}-17$

ZERO

$0.5300 \mathrm{E}-10$

$0.2247 \mathrm{E}-17$

$0.2150 \mathrm{E}-13$

ZERO

ZERO

ZERO

ZERO

ZERO

ZERO

ZERO

$0.1369 E-02$

$0.7343 \mathrm{E}+0.0$

$0.1670 \mathrm{E}-01$

ZERO

ZERO
$0.5734 \mathrm{E}-14$

$0.4734 \mathrm{E}-19$

$0.4506 \mathrm{E}-24$

TRACE

ZERO

$0.2287 \mathrm{E}-13$

$0.2312 \mathrm{E}-20$

ZERO

TRACE

ZERO

ZERO
$0.2193 \mathrm{E}-12$

$0.2193 \mathrm{E}-12$.

ZERO

$0.7391 \mathrm{E}-13$

$0.5006 \mathrm{E}-21$ ZERO

$0.1060 \mathrm{E}-13$

$0.4496 \mathrm{E}-21$

$0.4301 \mathrm{E}-17$

$0.4301 \mathrm{E}-17$

$0.1070 \mathrm{E}-09$

$0.1078 \mathrm{E}-02$

ZERO

ZERO

ZERO

ZERO

ZERO

ZERO

ZERO
198

Fan Exhaust 199 to stack Exit sump $0.1022 \mathrm{E}-23$

$1 \quad$ ZERO

$$
\begin{aligned}
& \text { ZERO } \\
& \text { ZERO }
\end{aligned}
$$

$0.5734 \mathrm{E}-14$

$0.4734 \mathrm{E}-19$

$0.4506 \mathrm{E}-24$

- TRACE

$0.4632 \mathrm{E}-09$

$0.2287 \mathrm{E}-13$

$0.2312 \mathrm{E}-20$

$0.6596 \mathrm{E}-23$

TRAC

ZERO

$0.2193 \mathrm{E}-12$

$0.7992 \mathrm{E}-21$

$0.7391 \mathrm{E}-13$

$0.5006 \mathrm{E}-21$

ZERO

$0.1060 \mathrm{E}-13$

$0.4496 \mathrm{E}-21$

$0.4301 \mathrm{E}-17$

$0.3655 \mathrm{E}+06$

$0.1070 \mathrm{E}-09$

$0.1078 \mathrm{E}-02$

ZERO

ZERO

TRACE

ZERO
0. $3785 \mathrm{E}-14$

$0.1017 \mathrm{E}-12$

$0.1015 \mathrm{E}-13$

$0.2215 E-12$

$0.6046 \mathrm{E}-18$

$0.3136 \mathrm{E}-01$

$0.4216 \mathrm{E}-22$

ZERO
$0.1774 \mathrm{E}-20$

$0.7601 \mathrm{E}-04$

ZERO

$0.7343 \mathrm{E}+00$
200

stack

Exhaust

ERO

ZERO

RRO

ZERO

ERO

ZERO

ZERO

ERO

EERO

ZERO

RO

ZERRO

ZERO

ZERO

ZERO

ZERO

ZERO

ERRO

ZERO

ZERO

ZERO

ZERO

ZERO

ERO

ZERO

ZERO

DRO

ZERO

ZERO

ZERO

ZERO

ZERO

ZERO

ZERO

ZERO

ZERO

ZTRO

ZRRO

TERO

ZERO

ZERO

ERO

ZERO

ZERO

ZERO

ZERO

$0.3785 \mathrm{E}-14$

$0.1017 \mathrm{E}-12$

$0.1015 \mathrm{~T}-13$

0.150-12

.

. $136 \mathrm{E}-18$

$0.4216 \mathrm{E}-22$

ZERO

$.1774 \mathrm{E}-20$

$.7601 \mathrm{E}-04$

$0.1369 \mathrm{E}-02$

$0.7343 E+00$

$0.1670 \mathrm{E}-01$

ZERO

$0.5734 \mathrm{E}-14$

$0.4734 \mathrm{E}-19$

$0.4506 \mathrm{E}-24$

ZERO

$0.2312 \mathrm{E}-20$

$0.6596 \mathrm{E}-23$

TRACE

ZERO

ZERO

$0.7391 E-13$

$0.5006 \mathrm{E}-21$ ZERO

$0.1060 \mathrm{E}-13$

$0.4496 \mathrm{E}-21$

$0.4301 \mathrm{E}-17$

$0.3655 E+06$

$0.1070 \mathrm{E}-09$

$0.1078 \mathrm{E}-02$

ZERO

ZERO 
Table A-1. HLW Flowsheet Material Balance (Tank 51/ITR Cycle 1) section 9 . Collection of Cell Vents and sand Filter

STREAM NUMBERS (CONT'D) $\rightarrow$ STREAM NAME

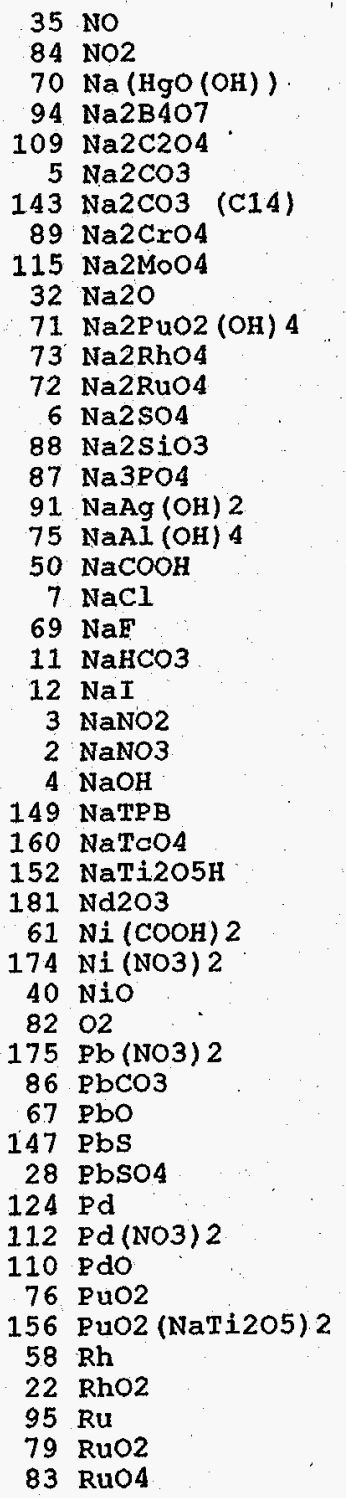

195 196 Sand Filter sand Filter sand Sand Filter Sand Filter Sand Filter
Accumulation Inlet Sump aftr sV rem ZERO aftr sV rem $0.7125 \mathrm{E}+01$ 198 Exhaust 199 stack Exit Sump Q. 7.125E+01

0.4239 22 $0.2733 \mathrm{E}-20$ $7 \mathrm{E}-17$ ZERO

$0.1366 \mathrm{E}-08$ ZERO ZERO

$0.1126 \mathrm{E}-10$

ZERO ZERO ZERO

$0.2038 \mathrm{E}-09$ $0.9955 \mathrm{E}-13$ $0.9955 \mathrm{E}-13$ $0.6741 \mathrm{E}-12$
ZERO $0.6314 \mathrm{E}-23$ $0.3414 \mathrm{E}-20$ $0.1041 E-16$ ZERO ZERO ZERO ZERO ZERO
$0.2908 \mathrm{E}-19$ 8E-19 $0.1159 \mathrm{E}-10$ ZERO ZERO

ZERO
$0.9803 E-13$ ZERO

$0.2504 \mathrm{E}-11$

$0.1288 \mathrm{E}-22$ ZERO

0 TRACE

$0.1464 \mathrm{E}-21$

$0.2455 \mathrm{E}-22$

TRACE

$0.9363 \mathrm{E}-20$ ZERO ZERO

WSRC-TR-95-0019 (Revision ZERO $0.1536 \mathrm{E}-09$
$0.5466 \mathrm{E}-24$

ZERO

ZERO

$0.51130 \mathrm{E}-20$

TERO

ZERO

ZERO

ZERO

ZERO

ZERO

ZERO

ZERO

ZERO

ZERO

ZERO

ZERO

ZERO
ZERO

ZERO

ZERO

ZERO

ZERO

ZERO

ZERO

ZERO

ZERO

ZERO

ZIRO

ZERO

ZERO

ZERO

ZERO

ZERO

ZERO

ZERO

ZERO

ZERO

ZERO

ZERO

ZERO

ZERO

ZERO

ZERO

ZERO

$.1130 \mathrm{E}-20$

ZERO

ZERO

$0.2732 \mathrm{E}-12$

ZERO

ZERO

$0.2253 \mathrm{E}-14$

ZERO

$0.1031 \mathrm{E}-16$

ZERO

$0.4077 \mathrm{E}-13$

$0.1991 \mathrm{E}-16$

$0.1557 \mathrm{E}-10$

ZERO

$0.6771 \mathrm{E}-1.4$

$0.6829 \mathrm{E}-24$

$0.2082 \mathrm{E}-20$

ZERO

ZERO

ZERO

$0.5817 \mathrm{E}-23$ ZERO

$0.2318 \mathrm{E}-14$

$0.1104 \mathrm{E}+06$ ZERO

$0.1961 \mathrm{E}-16$ ZERO

$0.5008 \mathrm{E}-15$

TRACE

ZERO

$0.3983 \mathrm{E}-16$

TRACE

TRACE

TRACE
$0.1873 \mathrm{E}-23$

ZERO
$0.1536 \mathrm{E}-09$

$0.1536 \mathrm{E}-09$

$0.1130 \mathrm{E}-20$

ZERO

ZERO

$0.2732 E-12$

ZERO
ZERO

ZERO

$0.2253 \mathrm{E}-1$

ZERO
0.1031 E-16

ZERO

$0.4077 \mathrm{E}-13$

$0: 1991 \mathrm{E}-16$

$0.1557 \mathrm{E}-10$ ZERO

$0.6771 \mathrm{E}-14$

$0.6829 \mathrm{E}-24$

$0.2082 \mathrm{E}-20$

ZERO

ZERO

ZERO

ZERO

$0.5817 \mathrm{E}-23$

ZERO

$0.2318 E-14$
$0.1104 E+06$

$0.1104 \mathrm{E}+06$

ZERO

$0.1961 E-16$

$0.5008 \mathrm{E}-15$

TRACE

ZERO

TRACE
$0.3983 E-16$

TRACE

TRACE

TRACE

$0.1873 \mathrm{E}-23$

ZERO

ZERO
ZERO

Date: January 1995
200

Stack

xhaust

$0.7125 \mathrm{E}+01$

ZERO

$0.1536 \mathrm{E}-09$

$0.5466 \mathrm{E}-24$

ZERO $0.1130 E-20$
ZERO

ZERO
ZERO ZERO
ZERO

ZERO.

ZERO $0.2732 E-12$

ZERO

ZERO

ZERO

ZERO

ZERO

ZERO

ZERO

TERO

ZERO

ZERO

ZERO

ZERO

ZERO

ZERO

ZERO

ZERO

ZERO

ZERO

ZERO

ZERO

2ERO

ZERO

ZERO

ZERO

ZERO

ZERO

ZERO

ZERO

ZERO

ZERO

ZERO

ZERO

ZFRO

ZERO

ZERO

ZERO

ZERO

Section 9, Page 12 of 13

2253E TERO

ZERO

ZERO

$0.4077 \mathrm{E}-13$

$0.1991 \mathrm{E}-16$

ZERO
ZERO

$.2082 \mathrm{E}-20$

ZERO

ZERO

ZERO

ZERO

ZERO

$0.1961 \mathrm{E}-16$ O8E-15
TRACE

TRACE

TRACE

TRACE

TRACE

ZERO 
Table A-1. HLW Flowsheet Material Balance (Tank 51/ITP Cycle 1) Section 9. Collection of Cell Vents and Sand Filter

STREAM NUMBERS (CONT'D) $\rightarrow$ STREAM NAME

$38 \mathrm{SO} 2$

$141 \mathrm{SO} 3 \mathrm{Vol} \mathrm{Cs} 2 \mathrm{O}$

136 Semi Vol CsCl

126 Semi Vol Group A

140 Semi Vol Na2B407

138 Semi Vol $\mathrm{NaCl}$

139 Semi Vol NaF

125 Semi Vol $\mathrm{NaI}$
23 Semi Vol RuO2

111 sio2

$48 \mathrm{Sr}$ (COOH) 2

$176 \mathrm{SI}$ (NO3) 2

$153 \mathrm{Sr}$ (NaT1205) 2

$127 \mathrm{Sr}(\mathrm{OH}) 2$

$30 \mathrm{sxCO3}$

46 sro

$159 \mathrm{TcO} 2$

92 ThO2

$\begin{array}{ll}107 & \text { TiO2 } \\ 119 & \text { Tritiun }\end{array}$

170308

$62 \mathrm{OO2}$ ( $\mathrm{COOH}) 2$

$177 \mathrm{VO2}$ (NO3) 2

16 VO2 (OH) 2

$27 \mathrm{Y}(\mathrm{COOH}) 3$

178 Y (NO3)

$31 \mathrm{Y} 2(\mathrm{CO} 3) 3$

31
47203

100 zeolite

$44 \mathrm{Zn}(\mathrm{COOH}) 2$

$179 \mathrm{zn}(\mathrm{NO}) 2$

$\begin{array}{rl}57 & \mathrm{Zno} \\ 104 & \mathrm{ZrO} 2\end{array}$

13 hydrate 120

1 water

TOTAL FLON, LB/GR

TEMPERATURE, DEG C

PRESSURE, ATM

PRESSURE, PSIG

PRESSURE, MM HG

ENTHALPY, PCU/HR

VAPOR FLOW, CFM

LIQUID FLOW, GPM

DENSITY, LBS/FT3

PHASE $\begin{array}{lll}195 & 196 & 197\end{array}$

Sand Filter Sand Filter Sand Filter
Accumulation Inlet Sump aftr SV rem

ZERO

ZERO

ZERO

$0.1024 \mathrm{E}-08$

$0.4407 \mathrm{E}-10$

$0.3056 \mathrm{E}-07$
ZERO

$0.3098 \mathrm{E}-08$

$0.1347 \mathrm{E}-11$

ZERO

$0.1276 \mathrm{E}-07$

$0.1258 \mathrm{E}-21$

$0.4686 \mathrm{E}-20$

ZERO

ZERO

$0.30695-12$

$0.1335 \mathrm{E}-12$

$0.1335 \mathrm{E}-12$

$0.1667 \mathrm{E}-10$ ZERO

$0.4167 \mathrm{E}-10$

$0.1464 \mathrm{E}-23$

YERO

ZERO

TRACE

ZERO

ZERO

$0.8457 \mathrm{E}-13$

ZERO

$0.3003 E-18$

ZERO
$0.6317 \mathrm{E}-11$

$0.6317 \mathrm{E}-11$

ZERO

$0.1730 \mathrm{R}-17$

$0,5328 \mathrm{E}-07$

$0.3501 \mathrm{E}+02$

$0.9926 \mathrm{E}+00$

$-0.1088 E+00$

$0.7544 \mathrm{E}+03$

$0.4161 \mathrm{E}-06$

$0.1490 \mathrm{E}+03$

SOLID

$\begin{array}{ll}\text { ZERO } & \text { ZERO } \\ \text { ZERO } & \text { ZERO }\end{array}$

ZERO ZERO

ZERO

ZERO

ZERO

ZERO

ZERO

ZERO
ZERO

ZERO

ZERO

ZERO

ZERO

ZERO

ZERO

ZERO

ZERO

ZERO

ZERO

ZERO

ZERO

ZERO

ZERO

ZERO

ZERO

ZERO

ZERO

ZERO

ZERO

ZERO

ZERO

ZERO

ZERO

ZERO

ZERO

$0.4750 \mathrm{E}+00$

$0.4750 \mathrm{E}+00$

$0.3500 E+02$

$0.1000 \mathrm{E}+01$

ZERO

$0: 1667 \mathrm{E}+02$

$0.9486 \mathrm{E}-03$

$0.6243 \mathrm{E}+02$

LIQUID

ZERO

ZERO

ZERO

ZERO

ZERO

ZERO

$0.2552 E-11$

TRACE

$0.9374 \mathrm{E}-24$

ZERO

ZERO

$0.6139 \mathrm{E}-16$

$0.5875 \mathrm{E}-16$

$0.1189 \mathrm{E}-15$

$0.3335 \mathrm{E}-14$

$0.1368 \mathrm{E}-09$

TRACE

ZERO

ZERO

TRACE

ZERO

ZERO

ZERO

$0.6006 \mathrm{E}-22$

ZERo

ZERO
ZER

$0.3460 \mathrm{E}-21$

$0.7965 \mathrm{E}+04$

$0.4840 E+06$

$0.3501 E+02$

$0.9680 \mathrm{E}+00$

$-0.4703 \mathrm{E}+00$

$0.7357 E+03$

$0.1014 \mathrm{E}+08$

$0.1181 \mathrm{E}+06$

$0.6830 \mathrm{E}-01$

VAPOR
198

an Exhaust

to stack

ZERO

ZERO

ZERO

ZERO

$0.8336 \mathrm{E}-14$

$0.1264 \mathrm{E}-14$

$0.7965 \mathrm{E}+04$

$0.4840 \mathrm{E}+06$

$0.3873 E+02$

$0.1000 \mathrm{E}+01$

ZERO

$0.1056 \mathrm{E}+08$

$0.1157 \mathrm{E}+06$

$0.6972 \mathrm{E}-01$

VAPOR
Sand Filter

$0.3460 \mathrm{E}-21$
199

Exit Sump

ZERO
ZERO

ZERO

ZERO

ZERO

ZERO

ZERO

ZERO.

ZERO

ZERO

ZERO

ZERO

ZERO

ZERO

ZERO

ZERO

ZERO

TERO

ERO

ZERO

ZERO

ZERO

ZERO

ZERO

ZERO

ZERO

ZERO

GERO

ZERO

ZERO

ERO

ZERO

200

stack

Exhaust

ZERO

ZERO

ZERO

ZERO

ZERO

ZERO

ZERO

ZERO

$0.2552 \mathrm{E}-11$

TRACE

$0.9374 \mathrm{E}-24$

ZERO

$0.6139 \mathrm{E}-16$

$0.5875 \mathrm{E}-16$

$0.1189 \mathrm{E}-15$

$0.3335 \mathrm{E}-14$

$0.1368 \mathrm{E}-09$

$0.8336 \mathrm{E}-14$

TRACE

ZERO

ZERO

ZERO

ZERO

$0.1692 \mathrm{E}-16$

0.600 ZERO

ZERO

$0.1264 \mathrm{E}-14$

$0.3460 \mathrm{E}-21$

$0.4750 \mathrm{E}+00$

$0.4750 \mathrm{E}+00$

$0.4840 \mathrm{E}+06$

$0.3500 E+02$

$0.3873 \mathrm{E}+02$

ZERO

$0.1667 \mathrm{E}+02$

$0.9486 \mathrm{E}-03$

$0.6243 E+02$

LIQUID

$$
\text { ZERO }
$$

$0.1056 \mathrm{E}+08$

$0.1157 \mathrm{E}+06$

$0.6972 E-01$
VAPOR 
WSRC-TR-95-0019-TL

WSRC-TR-95-0019

April 19, 1995

HLW FLOWSHEET MATERIAL BALANCE FOR

DWPF RADIOACTIVE OPERATION WITH

TANK 51 SLUDGE AND ITP CYCLE PRECIPITATE (U)

Distribution:

\section{HLWM-ENG}

S. F. Piccolo, 704-S

J. F. Ortaldo, 704-S (5)

H. H. Elder, 704-S

S. R. Wilson, 704-S

R. D. Reeves, 704-15S

J. T. Carter, 704-25S

R. E. Edwards, 704-25S

D. H. McGuire, 704-27S

W. A. Wagner, 704-27S

J. M. Gillam, 704-27S

J. E. Owen, 704-30S

R. W. Wilson, 724-2G

J. E. Marra, 703-H

G. K. Georgeton, 703-H

T. E. Pate, 703-H

E. D. Lee, 241-152H

W. B. Van Pelt, 241-152H

J. R. Fowler, 704-Z

\section{HLWM-PM}

N. R. Davis, 719-4A

J. N. Brooke, 719-4A

M. N. Wells, 719-4A
SRTC

L. M. Papouchado, 773-A

E. W. Holtzscheiter, 773-A

W. L. Tamosaitis, 773-A

M. J. Plodinec, 773-A

S. D. Fink, 773-A

N. E. Bibler, 773-A

M. S. Hay, 773-A

D. D. Walker, 773-A

L. F. Landon, 704-T

C. T. Randall, 704-T

R. A. Jacobs, 704-T

N. H. Kuehn, 704-T

L. M. Lee, 704-T

D. A. Crowley, 704-1T

F. G. Smith, 704-1T

A. S. Choi, 704-1T (15)

TIM, 703-43A, Rm. 26 (4)

E\&PD

R. P. Blundy, 730-B 